PNL-2433

UC-11

\title{
The Hanford 67-Series: A Volume of Atmospheric Field Diffusion Measurements
}

by

P. W. Nickola

November 1977

Prepared Jointly for the

U.S. Department of Energy and

U.S. Nuclear Regulatory Commission

under Contract EY-76-C-06-1830 


\title{
NOTICE
}

This report was prepared as an account of work sponsored by the United States Government. Nether the United States nor the Department of Energy, nor any of their empioyees, nor any of their contractors. subcontractors, or their employees, makes any warranty, express or implied, or assumes any legul liats lity or responsibility for the accuracy, completeness or usefulness of any information, apparatus, product or prot.ass disclosed, or represents that its use would not infringe privately owned rights.

The views, opinions and conclusions contained in this report are those of the contractor and do rot necessarily represent those of the United States Government or the Unitad States Departmant of Energy.

\author{
PACIFIC NORTHWEST LABORATORY \\ operated by \\ BATTELLE \\ for the \\ UNITED STATES DEPARTMENT OF ENERGY \\ Under Contract EY-76-C-06-1830
}

Printed in the United Staim of America

Ivailable irom

National Technical Information Service United States Department of Commerce

5235 Port Royal Road

Springfield, Virginia 22151

Price: Printed Copy s___: Microfiche 53.m

NTis

-Pages Selling Price

$001-025 \quad \$ 4.50$

$026-050 \quad 35.00$

057-975 $\quad 35.50$

676- $100 \quad \$ 5.00$

$107.725 \quad 56.50$

$126-750 \quad 5700$

$151-175 \quad 57.75$

$176-200$

$207-225 \quad \cdot \quad 53.75$

$226-250 \quad 59.00$

$257-275 \quad 510.60$

$276-300 \quad \$ 70.25$ 
PNL-24.33

$\mathrm{UC}-11$

THE HANFORD 67-SERIES:

A VOLUME OF ATMOSPHERIC FIELD

DIFFUSION MEASUREMENTS

by

P. W. Nickola

Atmospheric Sciences Department

Prepared under Contract No. EY-76-C-06-1830

for

The Division of Biomedical and

Environmental Reasearch,

Department of Energy

and

The Division of Reactor Safety Research, Nuclear Regulatory Commission

November 1977

BATTELLE

Pacific Northwest Laboratories

Richland, Washington 99352 
The field work summarized in this report was conducted under the auspices of the former Atomic Energy Commission's Division of Biology and Medicine and the former Energy Research and Development Administration's Division of Biomedical and Environmental Research, the functions of which have now been transferred to the Department of Energy.

The assembling of data and publication of this document is jointly sponsored by the above-mentioned ERDA Division and by the Division of Reactor Safety Research of the Nuclear Regulatory Commission. 


\begin{abstract}
$\underline{\text { ABSTRACT }}$
This volume documents atmospheric diffusion experiments carried out at the Hanford reservation during the period 1967 to 1973. A total of 103 tracer releases during 54 release periods is tabulated. Multi-tracer releases (generally from different elevations) were made during most of the experimental periods. Release heights varied from ground level to an elevation of $111 \mathrm{~m}$.

Tracers were sampled simultaneously on as many as 10 arcs at distances of up to $12.8 \mathrm{~km}$ from the tracer release point. As many as 718 field sampling locations were employed during some of the experiments. Vertical profiles of concentration were monitored on towers during 23 of the 54 release periods. Concurrent vertical profiles of mean temperature, of mean wind speed and direction, and of direction standard deviation are also tabled for elevations up to $122 \mathrm{~m}$.
\end{abstract}




\section{CONTENTS}

ABSTRACT. . . . . . . . . . . . . . . . iii

FIGURES . . . . . . . . . . . . . . . vi vi

TABLES. . . . . . . . . . . . . . . vi vi

INTRODUCTION. . . . . . . . . . . . . . 1

THE FIELD GRID. . . . . . . . . . . . . . . . 3

TRACER RELEASE, SAMPLING AND ASSAY. . . . . . . . 13

Zinc Sulfide Fluorescent Particulate 2210. . . . 13

Fluorescein. . . . . . . . . . . 22

Rhodamine B. . . . . . . . . . . . . 24

Krypton-85 . . . . . . . . . . . . 26

DESCRIPTION OF EXPERIMENTS AND DATA . . . . . . 28

The Appendix A Diffusion Data. . . . . . . . 31

The Appendix B Diffusion Summaries . . . . . . . 34

The Appendix C Meteorological Data . . . . . 36

ACKNOWLEDGMENTS . . . . . . . . . . . . . . 40

REFERENCES. . . . . . . . . . . . . 41

APPENDIX A: DIFFUSION DATA ........... . A-l

APPENDIX B: DIFFUSION SUMMARIES. . . . . . . . . B-2

APPENDIX C: METEOROLOGICAL DATA. . . . . . . . . C C-1 
1 View looking south along arc $1600 \mathrm{~m}$ from S-source. The $62-\mathrm{m}$ towers are spaced at $8^{\circ}$ intervals. Vacuum hose and stakes at two ground-level sampling locations are also visible . . . . . . . . . . . . . . . .

2 Aircraft view of near-source arcs on Hanford Diffusion Grid. . . . . . . . . . . . . . .

3 Aircraft view showing sampling arcs to distances of $1600 \mathrm{~m}$ from U- and S-sources. The s-source and associated arcs have been retouched to aid in their identification. . . . . . . . .

4 Insecticidal sprayers used in dispersal of $\mathrm{ZnS}$ and fluorescein tracers. View looks upwind from the s-source ... . . . . . . . . .

5 The diffusion grids superimposed on a contour map. Contour interval is $6.1 \mathrm{~m}(20 \mathrm{ft}$ ). (The 200-m arcs are not diagrammed.) . . . . . .

6 Assembled Rankin counter (right) and disassembled Rankin counter (left) with several field filter assemblies in place for assessment. . . . . . . . . . . . . . .

C-1 Vertical profiles of temperature. Letters designate height and type of tracer release . . . C-3

C-2 Vertical profiles of wind speed. Letters designate height and type of tracer release . . C -5

C-3 Vertical profiles of wind direction. Letters designate height and type of tracer release. Dashed curves are from Beckman and Whitley vanes; solid curves are from Aerovanes. . . . . C-8

C-4 Vertical profiles of wind direction standard deviation. Letters designate height and type of tracer release. Dashed curves are from Beckman and Whitley vanes; solid curves are from Aerovanes. . . . . . . . . . . . . C-ll 
1 Design Specifications of Hanford "U" and "S" Diffusion Grids . . . . . . . . . . . . . .

2 General Grid, Source, Release and Meteoro-

logical Information . . . . . . . . . .

3 Frequency of Tracer Release and Sampling at

1.5-m Elevation... . . . . . . . . .

4 Frequency of Tracer Release and Tower

Sampling. . . . . . . . . . . • . . . • . •

5 a Location and Quality of Sampling for the 28

Surface Releases of the 67-Series.......

5b Location and Quality of Sampling for 46

Releases from Elevation of 26 Meters during the 67-Series . . . . . . . . . . . . . .

5c Location and Quality of Sampling for 20

Releases from Elevation of 56 Meters during

the 67-Series . . . . . . . . . . . . .

5d Location and Quality of Sampling for 9

Releases from Elevation of 111 Meters during

the 67-Series................

C-1 Temperature (deg F) for Period of Tracer

Release

C-2 Wind speed ( $\mathrm{m} / \mathrm{sec})$ for Period of Tracer Release

C-3 Wind Direction (deg) for Period of Tracer

Release

C -6

C-4 Wind Direction Standard Deviation (deg) for

Period of Tracer Release. . . . . . . . . 

PNL-2433

THE HANFORD 67-SERIES:

A VOLUME OF ATMOSPHERIC FIELD DIFFUSION MEASUREMEINTS

\section{INTRODUCTION}

During the period 1959 to 1973, more than 300 atmospheric field diffusion experiments have been conducted at the Hanford reservation* near Richland, Washington. This volume documents. 103 of the more recent of these experiments. Both diffusion and concurrent meteorological data are presented in, hopefully, user-oriented format.

Prior to this volume, some of the earlier of the 300 experiments have been described in reports or journal articles. The 1959 experiments, dubbed the Green Glow diffusion program, were documented by Barad and Fuquay ${ }^{1}$. They included detailed diffusion and meteorological data for the 27 Green Glow field tests. Green Glow tracer releases were from an elevation of 2 to 3 meters. Sampling included both ground-level and tower arrays.

These near ground-level tracer releases with both horizontal and vertical sampling arrays continued at Hanford with a series of 42 field experiments in 1960, 1961, and 1.962 known as the Hanford 30-Series. Selected ground-level diffusion data and meteorological data from both the Green Glow and 30-Series experiments were tabled by Fuquay, simpson and Hinds in a journal article in 1964.2 only the more "reliable" tests were considered in the journal article -- 16 Green Glow and 30 series -30 experiments.

Concurrent with the 30-Series, another group of field experiments began at Hanford. These more than 200 tracer

\footnotetext{
*Currently under the jurisdiction of the Department of Energy.
} 
releases, beginning in the fall of 1960, were primarily elevated source experiments. The individual experiments (or subgroups of experiments) in this total of 200 were designed to investigate a variety of specific areas in the more general realm of diffusion. Results of these investigations have been presented in a variety of forums -- including annual reports ${ }^{3}$ to the sponsors, the Atomic Energy Commission and more recently the Environmental Research and Development Administration. However, measurements made during these field experiments have pertinence in areas beyond the narrower original objectives. It is with this thought in mind that this current data volume is published.

The diffusion experiments documented in this volume are the portion of those described in the preceding paragraph which were carried out at Hanford since July 1967. These have been rather arbitrarily labeled the Hanford 67-Series. Following publication of this report, there remain approximately 100 Hanford field diffusion experiments (carried out between 1960 and June 1967) which have not been documented in a fashion convenient for general research use. The experiments considered in the 67-Series have been selected primarily on the basis of being more recent, and hence having pertinent diffusion/ meteorology data more readily accessible to the author than the pre-July 1967 experiments.

The 103 tracer releases of the Hanford 67-Series were carried out during 54 different experimental periods. Multitracer releases (generally from two different elevations) account for the fact that the number of tracer releases is greater than the number of experimental periods. Meteorological measurements made during the tracer releases include vertical profiles of.wind speed, wind direction and temperature. Release duration was generally 30 minutes. As few as 63 and as many as 718 field locations were employed in 
sampling tracer concentration during a given release. In 32 of the 103 releases, ten or more towers were employed downwind of the source in an attempt to define vertical concentration distributions. Tower height varied from $27 \mathrm{~m}$ to $62 \mathrm{~m}$. As few as two and as many as ten sampling arcs, concentric about the release point, were used in the deployment of tracer samplers. The radii of these arcs varied from $0.2 \mathrm{~km}$ to $12.8 \mathrm{~km}$ from the source. Tracer was released at an elevation of $1 \mathrm{~m}, 2 \mathrm{~m}, 26 \mathrm{~m}$, $56 \mathrm{~m}$, or $111 \mathrm{~m}$. Details of the meteorology and of tracer dispersal and sampling for each experiment follow in the body and appendices of this report.

THE FIELD GRID

The Hanford reservation is located in a semi-arid region in the southeast of the state of Washington. The natural vegetation in the area is sagebrush 1 to $2 \mathrm{~m}$ in height interspersed with steppe grasses. Figures $1,2,3$ and 4 give some idea of the nature and density of the vegetation.

The center of the reservation is about $200 \mathrm{~m}$ above mean sea level. Although the reservation is nearly surrounded by hills or bluffs on all sides (some reaching as high as $1000 \mathrm{~m}$ msl), the field diffusion grids are located near the center of this approximately $40-\mathrm{km}$ diameter basin.

Figure 5 shows the diffusion sampling grids superimposed on a contour map. The bulk of the sampling arcs are located on a relatively flat area where the extremes in elevation range from $200 \mathrm{~m}$ to $230 \mathrm{~m} \mathrm{msl}$. The most distant tracer sampling arc, $12.8 \mathrm{~km}$ from the source, is at an elevation about $35 \mathrm{~m}$ lower than the nearer-source sampling arcs.

The primary or "ground-level" sampling on the Hanford diffusion grids is done at an elevation of $1.5 \mathrm{~m}$, an elevation that approximates the breathing height of man. About 1000 


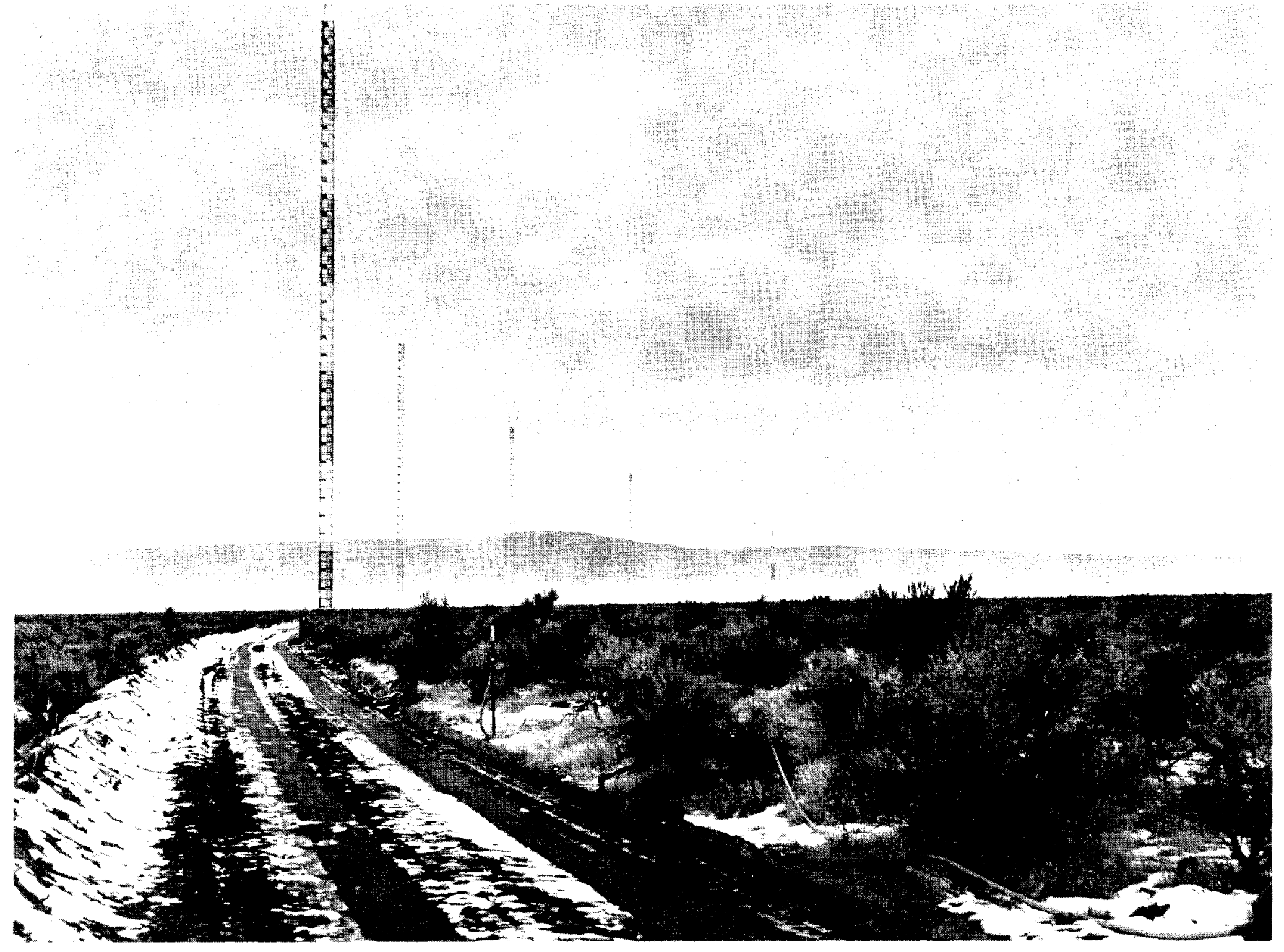

Figure 1. View looking south along arc $1600 \mathrm{~m}$ from s-source. The 62-m towers are spaced at $8^{\circ}$ intervals. Vacuum hose and stakes at two ground-level sampling locations are also visible. 


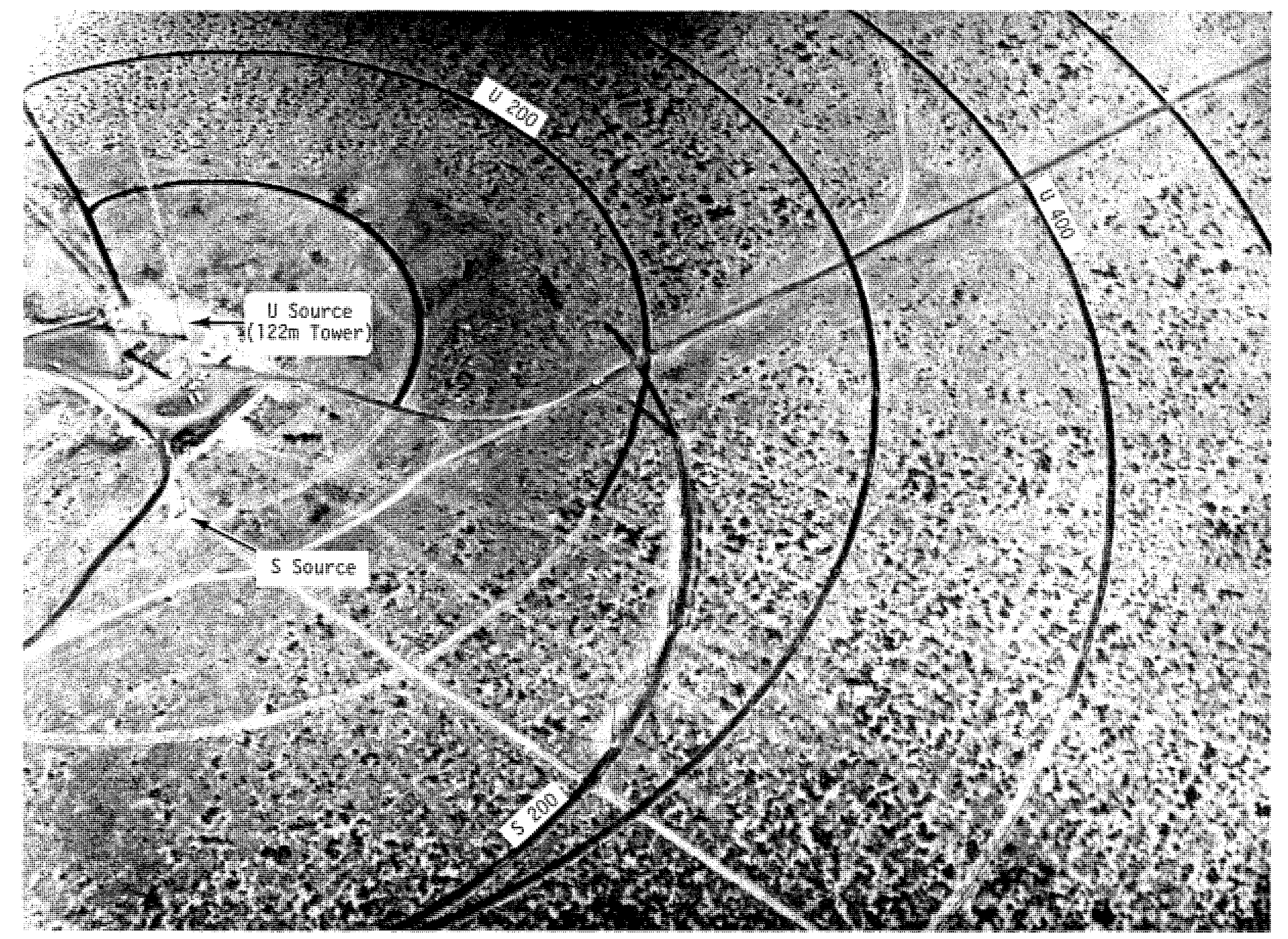

Figure 2. Aircraft view of near-source arcs on Hanford Diffusion Grid. 


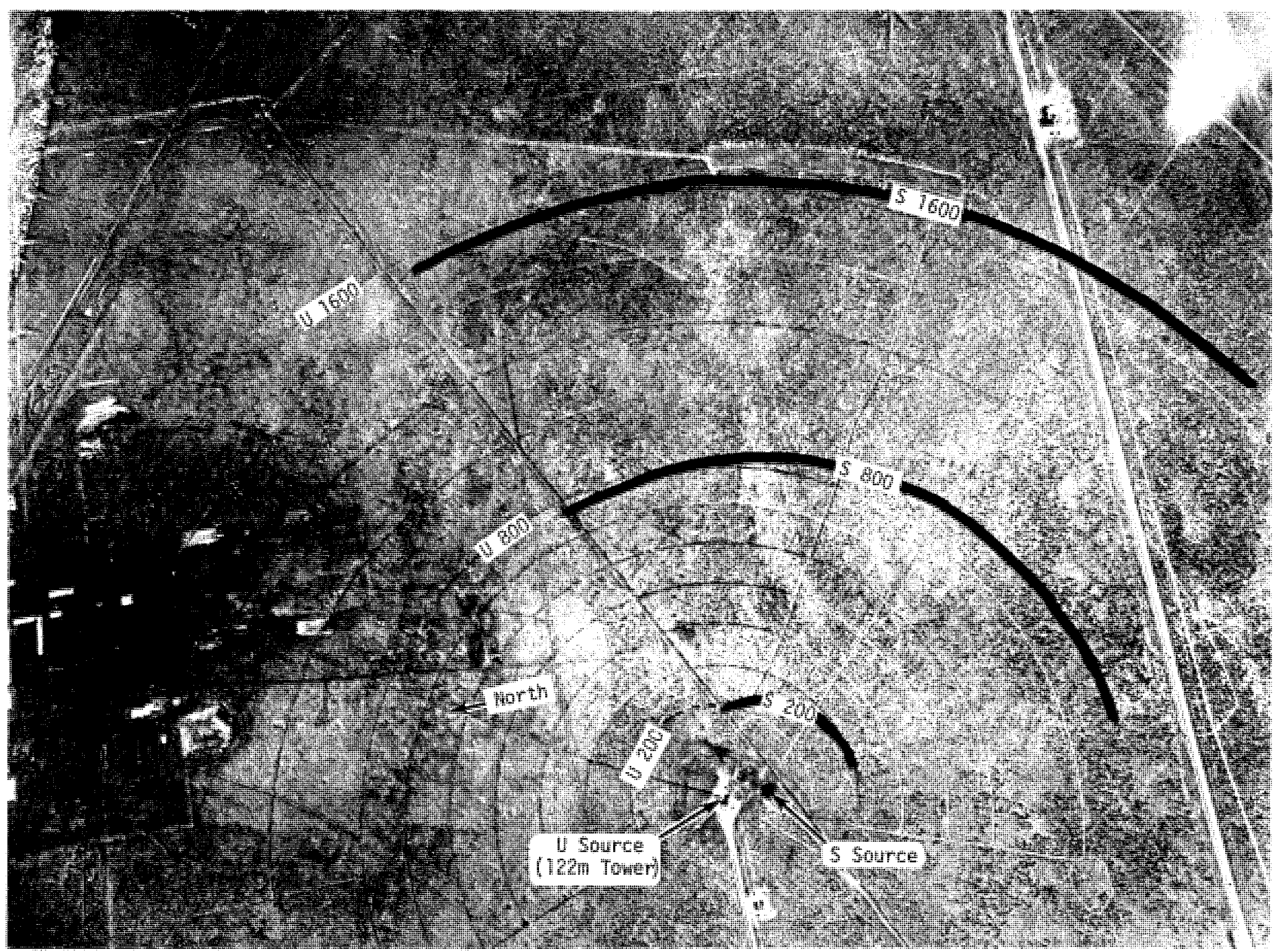

Figure 3. Aircraft view showing sampling arcs to distances of $1600 \mathrm{~m}$ from $\mathrm{U}-$ and S-sources. The S-source and associated arcs have been retouched to aid in their identification. 


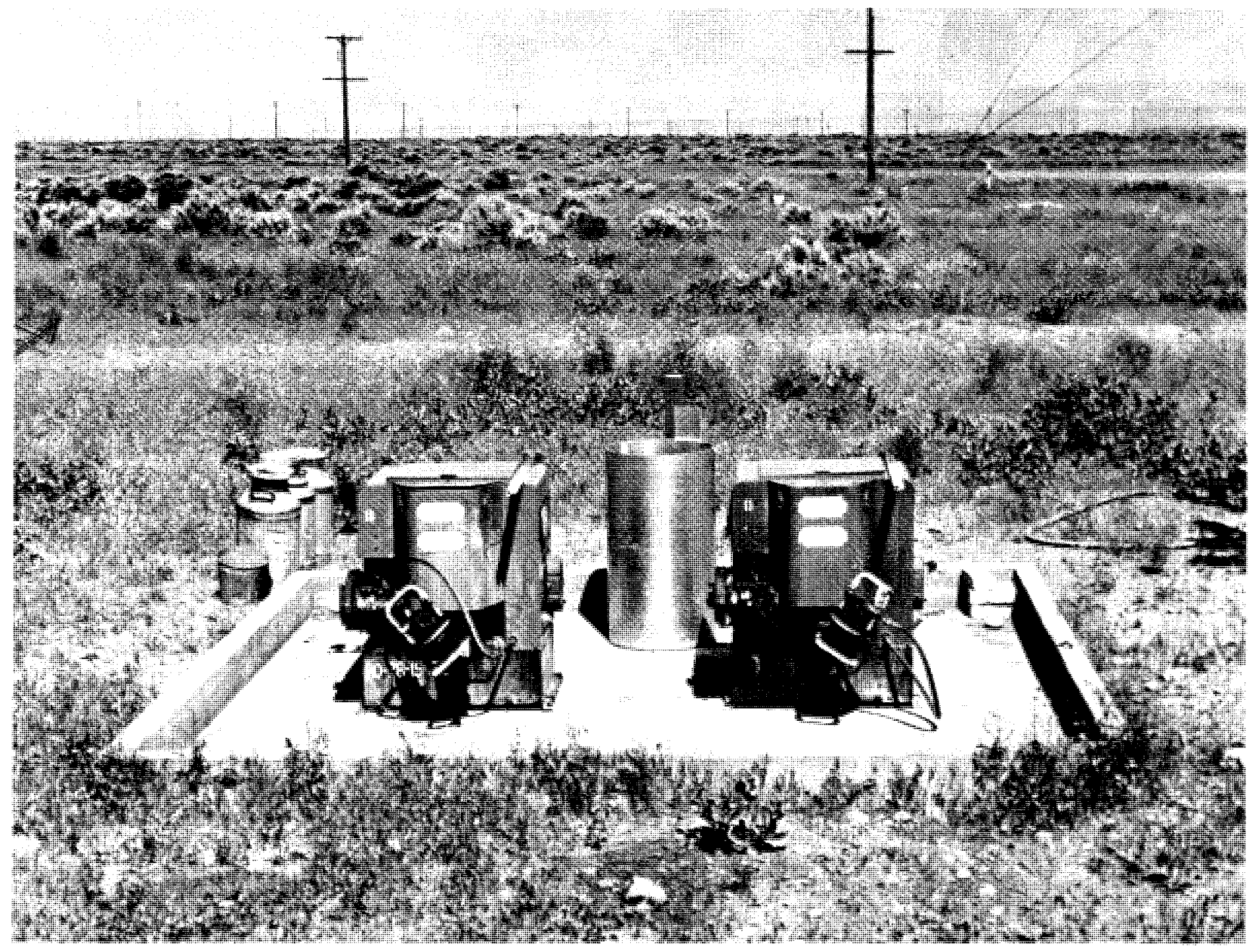

Figure 4. Insecticidal sprayers used in dispersal of $\mathrm{ZnS}$ and fluorescein tracers. View looks upwind from the s-source. 


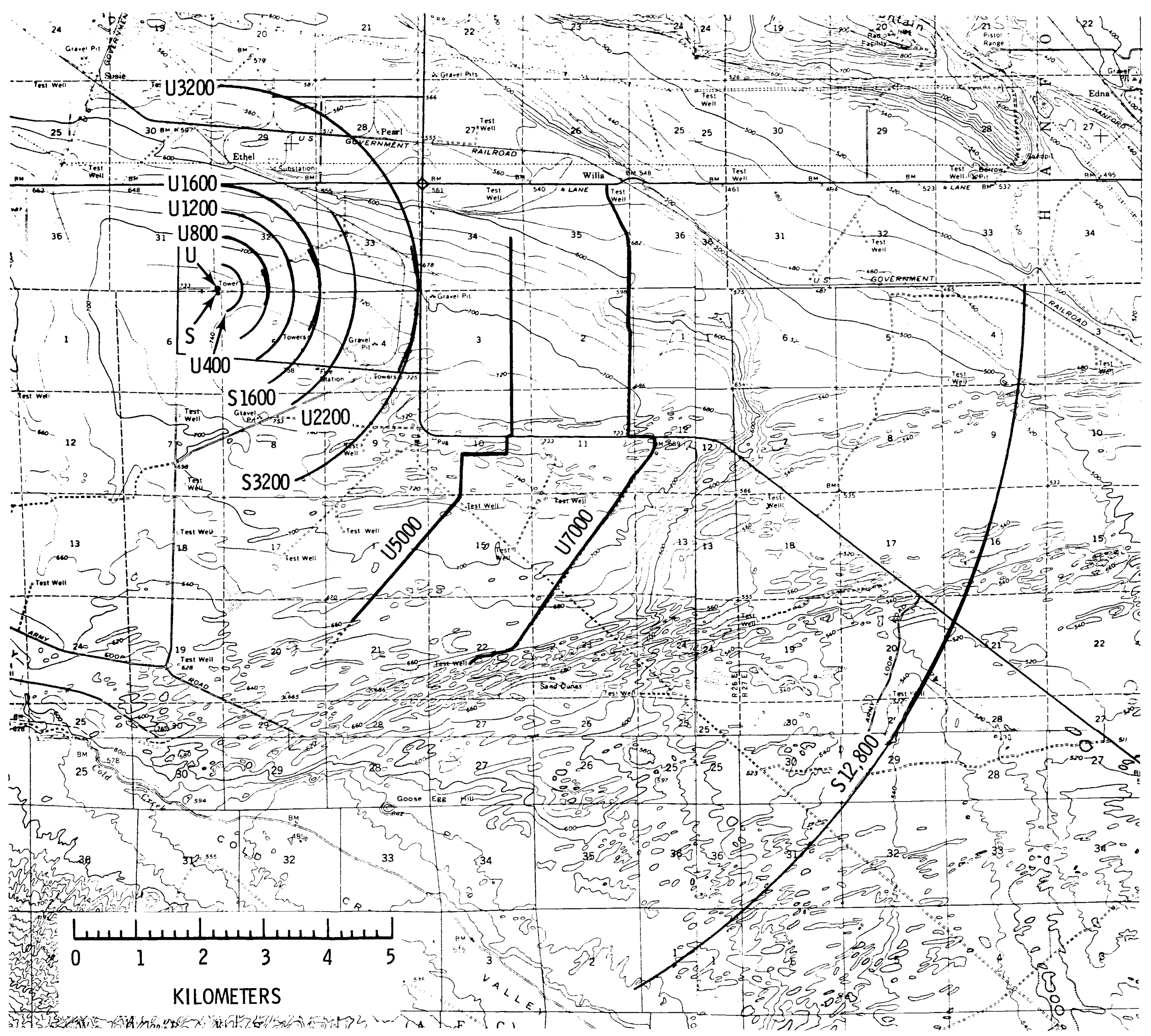

Figure 5. The diffusion grids superimposed on a contour map. Contour interval is $6.1 \mathrm{~m}$ (20 ft). (The 200-m arcs are not diagrammed.) 
ground-level sampling locations are instrumented with vacuum sources.

Power for field vacuum pumps is Supplied by hundreds of gasoline or propane fueled internal combustion engines. A single engine/pump assembly draws vacuum for 1 to 9 sampling stations, with the number depending on the flow rate required at the stations. Flow at each sampling station is controlled by inserting a critical flow orifice in the vacuum line just downstream of the filter-filter holder assembly upon which the particulate tracers are collected. Flow through each sampler is constant as long as the pressure drop across the control orifice is greater than half an atmosphere. This pressure drop is monitored for each engine/pump assembly by inserting a vacuum gauge in the vacuum line immediately downstream of the orifice at the most remote sampler serviced by that assembly.

The ground-level sampling can be supplemented by 365 tower-mounted sampling stations. The towers, as tall as $62 \mathrm{~m}$ and as far removed as $3.2 \mathrm{~km}$ from the tracer release point, are discussed in more detail later in this section. Vacuum and flow control to the towers are accomplished in a manner similar to that already described for the ground-level sampling.

The vacuum/filter field system described in the preceding paragraphs is employed in the collection of particulate tracers. A much less extensive but more sophisticated sampling network was also deployed on portions of the Hanford field grids during the 67-Series. This system $4,5,6$ employed Geiger-Müller tubes at up to 127 field locations to monitor concentrations of the inert gas krypton-85 during nine field experiments. Although the inert gas system recorded the realtime history of tracer concentration at all Geiger tube locations, only the time-integrated concentrations (exposures) are reported in this volume. An earlier data volume ${ }^{7}$ reported 
real-time concentration measurements for five of the nine krypton releases summarized in the current volume.

The sampling grid(s) used during the 67-Series evolved from grids laid out in 1959 and 1960. The grids were designated the "U" and the "S" grids because their original use was restricted to either thermally unstable (U) or thermally stable (S) atmospheres. The U-grid is laid out in a series of arcs of circles concentric about a $122-\mathrm{m}$ tower. This configuration is evident in Figures 2 and 3. Several arcs concentric about the U-source on these figures are labeled with the letter "U" followed by the radial distance in meters from source to arc. The arcs of the U-grid actually used in one or more of the 67-Series experiments, the crosswind extent of those arcs and other grid design specifications are given in Table 1. The intent is not to imply that all arcs or even the complete angular extent of a selected arc were employed during each field experiment. Experimental objectives, meteorological conditions, and manpower available all were factored into decisions as to which samplers should be activated.

The S-grid source, used with only near ground-level tracer releases, is located $100 \mathrm{~m}$ due south of the U-grid source. This location was selected so as to minimize the wake effect of buildings at the base of the 122-m. tower. Figure 4 is a view looking "upwind" from the S-source. The S-source is also indicated on Figures 2 and 3 . Fewer concentric arcs were instrumented about the S-source. The three arcs closest to the s-source are darkened and labeled on Figure 3. S-grid arcs used during the 67-Series were s200, S800, S1600, S3200 and S12800. The number designation, as with the U-arcs, gives the radial distance in meters from $s$-source to sampling arc. Further detail on the S-grid is given in Table 1 .

Two more "arcs" of opportunity were laid out with azimuths related to the U-source. These so-called arcs were not arcs 
TABLE 1

Design Specifications of Hanford "U" and "S" Diffusion Grids

\begin{tabular}{|c|c|c|c|c|c|c|}
\hline \multirow{2}{*}{$\begin{array}{l}\text { Grid Sourcel and } \\
\text { Distance from } \\
\text { Source (m) }\end{array}$} & \multirow{2}{*}{$\begin{array}{c}\text { Range }^{2} \text { of } \\
1.5 \mathrm{~m} \text { Sampling } \\
\text { Azimuths }\end{array}$} & \multirow[b]{2}{*}{$\begin{array}{l}\text { Sampler } \\
\text { Spacing }\end{array}$} & \multirow{2}{*}{$\begin{array}{c}\text { Sampler Flow } \\
\text { Rate } \\
\left(x 10^{-4} \mathrm{~m}^{3} / \mathrm{sec}\right)\end{array}$} & \multicolumn{3}{|c|}{ Towers } \\
\hline & & & & $\begin{array}{c}\text { Samples/ } \\
\text { Tower }\end{array}$ & Height (m) & Azimuths \\
\hline $\mathrm{U} 200$ & $342^{\circ}$ to $160^{\circ}$ & $4^{\circ}$ & $5.07^{4}$ & 17 & 33 & $\begin{array}{l}102^{\circ}, 110^{\circ}, 118^{\circ}, \\
126^{\circ}, 134^{\circ}\end{array}$ \\
\hline U300 & $340^{\circ}$ to $120^{\circ}$ & $4^{\circ}$ & 5.07 & & & \\
\hline $\mathrm{U} 400$ & $342^{\circ}$ to $154^{\circ}$ & $4^{\circ}$ & 5.07 & & & \\
\hline U500 & $340^{\circ}$ to $120^{\circ}$ & $4^{\circ}$ & 5.07 & & & \\
\hline $\mathrm{U} 600$ & $342^{\circ}$ to $122^{\circ}$ & $4^{\circ}$ & 5.07 & & & \\
\hline U 700 & $340^{\circ}$ to $120^{\circ}$ & $4^{\circ}$ & 5.07 & & & \\
\hline U800 & $340^{\circ}$ to $121^{\circ}$ & $3^{\circ}$ & 5.07 & & & \\
\hline U1200 & $360^{\circ}$ to $156^{\circ}$ & $2^{\circ}$ & 9.09 & & & \\
\hline $\mathrm{U} 1600$ & $360^{\circ}$ to $122^{\circ}$ & $2^{\circ}$ & 9.09 & & & \\
\hline U2200 & $48^{\circ}$ to $156^{\circ}$ & $2^{\circ}$ & $17.5^{5}$ & & & \\
\hline U3200 & $360^{\circ}$ to $114^{\circ}$ & $2^{\circ}$ & 17.5 & & & \\
\hline $\mathrm{U} 5000^{3}$ & $82^{\circ}$ to $156^{\circ}$ & $2^{\circ}$ & 17.5 & & & \\
\hline $\mathrm{U} 7000^{3}$ & $76^{\circ}$ to $146^{\circ}$ & $2^{\circ}$ & 17.5 & & & \\
\hline S200 & $68^{\circ}$ to $158^{\circ}$ & $2^{\circ}$ & 1.48 & 17 & 27 & $\begin{array}{l}98^{\circ}, 106^{\circ}, 114^{\circ}, \\
122^{\circ}, 130^{\circ}\end{array}$ \\
\hline 5800 & $70^{\circ}$ to $160^{\circ}$ & $2^{\circ}$ & 1.53 & 18 & 42 & $\begin{array}{l}98^{\circ}, 106^{\circ}, 114^{\circ}, \\
122^{\circ}, 130^{\circ}\end{array}$ \\
\hline$S 1600$ & $70^{\circ}$ to $154^{\circ}$ & $1^{\circ}$ & 2.32 & 19 & 62 & $\begin{array}{l}98^{\circ}, 106^{\circ}, 114^{\circ}, \\
122^{\circ}, 130^{\circ}\end{array}$ \\
\hline S3200 & $72^{\circ}$ to $157^{\circ}$ & $1^{\circ}$ & 5.13 & 19 & 62 & $\begin{array}{l}98^{\circ}, 106^{\circ}, 114^{\circ}, \\
122^{\circ}, 130^{\circ}\end{array}$ \\
\hline $\mathbf{S 1 2 8 0 0}$ & $91^{\circ}$ to $151^{\circ}$ & $1^{\circ}$ & 17.5 & & & \\
\hline
\end{tabular}


of circles, but were merely tracer sampling stations set out along existing roads or trails. They were dubbed the U5000 and $\mathrm{U} 7000$ arcs in correspondence with the approximate sourceto-sampler distances involved. The specific source-to-sampler distance is tabled in Appendix A each time a U5000 or U7000 sampler intercepted tracer. Figure 5 shows the configuration of the U5000 and U7000 arcs.

For reasons of economy, efficiency or experimental design, it frequently became advantageous to activate parts of the sand U-grids simultaneously. (For instance, a wider range of acceptable experiment wind directions was possible.) However, tracer sampling stations located at a constant distance and evenly spaced in azimuth on, say, the S-course, were at varying distances and azimuth spacing with respect to the U-source. This non-concentric effect is most significant at distances close to the source, as is evident in comparing locations of the U200 and S200 arcs on Figures 2 and 3. U and S sampling arcs become more nearly congruent at greater distances as is exemplified by the U1600 and S1600 arcs in Figure 3. When displacement of the tracer release source from the center of the employed sampling grid occurred, it was considered in the azimuths and distances reported -- with the exception of the sampling at the S12800 arc. Even when release was from the U-source, the S12800 diffusion data were reported without correction since the 100-meter maximum error in distance and the less than one-half degree maximum error in stated azimuth were deemed of minimal importance.

Twenty towers were instrumented for tracer sampling on the s-grid. These towers were placed at azimuths of $98^{\circ}, 106^{\circ}$, $114^{\circ}, 122^{\circ}$ and $130^{\circ}$ on the s200, s800, s1600 and s3200 arcs. Tower heights were $27 \mathrm{~m}, 42 \mathrm{~m}, 62 \mathrm{~m}$ and $62 \mathrm{~m}$ at the s200, s800, S1600 and $\$ 3200$ arcs, respectively. 
The 100-m separation of the $\mathrm{S}-$ and U-sources caused some complication when vertical sampling was desired with an elevated release. Elevated release was not possible from the Ssource, and the geometry of the field grids was such that a release of tracer at the U-source could likely not be sampled on both the $\mathbf{5} 200$ towers and the more distant s-grid towers. A curved trajectory would have been necessary. The problem was solved to a great extent by the erection of five towers on the U200 arc. These towers at azimuths of $102^{\circ}, 110^{\circ}, 118^{\circ}, 126^{\circ}$ and $134^{\circ}$ align reasonably well on a radial from the U-source through the S-tower arrays at the greater distances. The U200 towers, 33 meters in height, were used in only the eight " $V$ " experiments conducted after the summer of 1972 .

TRACER RELEASE, SAMPLING AND ASSAY

Four different tracers were released during the Hanford 67-Series. Small particulate tracers employed were zinc sulfide fluorescent powder ( $\mathrm{ZnS}$ FP), fluorescein and rhodamine $B$. The fourth tracer, krypton-85, is an inert gas. The three particulate tracers were collected on membrane filters, and concentrations determined in laboratory procedures which depended upon the fluorescent properties of the tracers. Assessment techniques were discriminatory to the extent that collection of $\mathrm{ZnS}$ and fluorescein or $\mathrm{ZnS}$ and rhodamine on a common filter proved no problem. Krypton-85, by virtue of its radioactivity, was monitored in situ by Geiger-Müller tubes.

Zinc Sulfide Fluorescent Particulate 2210

Use of fluorescent paint pigment (FP) as an atmospheric tracer was initiated in 1946 and documented in $1955^{8}$ at Stanford University under the auspices of the U.S. Army Chemical corps. The Hanford meteorology group began investigation of the use of such fluorescent pigments in 1952, and made use of FP field techniques on a relatively modest scale 
through 1958. The development in 1958 of an optical-electronic device 1,9 (which obviated the need for a tedious "man-andmicroscope" sample assay procedure) facilitated the laboratory assay of the large number of FP samples collected during the Green Glow and subsequent Hanford field diffusion programs.

The FP selected for use in the Hanford technique is Helecon Fluorescent Pigment 2210 manufactured by U.S. Radium Corp., Morristown, New Jersey. It is zns with an activator placed interstitially in its crystalline structure. These particulates have a specific gravity of 4.1. Based on optical microscope sizings at $1000 x$ magnification, the number median (geometric mean) diameter of FP 2210 is about $2.1 \mu \mathrm{m}$. Using methods detailed by Green and Lane ${ }^{10}$, the geometric standard deviation $\left(\sigma_{g}\right)$ and the mass median diameter can be computed. ( $\sigma_{g}$ is defined as the standard deviation of the logarithms of the particle radii about the mean. The mass and number $\sigma_{\mathrm{g}}$ values are identical for log normal size distributions.) The mass median diameter and $\sigma_{g}$ for the FP 2210 used in the 67Series are $4.1 \mu \mathrm{m}$ and $1.6 \mu \mathrm{m}$, respectively. Presuming stokes law for spheres applies, the number and mass diameters translate to terminal fall velocities of $1.9 \mathrm{~m} / \mathrm{hr}$ and $7.6 \mathrm{~m} / \mathrm{hr}$, respectively.

The ZnS tracer was dispersed to the atmosphere through a commercial insecticidal sprayer. Two of these dispersal devices are shown on Figure 4. A measured quantity of the tracer (generally 1 to $4 \mathrm{~kg}$ ) was added to a known volume of liquid carrier (generally about 150 l). Zns is insoluble in the liquid carrier. The tracer was maintained in suspension by insertion of a heavy-duty industrial propeller into the approximately 200 \& cylindrical tank (Figure 4) in which the tracer-liquid carrier was mixed. The tracer-carrier suspension was drawn directly from the cylindrical tank by the commercial sprayer unit. In the sprayer, the suspension was 
pumped to a nozzle assembly where it was atomized by mixing with a jet of heated air and dispersed to the atmosphere. The total tracer dispersed was determined by measuring liquid level in the cylindrical tank before and after tracer dispersal.

The temperature of the air used in the atomization was approximately $400^{\circ} \mathrm{C}$. This high temperature was instrumental in producing a spray that was sensibly dry a few meters from the generator nozzle. The evaporation of the liquid carrier aided in the dissipation of heat so that the effluent from the generator felt dry and thermally comfortable to the hand within 1 to $2 \mathrm{~m}$ from the nozzle.

In the early years of use of this Hanford tracer dispersal technique, the liquid carrier used was water. Some concern developed over the possibility that, under high humidity conditions, the evaporation of the water carrier in the nozzle spray might take place so slowly that there would be a significant gravitational settling. Therefore, a more volatile carrier, trichloroethane $\left(\mathrm{CH}_{3} \mathrm{CCl}_{3}\right)$, was frequently employed as the liquid carrier in many of the later experiments. The difficulty in use of trichloroethane was that it did not act as a lubricant (as water apparently did) in the insecticidal sprayer. Many more mechanical difficulties or failures of the tracer dispersal equipment occurred when trichloroethane was used. Although it is difficult to assess any field differences that might be due to a difference in carrier used, it can be qualitatively stated that at Hanford there was no obvious effect attributable to the carrier used in the dispersal process.

In the 67-Series, trichloroethane was used in the dispersal of $\mathrm{ZnS}$ in all experiments except four. In Tests V5, V6 and V7, water was used as the carrier. In the final test of the series, V8, a commercially available dry FP tracer dispenser was used. 
This dry dispenser, manufactured by Metronics Associates, Incorporated, of Palo Alto, California, is described by Leighton et al. in a 1965 journal article. ${ }^{11}$ This device evolved from the early stanford University work with FP. In the early 1950's, a Hanford dry dispenser was built from prints obtained from the stanford group. Hanford personnel were unable to obtain a constant tracer dispersal rate with this early model dry dispenser. This problem led to the more cumbersome wet dispersal technique which has already been described. A constant dispersal rate was demonstrated with the wet dispersal technique. Further details of the dispersal rate determination -- and of the wet dispersal technique -are given in chapter $\mathrm{V}$ of the Green Glow documentation. ${ }^{1}$

The possibility was considered that the wet dispersal technique would result in a significant agglomoration of individual tracer particles. However, in the years preceding development of a semi-automated device for assessment of FP 2210 at Hanford, a great number of filters were examined and particles were visually counted with the aid of a microscope and ultra-violet illumination. Very few agglomorates were observed during these microscopic examinations. The wet dispersal technique was in use at that time.

A comment should be made regarding the high temperatures to which the $\mathrm{ZnS}$ fluorescent particulates were subjected during the dispersal process. There was concern that the $400^{\circ} \mathrm{C}$ temperature might alter the fluorescent properties of the tracer even though the high temperature was experienced for only a fraction of a second. Nickolal2, in 1963, subjected samples of FP 2210 to temperatures of $1000^{\circ} \mathrm{C}$ for periods up to $20 \mathrm{sec}$ without discernible changes between the pre-heated and post-heated masses indicated when the samples were assayed on the soon-to-be-discussed Rankin counter assay device. 
It was also demonstrated in $1963^{12}$ that FP 2210 was not affected by exposure to bright sunlight -- as has been observed $^{13}$ for other fluorescent powders. Filters were selected from a field experiment in which tracer dispersal, field sampling and filter retrieval from the field all occurred during hours of darkness. The filters were stored in an opaque box until they were assayed for FP 2210. Subsequent to the original assessment, the filters were exposed to bright sunlight for more than 7 hours before reassessment. (The Rankin counter assessment used does not destroy or alter the field samples.) There was no reduction in the measured mass of FP on the filters.

The filter employed in collecting particulate tracers was a polyvinyl chloride membrane filter designated type VM-l by the manufacturer, the Gelman Instrument Company of Ann Arbor, Michigan. This filter offers a compromise between the opposing requirements of minimal restriction to flow through the filter and of a flat surface upon which to retain the sampled $\mathrm{ZnS}$. (The latter requirement is germane to the $\mathrm{ZnS}$ assay procedure which will be discussed presently.)

The $47 \mathrm{~mm}$ diameter filter is inserted into a polyethylene filter holder assembly which leaves a circular area $41 \mathrm{~mm}$ in diameter exposed for tracer collection. Figure 6 shows several of these assemblies in place in the turntables of the assay device (Rankin counter). Between the counters, two of the filter assemblies are shown turned face down to display the ribbed nozzle which can be inserted in a neoprene grommet at each field sampling location. A dust cap (as on the assembly marked "1-122" on Figure 6) is placed over the filter-filter holder assembly during handling and storage. In order to minimize tracer contamination from experiment to experiment, all filter-filter holder assemblies are used only once before being discarded. 


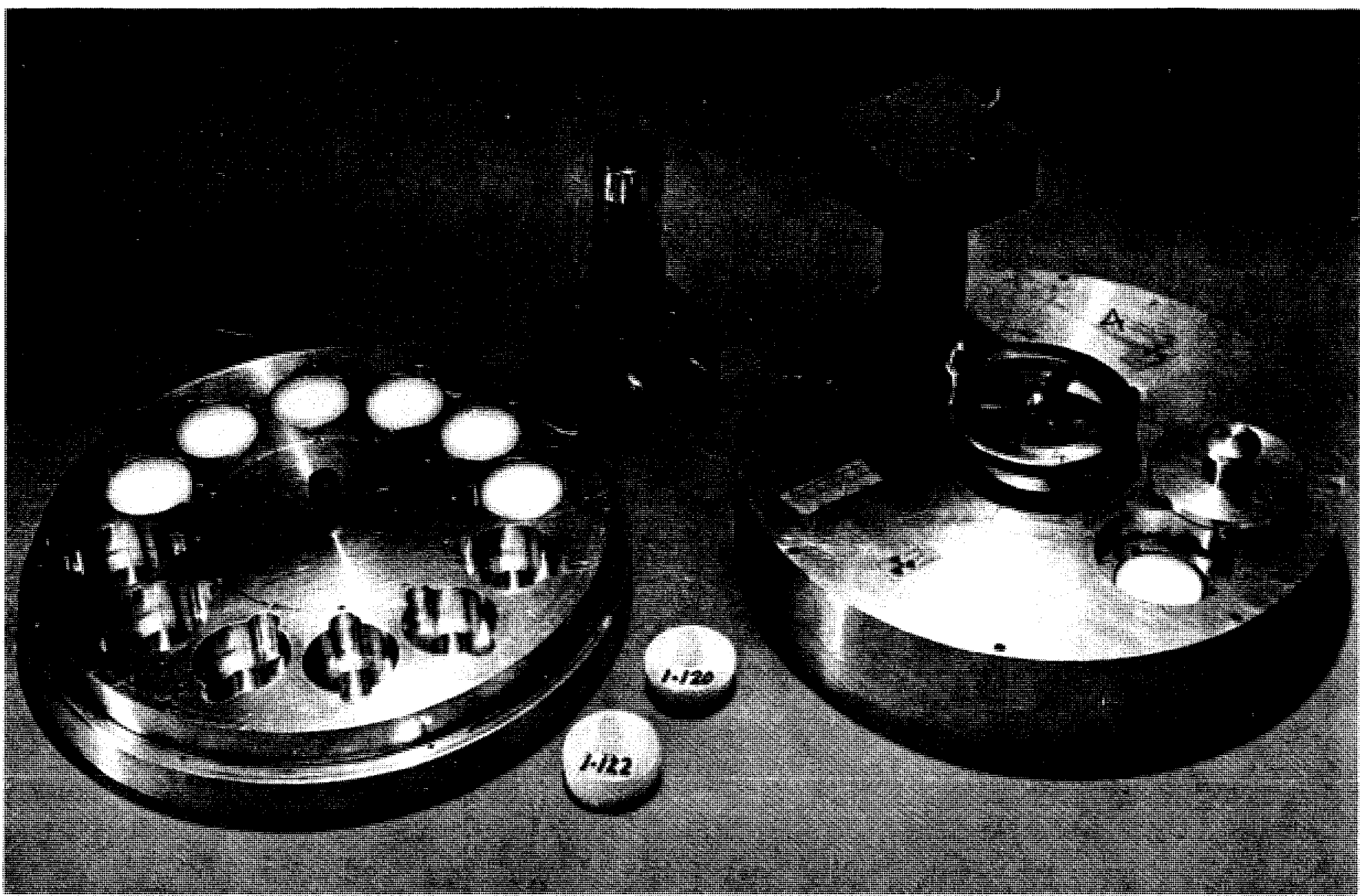

Figure 6. Assembled Rankin counter (right) and disassembled Rankin counter (left) with several field filter assemblies in place for assessment. 
Membrane filters from the field were assayed for $\mathrm{ZnS}$ FP 2210 by the Rankin counting method ${ }^{1,9}$ developed at Hanford in 1958. An assembled Rankin counter is shown at the right in Figure 6. A Rankin counter with top removed to expose a turntable is shown at the left. After the dust cap is removed from a field sampler, the remaining filter-filter holder assembly is inserted into a circular cavity in the turntable. Several filters can be seen in these cavities on Figure 6 . The exposed filter is rotated until it lies directly below a multiplier phototube. Here a 200-microcurie plutonium source, in the shape of an annulus about the face of the phototube, bombards the face of the filter with alpha particles. If there is any FP on the filter face, it is excited to fluorescence by the alpha bombardment, and the scintillations are monitored by the phototube, amplified and counted by a scaler. The VM-l filter, which retains the bulk of the FP on its surface rather than allowing deeper penetration, enhances the probability of the short-range alpha particles reaching the FP and of the resulting scintillations being seen by the photomultiplier. The Rankin counter calibration is specific for the type filter employed.

Design of the counting pig permits the operator to insert or remove a filter from the turntable at the same time another filter is being counted under the phototube. This procedure was a significant time saver in view of the large number of filters assayed for each experiment.

Normal counting time employed during the 67-Series was one minute for each filter. The, count rate on unexposed filters was generally zero. Field exposed background filter count rates were from 0 to about 6 counts/min due, presumably, to some foreign fluorescent material in the ambient atmosphere. Despite precautions, infrequently there was strong evidence of contamination of some exposed filters with the $\mathrm{ZnS}$ tracer. This contamination was generally associated with tower samples 
where it was more difficult to minimize the handling of filter assemblies during field deployment and collection.

The Rankin counter underwent a primary calibration against a series of filters of well established mass several times during the Hanford 67-Series. The calibration in effect at the end of the series was

$$
M=\left(2.06 \times 10^{-10}\right) \mathrm{C},
$$

where M is mass of ZnS FP 2210 in grams and C is Rankin counts/ min. If the level of detection with confidence is considered as 20 counts/min (about 3 times the maximum field background), the corresponding mass was about $4 \times 10^{-9}$ grams. Count rate reproducibility is good with the Rankin counter, particularly at the higher count rates. The ratio of count rate standard deviation to mean count rates of $100,1000,10,000$ and 100,000 counts/min are $0.16,0.048,0.038$ and 0.012 , respectively.

The Rankin counter was also checked (and tuned electronically, if necessary) against a standard filter left continually in one of the twelve turntable cavities. Inasmuch as the standard filter was counted each time it passed beneath the photomultiplier, this secondary calibration occurred once for each 10 field filters assayed. (A background filter occupied the twelfth turntable cavity.)

One difficulty with the Rankin counting technique is that atmospheric dust -- or carbon from the internal combustion engines associated with the field vacuum system -- can collect on the filter face and degrade the scintillations monitored by the photomultiplier. Accordingly, a series of previously assayed filters with zns thereon, but which had a clean appearance, were intentionally subjected to tracer-free but dustladen air. The filters were ranked subjectively from 1 (clean) to 10 (extremely heavy dust load) according to their post-dust 
visual appearance. It was found that assay of filters with dust nomenclature of 4 or less was essentially unaffected by the dust. With successively higher dust nomenclature, increasingly greater count rate degradation was observed. Therefore, in the exposures for $\mathrm{ZnS}$ listed in Appendix $\mathrm{A}$ of this report, dust nomenclature is indicated for filters graded 5 or greater. This point will be discussed further in the section entitled "The Experiments".

Perhaps it should be pointed out that a liquid scintillation counting technique $e^{1,14}$ is available which to a large extent overcomes the ZnS assay problems caused by dust-laden filters. However, the laboratory procedure is a much more tedious "wet" procedure than the simple Rankin counting approach. This fact, plus the relatively few dusty filters encountered during the 67-Series, led to the decision not to employ this more elaborate assay technique.

In order for a filter to sample particles carried in a fluid stream properly, the fluid velocity at the filter face should equal the ambient fluid stream velocity. In this isokinetic flow situation, the fluid streamlines neither diverge nor converge at the filter. Therefore, the particles imbedded in the fluid are sampled properly. However, if the face velocity at the filter is substantially greater or less than the ambient fluid velocity, the particles carried by the fluid will not (by virtue of their greater density than the fluid) faithfully follow the fluid streamlines in the vicinity of the filter. In the case of the Hanford field vacuum grid, the filter face velocities were essentially always less than ambient wind speed, resulting in sub-isokinetic sampling.

Sehmel ${ }^{15}$, in a 1966 wind tunnel study, investigated nonisokinetic sampling effects using ZnS FP 2210 and the standard Hanford field filter. He derived corrections for nonisokinetic flow which are functions of wind speed and filter flow rate. 
Sehmel's corrections have been applied to all the ZnS data presented in this report.

\section{Fluorescein}

An atmospheric tracer technique using uranine dye, the sodium salt of fluorescein, was reported by Robinson, et al. in 1958. ${ }^{16}$ The possibility of using this dye as a tracer to complement the existing ZnS FP 2210 technique was first investigated at Hanford in 1961,17 with early field results reported by Nickola ${ }^{18}$ and by Ludwick..$^{20}$ The term fluorescein, rather than the specific salt, uranine, has been applied to the dye in Hanford nomenclature. This dye is available through industrial chemical suppliers.

The fluorescein used during the Hanford 67-Series was labeled "Uranine Conc. Code 1801" by the vendor, Allied Chemical Company, San Francisco, California. These particulates have a specific gravity of 1.53. Based on microscope sizing at $1000 x$ magnification, the specific batch of fluorescein used had a number median diameter of $1.4 \mathrm{\mu m}$, a mass median diameter of $18.6 \mu \mathrm{m}$ and $\mathrm{a} \sigma_{\mathrm{g}}$ of 2.5 . The number and mass median diameters translate to terminal fall velocities of about 0.4 and $64 \mathrm{~m} / \mathrm{hr}$ if stokes law is applied.

As with the $\mathrm{ZnS}$, dispersal of fluorescein was by means of a commercial insecticidal sprayer. The liquid carrier used in the dispersal tank was trichloroethane. Fluorescein is insoluble in this liquid. The dispersal process used in all but two 67-Series fluorescein releases was as described for the $\mathrm{Zns}$ releases.

During Tests U91 and U92, the fluorescein dispersal technique was altered. In these two tests, the liquid in the mixing tank was water. Fluorescein was added to give an approximate $2.6 \%$ solution of fluorescein in water. This solution was dispersed to the atmosphere at a rate of about $95 \mathrm{l} / \mathrm{hr}$. 
Although no particle-size measurements were made during field tests U91 and U92, the manufacturer specifications on the insecticidal sprayer indicate that a droplet size of about $25 \mu \mathrm{m}$ diameter should be generated with the sprayer control settings employed and the liquid consumption rate observed. Stein et al. ${ }^{19}$ measured the density of fluorescein particles generated through nebulization as $0.58 \mathrm{~g} / \mathrm{cc}$. Presuming this density and the parent droplet size of $25 \mu \mathrm{m}$ apply, the resultant diameter of the dry fluorescein particle during T'ests U9l and 092 was $8.8 \mu \mathrm{m}$ with an associated Stokes terminal velocity of $5.0 \mathrm{~m} / \mathrm{hr}$. The lack of a measured size distribution of particles or droplets precludes further detail.

The filter employed for collection of the fluorescein tracer has already been described in the discussion of the $\mathrm{znS}$ tracer. In the cases where both $\mathrm{ZnS}$ and fluorescein were dispersed and sampled, the $\mathrm{ZnS}$ assay on the dry filter was carried out first. The filters were then placed individually in glass vials. Distilled water was added to dissolve the fluorescein particulates on the filter, leaving the insoluble $\mathrm{zns}$ imbedded on the filter. The fluorescein solution was then assayed with a previously calibrated spectrophotofluorometer with excitation and emission wave lengths tuned for optimum performance. The fluorometer employed was model number 4-8202 manufactured by the American Instrument Company of Silver Spring, Maryland. The analytical technique is explained in greater detail by Ludwick. 17,20

Although less than $1 \times 10^{-11}$ gram of fluorescein was detectable under laboratory conditions, field pollutants and filter-to-filter background variance resulted in a more realistic field-detection limit of about $5 \times 10^{-9}$ grams. The greater variance in background made definition of the tails of crosswind or vertical tracer distributions less certain with fluorescein than with zns. 
In contrast to the deleterious effect that the dust-zns combination displayed with the ZnS assay, the laboratory assessment of the fluorescein in solution was minimally affected by dust.

Although no laboratory tests were made to directly investigate the possible effects of the subjection of fluorescein to high temperatures during dispersal, Nickola ${ }^{18}$ found that $\mathrm{ZnS}$ and fluorescein released from the same location gave compatible downwind field concentrations. Reduction of fluorescence due to mixture for a fraction of a second with air at $400^{\circ} \mathrm{C}$ caused no obvious problems in these 1964 field tests.

No corrections have been attempted for non-isokinetic sampling of fluorescein on field filters.

$\underline{\text { Rhodamine B }}$

Use of another soluble fluorescent dye, Rhodamine B, began at Hanford in 1968. 21 This dye, dissolved in methanol, was dispersed in only six of the Hanford 67-Series experiments. The $1 \%$ rhodamine solution was dispersed to the atmosphere by means of a pair of ultrasonic nozzles. This technique, described by Dana, 22 generates particles of small diameter. No heat was supplied during the dispersal process.

Measurements made during Test's V1, V2 and V3 with Anderson cascade impactors revealed a mass median particle size of about $1 \mu \mathrm{m}$ and $\mathrm{a} \sigma_{\mathrm{g}}$ of 3.2. Although no measurement of the specific gravity of the ultrasonicly-generated particles was made, the specific gravity of the parent powder particulate (before solution) was 1.38. This contrasts with the specific gravity of 1.53 of the fluorescein parent particulate. Assuming the 1.38 specific gravity is applicable to the $1 \mu \mathrm{m}$ particles, and that stokes law applies (electron micrography displayed spherical particles), the terminal velocity of the mass median rhodomine particles was $0.14 \mathrm{~m} / \mathrm{hr}$. If it is 
presumed that the density of the nebulized particles is only 388 of the parent powder (as was the case with fluorescein), the $1 \mathrm{\mu m}$ rhodamine particle should have a stokes fall velocity of $0.05 \mathrm{~m} / \mathrm{hr}$. In either event; the terminal velocity of the rhodamine tracer particles should be negligibly small.

Rhodamine was sampled on the same filter as was ZnS. The assessment procedure for rhodamine was essentially identical to the procedure employed for fluorescein. Since fluorescein and rhodamine were not paired in field release, no tracer discrimination was required from the fluorometer.

As with fluorescein, the detection limit for rhodamine under ideal laboratory conditions was much lower than for field exposed filters. The respective laboratory and field detection limits were approximately $5 \times 10^{-11}$ and $2 \times 10^{-8}$ grams. Again, as with fluorescein, specification of the tails of field tracer distributions was more difficult with rhodamine than with ZnS.

Also, as with fluorescein, dust collected on field samples offered no obvious assay problem with rhodamine.

No corrections for non-isokinetic flow were applied to the masses of rhodamine collected on field filters. However, the small particle size would have made such corrections minimal in any event.

To some degree with all the particulate tracers, there are opposing objectives in dispersal. On the one hand there is the desire to disperse large masses of tracer to the atmosphere so that downwind sampling problems will be minimized. On the other hand, the desire is to disperse the particulate as individual small particles and to avoid effects due to dispersal technique (as opposed to effects due to atmospheric turbulence and diffusion). The dispersal of rhodamine $B$ as $1 \mu \mathrm{rn}$ particles tended to minimize dispersal problems and to maximize field detection problems. About $100 \mathrm{~g}$ of rhodamine were released 
during each test, whereas typical $\mathrm{ZnS}$ or fluorescein releases were from $1000 \mathrm{~g}$ to $3000 \mathrm{~g}$.

\section{Krypton-85}

A field system for measuring atmospheric concentrations of the inert radioactive gas krypton-85 was developed and deployed in the field at Hanford in 1967. Ludwick et al. ${ }^{4}$ and Nickola et al. ${ }^{5}$ have described this system in reasonable detail. A data volume including time-histories of concentration at 63 field locations for eight instantaneous (puff) releases and for five continuous releases (Tests $\mathrm{Cl}$ to $\mathrm{C5}$ of the 67-Series) has been published. ${ }^{7}$

Among advantages of this noble gas tracer technique are that krypton has minimal interaction with structures and vegetation, and will not react with other atmospheric constituents. And although only "continuous" releases are considered in this report, the field technique does include the capability of releasing instantaneous puffs by the simple procedure of dropping a brick on a quartz vial of the gaseous tracer.

Among disadvantages of this tracer technique are the cost of each field sampling unit and the necessity of running a signal cable from each sampler to a central signal processing station.

During the five experiments of the 67-Series prefixed with the letter " $C$ ", krypton was released from a pressurized cylinder. A minimal volume of krypton-85 had been inserted into the argon carrier in the cylinder. Release rate of the krypton was about $1 \mathrm{Ci} / \mathrm{min}$. The rate of the krypton/carrier gas dispersal was monitored by a rotameter, and valving was manually adjusted to maintain a nearly constant rate of release.

During the six krypton-release experiments prefixed with the letter " $V$ ", the flow rate from the pressurized source 
cylinder was automatically held to a constant rate by an electronic mass flow control device. Dispersal rate during the v-tests was about $0.6 \mathrm{Ci} / \mathrm{min}$.

Airborne krypton concentration was monitored by GeigerMüller tubes (Model 18546) manufactured by Amperex Electronic Corporation, Hicksville, New York. These detectors are of the end window type with a window diameter of greater than $50 \mathrm{~mm}$. These field detectors were calibrated by a procedure involving support of the detector inside a large meteorological balloon into which a known amount of krypton-85 had been inserted. The Geiger counters were calibrated in two modes. In the mode where the open face of the detector was exposed to the atmosphere, a krypton concentration of $1 \mu \mathrm{Ci} / \mathrm{m}^{3}$ corresponded to a count rate of 9.7 counts/sec. In a mode where a remote control valve partially shielded the face of the tube (even when the valve was open), a concentration of $1 \mu \mathrm{Ci} / \mathrm{m}^{3}$ resulted in a count rate of 5.5 counts/sec. (The weather-proof valves were used primarily on tower-mounted tubes where manual removal of between-experiment protective covers was impractical.)

During the prefix $C$ experiments, a total of 63 detectors were deployed on portions of arcs S200 and S800. Three S200 towers were instrumented to elevations of $10.7 \mathrm{~m}$, and three S800 towers were instrumented to elevations of $21.3 \mathrm{~m}$. A series of 38.4-sec end-to-end concentration measurements was made.

During the prefix $V$ experiments, a total of 127 detectors were deployed on portions of the U200, S800 and S1600 arcs. All ten towers on the $U 200$ and $S 800$ arcs were instrumented to their tops -- $32.8 \mathrm{~m}$ and $42.0 \mathrm{~m}$ at $\mathrm{s} 200$ and $\mathrm{s} 800$, respectively. The time increment for which short-period concentrations were recorded during the $\mathrm{V}$-tests was $10.0 \mathrm{sec}$. 
DESCRIPTION OF EXPERIMENTS AND DATA

The Hanford 67-Series experiments were carried out during 54 separate days. During most of the experiments, two or more tracers were released -- generally from different elevations above the same point. Zinc sulfide tracer was released on 50 occasions. Fluorescein, rhodamine B, and krypton-85 were released on 38, 6 and 9 occasions, respectively. Tracer sampling equipment was activated before tracer dispersal began and was continued for a period deemed long enough for the bulk of the tracer to pass before deactivation. (The deactivation time was based on the wind speed at 15 meters. A time period 2-1/2 times as long as necessary for uninhibited transport to the sampling arc of interest was generally allowed.)

The cataloging of the field diffusion and meteorological measurements in this volume follows (with three exceptions) the chronological sequence of experiments. Table 2 introduces the 54 experiments/103 releases of the 67-Series in the near chronological order. This table identifies the tracer release point, the type tracer, the release elevation, several pertinent meteorological measurements, and the extent of sampling during each test.

Tables 2 to 5 and Tables/Figures $\mathrm{C}-1$ to $\mathrm{C}-4$ are intended to be largely self-explanatory. The intent of these tables and figures is to enable a researcher to conveniently pinpoint the specific experiments in the appendices that are pertinent to his area of interest. If these tables and figures do their intended job, much of the narrative in this section can be considered of minimal importance.

Tables 3 and 4 give the frequency of releases from various elevations and show the number of times sampling was attempted at various distances. These tables also place a qualitative specification on the overall sampling results for specific locations. 
Table 5 is divided into four parts on the basis of tracer release height. These four tables give some detail on the location and quality of sampling for each field tracer release. The symbols indicating the degree of success at each sampling arc are based on slightly less severe standards than are the symbols which are associated with the quantitative summaries listed in Appendix B.

For reasons which will be pointed out later, there are many instances when it was felt that estimates should be supplied for "bad" or missing data. However, no effort has been made to change the general character of the observations merely because the observed data were unexplainable. For instance, consider field test D4. Both fluorescein and $\mathrm{ZnS}$ were released from the same point. Yet on three of the towers at a distance of $200 \mathrm{~m}$, complete vertical distributions of $\mathrm{ZnS}$ were observed, but no fluorescein was detected at all.

As has been mentioned in the section describing the ZnS tracer technique, sampling of particulates on filters opens the door to non-isokinetic sampling errors. Even though corrections have been applied to the $\mathrm{ZnS}$ data in an attempt to compensate for this error, it is felt that the wind tunneldetermined empirical corrections do not always perform an adequate adjustment. Furthermore, no isokinetic corrections are attempted with the fluorescein or rhodamine assessments. The result can be a "roller-coaster" effect in the situation where arcs of relatively low flow rate are interspersed with those of higher flow rate. Both $\mathrm{ZnS}$ and (especially) fluorescein for field test $\mathrm{U} 83$ offer such an example. The U400 (high flow rate), $\$ 800$ (low flow rate), U1200 (high), S3200 (low), U2200 (high) sequence of sampling results in normalized measured concentrations at these arcs which appear to be low, high, low, high and low, respectively. Let it be stressed that flow rate has been factored into the calculations. 
The uncertainty as to which values of concentration are most proper is disconcerting. (It is the author's opinion that inasmuch as the higher flow rates required a smaller correction, they generate more nearly correct values.) In defense of what may seem to be poor field technique, the roller coaster effect would never have been observed had the same flow rate been used at all sampling arcs -- or had a higher flow rate been used at each succeeding arc. Such flow arrangements would not necessarily have made the measured concentrations any closer to the correct values, but merely would have given results more pleasing to the eye.

Before proceeding with the description of the method of presentation of the individual field experiments, a mention of near-source wake effects seems in order. Although the ideal experiment would entail a point source release into an undisturbed ambient atmosphere, such releases are impossible. Perhaps the experiments with the least disturbance in the upwind fetch were the four prefix D experiments. Figure 4 shows the upwind fetch for these four experiments. During the $\mathrm{C}$ experiments, the particulate dispersal equipment shown in Figure 4 was placed on a low, flatbed trailer about $6 \mathrm{~m}$ in length which was parked about $4 \mathrm{~m}$ upwind of the source. The $\mathrm{zns}$ during Test C5 was dispersed from this trailer. The krypton during the $C$ experiments was released from the cement pad shown in Figure 4, and thus had the trailer and associated equipment forming a lattice-like cross section of about 8 to $10 \mathrm{~m}^{2}$ in the upwind fetch.

All elevated releases were undoubtedly affected to some degree by the wake induced by the 122-m tower from which release took place. This tower is a rather sturdy structure, triangular in cross section, and about $3.6 \mathrm{~m}$ on each side. Some idea of the appearance of this structure can be gained by examination of Figure 2 . 
The near ground-level releases from the U-source (Tests U56 to U70) were subjected to perhaps the worst wake situation. First, the dispersal equipment (Figure 4) was mounted on a small trailer; secondly, the wake of the $122-\mathrm{m}$ tower a few meters away no doubt added to the turbulence; and third, a small $4 \mathrm{~m} \times 4 \mathrm{~m} \times 3 \mathrm{~m}$ building stood near the base of the tower. (The bulk of the building complex shown in Figfure 4 at the base of the tower was dismantled prior to the start of the 67-Series.)

The Appendix A Diffusion Data

Appendix A gives the individual field diffusion measurements. These data form the bulk of this data volume. The 54 experiments are presented in the order given in Table 2. Dual tracer release data are presented side-by-side. Some general comments pertinent to each field experiment precede listing of the individual field measurements. The date and time of release, tracer release elevation and wind speed at release height are included in a heading preceding each arc or tower of measurements.

The body of the Appendix A tables gives AZIMUTH with respect to the source in the first column, and DISTANCE from the source in the last column. In instances where s-grid arcs (or portions of S-grid arcs combined with portions of U-grid arcs) were employed with U-source releases, the nonuniformity of the samplers with respect to these sources is reflected in these first and last columns. For example, during field test U71, release was from the U-source (Table 2). The Appendix A data for Test U7l fluorescein indicate that all sampling at the $400 \mathrm{M}$ arc was actually done on the U-grid inasmuch as azimuths increment evenly by $4^{\circ}$, and all distances are listed as $400 \mathrm{~m}$. However, on the $800 \mathrm{M}$ arc, the samplers exposed between $61.0^{\circ}$ and $97.0^{\circ}$ were on the U-grid $\left(3^{\circ}\right.$ increments and $800 \mathrm{~m}$ distance), while those exposed between $97.1^{\circ}$ and $110.7^{\circ}$ were 
on the S-grid (uneven azimuth increments and distances not $800 \mathrm{~m})$. As mentioned earlier in description of the grid, the data from the S12800 arc are not "corrected" for the variation in distance and direction resulting from release from the $\mathrm{U}$-source. The 100-meter separation of $\mathrm{U}$ - and S-sources is considered of minimal importance at this distance.

The second column in the Appendix A tables lists EXPOSURE. (For towers, a column specifying sampling HEIGHT precedes the EXPOSURE column.) The EXPOSURE data for particulate tracers evolves from division of the mass of tracer measured on each filter by the flow rate through the filter (Table 1). Inasmuch as no normalization to source strength is made in the EXPOSURE column, the magnitude of the individual numbers are directly related to the mass of tracer collected on each filter, and are therefore related to the confidence that can be placed in each sample. For the krypton tracer, the EXPOSURE column is the integral of concentrations measured over all the shorter time increments. Magnitude here is also related to the confidence that can be placed in an individual sample.

The EXPOSURE column has been left in digital format (as opposed to scientific notation as in the subsequent 2 columns) in order to give an analog appearance to concentration distribution across an arc (or up a tower). In most cases the shape of the crosswind (or vertical) distribution is relatively obvious with a glance at the column.

In the column headed $\mathrm{E} / \mathrm{Q}$, exposure has been normalized by dividing by the total mass emitted (or total curies in the case of the krypton emissions).

EU/Q is the exposure normalized to both unit emission and unit wind speed. The mean wind speed (U) used in this normalization is that listed in the heading immediately preceding each arc (tower) of data. It is the mean wind speed at the release height during the period of release. (In the cases 
where more than one tracer was released at the same time but release periods differed, the $U$ used in the $\mathrm{EU} / \mathrm{Q}$ column resulted from measurements over the longer release period.)

In many cases, a one-character symbol precedes the azimuth column in Appendix A. These symbols indicate that something less than ideal was associated with the sample. A detailed explanation of the alphabetical symbols is given at the beginning of Appendix A. The numerical symbols were mentioned earlier in the subsection entitle "zinc Sulfide Fluorescent Particulate 2210". It was stated that filters with visual dust nomenclature of 4 or less appeared to give no problems. However, the indicated mass of zinc sulfide on a filter was reduced when a visual dust rating of 5 or more was observed. The experiment upon which this conclusion was based was severe, however. It involved collecting tracer on a filter followed by the collection of dust. If the dust had been collected first, leaving the tracer "on top" of the dust, the degradation of the zinc sulfide assessment might not have been as severe. In any event, the observation of a 5 or greater dust loading can be associated with a reduction in indicated $\mathrm{ZnS}$, but it is not necessarily so in all cases. It should be pointed out that in many cases dust nomenclature of less than 5 is indicated on the Appendix A data. These are vestiges of laboratory assessment notes made before the mentioned dust loading experiments were made, and can thus be ignored.

Since each $1.5-\mathrm{m}$ sample was used in the computation of CROSSWIND INTEGRATED concentrations following each arc of data (and in other statistics to be found in Appendix B), it was deemed wiser to substitute a reasonable estimate for an obviously erroneous sample than to have an erroneous number go into the computations. These estimates are frequently merely interpolations or extrapolations of data collected on the same arc. At times it was necessary to plot areal distributions of 
tracer and to interpolate concentrations on a mid arc from arcs closer to or farther from the source than the arc in question.

In some instances, the amount or quality of data on an arc is so low that no estimations were attempted. Also, since no vertical moments or vertical crosswind sums were computed, the incentive to supply estimates for poor tower samples was not as great as it was for the poor $1.5-\mathrm{m}$ samples. Thus the poorer tower measurements are frequently reported directly with an accompanying remark symbol other than "E" for "estimate".

The Appendix B Diffusion Summaries

Appendix $B$ summarizes the ground-level data for each of the 103 releases. Presented first is a repeat of the specific experiment remarks from Appendix A. A heading then identifies the specific tracer and gives pertinent release information.

The first column of the tabular data indicates DISTANCE FROM SOURCE. The next four columns give statistics relating to the CROSSWIND DISTRIBUTION. The first of these columns gives the location of the MEAN of the crosswind distribution. The STANDARD DEVIATION $\left(\sigma_{y}\right)$ and COEFFICIENT of SKEWNESS and KURTOSIS for the crosswind distribution follow.

The next three columns give the AZIMUTH and the magnitude of the three largest exposures measured at each arc. $\mathrm{E} / \mathrm{Q}$ is exposure normalized to source strength, and EU/Q is exposure further normalized to wind speed, $U$, at tracer release height. Three values of exposure are given in order to reduce the chance of considering a "sport" or somehow erroneous measurement as representative of plume centerline.

The last two columns give CROSSWIND INTEGRATED values for the source and source/wind speed-normalized EXPOSURES.

Tabulated data followed by the symbol "?" indicate some uncertainty in the data. The uncertainty may involve estimates 
in the Appendix A data. Perhaps there was some question on the performance of the laboratory assay equipment. The symbol is intended to alert a user of the data to the fact that something not completely routine went into the generation of the associated number. An examination of the remarks preceding the table or scanning of the appropriate Appendix A field data should display the reason for the symbol. Where estimates of missing data were on the tails of a distribution, or where a broad distribution made one large estimate of minimal consequence, a "?" may not have been appended in the Appendix B data. Admittedly some subjectivity went into the decision as to whether to append the symbol or not, and a second review of the data would not result in precisely the same tagging. The symbol is intended primarily as a convenience to the data user.

Appending of the symbol " $\mathrm{X}$ " to Appendix $\mathrm{B}$ data symbolizes a much more serious difficulty with the data. The data are either incomplete or invalid. For instance, the computation of a mean, $\sigma_{y}$, skewness, kurtosis and crosswind integrated exposure from a badly truncated distribution lacks significance. An " $X$ " is then appended. (However, if the truncated distribution is such that there is little doubt that the plume centerline was observed, no " $X$ " is appended in the MAXIMUM FXPOSURE columns.)

The qualification for a "clean bill of health" in Appendix B was a bit more strict than was the case in Table 5. Thus a "?" may be found in Appendix B whereas a "C", indicating no problems, may have been listed for the same data in Table 5 . 
The Appendix C Meteorological Data

Vertical profiles of temperature, wind speed and wind direction were measured on the $122-\mathrm{m}$ tower at the U-source during the Hanford 67-Series. In addition, wind speed and direction were measured on a second tower -- also generally near the tracer release point.

Temperature measurements on the 122-m tower were made by Foxboro thermohms. The aspirated thermohms were exposed at elevations of $0.9,15.2,30.5,45.7,61.0,76.2,91.4$ and $122 \mathrm{~m}(3,50,100,150,200,250,300$ and $400 \mathrm{ft})$. During the eight prefix-V tests, an additional thermohm was exposed at an elevation of $6.1 \mathrm{~m}(20 \mathrm{ft})$. Recording was a series of points on a strip chart. About $3-1 / 2$ minutes were required to cycle through all temperature sensors. Inasmuch as most field tests were $30 \mathrm{~min}$ in duration, eight or nine measurements were available to compute the mean temperature for a given elevation as presented in Table $\mathrm{C}-1$ and Figure $\mathrm{C}-1$ of Appendix C.

Wind speed and direction on the $122-\mathrm{m}$ tower were measured by seven Aerovane anemometers mounted at elevations of 2.1 , $15.2,30.5,45.7,61.0,91.4$ and $122 \mathrm{~m}(7,50,100,150,200$, 300 and $400 \mathrm{ft}$ ). The starting speed for Aerovane propellers is approximately $1 \mathrm{~m} / \mathrm{sec}$. The Aerovane is a rather large streamlined vane/anemometer assembly about $80 \mathrm{~cm}$ in length. The assembly has a distance constant of about $4.5 \mathrm{~m}$ for speed and about $10 \mathrm{~m}$ for direction.

The Aerovane outputs were recorded on strip charts moving at the rate of $7.6 \mathrm{~cm} / \mathrm{min}$ ( 3 inches $/ \mathrm{min}$ ). The strip chart traces were averaged (means) by eye for 20-sec increments, and the resulting digitized data were used in computing mean speeds, mean directions, and standard deviations of wind direction for the periods of tracer release. 
For the bulk of the field tests, wind speed and direction were measured at six levels on a 25-m tower located about $25 \mathrm{~m}$ west of the S-source. (During Tests U84 to U92, these sensors were mounted on the tower at $106^{\circ}$ on arc sl600.) The system for measuring and recording winds on this tower has been described by Ratcliffe and Sheen. ${ }^{23}$ The wind speed sensors were Beckman and Whitley Model Ml564 three-cup anemometers. These cups have a starting threshold of less than $0.4 \mathrm{~m} / \mathrm{sec}$ and a distance constant of about $1.5 \mathrm{~m}$. Circuitry was arranged to permit accumulation of an integrated wind speed for $3.5 \mathrm{sec}$, after which $1.5 \mathrm{sec}$ were consumed in signal processing and recording. In each minute, 12 such recorded segments of wind speed were accumulated for each anemometer.

Wind direction transducers employed on the 25-m tower were Beckman and Whitley Model M1565 vanes. These vanes respond to winds of less than $0.4 \mathrm{~m} / \mathrm{sec}$ and have a distance constant of about $1.5 \mathrm{~m}$. The output signals from these lightweight vanes were smoothed by a filter having a 5-sec time constant. The filter output was sampled for $60 \mathrm{msec}$ once each 5 sec, digitized and recorded. The digitized data were used for computing mean directions and standard deviations of direction, $\sigma_{\theta}$.

The Beckman and Whitley cups and vanes were mounted at elevations of $0.76,1.5,3.0,6.1,12.2$ and $24.4 \mathrm{~m}(2.5,5$, $10,20,40$ and $80 \mathrm{ft}$ ) during most of the field experiments. During Tests U 84 to U92 they were mounted at elevations of $2,4,8,16,32$ and $36 \mathrm{~m}$. Thus, the spacing between wind sensors on the shorter towers was closer than was the spacing between Aerovanes on the 122-m tower. Wind speeds indicated by the Beckman and Whitley cups frequently averaged slightly higher than those computed from the Aerovanes, although the shapes of the profiles in the area of common measurement were in excellent agreement. The wind speeds reported on Table C-2 
and Figure $\mathrm{C}-2$ of Appendix $\mathrm{C}$ are based primarily on the cup measurements at the lower elevations, and on the Aerovane measurements above the measurement level of the cups.

Two sets of wind direction and wind direction standard deviation $\left(\sigma_{\theta}\right)$ data were developed. One set is based on the Aerovane measurements, and one set follows the Beckman and Whitley vane measurements. These measurements are found in Tables $\mathrm{C}-3$ and $\mathrm{C}-4$ of Appendix $\mathrm{C}$, and are graphed in Figures C-3 and C-4. With a few exceptions during light wind cases, these data show that agreement between Aerovane and Beckman and Whitley data is reasonably good. The agreement in mean direction profiles is rather expected. However, in view of the difference in physical characteristics of the large Aerovane and the relatively lightweight Beckman and Whitley vane, and the difference in the method of digitizing direction, the agreement in $\sigma_{\theta}$ profiles is more surprising.

It has been the author's experience that the orientation of a wind vane is not a simple matter. If only one vane is exposed, one may feel confidence in the orientation. Exposure of a second vane at an elevation differing from the first essentially always results in a difference in mean direction that can logically be explained by wind direction shear with height. However, addition of a third, a fourth, and more vanes at additional elevations seems to inevitably lead to mean direction profiles that have repetitive kinks that should not logically be there. This observation is made as a partial or likely explanation of some of the similarly-shaped wind profiles observed at times in the 67-Series. Aerovane direction profiles for the upper elevations during Tests U84, U85 and U86 are an example (Figure $C-3$ ).

The data presented on Tables $\mathrm{C}-1$ to $\mathrm{C}-4$ and Figures $\mathrm{C}-1$ to $\mathrm{C}-4$ are based on measurements made during tracer release. In the event that two or more tracers were released during the 
same experiment and the periods of release did not coincide, the meteorological data apply to the longer tracer release period.

Note that on Figure C-1, which depicts vertical profiles of temperature, the vertical scale is linear. On the companion Figures $\mathrm{C}-2, \mathrm{C}-3$ and $\mathrm{C}-4$, the vertical scale is logarithmic. Heights of tracer release are indicated on all these figures by the symbols $F, K, R$ and $Z$ for fluorescein, krypton-85, rhodamine $B$ and zinc sulfide, respectively.

It is recognized that use of Figures $\mathrm{C}-1$ to $\mathrm{C}-4$ in a quantitative sense would be difficult. However, the intent of these figures is to aid in picking out features in the vertical profiles that otherwise might not be obvious. Once a characteristic of interest is observed on the analogs of Figures $\mathrm{C}-1$ to $\mathrm{C}-4$, absolute values can be obtained from Tables $\mathrm{C}-1$ to $\mathrm{C}-4$. 


\section{ACKNOWLEDGEMENTS}

It would be impossible to acknowledge individually the great number of people involved in the field work, laboratory assessment, and data reduction associated with the Hanford 67-Series. However, thanks are particularly due those unnamed individuals who were frequently roused from warm beds to "head for the tules" in the rather unorthodox hours required by most of the 67-Series experiments.

For the data volume specifically, thanks are due Mr. G. H. Clark of the Australian AEC who, during his temporary assignment at Hanford, developed the parent computer program from which the Appendix A and B programs evolved.

The field work summarized in this report was conducted under the auspices of the former Atomic Energy Commission's Division of Biology and Medicine and the former Energy Research and Development Administration's Division of Biomedical and Environmental Research, the functions of which have now been transferred to the Department of Energy.

The assembling of data and publication of this document is jointly sponsored by the above-mentioned ERDA Division and by the Division of Reactor Safety Research of the Nuclear Regulatory Commission. 


\section{REFERENCES}

1. M. L. Barad and J. J. Fuquay (Eds.), The Green Glow Diffusion Program. Geophysical Research Papers No. 73, Vols. I and II, AFCRL-62-251 (I and II), Air Force Cambridge Research Laboratories, Bedford, MA, (also as HW-71400 (I and II), Hanford Laboratories, General Electric Co., Richland, WA), (I) Jan. and (II) Apr. 1962.

2. J. J. Fuquay, C. L. Simpson and W. T. Hinds, "Prediction of Environmental Exposures from Sources Near the Ground Based on Hanford Experimental Data." J. of Appl. Meteorol. 3 (6): 761-770, Dec. 1962 .

3. J. M. Hales and Staff, Pacific Northwest Laboratory Annual Report for 1976, to the ERDA Assistant Administrator for Environment and Safety, Part 3, Atmospheric Sciences. BNWL-2100 PT3, Battelle Pacific Northwest Laboratories, Richland, WA 99352, August 1977. Pertinent reports previously issued in this series are as follows:

\section{Annual Report for}

1975
1974
1973
1972
1971
1970
1969
1968
1967
1966
1965
1964
1963
1962
1961
1960

BNWL-2000, PT 3
BNWL-1950, PT 3
BNWL-1850, PT 3
BNWL-1751, Vo1. II, PT 1
BNWL-1651, Vo1. II, PT 1
BNWL-1551, Vo1. II, PT 1
BNWL-1307, Vo1. II, PT 1
BNWL-1051, Vo1. II, PT 1
BNWL-715, Vo1. II, PT 3
BNWL-481, Vo1. II, PT 1
BNWL-235, Vol. I
BNWL-36-I.
HW-81746
HW-77609
HW-73337
HW-70050

4. J. D. Ludwick, J. J. Lashock, R. E. Connally and

P. W. Nickola, "Automatic Real Time Air Monitoring of ${ }^{85} \mathrm{Kr}$ Utilizing the 4096 Memory of a Multiparameter Analyzer." Rev. Sci. Inst. 39 (6): 853-859, June 1968.

5. P. W. Nickola, J. D. Ludwick and J. V. Ramsde11, Jr., "An Inert Gas Tracer system for Monitoring the Real-Time History of a Diffusing Plume or Puff." J. App. Meteorol. 9. (4): 621-626, Aug. 1970 . 
6. P. W. Nickola, "Measurements of the Movement, Concentration and Dimensions of Clouds Resulting from Instantaneous Point Sources." J. Appl. Meteorol. 10 (5) : 967-973, oct. 1971 .

7. P. W. Nickola, J. V. Ramsdell, Jr., and J. D. Ludwick, Detailed Time-Histories of Concentrations Resulting from Puff and Short-Period Releases of an Inert Radioactive Gas: A Volume of Atmospheric Diffusion Data. BNWL-1272, Battelle, Pacific Northwest Laboratories, Richland, WA 99352 , Feb. 1970 .

8. P. A. Leighton, The Stanford Fluorescent-Particle Tracer Technique. [Defense Documentation Center, AD 91-94], Dept. of Chemistry, Stanford University, Palo Alto, CA, June 1955.

9. M. O. Rankin, A Zinc Sulfide Particle Detector. HW-55917, Hanford Laboratories, General Electric Co., Richland, WA, 1958 .

10. H. L. Green and W. R. Lane, Particulate Clouds: Dusts, Smokes and Mists. E. \& F. N. Spon, Ltd., London, England, 1957.

11. P. A. Leighton, W. A. Perkins, S. W. Grinnell and F. X. Webster, "The Fluorescent Particle Atmospheric Tracer." J.Appl. Meteorol. 4 (3): 334-348, June 1965.

12. P. W. Nickola and M. F. Scoggins, "Atmospheric Tracer Technology." In: Hanford Radiological Sciences Research and Development Annual Report. for 1963, HW-81746: 1.431.51, Hanford Atomic Products Operation, General Electric Co., Richland, WA, Jan. 1964.

13. A. E. J. Eggleton and N. Thompson, "Loss of Fluorescent Particles in Atmospheric Diffusion Experiments by Comparison with Radio-xenon Tracer." Nature 192: 935-936, Dece. 1961.

14. J. D. Ludwick and R. W. Perkins, "Liquid Scintillation Techniques Applied to Counting Phosphorescence Emission." Anal. Chem. 33 (9) : 1230-1235, Aug. 1961 .

15. G. A. Sehmel, Subisokinetic Sampling of Particles in an Air stream. BNWL-217, Battelle Pacific Northwest Laboratories, Richland, WA 99352, Mar. 1966. 
16. E. Robinson, J. A. Macleod and C. E. Lapple, "A Meteorological Tracer Technique Using Uranine Dye." J. of Meteorol. 16: 63-67, Feb. 1959 .

17. J. D. Ludwick, Dual Atmospheric Tracer Techniques for Diffusion Studies Using Phosphorescence - Fluorescence Analysis. HW-70892, Hanford Atomic Products Operation, General Electric Co., Richland, WA, March 1961.

18. P. W. Nickola, Field Testing of a Fluorescein-Zinc Sulfide Dual Atmospheric Tracer Technique. BNWL-I0 3 , Battelle, Pacific Northwest Laboratories, Richland, WA 99352, Aug. 1965.

19. F. Stein, N. Esmen and M. Corn, "The Density of Uranine Aerosol Particles." Am. Indust. Hyg. Ass. J. 27 (5): 428-430, sept.-Oct. I $\overline{966 .}$

20. J. D. Ludwick, "Atmospheric Diffusion Studies with Fluorescein and Zinc Sulfide Particles as Dual Tracers." J. Geophys. Res. 71 (6): 1553-1558, March 1966.

21. M. A. Wolf and M. T. Dana, "Experimental Studies in Precipitation Scavenging." In: Pacific Northwest Laboratory Annual Report for 1968 to the USAEC Division of Biology and Medicine, Vol. II: Physical Sciences, Part 1, Atmospheric Sciences, BNWL-1051, Part 1: 18-25, Battelle, Pacific Northwest Laboratories, Richland, WA, 99352, Nov. 1969.

22. M. T. Dana, "Calibration of an Ultrasonic Nozzle for Aerosol Generation." In: Pacific Northwest Laboratory Annual Report for 1970 to the USAEC Division of Biology and Medicine, Vol. II: Physical Sciences, Part I, Atmospheric Sciences, BNWL-155I, Vol. II, Part 1: 98-101, Battelle, Pacific Northwest Laboratories, Richland, WA, 99352, June 1971 .

23. C. A. Ratcliffe and E. M. Sheen, "An Automatic Data Collection System for Meteorological Tower Instrumentation." J. Appl. Meteorol. 3 (6): 807-809, Dec. 1964 . 
tABLE 2. GENERAL GRID. SOURCE, RELEASE AND METECRLLCGICAL INFORMATION.

\begin{tabular}{|c|c|c|c|c|c|c|c|c|c|c|c|c|c|c|c|c|}
\hline & GRIO & $\begin{array}{r}T Y \\
d F \\
---\end{array}$ & $\begin{array}{l}P E^{\prime} \\
=T R A\end{array}$ & $\begin{array}{l}\mathcal{E} \text { DU } \\
A C E R\end{array}$ & $\begin{array}{l}\text { JRAT I } \\
\text { RELE }\end{array}$ & $\begin{array}{l}\text { IUN" } \\
\text { EASE } \\
-2-\end{array}$ & UBAR & D $M / S$ & $\operatorname{sig} \theta$ & $\begin{array}{r}\text { OEG } \\
-\end{array}$ & \begin{tabular}{l} 
DELTA T \\
\hdashline 0.9 TO
\end{tabular} & $\frac{\text { F DEC }}{15 \mathrm{TC}}$ & $\begin{array}{l}\text { SAMPL INC } \\
\text { FRDM SL }\end{array}$ & $\begin{array}{l}\text { DISTANCE } \\
\text { URCE, M }\end{array}$ & $\begin{array}{l}\text { TUTAL } \\
\text { TUWERS }\end{array}$ & \\
\hline TEST & STURCE & $1 M$ & $2 M$ & $26 M$ & $56 M$ & $111 \mathrm{M}$ & $1.5 M$ & $61 M$ & $1.5 M^{3}$ & $61 M^{\circ}$ & $3 U M$ & $61 M$ & NEAREST & FARTHEST & $A C T I V E$ & TEST \\
\hline$\cdots$ & $\ldots \ldots$ & $\cdots$ & --- & -- & --- & $\cdots$ & $\cdots$ & $\cdots$ & $\cdots$ & $-\cdots$ & 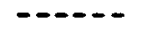 & $-\cdots$ & $\cdots$ & ------ & $\ldots-\cdots$ & --- \\
\hline$v_{1}$ & $S$ & & $2 \mathrm{~F}$ & & & & 1.1 & 3.9 & 22 & 10 & 1.7 & 0.7 & 200 & 3200 & 20 & U1 \\
\hline 42 & $S$ & & $2 \mathrm{~F}$ & & & & 1.6 & 6.6 & 10 & 5 & 6.4 & 2.0 & 200 & 3200 & 20 & 02 \\
\hline D 3 & 5 & & $2 \mathrm{~F}$ & & & & 2.4 & 4.7 & 33 & 17 & 1.9 & 0.6 & 200 & 3200 & 20 & 03 \\
\hline D4 & $s$ & & $16 . F 1$ & & & & 1.2 & 4.0 & 37 & 4 & 1.3 & 0.6 & 200 & 3200 & 20 & D 4 \\
\hline & & $k 1 \dot{5}$ & • & • & - & • & & & & & & & & & & \\
\hline C 1 & $s$ & K 15 & & & & & 1.2 & $5 . \forall$ & 7 & 3 & 6.9 & 6.9 & 200 & 800 & 6 & C1 \\
\hline $\mathrm{C} 2$ & $s$ & K 15 & & & & & 3.9 & $M$ & 6 & $M$ & -1.6 & -1.2 & 200 & 800 & 6 & C 2 \\
\hline $\mathrm{C}_{3}$ & $s$ & $K 14$ & & & & & 7.6 & M & 10 & M & -3.2 & -2.4 & 200 & 800 & 6 & C 3 \\
\hline$C_{4}$ & $S$ & $K 10$ & & & & & 3.8 & $M$ & 13 & M & -4.6 & -2.3 & 200 & 800 & 6 & C 4 \\
\hline$C .5$ & 5 & $K 20$ & 220 & & & & 2.6 & 7.5 & 7 & 3 & 1.0 & $M$ & 200 & 800 & 6.10 & $C 5$ \\
\hline & & - & - & - & - & - & & & & & & & & & & \\
\hline 456 & $u$ & & 2 & $\mathrm{~F}$ & & & 1.6 & 6.0 & 22 & 5 & 2.1 & $2 \cdot 3$ & 200 & 3200 & NUNE & $\cup 56$ \\
\hline U57 & $u$ & & 2 & $\mathrm{~F}$ & & & 1.3 & 3.4 & 9 & 4 & 5.9 & 0.7 & 400 & 3200 & NUNE & US7 \\
\hline 458 & 11 & & 2 & $\mathrm{~F}$ & & & 2.9 & 6.9 & 8 & 6 & 1.7 & 0.6 & 400 & 3200 & NUNE & 458 \\
\hline 459 & u & & $F$ & 7 & & & $1 \cdot \theta$ & 3.9 & 15 & $\theta$ & 1.3 & 0.1 & 400 & 12800 & NUNE & U59 \\
\hline 060 & U & & $F$ & 7 & & & 1.2 & 5.7 & 14 & 6 & 4.1 & 1.5 & 800 & 12800 & NDNE & U $6 J$ \\
\hline$(\div 61$ & U & • & $\dot{F i s}$ & $\dot{z}$ & - & $\cdot$ & 5.0 & 10.1 & 6 & 3 & 0.4 & 0.0 & 800 & 12800 & NIJNE & U61 \\
\hline 162 & $\mathrm{u}$ & & $\mathrm{F} 20$ & 7 & & & 5.2 & 9.2 & 6 & 6 & -0.4 & -0.5 & 800 & 12800 & NUNE & 1462 \\
\hline 663 & $U$ & & F 25 & 2 & & & 2.9 & 7.5 & 6 & 2 & 1.2 & 0.6 & 400 & 12800 & NUNE & U6 3 \\
\hline 164 & $u$ & & $F>0$ & 2 & & & 1.4 & 5.3 & 5 & 2 & 0.7 & 3.5 & 400 & 7000 & NUNE & U64 \\
\hline 165 & $U$ & & $F$ & 7 & & & 2.7 & 6.7 & 15 & 7 & 0.5 & 0.1 & 400 & 12800 & NLNE & U 5 \\
\hline U66 & U & - & $\dot{F}$ & $\dot{i}$ & - & • & 1.3 & 3.4 & 31 & 9 & 2.6 & 1.7 & 400 & 7000 & NUNE & U66 \\
\hline 1167 & u & & $F$ & 7 & & & 2.1 & 5.3 & 11 & 5 & 0.2 & 0.4 & 400 & 7000 & NONE & U67 \\
\hline 1,68 & U) & & $F$ & 2 & & & 2.1 & 6.0 & 13 & 4 & 1.4 & 0.5 & 400 & 12800 & NUNE & U68 \\
\hline 169 & U & & & 7 & & & 2.6 & 5.8 & 9 & 5 & 0.6 & 0.1 & 400 & 12800 & NUNE & 469 \\
\hline 176 & $u$ & & $\mathbf{F}$ & 2 & & & 4.6 & 8.7 & 8 & 5 & 0.1 & 0.0 & 400 & 12800 & NUNE & U70 \\
\hline 171 & U & - & - & $i$ & Fis & - & 1.5 & $4 \cdot 3$ & 15 & 11 & 1.3 & 0.3 & 400 & 12800 & NUNE & U71 \\
\hline 072 & u & & & 2 & F 20 & & 1.9 & 6.2 & 20 & $=5$ & 2.1 & 1.1 & 400 & 7000 & NUNE & U72 \\
\hline 173 & U & & & 2 & $F 20$ & & 2.7 & 6.7 & 0 & 4 & 0.9 & 0.7 & 400 & 12800 & NUNE & U73 \\
\hline U7\% & u & & & 2 & $\mathrm{~F}$ & & 1.7 & 6.5 & 10 & 3 & 2.3 & 1.3 & 400 & 7000 & NLINE & 074 \\
\hline 175 & U & & & 7 & $F$ & & 1.3 & 2.5 & 6 & 9 & 5.4 & 3.9 & 400 & 12800 & NUNE & U75 \\
\hline 1.78 & U & - & - & $i$ & $\dot{F}$ & - & 3.2 & 7.2 & 9 & 5 & 0.8 & 0.8 & 400 & 7000 & NUNE & U76 \\
\hline 1177 & u & & & 7. & $\mathrm{~F}$ & & 1.6 & 3.7 & 9 & 4 & 0.3 & 0.0 & 400 & 12800 & NUNE & 077 \\
\hline U7. & U & & & 2 & $\mathrm{~F}$ & & 3.8 & 7.4 & 6 & 2 & -0.5 & -0.3 & 400 & 7000 & NUNE & 078 \\
\hline 197\%, & U & & & 2 & $\mathrm{~F}$ & & 1.9 & $6 . d$ & 7 & 4 & 2.5 & 2.1 & 400 & 12800 & NUNE & U79 \\
\hline$(1800$ & $u$ & & & $z$ & $F$ & & 0.4 & 2.9 & 16 & 7 & 6.7 & 3.4 & 400 & 3200 & NUNE & Uui \\
\hline 1101 & U & - & - & i & $\dot{F}$ & - & 2.8 & 7.1 & 14 & 11 & 1.3 & 1.3 & 400 & 12800 & NuIVE & U४ 1 \\
\hline 162 & U & & & 7 & $\mathrm{~F}$ & & 1.1 & 4.5 & 22 & 5 & 6.3 & 3.1 & 400 & 12800 & NONE & U 82 \\
\hline $18: 3$ & U & & & 2 & $\mathrm{~F}$ & & 1.5 & 6.7 & 7 & 3 & 6.1 & 5.2 & 400 & 7000 & NLNE & U83 \\
\hline $\mathrm{UH}_{4} \mathrm{H}_{4}$ & v & & & & & 2 & 0.9 & 5.1 & 11 & 5 & $\therefore . c$ & 1.2 & 400 & 17800 & NONE & U84 \\
\hline U: 5 & u & & & & $F$ & 2 & $3 . i^{1}$ & 8.3 & 13 & 6 & -0.4 & 1.3 & 400 & $1>800$ & NUNE & U85 \\
\hline & & - & - & - & $\dot{F}$ & i & & & & & & & & & & \\
\hline Ue $\theta_{2}$ & U & & & & $F$ & 2 & 0.3 & 5.0 & 11 & 5 & 5.4 & 2.3 & 400 & 12800 & NUNE & U86 6 \\
\hline $1: 87$ & $u$ & & & & & 226 & 1.0 & 3.6 & 30 & 17 & 5.4 & 1.7 & 400 & 12800 & 1 & UE 7 \\
\hline UEP & U & & & & $F 16$ & 2 & 1.3 & 7.6 & 11 & 3 & 2.0 & 4.2 & 400 & 12800 & 1 & UB\& \\
\hline 1189 & U & & & & $\mathrm{F}$ & 2 & 0.9 & 3.1 & 26 & 14 & 5.9 & 1.0 & 400 & 12800 & 1 & U8 9 \\
\hline 190 & u & & & & $F 4$ & 2 & 0.9 & 4.3 & 13 & 6 & 6.6 & 0.5 & 400 & 12000 & 1 & U90 \\
\hline ᄂ9! & U & . & . & . & $\dot{F}$ & $\dot{z}$ & 0.3 & 3.8 & 12 & 10 & 2.8 & 1.2 & 400 & $12 \forall C U$ & 1 & U9 I \\
\hline $49 i$ & U & & & & $\mathrm{F}$ & 2 & 0.9 & 4.5 & $\theta$ & 4 & 3.4 & 1.1 & 400 & 12000 & 1 & U92 \\
\hline & & - & - & 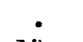 & - & - & & & & & & & & & & \\
\hline V I & U & & & $2 k$ & & & 3.5 & 6.1 & 14 & $\varepsilon$ & -3.4 & -1.6 & 200 & 3200. & 20 & $v_{1}$ \\
\hline V? & U & & & $Z F K$ & & & 2.9 & 4.9 & 12 & 11 & $-1 . c$ & -1.0 & 206 & 3200 & 20 & v 2 \\
\hline$\sqrt{3}$ & u. & & & 28 & & & 2.5 & 4.3 & 10 & 8 & -2.1 & -1.4 & 200 & 3200 & 20 & v 3 \\
\hline 14 & U & & & Q. 9.3 & & & 1.8 & 2.4 & 11 & 5 & -0.2 & -1.2 & 200 & 3200 & 17 & $v 4$ \\
\hline & U & • & • & $2 \dot{R K}$ & - & - & 1.2 & 7.4 & 14 & 3 & 4.9 & 3.3 & 200 & 3200 & 20 & V5 \\
\hline VB & u & & & ZRK & & & 2.9 & 8.6 & 6 & 4 & 1.7 & 2.1 & 200 & 3200 & 20 & Y 6 \\
\hline V7 & $\mathrm{U}$ & & & L K & & . & 3.2 & 4.6 & 20 & 19 & -4.1 & -2.0 & 200 & 3200 & 20 & v7 \\
\hline$v_{t}$ & $u$ & & & 210 & & & 1.8 & 3.4 & 9 & 4 & -0.9 & -0.7 & 200 & 3200 & 20 & V8 \\
\hline
\end{tabular}

'Z,F,R AMD K INDICATE ZINC SUIFIDE, FLUURESCEIN, RHIUAMINE B AND KRYPTUN-ES RESPECTIVELY.

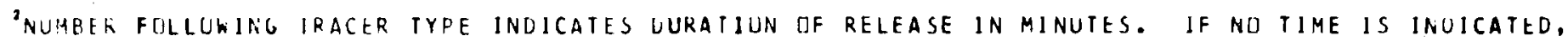
DURATION IS 30 MINUTFS.

JEECKMAN E WHITLEY VANE.

AAERDVANE. 
TABLE 3. FRFUUENCY DF tRACER RELEASE ANO SAMPLING AT 1.5-M ELEVATIUN.

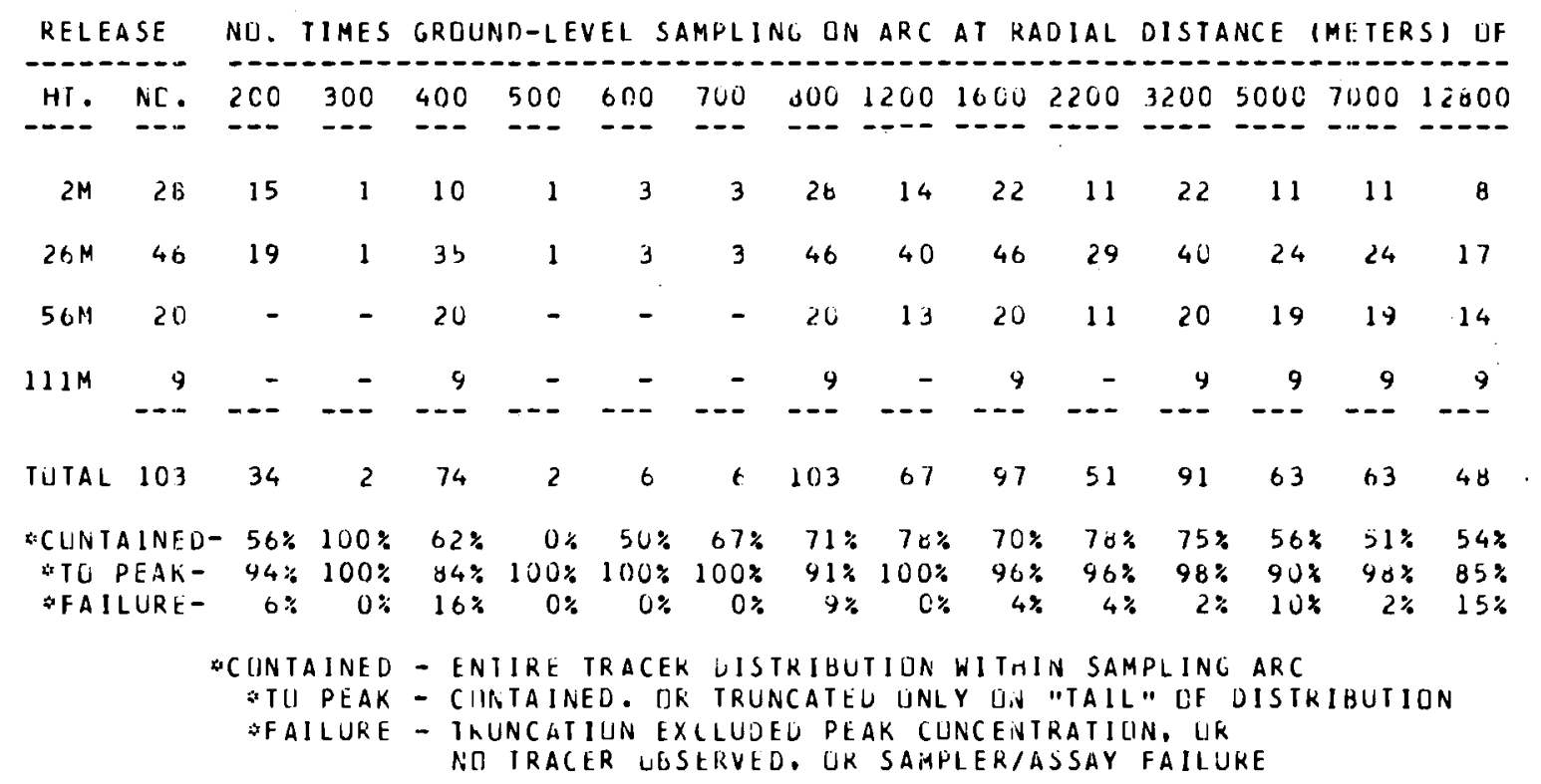

TALIE 4. FRFQUENCY LF TRACER REILASE AINO TURER SAMPLING.

NU. TIMES VERTICAL SAMPLING

TIN ARC AT RAUIAL IISTANCE DFF

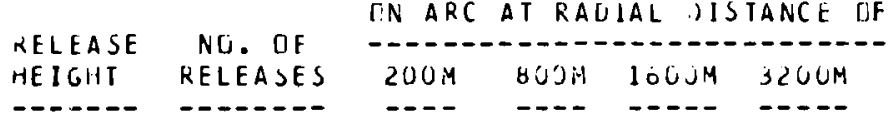

\begin{tabular}{|c|c|c|c|c|c|c|}
\hline $2 M$ & 28 & 14 & 14 & 6 & 9 & BASED \\
\hline $20 M$ & 46 & 18 & 18 & 14 & 14 & $\begin{array}{c}\text { LN } \\
\text { NUMBER }\end{array}$ \\
\hline $56 \mathrm{H}$ & 20 & - & - & - & 5 & $\begin{array}{l}\text { UF } \\
\text { ARCS }\end{array}$ \\
\hline $111 \mathrm{M}$ & $\begin{array}{r}9 \\
--2\end{array}$ & - & - & -- & --- & $\begin{array}{l}\text { ACTIVATED } \\
\text { FDR } \\
\text { SAMPLING }\end{array}$ \\
\hline IUTAL & 103 & 32 & 32 & 22 & -33 & \\
\hline & $\begin{array}{l}\text { ONIAINED- } \\
\text { O TU PEAK- } \\
\text { OFAILURE- }\end{array}$ & $\begin{array}{l}2 \% \\
80 \% \\
20 \%\end{array}$ & $\begin{array}{l}3 \% \\
69 \% \\
31 \%\end{array}$ & $\begin{array}{l}12 \% \\
66 \% \\
34 \%\end{array}$ & $\begin{array}{l}9 \% \\
63 \% \\
37 \%\end{array}$ & $\begin{array}{l}\text { BASED UH WUMUER IIF } \\
\text { ILWERS } \\
\text { ACTIVATED FLR SAMPLING }\end{array}$ \\
\hline
\end{tabular}

- CCNTAINFD - ENTIRE TRACER DISTRIBUTION WITHIN SAMPLING HEIGHT

-TL PEAK - CINTAINED. UR TRUNCATEU JNLY ON "IAIL" OF UISTRIBUTICN

WFAII.URE - TRUNCATIUN EXCLUDE L PEAK CONCEITRATIUN, GR NO TRACER CBSEKVED, UR SAMPLER/ASSAY FAILURE 
TABLE 5A. LOCATION AND DUALITY TF SAMPLING FOR THE 28 SURFACE RELEASES IIF THE 67-SERIES.

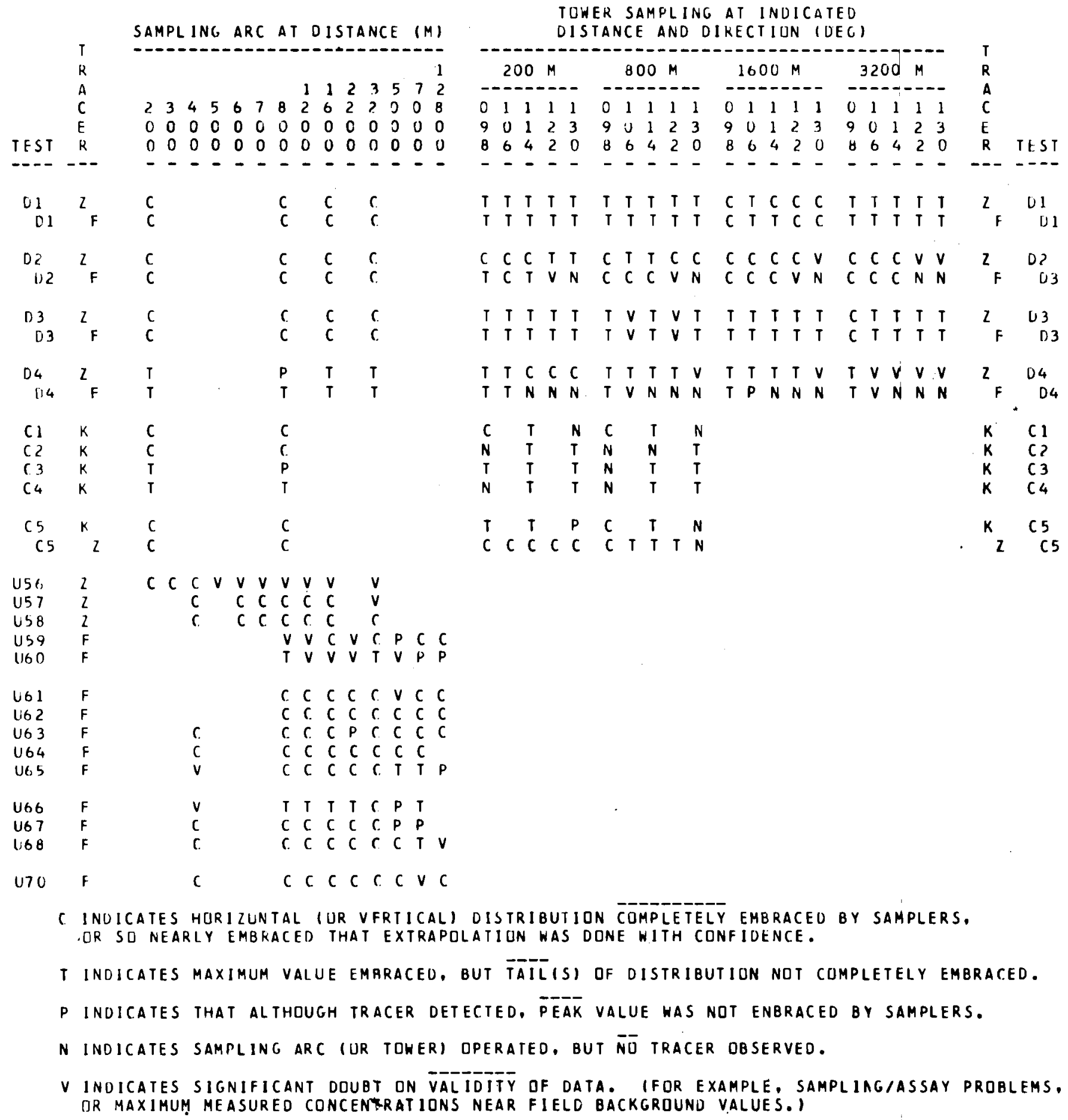


TABLE 5B. LLCATION AND QUAL ITY OF SAMPLING FOR 46 RELEASES FRUM ELEVATILIN UF 26 METERS DURING THE 67-SERIES.

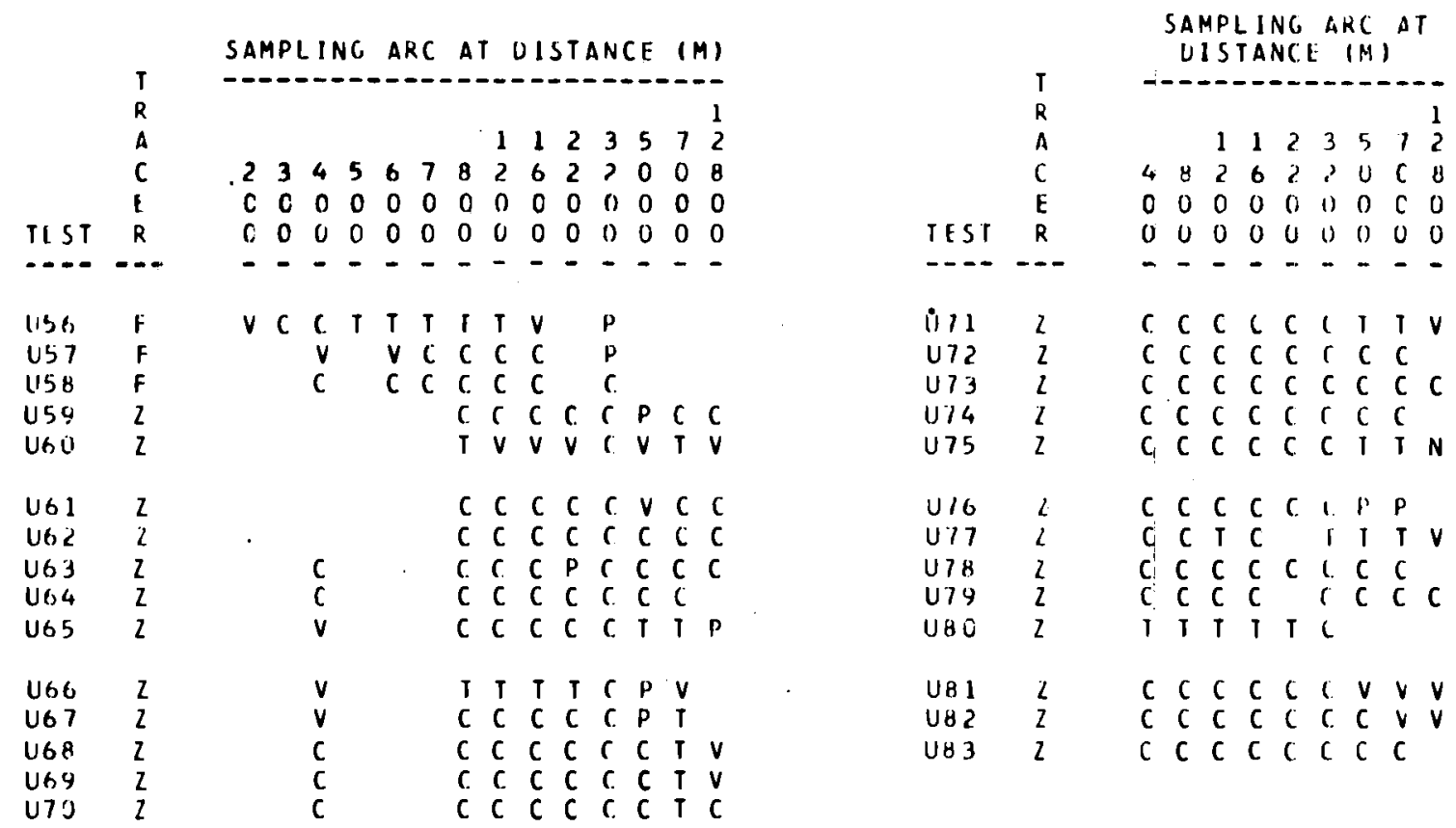

\begin{tabular}{|c|c|c|c|c|c|c|c|c|c|c|c|c|c|c|c|c|c|c|c|c|c|c|c|c|c|c|c|c|}
\hline & $T$ & & & $\begin{array}{l}{ }^{2} \mathrm{~L} \\
\mathrm{ST}\end{array}$ & $\begin{array}{l}\text { VG } \\
\text { VCE }\end{array}$ & $\begin{array}{l}A R C \\
(M)\end{array}$ & $\Delta T$ & . & & 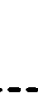 & & & & Af & & & - & & & & 80 & N & & EG & & 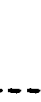 & - & \\
\hline & $\begin{array}{l}T \\
R\end{array}$ & --- & 0 & - & $=$ & $=-$ & & -- & & 20 & 10 & $M$ & & & 82 & 0 & $M$ & & & 161 & 0 & $M$ & & & 320 & 10 & M & \\
\hline & A & & & & 1 & 1 & 2 & 3 & & - & 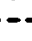 & $\cdots$ & & & - & & $\cdots$ & & & & $=-$ & $\ldots$ & & & & 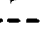 & & \\
\hline & C & 2 & 4 & 8 & 2 & 6 & 2 & 2 & 1 & 1 & 1 & 1 & 1 & 1 & 1 & 1 & 1 & 1 & 1 & 1 & 1 & 1 & 1 & & 1 & 1 & 1 & \\
\hline & E & 0 & 0 & 0 & 0 & 0 & 0 & 0 & 0 & 1 & 1 & 2 & 3 & 0 & 1 & 2 & 2 & 3 & 0 & 1 & 1 & 2 & 3 & 0 & 0 & 1 & 2 & 3 \\
\hline TEST & $k$ & 0 & 0 & 0 & 0 & 0 & 0 & 0 & 2 & 0 & 8 & 6 & 4 & 5 & 3 & 0 & 8 & 5 & 2 & 0 & 7 & 5 & 2 & 0 & $y$ & 6 & 3 & 1 \\
\hline--- & $-\infty$ & - & - & - & - & - & - & - & - & - & - & - & - & - & - & - & - & - & - & - & - & - & - & - & - & - & - & - \\
\hline$V_{1}$ & $z$ & C & C & $C$ & C & C & C & $C$ & $T$ & $\mathbf{T}$ & $\mathbf{T}$ & $T$ & $T$ & $T$ & $\mathbf{T}$ & $T$ & $\mathbf{T}$ & $C$ & $T$ & $\begin{array}{r}T \\
T\end{array}$ & $T$ & $T$ & $\mathbf{N}$ & $T$ & $T$ & $T$ & $\mathrm{~T}$ & $\mathbf{N}$ \\
\hline VI & $R$ & C & C & C & C & $v$ & $v$ & $v$ & $T$ & $T$ & $T$ & $T$ & $v$ & $T$ & $T$ & $T$ & $v$ & $N$ & $\mathbf{N}$ & v & $v$ & v & $\mathbf{N}$ & $N$ & $v$ & $v$ & v & $N$ \\
\hline$\sqrt{2}$ & $z$ & c & C & C & C & C & C & C & $N$ & $T$ & $T$ & $T$ & $\mathbf{T}$ & $\mathbf{N}$ & $P$ & $T$ & $T$ & $T$. & $N$ & $N$ & $T$ & $T$ & $T$ & $N$ & $N$ & $N$ & $T$ & $T$ \\
\hline v2 & $R$ & C & C & C & C & C & C & C & $\mathbf{N}$ & $T$ & $T$ & $T$ & T & $\mathbf{N}$ & v & $T$ & $T$ & $T$ & $N$ & $\mathbf{N}$ & $v$ & $T$ & $T$ & $N$ & $N$ & N & P & $T$ \\
\hline V 2 & K & $T$ & & $\mathbf{P}$ & & $P$ & & & $N$ & $T$ & P & $\mathrm{P}$ & $T$ & $\mathbf{N}$ & $\mathbf{N}$ & $T$ & $T$ & $\mathbf{T}$ & & & & & & & & & & \\
\hline$v_{3}$ & $z$ & $T$ & $T$ & $T$ & $T$ & $T$ & $T$ & C & $\mathbf{N}$ & $\mathrm{N}$ & $T$ & $T$ & $T$ & $\mathbf{N}$ & $\mathbf{N}$ & $\mathbf{N}$ & P & 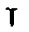 & $N$ & $\mathbf{N}$ & $\mathbf{N}$ & v & $T$ & $N$ & $\mathbf{N}$ & $\mathbf{N}$ & N & $T$ \\
\hline V3 & $R$ & $T$ & $T$ & $T$ & $T$ & $T$ & $T$ & C & $N$ & $\mathbf{N}$ & $T$ & $T$ & $T$ & N & $\mathbf{N}$ & $N$ & $v$ & $T$ & $\mathbf{N}$ & $N$ & N & $v$ & $v$ & $\mathbf{N}$ & $\mathbf{N}$ & N & $\mathbf{N}$ & $v$ \\
\hline$\sqrt{ } 4$ & $z$ & C & & C & & C & & & $\mathbf{N}$ & N & $T$ & T & T & $\mathbf{N}$ & $\mathbf{N}$ & $T$ & $T$ & T & $\mathbf{N}$ & $\mathbf{n}$ & $T$ & $T$ & $T$ & & & & & $v$ \\
\hline V4 & $\mathbf{R}$ & v & & C & & C & & & N & $\mathbf{N}$ & $T$ & $\mathrm{~T}$ & $T$ & $\mathbf{N}$ & $N$ & v & $\mathrm{T}$ & $\mathrm{T}$ & $\mathbf{N}$ & $\mathbf{N}$ & v & $T$ & $T$ & & & & v & v \\
\hline v5 & $z$ & C & C & C & C & C & & $C$ & $\boldsymbol{T}$ & $T$ & $T$ & $T$ & $\mathbf{N}$ & $T$ & $T$ & $v$ & iv & $N$ & C & $T$ & $T$ & $\mathbf{N}$ & $\mathbf{N}$ & $c$ & $C$ & T & $T$ & $\mathbf{N}$ \\
\hline V5 & $R$ & C & C & C & C & $C$ & & C & T & $T$ & $\mathbf{T}$ & $\mathbf{N}$ & $\mathbf{N}$ & $\mathbf{T}$ & $T$ & V & $N$ & $\mathbf{N}$ & C & C & v & $N$ & $\mathbf{N}$ & $v$ & $C$ & $T$ & N & $N$ \\
\hline V5 & K & $T$ & & $T$ & & $p$ & & & $T$ & $P$ & $P$ & $\mathbf{N}$ & $\mathbf{N}$ & $T$ & $T$ & $T$ & $N$ & $N$ & & & & & & & & & & \\
\hline$v_{6}$ & 2 & $C$ & C & $C$ & C & C & & C & N & $\mathbf{T}$ & $T$ & $T$ & n & $\mathbf{N}$ & $T$ & $T$ & $T$ & $\mathrm{~N}$ & $\mathbf{N}$ & C & C & $T$ & $\mathbf{N}$ & $\mathbf{N}$ & $N$ & C & $T$ & $N$ \\
\hline V6 & $R$ & C & & C & C & C & & C & N & $T$ & $T$ & $T$ & $\mathbf{N}$ & $N$ & $T$ & $T$ & C & $\mathbf{N}$ & $N$ & $v$ & C & C & $\mathbf{N}$ & $\mathbf{N}$ & $N$ & C & C & $N$ \\
\hline V6 & K & c & & C & & C & & & $\mathbf{N}$ & $T$ & $T$ & $\mathbf{P}$ & $\mathbf{N}$ & $N$ & $T$ & $T$ & $T$ & N & & & & & & & & & & \\
\hline V7 & $z$ & C & C & C & C & C & & C & $\mathbf{T}$ & $T$ & $\mathbf{T}$ & $T$ & $\mathbf{T}$ & $T$ & $T$ & $T$ & $T$ & $T$ & $\mathbf{N}$ & $T$ & $T$ & $T$ & $T$ & $N$ & $N$ & $T$ & $T$ & $T$ \\
\hline V7 & $K$ & $T$ & & & & & & & $T$ & $T$ & $T$ & $T$ & $T$ & & & & & & & & & & & & & & & \\
\hline V8 & 2 & C & C & C & C & C & & C & $\mathbf{N}$ & $v$ & $v$ & $T$ & $T$ & $N$ & $\mathbf{N}$ & $v$ & $T$ & $T$ & $\mathbf{N}$ & $\mathbf{N}$ & $N$ & C & $T$ & $\mathbf{N}$ & $\mathbf{N}$ & $\mathbf{N}$ & $\mathbf{N}$ & $T$ \\
\hline
\end{tabular}


TABLE 5C. LOCATIUN AND QUALITY OF SAMPLING FOR 20 RELEASES FROM ELEVATION OF 56 METERS DURING THE G7-SERIES.

\begin{tabular}{|c|c|c|c|c|c|c|c|c|c|}
\hline & & & & L I & & & (M) & AT & \\
\hline & $\begin{array}{l}T \\
R\end{array}$ & $\cdots$ & -- & -- & -- & . & - & -- &. \\
\hline & A & & & 11 & 2 & 3 & 5 & 7 & \\
\hline & C & 48 & 82 & 26 & 2 & 2 & 0 & 0 & 0 \\
\hline & $E$ & $\cap 0$ & 00 & 0 & 0 & 0 & 0 & 0 & 0 \\
\hline TEST & $R$ & 0 & 00 & 00 & 0 & 0 & 0 & 0 & 0 \\
\hline-- & --- & - & - - & -- & - & - & - & - & - \\
\hline 71 & $F$ & C & $C \mathrm{C}$ & $C \mathrm{C}$ & $C$ & $c$ & $\mathbf{T}$ & $T$ & \\
\hline U72 & $F$ & c & C $\mathrm{C}$ & C. C & C & $c$ & $r$ & $c$ & \\
\hline U73 & $F$ & & $C \mathrm{C}$ & $C \quad C$ & C & c & C. & $c$ & 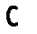 \\
\hline U74 & $F$ & N & $C \&$ & C. C & C & $c$ & C & $C$ & \\
\hline U 75 & $F$ & C & $C<$ & C $\mathrm{C}$ & $C$ & C & $T$ & $T$ & \\
\hline 07 & $\mathbf{F}$ & C & $C r$ & c. $c$ & $c$ & C & $\mathbf{P}$ & $C$ & \\
\hline U 77 & $F$ & C & $C T$ & $T T$ & & $T$ & $T$ & $T$ & \\
\hline U7H & $\mathrm{F}$ & C & $c c$ & C C & C & C & C & $C$ & \\
\hline U79 & $F$ & C & $c 8$ & C. & & & & $c$ & \\
\hline & $F$ & C & & & & & & & \\
\hline
\end{tabular}

\begin{tabular}{|c|c|c|c|c|c|c|c|c|c|c|}
\hline & & & & $\begin{array}{l}\text { LIN } \\
\text { TAS }\end{array}$ & & & $\begin{array}{l}C \\
M I\end{array}$ & AT & & \\
\hline & $T$ & -- & $\cdots$ & $-\cdots$ & -- & & - & -- & & TLWER \\
\hline & $\begin{array}{l}R \\
A\end{array}$ & & 1 & 1 & 2 & 3 & 5 & 7 & $\begin{array}{l}1 \\
2\end{array}$ & $\begin{array}{c}\Delta T \\
115.6\end{array}$ \\
\hline & C & 48 & 2 & 6 & 2 & 2 & 0 & 0 & 8 & DEG ON \\
\hline & $\mathbf{E}$ & 00 & 0 & 0 & 0 & 0 & 0 & & 0 & $3200 \mathrm{M}$ \\
\hline TEST & $R$ & 00 & 0 & 0 & 0 & 0 & 0 & 0 & 0 & $A R C$ \\
\hline$-\cdots$ & -- & $=$ & - - & - & - & - & - & - & - & $-\cdots-$ \\
\hline Uष1 & $F$ & C & : C & $C$ & C & C & C & C & C & \\
\hline Uy 2 & $F$ & $N C$ & C & C & $C$ & C & C & C & C & \\
\hline$U \otimes 3$ & $F$ & $C$ & C & C & $C$ & C & C & C & & \\
\hline U 45 & $F$ & C & & C & & C & C & C & C & \\
\hline U⿺6 & $F$ & C & & $T$ & & C & C & C & C & \\
\hline U8 8 & $F$ & v & & C & & C & C & C & C & C \\
\hline U89 & $F$ & $v 0$ & & C & & $T$ & $T$ & C & $N$ & $\mathrm{~N}$ \\
\hline U90 & $F$ & v & & $v$ & & $v$ & $v$ & $v$ & $v$ & v \\
\hline U91 & $F$ & C & & C & & $\mathbf{T}$ & $T$ & $T$ & T & $T$ \\
\hline U92 & $F$ & v & v & C & & C & C & C & $T$ & T \\
\hline
\end{tabular}

TABLE 50. LOCATIUN AND QUALITY MF SAMPLING FOR 9 RELEASES FROM ELEVATION DF 111 METERS DURING 67-SERIES.

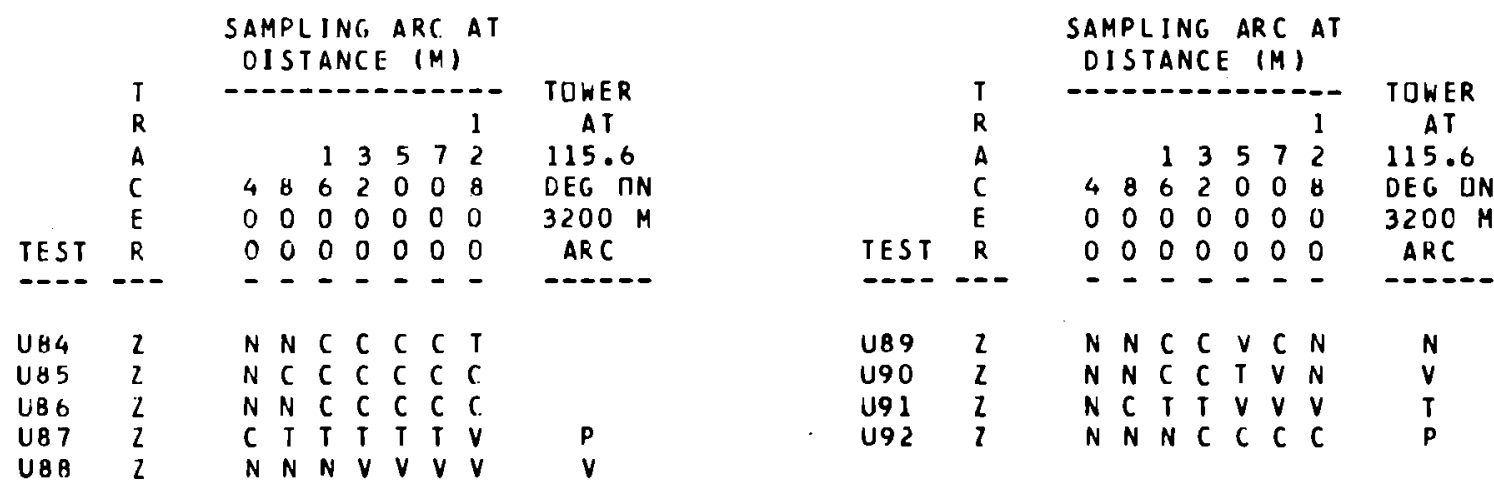

C INDICATES HORIZUNTAL (OR VFRTICAL) DISTRIBUTIDN COMPLETEL̄Y EMBRACED BY SAMPLERS, UR SO NEARLY SD THAT EXTRAPOLATIDN WAS DDNE WITH CONFIDENCE.

T INOICATESMAXIMUM VALUE EMBRACED, BUT TÄĹS CF DISTRIBUTION NOT CUMPLETELY EMBRACED.

P indicates that althuUgh tracer detected. PEÁ Value has not embraced BY SAMPLERS.

N INDICATES SAMPLING ARC (OR TOHERI OPERATED. BUT NO TRACER CBSERVED.

$\checkmark$ INDICATES SIGNIFICANT DOUBT ON VALIOITTY OF DATA. 
APPENDIX A

DIFFUSION DATA 
MEANING OF REMARK SYMBOLS WHICH MAY

PRECEDE AZIMUTH COLUMN IN APPENDIX A TABLES

Symbol

E

$\mathrm{H}$

W

C

$\mathbf{P}$

$\mathrm{x}$

Q

I

Significance

ESTIMATED values.

DOA (dead on arrival), HOT. At time of filter pickup, vacuum supply to filter was found to have failed due to dead engine. Engine hot, however, indicating vacuum missing only a short time and hence likely a valid sample.

DOA, WARM. Same as $\mathrm{H}$, except engine only warm to touch. Possibly valid sample, but more doubt than with $\mathrm{H}$.

DOA, COLD. Same as $H$, except engine cold to touch, and no fuel in tank. Sampling occurred as long as fuel lasted, but ended considerably before filter pickup.

DOA, cold, PARTIAL tank of gasoline. Same as C except sampling was likely of shorter duration since engine failed before burning all the fuel.

DOA, cold, full tank. Engine failed shortly after starting. Sample is essentially due to impaction on filter rather than to flow through filter.

$D O A$, other. Engine $D O A$, but no other field note given.

IMPACTION sample. Engine for vacuum supply was never started. Sample is due to impaction on filter rather than flow through filter. 
L

M

D

S

$\mathbf{T}$

$\mathbf{F}$

R

G

$\mathrm{V}$

B

$\mathrm{N}$

Filter LOST or, in the case of krypton, GeigerMüller tube not operating.

Filter was MISSING from holder assembly. The supporting crepe paper roll was often assayed in the case of the zns tracer, but it is a much less efficient sampler than the membrane filter.

Filter assembly DROPPED. Effect unknown.

Filter SLIGHTLY cracked. Effect likely minimal. Filter TORN badly. Effect likely significant. Apparent FINGERPRINT on filter. Time of placement and effect, if any, unknown.

For Rankin counting of ZnS, a RECOUNT of a filter with an unexpected mass assessment, but with essentially unchanged mass indicated on the repeat assessment.

GEIGER-MULLER tube noisey or questionable.

Low VOLTAGE on Geiger-Müller tube. Likely low exposures indicated.

Tracer likely extends BEYOND this last active sampler. (The symbol was not always appended to top vertical samplers even though tracer obviously extended higher.)

Samplers operated, but No tracer observed on the arc (tower). Possibly no tracer passed samplers or possibly concentration below detection limit. 
Symbol

Z

Any other field or assessment remark. (A check of field notes or laboratory assay notes would be needed to elaborate on each $\mathrm{Z}$ remark.)

$\left.\begin{array}{l}1 \\ 2 \\ 3 \\ 4\end{array}\right\}$

\section{Visible dust on filter, but of minimal concern.*} (1)

\author{
Increasing
}

significance

of

visible

dust on

filters. *

\footnotetext{
*The numerical remark symbols are a qualitative ranking of visible dust on field filters. Larger numbers indicate darker filters -- except " 0 " indicates the most severe loading. Zns assay is degraded most severely by dust, al though a nomenclature of less than 5 can be considered of minimal importance. See text under "zinc Sulfide Fluorescent: Particulate 2210" and "The Appendix A Diffusion Data" for further detail.
} 
GHOUND-LEVEL AND TOHER SAHPLING 200K TO 3200M. INYERSION OF 3F DEG IIM TO $15 \%$ IT O445: ISOTHERMAL AT O5IS. ALL ARCS ERBRACE CROSSWIHD EXTENT OF PRACER DISTRIBUTION--EXCEPT MINOR EXTRAPULATION AT MURTH END OF $200 M$ ARC. VERTICAL DISTRIBUTIONS OF TRACER OH ALL 20 TOHERSI PEAK VALUES MEASURED. BUT TRUNCATED AT TOP IH ALL CASES.

\begin{abstract}
TEST DI DUE JULY 7,1967 O445 TU OSIS RST ZINC SULEIDE RELEASE FROM ELEYATIUN UF $2 M$
2OOH ARC SAMPLER HT $1.5 \mathrm{SM}$ U. 1.2 M/SEC AT
\end{abstract}

\begin{tabular}{|c|c|c|c|c|}
\hline $\begin{array}{l}\text { ALIMUTH } \\
\text { DEGREES }\end{array}$ & $\begin{array}{l}\text { EXPUSURE } \\
\text { GH-SEC/CU.H } \\
\times 10 E+6\end{array}$ & $\begin{array}{c}\text { E/O } \\
\text { SEC/CU!H }\end{array}$ & $\begin{array}{l}\text { EU/O } \\
\text { I/SU.H }\end{array}$ & $\begin{array}{l}\text { DISTANCE } \\
\text { METERS }\end{array}$ \\
\hline
\end{tabular}

\begin{tabular}{|c|c|c|}
\hline \multirow{2}{*}{$\begin{array}{l}58.0 \\
60.0\end{array}$} & \multirow{4}{*}{$\begin{array}{r}23 \\
57 \\
171 \\
481\end{array}$} & $2.074 E-08$ \\
\hline & & $5.036 E-08$ \\
\hline 62.0 & & $1.511 E-07$ \\
\hline 64.0 & & $4.236 E-07$ \\
\hline $\begin{array}{l}66.0 \\
68.0\end{array}$ & $\begin{array}{l}1346 \\
5919\end{array}$ & $\begin{array}{l}1.185 E-06 \\
5.211 E-06\end{array}$ \\
\hline 70.0 & 8604 & $7.575 E-06$ \\
\hline 72.0 & 17469 & $1.538 \mathrm{E}-05$ \\
\hline 74.0 & 25559 & $2.250 E-05$ \\
\hline 76.0 & 45842 & $4.035 E-05$ \\
\hline 78.0 & 64761 & $5.701 E-05$ \\
\hline $\begin{array}{l}80.0 \\
82.0\end{array}$ & $\begin{array}{r}97082 \\
111834\end{array}$ & $\begin{array}{r}7.666 t-05 \\
9.845 E-05\end{array}$ \\
\hline 84.0 & 110312 & $1.041 E-04$ \\
\hline 86.0 & 129653 & $1.141 E-04$ \\
\hline 88.0 & .175942 & $1.544 E-04$ \\
\hline 90.0 & 163676 & $1.441 E-04$ \\
\hline $92: 0$ & 199980 & $1.760 E-04$ \\
\hline 94.0 & 198570 & $1.748 E-04$ \\
\hline 96.0 & 232409 & $2.050 E-04$ \\
\hline $\begin{array}{r}98.0 \\
100.0\end{array}$ & $\begin{array}{l}315008 \\
278189\end{array}$ & $\begin{array}{l}2.773 E-04 \\
2.449 E-04\end{array}$ \\
\hline 102.0 & 310222 & $2.731 E-04$ \\
\hline 104.0 & 216278 & $1.904 E-04$ \\
\hline $10 t .0$ & $2 \cos 83$ & $1.819 E-04$ \\
\hline 108.0 & 205923 & $1.813 E-04$ \\
\hline 110.0 & 192307 & $1.693 \mathrm{E}-04$ \\
\hline 112.0 & 123306 & $1.085 E-04$ \\
\hline 114.0 & 00900 & $7.122 E-05$ \\
\hline 116.0 & 61197 & $5.387 E-05$ \\
\hline 118.0 & 913 & $7.164 E-05$ \\
\hline 120.0 & & $5.772 \mathrm{E}-05$ \\
\hline 122.0 & 70825 & $6.235 E-05$ \\
\hline 124.0 & 60995 & $5.369 E-05$ \\
\hline 126.0 & 53679 & $4.725 E-05$ \\
\hline 128.0 & 18451. & $1.624 E-05$ \\
\hline 130.0 & 4122 & $3.629 E-06$ \\
\hline 132.0 & 2126 & $1.872 \mathrm{E}-06$ \\
\hline 134.0 & 3294 & $2.900 \mathrm{E}-06$ \\
\hline 136.0 & 1453 & $1.280 E-O E$ \\
\hline & 4586 & $4.03 \mathrm{dE}-06$ \\
\hline 140.0 & 2887 & $2.542 E-06$ \\
\hline 142.0 & 1436 & $1.265 \mathrm{E}-06$ \\
\hline 144.0 & 296 & $2.607 E-07$ \\
\hline 146. & 124 & $1.046 E-07$ \\
\hline 148.0 & 3 & $2.962 E-09$ \\
\hline 150.0 & 60 & $5.332 E-00$ \\
\hline 152. & 43 & $3.051 \mathrm{E}-08$ \\
\hline 154.0 & 10 & $0.087 E-09$ \\
\hline 156. & 0 & \\
\hline
\end{tabular}

CROSSIIMD INTEGRATED: $2.427 E-02$ 2.913E-02 SEC/50.M

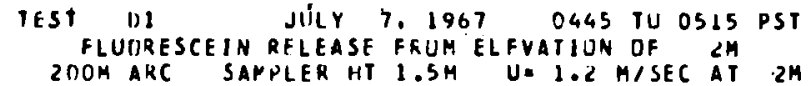

AZIMUTH EXPUSURL EIO EU/O DISTANCE $\times 10 E+6$

\begin{tabular}{|c|c|c|c|c|}
\hline $\begin{array}{l}54.0 \\
60.0\end{array}$ & $\begin{array}{l}33 \\
76\end{array}$ & $\begin{array}{l}? .433 \mathrm{E}-08 \\
6.714 \mathrm{E}-108\end{array}$ & $\begin{array}{l}3.320 E-08 \\
8.057 E-08\end{array}$ & $\begin{array}{l}20.0 \\
200\end{array}$ \\
\hline $62 . c$ & 235 & $2.073 E-07$ & $2.488 E-07$ & \\
\hline 64.0 & 635 & $5.593 E-07$ & $6.712 E-07$ & 200 \\
\hline 66.0 & 1644 & $1.497 k-06$ & $1.790 E-06$ & 200 \\
\hline 63.0 & 5989 & $5.272 \mathrm{E}-0 \mathrm{~S}$ & $6.327 E-06$ & 200 \\
\hline 70.0 & 11458 & $1.053 t-65$ & $1.263 E-05$ & 200 \\
\hline $7 \ldots 0$ & 19063 & $1.67 \mathrm{BE}-05$ & $2.014 \mathrm{E}-\mathrm{Ob}$ & 200 \\
\hline$\pi 4.0$ & 28413 & $2.501 E-05$ & $3.001 \mathrm{E}-05$ & 200 \\
\hline 76.0 & 57208 & $3.036 E-05$ & $6.043 \mathrm{E}-0 \mathrm{~S}$ & 200 \\
\hline $7 \mathrm{H} .0$ & 90769 & $7.110 E-\cup 5$ & $4.532 E-05$ & 200 \\
\hline 80.0 & 96476 & $8.493 E-J 5$ & $1.019 \mathrm{E}-64$ & 200 \\
\hline 92.0 & 166747 & $1.415 t-04$ & $1.698 E-04$ & 200 \\
\hline A4.0 0 & 145118 & $1.277 \mathrm{E}-04$ & $1.533 E-04$ & 200 \\
\hline$B 6.0$ & 140653 & $1.23 B E-04$ & $1.4 \forall 6 E-04$ & 200 \\
\hline$\forall 8.0$ & 198704 & $1.749 E-04$ & $2.099 E-04$ & 200 \\
\hline 90.0 & 223264 & $1.965 E-64$ & $2.35 \forall E-04$ & 200 \\
\hline 92.0 & 245591 & $2.162 t-04$ & $2.574 E-0$. & 200 \\
\hline 94.0 & $291 ; 245$ & $2.555 E-04$ & $3.0 B G E-04$ & 200 \\
\hline 96.0 & 401482 & $3.53 \mathrm{Bt}-114$ & $4.245 E-04$ & 200 \\
\hline $9+.0$ & 502355 & $4.422 \mathrm{E}-04$ & $5.307 E-04$ & 200 \\
\hline 100.0 & 346064 & $3.046 E-O t_{t}$ & $3.656 E-04$ & 200 \\
\hline 102.0 & 390718 & $3.437 t=04$ & $4.127 E-04$ & 200 \\
\hline 104.0 & 323736 & $2.850 E-04$ & $3.42 U E-04$ & 200 \\
\hline 3.6 .0 & 251173 & $2.211 E-04$ & $2.653 E-04$ & 200 \\
\hline 138.0 & 291362 & $2.565 E-04$ & $3.67 \mathrm{BE}-04$ & 200 \\
\hline 110.0 & 267918 & $2.35 A E-04$ & $E-04$ & 200 \\
\hline .0 & 197 & $1.739 \mathrm{E}$ & 2.0 & 200 \\
\hline 114.0 & 111627 & $9.8<6 E-05$ & 1.174 & 200 \\
\hline 116.0 & 87067 & $4 E-05$ & 9.19 & 200 \\
\hline & 116 & 1.0 & 1.2 & 200 \\
\hline $1<0.0$ & 91533 & B. $057 t-05$ & $9.66 y \mathrm{E}-0\}$ & 200 \\
\hline 12.2 .0 & 82602 & $7.271 E-05$ & 0.7 & 200 \\
\hline 124.0 & $919 \mathrm{HA}$ & $\therefore .04 B t-05$ & $9.717 \mathrm{E}-05$ & 200 \\
\hline $1,6.0$ & 93110 & $6.193 t-05$ & $9.836 \mathrm{E}-05$ & 200 \\
\hline 128. & 336 & 2.96 & 3.5 & 200 \\
\hline 130.0 & 5378 & $5.263 E-06$ & $6.315 E-06$ & 20 \\
\hline 132.0 & 6423 & $3.894 \mathrm{t}-06$ & $4.672 \mathrm{E}-\mathrm{VE}$ & 200 \\
\hline 134.0 & 2790 & $2.456 \mathrm{E}$ & 2.9 & 200 \\
\hline 146.0 & 835 & $7.35, E-07$ & $0.824 E-07$ & 200 \\
\hline $1, H .0$ & 779 & $0.064 E-07$ & $\forall .237 E-07$ & 200 \\
\hline 140.0 & 346 & $3.051 \mathrm{E}$ & & 200 \\
\hline 142.0 & 6o & 0.0 & A & 200 \\
\hline 144.0 & 161 & 1.4 & & 200 \\
\hline 146.0 & 38 & 3.4 & 4.10 & 200 \\
\hline 144.0 & 33 & $2.933 E$ & $3.520 \mathrm{E}$ & 200 \\
\hline $15 \mathrm{i} .0$ & 16 & $1.467 \mathrm{E}-0 \mathrm{~B}$ & $1.760 E-O \theta$ & 200 \\
\hline 152.0 & 12 & & & 200 \\
\hline $1>4.0$ & 7 & $6.519 E-09$ & $7.823 E-04$ & 200 \\
\hline & 8 & $7.171 \mathrm{E}-09$ & $8.605 E-09$ & 200 \\
\hline & U & & & \\
\hline
\end{tabular}

CRTISSNIND INIEGRATED= 3.320E-02 3.984E-02 $1 / 4$

TEST C1
FLUTRESCEIN. RELEASE FRUM ELFVATIUN OF

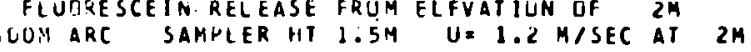

ALIMUTH EXPOSURE E/O FU/O OLSTANCE DEGFFES GM-SEC/CU.M SEG/CU.H I/SO.M METERS $\times 10 E+6$

$\begin{array}{lrrl}74.0 & 0 & 0 . & 0 . \\ 76.0 & 26 & 2.292 E-08 & 2.751 E-08 \\ 74.0 & 58 & 5.158 E-08 & 6.140 E-08 \\ 80.0 & 48 & 4.298 E-08 & 5.158 E-00 \\ 82.0 & 107 & 9.456 E-08 & 1.135 E-07 \\ 84.0 & 367 & 3.238 E-07 & 3.866 E-07 \\ 86.0 & 651 & 5.731 E-07 & 6.877 E-07 \\ 88.0 & 1490 & 1.312 E-06 & 1.575 E-06 \\ 90.0 & 4023 & 3.542 E-06 & 4.250 E-06\end{array}$

800
800
800
800
800
800
800
800
800
800

76.0
78.0
20.0
82.0
84.0
86.0
88.0
90.0
92.0

109
35
137
270
721
1745
2677
3859

0 .

9.601E-U8 $3.169 \mathrm{E}-0 \mathrm{O}$ $1.212 E-U 7$ $2.379 E-07$ $6.352 \mathrm{E}-07$

$1.537 E-06$

$2.357 E-06$ $3.397 E-06$
0.

$1.152 \mathrm{E}-07$ $3.802 \mathrm{E}-0 \mathrm{~B}$ $1.455 E-07$ $2.055 E-07$ $7.622 \mathrm{E}-07$

$1.844 E-06$

$2.820 \mathrm{E}-06$ 4.077 E-06
800
800
800
100
800
800
800
800
800 


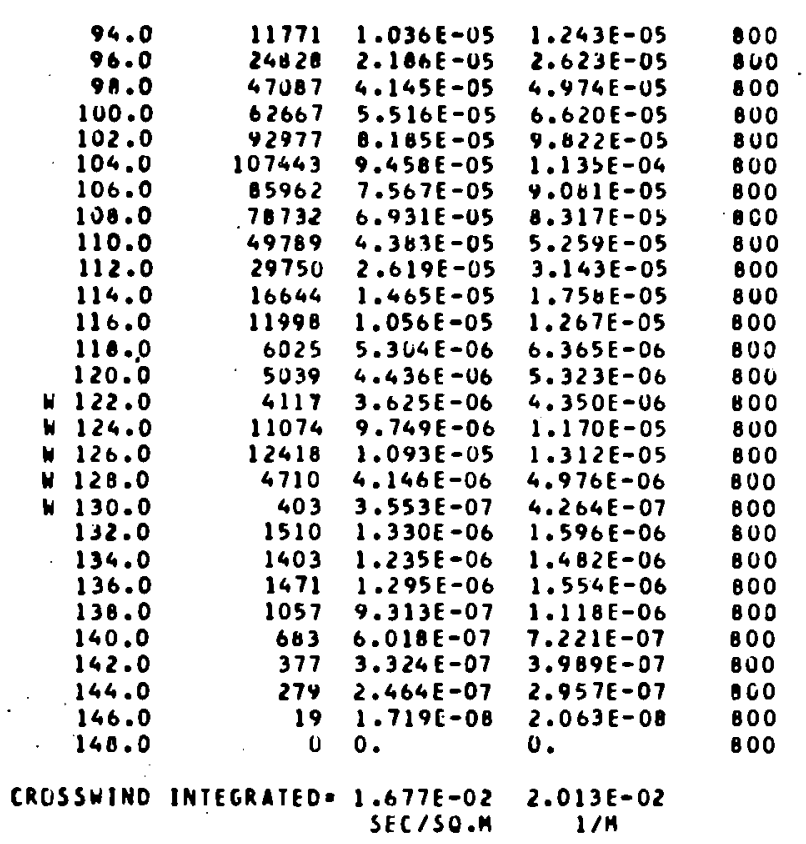

TEST DI JULY 7, 1967 0445 TO 0515 PST ZINC SULF1OE RELEASE FROM ELEVATION OF $2 \mathrm{H}$
$1600 \mathrm{M}$ ARC SAMPLER HT $1.5 \mathrm{GM}$ U: $1.2 \mathrm{H} / \mathrm{SEC}$ AT $2 \mathrm{M}$

\section{AzIAUTH EXPOSURE E/O EU/O DISTANCE DEGKEES GH-SEC/CU.M SEC/CU.M I/SO.M. METERS $\times 10 E+6$}

\begin{tabular}{|c|c|c|c|c|c|}
\hline & $\begin{array}{l}82.0 \\
83.0 \\
84.0 \\
85.0\end{array}$ & $\begin{array}{r}0 \\
98 \\
25 \\
83\end{array}$ & $\begin{array}{l}0 . \\
8.693 \mathrm{E}-08 \\
2.26 \mathrm{E}-08 \\
7.370 \mathrm{E}-\mathrm{UB}\end{array}$ & $\begin{array}{l}0 . \\
1.043 E-07 \\
2.721 E-08 \\
8.844 E-08\end{array}$ & $\begin{array}{l}1600 \\
1600 \\
1600 \\
1600\end{array}$ \\
\hline & 86.0 & 83 & $7.370 E-08$ & $6.044 E-08$ & 1600 \\
\hline & 87.0 & 139 & $1.228 E-07$ & $1.474 E-07$ & 1600 \\
\hline & B 8.0 & 124 & $1.096 \mathrm{E}-07$ & $1.315 E-07$ & 1600 \\
\hline & 89.0 & 176 & $1.550 E-07$ & $1.660 E-07$ & 1600 \\
\hline & 90.0 & 85 & $7.559 E-08$ & $9.071 \mathrm{E}-0 \mathrm{~B}$ & 1600 \\
\hline & 91.0 & 341 & $3.005 E-07$ & $3.606 E-07$ & 1600 \\
\hline & 92.0 & 435 & $3.836 E-07$ & $4.604 E-07$ & 1600 \\
\hline & 93.0 & 633 & $5.575 E-07$ & $6.69 \mathrm{JE}-07$ & 1600 \\
\hline & 94.0 & 880 & $7.748 E-07$ & $9.240 E-07$ & 1600 \\
\hline & 95.0 & 1180 & $1.039 \mathrm{E}-\mathrm{U} 6$ & $1.247 \mathrm{E}-06$ & 1600 \\
\hline & 96.0 & 1524 & $1.342 E-06$ & $1.610 E-06$ & 1600 \\
\hline & $\begin{array}{l}97.0 \\
98.0\end{array}$ & $\begin{array}{l}2146 \\
3518\end{array}$ & $\begin{array}{l}1.890 E-06 \\
3.097 E-06\end{array}$ & $\begin{array}{l}2.260 E-06 \\
3.717 E-06\end{array}$ & $\begin{array}{l}1600 \\
1600\end{array}$ \\
\hline & 99.0 & 18404 & $7.399 \mathrm{E}-06$ & $8.87 \theta E-06$ & 1600 \\
\hline & 100.0 & 14510 & $1.277 E-05$ & $1.533 \mathrm{E}-05$ & 1600 \\
\hline & 101.0 & 20446 & $1.844 t-05$ & $2.213 \mathrm{E}-05$ & 1600 \\
\hline & 102.0 & 28039 & $2.468 E-05$ & $2.962 \mathrm{E}-05$ & 1600 \\
\hline & 103.0 & 28016 & $2.537 \mathrm{E}-05$ & $3.044 E-05$ & 1600 \\
\hline & 104.0 & 24578 & $2.164 E-05$ & $2.590 t-05$ & 1600 \\
\hline & 105.0 & 24325 & $2.141 E-05$ & $2.570 E-05$ & 1600 \\
\hline & 106.0 & .26274 & $2.313 E-05$ & $2.776 \mathrm{E}-05$ & 1600 \\
\hline & 107.0 & 23659 & $2.003 E-05$ & $2.499 \mathrm{E}-05$ & 1600 \\
\hline & 108.0 & 21586 & $1.900 E-05$ & $2.280 E-05$ & 1600 \\
\hline & 109.0 & 19097 & $1.681 E-05$ & $2.017 E-05$ & 1600 \\
\hline & 110.0 & 21272 & $1.873 E-05$ & $2.247 E-.05$ & 1600 \\
\hline & 111.0 & 2.3563 & $2.074 E-05$ & $2.489 F-05$ & 1600 \\
\hline & 112.0 & 20242 & $1.782 E-05$ & $2.130 \mathrm{E}-0 \mathrm{~b}$ & 1600 \\
\hline & 113.0 & 17547 & $1.545 t-05$ & $1.854 E-05$ & 1500 \\
\hline & 114.0 & 13089 & $1.152 E-05$ & $1.383 E-05$ & 1600 \\
\hline E & 115.0 & 9725 & B.561E-06 & $1.027 E-05$ & 1600 \\
\hline & 116.0 & 1797 & $6.864 E-06$ & $8.236 E-06$ & 1600 \\
\hline & 117.0 & 6633 & 5. $839 \mathrm{E}$ & $7.007 E-06$ & 1600 \\
\hline 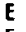 & 118.0 & 5011 & $5.291 \mathrm{t}-06$ & $6.350 E-06$ & 1600 \\
\hline & 119.0 & 5367 & $4.724 E-06$ & $5.669 \mathrm{k}-06$ & 1500 \\
\hline & 120.0 & 4937 & $4.347 E-06$ & $5.216 \mathrm{E}-06$ & 1600 \\
\hline & 121.0 & 4306 & $3.969 E-06$ & $4.762 E-06$ & 1600 \\
\hline E & 122.0 & 3756 & $3.307 E-06$ & $3.969 \mathrm{E}-06$ & 1600 \\
\hline & 123.0 & 3097 & $2.727 E-06$ & $3.272 E-06$ & 1600 \\
\hline p & 124.0 & 2155 & $1.097 E-06$ & $2.277 E-06$ & 1600 \\
\hline $\mathbf{P}$ & 125.0 & 2908 & $2.561 E-06$ & $3.073 E-06$ & 1600 \\
\hline $\mathbf{P}$ & & 4622 & $4.069 E-06$ & $4.882 E-06$ & 1600 \\
\hline & 127.0 & 747 & $6.576 E-07$ & $7.892 E-07$ & 1600 \\
\hline
\end{tabular}

94.0
96.0
98.0
100.0
102.0
114.0
106.0
134.0
113.0
112.0
114.0
116.0
118.0
120.0
122.0
124.0
126.0
128.0
130.0
132.0
134.0
136.0
134.0
140.0
142.0
144.0
146.0
140.0

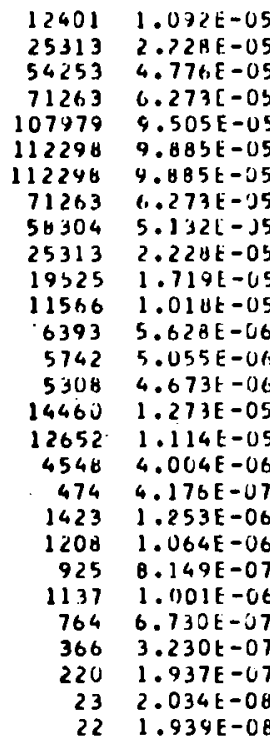

$1.310 E-0 S$

$2.674 E-05$

$5.731 E-05$

$7.526 E-05$

$1.141 \mathrm{E}-04$

$1.186 \mathrm{E}-04$

$1.196 E-04$

$7.52+1-05$

$0.159 \mathrm{E}-0 \mathrm{~S}$
$2.674 \mathrm{E}-05$

$2.063 E-05$

$1.222 \mathrm{E}-05$

$0.754 E-06$

$6.066 \mathrm{E}-06$

$5,608 E-00$

$1,524 E-05$

$1,336 E-05$

$4.805 E-06$

$5.011 E-07$

$1.504 E-06$

$1.277 E-06$

$9.776 E-07$

$1.201 E-06$

$3.876 \mathrm{E}-\mathrm{CO}$

$2.325 \mathrm{E}-07$

$2.440 E-0 B$

$2.327 E-08$

CKITSSIAINO INTEGRATFU 1. H36E-02 2.204E-02

SHC/50.M

$1 / \mathrm{H}$

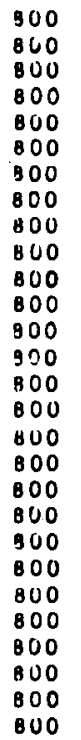

800
TEST DI
FLULRESCEIN RELEASE FRUM ELEVATION OF $1600 \%$ ARC SAMPLER HI $1.5 \mathrm{M} \quad U=1.2 \mathrm{M} / \mathrm{SEC}$ AT $2 \mathrm{H}$

AZIIUTH EXPOSURE $\mathrm{T} / O$ EU/O OISTANCE OEGKEES GM-SEC/CU.M SEC/CU.M I/SU.M METERS $\times 10 E+6$

1

\begin{tabular}{|c|c|c|c|c|}
\hline 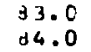 & $\begin{array}{r}105 \\
70\end{array}$ & $\begin{array}{l}9.313 E-U 4 \\
6.194 E-U B\end{array}$ & $\begin{array}{l}1.110 E-01 \\
7.49 Y E-C O B\end{array}$ & $\begin{array}{l}1690 \\
1600\end{array}$ \\
\hline $8 \pm .0$ & 35 & $3.1] 7 E-00$ & $3.740 E-O B$ & 1600 \\
\hline 46.0 & 70 & h. $144 \mathrm{E}-08$ & $7.433 \mathrm{E}-08$ & 1600 \\
\hline 97.0 & 143 & $1.254 E-07$ & $1.517 E-07$ & 1500 \\
\hline Bj. 0 & $<00$ & $1.763 E-07$ & $2.116 E-07$ & 1600 \\
\hline 89.0 & 174 & $1.534 E-07$ & $1.841 \mathrm{E}-07$ & 1600 \\
\hline 90.0 & 245 & $2.158 E-07$ & $2.54 \cup E-07$ & 1600 \\
\hline 91.0 & 287 & $2.532, E-07$ & $3.039 E-07$ & 1600 \\
\hline 92.0 & 280 & $3.470 E-07$ & $2.464 t-07$ & 1600 \\
\hline 93.6 & 436 & $3.042 E-U 7$ & $4.611 E-07$ & 1500 \\
\hline 34.0 & 500 & $4.4134 F-37$ & $5.2 \pm 4[-01$ & 1600 \\
\hline 45.0 & 549 & $4 . H 4 O E-J 7$ & $5.800 E-67$ & 1600 \\
\hline 73.0 & 20.30 & $1.7 B H E-06$ & $2.145 E-06$ & 1600 \\
\hline 97.0 & 25,6 & $3.224[-66$ & $2.669 \mathrm{E}-06$ & 1600 \\
\hline 9.1 .0 & 366.7 & $3.229 t-66$ & $3.874 r-06$ & 1600 \\
\hline 99.0 & 39361 & $3.465 t-05$ & $4.158 t-05$ & 1500 \\
\hline 10.0 & 61547 & $5.41 \mathrm{HE}-J 5$ & E.S & 1600 \\
\hline 101.0 & 21468 & 1.H9G,E-US & $2.260 t-0 s$ & 1600 \\
\hline 102.0 & 24331 & $2.142 E-05$ & $2.5701-03$ & 1600 \\
\hline 103.0 & 25046 & $2.205 E-05$ & $2.640 E-05$ & 1600 \\
\hline 104.0 & 22849 & $2.015 . t-15$ & $\angle .419 E-05$ & 1500 \\
\hline 1115.0 & 23615 & $2.079 E-05$ & $2.49 D E-O B$ & 1600 \\
\hline 106.0 & 19321 & $1.701 \mathrm{E}-\mathrm{US}$ & $2.041 \mathrm{E}-05$ & 1600 \\
\hline 107.0 & 19321 & $1.701 \mathrm{E}-05$ & 2.0411 & 1600 \\
\hline 108.0 & 18600 & $1.638 L-05$ & $1.965 t-05$ & 1600 \\
\hline $1 u^{\prime \prime .00}$ & 12164 & $1.071 E-05$ & 1.2LSE-Uइ & 1600 \\
\hline 110.0 & 17089 & $1.575 k-05$ & $1.040 t-25$ & 1500 \\
\hline 111.0 & 17412 & $1.533 E-05$ & $1.834 t-05$ & 1600 \\
\hline 112.0 & 16696 & $1.470 \mathrm{E}-05$ & $1.764 \mathrm{E}-05$ & 1600 \\
\hline 113.0 & 13357 & $1.176 E-05$ & $1.411 f-05$ & 1600 \\
\hline 114.8 & 10562 & $9.29 \mathrm{BE}-06$ & $1.116 E-05$ & 1600 \\
\hline 115.0 & 7437 & $6.547 E-06$ & $\because .857 E-06$ & 1600 \\
\hline 116.0 & 6544 & $5.76] E-06$ & $0.914 E-06$ & 1300 \\
\hline 117.0 & 6442 & $6.023 \mathrm{E}-36$ & $7.220 E-06$ & 1600 \\
\hline 116.0 & 6,693 & $5.89>t-06$ & $7.071 \mathrm{E}-00$ & 1640 \\
\hline 119.0 & BUYU & $3.36+E-06$ & $0.4421-06$ & 1600 \\
\hline $1<0.0$ & 5651 & $4.975 t-66$ & $5.970 t-00$ & 1600 \\
\hline $1<1.0$ & 4907 & $4.320 E-03$ & $5.134 E-06$ & 1500 \\
\hline $1<2.0$ & 4312 & $3.796 \mathrm{E}-06$ & $4.556 E-06$ & 1600 \\
\hline 123.0 & 2642 & $2.326 \mathrm{E}-06$ & $2.79 \angle E-06$ & 1600 \\
\hline $1<4.0$ & 2824 & $7.48 \mathrm{BE}-06$ & $2.984 E-06$ & 1600 \\
\hline $1<5.0$ & 3022 & $2.661 E-06$ & $3.193 E-06$ & 1600 \\
\hline & 2028 & $1.785 E-06$ & $\therefore .143 E-06$ & 1600 \\
\hline & 549 & $.040 \mathrm{E}-07$ & $5.808 E-07$ & 1600 \\
\hline
\end{tabular}




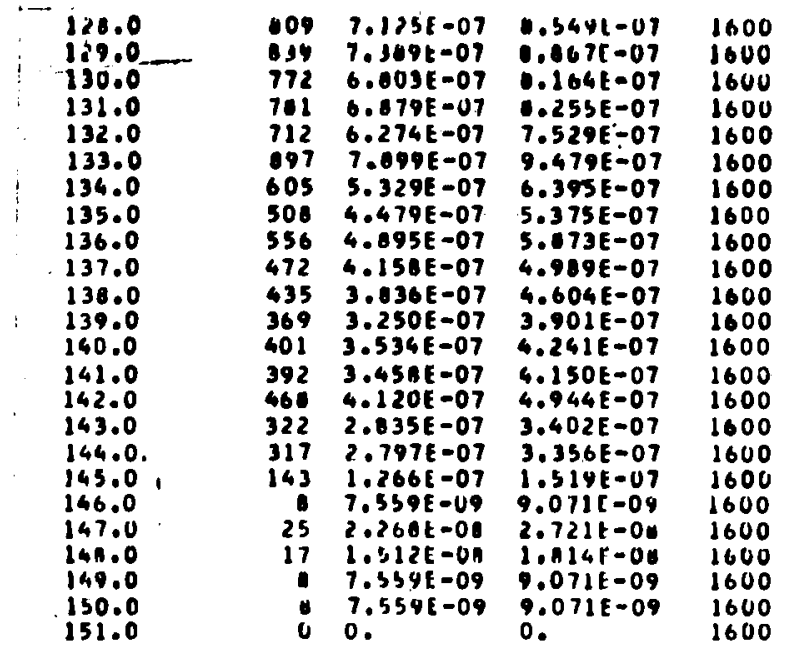

ROSSWIMO INTEGRATED 1.031 E-02 1.230 E-02 SEC/SO.M I IM

TEST D1 JULY 7, 1967 0445 TO 0515 PST

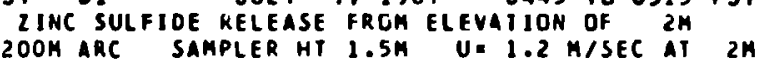
ALIMUTH EXPOSURE
DEGRES GH-SEC/CU.H SEC/CU.M IISU.M OISTIANCE
XIOE 46

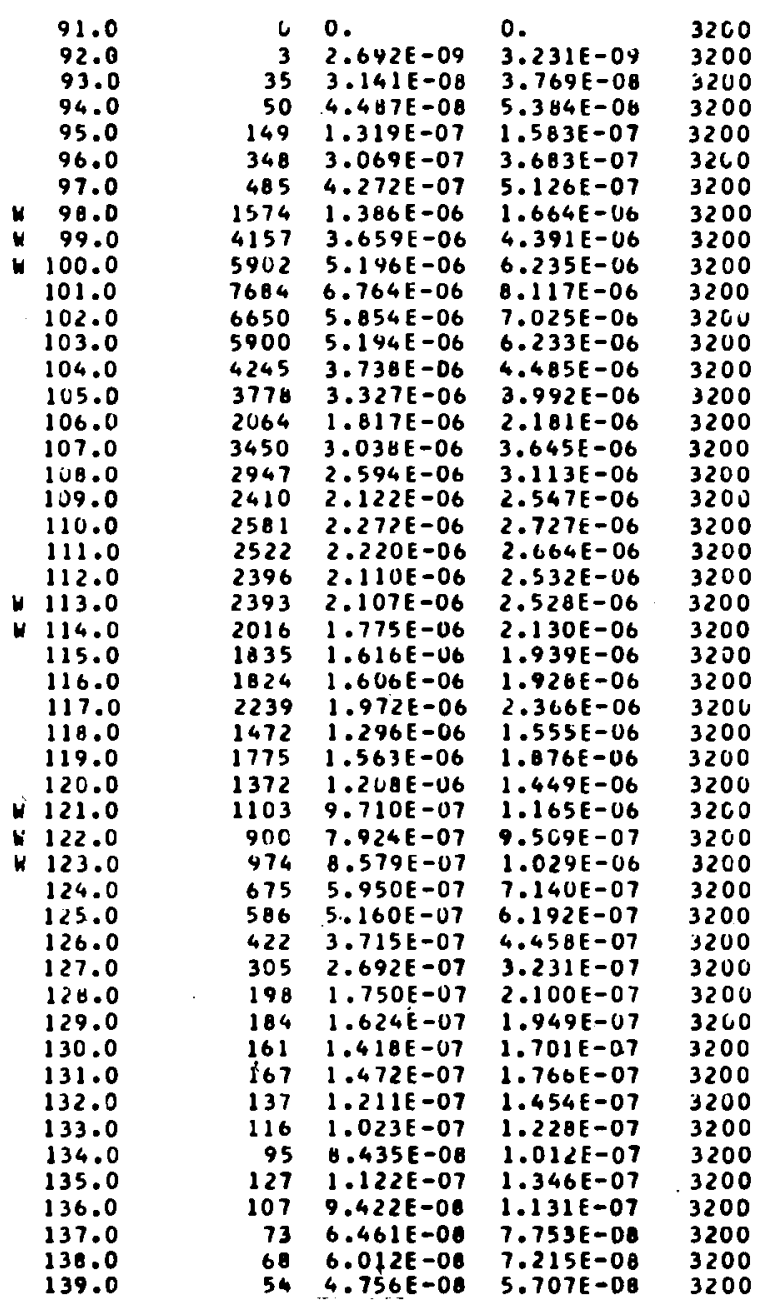

$$
\begin{aligned}
& 1.1100 \\
& 111.0 \\
& 111.0 \\
& 111.0 \\
& 112.0 \\
& 133.0 \\
& 134.0 \\
& 135.0 \\
& 136.0 \\
& 137.0 \\
& 1313.0 \\
& 139.0 \\
& 119.0 \\
& 141.0 \\
& 147.0 \\
& 143.0 \\
& 144.0 \\
& 141.01 \\
& 141.01 \\
& 117.0 \\
& 119.11 \\
& 119.0 \\
& 151.0
\end{aligned}
$$

CRUSSHINO

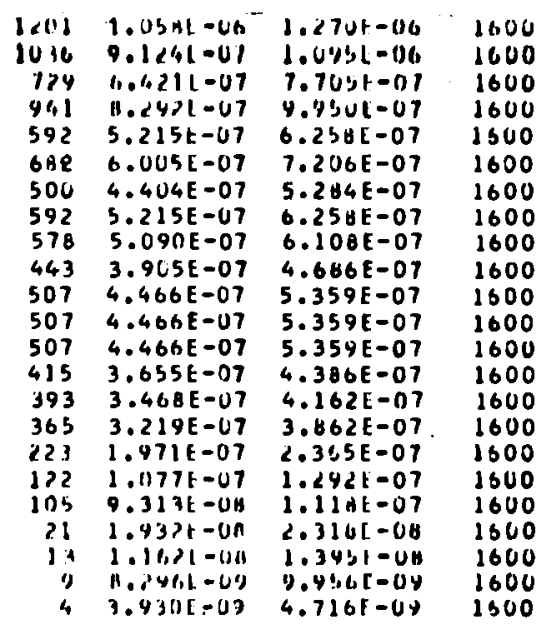

1300
1600

1600

\begin{tabular}{|c|c|c|c|c|}
\hline $\begin{array}{l}92.0 \\
43.0\end{array}$ & $\begin{array}{l}10 \\
25\end{array}$ & $\begin{array}{l}9.253 t=09 \\
2.251 t=00\end{array}$ & $\begin{array}{l}1.110 \mathrm{E}-00 \\
2.701 \mathrm{E}-0 \mathrm{O}\end{array}$ & $\begin{array}{l}3200 \\
3200\end{array}$ \\
\hline $\begin{array}{l}43.0 \\
44.0\end{array}$ & 69 & $6.154 E-08$ & $7.334 E-08$ & 3200 \\
\hline 95.0 & 142 & $1.255 \mathrm{E}-07$ & $1.506 \mathrm{E}-07$ & 3200 \\
\hline 96.0 & 324 & 2.9 JOE-U7 & $3.480 E-07$ & 3200 \\
\hline 47.0 & 484 & $4.311 E-07$ & $3.173 E-07$ & 3200 \\
\hline $9 R .0$ & 1478 & $1.302 \mathrm{t}-06$ & $1.562 \mathrm{E}-06$ & 3200 \\
\hline 44.0 & 4036 & $3.553 E-06$ & $4.264 \mathrm{E}-06$ & 3200 \\
\hline 100.0 & 5651 & $4.975 E-06$ & $5.970 E-06$ & 3200 \\
\hline 101.0 & 6952 & $6.120 \mathrm{E}-06$ & $7.344 E-06$ & 3200 \\
\hline 1.2 .0 & 5853 & $5.153 E-06$ & $6.18 \triangle E-U_{0}$ & 3200 \\
\hline 103.0 & 4843 & 4.264 E-06 & $5.117[-06$ & 3200 \\
\hline $111 \% .0$ & 3767 & $3.316 E-06$ & $3.974 E-06$ & 3200 \\
\hline ius.o & 3565 & $3.13 \mathrm{HE}-\mathrm{C} 6$ & $3.766 \mathrm{E}-06$ & 3200 \\
\hline jut.n & 3497 & $3.079 \mathrm{E}-06$ & $3.695 F-06$ & 3200 \\
\hline 1.7 .0 & $322 \theta$ & $2.042 t-06$ & $3.4111-06$ & 3200 \\
\hline ivi.u & 2754 & $2.605 E-06$ & $3 \times 26 E-06$ & 3200 \\
\hline 109.0 & 2959 & $2.605 E-06$ & $3.126 \mathrm{E}-06$ & 3200 \\
\hline 110.0 & 2488 & $1.190 \mathrm{E}-06$ & $2.629 \mathrm{E}-06$ & 3200 \\
\hline 111.0 & 2151 & $1.094 E-06$ & $2.273 \mathrm{E}-06$ & 3200 \\
\hline 112.0 & 2353 & $2.072 E-06$ & $2.406 E-06$ & 3200 \\
\hline 113.6 & 2017 & $1.77 \mathrm{hE}-J 6$ & $2.131 \mathrm{E}-06$ & 3260 \\
\hline 114.0 & CUA4 & $1.035 \mathrm{E}-66$ & $2.202 E-06$ & $320 \mathrm{C}$ \\
\hline 115.0 & 1591 & $1.401 E-06$ & $1.681 \mathrm{E}-06$ & 3200 \\
\hline 116.0 & 2353 & $2.072 \mathrm{E}-06$ & $2.406 E-06$ & 3200 \\
\hline 117.0 & 2421 & $2.131 t-06$ & $2.557 E-0.6$ & 3200 \\
\hline ii 0.0 & 1658 & $1.460 \mathrm{E}-06$ & $1.752 \mathrm{E}-06$ & 3200 \\
\hline 119.0 & 1635 & $1.440 \mathrm{E}-06$ & $1.728 \mathrm{E}-06$ & 3200 \\
\hline 120.0 & $156 \mathrm{~A}$ & $1.301 E-06$ & $1.657 E-06$ & 3200 \\
\hline 121.0 & 873. & $7.687 E-07$ & $9.224 \mathrm{E}-07$ & 3200 \\
\hline $1: 2.0$ & 917 & $H .072 \mathrm{E}-07$ & $9.687 \mathrm{E}-07$ & 3200 \\
\hline 123.0 & 756 & $0.667 t-07$ & $7.994 F-07$ & $\$ 200$ \\
\hline $124.0^{\circ}$ & 479 & $4.217 \mathrm{E}-07$ & $5.050 E-07$ & 3200 \\
\hline 123.0 & 585 & $5.157 \mathrm{E}-07$ & $0.1 \forall 4 E-07$ & 3200 \\
\hline $1<h .0$ & 329 & $2.4 \cup 0 E-J 7$ & $3.4 \triangle \cup E-67$ & $\$ 200$ \\
\hline $1<7.0$ & 206 & $1.819 E-07$ & $2.183 E-07$ & 9200 \\
\hline $1,3.0$ & 171 & $1.504 E-07$ & $1.810 E-07$ & 3200 \\
\hline $1<4.0$ & 132 & $1.170 \mathrm{t}-07$ & $1.404 \mathrm{E}-07$ & 3200 \\
\hline 130.0 & 116 & $1.029 E-07$ & $1.235 E-07$ & 3200 \\
\hline 131.0 & 132 & $1.170 E-07$ & $1.404 \mathrm{E}-07$ & 3200 \\
\hline 132.0 & 107 & $9.445 \mathrm{E}-08$ & $1.133 \mathrm{E}-07$ & 3200 \\
\hline 133.0 & 107 & $9.445 \mathrm{E}-00$ & $1.133 \mathrm{E}-07$ & 3200 \\
\hline 134.0 & 53 & $4.743 \mathrm{E}-08$ & $5.692 \mathrm{E}-08$ & 3200 \\
\hline 135.0 & 95 & $0.410 \mathrm{E}-08$ & $1.009 \mathrm{E}-07$ & 3200 \\
\hline 136.0 & 70 & $6.24 A E-08$ & $7.497 \mathrm{E}-08$ & 3200 \\
\hline 137.0 & 83 & $7.376 E-08$ & $0.851 \mathrm{E}-08$ & 3200 \\
\hline 138.0 & 41 & $3.615 E-08$ & $4.336 \mathrm{E}-0 \mathrm{~B}$ & 3200 \\
\hline 139.0 & 48 & $4.273 E-00$ & $5.127 E-08$ & 3200 \\
\hline
\end{tabular}

(16)

600

1600

1600

1600

600

1600

1600

600

660

6000

SEC/SO.M $1,31 / \mathrm{H}$

IEST UL JULY 7. 1967 U445 TU 0515 PST FLUORESCEIN RELEASE FROM ELFVATION OF $2 M$ 32 NOH AHC SAMPLFR HT $1.5 \mathrm{M}$ H= $1.2 \mathrm{M} / \mathrm{SEC}$ AT $2 \mathrm{H}$ AZIMUTH EXPUSURT E/G EU/O DISIANCE DECREES GH-SEC/CU.M SEC/CU.M I/SO.M METERS $\times 10 E+6$ 


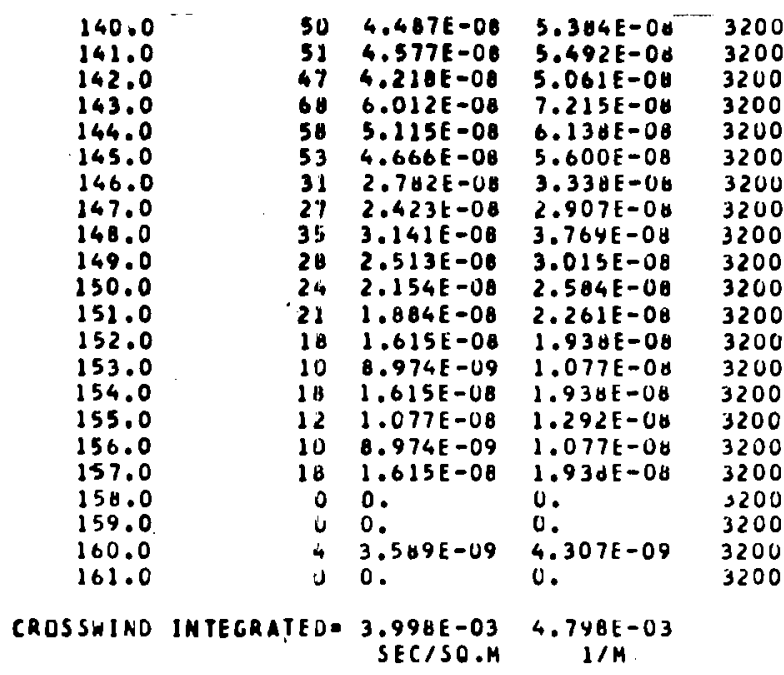

TOWEK DATA FOLLQH....

TEST DI JULY 7,1967 0445 IU 0515 PST IINC SULFIOE RELEASE FRUM ELFVATIUN OF $2 M$
2OUM ARC
U. 1.2 M/SEC AT $2 M$

\begin{tabular}{|c|c|c|c|c|c|}
\hline $\begin{array}{l}\text { AZIMUTH } \\
\text { OECKEESS }\end{array}$ & $\begin{array}{l}\text { HE IGHT } \\
\text { ME TERS }\end{array}$ & $\begin{array}{c}\text { EXPDSURE } \\
\text { GM-SEC/CU,H } \\
\times 1 U E+6\end{array}$ & $\begin{array}{c}F / O \\
S E C / / C U . M\end{array}$ & $\begin{array}{l}\text { EU/O } \\
\text { I/SO.M }\end{array}$ & $\begin{array}{l}\text { UISTANCE } \\
\text { METERS }\end{array}$ \\
\hline $\begin{array}{l}98.0 \\
98.0\end{array}$ & .3 & $\begin{array}{l}113506 \\
113213\end{array}$ & $\begin{array}{r}9.992 E-05 \\
9.966 E-05\end{array}$ & $\begin{array}{l}1.199 \mathrm{E}-04 \\
1.196 \mathrm{E}-04\end{array}$ & $\begin{array}{l}200 \\
200\end{array}$ \\
\hline & $\begin{array}{l}1.4 \\
1.5\end{array}$ & $\begin{array}{l}122172 \\
315000\end{array}$ & $\begin{array}{l}1.075 E-04 \\
2.773 E-04\end{array}$ & $\begin{array}{l}1.241 E-04 \\
3.328 E-04\end{array}$ & $\begin{array}{l}200 \\
200\end{array}$ \\
\hline $\begin{array}{l}98.0 \\
98.0 \\
98.0\end{array}$ & $\begin{array}{l}2.7 \\
4.1 \\
5.4\end{array}$ & $\begin{array}{r}328283 \\
17322 \\
12591\end{array}$ & $\begin{array}{l}2.890 E-04 \\
1.525 E-05 \\
1.100 E-05\end{array}$ & $\begin{array}{l}3.460 E-04 \\
1.830 E-05 \\
1.330 E-05\end{array}$ & $\begin{array}{l}200 \\
200 \\
200\end{array}$ \\
\hline 98.0 & 6.4 & 13584 & $1.196 E-05$ & $1.433 E-05$ & 200 \\
\hline 98.0 & & 18919 & $1.665 \mathrm{E}-05$ & 1.99 ut -05 & 200 \\
\hline 98.0 & 9.5 & 8680 & $7.641 \mathrm{E}-U 6$ & $9.170 \mathrm{E}-06$ & 200 \\
\hline $\begin{array}{l}98.0 \\
98.0\end{array}$ & $\begin{array}{l}10.8 \\
13.5\end{array}$ & $\begin{array}{l}13153 \\
11216\end{array}$ & $\begin{array}{l}1.154 \mathrm{E}-05 \\
9.874 \mathrm{E}-00\end{array}$ & $\begin{array}{l}1.389 \mathrm{t}-05 \\
1.145 \mathrm{~L}-0 \mathrm{~S}\end{array}$ & $\begin{array}{l}200 \\
200\end{array}$ \\
\hline $\begin{array}{l}98 . \\
98 .\end{array}$ & 16.2 & 7569 & $0.664 E-06$ & $7.946 E-U 6$ & 200 \\
\hline & & 3879 & $3.415 E-06$ & $4.098 t-06$ & 200 \\
\hline & & 2764 & $2.451 \mathrm{E}$ & $2.941 \mathrm{E}-06$ & \\
\hline & & 08 & & $4.334 E-07$ & 200 \\
\hline 98.0 & 27.0 & 493 & $4.347 E-U 7$ & $5.216 t-07$ & 200 \\
\hline
\end{tabular}

AIIHUTH HEIGHT EXPOSURE E/O EUIO DISTANCE OEGREES METERS GM-SEC/CU.M SECICU.M - I/SO.M METERS $\times 10 E+6$

$\begin{array}{lrrrr}106.0 & .3 & 77269 & 6.797 E-05 & 8.136 E-05 \\ 106.0 & .7 & 68183 & 6.002 E-05 & 7.202 E-05 \\ 106.0 & 1.4 & 58545 & 5.154 E-0 S & 6.164 E-05 \\ 106.0 & 1.5 & 206583 & 1.819 E-04 & 2.182 E-04 \\ 106.0 & 2.7 & 193142 & 1.700 E-04 & 2.040 E-04 \\ 106.0 & 4.1 & 283526 & 2.496 E-04 & 2.995 E-04 \\ 106.0 & 5.4 & 333293 & 2.934 E-04 & 3.521 E-04 \\ 106.0 & 6.8 & 291169 & 2.563 E-04 & 3.076 E-04 \\ 106.0 & 8.1 & 373549 & 3.286 E-04 & 3.946 E-04 \\ 106.0 & 9.5 & 304365 & 2.679 E-04 & 3.215 E-04 \\ 106.0 & 10.8 & 416864 & 3.07 U E-04 & 4.404 E-04 \\ 106.0 & 13.5 & 299851 & 2.640 E-04 & 3.167 E-04 \\ 106.0 & 16.2 & 196735 & 1.732 E-04 & 2.078 E-04 \\ 106.0 & 18.9 & 121602 & 1.070 E-04 & 1.285 E-04 \\ 106.0 & 21.6 & 63953 & 3.630 E-05 & 6.756 E-05 \\ 106.0 & 24.3 & 37555 & 3.306 E-05 & 3.967 E-0 S \\ 106.0 & 27.0 & 21662 & 1.907 E-05 & 2.288 E-05\end{array}$

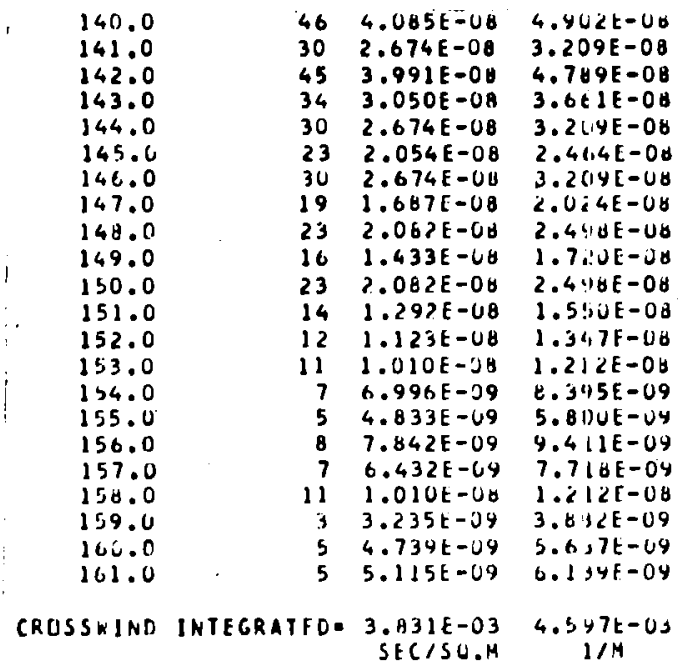

3200 3200 3200 3200 3200 3200 3200 3200 1200 3200 1200 3200 3200 3200 3200 3200 3200 3200 3200 3200
TOHER DATA FULLOH....

TEST JULY 7, 1967 U445 TU 0515 PST
FLUOKESCEIN RELEASE FKUM ELFVATIUN DF LM
2OUH ARC

$\begin{array}{lcccc}\text { AIIMUTH HEIGHT EXPUSURE } & \text { FIU } & \text { EU/O UISTANCE } \\ \text { DEGKEES HETERS GH-SLCICU.M SEF/CU.M } & \text { I/SO.M METERS }\end{array}$ $\times 10 E+6$

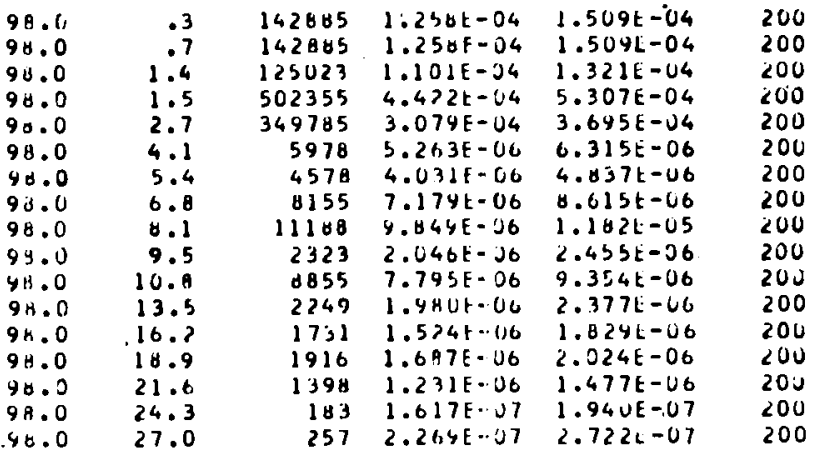

AZIMUTH HEIGHT EXPOSURE F/O EU/O DISTANCE DECKEES METERS GM-SEC/CU.M SEC/CU.M I/SO.M METERS $\times 10 E+6$

$\begin{array}{lrrrrr}106.0 & .3 & 78525 & 6.912 E-05 & 8.295 E-05 & 200 \\ 106.0 & .7 & 97598 & 8.541 E-05 & 1.031 E-04 & 200 \\ 106.0 & 1.4 & 93110 & 0.190 E-05 & 9.836 E-05 & 100 \\ 106.0 & 1.5 & 251173 & 2.211 E-04 & 2.653 E-04 & 200 \\ 106.0 & 2.7 & 192005 & 1.690 E-04 & 2.028 E-04 & 200 \\ 106.0 & 4.1 & 334900 & 2.946 E-04 & 3.530 E-04 & 200 \\ 106.0 & 5.4 & 394440 & 3.472 E-04 & 4.167 E-04 & 200 \\ 106.0 & 6.8 & 275361 & 2.474 E-04 & 2.909 E-04 & 200 \\ 106.0 & 8.1 & 498634 & 4.389 E-04 & 3.267 t-04 & 200 \\ 106.0 & 9.5 & 296245 & 2.555 E-04 & 3.066 E-04 & 200 \\ 106.0 & 10.8 & 513518 & 4.570 E-04 & 5.424 E-04 & 20 J \\ 106.0 & 13.5 & 334900 & 2.940 E-04 & 3.538 E-04 & 200 \\ 106.0 & 16.2 & 212100 & 1.067 E-04 & 2.240 E-04 & 200 \\ 106.0 & 18.9 & 138420 & 1.218 E-04 & 1.452 E-04 & 200 \\ 110.0 & 21.6 & 38136 & 3.357 E-05 & 4.028 E-05 & 200 \\ 106.0 & 24.3 & 44867 & 3.950 E-05 & 4.740 E-05 & 20 U \\ 106.0 & 27.0 & 23925 & 2.106 E-05 & 2.527 E-05 & 200\end{array}$


laZ IMUTh HEIGHT

114.0
114.0
114.0
114.0
114.0
114.0
114.0
114.0
114.0
114.0
114.0
114.0
114.0
114.0
114.0
114.0
114.0

.3
1.7
1.4
2.7
4.1
5.4
6.8
0.1
9.5
10.8
13.5
16.2
10.9
21.6
24.3
27.0

EXPOSURE $x+3 E C / C O$

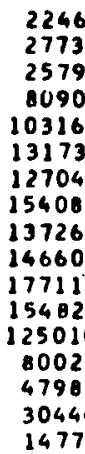

E 10 SEC/CU.H

1.970E-05 $2.442 E-05$ $2.271 E-05$ $9.002 \mathrm{E}-05$ $1.260 E-04$ $1.110 \mathrm{E}-04$ $1.356 E-04$ $1.208 E-04$ $1.291 E-04$ $1.559 \mathrm{E}-04$ $1.363 E-04$ $1.100 E-04$ $7.044 E-05$ $4.224 E-05$ $2.600 E-05$ 1.301E-05 EU/O DISTANCE
I/SO.M METERS

$2.373 E-05$ $2.930 E-05$ $2.725 E-05$ $0.546 E-05$ $1.090 E-04$ $1.392 \mathrm{E}-04$ $1.342 \mathrm{E}-04$
$1.628 \mathrm{E}-04$ $1.450 t-04$ $1.549 E-04$ $1.871 E-04$ $1.635 \mathrm{E}-04$ $1.321 E-04$ $0.453 E-05$ $5.069 E-05$ $3.216 E-05$ $1.5615-05$

200
200
200
200
200
200
200
200
200
200
200
200
200
200
200
200
200

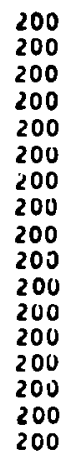

ALIMUTH HEIGHT $-S E C / C$
$X 10 E+6$

$\begin{array}{lllll}122.0 & .3 & 1130 & 9.954 E-07 & 1.194 E-06 \\ 122.0 & .7 & 18619 & 1.639 E-05 & 1.967 E-05 \\ 122.0 & 1.4 & 22513 & 1.982 E-05 & 2.378 E-05 \\ 122.0 & 1.5 & 70825 & 6.235 E-05 & 7.482 E-05 \\ 122.0 & 2.7 & 75379 & 6.636 E-05 & 7.963 E-05 \\ 122.0 & 4.1 & 73082 & 6.433 E-05 & 7.720 E-05 \\ 122.0 & 5.4 & 80831 & 7.115 E-05 & 8.539 E-05 \\ 122.0 & 6.8 & 70943 & 6.245 E-05 & 7.494 E-05 \\ 122.0 & 6.1 & 65169 & 5.737 E-05 & 6.884 E-05 \\ 122.0 & 9.5 & 62849 & 5.533 E-05 & 6.639 E-05 \\ 122.0 & 10.8 & 60954 & 5.366 E-05 & 6.439 E-05 \\ 122.0 & 13.5 & 65245 & 5.743 E-05 & 6.892 E-05 \\ 122.0 & 16.2 & 57544 & 5.066 E-05 & 6.079 E-05 \\ 122.0 & 18.9 & 60245 & 5.303 E-05 & 6.364 E-05 \\ 122.0 & 21.6 & 52023 & 4.580 E-05 & 5.495 E-05 \\ 122.0 & 24.3 & 32244 & 2.838 E-05 & 3.406 E-05 \\ 122.0 & 27.0 & 22621 & 1.991 E-05 & 2.390 E-05\end{array}$
$\begin{array}{ccc}\text { E/O } & \text { EU/W DISTANCE } \\ \text { SECICU.M I/SO.M METERS }\end{array}$

\section{0} 200 200

200

200

200

200

200

200

200

200

200

200

200

200

200
AL IMUTH HEIGHT DEGKEES METERS GM

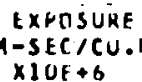

114.0

114.0

114.0

2114.6

114.0

114.0

114.0

114.0

114.0

114.0

114.0

114.0

114.0

114.0

114.0

114.0

114.0$$
\begin{aligned}
& 31 \\
& 29 \\
& 31 \\
& 111 \\
& 230 \\
& 120 \\
& 100 \\
& 167 \\
& 156 \\
& 156 \\
& 223 \\
& 171 \\
& 147 \\
& 89 \\
& 35 \\
& 3364 \\
& 1564 \\
& 1568
\end{aligned}
$$$$
314042.765 t-05
$$$$
31404 \quad 2.765 E-05
$$$$
9.026 \mathrm{E}-05
$$$$
2.031 E-04
$$$$
\text { 1.061E-04 }
$$$$
0.844 E-05
$$$$
1.474 E-04
$$$$
1.376 E-04
$$$$
1.376 E-04
$$$$
1.965 E-04
$$$$
1.513 \text { E-04 }
$$$$
1.297 E-04
$$$$
7.861 E-05
$$$$
3.16, U E-05
$$$$
2.962 \mathrm{E}-05
$$$$
106891.645 \mathrm{E}-05
$$

.3
.7
1.4
1.5
2.7
4.1
5.4
6.8
0.1
9.5
10.8
13.5
16.2
18.4
21.6
24.8
27.0

3.15E-05

$1.174 t-144$

$2.437 \mathrm{E}-04$

1.274 E- 04

$1.061 E-04$

$1.769 E-04$

$1.651 \mathrm{E}-04$

$1.651 \mathrm{E}-04$

$2.358 \mathrm{E}-04$

$1.816 E-04$

$1.557 E-04$

$9.433 E-05$

$3.791 E-05$

200

AZIMUTII HEIGH

EXPOSURE

- F/O

EUIU DISTANCE $\times 10 \mathrm{E}+6$

$\begin{array}{lr}122.0 & .3 \\ 122.0 & .7 \\ 122.0 & 1.4 \\ 122.0 & 1.5 \\ 122.0 & 2.7 \\ 122.0 & 4.1 \\ 122.0 & 5.4 \\ 122.0 & 6.8 \\ 122.0 & 8.1 \\ 122.0 & 9.5 \\ 122.0 & 10.8 \\ 122.0 & 13.5 \\ 122.0 & 16.2 \\ 122.0 & 18.9 \\ 122.0 & 21.6 \\ 122.0 & 24.3 \\ 122.0 & 27.0\end{array}$

$\begin{array}{ll}2160 & 1.902 E-06 \\ 21307 & 1.876 E-05 \\ 31404 & 2.765 E-05 \\ 82602 & 7.271 E-05 \\ 89300 & 7.861 E-05 \\ 50042 & 5.109 E-05 \\ 82632 & 7.271 E-05 \\ 7143 B & 6.289 E-05 \\ 55809 & 4.913 E-05 \\ 71438 & 6.289 E-05 \\ 58042 & 5.109 E-05 \\ 61019 & 5.371 E-05 \\ 55804 & 4.913 E-05 \\ 66228 & 5.830 E-05 \\ 59530 & 5.240 E-05 \\ 26917 & 2.369 E-05 \\ 27665 & 2.435 E-05\end{array}$

$2.282 E-06$

$2.251 t-05$

$3.317 E-05$

$8.726 E-05$

$9.433 r-05$

$6.131 E-U 5$

$8.726 E-05$

$7.546 E-05$

$5.895 t-65$

$7.546 E-05$

$6.131 E-05$

$6.446 t-05$

$5.895 E-05$

$6.996 E-05$

$6.288 E-05$

$2.843 t-05$

$2.922 E-05$
ALIMUTH HEIGHT EXPOSURE F/O EU/O DISTANCE DEGKEES METERS GM-SEC/CU.M SEC/CU.M I/SO.M METERS. $\times 10 E+6$

$\begin{array}{ll}130.0 & .3 \\ 130.0 & .7 \\ 130.0 & 1.4 \\ 130.0 & 1.5 \\ 130.0 & 2.7 \\ 130.0 & 4.1 \\ 130.0 & 5.4 \\ 130.0 & 6.8 \\ 130.0 & 8.1 \\ 130.0 & 9.5 \\ 130.0 & 10.8 \\ 130.0 & 13.5 \\ 130.0 & 16.2 \\ 130.0 & 18.9 \\ 130.0 & 21.6 \\ 130.0 & 24.3 \\ 130.0 & 27.0\end{array}$

$1.138 E-06$

$1.294 E-06$

$1.098 E-06$

$4.355 E-06$

$8.960 \mathrm{E}-06$

$2.660 \mathrm{E}-05$

$5.663 E-05$

$1.113 \mathrm{E}-04$

$1.091 \mathrm{E}-04$

$8.143 E-05$

$5.986 \mathrm{E}-05$

$1.727 E-05$

$1.254 E-05$

$1.268 \mathrm{E}-05$

$1.527 E-05$

1.125E-05

$1.253 E-05$

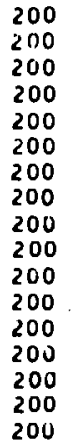

A2 IMUTH HEICHT EXPOSURF OEGRFES METERS GM-SEC/CU XIUE +6
130.0
130.0
130.0
130.0
130.0
130.0
130.0
130.0
130.0
130.0
130.0
130.0
130.0
130.0
130.0
130.0
130.0

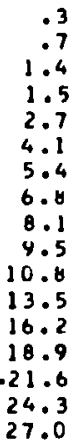

$\begin{array}{rl}3990 & 3.512 E-06 \\ 3545 & 3.121 E-06 \\ 3693 & 3.252 E-06 \\ 597 \mathrm{H} & 3.263 E-06 \\ 408 \mathrm{~A} & 8.001 E-06 \\ 25047 & 2.205 E-05 \\ 55809 & 4.913 E-05 \\ 80309 & 7.075 E-05 \\ 116093 & 1.072 E-04 \\ 55809 & 4.913 E-05 \\ 71438 & 6.289 E-05 \\ 15853 & 1.396 E-05 \\ 13520 & 1.19 U E-05 \\ 11887 & 1.046 E-05 \\ 15386 & 1.354 E-05 \\ 10721 & 9.438 E-06 \\ 14453 & 1.272 E-05\end{array}$

14453
F/O

EU/N DISTANCE

1/SO.M METERS

$4.215 E-06$ $3.745 E-06$ $3.902 E-06$ $6.315 t-06$ $4.601 \mathrm{t}-06$ $2.646 \mathrm{E}-05$ $2.646 E-05$ $3.895 E-05$
$0.490 E-05$ $1.226 E-04$ $5.895 \mathrm{E}-0 \mathrm{~F}$

$7.546 \mathrm{E}-05$ $1.675 \mathrm{t}-05$ $1.428 E-05$ $1.256 E-05$ $1.625 \mathrm{E}-05$

$1.133 E-05$ $1.527 E-05$
200
200
200
200
200
200
200
200
200
200
200
200
200
200
200
200
200 
TOWER DATA FOLLOW....

TEST DI JULY 7,1867 O445 TU OS15 PST
IINC SULFIOE RELEASE FROA ELFVATIUN OF LM
OOOH ARC

AZIMUTH HEIGHT EXPOSURE
DEGREES METERS GM-SECICU.M SECICU.A IISO.M HETERS $\times 10 E+6$

$\begin{array}{rr}98.0 & .3 \\ 98.0 & .5 \\ 98.0 & 1.1 \\ 98.0 & 1.5 \\ 98.0 & 2.1 \\ 98.0 & 4.2 \\ 98.0 & 6.3 \\ 98.0 & 8.4 \\ 98.0 & 10.5 \\ 98.0 & 12.6 \\ 98.0 & 14.7 \\ 98.0 & 16.0 \\ 98.0 & 21.0 \\ 98.0 & 25.2 \\ 98.0 & 29.4 \\ 98.0 & 33.6 \\ 98.0 & 37.8 \\ 98.0 & 42.0\end{array}$

$\begin{array}{rl}29070 & 2.559 E-05 \\ 47747 & 4.207 E-05 \\ 52530 & 4.624 E-05 \\ 47087 & 4.145 E-05 \\ 49024 & 4.316 E-05 \\ 47507 & 4.182 E-05 \\ 42609 & 3.751 E-05 \\ 35541 & 3.129 E-05 \\ 22299 & 1.963 E-05 \\ 19432 & 1.746 E-05 \\ 15034 & 1.323 E-05 \\ 11563 & 1.018 E-05 \\ 7647 & 6.732 E-06 \\ 4552 & 4.007 E-06 \\ 2491 & 2.193 E-06 \\ 1516 & 1.335 E-06 \\ 1160 & 1.021 E-06 \\ 551 & 4.855 E-07\end{array}$

$3.0716-05$ $5.048 \mathrm{E}-0.5$ 5. $549 E-05$ $4.974 E-05$ 5.179E-05 $5.018 t-05$ $4.501 E-05$ $3.754 E-05$ $2.350 E-05$ $2.095 \mathrm{t}-05$ $1.568 E-05$ $1.221 \mathrm{E}-05$ $8.078 \mathrm{E}-06$ $4.809 t-06$ $2.632 E-06$ $1.601 E-06$ $1.225 E-06$

$5514.855 \mathrm{E}-07 \quad 5.826 \mathrm{E}-07$

800
800
800
800
800
800
800
800
800
800
800
800
800
800
800
800
800
800

AIIMUTH HE IGH

EXPIISURE

F/O $x \perp O E+6$

$\begin{array}{lr}106.0 & .3 \\ 106.0 & .5 \\ 106.0 & 1.1 \\ 106.0 & 1.5 \\ 106.0 & 2.1 \\ 106.0 & 4.2 \\ 106.0 & 6.3 \\ 106.0 & 8.4 \\ 106.0 & 10.5 \\ 106.0 & 12.6 \\ 106.0 & 14.7 \\ 106.0 & 16.8 \\ 106.0 & 21.0 \\ 106.0 & 25.2 \\ 106.0 & 29.4 \\ 106.0 & 33.6 \\ 106.0 & 37.8 \\ 106.0 & 42.0\end{array}$

$\begin{array}{rl}93281 & 8.211 E-05 \\ 87615 & 7.713 E-05 \\ 100077 & 0.810 E-05 \\ 85962 & 7.567 E-05 \\ 113929 & 1.003 E-04 \\ 117282 & 1.032 E-04 \\ 95743 & 8.428 E-05 \\ 87069 & 7.645 E-05 \\ 82470 & 7.260 E-05 \\ 69780 & 6.143 E-05 \\ 53232 & 4.6166 E-05 \\ 42233 & 3.718 E-05 \\ 22891 & 2.015 E-05 \\ 11594 & 1.021 E-05 \\ 6691 & 5.890 E-06 \\ 3799 & 3.344 E-06 \\ 1843 & 1.673 E-06 \\ 1313 & 1.156 E-06\end{array}$

EUIU DISTANCE I/SU.M METERS

$9.854 \mathrm{E}-05$ $9.255 t-05$ $1.057 \mathrm{E}-04$ 9.081E-05 1. $203 i-04$ $1.239 \mathrm{E}-04$ $1.011 E-04$ $9.197 \mathrm{E}-05$ 0.712E-05 $7.371 E-05$ $5.623 E-05$ $4.461 t=05$ $2.418 E-05$ $1.225 \mathrm{E}-05$ 7. $068 \mathrm{E}-06$ $4.013 E-06$ $1.947 t-06$ 1.388E-06
ALIMUTH HEIGHT EXPOSURE F/O
DEGREES METERS GH-SECICU.M SEC/CU.M IVUN UISTANCE

ALIMUTH HEIGHT EXPOSURE F/O
DEGREES METERS GH-SECICU.M SEC/CU.M IVUN UISTANCE

ALIMUTH HEIGHT EXPOSURE F/O
DEGREES METERS GH-SECICU.M SEC/CU.M IVUN UISTANCE

TUHER DATA FILLUH....

TEST DI JULY 7. 1967 0645 TU 0515 PST FLUURESCEIN RELFASE FROM ELFVITION OF ¿M OOOM AKC U $=1.2 \mathrm{M} / \mathrm{SEC}$ AT $2 \mathrm{M}$

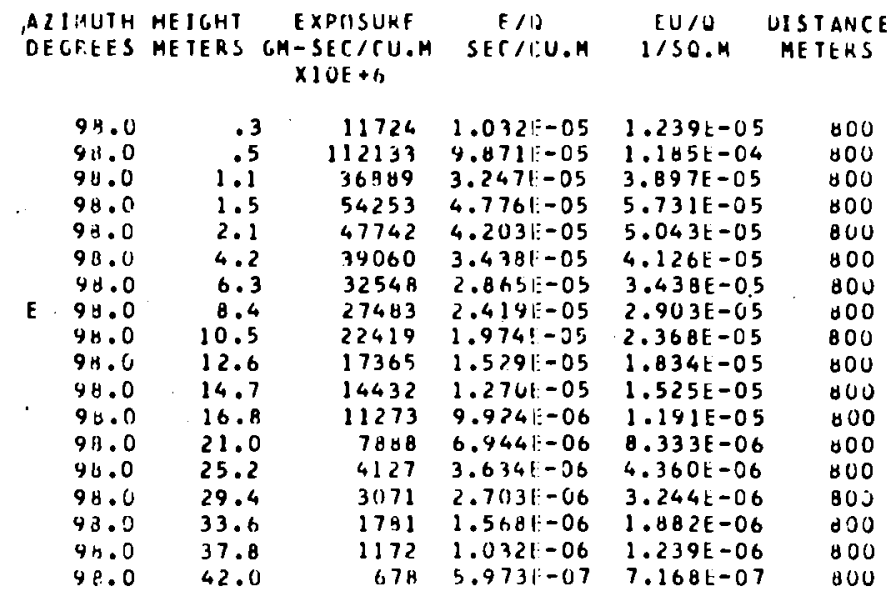

\begin{tabular}{|c|c|c|c|c|c|c|}
\hline \multicolumn{2}{|c|}{$\begin{array}{l}\text { ALIMUIH } \\
\text { DEG,KHES }\end{array}$} & $\begin{array}{l}\text { HEIGHT } \\
\text { ME TEKS }\end{array}$ & $\begin{array}{c}\text { EXPOSURF } \\
G M-S E C / C U . M \\
x 1 \cup F+6\end{array}$ & $\begin{array}{c}F / 6) \\
S[6 / 6, . M\end{array}$ & $\begin{array}{l}\text { EU/O } \\
1 / S U . M\end{array}$ & $\begin{array}{l}\text { DISTANCE } \\
\text { METERS }\end{array}$ \\
\hline E & 106.0 & .3 & 97100 & $0.555 E-05$ & $1.027 t-04$ & 400 \\
\hline & 1,h.? & .5 & $\begin{array}{r}97180 \\
105819\end{array}$ & $\begin{array}{l}4.5551-05 \\
4.51-05\end{array}$ & $1.027 E-04$ & 800 \\
\hline & $1 \mathrm{j} \theta .0$ & 1.5 & 1122298 & $\begin{array}{l}9.315 T-05 \\
4 . \forall B \leq E-U S\end{array}$ & $\begin{array}{l}1.118 E-04 \\
1.186 E-04\end{array}$ & $\begin{array}{l}000 \\
800\end{array}$ \\
\hline & 106.0 & 2.1 & 110138 & $9.6931-05$ & $1.163 E-04$ & 000 \\
\hline & 106.0 & 4.2 & (13541 & $7.7941-05$ & $9.353 k-05$ & 800 \\
\hline & $1 \cup 6.0$ & 6.3 & 90700 & $7.944 E-05$ & $9.5 \forall 1 E-05$ & 800 \\
\hline$F$ & 110.6 & 8.4 & 90700 & $7.9 A \div[-05$ & $9.581 t-05$ & 600 \\
\hline & 100.0 & 10.5 & 90700 & 7.5 H $4 E-05$ & $9.3 \theta 1 E-05$ & $\forall 00$ \\
\hline & 106.0 & 12.6 & B 2061 & $7.274 E-05$ & $8.669 E-05$ & 600 \\
\hline & 6.0 & 14.7 & $564<4$ & $4.9671-05$ & $5.960 t-65$ & $\forall 00$ \\
\hline & 100.0 & 16.8 & 46627 & $4.29 \cup E-05$ & $5.158 E-U 5$ & 800 \\
\hline & 106.0 & 21.0 & 26760 & $2.356 E-05$ & $2.827 t-05$ & 000 \\
\hline & 106.0 & 5.2 & 13980 & $1.231 E-05$ & $1.477 E-05$ & 800 \\
\hline & 106.0 & $.24,4$ & 6985 & $6.150 E-00$ & $7.379 t-06$ & $\forall 00$ \\
\hline & 1116.0 & 33.6 & 45,03 & $3.9651-06$ & $4.75 B E-06$ & d0u \\
\hline & 0.0 & 37.8 & 1437 & $1.2 \mathrm{~h}, 1-06$ & $1.540 t-06$ & 000 \\
\hline & 1.6 .0 & 42.0 & 1277 & $1.1 \geqslant 4 E-26$ & $1.349 t-06$ & 800 \\
\hline
\end{tabular}
$\times \perp 0 E+6$

\begin{tabular}{|c|c|c|c|c|c|}
\hline $\begin{array}{l}114.0 \\
114.0 \\
114.0 \\
114.0 \\
114.0 \\
114.0 \\
114.0 \\
114.0 \\
114.0 \\
1114.0 \\
114.0 \\
114.0 \\
114.0 \\
114.0 \\
114.0 \\
114.0 \\
114.0 \\
114.0\end{array}$ & $\begin{array}{l}.3 \\
.5 \\
1.1 \\
2.1 \\
1.5 \\
4.2 \\
6.3 \\
8.4 \\
10.5 \\
12.6 \\
14.7 \\
16.8 \\
21.0 \\
25.2 \\
29.4 \\
33.6 \\
37.8 \\
42.0\end{array}$ & $\begin{array}{l}14591 \\
13528 \\
16917 \\
17285 \\
16644 \\
19731 \\
19592 \\
27284 \\
31826 \\
29165 \\
21291 \\
18925 \\
11297 \\
9284 \\
5717 \\
4392 \\
2021 \\
927\end{array}$ & $\begin{array}{l}1.2 \mathrm{BHE}-05 \\
1.191 \mathrm{E}-05 \\
1.489 \mathrm{E}-05 \\
1.572 \mathrm{E}-05 \\
1.465 \mathrm{E}-05 \\
1.737 \mathrm{E}-05 \\
1.725 \mathrm{E}-05 \\
2.402 \mathrm{E}-05 \\
2.802 \mathrm{E}-05 \\
2.567 \mathrm{E}-05 \\
1.874 \mathrm{E}-05 \\
1.666 \mathrm{E}-05 \\
9.945 \mathrm{E}-06 \\
8.173 \mathrm{E}-06 \\
5.033 \mathrm{E}-06 \\
3.867 \mathrm{E}-06 \\
1.780 \mathrm{E}-06 \\
6.161 \mathrm{E}-07\end{array}$ & $\begin{array}{l}1.541 E-0 b \\
1.429 E-05 \\
1.767 t-05 \\
1.826 E-05 \\
1.75 H E-05 \\
2.084 t-05 \\
2.070 E-05 \\
2.862 E-05 \\
3.362 t-05 \\
3.081 E-05 \\
2.249 E-05 \\
1.999 E-05 \\
1.193 t-05 \\
9.807 t-06 \\
6.040 t-06 \\
4.640 E-06 \\
2.136 t-06 \\
9.794 t-07\end{array}$ & $\begin{array}{l}300 \\
800 \\
000 \\
000 \\
000 \\
800 \\
800 \\
600 \\
400 \\
800 \\
800 \\
800 \\
800 \\
000 \\
800 \\
800 \\
800 \\
800\end{array}$ \\
\hline
\end{tabular}

A7 I IJTH HEIGH

EXUNSURF

$F / 6$

EU/A UISTANCE DEGMIES MLIERS GH-SEC/CU.M

114.0
114.0
114.0
114.0
114.0
114.0
114.0
114.0
111.00
114.0
114.0
114.0
114.01
114.0
114.0
114.0
114.0
114.0

.3
.5
1.1
1.5
2.1
4.2
6.3
1.4
10.5
12.6
14.7
16.8
21.0
25.8
29.4
33.6
37.6
42.0
$x \mid$ Ut +

\begin{tabular}{|c|c|c|}
\hline $1307 \mathrm{~A}$ & $1.151 E-J 5$ & $1.38 a 5-05$ \\
\hline 12857 & $1 E-05$ & $8 t-05$ \\
\hline 14437 & $1.27 u t-0 s$ & $25 k-05$ \\
\hline 19525 & $1.719 \mathrm{~F}-05$ & $2.063 E-05$ \\
\hline $14 \mathrm{H}$ is 3 & $1.310 E-35$ & 1.5 \\
\hline 11498 & $1.312 \mathrm{~F}-15$ & $1.215 E-05$ \\
\hline 12857 & $1.131 \mathrm{E}-05$ & $1.358 t-05$ \\
\hline 16648 & 1.4 hyt -05 & $3[-05$ \\
\hline 24 & & -05 \\
\hline 18945 & $1.66 \cup \mathrm{E}-05$ & $2.001 E-05$ \\
\hline 17160 & $1.5 \cap Y E-05$ & $1.011 k-05$ \\
\hline 17416 & 1. Shut -2.5 & $1.382 E-05$ \\
\hline 10370 & $.124 E-06$ & $1.095 t-0 b$ \\
\hline $\begin{array}{l}9464 \\
6750\end{array}$ & $\begin{array}{l}0.335 t-00 \\
5.451 E-06\end{array}$ & $\begin{array}{l}1.000 E-05 \\
7.1416-06\end{array}$ \\
\hline$C O O A$ & $5.289[-J 6$ & $367 E-06$ \\
\hline 2713 & 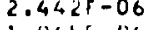 & 06 \\
\hline 1704 & $1.065 E=00$ & $.277 t-06$ \\
\hline
\end{tabular}




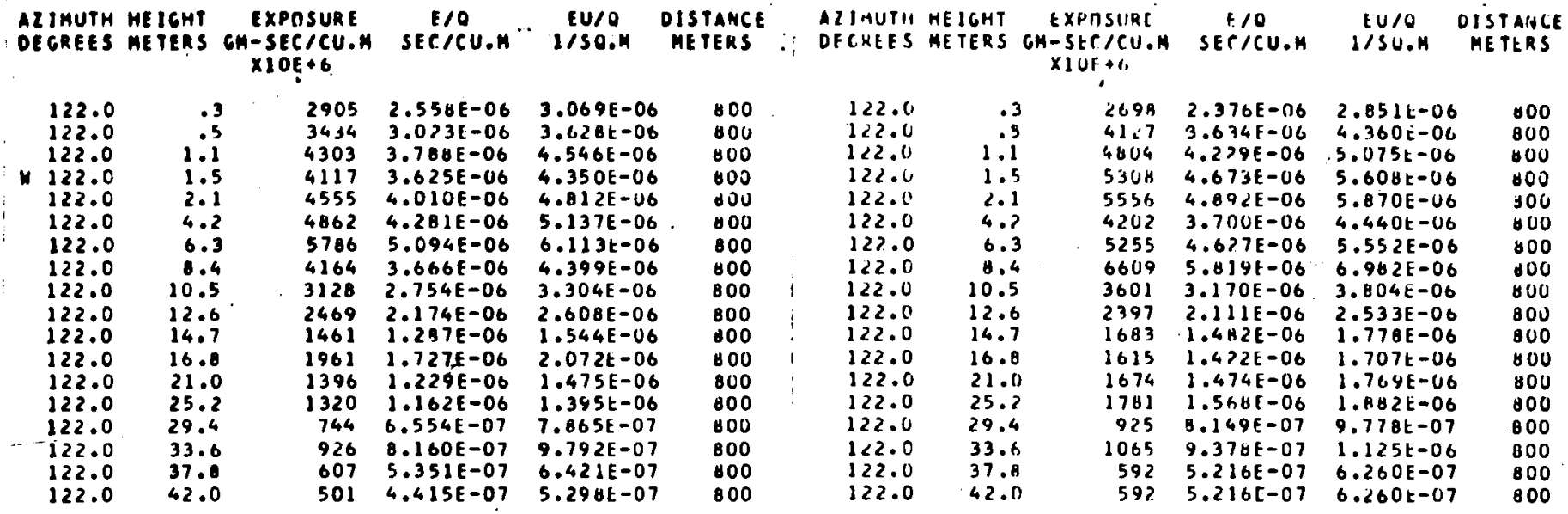

\section{AZIMUTH HEIGHT EXPOSURE
DEGREES METERS GM-SEC/CU.H SEC/CU.H I/SU.A DISTANCE $\times 10 E \div 6$}

$\begin{array}{rrrrrr}130.0 & .3 & 471 & 4.152 E-07 & 4.983 E-07 & 800 \\ 130.0 & .5 & 240 & 2.1212-07 & 2.545 E-07 & 800 \\ 130.0 & 1.1 & 699 & 6.161 E-07 & 7.393 E-07 & 800 \\ 130.0 & 1.5 & 403 & 3.553 E-07 & 4.264 E-07 & 800 \\ 130.0 & 2.1 & 717 & 6.319 E-07 & 7.582 E-07 & 800 \\ 130.0 & 4.2 & 935 & 8.233 E-07 & 9.880 E-07 & 800 \\ 130.0 & 6.3 & 3088 & 2.718 E-06 & 3.262 E-06 & 800 \\ 130.0 & 8.4 & 3691 & 3.250 E-06 & 3.899 E-06 & 800 \\ 130.0 & 10.5 & 2811 & 2.474 E-06 & 2.969 E-06 & 800 \\ 130.0 & 12.6 & 2409 & 2.121 E-06 & 2.545 E-06 & 800 \\ 130.0 & 14.7 & 1756 & 1.546 E-06 & 1.855 E-06 & 800 \\ 130.0 & 16.8 & 1088 & 9.582 E-07 & 1.150 E-06 & 800 \\ 130.0 & 21.0 & 775 & 6.823 E-07 & 8.187 E-07 & 600 \\ 130.0 & 25.2 & 1015 & 8.940 E-07 & 1.073 E-06 & 800 \\ 130.0 & 29.4 & 908 & 7.997 E-07 & 9.596 E-07 & 800 \\ 130.0 & 33.6 & 707 & 6.231 E-07 & 7.477 E-07 & 800 \\ 130.0 & 37.8 & 480 & 4.234 E-07 & 5.080 E-07 & 800 \\ 130.0 & 42.0 & 306 & 2.695 E-07 & 3.234 E-07 & 800\end{array}$

TOLER DATA FDLLLW....

TEST DI JULY 7. 1967 O O445 TO 0515 PST
ZINC SULFIDE RELEASE FROM ELFYATION OF $2 \mathrm{H}$ $1600 \mathrm{M} A R C$ U $1.2 \mathrm{M} / \mathrm{SEC} A \mathrm{~T}^{2 \mathrm{H}} 2 \mathrm{M}$

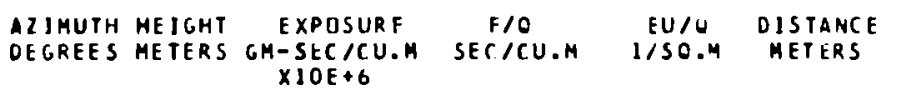

$\begin{array}{lrrrrr}98.0 & .2 & 3642 & 3.207 E-06 & 3.848 E-06 & 1600 \\ 98.0 & .4 & 3520 & 3.094 E-06 & 3.719 E-06 & 1000 \\ 98.0 & .8 & 4952 & 4.360 E-06 & 5.232 E-06 & 1600 \\ 94.0 & 1.5 & 3518 & 3.097 E-06 & 3.717 E-06 & 1600 \\ 98.0 & 1.6 & 4456 & 3.923 E-06 & 4.708 E-06 & 1600 \\ 98.0 & 3.1 & 4282 & 3.770 E-06 & 4.524 E-06 & 1600 \\ 94.0 & 6.2 & 3977 & 3.501 E-06 & 4.202 E-06 & 1600 \\ 98.0 & 9.3 & 3723 & 3.278 E-06 & 3.933 E-06 & 1600 \\ 98.0 & 12.4 & 2548 & 2.243 E-06 & 2.692 E-06 & 1600 \\ 98.0 & 15.5 & 2473 & 2.178 E-06 & 2.613 E-06 & 1600 \\ 98.0 & 18.6 & 1730 & 1.524 E-06 & 1.828 E-06 & 1600 \\ 98.0 & 21.7 & 1438 & 1.266 E-06 & 1.520 E-06 & 1600 \\ 98.0 & 24.8 & 853 & 7.511 E-07 & 9.013 E-07 & 1600 \\ 94.0 & 31.0 & 350 & 3.089 E-07 & 3.706 E-07 & 1600 \\ 94.0 & 37.2 & 135 & 1.194 E-07 & 1.433 E-07 & 1600 \\ 98.0 & 43.4 & 80 & 7.103 E-08 & 8.523 E-08 & 1600 \\ 98.0 & 49.6 & 46 & 4.056 E-08 & 4.867 E-08 & 1600 \\ 98.0 & 55.8 & 9 & 8.507 E-09 & 1.0211 E-08 & 1600 \\ 94.0 & 62.0 & 6 & 5.551 E-09 & 6.061 E-09 & 1600\end{array}$

AZ 1 MUTH
DEGKEES

130.0
130.0
130.0
135.0
130.0
130.0
139.0
130.0
130.0
130.0
130.0
130.0
135.0
130.0
130.0
130.0
130.0
130.0

\begin{tabular}{|c|c|}
\hline \multirow{2}{*}{\multicolumn{2}{|c|}{$\begin{array}{r}130.0 \\
130.0\end{array}$}} \\
\hline & \\
\hline 130.0 & 1.1 \\
\hline 130.0 & 1.5 \\
\hline 130.0 & 2.1 \\
\hline 130.0 & 4.2 \\
\hline 132.0 & 6.3 \\
\hline 130.0 & 8.4 \\
\hline 130.0 & 10.5 \\
\hline 130.0 & 12.6 \\
\hline 130.0 & 14.7 \\
\hline 130.0 & 16.8 \\
\hline 130.0 & 21.0 \\
\hline 130.0 & 25.2 \\
\hline 130.0 & 29.4 \\
\hline $\begin{array}{l}130.0 \\
130.0\end{array}$ & 33.6 \\
\hline 130.0 & \\
\hline 130.0 & 42.0 \\
\hline
\end{tabular}

EXPOSURE $X I O E+h$
EU/O OISTANCE SEC/OU.M 1/50.M METERS

$\begin{array}{rlll}420 & 3.703 E-07 & 4.444 E-07 & 800 \\ 263 & 2.316 E-07 & 2.779 E-07 & 800 \\ 313 & 2.757 E-07 & 3.309 E-07 & 800 \\ 474 & 4.176 E-07 & 5.011 E-67 & 800 \\ 649 & 6.068 E-07 & 7.281 E-07 & 800 \\ 474 & 4.176 E-07 & 5.011 E-07 & 800 \\ 2820 & 2.483 E-06 & 2.979 E-06 & 800 \\ 2467 & 2.167 E-06 & 2.601 E-06 & 800 \\ 2668 & 1.821 E-06 & 2.185 E-06 & 800 \\ 1781 & 1.564 E-06 & 1.682 E-06 & 000 \\ 1351 & 1.190 E-06 & 1.428 E-06 & 800 \\ 1280 & 1.127 E-06 & 1.352 E-06 & 800 \\ 592 & 5.216 E-07 & 6.260 E-07 & 800 \\ 1065 & 9.370 E-07 & 1.125 E-06 & 800 \\ 737 & 6.446 E-07 & 7.735 E-07 & 800 \\ 646 & 5.689 E-07 & 6.827 E-07 & 800 \\ 786 & 6.919 E-07 & 0.303 E-07 & 800 \\ 431 & 3.798 E-07 & 4.557 E-07 & 800\end{array}$

TULER UATA FILLLW....

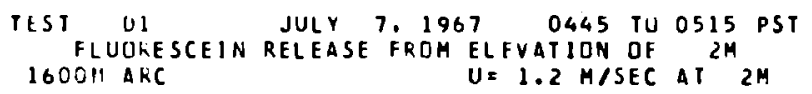

AZIIIUTH HEIGHT EXPTSURE F/O EU/O OISTANGE DEGFES METERS GM-SEC/CU.M SEC/CU.M I/SO.M METERS

\begin{tabular}{|c|c|c|c|}
\hline 94.0 & .2 & 2973 & $2.617 E-06$ \\
\hline $9 \pi .0$ & .4 & 3221 & $2.836 F-06$ \\
\hline $9 \mathrm{~m} .0$ & .8 & 3370 & $2.967 E-06$ \\
\hline 48. & 1.5 & 3667 & $3.279 \mathrm{E}-06$ \\
\hline 45.0 & 1.6 & 3320 & $2.923 E-06$ \\
\hline 90.0 & 3.1 & 2311 & $2.035 \mathrm{E}-06$ \\
\hline $\begin{array}{l}9 \text { त. } \\
98.0\end{array}$ & $\begin{array}{l}6.2 \\
9.3\end{array}$ & 3816 & $\begin{array}{l}3.3+0 E-06 \\
2.792 E-06\end{array}$ \\
\hline 90.2 & 12.4 & $\begin{array}{l}317 \\
1733\end{array}$ & $1.526 E-06$ \\
\hline $9+.0$ & 15.5 & 1390 & $1.224 E-06$ \\
\hline 98.0 & 18.6 & 1225 & $1.079 E-06$ \\
\hline $9+0$ & 21.7 & 266 & $2.345 E-07$ \\
\hline 98.0 & 24.8 & 486 & $4 . \geqslant 49 F-07$ \\
\hline $9 i .0$ & 31.0 & 252 & $2.221 E-07$ \\
\hline 98.0 & 37.2 & 160 & $1.410 \mathrm{E}-07$ \\
\hline $9 \mathrm{H} . \mathrm{O}$ & 43.4 & 171 & $1.514 E-07$ \\
\hline 98.0 & 49.6 & 42 & $3.743 E-08$ \\
\hline 90.0 & 55.8 & b & $5.406 E-09$ \\
\hline 98.0 & 62.0 & 0 & 0. \\
\hline
\end{tabular}

$3.141 t=06 \quad 1600$ $3.403 E-06 \quad 1000$ $3.560 \mathrm{E}-06 \quad 1000$ $3.874 E-06 \quad 1600$ $3.874 E-06$
$3.500 E-06$ $2.442 t-06 \quad 1000$ $4.032 E-06 \quad 1600$ $3.350 t-06 \quad 1600$ $1.831 \mathrm{E}-06 \quad 1600$ $1.469 E-06 \quad 1600$ $1.294 E-06 \quad 3600$ $2.815 E-07 \quad 1600$ $5.135 E-07 \quad 1600$ $2.665 \mathrm{E}-07 \quad 1600$ $1.692 \mathrm{E}-07 \quad 1600$ $1.816 E-07 \quad 1600$ $4.491 E-08 \quad 1600$ $6.487 \mathrm{E}-09 \quad 1600$ 1600 


ALIMUTH HE
DE CREES HE

106.0
106.0
106.0
106.0
106.0
106.0
106.0
106.0
106.0
106.0
106.0
106.0
106.0
106.0
106.0
106.0
106.0
106.0
106.0

T 6 EXPOSURE
GH-SEC/CU.H SEC/CU.M
XJOE+6

.2
.4
1.5
1.6
3.1
6.2
9.3
12.4
15.5
16.6
21.7
24.8
31.0
37.2
43.4
49.6
55.8
62.0

19187
2317
22927
26279

1.689E-0b $2.097 E-05$

$262792.313 E-05$

$26508 \quad 2.334 E-05$

$31433 \quad 2.767 E-05$

$20029 \quad 1.763 E-05$

$27758 \quad 2.444 E-05$

$257812.269 E-05$

$21596 \quad 1.901 E-05$

$210721.055 E-05$

$14379 \quad 1.266 E-05$

$11760 \quad 1.035 E-05$

$6005 \quad 5.296 E-06$

$3999 \quad 3.521 E-06$

$24912.193 \mathrm{~F}=06$

$376 \quad 3.318 E-07$

222

$1.957 \mathrm{E}-07$

EU/O DISTANCE

1/SO.M

$\begin{array}{ll}2.027 E-05 & 1600 \\ 2.516 E-05 & 1600 \\ 2.422 E-05 & 1600 \\ 2.776 E-05 & 1600 \\ 2.000 E-05 & 1600 \\ 3.320 E-05 & 1600 \\ 2.116 E-05 & 1600 \\ 2.932 E-05 & 1600 \\ 2.723 E-05 & 1600 \\ 2.281 E-05 & 1600 \\ 2.226 E-05 & 1600 \\ 1.519 E-05 & 1600 \\ 1.242 E-05 & 1600 \\ 6.344 E-06 & 1600 \\ 4.225 E-06 & 1600 \\ 2.632 E-06 & 1600 \\ 9.261 E-07 & 1600 \\ 3.961 E-07 & 1600 \\ 2.348 E-07 & 1600\end{array}$

AZIHUTH HEIGHT EXPQSURE

DEGREES METERS GM-SECICU H SIO

114.0
114.0

114.0

114.0

114.0

114.0

114.0

114.0

114.0

114.0

114.0

114.0

114.0

114.0

114.0

114.0

114.0

114.0

114.0 $\times 10 E+6$

.2
.4
.8
1.5
1.6
3.1
6.2
9.3
12.4
15.5
18.6
21.7
24.8
31.0
37.2
43.4
49.6
55.6
62.0$$
8951
$$

$110311.042 \mathrm{E}-05^{\circ}$

$\begin{array}{ll}11068 & 9.744 \mathrm{E}-06 \\ 13089 & 1.152 \mathrm{E}-05\end{array}$

$13192 \quad 1.161 \mathrm{E}-05$

$11178 \quad 9.840 E-06$

$13344 \quad 1.175 \mathrm{E}-05$

$12199 \quad 1.074 E-05$

$10416 \quad 9.169 \mathrm{E}-06$

$8376 \quad 7.374 E-06$

$.6889 \quad 6.063 E-06$

$5706 \quad 4.653 \mathrm{E}-06$

$3130 \quad 2.763 E-06$

$13531.191 \mathrm{E}-06$

$969 \quad 0.538 \mathrm{E}-07$

$597 \quad 5.256 E-07$

$14 \quad 1.249$ E-08

EU/O DISTANCE I/SO.M METERS $1.169 t-05$ $1.394 E-05$ $1.161 E-05$ $1.410 \mathrm{E}-05$ $1.404 t-05$ $1.269 E-05$ $1.100 E-05$ $0.849 t-06$ $7.278 \mathrm{t}-06$ $3.315 t-06$ $1.429 E-06$ $1.025 E-06$ $6.309 E-07$ $9.86 B E-08$

1600 1600
1600 1600

1600

1600

1600

1600

1600

1600

1600

1600

1600

1600
1600

1600

1600

1600

1600

$1.499 t-08$
$13295 \quad 1.17 U E-05$

\section{$9.455 \mathrm{E}-06$} $1.250 E-05$
Q 2 INUTH HEICHT DEGKEES METERS

EXPOSUKF F/U $x \perp \cup E+6$

100.0
106.0
106.0
106.0
106.0
106.0
106.0
106.0
106.0
106.0
106.0
106.0
106.0
106.0
106.0
106.0
106.0
106.0
106.0

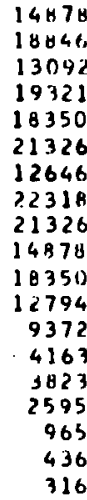

.2
.4
.8
1.5
1.6
3.1
6.2
9.3
12.4
15.5
18.6
21.7
24.8
31.0
37.2
43.4
49.6
55.8
62.0

$$
\begin{aligned}
& 1.310 E-05 \\
& 1.659 E-U 5 \\
& 1.153 E-05 \\
& 1.701 E-05 \\
& 1.615 E-05 \\
& 1.877 E-05 \\
& 1.113 E-05 \\
& 1.965 E-05 \\
& 1.877 E-05 \\
& 1.310 E-05 \\
& 1.615 E-35 \\
& 1.126 E-05 \\
& 8.250 E-06 \\
& 3.665 E-06 \\
& 3.366 E-06 \\
& 2.295 E-36 \\
& 0.50 J E-07 \\
& 3.843 E-07 \\
& 2.712 E-07
\end{aligned}
$$

AZ I AUTH HE IGHT $x \perp \cup E+6$

114.0
114.0
114.0
114.0
114.0
114.0
114.0
114.0
114.0
114.0
114.0
114.0
114.0
114.0
114.0
114.0
114.0
114.0
114.0

.2
.4
.8
1.5
1.6
3.1
6.2
9.3
12.4
15.5
18.1
21.7
24.8
31.0
37.2
43.4
49.6
55.8
62.0

$$
\begin{array}{r}
6247 \\
847 \\
758 \\
1056 \\
1136 \\
877 \\
11604 \\
952 \\
773 \\
741 \\
664 \\
684 \\
475 \\
292 \\
141 \\
989 \\
42 \\
169 \\
108
\end{array}
$$

$6247 \quad 5.444 E-06$ $84797.464 \mathrm{E}-106$ $\begin{array}{rl}7586 & 6.678 E-106 \\ 10562 & 9.298 E-16\end{array}$

$1136.69 .953 E-06$

$8776 \quad 7.776 E-06$

$11604 \quad 1.022 \mathrm{~F}-05$

$\$ 521 \quad B .3 \mathrm{BIE}=06$

6.AD9E- 36

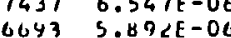

$68426.023 E-06$

4759 4.1B9E-06

$2923 \quad 2.574 E-06$

$1414 \quad 1.245 E-06$

989 8.708E-07

$422 \quad 3.716 \mathrm{E}-07$

$1.493 \mathrm{E}-07$

$9.5>3 E-08$ DEGIEES METERS GM-SECICU.M SECICU.M
EU/O UISTANCE 1/SO.M METERS

$1.572 t-05$

$1.383 t-05 \quad 1600$

$2.041 E-05,1600$

$1.938 E-05^{\circ} 1600$

$2.253 E-05 \quad 1600$

$1.336 E-05 \quad 1600$

$2.35 B E-05 \quad 1600$

$2.253 \mathrm{E}-05 \quad 1600$

$1.572 E-05 \quad 1600$

$1.938 E-05 \quad 1600$

$1.352 \mathrm{E}-05 \quad 1600$

$9.900 \mathrm{E}-06 \quad 1600$

$4.398 \mathrm{t}-06 \quad 1600$

$4.039 E-06 \quad 1600$

$2.742 \mathrm{c}-06 \quad 1600$

$1.020 E=06 \quad 1600$

$4.611 E-07 \quad 1000$

$3.339 E-07 \quad 1600$ $\begin{array}{cccc}\text { AZIMUTH HEIGHT EXPOSURE } & \text { F/O } & \text { EU/O, DISTANCE } \\ \text { DEGREES METERS GH-SEC/CU.H SECICU.M } & 1 / 50 . H \\ \text { XIOE+6 } & & \end{array}$

\begin{tabular}{l}
122.0 \\
122.0 \\
122.0 \\
\hline 122.0 \\
122.0 \\
122.0 \\
122.0 \\
122.0 \\
122.0 \\
122.0 \\
122.0 \\
122.0 \\
122.0 \\
122.0 \\
122.0 \\
122.0 \\
122.0 \\
122.0 \\
122.0
\end{tabular}

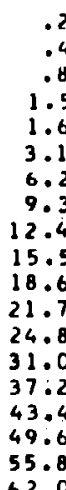

.2
.4
.8
1.5
1.6
3.1
6.2
9.3
12.4
15.5
18.6
21.7
24.8
31.0
37.2
43.4
49.6
55.8
62.0

$$
\begin{array}{r}
142 \\
394 \\
562 \\
3756 \\
5628 \\
3643 \\
5618 \\
4932 \\
3753 \\
2506 \\
1308 \\
1417 \\
1323 \\
368 \\
570 \\
215 \\
98 \\
48 \\
15
\end{array}
$$

$1421.250 E-07$

$1.500 \mathrm{E}-07$

$562 \quad 3.472 E-07 \quad 4.167 E-07$

$3756 \quad 3.307 E-06 \quad 3.969 E-06$

$5628 \quad 4.955 E-06 \quad 5.946 t-06$

$3643 \quad 3.207 E-06 \quad 3.848 E-061$

$5618 \quad 4.946 E-06 \quad 5.936 \mathrm{E}-06$

$4.342 E-06 \quad 5.210 E-06$

$753 \quad 3.304 E=06 \quad 3.965 t-06$

$2506 \quad 2.207 \mathrm{E}-06 \quad 2.648 \mathrm{E}-06$

$\begin{array}{lll}1308 & 1.152 E-06 & 1.382 E-06 \\ 1417 & 1.246 E-06 & 1.498 E-06\end{array}$

$13231.165 E-06$

$30301.398 E-06$

$3030.243 E-07 \quad 3.892 \mathrm{E}-07$

$215 \quad 1.894 E-07 \quad 2.273 \mathrm{~L}-07$

$98 \quad 1.691 E-08 \quad 1.043 E-07$

$48 \quad 4.253 E-08 \quad 5.104 E-08$

$15 \quad 1.388 E-08$

$1.665 E-08$
1600
1600
1000
1600
1600
1600
1600
1600
1600
1600
1600
1600
1600
1600
1600
1600
1600
1600
1600
A IIAUTH HEIGHT. EXPOSURF F/O DFCHEES HE IERS GH-SEC/CU

122.0
122.0
122.0
122.0
122.0
122.0
122.0
122.0
122.0
122.0
122.0
122.0
122.0
122.0
12210
122.0
122.0
122.0
122.0
$X 10 F+6$

.2
.4
.8
1.5
1.6
3.1
6.2
9.3
12.4
15.5
18.6
21.7
24.1
31.0
37.2
43.4
49.6
55.8
62.0

$\begin{array}{rrr}284 & 2.506 E-07 & 3 \\ 244 & 2.500 E-07 & 3 \\ 532 & 4.691 E-07 & 5 \\ 4312 & 3.796 E-06 & 4 \\ 4362 & 3.840 E-00 & 4 \\ 2477 & 2.131 E-06 & 2 \\ 3465 & 3.491 E-06 & 4 \\ 4015 & 3.534 E-06 & 4 \\ 1584 & 1.395 E-06 & 1 \\ 1886 & 1.661 E-06 & 1 \\ 705 & 6.213 E-07 & 7 \\ 941 & 1.292 E-07 & 9 \\ 753 & 6.629 E-07 & 7 \\ 287 & 2.533 E-07 & 3 \\ 330 & 2.907 E-07 & 3 \\ 216 & 1.909 E-07 & 2 \\ 119 & 1.056 E-07 & 1 \\ 68 & 5.988 E-08 & 7 \\ 23 & 2.058 E-08 & 2\end{array}$

$23 \quad 2.058 E-08$
EU/O DISTANCE 1/SO.H. METERS

$6.599 t-06$

0.014E-06 1600

$1.116 E-05.160 \mathrm{~J}$

$1.194 \mathrm{E}-0 \mathrm{~S} \quad 160 \mathrm{~J}$

$9.271 \mathrm{E}-06 \quad 1600$

$1.226 E-05 \quad 1600$

$1.006 \mathrm{E}-05 \quad 1600$

$8.171 E-06 \quad 1600$

$7.057 E-06 \quad 1600$

$7.071 \mathrm{t}-06 \quad 1600$

$7.228 \mathrm{~L}-06 \quad 1600$

$5.027 L-06 \quad 1000$

$3.088 E-06 \quad 1600$

$1.494 \mathrm{E}-06 \quad 1600$

$1.045 E-06 \quad 1600$

$4.461 E-07 \quad 1600$

$1.792 \mathrm{E}-07 \quad 1600$ 


\begin{tabular}{|c|c|c|c|c|c|}
\hline $\begin{array}{l}\text { AZIMUTH } \\
\text { DEGREES }\end{array}$ & $\begin{array}{l}\text { HE ICHT } \\
\text { METERS }\end{array}$ & $\begin{array}{l}\text { EXPOSURE } \\
\text { GH-SEC/CU.H } \\
\text { X1OE } 6\end{array}$ & $\begin{array}{l}E / O \\
\text { SEC/CU.N }\end{array}$ & $\begin{array}{l}\text { EU/U. } \\
\ldots 1 / 50 . \mathrm{H} \\
. .\end{array}$ & $\begin{array}{c}\text { DISTANCE } \\
\text { METERS }\end{array}$ \\
\hline $\begin{array}{l}130.0 \\
130.0 \\
130.0 \\
130.0 \\
130.0 \\
130.0 \\
130.0 \\
130.0 \\
130.0 \\
130.0 \\
130.0 \\
130.0 \\
130.0 \\
130.0 \\
130.0 \\
130.0 \\
130.0 \\
130.0 \\
130.0\end{array}$ & $\begin{array}{l}.2 \\
.4 \\
.6 \\
1.5 \\
1.6 \\
3.1 \\
6.2 \\
9.3 \\
12.4 \\
15.5 \\
18.6 \\
21.7 \\
24.8 \\
31.0 \\
37.2 \\
43.4 \\
49.6 . \\
55.8 \\
62.0\end{array}$ & $\begin{array}{r}601 \\
065 \\
1182 \\
772 \\
725 \\
794 \\
959 \\
042 \\
770 \\
764 \\
701 \\
660 \\
313 \\
228 \\
125 \\
122 \\
62 \\
43 \\
6\end{array}$ & $\begin{array}{l}5.298 E-07 \\
7.670 E-07 \\
1.041 E-06 \\
6.803 E-07 \\
6.387 E-07 \\
6.992 E-07 \\
8.442 E-07 \\
7.414 E-07 \\
6.850 E-07 \\
6.729 E-07 \\
6.171 E-07 \\
5.813 E-07 \\
2.756 E-07 \\
2.008 E-07 \\
1.104 E-07 \\
1.080 E-07 \\
7.242 E-08 \\
3.828 E-08 \\
5.551 E-09\end{array}$ & $\begin{array}{l}.6 .358 E-07 \\
9.144 E-07 \\
1.250 E-06 \\
8.164 E-07 \\
7.665 E-07 \\
1.391 E-07 \\
1.013 E-06 \\
1.897 E-07 \\
8.220 E-07 \\
8.075 E-07 \\
7.406 E-07 \\
6.975 E-07 \\
3.307 E-07 \\
2.409 E-07 \\
1.324 E-07 \\
1.296 E-07 \\
8.691 E-08 \\
4.594 E-08 \\
6.661 E-09\end{array}$ & $\begin{array}{l}1600 \\
1600 \\
1600 \\
1600 \\
1600 \\
1600 \\
1600 \\
1600 \\
1600 \\
1600 \\
1600 \\
1600 \\
1600 \\
1600 \\
1600 \\
1600 \\
1600 \\
1600 \\
1600\end{array}$ \\
\hline
\end{tabular}

OHER OATA FDLLOH....

TEST DI JULY 7,1967 O445 TO 0515 PST $3200 M$ ARC

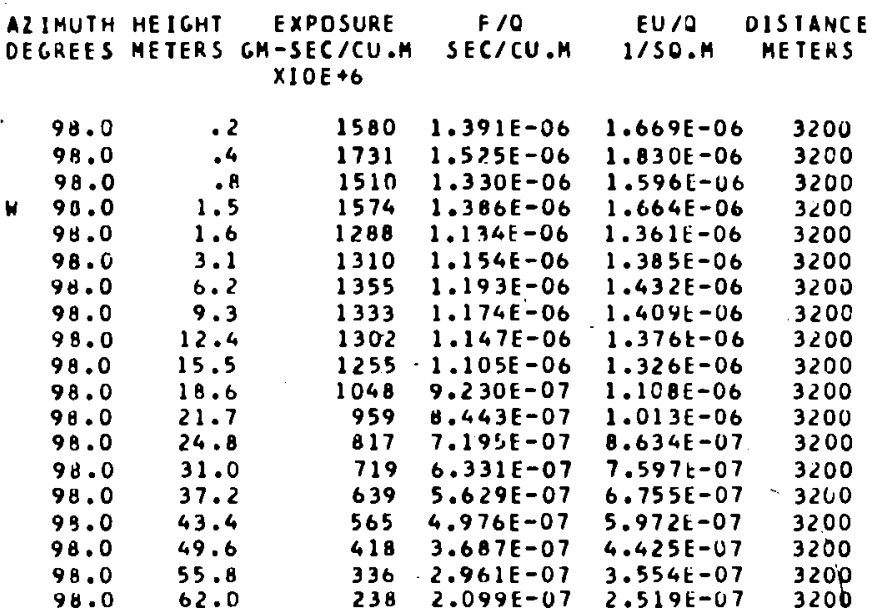

AZIMUTH HEJCHT EXPOSURE E/O EU/O UISTANCE DEGREES METERS CM-SECICU.M SECICU.H I/SO.M METERS $X 1 O E+6$

\begin{tabular}{|c|c|c|c|c|c|}
\hline 106.0 & .2 & $\begin{array}{l}2204 \\
2342\end{array}$ & $\begin{array}{l}1.941 \mathrm{~F}=06 \\
2.062 \mathrm{E}-06\end{array}$ & $2.329 t-06$ & 3200 \\
\hline $10 b .0$ & .8 & 2215 & $1.950 E-06$ & $2.340 E-06$ & 3200 \\
\hline 106.0 & 1.5 & 2064 & $1.817 \mathrm{E}-06$ & $2.181 E-06$ & 3200 \\
\hline 106.0 & 1.6 & 1215 & $1.070 E-06$ & $1.284 t-06$ & 3200 \\
\hline 106.0 & 3.1 & 1964 & $1.729 E-06$ & $2.075 t-66$ & 3200 \\
\hline 106.0 & 6.2 & 2671 & $2.352 E-06$ & $2.822 \mathrm{E}-06$ & $\$ 200$ \\
\hline 106.0 & $9 \cdot 3$ & 3524 & $102 E-06$ & $3.723 L-06$ & 3200 \\
\hline 106.0 & 12.4 & 3827 & $3.369 E-06$ & $4.043 \mathrm{E}-06$ & 3200 \\
\hline 106.0 & 15.5 & 3811 & $3.355 E-06$ & $4.026 E-06$ & 3200 \\
\hline 306.0 & .18 .6 & 3370 & $7 E-06$ & $1 E-06$ & 3200 \\
\hline 106.0 & 21.7 & 2780 & $.7 E-06$ & $2.937 t-06$ & 3200 \\
\hline 106.0 & 24.8 & 2594 & $21284 E-06$ & $2.741 E-06$ & 3200 \\
\hline 106.0 & 31.0 & 2316 & $2.039 E-06$ & $2.447 t-06$ & 3200 \\
\hline 106.0 & 37.2 & 1946 & $1.713 \mathrm{E}-06^{\circ}$ & $2.056 t-06$ & 3200 \\
\hline 106.0 & 43.4 & 1849 & $1.628 E-06$ & $1.954 E-06$ & 3200 \\
\hline 106.0 & 49.6 & 1845 & $1.624 E-06$ & $1.949 t-06$ & 3200 \\
\hline 106.0 & 55.8 & 1838 & $.619 E-06$ & $1.942 E-06$ & 3200 \\
\hline 106.0 & 62.0 & 61 & $384 E-0 B$ & $6.461 E-08$ & 3200 \\
\hline
\end{tabular}

AZIMUTH HEICHT EXPOSUKE F/D EU/U UISTANCE -SECICU.M SECICU.M IISO.M METERS

$\begin{array}{rr}132.0 & .2 \\ 130.0 & .4 \\ 130.0 & .4 \\ 130.0 & 1.5 \\ 130.0 & 1.6 \\ 130.0 & 3.1 \\ 130.0 & 6.2 \\ 130.0 & 9.3 \\ 130.0 & 12.4 \\ 130.0 & 15.5 \\ 130.0 & 18.6 \\ 130.0 & 21.7 \\ 130.0 & 24.8 \\ 130.0 & 31.0 \\ 130.0 & 37.2 \\ 130.0 & 43.4 \\ 130.0 & 49.6 \\ 130.0 & 55.8 \\ 130.0 & 62.0\end{array}$

$\begin{array}{llll}231 & 2.034 E-07 & 2.440 E-07 & 1600 \\ 535 & 4.716 E-07 & 5.654 E-07 & 1600 \\ 574 & 5.090 E-07 & 6.108 E-07 & 1600 \\ 729 & 6.421 E-37 & 7.755 E-07 & 1600 \\ 493 & 4.342 E-07 & 5.210 E-07 & 1600 \\ 823 & 7.253 E-07 & 8.703 E-07 & 1600 \\ 847 & 7.460 E-07 & 8.953 E-07 & 1600 \\ 571 & 5.078 E-07 & 6.033 E-07 & 1600 \\ 606 & 5.340 E-07 & 6.408 E-07 & 1600 \\ 556 & 4.903 E-07 & 5.884 E-07 & 1600 \\ 450 & 3.967 E-07 & 4.761 E-07 & 1600 \\ 599 & 5.277 E-07 & 6.333 E-07 & 1600 \\ 323 & 2.844 E-07 & 3.413 E-07 & 1600 \\ 280 & 2.470 E-07 & 2.964 E-07 & 1600 \\ 105 & 9.315 E-08 & 1.118 E-07 & 1600 \\ 91 & 8.068 E-08 & 9.681 E-08 & 1600 \\ 38 & 3.368 E-08 & 4.042 \mathrm{E}-08 & 1600 \\ 29 & 2.358 E-08 & 3.069 E-08 & 1600 \\ 12 & 1.102 E-08 & 1.322 E-08 & 1600\end{array}$

TULER DATA FDLLOW....

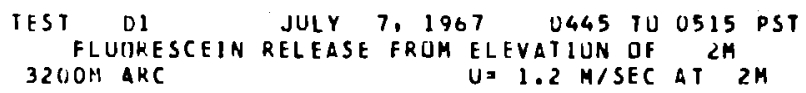




\begin{tabular}{|c|c|c|c|c|c|c|c|c|c|c|c|c|}
\hline $\begin{array}{l}2 \text { IMUTH } \\
\text { EGREES } \\
1\end{array}$ & $\begin{array}{l}\text { HE IGHT } \\
\text { METERS }\end{array}$ & $\begin{array}{l}\text { EXPQSURE } \\
\text { GM-SECICU.M } \\
\text { XIOE } 6\end{array}$ & $\begin{array}{c}F / O \\
\text { SEC } C / C U . M\end{array}$ & $\begin{array}{c}E U / 0 \\
1 / 50.4\end{array}$ & $\begin{array}{l}\text { DISTANCE } \\
\text { METERS }\end{array}$ & & $\begin{array}{l}\text { A2 l WUTH } \\
\text { DECNES }\end{array}$ & $\begin{array}{l}\text { HE IGIIT } \\
\text { ME TERS }\end{array}$ & $\begin{array}{l}\text { EXHOSIJKF } \\
\text { GH-SECICU.H } \\
X 1 \cup E+6\end{array}$ & $\begin{array}{c}\text { F/O } \\
\text { SECICU.M }\end{array}$ & $\begin{array}{l}E \cup / O \\
1 / S O . M\end{array}$ & $\begin{array}{l}\text { DIS IANCE } \\
\text { ME TERS }\end{array}$ \\
\hline $\begin{array}{l}114.0 \\
114.0 \\
114.0 \\
114.0 \\
114.0 \\
114.0 \\
114.0 \\
114.0 \\
114.0 \\
114.0 \\
114.0 \\
114.0 \\
114.0 \\
114.0 \\
114.0 \\
114.0 \\
114.0 \\
114.0 \\
114.0\end{array}$ & $\begin{array}{r}.2 \\
.4 \\
.8 \\
1.5 \\
1.6 \\
3.1 \\
6.2 \\
9.3 \\
12.4 \\
15.5 \\
18.6 \\
21.7 \\
24.8 \\
31.0 \\
37.2 \\
43.4 \\
49.6 \\
55.8 \\
62.0\end{array}$ & $\begin{array}{l}2123 \\
2012 \\
1791 \\
2016 \\
1829 \\
1855 \\
1786 \\
1753 \\
2115 \\
1673 \\
1477 \\
1386 \\
1303 \\
1148 \\
1156 \\
1202 \\
1274 \\
1407 \\
1491\end{array}$ & $\begin{array}{l}1.069 \mathrm{E}-06 \\
1.771 \mathrm{E}-06 \\
1.577 \mathrm{E}-06 \\
1.775 \mathrm{E}-06 \\
1.611 \mathrm{E}-06 \\
1.633 \mathrm{E}-06 \\
1.573 \mathrm{E}-06 \\
1.543 \mathrm{E}-06 \\
1.862 \mathrm{E}-06 \\
1.473 \mathrm{E}-06 \\
1.301 \mathrm{E}-06 \\
1.220 \mathrm{E}-06 \\
1.146 \mathrm{E}-06 \\
1.011 \mathrm{E}-06 \\
1.018 \mathrm{E}-06 \\
1.059 \mathrm{E}-06 \\
1.122 \mathrm{E}-06 \\
1.239 \mathrm{E}-06 \\
1.313 \mathrm{E}-06\end{array}$ & $\begin{array}{l}2.243 E-06 \\
2.126 E-06 \\
1.092 E-06 \\
2.130 E-06 \\
1.933 E-06 \\
1.960 E-06 \\
1.887 E-06 \\
1.852 E-06 \\
2.235 E-06 \\
1.768 E-06 \\
1.561 E-06 \\
1.465 E-06 \\
1.377 E-06 \\
1.213 E-06 \\
1.222 E-06 \\
1.271 E-06 \\
1.346 E-06 \\
1.467 E-06 \\
1.575 E-06\end{array}$ & $\begin{array}{l}3200 \\
3200 \\
3200 \\
3200 \\
3200 \\
3200 \\
3200 \\
3200 \\
3200 \\
3200 \\
3200 \\
3200 \\
3200 \\
3200 \\
3200 \\
3200 \\
3200 \\
3200 \\
3200\end{array}$ & . & $\begin{array}{l}114.0 \\
114.0 \\
114.0 \\
114.0 \\
114.0 \\
114.0 \\
114.0 \\
114.0 \\
114.0 \\
114.0 \\
114.0 \\
114.0 \\
114.0 \\
114.0 \\
114.0 \\
114.0 \\
114.0 \\
114.0 \\
114.0\end{array}$ & $\begin{array}{r}.2 \\
.4 \\
.8 \\
1.5 \\
1.6 \\
3.1 \\
6.2 \\
9.3 \\
12.4 \\
15.5 \\
18.6 \\
21.7 \\
24.8 \\
31.0 \\
37.2 \\
43.4 \\
49.6 \\
55.8 \\
62.0\end{array}$ & $\begin{array}{l}1760 \\
1760 \\
1614 \\
2684 \\
1690 \\
1619 \\
1514 \\
1302 \\
1373 \\
1408 \\
2092 \\
1835 \\
1857 \\
1686 \\
895 \\
100 ? \\
981 \\
1047 \\
1260\end{array}$ & $\begin{array}{l}1.550 E-06 \\
1.550 E-06 \\
1.476 E-06 \\
1.835 E-06 \\
1.486 F-06 \\
1.426 E-06 \\
1.333 E-06 \\
1.147 E-06 \\
1.209 E-06 \\
1.240 E-06 \\
1.842 E-06 \\
1.616 E-06 \\
1.635 E-06 \\
1.484 E-06 \\
7.8 B 4 E-07 \\
8.874 F-07 \\
1.636 E-07 \\
9.576 E-07 \\
1.177 E-06\end{array}$ & $\begin{array}{l}1.860 E-06 \\
1.860 E-06 \\
1.711 E-06 \\
2.202 E-06 \\
1.786 E-06 \\
1.711 E-06 \\
1.599 E-06 \\
1.376 E-06 \\
1.450 E-06 \\
1.488 E-06 \\
2.210 E-06 \\
1.939 E-06 \\
1.962 E-06 \\
1.781 E-06 \\
9.461 E-07 \\
1.059 E-06 \\
1.036 E-06 \\
1.149 E-06 \\
1.352 E-06\end{array}$ & $\begin{array}{l}3200 \\
3200 \\
3200 \\
3200 \\
3200 \\
3200 \\
3200 \\
3200 \\
3200 \\
3200 \\
3200 \\
3200 \\
3200 \\
3200 \\
3200 \\
3200 \\
3200 \\
3200 \\
3200\end{array}$ \\
\hline
\end{tabular}

\begin{tabular}{|c|c|c|c|c|c|c|c|c|c|c|c|}
\hline $\begin{array}{l}\text { AZ IMUTH } \\
\text { DEGKEES }\end{array}$ & $\begin{array}{l}\text { HE IGHT } \\
\text { HE TERS }\end{array}$ & $\begin{array}{c}\text { EXPOSURE } \\
\text { GN-SEC/CU.H } \\
\text { X1OE }+6\end{array}$ & $\begin{array}{c}\text { E/O } \\
\text { SECICU.M }\end{array}$ & $\begin{array}{l}\text { EU/O } \\
\text { IISO.H }\end{array}$ & $\begin{array}{l}\text { DISTANCE } \\
\text { METERS }\end{array}$ & $\begin{array}{l}\text { ALIIAIITH } \\
\text { DFC,KEES }\end{array}$ & $\begin{array}{l}\text { HE IGHT } \\
\text { AE TERS }\end{array}$ & $\begin{array}{l}\text { EXPOSUKE } \\
\text { GM-SEC/CU.H } \\
\times 10 F+t\end{array}$ & $\begin{array}{c}E / \theta \\
S E C / C U . M\end{array}$ & $\begin{array}{l}E U / 0 \\
1 / 50 . M\end{array}$ & $\begin{array}{l}\text { DISTANCE } \\
\text { METERS }\end{array}$ \\
\hline $\begin{array}{l}122.0 \\
122.0 \\
122.0 \\
1122.0 \\
122.0 \\
122.0 \\
122.0 \\
122.0 \\
122.0 \\
122.0 \\
122.0 \\
122.0 \\
122.0 \\
122.0 \\
122.0 \\
122.0 \\
122.0 \\
122.0 \\
122.0\end{array}$ & $\begin{array}{r}.2 \\
.4 \\
.8 \\
1.5 \\
1.6 \\
3.1 \\
6.2 \\
9.3 \\
12.4 \\
15.5 \\
18.6 \\
21.7 \\
24.8 \\
31.0 \\
37.2 \\
43.4 \\
49.6 \\
55.8 \\
62.0\end{array}$ & $\begin{array}{r}967 \\
1004 \\
928 \\
900 \\
901 \\
990 \\
1122 \\
1127 \\
833 \\
574 \\
415 \\
444 \\
367 \\
433 \\
368 \\
321 \\
306 \\
366 \\
442\end{array}$ & $\begin{array}{l}8.696 E-07 \\
8.639 E-07 \\
8.175 E-07 \\
7.924 E-07 \\
7.933 E-07 \\
6.772 E-07 \\
9.880 E-07 \\
9.922 E-07 \\
7.333 E-07 \\
5.059 E-07 \\
3.65 \forall E-07 \\
3.916 E-07 \\
3.410 E-07 \\
3.818 E-07 \\
3.247 E-07 \\
2.831 E-07 \\
2.700 E-07 \\
3.222 E-07 \\
3.894 E-07\end{array}$ & $\begin{array}{l}1.043 E-06 \\
1.061 E-06 \\
9.010 E-07 \\
9.509 E-07 \\
9.519 E-07 \\
1.047 E-06 \\
1.166 E-06 \\
1.191 E-06 \\
8.799 E-07 \\
6.071 E-07 \\
4.389 E-07 \\
4.699 E-07 \\
4.092 E-07 \\
4.582 E-07 \\
3.896 E-07 \\
3.397 E-07 \\
3.240 E-07 \\
3.867 E-07 \\
4.673 E-07\end{array}$ & $\begin{array}{l}3200 \\
3200 \\
3200 \\
3200 \\
3200 \\
3200 \\
3200 \\
3200 \\
3200 \\
3200 \\
3200 \\
3200 \\
3200 \\
3200 \\
3200 \\
3200 \\
3200 \\
3200 \\
3200\end{array}$ & $\begin{array}{l}122.01 \\
122.0 \\
122.0 \\
122.0 \\
122.0 \\
122.0 \\
122.0 \\
122.0 \\
122.0 \\
122.0 \\
122.0 \\
122.0 \\
122.0 \\
122.0 \\
122.0 \\
122.0 \\
122.0 \\
122.0 \\
122.0\end{array}$ & $\begin{array}{r}.7 \\
.4 \\
.8 \\
1.5 \\
1.6 \\
3.1 \\
6.8 \\
9.3 \\
12.4 \\
13.5 \\
18.6 \\
21.7 \\
24.6 \\
31.0 \\
37.2 \\
43.4 \\
49.6 \\
55.8 \\
62.0\end{array}$ & $\begin{array}{l}831 \\
131 \\
756 \\
916 \\
746 \\
906 \\
768 \\
406 \\
396 \\
446 \\
340 \\
372 \\
273 \\
267 \\
318 \\
283 \\
286 \\
273 \\
277\end{array}$ & $\begin{array}{l}7.370 E-07 \\
7.370 E-07 \\
6.661 E-07 \\
1.072 E-07 \\
6.567 E-07 \\
7.97 H E-07 \\
6.943 E-07 \\
7.978 E-07 \\
5.251 E-07 \\
3.934 E-07 \\
2.944 E-07 \\
3.176 E-07 \\
2.411 E-07 \\
2.355 E-07 \\
2.806 E-07 \\
2.496 E-07 \\
2.524 E-07 \\
2.411 E-07 \\
2.439 E-07\end{array}$ & $\begin{array}{l}8.764 t-07 \\
6.764 E-07 \\
7.994 E-07 \\
9.686 E-07 \\
7.881 E-07 \\
9.573 t=07 \\
6.332 E-07 \\
9.573 t-07 \\
6.301 t-07 \\
4.721 E-07 \\
3.593 t-07 \\
3.931 E-07 \\
2.893 E-07 \\
2.826 E-07 \\
3.367 E-07 \\
2.995 E-07 \\
3.029 E-07 \\
2.893 E-07 \\
2.927 E-07\end{array}$ & $\begin{array}{l}3200 \\
3200 \\
3200 \\
3200 \\
3200 \\
3200 \\
3200 \\
3200 \\
3200 \\
3200 \\
3200 \\
3200 \\
3200 \\
3200 \\
3200 \\
3200 \\
3200 \\
3200 \\
3200\end{array}$ \\
\hline
\end{tabular}

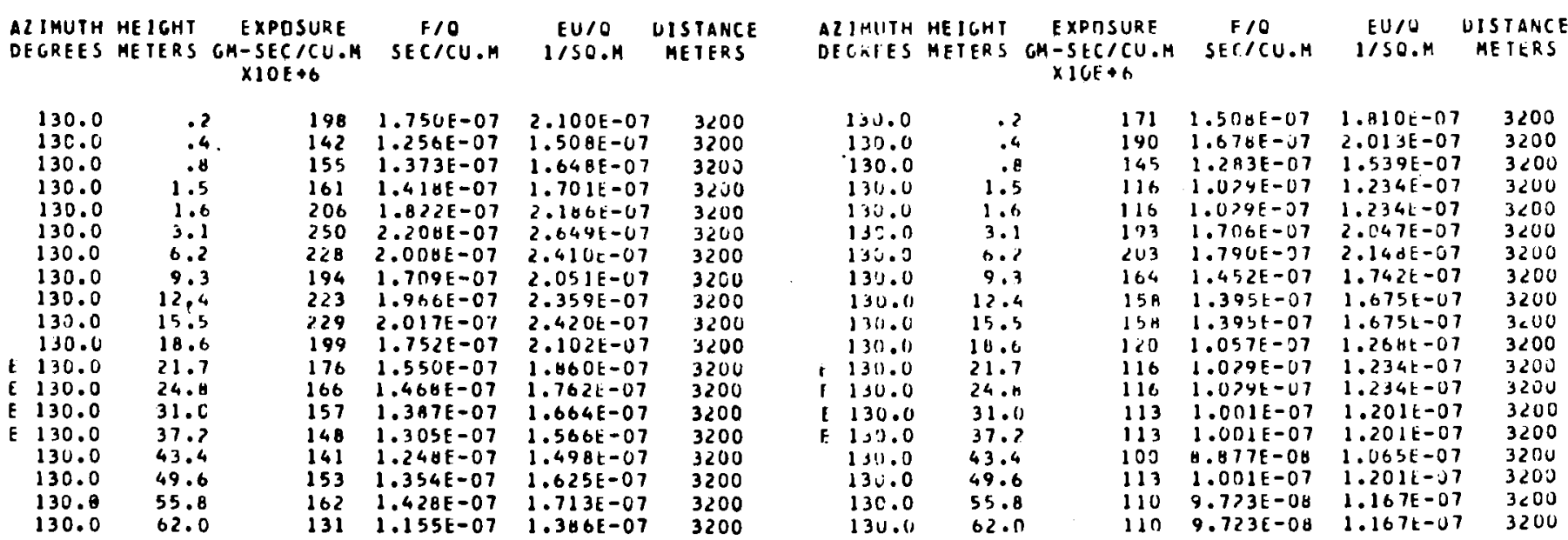




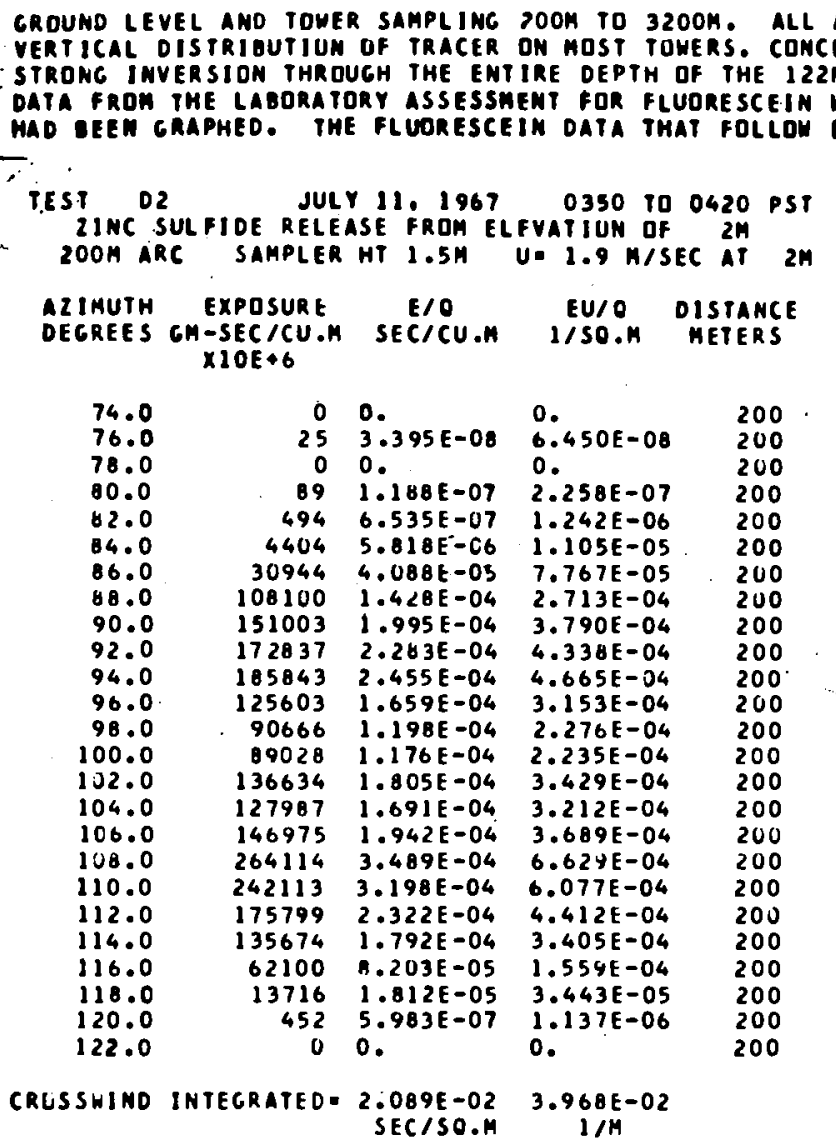

TEST D2 JULY 11.1967 0350 TU 0420 PST. 2 INC SULFIOE RELEASE FRDM ELEVATION OF $2 M$
OOOM ARC SAMPLER HT $1.5 M \quad$ U. 1.9 MISEC AT $2 M$

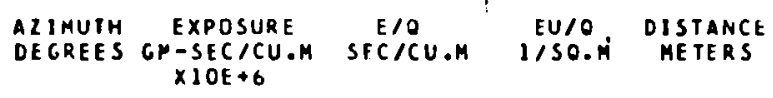

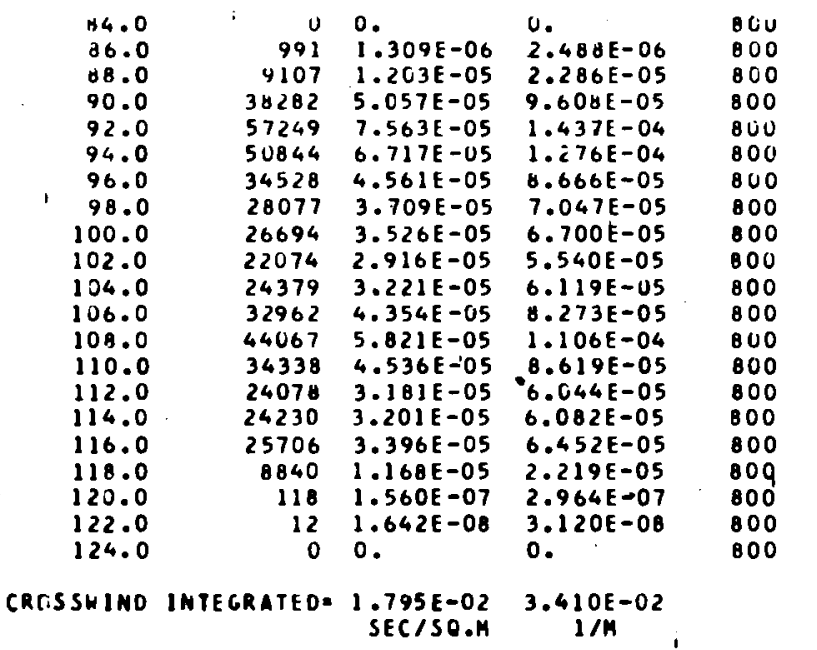

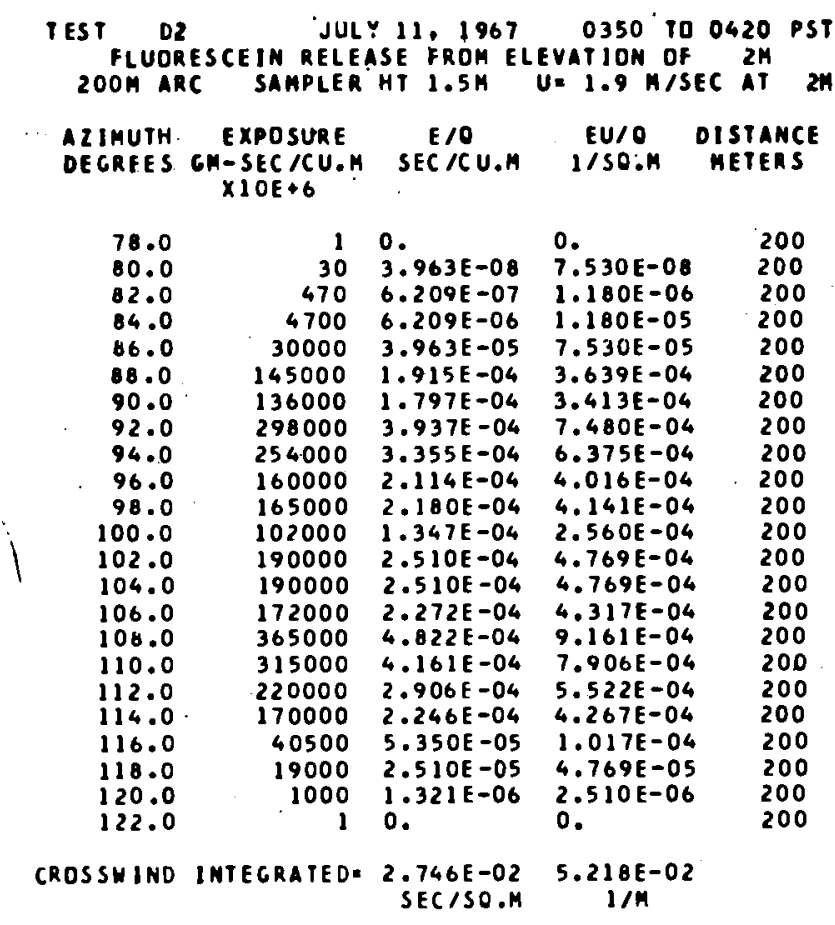

TEST D2 JULY11.1967 0350 TO 0420 PST FLUORESCEIN RELEASE FROA ELEVATION OF $2 \mathrm{M}$
OOM ARC SAMPLER HT $1.5 \mathrm{U}$ UIF $1.9 \mathrm{M} / \mathrm{SEC}$ AT $2 \mathrm{M}$ $\begin{array}{lccc}\text { AZIMUTH EXPOSURE } & \text { EIO } & \text { EU/O OISTANCE } \\ \text { DECREES CM-SECICU.M SECICU.M IISO.M METERS }\end{array}$ $\times 10 E+6$

$\therefore 4.0$

84.0

88.0

90.0

92.0

94.0

9.6 .0

100.0

102.0

104.0

106.0

108.0

110.0

112.0

114.0

116.0

118.0

120.0

122.0
124.0

10.0000

CROSSWIND INTEGRATED $2.214 E-02$ 4.207E-02

800

800

800

800

800

000

800

800
600

80

800
800
800

800

800

$B 00$

800

800

800

800 
TEST D2 JULY $11.1967 \quad 0350$ TU 0420 PST 2INC SULFIDE RELEASE FROM ELEVATION OF $2 \mathrm{H}$ $1600 M$ ARC SAMPLER HT $1.5 \mathrm{M} U=1.9 \mathrm{M} / \mathrm{SEC}$ AT $2 \mathrm{M}$

AZIMUTH EXPOSURE
DEGRES EM-SEC/CU.M
XIOE+6

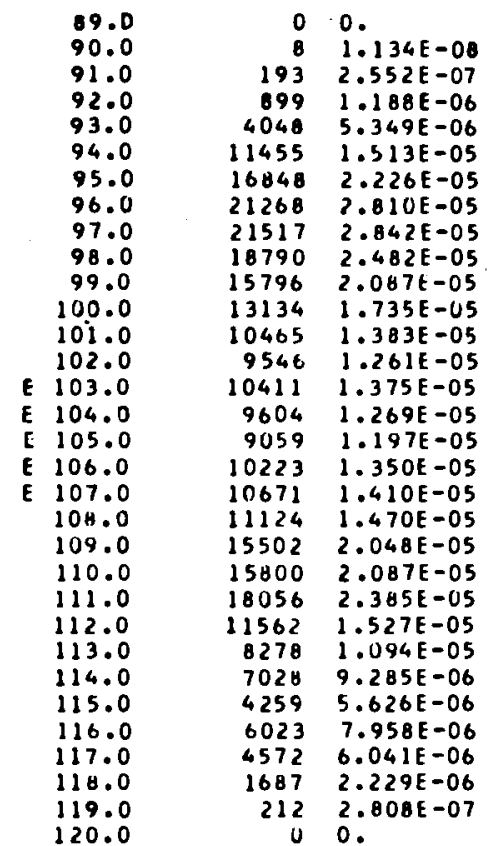

0.

1600

$2.250 \mathrm{E}-06 \quad 1600$

$1.016 \mathrm{E}-05 \quad 1600$

$2.875 E-05 \quad 1600$

$4.229 E-05 \quad 1600$

$5.33 d E-05 \quad 1600$

$5.401 E-05 \quad 1600$

$4.716 \mathrm{E}-05 \quad 1600$

$3.965 \mathrm{~F}-05 \quad 1600$

$3.297 E-05 \quad 1600$

$2.627 E-05 \quad 1600$

$2.396 E-05 \quad 1600$

$2.613 E-05 \quad 1600$

$2.411 E-05 \quad 1600$

$2.274 E-05 \quad 1600$

$2.566 \mathrm{E}-05 \quad 1600$

$2.674 \mathrm{E}-05 \quad 1600$

$2.792 \mathrm{E}-05 \quad 1600$

$3.891 E-05 \quad 1600$

$3.966 E-05 \quad 1600$

$4.532 E-05 \quad 1600$

$2.902 E-05 \quad 1600$

$2.078 E-05 \quad 1600$

$1.764 \mathrm{E}-05 \quad 1600$

$1.069 E-05 \quad 1600$

$1.512 \mathrm{E}-05 \quad 1600$

$1.148 E-05 \quad 1600$

$4.235 E-06 \quad 1600$

$5.334 \mathrm{E}-07 \quad 1600$

CROSSHINO INTEGRAIEUE $1.099 E-02$ 2.089E-02 SEC/SO.M
TEST D2 D2 JULY 11,1967 CEIN RELEASE FROM ELEVATION OF $2 \mathrm{M}$ $1600 M$ ARC SAMPLER HT $1.5 \mathrm{M} U=1.9 \mathrm{M} / \mathrm{SEC}$ AT $2 \mathrm{H}$

$\begin{array}{lcc}\text { AZIMUTH EXPOSURE } & \text { E/O } & \text { EU/O OISTANCE } \\ \text { DEGRES GM-SEC/CU.M SECICU.M } & \text { I/SO.M METERS }\end{array}$ $\times 10 E+6$

89.0
90.0
91.0
92.0
93.0
94.0
95.0
96.0
97.0
98.0
99.0
100.0
101.0
102.0
103.0
104.0
105.0
1106.0
107.0
108.0
109.0
110.0
111.0
112.0
113.0
114.0
115.0
116.0
117.0
118.0
119.0
120.0

$\begin{array}{rl}1 & 0 . \\ 50 & 6.605 E-08 \\ 260 & 3.435 E-07 \\ 930 & 1.229 E-06 \\ 4200 & 5.548 E-06 \\ 9700 & 1.281 E-05 \\ 19500 & 2.576 E-05 \\ 19500 & 2.576 E-05 \\ 24500 & 3.236 E-05 \\ 21000 & 2.774 E-05 \\ 18000 & 2.378 E-05 \\ 12200 & 1.612 E-05 \\ 10400 & 1.374 E-05 \\ 10800 & 1.427 E-05 \\ 11000 & 1.453 E-05 \\ 11000 & 1.453 E-05 \\ 11500 & 1.519 E-05 \\ 11000 & 1.453 E-05 \\ 13000 & 1.717 E-05 \\ 13500 & 1.783 E-05 \\ 16300 & 2.153 E-05 \\ 15800 & 2.087 E-05 \\ 16800 & 2.219 E-05 \\ 12800 & 1.691 E-05 \\ 9700 & 1.281 E-05 \\ 6500 & 8.587 E-06 \\ 4500 & 5.945 E-06 \\ 7000 & 9.247 E-06 \\ 4800 & 6.341 E-06 \\ 2150 & 2.840 E-06 \\ 250 & 3.303 E-07 \\ 1 & 0 .\end{array}$

0 .

1.255E-07

$6.526 \mathrm{E}-07$

$2.334 E-06$

$1.054 E-05$

$2.435 E-05$

$4.894 E-05$

4.894 E-05

$6.149 E-05$

$5.271 E-05$

$4.518 E-05$

$3.062 E-05$

$2.610 E-05$

2.711E-05

$2.761 E-05$

2.761E-05

$2.806 E-05$

$2.761 E-05$

$3.263 E-05$

$3.388 E-05$

$4.091 E-05$

$3.966 \mathrm{E}-05$

$4.217 E-05$

$3.213 E-05$

$2435 E-05$

1.631E-05

$1.129 \mathrm{E}-05$

$1.757 E-05$

$1.205 E-05$

$5.396 E-06$

6 .

1600

1600

1600

1600

600

1600

1600

1600

1600

1600

1600

1600
1600

1600

1600

1600

1600

1600

1600
1600

1600

1600

1600

1600
1600

1600

1600

1600

1600

CROSSHIND INTEGRATEO= $1.175 E-02 \quad 2.233 E-02$
TEST 02 JULY 11, $1967 \quad 0350 \quad$ TO 0420 PST ZIMC SULFIDE RELEASE BROM ELEVATIUN OF $2 \mathrm{M}$ $3200 \mathrm{M}$ ARC SAMPLERHT $1.5 \mathrm{M}$ U. $1.9 \mathrm{M} / \mathrm{SEC}$ AT $2 \mathrm{M}$
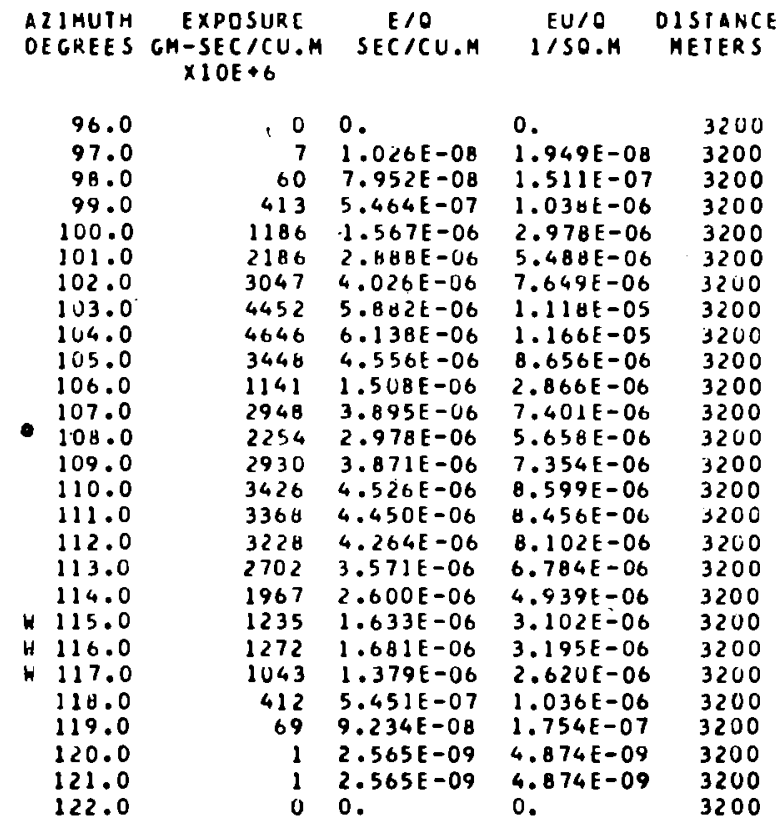

0.

$1.949 \mathrm{E}-08$

$1.511 \mathrm{E}-07$

$1.038 \mathrm{E}-06$

$2.978 \mathrm{E}-06$

$5.488 E-06$

$7.649 \mathrm{E}-06$

1.11 t -05

$1.166 E-05$

$8.656 \mathrm{E}-06$

$2.866 \mathrm{E}-06$

$7.401 E-06$

$5.658 \mathrm{E}-06$

$7.354 \mathrm{E}-06$

$8.599 \mathrm{E}-06$

$8.456 E-06$

B. $102 E-06$

$6.784 E-06$

$4.939 t-06$

$3.102 E-06$

$3.195 E-06$

$2.620 \mathrm{E}-06$

$1.036 \mathrm{E}-06$

$1.754 E-07$

$4.874 E-09$

$4.874 E-09$

0 .

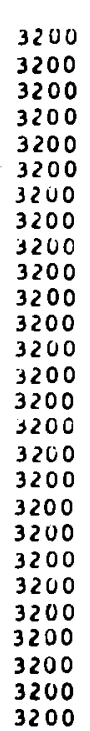

3200
3200
3200
3200
3200
3200
3200
3200
3200
3200
3200
3200
3200
3200
3200
3200
3200
3200
3200
3200
3200
3200
3200
3200
3200
3200
3200

CROSSHIND INTEGRATED= $\begin{gathered}3.501 E-03 \\ \text { SEC/SO.M }\end{gathered}$

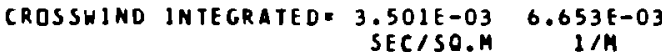

SECISO.M
TEST D2 DULY 11 . 1967 T 0350 TO 0420

0350 TO 0420 PST $3200 \mathrm{M}$ ARC SAHPLER HT $1.5 \mathrm{M} U=1.9 \mathrm{M} / \mathrm{SEC}$ AT $2 \mathrm{H}$

$\begin{array}{lcc}\text { ALIMUTH EXPOSURE } & \text { E/O } & \text { EU/O OISTANCE } \\ \text { OEGRES CH-SEC/CU.K SECICU.M I/SO.M METERS }\end{array}$ $X 10 E+6$

$$
\begin{array}{r}
97.0 \\
98.0 \\
99.0 \\
100.0 \\
101.0 \\
102.0 \\
103.0 \\
104.0 \\
105.0 \\
106.0 \\
107.0 \\
108.0 \\
109.0 \\
110.0 \\
111.0 \\
112.0 \\
113.0 \\
114.0 \\
115.0 \\
116.0 \\
117.0 \\
118.0 \\
119.0 \\
120.0 \\
121.0
\end{array}
$$

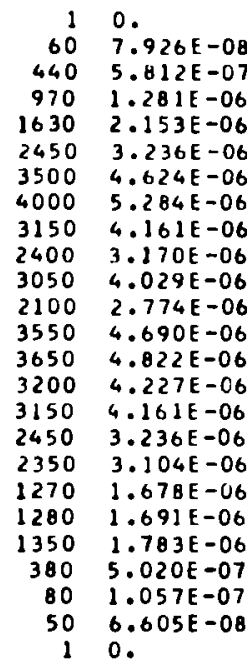

CROSSHIND INTEGRATED= $3.431 E-03 \quad 6.520$ SE-03
0.

$1.506 E-07$

$1.104 E-06$

$2.435 E-06$

$4.091 E-06$

$6.149 E-06$

8.785E-06

$1.004 E-05$

$7.906 E-06$

$6.024 E-06$

$7.655 \mathrm{E}-06$

$5.271 E-06$

8.910E-06

$9.161 E-06$

$8.032 E-06$

$7.906 E-06$

$6.149 \mathrm{E}-06$

$5.898 E-06$

$3.188 E-06$

$3.213 E-06$

$3.388 E-06$

$9.538 \mathrm{E}-07$

$2.008 E-07$

3200
3200
3200
3200
3200
3200
3200
3200
3200
3200
3200
3200
3200
3200
3200
3200
3200
3200
3200
3200
3200
3200
3200
3200
3200

$1 / \mathrm{M}$

$+2$

$$
\text { ( }
$$


TOHER DATA FOLLDH....

TEST D2 JULY 11,1967 O 0350 TU O420 PST 2 INC SULFIDE RELEASE FROM ELEVATION OF
$2 \mathrm{U}$. $1.9 \mathrm{M}$ MSEC AT $2 \mathrm{M}$

AZIKUTH HEIGHT DEGREES METERS GM EXPOSUR $-5 L C / C l$
$X 2 O E+6$

$\begin{array}{ll}98.0 & \\ 98.0 & \\ 98.0 & 1 . \\ 98.0 & 1.5 \\ 98.0 & 2 . \\ 98.0 & 4 . \\ 98.0 & 5.4 \\ 98.0 & 6.0 \\ 98.0 & 8 . \\ 98.0 & 9.5 \\ 98.0 & 10 . \\ 98.0 & 13 . \\ 98.0 & 16 . \\ 98.0 & 18.9 \\ 98.0 & 21.0 \\ 98.0 & 24 . \\ 98.0 & 27.0\end{array}$

$\begin{array}{rl}23684 & 3.129 E-05 \\ 22675 & 2.995 E-05 \\ 14815 & 1.957 E-05 \\ 90666 & 1.198 E-04 \\ 125116 . & 1.653 E-04 \\ 178714 & 2.361 E-04 \\ 206449 & 2.727 E-04 \\ 164811 & 2.177 E-04 \\ 185452 & 2.450 E-04 \\ 165867 & 2.191 E-04 \\ 140411 & 1.855 E-04 \\ 41314 & 5.458 E-05 \\ 7351 & 9.711 E-06 \\ 566 & 7.487 E-07 \\ 38 & 5.085 E-08 \\ 0 & 0 . \\ 0 & 0 .\end{array}$

FU/U DISTANCE

$E / 0$ $1 / 50.4$

$5.945 t-05$ $5.691 E-05$ $3.718 E-05$ $2.276 E-04$ $3.140 E-04$ $4.486 E-04$ $5.182 E-04$ $4.137 E-04$ $4.655 E-04$ $4.163 E-04$ $3.524 E-04$ $1.037 \mathrm{E}-04$ $1.845 E-05$ $1.423 E-06$ $9.661 E-0 B$ 0.

200
200
200
200
200
200
200
200
200
200
200
200
200
200
200
200
200

AZIMUTH HEIGHT DEGKEES METERS GEXPOSURE $X E C / C$
$X I O E+6$

$\begin{array}{lr}106.0 & .3 \\ 106.0 & .7 \\ 106.0 & 1.4 \\ 106.0 & 1.5 \\ 106.0 & 2.7 \\ 106.0 & 4.1 \\ 106.0 & 5.4 \\ 106.0 & 6.8 \\ 106.0 & 8.1 \\ 106.0 & 9.5 \\ 106.0 & 10.8 \\ 106.0 & 13.5 \\ 106.0 & 16.2 \\ 106.0 & 18.9 \\ 106.0 & 21.6 \\ 106.0 & 24.3 \\ 106.0 & 27.0\end{array}$

$$
\begin{array}{r}
53433 \\
49735 \\
52980 \\
146975 \\
189773 \\
236672 \\
236243 \\
163436 \\
194187 \\
145057 \\
123157 \\
30603 \\
23859 \\
6195 \\
1795 \\
118
\end{array}
$$

$1.942 \mathrm{E}-04$

$1897732.507 E-04$

$2366723.126 E-04$

$1634362.159 E-04$

145057 b. $16 E-04$

$123157 \quad 1.627 E-04$

$30603 \quad 4.043 E-09$

$238593.152 E-J 5$

$6195 \quad 8.184 \mathrm{E}-06$

$17952.371 \mathrm{E}-0 \mathrm{~B}$

$1.567 E-07$

$7.544 E-09$

200
TOWER DATA FOLLON

TEST D2 JULY 11. 19670350 TO 0420 PST FLUORESCEIN RELEASE FROM ELEVATION OF $2 \mathrm{M}$ 20OM ARC UE 1.9 M/SEC AT $2 M$

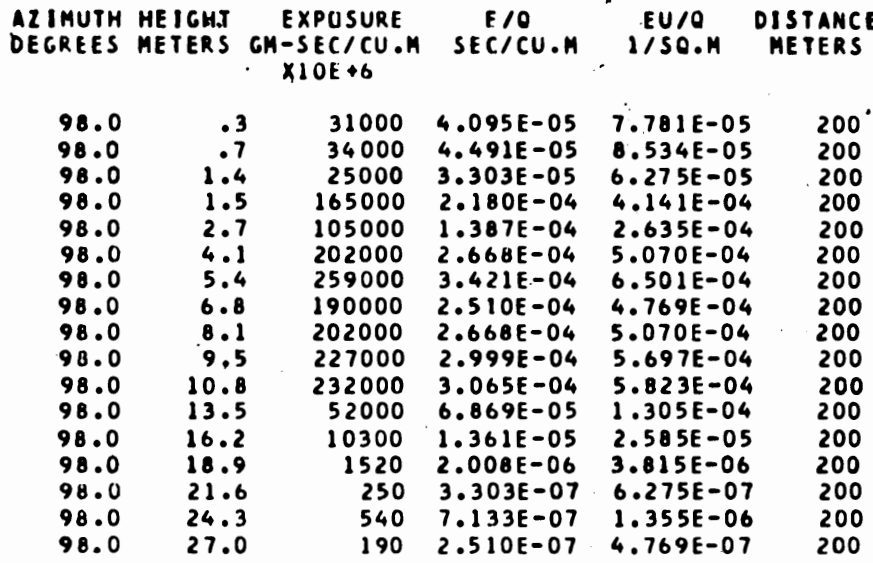

AL IMUTH HEIGHT EXPUSURE -SEC /CU.
$X 10 E+6$

106.0
106.0
106.0
106.0
106.0
106.0
106.0
106.0
106.0
106.0
106.0
106.0
106.0
106.0
106.0
106.0
106.0

.3
1.7
1.4
1.5
2.7
4.1
5.4
6.8
8.1
9.5
10.8
13.5
16.2
18.9
21.6
24.3
27.0

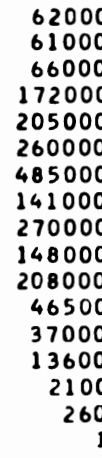

$$
8 .
$$

8.190E-05 B. $05 B E-05$ $0.719 E-05$ $2.272 E-04$ $2.708 E-04$ $3.435 E-04$ $6.407 E-04$ $1.863 E-04$ $3.567 E-04$ $1.955 E-04$ $2.748 E-04$ $6.143 E-05$ $4.888 E-05$ $1.797 E-05$ $2.774 E-06$ $3.435 E-07$ 0.

AZIMUTH HEIGHT EXPOSURE DEGREES METERS GM-SEC/CU.M SECICU.M $\times 10 E+6$

AZIMUTH HEIGHT EXPUSURE F/U DEGREES METERS GM-SEC/CU.M SEC/CU.M I/SU.M METERS $\times 10 E+6$

$\begin{array}{lr}114.0 & .3 \\ 114.0 & .7 \\ 114.0 & 1.4 \\ 114.0 & 1.5 \\ 114.0 & 2.7 \\ 114.0 & 4.1 \\ 114.0 & 5.4 \\ 114.0 & 6.8 \\ 114.0 & 8.1 \\ 114.0 & 9.5 \\ 114.0 & 10.8 \\ 114.0 & 13.5 \\ 114.0 & 16.2 \\ 114.0 & 18.9 \\ 114.0 & 21.6 \\ 114.0 & 24.3 \\ 114.0 & 27.0\end{array}$

$\begin{array}{rr}45437 & 6.002 E-05 \\ 30132 & 3.981 E-05 \\ 29051 & 3.838 E-05 \\ 135674 & 1.792 E-04 \\ 117845 & 1.557 E-04 \\ 182046 & 2.405 E-04 \\ 122477 & 1.618 E-04 \\ 179465 & 2.371 E-04 \\ 86615 & 1.144 E-04 \\ 91695 & 1.211 E-04 \\ 82671 & 1.092 E-04 \\ 20669 & 2.730 E-05 \\ 4397 & 5.804 E-06 \\ 473 & 6.257 E-07 \\ 7 & 1.017 E-00 \\ 2 & 3.868 E-09 \\ 4 & 5.658 E-09\end{array}$

$1.140 E-04$ $7.563 E-05$ $7.292 t-05$ $3.405 t-04$ $2.958 t-04$ $4.569 E-04$ $3.074 E-04$ $4.504 t-04$ $2.174 E-04$ $2.301 E-104$ 2.075E-04 . $5.188 E-05$ 1.104E-05 $1.104 \mathrm{E}-06$ $1.932 t-00$ $\pi .350 t=09$ $1.075 E-08$

200
200
200
200
200
200
200
200
200
200
200
200
200
200
200
200
200

114.0
114.0
114.0
114.0
114.0
114.0
114.0
114.0
114.0
114.0
114.0
114.0
114.0
114.0
114.0
114.0
114.0

$$
\begin{aligned}
& .3 \\
& .7 \\
& 1.4 \\
& 1.5 \\
& 2.7 \\
& 4.1 \\
& 5.4 \\
& 6.8 \\
& 8.1 \\
& 9.5 \\
& 10.8 \\
& 13.5 \\
& 16.2 \\
& 18.9 \\
& 21.6 \\
& 24.3 \\
& 27.0
\end{aligned}
$$

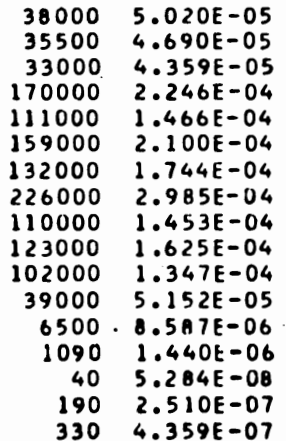

$9.538 E-05$ $8.910 E-05$ B. $283 E-05$ $4.267 E-04$ 2.786E-04 $3.991 E-04$ $3.313 E-04$ $5.672 E-04$ $2.761 E-04$ 3.0B7E-04 $2.560 E-04$ $9.789 E-05$ $1.631 E-05$ $2.736 E-06$ $1.004 E-07$ $4.769 E-07 \quad 200$

$0.283 E-07 \quad 200$ 


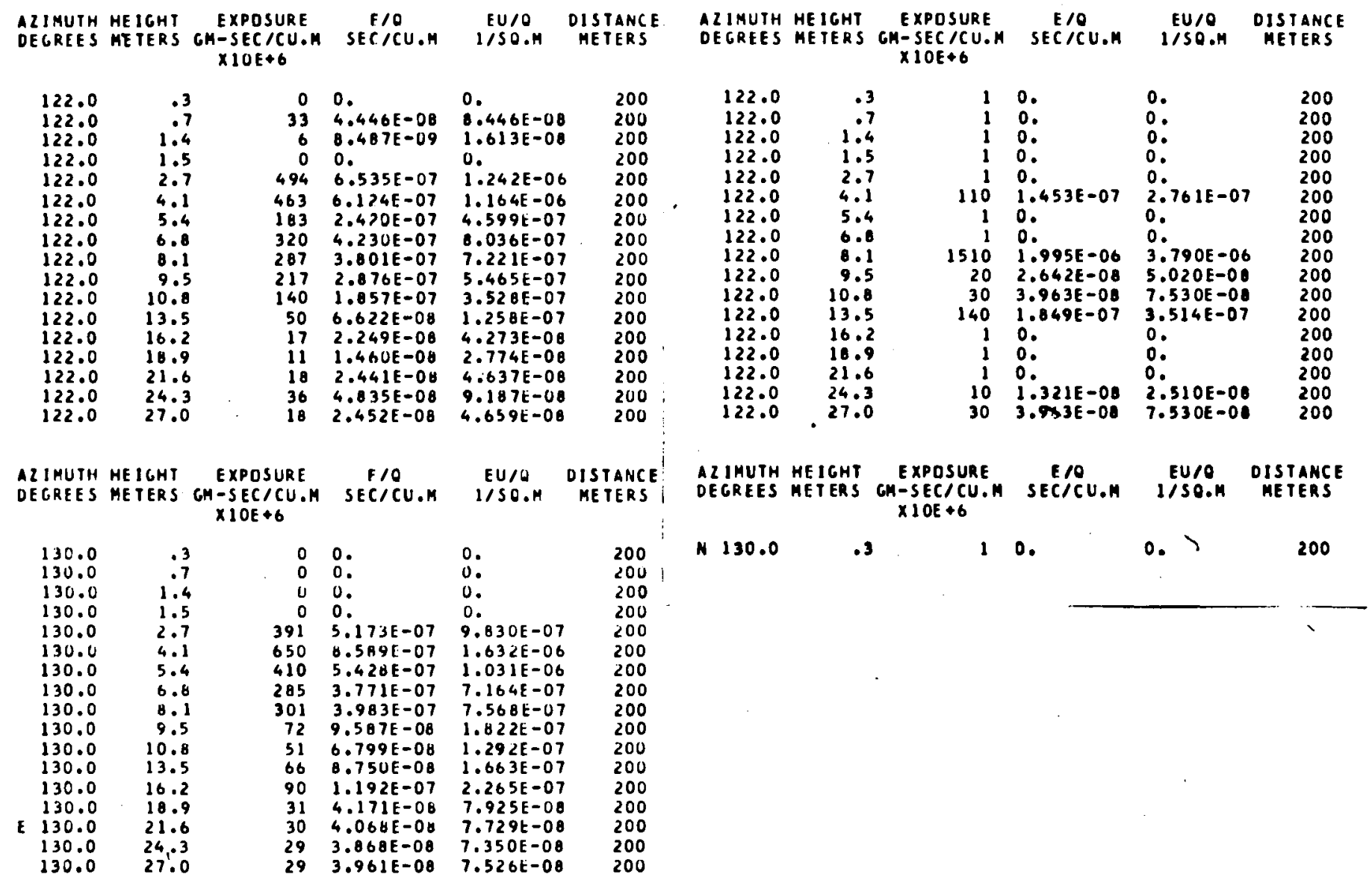

TDHER DATA FOLLOH....

TOWER DATA FOLLOH....
TEST D2 JULY 11, 1967 T2 0350 TU 0420 PST IINC SULFIDE RELEASE FRDH ELFVAIION OF $2 M$ BOOM ARC UE 1.9 MISEC AT $2 N$

$\begin{array}{lccc}\text { AZIMUTH HEIGHT EXPOSURF } & \text { F/O } & \text { EU/O DISTANCE } \\ \text { OECREES METERS GM-SECICU.M SECICU.M IISO.M METERS }\end{array}$ $\times 10 E+6$

$\begin{array}{lrrrrr}9 H .0 & .3 & 14564 & 1.924 E-0 S & 3.656 E-05 & 800 \\ 98.0 & .5 & 25811 & 3.410 E-05 & 6.478 E-05 & 800 \\ 98.0 & 1.1 & 29106 & 3.845 E-05 & 7.305 E-05 & 800 \\ 9 H .0 & 1.5 & 28077 & 3.709 E-05 & 7.047 E-05 & 800 \\ 98.0 & 2.1 & 27920 & 3.688 E-05 & 7.008 E-05 & 800 \\ 98.0 & 4.2 & 29001 & 3.831 E-05 & 7.279 E-05 & 800 \\ 98.0 & 6.3 & 29093 & 3.843 E-05 & 7.302 E-05 & 800 \\ 98.0 & 8.4 & 2744 & 3.625 E-06 & 6.888 E-06 & 800 \\ 98.0 & 10.5 & 23698 & 3.131 E-05 & 5.948 E-05 & 300 \\ 98.0 & 12.6 & 11047 & 1.459 E-05 & 2.773 E-05 & 800 \\ 98.0 & 14.7 & 9566 & 1.264 E-05 & 2.401 E-05 & 800 \\ 98.0 & 16.8 & 4291 & 5.668 E-06 & 1.077 E-05 & 800 \\ 98.0 & 21.0 & 872 & 1.153 E-06 & 2.190 E-06 & 800 \\ 98.0 & 25.2 & 160 & 2.114 E-07 & 4.017 E-07 & 800 \\ 98.0 & 29.4 & 199 & 2.603 E-08 & 4.945 E-08 & 800 \\ 98.0 & 33.6 & 4 & 6.600 E-09 & 1.254 E-08 & 800 \\ 98.0 & 37.8 & 0 & 0.0 & 0.0 & 800 \\ 98.0 & 42.0 & 0 & 0.0 & 0.0 & 800\end{array}$
TEST D2 JULY 11.1967 0350 TO 0420 PST FLUORESCEIN RELEASE FROM ELEVATION DF $2 \mathrm{H}$ OOOM ARC U. 1.9 HISEC AT $2 M$ $\begin{array}{lcccc}\text { AZIMUTH HEICHT EXPOSURE } & \text { E/O } & \text { EU/O OISTANCE } \\ \text { OEGREES METERS GM-SEC/CU.M SEC/CU.M } & 1 / \text { SO.M METERS }\end{array}$

$\begin{array}{lrrl}98.0 & .3 & 15000 & 1.982 \mathrm{E}-05 \\ 98.0 & .5 & 27500 & 3.633 \mathrm{E}-05 \\ 98.0 & 1.1 & 23500 & 3.104 \mathrm{E}-05 \\ 98.0 & 1.5 & 36000 & 4.756 \mathrm{E}-05 \\ 98.0 & 2.1 & 28500 & 3.765 \mathrm{E}-05 \\ 98.0 & 4.2 & 36000 & 4.756 \mathrm{E}-05 \\ 98.0 & 6.3 & 37000 & 4.808 \mathrm{E}-05 \\ 98.0 & 8.4 & 13800 & 1.023 \mathrm{E}-05 \\ 98.0 & 10.5 & 34500 & 4.557 \mathrm{E}-05 \\ 98.0 & 12.6 & 18800 & 2.483 \mathrm{E}-05 \\ 98.0 & 14.7 & 12600 & 1.664 \mathrm{E}-05 \\ 98.0 & 16.8 & 6100 & 8.058 \mathrm{E}-06 \\ 98.0 & 21.0 & 1900 & 2.510 \mathrm{E}-06 \\ 98.0 & 25.2 & 190 & 2.510 \mathrm{E}-07 \\ 98.0 & 29.4 & 10 & 1.321 \mathrm{E}-08 \\ 98.0 & 33.6 & 1 & 0.0 \\ 98.0 & 37.8 & 1 & 0 . \\ 98.0 & 42.0 & 1 & 0 .\end{array}$

$\begin{array}{ll}3.765 E-05 & 000 \\ 6.902 E-05 & 800 \\ 5.898 E-05 & 800 \\ 9.036 E-05 & 800 \\ 7.153 E-05 & 000 \\ 9.036 E-05 & 000 \\ 9.287 E-05 & 800 \\ 3.464 E-05 & 800 \\ 0.659 E-05 & 800 \\ 4.719 E-05 & 800 \\ 3.162 E-05 & 000 \\ 1.531 E-05 & 800 \\ 4.769 E-06 & 800 \\ 4.769 E-07 & 800 \\ 2.510 E-08 & 800 \\ 0.0 & 000 \\ 0.0 & 800 \\ 0 . & 800\end{array}$




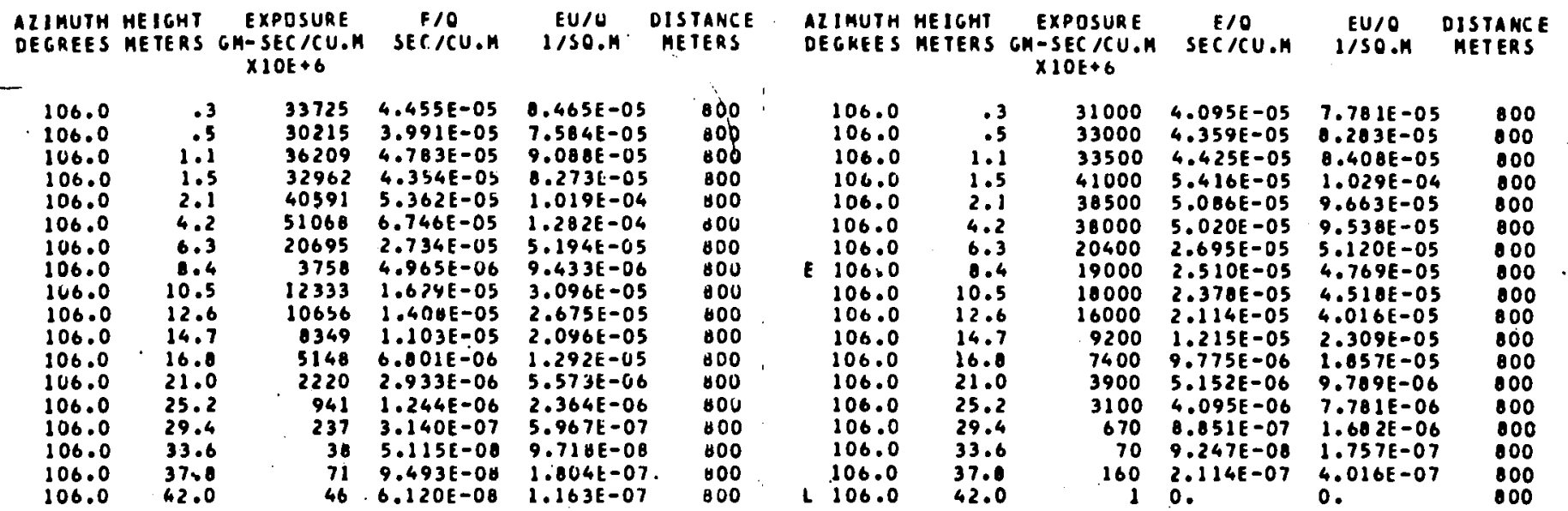

\begin{tabular}{|c|c|c|c|c|c|c|c|c|c|c|c|}
\hline $\begin{array}{l}\text { II IMUTH } \\
\text { DEGREES }\end{array}$ & $\begin{array}{l}\text { HE IGHT } \\
\text { ME TERS }\end{array}$ & $\begin{array}{l}\text { EXPDSURE } \\
\text { GH - SEC / CU A } \\
\times 10 E+6\end{array}$ & $\begin{array}{c}E / O \\
\text { S } E C / C U . M\end{array}$ & $\begin{array}{l}\text { EU/O } \\
1 / S O . M\end{array}$ & $\begin{array}{c}\text { UISTANCE } \\
\text { METERS }\end{array}$ & $\begin{array}{l}\text { AZ IMUTH } \\
\text { DEGREES }\end{array}$ & $\begin{array}{l}\text { HE IGHT } \\
\text { METERS }\end{array}$ & $\begin{array}{l}\text { EXPOSURE } \\
\text { GH-SEC/CU.H } \\
\times 1 O E+6\end{array}$ & $\begin{array}{c}E / O \\
S E C / C U . M\end{array}$ & $\begin{array}{l}\text { EU/O } \\
1 / 50 . \mathrm{H}\end{array}$ & $\begin{array}{l}\text { DISTANCE } \\
\text { METERS }\end{array}$ \\
\hline $\begin{array}{l}114.0 \\
114.0 \\
114.0 \\
114.0 \\
114.0 \\
114.0 \\
114.0 \\
114.0 \\
114.0 \\
114.0 \\
114.0 \\
114.0 \\
114.0 \\
114.0 \\
114.0 \\
114.0 \\
114.0 \\
114.0\end{array}$ & $\begin{array}{l}.3 \\
: .5 \\
1.1 \\
1.5 \\
2.1 \\
4.2 \\
6.3 \\
8.4 \\
10.5 \\
12.6 \\
14.7 \\
16.8 \\
21.0 \\
25.2 \\
29.4 \\
33.6 \\
37.8 \\
42.0\end{array}$ & $\begin{array}{r}19120 \\
18340 \\
21381 \\
24230 \\
20549 \\
20847 \\
15830 \\
16082 \\
14302 \\
8587 \\
3699 \\
2190 \\
248 \\
73 \\
43 \\
2 \\
7 \\
42\end{array}$ & $\begin{array}{l}2.576 E-05 \\
2.473 E-05 \\
2.874 E-05 \\
3.201 E-05 \\
2.715 E-05 \\
2.754 E-05 \\
2.0911 E-05 \\
2.175 E-05 \\
1.849 E-05 \\
1.134 E-05 \\
4.8 B 8 E-06 \\
2.894 E-06 \\
3.285 E-07 \\
9.729 E-08 \\
5.726 E-08 \\
3.300 E-09 \\
9.654 E-09 \\
5.661 E-08\end{array}$ & $\begin{array}{l}4.799 t-05 \\
4.603 E-05 \\
5.367 t-05 \\
6.082 E-05 \\
5.158 t-05 \\
5.232 E-05 \\
3.973 E-05 \\
4.037 t-05 \\
3.590 L-05 \\
2.155 t-05 \\
9.287 E-06 \\
5.498 E-06 \\
6.242 E-07 \\
1.848 E-07 \\
1.086 E-07 \\
6.270 E-09 \\
1.834 E-08 \\
1.076 E-07\end{array}$ & $\begin{array}{l}800 \\
800 \\
600 \\
800 \\
400 \\
200 \\
800 \\
800 \\
000 \\
600 \\
000 \\
800 \\
600 \\
800 \\
000 \\
800 \\
800 \\
800\end{array}$ & $\begin{array}{l}114.0 \\
114.0 \\
114.0 \\
114.0 \\
114.0 \\
114.0 \\
114.0 \\
114.0 \\
1114.0 \\
114.0 \\
114.0 \\
114.0 \\
114.0 \\
114.0 \\
114.0 \\
114.0 \\
114.0 \\
1114.0\end{array}$ & $\begin{array}{r}.3 \\
.5 \\
1.1 \\
1.5 \\
2.1 \\
4.2 \\
6.3 \\
6.4 \\
10.5 \\
12.6 \\
14.7 \\
16.0 \\
21.0 \\
25.2 \\
29.4 \\
33.6 \\
37.8 \\
42.0\end{array}$ & $\begin{array}{r}19100 \\
21600 \\
24500 \\
24500 \\
17300 \\
26700 \\
16000 \\
16500 \\
16000 \\
12200 \\
5900 \\
3030 \\
410 \\
100 \\
70 \\
1 \\
80 \\
1\end{array}$ & $\begin{array}{l}2.523 E-05 \\
2.053 E-05 \\
3.236 E-05 \\
3.236 E-05 \\
2.285 E-05 \\
3.527 E-05 \\
2.114 E-05 \\
2.180 E-05 \\
2.114 E-05 \\
1.612 E-05 \\
7.794 E-06 \\
4.003 E-06 \\
5.416 E-07 \\
1.321 E-07 \\
9.247 E-08 \\
0.0 \\
1.057 E-07 \\
0.0\end{array}$ & $\begin{array}{l}4.794 E-05 \\
5.421 E-05 \\
6.149 E-05 \\
6.149 E-05 \\
4.342 E-05 \\
6.701 E-05 \\
4.016 E-05 \\
4.141 E-05 \\
4.016 E-05 \\
3.062 E-05 \\
1.481 E-05 \\
7.605 E-06 \\
1.029 E-06 \\
2.510 E-07 \\
1.757 E-07 \\
0 . \\
2.008 E-07 \\
0.0\end{array}$ & $\begin{array}{l}800 \\
800 \\
800 \\
800 \\
800 \\
600 \\
800 \\
800 \\
800 \\
800 \\
800 \\
800 \\
800 \\
000 \\
800 \\
800 \\
800 \\
800\end{array}$ \\
\hline
\end{tabular}

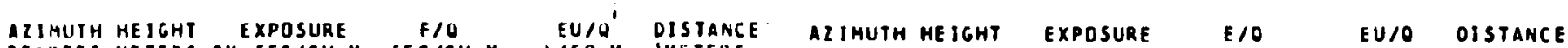
DEGRES METERS GM-SECICU.M SECICU.M JISO.M IMETERS DEGRES METERS CM-SECICU.M SECICU.M IISO.M METERS $\times 10 E+6$

\begin{tabular}{|c|c|c|c|c|c|c|c|}
\hline $\begin{array}{l}122.0 \\
122.0 \\
122.0 \\
122.0 \\
122.0 \\
122.0 \\
122.0 \\
122.0 \\
122.0 \\
122.0 \\
122.0 \\
122.0 \\
122.0 \\
122.0 \\
122.0 \\
122.0 \\
122.0 \\
122.0\end{array}$ & $\begin{array}{r}.3 \\
.5 \\
1.1 \\
1.3 \\
2.1 \\
4.2 \\
6.3 \\
8.4 \\
10.5 \\
12.6 \\
14.7 \\
16.8 \\
21.0 \\
25.2 \\
29.4 \\
33.6 \\
37.8 \\
42.0\end{array}$ & : & $\begin{array}{r}3 \\
0 \\
3 \\
12 \\
2 \\
305 \\
189 \\
130 \\
46 \\
79 \\
128 \\
51 \\
32 \\
11 \\
13 \\
21 \\
19 \\
0\end{array}$ & $\begin{array}{l}4.513 E-09 \\
0 . \\
4.105 E-04 \\
1.642 \mathrm{E}-08 \\
3.926 E-04 \\
5.093 E-07 \\
2.504 E-07 \\
1.721 E-07 \\
1.141 E-07 \\
1.056 E-07 \\
1.695 E-07 \\
6.789 E-08 \\
4.370 E-08 \\
1.497 E-08 \\
1.735 E-08 \\
2.805 E-08 \\
2.574 E-08 \\
0.5\end{array}$ & $\begin{array}{l}A .579 t-09 \\
0.9 \\
7.749 E-09 \\
3.120 E-08 \\
7.460 E-09 \\
9.677 t-07 \\
4.758 E-07 \\
3.270 E-07 \\
2.169 E-07 \\
2.006 t-07 \\
3.221 E-07 \\
1.290 E-07 \\
8.224 E-08 \\
2.844 E-08 \\
3.297 E-08 \\
5.329 E-08 \\
4.891 E-08 \\
0 .\end{array}$ & $\begin{array}{l}800 \\
800 \\
8110 \\
000 \\
800 \\
800 \\
100 \\
600 \\
800 \\
800 \\
800 \\
000 \\
800 \\
100 \\
800 \\
800 \\
800 \\
800\end{array}$ & $\begin{array}{l}122.0 \\
122.0 \\
122.0 \\
122.0 \\
122.0 \\
122.0 \\
122.0 \\
122.0 \\
122.0 \\
122.0 \\
122.0 \\
122.0 \\
122.0 \\
122.0 \\
122.0 \\
122.0 \\
122.0 \\
122.0\end{array}$ \\
\hline
\end{tabular}
$\times 10 E+6$ 


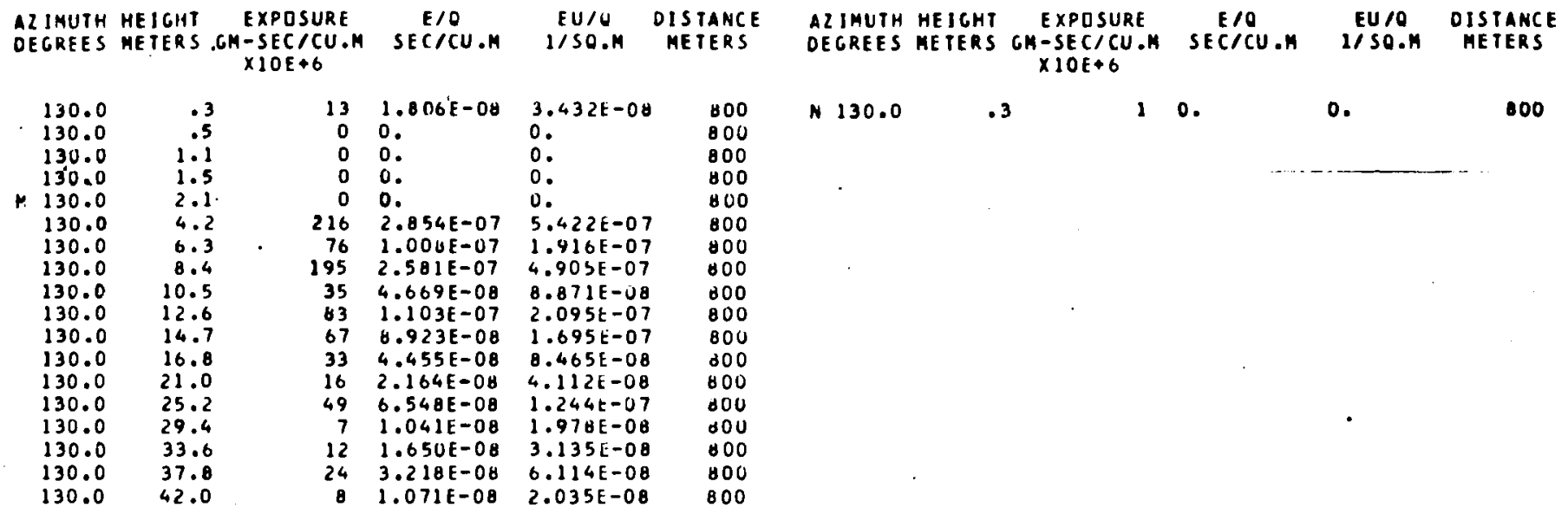

TOWER OAIA FOLLEH....

\begin{abstract}
TEST D2 JULY 11.1967 0350 TO 0420 PST IINC SULFIOE RELEASE FROA ELFVATION UF $2 \mathrm{M}$ $1600 M$ AKC $U=1.9 \mathrm{M} / \mathrm{SEC}$ AI $2 \mathrm{M}$
\end{abstract}

TOWER DATA FDLLDW,...

TEST D2 JULY 11,1967 0350 TO 0420 PST FLUURESCEIN RELEASE FRDA ELEVATION OF $2 \mathrm{M}$ 1600 ARC $U=1.9$ M/SEC AT $2 \mathrm{M}$

ALIMUTH HEIGHT EXPOSURE E/O EU/O DISTANCE DEGREES METERS IM-SEC/CU.M SECICU.M I/SO.M METERS $\mathrm{X} 1 \mathrm{DE}+6$

\begin{tabular}{|c|c|c|c|c|c|}
\hline 98.0 & .2 & 15800 & $2.087 E-05$ & $3.966 E-05$ & 1600 \\
\hline 98.0 & .4 & 16700 & $2.206 E-05$ & $4.192 E-05$ & 1600 \\
\hline $9 B .0$ & .6 & 18300 & $2.417 \mathrm{E}-05$ & $4.593 E-05$ & 1600 \\
\hline 98.0 & 1.5 & 21000 & $2.774 E-05$ & $5.271 E=05$ & 1600 \\
\hline 98.0 & 1.6 & 19800 & $2.616 E-05$ & $4.970 E-05$ & 1600 \\
\hline 98.0 & 3.1 & 22200 & $2.933 E-05$ & $5.572 E-05$ & 1600 \\
\hline 98.0 & 6.2 & 18100 & $2.391 E-05$ & $4.543 E-05$ & 1600 \\
\hline 98.0 & 9.3 & 16100 & $2.127 E-05$ & $4.041 E-05$ & 1600 \\
\hline $\begin{array}{l}98.0 \\
98.0\end{array}$ & $\begin{array}{l}12.4 \\
15.5\end{array}$ & $\begin{array}{r}5500 \\
11500\end{array}$ & $\begin{array}{l}7.266 E-06 \\
1.519 E-05\end{array}$ & $\begin{array}{l}1.380 E-05 \\
2.806 E-05\end{array}$ & $\begin{array}{l}1600 \\
1600\end{array}$ \\
\hline 98.0 & 18.6 & 1380 & $1.823 E-06$ & $3.464 E-06$ & 1600 \\
\hline 98.0 & 21.7 & 590 & $7.794 E-07$ & $1.481 E-06$ & 1600 \\
\hline 98.0 & 24.8 & 220 & $2.906 E-07$ & $5.522 \mathrm{E}-07$ & 1600 \\
\hline 98.0 & 31.0 & 10 & $1.321 E-08$ & $2.510 E-08$ & 1600 \\
\hline 98.0 & 37.2 & 1 & 0. & 0. & 1600 \\
\hline 98.0 & 43.4 & 1 & 0. & 0 . & 1600 \\
\hline 98.0 & 49.6 & 1 & 0 . & 0. & 1600 \\
\hline 98.0 & 55.8 & 1 & 0 . & 0 . & 1600 \\
\hline 98.0 & 62.0 & 1 & 0 . & 0 . & 1600 \\
\hline
\end{tabular}

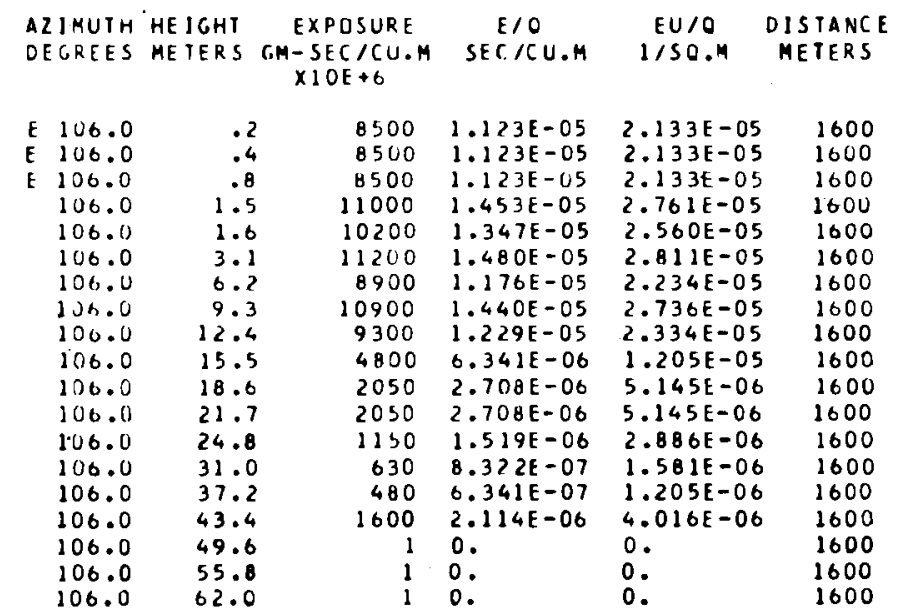




\begin{tabular}{|c|c|c|c|c|c|c|c|c|c|c|c|}
\hline $\begin{array}{l}\text { AZ IMUTH } \\
\text { DE GREES }\end{array}$ & $\begin{array}{l}\text { HE IGHT } \\
\text { HETERS }\end{array}$ & $\begin{array}{l}\text { EXPDSURE } \\
\text { GH -SECICU H } \\
\text { XIOE }+6\end{array}$ & $\begin{array}{c}E / 0 \\
\text { SEC/CU.H }\end{array}$ & $\begin{array}{l}E U / 0 \\
1 / 50 . \mathrm{H}\end{array}$ & $\begin{array}{l}\text { DISTANCE } \\
\text { METERS }\end{array}$ & $\begin{array}{l}\text { AZ JMUTH } \\
\text { DEGREES }\end{array}$ & $\begin{array}{l}\text { HE ICHT } \\
\text { HETERS }\end{array}$ & $\begin{array}{l}\text { EXPOSURE } \\
\text { GH - SEC / CU } \\
\times 1 O E+6\end{array}$ & $\begin{array}{c}\text { E } / 0 \\
\text { SEC/CU.M }\end{array}$ & $\begin{array}{l}E U / 0 \\
1 / 30 . \mathrm{H}\end{array}$ & $\begin{array}{c}\text { DISTAMCE } \\
\text { HETERS }\end{array}$ \\
\hline $\begin{array}{l}114.0 \\
114.0 \\
114.0 \\
114.0 \\
114.0 \\
114.0 \\
114.0 \\
114.0 \\
114.0 \\
114.0 \\
114.0 \\
114.0 \\
114.0 \\
114.0 \\
114.0 \\
114.0 \\
114.0 \\
114.0 \\
114.0\end{array}$ & $\begin{array}{l}.2 \\
.4 \\
.8 \\
1.5 \\
1.6 \\
3.1 \\
6.2 \\
9.3 \\
12.4 \\
15.5 \\
18.6 \\
21.7 \\
24.0 \\
31.0 \\
37.2 \\
43.4 \\
49.6 \\
55.0 \\
62.0\end{array}$ & $\begin{array}{l}6207 \\
5723 \\
6395 \\
7020 \\
6603 \\
7299 \\
6047 \\
6033 \\
2910 \\
1530 \\
794 \\
292 \\
119 \\
41 \\
21 \\
10 \\
1 \\
0 \\
0\end{array}$ & $\begin{array}{l}9.201 E-06 \\
7.561 E-06 \\
8.448 E-06 \\
9.205 E-06 \\
8.723 E-06 \\
9.642 E-06 \\
9.045 E-06 \\
7.971 E-06 \\
3.045 E-06 \\
2.022 E-06 \\
1.049 E-06 \\
3.867 E-07 \\
1.575 E-07 \\
5.502 E-08 \\
2.852 E-08 \\
1.401 E-08 \\
2.441 E-09 \\
1.195 E-09 \\
0.1\end{array}$ & $\begin{array}{l}1.558 E-05 \\
1.437 E-05 \\
1.605 E-05 \\
1.764 E-05 \\
1.657 E-05 \\
1.832 E-05 \\
1.719 E-05 \\
1.514 E-05 \\
7.305 E-06 \\
3.042 E-06 \\
1.994 E-06 \\
7.340 E-07 \\
2.992 E-07 \\
1.045 E-07 \\
5.419 E-08 \\
2.662 E-08 \\
4.637 E-09 \\
2.270 E-08 \\
0.2\end{array}$ & $\begin{array}{l}1600 \\
1600 \\
1600 \\
1600 \\
1600 \\
1600 \\
1600 \\
1600 \\
1600 \\
1600 \\
1600 \\
1600 \\
1600 \\
1600 \\
1600 \\
1600 \\
1600 \\
1600 \\
1600\end{array}$ & $\begin{array}{l}114.0 \\
114.0 \\
114.0 \\
114.0 \\
114.0 \\
114.0 \\
114.0 \\
114.0 \\
114.0 \\
114.0 \\
114.0 \\
114.0 \\
114.0 \\
114.0 \\
114.0 \\
114.0 \\
114.0 \\
114.0 \\
114.0\end{array}$ & $\begin{array}{r}.2 \\
.4 \\
1.0 \\
1.5 \\
3.1 \\
6.2 \\
9.3 \\
12.4 \\
15.5 \\
18.6 \\
21.7 \\
24.8 \\
31.0 \\
37.2 \\
43.4 \\
49.6 \\
55.8 \\
62.0\end{array}$ & $\begin{array}{r}5650 \\
5600 \\
4400 \\
6500 \\
4850 \\
4100 \\
3900 \\
3650 \\
3200 \\
1450 \\
840 \\
260 \\
160 \\
70 \\
10 \\
20 \\
40 \\
20 \\
1\end{array}$ & $\begin{array}{l}7.464 E-06 \\
7.396 E-06 \\
5.012 E-06 \\
1.587 E-06 \\
6.407 E-06 \\
5.416 E-06 \\
5.152 E-06 \\
4.822 E-06 \\
4.227 E-06 \\
1.915 E-06 \\
1.110 E-06 \\
3.435 E-07 \\
2.114 E-07 \\
9.247 E-08 \\
1.321 E-08 \\
2.642 E-08 \\
5.284 E-08 \\
2.642 E-08 \\
0 .\end{array}$ & $\begin{array}{l}1.418 E-05 \\
1.406 E-05 \\
1.104 E-05 \\
1.631 E-05 \\
1.217 E-05 \\
1.029 E-05 \\
9.789 E-06 \\
9.161 \mathrm{E}-06 \\
1.032 E-06 \\
3.639 E-06 \\
2.100 E-06 \\
6.526 E-07 \\
4.016 E-07 \\
1.757 E-07 \\
2.510 E-08 \\
5.020 E-08 \\
1.004 E-07 \\
5.020 E-08 \\
0.0\end{array}$ & $\begin{array}{l}1600 \\
1600 \\
1600 \\
1600 \\
1600 \\
1600 \\
1600 \\
1600 \\
1600 \\
1600 \\
1600 \\
1600 \\
1600 \\
1600 \\
1600 \\
1600 \\
1600 \\
1600 \\
1600\end{array}$ \\
\hline $\begin{array}{l}\text { AL IMUTH } \\
\text { DE GREES }\end{array}$ & $\begin{array}{l}\text { HE ICHT } \\
\text { ME TERS }\end{array}$ & $\begin{array}{c}\text { EXPOSURE } \\
\text { GN-SEC/CU.M } \\
\times 1 O E+6\end{array}$ & $\begin{array}{c}\text { E } / 0 \\
S E C / C U . M\end{array}$ & $\begin{array}{l}E \cup / 0 \\
1 / S O . H\end{array}$ & $\begin{array}{l}\text { OISTANCE } \\
\text { METERS }\end{array}$ & $\begin{array}{l}\text { ALIMUTH } \\
\text { OEGREES }\end{array}$ & $\begin{array}{l}\text { HE IGHT } \\
\text { METERS }\end{array}$ & $\begin{array}{c}\text { EXPOSURE } \\
\text { GH-SECICU.H } \\
\times 10 E * 6\end{array}$ & $\begin{array}{c}E / 0 \\
\text { SEC/CU.M }\end{array}$ & $\begin{array}{l}\text { EU70 } \\
1150 . \mathrm{H}\end{array}$ & $\begin{array}{l}\text { DISTANCE } \\
\text { HETERS }\end{array}$ \\
\hline $\begin{array}{l}122.0 \\
122.0 \\
122.0 \\
122.0 \\
122.0 \\
122.0 \\
122.0 \\
122.0 \\
122.0 \\
122.0 \\
122.0 \\
122.0 \\
122.0 \\
122.0 \\
122.0 \\
122.0 \\
122.0 \\
122.0 \\
122.0\end{array}$ & $\begin{array}{l}.12 \\
.4 \\
.6 \\
1.5 \\
1.6 \\
3.1 \\
6.2 \\
9.3 \\
12.4 \\
15.5 \\
18.6 \\
21.7 \\
24.8 \\
31.0 \\
37.2 \\
43.4 \\
49.6 \\
55.8 \\
62.0\end{array}$ & $\begin{array}{r}2 \\
0 \\
0 \\
0 \\
0 \\
59 \\
61 \\
19 \\
0 \\
29 \\
2 \\
36 \\
0 \\
55 \\
20 \\
11 \\
7 \\
0 \\
0\end{array}$ & $\begin{array}{l}2.978 E-09 \\
0 . \\
0 . \\
0 . \\
0 . \\
7.050 E-08 \\
9.088 E-08 \\
2.608 E-08 \\
0 . \\
3.861 E-08 \\
3.363 E-09 \\
4.834 E-08 \\
1.081 E-08 \\
7.385 E-08 \\
2.716 E-08 \\
1.528 E-08 \\
9.763 E-09 \\
0 . \\
0 .\end{array}$ & $\begin{array}{l}5.658 E-09 \\
0 . \\
0 . \\
0 . \\
0 . \\
1.492 E-07 \\
1.727 E-07 \\
4.956 E-08 \\
0 . \\
7.337 E-08 \\
6.391 E-09 \\
9.185 E-08 \\
2.053 E-08 \\
1.403 E-07 \\
5.161 E-08 \\
2.904 E-08 \\
1.855 E-08 \\
0 . \\
0 .\end{array}$ & $\begin{array}{l}1600 \\
1600 \\
1600 \\
1600 \\
1600 \\
1600 \\
1600 \\
1600 \\
1600 \\
1600 \\
1600 \\
1600 \\
1600 \\
1600 \\
1600 \\
1600 \\
1600 \\
1600 \\
1600 \\
1600 \\
1600\end{array}$ & $\begin{array}{l}122.0 \\
122.0 \\
122.0 \\
122.0 \\
122.0 \\
122.0 \\
122.0 \\
122.0 \\
122.0 \\
122.0 \\
122.0 \\
122.0 \\
122.0 \\
122.0 \\
122.0 \\
122.0 \\
122.0 \\
122.0 \\
122.0\end{array}$ & $\begin{array}{r}.2 \\
.4 \\
1.8 \\
1.5 \\
1.6 \\
3.1 \\
6.2 \\
9.3 \\
12.4 \\
15.5 \\
18.6 \\
21.7 \\
24.8 \\
31.0 \\
37.2 \\
43.4 \\
49.6 \\
55.8 \\
62.0\end{array}$ & $\begin{array}{l}1 \\
1 \\
1 \\
1 \\
1 \\
1 \\
00 \\
1 \\
1 \\
1 \\
1 \\
30 \\
40 \\
10 \\
20 \\
60 \\
1 \\
1 \\
1\end{array}$ & $\begin{array}{l}0 . \\
0 . \\
0 . \\
0 . \\
0 . \\
0 . \\
1.057 E-07 \\
0 . \\
0 . \\
0 . \\
0 . \\
3.963 E-08 \\
5.284 E-08 \\
1.321 E-08 \\
2.642 E-08 \\
7.926 E-08 \\
0 . \\
0 . \\
0 .\end{array}$ & $\begin{array}{l}0 . \\
0 . \\
0 . \\
0 . \\
0 . \\
0 . \\
2.008 E-07 \\
0 . \\
0 . \\
0 . \\
0 . \\
7.530 E-08 \\
1.004 E-07 \\
2.510 E-08 \\
5.020 E-00 \\
1.506 E=07 \\
0 . \\
0 . \\
0 .\end{array}$ & $\begin{array}{l}1600 \\
1600 \\
1600 \\
1600 \\
1600 \\
1600 \\
1600 \\
1600 \\
1600 \\
1600 \\
1600 \\
1600 \\
1600 \\
1600 \\
1600 \\
1600 \\
1600 \\
1600 \\
1600\end{array}$ \\
\hline $\begin{array}{l}\text { AZIMUTH } \\
\text { DEGREES }\end{array}$ & $\begin{array}{l}\text { HE IGHT } \\
\text { METERS }\end{array}$ & $\begin{array}{l}\text { EXPOSURE } \\
\text { GH-SEC/CU.H } \\
\text { XIOE+6 }\end{array}$ & $\begin{array}{c}E / O \\
S E C / C U, H\end{array}$ & $\begin{array}{l}E U / O \\
1 / S O . M\end{array}$ & $\begin{array}{l}\text { DISTANCE } \\
\text { METERS }\end{array}$ & $\begin{array}{l}\text { AZIMUTH } \\
\text { DECREES }\end{array}$ & $\begin{array}{l}\text { HE ICHT } \\
\text { METERS }\end{array}$ & $\begin{array}{l}\text { EXPOSURE } \\
\text { CH-SEC/CU.H } \\
\times 1 O E+6\end{array}$ & $\begin{array}{c}E / 0 \\
S E C / C U . H\end{array}$ & $\begin{array}{l}E U / 0 \\
1 / 50.4\end{array}$ & $\begin{array}{c}\text { DISTANCE } \\
\text { METERS }\end{array}$ \\
\hline $\begin{array}{l}130.0 \\
130.0 \\
130.0 \\
130.0\end{array}$ & $\begin{array}{r}.2 \\
.4 \\
.8 \\
1.5\end{array}$ & $\begin{array}{r}0 \\
8 \\
23 \\
0\end{array}$ & $\begin{array}{l}0.134 E-08 \\
1.1340 \mathrm{E}-08 \\
3.120 \mathrm{l} \\
0 .\end{array}$ & $\begin{array}{l}0 . \\
2.155 E-08 \\
5.927 t-08 \\
0 .\end{array}$ & $\begin{array}{l}1600 \\
1600 \\
1600 \\
1600\end{array}$ & N 130.0 & .2 & 1 & 0. & $\ldots$ & 1600 \\
\hline $\begin{array}{l}130.0 \\
130.0 \\
130.0 \\
130.0 \\
130.0 \\
130.0 \\
130.0 \\
130.0 \\
130.0 \\
130.0 \\
130.0 \\
130.0 \\
130.0 \\
130.0 \\
130.0\end{array}$ & $\begin{array}{l}1.6 \\
3.1 \\
6.2 \\
9.3 \\
12.4 \\
15.5 \\
18.6 \\
21.7 \\
24.8 \\
31.0 \\
3.7 .2 \\
43.4 \\
49.6 \\
55.8 \\
62.0\end{array}$ & $\begin{array}{r}21 \\
153 \\
15 \\
6 \\
57 \\
5 \\
40 \\
19 \\
30 \\
6 \\
0 \\
47 \\
7 \\
0 \\
22\end{array}$ & $\begin{array}{l}2.836 E-08 \\
2.030 E-07 \\
2.020 E-08 \\
8.694 E-09 \\
7.648 E-08 \\
7.021 E-09 \\
5.382 E-08 \\
2.578 E-08 \\
4.014 E-08 \\
4.688 E-09 \\
0 . \\
6.241 E-08 \\
9.763 E-09 \\
0.0 \\
2.976 E-08\end{array}$ & $\begin{array}{l}5.38 B E-08 \\
3.85 B E-07 \\
3.837 E-08 \\
1.652 E-08 \\
1.453 E-07 \\
1.334 E-08 \\
1.023 E-07 \\
4.899 E-08 \\
7.627 E-08 \\
1.651 E-08 \\
0.1 \\
1.186 E-07 \\
1.855 E-08 \\
0.1 \\
5.654 E-08\end{array}$ & $\begin{array}{l}1600 \\
1600 \\
1600 \\
1600 \\
1600 \\
1600 \\
1600 \\
1600 \\
1600 \\
1600 \\
1600 \\
1600 \\
1600 \\
1600 \\
1600\end{array}$ & • & & 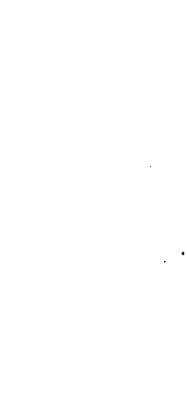 & & . & . \\
\hline
\end{tabular}


TOWER OATA FOLLOH....

TEST D2 SULY 11,1967 O 1950 TO 0420 PST

IINC SULFJOE RELEASE FROA ELEVATION UF AT
$3200 \mathrm{M}$ AKC

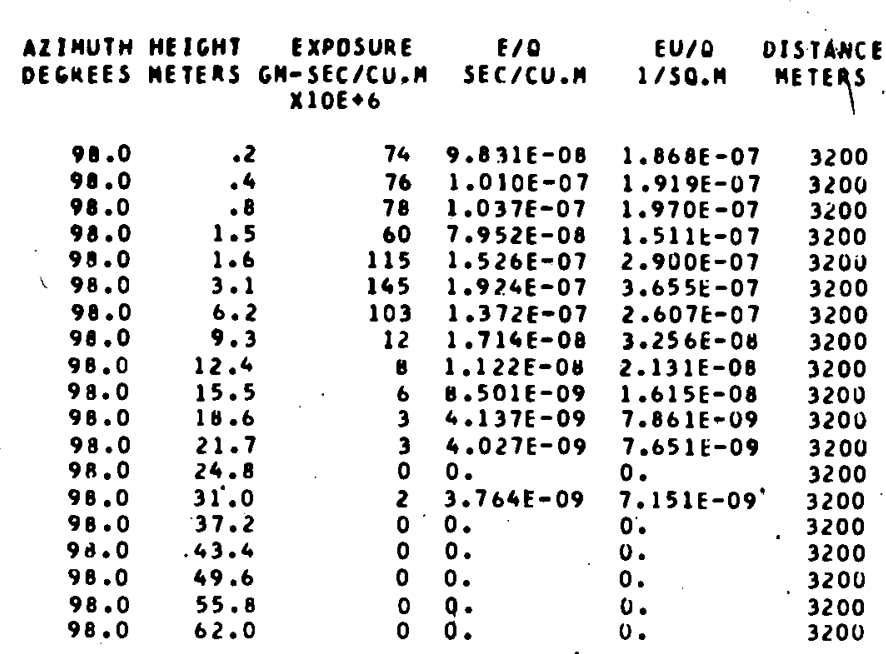

A2 IMUTH HE IGHT DEGREES METERS GM-SEC/CU.M SEC/CU.M I/SO.M MEIERS

106.0
106.0
106.0
106.0
106.0
106.0
106.0
106.0
106.0
106.0
106.0
106.0
106.0
106.0
106.0
106.0
106.0
106.0
106.0

.2
.4
.8
1.5
1.6
3.1
6.2
9.3
12.4
15.5
18.6
21.7
24.8
31.0
37.2
43.4
49.6
55.8
62.0
$\times 10 E+6$
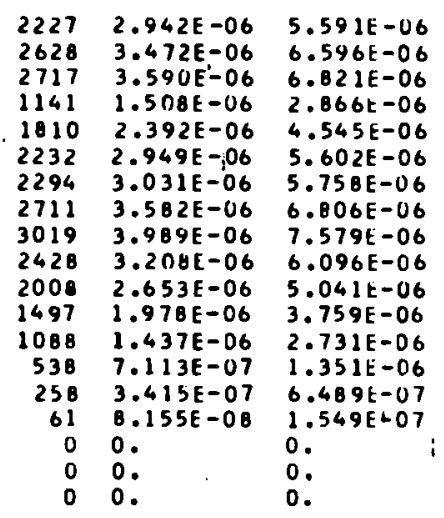

3200
3200
3200
3200
3200
3200
3200
3200
3200
3200
3200
3200
3200
3200
3200
3200
3200
3200
3200

AZ I HUTH HE IGH

EXPOSURE SEC/C IO EUIO DISTANCE
ICU.M ISO.M METERS \begin{tabular}{l}
$X \perp O E+6$ \\
\hline $10 C$
\end{tabular}

114.0
114.0
114.0
114.0
114.0
114.0
114.0
114.0
114.0
114.0
114.0
114.1
114.0
114.0
114.0
114.0
114.0
114.0
114.0

.2
.4
.8
1.5
1.6
3.1
6.2
9.3
12.4
15.5
18.6
21.7
24.8
31.0
37.2
43.4
49.6
55.8
62.0

$4.946 E-06 \quad 3200$ $4.585 \mathrm{t}-06$ 5.762E-06 $4.939 E=06$ $4.939 E-06$
$5.532 E-06$ $5.532 E-06$
$7.091 E-06$ $7.091 E-06$
$6.216 E-06$ $6.292 \mathrm{~L}=06$ 4.63 Bt -06 $4.254 E-06$ $4.040 \mathrm{E}-06$ $3.072 t-06$ $2.470 E-06$ $1.183 \mathrm{E}-06$ $4.206 E-07$ $4.206 E-07$
$2.654 E-07$ $7.380 \mathrm{E}-08$ $5.540 E-08$

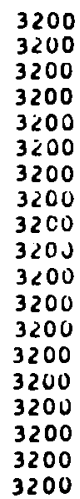

TOWER DATA FOLLOW....

TEST D2 JULY 11. $1967 \quad 0350$ TU 0420 PST FLUORESCEIN RELEASE FROH ELEVATION DF $2 \mathrm{H}$ $3200 \mathrm{~A}$ ARC U. $1.9 \mathrm{M} / \mathrm{SEC}$ AT $2 \mathrm{M}$

ALIMUTH HEIGHT EXPOSURE E/O EU/O DISTANCE DEGREES METERS GH-SEC/CU.H SEC/CU.M

I/SO.M METERS $\times 10 E+6$

$\begin{array}{llllll}98.0 & .2 & 50 & 6.605 E-08 & 1.255 E-07 & 3200 \\ 98.0 & .4 & 70 & 9.247 E-08 & 1.757 E-07 & 3200 \\ 98.0 & .8 & 70 & 9.247 E-08 & 1.757 E-07 & 3200 \\ 98.0 & 1.5 & 60 & 7.926 E-08 & 1.506 E-07 & 3200 \\ 98.0 & 1.6 & 70 & 9.247 E-08 & 1.757 E-07 & 3200 \\ 98.0 & 3.1 & 90 & 1.189 E-07 & 2.259 E-07 & 3200 \\ 98.0 & 6.2 & 60 & 7.926 E-08 & 1.506 E-07 & 3200 \\ 98.0 & 9.3 & 1 & 0 . & 0 . & 3200 \\ 98.0 & 12.4 & 1 & 0 . & 0 . & 3200 \\ 98.0 & 15.5 & 1 & 0 . & 0 . & 3200 \\ 98.0 & 18.6 & 1 & 0 . & 0 . & 3200 \\ 98.0 & 21.7 & 1 & 0 . & 0 . & 3200 \\ 98.0 & 24.8 & 1 & 0 . & 0 . & 3200 \\ 98.0 & 31.0 & 1 & 0 . & 0 . & 3200 \\ 98.0 & 37.2 & 1 & 0 . & 0 . & 3200 \\ 98.0 & 43.4 & 1 & 0 . & 0 . & 3200 \\ 98.0 & 49.6 & 1 & 0 . & 0 . & 3200 \\ 98.0 & 55.8 & 1 & 0 . & 0 . & 3200 \\ 98.0 & 62.0 & 1 & 0 . & 0 . & 3200\end{array}$

AIIMUTH HEIGHT EXPOSURE E/O EU/O DISTANCE DEGRES HETERS GH-SEC/CU.M SEC/CU.M I/SO.M METERS $X 10 E+6$

$\begin{array}{lr}106.0 & .2 \\ 106.0 & .4 \\ 106.0 & .8 \\ 106.0 & 1.5 \\ 1106.0 & 1.6 \\ 106.0 & 3.1 \\ 106.0 & 6.2 \\ 106.0 & 9.3 \\ 106.0 & 12.4 \\ 106.0 & 15.5 \\ 106.0 & 18.6 \\ 106.0 & 21.7 \\ 106.0 & 24.8 \\ 106.0 & 31.0 \\ 106.0 & 37.2 \\ 106.0 & 43.4 \\ 106.0 & 49.6 \\ 106.0 & 55.0 \\ 106.0 & 62.0\end{array}$

$\begin{array}{rl}3300 & 4.359 E-06 \\ 3050 & 4.029 E-06 \\ 3350 & 4.425 E-06 \\ 2400 & 3.170 E-06 \\ 2650 & 3.765 E-06 \\ 4300 & 5.680 E-06 \\ 3550 & 4.690 E-06 \\ 3700 & 4.868 E-06 \\ 3600 & 4.756 E-06 \\ 2900 & 3.831 E-06 \\ 2600 & 3.435 E-06 \\ 2000 & 2.642 E-06 \\ 2050 & 2.708 E-06 \\ 680 & 8.983 E-07 \\ 240 & 3.170 E-07 \\ 90 & 1.189 E-07 \\ 1 & 0.1 \\ 1 & 0 .\end{array}$

$\begin{array}{ll}6.283 E-06 & 3200 \\ 7.655 E-06 & 3200 \\ 8.408 E-06 & 3200 \\ 6.024 E-06 & 3200 \\ 7.153 E-06 & 3200 \\ 1.079 E-05 & 3200 \\ 6.910 E-06 & 3200 \\ 9.287 E-06 & 3200 \\ 9.036 E-06 & 3200 \\ 7.279 E-06 & 3200 \\ 6.526 E-06 & 3200 \\ 5.020 E-06 & 3200 \\ 5.145 E-06 & 3200 \\ 1.707 E-06 & 3200 \\ 6.024 E-07 & 3200 \\ 2.259 E-07 & 3200 \\ 0 . & 3200 \\ 0 . & 3200 \\ 0 . & 3200\end{array}$

AZ IMUTH HEIGHT

EXPOSURE

$F / 0$

EUIO DISTANCE DEGRES HETERS GH-SEC/CU.M SEC/CU.H $\times 10 E+6$

114.0
114.0
114.0
114.0
114.0
114.0
114.0
114.0
114.0
114.0
114.0
114.0
114.0
114.0
114.0
114.0
114.0
114.0
114.0

.2
.4
.8
1.5
1.6
3.1
6.2
9.3
12.4
15.5
18.6
21.7
24.8
31.0
37.2
43.4
49.6
55.8
62.0

1750
1750
1580
2350
1660
1650
2040
2230
1830
1760
1700
1650
1350
1050
740
570
240
80
50

$2.312 E-06$ $2.312 E-06$ $2.087 E-06$ 3. $104 \mathrm{E}-06$ 2.193 E-06 $2.180 E-06$ $2.695 \mathrm{E}-06$ $2.946 E-06$ $2.417 E-06$ 2. $325 E-06$ $2.246 E-06$ 2.180E-06 $1.783 E-06$ $1.387 E-06$ $9.775 E-07$ $9.775 E=07$
$3.530 E-07$ $7.530 E-07$
$3.170 E-07$ $1.057 E-07$
$6.605 E-08$

$\begin{array}{ll}4.392 E-06 & 3200 \\ 4.392 E-06 & 3200 \\ 3.966 E-06 & 3200 \\ 5.89 B E-06 & 3200 \\ 4.166 E-06 & 3200 \\ 4.141 E-06 & 3200 \\ 5.120 E-06 & 3200 \\ 5.597 E-06 & 3200 \\ 4.593 E-06 & 3200 \\ 4.417 E-06 & 3200 \\ 4.267 E-06 & 3200 \\ 4.141 E-06 & 3200 \\ 3.388 E-06 & 3200 \\ 2.635 E-06 & 3200 \\ 1.857 E-06 & 3200 \\ 1.431 E-06 & 3200 \\ 6.024 E-07 & 3200 \\ 2.008 E-07 & 3200 \\ 1.255 E-07 & 3200\end{array}$

1/50.M METERS 


\begin{tabular}{|c|c|c|c|c|c|c|c|c|c|c|c|}
\hline $\begin{array}{l}\text { ALIMUTH } \\
\text { DEGREES }\end{array}$ & $\begin{array}{l}\text { HE IGHT } \\
\text { METERS }\end{array}$ & $\begin{array}{c}\text { EXPOSURE } \\
\text { GH-SEC/CU.H } \\
\times 1 O E+6\end{array}$ & $\begin{array}{c}\text { E/O } \\
\text { SEC/CU.A }\end{array}$ & $\begin{array}{c}E U / O \\
1 / 5 O . M\end{array}$ & $\begin{array}{l}\text { OISTANCE } \\
\text { ME TERS }\end{array}$ & $\begin{array}{l}\text { AZ I HUTH } \\
\text { DE GREES }\end{array}$ & $\begin{array}{l}\text { HE IGHT } \\
\text { NETERS }\end{array}$ & $\begin{array}{l}\text { EXPOSURE } \\
\text { GH-SEC /CU.H } \\
\times 1 O E+6\end{array}$ & SEC/CU.H & $\begin{array}{l}E U / O \\
1 / S O \cdot A\end{array}$ & $\begin{array}{c}\text { OISTANCE } \\
\text { HETERS }\end{array}$ \\
\hline $\begin{array}{l}122.0 \\
122.0 \\
122.0 \\
122.0 \\
122.0 \\
122.0 \\
122.0 \\
122.0 \\
122.0 \\
122.0 \\
122.0 \\
122.0 \\
122.0 \\
122.0 \\
122.0 \\
122.0 \\
122.0 \\
122.0 \\
122.0\end{array}$ & $\begin{array}{l}.2 \\
.4 \\
.8 \\
1.5 \\
1.6 \\
3.1 \\
6.2 \\
9.3 \\
12.4 \\
15.5 \\
10.6 \\
21.7 \\
24.0 \\
31.0 \\
37.2 \\
43.4 \\
49.6 \\
55.1 \\
62.0\end{array}$ & $\begin{array}{r}0 \\
0 \\
0 \\
0 \\
0 \\
17 \\
22 \\
25 \\
16 \\
1 \\
7 \\
11 \\
1 \\
2 \\
1 \\
13 \\
5 \\
6 \\
0\end{array}$ & $\begin{array}{l}0 . \\
0 . \\
0 . \\
0 . \\
0 . \\
2.309 E-08 \\
2.95 U E-08 \\
3.305 E=08 \\
2.131 E-08 \\
2.125 E=09 \\
9.309 E-09 \\
1.510 E-08 \\
1.176 E-08 \\
3.764 E-09 \\
1.807 E-09 \\
1.735 E-08 \\
6.755 E-09 \\
0.331 E-09 \\
0.3\end{array}$ & $\begin{array}{l}0 . \\
0 . \\
0 . \\
0 . \\
0 . \\
4.386 E-08 \\
5.605 E-08 \\
6.280 E-08 \\
4.049 E-08 \\
4.038 E-09 \\
1.769 E-08 \\
2.869 E-08 \\
2.234 E-08 \\
7.151 E-09 \\
3.433 E-09 \\
3.297 E-08 \\
1.283 E-08 \\
1.583 E-08 \\
0 .\end{array}$ & $\begin{array}{l}3200 \\
3200 \\
3200 \\
3200 \\
3200 \\
3200 \\
3200 \\
3200 \\
3200 \\
3200 \\
3200 \\
3200 \\
3200 \\
3200 \\
3200 \\
3200 \\
3200 \\
3200 \\
3200\end{array}$ & $N 122.0$ & $\ldots$ & 2 & 0. & 0. & 3200 \\
\hline $\begin{array}{l}\text { AZIMUTH } \\
\text { OEGREES }\end{array}$ & $\begin{array}{l}\text { HE IGNT } \\
\text { ME TERS }\end{array}$ & $\begin{array}{l}\text { EXPOSURE } \\
\text { GH-SECICU.H } \\
\text { X1OE } \$ 6\end{array}$ & $\begin{array}{c}E / O \\
S E C / C U . M\end{array}$ & $\begin{array}{l}E U / 0 \\
1 / 50 . M\end{array}$ & $\begin{array}{l}\text { OISTANCE } \\
\text { METERS }\end{array}$ & $\begin{array}{l}\text { AZIMUTH } \\
\text { DEGREES }\end{array}$ & $\begin{array}{l}\text { HEICHT } \\
\text { HETERS }\end{array}$ & $\begin{array}{l}\text { EXPOSURE } \\
\text { GH-SEC /CU.H } \\
\times 1 O E \$ 6\end{array}$ & SEC/CU.H & $\begin{array}{l}\text { EU/O } \\
1 / 50.4\end{array}$ & $\begin{array}{c}\text { DISTANCE } \\
\text { METERS }\end{array}$ \\
\hline $\begin{array}{l}130.0 \\
130.0 \\
130.0 \\
130.0 \\
130.0 \\
130.0 \\
130.0 \\
130.0 \\
130.0 \\
130.0 \\
130.0 \\
130.0 \\
130.0 \\
130.0 \\
130.0 \\
130.0 \\
130.0 \\
130.0 \\
130.0\end{array}$ & $\begin{array}{r}.2 \\
.4 \\
.8 \\
1.3 \\
1.6 \\
3.1 \\
6.2 \\
9.3 \\
12.4 \\
15.3 \\
18.6 \\
21.7 \\
24.8 \\
31.0 \\
37.2 \\
43.4 \\
49.6 \\
55.8 \\
62.0\end{array}$ & $\begin{array}{r}0 \\
0 \\
4 \\
0 \\
0 \\
19 \\
19 \\
6 \\
6 \\
0 \\
0 \\
0 \\
0 \\
12 \\
12 \\
6 \\
0 \\
0 \\
0\end{array}$ & $\begin{array}{l}0 . \\
0 . \\
5.387 E-09 \\
0 . \\
0 . \\
2.565 E-08 \\
2.565 E-08 \\
0.570 E-09 \\
8.974 E-09 \\
0 . \\
0 . \\
0 . \\
0 . \\
1.694 E-08 \\
1.626 E-08 \\
8.676 E-09 \\
0 . \\
0 . \\
0 .\end{array}$ & $\begin{array}{l}0 . \\
0 . \\
1.023 E-08 \\
0 . \\
0 . \\
4.674 E-08 \\
4.874 E-08 \\
1.628 E-08 \\
1.705 E-08 \\
0 . \\
0 . \\
0 . \\
0 . \\
3.218 E-08 \\
3.090 E-08 \\
1.648 E-08 \\
0 . \\
0 . \\
0 .\end{array}$ & $\begin{array}{l}3200 \\
3200 \\
3200 \\
3200 \\
3200 \\
3200 \\
3200 \\
3200 \\
3200 \\
3200 \\
3200 \\
3200 \\
3200 \\
3200 \\
3200 \\
3200 \\
3200 \\
3200 \\
3200\end{array}$ & N 130.0 & .2 & $\ldots$ & 0. & $>-$ & 3200 \\
\hline
\end{tabular}


GRDUNO LEVEL AHO TONER SAMPLING 200M TO $3200 \mathrm{M}$.

20OM ARC EXTRAPOLATED AT BUTH EDGES. FLAT OR MULTI-MODE CROSSHIND OISTRIBUTIONS DN ALL ARCS. INYALID SAMPLES ABDVE 4.2M LEVEL DN TOWER AT 122 DEG ON BOOH ARC. VERTICAL OISTRIBUTION OF TRACERS ON ALL 20 TOWERSI PEAK VALUES MEASURED. BUT TRUNCATED AT TOP IN ALL CASES.

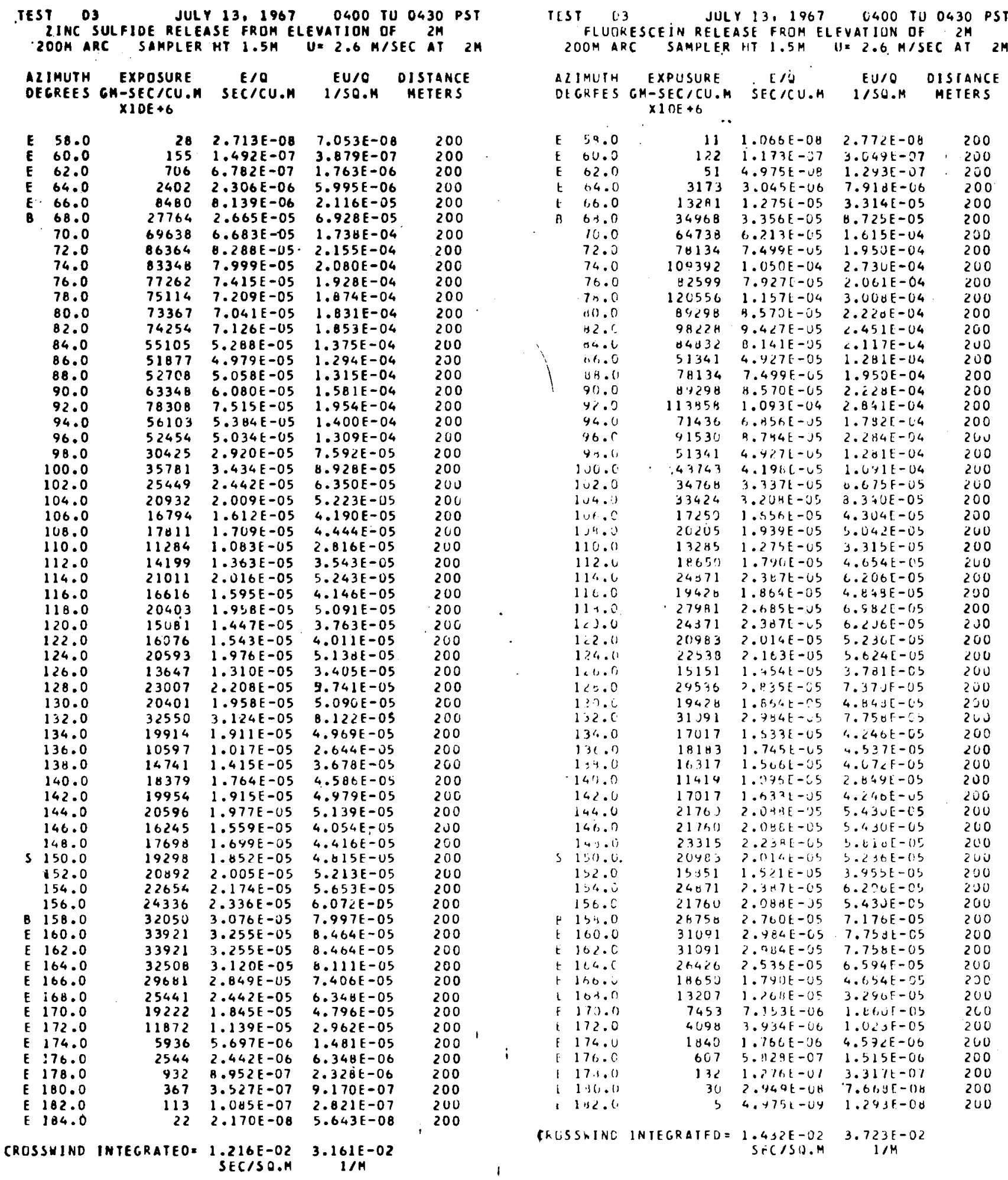


TEST D3 JULY 13, 1967 0400 TU 0430 PST 2 INC SULFIOE RELEASE FROH ELEVATION OF $2 M$ DOOM ARC SAMPLER HT $1.5 \mathrm{M}$ U. $2.6 \mathrm{H} / \mathrm{SEC}$ AT $2 \mathrm{M}$

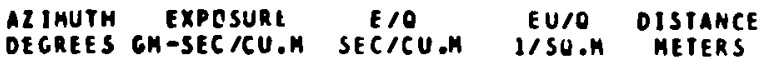
$\times 10 E+6$

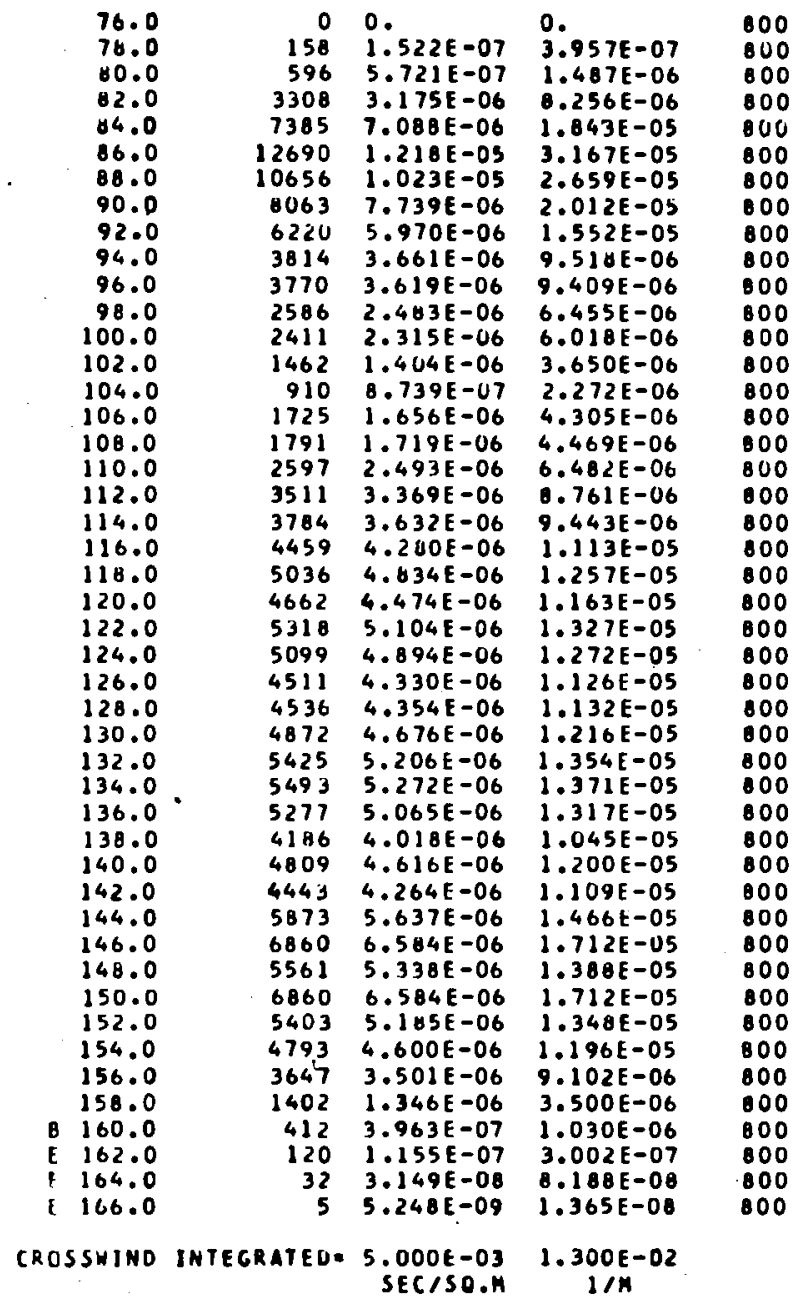
TEST D D
FLUURESCEIN RELEASE FRUM ELFVATION OF 13,1967 OM PST SAMPLER HT $1.5 \mathrm{M} U=2.6 \mathrm{H} / 5 E \mathrm{C}$ AT $2 \mathrm{M}$

AZIMUTH EXPOSURE $T / O$ CU/O OISTANCE OEGKES CH-SEC/CU.H SEC/CU.M I/SO.M METERS $\times 10 \mathrm{t}+6$

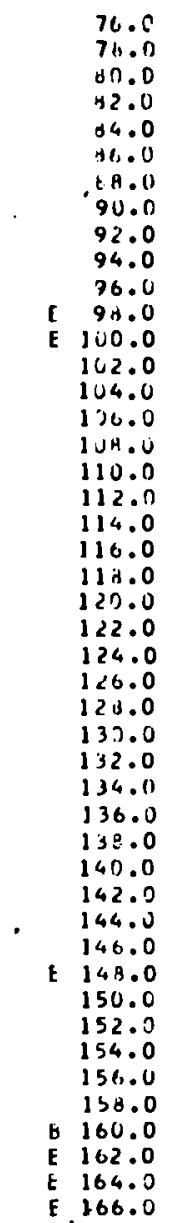

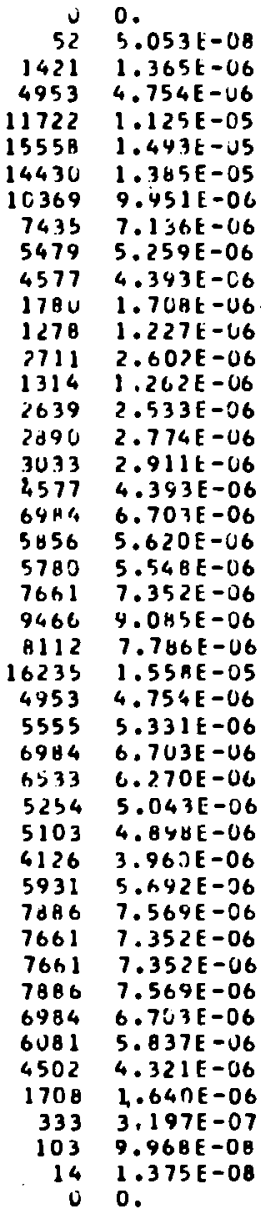

i.

$1.314 E-07$ $3.34 \forall E-06$ $1.236 E-05$ $2.925 \mathrm{~F}-05$ 3. $H$ B LE-OS $3.601 E-05$ $2.597 E-05$ $1.855 \mathrm{E}-05$ $1.367 E-05$ $1.142 E-05$ $4.442 \mathrm{E}-06$ $3.191 \mathrm{E}-06$ $6.703 E-06$ $3.29 \cup E-06$ 6. $587 \mathrm{E}-06$ $7.21<E-06$ $7.5 \times \mathrm{t}-06$ $1.142 \mathrm{E}-05$ $1.743 \mathrm{E}-05$ $1.661 E-05$ $1.442 \mathrm{E}-05$ $1.91<E-05$ $2.362 E-05$

$2.024 E-05$ $4.051 E-05$ $1.236 \mathrm{E}-05$ $1.306 E-05$

$1.743 E-05$

$1.630 E-05$

$1.311 E-05$ $1.274 \mathrm{E}-05$ $1.030 \mathrm{E}-05$ $1.480 E-05$ 1.960 E-CS $1.912 E-05$ $1.912 \mathrm{E}-05$

$1.966 E-05$

$1.743 E-05$

$1.518 \mathrm{E}-05$

1. $123 \mathrm{E}-05$ $4.263 t-06$ $8.311 E-07$ $2.592 E-07$ $3.575 \mathrm{E}-08$ 0 .

CRUSSHIND INTEGRATED * $6.743 E-03$ 1.753E-02
800
800
800
800
800
800
800
800
800
800
800
100
800
800
800
800
800
800
800
800
800
800
800
800
800
800
800
800
800
800
800
800
800
800
800
800
800
800
800
800
800
800
800
800
800
800 
TEST D3 LIME SULFIDE RELEASE FROM ELEVATION OF 2430 PST $1600 \mathrm{M}$ ARC SAMPLER HT $1.5 \mathrm{H}$ U. 2.6 M/SEC AT 21

$\begin{array}{lcc}\text { ALIMUTH EXPOSURE } & \text { E/O } & \text { EU/O OISTANCE } \\ \text { DEGREES GH-SEC/CU.M SEC/CU.M TISO.M METERS }\end{array}$ $\times 10 E+6$

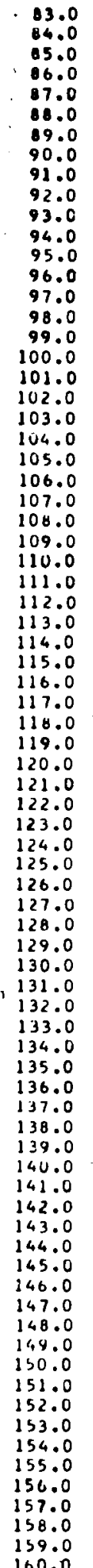

0.

$7.336 E-06$

$5.380 E-08$

$1.467 E-07$

$6.358 \mathrm{E}-08$

$1.418 \mathrm{E}-07$

$1.859 \mathrm{E}-07$

$3.032 E-07$

$2.220 E-06$

3. $345 E-06$

$6.505 E-06$

$9.361 E-06$

$1.047 E-05$

$1.143 E-05$

$1.095 E-05$

$9.094 E-06$

$9.156 E-06$

$9.439 \mathrm{E}-06$

$8.931 E-06$

$8.725 E-06$

8.838E-06

$1.096 E-05$

$1.011 E-05$

$7.644 \mathrm{E}-06$

$1.168 E-05$

$1.137 E-05$
$1.097 E-05$
$1.301 E-05$

1.301E-0S

1.166 E-0S

$8.344 \mathrm{E}-06$

$6.950 E-06$

$9.601 E-06$

$9.004 \mathrm{E}-06$
$8.251 \mathrm{E}-06$

6.720 E-06

$6.730 \mathrm{~F}-06$

$5.220 E-06$

$4.441 E-06$
$3.629 E-06$

$3.433 E-06$

$3.600 \mathrm{E}-06$

$3.81 S E-U 6$

$3.981 E-06$

$3.248 \mathrm{E}-06$

$4.705 t-06$

$5.5 C 2 \mathrm{E}-06$

$5.140 E-06$

$5.414 E-06$

$6.564 \mathrm{E}-06$

$6.63 \angle E-06$

$6.5101-06$

$6.192 \mathrm{E}-06$

$6.495 E-06$

$6.079 \mathrm{E}-06$

$7.229 E-06$
$8.403 E-06$

$8.403 \mathrm{E}-06$
$7.424 \mathrm{E}-06$

$8.466 \mathrm{~F}-06$

$7.517 E-06$

$7.821 E-00$

6.56日E-U6

$6.172 E-06$

$4.651 \mathrm{E}-06$

$5.331 E-06$

3.164 E-O6

$2.573 \mathrm{E}-06$

$1.893 \mathrm{E}-06$
$1.203 \mathrm{E}-06$

$6.211 E-07$

$3.766 \mathrm{E}-07$

$2.250 E-07$

1.174F-07

$5.300 E-08$

$\begin{array}{lll}7 & 7.524 \mathrm{E}-09 & 1.956 \mathrm{E}-08 \\ 3 & 3.762 \mathrm{E}-09 & 9.702 \mathrm{E}-09\end{array}$

1600

1600

CROSSHIND INTEGRAIED=4.757E-03 1.237E-02 SECISO.K 114

TEST D3 JUTY 13. 1967 OSOO TU 0430 PST FLUURESCEIA RELEASE FRUM ELEYATION OF 2M AZIAUTH EXPUSURE
OEGKEES GM-SECICU.M SECICU.M IISLOM DISIANCE $X 1 O E+6$

B3.0

84.0

o5.0

ib. 0

47.1

88.0

89.0

90.0

$\rightarrow 1.0$

92.0

93.0

94.0

45.0

96.0

97.0

94.0
100.0

101.0

122.0

103.0

104.0

165.0

$11) 6.0$

107.0

1 Us. 0

159.0

110.0

111.0

112.0

113.0

114.0

115.0

116.0

117.0

120.0

$1<1.0$

122.0

123.0

124.0

$1<5.0$

$1<0.0$

147.0

125.0

124.6

$13 i) .0$

131.0

$1,2.3$

$+33.0$

194.0

131.0

135.0

131.0

$13+.0$

119.0

11.0 .1 ,

1.1 .0

142.0

$1,3.11$

144.1

145.0

161.01

147.6

142.0

164.6

11.1 .0

131.11

$1>2.6$

$1 \cdot 3.01$

134.0

E 155.0

151.00

151.0

$10 H .0$
1

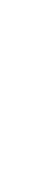

$\begin{array}{rr}1 & 1.360 E-09 \\ 21 . & 2.0140 E-08 \\ B \quad & .347 E-09\end{array}$

S. $5.40 E-09$ 1.4QISt-Od

$141.42 \pi t-U \theta 3.713 E-U C$

$514.987 \mathrm{E}-60 \quad 1.297 \mathrm{E}-07$

$26 \quad 2.516 E-5 B \quad 6.542 E-08$

$226 \quad 2.176 E-07 \quad 5.6385-07$

$545 \quad 5.236 E-J 7$ 1.361E-06

$2000 \quad 1.920 E-06 \quad 4.992 E-06$

$2420 \quad 2.707 \mathrm{E}-06 \quad 7.037 \mathrm{~F}-06$

$4159 \quad 3.99 / F-U 6 \quad 1.034 F-05$

$4754 \quad 4.563 E-06 \quad 1.196 E-05$

5052 4.049E-U6 1.261E=05

$5647 \quad 5.420 t-06 \quad 1.404 E-05$

$7731 \quad 7.419 \mathrm{E}-06 \quad 1.929 \mathrm{E}-05$

$5052 \quad 4.849 E-06 \quad 1.2 C 1 E-05$

$4457 \quad 4.2744-06 \quad 1.1225-05$

$3061 \quad 3.706 \mathrm{E}-06 \quad 9.636 \mathrm{E}-00$

$2572 \quad 2.469 E-06 \quad 6.416 E-06$

$3266 \quad 3.135 E-156 \quad 8.151 E-06$

$3167 \quad 3.04 \cap E-06 \quad 7.904 E-00$

4010 3.1149t-06, 1.001t-0j

$4606 \quad 4.420 \mathrm{t}-06, \quad 1.144 \mathrm{E}-0 \mathrm{~S}$

$2919 \quad 2.4021-56 \quad 7.285 E-06$

$44034.706 \mathrm{~L}-U 6 \quad 1.224 \mathrm{E}-\mathrm{US}$

$4703 \quad 4.7$ UBE-JG $1.224 E-05$

4457 4.27At-J6 1.112t-05

4159 3.49?L-J6 1.03UE-0S

$430 \mathrm{~B} \quad 4.135 \mathrm{t}-66 \quad 1.075 \mathrm{~F}-05$

$5 J 52 \quad 4.449 E-05$

$37133.56 .4 \mathrm{~F}-\mathrm{CH}$

$31172.942 \mathrm{E}-06$

$350,4 \quad 3.421 \mathrm{~L}-U \mathrm{E}$

$43004.135 \mathrm{~L}-0 \mathrm{~B}$

$26712.5165-06$

$2423 \quad 2.3<6 E-06$

$21>5 \quad 2.0405-06$

$1 \% 77 \quad 1 . A 1: 2 F-36$

13511.4 yyt-ve

1334 1.215E-U6

$15281.467 t-60$

$18111.7395-35$

2071 1.9UAE-UC

$14101.333 E-66$

T213 2.124E-06

$1435 \quad 1.761 E-U B$

$1764 \quad 1.643 t-36$

$14581.784 \mathrm{E}-06$

$2307 \quad 2.215 E-26$

1760 1.671t-06

$2071 \quad 1.404 \mathrm{t}-06$

$2420 ? .7075-06$

$23072.215 t-0 s$

(32) $2.7078-36$

$2770 \quad 2.0545-06$

$23732.278 t-06$

$26712.516[-06$

$26712.5161-06$

2919
2572
$3.40254-66$

2572
33093
$2.364 t-36$

$23732.2791-i 0$

$26712.466 t-06$

$26712.564 t-06$.

126j $2.1041-130$

$2324 \quad 1.443 \mathrm{E}-\mathrm{Jh}$

$1929 \quad 1.0421-30$

$1524 \quad 1.4671-06$

$1.321 .376 .1-56$

औ66 ०. $3198-67$

$3112.4928-67$

$148 \quad 1.428 \mathrm{E}-07$

$6154345-04$

$30 \quad 2.947 \mathrm{~L}-\mathrm{OH}_{\mathrm{B}}$

$141.36 \% 5-0 \mathrm{H}$

$50.0+1 t-09$

$1.261 E-0 S$

$9.265[-U S$

$7.7 \triangle U E-00$

$6.844 E-06$

$1.0751-05$

c. $542 \mathrm{E}-06$

$6.047 E-06$

5. 3.) 45-06

$4.6 H O 1-U C$
$3.0721-06$

$3.34<E-16$

3. $813 E-06$

$4.521 E-0 b$

5.16\%t-0s

3.519E-06

$5.523 L-06$

4.580 E-O6

$4.4 J 3 \mathrm{E}-06$

$4.638 \mathrm{E}-06$

$5.750 t-06$

$4.344 F-166$

3.169 E-06

7.037 E-U6

$3.730 E-06$

$7.031 L-U h$

$0.913 t-J 6$
$5.923[-c 6$

$6.54<E-16$

$7.20,1-60$

$6.418 \mathrm{E}-06$

$.656 \mathrm{~F}-\mathrm{i6}$

$3.92 د E-U h$

c. $6664-06$

c.660L-00

S. Sं। $1 E-00$

$4.215 t-6 t$

s.

9.57ut-00

$2.1635-06$

1.7 UE -01

$3.713 E-07$

$1.532 \mathrm{f}-0)$

$7.65 c t-0 y$

3.536t-Ut

$1.297 E-08$
$. .844 E-09$

1600 .

1600

1600

1600
1600

1 ค०OO

1600

1500

1600

1600
1600

1600

1600 


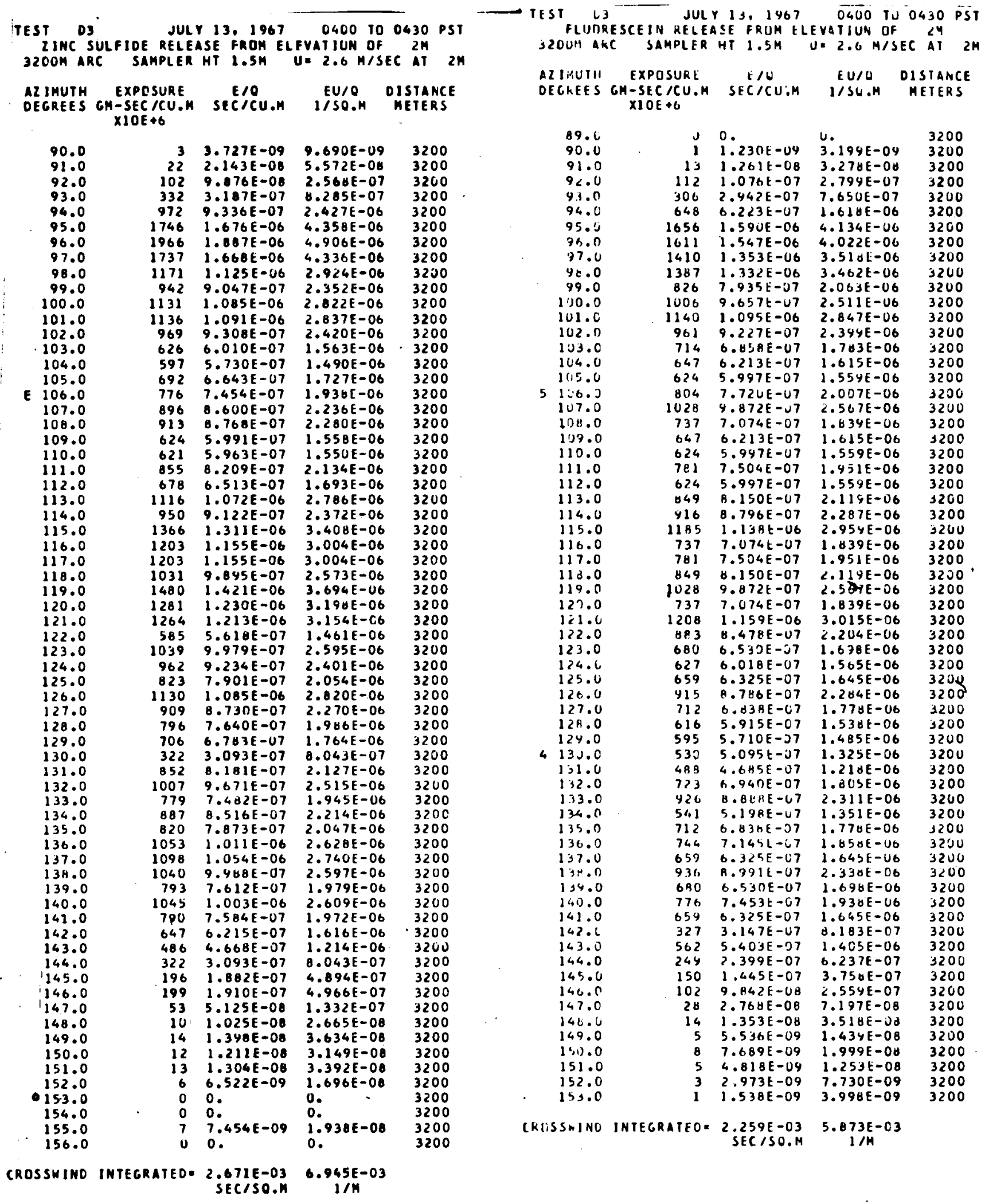


TOHER DATA FULLDW....

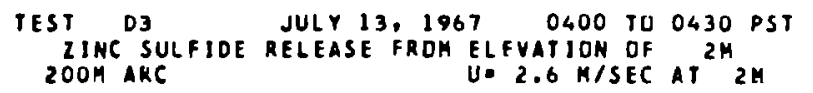

TDWER DATA F RLLOH....

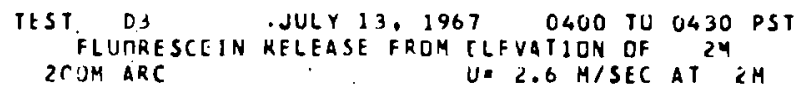

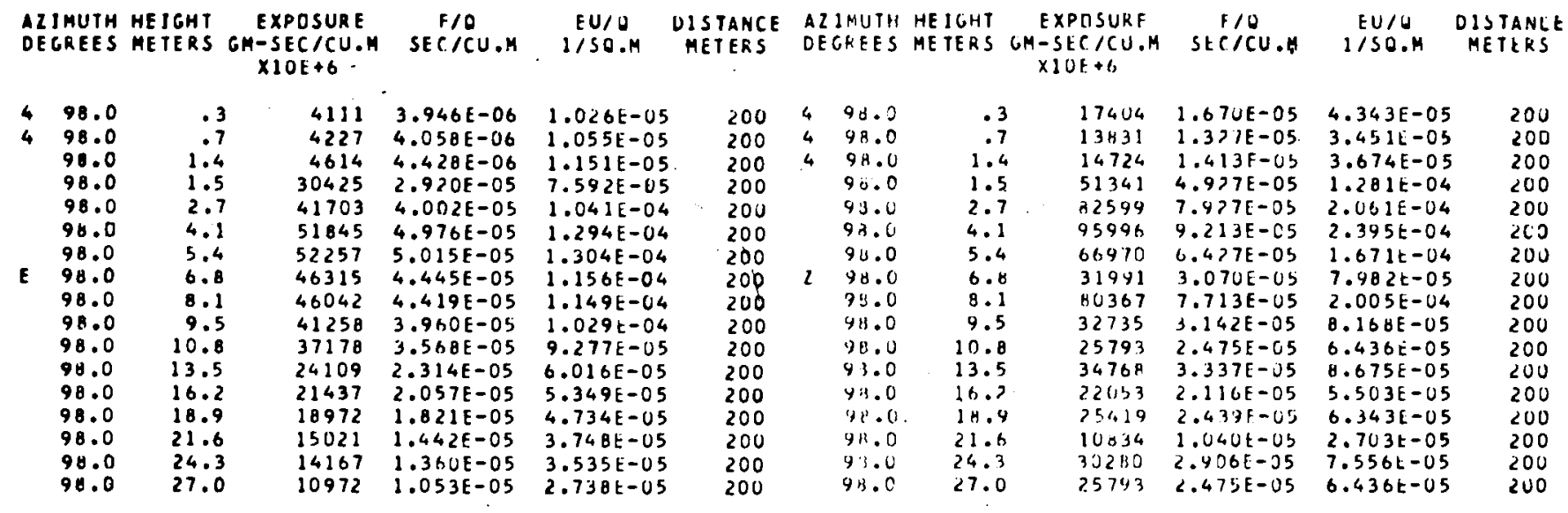

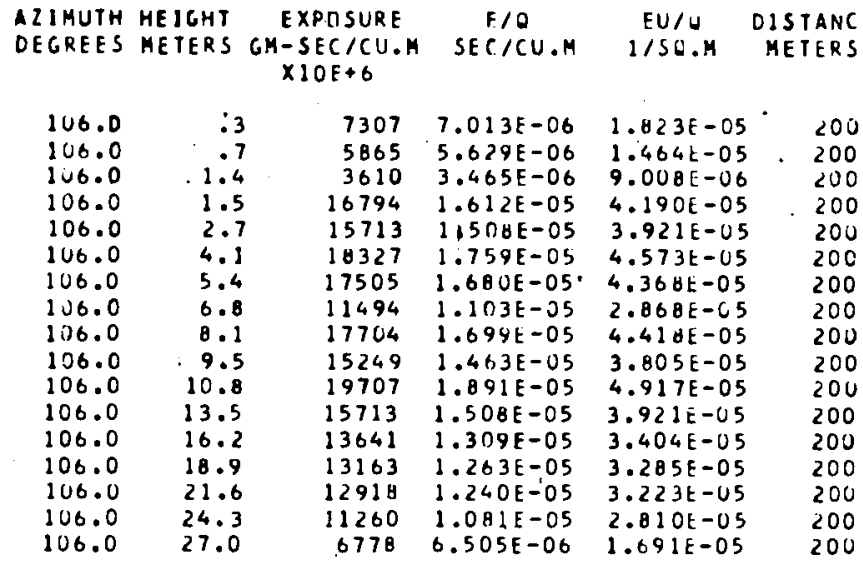
ALIMUTH HELGHT
DEGKEES METERS
EXPUSHRF E
$\times 101+6$

6597
7920
4809
17250
16317
17017
16044
13751
27203
18650
17484
16084
15617
34701
15151
40427
9786

$\begin{array}{lr}106.0 & .3 \\ 1110.0 & .7 \\ 106.0 & 1.4 \\ 106.0 & 1.5 \\ 106.0 & 2.7 \\ 106.0 & 4.1 \\ 106.0 & 5.4 \\ 1116.0 & 6.8 \\ 106.0 & 8.1 \\ 110.0 & 9.5 \\ 106.0 & 10.8 \\ 1116.01 & 13.5 \\ 106.0 & 16.2 \\ 110.0 & 14.9 \\ 106.0 & 21.6 \\ 106.0 & 24.3 \\ 106.0 & 27.0\end{array}$

$\mathrm{F} / \mathrm{O}$ StC. CU.M
FU/O OISTANCE IISE.M METEKS
200
200
200
200
200
200
200
200
200
200
200
200
200
200
200
200
200

\begin{tabular}{|c|c|c|c|c|c|c|c|c|c|c|c|}
\hline $\begin{array}{l}2 \text { I MUTH } \\
\text { EGREES }\end{array}$ & $\begin{array}{l}\text { HE JGHT } \\
\text { ME TERS }\end{array}$ & $\begin{array}{c}\text { EXPOSURE } \\
\text { GM-SEC/CU.M } \\
\times 10 L+6\end{array}$ & $\begin{array}{c}F / O \\
S E C / C U . M\end{array}$ & $\begin{array}{l}E \cup / 0 \\
1 / S O . M\end{array}$ & $\begin{array}{l}\text { OISTANCE } \\
\text { METERS }\end{array}$ & $\begin{array}{l}\text { AZIYUTH } \\
\text { DERGES }\end{array}$ & $\begin{array}{l}\text { HE IGHT } \\
\text { METERS }\end{array}$ & $\begin{array}{c}\text { EXPOSUIRE } \\
\text { GM-SEC } / C U . M \\
\text { XJOF+C }\end{array}$ & $\begin{array}{c}F / O \\
\operatorname{SEC} / \mathrm{CU}, \mathrm{M}\end{array}$ & $\begin{array}{l}E U / A \\
1 / 50.4\end{array}$ & $\begin{array}{l}\text { OISTANCE } \\
\text { METERS }\end{array}$ \\
\hline $\begin{array}{l}114.0 \\
114.0 \\
114.0 \\
114.0 \\
114.0 \\
114.0 \\
114.0 \\
114.0 \\
114.0 \\
114.0 \\
114.0 \\
114.0 \\
114.0 \\
114.0 \\
114.0 \\
114.0 \\
114.0\end{array}$ & $\begin{array}{l}.3 \\
.7 \\
1.4 \\
1.5 \\
2.7 \\
4.1 \\
5.4 \\
6.8 \\
8.1 \\
9.5 \\
10.8 \\
13.5 \\
16.2 \\
18.9 \\
21.6 \\
24.3 \\
27.0\end{array}$ & $\begin{array}{r}5277 \\
6163 \\
5900 \\
21011 \\
19075 \\
22659 \\
19308 \\
18305 \\
23316 \\
33194 \\
31360 \\
27207 \\
30381 \\
18368 \\
13260 \\
6283 \\
3500\end{array}$ & $\begin{array}{l}5.065 E-06 \\
5.915 E-06 \\
5.663 E-06 \\
2.016 E-05 \\
1.031 E-05 \\
2.175 E-05 \\
1.853 E-05 \\
1.757 E-05 \\
2.238 E-05 \\
3.186 E-05 \\
3.010 E-05 \\
2.611 E-05 \\
2.916 E-05 \\
1.763 E-05 \\
1.273 E-05 \\
6.031 E-06 \\
3.360 E-06\end{array}$ & $\begin{array}{l}1.317 E-05 \\
1.538 t-05 \\
1.472 t-05 \\
5.243 E-05 \\
4.760 E-05 \\
5.654 t-05 \\
4.818 t-05 \\
4.567 E-05 \\
5.818 t-05 \\
8.283 t-05 \\
71.825 t-05 \\
6.789 t-05 \\
7.581 E-05 \\
4.583 E-05 \\
3.309 E-05 \\
1.568 t-05 \\
8.736 E-06\end{array}$ & $\begin{array}{l}200 \\
200 \\
200 \\
200 \\
200 \\
200 \\
200 \\
200 \\
200 \\
200 \\
200 \\
200 \\
200 \\
200 \\
200 \\
200 \\
200\end{array}$ & $\begin{array}{l}114.01 \\
114.00 \\
114.0 \\
114.0 \\
114.0 \\
114.0 \\
114.0 \\
114.0 \\
114.0 \\
114.0 \\
114.0 \\
114.0 \\
114.0 \\
114.0 \\
114.0 \\
114.0 \\
114.0\end{array}$ & $\begin{array}{r}.3 \\
.7 \\
1.4 \\
1.5 \\
2.7 \\
4.1 \\
1.04 \\
6.8 \\
6.1 \\
9.5 \\
10.8 \\
13.5 \\
16.2 \\
18.9 \\
21.5 \\
24.3 \\
27.0\end{array}$ & $\begin{array}{l}5354 \\
6447 \\
5176 \\
24871 \\
19350 \\
23315 \\
17017 \\
19103 \\
20426 \\
38194 \\
40377 \\
38134 \\
23923 \\
24871 \\
21700 \\
6986 \\
5509\end{array}$ & 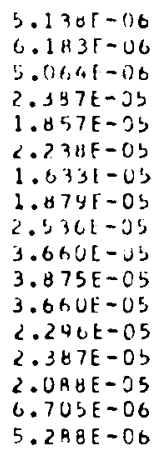 & 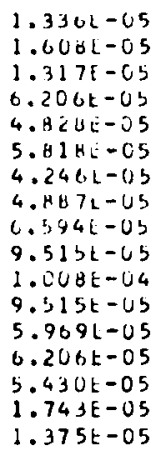 & $\begin{array}{l}200 \\
200 \\
200 \\
200 \\
200 \\
200 \\
200 \\
200 \\
200 \\
200 \\
200 \\
200 \\
200 \\
200 \\
200 \\
200 \\
200\end{array}$ \\
\hline
\end{tabular}




\begin{tabular}{|c|c|c|c|c|c|c|c|c|c|c|c|}
\hline $\begin{array}{l}\text { LIMUTH } \\
\text { JEGREES }\end{array}$ & $\begin{array}{l}\text { HE IGHT } \\
\text { METERS }\end{array}$ & $\begin{array}{l}\text { EXPDSURE } \\
\text { CH -SECICU.H } \\
\text { XIOE } \odot 6\end{array}$ & $\begin{array}{c}F / O \\
S E C / C U, H\end{array}$ & $\begin{array}{l}E U / 0 \\
1 / 50 . H\end{array}$ & $\begin{array}{c}\text { DISTANCE } \\
\text { HETERS }\end{array}$ & $\begin{array}{l}\text { ALI:AUTH } \\
\text { DEGKEES }\end{array}$ & $\begin{array}{l}\text { ME IGHT } \\
\text { ME TERS }\end{array}$ & $\begin{array}{l}\text { EXPTSURE } \\
\text { GH-SECICU.H } \\
\times 1 O E+6\end{array}$ & $\begin{array}{c}E / O \\
\text { SEC /CU.H }\end{array}$ & $\begin{array}{l}E U / 0 \\
1 / 50 . M\end{array}$ & $\begin{array}{l}\text { DIST ANCE } \\
\text { METERS }\end{array}$ \\
\hline $\begin{array}{l}122.0 \\
122.0 \\
122.0 \\
122.0 \\
122.0 \\
122.0 \\
122.0 \\
122.0 \\
122.0 \\
122.0 \\
122.0 \\
122.0 \\
122.0 \\
122.0 \\
122.0 \\
122.0 \\
122.0\end{array}$ & $\begin{array}{r}.3 \\
.7 \\
1.4 \\
1.5 \\
2.7 \\
4.1 \\
5.4 \\
6.8 \\
8.1 \\
9.5 \\
10.8 \\
13.5 \\
16.2 \\
18.9 \\
21.6 \\
24.3 \\
27.0\end{array}$ & $\begin{array}{l}350 \\
3250 \\
4007 \\
16076 \\
16433 \\
18591 \\
19888 \\
13522 \\
15207 \\
14006 \\
16783 \\
12443 \\
9882 \\
9684 \\
9903 \\
6242 \\
5880\end{array}$ & $\begin{array}{l}3.360 E-07 \\
3.120 E-06 \\
3.846 E-06 \\
1.543 E-05 \\
1.577 E-05 \\
1.784 E-05 \\
1.909 E-05 \\
1.298 E-05 \\
1.459 E-05 \\
1.344 E-05 \\
1.611 E-05 \\
1.194 E-05 \\
9.484 E-06 \\
9.294 E-06 \\
9.505 E-06 \\
5.991 E-06 \\
5.644 E-06\end{array}$ & $\begin{array}{l}0.737 E-07 \\
8.111 E-06 \\
1.000 E-05 \\
4.011 E-05 \\
4.101 E-05 \\
4.639 E-05 \\
4.962 E-05 \\
3.374 E=05 \\
3.795 E-05 \\
3.495 E-05 \\
4.188 E-05 \\
3.105 E-05 \\
2.466 E-05 \\
2.416 E-05 \\
2.471 E-05 \\
1.558 E-05 \\
1.467 E-05\end{array}$ & $\begin{array}{l}200 \\
200 \\
200 \\
200 \\
200 \\
200 \\
200 \\
200 \\
200 \\
200 \\
200 \\
200 \\
200 \\
200 \\
200 \\
200 \\
200\end{array}$ & $\begin{array}{l}122.0 \\
122.0 \\
122.0 \\
122.0 \\
1122.0 \\
122.0 \\
122.0 \\
122.0 \\
122.0 \\
122.0 \\
122.0 \\
122.0 \\
122.0 \\
122.0 \\
122.0 \\
122.0 \\
122.0\end{array}$ & $\begin{array}{l}.3 \\
1.7 \\
1.5 \\
2.7 \\
4.1 \\
5.4 \\
6.8 \\
8.1 \\
9.5 \\
10.8 \\
13.5 \\
16.2 \\
18.9 \\
21.6 \\
24.3 \\
27.0\end{array}$ & $\begin{array}{r}522 \\
6209 \\
11685 \\
20983 \\
0 \\
20963 \\
14918 \\
12352 \\
24871 \\
12352 \\
15851 \\
19428 \\
11845 \\
20205 \\
10252 \\
12818 \\
13518\end{array}$ & $\begin{array}{l}5.010 E-07 \\
5.959 E-06 \\
1.141 E-05 \\
2.014 E-05 \\
0 . \\
2.014 E-05 \\
1.432 E-05 \\
1.135 E-05 \\
2.387 E-05 \\
1.185 E-05 \\
1.571 E-05 \\
1.8 B 4 E-05 \\
1.141 E-05 \\
1.939 E-05 \\
9.840 E-06 \\
1.23 U E-0 S \\
1.297 E-05\end{array}$ & $\begin{array}{l}1.303 E-06 \\
1.549 E-05 \\
2.966 E-05 \\
5.236 E-05 \\
0 . \\
5.236 E-05 \\
3.722 E-05 \\
3.082 E-05 \\
6.206 E-05 \\
3.082 E-05 \\
3.955 E-05 \\
4.848 E-05 \\
2.966 E-05 \\
5.042 E-05 \\
2.558 E-05 \\
3.199 E-05 \\
3.373 E-05\end{array}$ & $\begin{array}{l}200 \\
200 \\
200 \\
200 \\
200 \\
200 \\
200 \\
200 \\
200 \\
200 \\
200 \\
200 \\
200 \\
200 \\
200 \\
200 \\
200\end{array}$ \\
\hline
\end{tabular}

AZIMUTH HEIGHT EXPOSURE F/O EU/O OISTANCE OEGREES METERS GM-SEC/CU.M SEC/CU.M I/SO.M METERS $\times 10 E+6$

$\begin{array}{lrrrrr}130.0 & .3 & 7992 & 7.670 E-06 & 1.994 E-05 & 200 \\ 130.0 & .7 & 6025 & 5.783 E-06 & 1.503 E-05 & 200 \\ 130.0 & 1.4 & 6322 & 6.067 E-06 & 1.577 E-05 & 200 \\ 130.0 & 1.5 & 20401 & 1.958 E-05 & 5.090 E-05 & 200 \\ 130.0 & 2.7 & 18617 & 1.806 E-05 & 4.695 E-05 & 200 \\ 130.0 & 4.1 & 16819 & 1.614 E-05 & 4.197 E-05 & 200 \\ 130.0 & 5.4 & 32703 & 3.138 E-05 & 8.160 E-05 & 200 \\ 130.0 & 6.8 & 27844 & 2.672 E-05 & 6.948 E-05 & 200 \\ 130.0 & 8.1 & 34902 & 3.350 E-05 & 1.709 E-05 & 200 \\ 130.0 & 9.5 & 24433 & 2.345 E-05 & 6.097 E-05 & 200 \\ 130.0 & 10.8 & 22045 & 2.116 E-05 & 5.501 E-05 & 200 \\ 130.0 & 13.5 & 21258 & 2.040 E-05 & 5.304 E-05 & 200 \\ 130.0 & 16.2 & 21263 & 2.041 E-05 & 5.306 E-05 & 200 \\ 130.0 & 18.9 & 15734 & 1.510 E-05 & 3.926 E-05 & 200 \\ 130.0 & 21.6 & 14107 & 1.354 E-05 & 3.520 E-05 & 200 \\ 130.0 & 24.3 & 9573 & 9.187 E-06 & 2.389 E-05 & 200 \\ 130.0 & 27.0 & 6009 & 5.760 E-06 & 1.500 E-05 & 200\end{array}$

TOWER UATA FOLLOH....

TEST 03 JULY 13.1967. 0400 TO 0430 PST ZINC SULFIOE RELEASE FROM ELFVATION UF $2 M$ SOOM ARC U. 2.6 M/SEC AT $2 \mathrm{H}$

AZIMUTH HEIGH E XPOSURE
OEGREES METERS GM-SEC/CU.M SEC/CU.M I/SO.M METERS $\times 10 E+6$

$\begin{array}{lrrrrr}98.0 & .3 & 618 & 5.937 E-07 & 1.544 E-06 & 800 \\ 98.0 & .5 & 2306 & 2.213 E-06 & 5.755 E-06 & 800 \\ 98.0 & 1.1 & 2563 & 2.460 E-06 & 6.397 E-06 & 800 \\ 98.0 & 1.5 & 2586 & 2.483 E-06 & 6.455 E-06 & 800 \\ 98.0 & 2.1 & 432 & 4.146 E-07 & 1.078 E-06 & 800 \\ 98.0 & 4.2 & 3230 & 3.100 E-06 & 0.061 E-06 & 800 \\ 98.0 & 6.3 & 2807 & 2.694 E-06 & 7.005 E-06 & 800 \\ 98.0 & 8.4 & 2853 & 2.738 E-06 & 7.120 E-06 & 800 \\ 98.0 & 10.5 & 3101 & 2.977 E-06 & 7.740 E-06 & 800 \\ 98.0 & 12.6 & 3357 & 3.222 E-06 & 8.378 E-06 & 800 \\ 98.0 & 14.7 & 1667 & 1.600 E-06 & 4.160 E-06 & 800 \\ 98.0 & 16.8 & 2559 & 2.456 E-06 & 6.386 E-06 & 800 \\ 98.0 & 21.0 & 4449 & 4.271 E-06 & 1.110 E-05 & 800 \\ 98.0 & 25.2 & 2812 & 2.699 E-06 & 7.017 E-06 & 800 \\ 98.0 & 29.4 & 2420 & 2.323 E-06 & 6.041 E-06 & 800 \\ 98.0 & 33.6 & 3203 & 3.074 E-06 & 7.994 E-06 & 800 \\ 98.0 & 37.8 & 3607 & 3.462 E-06 & 9.001 E-06 & 800 \\ 98.0 & 42.0 & 2735 & 2.625 E-06 & 6.825 E-06 & 800 \\ 98 & & & & & \end{array}$

AZ I HUTH HEIGHT DEGREES ME TERS

130.0
130.0
130.0
133.0
130.0
130.0
130.0
135.0
131.0
130.0
130.0
130.0
130.0
130.0
130.0
130.0
130.0

EXPOSURE $-S E C / C U$
$\times 1 O E+6$

.3
.7
1.4
1.5
2.7
4.1
5.4
6.8
0.1
9.5
10.8
13.5
16.2
18.9
21.6
24.3
27.0

$\begin{array}{rl}13985 & 1.342 E-05 \\ 8853 & 8.496 E-06 \\ 6853 & 8.496 E-06 \\ 19428 & 1.864 E-05 \\ 34979 & 3.357 E-05 \\ 19428 & 1.864 E-05 \\ 47109 & 4.571 E-05 \\ 34768 & 3.337 E-05 \\ 38134 & 3.660 E-05 \\ 28758 & 2.76 U E-05 \\ 26426 & 2.536 E-05 \\ 38867 & 3.730 E-05 \\ 34201 & 3.282 E-05 \\ 23315 & 2.234 E-05 \\ 23315 & 2.238 E-05 \\ 13985 & 1.342 E-05 \\ 8619 & 8.272 E-06\end{array}$

$3.490 \mathrm{t}-05$ $2.209 \mathrm{E}-05$ $2.209 E-05$ $4.848 E-05$ $8.728 E-05$ $4.848 \mathrm{E}-05$ $1.175 \mathrm{E}-04$ $0.675 E-05$ $9.515 E-05$ $6.594 E-05$ $9.698 E-05$ $9.698 E-05$
$8.534 E-05$ $5.818 E-05$ $5.818 E-05$ $3.490 \mathrm{E}-05$ $2,151 \mathrm{E}-05$
200
200
200
200
200
200
200
200
200
200
200
200
200
200
200
200
200

ANCE

200
200
200
200
200
200
200
200
200
200
200
200
200
200
200
200
200
ITKEF TATA FULLOW....

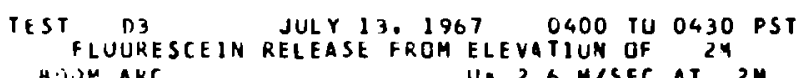
HJUM AKC U. 2.6 M/SEC AT $2 M$

ALIAUTH HEIGHT EXPUSURE F/C EUIO DISTANCE OEGEFES METERS GM-SEC/CU.H SECICU.H I/SO.H METERS $\times 10 \mathrm{~F}+6$

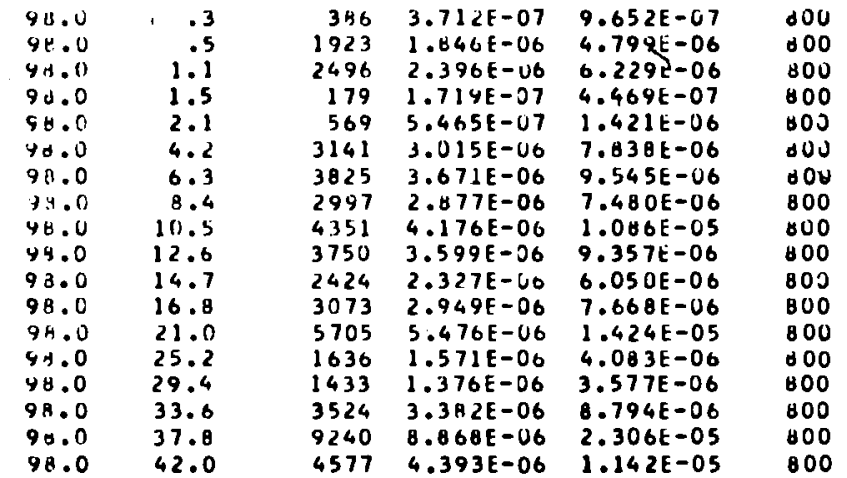




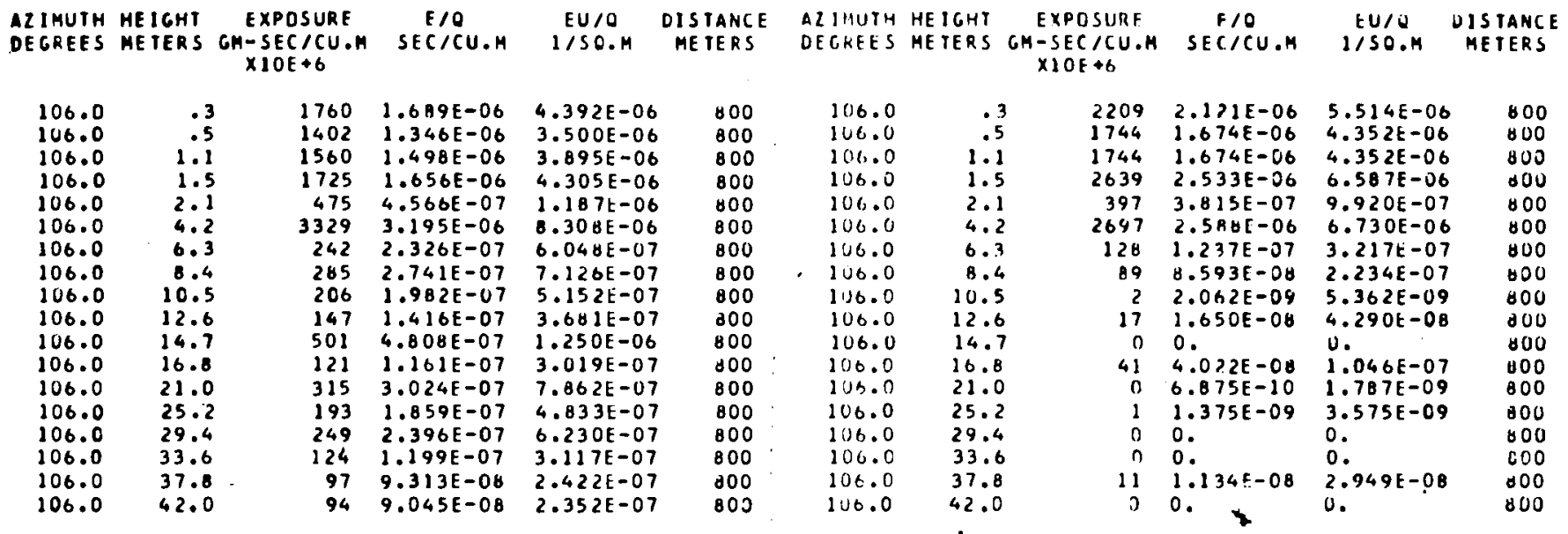

AZIMUTH HEIGHT EXPOSURE E/O FU/O OISTANCE ALIMUTH HEIGHT EXPOSURF F/O DE DEGRES HETERS GM-SECICU.M SES/CU.M I/SO.M HETERS DEGREES METERS GM-SEC/CU.M SEC/CU.M I/SG.M METERS $\times 10 E+6$

$\begin{array}{lrrrrr}114.0 & .3 & 4484 & 4.304 \mathrm{E}-06 & 1.119 \mathrm{E}-05 & 800 \\ 114.0 & .5 & 3025 & 2.904 \mathrm{E}-06 & 7.550 \mathrm{E}-06 & 800 \\ 114.0 & 1.1 & 4070 & 3.906 \mathrm{E}-06 & 1.016 \mathrm{E}-05 & 800 \\ 114.0 & 1.5 & 3784 & 3.632 \mathrm{E}-06 & 9.443 \mathrm{E}-06 & 800 \\ 114.0 & 2.1 & 792 & 7.610 \mathrm{E}-07 & 1.979 \mathrm{E}-06 & 800 \\ 114.0 & 4.2 & 3342 & 3.207 \mathrm{E}-06 & 8.339 \mathrm{E}-06 & 80 \\ 114.0 & 6.3 & 3014 & 2.893 \mathrm{E}-06 & 7.522 \mathrm{E}-06 & 800 \\ 114.0 & 8.4 & 336 \mathrm{~B} & 3.232 \mathrm{E}-06 & 8.404 \mathrm{E}-06 & 800 \\ 114.0 & 10.5 & 3421 & 3.283 \mathrm{E}-06 & 8.537 \mathrm{E}-06 & 800 \\ 114.0 & 12.6 & 2886 & 2.770 \mathrm{E}-06 & 7.203 \mathrm{E}-06 & 800 \\ 114.0 & 14.7 & 1830 & 1.757 \mathrm{E}-06 & 4.568 \mathrm{E}-06 & 800 \\ 114.0 & 16.8 & 1922 & 1.845 \mathrm{E}-06 & 4.791 \mathrm{E}-06 & 800 \\ 114.0 & 21.0 & 2137 & 2.051 \mathrm{E}-06 & 5.332 \mathrm{E}-06 & 800 \\ 114.0 & 25.2 & 1565 & 1.502 \mathrm{E}-06 & 3.906 \mathrm{E}-06 & 800 \\ 114.0 & 29.4 & 1159 & 1.113 \mathrm{E}-06 & 2.893 \mathrm{E}-06 & 800 \\ 114.0 & 33.6 & 2229 & 2.140 \mathrm{E}-06 & 5.563 \mathrm{E}-06 & 800 \\ 114.0 & 37.0 & 1214 & 1.165 \mathrm{E}-06 & 3.029 \mathrm{E}-06 & 800 \\ 114.0 & 42.0 & 1612 & 1.547 \mathrm{E}-06 & 4.023 \mathrm{E}-06 & 800\end{array}$

\begin{tabular}{|c|c|c|c|c|c|c|}
\hline $\begin{array}{l}800 \\
800 \\
800\end{array}$ & $\begin{array}{l}114.0 \\
114.0 \\
114.0\end{array}$ & $\begin{array}{r}.3 \\
.5 \\
1.1\end{array}$ & $\begin{array}{l}5028 \\
3900 \\
5254\end{array}$ & $\begin{array}{l}4.826 E-06 \\
3.743 E-06 \\
5.043 E-06\end{array}$ & $\begin{array}{l}1.255 \mathrm{E}-05 \\
9.732 \mathrm{E}-06 \\
1.311 \mathrm{E}-05\end{array}$ & $\begin{array}{l}800 \\
100 \\
800\end{array}$ \\
\hline $\begin{array}{l}800 \\
800\end{array}$ & $\begin{array}{l}114.0 \\
114.0\end{array}$ & $\begin{array}{l}1.5 \\
2.1\end{array}$ & $\begin{array}{l}6984 \\
1565\end{array}$ & $\begin{array}{l}6.703 \mathrm{E}-06 \\
1.502 \mathrm{E}-06\end{array}$ & $\begin{array}{l}1.743 \mathrm{E}-05 \\
3.905 \mathrm{E}-06\end{array}$ & $\begin{array}{l}\text { UDO } \\
\text { UDO }\end{array}$ \\
\hline $\begin{array}{lll}4 & 0 & 0 \\
0 & 0 & 0\end{array}$ & $\begin{array}{l}114.0 \\
114.0\end{array}$ & $\begin{array}{l}4.2 \\
6.3\end{array}$ & $\begin{array}{l}4126 \\
4803\end{array}$ & $\begin{array}{l}3.960 E-06 \\
4.609 E-06\end{array}$ & $\begin{array}{l}1.030 E-05 \\
1.198 \mathrm{E}-05\end{array}$ & $\begin{array}{l}800 \\
000\end{array}$ \\
\hline $\begin{array}{l}800 \\
800\end{array}$ & $\begin{array}{l}114.0 \\
114.0\end{array}$ & $\begin{array}{r}9.4 \\
10.5\end{array}$ & $\begin{array}{l}3900 \\
6307\end{array}$ & $\begin{array}{l}3.743 E-06 \\
6.053 E-06\end{array}$ & $\begin{array}{l}9.732 E-06 \\
1.574 t-05\end{array}$ & $\begin{array}{l}\forall 00 \\
\forall 00\end{array}$ \\
\hline 300 & 114.0 & 12.6 & 2922 & $2.8050-06$ & $7.293 \mathrm{~F}-06$ & 800 \\
\hline 800 & 114.0 & 14.7 & 2711 & $2.6 \cap \angle E-06$ & $6.765 E-06$ & 000 \\
\hline 800 & 114.0 & $16 \cdot A$ & 2446 & $2.396 E-06$ & $6.229 t-06$ & 800 \\
\hline 800 & 114.0 & $21 \cdot C$ & 2424 & $2.327 \mathrm{~F}-06$ & $6.050 E-06$ & duo \\
\hline 30 & 114.0 & 25.2 & $1 H 87$ & $1.611 E-06$ & $4.710 t-06$ & 800 \\
\hline 30 & 114.0 & 29.4 & 2281 & $2.290 E-06$ & $5.693 E-06$ & 800 \\
\hline & 114.0 & 33.6 & 1869 & $1.794 \mathrm{E}-06$ & $4.665 E-06$ & 800 \\
\hline & 114.0 & 37.8 & 2209 & $2.121 E-36$ & $.514 E-06$ & 800 \\
\hline & 114.0 & 42.0 & 2066 & $1.983 E-06$ & $5.157 E-06$ & 800 \\
\hline
\end{tabular}

\begin{tabular}{|c|c|c|c|c|c|c|}
\hline $\begin{array}{l}800 \\
800 \\
800\end{array}$ & $\begin{array}{l}114.0 \\
114.0 \\
114.0\end{array}$ & $\begin{array}{r}.3 \\
.5 \\
1.1\end{array}$ & $\begin{array}{l}5028 \\
3900 \\
5254\end{array}$ & $\begin{array}{l}4.826 E-06 \\
3.743 E-06 \\
5.043 E-06\end{array}$ & $\begin{array}{l}1.255 \mathrm{E}-05 \\
9.732 \mathrm{E}-06 \\
1.311 \mathrm{E}-05\end{array}$ & $\begin{array}{l}800 \\
100 \\
800\end{array}$ \\
\hline 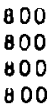 & $\begin{array}{l}114.0 \\
114.0 \\
114.0 \\
114.0\end{array}$ & $\begin{array}{l}1.5 \\
2.1 \\
4.2 \\
0.3\end{array}$ & $\begin{array}{l}6984 \\
1565 \\
4126 \\
4803\end{array}$ & $\begin{array}{l}6.703 E-06 \\
1.502 \mathrm{E}-06 \\
3.960 \mathrm{E}-06 \\
4.609 \mathrm{E}-06\end{array}$ & $\begin{array}{l}1.743 E-05 \\
3.905 E-06 \\
1.030 E-05 \\
1.198 E-05\end{array}$ & $\begin{array}{l}400 \\
\text { UDO } \\
400 \\
000\end{array}$ \\
\hline $\begin{array}{l}800 \\
800\end{array}$ & $\begin{array}{l}114.0 \\
114.0\end{array}$ & $\begin{array}{r}0.4 \\
10.5\end{array}$ & $\begin{array}{l}3900 \\
6307\end{array}$ & $\begin{array}{l}3.743 E-06 \\
6.053 E-06\end{array}$ & $\begin{array}{l}9.732 E-06 \\
1.574 t-05\end{array}$ & $\begin{array}{l}\forall 00 \\
\forall 00\end{array}$ \\
\hline$\$ 00$ & 114.0 & 12.6 & 2922 & $2.805 E-06$ & $7.293 \mathrm{~F}-06$ & 800 \\
\hline 800 & 114.0 & 14.7 & 2711 & $2.60 \angle E-06$ & $6.765 E-06$ & 000 \\
\hline 800 & 114.0 & $21 . c$ & 2424 & $2.327 \mathrm{~F}-06$ & $6.050 E-06$ & dUO \\
\hline 800 & 114.0 & 25.2 & $1 \mathrm{HB7}$ & $1.611 E-06$ & $4.710 t-06$ & $\forall 00$ \\
\hline 800 & 114.0 & 29.4 & 2281 & $2.190 E-06$ & $5.693 E-06$ & 800 \\
\hline 300 & 114.0 & 33.6 & 1869 & $1.794 \mathrm{E}-06$ & $4.665 E-06$ & 800 \\
\hline 300 & 114.0 & 37.8 & 2209 & $2.121 E=26$ & $5.514 E-06$ & 800 \\
\hline & 114.0 & 42.0 & 2066 & $1.983 E-06$ & $.157 E-06$ & 800 \\
\hline
\end{tabular}
$\times 10 \mathrm{~F}+6$

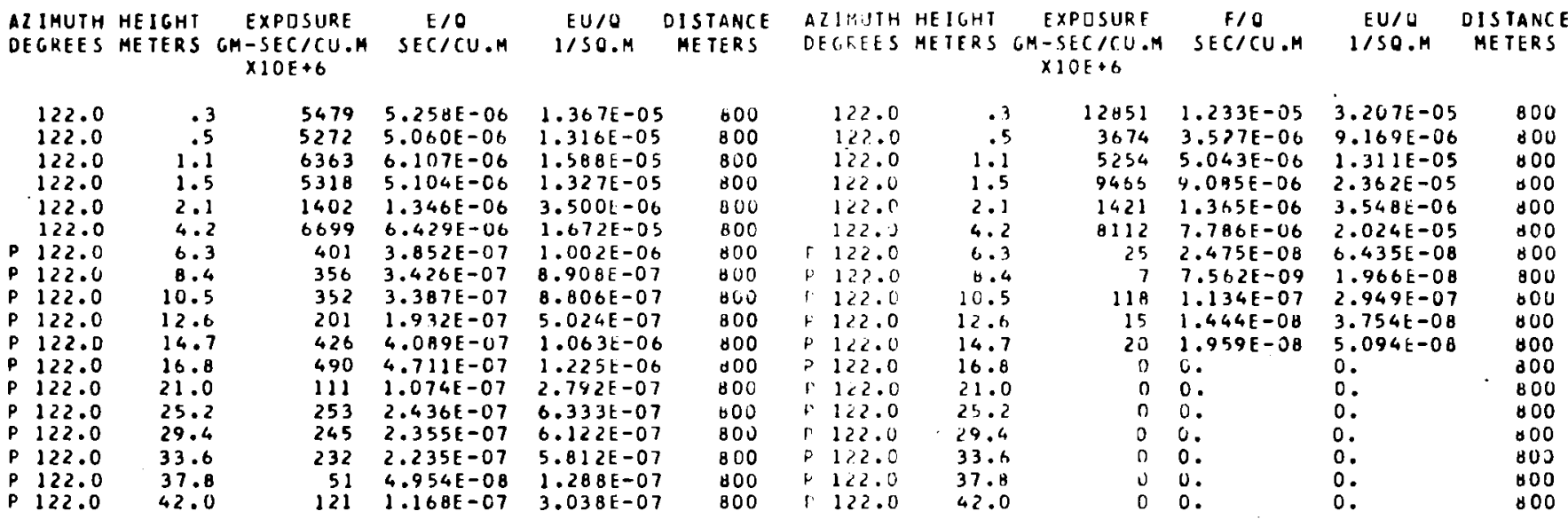


ALI IMUTH HEIGHT

EXPOSURE

E 10

EU/O DISTANCE $O M-S E C / C U$
$\times 1 O E+6$

$\begin{array}{lr}130.0 & .3 \\ 130.0 & .5 \\ 130.0 & 1.1 \\ 130.0 & 1.5 \\ 130.0 & 2.1 \\ 130.0 & 4.2 \\ 130.0 & 6.3 \\ 130.0 & 1.4 \\ 130.0 & 10.5 \\ 130.0 & 12.6 \\ 130.0 & 14.7 \\ 130.0 & 16.0 \\ 130.0 & 21.0 \\ 130.0 & 25.2 \\ 130.0 & 29.4 \\ 130.0 & 33.6 \\ 130.0 & 37.8 \\ 130.0 & 42.0\end{array}$

$\begin{array}{lll}1438 & 1.381 E-06 & 3.591 E-06 \\ 2573 & 2.470 E-06 & 6.422 E-06 \\ 5893 & 5.656 E-06 & 1.470 E-05 \\ 4872 & 4.676 E-06 & 1.216 E-05 \\ 1643 & 1.577 E-06 & 4.101 E-06 \\ 4929 & 4.731 E-06 & 1.230 E-05 \\ 4665 & 4.478 E-06 & 1.164 E-05 \\ 3025 & 2.904 E-06 & 7.550 E-06 \\ 2624 & 2.518 E-06 & 6.548 E-06 \\ 2542 & 2.440 E-06 & 6.344 E-06 \\ 1887 & 1.811 E-06 & 4.709 E-06 \\ 1664 & 1.597 E-06 & 4.153 E-06 \\ 1525 & 1.464 E-06 & 3.806 E-06 \\ 1540 & 1.479 E-06 & 3.845 E-06 \\ 1348 & 1.294 E-06 & 3.364 E-06 \\ 1128 & 1.083 E-06 & 2.816 E-06 \\ 1170 & 1.124 E-06 & 2.921 E-06 \\ 1091 & 1.048 E-06 . & 2.724 E-06\end{array}$

$\begin{array}{ll}800 & 130.0 \\ 800 & 130.0 \\ 800 & 130.0 \\ 800 & 130.0 \\ 800 & 130.0 \\ 800 & 131.0 \\ 800 & 110.0 \\ 800 & 130.0 \\ 800 & 130.0 \\ 800 & 130.0 \\ 800 & 130.0 \\ 800 & 130.0 \\ 800 & 130.0 \\ 800 & 130.0 \\ 800 & 131.0 \\ 800 & 130.0 \\ 800 & 130.0 \\ 800 & 130.0\end{array}$

.3
.5
1.1
1.5
2.1
4.2
$6: 3$
1.4
10.5
12.6
14.7
16.8
21.0
25.2
29.4
33.6
37.8
42.0

EXPDSIIRE

E $/ 0$

EUIO DISTAHCE $\times 1 O E+0$

$\begin{array}{ll}1457 & 1.399 E-06 \\ 1529 & 1.466 E-06 \\ 4878 & 4.682 E-06 \\ 5555 & 5.331 E-06 \\ 1529 & 1.464 E-06 \\ 3223 & 3.094 E-06 \\ 3524 & 3.382 E-06 \\ .2245 & 2.155 E-06 \\ 2922 & 2.805 E-06 \\ 2847 & 2.733 E-06 \\ 1831 & 1.777 E-06 \\ 1867 & 1.011 E-06 \\ 1027 & 9.865 E-07 \\ 1135 & 1.090 E-06 \\ 1457 & 1.399 E-06 \\ 1457 & 1.399 E-06 \\ 1242 & 1.193 E-06 \\ 1027 & 9.865 E-07\end{array}$

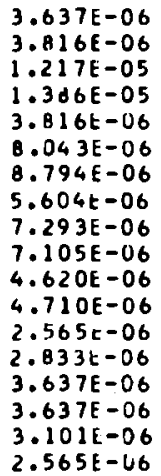

800
800
800
100
100
800
600
800
800
800
800
800
800
800
800
800
800
800

TOWER DATA FOLLDH....

TOWEK DATA FILLOW....

TEST 03 JULY 13,1967 0400 TU 0430 PST 2INC SULF IOE RELEASE FROM ELEVATION DF $2 \mathrm{M}$ $1600 H$ ARC UE $2.6 \mathrm{M} / \mathrm{SEC}$ AT $2 \mathrm{M}$
IEST UI JULY 13,1967 . 19400 TU 0430 PST
FLUURESCEIN RELEASE FRUA ELEVATIUN OF L4 $160 \mathrm{JM} A K C \quad U=2.6 \mathrm{M} / \mathrm{SEC}$ AT $2 \mathrm{M}$

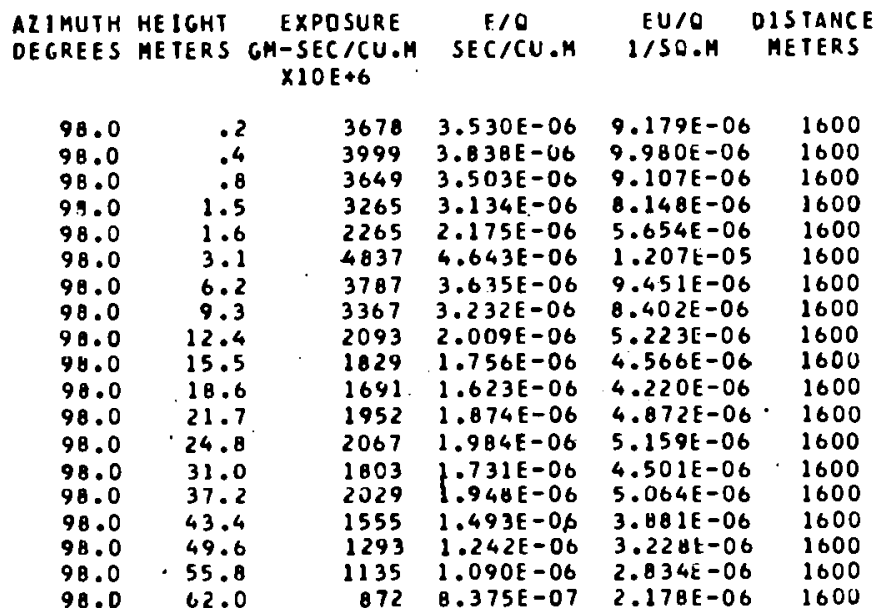
ALIMUTH HEIGHT EXPOSURE
DEGREES HETERS CH-SECICU.M SECICU.M IISO.M MEIERS $\times 10 E+0$

$\begin{array}{lrrrrr}106.0 & .2 & 3378 & 3.243 E-06 & 0.431 E-06 & 1600 \\ 106.0 & .4 & 3999 & 3.838 E-06 & 9.980 E-06 & 1600 \\ 106.0 & .8 & 3895 & 3.739 E-06 & 9.720 E-06 & 1600 \\ 106.0 & 1.5 & 4051 & 3.888 E-06 & 1.011 E-05 & 1600 \\ 106.0 & 1.6 & 3937 & 3.779 E-06 & 9.826 E-06 & 1600 \\ 106.0 & 3.1 & 4161 & 3.994 E-06 & 1.038 E-05 & 1600 \\ 106.0 & 6.2 & 2441 & 2.392 E-06 & 6.218 E-06 & 1600 \\ 106.0 & 19.3 & 803 & 7.714 E-07 & 2.006 E-06 & 1600 \\ 106.0 & 12.4 & 1455 & 1.397 E-06 & 3.631 E-06 & 1600 \\ 106.0 & 15.5 & 1225 & 1.177 E-06 & 3.059 E-06 & 1600 \\ 106.0 & 18.6 & 1410 & 1.354 E-06 & 3.520 E-06 & 1600 \\ 106.0 & 21.7 & 1064 & 1.072 E-06 & 2.657 E-06 & 1600 \\ 106.0 & 24.8 & 1102 & 1.058 E-06 & 2.751 E-06 & 1600 \\ 106.0 & 31.0 & 1171 & 1.124 E-06 & 2.923 E-06 & 1600 \\ 106.0 & 37.2 & 1093 & 1.050 E-06 & 2.729 E-06 & 1600 \\ 106.0 & 43.4 & 734 & 7.050 E-07 & 1.833 E-06 & 1600 \\ 106.0 & 49.6 & 830 & 7.975 E-07 & 2.073 E-06 & 1600 \\ 106.0 & 55.8 & 929 & 8.917 E-07 & 2.318 E-06 & 1600 \\ 106.0 & 62.0 & 840 & 8.063 E-07 & 2.096 E-06 & 1600\end{array}$

\begin{tabular}{|c|c|}
\hline $\begin{array}{l}\text { AZ J, IUTH } \\
\text { OECKEES }\end{array}$ & $\begin{array}{l}\text { HE IGHT } \\
\text { METERS }\end{array}$ \\
\hline 93.0 & .2 \\
\hline Y 0.0 & .4 \\
\hline $\begin{array}{l}98.0 \\
98.0\end{array}$ & $\begin{array}{r}.8 \\
1.5\end{array}$ \\
\hline $\begin{array}{l}28.0 \\
99.0\end{array}$ & $\begin{array}{l}1.6 \\
3.1\end{array}$ \\
\hline $\begin{array}{l}7 A .0 \\
Y 4.0\end{array}$ & $\begin{array}{l}6.2 \\
9.3\end{array}$ \\
\hline 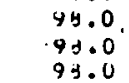 & $\begin{array}{l}12.4 \\
15.5 \\
18.6\end{array}$ \\
\hline Y:.0 & 21.7 \\
\hline 95.0 & 24.8 \\
\hline $\begin{array}{l}9.3 .0 \\
9.9 .1\end{array}$ & $\begin{array}{l}31.0 \\
37.2\end{array}$ \\
\hline 94.0 & 43.4 \\
\hline+4.0 & 49.6 \\
\hline $\begin{array}{l}94.0 \\
y+.0\end{array}$ & $\begin{array}{l}55.8 \\
62.0\end{array}$ \\
\hline
\end{tabular}

EXPOSURF

$F / 0$

$$
\times 10 \mathrm{~F}+6
$$

$\begin{array}{llll}3266 & 3.135 E-06 & 8.151 E-06 & 1600 \\ 3465 & 3.325 E-06 & 8.646 E-06 & 1600 \\ 3415 & 3.270 E-06 & 8.522 E-06 & 1600 \\ 7731 & 7.419 E-06 & 1.929 E-05 & 1600 \\ 2623 & 2.376 E-06 & 6.047 E-06 & 1600 \\ 4903 & 4.706 E-06 & 1.224 E-05 & 1600 \\ 3613 & 3.466 E-06 & 9.018 E-06 & 1600 \\ 2274 & 2.191 E-06 & 5.676 E-06 & 1000 \\ 1361 & 1.326 E-06 & 3.448 E-06 & 1600 \\ 1163 & 1.136 E-06 & 2.953 E-06 & 1600 \\ 1580 & 1.517 E-06 & 3.943 E-06 & 1600 \\ 4754 & 4.563 E-06 & 1.186 E-05 & 1600 \\ 1049 & 1.007 E-06 & 2.619 E-06 & 1600 \\ 1629 & 1.564 E-06 & 4.067 E-06 & 1600 \\ 1877 & 1.802 E-06 & 4.666 E-06 & 1600 \\ 1138 & 1.093 E-06 & 2.8411-06 & 1000 \\ 1549 & 1.535 E-06 & 3.990 E-06 & 1000 \\ 1079 & 1.036 E-06 & 2.693 E-06 & 1600 \\ 640 & 6.546 E-07 & 2.222 E-06 & 1600\end{array}$

EU/O DISTANCE

I/SO.M METERS 


\begin{tabular}{|c|c|c|c|c|c|c|c|c|c|c|c|}
\hline $\begin{array}{l}\text { PZIMUTH } \\
\text { DEGREES }\end{array}$ & $\begin{array}{l}\text { HE ICHT } \\
\text { HE TERS }\end{array}$ & $\begin{array}{c}\text { EXPDSURE } \\
\text { GH-SEC/CU,H } \\
\times 10 E+6\end{array}$ & $\begin{array}{c}F / O \\
\text { SEC } / \text { CU.M }\end{array}$ & $\begin{array}{l}\text { EU/U } \\
1 / 50 . M\end{array}$ & $\begin{array}{l}\text { DISTANCE } \\
\text { METERS }\end{array}$ & $\begin{array}{l}\text { AZI I UUTH } \\
\text { DECKFES }\end{array}$ & $\begin{array}{l}\text { HEICHT } \\
\text { MFTERS }\end{array}$ & $\begin{array}{c}\text { EXPOSUKE } \\
\text { GH-SECICU.H } \\
\times 1 U E+6\end{array}$ & $\begin{array}{c}F / U \\
\text { SEC } / C U . M\end{array}$ & $\begin{array}{l}E U / 0 \\
1 / 50.4\end{array}$ & $\begin{array}{l}\text { UIS TANCE } \\
\text { METERS }\end{array}$ \\
\hline $\begin{array}{l}114.0 \\
114.0 \\
114.0 \\
114.0 \\
114.0 \\
114.0 \\
114.0 \\
114.0 \\
114.0 \\
114.0 \\
114.0 \\
114.0 \\
114.0 \\
114.0 \\
114.0 \\
114.0 \\
114.0 \\
114.0 \\
114.0\end{array}$ & $\begin{array}{l}.2 \\
.4 \\
.8 \\
1.5 \\
1.6 \\
3.1 \\
6.2 \\
9.3 \\
12.4 \\
15.5 \\
18.6 \\
21.7 \\
24.8 \\
31.0 \\
37.2 \\
43.4 \\
49.6 \\
55.8 \\
62.0\end{array}$ & $\begin{array}{l}2668 \\
2464 \\
2014 \\
2765 \\
2367 \\
4016 \\
4081 \\
3513 \\
3324 \\
3258 \\
2705 \\
3017 \\
2479 \\
2101 \\
1645 \\
1445 \\
1104 \\
633 \\
686\end{array}$ & $\begin{array}{l}2.561 E-06 \\
2.363 E-06 \\
1.933 E-06 \\
2.673 E-06 \\
2.272 E-06 \\
3.854 E-06 \\
3.917 E-06 \\
3.372 E-06 \\
3.191 E-06 \\
3.127 E-06 \\
2.596 E-06 \\
2.896 E-06 \\
2.380 E-06 \\
2.017 E-06 \\
1.628 E-06 \\
1.388 E-06 \\
1.060 E-06 \\
7.998 E-07 \\
6.590 E-07\end{array}$ & $\begin{array}{l}6.659 \mathrm{E}-06 \\
6.149 \mathrm{E}-06 \\
5.026 \mathrm{E}-06 \\
6.950 \mathrm{E}-06 \\
5.908 \mathrm{E}-06 \\
1.002 \mathrm{E}-05 \\
1.019 \mathrm{E}-05 \\
1.768 \mathrm{E}-06 \\
8.296 \mathrm{E}-06 \\
8.130 \mathrm{E}-06 \\
6.750 \mathrm{E}-06 \\
7.529 \mathrm{E}-06 \\
6.188 \mathrm{E}-06 \\
5.243 \mathrm{E}-06 \\
4.232 \mathrm{E}-06 \\
3.608 \mathrm{E}-06 \\
2.756 \mathrm{E}-06 \\
2.080 \mathrm{E}-06 \\
1.713 \mathrm{E}-06\end{array}$ & $\begin{array}{l}1600 \\
1600 \\
1600 \\
1600 \\
1600 \\
1600 \\
1600 \\
1600 \\
1600 \\
1600 \\
1600 \\
1600 \\
1600 \\
1600 \\
1600 \\
1600 \\
1600 \\
1600 \\
1600\end{array}$ & $\begin{array}{l}114.0 \\
114.0 \\
1114.0 \\
114.0 \\
114.0 \\
114.0 \\
114.0 \\
114.0 \\
114.0 \\
114.0 \\
114.0 \\
114.0 \\
114.0 \\
114.0 \\
114.0 \\
114.0 \\
114.0 \\
114.0 \\
114.0\end{array}$ & $\begin{array}{l}.2 \\
.4 \\
.8 \\
1.5 \\
1.6 \\
3.1 \\
6.2 \\
9.3 \\
12.4 \\
15.5 \\
18.6 \\
21.7 \\
24.0 \\
31.0 \\
37.2 \\
43.4 \\
49.6 \\
55.8 \\
62.0\end{array}$ & $\begin{array}{l}2225 \\
2175 \\
2274 . \\
3713 \\
2373 \\
2721 \\
2721 \\
2770 \\
3415 \\
2969 \\
2770 \\
2076 \\
1679 \\
1877 \\
1877 \\
1074 \\
1150 \\
1126 \\
1150\end{array}$ & $\begin{array}{l}2.135 E-06 \\
2.088 E-06 \\
2.183 E-06 \\
3.514 E-06 \\
2.278 E-06 \\
2.611 E-06 \\
2.611 E-06 \\
2.659 E-06 \\
3.27 \forall E-06 \\
2.849 E-06 \\
2.659 E-06 \\
1.993 E-06 \\
1.612 E-06 \\
1.502 E-06 \\
1.602 E-06 \\
1.036 E-06 \\
1.104 E-06 \\
1.081 E-06 \\
1.104 E-06\end{array}$ & $\begin{array}{l}5.552 E-06 \\
5.428 E-06 \\
5.676 E-06 \\
9.265 E-06 \\
5.923 E-06 \\
6.790 E-06 \\
6.790 E-06 \\
6.913 E-06 \\
8.522 E-06 \\
7.409 E-06 \\
6.913 E-06 \\
5.161 E-06 \\
4.191 E-06 \\
4.666 E-06 \\
4.666 E-06 \\
2.693 E-06 \\
2.87 U E-06 \\
2.811 E-06 \\
2.870 E-06\end{array}$ & $\begin{array}{l}1600 \\
1600 \\
1600 \\
1600 \\
1600 \\
1600 \\
1600 \\
1600 \\
1600 \\
1600 \\
1600 \\
1600 \\
1600 \\
1600 \\
1600 \\
1600 \\
1600 \\
1600 \\
1600\end{array}$ \\
\hline
\end{tabular}

\begin{tabular}{|c|c|c|c|c|c|c|c|c|c|c|c|}
\hline $\begin{array}{l}\text { AZIMUTH } \\
\text { DEGKEES }\end{array}$ & $\begin{array}{l}\text { HE JGHT } \\
\text { ME TERS }\end{array}$ & $\begin{array}{l}\text { EXPOSURE } \\
\text { CH-SEC/CU.M } \\
\times 1 O E+6\end{array}$ & $\begin{array}{c}\text { E } / 0 \\
\text { SEC/CU.H }\end{array}$ & $\begin{array}{l}\text { EU/O } \\
1 / S O . M\end{array}$ & $\begin{array}{l}\text { DISTANCE } \\
\text { METERS }\end{array}$ & $\begin{array}{l}\text { AZIA,UIH } \\
\text { DECHEES }\end{array}$ & $\begin{array}{l}\text { HE IGHT } \\
\text { ME IERS }\end{array}$ & $\begin{array}{c}\text { EXPOSURF } \\
\text { CH-SECICU.H } \\
\times 1 O E+6\end{array}$ & $\begin{array}{c}F / O \\
S E C /[U . M\end{array}$ & $\begin{array}{c}E U / U \\
1 / S Q . M\end{array}$ & $\begin{array}{l}\text { DISTANCE } \\
\text { METERS }\end{array}$ \\
\hline $\begin{array}{l}122.0 \\
122.0 \\
122.0 \\
122.0 \\
122.0 \\
122.0 \\
122.0 \\
122.0 \\
122.0 \\
122.0 \\
122.0 \\
122.0 \\
122.0 \\
122.0 \\
122.0 \\
122.0 \\
122.0 \\
122.0 \\
122.0\end{array}$ & $\begin{array}{l}.2 \\
.4 \\
.8 \\
1.5 \\
1.6 \\
3.1 \\
6.2 \\
9.3 \\
12.4 \\
15.5 \\
18.6 \\
21.7 \\
24.8 \\
31.0 \\
37.2 \\
43.4 \\
49.6 \\
55.8 \\
62.0\end{array}$ & $\begin{array}{l}1377 \\
1511 \\
1288 \\
1454 \\
1530 \\
1364 \\
1882 \\
1235 \\
1401 \\
1752 \\
1284 \\
1489 \\
1063 \\
1050 \\
866 \\
737 \\
419 \\
531 \\
273\end{array}$ & $\begin{array}{l}1.322 \mathrm{E}-06 \\
1.450 \mathrm{E}-06 \\
1.237 \mathrm{E}-06 \\
1.396 \mathrm{E}-06 \\
1.469 \mathrm{E}-06 \\
1.309 \mathrm{E}-06 \\
1.806 \mathrm{E}-06 \\
1.186 \mathrm{E}-06 \\
1.345 \mathrm{E}-06 \\
1.662 \mathrm{E}-06 \\
1.232 \mathrm{E}-06 \\
1.429 \mathrm{E}-06 \\
1.020 \mathrm{E}-06 \\
1.008 \mathrm{E}-06 \\
1.316 \mathrm{E}-07 \\
7.079 \mathrm{E}-07 \\
4.030 \mathrm{E}-07 \\
5.097 \mathrm{E}-07 \\
2.671 \mathrm{E}-07\end{array}$ & $\begin{array}{l}3.437 E-06 \\
3.771 E-06 \\
3.216 E-06 \\
3.629 E-06 \\
3.820 E-06 \\
3.404 E-06 \\
4.697 E-06 \\
3.084 E-06 \\
3.497 E-06 \\
4.374 E-06 \\
3.204 E-06 \\
3.717 E-06 \\
2.652 E-06 \\
2.621 E-06 \\
2.162 E-06 \\
1.841 E-06 \\
1.048 E-06 \\
1.325 E-06 \\
6.615 E-07\end{array}$ & $\begin{array}{l}1600 \\
1600 \\
1600 \\
1600 \\
1600 \\
1600 \\
1600 \\
1600 \\
1600 \\
1600 \\
1600 \\
1600 \\
1600 \\
1600 \\
1600 \\
1600 \\
1600 \\
1600 \\
1600\end{array}$ & $\begin{array}{l}122.0 \\
122.0 \\
122.0 \\
122.0 \\
122.0 \\
122.0 \\
122.0 \\
122.0 \\
122.0 \\
122.0 \\
122.0 \\
122.0 \\
122.0 \\
122.0 \\
122.0 \\
122.0 \\
122.0 \\
122.0 \\
122.0\end{array}$ & $\begin{array}{l}.2 \\
.4 \\
.14 \\
1.5 \\
1.6 \\
3.1 \\
6.2 \\
9.3 \\
12.4 \\
15.5 \\
18.6 \\
21.7 \\
24.8 \\
31.0 \\
37.2 \\
43.4 \\
49.6 \\
55.8 \\
62.0\end{array}$ & $\begin{array}{l}1008 \\
1126 \\
1247 \\
1551 \\
1339 \\
1079 \\
1197 \\
1292 \\
1173 \\
964 \\
961 \\
630 \\
630 \\
583 \\
439 \\
725 \\
311 \\
263 \\
134\end{array}$ & $\begin{array}{l}9.679 E-07 \\
1.0 A 1 E-06 \\
1.240 E-06 \\
1.4 B 9 E-06 \\
1.2 B 5 E-06 \\
1.036 E-06 \\
1.149 E-06 \\
1.240 E-06 \\
1.127 E-06 \\
9.453 E-07 \\
9.2705-07 \\
6.053 E-07 \\
6.053 E-07 \\
5.599 E-07 \\
4.216[-07 \\
0.954 E-07 \\
2.992 E-07 \\
2.770 E-07 \\
1.768 E-07\end{array}$ & $\begin{array}{l}2.517 E-06 \\
2.811 E-06 \\
3.224 E-06 \\
3.672 E-06 \\
3.342 E-06 \\
2.693 E-06 \\
2.988 E-06 \\
3.224 E-06 \\
2.929 E-06 \\
2.45 B E-06 \\
2.399 E-06 \\
1.574 E-06 \\
1.574 E-06 \\
1.456 E-06 \\
1.096 E-06 \\
1.809 E-06 \\
7.780 E-07 \\
7.073 E-07 \\
4.597 t-07\end{array}$ & $\begin{array}{l}1600 \\
1600 \\
1600 \\
1600 \\
1600 \\
1600 \\
1600 \\
1600 \\
1600 \\
1600 \\
1600 \\
1600 \\
1000 \\
1600 \\
1600 \\
1600 \\
1600 \\
1600 \\
1000\end{array}$ \\
\hline
\end{tabular}

\begin{tabular}{|c|c|c|c|c|c|c|c|c|c|c|c|}
\hline $\begin{array}{l}\text { ALIMUTH } \\
\text { DECREES }\end{array}$ & $\begin{array}{l}\text { HE IGHT } \\
\text { METERS }\end{array}$ & $\begin{array}{c}\text { EXPDSURE } \\
\text { GH-SEC/CU.M } \\
\times 1 O E+6\end{array}$ & $\begin{array}{c}\text { E/O } \\
\text { SEC / CU.H }\end{array}$ & $\begin{array}{l}\text { EU/O } \\
1 / S O . H\end{array}$ & $\begin{array}{l}\text { DISTANCE } \\
\text { METERS }\end{array}$ & $\begin{array}{l}\text { ALIMUTH } \\
\text { DEGKEES }\end{array}$ & $\begin{array}{l}\text { HE JGHT } \\
\text { ME TERS }\end{array}$ & $\begin{array}{c}\text { EXPOSURE } \\
\text { GM- SEC /CU.M } \\
\times 10 F+6\end{array}$ & $\begin{array}{l}F / O \\
\text { SEC } / \text { CO.M }\end{array}$ & $\begin{array}{l}\text { EU/O } \\
1 / 50.4\end{array}$ & $\begin{array}{l}\text { DISIANCE } \\
\text { METERS }\end{array}$ \\
\hline $\begin{array}{l}130.0 \\
130.0 \\
130.0 \\
130.0 \\
130.0 \\
130.0 \\
130.0 \\
130.0 \\
130.0 \\
130.0 \\
130.0 \\
130.0 \\
130.0 \\
130.0 \\
130.0 \\
130.0 \\
130.0 \\
130.0 \\
130.0\end{array}$ & $\begin{array}{l}.2 \\
.4 \\
.8 \\
1.5 \\
1.6 \\
3.1 \\
6.2 \\
9.3 \\
12.4 \\
15.5 \\
18.6 \\
21.7 \\
24.8 \\
31.0 \\
37.2 \\
43.4 \\
49.6 \\
55.8 \\
62.0\end{array}$ & $\begin{array}{l}2335 \\
1764 \\
1442 \\
2205 \\
1452 \\
1650 \\
1729 \\
1495 \\
1283 \\
1156 \\
857 \\
689 \\
990 \\
697 \\
645 \\
533 \\
431 \\
388 \\
334\end{array}$ & $\begin{array}{l}2.241 E-06 \\
1.694 E-06 \\
1.385 E-06 \\
2.116 E-06 \\
1.394 E-06 \\
1.584 E-06 \\
1.660 E-06 \\
1.436 E-06 \\
1.232 E-06 \\
1.1199 E-06 \\
8.233 E-07 \\
0.536 E-07 \\
9.509 E-07 \\
6.695 E-07 \\
6.198 E-07 \\
5.124 E-07 \\
4.143 E-07 \\
3.726 E-07 \\
3.208 E-07\end{array}$ & $\begin{array}{l}5.027 E-06 \\
4.403 E-06 \\
3.600 E-06 \\
5.502 E-06 \\
3.024 E-06 \\
4.118 E-06 \\
4.315 E-06 \\
3.733 E-06 \\
3.202 E-06 \\
2.865 E-06 \\
2.141 E-06 \\
2.219 E-06 \\
2.472 E-06 \\
1.741 E-06 \\
1.612 E-06 \\
1.332 E-06 \\
1.077 E-06 \\
9.689 E-07 \\
.8 .340 E-07\end{array}$ & $\begin{array}{l}1600 \\
1600 \\
1600 \\
1600 \\
1600 \\
1600 \\
1600 \\
1600 \\
1600 \\
1600 \\
1600 \\
1600 \\
1600 \\
1600 \\
1600 \\
1600 \\
1600 \\
1600 \\
1600\end{array}$ & $\begin{array}{l}130.0 \\
130.0 \\
130.0 \\
130.0 \\
130.0 \\
130.0 \\
130.0 \\
130.0 \\
130.0 \\
130.0 \\
130.0 \\
130.0 \\
130.0 \\
130.0 \\
130.0 \\
130.0 \\
130.0 \\
130.0 \\
130.0\end{array}$ & $\begin{array}{l}.8 \\
.4 \\
.8 \\
1.5 \\
1.6 \\
3.1 \\
6.2 \\
9.3 \\
12.4 \\
15.5 \\
18.6 \\
21.7 \\
24.8 \\
31.0 \\
37.2 \\
43.4 \\
49.6 \\
55.8 \\
62.0\end{array}$ & $\begin{array}{l}1207 \\
1094 \\
1433 \\
1764 \\
937 \\
746 \\
989 \\
736 \\
517 \\
725 \\
677 \\
607 \\
725 \\
677 \\
460 \\
255 \\
347 \\
181 \\
226\end{array}$ & $\begin{array}{l}1.231 E-06 \\
1.050 E-06 \\
1.376 E-06 \\
1.693 E-06 \\
6.949 E-07 \\
7.639 E-07 \\
9.500 E-07 \\
7.639 E-07 \\
4.964 E-07 \\
6.959 E-07 \\
6.506 E-07 \\
5.876 E-07 \\
6.954 E-07 \\
6.500 E-01 \\
4.420 E-07 \\
2.448 E-07 \\
3.332 E-07 \\
1.745 E-07 \\
2.176 E-07\end{array}$ & $\begin{array}{l}3.200 E-06 \\
2.73 C E-06 \\
3.578 E-06 \\
4.403 E-06 \\
2.340 E-06 \\
1.986 E-06 \\
2.470 E-06 \\
1.946 E-06 \\
1.291 E-06 \\
1.809 E-06 \\
1.692 E-06 \\
1.515 E-06 \\
1.809 E-06 \\
1.692 L-06 \\
1.149 E-06 \\
6.365 E-07 \\
8.664 E-07 \\
4.538 E-07 \\
5.658 E-07\end{array}$ & $\begin{array}{l}1600 \\
1600 \\
1600 \\
1600 \\
1000 \\
1000 \\
1600 \\
1600 \\
1600 \\
1000 \\
1600 \\
1600 \\
1600 \\
1600 \\
1600 \\
1600 \\
1600 \\
1600 \\
1600\end{array}$ \\
\hline
\end{tabular}


TOHER DATA FOLLDW....

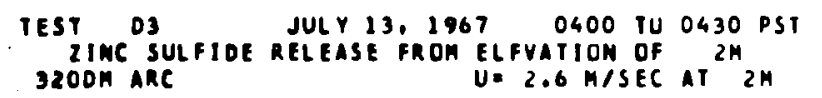

\begin{tabular}{|c|c|c|c|c|c|c|}
\hline \multicolumn{2}{|c|}{$\begin{array}{l}\text { ALIHUTH } \\
\text { DECREES }\end{array}$} & $\begin{array}{l}\text { ME IGHT } \\
\text { METERS }\end{array}$ & $\begin{array}{c}\text { EXPOSURE } \\
\text { GM-SEC/CU. } \\
\times 10 E \bullet 6\end{array}$ & $\begin{array}{c}F / 0 \\
\text { SEC/CU.M }\end{array}$ & $\begin{array}{l}\text { EU/O } \\
1 / 50 . \mathrm{H}\end{array}$ & $\begin{array}{l}\text { DISTANC } \\
\text { METERS }\end{array}$ \\
\hline & $\begin{array}{l}98.0 \\
98.0 \\
91.0 \\
94.0 \\
96.0 \\
96.0 \\
98.0\end{array}$ & $\begin{array}{l}.2 \\
.4 \\
.15 \\
1.5 \\
1.6 \\
3.1 \\
6.2 \\
9.3\end{array}$ & $\begin{array}{l}1315 \\
1417 \\
1359 \\
1319 \\
2278 \\
1657 \\
1579 \\
196\end{array}$ & $\begin{array}{l}1.262 E-06 \\
1.360 E-06 \\
1.304 E-06 \\
1.125 E-06 \\
1.227 E-06 \\
1.619 E-06 \\
1.516 E-06 \\
1.886 E-07\end{array}$ & $\begin{array}{l}3.281 E-06 \\
3.537 E-06 \\
3.392 E-06 \\
2.924 E-06 \\
3.190 E-06 \\
4.210 E-06 \\
3.941 E-06 \\
4.902 E-07\end{array}$ & $\begin{array}{l}3200 \\
3200 \\
3200 \\
3200 \\
3200 \\
3200 \\
3200 \\
3200\end{array}$ \\
\hline 5 & $\begin{array}{l}96.0 \\
98.0 \\
96.0 \\
98.0 \\
98.0 \\
98.0\end{array}$ & $\begin{array}{l}12.4 \\
15.5 \\
14.6 \\
21.7 \\
24.8 \\
31.0\end{array}$ & $\begin{array}{r}1315 \\
661 \\
570 \\
311 \\
102 \\
.63\end{array}$ & $\begin{array}{l}1.263 E-06 \\
6.350 E-07 \\
5.479 E-07 \\
2.998 E-07 \\
9.872 E-08 \\
6.137 E-08\end{array}$ & $\begin{array}{l}3.284 E-06 \\
1.651 E-06 \\
1.424 E-06 \\
7.770 E-07 \\
2.567 E-07 \\
1.596 E-07\end{array}$ & $\begin{array}{l}3200 \\
3200 \\
3200 \\
3200 \\
3200 \\
3200\end{array}$ \\
\hline & $\begin{array}{l}98.0 \\
98.0 \\
98.0 \\
98.0 \\
98.0\end{array}$ & $\begin{array}{l}37.2 \\
43.4 \\
49.6 \\
55.8 \\
62.0\end{array}$ & $\begin{array}{l}65 \\
28 \\
32 \\
14 \\
1\end{array}$ & $\begin{array}{l}6.315 E-08 \\
2.716 E-0 B \\
3.139 E-0 B \\
1.348 E-08 \\
1.523 E-09\end{array}$ & $\begin{array}{l}1.642 E=07 \\
7.061 E=08 \\
8.161 E=08 \\
3.506 E-08 \\
3.961 E-09\end{array}$ & $\begin{array}{l}3200 \\
3200 \\
3200 \\
3200 \\
3200\end{array}$ \\
\hline
\end{tabular}

TDHEK UATA FIILLOH....

$$
\begin{aligned}
& \text { ILST D3 JULY 13.1967 0400 TU 0430 PST } \\
& \text { FLUOKESCEJN RELEASE FRUM ELEVATION OF } 24 \\
& 32 \mathrm{COM} A R C \text { U. } 2.6 \mathrm{M} / \mathrm{SEC} A T^{24}
\end{aligned}
$$

AzIIUUTH HEIGHT EXPIISURT F/O IU/U DISTANCE DEGRFES MFTERS GM-SEC/CU.M SEC/CU.M I/SO.M METERS $\times 10 t+6$

98.0
94.0
90.0
98.0
90.0
40.0
97.0
96.0
96.0
99.0
94.0
99.0
98.0
94.0
47.0
99.0
78.0
70.0
94.0

.2
.4
.8
1.5
1.6
3.1
6.2
9.3
12.4
15.5
18.6
21.7
24.8
31.0
37.2.
43.4
49.6
55.8
62.0

$\begin{array}{rl}1247 & 1.246 E-06 \\ 1320 & 1.267 E-06 \\ 1297 & 1.246 E-06 \\ 1387 & 1.332 E-06 \\ 1522 & 1.461 E-06 \\ 1611 & 1.547 E-06 \\ 1432 & 1.375 E-06 \\ 157 & 1.507 E-07 \\ 849 & 0.150 E-07 \\ 781 & 7.504 E-07 \\ 509 & 4.890 E-07 \\ 295 & 2.840 E-07 \\ 147 & 1.415 E-07 \\ 50 & 4.816 E-00 \\ 50 & 4.818 E-00 \\ 54 & 5.228 E-08 \\ 14 & 1.384 E-08 \\ 6 & 6.151 E-09 \\ 20 & 1.968 E-08\end{array}$

$3.239 E-06$ $3.244 E-06$ $3.239 E-06 \quad 3200$ $3.462 E-06 \quad 3200$ $3.7 \rightarrow 6 E-06 \quad 3200$ $3.574 \mathrm{E}-66 \quad 320 \mathrm{~J}$ $3.918 k-07 \quad 3200$ $2.119 \mathrm{E}-06 \quad 3200$ $1.951 E-06 \cdot 3200$ $1.271 E-06 \quad 3200$ $7.363 E-07 \quad 3200$ $3.678 E-07 \quad 3200$ $1.253 \mathrm{E}-07 \quad 3200$ $1.253 E-07 \quad 3200$ $1.359 E-07 \quad 3200$ $3.598 E-08 \quad 3200$ $1.399 E-08 \quad 3200$ $5.11 d E-O H \quad 3200$

EXPOSURF

$F / 0$

\begin{tabular}{|c|c|}
\hline $1 \cup 6.0$ &. \\
\hline 160.0 & .4 \\
\hline 126.0 & : \\
\hline $1 \cup 6.0$ & 1.5 \\
\hline $1,0,1$ & . \\
\hline $1 \div 6.0$ & 3.1 \\
\hline 1010.0 & \\
\hline 11.6 .0 & 9.3 \\
\hline Ius.u & 12.4 \\
\hline list. 0 & 3. \\
\hline $1 \cup 5.0$ & 18.6 \\
\hline 136.0 & 21.7 \\
\hline 116.6 & 24.0 \\
\hline 1 1 th. & 31.0 \\
\hline 100.0 & 37.2 \\
\hline LUt.0 & 43 \\
\hline $1 \cup t .0$ & \\
\hline 1 1 & 5.8 \\
\hline 1.30 .0 & 2 \\
\hline
\end{tabular}

EU/U UISTANCE $X] \cup E+\infty$

$\begin{array}{ll}1.351 t-06 & 3200 \\ 1.645 t-06 & 3200 \\ 1.511 t-06 & 3200 \\ 2.007 t-06 & 3200 \\ 1.465 t-06 & 3200 \\ 1.725 t-06 & 3200 \\ 1.858 t-06 & 3200 \\ 1.431 t-06 & 3200 \\ 1.405 E-06 & 3200 \\ 1.271 E-06 & 3<00 \\ 1.138 t-06 & 3200 \\ 1.245 t-06 & 3200 \\ 6.85 U t-07 & 3200 \\ 6.584 E-07 & 3200 \\ 6.584 t-07 & 3200 \\ 5.2781-07 & 3200 \\ 5.038 E-07 & 3200 \\ 6.797 E-07 & 3200 \\ 2.079 t-08 & 3200\end{array}$

AZIMUTH HEIGHI EXPOSURE F/O DEGREES MEIERS GM-SECICU.H SET/CU.M $X] 0 E+6$

$\begin{array}{lr}114.0 & .2 \\ 114.0 & .4 \\ 114.0 & .8 \\ 114.0 & 1.5 \\ 114.0 & 1.4 \\ 114.0 & 3.1 \\ 114.0 & 6.2 \\ 114.0 & 9.3 \\ 114.0 & 12.4 \\ 114.0 & 15.5 \\ 114.0 & 18.0 \\ 114.0 & 21.7 \\ 114.0 & 24.8 \\ 114.0 & 31.0 \\ 114.0 & 37.2 \\ 114.0 & 43.4 \\ 114.0 & 49.6 \\ 114.0 & 55.8 \\ 114.0 & 62.0\end{array}$

$\begin{array}{rlll}753 & 7.230 E-07 & 1.880 E-06 & 3200 \\ 999 & 9.588 E-07 & 2.493 E-06 & 3200 \\ 656 & 6.299 E-07 & 1.638 E-06 & 3200 \\ 950 & 4.122 E-07 & 2.372 E-06 & 3200 \\ 919 & 5.824 E-07 & 2.294 E-06 & 3200 \\ 1574 & 1.511 E-06 & 3.929 E-06 & 3200 \\ 1492 & 1.432 E-06 & 3.723 E-06 & 3200 \\ 1867 & 1.742 E-06 & 4.660 E-06 & 3200 \\ 1820 & 1.747 E-06 & 4.542 E-06 & 3200 \\ 175 B & 1.687 E-06 & 4.387 E-06 & 3200 \\ 1648 & 1.582 E-06 & 4.114 E-06 & 3200 \\ 1853 & 1.779 E-06 & 4.625 E-06 & 3200 \\ 1657 & 1.590 E-06 & 4.135 E-06 & 3200 \\ 1230 & 1.181 E-06 & 3.071 E-06 & 3200 \\ 1192 & 1.145 E-06 & 2.976 E-06 & 3200 \\ 1144 & 1.098 E-06 & 2.855 E-06 & 3200 \\ 1035 & 9.937 E-07 & 2.584 E-06 & 3200 \\ 586 & 5.632 E-07 & 1.464 E-06 & 3200 \\ 522 & 5.012 E-07 & 1.303 E-06 & 3200\end{array}$

ALIVITA HEIGHT

114.0
1114.0
114.01
114.0
1114.0
114.0
114.0
1114.0
114.0
1114.0
114.0
1114.0
114.0
114.0
114.0
114.0
114.0
114.0
114.0
.2
.1
.8
1.5
1.6
3.1
6.2
9.3
12.4
15.5
18.6
21.7
24.8
31.0
37.2
43.4
49.6
55.8
62.0 EXPOSUR: F/O EU/O OISTANCE xivito

.2
.8
.8
1.5
1.6
3.1
6.7
9.3
2.4
5.5
8.6
1.7
4.8
1.0
7.2
3.4
9.6
5.8
2.0

$\begin{array}{rl}6 Y 1 & 0.633 E-D 7 \\ 1275 & 1.274 E-06 \\ 108 & 7.761 E-07 \\ 913 & 9.796 E-07 \\ 979 & 9.401 E-07 \\ 1073 & 1.030 E-06 \\ 2003 & 1.499 E-06 \\ 1253 & 1.203 E-06 \\ 1926 & 1.84 E-06 \\ 1477 & 1.418 E-06 \\ 1636 & 1.569 E-06 \\ 1233 & 1.203 E-06 \\ 3159 & 3.032 E-06 \\ 1454 & 1.396 E-06 \\ 1028 & 9.872 E-07 \\ 1230 & 1.181 E-06 \\ 903 & 9.442 E-07 \\ 573 & 5.505 E-07 \\ 552 & 5.300 E-07\end{array}$
$3.183 E-46$ $2.010 t-66$ $2.247 t-06$ $2.444 E-06$ $2.679 t-06$ $5.198 t-06$ $3.127 t-00$ 4. BU6E- 06 $3.686 \mathrm{E}-06$ $4.078 E-U O$ $3.127 t-06$ $7.864 E-06$ $3.630 t-06$ $2.567 E-06$ $3.071 \mathrm{E}-06$ $2.455 t-06$ $1.378 \mathrm{E}-06 \quad 3200$ 


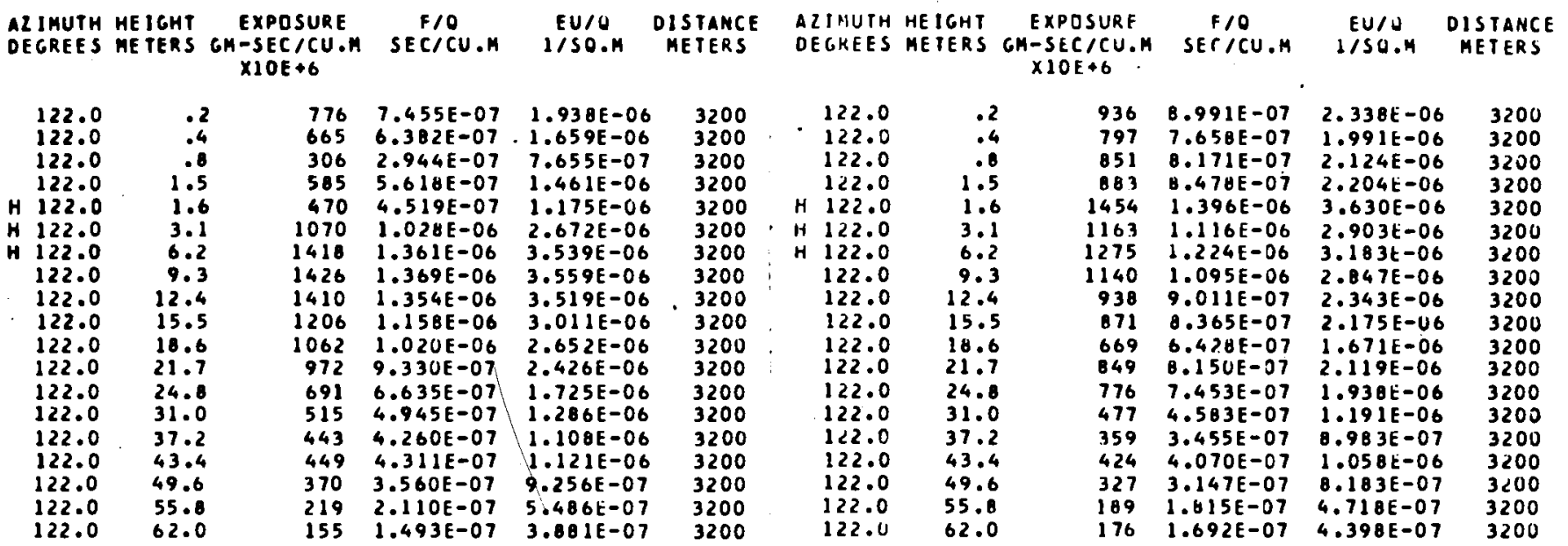

\begin{tabular}{|c|c|c|c|c|c|c|c|c|c|c|c|c|}
\hline $\begin{array}{l}2 \text { IMUTH } \\
\text { EGREES }\end{array}$ & $\begin{array}{l}\text { HE IGHT } \\
\text { METERS }\end{array}$ & $\begin{array}{l}\text { EXPQSURE } \\
\text { GH-SEC/CU.H } \\
\times 10 E+6\end{array}$ & $\begin{array}{c}E / O \\
\text { SEC/CU.M }\end{array}$ & $\begin{array}{c}E U / O \\
1 / 50 . M\end{array}$ & $\begin{array}{l}\text { DISTANCE } \\
\text { HE TERS }\end{array}$ & & $\begin{array}{l}\text { AI IMUTH } \\
\text { OEGREES }\end{array}$ & $\begin{array}{l}\text { HEICHT } \\
\text { METERS }\end{array}$ & $\begin{array}{l}\text { EXPDSURE } \\
\text { CH-SEC/CU.H } \\
\text { X1OE }+6\end{array}$ & $\begin{array}{c}E / O \\
\text { SEC/CU.M }\end{array}$ & $\begin{array}{l}E \cup / 0 \\
1 / S 0 . M\end{array}$ & $\begin{array}{c}\text { DISTANCE } \\
\text { METERS }\end{array}$ \\
\hline $\begin{array}{l}130.0 \\
130.0 \\
130.0 \\
130.0 \\
130.0 \\
130.0 \\
130.0 \\
130.0 \\
130.0 \\
130.0 \\
130.0 \\
130.0 \\
130.0 \\
130.0 \\
130.0 \\
130.0 \\
130.0 \\
130.0 \\
130.0\end{array}$ & $\begin{array}{l}.2 \\
.4 \\
.8 \\
1.5 \\
1.6 \\
3.1 \\
6.2 \\
9.3 \\
12.4 \\
15.5 \\
18.6 \\
21.7 \\
24.8 \\
31.0 \\
37.2 \\
43.4 \\
49.6 \\
55.8 \\
62.0\end{array}$ & $\begin{array}{l}529 \\
507 \\
333 \\
322 \\
213 \\
602 \\
940 \\
907 \\
865 \\
782 \\
793 \\
613 \\
711 \\
556 \\
369 \\
482 \\
265 \\
360 \\
253\end{array}$ & $\begin{array}{l}5.07 \forall E-07 \\
4.873 E-07 \\
3.196 E-07 \\
3.093 E-07 \\
2.050 E-07 \\
5.786 E-07 \\
9.411 E-07 \\
8.707 E-07 \\
8.307 E-07 \\
7.506 E-07 \\
7.613 E-07 \\
5.8 B 8 E-07 \\
6.831 E-07 \\
5.345 E-07 \\
3.549 E-07 \\
4.634 E-07 \\
2.552 E-07 \\
3.458 E-07 \\
2.437 E-07\end{array}$ & $\begin{array}{l}1.320 E-06 \\
1.267 E-06 \\
8.309 E-07 \\
8.043 E-07 \\
5.330 E-07 \\
1.504 E-06 \\
2.447 E-06 \\
2.264 E-06 \\
2.160 E-06 \\
1.952 E-06 \\
1.979 E-06 \\
1.531 E-06 \\
1.776 E-06 \\
1.390 E-06 \\
9.227 E-07 \\
1.205 E-06 \\
6.636 E-07 \\
8.991 E-07 \\
6.337 E-07\end{array}$ & $\begin{array}{l}3200 \\
3200 \\
3200 \\
3200 \\
3200 \\
3200 \\
3200 \\
3200 \\
3200 \\
3200 \\
3200 \\
3200 \\
3200 \\
3200 \\
3200 \\
3200 \\
3200 \\
3200 \\
3200\end{array}$ & $!^{4}$ & $\begin{array}{l}130.0 \\
130.0 \\
130.0 \\
130.0 \\
130.0 \\
130.0 \\
130.0 \\
130.0 \\
130.0 \\
130.0 \\
130.0 \\
130.0 \\
130.0 \\
130.0 \\
130.0 \\
130.0 \\
130.0 \\
130.0 \\
130.0\end{array}$ & $\begin{array}{l}.2 \\
.4 \\
.8 \\
1.5 \\
1.6 \\
3.1 \\
6.2 \\
.9 .3 \\
12.4 \\
15.5 \\
18.6 \\
21.7 \\
24.8 \\
31.0 \\
37.2 \\
43.4 \\
49.6 \\
55.8 \\
62.0\end{array}$ & $\begin{array}{l}530 \\
424 \\
262 \\
530 \\
562 \\
712 \\
1230 \\
781 \\
647 \\
616 \\
509 \\
584 \\
573 \\
424 \\
338 \\
306 \\
306 \\
240 \\
243\end{array}$ & $\begin{array}{l}5.095 E-07 \\
4.070 E-07 \\
2.522 E-07 \\
5.095 E-07 \\
5.403 E-07 \\
6.830 E-07 \\
1.185-06 \\
7.504 E-07 \\
6.213 E-07 \\
5.915 E-07 \\
4.090 E-07 \\
5.608 E-07 \\
5.505 E-07 \\
4.070 E-07 \\
3.250 E-07 \\
2.942 E-07 \\
2.942 E-07 \\
2.307 E-07 \\
2.337 E-07\end{array}$ & $\begin{array}{l}1.325 E-06 \\
1.058 E-06 \\
6.557 E-07 \\
1.325 E-06 \\
1.405 E-06 \\
1.77 B E-06 \\
3.071 E-06 \\
1.951 E-06 \\
1.615 E-06 \\
1.538 E-06 \\
1.271 E-06 \\
1.458 E-06 \\
1.431 E-06 \\
1.058 E-06 \\
8.449 E-07 \\
7.650 E-07 \\
7.650 E-07 \\
5.997 E-07 \\
6.077 E-07\end{array}$ & $\begin{array}{l}3200 \\
3200 \\
3200 \\
3200 \\
3200 \\
3200 \\
3200 \\
3200 \\
3200 \\
3200 \\
3200 \\
3200 \\
3200 \\
3200 \\
3200 \\
3200 \\
3200 \\
3200 \\
3200\end{array}$ \\
\hline
\end{tabular}


GROUND LEVEL AND TOWER SAMPLING 200 TO $3200 \mathrm{M}$. ALL GROUNO LEVEL SAMPLING TRUNCATED SIGHIFICANTLY ON NORTH. TRACER ON MOST TOWERS. .QUT FREOUENTLY LIGHT ANO SPOTTY.

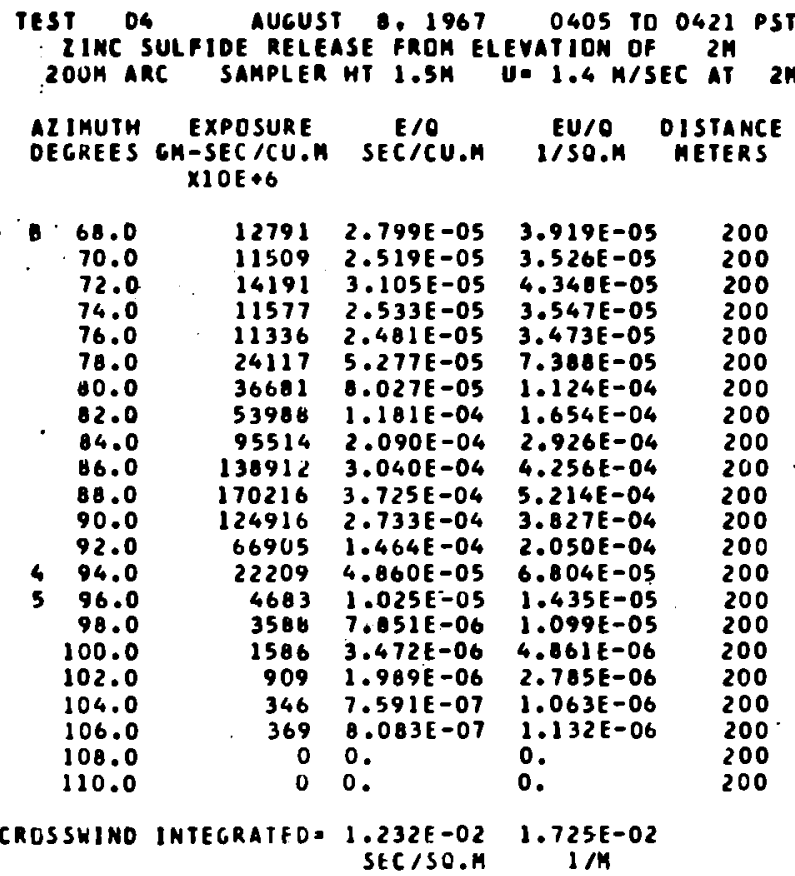
TEST U4 AUGUST H, 1967 O405 TU 0421 PST

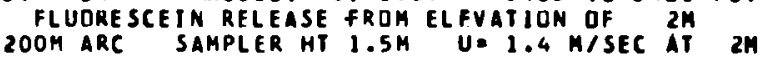

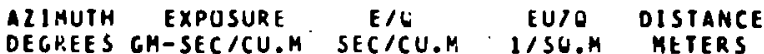
$\times 10 E+6$

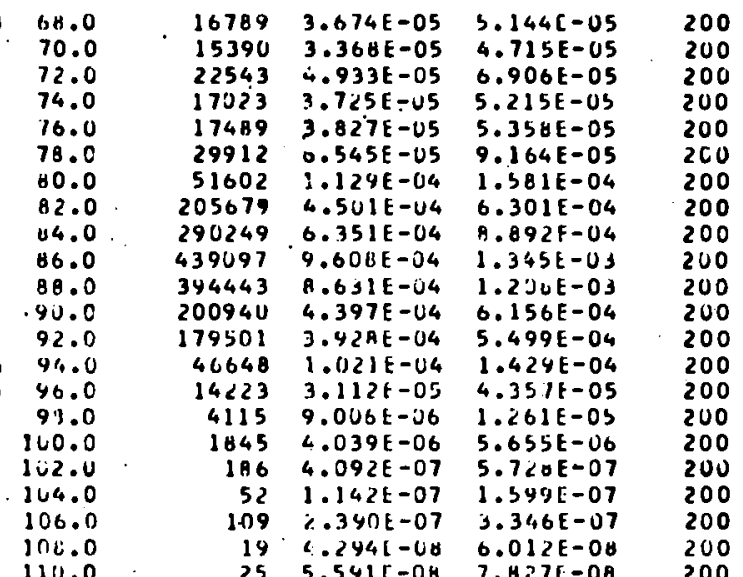

KKOSSHIND INTEGRATED $=2.976 E-02$
SEC/SO.A

200

TEST O4 AUGUST 0.1967 O405 TU 0421 PST ZINC SULFIDE KELEASE FRDM ELEVATION OF $2 M$ BOOH ARC SAMPLER HT $1.5 \mathrm{M}$ U $1.4 \mathrm{M} / \mathrm{SEC}$ AT $2 \mathrm{M}$

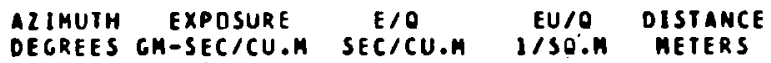

B 70.0

$\begin{array}{lll}70.0 & 5633 & 1.233 E-05 \\ 72.0 & 4129 & 9.036 E-06\end{array}$

$76.0 \quad 5064 \quad 1.108 E-05 \quad 1.552 E-05$

$78.0 .43199 .451 E-06 \quad 1.323 E-05$

$80.0 \quad 2423 \quad 5.304 E-06 \quad 7.425 E-06$

$82.0 \quad 3076 \quad 6.731 E-06 \quad 9.424 E-06$

\begin{tabular}{lll|l}
$04.0 \quad 2178$ & $4.766 E-06$ & $\$ 6.673 E-06$
\end{tabular}

$86.0 \quad 2510 \quad 5.494 E-06 \quad 7.691 E-06$

$88.0 \quad 1301 \quad 2.849 E-06 \quad 3.989 E-06$

$90.0 \quad 341 \quad 7.479 E-07 \quad 1.047 E-06$

$92.0 \quad 354 \quad 7.751 E-07$ 1.085E-06

$94.0 \quad \therefore \quad 198 \quad 4.352 E-07 \quad 6.092 E-07$

$96.0 \quad 139 \quad 3.060 E-07 \quad 4.284 E-07$

$98.0 \quad 257 \quad 5.643 E-07 \quad 7.901 E-07$

100.0

$27.643 E-07$

iv 0 .

102.0

1108.0

0.5

$2.380 E-07$

u.

$12 \quad 2.720 E-08$ 3.008E-08

$3.008 E-08$

0 0..

0 .

800

800

800

00

800
800
800

.00

800

100

000

000
800

.00

100

000

000

800

CROSSHINO INTEGRATED $=2.238 E-03 \quad 3.133 E-03$

800 SEC/SO.H

0405 TU 0421 PST FLUL'RESCE IN RFLEASE FKUM ELFVATIUN OF $2 M$ DOOM ARC SAMPLER HT $1.5 \mathrm{M} U=1.4 \mathrm{M} / \mathrm{SEC}$ AT $2 \mathrm{H}$

AZIITUTH EXPOSURE $T / O$ EU/O DISIANCE DEGPEES GH-SECICU.M SECICU.M I/SO.M MEIERS $\times 10 E+6$

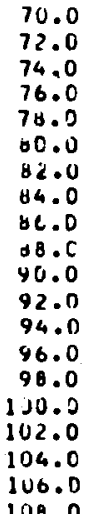

$\begin{array}{rl}6011 & 1.316 E-U 5 \\ 5034 & 1.102 E-05 \\ 4432 & 9.699 E-06 \\ 556 J & 1.217 E-05 \\ 6764 & 1.480 E-05 \\ 3076 & 6.736 E-06 \\ 383 U & 0.382 E-06 \\ 3604 & 7.04 B E-06 \\ 2627 & 5.749 E-U 6 \\ 2107 & 4.612 E-06 \\ 1176 & 2.575 E-06 \\ 132 & 1.822 E-U 6 \\ 316 & 6.936 E-U 7 \\ 145 & 3.174 E-07 \\ 256 & 5.604 E-07 \\ 170 & 3.723 E-07 \\ 17 & 3.919 E-08 \\ 1 & 2.536 E-04 \\ 25 & 5.643 E-08 \\ 0 & 1.176 E-09\end{array}$

$1.642 \mathrm{E}-05$

$1.54<E-05$

$1.350 \mathrm{E}-05$

$1.703 E-05$

2. $672 E-05$

$9.431 E-06$

$1.173 \mathrm{E}-05$

$1.104 \mathrm{E}-05$

$4.04+E-06$

$6.457 E-06$

$3.604 E-06$

$2.551 E-06$

Y.711E-07

$4.444 E-07$

$7.845 \mathrm{E}-07$

S. 212E-07

$5.486 E-U B$

$3.621 E-09$

$7.900 E-00$

$1.646 \mathrm{E}-09$

$B 00$

800

300
600

. 300

800

$\triangle 00$

800

$B \cup 0$
900

400

800

800

800
800

800

$B 00$

106.0

800
800

000

CRESSININD INTEGRATED= $2.811 E-03 \quad 3.935 E-03$

SEC/SO.M

$1 / 4$ 


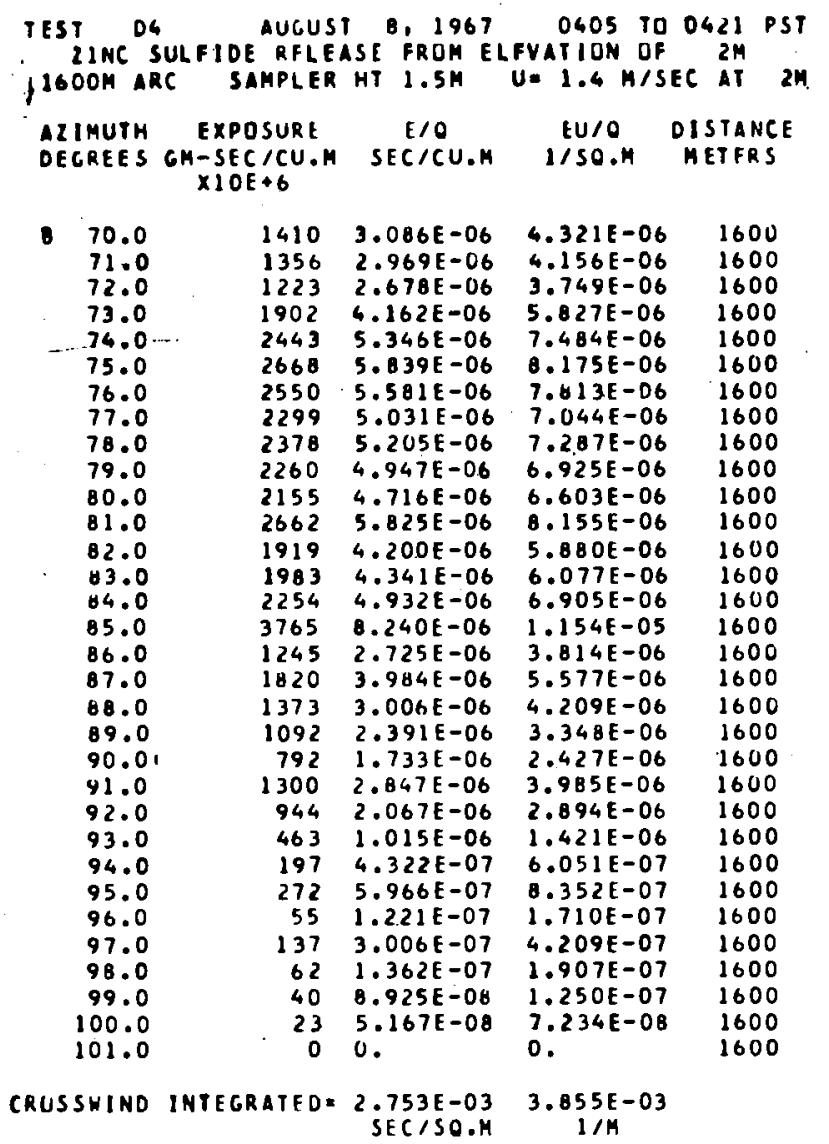

TEST D4 AUGUST $8,1967 \quad 0405$ TO 0421 PST

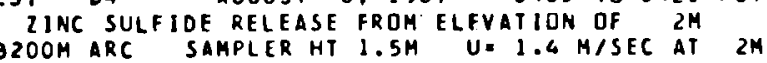
AZIMUTH EXPOSURE
OEGREES GM-SEC/CU.M SEC/CU.M I/SO.M DISIANCE $X 10 E+6$

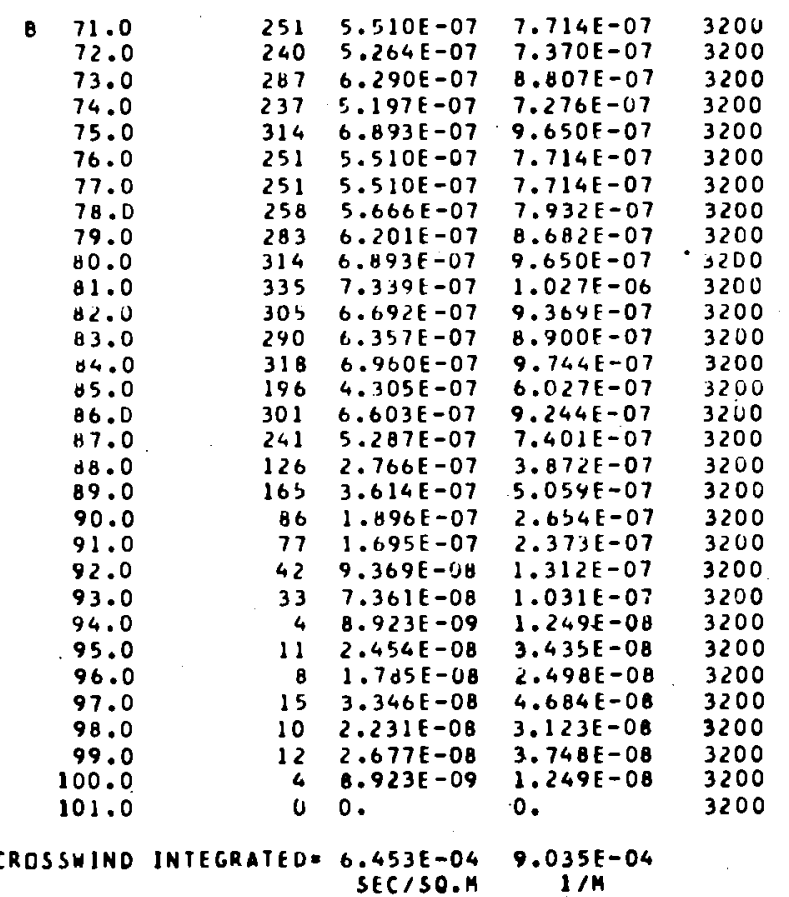

TEST RIA AIICUSI H. 1967 OUUS IH O4RL PST 160OM ARC SAMHLER HT $1.5 \mathrm{M} U$ U $1.6 \mathrm{M} / \mathrm{SEC}$ AT $2 M$

\begin{tabular}{|c|c|c|c|}
\hline $\begin{array}{l}A<1 \text { I } \\
\text { OECK }\end{array}$ & $\begin{array}{r}E X P \\
G M-S E\end{array}$ & $\begin{array}{c}t / 0 \\
S t C / C U, M\end{array}$ & $\begin{array}{c}E U / Q \\
1 / S U . M\end{array}$ \\
\hline
\end{tabular}

\begin{tabular}{|c|c|c|c|c|}
\hline $\begin{array}{l}70.0 \\
71.0 \\
72.0 \\
73.0\end{array}$ & $\begin{array}{l}1500 \\
1720 \\
1697 \\
2027\end{array}$ & $\begin{array}{l}3.3010 t-06 \\
3.765 t-06 \\
3.714 E-06 \\
4.437 E-06\end{array}$ & $\begin{array}{l}4.620 E-06 . \\
5.271 E-06 \\
5.19 Y E-06 \\
6.212 E-06\end{array}$ & $\begin{array}{l}1600 \\
1600 \\
1600 \\
1600\end{array}$ \\
\hline 74.0 & 1980 & $4.334 E-06$ & $6.067 E-06$ & 1600 \\
\hline 75.0 & 2724 & $3.962 t-06$ & $0.347 t-06$ & 1600 \\
\hline $\begin{array}{l}16.0 \\
77.0\end{array}$ & $\begin{array}{l}2327 \\
2573\end{array}$ & $\begin{array}{l}5.044 E-06 \\
5.636 E-06\end{array}$ & $\begin{array}{l}7.131 \mathrm{E}-06 \\
7.891 \mathrm{E}-06\end{array}$ & $\begin{array}{l}1600 \\
1600\end{array}$ \\
\hline $\begin{array}{l}74.0 \\
79.0\end{array}$ & $\begin{array}{l}2625 \\
2278\end{array}$ & $\begin{array}{l}5.745 t-06 \\
4.985 t-06\end{array}$ & $\begin{array}{l}8.043 E-06 \\
6.979 E-06\end{array}$ & $\begin{array}{l}1600 \\
1600\end{array}$ \\
\hline $\begin{array}{r}40.0 \\
+1.0\end{array}$ & $\begin{array}{l}2774 \\
2675\end{array}$ & $\begin{array}{l}5.071 t-06 \\
5.853 E-06\end{array}$ & $\begin{array}{l}8.499 E-06 \\
8.195 E-06\end{array}$ & $\begin{array}{l}1600 \\
1600\end{array}$ \\
\hline 42.0 & 2179 & $+.76 A=-06$ & $6.675 \mathrm{E}-00$ & 1600 \\
\hline$\$ 3.0$ & $21>4$ & $\because . B O O E-O B$ & $6.523 \mathrm{E}-06$ & 1600 \\
\hline d4.0 & 1081 & $4.117 E-06$ & $5.764 t-06$ & 1600 \\
\hline 45.0 & $2030^{\circ}$ & $4.442 \mathrm{E}-06$ & $6.219 t-06$ & 1300 \\
\hline 46.0 & 2145 & $\therefore .696 \mathrm{E}-06$ & $6.574 E-06$ & 1600 \\
\hline 37.0 & 2027 & $4.437 E-06$ & $6.212 \mathrm{E}-00$ & 1600 \\
\hline 88.0 & 1741 & $3.920 \mathrm{E}-06$ & $5.489 t-06$ & 1600 \\
\hline 89.0 & 1201 & $2.620 E-06$ & $3.6005-06$ & 1600 \\
\hline 90.0 & 1413 & $3.093 t-06$ & $4.331 \mathrm{E}-06$ & 1600 \\
\hline 91.0 & 1059 & $2.31 \mathrm{HL}-\mathrm{CS}$ & $3.245 t-06$ & 1500 \\
\hline 92.0 & 775 & $1.698 t-06$ & $2.377 \mathrm{E}-06$ & 1600 \\
\hline 93.0 & 435 & $9.536 E-J 7$ & $1.335 \mathrm{E}-06$ & 1600 \\
\hline 94.0 & 442 & $1.07 \forall E-06$ & $1.509 E-06$ & 1600 \\
\hline 95.0 & 126 & $2.765 \mathrm{E}-67$ & $3.071 E-07$ & 1600 \\
\hline 96.0 & 135 & $2.972 E-07$ & $4.161 \mathrm{E}-07$ & 1600 \\
\hline 97.0 & 37 & $8.218 \mathrm{E}-0 \mathrm{~s}$ & $1.151 E-07$ & ¿s00 \\
\hline 98.0 & 12 & $2.739 E-0 B$ & $3.835 \mathrm{E}-00$ & 1600 \\
\hline 99.1 & 76 & $1.600 \mathrm{t}-07$ & $2.352 \mathrm{E}-07$ & 1600 \\
\hline 100.0 & $u$ & i. OUSE-09 & $1.5201-09$ & 1600 \\
\hline 101.0 & 0 & 0. & 0 . & 1600 \\
\hline 102.0 & 0 & $t .202 \mathrm{E}-10$ & $8.683 E-10$ & 1600 \\
\hline 103.0 & 0 & 0 & 0. & 1600 \\
\hline
\end{tabular}

CRESSAIND INTEGRATED $=2.864 E-03$ S.010E-03

IEST DS AUGUST B. 1967 O405 TU 0421 PST FLUTRESCEIN RFLEASE FRUM ELFVATIUN UF $2 M$
ZUUN AKC SAMPLER HT $1.5 \%$ U 1.4 M/SEC AT 2 M

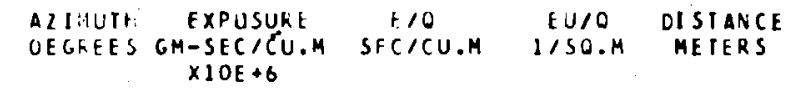

\author{
0
}

71.0
72.0

73.0

74.0

75.0

76.0

77.0

70.0

79.0

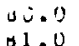

$3 \rightarrow 0$

39.0

04.0

05.0

0.96
B

14.

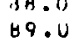

40.0

40.0
41.0

92.0

93.0

94.0

95.0

96.0

97.0

Y. 9.0

99.0

100.0

101.0

$246 \quad 0.276 E-07 \quad 6.787[-07$

$9.114 \mathrm{E}-07 \quad 3200$

$31 甘 \quad 3.977 E-07 \quad 9.764 E-07 \quad 3200$

$286 \quad 6.27 \mathrm{HE}-07 \quad 0.787 E-07 \quad 3200$

$276 \quad 6.042 E-07 \quad 8.459 E-U 7 \quad 3200$

$241 \quad 3.294 E-07 \quad 7.412 E-07 \quad 3200$

$340 \quad 7.445 \mathrm{E}-07 \quad 1.042 \mathrm{E}-06 \quad 3200$

372 B.146E-U7 1.14JE-06 3200

$129 \quad 2.840 \mathrm{E}-\mathrm{U}, \quad 3.976 \mathrm{~F}-07 \quad 3200$

$\begin{array}{llll}361 & 7.917 \mathrm{t}-07 & 1.100 E-06 & 3200 \\ 343 & 0.614 E-07 & 1.206 E-06 & 3200 .\end{array}$

$2700.0425-07$ O.459t-07 3200

O.

265 ..AU9E-07 B.132E-07 3200

$200 \quad 1.343 E-07 \quad 6.136 E-07 \quad 3200$

$\begin{array}{llll}234 & 5.226 E-07 & 7.314 E-07 & 3200 \\ 270 & 5.925 E-07 & 8.296 E-07 & 3200\end{array}$

$123 \quad 3.700 E-U 7 \quad 3.786 E-07 \quad 3200$

$1613.541 t-07 \quad 4.956 E-07 \quad 3200$

52 1.157t-07 1.620E-07 3200

$1503.471 E-07 \quad 4.860 E-07 \quad 3200$

129 2. $140 \mathrm{E}-07 \quad 3.970 \mathrm{E}-07 \quad 3200$

$25 \quad 5.540 E-08 \quad 7.756 E-08 \quad 3200$

$18 \quad 4.0367 E-08 \quad 5.694 E-U 8 \quad 3200$

$113.454 E-08 \quad 3.436 E-08 \quad 3200$

$40 \quad 8.766 \mathrm{E}-\mathrm{UB} \quad 1.227 \mathrm{E}-07 \quad 3200$

$2 \quad 5.844 E-09$ 8.1H1E-OY 3200

U $1 . .909 E-10 \quad 6.872 E-10 \quad 3200$

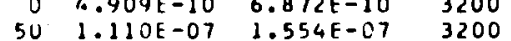

$92.174 \mathrm{E}-\mathrm{UB} \quad 3.043 \mathrm{E}-\mathrm{OB} \quad 3200$

CRUSSHIND INTEGRATED= $0.553 E-04 \quad 9.174 E-04$ 
TOUER DATA FOLLOW....

TEST D4 AUGUST B, 1967 0405 TO D42I PSTI IINC SULFIDE RELEASE FROM ELEVATION OF $2 M$

- $200 M$ ARC UE 1.4 M/SEC IT $2 \mathrm{P}$
TOWER DATA FOLLOW....

TEST D4 AUGUST 8, 1967 O405 TU 0421 PST FLUORESCEIN RELEASE FROH ELFVATIUN DF $2 \mathrm{M}$ 2OUM ARC UE 1.4 M/SEC AT $2 \mathrm{M}$

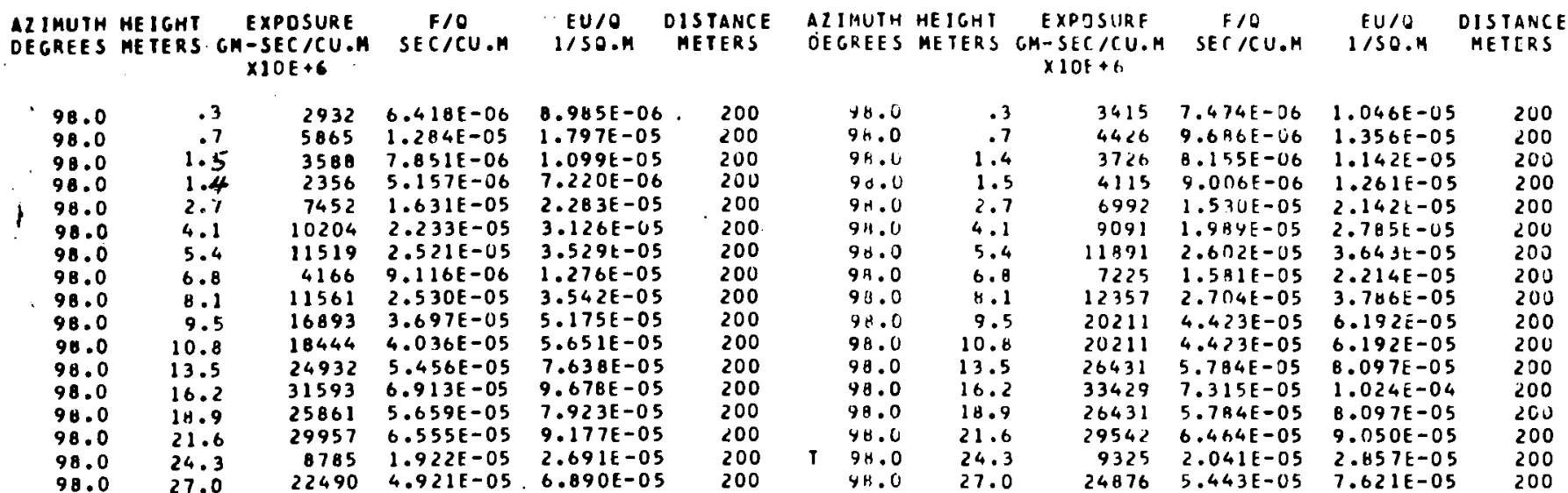

\begin{tabular}{|c|c|c|c|c|}
\hline $\begin{array}{l}\text { AL IMUTH } \\
\text { DEGREES }\end{array}$ & $\begin{array}{l}\text { HE IGHT } \\
\text { METERS C }\end{array}$ & $\begin{array}{c}\text { EXPOSURE } \\
\text { CM-SEC/CU.H } \\
\times 1 O E \$ 6\end{array}$ & $\begin{array}{c}F / O \\
\text { SEC/CU.H }\end{array}$ & $\begin{array}{l}E U / 0 \\
1 / 50 . M\end{array}$ \\
\hline $\begin{array}{ll}\text { E } & 106.0 \\
\text { E } & 106.0 \\
106.0 \\
106 \\
106.0 \\
106.0 \\
106.0 \\
106.0 \\
106.0 \\
106.0 \\
106.0 \\
106.0 \\
106.0 \\
106.0 \\
106.0 \\
106.0 \\
106.0 \\
106.0\end{array}$ & $\begin{array}{l}.3 \\
.7 \\
1.5 \\
1.4 \\
2.7 \\
4.1 \\
5.4 \\
6.8 \\
1.1 \\
9.5 \\
10.8 \\
13.5 \\
16.2 \\
18.9 \\
21.6 \\
24.3 \\
27.0\end{array}$ & $\begin{array}{l}70 \\
176 \\
369 \\
353 \\
551 \\
402 \\
268 \\
392 \\
194 \\
106 \\
632 \\
628 \\
1088 \\
1872 \\
1459 \\
1427 \\
1084\end{array}$ & $\begin{array}{l}1.546 E-07 \\
3.866 E-07 \\
0.083 E-07 \\
7.732 E-07 \\
1.206 E-06 \\
8.814 E-07 \\
5.876 E-07 \\
8.583 E-07 \\
4.253 E-07 \\
2.320 E-07 \\
1.384 E-06 \\
1.376 E-06 \\
2.381 E-06 \\
4.098 E-06 \\
3.193 E-06 \\
3.124 E-06 \\
2.374 E-06\end{array}$ & $\begin{array}{l}2.165 E-07 \\
5.412 E-07 \\
1.132 E-06 \\
1.082 E-06 \\
1.689 E-06 \\
1.234 E-06 \\
8.227 E-07 \\
1.202 E-06 \\
5.954 E-07 \\
3.247 E-07 \\
1.938 E-06 \\
1.927 E-06 \\
3.334 E-06 \\
5.737 E-06 \\
4.471 E-06 \\
4.373 E-06 \\
3.323 E-06\end{array}$ \\
\hline
\end{tabular}

DISTANCE METERS

$\begin{array}{ll}200 & 1106.0 \\ 200 & 1106.0 \\ 200 & 1106.0 \\ 200 & 100.0 \\ 200 & 1106.0 \\ 200 & 106.0 \\ 200 & 106.0 \\ 200 & 106.0 \\ 200 & 106.0 \\ 200 & 106.0 \\ 200 & 166.0 \\ 200 & 106.0 \\ 200 & 136.0 \\ 200 & 106.0 \\ 200 & 106.0 \\ 200 & 106.0 \\ 200 & 106.0\end{array}$$$
27 .
$$

24876

$7.621 \mathrm{E}-0.05$

AIIMUTH HEIGHT EXPOSURE $M-S E C / C U, H$
$X 1 O E+B$

$$
X 10 E+B
$$

.3
.7
1.4
1.5
2.7
4.1
5.4
6.8
8.1
9.5
10.8
13.5
16.2
14.9
21.6
24.3
27.0

$$
0 .
$$$$
0 .
$$$$
0 .
$$$$
1092.39
$$$$
0 .
$$$$
09.72
$$$$
201
$$$$
0 .
$$

$2014.416 E-07$

$4.416 \mathrm{E}-07$ $2.431 E-09$

$124 \quad 2.714 \mathrm{E}-07$

$\begin{array}{ll}124 & 2.714 E-07 \\ 242 & 5.307 \mathrm{E}-07\end{array}$

$\begin{array}{ll}242 & 5.307 E-07 \\ 794 & 1.738 E-06\end{array}$

$805 \quad 1.762 \mathrm{E}-06$

$1216 \quad 2.642 \mathrm{E}-06$

$1216 \quad 2.652 \mathrm{E}-06$

$572 \quad 1.252 \mathrm{E}-06$
EU/O OISTANCE

200

200 $3.346 E-07 \quad 200$ $0.346 E-07 \quad 200$

$1.361 E-09$

$6.182 \mathrm{E}-07$ $3.403 E-09$ $6.409 t-07$ $3.800 E-07$ $7.430 E-07$ $2.433 \mathrm{E}-06$ $2.467 E-06$ $3.726 E-06$ $3.726 \mathrm{E}-06$

\begin{tabular}{|c|c|c|c|c|c|}
\hline $\begin{array}{l}\text { AZIMUTH } \\
\text { DEGREES }\end{array}$ & $\begin{array}{l}\text { HE ICHT } \\
\text { ME TERS }\end{array}$ & $\begin{array}{c}\text { EXPOSURE } \\
\text { GM-SEC/CU.H } \\
\times 1 O E+6\end{array}$ & $\begin{array}{l}E / O \\
S E C / C U . M\end{array}$ & $\begin{array}{l}\text { EU/O } \\
\text { I/SO.M }\end{array}$ & $\begin{array}{l}\text { DISTANCE } \\
\text { METERS }\end{array}$ \\
\hline $\begin{array}{ll}1 & 114.0 \\
1 & 114.0 \\
1 & 114.0 \\
1 & 114.0 \\
114.0 \\
114.0 \\
114.0 \\
114.0 \\
114.0 \\
114.00 \\
114.0 \\
114.0 \\
114.0 \\
114.0 \\
114.0 \\
114.0 \\
114.0\end{array}$ & $\begin{array}{r}.3 \\
.7 \\
1.4 \\
2.7 \\
4.1 \\
5.4 \\
6.8 \\
8.1 \\
9.5 \\
10.8 \\
13.5 \\
16.2 \\
14.9 \\
21.6 \\
24.3 \\
27.0\end{array}$ & $\begin{array}{r}0 \\
0 \\
0 \\
477 \\
512 \\
346 \\
310 \\
349 \\
38 \\
141 \\
63 \\
109 \\
45 \\
0 \\
0 \\
67\end{array}$ & $\begin{array}{l}0 . \\
0 . \\
0 . \\
1.044 E-06 \\
1.1211 E-06 \\
7.577 E-07 \\
6.804 E-07 \\
7.655 E-07 \\
8.505 E-08 \\
3.093 E-07 \\
1.392 E-07 \\
2.397 E-07 \\
1.005 E-07 \\
0 . \\
0 . \\
1.469 E-07\end{array}$ & $\begin{array}{l}0 . \\
0 . \\
0 . \\
1.461 E-06 \\
1.570 E-06 \\
1.061 E-06 \\
9.526 E-07 \\
1.072 E-06 \\
1.191 E-07 \\
4.330 E-07 \\
1.948 E-07 \\
3.356 E-07 \\
1.407 E-07 \\
0 . \\
0 . \\
2.057 E-07\end{array}$ & $\begin{array}{l}200 \\
200 \\
200 \\
200 \\
200 \\
200 \\
200 \\
200 \\
200 \\
200 \\
200 \\
200 \\
200 \\
200 \\
200 \\
200\end{array}$ \\
\hline
\end{tabular}
$1.753 \mathrm{E}-06$
ALIMUTH HEIGHT EXPOSURE
DEGRES METERS GM-SEC/CU.M SEC/CU.M I/SO.M DISTANCE DEGREES METERS GM-SEC/CU

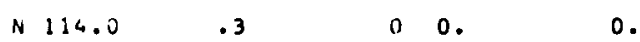

200 


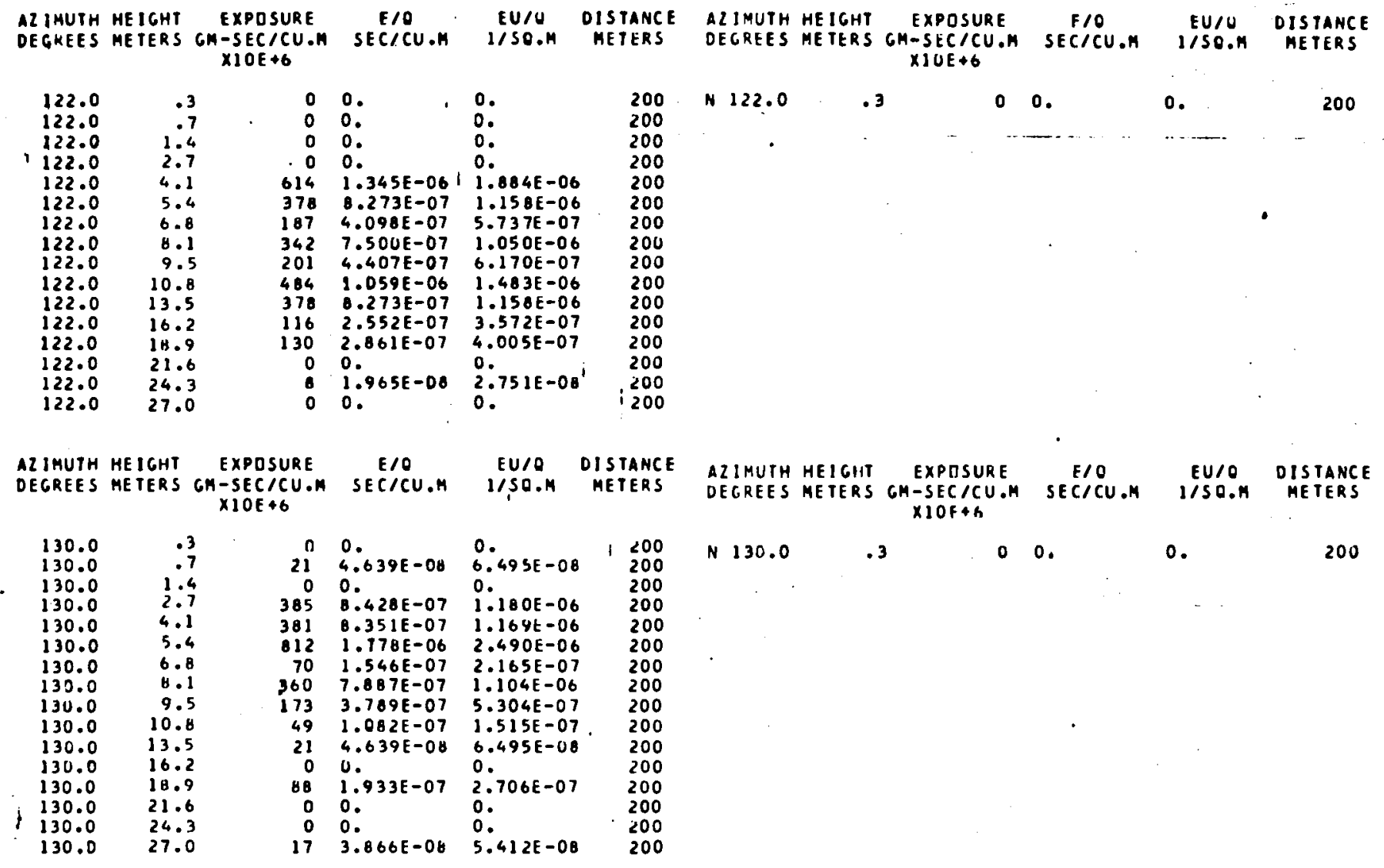

TOWER OATA FDLLOH....

TOHER DATA FRLLOW....

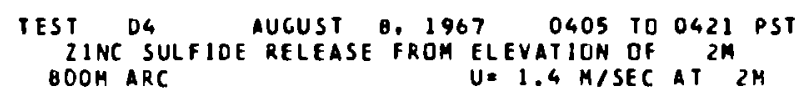
TEST D4 AUGUST B. 1967 0405 TU 0421 PST FLURRESCEIN RELEASE FROH ELFVATION DF $\angle H$ BCON ARC UE $1.4 \mathrm{M} / \mathrm{SEC}$ AT $2 \mathrm{M}$
AZIMUTH HEIGHT EXPOSURF F/O EU/O DISTANCE DEGREES METERS GM-SEC/CU.M SEC/CU.H I/SO.A METERS $\times 10 E+6$

$\begin{array}{lrrrrr}98.0 & .3 & 51 & 1.122 \mathrm{E}-07 & 1.571 \mathrm{E}-07 & 800 \\ 98.0 & .5 & 107 & 2.351 \mathrm{E}-07 & 3.291 \mathrm{E}-07 & 800 \\ 96.0 & 1.1 & 263 & 5.770 \mathrm{E}-07 & 8.078 \mathrm{E}-07 & 800 \\ 98.0 & 1.5 & 257 & 5.643 \mathrm{E}-07 & 7.901 \mathrm{E}-07 & 800 \\ 98.0 & 2.1 & 251 & 5.507 \mathrm{E}-07 & 7.710 \mathrm{E}-07 & 800 \\ 98.0 & 4.2 & 906 & 2.115 \mathrm{E}-06 & 2.960 \mathrm{E}-06 & 800 \\ 93.0 & 6.3 & 1052 & 2.302 \mathrm{E}-06 & 3.223 \mathrm{E}-06 & 800 \\ 98.0 & 8.4 & 686 & 1.502 \mathrm{E}-06 & 2.103 \mathrm{E}-06 & 800 \\ 98.0 & 10.5 & 1193 & 2.612 \mathrm{E}-06 & 3.656 \mathrm{E}-06 & 000 \\ 98.0 & 12.6 & 1489 & 3.260 \mathrm{E}-06 & 4.564 \mathrm{E}-06 & 800 \\ 98.0 & 14.7 & 1536 & 3.363 \mathrm{E}-06 & 4.708 \mathrm{E}-06 & 800 \\ 98.0 & 16.8 & 1893 & 4.142 \mathrm{E}-06 & 5.799 \mathrm{E}-06 & 800 \\ 98.0 & 21.0 & 3289 & 7.198 \mathrm{E}-06 & 1.008 \mathrm{E}-05 & 800 \\ 98.0 & 25.2 & 4321 & 9.457 \mathrm{E}-06 & 1.324 \mathrm{E}-05 & 800 \\ 98.0 & 29.4 & 3653 & 7.995 \mathrm{E}-06 & 1.119 \mathrm{E}-05 & 800 \\ 98.0 & 33.6 & 4289 & 9.387 \mathrm{E}-06.06 & 1.314 \mathrm{E}-05 & 800 \\ 98.0 & 37.8 & 3916 & 8.570 \mathrm{E}-06 & 1.200 \mathrm{E}-05 & 800 \\ 98.0 & 42.0 & 4109 & .0 .992 \mathrm{E}-06 & 1.259 \mathrm{E}-05 & 800\end{array}$


AZ IHUTH HEIGHT
OEGRES METERS

EXPOSURE F/O EU/O DISTANCE $\times 10 \mathrm{E}+6$

$\begin{array}{ll}106.0 & .3 \\ 106.0 & .5 \\ 106.0 & 1.1 \\ 106.0 & 1.5 \\ 106.0 & 2.1 \\ 106.0 & 4.2 \\ 106.0 & 6.3 \\ 106.0 & 0.4 \\ 106.0 & 10.5 \\ 106.0 & 12.6 \\ 106.0 & 14.7 \\ 106.0 & 16.8 \\ 106.0 & 21.0 \\ 106.0 & 25.2 \\ 106.0 & 29.4 \\ 106.0 & 33.6 \\ 106.0 & 37.8 \\ 106.0 & 42.0\end{array}$

.

$\begin{array}{rll}68 & 1.496 E-07 & 2.094 E-07 \\ 16 & 3.562 E-08 & 4.986 E-08 \\ 0 & 0.0 & 0 . \\ 12 & 2.720 E-08 & 3.808 E-08 \\ 0 & 0 . & 0 . \\ 620 & 1.795 E-06 & 2.513 E-06 \\ 425 & 9.300 E-07 & 1.302 E-06 \\ 199 & 4.356 E-07 & 6.101 E-07 \\ 515 & 1.128 E-06 & 1.579 E-06 \\ 153 & 3.366 E-07 & 4.712 E-07 \\ 360 & 7.898 E-07 & 1.106 E-06 \\ 73 & 1.611 E-07 & 2.255 E-07 \\ 58 & 1.271 E-07 & 1.779 E-07 \\ 38 & 8.406 E-08 & 1.177 E-07 \\ 189 & 4.141 E-07 & 5.798 E-07 \\ 71 & 1.575 E-07 & 2.205 E-07 \\ 130 & 2.864 E-07 & 4.010 E-07 \\ 82 & 1.802 E-07 & 2.522 E-07\end{array}$

800
800
800
800
800
800
800
800
800
800
800
800
800
800
800
400
800
800
A2IMUTH HEIGH DECREES METER

EXPOSURE E/O EU/O DISTANCE GM-SEC/CU.M SEC/CU.M I/SO.M METERS $X 10 E+6$

\begin{tabular}{|c|c|c|c|c|}
\hline $\begin{array}{l}114.0 \\
114.0 \\
114.0 \\
114.0 \\
114.0 \\
114.0 \\
114.0 \\
114.0 \\
114.0 \\
114.0 \\
114.0 \\
114.0 \\
114.0 \\
114.0 \\
114.0 \\
114.0 \\
114.0\end{array}$ & $\begin{array}{l}.3 \\
.5 \\
1.1 \\
2.1 \\
4.2 \\
6.3 \\
8.4 \\
10.5 \\
12.6 \\
14.7 \\
16.8 \\
21.0 \\
25.2 \\
29.4 \\
33.6 \\
37.8 \\
42.0\end{array}$ & $\begin{array}{r}27 \\
13 \\
0 \\
0 \\
472 \\
297 \\
315 \\
273 \\
253 \\
73 \\
78 \\
224 \\
24 \\
89 \\
12 \\
16 \\
30\end{array}$ & $\begin{array}{l}5.983 E-08 \\
2.849 E-08 \\
0 . \\
0 . \\
1.034 E-06 \\
6.504 E-07 \\
6.894 E-07 \\
5.963 E-07 \\
5.547 E-07 \\
1.616 E-07 \\
1.726 E-07 \\
4.917 E-07 \\
5.254 E-08 \\
1.949 E-07 \\
2.760 E-08 \\
3.525 E-08 \\
6.704 E-08\end{array}$ & $\begin{array}{l}8.377 E-08 \\
3.989 E-08 \\
0 . \\
0 . \\
1.447 E-06 \\
9.105 E-07 \\
9.652 E-07 \\
0.377 E-07 \\
7.766 E-07 \\
2.262 E-07 \\
2.416 E-07 \\
6.864 E-07 \\
7.355 E-08 \\
2.728 E-07 \\
3.892 E-08 \\
4.935 E-08 \\
9.366 E-08\end{array}$ \\
\hline
\end{tabular}

ALIMUTH HEIGHT EXPOSURE DEGREES METERS CH-SECIC

$\begin{array}{lr}122.0 & .3 \\ 122.0 & .5 \\ 122.0 & 1.1 \\ 122.0 & 2.1 \\ 122.0 & 4.2 \\ 122.0 & 6.3 \\ 122.0 & 8.4 \\ 122.0 & 10.5 \\ 122.0 & 12.6 \\ 122.0 & 14.7 \\ 122.0 & 16.0 \\ 122.0 & 21.0 \\ 122.0 & 25.2 \\ 122.0 & 29.4 \\ 122.0 & 33.6 \\ 122.0 & 37.0 \\ 122.0 & 42.0\end{array}$
$\times 10 E+6$

$$
\begin{array}{r}
58 \\
0 \\
0 \\
0 \\
99 \\
267 \\
109 \\
37 \\
93 \\
139 \\
181 \\
68 \\
151 \\
109 \\
88 \\
38 \\
42
\end{array}
$$$$
\begin{array}{ll}
0 & 0 \\
0 & 0 \\
0 & 0 \\
9 & 2 \\
7 & 5 \\
9 & 2 \\
7 & 6 \\
3 & 2 \\
9 & 3.0 \\
1 & 3 \\
8 & 1 \\
1 & 3 \\
9 & 2.3 \\
8 & 1.9 \\
8 & 8.3 \\
2 & 9.2
\end{array}
$$$$
1.271 E-07
$$

EU/O DISTANCE I/SO.M METERS

$1.780 \mathrm{E}-07$

0.

$.176 \mathrm{E}-07 \quad 3.046 \mathrm{E}-07$ $5.853 E-07 \quad 0.195 E-07$ $2.45 E E-07$ 3.369E-07 $0.103 E-08 \quad 1.134 E-07$ $2.057 \mathrm{E}-07$ 2.08 $3.052 \mathrm{E}-07 \quad 4.272 \mathrm{E}-07$ $3.970 \mathrm{E}-07 \quad 5.55 \mathrm{BE}-07$ $1.492 \mathrm{E}-07 \quad 2.088 \mathrm{E}-67$ $3.310 E-07 \quad 4.634 E-07$ $2.387 E-07 \quad 3.342 E-07$ $8.372 E-08 \quad 1.172 E-07$ $9.218 \mathrm{E}-08$ 1.291E-U? $1.946 E-072.724 E-07$

800

400
$\square 00$

600

800

800

800

600

800

800

800

800

$\triangle 00$
A2 II.UTH HEIGHT F/O EUIO OISTANCE DEGREES METERS GM-SEC/C
$\times 10 E+6$

$\begin{array}{lr}106.0 & .3 \\ 106.0 & .5 \\ 106.0 & 1.1 \\ 106.0 & 1.5 \\ 106.0 & 2.1 \\ 106.0 & 4.2 \\ 106.0 & 6.3 \\ 106.0 & 8.4 \\ 106.0 & 10.5 \\ 106.0 & 12.0 \\ 106.0 & 14.7 \\ 136.0 & 16.8 \\ 106.0 & 21.0 \\ 106.0 & 25.2 \\ 106.0 & 29.4 \\ 106.0 & 33.6 \\ 106.0 & 37.8 \\ 106.0 & 42.0\end{array}$

$$
\begin{array}{r}
0 \\
0 \\
0 \\
25 \\
0 \\
0 \\
0 \\
1 \\
6 \\
2 \\
17 \\
0 \\
2 \\
32 \\
16 \\
130 \\
94 \\
220
\end{array}
$$

0.

0.

$5.643 E-08$

0 .

$1.881 \mathrm{E}-0 \mathrm{O}$

1. $484 E-08$

$0.270 E-09$

3.84 UE - 08

3.

$5.643 E-09$

$7.054 \mathrm{E}-08$

$3.527 \mathrm{E}-\mathrm{OH}$

$2.861 E-07$

$.2 .077 \mathrm{E}-07$

$4.820 E-07$

0 .

0.

0.

0 .

$2.633 E-08$

$2.085 E-08$

$0.778 E-09$

$5.376 E-O B$

$7.900 E-09$

$9.875 E-08$

$4.938 E-08$

$4.005 E-07$

$2.90 \mathrm{BE}-07$

$6.748 E-07$

ALIYUTH HEIGHT EXPOSURE F/O EU/O DISTANCE OEGREES METERS GM-SECICU.M SECICU.M IISO.M METERS XIUE +6

$\begin{array}{llllll}N 114.0 & .3 & 0 & 0 . & 0 .\end{array}$

800 


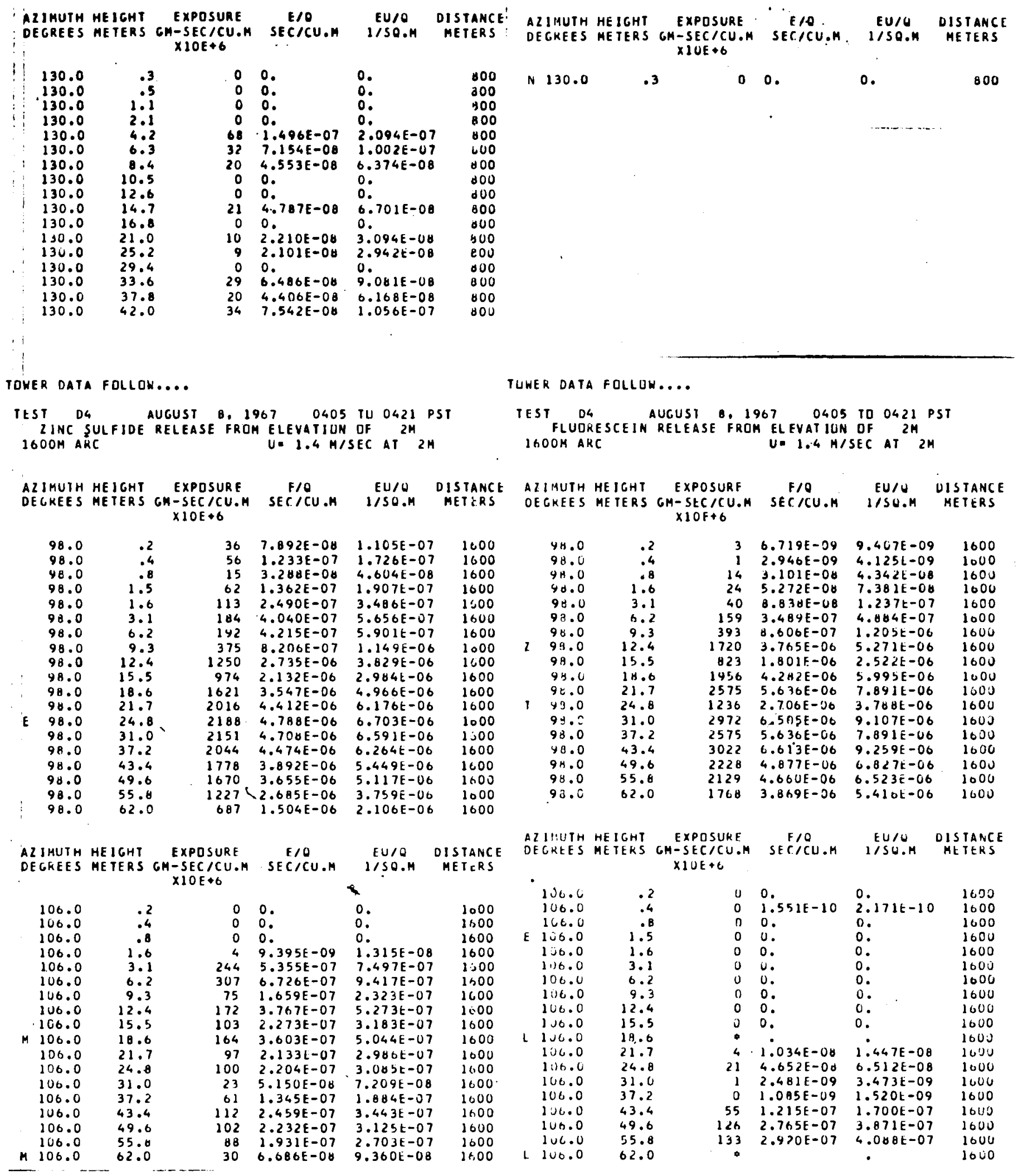




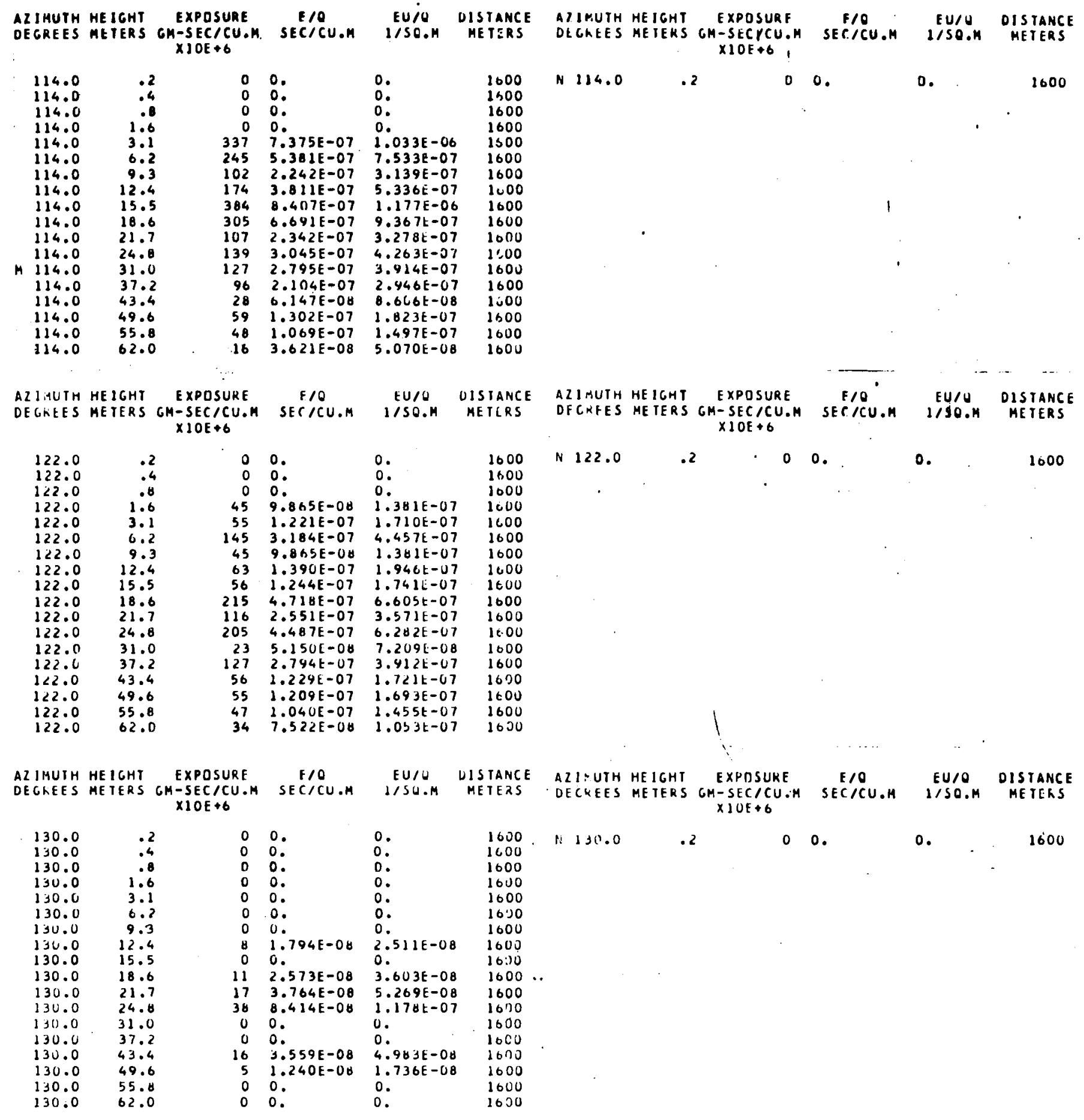


TOWER DATA FOLLEH....

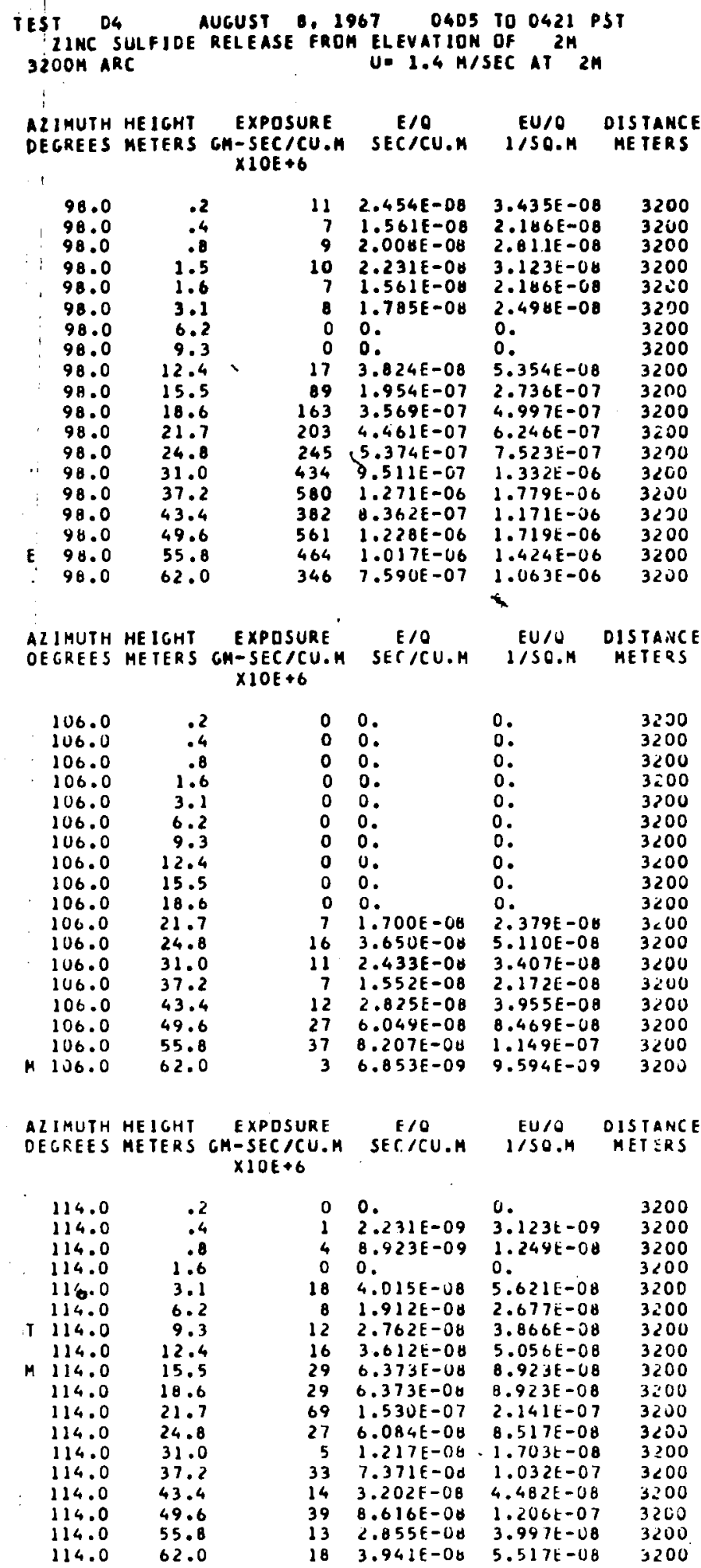

TUHEK DATA FOLLOH....

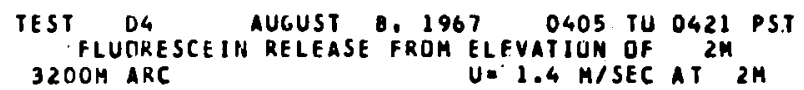

ALIHUTH HEIGHT EXPOSURE EIO EU/O DISTANCE DEGKEES METERS CM-SEC/CU.M SEC/CU.M I/SO.H METERS $X \perp \cup E+6$

$\begin{array}{ll}98.0 & .2 \\ 98.0 & .4 \\ 98.0 & .8 \\ 98.0 & 1.5 \\ 96.0 & 1.6 \\ 98.0 & 3.1 \\ 90.0 & 6.2 \\ 98.0 & 9.3 \\ 98.0 & 12.4 \\ 98.0 & 15.5 \\ 90.0 & 18.6 \\ 96.0 & 21.7 \\ 98.0 & 24.8 \\ 98.0 & 31.0 \\ 98.0 & 37.2 \\ 90.0 & 43.4 \\ 98.0 & 49.6 \\ 98.0 & 55.8 \\ 98.0 & 62.0\end{array}$

\begin{tabular}{|c|c|c|c|}
\hline $\begin{array}{l}3 \\
0 \\
0 \\
0 \\
0 \\
0 \\
0 \\
0\end{array}$ & $\begin{array}{l}0.779 E-09 \\
1.192 E-09 \\
1.402 E-10 \\
4.909 E-10 \\
0 . \\
0 . \\
0 . \\
0 .\end{array}$ & $\begin{array}{l}9.490 \mathrm{t}-09 \\
1.669 \mathrm{t}-09 \\
1.963 \mathrm{E}-10 \\
6.872 \mathrm{E}-10 \\
0 . \\
0 . \\
0 . \\
0 .\end{array}$ & $\begin{array}{l}3200 \\
3200 \\
3200 \\
3200 \\
3200 \\
3200 \\
3200 \\
3200\end{array}$ \\
\hline $\begin{array}{l}40 \\
13\end{array}$ & $\begin{array}{l}0.766 E-08 \\
2.945 E-0 d\end{array}$ & $\begin{array}{l}1.227 \mathrm{E}-07 \\
4.123 \mathrm{E}-08\end{array}$ & $\begin{array}{l}3<00 \\
3<00\end{array}$ \\
\hline $\begin{array}{l}149 \\
254 \\
222 \\
372 \\
382\end{array}$ & $\begin{array}{l}3.261 E-07 \\
5.575 E-07 \\
4.874 E-07 \\
8.146 E-07 \\
0.380 E-07\end{array}$ & $\begin{array}{l}4.565 E-07 \\
7.805 E-07 \\
6.823 E-07 \\
1.140 E-06 \\
1.173 E-06\end{array}$ & $\begin{array}{l}3200 \\
3200 \\
3200 \\
3200 \\
3200\end{array}$ \\
\hline $\begin{array}{l}361 \\
607\end{array}$ & $\begin{array}{l}7.912 E-07 \\
1.329 E-06\end{array}$ & $\begin{array}{l}1.108 E-06 \\
1.060 E-06\end{array}$ & $\begin{array}{l}3<00 \\
3<00\end{array}$ \\
\hline 415 & $9.001 E-07$ & $1.271 \div-06$ & $\begin{array}{l}3<00 \\
3200\end{array}$ \\
\hline
\end{tabular}

AZIMUTH HEIGHT EXPOSURE E/O. EU/O DISTANCE DECREES METERS GH-SECICU.M SECICU.M I/SO.H HETERS XIOE +6

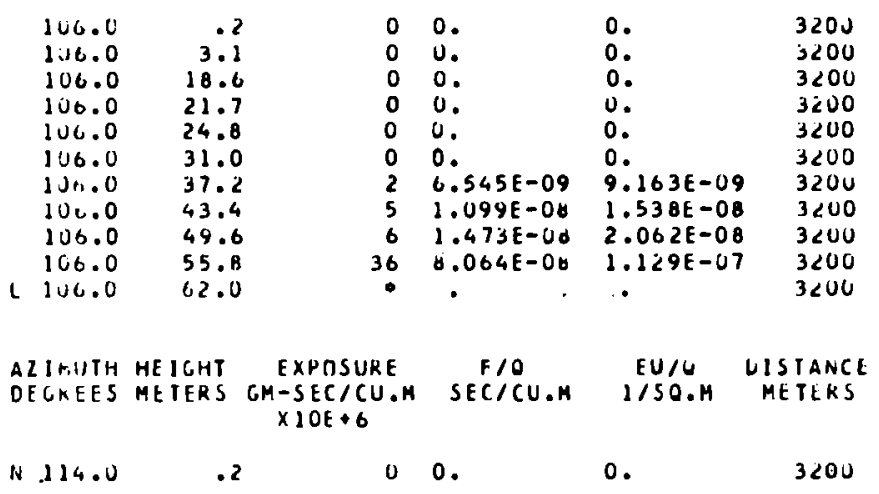

$N .114 .0 \quad .2000 .3200$ 


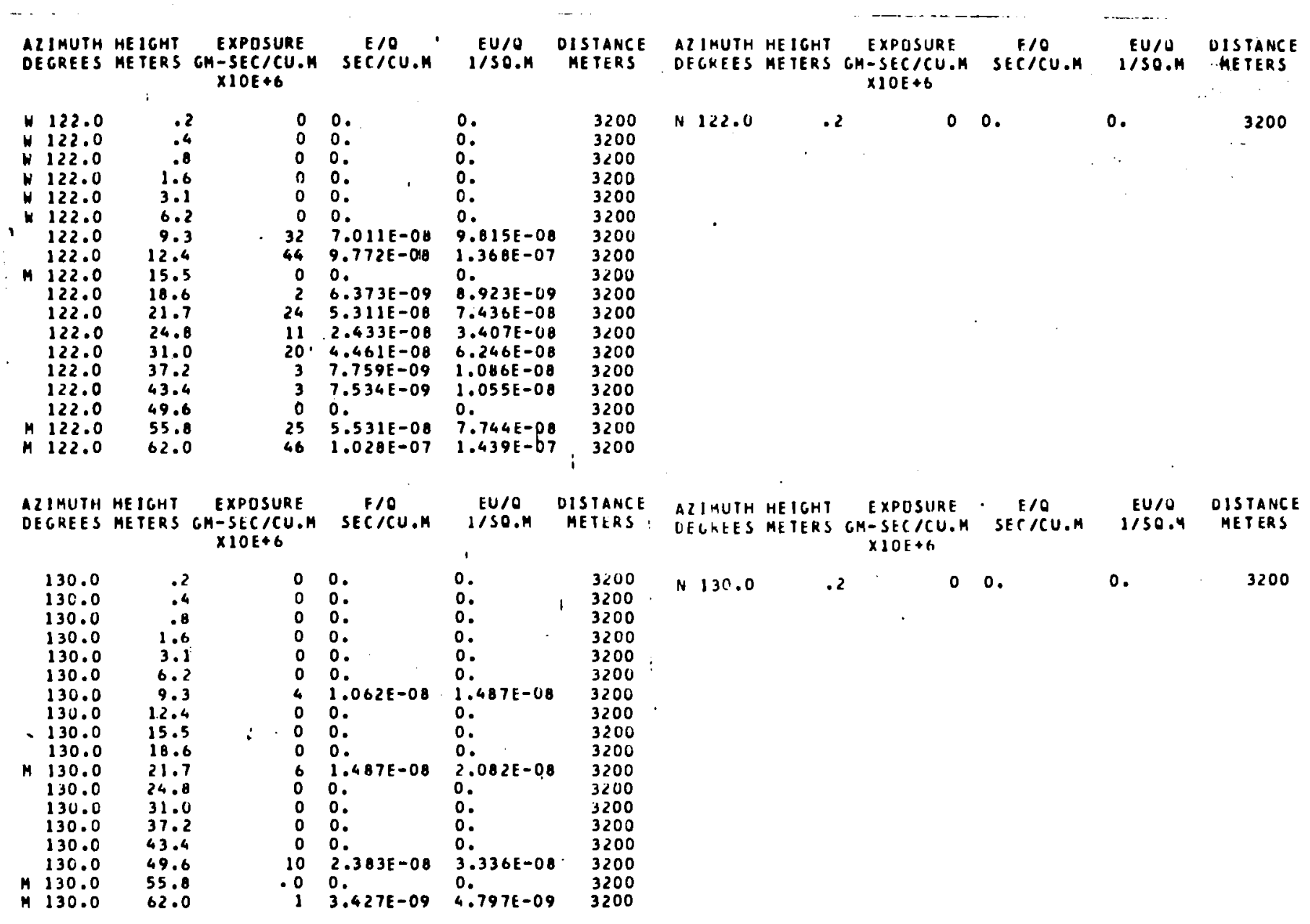


GKOUNG LEVEL ANU TDWEK SAMPLING AT 2OOM AND BUOH UNLY. AFTER RELATIVELY NINOK EXTKAPOLATIUN DN NOKTH. BUTH ARCS EMORACE CRLSSWINU EXTENT DF TRACER. DNLY 5 IOWERS SAHPLING AT EACI ARCI 3 OF 4 TOWERS "HIT" EMBRALE THE VERTICAL EXTEHT DF TRACER. OIRECIIUN SHEAR WITH HEIGHT IS UBVIUUS FRUM TOHER SAMPLES.

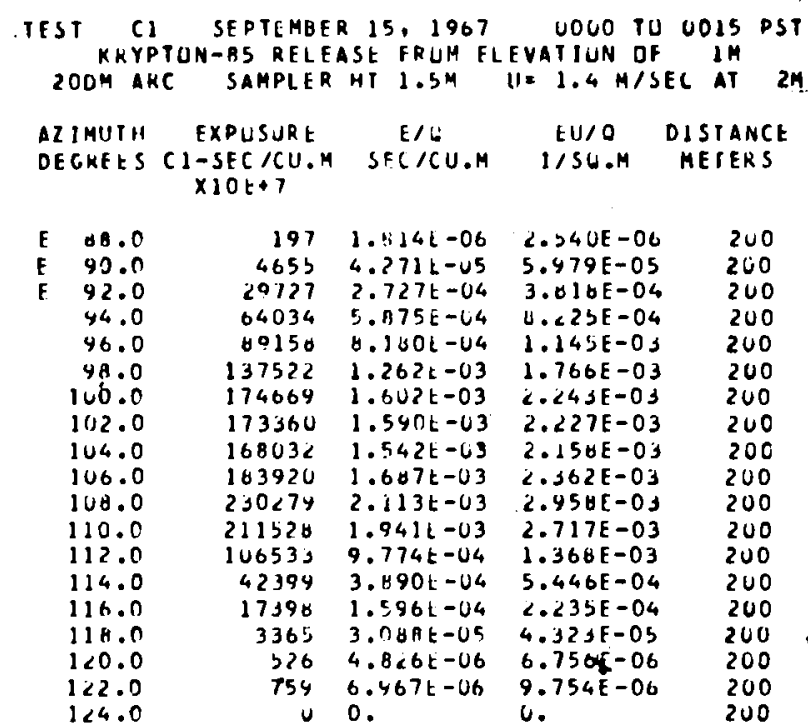

CRISSWIND INTEGKATEU: $1.049 \mathrm{t}-011.469 E-01$

$S T C S 0 . M \quad 1 / M$

TEST CI SEPTEMEFR 15, 1967 UOOO TU OOI5 PST
KKYPTCN-BS RFLEASE FRUM ELEVATION DF IM ONOM AKC SAMPLER HT $1.3 \mathrm{M} U=1.4 \mathrm{M} / S E C$ AT $2 \mathrm{M}$

AIIHUTH EXPLSURE L/ EU/O DISIANCE

DEGKFES CI-SECICU.M SECICH.M I/SG.M MEIEKS $\times 10 c+7$

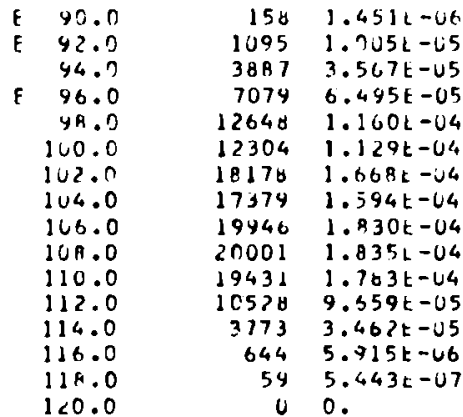

CRCSSWIAT INTECKATEU. $3.769 \mathrm{c}-02 \quad 5.277 \mathrm{~L}-0 \mathrm{C}$

SEC $150 . M \quad 1 / M$ 


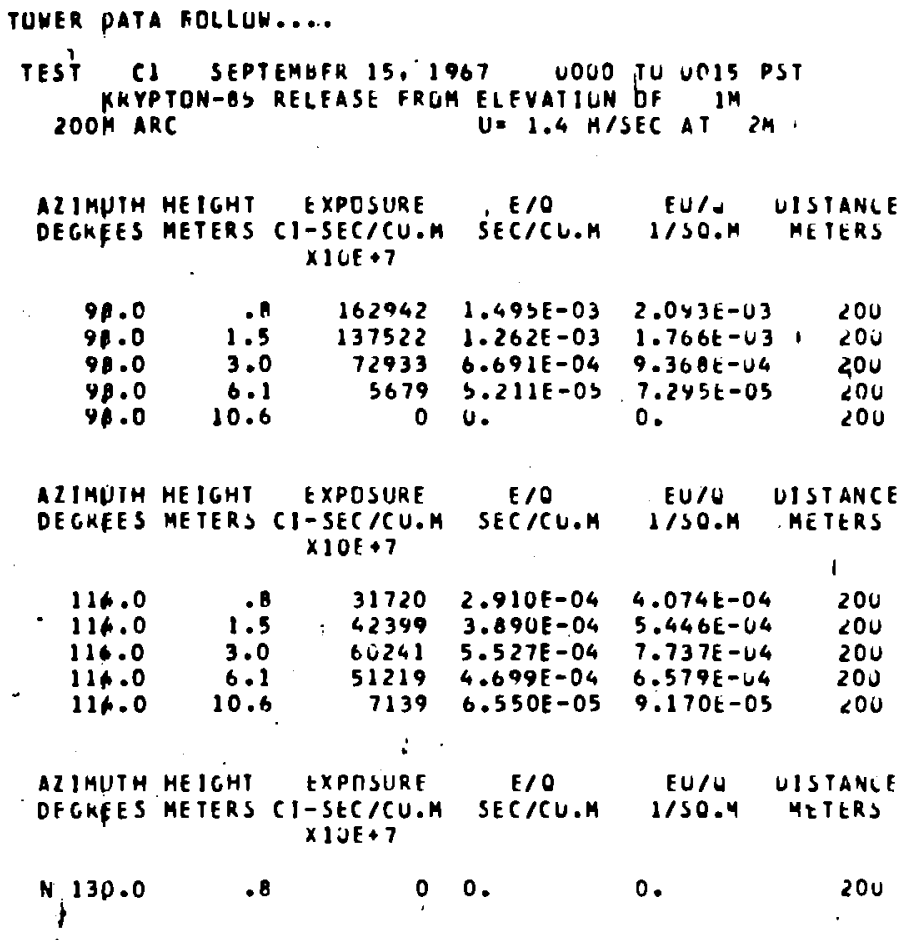

L $\quad 9 B .0$

9 B. 0

98.0

98.0

.8
1.5
4.6
10.6

10.6

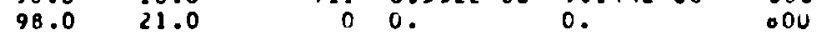

0.

0.000

$1.625 E-04 \quad 000$

$80447.381 E-05$ 1.033E-U4 690

711 6.53<E-06 $9.144 E-66$

ALIHUTH HEIGHT EXPQSURE F/O EU/U DISTANCE DEGREES METERS CI-SEC/CU.M SEC/CU.M I/SO.M METERS $\times 10 \mathrm{O}+7$

$\begin{array}{rrrrrr}114.0 & .8 & 3841 & 3.571 E-05 & 4.940 E-05 & 80 U \\ 114.0 & 1.5 & 3773 & 3.462 E-05 & 4.846 E-U 5 & \text { ECU } \\ 114.0 & 4.6 & 5996 & 5.501 E-05 & 7.701 E-05 & 80 U \\ 114.0 & 10.6 & 6158 & 5.650 E-05 & 7.910 E-U 5 & 000 \\ 114.0 & 21.0 & 59 & 5.443 E-07 & 7.620 E-07 & 800\end{array}$

AZIHUTH HEIGHT EXPOSURE E/O EU/O UISTANCE DECREES METERS CI-SEC/CU.M SEC/CU.M I/SO.M METERS $\times 10 E+7$
N 130.0
.8
00.
0.
800 


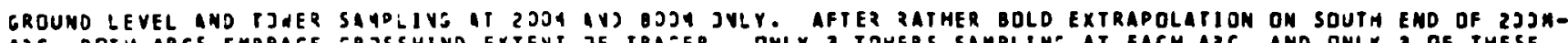
AZC. BOTH ARCS EMBRAEE ERJSSHIND EXTEVT JF TRAEER. DNLY 3 TOMERS SAMPLINS AT EACH AZC. AND ONLY 3 OF THESE INTERCEPT TRACER. VJVE JF THE TRAERS EMBRACE THE VERTICAL EXTENT DF THE PLUAE DURING THIS UNSTABLE RELEASE.

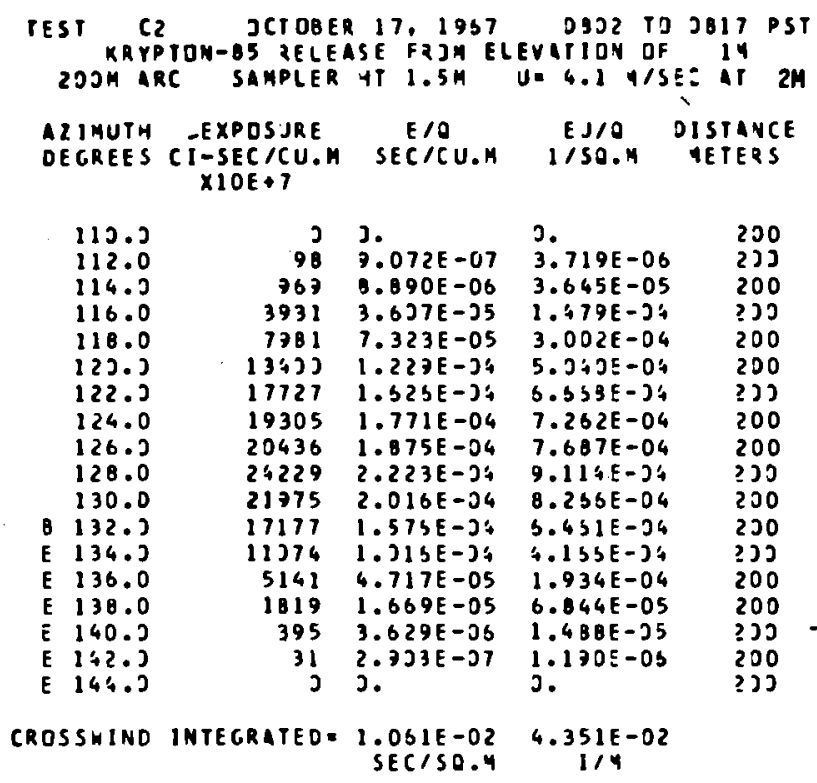

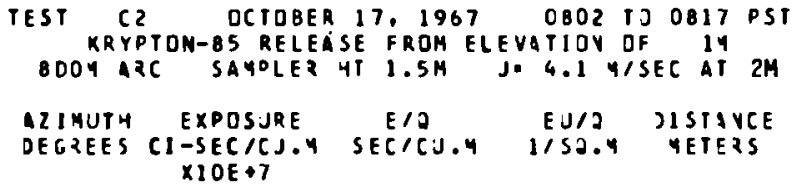

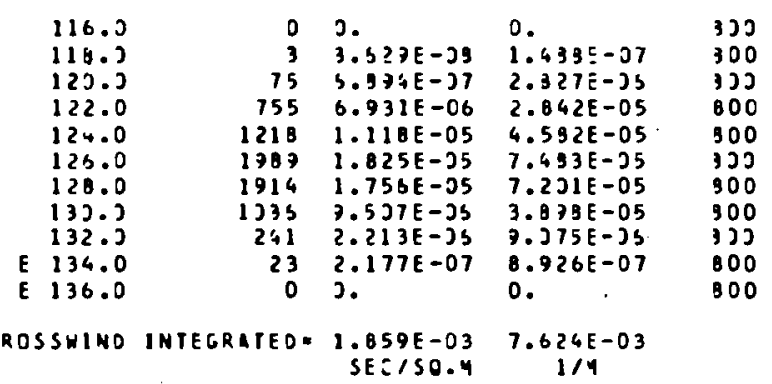




\begin{tabular}{|c|c|c|c|c|c|}
\hline $\begin{array}{c}\text { TEST } \\
\text { } \mathrm{C} \\
200 \mathrm{~K}\end{array}$ & ton-as & $\begin{array}{l}\text { TOBER 17. } 1 \\
\text { RELEASE FRO }\end{array}$ & $\begin{array}{l}1967 \\
0802 \\
\text { OH ELEVATIOH } \\
\text { UE } 4.2 \mathrm{H}\end{array}$ & $\begin{array}{l}\text { TO } 08270 \\
\text { OF } \\
\text { SEC AT } 24\end{array}$ & ST \\
\hline $\begin{array}{l}\text { ALIMUTH } \\
\text { DEGREES }\end{array}$ & $\begin{array}{l}\text { ME IGHT } \\
\text { METERS }\end{array}$ & $\begin{array}{l}\text { EXPJSULE } \\
\text { CI-SEC ICU:W } \\
\times 10 E \bullet 7\end{array}$ & $\begin{array}{c}\text { E/O } \\
\text { SEC/CU.H }\end{array}$ & $\begin{array}{l}E J / O \\
2 / S O .4\end{array}$ & $\begin{array}{l}\text { DISTANCE } \\
\text { AETEAS }\end{array}$ \\
\hline 100.0 & .6 & 0 & 0. & 0. & 200 \\
\hline $\begin{array}{l}\text { AZ I HUT } 4 \\
\text { DEGREES }\end{array}$ & $\begin{array}{c}\text { HE IGHT } \\
\text { METERS } \\
\text {. }\end{array}$ & $\begin{array}{l}\text { EXPSSJRE } \\
\text { CI-SEC/CU.H } \\
\text { XIOE } \$\end{array}$ & $\begin{array}{c}\text { E/O } \\
\text { SEC/CU.H }\end{array}$ & $\begin{array}{l}E 0 / 2 \\
1 / 50.4\end{array}$ & $\begin{array}{l}\text { DISTANEE } \\
\text { METERS }\end{array}$ \\
\hline $\begin{array}{l}114.0 \\
114.0 \\
114.0 \\
114.0 \\
114.0\end{array}$ & $\begin{array}{r}. .9 \\
1.5 \\
3.0 \\
6.1 \\
10.6\end{array}$ & $\begin{array}{r}957 \\
769 \\
996 \\
1194 \\
1127\end{array}$ & $\begin{array}{l}3.731 E-36 \\
.830 E-25 \\
9.144 E-06 \\
1.096 E-05 \\
1.034 E-35\end{array}$ & $\begin{array}{l}3.600 E-05 \\
3.545 E-35 \\
3.749 E-05 \\
4.493 E-05 \\
4.2 \div 0 E-05\end{array}$ & $\begin{array}{l}203 \\
233 \\
200 \\
200 \\
203\end{array}$ \\
\hline $\begin{array}{l}\text { AZIMUTH } \\
\text { OEGREES }\end{array}$ & $\begin{array}{l}\text { ME IGHT } \\
\text { METEZS }\end{array}$ & 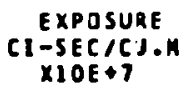 & $\begin{array}{c}E / 2 \\
\text { SEC/CU.H }\end{array}$ & $\begin{array}{c}5 J / 0 \\
1 / 50.4\end{array}$ & $\begin{array}{l}\text { OISTANEE } \\
\text { METERS }\end{array}$ \\
\hline $\begin{array}{l}130.0 \\
130.0 \\
130.0 \\
130.0 \\
130.0\end{array}$ & $\begin{array}{r}.1 .5 \\
1.5 \\
6.1 \\
10.6\end{array}$ & $\begin{array}{l}22081 \\
21775 \\
27575 \\
12454 \\
16512\end{array}$ & $\begin{array}{l}2.026 E-04 \\
2.016 E-24 \\
1.717 E-34 \\
1.143 E-04 \\
1.515 E-04\end{array}$ & $\begin{array}{l}.306 E-04 \\
B .266 E-04 \\
7.350 E-34 \\
4.685 E-04 \\
6.211 E-04\end{array}$ & $\begin{array}{l}200 \\
200 \\
253 \\
200 \\
200\end{array}$ \\
\hline
\end{tabular}

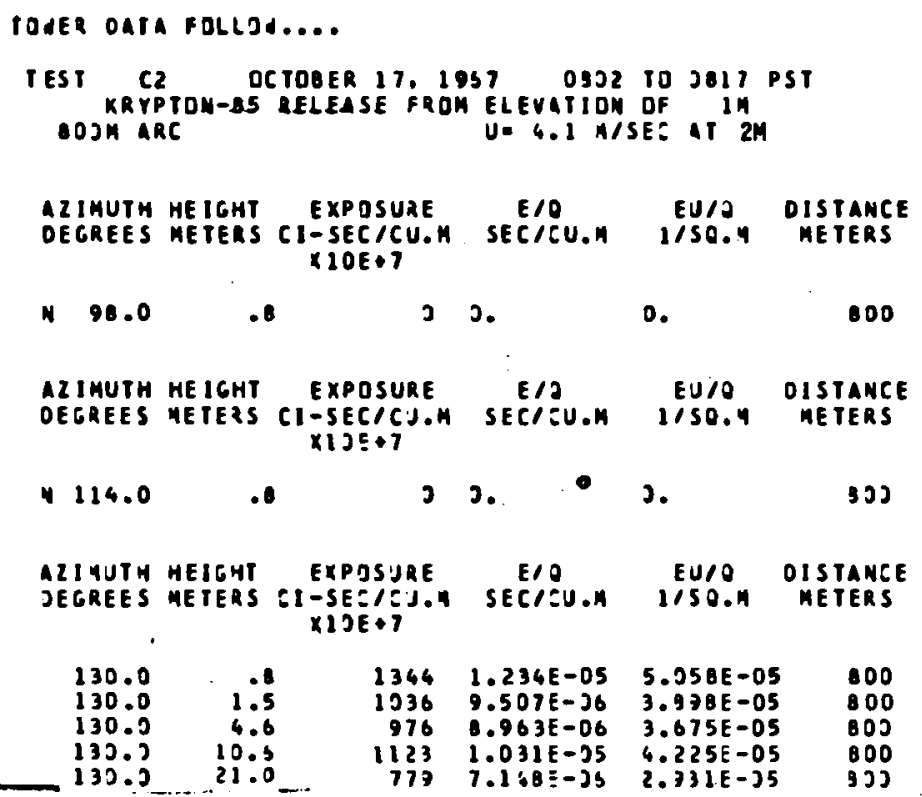


GROUMO LEVEL AMO TOHER SAMPLIMG IT 2 JOY IV DOOY JMLY. BOTH ARCS SEVERELY TRUMCATED AT SOUTH EMD. JWLY 3 T JAERS SAMOLIMG AT EACH ARCI 5 OFTHE G TOHERS INTEREEPT TRACER. MO TONEZ GOAPLETELY EABZACES THE VERTICAL EXTENT OF TRAEER DURIHG THIS RELEASE INTO AN UNSTAGLE ATHOSPHERE.

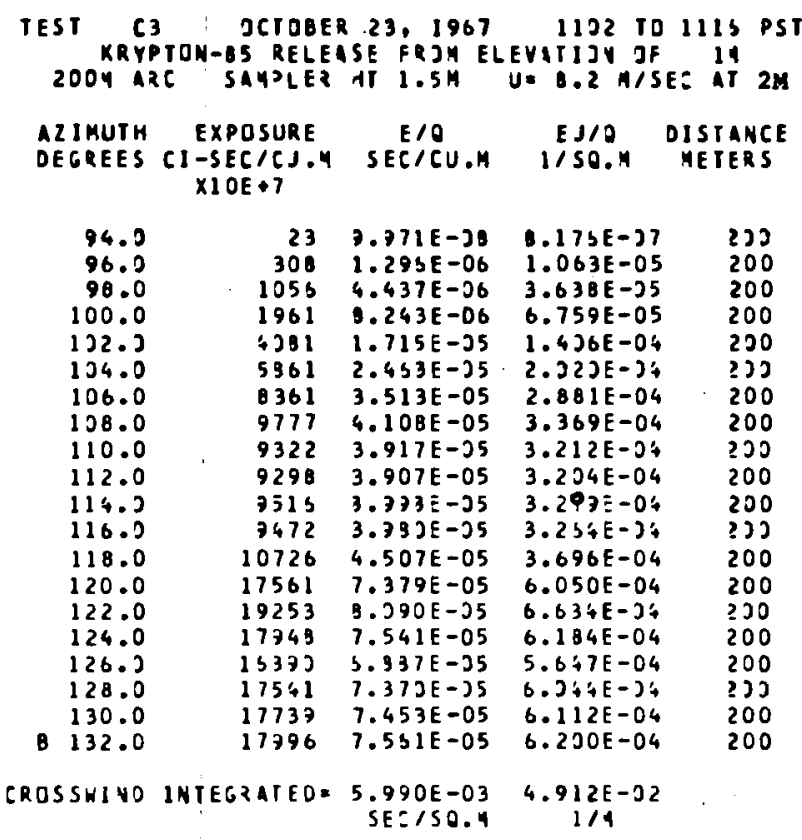

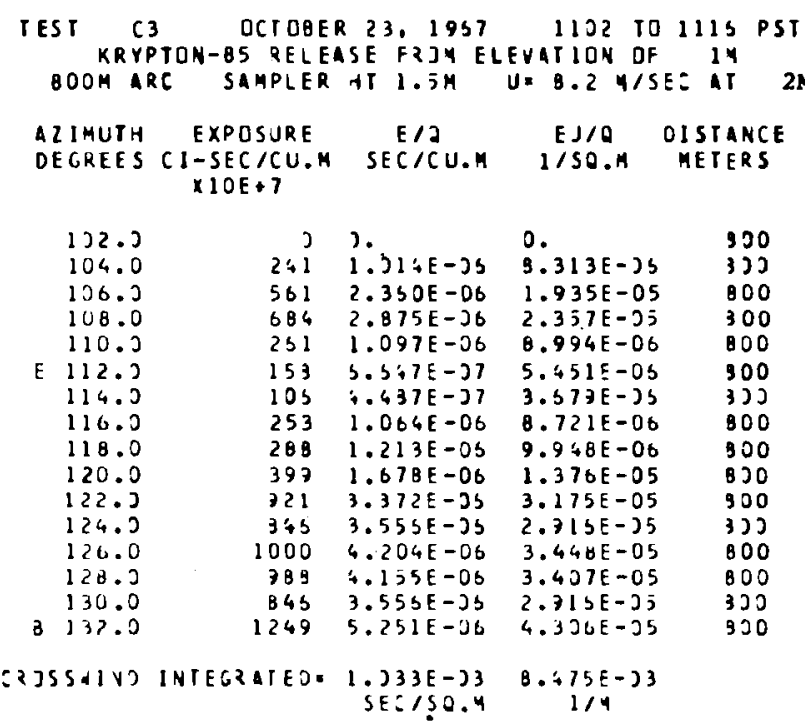




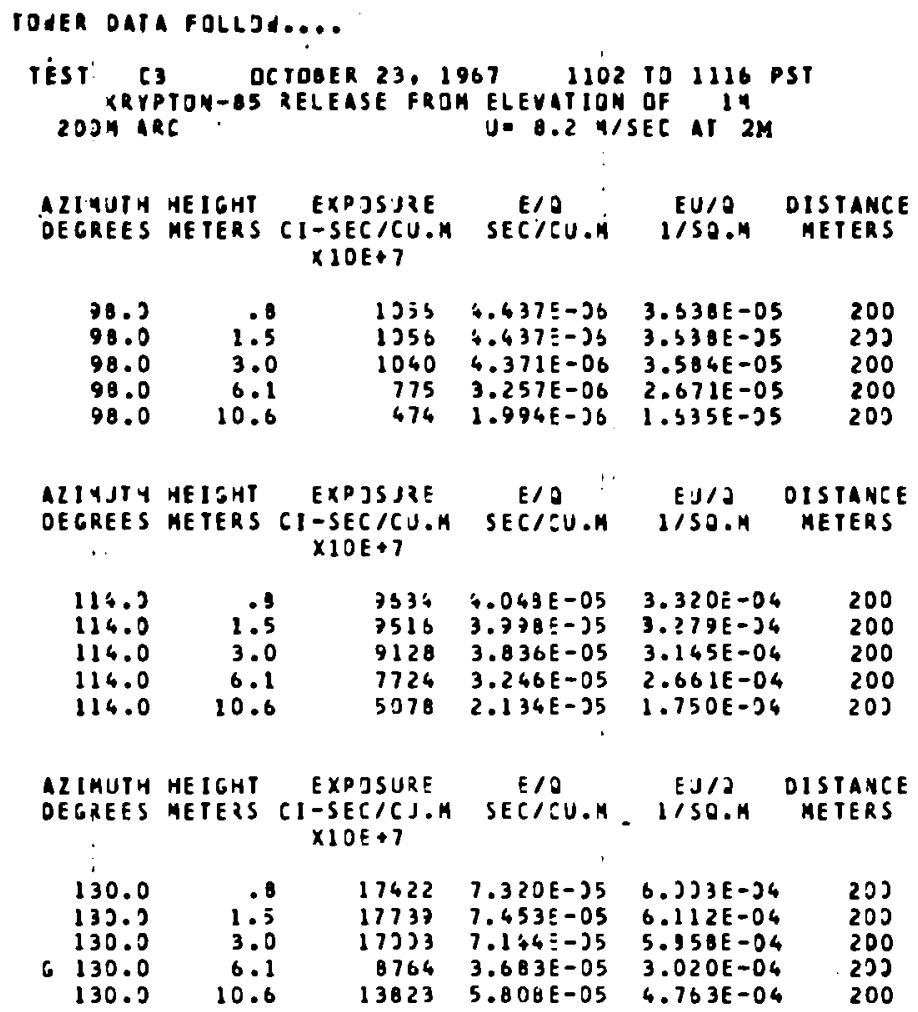




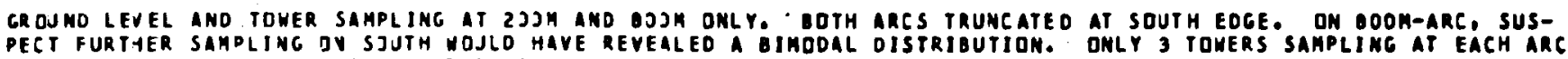
PECT FURTHER SAMPLING DY SJUTH NOJLO HAVE REVEALED A BIMODAL DISTRIBUTIOH, ONL
FDUR TOWERS INTERCEPTED TRACER DURINO THIS RELSASE. INTO AN UHSTABLE ATMOSPHERE.

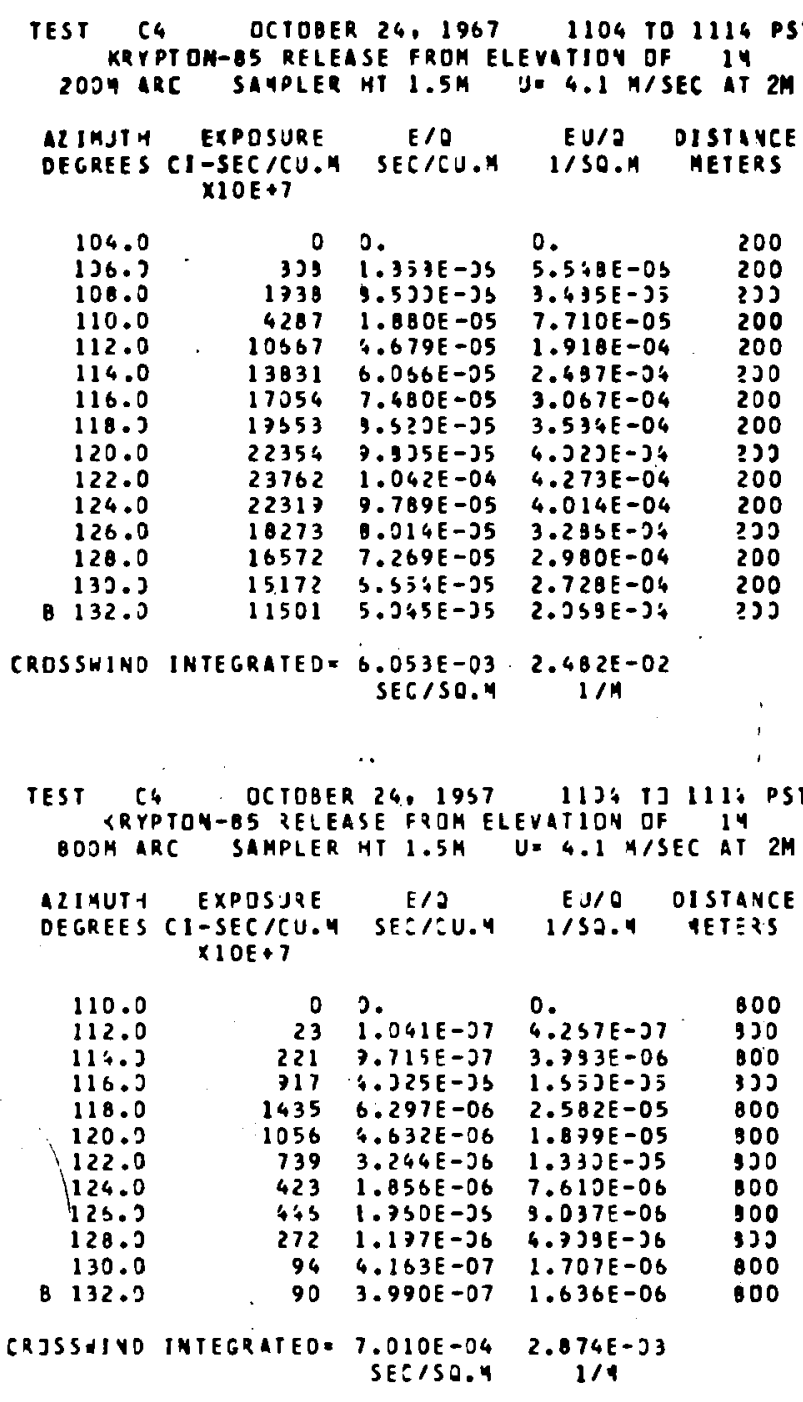




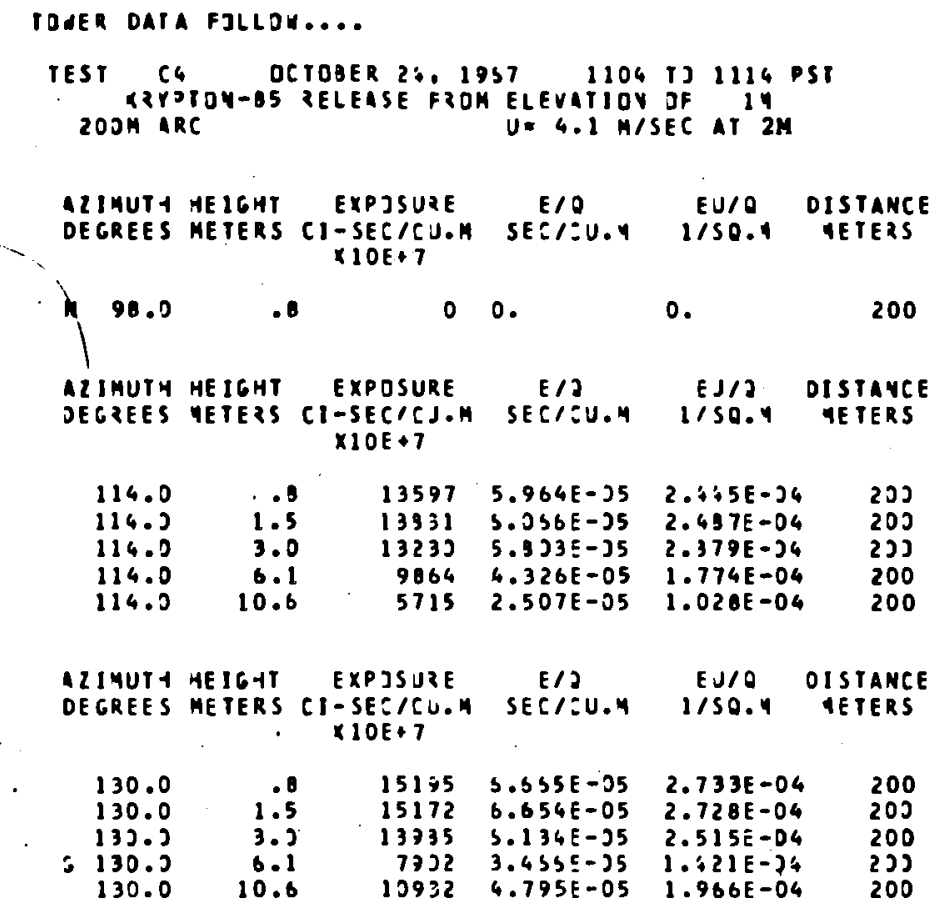


GROUND LEYEL AND TOWER SAMPLING AT 2OOM AND BOOM DNLY. KRYPTON RELEASED ABDUT 2H TO RIGHT. (FACING DOMNMINOI AND IM BELOH ZNS RELEASE PDINT. BDTH ARCS EMBRACE THE CROSSWIND DISTRIBUTION DF TRACER. BOTH TRACER DISTRIBUTIONS EXTENDED ABOVE TOHER TOPS, BUT MAXIMUA CONCENTRATIONS WERE GENERALLY BELDH TDHER TOPS.

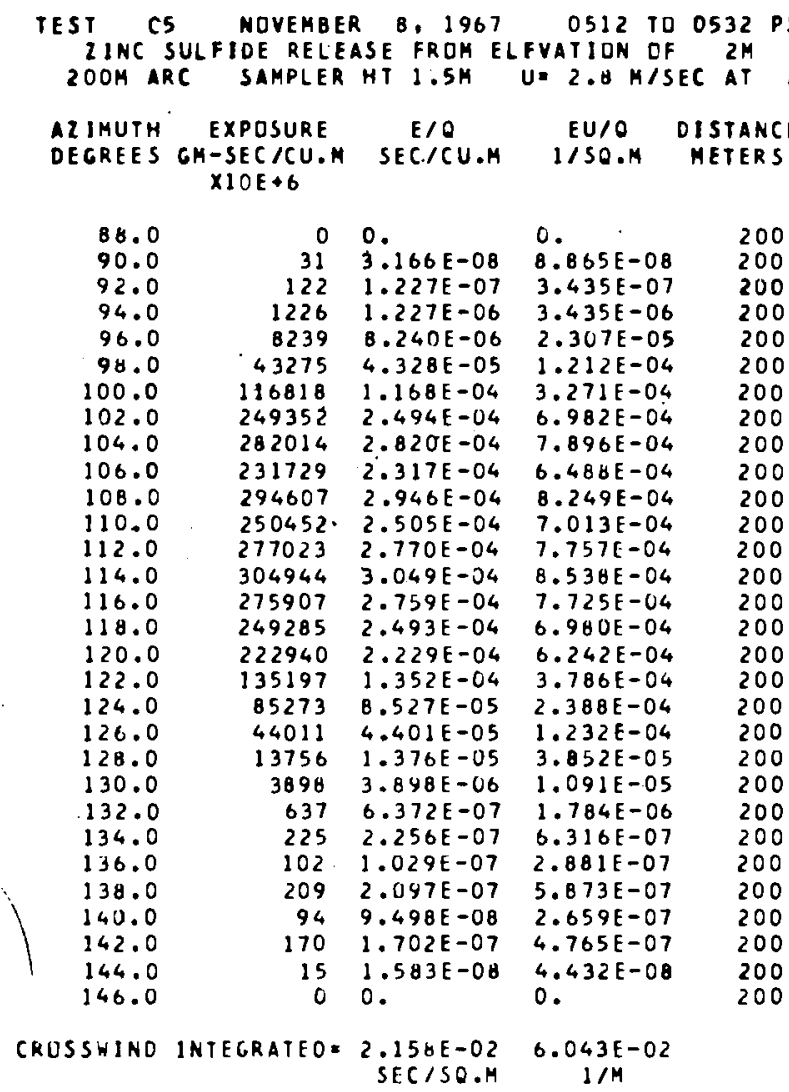
TEST C5 NUVEMBFR 8,1967 USI2 TU USS2 PST KRYPTUN-RS RELEASE FRUM ELFVATIUN DF IM POOM ARC SAMPLER HT I.SM UE LOU MISEC AT 2 H

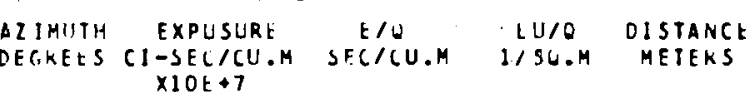

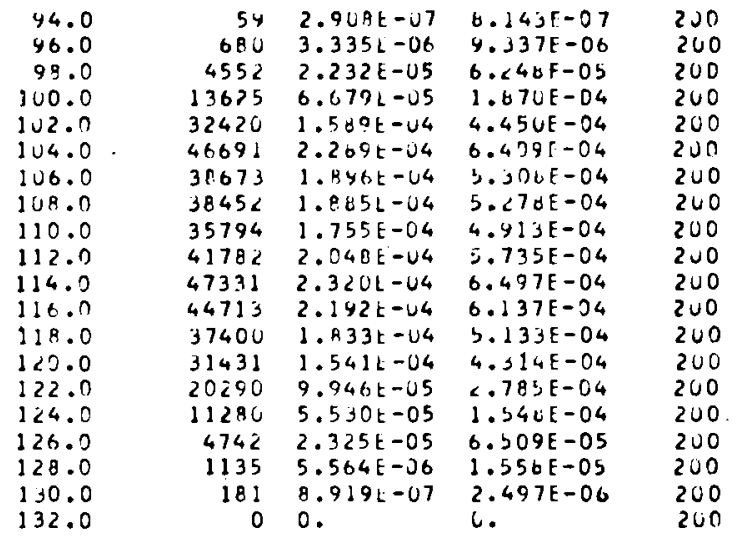

CROSSHIND INTEGRATEU: $1.544 \mathrm{~L}=02 \quad 4.324 \mathrm{~F}-02$ SEC $130.4 \quad 1 / 4$

TEST CS NQVEMBER 8.1967 OS12 TU OS32 PST 2 INC SULFIDE RELEASE FRUM ELEVATION DF $2 M$ GOOM ARC SAMPLER HT $1.5 \mathrm{M} U=2.8 \mathrm{M} / \mathrm{SEC}$ AT $2 \mathrm{M}$

ALIMUTH EXPLSURE E/O EU/O DISTANCE OEGREES CM-SEC/CU.H SEC/CU.M IISO.M METERS $\times \cdot 10 \mathrm{O}+6$

$\begin{array}{rrlll}96.0 & 0 & 0 . & 0 . & 800 \\ 93.0 & 914 & 9.149 \mathrm{E}-07 & 2.562 \mathrm{E}-06 & 800 \\ 100.0 & 7928 & 7.928 \mathrm{E}-06 & 2.220 \mathrm{E}-05 & 800 \\ 102.0 & 9042 & 9.042 \mathrm{E}-06 & 2.532 \mathrm{E}-05 & 800 \\ 104.0 & 11365 & 1.137 \mathrm{E}-05 & 3.182 \mathrm{E}-05 & 800 \\ 106.0 & 11802 & 1.180 \mathrm{E}-05 & 3.305 \mathrm{E}-05 & 800 \\ 108.0 & 14581 & 1.458 \mathrm{E}-05 & 4.083 \mathrm{E}-05 & 800 \\ 110.0 & 9486 & 9.486 \mathrm{E}-06 & 2.656 \mathrm{E}-05 & 800 \\ 112.0 & 12039 & 1.204 \mathrm{E}-05 & 3.371 \mathrm{E}-05 & 800 \\ 114.0 & 22184 & 2.218 \mathrm{E}-05 & 6.212 \mathrm{E}-05 & 800 \\ 116.0 & 14692 & 1.469 \mathrm{E}-05 & 4.114 \mathrm{E}-05 & 800 \\ 118.0 & 7158 & 7.159 \mathrm{E}-06 & 2.004 \mathrm{E}-05 & 800 \\ 120.0 & 593 & 5.934 \mathrm{E}-07 & 1.661 \mathrm{E}-06 & 800 \\ 122.0 & 103 & 1.034 \mathrm{E}-07 & 2.894 \mathrm{E}-07 & 800 \\ 124.0 & 38 & 3.828 \mathrm{E}-08 & 1.072 \mathrm{E}-07 & 800 \\ 126.0 & 0 & 0.0 & 0.07 & 800\end{array}$

CROSSHIND INTEGRATED= $3.405 E-03 \quad 9.534 E-03$

TEST C5 NUVEMBFK 8,1967 U512 TU OS32 PST
KRYPTUN-85 RELEASE FRUM ELEVAIJUN OF IM KRYPTUN-85 RELEASE FRUM ELEVAIJUN OF IM

AZIMUIH EXPUSURE E/U EUIO DISTANCE DECKEES CI-SEC/CU.M STCILU.M IISU.M MEIEKS $\times 10 \mathrm{E}+7$

\begin{tabular}{|c|c|c|c|}
\hline $\begin{array}{l}46.0 \\
93.0\end{array}$ & $\begin{array}{r}U \\
55\end{array}$ & $\begin{array}{l}3.714 E-J 7 \\
2.714 E-3\end{array}$ & 7. $C D \cup E-07$ \\
\hline 100.0 & $11>7$ & $5.526 t=06$ & 1. $347 \mathrm{~F}-0 \mathrm{~b}$ \\
\hline 102.0 & $2 \cup 2 b$ & $9.927 L-\cup 6$ & $2.7795=0 b$ \\
\hline $1 \cup 4.0$ & 1284 & $6.3<1 t-46$ & $1.17 \cup 1-05$ \\
\hline 166.0 & 747 & $3.604 t-06$ & 1. $\cup 26 !-05$ \\
\hline $\begin{array}{l}108.0 \\
110.0\end{array}$ & $\begin{array}{l}1635 \\
2673\end{array}$ & $\begin{array}{l}8.976 t-06 \\
1.311 E-05\end{array}$ & $\begin{array}{l}2.31 Y E-05 \\
3.67 \cup E-05\end{array}$ \\
\hline 112.0 & 3574 & $1.755 t-05$ & $4.913 E-05$ \\
\hline 114.0 & 4038 & $1.960 \mathrm{t}-0 \mathrm{~s}$ & $5.343 E-O 5$ \\
\hline 116.0 & 5517 & $2.705 t-05$ & $7.573 E-05$ \\
\hline 118.0 & 6526 & $3.199 k-05$ & $6.957 \mathrm{~F}-0 \mathrm{~s}$ \\
\hline 120.0 & 2027 & $1.386 t-05$ & $3.882 E-05$ \\
\hline 22.0 & B 14 & $3.944 t-06$ & $1.1 ! \forall E-O S$ \\
\hline 124.0 & 55 & $2.714 t-07$ & $7.0 D \cup F-07$ \\
\hline $1<6.0$ & $u$ & 0. & 0. \\
\hline
\end{tabular}

$\begin{array}{rl}\text { CROSSHIND INTEGKATED = } 4.533 t-03 & 1.264 E-02 \\ \text { SEC } 150.4 & 1 / 4\end{array}$
$B \cup 0$
$B \cup 0$
$B \cup 0$
$8 \cup 0$
$P \cup 0$
$8 \cup 0$
$8 \cup 0$
$B \cup 0$
$8 \cup 0$
$8 \cup 0$
800
$8 \cup 0$
900
$9 \cup 0$
$8 \cup 0$
$8 \cup 0$ 
TULER DATA FOLLD....

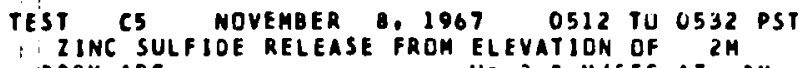
IOOH ARC

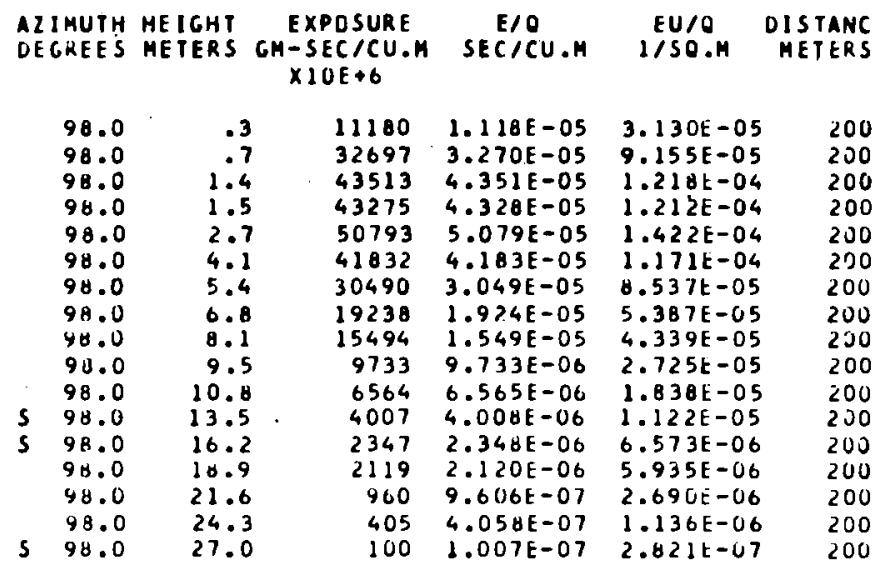

AZIHUIH HEICHT EXPOSURE F/O EU/U DISTANCE DECKEES METERS CH-SEC/CU.M SEC/CU.H I/SO.M METERS $X 10 E+6$

$\begin{array}{lrrrrr}106.0 & .3 & 172967 & 1.730 \mathrm{E}-04 & 4.843 \mathrm{E}-04 & 200 \\ 106.0 & .7 & 261847 & 2.618 \mathrm{E}-04 & 7.332 \mathrm{E}-04 & 200 \\ 106.0 & 1.4 & 222809 & 2.228 \mathrm{E}-04 & 6.239 \mathrm{E}-04 & 200 \\ 106.0 & 1.5 & 231729 & 2.317 \mathrm{E}-04 & 6.488 \mathrm{E}-04 & 200 \\ 106.0 & 2.7 & 260762 & 2.608 \mathrm{E}-04 & 7.301 \mathrm{E}-04 & 200 \\ 106.0 & 4.1 & 229356 & 2.294 \mathrm{E}-04 & 6.422 \mathrm{E}-04 & 200 \\ 106.0 & 5.4 & 244858 & 2.449 \mathrm{E}-04 & 6.856 \mathrm{E}-04 & 200 \\ 106.0 & 6.8 & 199451 & 1.995 \mathrm{E}-04 & 5.585 \mathrm{E}-04 & 200 \\ 5106.0 & 8.1 & 153189 & 1.532 \mathrm{E}-04 & 4.289 \mathrm{E}-04 & 200 \\ 106.0 & 9.5 & 154614 & 1.546 \mathrm{E}-04 & 4.329 \mathrm{E}-04 & 200 \\ 106.0 & 10.8 & 125396 & 1.254 \mathrm{E}-04 & 3.511 \mathrm{E}-04 & 200 \\ 106.0 & 13.5 & 112442 & 1.124 \mathrm{E}-04 & 3.148 \mathrm{E}-04 & 200 \\ 106.0 & 16.2 & 79579 & 7.958 \mathrm{E}-05 & 2.228 \mathrm{E}-04 & 200 \\ 106.0 & 10.9 & 39374 & 3.937 \mathrm{E}-05 & 1.102 \mathrm{E}-04 & 200 \\ 106.0 & 21.6 & 23339 & 2.334 \mathrm{E}-05 & 6.535 \mathrm{E}-05 & 200 \\ 106.0 & 24.3 & 10859 & 1.086 \mathrm{E}-05 & 3.041 \mathrm{E}-05 & 200 \\ 106.0 & 27.0 & 7913 & 7.913 \mathrm{E}-06 & 2.216 \mathrm{E}-05 & 200\end{array}$

AZIMUTH HEICHT EXPOSURE EIO EU/G DISTANCE DEGREES METERS GH-SEC/CU.M SECICU.H I/SE.M METERS $\times 10 E+6$

\begin{tabular}{|c|c|c|c|c|}
\hline $\begin{array}{l}114.0 \\
114.0 \\
114.0 \\
114.0 \\
114.0 \\
114.0 \\
114.0 \\
114.0 \\
114.0 \\
114.0 \\
1114.0 \\
114.0 \\
114.0 \\
114.0 \\
114.0 \\
114.0 \\
114.0\end{array}$ & $\begin{array}{l}.3 \\
.7 \\
1.4 \\
1.5 \\
2.7 \\
4.1 \\
5.4 \\
6.6 \\
8.1 \\
9.5 \\
10.8 \\
13.5 \\
10.2 \\
18.9 \\
21.6 \\
24.3 \\
27.0\end{array}$ & $\begin{array}{l}277221 \\
296363 \\
256575 \\
304944 \\
254959 \\
189984 \\
204113 \\
163265 \\
153006 \\
146280 \\
120610 \\
98083 \\
64340 \\
47059 \\
21217 \\
13606 \\
7710\end{array}$ & $\begin{array}{l}2.772 E-04 \\
2.964 E-04 \\
2.566 E-04 \\
3.049 E-04 \\
2.550 E-04 \\
1.900 E-04 \\
2.041 E-04 \\
1.633 E-04 \\
1.530 E-04 \\
1.463 E-04 \\
1.206 E-04 \\
9.8 B 8 E-05 \\
6.934 E-05 \\
4.706 E-05 \\
2.122 E-05 \\
1.361 E-05 \\
7.710 E-06\end{array}$ & $\begin{array}{l}7.762 E-04 \\
8.29 \forall E-04 \\
7.184 E-04 \\
8.53 \text { E-04 } \\
7.139 t-04 \\
5.320 t-04 \\
5.715 E-04 \\
4.571 t-04 \\
4.284 E-04 \\
4.096 t-04 \\
3.377 t-04 \\
2.764 E-04 \\
1.442 L-04 \\
1.318 t-04 \\
5.941 E-05 \\
3.810 E-05 \\
2.159 E-05\end{array}$ \\
\hline
\end{tabular}

TOLER DATA FOLLUH....

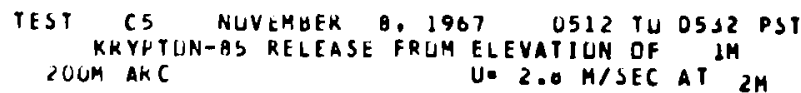

AZ JHUIH HE IGHT

EXPOSURE

E $/ 0$

EUTO DISTANLE DECKEES HETERS CI-SECICU
XIUE +7

$\begin{array}{lrrrrr}48.0 & .8 & 4718 & 2.313 E-05 & 6.476 t-05 & 20 U \\ 98.0 & 1.5 & 4552 & 2.232 E-05 & 6.248 t-05 & 20 U \\ 43.0 & 3.0 & 3121 . & 2.511 E-05 & 7.030 t-U 5 & 20 J \\ 48.0 & 6.1 & 2808 & 1.377 E-05 & 3.854 E-U 5 & 20 U \\ 98.0 & 10.6 & 834 & 4.091 E-06 & 1.145 E-05 & 20 U\end{array}$

AIIMUTH HEIGHT EXPOSURE E/O DEGREES METERS CI-SECICU.M SECICL.M LIYO.M METCRS $\times 10 \mathrm{E}+7$

\begin{tabular}{|c|c|c|c|c|c|}
\hline $\begin{array}{l}114.0 \\
114.0 \\
114.0 \\
114.0 \\
114.0\end{array}$ & $\begin{array}{r}1.8 \\
1.5 \\
3.0 \\
6.1 \\
10.6\end{array}$ & $\begin{array}{l}40289 \\
47331 \\
46153 \\
36045 \\
23319\end{array}$ & $\begin{array}{l}2.367 E-04 \\
2.320 E-04 \\
2.262 E-04 \\
1.865 E-04 \\
1.144 E-04\end{array}$ & $\begin{array}{l}6.668 t-U 4 \\
6.497 t-U 4 \\
6.335 E-U 4 \\
5.222 E-U 4 \\
3.2 U 3 L-U 4\end{array}$ & $\begin{array}{l}200 \\
\angle 00 \\
200 \\
200 \\
200\end{array}$ \\
\hline
\end{tabular}




\begin{tabular}{|c|c|c|c|c|c|c|c|c|c|c|c|}
\hline $\begin{array}{l}\text { ALIMUTH } \\
\text { DECREES }\end{array}$ & $\begin{array}{l}\text { HE IGHT } \\
\text { METERS }\end{array}$ & $\begin{array}{l}\text { EXPOSURE } \\
\text { CH-SEC/CU.H } \\
\times 10 E+6\end{array}$ & $\begin{array}{c}F / O \\
S E C / C U . H\end{array}$ & $\begin{array}{l}\text { EU/O } \\
1 / S 0 . H\end{array}$ & $\begin{array}{c}\text { DIS IANCE } \\
\text { METERS }\end{array}$ & & & & & & \\
\hline $\begin{array}{l}122.0 \\
122.0 \\
122.0 \\
122.0 \\
122.0 \\
122.0 \\
122.0 \\
122.0 \\
122.0 \\
122.0 \\
122.0 \\
122.0 \\
122.0 \\
122.0 \\
122.0 \\
122.0 \\
122.0\end{array}$ & $\begin{array}{l}.3 \\
.7 \\
1.4 \\
1.5 \\
2.7 \\
4.1 \\
5.4 \\
6.8 \\
6.1 \\
9.5 \\
10.8 \\
13.5 \\
16.2 \\
18.9 \\
21.6 \\
24.3 \\
27.0\end{array}$ & $\begin{array}{r}16869 \\
107163 \\
99429 \\
135197 \\
115578 \\
95171 \\
96032 \\
88425 \\
80018 \\
65495 \\
59799 \\
43641 \\
23803 \\
14369 \\
11890 . \\
9372 \\
3644\end{array}$ & $\begin{array}{l}1.607 E-05 \\
1.072 E-04 \\
9.943 E-05 \\
1.352 E-04 \\
1.156 E-04 \\
9.517 E-05 \\
9.603 E-05 \\
8.843 E-05 \\
1.802 E-05 \\
6.550 E-05 \\
5.980 E-05 \\
4.364 E-05 \\
2.380 E-05 \\
1.437 E-05 \\
1.189 E-05 \\
9.373 E-06 \\
3.644 E-06\end{array}$ & $\begin{array}{l}4.723 E-05 \\
3.001 E-04 \\
2.784 E-04 \\
3.786 E-04 \\
3.236 E-04 \\
2.665 E-04 \\
2.664 E-04 \\
2.476 E-04 \\
2.465 E-04 \\
1.834 E-04 \\
1.674 E-04 \\
1.222 E-04 \\
6.665 E-05 \\
4.023 E-05 \\
3.329 E-05 \\
2.624 E-05 \\
1.020 E-05\end{array}$ & $\begin{array}{l}200 \\
200 \\
200 \\
200 \\
200 \\
200 \\
200 \\
200 \\
200 \\
200 \\
200 \\
200 \\
200 \\
200 \\
200 \\
200 \\
200\end{array}$ & - & & . & $\cdot$ & & . \\
\hline $\begin{array}{l}\text { AZIHUTH } \\
\text { DECREES }\end{array}$ & $\begin{array}{l}\text { HE ICHT } \\
\text { METERS }\end{array}$ & $\begin{array}{l}\text { EXPOSURE } \\
\text { CH-SEC/CU.M } \\
\text { X } 10 E+6\end{array}$ & $\begin{array}{c}E / O \\
\text { SEC/CU.M }\end{array}$ & $\begin{array}{l}\text { EU/O } \\
1 / S O . M\end{array}$ & $\begin{array}{l}\text { DISTANCE } \\
\text { METERS }\end{array}$ & $\begin{array}{l}\text { ALIMUTH } \\
\text { DEGREES }\end{array}$ & $\begin{array}{l}\text { HE IGHT } \\
\text { METERS }\end{array}$ & $\begin{array}{c}\text { EXPOSURE } \\
\text { CI-SEC /CU.M } \\
\text { XIUE }+7\end{array}$ & $\begin{array}{c}E / O \\
\text { SEC/CU.M }\end{array}$ & $\begin{array}{l}\text { EU/O } \\
1 / 50.4\end{array}$ & $\begin{array}{l}\text { UISTANCE } \\
\text { METERS }\end{array}$ \\
\hline $\begin{array}{l}130.0 \\
130.0 \\
130.0 \\
130.0 \\
130.0 \\
130.0 \\
130.0 \\
130.0 \\
130.0 \\
130.0 \\
130.0 \\
130.0 \\
130.0 \\
130.0 \\
130.0 \\
130.0 \\
130.0\end{array}$ & $\begin{array}{r}.3 \\
.7 \\
1.4 \\
1.5 \\
2.7 \\
4.1 \\
5.4 \\
6.8 \\
8.1 \\
9.5 \\
10.8 \\
13.5 \\
16.2 \\
18.9 \\
21.6 \\
24.3 \\
27.0\end{array}$ & $\begin{array}{l}1650 \\
2550 \\
2588 \\
1698 \\
2857 \\
4137 \\
4582 \\
4232 \\
4502 \\
3530 \\
4032 \\
3037 \\
2762 \\
2163 \\
1488 \\
997 \\
486\end{array}$ & $\begin{array}{l}1.650 E-06 \\
2.551 E-06 \\
2.588 E-06 \\
3.898 E-06 \\
2.857 E-06 \\
4.137 E-06 \\
4.5 B 2 E-06 \\
4.232 E-06 \\
4.502 E-06 \\
3.531 E-06 \\
4.032 E-06 \\
3.038 E-06 \\
2.762 E-06 \\
2.164 E-06 \\
1.488 E-06 \\
9.974 E-07 \\
4.866 E-07\end{array}$ & $\begin{array}{l}4.621 \mathrm{E}-06 \\
7.143 \mathrm{E}-06 \\
7.247 \mathrm{E}-06 \\
1.091 \mathrm{E}-05 \\
1.001 \mathrm{E}-06 \\
1.15 \mathrm{E}-05 \\
1.283 \mathrm{E}-05 \\
1.165 \mathrm{E}-05 \\
1.261 \mathrm{E}-05 \\
9.866 \mathrm{E}-06 \\
1.129 \mathrm{E}-05 \\
8.505 \mathrm{E}-06 \\
7.735 \mathrm{E}-06 \\
6.059 \mathrm{E}-06 \\
4.167 \mathrm{E}-06 \\
2.793 \mathrm{E}-06 \\
1.363 \mathrm{E}-06\end{array}$ & $\begin{array}{l}200 \\
200 \\
200 \\
200 \\
200 \\
200 \\
200 \\
200 \\
200 \\
200 \\
200 \\
200 \\
200 \\
200 \\
200 \\
200 \\
<00\end{array}$ & $\begin{array}{l}130.0 \\
130.0 \\
130.0 \\
130.0 \\
131.0\end{array}$ & $\begin{array}{r}.8 \\
1.5 \\
3.0 \\
6.1 \\
10.2\end{array}$ & $\begin{array}{l}106 \\
101 \\
253 \\
502 \\
605\end{array}$ & $\begin{array}{l}5.235 E-07 \\
0.41 \times E-07 \\
1.241 E-06 \\
2.462 E-06 \\
2.466 E-06\end{array}$ & $\begin{array}{l}1.466 E-06 \\
2.447 t-06 \\
3.474 t-06 \\
6.894 t-06 \\
8.316 t-06\end{array}$ & $\begin{array}{l}200 \\
200 \\
200 \\
200 \\
200\end{array}$ \\
\hline
\end{tabular}

TDHER DATA FQLLOH....

TIIWER DATA FRLLUW...

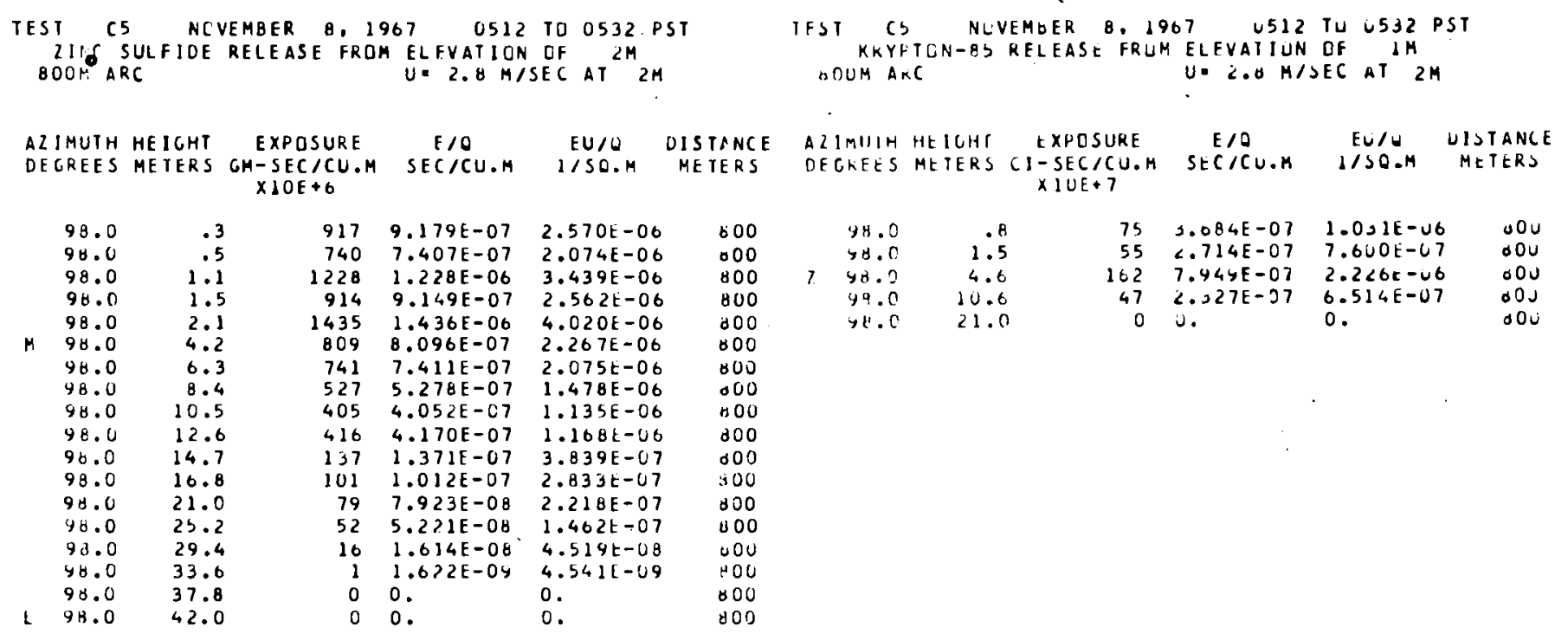




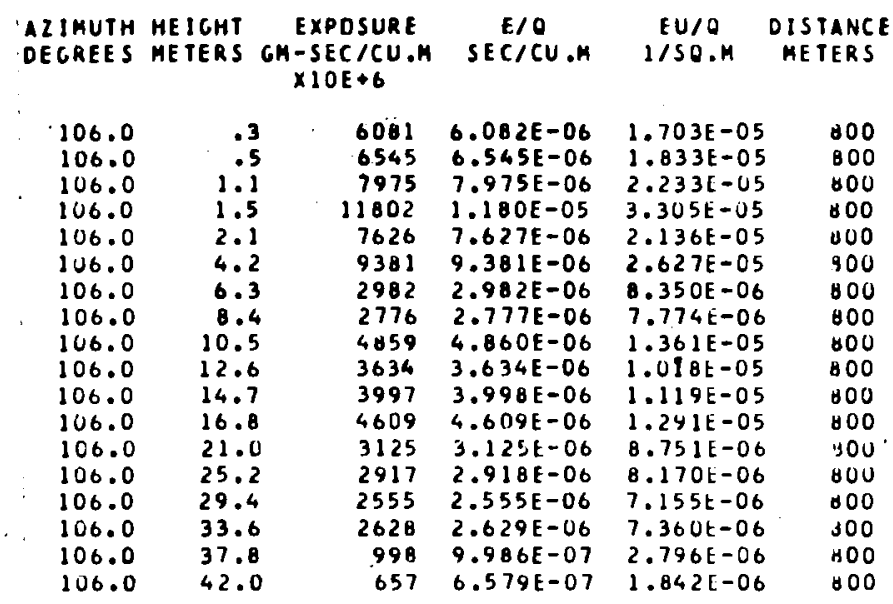

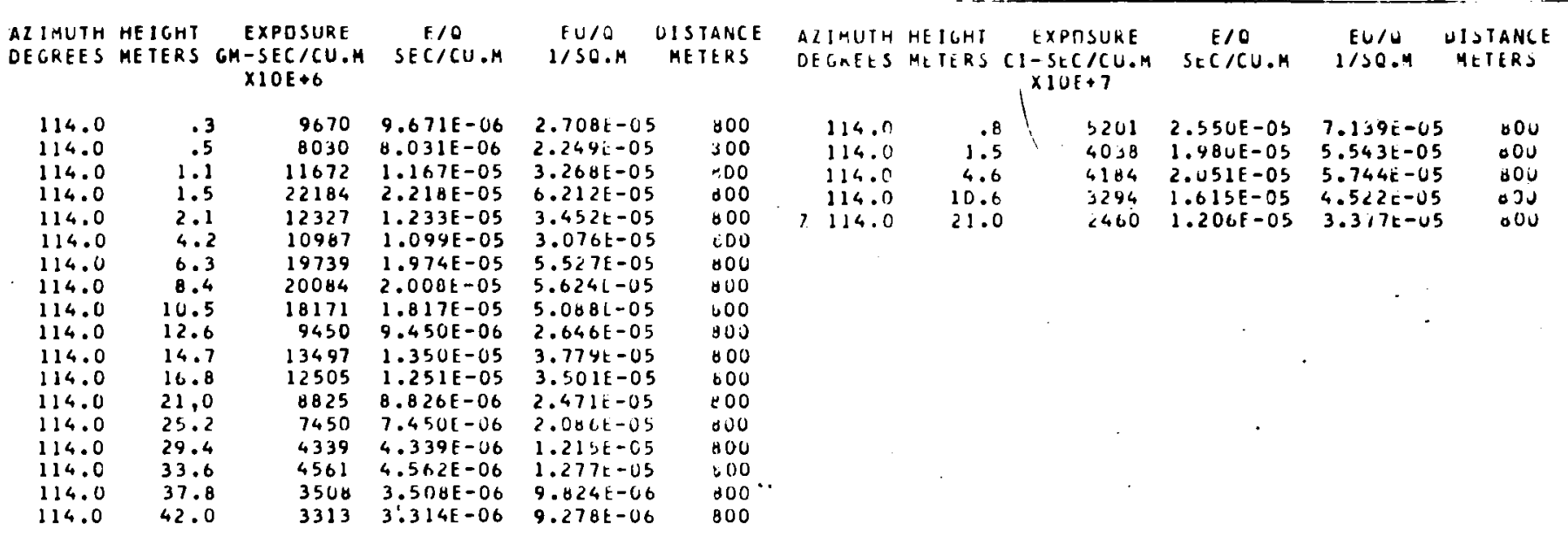

ALIHUTH HEIGHT EXPOSURE E/O EU/O OISIANCE DECREES METEKS GH-SEC/CU.M SEC/CU.M I/SC.H METERS $\times 10 E+6$

$\begin{array}{lrrrrl}122.0 & .3 & 39 & 3.915 E-08 & 1.096 E-07 & 800 \\ 122.0 & .5 & 83 & 0.322 E-08 & 2.330 E-07 & 500 \\ 122.0 & 1.1 & 386 & 3.868 E-07 & 1.083 E-06 & 800 \\ 122.0 & 1.5 & 103 & 1.034 E-07 & 2.894 E-07 & 800 \\ 122.0 & 2.1 & 599 & 6.000 E-07 & 1.660 E-06 & 800 \\ 122.0 & 4.2 & 944 & 9.440 E-07 & 2.643 E-06 & 800 \\ 122.0 & 6.3 & 886 & 8.863 E-07 & 2.482 E-06 & 000 \\ 122.0 & 1.4 & 1058 & 1.058 E-06 & 2.963 E-06 & 800 \\ 122.0 & 10.5 & 645 & 6.457 E-07 & 1.808 E-06 & 800 \\ 122.0 & 12.6 & 1733 & 1.733 E-06 & 4.854 E-06 & 900 \\ 122.0 & 14.7 & 1113 & 1.114 E-06 & 3.118 E-06 & 000 \\ 122.0 & 16.8 & 1656 & 1.657 E-06 & 4.64 C E-06 & 800 \\ 122.0 & 21.0 & 1184 & 1.184 E-06 & 3.316 E-06 & 800 \\ 122.0 & 25.2 & 2273 & 2.274 E-06 & 6.367 E-06 & 800 \\ 122.0 & 29.4 & 1316 & 1.316 E-06 & 3.685 E-06 & 000 \\ 122.0 & 33.6 & 758 & 7.589 E-07 & 2.125 E-06 & 8110 \\ 122.0 & 37.8 & 479 & 4.797 E-07 & 1.343 E-06 & 800 \\ 122.0 & 42.0 & 463 & 4.634 E-07 & 1.297 E-06 & 830\end{array}$
ALIMUTH HEIGHT EXPOSURE
OECREES METERS CM-SEC/CU.M SECIO $\times 10 E+6$

EIO
EUIU UISTANCE $-S E C / C O A P$
0.

400
$\begin{array}{llll}130.0 & .3 & 0 & 0\end{array}$
00.

0.

.00 
SAMPLING $200 M$ TD JPUUMI NU TUWER SAMPLING. CROSSWIND DISTRIBUTJOIS RATHER BULOLY TXIKAPLILATEU UN NURTH FUR ALL AKCS HEYIIND 4OOM. KELATIVELY FLAT OR MULTIMODAL CROSSWINO UISIRIBUTIUNS. INTERRUPTHD FUR AUTUT 2 MIN SIARTING AT DSO2: FLUURESCEIN ALSU INIERRUPTEO FDR ABUUT 45 SEC.

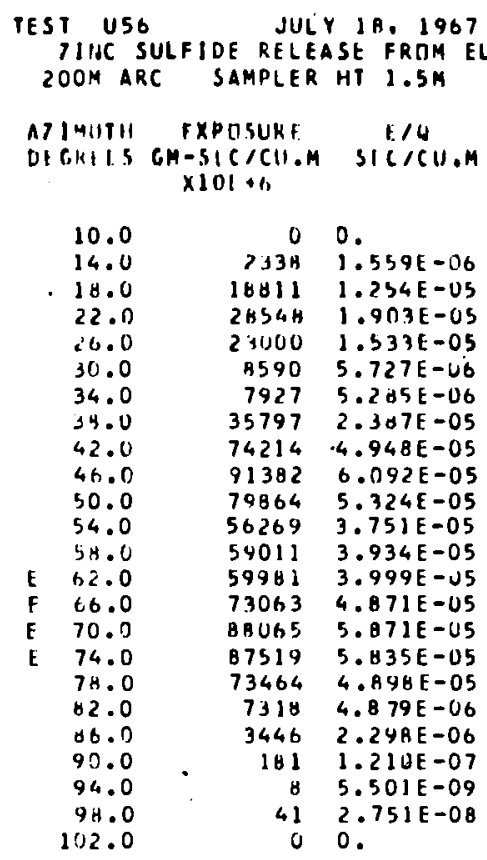
CRLISSAIND INTEGRLTEU= B.1YIE-03
0445 TO 0515 PST GVATIUN DF $2 M$ VE $1.8 \mathrm{M} / \mathrm{SEC}$ AT $2 \mathrm{H}$

EUIU DISIANCE

I/SU.M MEIERS

0.

$2.257 E-U S$

$3.426 E-05$

$2.760 E-0 S$

$1.031 \mathrm{E}-05$

$9.513 E-06$

$9.906 \mathrm{E}-05$

$1.097 E-04$

$9.584 E-05$

$6.752 E-05$

$7.081 E-05$

$7.194 E-05$

$8.768 E-05$

$1.057 E-04$

$1.050 E-04$

8.816E-05

$0.782 E-06$

$4.136 E-06$

$2.178 \mathrm{E}-07$

$4.951 \mathrm{E}-0 \mathrm{~B}$

0 .

$1.473 E-02$

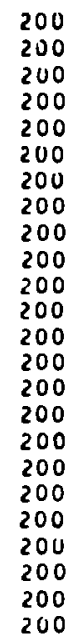

200
200
200
200
200
200
200
200
200
200
200
200
200
200
200
200
200
200
200
200
200
200
200
200
$2.006 E-06$

TEST. U5 UDRESCEIN RELEASE FRUM ELEVATION DF $26 \mathrm{M}$ 20OM ARC SAKPLER HT $1.5 \mathrm{M}$ U. $4.3 \mathrm{M} /$ SEC AT $26 \mathrm{M}$

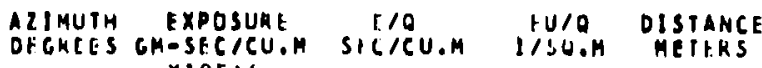

SEC/SO.M I/M

TEST US6

JULY 18,1967

0445 TO 0515 PST

2INC SULFIDE RELEASE FROM ELFVATIUN OF
$300 M$ ARC SAMPLER HT $1.5 M$
UE I.O H/SEC AT

$\begin{array}{lcc}\text { AZIMUTH EXPOSURL LI/O } & \text { EU/O DISTANCE } \\ \text { DEGKEES GM-SECICU.M SECICU.M I/SO.M METERS }\end{array}$ $X 10 E * 6$

\begin{tabular}{|c|c|c|}
\hline 8.0 & i & 0. \\
\hline 12.6 & 145 & $9.719 E-0 A$ \\
\hline 16.0 & 8264 & $5.5041-06$ \\
\hline 20.0 & 24014 & $1.601 E-05$ \\
\hline $24 \cdot 0$ & 34.97 & $? .333 \mathrm{E}-\mathrm{US}$ \\
\hline$\angle 3.0$ & 20474 & $1.365 E-05$ \\
\hline 32.0 & 7485 & $4.991 \mathrm{E}-\mathrm{Cb}$ \\
\hline 36.0 & 7488 & $4.997 E-06$ \\
\hline 40.0 & 349 AU & $2.332 \mathrm{~L}-\mathrm{Cb}$ \\
\hline 44.0 & 68423 & 4.5 YBE -05 \\
\hline 48.0 & 75273 & $5.018 E-35$ \\
\hline 52.0 & 50534 & $3.369 E-05$ \\
\hline $5 i, .0$ & 38060 & $2.537 \mathrm{E}-05$ \\
\hline bo. 0 & 32615 & $2.174 E-05$ \\
\hline 64.0 & 54368 & $3.625 E-05$ \\
\hline 68.0 & 40876 & $2.725 E-05$ \\
\hline 72.0 & 30,470 & $2.431 t-05$ \\
\hline 76.0 & 35030 & $2.335 E-U 5$ \\
\hline BO. 0 & 33505 & $7.234 \mathrm{E}-05$ \\
\hline 04.0 & 5838 & $3.892 E-06$ \\
\hline 49.0 & 94 & $6.32 \mathrm{bE}-0 \mathrm{~B}$ \\
\hline 92.0 & 1 & $9.169 \mathrm{E}-10$ \\
\hline 30.0 & 8 & $5.501 E-09$ \\
\hline 30.6 & v & \\
\hline
\end{tabular}

$0 . x$

$1.749 \mathrm{E}-07$

$9.917 E-06$

$2.802 E-05$

$4.200 \mathrm{E}-05$

$2.457 E-05$

$B .9 B 3 E-06$

$8.986 E-06$

$4.196 \mathrm{E}-05$

$8.259 E-05$

$9.033 E-05$

$6.064 E-U 5$

$4.567 E-05$

$3.914 \mathrm{E}-05$

$6.524 \mathrm{E}-05$

$4.905 E-05$

$4.376 E-05$

$4.204 E-05$

$7.006 \mathrm{E}-06$

$1.134 E-07$

$1.65 \cup E-09$

$9.902 \mathrm{E}-09$

u.

CRISSRINU INTEGRATED $=0.50 \mathrm{HE}-03 \quad 1.531 \mathrm{E}-02$

\section{$\times 10 E+6$}

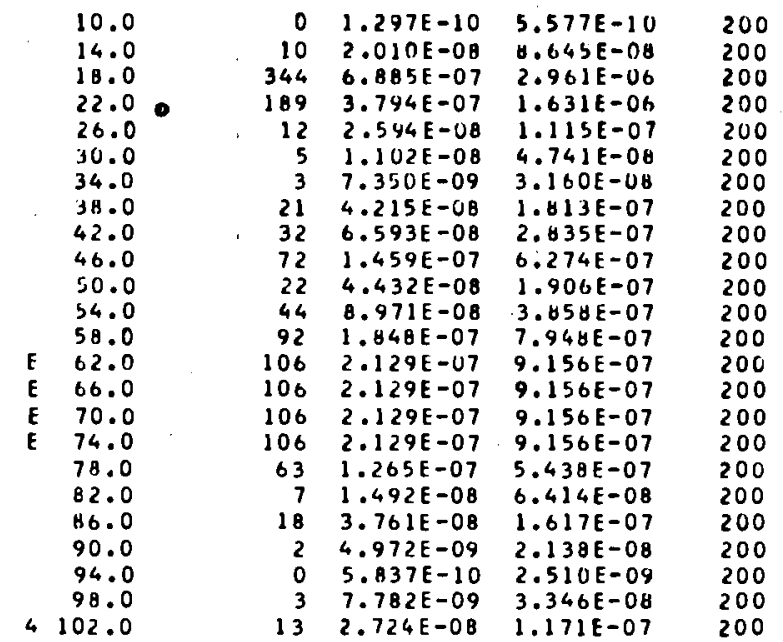

CROSSHIND INTECRATED = $\begin{gathered}3.877 E-05 \\ \text { SECISO.M }\end{gathered}$

TEST US6 JULY 18, 19670445 TU OS15 PST FLUDRESCEIN RELEASE FROM ELEVATION OF 2GM

$300 \mathrm{M}$ ARC SAMPLER HT $1.5 \mathrm{M} U$ U $4.3 \mathrm{M} / \mathrm{SEC}$ AT $26 \mathrm{M}$

$\begin{array}{lcc}\text { AZIMUTH EXPOSURE } & \text { E/O } & \text { EU/O DISTANCE } \\ \text { DEGKEES GM-SEC/CU.M SECICU.M IISO.M METERS }\end{array}$ $\times 10 E * 6$
8.0
12.0
16.0
20.0
24.0
28.0
32.0
40.0
44.0
48.0
52.0
56.0
60.0
64.0
68.0
72.0
72.0
80.0
84.0
88.0
92.0
96.0

100.0

74
240
333
196
67
24
19
26
111
205
29
236
170
268
409
176
11
176

$\begin{array}{rl}0 & 6.485 E-11 \\ 0 & 1.247 E-10 \\ 74 & 1.461 E-07 \\ 248 & 4.961 E-07 \\ 333 & 6.669 E-07 \\ 196 & 3.924 E-07 \\ 67 & 1.351 E-07 \\ 24 & 4.864 E-08 \\ 19 & 3.891 E-08 \\ 26 & 5.260 E-08 \\ 55 & 1.102 E-07 \\ 111 & 2.237 E-07 \\ 205 & 4.118 E-07 \\ 290 & 5.804 E-07 \\ 236 & 4.723 E-07 \\ 170 & 3.405 E-07 \\ 268 & 5.372 E-07 \\ 409 & 8.182 E-07 \\ 176 & 3.534 E-07 \\ 11 & 2.335 E-08 \\ 176 & 3.534 E-07 \\ 0 & 7.782 E-10 \\ 0 & 0.9 \\ 2 & 5.188 E-09\end{array}$

CROSSHINO INTEGRATEDE $1.301 E-04 \quad 5.592 E-04$
$2.789 E-10$

$5.577 E-10$

$6.367 E-07$

$2.133 E-06$

$2.868 E-06$

$1.687 E-06$

$5.810 \mathrm{E}-07$

$2.091 E-07$

$1.673 E-07$

$2.262 \mathrm{E}-07$

$4.741 E-07$

$9.621 E-07$

$1.771 E-00$

$2.496 \mathrm{E}-06$

$2.031 E-06$

$1.464 E-06$

$2.310 E-06$

$3.518 E-06$

$1.520 E-06$

$1.004 \mathrm{E}-07$

$1.520 E-06$

$3.346 E-09$

0.

300
300
300
300
300
300
300
300
300
300
300
300
300
300
300
300
300
300
300
300
300
300
300
300

SEC/SO.M I/M 


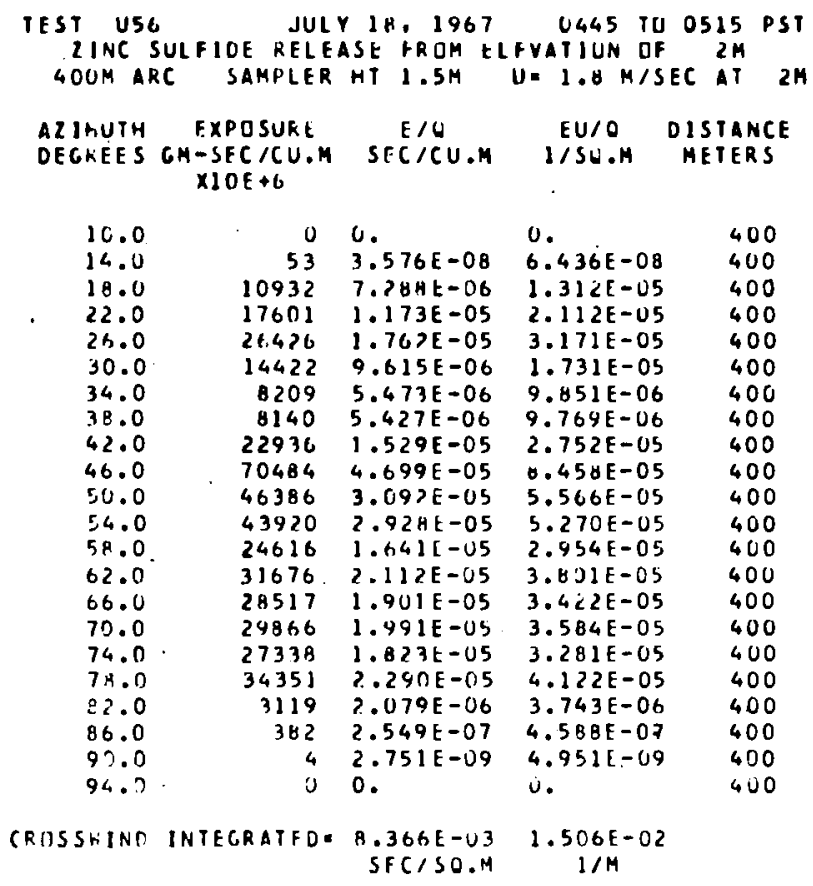

TEST USG JULY 1 H. 1907 U445 IU OS15 PST

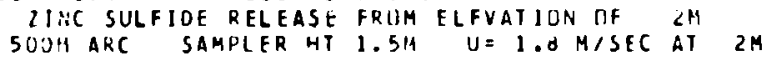

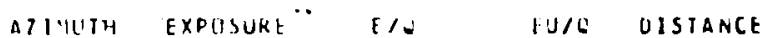
OEGLES GM-SFCICU.H SEL/CU.M IISU.M MEIERS $\times 1 C E+C$

\begin{tabular}{|c|c|c|c|}
\hline E & 12.0 & 0 & 0 . \\
\hline r & 16.0 & 478 & $3.141 E-0$ \\
\hline f & $2 n .0$ & 10312 & $6.875 \mathrm{~L}-06$ \\
\hline E & 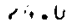 & 1856.3 & 1.73HE-OS \\
\hline & $2 \therefore .0$ & 16,500 & 1. IUnt-us \\
\hline & 32.0 & $B 5>4$ & $5.6,63 E-06$ \\
\hline [ & $3 e .2$ & 7974 & $5.315[-06$ \\
\hline is & $\begin{array}{l}40.0 \\
44.0\end{array}$ & $\begin{array}{l}16544 \\
54751\end{array}$ & $\begin{array}{l}1.1 U B E-05 \\
3.430 E-05\end{array}$ \\
\hline & $1 .+1$ & $234>0$ & $1.5 t A L-U S$ \\
\hline & 52.0 & 51671 & $3.445 t-05$ \\
\hline & 56.0 & 53122 & $3.54>E-U S$ \\
\hline & 61.9 & 33417 & $2.224[-.35$ \\
\hline & 64.0 & 41627 & $2.77 S E-U S$ \\
\hline & $\begin{array}{l}08.0 \\
72.0\end{array}$ & $\begin{array}{l}31069 \\
24371\end{array}$ & $\begin{array}{l}2.071 E-0 S \\
1.9 S S E-U S\end{array}$ \\
\hline & $7 t .0 \mathrm{C}$ & 30034 & $7.007 t-05$ \\
\hline & y & 10276 & $6.351 t-26$ \\
\hline & 154.0 & 524 & $3.53 \cap E-J 7$ \\
\hline & ה 8.0 & 16 & $1.1 U Q E-J 4$ \\
\hline & $9<.0$ & 28 & $1.925 E-08$ \\
\hline & 96.11 & 6 & 0 . \\
\hline
\end{tabular}

6.

$5.7435-07$ $1.397 \mathrm{E}-\mathrm{US}$ $2.2 \angle O E-U S$ 1. $\rightarrow \mathrm{HOL}-\mathrm{US}$ 1. $U \angle A H-O S$ $4.50,1-C t$ 1.491E-US $1.491 E-U b$
$7.074 t-0 S$ C. U S tE-US c. $201 \mathrm{E}-0 \mathrm{~S}$ $6.375 E-05$ $4.010 \mathrm{E}-05$ $4.995 \mathrm{E}-05$ $3.728 E-05$ $3.519 E-015$ $3.61<F-U S$ $1.233 \mathrm{E}-U \mathrm{~S}$ 6. $334 t-U 1$ $1.4 H O E-J G$ $3.400 E-06$ 0 . 500
500
500
500
500
500
500
500
500
500
500
500
500
500
500
500
500
500
500
500
500
500

(KHSSWIIVL INTEGRATEU = $1.031 \mathrm{E}-02 \quad 1.655 \mathrm{E}-0 \mathrm{C}$

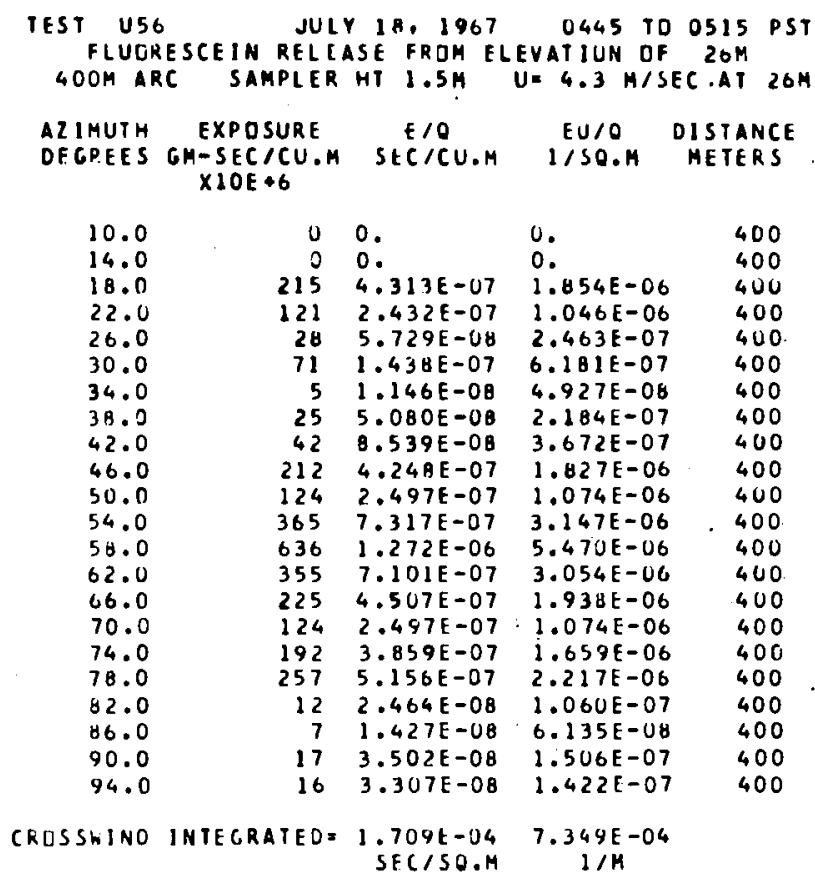

CRUSSHIND INTEGRATHD $=5.765 E-04 \quad 2.479 E-03$ 


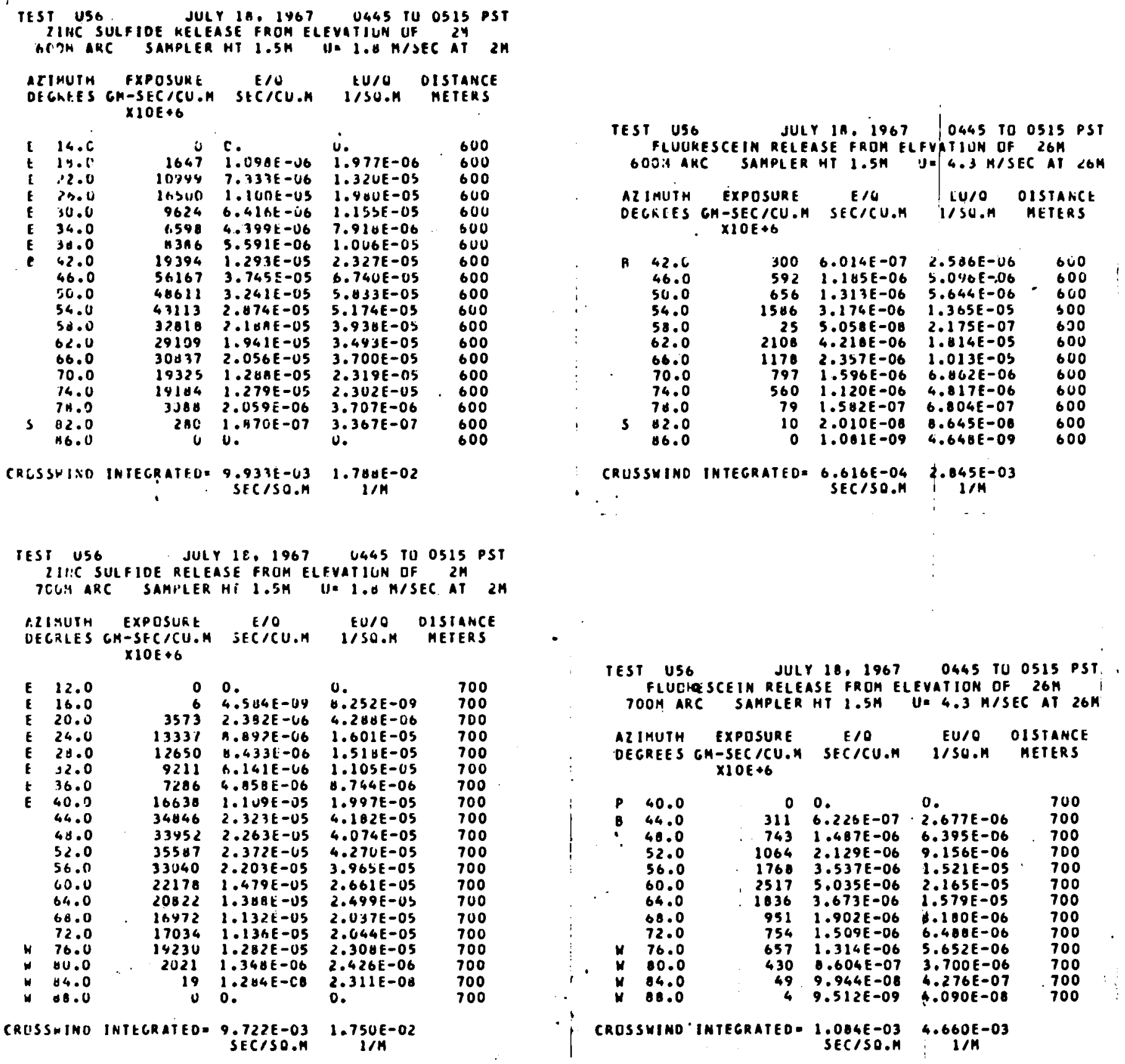




\begin{tabular}{|c|c|c|c|}
\hline \multicolumn{4}{|c|}{$\begin{array}{l}\text { TEST U56 JULY } 18,1967 \\
\text { ZINC SULFIDE RELEASE FROH }\end{array}$} \\
\hline \multicolumn{2}{|c|}{$\begin{array}{l}\text { AIIMUTH } \\
\text { DEGREES }\end{array}$} & $\begin{array}{l}\text { EXPUSURE } \\
\text { CH-SEC/CU.M } \\
\times \perp O E+O\end{array}$ & $\begin{array}{cl}E / J \\
S H C / C U . M\end{array}$ \\
\hline & & 0 & 0. \\
\hline & & 10 & \\
\hline & & & \\
\hline & & & \\
\hline$E$ & & & \\
\hline & & & \\
\hline & & & \\
\hline & & & \\
\hline 3 & & & \\
\hline & & & \\
\hline & & & \\
\hline & & & 2. \\
\hline & & & \\
\hline & & & \\
\hline & & & \\
\hline & $t 1$ & 160 & \\
\hline & & & \\
\hline & & & \\
\hline & 70 & & \\
\hline & & 12 & $\mathrm{H.}$ \\
\hline & & & \\
\hline & & & \\
\hline & 32.0 & 41 & $5.410 E-6$ \\
\hline
\end{tabular}

CROSSWINO. INTEGRATED=9.17hL-U3 SEC/SO:M
O4G5 TO 0515 PST

EVATIUN DF 2 H EU/O OISIANCE 1/SO.H METERS
ט.

$1.317 E-06$ $6.598 E-06$ $1.630 E-05$ $1.485 E-05$ $9.899 E-06$ $8.579 E-06$ $8.579 E-06$ 1.11SE-05 $2.09 S E-05$ $4.547 E-05$ $4.760 E-05$

$3.790 E-05$ $3.354 E-05$ $2.247 E-05$ $1.994 E-05$ $1.025 E-05$

$1.820[-05$ C.011E-05 $1.46 \forall[-05$ $1.464 E-05$ $3.007 E-06$ 0.

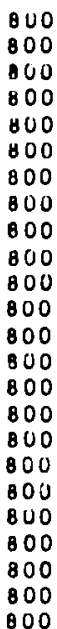

$1.652 \mathrm{E}-02$
TEST U56 JULY 18,1967 GECCEN RFLEASE FROM ELFVATION DF $26 \mathrm{M}$ BOUM ARC SAMPLER HT $1.5 \mathrm{M} U=.4 .3 \mathrm{H} / \mathrm{SEC}$ AT $26 \mathrm{M}$ AZIMUTH EXPOSURF $1 / O$ EU/O DISTANCE DEGREES GH-SECICU.M SEC/CU.M I/SH.M METERS $X 1 O E+6$

D

$\begin{array}{rrrrr}60.0 & 224 & 4.494 E-07 & 1.933 E-06 & 800 \\ 43.0 & 365 & 7.305 E-07 & 3.141 E-06 & 800 \\ 46.0 & 646 & 1.293 E-06 & 5.55 B E-06 & 800 \\ 49.0 & 927 & 1.055 E-06 & 7.975 E-06 & 800 \\ 52.0 & 1132 & 2.265 E-06 & 9.741 E-06 & 800 \\ 55.0 & 2108 & 4.217 E-06 & 1.013 E-05 & 800 \\ 58.0 & 3061 & 6.124 E-06 & 2.633 E-05 & 800 \\ 61.0 & 44 & 8.807 E-08 & 3.787 E-07 & 800 \\ 64.0 & 22 & 4.449 E-08 & 1.913 E-07 & 800 \\ 67.0 & 1155 & 2.311 E-06 & 9.936 E-06 & 800 \\ 70.0 & 882 & 1.766 E-06 & 7.593 E-06 & 800 \\ 73.0 & 701 & 1.403 E-06 & 6.032 E-06 & 800 \\ 76.0 & 700 & 1.401 E-06 & 6.023 E-06 & 800 \\ 79.0 & 365 & 7.305 E-07 & 3.141 E-06 & 800 \\ 82.0 & 153 & 3.068 E-07 & 1.319 E-06 & 800 \\ 85.0 & 17 & 3.567 E-08 & 1.534 E-07 & 800\end{array}$

CKDSSHINU INTEGRATED= $1.048 E-03 \quad 4.506 E-03$

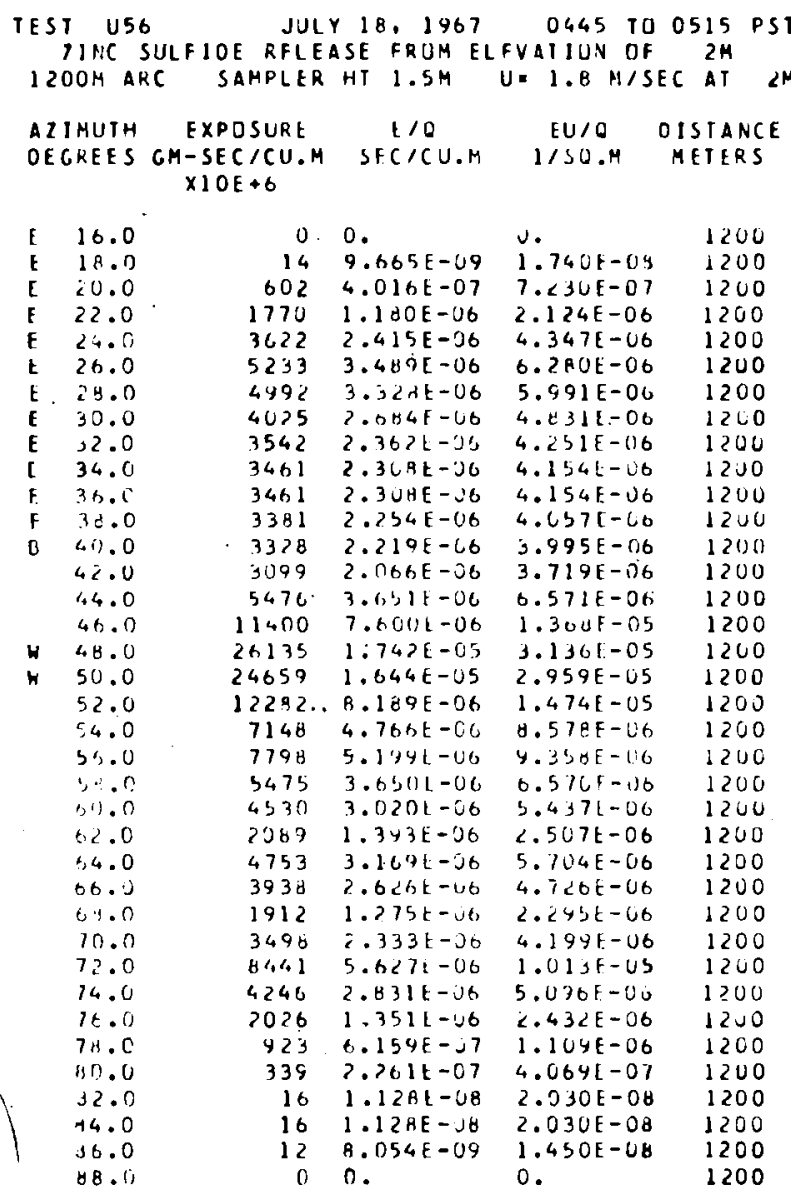

CRISSWIHO INTECRATED $=4.961 E-33 \quad 8.930 E-0.3$

$$
\text { SECISO.M } \quad 1 / M
$$

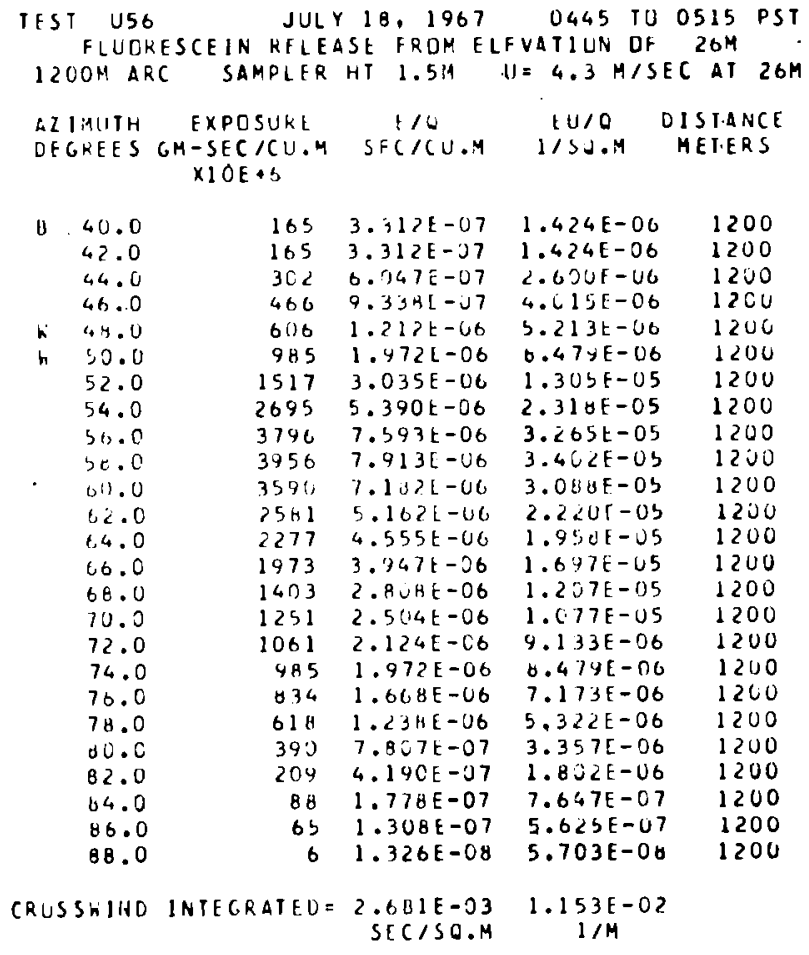




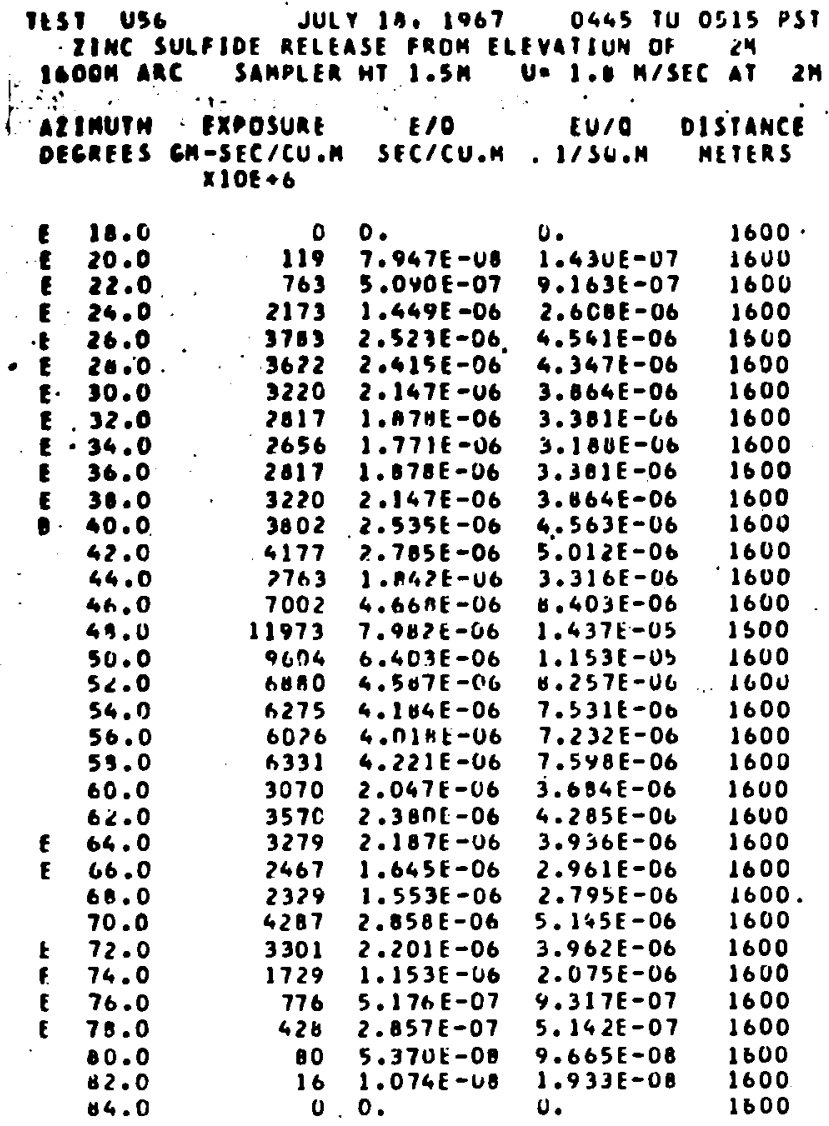

CROSSWIHD INTEGRATEUE $4.246 E-03 \quad 7.732 E-03$
TEST US6 NUIY 18.1967 O445 TO OSIS PST 7 INC SULFIDE RELEASE FROH ELFVATIUN UF $2 M$
$20 O M$ ARC SAMPLER HI $1.5 \mathrm{M} U=1.3 \mathrm{M} / S E C$ AT $2 \mathrm{M}$

\section{ALIMUTH EXPOSURE E/O EU/O DISTAHCE \\ DFGKEES CH-SECICU.M SECICU.H I/SU.M METERS} $\times 10 E+6$
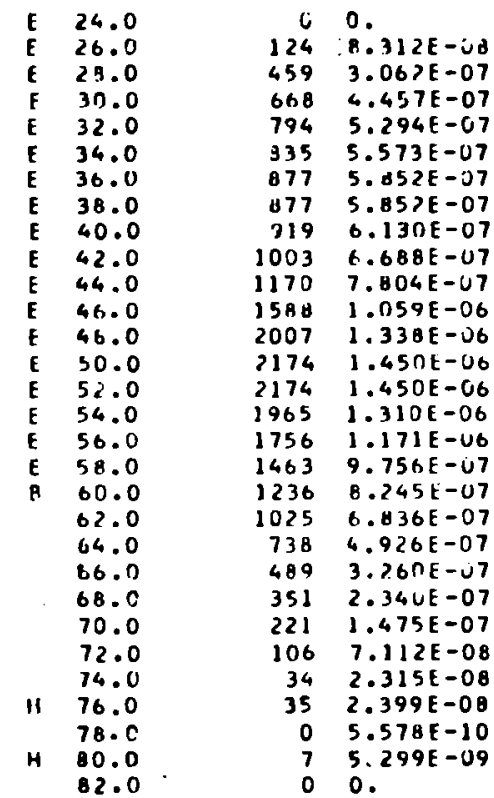

0.

$1.496 \mathrm{E}-07$

$5.51<E-07$

$6.023 \mathrm{E}-07 \quad 3200$

$4.529 \mathrm{E}-07 \quad 3200$

1. $003 E-06 \quad 3200$

$1.053 \mathrm{E}-06$

$1.053 \mathrm{E}-06 \quad 3200$

$1.103 \mathrm{E}-06 \quad 3200$

1.204 E-U6 3200

$1.405 E-06 \quad 3200$

$1.907 E-06 \quad 3200$

$2.409 E-06 \quad 3200$

2. 010 E-06 3200

2.61UE-U6 3200

$2.10 \mathrm{E}-06 \quad 3200$

$1,756 E-06 \quad 3200$

$1.484 \mathrm{E}-06 \quad 3200$

$1.231 \mathrm{E}-06 \quad 3200$

$0.066 E-07 \quad 3200$

$5.869 E-07 \quad 3200$

$4.212 \mathrm{E}-07 \quad 3200$

$2.656 E-07 \quad 3200$

$1.280 E-07 \quad 3200$

$4.167 \mathrm{E}-08 \quad 3200$

$4.31 B E-08 \quad 3200$

$1.004 E-09 \quad 3200$

$9.534 E-04 \quad 3200$

CROSSHINO INTEGRATED= 1.870E-03 3.366E-03

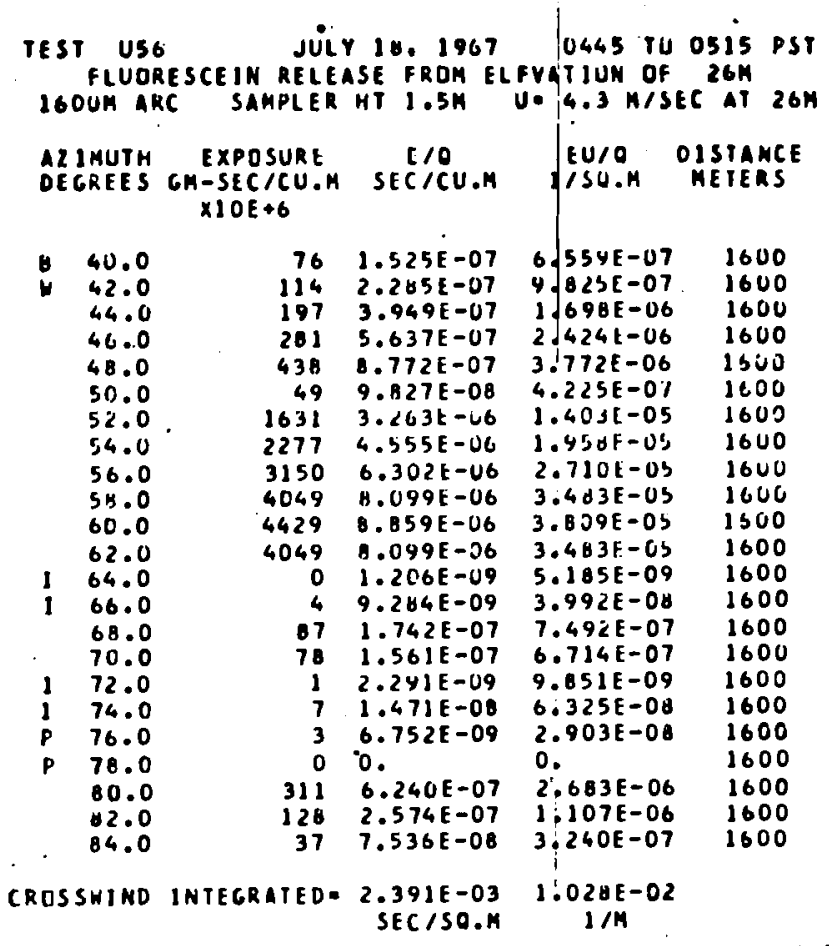

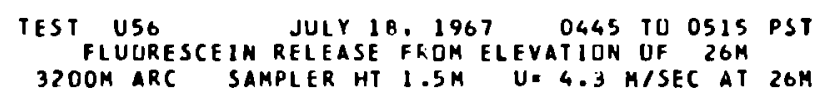

AZIMUTH EXPOSURE E/O EUIO DISTINCE DEGREES CH-SECICU.M SECICU.M I/SO.M METERS $X \perp O E+6$

$\begin{array}{rrrrr}60.0 & 1103 & 2.208 \mathrm{E}-06 & 9.494 \mathrm{E}-06 & 3200 \\ 62.0 & 985 & 1.971 \mathrm{E}-06 & 8.476 \mathrm{E}-06 & 3200 \\ 64.0 & 985 & 1.971 \mathrm{E}-06 & 1.476 \mathrm{E}-06 & 3200 \\ 66.0 & 768 & 1.537 \mathrm{E}-06 & 6.610 \mathrm{E}-06 & 3200 \\ 68.0 & 610 & 1.222 \mathrm{E}-06 & 5.253 \mathrm{E}-06 & 3200 \\ 70.0 & 452 & 9.059 \mathrm{E}-07 & 3.895 \mathrm{E}-06 & 3200 \\ 72.0 & 308 & 6.166 \mathrm{E}-07 & 2.651 \mathrm{E}-06 & 3200 \\ 74.0 & 218 & 4.360 \mathrm{E}-07 & 1.678 \mathrm{E}-06 & 3200 \\ 76.0 & 115 & 2.302 \mathrm{E}-07 & 9.897 \mathrm{E}-07 & 3200 \\ 78.0 & 51 & 1.037 \mathrm{E}-07 & 4.457 \mathrm{E}-07 & 3200 \\ 80.0 & 16 & 3.351 \mathrm{E}-08 & 1.441 \mathrm{E}-07 & 3200 \\ 82.0 & 3 & 6.325 \mathrm{E}-09 & 2.720 \mathrm{E}-08 & 3200\end{array}$

CROSSHIND INTEGRATEO= $1.256 E-03$ 5.400E-03

SECISO.M T. $1 / \mathrm{H}$ 
SAMPLIMG 400N TO $3200 M I$ MO TOHER SAMPLIMC.

TRACER DISTRIOUTION EXIENUS GEYOND SAMPLERS AT 320OM. AND TO LESSER EXTENT AT IGOOM. ZNS EXTRAPOLATED AT 3200N. STRTAG LOW-LEVEL TEMPERATURE INVERSION.

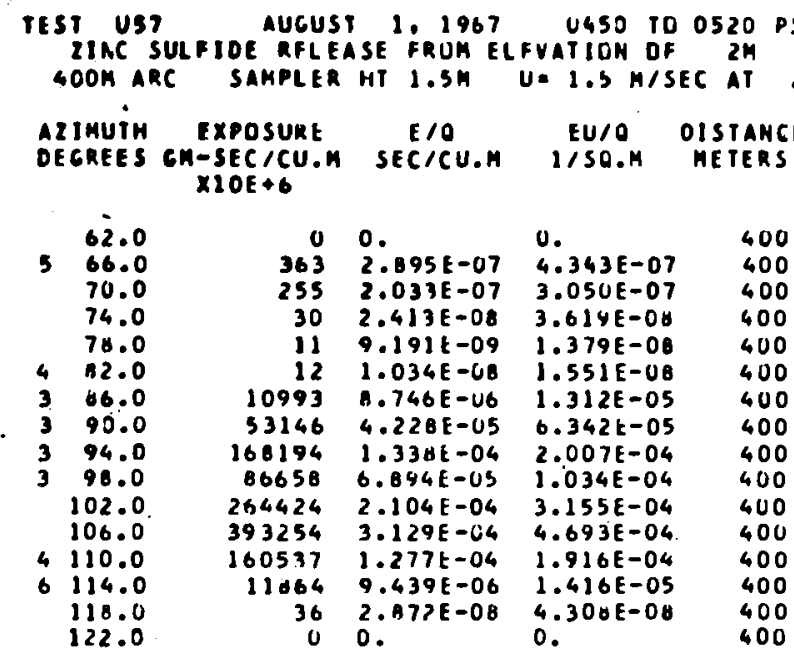

CRUSSHINO JNTEGKATFOE 2 STS4E-02 $3.832 \mathrm{E}-02$

TEST US7 AUCUST 1.1967 U450 IU 0520 PST

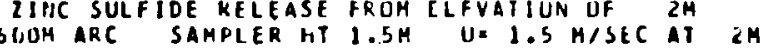

AIIHUTH EXPOSURE E/O EU/O DISTANCE OFGKFES CM-SECICU.M SFCICU.M IISU.K METERS $\times 105+6$

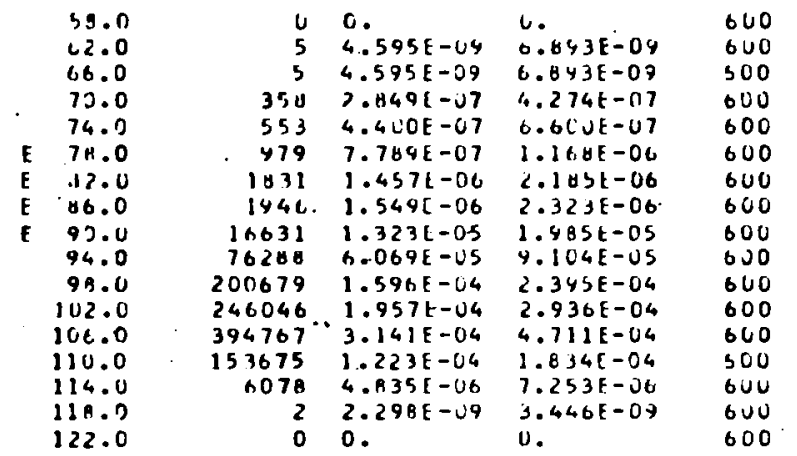

CRRSSHINO INTEGRATFD= 3.665E-U2 $5.490 \mathrm{~F}-02$

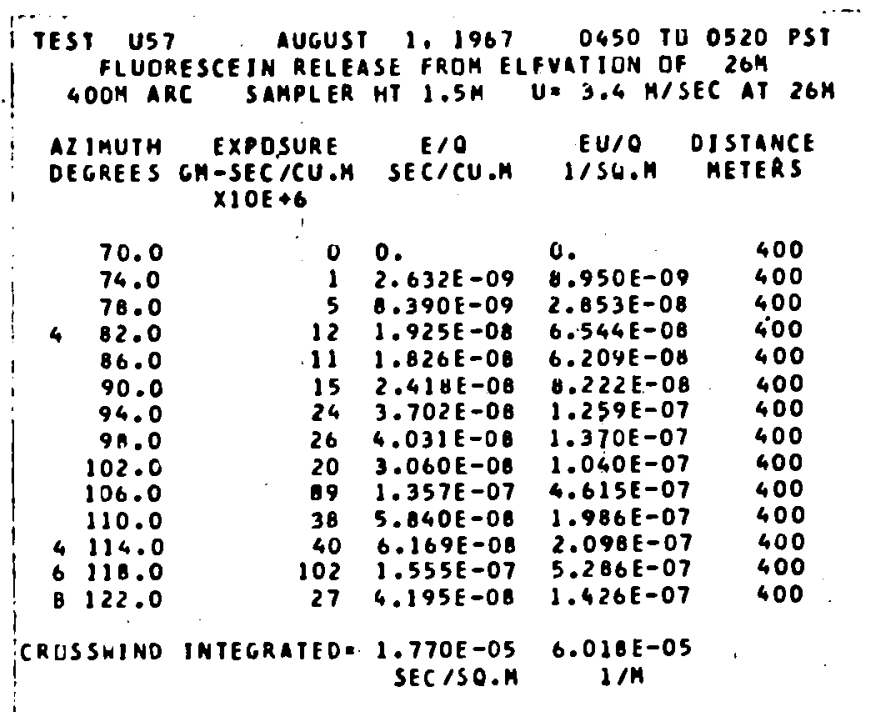

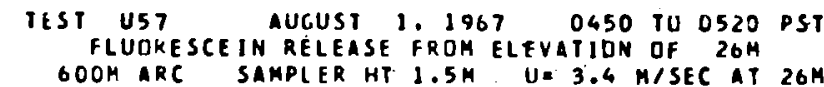
AZIMUTH EXPQSURE EIO EU/O OISTANCE - OECREES CM-SECICU.M SECICU.M MISO.M METERS

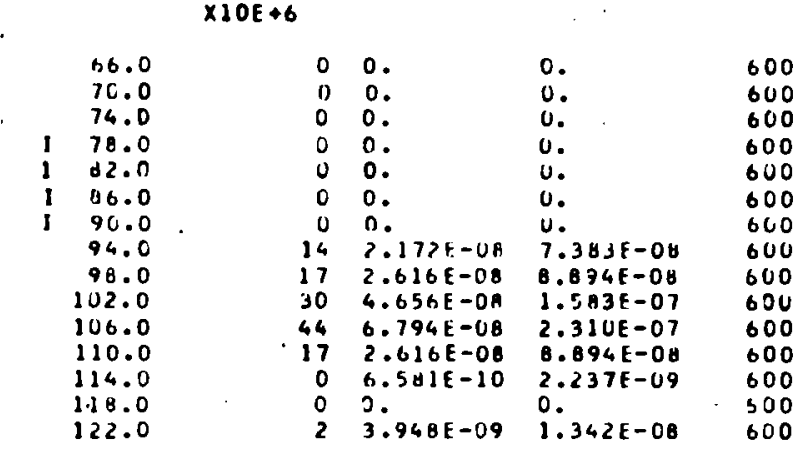

CROSSHINO INTEGRATED= B.090E-06 2.751E-US 


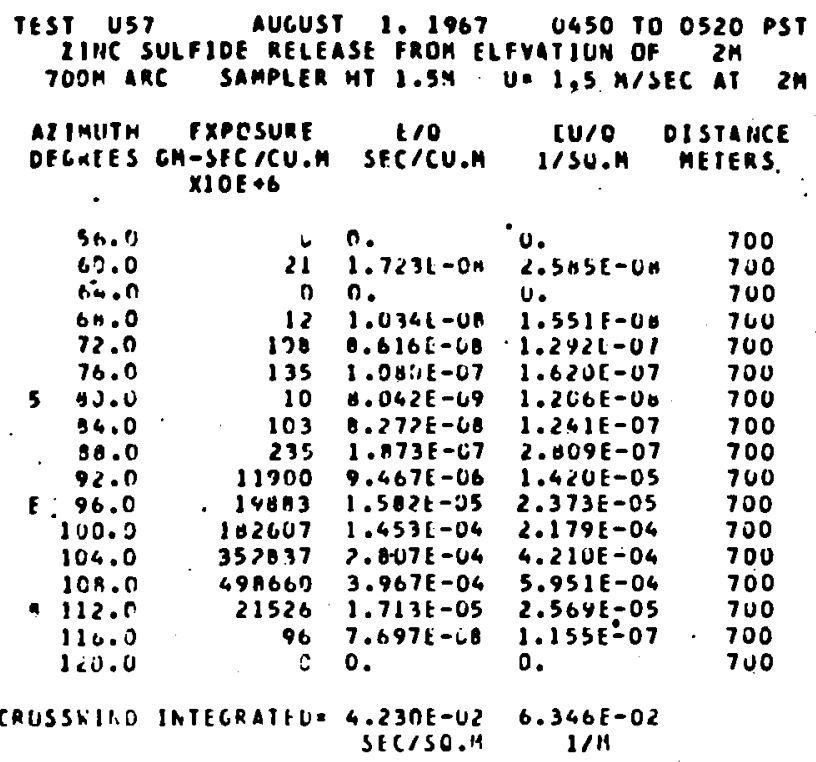

TEST UST AUR.UST 1. $1967 \quad 0450$ TO 0520 PST ZIIE SULFIOE RFLEASE FEUH ELFVATION OF LM AIIMUTH EXPUSURE E/U EU/O OISTANCE OFCREES GM-SFC/CU.M SFCICU.M I/SO.M METERS $\times 10 E+6$

\begin{tabular}{|c|c|c|c|c|}
\hline $\begin{array}{l}59.0 \\
61.0\end{array}$ & $\begin{array}{l}0 \\
\text { C }\end{array}$ & $\begin{array}{l}0 . \\
n .\end{array}$ & $\begin{array}{l}\text { u. } \\
\text { u. }\end{array}$ & $\begin{array}{l}300 \\
B 00\end{array}$ \\
\hline 64.0 & 0 & 0. & u. & ous \\
\hline 67.0 & 17 & $1.379 t-08$ & $2.06 \mathrm{dE}-08$ & 000 \\
\hline 10.0 & 122 & $9.765 t-68$ & $1.46 .5 E-07$ & 000 \\
\hline $7 \% .0$ & $436^{\circ}$ & $3.469 E-07$ & $5.204 E-07$ & $B 00$ \\
\hline 76.0 & 441 & $3.515 \mathrm{E}-07$ & $5.2736-07$ & 300 \\
\hline 79.0 & 46 & $3.67 b[-08$ & $5.514 E-08$ & 800 \\
\hline $82 . r$ & 36 & $2.472 E-v \theta$ & $4.3 \cup d E-O d$ & BuU \\
\hline 115.0 & 63 & $5.055 E-08$ & $1.582 \mathrm{t}-00$ & 000 \\
\hline$\forall 0.0$ & 73 & S.AS $9 E-00$ & 6.789E-Ud & 800 \\
\hline 91.0 & 1260 & $1.003[-06$ & $1.504 E-06$ & 100 \\
\hline 94.0 & 13441 & $1.109 E-05$ & $1.664 E-05$ & 900 \\
\hline 97.0 & 93531 & $7.441 E-C 5$ & $1.116 E-04$ & 900 \\
\hline 1. 100.0 & 104353 & $H .302 E-05$ & $1.245 \mathrm{E}-04$ & Buo \\
\hline$h 103.0$ & 30439 & $2.422 E-05$ & $3.63<t-05$ & 800 \\
\hline+100.0 & 191731 & $1.525 \mathrm{E}-04$ & $2.284[-04$ & 800 \\
\hline 104.0 & 95232 & $7.576 E-05$ & $1.136 E-04$ & 800 \\
\hline 112.0 & 61768 & $4.914 E-05$ & $7.371 \mathrm{E}-05$ & 800 \\
\hline 115.0 & 4057 & $3.22 \mathrm{AE}-06$ & $4.842 E-06$ & 800 \\
\hline 112.0 & 2 & $2.248 \mathrm{E}-09$ & $3.446 E-07$ & 000 \\
\hline 121.0 & 0 & & 0. & 800 \\
\hline
\end{tabular}

CRIISSWINU INTECRATED $1.9914-02$ 2.987E-02

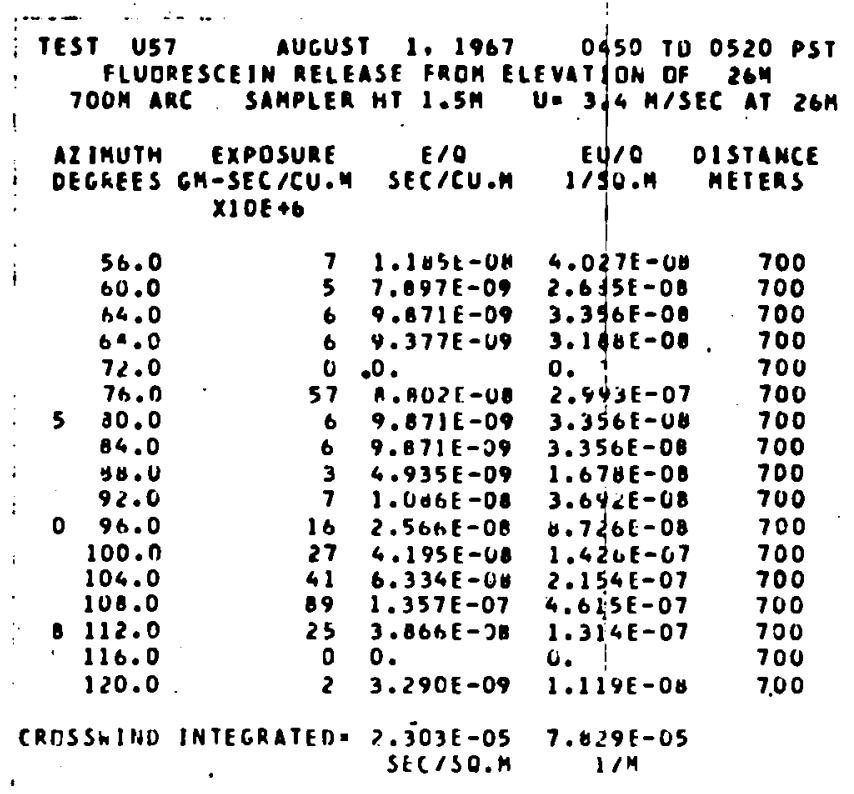

\begin{tabular}{|c|c|c|c|c|}
\hline $\begin{array}{l}\text { IEST UST } \\
\text { FLUDR } \\
\text { OOOY AR }\end{array}$ & $\begin{array}{l}7 \\
\text { RESCE AUGUS } \\
R C \text { SN RELE } \\
\text { RC SAMPLER }\end{array}$ & $\begin{array}{l}\text { T. } 1967 \\
\text { ASE FRUN } \\
\text { HI } 1.5 \mathrm{H}\end{array}$ & $\begin{array}{r}0450 \text { TO } \\
\text { EVATIUN OF } \\
\text { U. } 3.4 \mathrm{M} / \mathrm{S}\end{array}$ & 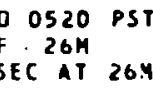 \\
\hline $\begin{array}{l}\text { ALIKUTH } \\
\text { DEGREES }\end{array}$ & $\begin{array}{c}\text { EXPOSURE } \\
\text { GM-SEC/CU.H } \\
\times 1 O E \& 6\end{array}$ & $\begin{array}{c}E / O \\
S E C / C U . H\end{array}$ & $\begin{array}{l}\text { EU/O } \\
\text { I/SU.h }\end{array}$ & $\begin{array}{l}\text { OISTANCE } \\
\text { METERS }\end{array}$ \\
\hline $\begin{array}{r}64.0 \\
67.0 \\
70.0 \\
73.0 \\
76.0 \\
79.0 \\
82.0 \\
85.0 \\
80.0 \\
491.0 \\
94.0 \\
97.0 \\
W 100.0 \\
1103.0 \\
W 106.0 \\
109.0 \\
112.0 \\
115.0 \\
118.0 \\
121.0\end{array}$ & $\begin{array}{r}1 \\
0 \\
5 \\
0 \\
0 \\
0 \\
0 \\
0 \\
0 \\
0 \\
0 \\
14 \\
34 \\
11 \\
39 \\
23 \\
18 \\
2 \\
8 \\
10\end{array}$ & $\begin{array}{l}1.645 E-09 \\
0 . \\
0.390 E-09 \\
0 . \\
0 . \\
1.316 E-09 \\
0 . \\
0 . \\
4.935 E-10 \\
0 . \\
0 . \\
2.270 E-08 \\
5.162 E-08 \\
1.826 E-08 \\
6.005 E-08 \\
3.537 E-08 \\
2.764 E-08 \\
3.290 E-09 \\
1.234 E-08 \\
1.629 E-00\end{array}$ & $\begin{array}{l}5.594 E-09 \\
0.0 \\
2.053 E-08 \\
0.1 \\
0 . \\
4.475 E-09 \\
0 . \\
0 . \\
1.678 E-09 \\
0 . \\
0 . \\
7.719 E-00 \\
1.762 E-07 \\
6.204 E-08 \\
2.042 E-07 \\
1.203 E-07 \\
9.397 E-08 \\
1.119 E-08 \\
4.195 E-08 \\
5.530 E-08\end{array}$ & $\begin{array}{l}800 \\
800 \\
800 \\
800 \\
800 \\
800 \\
000 \\
800 \\
800 \\
800 \\
800 \\
800 \\
800 \\
800 \\
800 \\
800 \\
800 \\
000 \\
800 \\
800\end{array}$ \\
\hline SSRIND & TEGRATED & $\begin{array}{r}1.087 E-0 S \\
\text { SEC/SO.M }\end{array}$ & $\begin{array}{c}3.697 \mathrm{E}-05 \\
1 / \mathrm{M}\end{array}$ & \\
\hline
\end{tabular}




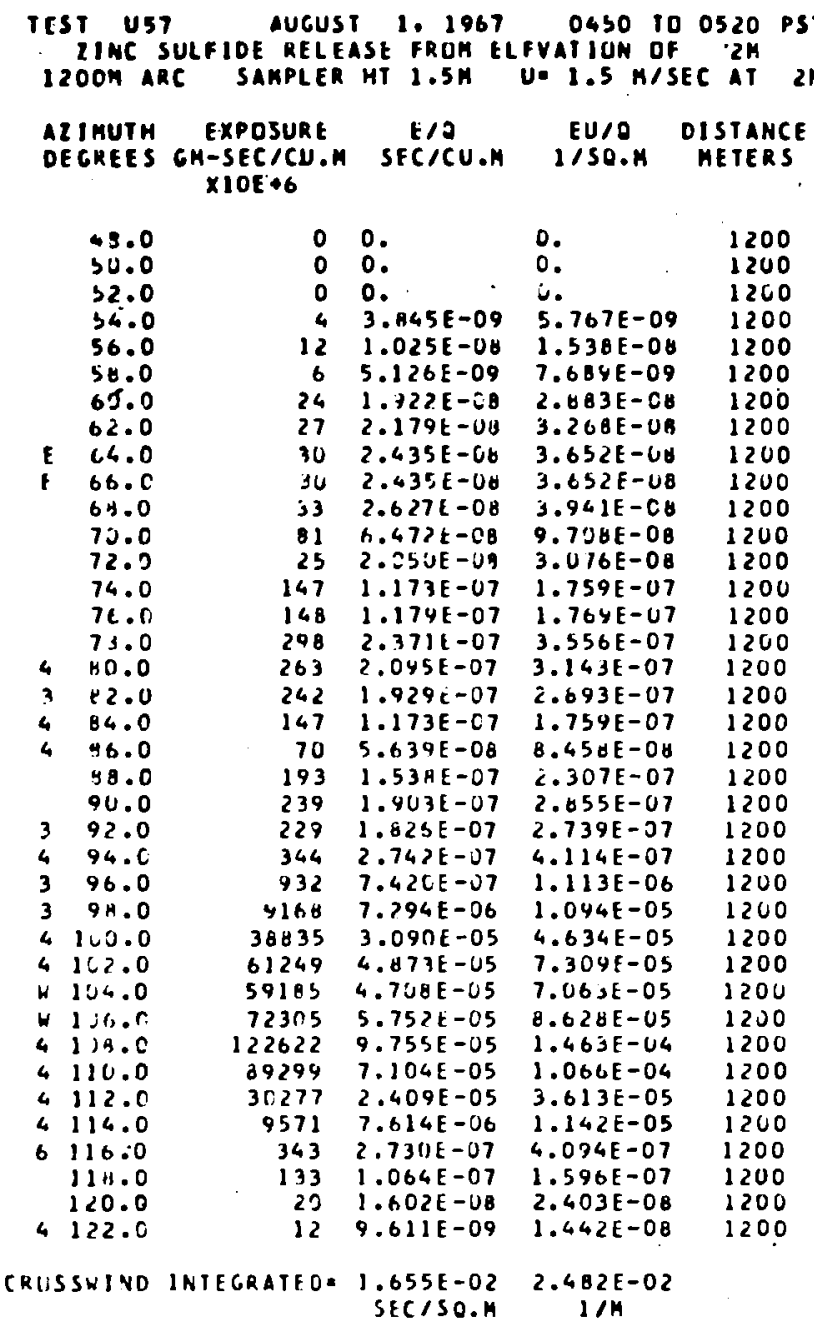

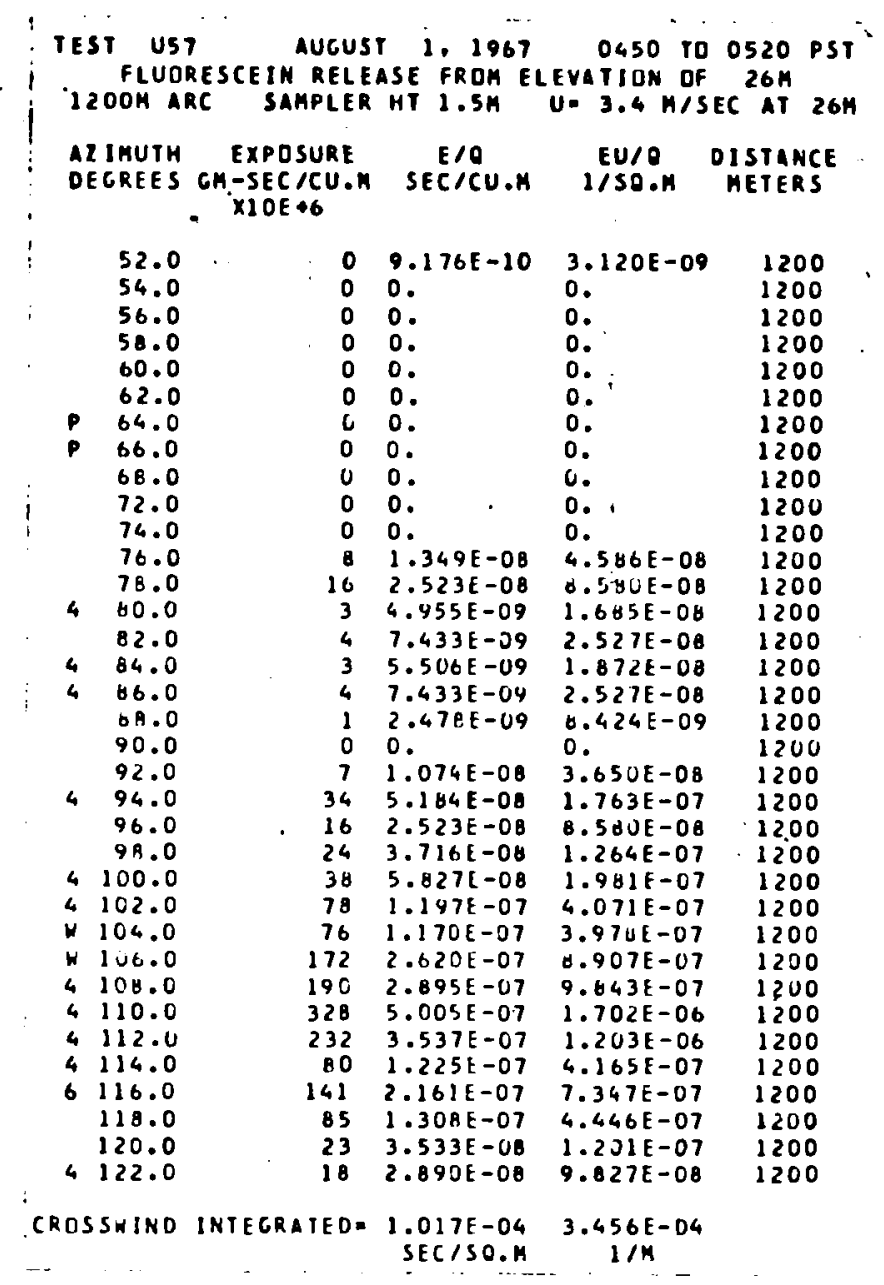




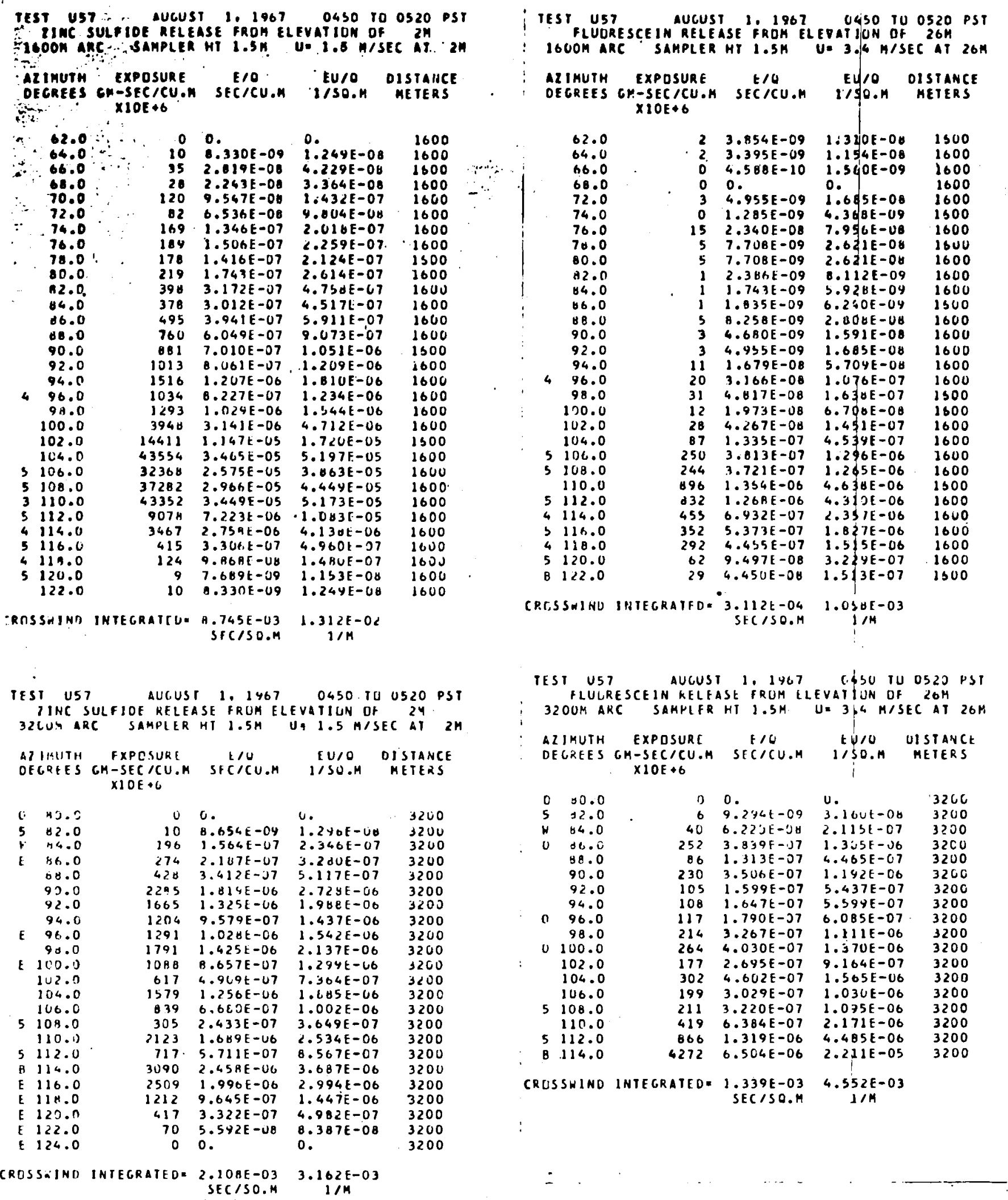


SAMPLING 4OOM TO 320OM: NU TOEER SAMPLING.

ALL AKCS FMBRACE CRCSSWINU EXTENT DF TRACER DISTRIBUTIONS. UNUSUAL APPEARANCE DF ZNS REMAINING IN DISPERSAL APPARATUS

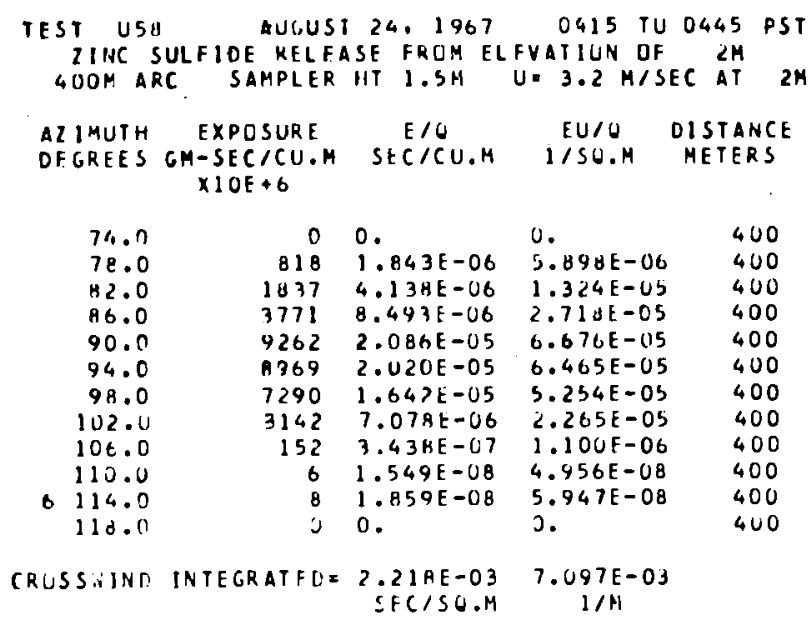

TEST USB AUCUST 24,1967 1445 TU 0445 PST 21 iNC SULFIDE KELFASE FFIM ELFVATION OF $2 M$ GUUI" ARC SAMPLER HI $1.5 \mathrm{M} U=3.2 \mathrm{M} / \mathrm{SEC}$ AT $2 M$ $\begin{array}{cccc}\text { AZIMUTH EXPOSURE } & \text { EIO } & \text { EU/O OISIANCE } \\ \text { OFGHES GM-SECICU.M SFCICU.M } & \text { IISU.M METERS } \\ \text { XIOE } & \end{array}$

$\begin{array}{rrl}66.0 & 0 & 0 . \\ 70.0 & 9 & 2.16+E-U 0 \\ 74.0 & 9 & 2.161 \mathrm{E}-08 \\ 74.0 & 1 & 0.0 \\ 82.0 & 15 & 3.407 E-U 6 \\ 156.0 & 244 & 5.514 E-07 \\ 40.0 & 308 & 6.938 E-U 7 \\ 94.0 & 5637 & 1.270 E-05 \\ 99.0 & 1796 & 4.045 E-06 \\ 102.0 & 1488 & 3.352 E-U 6 \\ 116.0 & 27 & b .145 E-08 \\ 110.0 & 0 & 0 .\end{array}$

$\begin{array}{ll}6.93 J E-08 & 600 \\ 6.538 E-08 & 600 \\ 5.9 & 600 \\ 1.040 E-07 & 600 \\ 1.764 E-06 & 600 \\ 2.220 E-06 & 600 \\ 4.063 E-05 & 600 \\ 1.295 E-05 & 600 \\ 1.072 E-05 & 600 \\ 1.482 E-07 & 600 \\ U .96 & 600\end{array}$

CRESSHIHO DNTEGKATFU = B.997E-04 2. B7YE-03 SFCISU.M $\quad 1 / M$ follohing tracer release leaves some doubt on "O".

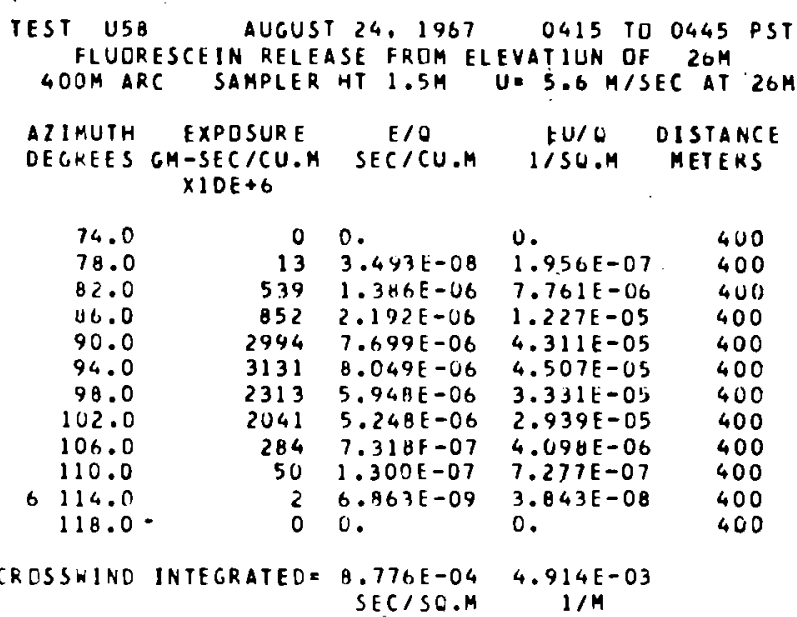

TEST U5A AUGUST 24.1967 0415 TO 0445 PST FLUDRESCEIN RELEASE FROM ELFVATILN OF $26 \mathrm{M}$ GOOM ARC SAMPLER HT $1.5 \mathrm{M} U=5.6 \mathrm{M} / \mathrm{SEC}$ AT $26 \mathrm{M}$

ALIHUTH EXPOSURE $t / 0$ DEGKES GM-SECICU.M SEC/CU.M IISL.M METERS $X 10 E+6$

$$
\begin{array}{r}
70.0 \\
14.0 \\
73.0 \\
72.0 \\
00.0 \\
90.0 \\
94.0 \\
98.0 \\
102.0 \\
106.0 \\
110.0
\end{array}
$$$$
\begin{array}{rl}
0 & 0 . \\
0 & 1.044 E-09 \\
0 & 8.336 E-11 \\
3 & 9.419 \mathrm{~L}-09 \\
516 & 1.327 \mathrm{E}-06 \\
2994 & 7.649 \mathrm{E}-06 \\
4288 & 1.102 \mathrm{E}-05 \\
2245 & 5.773 \mathrm{E}-06 \\
2313 & 5.94 \mathrm{BE}-06 \\
121 & 3.133 \mathrm{E}-07 \\
10 & 2.659 \mathrm{E}-0 \mathrm{~B}
\end{array}
$$$$
0 .
$$$$
6.063 E-09
$$$$
4.668 \mathrm{E}-10
$$$$
5.275 E-06
$$$$
7.431 E-06
$$$$
4.311 \mathrm{E}-05
$$$$
6.174 \mathrm{E}-0 \mathrm{~b}
$$$$
3.233 \mathrm{E}-05
$$$$
3.331 \mathrm{E}-05
$$$$
\text { 1. } 75 S E-06
$$$$
10 \quad 2.659 \mathrm{E}-0 \mathrm{~B} \quad 1.489 \mathrm{E}-07
$$

CEUSSIINO INTEGRATFD $=$
SEC/SO.M

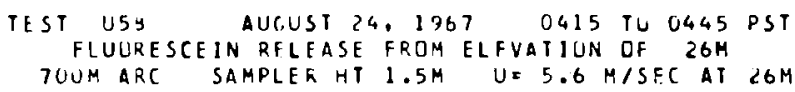

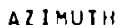
EXPOSURE $E / 0$ EUIO OISTANCE DEGKEES GH-SEC/CU.M SECICU.M I/SO.M METERS $\times 10 \mathrm{E}+6$
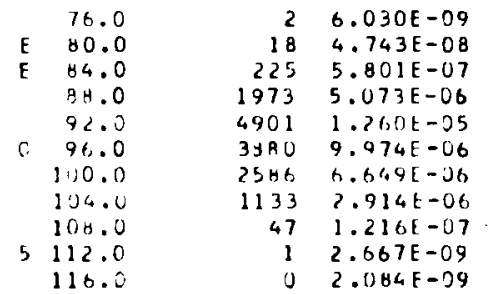

$3.377 \mathrm{E}-0.0$ $3.377 E-08$
$2.656 E-07$ $2.656 E-07$
$3.246 E-06$ $2.841 \mathrm{E}-05$ $7.056 F-05$ 5. 5 HEE-US $3.723 t-0 b$ 1. $632 \mathrm{E}-\mathrm{US}$ 1. $632 \mathrm{E}-\mathrm{US}$
$6.811 \mathrm{E}=07$ $1.474 \mathrm{E}-0 \mathrm{~B}$ $1.167 \mathrm{E}-\mathrm{OH}$

600
500
600
600
600
600
600
600
600
600
600
600 
TEST US. AUGUSI 24,1967 O415 TU 0445 PST 2 IHC SULFIDE RELEASE FRUM ELFVAIIUN UF LM
SCOH AKC SAMPLER H? $1.5 \mathrm{M} W=3.2 \mathrm{M} / \mathrm{SEC}$ AT $\begin{array}{lcc}\text { AZIHUTH EXPDSURE } & \text { E/O } & \text { EU/O OISIANCE } \\ \text { DEGHEES GM-SECICU.M S SECICU.M IISO.M METERS }\end{array}$ $\times 10 \mathrm{E}+6$

67.0
70.0
73.0
76.0
79.0
82.0
05.0
33.0
91.0
94.0
97.0
100.0
103.0
196.0

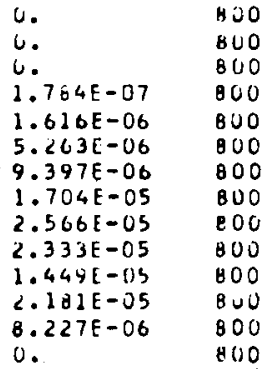

CRLSSHINU INTEGRATFO- $1.663 E-03 \quad 5.320 E-03$
TEST USA AUCUSI 24,1967 O4 15 IU U445 PST IIA:C SULFIDE RFLEASE FRLM ELFVATIUN LIF 24 $120 \mathrm{H}$ MKC SAMPLEK H: $1.5 \mathrm{M} \mathrm{N}=3.2 \mathrm{M} / \mathrm{SEC}$ AT $2 \mathrm{M}$

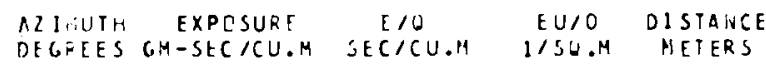
$\times 10 E+6$

\begin{tabular}{|c|c|c|}
\hline $7 ? . \mathrm{C}$ & $u$ & 0. \\
\hline 13.0 & 56 & $1.27 B E-J 7$ \\
\hline $0,2,0$ & 200 & $4.526 E-07$ \\
\hline 34.0 & 279 & $6.284 \mathrm{t}-U 7$ \\
\hline 10.0 & 605 & $1.363 t-015$ \\
\hline 5.6 .0 & 386 & $\mathrm{H} .74>\mathrm{E}-\cup 7$ \\
\hline $4 \mathrm{J.0}$ & 1244 & $2 . H 04 E-J 6$ \\
\hline 92.0 & 1010 & $2.775 t-06$ \\
\hline 34.0 & 836 & $1.8 \Delta 35-06$ \\
\hline 96,0 & 378 & $8.5171-07$ \\
\hline 98.0 & 276 & $6.237 E-07$ \\
\hline 30.0 & 471 & $2.189 E-06$ \\
\hline$\therefore 0$ & 642 & $1.448 E-06$ \\
\hline 04.0 & 58 & $1.313 E-07$ \\
\hline 66.0 & 6 & $1.555 \mathrm{E}-08$ \\
\hline $0 n .0$ & 31 & $7.08 \$ 1-U 8$ \\
\hline 10.0 & 0 & 0. \\
\hline
\end{tabular}

CK.SSAIND INIEGRATED $=6.59 .3 E-34$ 2.110E-03 SFCISU.M $1 / M$

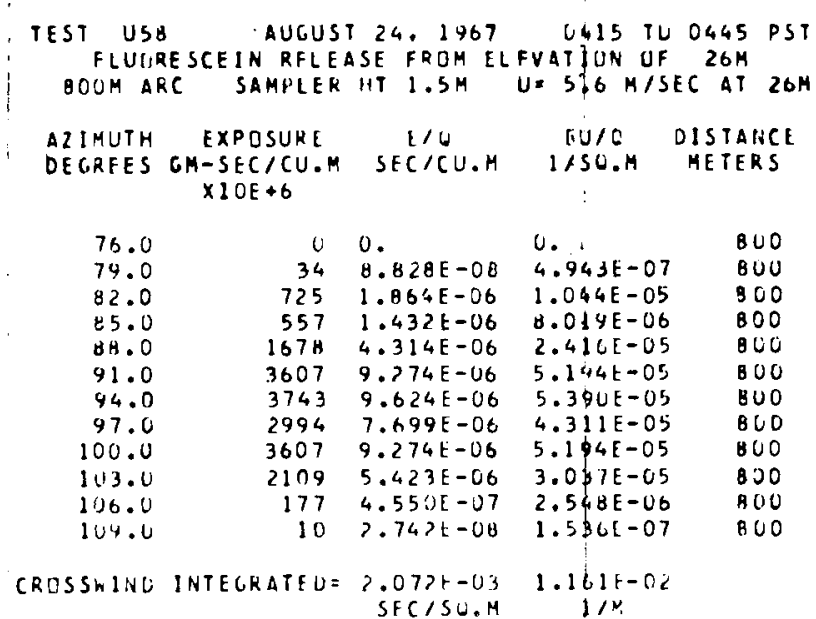

TEST USA AUCUST 24.1967 0415 TO 0445 PST FLUUKESCEIN RFLEASI FHJM [LFVAT]UN DF $26 M$ $120 U M$ AKC SAMPLER HT $1.5 \mathrm{M} U=3.0 \mathrm{M} / 5 E C$ AT $2 G \mathrm{M}$ $\begin{array}{lccc}\text { AZIHIITH EXPOSURE } & \text { EIE } & \text { EJPO DISTALCE } \\ \text { DFCKEES GM-SECICU.M SECICU.M IISG.M METERS }\end{array}$ $\times 10 \mathrm{~L}+6$

\begin{tabular}{|c|c|c|c|c|c|c|}
\hline & $7 \cdot .0$ & & 19 & $4.424 \mathrm{c}-0 \mathrm{~B}$ & $2.7371-07$ & 1200 \\
\hline$E$ & SU.C & & 55 & $1.422 \mathrm{E}-07$ & $7.964 t-07$ & 1200 \\
\hline & $d 2 . c$ & & 134 & 3.46 HE $-1,7$ & $1.942 E-06$ & 1200 \\
\hline & $\mathrm{N}_{4} . \mathrm{C}$ & & 276 & $7.110[-07$ & $3.4 d<[-06$ & 1200 \\
\hline E & $B E, 0$ & & 391 & $1.005 E-66$ & $3.630 E-06$ & 1200 \\
\hline & 16.0 & & 276 & $7.10 N E-07$ & $3.961 \mathrm{E}-06$ & 1200 \\
\hline & 9.3 .0 & & 2316 & $5.9541-6 t$ & $3.3341-05$ & 1200 \\
\hline & 92.0 & & 2733 & $7.02 B E-(26)$ & $3.936, \mathrm{~F}=05$ & $1<00$ \\
\hline & +4.0 & & 2164 & $5.563 \mathrm{~L}-06$ & $3.113 f-05$ & 1200 \\
\hline & 46.0 & & 1556 & $4.001 E-06$ & $2 \cdot 24+1 k-U 3$ & 1200 \\
\hline & 99.0 & & 1328 & $3.415 t-06$ & $1.913 E-05$ & 1200 \\
\hline & 100.0 & & 1822 & $4.685 E-05$ & $2.623 E-05$ & 1200 \\
\hline & 102.0 & & 1936 & $4.97+[-06$ & $2.787 E-05$ & 1200 \\
\hline & 134.0 & & 330 & $0.505 E-07$ & $4.763 E-06$ & 1200 \\
\hline & 106.0 & & 20 & $5.389 E-0 \theta$ & $3.016 E-07$ & 1200 \\
\hline & 108.0 & & 5 & $1.437 \mathrm{E}-08$ & $8.043 E-00$ & 1200 \\
\hline & 110.0 & & 0 & $3.255 E-10$ & $1.823 t-09$ & 1200 \\
\hline & & & & $\begin{array}{l}1.655 E-03 \\
\text { SECISU.M }\end{array}$ & $\begin{array}{c}9 .<68 E-03 \\
1 / H\end{array}$ & \\
\hline
\end{tabular}

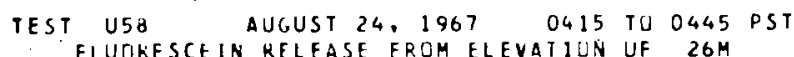
FLUTKESCE IN KELEASE FROM ELEVATIUN UF $26 \mathrm{M}$
6OUH ARC SAMPLER HT $1.5 \mathrm{M}$ U $=5.6 \mathrm{H} / \mathrm{SEC}$ AT $26 \mathrm{M}$

TEST USP AUGUST 24. 1967 0415 TU 0445 PST

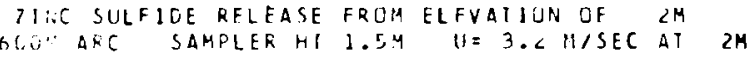

AIITHTH EXPOSURT E/U CU/O OISTANCE DEGHES GM-SECICU.M SECICU.M 1/SW.Y MEIERS $X \perp O E+\ell$

$\begin{array}{lccc}\text { AZIMUTH EXPOSURE } & \text { E/O } & \text { EUIO DISTANCE } \\ \text { DEGKEES CM-SECICU.M SECICU.M TISO.M MEIERS }\end{array}$ $\times 1 O E+6$

\begin{tabular}{|c|c|c|}
\hline 七刀. & j & c. \\
\hline$\therefore .0$ & 42 & $9.502 \mathrm{E}-06$ \\
\hline 114.6 & 154 & $3.473 L=67$ \\
\hline$r+.0$ & 113 & $? .609 E-07$ \\
\hline$A H . O$ & 212 & 4.7 जBt $-U 7$ \\
\hline$\Rightarrow 1.0$ & 612 & 1. $\left.3 M_{1}\right) E-30$ \\
\hline 92.0 & 596 & $1.344 E-06$ \\
\hline 94.0 & 499 & $1.125 \mathrm{E}-00$ \\
\hline 96.0 & 470 & $1.059 E-06$ \\
\hline 98.0 & 562 & $1.266 \mathrm{E}-06$ \\
\hline 1.20 .0 & 545 & $1.2 .28 E-06$ \\
\hline 102.0 & 373 & $8.414 E-U 7$ \\
\hline 104.0 & 75 & $1.710 \mathrm{E}-07$ \\
\hline 100.0 & 10 & $2.419 E-08$ \\
\hline 104.0 & 28 & 6.397 \\
\hline 110.3 & 0 & 0. \\
\hline
\end{tabular}

i.

3. $041+-07$

1600

$8.340 E-07$

$1.531 \mathrm{E}-06$

$4.417 E-C 6$

$4.30 .1 E-06$

$3.599 E-06$

$4.052 E-06$

$3.931 \mathrm{E}-0 \mathrm{~B}$

2.692E-06

$5.473 E-07$

7.74UE-08

$2.046 E-07 \quad 1600$

0 .

1600

1600

1600
1600

1600

1600

1500

1600

1600

1600
1600

CPISSHINO INTEGRATE D $=5.409 E-04$

SEC $150 . M$

1. $731 \mathrm{E}-03$

$1 / 4$

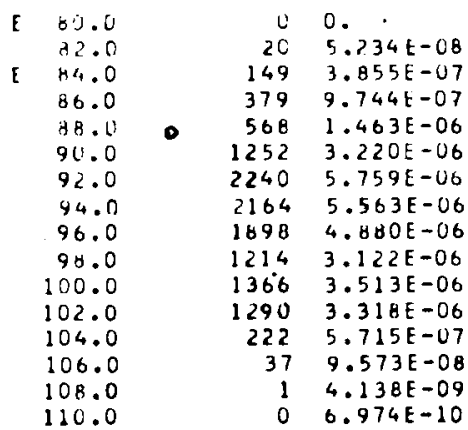

0.

CROSSHIND INTECRATEDE $2.839 E-03$

$S F C / S O . M$

2. $431 \mathrm{E}-07$

$5.457 E-06$

8.191E-06

$1.803 \mathrm{E}-05$

$3.225 \mathrm{E}-0 \mathrm{~S}$

$3.115 E-05$

$2.733 E-05$

$1.749 E-05$

$1.967 E-05$

$1.850 \mathrm{E}-05$

3. $200 E-06$

$5.361 E-07$

2.317E-0B

$3.905 E-09$

1600

1600

1600

1600

1600

1600

1600
1600

1600

1500

1600

1600

1600

1600

1600
1600
$2.159 \mathrm{t}-00$ 


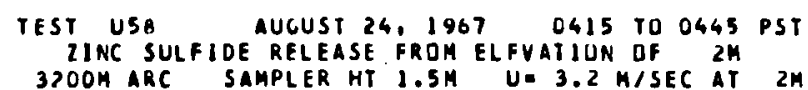

ALIMUTH EXPUSURE

E10. EU/O OISIANCE $\times \perp O E+6$

\begin{tabular}{|c|c|c|c|c|}
\hline $\begin{array}{l}84.0 \\
86.0 \\
88.0 \\
90.0 \\
92.0 \\
94.0 \\
96.0 \\
98.0 \\
100.0 \\
102.0 \\
104.0 \\
106.0 \\
108.0\end{array}$ & $\begin{array}{r}0 \\
22 \\
38 \\
117 \\
212 \\
137 \\
135 \\
145 \\
97 \\
94 \\
54 \\
1 \\
0\end{array}$ & $\begin{array}{l}0 . \\
4.994 E-08 \\
8.763 E-08 \\
2.638 E-07 \\
4.777 E-07 \\
3.100 E-07 \\
3.044 E-07 \\
3.279 E-07 \\
2.265 E-07 \\
2.120 E-07 \\
1.225 E-07 \\
3.769 E-09 \\
0.7\end{array}$ & $\begin{array}{l}0 . \\
1.598 E-07 \\
2.804 E-07 \\
8.443 E-07 \\
1.529 E-06 \\
9.920 E-07 \\
9.739 E-07 \\
1.049 E-06 \\
7.056 E-07 \\
6.784 E-07 \\
3.920 E-07 \\
1.206 E-08 \\
0 .\end{array}$ & $\begin{array}{l}3200 \\
3200 \\
3200 \\
3200 \\
3200 \\
3200 \\
3200 \\
3200 \\
3200 \\
3200 \\
3200 \\
3200 \\
3200\end{array}$ \\
\hline
\end{tabular}

CROSSHIND INTEGRAIED= $2.659 E-04$ B.508E-04 SEC/SO.H I/M

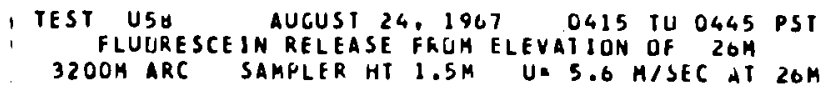

AII MUTH

EXPOSURE

$E / 4$

EU/O DISTANCE DEGREES GM-SEC/CU.M SECICU.M I/SO.H MEIERS $\times 10 E+6$

82.0
84.0
86.0
48.0
90.0
92.0
94.0
96.0
98.0
100.0
102.0
104.0
106.0
108.0

$\begin{array}{rl}U & 0 . \\ 2 & 7.221 E-09 \\ 24 & 6.341 E-00 \\ 81 & 2.063 E-07 \\ 361 & 9.288 E-07 \\ 315 & 0.104 E-07 \\ 749 & 1.926 E-06 \\ 611 & 1.571 E-06 \\ 413 & 1.064 E-06 \\ 519 & 1.334 E-06 \\ 571 & 1.470 E-06 \\ 246 & 6.350 E-07 \\ 29 & 7.629 E-08 \\ 2 & 7.221 E-09\end{array}$

1.

$4.044 E-00$ $3.551 E-07$

$1.107 E-36$ $5.201 E-16$

$4.538 E-16$

1. $079 E-05$ H. 749E-06

$5.950 E-06$ $7.473 E-08$ $8.231 E-C 6$

$3.556 E-06$

$4.272 \mathrm{E}-07$

4. $C 44 E-C B$

3200

3200

3200

3200

3200

3200

3200

3200

3200

3200

3200

3200

CRUSSHIND INTEGRATEO= $1.128 E-C_{3} 6.319 E-03$

SEC/SO.M

$1 / 4$ 
, SAMPLIHG DOON TO $12800 \%$, NO TOHER SAHPLING.

SOUIPMENT OIFFICULTIES DURING ASSAY OF FLUORESCEIM FILTERS FDR GULM. 120OH. ANO $2200 \mathrm{~A}$ ARCS. 500OP ARC TRUNCATEO ON NORTH. 7000 ANO 12600 AKC OATA EXIRAPOLATEO SLIGHTLY TO NURTH.

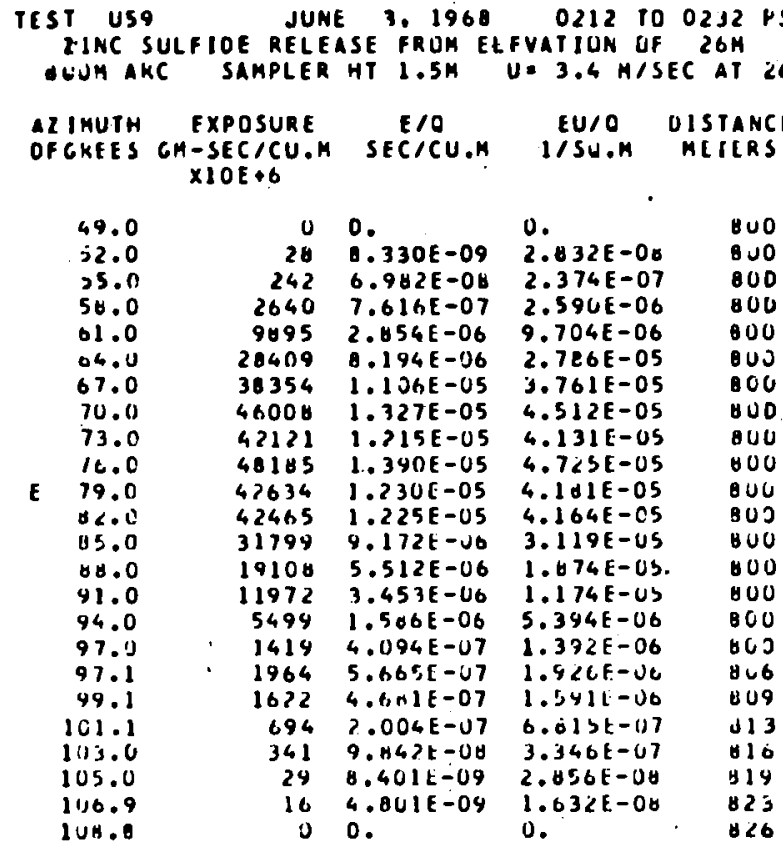

CRIJSSWIND INTEGRATHD. $4.502 E-03 \quad 1.531 \mathrm{~F}-02$ SECISU.H

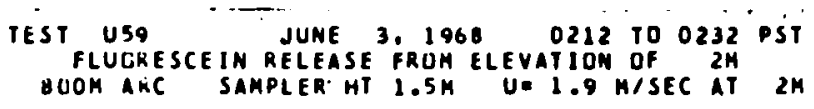 ALIMUTH EXPUSURE
DECKLES CM-SECICU.M SECICU.H IISO.H METERS $\times 10 E+6$

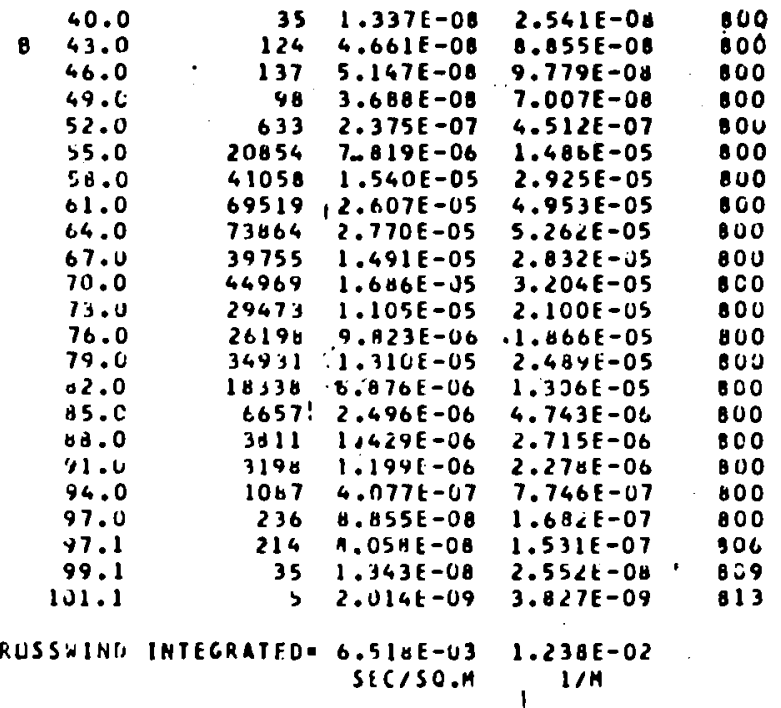

TEST USH JUNE 3, 1968 .0212 TO 0232 PST
FLUURESCEIN RELEASE FROM ELEVATION OF 24
12OOM ARC SAHPLER HT 1.5H U. 1.9 M/SEC AT $2 M$

AZIMUTH EXPOSURE EIO EJIO DISTANCE

DECKEES GH-SEC/CU.M SECICU.M I/SO.M HETERS $\times 10 E+6$

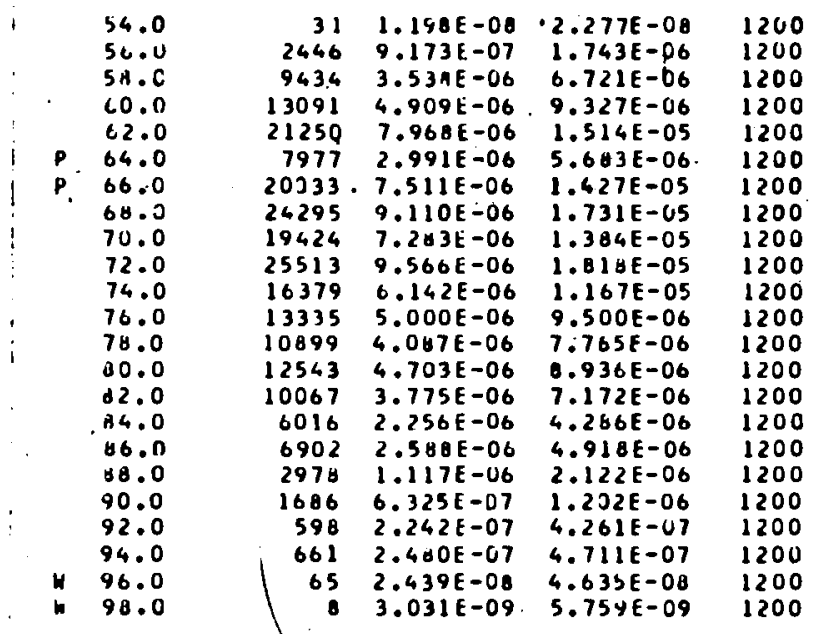

CRUSSHIND INTEGRATEDE $3.544 E-03 \quad 6.733 E-03$

$1 / n$ 

TEST US9
ZINC SULFIOE RELEASE FROM ELFVATION OF 226 PST $1600 \mathrm{M}$ ARC SAMPLER HT $1.5 \mathrm{M}$ U $3.4 \mathrm{M} / \mathrm{SEC}$ AT $26 \mathrm{M}$ AZIMUTH EXPOSURE EXO
DEGRES CH-SECICU.M SECICU.M IEU/O OISTANCE $\times 10 E+6$

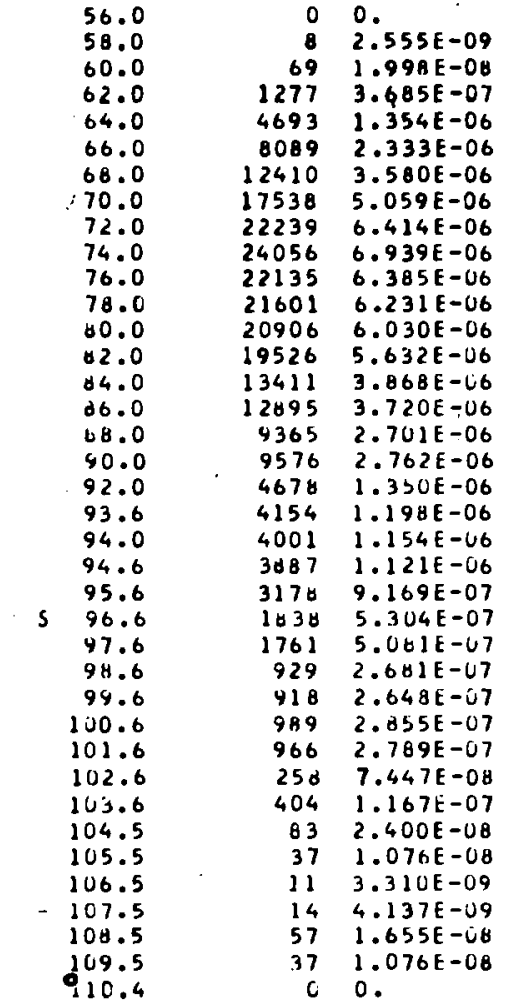

$\begin{array}{ll}0 . & 1600 \\ 8.609 E-09 & 1600 \\ 6.792 E-00 & 1600 \\ 1.253 E-06 & 1600 \\ 4.603 E-06 & 1600 \\ 7.933 E-06 & 1600 \\ 1.217 E-05 & 1600 \\ 1.72 U E-05 & 1600 \\ 2.181 E-05 & 1600 \\ 2.354 E-05 & 1600 \\ 2.171 E-05 & 1600 \\ 2.118 E-05 & 1600 \\ 2.05 U E-05 & 1600 \\ 1.915 E-05 & 1660 \\ 1.315 E-05 & 1600 \\ 1.265 E-05 & 1600 \\ 9.185 E-06 & 1600 \\ 9.392 E-06 & 1600 \\ 4.506 E-16 & 1600 \\ 4.074 E-06 & 1603 \\ 3.924 E-06 & 1600 \\ 3.812 E-06 & 1604 \\ 3.117 E-06 & 1606 \\ 1.003 E-06 & 1600 \\ 1.727 E-06 & 1610 \\ 9.116 E-07 & 1611 \\ 9.003 E-07 & 1613 \\ 9.706 E-07 & 1615 \\ 9.481 E-07 & 1617 \\ 2.532 E-07 & 1618 \\ 3.467 E-07 & 1620 \\ 8.159 E-08 & 1622 \\ 3.650 E-08 & 1623 \\ 1.125 E-08 & 1625 \\ 1.407 E-08 & 1627 \\ 5.627 E-00 & 1628 \\ 3.658 E-00 & 1630 \\ 0.0 & 1632\end{array}$

CRUSSHIND INTEGRATED= $3.776 E-03$ 1.284E-02 SFC/SO.M I/M

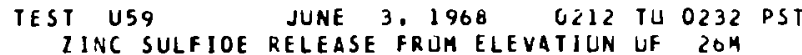
$2200 M$ ARC SAMPLER HT $1.5 \mathrm{M} U=3.4 \mathrm{M} / \mathrm{SEC}$ AT $26 \mathrm{M}$

AZIMUTH EXPOSURE E/O EU/O DISTANCE DEGREES GM-SECICU.M SECICU.M IISU.M MEIEKS $\times 10 E+6$

\begin{tabular}{|c|c|c|c|}
\hline & 60.0 & 0 & 0. \\
\hline & $\begin{array}{l}t 2.0 \\
t 4.0\end{array}$ & $\begin{array}{r}38 \\
711\end{array}$ & $\begin{array}{l}1.098 E-08 \\
2.051 E-07\end{array}$ \\
\hline & $6 t .0$ & 2622 & $7.565 \mathrm{E}-07$ \\
\hline & 68.0 & 3074 & $8.867 \mathrm{E}-07$ \\
\hline & $\begin{array}{l}70.0 \\
72.0\end{array}$ & $\begin{array}{r}7512 \\
12205\end{array}$ & $\begin{array}{l}2.167 \mathrm{E}-06 \\
3.521 \mathrm{E}-06\end{array}$ \\
\hline & 74.0 & 14065 & $4.057 \mathrm{E}-06$ \\
\hline & 76.0 & 10319 & $2.977 E-06$ \\
\hline c & 78.0 & 15216 & $4.389 \mathrm{E}-06$ \\
\hline & 80.0 & 10131 & $2.922 E-06$ \\
\hline & 82.0 & 5803 & $1.696 \mathrm{E}-06$ \\
\hline & .74 .0 & 5020 & $1.448 E-06$ \\
\hline 5 & $d \epsilon . U$ & 4355 & $1.256 t-06$ \\
\hline & HA.O & 5788 & $1.664 E-06$ \\
\hline & 90.0 & 6527 & $1.003 E-06$ \\
\hline & 42.0 & 5251 & $1.457 E-06$ \\
\hline$s$ & 94.0 & 3120 & 9.0 UOE - U7 \\
\hline & $9 i .0$ & 2133 & $6.154 E-67$ \\
\hline L & 92.0 & 1213 & $3.439 E-6 i 7$ \\
\hline & $1 \cup 0.0$ & 727 & $2.097 E-67$ \\
\hline & $1 \cup 2.0$ & 702 & $2.027 E-07$ \\
\hline & 134.6 & 267 & $7.711 E-\cup \theta$ \\
\hline [ & 106.0 & 230 & $6.637 t-0 t$ \\
\hline & 10.3 .0 & 179 & $5.105 E-08$ \\
\hline & 110.0 & 19 & $5.551 E-69$ \\
\hline & 112.0 & 4 & $1.207 E-09$ \\
\hline & 114.0 & 1 & $4.827 E-10$ \\
\hline
\end{tabular}

0

$3.734 E-00 \quad 2200$

$0.9755-07-2200$

$2.572 \mathrm{E}-00 \quad 2200$

$3.015 \mathrm{E}-06 \quad 2200$

$7.367 \mathrm{E}-06 \quad 2200$

$1.197 E-05$ <2UU

$1.379 \mathrm{E}-0 \mathrm{~S} \quad 2200$

$1.01 \angle E-C 5 \quad \angle 200$

$1.4921-C 3 \quad 2200$

y.935E-06 2200

$5.767 E-06 \quad 2200$

$4.9 \angle 3 E-06 \quad 2200$

$4.271 E-16 \quad 2200$

$5.676 \mathrm{E}-06 \quad 2200$

$0.401 \mathrm{E}-06 \quad 2200$

$4.954 t-10 \quad \angle 200$

3. $00 J E-00$ LCLU

2. LC $\angle E-06 \quad \angle 200$

$1.19 L \mathrm{E}-0 \mathrm{CO} \quad 2 \mathrm{LO}$

$7.131 E-07 \quad 2200$

Q.EYJt-U1 2200

$2.622 \mathrm{E}-47 \quad 220 \mathrm{O}$

$2.25 / 1-47 \quad 2200$

$1.756[-07 \quad 2200$

$1.807 E-00$
4.1031500 2200

$1.641 E-09 \quad 2200$

2200
TEST US9 FUNE 3 , 1968 JUNE 0212 TO 0232 PST $1600 M$ ARC SAMPLER HT $1.5 \mathrm{SM}$ U $1.9 \mathrm{M} / \mathrm{SEC}$ AT $2 \mathrm{M}$

AZIMUTH EXPOSURE E/O EU/O DISTANCE DEGREES GH-SEC/CU.A SECICU.M I/SO.M METERS $X \perp U E+6$

56.0
58.0
60.0
62.0
64.0
66.0
68.0
70.0
72.0
74.0
76.0
78.0
80.0
82.0
84.0
86.0
88.0
90.0
96.0
43.6
94.0
94.6
95.6
96.6
97.6
98.6
99.6

$\begin{array}{rr}25 & 9.607 E-09 \\ 598 & 2.246 E-07 \\ 3244 & 1.217 \mathrm{E}-06 \\ 7549 & 2.831 \mathrm{E}-06 \\ 12922 & 4.845 \mathrm{E}-06 \\ 16393 & 6.147 \mathrm{E}-06 \\ 19437 & 7.288 \mathrm{E}-06 \\ 20655 & 7.745 \mathrm{E}-06 \\ 26135 & 9.800 \mathrm{E}-06 \\ 8538 & 3.202 \mathrm{E}-06 \\ 5172 & 1.939 \mathrm{E}-06 \\ 11095 & 4.160 \mathrm{E}-06 \\ 6711 & 2.517 \mathrm{E}-06 \\ 7807 & 2.928 \mathrm{E}-06 \\ 5270 & 1.976 \mathrm{E}-06 \\ 3371 & 1.264 \mathrm{E}-06 \\ 2611 & 9.743 \mathrm{E}-07 \\ 1890 & 7.088 \mathrm{E}-07 \\ 510 & 1.913 \mathrm{E}-07 \\ 355 & 1.334 \mathrm{E}-07 \\ 184 & 6.935 \mathrm{E}-08 \\ 157 & 5.899 \mathrm{E}-08 \\ 235 & 8.821 \mathrm{E}-08 \\ 178 & 6.696 \mathrm{E}-08 \\ 62 & 2.356 \mathrm{E}-08 \\ 16 & 6.111 \mathrm{E}-09 \\ 2 & 1.063 \mathrm{E}-09\end{array}$

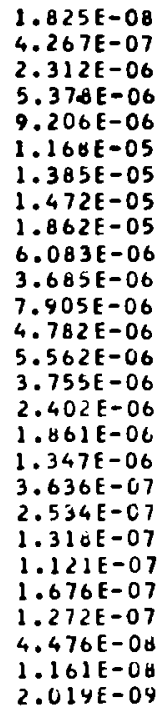

1600

1600

1600

1600

1600

1500

1600

1600

1600

1600

1600

1300

1600

1600

1600

1600

1600

1500

1600

1603

1600

1604

1606

1508

1610

1611

CRUSSHIND INTEGRATED $=3.360 \mathrm{E}-03 \quad 6.303 \mathrm{BE}-03$

1613

CRIISSHINU INTEGRATEU $=2.5 Y 45-03 \quad 0.0 \angle U E-U 3$

$\begin{array}{llllll}\text { IEST U59 JUNE } & 391968 & 0212 & \text { TO } 0232 & \text { PST } \\ \text { FLUORESCEIN RELEASE FROM ELEVATIUN DF } 2 M & \end{array}$ 22 COM ARC SAMPLER HT 1.5M UE 1.9 M/SEC AT $2 \mathrm{H}$

\section{AZIMUTH EXPOSURE EIO EUIO DISTANCE DECREES GM-SEC/CU.M SEC/CU.K I/SO.M HETERS $\times 10 \mathrm{~F}+6$}

\begin{tabular}{|c|c|c|c|c|c|}
\hline \multirow[t]{5}{*}{ B } & 49.0 & 33 & $1.259 E-08$ & $2.391 E-00$ & 2200 \\
\hline & 50.0 & 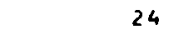 & $9.064 E-09$ & $1.722 \mathrm{E}-0 \mathrm{~b}$ & 2200 \\
\hline & 52.0 & 11 & $4.465 E-09$ & $8.522 E-09$ & 2200 \\
\hline & 54.0 & 4 & $1.550 E-09$ & $2.945 E-09$ & 2200 \\
\hline & $5 e .0$ & 13 & $5.190 E-09$ & $9.860 \mathrm{E}-09$ & 2200 \\
\hline \multirow[t]{2}{*}{2} & 50.0 & 3 & $1.148 E-09$ & $2.275 E-09$ & 2200 \\
\hline & 6\%. 0 & 28 & $1.003 \mathrm{E}-08$ & $2.057 \mathrm{E}-0 \mathrm{\theta}$ & 2200 \\
\hline \multirow[t]{3}{*}{$s$} & 62.0 & 938 & $3.518 E-07$ & $6.634 E-07$ & 2200 \\
\hline & 64.0 & 5302 & $1.988 \mathrm{E}-06$ & $3.778 E-06$ & 2200 \\
\hline & te.u & 8845 & $3.316 E-06$ & $0.301 E-06$ & 2200 \\
\hline 4 & 60.0 & 9793 & $3.672 \mathrm{E}-06$ & $0.977 E-06$ & 2200 \\
\hline \multirow[t]{3}{*}{0} & 70.0 & 9161 & $3.435 E-06$ & $6.527 E-06$ & 2200 \\
\hline & 72.0 & 12640 & $4.740 \vec{E}-06$ & $9.005 \mathrm{E}-06$ & 2200 \\
\hline & 74.0 & 7263 & $2.724 E-06$ & $5.175 E-06$ & 2200 \\
\hline 3 & 16.0 & 12956 & $4.858 E-06$ & $9.230 E-00$ & 2200 \\
\hline \multirow[t]{2}{*}{0} & 73.0 & 10426 & $3.909 E-06$ & $7.42 \cup E-06$ & 2200 \\
\hline & $d 0.0$ & 6947 & $2.605 E-06$ & $4.949 E-06$ & 2200 \\
\hline$t$ & 82.0 & 5302 & $1.98 \times E-06$ & $3.770 \mathrm{E}-06$ & 2200 \\
\hline$\theta$ & 14.0 & 4733 & $1.775 E-06$ & $3.372 E-06$ & 2200 \\
\hline 5 & 80.0 & 3211 & $1.2 V 4 E-U 6$ & $2.280 \mathrm{E}-06$ & 2200 \\
\hline \multirow[t]{3}{*}{4} & \&3.0 & 2154 & $H .0 \times 7 \mathrm{E}-07$ & $1.530 E=06$ & 2200 \\
\hline & 90.0 & 1232 & $4.620 E-C 7$ & 8.77 YE-07 & 2260 \\
\hline & 92.0 & 896 & $3.363 E-07$ & C.3S'UE -07 & 2200 \\
\hline$T$ & Y4.0 & 541 & $2.031 E-07$ & 3.OS UE-OT & $\angle 200$ \\
\hline L & 90.0 & 239 & A. $996 \mathrm{k}-08$ & $1.75 y E-67$ & 2260 \\
\hline $\mathrm{t}$ & 94.0 & 102 & $3.83 C E-08$ & $7.277 \mathrm{E}-\mathrm{Ua}$ & $\angle 200$ \\
\hline 0 & 100.0 & 49 & $1.857 t-08$ & $3.524 E-U d$ & $\angle 200$ \\
\hline \multirow[t]{2}{*}{ 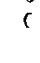 } & 102.0 & 42 & $1.611 \mathrm{E}-08$ & $3.001 E-08$ & 2200 \\
\hline & 164.0 & 25 & $9.417 E-09$ & $1.79 y E-0 y$ & 2200 \\
\hline$K L$ & $A$ & $160=$ & $\begin{array}{r}2.964 E-03 \\
\text { SEC/SO.M }\end{array}$ & $\begin{array}{c}5.631 \mathrm{E}-03 \\
1 / \mathrm{M}\end{array}$ & \\
\hline
\end{tabular}


TEST US9 JUNE 3,1968 O 0212 TU O232 PST ZINC SULF,IDE KELEASE FRUM ELEVATIUN UH $20 M$ $320 U 14$ AKC SAMPLER HT $1.5 \mathrm{M} \quad U=3.4 \mathrm{M} / \mathrm{SEC}$ AT $26 \mathrm{M}$ A2IMUTH EXPOSURE E/Q LU/O DISIAIACE DEGREES GM-SECICU.M SECICU.M IJSU.M MLIERS * $\times 10 E+6$

\begin{tabular}{|c|c|c|}
\hline 64.0 & 0 & 0. \\
\hline 06.0 & 3 & $1.006 E=04$ \\
\hline 64.0 & 33 & $9.533 E-09$ \\
\hline 70.0 & 482 & $1.391 E-07$ \\
\hline 72.7 & 3168 & $9.139 \mathrm{E}-07$ \\
\hline 73.7 & 2366 & $6 . \forall 26 \varepsilon-07$ \\
\hline .74 .7 & 3429 & $9.891 \mathrm{E}-07$ \\
\hline 75.7 & 5659 & $1.632 E-06$ \\
\hline 76.8 & 3762 & $1.085 E-06$ \\
\hline 77.8 & 4753 & $1.371 \mathrm{E}=06$ \\
\hline 78.8 & 5084 & $1.467 \mathrm{E}-06$ \\
\hline 79.8 & 4141 & $1.195 E-06$ \\
\hline d). 8 & 3680 & $1.062 E-06$ \\
\hline$B 1.8$ & 4367 & $1.260 E-66$ \\
\hline$\forall 2.8$ & 3806 & $1.049 E-06$ \\
\hline 09.8 & 7266 & $2.046[-06$ \\
\hline 74.8 & $546 \theta$ & $1.5776-06$ \\
\hline 85.8 & 3513 & $1.013 E-06$ \\
\hline 86.8 & $330 y$ & $9.546 E-07$ \\
\hline 87.8 & 3755 & $1.063 E-J 6$ \\
\hline 68.8 & 2487 & $7.174 E-07$ \\
\hline 89.8 & 3395 & $9.793 E-07$ \\
\hline 90.8 & 3478 & $1.003 E-06$ \\
\hline 91.6 & 4535 & $1.30 B E-0 E$ \\
\hline 92.8 & 1760 & $5.077 \varepsilon-07$ \\
\hline 93.6 & 1004 & $2.897 E-07$ \\
\hline 94.6 & 1005 & $2.901 E-07$ \\
\hline 95.8 & 1342 & $3.873 E-07$ \\
\hline 96.8 & 1491 & $4.301 E-07$ \\
\hline 97.8 & 1022 & $2.94 B E-\cup 7$ \\
\hline 98.8 & 626 & $1.8 \cup 7 E-07$ \\
\hline 99.8 & 1042 & $3.067 E-07$ \\
\hline 100.0 & 549 & $1.584 E-07$ \\
\hline 101.8 & 572 & $1.537 E-07$ \\
\hline 102.0 & 498 & $1.439 t-j 7$ \\
\hline 113.8 & 439 & $1.411 \mathrm{E}-07$ \\
\hline 104.9 & 352 & $1.015 E-07$ \\
\hline 105.8 & 183 & $5.243 \dot{i}-08$ \\
\hline 106.8 & 86 & $2.509 E-00$ \\
\hline 107.8 & 184 & $5.332 E-08$ \\
\hline 108.8 & 236 & $6.822 E-48$ \\
\hline 134.7 & 96 & $2.784 E-08$ \\
\hline 110.7 & 58 & $1.686 E-U 8$ \\
\hline 111.7 & 32 & $9.40+E-69$ \\
\hline 112.7 & $u$ & \\
\hline
\end{tabular}

$u$.

$3.241 E-U S \quad 3200$

$4.731 E-07 \quad 3200$

$3.107 t-66$ \$168

$2.321 E-06 \quad 3170$

$3.363 \mathrm{E}-06 \quad 3172$

$5.550 E-06 \quad 3173$

$3.690 E-06 \quad 3175$

$4.661 E-06 \quad \$ 177$

$4.987 t-06 \quad 3179$

$4.06 \angle E-00 \quad 3180$

$3.61 \cup E-36 \quad 3102$

$4.203 E-06 \quad 3184$

$3.735 E-06 \quad 3105$

$7.126[-60$ 316?

S. $30 \angle \mathrm{E}-\mathrm{GC}$ IIHY

$3.440 t-00$ 3141

$3.246 \mathrm{E}-U 6$ 3142

$3.643 E-66 \quad 3194$

$2.434 E-00 \quad 3146$

$3.330 E-O 6$ 3194

$3.411 E-60 \quad 3149$

$4.44 E E-06 \quad 3201$

$1.726 \mathrm{~F}-60 \quad 3<03$

$9.851 E-07 \quad 3205$

$9.064 \mathrm{E}-07 \quad 3200$

$1.317 E-06 \quad 3200$

$1.462 E-06 \quad 2216$

$1.002 \mathrm{E}-06 \quad 3212$

$6.14 b E-07$ \$213

$1.022 \mathrm{E}-00 \quad 3215$

$5.3 \cup S E-07$ 3217

S. $225 E-U 7$ 921

$4.8921-07 \quad 3220$

4.799E-07 2222

$3.452 \mathrm{t}-\mathrm{Ui} \quad 3224$

$1.79 Y E-67 \quad 3225$

$8.531 k-06 \quad 3227$

1.813E-U7 3224

2.319E-07 3230

$9.464 E-6 \theta \quad 3232$

$5.73 \angle E-U O \quad 3233$

$3.144 E-08$ 323:

4 .

237

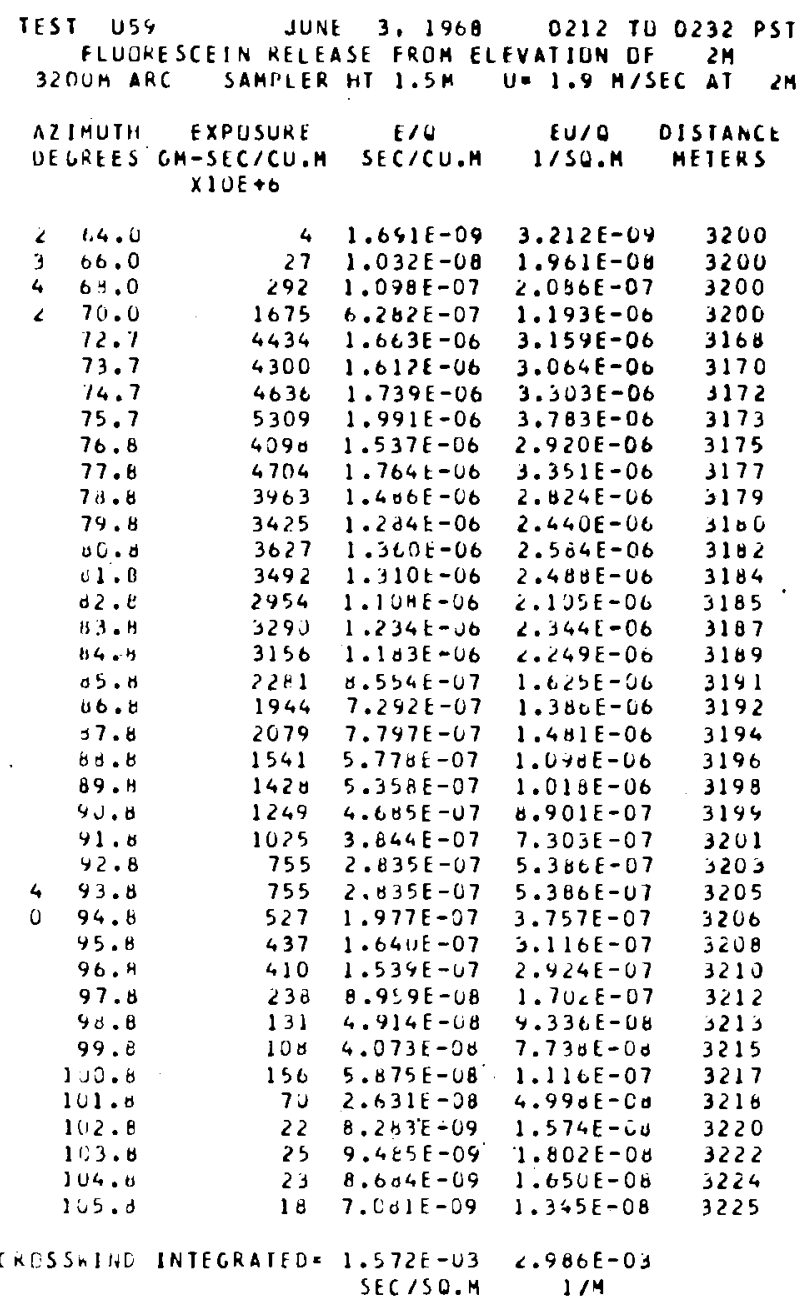

CRLSSWIHU INIEGRATED $=1.574 E-03$ 5.360L-CS

SEC/SO.M L

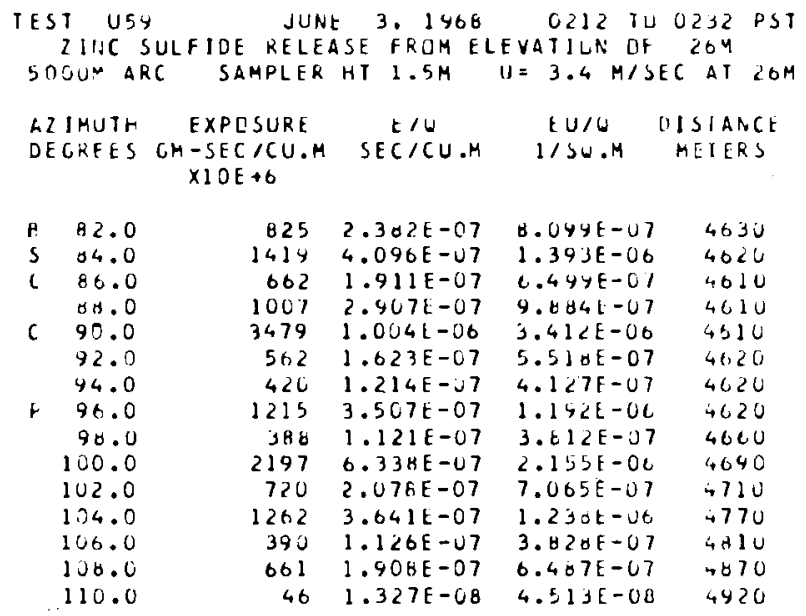

\begin{tabular}{|c|c|c|c|c|c|}
\hline & $\begin{array}{l}\text { T U5 } \\
\text { FLUD } \\
\text { COM A }\end{array}$ & $\begin{array}{l}\text { JUN } \\
\text { ESCEIN RELE } \\
\text { CSAMPLER }\end{array}$ & 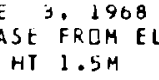 & $\begin{array}{l}0212 \text { JU } \\
\text { FVATION UE } \\
U=1.7 \mathrm{M} / \mathrm{S}\end{array}$ & $\begin{array}{rl}0232 & P S T \\
& 2 M \\
\text { SEC AT } & 2 M\end{array}$ \\
\hline & $\begin{array}{l}\text { JMUTh } \\
\text { GKEES }\end{array}$ & $\begin{array}{l}\text { EXPOSURE } \\
\text { GH-SEC/CU.H } \\
\text { XIUE }\end{array}$ & $\begin{array}{c}E / O \\
\text { SEC/CU.M }\end{array}$ & $\begin{array}{l}E U / 0 \\
1 / S O . M\end{array}$ & $\begin{array}{l}\text { DISTANCE } \\
\text { METERS }\end{array}$ \\
\hline B & $s<.0$ & 1201 & $4.505 E-07$ & $8.559 E-07$ & 4630 \\
\hline & $\$ 4.0$ & 1536 & $5.762 E-07$ & $1.095 E-C 6$ & 4620 \\
\hline c & 170.0 & $1<01$ & $4.5 \cup 5 \varepsilon-37$ & $8.55 Y E-07$ & 4610 \\
\hline & 69.0 & 1221 & $4.574 L-07$ & $\triangle .70 J E-07$ & 4010 \\
\hline ( & 90.0 & 1839 & $6.897 t-07$ & $1.310 E=06$ & 4610 \\
\hline & 92.0 & $127^{\circ}$ & $2.730 E-07$ & $5.106 E-07$ & 4620 \\
\hline & 94.0 & 491 & $1.042 E-07$ & $3.500 E-07$ & 4620 \\
\hline$r$ & 46.0 & 267 & $1.004 E-07$ & $1.907 E-07$ & 4020 \\
\hline & $9 H .0$ & 215 & $8.064 E-08$ & $1.532 E-07$ & 4560 \\
\hline & $1 \cup 0.0$ & 359 & $1.349 t-07$ & $2.503 \mathrm{E}-07$ & 4690 \\
\hline & $1,2.0$ & 221 & e.310E-UB & $1.579 E-07$ & 4710 \\
\hline & 104.0 & 247 & $9.296 E-68$ & $1.766 E-07$ & 4770 \\
\hline & 106.6 & 45 & $1.732 \mathrm{E}-08$ & $3.234 \mathrm{E}-0 \mathrm{E}$ & 4610 \\
\hline & 1 Us.0 & 45 & $1.702 t-00$ & $3.234 E-0 d$ & 4876 \\
\hline & 116.0 & 4 & $1.452 E-39$ & $3.51 \mathrm{dE}-09$ & 4720 \\
\hline
\end{tabular}

GRTSSLIIVO IHIEGRATFD: $\begin{gathered}5.8 J 2 E-04 \\ \text { SEC/SO.M }\end{gathered}$ 


\begin{tabular}{|c|c|c|c|c|c|}
\hline & $\begin{array}{l}112.0 \\
114.0 \\
116.0\end{array}$ & $\begin{array}{l}5 \\
9 \\
0\end{array}$ & $\begin{array}{l}1.948 E-69 \\
2.655 E-09 \\
0 .\end{array}$ & $\begin{array}{l}4.923 E-09 \\
9.026 \mathrm{E}-09 \\
0 .\end{array}$ & $\begin{array}{l}4990 \\
5080 \\
5020\end{array}$ \\
\hline \multicolumn{2}{|c|}{ CROSSHIND } & INTEGRATEDO & $\begin{array}{l}7.170 E-04 \\
\text { SEC/SO.H }\end{array}$ & $\begin{array}{c}2.438 E-03 \\
1 / K\end{array}$ & \\
\hline & \multicolumn{3}{|c|}{4} \\
\hline \multicolumn{3}{|c|}{$\begin{array}{l}\text { TEST USY } \\
\text { ZINC SULFIDE R } \\
\text { TODOM ARC SAMP }\end{array}$} & $\begin{array}{l}\text { E } 3,1968 \\
\text { ASE FROH EI } \\
\text { HT } 1.5 \mathrm{H}\end{array}$ & $\begin{array}{rl}0212 & T U \\
\text { FVATIUN OF } \\
U=3.4 \mathrm{~N} / \mathrm{SE}\end{array}$ & $\begin{array}{l}02 \$ 2 \text { PS } \\
264 \\
C A T 26 F\end{array}$ \\
\hline \multicolumn{2}{|c|}{$\begin{array}{l}\text { AZ,IHUTH } \\
\text { DEGKEESS }\end{array}$} & $\begin{array}{l}\text { EXPOSURE } \\
\text { GH-SEC/CU.M } \\
\text { XIOE } \$ 6\end{array}$ & $\begin{array}{l}\text { E/O } \\
\text { SEC/CU.H }\end{array}$ & $\begin{array}{l}\text { EU/O D } \\
\text { I/SH.M }\end{array}$ & $\begin{array}{l}\text { DISIANCE } \\
\text { METERS }\end{array}$ \\
\hline E & 70.0 & 14 & $4.103 E-09$ & $1.395 E-00$ & 0 \\
\hline $\begin{array}{l}E \\
E\end{array}$ & $\begin{array}{l}72.0 \\
74.0 \\
76.0\end{array}$ & $\begin{array}{l}28 \\
48 \\
75\end{array}$ & $\begin{array}{l}0.045 E-09 \\
1.400 E-08 \\
2.184 E-08\end{array}$ & $\begin{array}{l}2.744 E-08 \\
4.754 F-06 \\
7.426 E-06\end{array}$ & $\begin{array}{r}0 \\
0 \\
6280\end{array}$ \\
\hline \multirow{4}{*}{0} & 78.0 & 105 & $3.053 \mathrm{E}-08$ & $1.038 t-07$ & 6300 \\
\hline & $\begin{array}{l}00.0 \\
82.0 \\
44.0\end{array}$ & $\begin{array}{l}157 \\
384 \\
898\end{array}$ & $\begin{array}{l}4.537 E-0 B \\
1.110 E-07 \\
2.547 E-07\end{array}$ & $\begin{array}{l}1.543 E-07 \\
3.775 E-07 \\
6.813 E-07\end{array}$ & $\begin{array}{l}6400 \\
6490 \\
6500\end{array}$ \\
\hline & 80.0 & 346 & $9.992 \mathrm{E}-08$ & $3.397 E-07$ & 6500 \\
\hline & $\forall$ ४. 0 & 547 & 1.57AE -07 & $5.367 E-0.7$ & 6440 \\
\hline \multirow[t]{2}{*}{0} & 90.0 & 473 & $1.36 \mathrm{~h}$ & $4.644 E-07$ & 6500 \\
\hline & & 1672 & 4.826 & $1.64 J E-66$ & 6490 \\
\hline 4 & 94.0 & 79 & $2.305 E-02$ & $7.830 \mathrm{E}-0 \mathrm{~B}$ & 0500 \\
\hline & 96.0 & $\begin{array}{r}46 \\
191\end{array}$ & $\begin{array}{l}1.352 E-08 \\
5.527 E-08\end{array}$ & $\begin{array}{l}4.595 \mathrm{E}-06 \\
1.679 \mathrm{E}-07\end{array}$ & $\begin{array}{l}6510 \\
6520\end{array}$ \\
\hline & 100.0 & 210 & $6.002 E-0 B$ & $2.060 E-07$ & 0560 \\
\hline 0 & 102.0 & 241 & $6.963 E-08$ & $2.367 E-07$ & 0610 \\
\hline & 104.0 & 337 & & & 0650 \\
\hline & 106.0 & 134 & $3.874 E-08$ & $1.317 E-07$ & 6720 \\
\hline & & 71 & $2.063 \mathrm{E}=$ & $7.010 E-08$ & 6800 \\
\hline & 110.0 & 39 & $1.134 E-0 B$ & $3.657 \mathrm{E}-00$ & 7000 \\
\hline & ii 2.0 & 11 & $3.258 E-09$ & $1.100 E-00$ & 7300 \\
\hline & 114.0 & 9 & $2.775 E-09$ & $9.437 E-69$ & 7210 \\
\hline & 116.0 & 0 & 0. & 0. & .7220 \\
\hline \multicolumn{2}{|c|}{ CROS + INL } & NTEGRATFD= & $\begin{array}{l}3.960 E-04 \\
\text { SFCISO. }\end{array}$ & $\begin{array}{c}1.346 E-03 \\
1 / M\end{array}$ & \\
\hline
\end{tabular}

TEST U59 JUNE 3, 1968 0212 TU 0232 PST ZINC SULFIDE RELEASE FROM ELFVAT IUN OF ZUM $12800 \mathrm{M}$ AKC SAMPLER HT $1.5 \mathrm{M} U=3.4 \mathrm{M} / \mathrm{SEC}$ AT $20 \mathrm{M}$

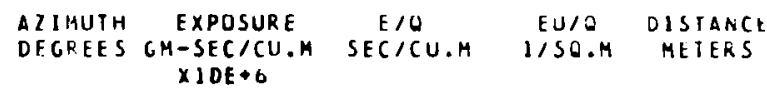

GKIISSAIND: INTEGRATFU. $1.027 E-U 4$ 3.4BUL-GL

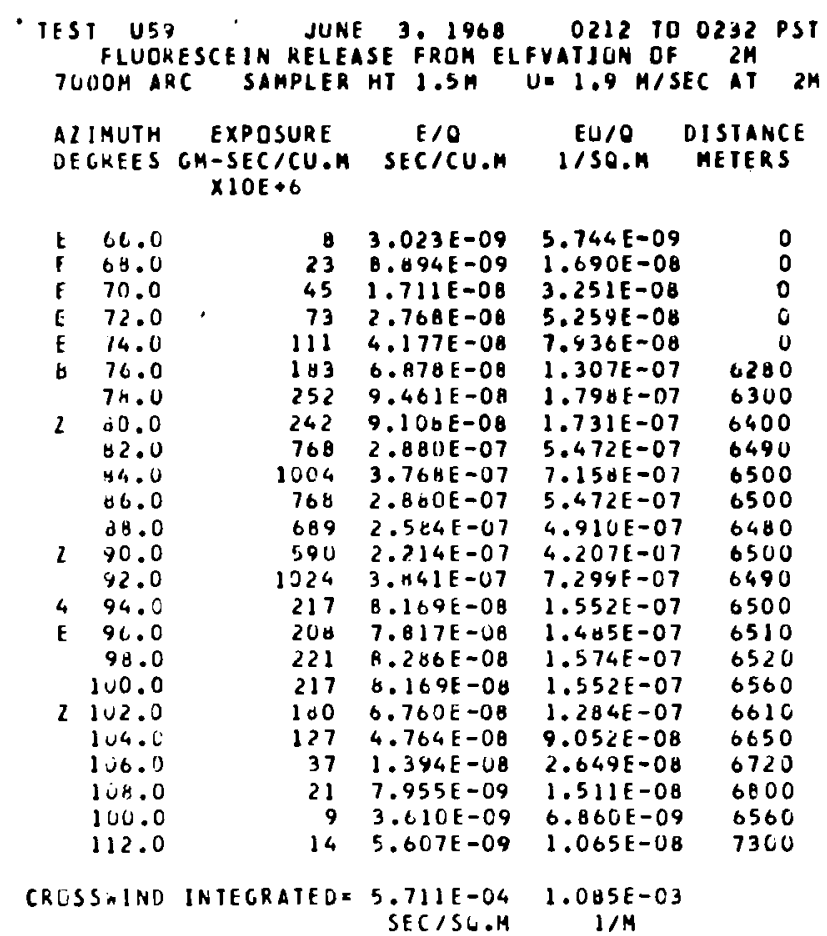

TEST U5Y JUKE 3, 1968 Q212 TO 0232 PST FLUOEESCEIN KELEASE FROM ELFVATION OF ZM $12 A C U M A R C$ SAMPLER HT $1.5 M \quad U=1.9$ H/SEC AT $2 \mathrm{H}$

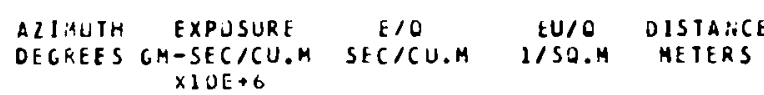

E 06.0

E 07.0

L 88.0

E 69.0

E 90.0

P 91.0

32.0

93.0

94.0

95.0

90.0

97.6

93.0

$\rightarrow 9.8$

130.0

$1 \mathrm{J1.0}$

102.6

1.19 .0

livi.

5 lus.o

1un.i

I)

icy.c

110.0

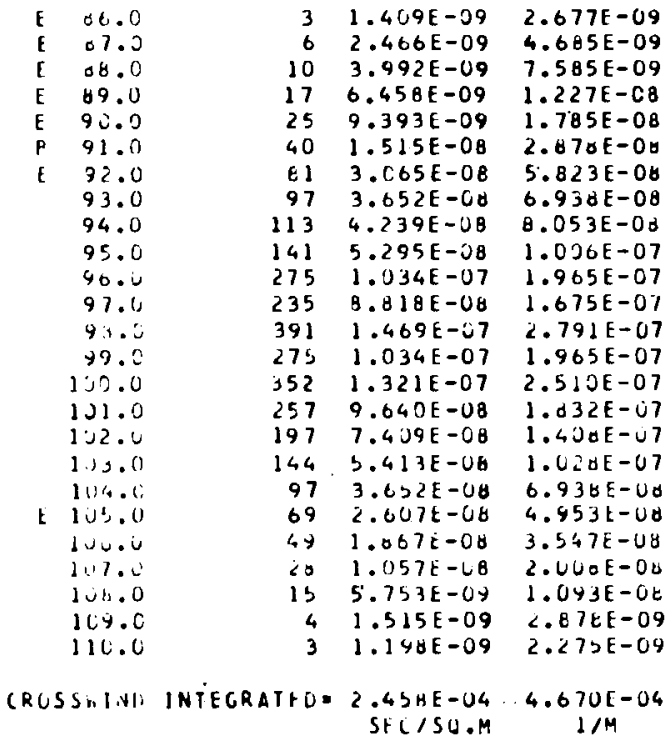

12800 12800 12800 12900 12300 $1230 J$ 12800 12860 12960 12800 12800 12000 12800 12800 12900 12800 12000 12000 $1<b 00$ 1200 $1 \angle 500$ 12000 12606 12800 12200 
SAMELING UOOM TO 12 GOUM, NO TOHER SAMFLING. MUCH LUST UN FILTERS. MANY FIELD PRUBLEMS, MINIMAL EFFORT MADE TU "SURRECT" DATA DUE IU THE IARGE NUMBIK OFF UNCERIAIHIIES. VERY SKEWED DISIRIBUTIIIN. MDST ARCS ARE TRUNCATEU IIN NIIRTH FNO.

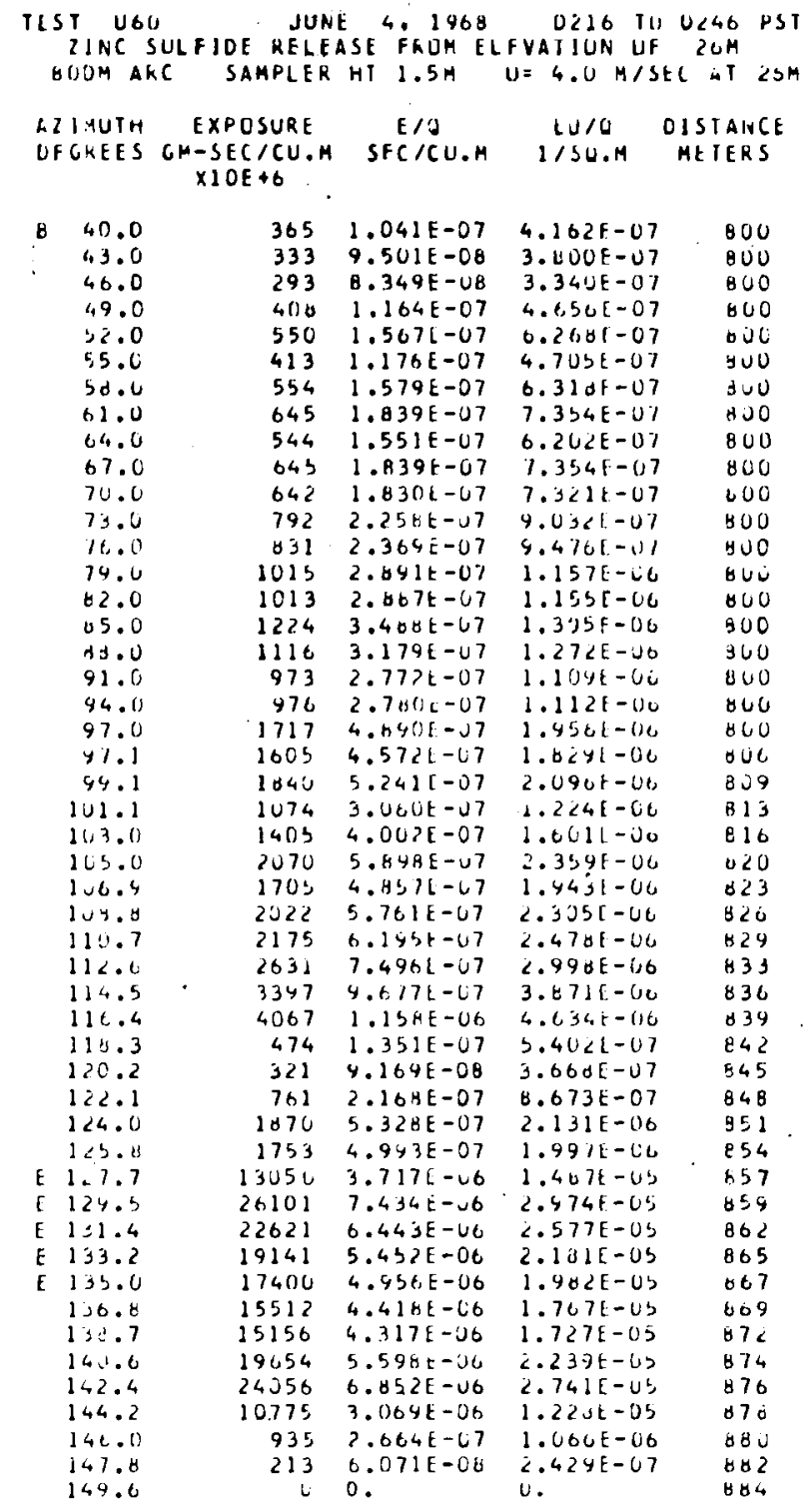

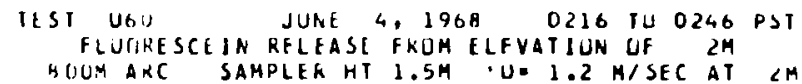

AZIMUIH EXPOSURE
DEGKFES GM-SECICU.M SECICU.M IISU.H MEIEKS
XIOE+G.

\section{$40.0 \quad 2565 \quad 8.749 E-07 \quad 1.050 E-06 \quad 800$}

$43.0 \quad 2634 \quad 4.981 E-07 \quad 1.070 E-06 \quad 800$

$4 C .0 \quad 2974 \quad 1.014 E-06 \quad 1.217 E-06 \quad B 00$

$49.0 \quad 2906 \quad 4.409 E-U 7$ 1.184E-06 810

4U6, $1.306 E-06 \quad 1.663 E-06 \quad 800$

$\begin{array}{lllll}4.0 & 4404 & 1.5021-06 & 1.802 \mathrm{E}-06 & 800 \\ 16.0 & 519 \mathrm{H} & 1.773 \mathrm{E}-06 & 2.127 \mathrm{E}-06 & 100\end{array}$

$\begin{array}{lllll}16.0 & 5198 & 1.773 E-06 & 2.127 E-06 & 100 \\ 6.0 & 4971 & 1.095 E-06 & 2.034 E-06 & 000\end{array}$

$04.0 \quad 5079 \quad 2.005 E-D 6 \quad 2.406 E-06 \quad 800$

$67.0 \quad 7460 \quad 2.546 t-U 6 \quad 3.050 E-60 \quad B 60$

(U.) $42001.437 t-06 \quad 1.7101-06 \quad 800$

$7310 \quad 54251.85010-60 \quad 2.220 \mathrm{E}-06$ 800

$76.0 \quad 5379 \quad 2.005 \mathrm{E}-66 \quad 2.466 \mathrm{E}-06 \quad \mathrm{HOO}$

$79.0 \quad 7406 ? .346 \mathrm{~F}-06 \quad 3.056 \mathrm{E}-06$ YUU

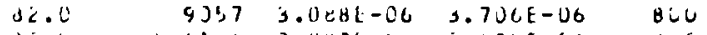

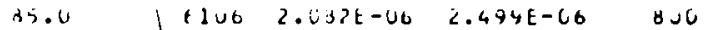

OH.1 $\quad 0376 \quad 2.356 L-06 \quad 3.427 E-06$ प0O

\#1.0 $\quad 1064 \mathrm{t} \quad 3.630 \mathrm{E}-06 \quad 4.356 \mathrm{E}-06$ 800

94.0 7O14 2.312E-06 2.87UE-00 800

$97.11 \quad 14061+.74 \div 5-33 \quad 5.753 E-06 \quad \forall 60$

$\begin{array}{lllll}97.1 & 14641 & 4.9421-66 & 5.99 J E-66 & B U 0 \\ 94.1 & 12304 & 4.2231-00 & 5.007 t-06 & \text { Büy }\end{array}$

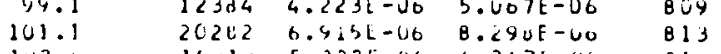

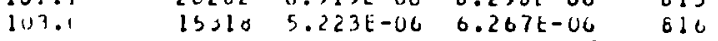

1.5.0 $35325 \quad 1.2 J 4 E-65 \quad 1.443 E-05 \quad 0<0$

116.4 (48OJ) $1.004 E-05 \quad 1.447 \mathrm{~F}-0 \mathrm{~S}-82 \mathrm{~J}$

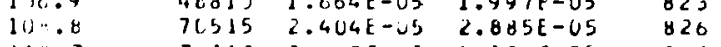

HE.? 7H112 $2.603 E-U 5$ 3.19EE-05 829

$112.0 \quad 41412$ 3.683E-05 $\$ .649 \mathrm{E}-\mathrm{US}$ B33

$114.5 \quad 97647 \quad 3.329 \mathrm{E}-\mathrm{J}^{5} \quad 3.995 \mathrm{E}-0 \mathrm{~S}$

$\begin{array}{rrrrr}116.4 & 202555 & 6.906 E-55 & 6.287 E-05 & 839 \\ 113.3 & 1335 & 4.553 E-07 & 5.463 E-07 & 842\end{array}$

$\begin{array}{lllll}113.3 & 1335 & 4.553 E-07 & 5.463 E-07 & 842 \\ 120.7 & 7266 & 7.728 E-07 & 9.273 E-07 & 845\end{array}$

$\begin{array}{lllll}12.1 & 5013 & 1.709 E-46 & 2.051 \mathrm{E}-06 & 840\end{array}$

$124.0 \quad 4457 \quad 1.530 E-16 \quad 1.036 E-60 \quad 851$

$12502589.027 \mathrm{E}-67 \quad 1.057 \mathrm{E}-06 \quad 854$

E $127.7 \quad 4411 \quad 1.544 E-06 \quad 1.805 E-C 6 \quad 857$

$12 \% .5 \quad 1247 \quad 2.812 \mathrm{E}-06 \quad 3.375 \mathrm{E}-60 \quad 859$

$131.4 \quad 12760 \quad 4.351 \mathrm{t}-06 \quad 3.221 \mathrm{E}-06 \quad 862$

$133.2 \quad$ IOU25 $6.146 \mathrm{E}-06 \quad 7.37 \supset \mathrm{E}-00 \quad 065$

$\begin{array}{lllll}135.0 & 26299 & 0.907 \mathrm{E}-06 & 1.076 \mathrm{E}-0 \mathrm{~L} & 607\end{array}$

$136.0 \quad 30012 \quad 1.051 \mathrm{E}-05 \quad 1.261 \mathrm{E}-05 \quad 964$

$133.7 \quad 23291 \quad 7.441 \mathrm{E}-06 \quad 9.524 \mathrm{E}-00 \quad 072$

$140.6 \quad 16521 \quad 5.633 t-06 \quad$ C.76UE-06 874

$\begin{array}{lllll}142.4 & 3659 & 1.248 \mathrm{E}-06 & 1.457 \mathrm{E}-06 & 876\end{array}$

$146.5 \quad 1054 \quad 0.3<31-07 \quad 7.5061-07 \quad 878$

$\begin{array}{lllll}14000 & 138 & 4.738 t-05 & 3.600 E-08 & 300\end{array}$

CRISS.1ITH INTEGRATEO= 9.379E-03 1.15LE-02

SFCISO.H N I/M

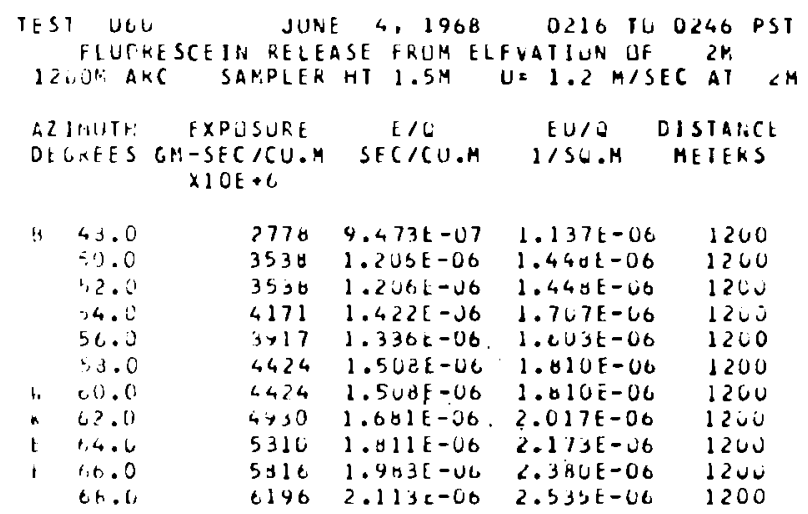

TEST U6J JUNE 4, 1968 OU 216 IU 0245 HST IIHC SULFIDE RELEASE FRUH ELFVATION OF $26 \mathrm{M}$ $1200 M A R C$ SAMPLER HI $1.5 \mathrm{MH} U=4.0 \mathrm{M} / \mathrm{SEC}$ AI $20 \mathrm{M}$

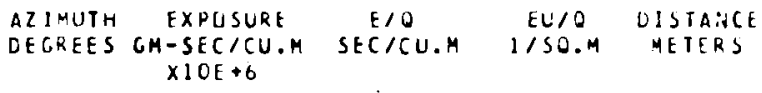

\begin{tabular}{|c|c|c|c|c|c|}
\hline \multirow[t]{6}{*}{8} & 48.0 & 116 & $3.326 E-08$ & $1.331 E-07$ & 1200 \\
\hline & 30.0 & 148 & $4.2 \angle 1 \mathrm{E}-\mathrm{OH}$ & $1.6 B E[-07$ & 1200 \\
\hline & 22.0 & 161 & $4.50 \forall E-0 B$ & 1.H3+E -0 & 1200 \\
\hline & 1,4.0 & 173 & $4.955 E-68$ & $1.982 E-07$ & 1200 \\
\hline & 56.0 & 102 & $5.207 E-08$ & $2.083[-07$ & 1200 \\
\hline & 54.0 & 139 & $3.909 t-00$ & $1.507 E-07$ & 1200 \\
\hline & 00.0 & 148 & $4.221 \mathrm{E}-\mathrm{OB}$ & $1.000 E-07$ & 1200 \\
\hline & 62.0 & 275 & $5.001 \mathrm{E}-08$ & $2.000 E-07$ & $12 v 0$ \\
\hline E & 04.0 & 193 & $5.506 E-08$ & $2.20<E-67$ & 1200 \\
\hline & $O B .0$ & 217 & $6.194 \mathrm{E}-0 \mathrm{E}$ & 2.47 OE -07 & 1200 \\
\hline & 69.0 & 237 & $67 E-08$ & $7 E-07$ & 1200 \\
\hline
\end{tabular}




\begin{tabular}{|c|c|c|c|}
\hline & 70.0 & 307 & $0.763 E-08$ \\
\hline & 72.0 & 208 & $5.942 E-08$ \\
\hline & 74.0 & 263 & $7.50 ? E-0 R$ \\
\hline & 76.0 & 361 & $1.030 E-07$ \\
\hline & 78.0 & 369 & $1.053 E-07$ \\
\hline & 80.0 & 331 & $9.451 k-08$ \\
\hline & $\begin{array}{l}82.0 \\
84.0\end{array}$ & $\begin{array}{l}372 \\
459\end{array}$ & $\begin{array}{l}1.060 E-07 \\
1.30 B E-07\end{array}$ \\
\hline & 36.0 & 513 & $1.464 E-07$ \\
\hline & 84.0 & 599 & $1.707 \mathrm{E}-07$ \\
\hline & 90.0 & 563 & $1.606 \mathrm{E}-07$ \\
\hline & 42.0 & 557 & $1.587 E-07$ \\
\hline & 94.0 & 791 & $2.255 E-67$ \\
\hline & 96.0 & 452 & $1.289 E-67$ \\
\hline & 98.13 & 491 & $1,399 E-07$ \\
\hline & 100.0 & 1055 & $3.005 E-07$ \\
\hline & 102.0 & $1<39$ & $3.531 E-07$ \\
\hline & 104.0 & 1171 & $3.338 E-07$ \\
\hline & 106.0 & 1544 & $4.348 E-07$ \\
\hline E & 1ขค.0 & 1610 & $4.5 \mathrm{dHL}-07$ \\
\hline & 110.0 & 2467 & $7.024 \mathrm{E}-07$ \\
\hline 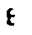 & 112.0 & 2496 & $7.112 t-07$ \\
\hline & 114.0 & 2672 & $\theta .1 \forall 1 E-07$ \\
\hline $\mathbf{E}$ & 116.0 & 3624 & $1.032 \mathrm{E}-06$ \\
\hline & 119.0 & 4367 & $1.244 E-C 6$ \\
\hline 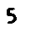 & 120.0 & 3466 & $9.874 t-67$ \\
\hline & $1<2.0$ & 7925 & $2.257 \mathrm{E}-06$ \\
\hline E & 124.0 & 13531 & $3.054 E-06$ \\
\hline$t$ & 126.0 & 18525 & $5.276 E-06$ \\
\hline$t$ & $1 \angle 4 . C$ & 23501 & $6.694 E-06$ \\
\hline b. & 190.0 & 25960 & $7,344 t-136$ \\
\hline & 132.0 & 20136 & $5.735 t-v t$ \\
\hline 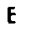 & 134.0 & 14497 & $4.124 E-06$ \\
\hline & $13 l .0$ & 10785 & $3.072 k-06$ \\
\hline & $13 B .0$ & 8171 & $2.3 \angle 7 E-0 b$ \\
\hline 5 & 140.0 & 6916 & $1.970 E-06$ \\
\hline$s$ & 142.0 & 3542 & $1.009 E-06$ \\
\hline 0 & 144.0 & 163 & $4.657 \mathrm{E}-06$ \\
\hline 9 & 146.6 & 0 & $n$. \\
\hline
\end{tabular}

CRISSSINL INTEGRATEO= $2.244 \ddot{E}-03 \quad 0.471 \mathrm{H}-U 3$

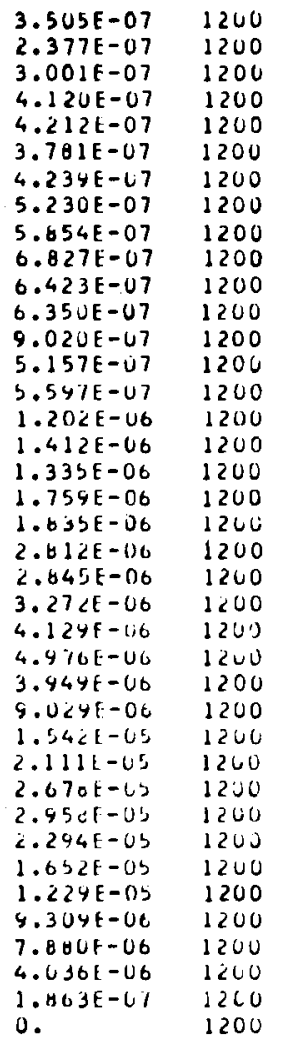

$1 / M$

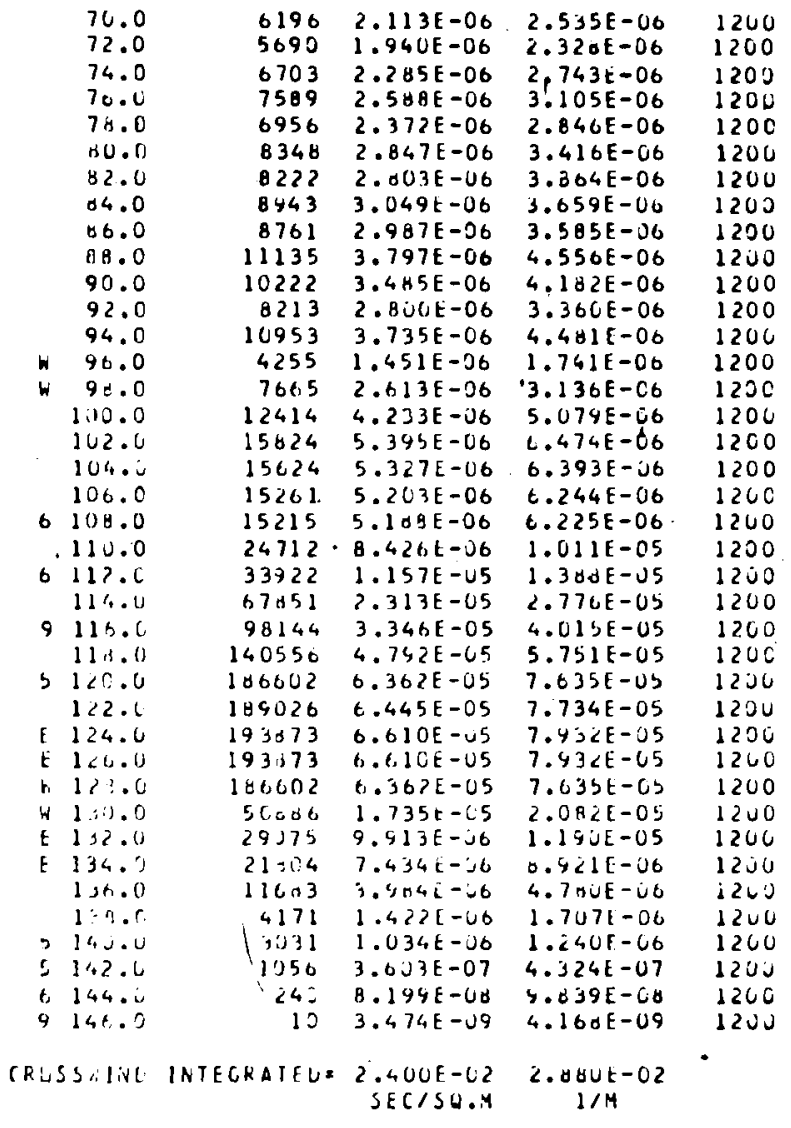

TEST U6U JUNE 4, 196 U 0216 TU $0 \angle 46$ PST 2INC SULFIDE RELEASE FROM ELEVAIILN DH 204 16CJM ARC. SAMPLER HT I.5M U $=4.0 \mathrm{M} / \mathrm{SEC}$ AT 264

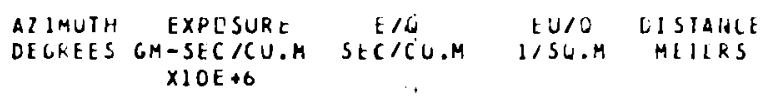

\begin{tabular}{|c|c|c|c|c|}
\hline 49.0 & 50 & $1.075 E-0 H$ & $0.697 E-00$ & 1000 \\
\hline 3.0 .0 & 35 & $1.009 E-08$ & 4.0130E-0४ & 1600 \\
\hline 52.0 & 70 & $1.996 E-08$ & $7.483 E-08$ & 1600 \\
\hline 54.0 & 44 & $1.422 \mathrm{E}-08$ & $5.689 \mathrm{k}-0 \mathrm{0}$ & 1600 \\
\hline 56.0 & 52 & $1.491 E-08$ & $5.965 t-00$ & 1600 \\
\hline 58.0 & 55 & $1.583 k-08$ & $6.332 \mathrm{E}-.06$ & 1600 \\
\hline 60.0 & 63 & $1.012 \varepsilon-0 \theta$ & $7.244 E-00$ & 1600 \\
\hline 42.0 & HE & $2.523 L-08$ & 1. CUYt-07 & 1060 \\
\hline$c \% .0$ & 95 & 2.73 OE-UE & $1.0925-07$ & 1600 \\
\hline 66.0 & 107 & $3.051 E-08$ & $1.2201-07$ & 3600 \\
\hline $6 \mathrm{H.0}$ & 120 & $3.4 \perp \mathrm{HE}-\cup \mathrm{O}$ & $1.307 \mathrm{E}-07$ & 1600 \\
\hline $7 \because .0$ & 116 & $3.3 \angle 6 E-U \theta$ & $1.331 \mathrm{E}-07$ & 1600 \\
\hline 72.6 & 156 & $4.450 E-08$ & 1.7 HCE -07 & 1600 \\
\hline 74.0 & 156 & $4.450 t-08$ & 1.7 UUt $-\cup 7$ & 1600 \\
\hline $7 \in .6$ & 128 & $3.670 k-08$ & $1.460[-67$ & 1600 \\
\hline $7+.0$ & 185 & $5.276 F-\cup B$ & $2.1111-07$ & 1600 \\
\hline 00.0 & 184 & $5.253 t-v 8$ & $2.1018-07$ & 1500 \\
\hline 02.0 & 205 & $5.8511 E-U 8$ & $2.36 \mathrm{JF}-07$ & 1600 \\
\hline 04.0 & 211 & h.J $33 E-U d$ & $2.413 k-j 1$ & 1000 \\
\hline 46.0 & 233 & $6.633 L-08$ & $0.0611-07$ & 1060 \\
\hline$t t .0$ & 313 & $8.424 t-J \theta$ & $3.570 E-07$ & 1600 \\
\hline 90.0 & 350 & $9.979 E-08$ & $3.992 \mathrm{E}-\mathrm{U} 2$ & 1000 \\
\hline 92.0 & 410 & $1.170 E-07$ & $4.680 E-07$ & ISUC \\
\hline $93 . t$ & 610 & $1.73 \partial E-07$ & $0.4 b 18-07$ & 1603 \\
\hline 94.0 & 414 & $1.181 E-07$ & $4.726 \mathrm{t}-07$ & 1600 \\
\hline 94.6 & 361 & $1.007 E-07$ & $4.344 E-07$ & dous \\
\hline 95.6 & 507 & $1.447 E-07$ & $5.787 \mathrm{E}-07$ & 1500 \\
\hline 90.6 & 601 & $1.712 \mathrm{E}-07$ & $6.640 E-07$ & 1608 \\
\hline 97.6 & 667 & $1.900 E-07$ & $7.60<E-07$ & 1610 \\
\hline$=8.6$ & 721 & $2.054 E-07$ & $0.2100-07$ & 1611 \\
\hline 99.6 & 613. & $1.746 \mathrm{E}-07$ & $0.985 E-07$ & 1613 \\
\hline 100.6 & 745 & $2.123 \mathrm{E}-47$ & $0.4921-07$ & $161 \%$ \\
\hline
\end{tabular}

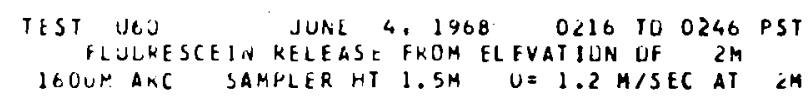

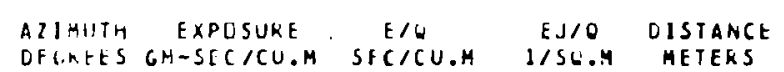
$X] \mathrm{OE}+6$

\begin{tabular}{|c|c|c|c|c|}
\hline $4 \pi .0$ & 1547 & $5.276 \mathrm{E}-07$ & $0.332 \mathrm{E}-07$ & 1600 \\
\hline $5 n .0$ & 1509 & $5.147 \mathrm{E}-07$ & $6.176 F-07$ & 1600 \\
\hline 52.0 & 1319 & $4.499 E-07$ & $5.399 E-07$ & 1600 \\
\hline $5,4.0$ & 1201 & $4.370 E-07$ & $5.244 E-07$ & 1600 \\
\hline bli.c & 1509 & $5.147 E-07$ & $0.17 G E-07$ & 1600 \\
\hline $3: . c$ & 2117 & $7.219 \mathrm{E}-07$ & $0.662 \mathrm{E}-07$ & 1600 \\
\hline 60.0 & 1727 & $6.5716-07$ & $7.0 \forall S E-07$ & 16uo \\
\hline$\because 00$ & $15 \mathrm{H}$ & $5.4 \cup 6 E-07$ & $6.487 E-U 7$ & 1603 \\
\hline (4..) & 2155 & $7.34 B E-U 7$ & $0.81 d E-U 7$ & 1600 \\
\hline $6,6.0$ & $2<04$ & $7.737 E-07$ & $9.284 E-01$ & 1600 \\
\hline 6.00 & 2447 & $8.514 t-67$ & $1.022 \mathrm{E}-00$ & ievo \\
\hline 70.6 & 2155 & $7.34 d E-07$ & $B .010 E-07$ & 1600 \\
\hline ial & 2610 & $8.402 k-07$ & $1.056 E-00$ & 1600 \\
\hline 14.3 & 2231 & $7.067 E-07$ & $9.124 E-C 7$ & 1000 \\
\hline 76.0 & 2535 & $8.643 E-\cup 7$ & $1.037 E-06$ & 1600 \\
\hline 73.0 & 2345 & $7.996 \mathrm{E}-07$ & $4.595 \mathrm{t}-07$ & 1600. \\
\hline 45.6 & 3035 & $1.205 t-06$ & $1.446 t-0 t$ & 1600 \\
\hline$=2.0$ & 2307 & $7.806 E-07$ & $9.43 \times E-67$ & 1060 \\
\hline 64.0 & 2269 & $7.797 E-07$ & $9.204 E-07$ & 1300 \\
\hline (ii). 0 & 2762 & $9.4<\cap E-U 7$ & 1.13 $3 \cup E-\cup 0$ & 1600 \\
\hline$n 3.0$ & 4041 & $1.3706-06$ & $1.654 E-06$ & 166.0 \\
\hline$\$ 0.0$ & 3155 & $1.07 h E-06$ & $1.291 E-00$ & 1600 \\
\hline 92.0 & 7960 & $2.716 \mathrm{E}-06$ & $3.25 y E-06$ & 1600 \\
\hline 93.6 & 4454 & $1.519 k-06$ & $1.023 t-00$ & 1063 \\
\hline 94.0 & 4041 & $1.378 E-06$ & $1.654 \mathrm{E}-00$ & 1600 \\
\hline 44.6 & 3412 & $1.164 t-66$ & $1.340 E=00$ & 1604 \\
\hline 95.6 & 3809 & $1.299 E-06$ & $1.55 Y E-06$ & 1606 \\
\hline 96.6 & 5198 & $1.772 \mathrm{E}-\mathrm{U6}$ & $2.127 \mathrm{E}-06$ & 1608 \\
\hline$y 7 . t$ & 5347 & $1.323 E-U 6$ & $2.100 E-00$ & 1610 \\
\hline 40.6 & 3016 & $1.02 b t-16$ & $1.234 E-06$ & 1611 \\
\hline 99.6 & 6091 & $2.077 \mathrm{E}-06$ & $2.49 \angle E-00$ & 1513 \\
\hline & 4157 & & & 161 \\
\hline
\end{tabular}




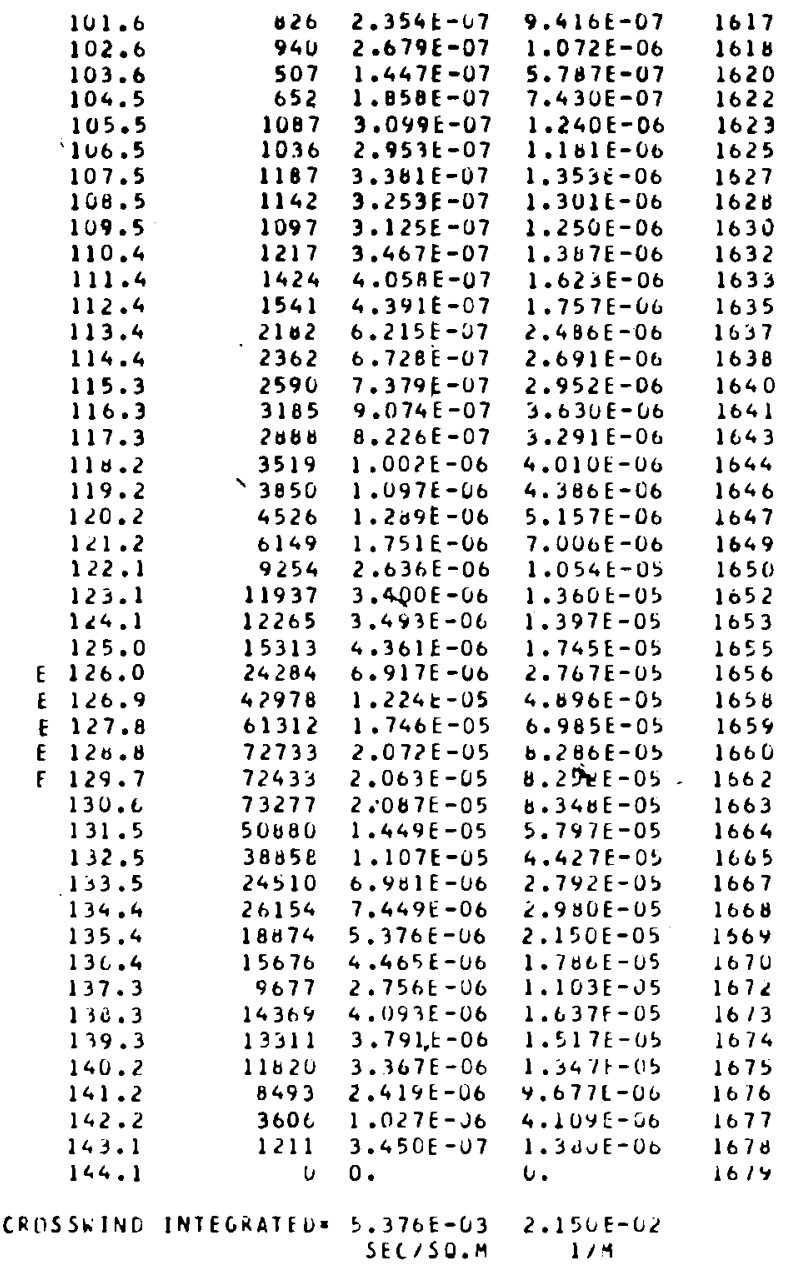

TEST U6O JUNE 4, 1968 O216 1110246 PST Z1RC SULFIDE RELEASE FROM ELFVATIUN DF ZOM Z2OOM ARC SAMPLER HT $1.5 \mathrm{M} U=4.0 \mathrm{M} / \mathrm{SEC}$ AT $26 \mathrm{M}$ AZIMUTH EXPOSURE
OECREES GM-SEC/CU.M SEC/CU.M IISU.M MISTANCE $\times 1 O E+6$

\begin{tabular}{|c|c|}
\hline & 48.0 \\
\hline & S.:0 \\
\hline & 52.0 \\
\hline & 54.0 \\
\hline & 56.0 \\
\hline & $\$ 8.0$ \\
\hline & 60.2 \\
\hline & $1, \angle C O$ \\
\hline & $8,4.0$ \\
\hline & te. 0 \\
\hline & 70.0 \\
\hline & 72.0 \\
\hline & 74.0 \\
\hline & 76.0 \\
\hline & 74.0 \\
\hline & 00.0 \\
\hline & 02.0 \\
\hline & $04 . \mathrm{C}$ \\
\hline & 10.0 \\
\hline & 40.0 \\
\hline & 42.0 \\
\hline & Y4.0 \\
\hline & 40.0 \\
\hline & $4: n$ \\
\hline
\end{tabular}

\begin{tabular}{|c|c|c|c|}
\hline 7 & $2.145 \mathrm{E}-09$ & $0.579 \mathrm{E}-07$ & 2200 \\
\hline 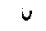 & 0 . & u. & 2200 \\
\hline 4 & 0 . & u. & 2200 \\
\hline i & 0 . & u. & 2200 \\
\hline 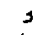 & $1.072[-09$ & $4.29 \cup E-09$ & 2200 \\
\hline a & 0. & u. & 2200 \\
\hline 30 & $0.644 \mathrm{E}-04$ & 3.47 YE-Ud & $\angle 200$ \\
\hline 16 & $4.766 E-09$ & $1.907 E-08$ & 2200 \\
\hline 29 & $8.341 \mathrm{E}-04$ & $3.39 \in E-O n$ & $\therefore 200$ \\
\hline 20 & $5.958 \mathrm{t}-6 \mathrm{y}$ & $2.303 E-60$ & $\angle 200$ \\
\hline 20 & $5.7 \angle 0 t-04$ & $\angle . \angle N \otimes E-C_{0}$ & $\angle 200$ \\
\hline 29 & $8.460 E=09$ & $3.384 \mathrm{E}-60$ & 2260 \\
\hline 17 & $5.005 t-09$ & $2.002[-0 t$ & $\angle 200$ \\
\hline 19 & $5.401 E-\cup 9$ & $2.193 E-U 8$ & 2200 \\
\hline & 0 . & u. & 2260 \\
\hline 76 & 2.1 & 6.77 & $\angle 200$ \\
\hline 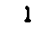 & $4.766 \bar{c}-10$ & $1.937 E-09$ & $\angle 200$ \\
\hline & 0 . & u. & 2200 \\
\hline 14 & 4.17 & $1.660 E-\cup d$ & 2200 \\
\hline 25 & $7.150 E-09$ & $2.060 E-00$ & 2200 \\
\hline & 0 . & u. & 2200 \\
\hline 0. & $2.336 E-U \theta$ & 9.34 & 2260 \\
\hline 97 & $2.776 \mathrm{E}-08$ & 1.11 & 2200 \\
\hline 1 & $2.804 E-08$ & $1.15, E-U 7$ & 2200 \\
\hline & $4.760 \mathrm{E}$ & $1.9 \cup 7 \mathrm{E}$ & $22 \mathrm{GJ}$ \\
\hline$c$ & 0 . & 0. & 2200 \\
\hline
\end{tabular}

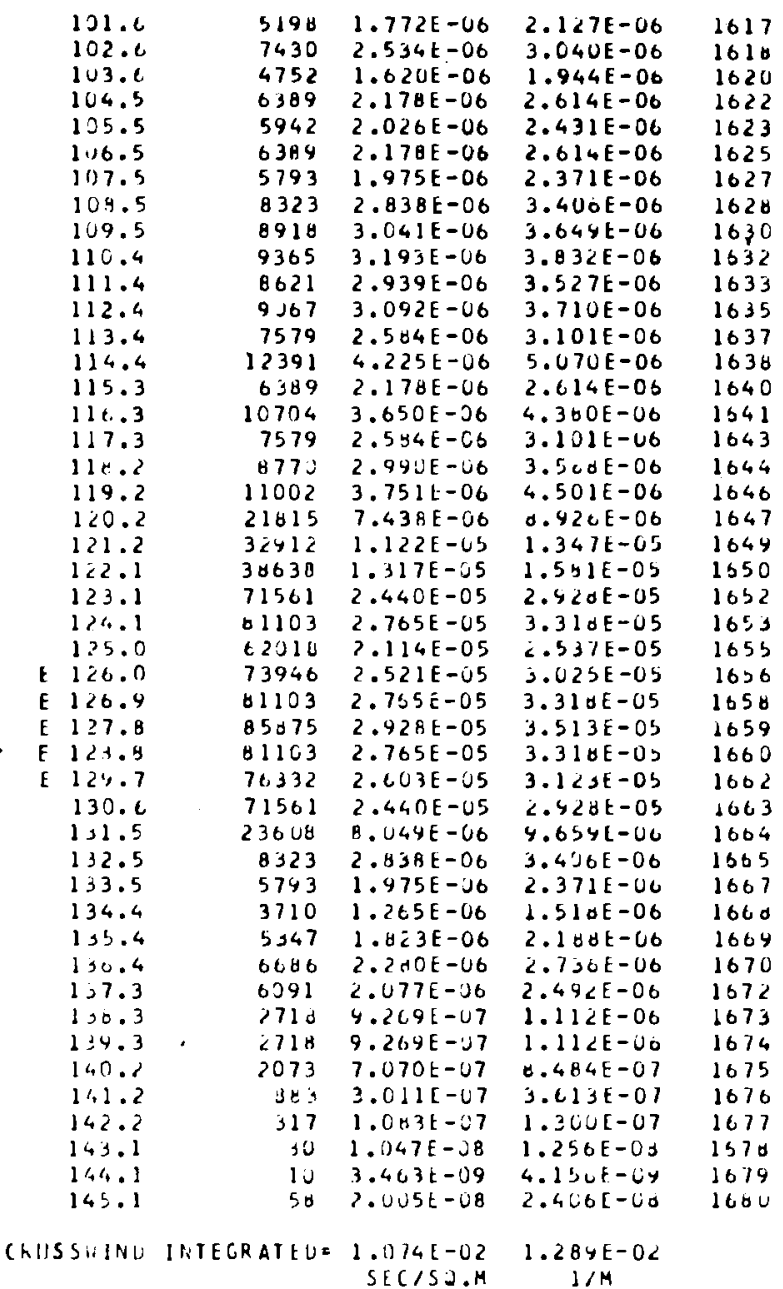

TEST UGU JUNE 4. 1968 O216 TO U246 PST FLULLESCEIN RELEASE FRLM ELEVATIUN CIF $2 \mathrm{M}$ ZZOUM AKC SAMPLER HT $1.5 \mathrm{M} U=1.2 \mathrm{M} / \mathrm{SEC}$ AT 24

HLIPISTH EXPOSURE E/O EJ/O UISIANCE DEGKFES GM-SECICU.M SECICU.M I/SO.M METERS $\times 10 E+0$

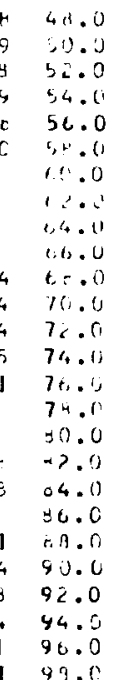

\begin{tabular}{|c|c|}
\hline 302 & $1.030 t-37$ \\
\hline 239 & $8.168 E-08$ \\
\hline & $9.983 E-J 8$ \\
\hline 145 & $6.673 t-68$ \\
\hline $3 \times 6$ & d. $317 t-07$ \\
\hline 392 & $1.340 E-07$ \\
\hline 491 & $1.676 t-07$ \\
\hline 496 & $1.6 .9<E-37$ \\
\hline 242 & $4.372[-0]$ \\
\hline 343 & $2.477:-07$ \\
\hline 012 & $2.771 E-07$ \\
\hline 411 & $1.404 \mathrm{E}-07$ \\
\hline 143 & $2.877 E-07$ \\
\hline & $5.33 \mathrm{HE}-11$ \\
\hline 63 & $3.625 t-07$ \\
\hline , & 1. DC UE -10 \\
\hline 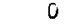 & $3.310 t-10$ \\
\hline 412 & $2.771 \mathrm{E}-07$ \\
\hline 6.5 & $2.077 E-07$ \\
\hline & \\
\hline 648 & $2.344 E-07$ \\
\hline 261 & $9.5 \subset 8 E-68$ \\
\hline & \\
\hline & $4.271 E-10$ \\
\hline & $1.00+E-10$ \\
\hline
\end{tabular}

$1.236 E-07 \quad 2260$ $9.0 J 1 E-00 \quad 2200$ $1.193 t-07 \quad 2200$

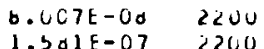
2200 $1.6 J \theta E-07 \quad 2200$ $2.011 \mathrm{E}-\mathrm{C} 2 \quad 2200$ $2.031 \mathrm{E}-\mathrm{C} .7$ 22U $5.2400-07 \quad 2200$ $3.453 E-07 \quad 2200$ $3.325 E-07 \quad 2200$ $1.635 E-072200$ $2.453 E-072200$ $0.466 E-112200$ $4.350 \mathrm{LE}-072200$ $1.281 F-10 \quad 2200$ $3.472 E-10 \quad 2200$ $3.325 E-07 \quad 2200$ $2.492 \mathrm{E}-\mathrm{0} 7 \quad 220 \mathrm{O}$

2. $619 E-07$ $1.151 \mathrm{E}-07$ $4.594 E-07$ $5.125 E-10 \quad 2200$ $1.281 E-10$
2200 2200 2200 2200 


\begin{tabular}{|c|c|c|c|c|c|}
\hline 5 & $\begin{array}{l}100.0 \\
102.0 \\
104.0\end{array}$ & $\begin{array}{l}311 \\
147 \\
358\end{array}$ & $\begin{array}{l}\text { H. H65E-08 } \\
4.2 U 6 E-08 \\
1.021 E-07\end{array}$ & $\begin{array}{l}3.546 E-07 \\
1.663 E-07 \\
4.065 E-07\end{array}$ & $\begin{array}{l}\angle 200 \\
2200 \\
2200\end{array}$ \\
\hline 1 & $\begin{array}{l}106.0 \\
108.0\end{array}$ & 674 & $0.921 E-07$ & $\begin{array}{l}0.003 \mathrm{E}-07 \\
7.603\end{array}$ & $\begin{array}{r}2200 \\
12200\end{array}$ \\
\hline & $\begin{array}{l}108.0 \\
110.0\end{array}$ & $\begin{array}{l}674 \\
893\end{array}$ & $\begin{array}{l}1.921 \mathrm{E}-07 \\
7.544 \mathrm{E}-07\end{array}$ & $\begin{array}{l}7.603 E-07 \\
1.010 E-06\end{array}$ & 2200 \\
\hline 0 & $i 12.0$ & 0 & $2.303 E-10$ & $9.533 t-10$ & $<200$ \\
\hline 6 & 114.0 & 645 & $1.840 E-07$ & $7.359 E-07$ & 2200 \\
\hline & $\begin{array}{l}116.0 \\
118.0\end{array}$ & 2603 & $\begin{array}{l}7.414 E-07 \\
0 .\end{array}$ & & $\begin{array}{l}2200 \\
2205\end{array}$ \\
\hline 0 & $\begin{array}{l}120.0 \\
122.0\end{array}$ & $\begin{array}{l}179 \\
830\end{array}$ & $\begin{array}{l}5.124 E-0 B \\
2.364 E-07\end{array}$ & $\begin{array}{l}2.050 E-07 \\
9.456 E-0.7\end{array}$ & $\begin{array}{l}2200 \\
2200\end{array}$ \\
\hline 1 & 124.0 & 23 & $6.673 E-09$ & $2.669 \mathrm{E}-08$ & 2200 \\
\hline & 126.0 & 13212 & $3.763 . E-06$ & $1.505 E-05$ & 2200 \\
\hline$\theta$ & 128.0 & 12526 & $3.568 \mathrm{E}-06$ & $1.427 E-05$ & 2200 \\
\hline 1 & 130.0 & 36 & $1.004 E-08$ & $4.337 E-08$ & 2200 \\
\hline 4. & 132.0 & 36013 & $1.040[-05$ & $4.194 t-05$ & $<200$ \\
\hline 1 & 134.0 & 3270 & $9.197 E-07$ & $3.679 E-06$ & 2260 \\
\hline 7 & 136.0 & 6096 & $1.736 \mathrm{E}-06$ & $6.946 E-06$ & 2200 \\
\hline & 136.0 & 927 & $2.642 E-07$ & $1.057 E-06$ & 2200 \\
\hline & 140.0 & 5092 & $1.450 E-06$ & $5.802 E-06$ & 2200 \\
\hline 1 & 342.0 & 0 & & & 2200 \\
\hline & 144.0 & 7 & $2.145 E-09$ & $8.579 E-09$ & 2200 \\
\hline & 146.0 & 0 & 0 . & & 2200 \\
\hline CROS & SSWIND & IIEGRATFDE & $\begin{array}{r}1.864 E-03 \\
\text { SFC/SO.M }\end{array}$ & $\begin{array}{c}7.455 E-03 \\
1 / M\end{array}$ & \\
\hline
\end{tabular}

TEST U6O JULA 4. 1968 O2216 TO C246 PST 2IISC SULFIOE RELEASE FROM ELFVAIIUN OF $26 \mathrm{M}$ $3200 M$ ARC SAMPLER HT $1.5 \mathrm{~K}$ UF $4.0 \mathrm{H} / \mathrm{SEC}$ A $26 \mathrm{M}$

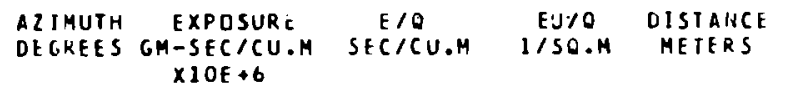

\begin{tabular}{|c|c|c|c|c|}
\hline 59.0 & $\begin{array}{l}0 \\
1\end{array}$ & 0.7 & 0. & $\begin{array}{l}3200 \\
3200\end{array}$ \\
\hline $\begin{array}{l}60.0 \\
62.0\end{array}$ & $\begin{array}{l}1 \\
0\end{array}$ & $\begin{array}{l}4.766 t-10 \\
0 .\end{array}$ & $\begin{array}{l}1.9 \cup 7 E-09 \\
0 .\end{array}$ & $\begin{array}{l}3200 \\
3200\end{array}$ \\
\hline 64.0 & 5 & $1.668 t-09$ & $0.673[-09$ & 3200 \\
\hline 66.0 & 5 & $1.668 E-09$ & $6.673 E-C 9$ & 3200 \\
\hline 68.0 & 6 & $1.787 t-09$ & $7.150 E-09$ & 3200 \\
\hline 70.0 & 1 & $4.766 E-10$ & $1.907 E-09$ & 3200 \\
\hline 72.7 & 25 & $7.317 E-69$ & $2.927 E-0 H$ & 3168 \\
\hline 73.7 & 35 & $1.015 E-08$ & $4.035 E-08$ & 3170 \\
\hline 74.7 & 42 & $1.219 \mathrm{E}-08$ & $4.87 d E-08$ & 3172 \\
\hline 75.7 & 34 & 9.75 BE -09 & $3.90<E-08$ & 9173 \\
\hline 76.8 & 51 & $1.463 E-08$ & $5.853 E-08$ & 3175 \\
\hline 77.8 & 25 & $7.317 \mathrm{E}-09$ & $6.927 \mathrm{E}-0 \mathrm{~d}$ & 3177 \\
\hline 78.8 & 47 & $1.341 \mathrm{E}-0 \mathrm{~B}$ & $5.360 E-08$ & 3179 \\
\hline 79.8 & 42 & $1.219 E-08$ & $4.870 t-08$ & 3180 \\
\hline$B \cup .8$ & 17 & $4.878 E-09$ & $1.451 \mathrm{E}-0 \mathrm{~d}$ & 3162 \\
\hline B1. 8 & 22 & $6.51) 4 E-09$ & $2.60<E-00$ & 318 \\
\hline 02.8 & 74 & R. $536 \mathrm{E}-09$ & $3.415 \mathrm{E}-08$ & 318 \\
\hline 03.8 & 21 & $0.1097 E-09$ & $2.434 E-00$ & 3167 \\
\hline 84.8 & 54 & $1.545 \mathrm{E}-00$ & $6.179 \mathrm{~F}-08$ & 3189 \\
\hline 85.8 & 24 & $6.910 E-09$ & $2.764 \mathrm{E}-0 \mathrm{~B}$ & 3191 \\
\hline 86.8 & 59 & $1.707 \mathrm{E}-68$ & $6.629 E-06$ & 3192 \\
\hline 87.8 & 47 & $1.341 E-68$ & $5.360 E-U 8$ & 3194 \\
\hline 68. 8 & 61 & $1.748 \mathrm{E}-68$ & $6.492 E-08$ & 3196 \\
\hline 89.8 & 74 & $2.114 E-60$ & $4.455 E-08$ & 3198 \\
\hline 90.8 & 82 & $2.35 \times E-U \theta$ & $9.431 \varepsilon-08$ & 3195 \\
\hline 91.6 & 74 & $2.114 E-08$ & $0.455 E-06$ & 3201 \\
\hline 92.6 & 100 & 3.UOSE- & $1.236 E-C 7$ & 32,3 \\
\hline 93.8 & 131 & $3.740 E-08$ & $1.496 E-07$ & 3205 \\
\hline 94.8 & $1>9$ & $3.644 E-08$ & $1.430 E-07$ & 3206 \\
\hline 95.8 & 131 & $3.740 c-08$ & $1.496 E-07$ & 3208 \\
\hline 96.8 & 176 & $5.040 E-08$ & $2.016 E-07$ & 321 \\
\hline 97.8 & 169 & 4. . $37 E-08$ & $1.935 E-07$ & 321 \\
\hline 90.8 & 232 & $6.626 E-08$ & $2.650 E-07$ & 321 \\
\hline 99.8 & 229 & $0.544 E-00$ & $2.618 \varepsilon-07$ & 321 \\
\hline 100.0 & 172 & $4.919 E-08$ & $1.967 E-07$ & 3217 \\
\hline 101.8 & 101 & $2.886 \mathrm{E}-08$ & $1.154 E-07$ & 321 \\
\hline 102.8 & 211 & $6.016 E-08$ & $2.406 \mathrm{E}-07$ & 322 \\
\hline 103.8 & 391 & $1.114 E-07$ & $4.455 E-07$ & 322 \\
\hline 104.8 & 313 & $8.943 \mathrm{E}-0 \mathrm{~B}$ & $3.577 E-07$ & 322 \\
\hline 105.8 & 560 & 1.59 AE-07 & $6.390 E-07$ & 322 \\
\hline 106.8 & 472 & $1.345 E-07$ & $5.382 E-07$ & 3227 \\
\hline 107.8 & 792 & $2.256 \mathrm{E}-07$ & $9.024 \mathrm{E}-07$ & 322 \\
\hline
\end{tabular}

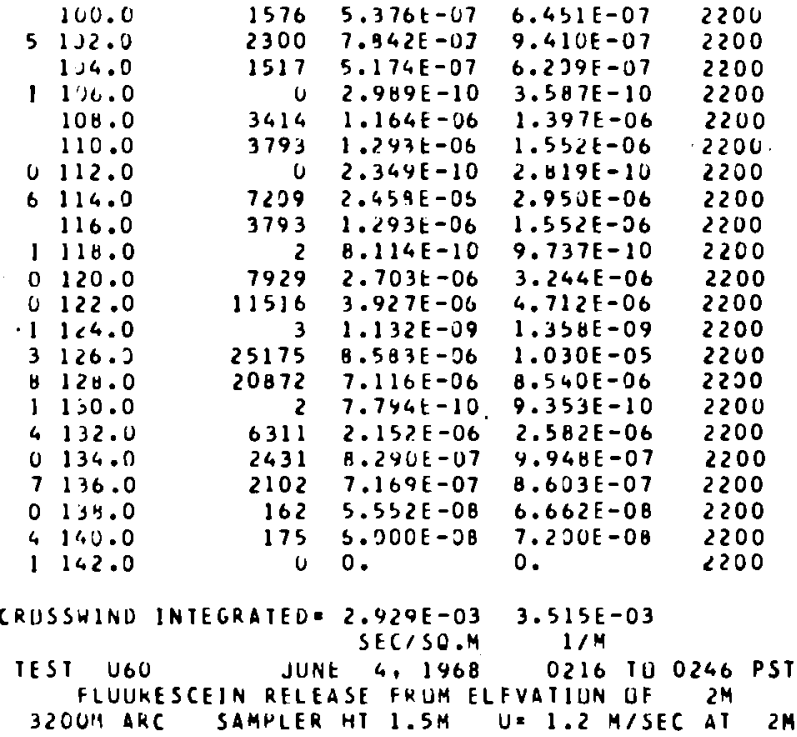

AZIMUIH EXPOSURE E/O EU/O OISTANCE DEGREES GM-SEC/CU.M SECICU.M I/SO.M METERS $X 10 E+6$

50.0

52.0

$7 \quad 34.0$

656.0

59.0

$$
\text { to. } 0
$$

562.0

64.0

06.0

68.0

70.0

72.7

73.7
74.7

75.7

76.8

77.8

77.8

78.8
19.8

BO. 3

$\Delta 1.8$

82.6

82.6
83.8

84.6

85.9

d6.

37.8

08.8

44.8

90.8

S $91 . \mathrm{A}$

92.8

93.8

44.8

95.8

96.5

97.8

F 48.8

99.8

100.0

101.9

102.8

103.6

104.8

106.8

106.8

\begin{tabular}{|c|c|c|c|}
\hline $\begin{array}{l}129 \\
160 \\
11\end{array}$ & $\begin{array}{l}4.409 E-08 \\
5.477 E-08 \\
3.876 E-08\end{array}$ & $\begin{array}{l}5.291 E-08 \\
6.572 E-08 \\
4.651 E-0 B\end{array}$ & $\begin{array}{l}3200 \\
3200 \\
3200\end{array}$ \\
\hline $\begin{array}{r}135 \\
96\end{array}$ & $\begin{array}{l}4.623 E-06 \\
3.34>E-08\end{array}$ & $\begin{array}{l}5.54 \forall E-08 \\
4.010 E-08\end{array}$ & $\begin{array}{l}3200 \\
3200\end{array}$ \\
\hline 129 & $4.409 E-08$ & $5.2 \geqslant 1 E-08$ & 3200 \\
\hline 132 & $4.516 E-08$ & $5.419 E-O \theta$ & 3200 \\
\hline $\begin{array}{l}166 \\
213\end{array}$ & $\begin{array}{l}5.671 E-03 \\
7.272 E-08\end{array}$ & $\begin{array}{l}6.829 E-06 \\
8.75 U E-08\end{array}$ & $\begin{array}{l}3200 \\
3200\end{array}$ \\
\hline 163 & $5.584 E-08$ & $6.701 E-08$ & 3200 \\
\hline 213 & $7.292 t-08$ & 8.75UE-OU & 3200 \\
\hline 132 & $4.516 E-08$ & $5.419 \mathrm{E}-08$ & 3200 \\
\hline 246 & $8.413 E-08$ & $1.010 E-07$ & 3168 \\
\hline $\begin{array}{l}447 \\
524\end{array}$ & $\begin{array}{l}1.533 E-07 \\
1.78 \theta E-37\end{array}$ & $\begin{array}{l}1.840 E-07 \\
2.146 E-07\end{array}$ & $\begin{array}{l}3170 \\
3172\end{array}$ \\
\hline 460 & $1.570 k-07$ & $1.884 E-07$ & 3173 \\
\hline 503 & $1.715 t-07$ & $2.059 E-07$ & 3175 \\
\hline 535 & $1.825 E-07$ & $2.190 \mathrm{E}-07$ & 3177 \\
\hline 449 & $1.533 E-07$ & $1.840 E-07$ & 3179 \\
\hline 449 & $1.533 E-07$ & $1.840 E-07$ & 3180 \\
\hline 567 & $1.934 E-97$ & $2.321 \mathrm{E}-37$ & 3182 \\
\hline 32 & $1.093 E-0 B$ & $1.311 \mathrm{E}-0 \mathrm{~B}$ & 3184 \\
\hline 33 & $1.129 E-08$ & $1.355 E-08$ & 3185 \\
\hline 22 & $7.648 E-09$ & 9.17 \&E-09 & 3187 \\
\hline 577 & $1.970 E-07$ & $2.364 E-07$ & 3189 \\
\hline $\begin{array}{l}513 \\
535\end{array}$ & $\begin{array}{l}1.752 E-07 \\
1.825 E-07\end{array}$ & $\begin{array}{l}2.1 \supset 2 E-07 \\
2.1 \ni 0 E-07\end{array}$ & $\begin{array}{l}3191 \\
3192\end{array}$ \\
\hline OB 4 & $2.335 E-07$ & $2.801 E-07$ & 3194 \\
\hline 951 & $3.245 E-07$ & $3.874 \mathrm{E}-07$ & 3196 \\
\hline 909 & $3.099 E-\cup 7$ & $3.719 E-07$ & 3198 \\
\hline 741 & $3.207 t-07$ & $3.850 E-07$ & 3199 \\
\hline 899 & $3.063 E-07$ & $3.676 \mathrm{E}-07$ & 3201 \\
\hline 748 & $2.553 E-07$ & $3.064 E-07$ & 3203 \\
\hline 569 & $1.941 \mathrm{E}-07$ & $2.329 E-07$ & 3205 \\
\hline 954 & $3.256 E-07$ & $3.907 E-07$ & 3206 \\
\hline 954 & $3.256 E-07$ & $3.907 E-07$ & 3208 \\
\hline 932 & $3.130 \mathrm{E}-07$ & $3.815 E-07$ & 3210 \\
\hline 1201 & $4.097 E-07$ & $4.917 E-07$ & 3212 \\
\hline 1089 & $3.715 E-07$ & $4.456 \mathrm{E}-07$ & 3213 \\
\hline 1470 & $5.015 t-07$ & $6.010 E-07$ & 3215 \\
\hline 1470 & $5.015 E-07$ & $6.01 \& E-07$ & 3217 \\
\hline 1112 & $3.791 \mathrm{E}-07$ & $4.550 E-07$ & 3218 \\
\hline 1403 & $4.786 E-07$ & $5.743 E-07$ & 3220 \\
\hline 1695 & $5.780 E-07$ & $6.936 E-07$ & 3222 \\
\hline 1605 & $5.474 E-07$ & $6.569 E-07$ & 3224 \\
\hline 1156 & $3.944 E-07$ & $4.733 \mathrm{E}-07$ & 3225 \\
\hline 2211 & $7.539 E-07$ & $9.047 \mathrm{E}-07$ & 3227 \\
\hline 2413 & B. $227 \mathrm{E}-07$ & $9.873 E-07$ & 3229 \\
\hline
\end{tabular}




\begin{tabular}{|c|c|c|c|c|c|}
\hline & & & & & \\
\hline & 168.8 & 1008 & $5.150 \mathrm{E}-07$ & $2.060 E-06$ & 3230 \\
\hline & $\begin{array}{l}109.7 \\
110.7\end{array}$ & $\begin{array}{r}2107 \\
886\end{array}$ & $\begin{array}{l}6.004 E-07 \\
2.524 E-07\end{array}$ & $\begin{array}{l}2.402 E-06 \\
1.010 E-06\end{array}$ & $\begin{array}{l}3232 \\
3233\end{array}$ \\
\hline & 111.7 & 1625 & $4.630 \mathrm{E}-07$ & $1.852 \mathrm{E}-00$ & 3235 \\
\hline & 112.7 & 1147 & $3.268 \mathrm{BE}-07$ & $1.307 E-06$ & 3237 \\
\hline E & 113.7 & 1141 & $3.252 E-U 7$ & $1.301 E-06$ & 3238 \\
\hline$=$ & 114.6 & 1184 & $3.374 E-07$ & $1.350 E-06$ & 3240 \\
\hline 4 & 115.6 & 1235 & $3.520 \mathrm{E}-07$ & $1.4005-06$ & 3241 \\
\hline H & 116.6 & 1746 & $4.975 E-07$ & $1.996 E-06$ & 3243 \\
\hline 8 & 117.6 & 1195 & $3.406 E-07$ & $1.363 E-06$ & 3245 \\
\hline H & 118.6 & 1341 & $3.821 \mathrm{E}-07$ & $1.528 E-06$ & 3246 \\
\hline & 119.5 & 2865 & $8.162 E-07$ & $3.265 \mathrm{E}-06$ & 3248 \\
\hline & 120.5 & 2974 & $0.471 E-07$ & $3.388 \mathrm{E}-06$ & 3249 \\
\hline & 121.5 & 2934 & B. 357 E-07 & $3.343 \mathrm{E}-06$ & 3251 \\
\hline & 122.4 & 2808 & B. $000 \mathrm{t}-07$ & $3.200 E-06$ & 3252 \\
\hline & 123.4 & 4636 & $1.321 t-06$ & $5.283 t-06$ & 3253 \\
\hline & 124.4 & 941 & $2.6 B 3 E-07$ & $1.073 E-0.6$ & 3255 \\
\hline & 125.4 & 2359 & $6.719 E-07$ & $2.600 \mathrm{E}-\mathrm{C6}$ & 3256 \\
\hline & 126.3 & 4452 & 1.26 AE -06 & $5.073 E-06$ & 3258. \\
\hline & 127.3 & 7976 & $2.772 \mathrm{E}-06$ & $9.087 E-06$. & 3259 \\
\hline & 123.3 & 6519 & $1.857 E-06$ & $7.427 E-06$ & 3261 \\
\hline & 129.3 & 7194 & $2.049 E-06$ & $8.196[-06$ & 3262 \\
\hline & 130.3 & 14271 & $4.065 E-06$ & $1.626 E-05$ & 3263 \\
\hline & 131.2 & 18070 & $5.375 E-06$ & $2.150 E-05$ & 3264 \\
\hline & 132.2 & 23278 & $6.630 E-06$ & $2.652 E-05$ & 3266 \\
\hline & 133.2 & 21838 & $6.220 E-06$ & $2.488 E-05$ & 3267 \\
\hline & 134.2 & 7087 & $2.019 E-06$ & $8.074 E-06$ & 3268 \\
\hline & 135.2 & 9616 & $2.739 E-06$ & $1.096 E-05$ & 3270 \\
\hline & 136.2 & 1632 & $4.650 E-07$ & $1.86 \cup E-06$ & 3271 \\
\hline & 137.1 & $727^{\circ}$ & $2.073 E-07$ & $8.292 E-07$ & $\$ 272$ \\
\hline & 138.1 & 104 & $2.967 E-0 \theta$ & $1.187 \mathrm{E}-07$ & 3273 \\
\hline & 139.1 & 67 & $1.910 E-0 B$ & $7.642 E-08$ & 3274 \\
\hline & 140.1 & 7 & $2.032 E-09$ & B.130E-09 & 3276 \\
\hline & 141.1 & 0 & $c$ & 0 . & $\$ 277$ \\
\hline CRES & SWIND & INTEGRATFU & $\begin{array}{l}2.607 E-03 \\
\text { SEC/SO.M }\end{array}$ & $\begin{array}{c}1.043 \mathrm{E}-02 \\
1 / \mathrm{M}\end{array}$ & \\
\hline & & & . & & \\
\hline TES & T U6 & JUNE & 4,1968 & & $\begin{array}{l}0246 \text { PST } \\
26 M\end{array}$ \\
\hline & $2 I N C S 4$ & $\begin{array}{l}\text { DE RELEA } \\
\text { SAMPIER }\end{array}$ & $\begin{array}{l}\text { ASE FROM EL } \\
\text { HT } 1.5 M\end{array}$ & $\begin{array}{l}\text { FVATILN OF } \\
U=4.0 \mathrm{M} / \mathrm{SE}\end{array}$ & $\begin{array}{c}26 M \\
C A T\end{array} 26 M$ \\
\hline & IMUIH & aSURE & $E / 0$ & $E \cup / O$ & I STANCE \\
\hline & GREES & $\begin{array}{c}G M-S E C / C U \cdot M \\
\times 1 O E+6\end{array}$ & SFC/CU.M & $1 / 50 . \mathrm{K}$ & METERS \\
\hline B & .2 .0 & 133 & $3.613 E-08$ & $1.525 E-07$ & 4630 \\
\hline 4 & 14.0 & 134 & $3 .+37 t-08$ & $1.535 E-07$ & $46<0$ \\
\hline$E$ & 06.0 & 106 & $3.027 t-C B$ & $1.211 E-07$ & 4610 \\
\hline & $\triangle A .0$ & 81 & $2.312 E-08$ & $9.247 E-00$ & 4610 \\
\hline & 90.0 & 156 & $4.457 E-08$ & $1.783 E-07$ & 4610 \\
\hline & 92.0 & 20 & 5.95 मE -09 & $2.383 E-08$ & 4620 \\
\hline & 94.0 & 123 & $3.527 E-08$ & $1.411 \mathrm{E}-07$ & 4620 \\
\hline & 96.0 & 146 & $4.171 E-08$ & $1.660 \mathrm{E}-07$ & 4620 \\
\hline & 93.0 & 193 & $5.517 E-O B$ & $2.2 \cup 7 E-07$ & 4660 \\
\hline 5 & 100.0 & 32 & $9.175 E-09$ & $3.670 E-08$ & 4690 \\
\hline & 102.0 & 837 & $2.387 E-07$ & $9.547 E-07$ & 4710 \\
\hline & 104.0 & 196 & $5.589 \mathrm{E}-08$ & $2.235 \mathrm{E}-07$ & 4770 \\
\hline & 166.0 & 1590 & $4.55>E-07$ & $1.821 \mathrm{E}-06$ & 4810 \\
\hline & 108.0 & 202 & $5.767 \mathrm{E}-08$ & $2.307 E-07$ & 4870 \\
\hline & 110.0 & 435 & $1.242 \mathrm{E}-07$ & $4.967 E-07$ & $49<0$ \\
\hline E & 112.0 & 304 & $8.81 B E-0 B$ & $3.527 E-07$ & 4990 \\
\hline & 114.0 & 184 & $5.243 E-08$ & $2.097 E-07$ & $>080$ \\
\hline & 116.0 & 1073 & $3.050 E-07$ & $1.223 E-00$ & 5020 \\
\hline & 118.0 & 1100 & $3.134 E-07$ & $1.254 E-06$ & 5100 \\
\hline & 120.0 & 280 & $8.210 E-08$ & $3.284 E-07$ & $\$ 130$ \\
\hline & 122.0 & 71 & $2.03+E-28$ & $0.151 \mathrm{E}-08$ & 4830 \\
\hline E & 124.0 & 204 & 5.95 AE -08 & $2.363 E-07$ & 4660 \\
\hline 5 & 126.0 & $53 \%$ & $1.524 E-07$ & $6.096 E-07$ & 4770 \\
\hline 6 & 128.0 & 1209 & $3.444 E-07$ & $1.377 \mathrm{E}-06$ & 4900 \\
\hline $\mathrm{E}$ & 130.0 & 711 & $2.026 E-07$ & $8.103 t-07$ & 4970 \\
\hline & 132.0 & 269 & $7.6866-08$ & $3.074 E-67$ & 4980 \\
\hline 5 & 134.0 & 64 & $2.407 E-08$ & $9.626 E-08$ & 4990 \\
\hline & 136.0 & 0 & 0. & 0. & 5000 \\
\hline CRO & SWIND & $A T E D=$ & $\begin{array}{l}5.059 E-04 \\
\text { SFCISO.M }\end{array}$ & $\begin{array}{c}2.028 E-03 \\
1 / M\end{array}$ & \\
\hline
\end{tabular}

\begin{tabular}{|c|c|c|c|c|c|}
\hline & 108.8 & 2951 & $1.006 \mathrm{E}-06$ & $1.200 E-06$ & 3230 \\
\hline & $1,49.7$ & $3<87$ & $1.121 \mathrm{E}-06$ & $1.345 E-06$ & 3232 \\
\hline 2 & 110.7 & 3355 & $1.144 E-06$ & $1.373 E-06$ & 3233 \\
\hline & 111.7 & 3624 & $1.236 E-06$ & $1.483 E-06$ & 3235 \\
\hline & 112.7 & 4499 & $1.534 t-06$ & $1.841 E-06$ & 3237 \\
\hline 0 & 113.7 & 3843 & $1.328 E-06$ & $1.593 \mathrm{E}-06$ & 3238 \\
\hline 8 & 114.6 & 3355 & $1.144 E-06$ & $1.373 E-06$ & 3240 \\
\hline & 115.6 & 3591 & $1.259 E-06$ & $1.510 E-U B$ & 3241 \\
\hline & 116.6 & 3826 & $1.305 E-06$ & $1.566 \mathrm{E}-0 \mathrm{~S}$ & 3243 \\
\hline$H$ & 117.6 & 2682 & $9.145 E-07$ & $1.097 E-06$ & 3245 \\
\hline & 118.6 & 1942 & $6.621 E-07$ & $7.91,6 E-07$ & 3246 \\
\hline & 119.5 & 2480 & $3.457 E-07$ & $1.015 E-06$ & 3248 \\
\hline & 120.5 & 3759 & $1.282 E-06$ & 1.538 & 3249 \\
\hline & 121.5 & 4095 & $1.396 \mathrm{E}-06$ & $1.676 E-06$ & 3251 \\
\hline & 122.4 & 3287 & $1.121 E-06$ & $1.345 E-06$ & 3252 \\
\hline & 123.4 & 3227 & $1.100 E-06$ & 1.326 & 3253 \\
\hline & $1<4.4$ & 4413 & $1.5 O S E-0 S$ & 1.8006 & 3255 \\
\hline & 125.4 & 2903 & $7.849 t-07$ & $1.188 E-06$ & 3256 \\
\hline & 126.3 & 7111 & 2.42 & & 3258 \\
\hline & 127.3 & 4563 & $1.556 t-06$ & $1.867 E-06$ & 3259 \\
\hline & 128.3 & 4090 & $1.395 E-06$ & 1.673 & 3261 \\
\hline & 129.3 & 7758 & $2.645 \mathrm{E}$ & 3.174 & 3262 \\
\hline & 130.3 & 12289 & $4.190 E-06$ & $5.02 \forall E-06$ & 3263 \\
\hline & 131.2 & 13908 & $4.742 E-06$ & $5.6 .90 E-06$ & 3264 \\
\hline & 132.2 & 6463 & $2.204 E-06$ & $2.645 E-06$ & 3266 \\
\hline & 133.2 & 1201 & $\therefore .097 E-07$ & $4.917 E-07$ & 3267 \\
\hline & 134.2 & 360 & $1.229 E-07$ & $1.475 E-07$ & 3268 \\
\hline & 135.2 & 151 & $5.179 \mathrm{E}-08$ & $0.215 E-08$ & 3270 \\
\hline & 136.2 & 31 & $1.072 \mathrm{E}-0 \mathrm{~B}$ & $1.286 E-08$ & 3271 \\
\hline & & 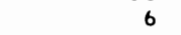 & $2.229 E-09$ & $2.675 E-09$ & 3272 \\
\hline & & ATED: & $\begin{array}{r}2.919 E-03 \\
\text { SEC/SO.M }\end{array}$ & $\begin{array}{c}3.503 E-03 \\
1 / M\end{array}$ & \\
\hline
\end{tabular}

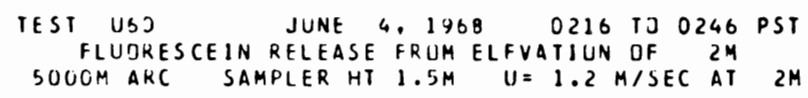
$\begin{array}{lcc}\text { ALIMUTH EXPOSURE } & \text { E/O } & \text { EU/O DISTANCE } \\ \text { OEGREES GM-SECICU.M SECICU.M I/SO.Y METERS }\end{array}$ $X 10 E+6$

\begin{tabular}{l}
$B \quad 02.0 \quad 957 \quad 3.265 E-07 \quad 3.918 E-07 \quad 4630$ \\
\hline
\end{tabular}

$\begin{array}{llllll}4 & 44.0 & 997 & 3.399 E-07 & 4.079 E-07 & 4620 \\ 0 & 46.0 & 376 & 1.282 E-07 & 1.539 E-07 & 4510\end{array}$

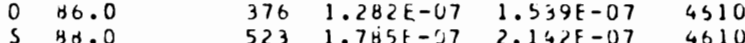

SO.0 523 . $1.785 E-572.142 E-07 \quad 4610$

$\begin{array}{lllll}90.0 & 661 & 2.256 E-J 7 & 2.7 J 7 E-07 & 4510 \\ 9<.0 & 661 & 2.256 E-07 & 2.707 E-07 & 4620\end{array}$

$9.4 .0 \quad 582 \quad 1.967 \mathrm{E}-07 \quad 2.384 \mathrm{E}-07 \quad 4620$

$96.0 \quad 661 \quad 2.256 F-U 7 \quad \angle .737 F-07 \quad 4520$

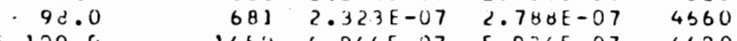

5100.0 145J $4.945 E-07$ 5.936E-07 4690

$\begin{array}{lllll}102.0 & 1391 & 4.745 E-J 7 & 5.634 E-37 & 4710 \\ 104.0 & 1371 & 4.677 E-07 & 5.613 E-07 & 4770\end{array}$

$\begin{array}{lllll}104.0 & 1371 & 4.677 \mathrm{E}-07 & 5.613 \mathrm{E}-07 & 4770 \\ 106.0 & 1414 & 4.822 \mathrm{E}-07 & 5.786 \mathrm{E}-07 & 4810\end{array}$

IUS.0 $\quad 1352 \quad 4.610 \mathrm{E}-07 \quad 3.532 \mathrm{t}-07 \quad 4870$

$\begin{array}{lllll}110.0 & 1016 & 3.467 E-U 7 & 4.16 U E-07 & 4920\end{array}$

E $112.0 \quad 997 \quad 3.379 \mathrm{E}-07 \quad 4.079 \mathrm{E}-07 \quad 4990$

$114.0 \quad 977 \quad 3.332 E-C 7 \quad 3.979 E-07 \quad 5080$

$116.0 \quad 937 \quad 3.194 E-07 \quad 3.837 E-07 \quad 5020$

$118.0 \quad 1115 \quad 3.803 E-07 \quad 4.564 E-07 \quad 5100$

120.0 $314 \quad 1.090 E-U 7 \quad 1.308 E-07 \quad 5130$

$\begin{array}{lllll}122.0 & 240 & 9.210 E-J 3 & 9.852 E-08 & 4330\end{array}$

$124.0 \quad 3191.090 \mathrm{E}-J 7$
5

$\begin{array}{llllll}5 & 120.0 & 438 & 1.494 E-07 & 1.792 E-07 & 4770\end{array}$

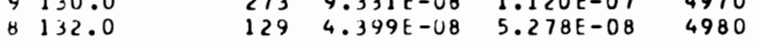

CRUSSMINO INTEGRATED $=1.166 E-03 \quad 1.399 E-03$ 


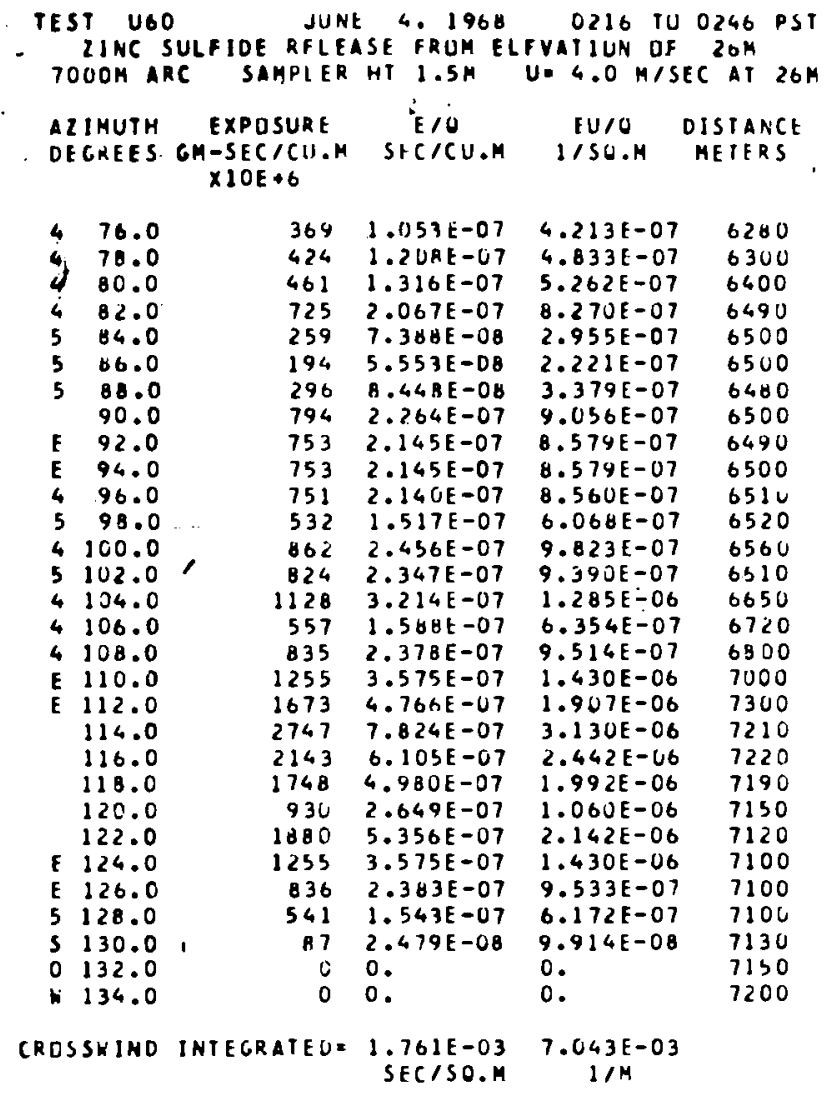

IEST UGD JUNE 4, 1968 O216 TO 0246 PST ZIINC SULFIDE RELEASE FROM ELEVAIIIN OF $\angle 6 \mathrm{M}$ $12000 \mathrm{H}$ ARC SARPLER HT $1.5 \mathrm{M}$ U: 4.0 M/SEC AT $26 \mathrm{M}$

AZIMUTH EXPOSURE E/O EUIO DISIANCE DEGREES GM-SECICU.M SECICU.M I/SO.M MEIERS $X 10 E+6$

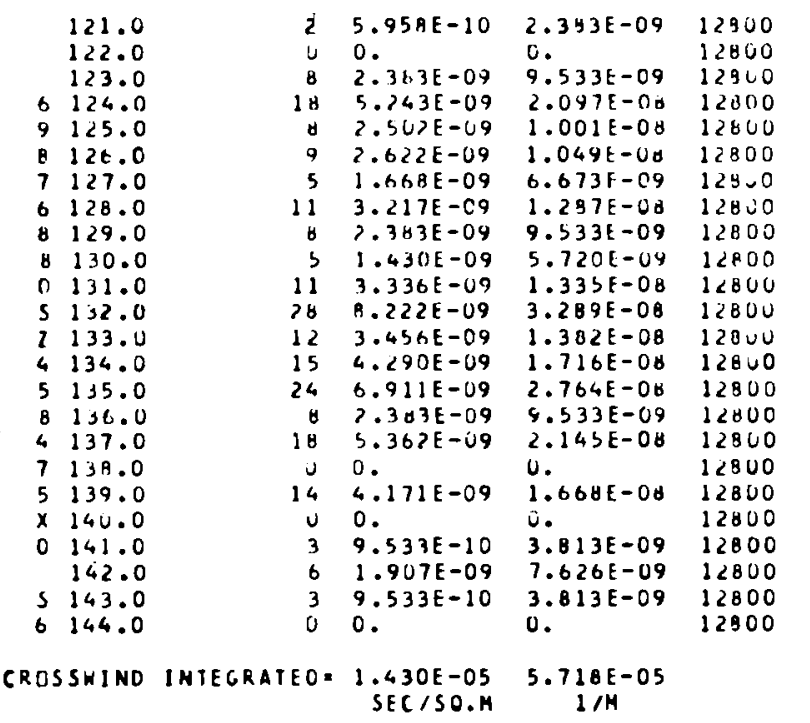

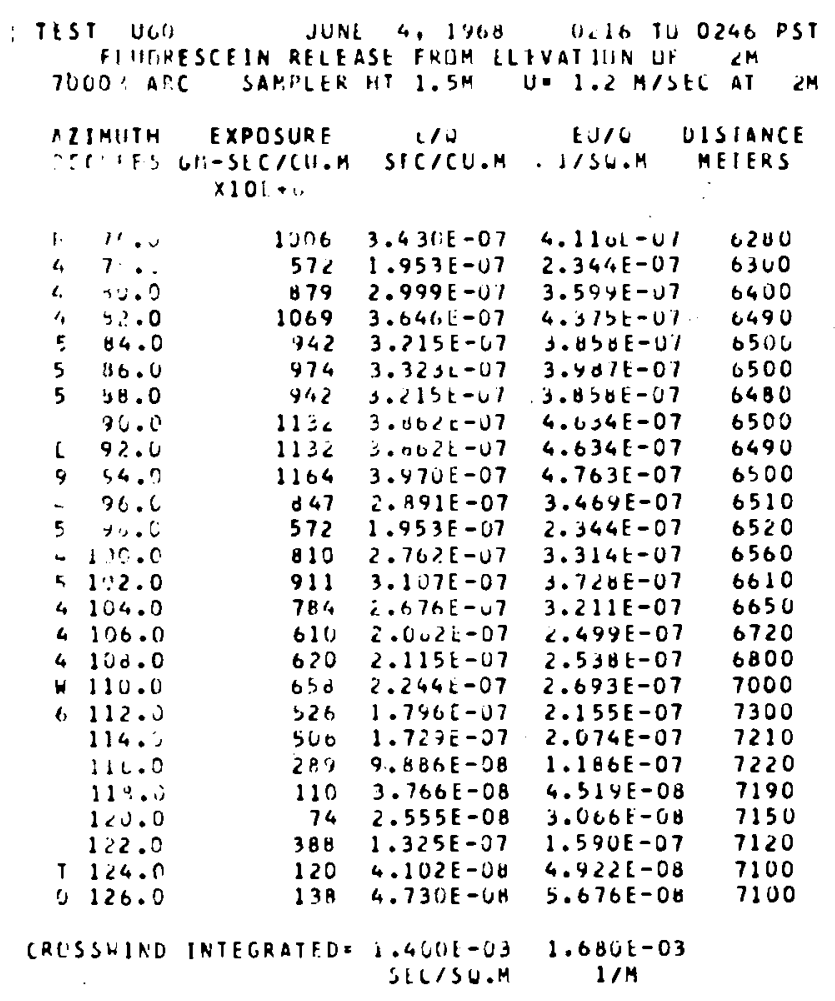
TLST UES JUNE 4. 1968 OLO 0216 TU 0246 PST FLUDRESCEIN RELEASE FRUM ELFVATIUN DF $2 M$ 12 AOIM ARC SAMPLER HT $1.5 M$ UE $1.2 \mathrm{M} / \mathrm{SEC}$ AT $2 \mathrm{M}$ 
SAMPLING GOOM TO 12 GOUM. NO TOHER SAMPLING. ALL AKCS EMGRACE CRUSSHIND EXIENT DF TRACER. NDT ENDUGH TRACER SAMPLED ON SUORM ARC TOCDNFIDENTLY INTERPDLATE UUESTIONABLE DATA POINTS. THIRTY MIN DF ZINC SULFIDE DISPERSAL. IS MIN DF FLDURESCEIN DISPIKSAL.

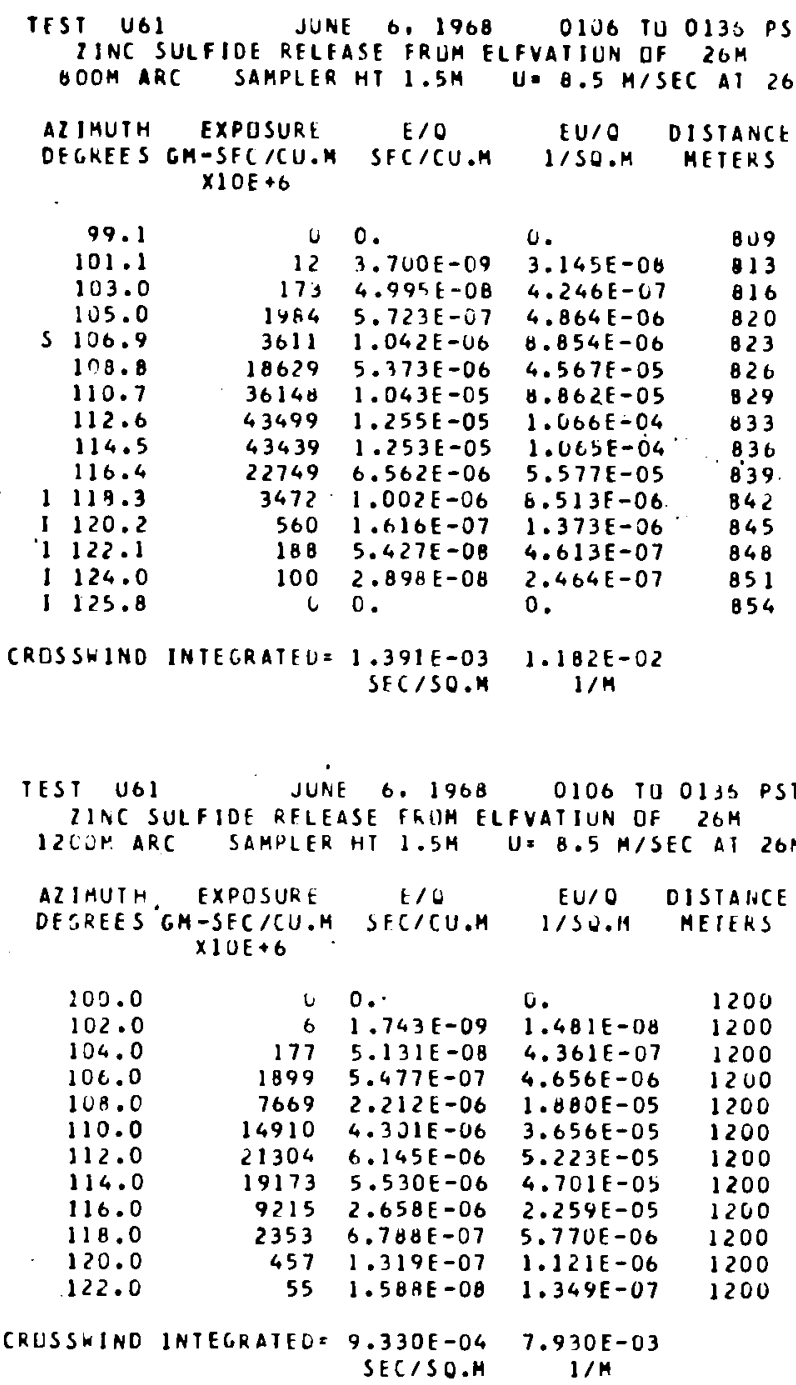

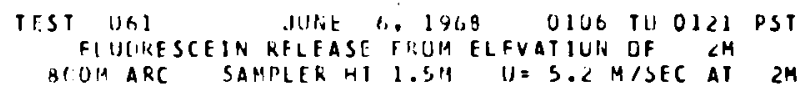

ATIMIITH FXPUSUKT IIO LU/O DISTAIACE DFGKHES GH-SECIGU.M SICICU.M IISL.M METERS $X 10 \mathrm{E}+6$

$$
\begin{array}{r}
99.1 \\
101.1 \\
193.0 \\
1115.0 \\
5116.0 \\
114.8 \\
110.7 \\
112.6 \\
111.5 \\
1116.4 \\
1114.3 \\
1120.2 \\
11122.1 \\
1124.0
\end{array}
$$

CRISSWING INTEGRATFD $=3.036 E-03$
SECISO.M

0.

$6.05315-0$

$8338 \quad 5.864 E-60$

11949 H. $403 E-06$

$46026 \quad 3.434 \mathrm{E}-05$

$455703.205[-05$

$303772.136 \mathrm{t}-45$

$11949 \quad H .4 U 3 E-06$

$25471.791[-06$

$64 \quad 4.509 E-0 B$

$10 \quad 7.304 \mathrm{E}-09$

$\begin{array}{cl}143 & 1.013 t-07 \\ & 0 .\end{array}$
0.

$3.563 E=07$

5. $033 \mathrm{~L}-07$

$3.049 E-05$

4.37UE-05

$1.785 t-04$

$1.666 \mathrm{E}-04$

$1.111 \mathrm{E}-04$

4. $370 E-05$

9.315E-06

$2.344 E-07$

$3.798 E-08$

$5.265 E-07$

0.

$1.61 \cup t-02$

$1 / 4$

$$
\begin{aligned}
& 809 \\
& 813 \\
& 816 \\
& 820 \\
& 823 \\
& 826 \\
& 829 \\
& 833 \\
& 836 \\
& 839 \\
& 842 \\
& 845 \\
& 848 \\
& 851
\end{aligned}
$$

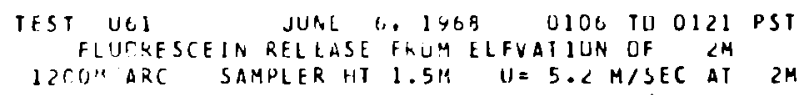

CRRSSAINT INIEGRATFD= 1.1 UTE-03 G.14) SIC/SC.A 


\begin{tabular}{|c|c|c|c|c|}
\hline $\begin{array}{l}\text { ALAMUTH } \\
\text { DECREES }\end{array}$ & $\begin{array}{l}\text { EXPOSURE } \\
\text { CH-SEC/CU.H } \\
\text { XIOE } \odot 6\end{array}$ & $\begin{array}{c}E / 0 \\
\text { SEC/CU.M }\end{array}$ & $\begin{array}{l}E U / O \\
\text { I SSO.M }\end{array}$ & $\begin{array}{c}\text { DISIANCE } \\
\text { MEIERS }\end{array}$ \\
\hline $\begin{array}{l}100.6 \\
101.5 \\
102.8 \\
103.6 \\
104.5 \\
105.5 \\
106.8 \\
107.3 \\
108.5 \\
109.5 \\
110.4 \\
111.4 \\
112.4 \\
113.4 \\
114.4 \\
115.3 \\
116.3 \\
117.3 \\
118.2 \\
119.2 \\
1120.2 \\
121.2\end{array}$ & $\begin{array}{r}0 \\
0 \\
10 \\
64 \\
369 \\
750 \\
2179 \\
5029 \\
7028 \\
9045 \\
904 \\
11858 \\
15462 \\
15305 \\
12870 \\
9467 \\
4900 \\
2259 \\
1660 \\
500 \\
129 \\
0 \\
0 \\
0\end{array}$ & $\begin{array}{l}0 . \\
0 . \\
3.019 E-09 \\
1.062 E-00 \\
1.067 E-07 \\
2.164 E-07 \\
6.205 E-07 \\
1.4511 E-06 \\
2.238 E-06 \\
2.609 E-06 \\
3.420 E-06 \\
4.460 E-06 \\
4.615 E-06 \\
3.712 E-06 \\
2.725 E-06 \\
1.414 E-06 \\
6.517 E-07 \\
1.927 E-07 \\
1.676 E-07 \\
3.724 E-00 \\
0 .\end{array}$ & $\begin{array}{l}0 . \\
0 . \\
2.566 E-00 \\
1.503 F-07 \\
9.060 E-07 \\
1.839 E-06 \\
5.362 E-06 \\
1.233 E-05 \\
1.919 E-05 \\
2.21 .0 E-05 \\
2.907 E-05 \\
3.791 E-05 \\
3.752 E-05 \\
3.155 E-05 \\
2.316 E-06 \\
1.201 E-05 \\
5.539 E-D 6 \\
1.636 E-06 \\
1.424 E-06 \\
3.165 E-07 \\
0 .\end{array}$ & $\begin{array}{l}1615 \\
1617 \\
1618 \\
1620 \\
1622 \\
1623 \\
1625 \\
1627 \\
1628 \\
1630 \\
1632 \\
1633 \\
1639 \\
1637 \\
1638 \\
1640 \\
1641 \\
1643 \\
1644 \\
1646 \\
1647 \\
1649\end{array}$ \\
\hline IS SWINO & IMTEGRATED O & $\begin{array}{l}7.970 E-04 \\
\text { SEC } / \mathrm{SO} \text {.M }\end{array}$ & $\begin{array}{c}6.774 \mathrm{E}- \\
1 / \mathrm{H}\end{array}$ & \\
\hline
\end{tabular}

TEST U61 JUNE 6. 1968 OS $0106^{\circ}$ TO 0136 PST IINC SULFIOE RELEASE FROM ELFVATION OF $26 \mathrm{M}$ $2200 M$ ARC SAMPLER HT $1.5 \mathrm{SH}, U=8.5$ M/SEC $A 1$ $26 \mathrm{H}$

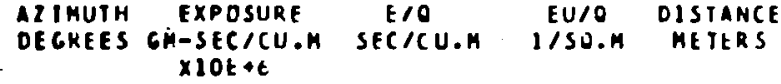

$\begin{array}{rrl}102.0 & 0 & 0 . \\ 104.0 & 126 & 3.661 E-08 \\ 106.0 & 323 & 9.334 E-08 \\ 108.0 & 7264 & 2.045 E-06 \\ 110.0 & 10721 & 3.042 E-06 \\ 112.0 & 7653 & 2.206 E-06 \\ 114.0 & 3595 & 1.037 E-06 \\ 116.0 & 718 & 2.071 E-07 \\ 118.0 & 109 & 3.159 E-08 \\ 4120.0 & 9 & 2.623 E-09 \\ 122.0 & 0 & 0 .\end{array}$

0.

2200

$7.934 E-07 \quad 2200$
$1.702 E-05 \quad 2200$

$1.701 E-05 \quad 2200$

$2.629 t-03 \quad 2200$

$1.876 t-05,2200$

$0.814 t-06 \quad 2200$

$1.760 \mathrm{E}-06 \quad 2200$

$2.645 \mathrm{E}-07 \quad 2200$

0 . CROSSHIND INTEGRATED. $6.761 E-04 \quad 5.747 E-03$

TEST U61 JUNE 6. 1968 UIUG TI O136 PST ZINC SULFIDE RFLEASE FRUA ELFVATION OF $26 \mathrm{M}$ $3200 M$ ARC SAMPLER HT $1.5 \mathrm{M}$ U $=0.5 \mathrm{M} / \mathrm{SEC}$ AT $26 \mathrm{M}$ NZIMUTH EXPOSURE T/O EU/O OISTANCE DEGRES GH-SEC/CU.M SEC/CU.M I/SO.H HETEKS $\times 10 E+6$

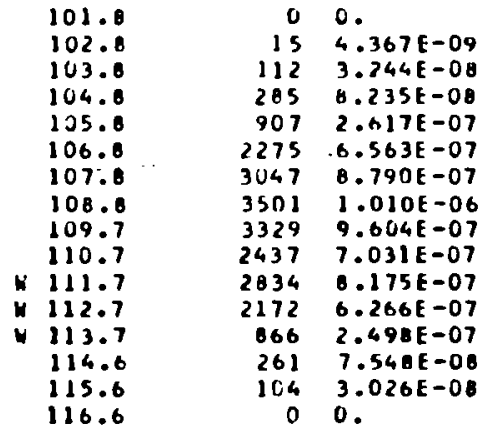

0.

3210

$3.712 \mathrm{E}-0 \mathrm{O} \quad 3220$

$2.757 E-07 \quad 3222$

$7.000 E-07 \quad 3224$

$2.224 E-06 \quad 3225$

$5.578 E-06 \quad 3227$

$7.471 E-06 \quad 3229$

$0.585 E-06 \quad 3230$

$8.163 E-06 \quad 3232$

$5.976 E-06 \quad 3233$

$6.949 E-06 \quad 3235$

$5.327 E-06 \quad 3237$

$2.124 E-06 \quad 3236$

$2.572 \mathrm{E}-07$

u.

3243

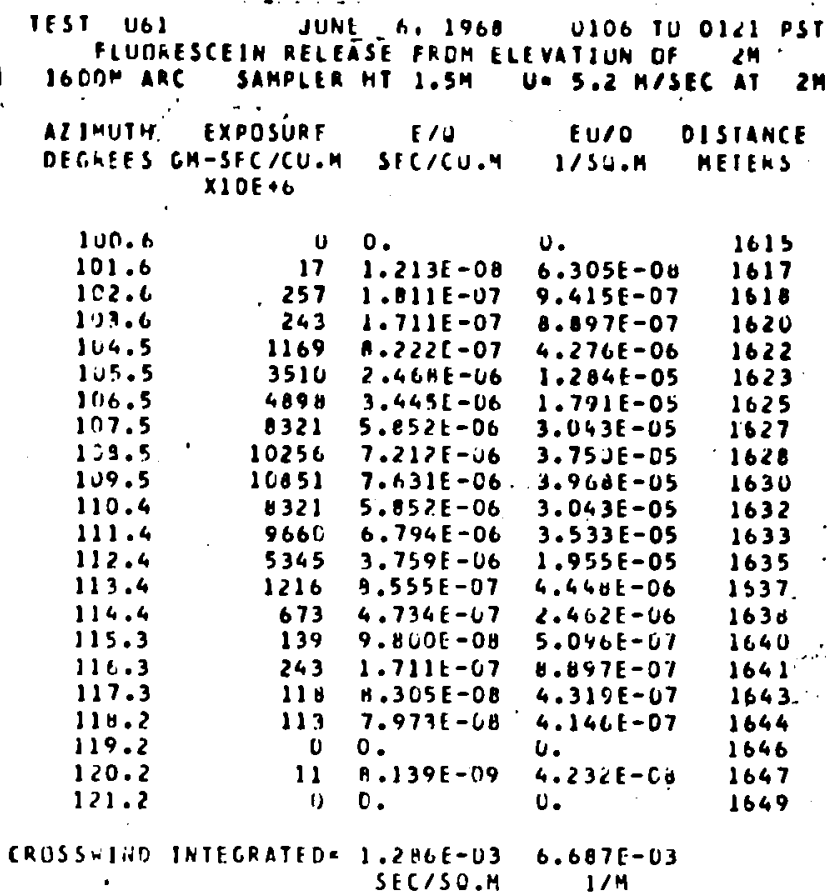

TEST U61 JUNE C. 196A O1UG TU 0121 PST FLURRESCEIN RFLEASE FH.CM. ELFVATIUN OF $2 \mathrm{H}$ $2200 M$ ARC SAKPLER HI $1.5 \mathrm{M}$ UE 3.2 M/SEC AT $2 \mathrm{M}$

AZIFUTH EXPOSURE E/O EUIO DISIANCE OHCREES GM-SFC/CII.M SFC/CII.M I/SO.Y MEIERS $\times 10 F+6$

\begin{tabular}{|c|c|c|c|c|c|}
\hline & $\begin{array}{l}102.0 \\
154.0\end{array}$ & $\begin{array}{r}0 \\
373\end{array}$ & $\begin{array}{l}0.0 \\
2.625 \varepsilon-07\end{array}$ & $1.365 \mathrm{E}-06$ & $\begin{array}{l}2200 \\
2200\end{array}$ \\
\hline & $111 \%, 0$ & $11 ?$ & $7.936,[-\cup n$ & $4.127 E-07$ & 2200 \\
\hline & IUK.0 & 0,403 & $1.455 t-\sigma b$ & $2.324 t-$ US & 2200 \\
\hline & 110.0 & 4404 & $3.0)+7[-00$ & $1.0111-0 b$ & 2200 \\
\hline & 112.0 & 748 & $5.26 \mathrm{EE}-07$ & $2.736 t-46$ & 2200 \\
\hline & 114.0 & 215 & J.513t -07 & $7.0701-07$ & $<200$ \\
\hline & $11 \mathrm{~h} .0$ & 13 & $9.4<9 E-<9$ & $4.924 E-08$ & $2200^{\circ}$ \\
\hline & 119.0 & 4 & $2.9 ; 9[-\cup 9$ & $1.5,231-08$ & $\angle 200$ \\
\hline & 120.0 & 1 & $1.019 E-09$ & $3.2671-04$ & 2200 \\
\hline & $\begin{array}{l}122.0 \\
124.0\end{array}$ & $\begin{array}{l}t \\
0\end{array}$ & $\begin{array}{l}4.404 \mathrm{E}-09 \\
3.523 \mathrm{E}-10\end{array}$ & $\begin{array}{l}2.240 \mathrm{E}-08 \\
1.632 \mathrm{E}-09\end{array}$ & $\begin{array}{l}2200 \\
2200\end{array}$ \\
\hline
\end{tabular}

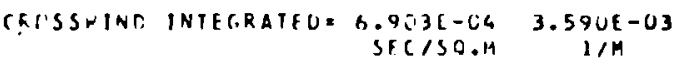

THST UQI JUHA $6.176 \mathrm{H}$ OIUO TU 0121 PST FLUGKESCEIH RFLEASE FRDM ELFVATIUN UF CM 32 ON GRC SAMPLER HT $1.3 M$ U $=3.2 \mathrm{MISEC}$ AT $2 M$ LIIAUTH EXPOSURE E/O LU/C DISIAITCE DESREES TM-SEC/CU.A SEC/CU.M J/SU.H METERS $\times 10 E+6$

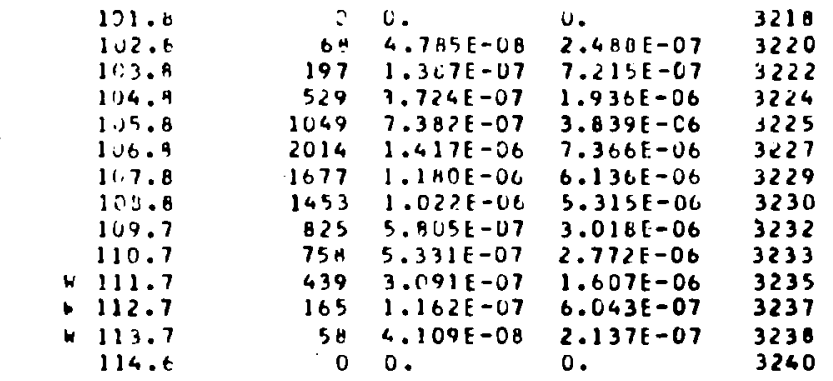

CRESSHIND INTEGRATED $=3.614$ E-O4 1.879 E-03 $1 / \mathrm{M}$ 


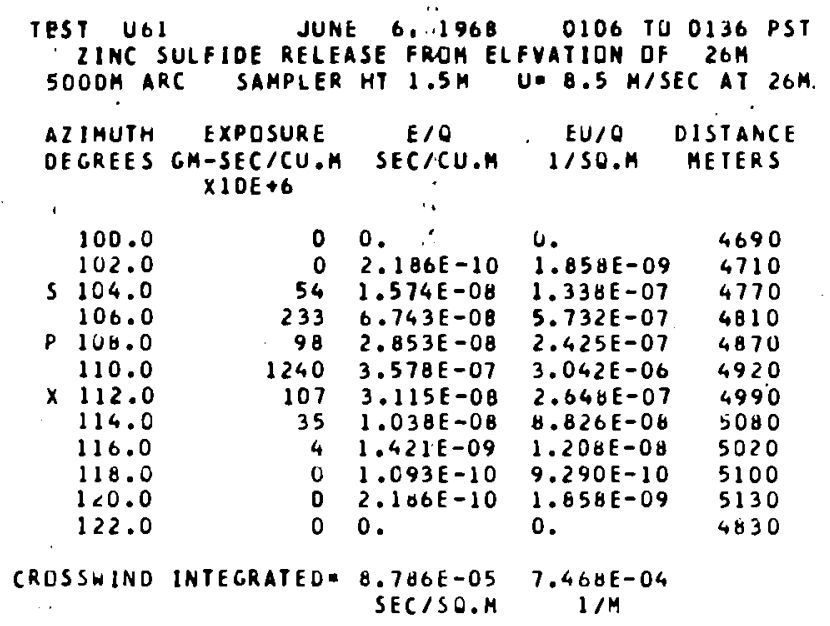

TEST U6I JUNE b. 1968 O $0106 \quad 100136$ PST 2 INC SULFIDE RELEASE FRUM ELEVATIUN OF $26 \mathrm{M}$ TOOUM ARC SAMPLER HT $1.5 \mathrm{M} U=8.5 \mathrm{M} / \mathrm{SEC}$ AT $26 \mathrm{M}$

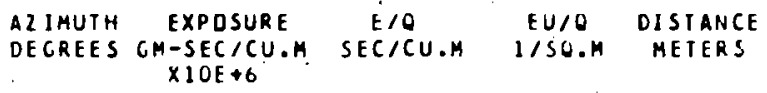

$\begin{array}{lrlll}102.0 & 0 & 0 . & 0 . & 6610 \\ 104.0 & 7 & 2.077 E-09 & 1.765 E-08 & 6650 \\ 106.0 & 70 & 2.033 E-08 & 1.720 E-07 & 6720 \\ 100.0 & 408 & 1.178 E-07 & 1.001 E-06 & 6800 \\ 110.0 & 505 & 1.459 E-07 & 1.240 E-06 & 7000 \\ 112.0 & 144 & 4.164 E-08 & 3.540 E-07 & 7300 \\ 114.0 & 73 & 2.131 E-08 & 1.812 E-07 & 7210 \\ 116.0 & 10 & 2.951 E-09 & 2.508 E-08 & 7220 \\ 118.0 & 0 & 0 . & 0 . & 7190 \\ 120.0 & 0 & 0 . & 0 . & 7150\end{array}$

CROSSWINO INTEGRATED= $0.559 E-05 \quad 7.275 E-04$ SEC/SO.M

TEST U61 JUNE 6. 1968 O106 TO 0136 PST 2INC SULFIDE RELEASE FRUM ELFVATIUN DF $26 \mathrm{M}$ $12800 \mathrm{M}$ ARC SAMPLER HT $1.5 \mathrm{M} U=0.5 \mathrm{M} / \mathrm{SEC}$ AT $26 \mathrm{M}$

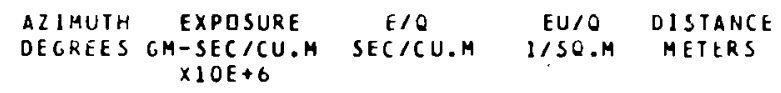

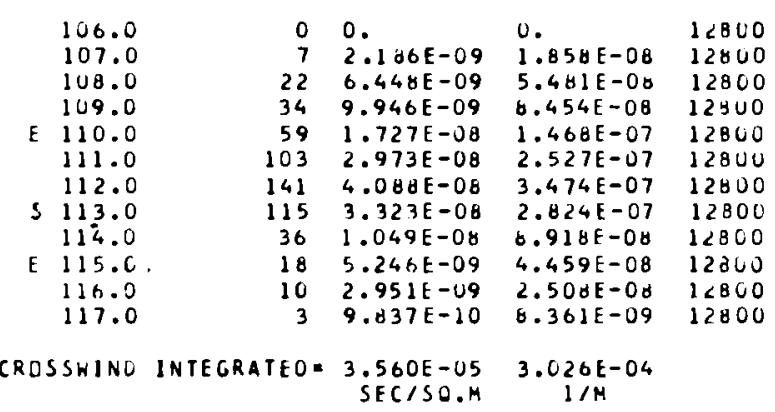

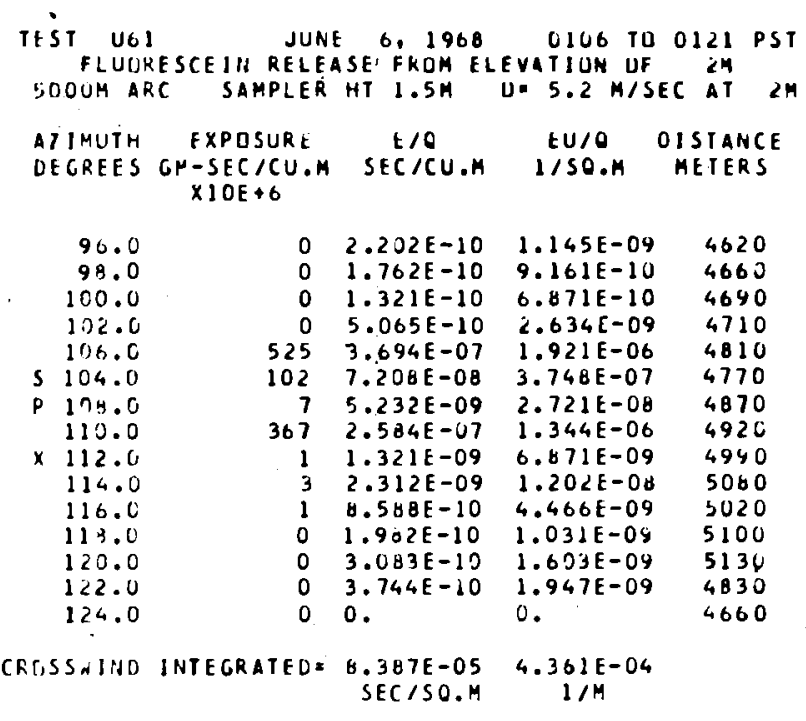

TEST U61 JUNE 6. 1968 O 0106 TU 0121 PST. FLUDRESCEIN RELEASE FROM ELEVATION UF $2 M$ TOUOM ARC SAMPLER HT $1.5 \mathrm{M} U=5.2 \mathrm{M} / \mathrm{SEC}$ AT $2 \mathrm{M}$

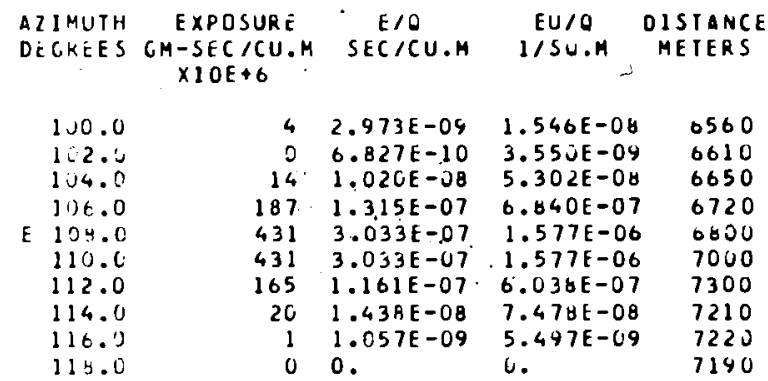

CRLSSIIHU INTEGRATEOE 2.136E-04 1.111E-O3
SECISO.M

IFST U6I JUNE 6,1968 0106 TU 0121 PST FLUTRESCEIN RELEASE FROM ELFVATIUN OF $2 \mathrm{M}$ $12 U$ OM ARC SAMPIER MI $1.5 \mathrm{M} U=5.2 \mathrm{~K} / \mathrm{SEC} A T$ TH $\begin{array}{lcccc}\text { AZIMUIH EXPOSURT } & \text { EIO } & \text { EU/O } & \text { DISTAHCE } \\ \text { DFCHEES CM-SECICU.M } & \text { SEC/CU.M } & \text { IISC.M } & \text { MEIEKS }\end{array}$ DFCFEES CM-SEC/CU
$\times 1 O E+6$
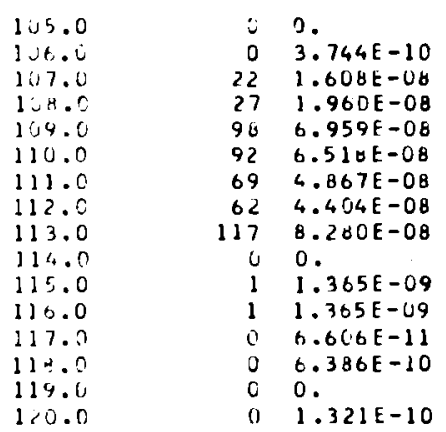

0 . $1<300$ $1.947 E-0912800$ $6.35 \% E-0012800$ $1.019 E-07 \quad 12800$ $3.619 E-07 \quad 1 \angle 860$ 3.39DE-07 12900 $2.531 \mathrm{E}-07 \quad 12800$ $2.290 E-07 \quad 12800$ $4.306 \mathrm{E}-07 \quad 12 d 00$

$7.100 E-09 \quad 12800$ $7.100 E-09 \quad 12500$ $3.435 \mathrm{E}-10 \quad 12 \mathrm{~B} 5 \mathrm{C}$ $3.321 \mathrm{E}-04 \quad 12860$ i. 12900 b. $71 E-10 \quad 12900$

CRLISSIINU INTEGRATED= $7.817 E-05$ 4.0S5E-04 
SAMPLINC HOOH TD 12 BOJM,ND IUHER SAMPLING. ALL ARCS EMBRACED CRUSSHINL EXIENT DF TRACLR.

21 HC SULFIOE DISPERSED FOR 30 MIN, DISPERSAL DIFFICULIIES RESIRILTED FLUIIKESCEIN DISPERSAL ID I9.5 MIN.

LICHT RAIN FELL AFTER TRACFR RELFASE ENUEU BUT GEFDRE ALL FILTEKS COLLECTEOE ND.DBVIUUS EFFECT DN TRACERS.

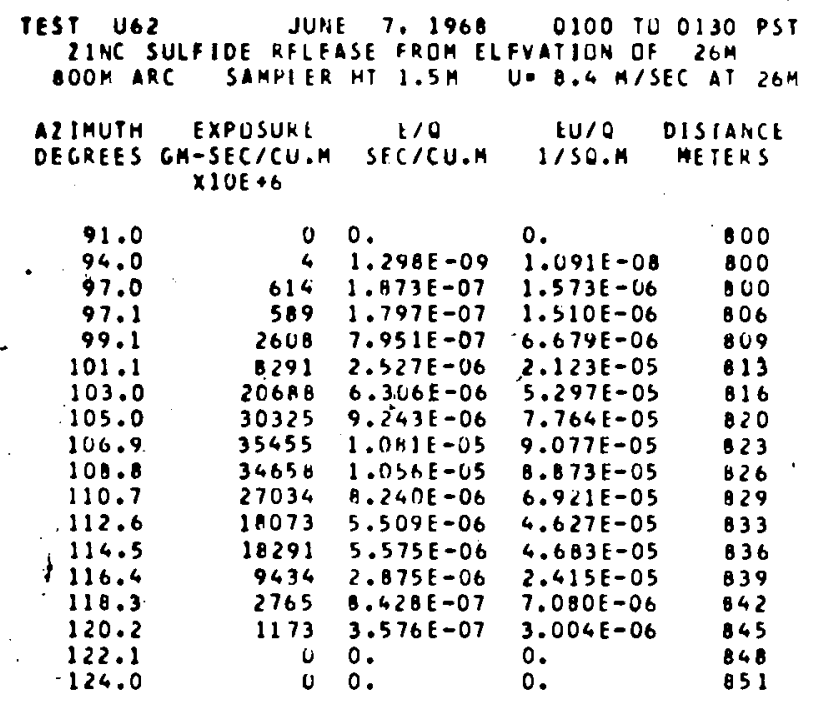

CRDSSWIND INTEGRATEOE $1.763 E-03 \quad 1.481 E-02$

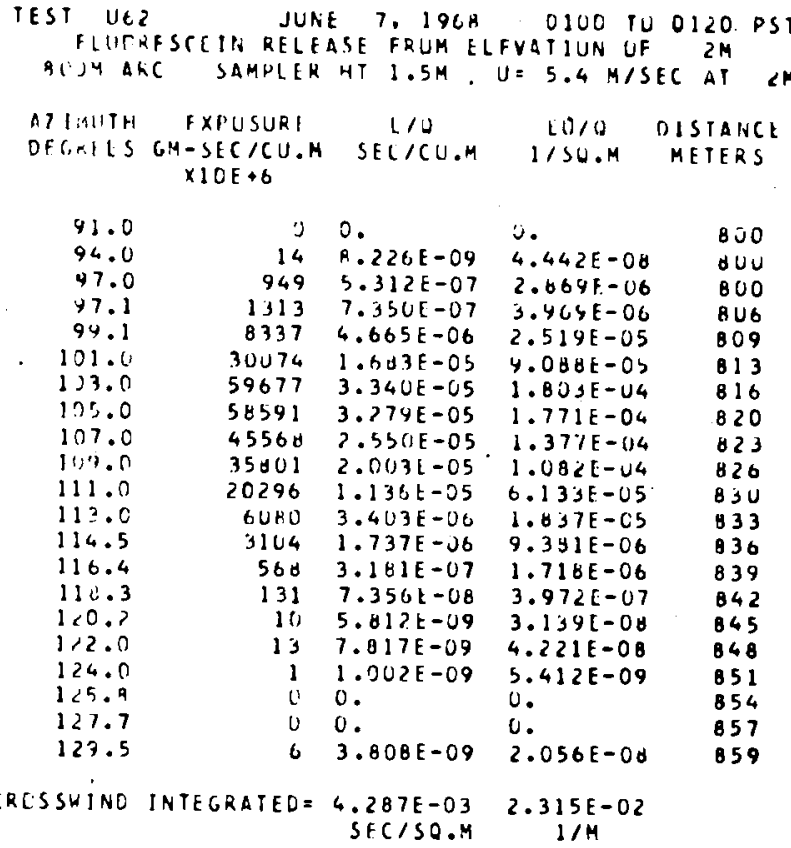

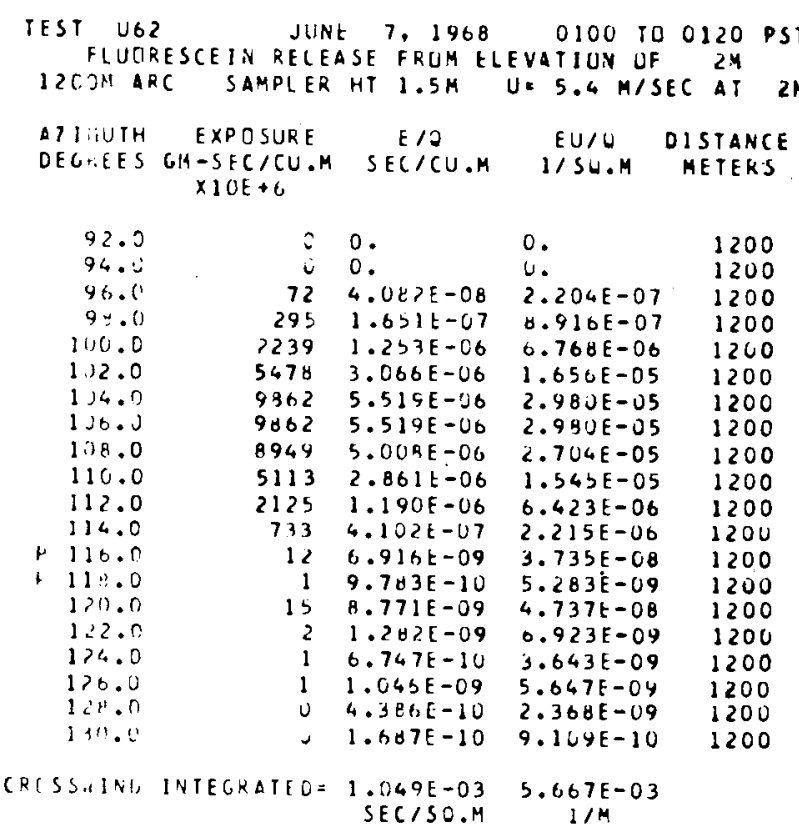

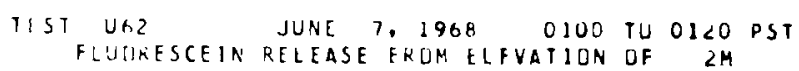

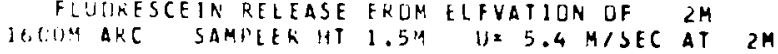

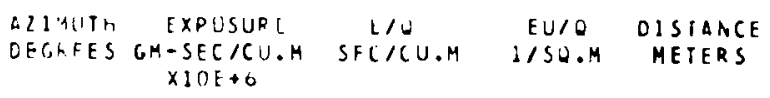

TEST U62 JUNE 7.1968 0100 TO 0130 PST 2INC SULFIOE RELEASE FROM ELEVATION DF $26 \mathrm{M}$
$1200 \mathrm{M}$ ARC SAMPLER HT $1.5 \mathrm{M}$ U 8.4 M/SEC AT $26 \mathrm{M}$ $\begin{array}{lccc}\text { AZIMUTH EXPOSURE } & \text { E/O } & \text { EU/O DISTANCE } \\ \text { DEGRES GP-SECICU.M SFCICU.M I/SO.M METERS }\end{array}$ $X 10 E+6$

\begin{tabular}{|c|c|c|c|c|}
\hline 94.0 & 0 & 0. & U. & 1200 \\
\hline 96.0 & 13 & $4.217 E-09$ & $3.542 \mathrm{E}-0 \mathrm{~B}$ & 1200 \\
\hline 98.0 & 191 & $3.843 E-98$ & $4.90 \Delta E-07$ & 1200 \\
\hline 100.0 & 1338 & $4.080 E-07$ & $3.427 E-06$ & 1200 \\
\hline 102.0 & 3866 & $1.178 E=06$ & $9.898 \mathrm{E}-06$ & 100 \\
\hline 104.0 & 9197 & $2.803 \mathrm{E}-06$ & $5 E-05$ & 200 \\
\hline 106.0 & 14043 & $4.280 E-06$ & $3.595 E-05$ & 1200 \\
\hline 108.0 & 17302 & $5.273 E-06$ & $4.430 E-05$ & 1200 \\
\hline 110.0 & 12061 & $3.676 E-06$ & $3.086 \mathrm{E}-05$ & 1200 \\
\hline 112.0 & 10443 & $3.183 E-06$ & $2.674 E-05$ & 1200 \\
\hline 114.0 & 6266 & $2.525 E-06$ & $2.121 \mathrm{E}-05$ & 1200 \\
\hline 116.0 & 4303 & $1.312 \mathrm{E}-06$ & $1.102 E=05$ & 1200 \\
\hline 118.0 & 1650 & $4.422 \mathrm{E}-07$ & $3.714 \mathrm{E}-06$ & 1200 \\
\hline 120.0 & 297 & $9.056 E-08$ & $7.607 E-07$ & 1200 \\
\hline 122.0 & 10 & $3.213 E-09$ & $2.699 E-08$ & 1200 \\
\hline 124.0 & 12 & $3.815 \mathrm{E}-09$ & $3.205 \mathrm{E}-08$ & 1200 \\
\hline & 0 & 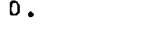 & & 1200 \\
\hline
\end{tabular}

CRCSSHINO INTECRATEOE $1.057 E-03 \quad 6.882 E-03$ SFCISO.H $\quad 1 / \mathrm{M}$

TEST U62 JUNE 7.1968 0100 TO 0130 PST IINC SULFIOE RELEASE FRUM ELFVATIDN UF $26 \mathrm{M}$ IOOOH ARC SAMPLFR HT $1.5 \mathrm{M}$ U $=8.4$ H/SEC AT $26 \mathrm{M}$

$\begin{array}{lccc}\text { AZIHUTH EXPOSURE } & \text { E/O } & \text { EU/O DISIANCE } \\ \text { DFGREES GM-SECICU.M SFCICU.H IISO.M METERS }\end{array}$ $X \perp O E+6$

$\begin{array}{lclll}94.6 & 0 & 0 . & 0 . & 1600 \\ 95.6 & U & 0 . & 0 . & 1606 \\ 96.6 & 15 & 4.545 E-09 & 3.852 E-00 & 1606 \\ 97.6 & 23 & 7.133 E-09 & 5.991 E-08 & 1610 \\ 98.6 & 23 & 7.133 E-09 & 5.991 E-08 & 1611 \\ 99.6 & 115 & 3.515 E-08 & 2.953 E-07 & 1613 \\ 100.6 & 142 & 5.553 E-00 & 4.665 E-07 & 1615 \\ 101.6 & 944 & 2.879 E-07 & 2.418 E-06 & 1617 \\ 102.6 & 1447 & 4.412 E-07 & 3.706 E-06 & 1618 \\ 103.6 & 2285 & 6.965 E-07 & 5.850 E-06 & 1620\end{array}$

\begin{tabular}{|c|c|c|c|c|}
\hline $9 \cdot .6$ & 0 & $n$. & u. & 1000 \\
\hline 31.1 & u & 0 . & 0. & 1500 \\
\hline 97.6 & 9 & $5.2+7 E-\cup 9$ & $2.655 E-08$ & 1610 \\
\hline 94.6 & 6 & 4. $891 E-09$ & $2.641 E-08$ & 1611 \\
\hline 49.6 & 44 & $2.49 \mathrm{AE}-08$ & $1.349 E-07$ & 1613 \\
\hline $\begin{array}{l}100.6 \\
101.6\end{array}$ & $\begin{array}{r}798 \\
3517\end{array}$ & $\begin{array}{l}4.47 \cup E-07 \\
1.96 B E-06\end{array}$ & $\begin{array}{l}2.414 E=06 \\
1.053 E-05\end{array}$ & $\begin{array}{l}1615 \\
1617 .\end{array}$ \\
\hline $\begin{array}{l}1) 2.6 \\
1 \cap 3.6\end{array}$ & $\begin{array}{r}2178 \\
.4162\end{array}$ & $\begin{array}{l}1.219 E-06 \\
2.329 E-C 0\end{array}$ & $\begin{array}{l}6.582 E-06 \\
1.38 E-05\end{array}$ & 1618 \\
\hline
\end{tabular}




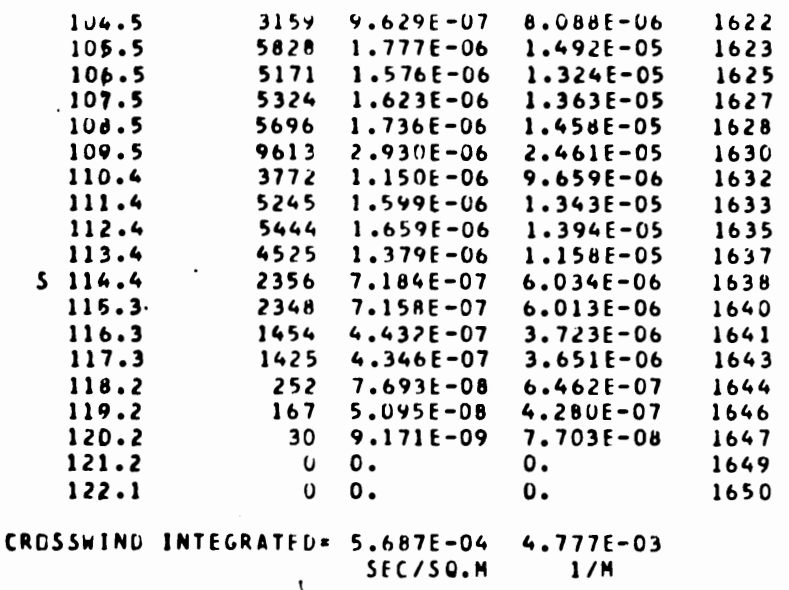

\begin{tabular}{|c|c|c|c|c|}
\hline $\begin{array}{l}\text { TEST UG } \\
\text { ZINC } \\
2200 \mathrm{M}\end{array}$ & $\begin{array}{l}2 \\
\text { SULFIDE RELE } \\
\text { RC SAMPLER }\end{array}$ & $\begin{array}{l}\text { E } 7,1968 \\
\text { ASE FRUM E } \\
\text { HT } 1.5 \mathrm{SH}\end{array}$ & $\begin{array}{r}0100 \text { TD } \\
\text { EVATION OF } \\
U=8.4 \mathrm{~m} / \mathrm{S}\end{array}$ & $\begin{array}{l}0 \quad 0130 \\
F \quad 26 M \\
S E C \quad A T\end{array}$ \\
\hline $\begin{array}{l}\text { AZ IMUTH } \\
\text { DEGREES }\end{array}$ & $\begin{array}{l}\text { EXPOSURE } \\
\text { GM-SEC/CU.M } \\
\text { XIOE+ }\end{array}$ & $\begin{array}{c}E / U \\
\text { SEC/CU.M }\end{array}$ & $\begin{array}{c}\text { EU/O } \\
1 / S O . M\end{array}$ & $\begin{array}{l}\text { DISTANCE } \\
\text { METERSS }\end{array}$ \\
\hline - 96.0 & 1 & $3.423 \mathrm{E}-10$ & $2.875 E-09$ & 2200 \\
\hline $\begin{array}{r}98.0 \\
100.0\end{array}$ & $\begin{array}{r}4 \\
57\end{array}$ & $\begin{array}{l}1.369 E-09 \\
1.757 E-08\end{array}$ & $\begin{array}{l}1.150 E-08 \\
1.476 E-07\end{array}$ & $\begin{array}{l}2200 \\
2200\end{array}$ \\
\hline 102.0 & 510 & $1.555 E-07$ & $1.306 E-06$ & 2200 \\
\hline 164.0 & 2029 & $6.185 E-07$ & $5.195 E-06$ & 2200 \\
\hline 106.0 & 4086 & $1.246 E-06$ & $1.046 E-05$ & 2200 \\
\hline E 108.0 & 5613 & $1.711 \mathrm{E}-06$ & $1.437 E-05$ & 2200 \\
\hline 110.0 & 5646 & $1.721 \mathrm{E}-06$ & $1.446 E-05$ & 2200 \\
\hline 112.0 & 3882 & $1.183 \mathrm{E}-06$ & $9.939 \mathrm{E}-06$ & 2200 \\
\hline 114.0 & 3071 & $9.362 \mathrm{E}-07$ & $7.064 E-06$ & 2200 \\
\hline 116.0 & 1339 & $4.083 E-07$ & $3.430 E-06$ & 2200 \\
\hline 118.0 & 391 & $1.192 \mathrm{E}$ & $1.001 E-06$ & 2200 \\
\hline 120.0 & 148 & $4.541 \mathrm{E}-08$ & $3.814 E-07$ & 2200 \\
\hline 122.0 & 6 & $1.939 E-09$ & $1.629 \mathrm{E}-08$ & 2200 \\
\hline 124.0 & 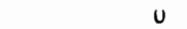 & $2.282 E-10$ & $1.917 E-09$ & 2200 \\
\hline 126. & c & 0 . & 0 . & 2200 \\
\hline Cos Sin INO & EGRAT & $\begin{array}{l}6.271 E-04 \\
\text { SEC/SO.M }\end{array}$ & $\begin{array}{c}5.267 E-03 \\
1 / M\end{array}$ & \\
\hline
\end{tabular}

TEST U62 JUNE 7. 1968 O 0100 TO 0130 PST ZINC SULFIDE RELEASE FRDM ELFVATIUN OF $26 \mathrm{M}$ $3200 \mathrm{M}$ ARC SAMPLER HT $1.5 \mathrm{M} U=8.4 \mathrm{M} / \mathrm{SEC}$ AT $26 \mathrm{M}$

AZIMUTH EXPOSURE E/O EU/O DISTANCE DEGREES GM-SEC/CU.M SECICU.M I/SO.M METERS $X 1 O E+6$

\begin{tabular}{|c|c|c|c|c|}
\hline 99.8 & 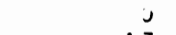 & 0. & 0. & 3200 \\
\hline 100.8 & 17 & $5.454 E-09$ & $4.581 E-08$ & 3217 \\
\hline 101.8 & 52 & $1.6 \cup 4 \mathrm{E}-08$ & $1.347 \mathrm{E}-07$ & 3218 \\
\hline 142.8 & 217 & $6.641 E-08$ & $5.570 E-07$ & 3220 \\
\hline 103.8 & 394 & $1.203 E-07$ & $1.011 E-06$ & 3222 \\
\hline 104.8 & 1330 & $4.055 E-07$ & $3.4 \cup 6 E-06$ & 3224 \\
\hline 105.8 & 2253 & $6.869 E-07$ & $5.770 E-06$ & 3225 \\
\hline 106.8 & 3212 & $9.791 E-07$ & $8.225 \mathrm{E}-06$ & 3227 \\
\hline 107.8 & $281 t^{\circ}$ & $8.591 E-07$ & $7.217 \mathrm{E}-06$ & 3229 \\
\hline 108.8 & 2830 & $8.627 \mathrm{E}-57$ & $7.246 t-06$ & 3230 \\
\hline 109.7 & 2319 & $7.071 E-07$ & 5.93 YE-06 & 3232 \\
\hline 110.7 & 3240 & $9.878 E-07$ & $8.297 E-06$ & 3233 \\
\hline iil. & 2958 & $9.018 \mathrm{E}-07$ & $7.575 E-06$ & 3235 \\
\hline 112.7 & 4022 & $1.226 E-06$ & $1.030 E-05$ & 3237 \\
\hline 113.7 & 2892 & $8.816 E-07$ & $7.405 E-06$ & 3238 \\
\hline 114.6 & 1270 & $3.872 \mathrm{E}-07$ & $3.253 E-06$ & 3240 \\
\hline 115.6 & 651 & $1.983 E-07$ & $1.665 E-06$ & 3241 \\
\hline 116.6 & 482 & $1: 469 E-07$ & $1.234 E-06$ & 3243 \\
\hline 117.6 & 87 & $2.663 E-08$ & $2.237 E-07$ & 3245 \\
\hline 118.6 & 38 & $1.187 E-08$ & $9.971 \mathrm{E}-08$ & 3246 \\
\hline 119.5 & & $6.416 E-10$ & $5.390 E-09$ & 3248 \\
\hline 120.5 & 13 & $4.171 \mathrm{E}-09$ & $3.503 \mathrm{E}-00$ & 3249 \\
\hline 121.5 & 4 & $1.283 \mathrm{E}-09$ & $1.078 \mathrm{E}-08$ & 3251 \\
\hline & 0 & 0. & 0 . & 3252 \\
\hline IN & 0 & $\begin{array}{r}5.269 E-04 \\
\text { SEC/SO.M }\end{array}$ & $\begin{array}{c}4.426 E-03 \\
1 / M\end{array}$ & \\
\hline
\end{tabular}

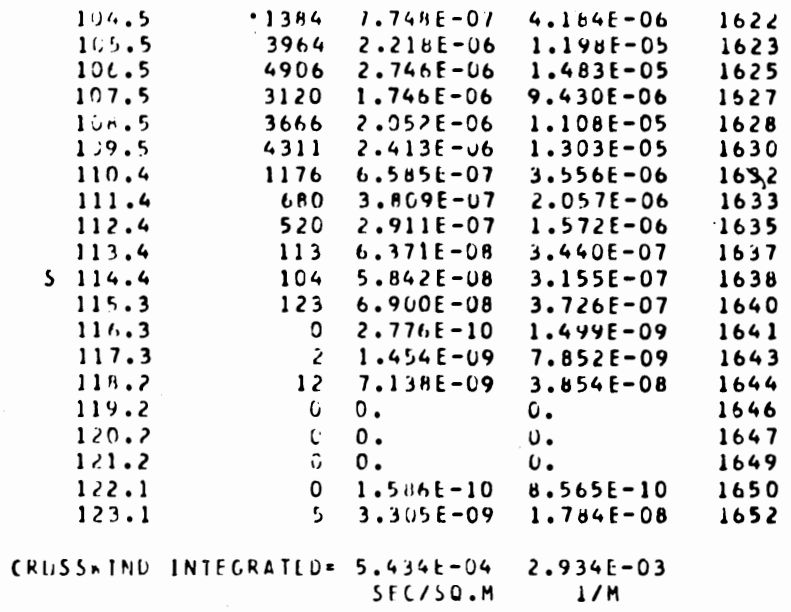

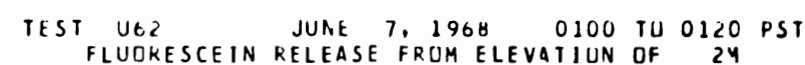
$220 D M$ ARC SAMPLER HT $1.5 \mathrm{M} U=5.4 \mathrm{M} / \mathrm{SEC}$ AT $2 \mathrm{M}$

AzIIIITR EXPQSURE E/O EU/O DISTANCE DFGREES GM-SECICU.M SFCICU.M I/SU.M MEIERS $X I O E+6$

96.0
98.0
1100.0
102.0
104.0
110.0
119.0
110.0
112.0
114.0
116.0
119.0
1120.0
122.0
124.0
$B 1<6.0$
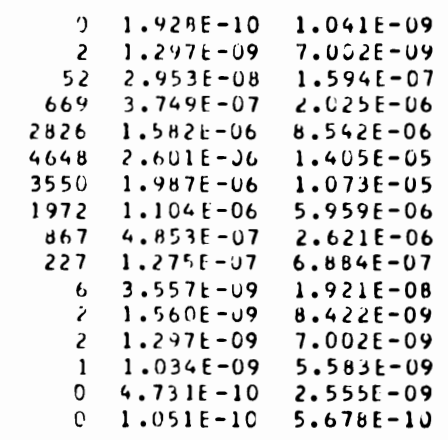

2200

2200

2200

2200

2200

2200

2200

2200

2200

2200

2200

2200

2200

2200

2200

CROSSMINO INTEGRATHO $=6.374 E-04 \quad 3.442 E-03$

\section{TEST U62 JUNE 7. 1968 O100 TO 0120 PST \\ FLUNRESCFIN RELEASE FROM ELEVATIUN UF LM} $320 G 11$ ARC SAMPLER HT $1.5 \mathrm{M} U=5.4 \mathrm{M} / \mathrm{SEC}$ AT $2 \mathrm{M}$

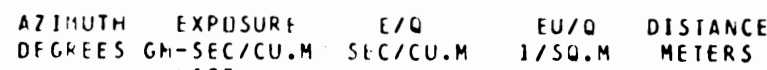




\begin{tabular}{|c|c|c|c|c|}
\hline \multirow{2}{*}{$\begin{array}{l}\text { TEST U6Z } \\
2 \text { INC S } \\
5000 M \text { AR } \\
\text { ALIMUTH } \\
\text { DEGREES }\end{array}$} & $\begin{array}{l}\text { JUNE } \\
\text { ULF IOE RELEA } \\
\text { SAMPLER }\end{array}$ & $\begin{array}{l}\text { T. } 1960 \\
\text { SL FROM Et } \\
\text { HT } 1.5 \mathrm{H}\end{array}$ & $\begin{array}{r}0100 \\
\text { FVATION }\end{array}$ & $\begin{array}{l}110230 \text { PST } \\
\text { F } 26 \mathrm{M} \\
\text { SEC AT } 26 \mathrm{C}\end{array}$ \\
\hline & $\begin{array}{c}\text { EXPOSURE } \\
\text { CH-SEC } / C U . H \\
\times 1 O E+6\end{array}$ & $\begin{array}{c}\text { ER/O } \\
\text { SEC/CU.M }\end{array}$ & $\begin{array}{l}E U / O \\
1 / S O . M\end{array}$ & $\begin{array}{l}\text { OLSTANCE } \\
\text { METERS }\end{array}$ \\
\hline $\begin{array}{l}100.0 \\
102.0\end{array}$ & $\begin{array}{l}0 \\
4\end{array}$ & $0.369 E-09$ & $\begin{array}{l}0.150 E-08 \\
1.150\end{array}$ & $\begin{array}{l}4690 \\
4710\end{array}$ \\
\hline $\begin{array}{l}104.0 \\
106.0\end{array}$ & $\begin{array}{r}53 \\
589\end{array}$ & $\begin{array}{l}1.620 E-08 \\
1.796 E-07\end{array}$ & $\begin{array}{l}1.361 E-07 \\
1.508 E-06\end{array}$ & $\begin{array}{l}4770 \\
4810\end{array}$ \\
\hline 108.0 & 1266 & $3.861 \mathrm{f}-07$ & $3.243 \mathrm{E}-06$ & $\begin{array}{l}4810 \\
4070\end{array}$ \\
\hline $\begin{array}{l}110.0 \\
112.0\end{array}$ & $\begin{array}{l}2025 \\
1877\end{array}$ & $\begin{array}{l}6.174 E-07 \\
5.723 E-07\end{array}$ & $\begin{array}{l}5.186 E-06 \\
4.807 E-06\end{array}$ & 4920 \\
\hline 114.0 & 928 & $\begin{array}{l}3.123 E=07 \\
2.829 E-07\end{array}$ & $\begin{array}{l}2.807 E-06 \\
2.377 E-06\end{array}$ & $\begin{array}{l}4990 \\
5080\end{array}$ \\
\hline 116.0 & 130 & $3.970 E-08$ & $3.335 E-07$ & 5020 \\
\hline 119.0 & 12 & $3.765 E-09$ & $3.162 \mathrm{E}-08$ & 5100 \\
\hline 120.0 & 12 & $3.879 E-09$ & $3.258 \mathrm{E}-0 \mathrm{~d}$ & 5130 \\
\hline $1<2$. & 2 & $6.845 E-10$ & $5.750 E-09$ & 4830 \\
\hline & 0 & 0. & 0. & 4660 \\
\hline DSSWIND & INIEGRATED: & $\begin{array}{l}3.630 E-04 \\
\text { SEC } 150 . M\end{array}$ & $\begin{array}{c}3.049 E-D S \\
1 / M\end{array}$ & \\
\hline
\end{tabular}

IEST UG2
2INC SULFIOE RELEASE FROA 1968 OELFATION OF 0130 PST $7000 \mathrm{M}$ ARC SAMPLER HT $1.5 \mathrm{M}$ UE $8.4 \mathrm{M} / \mathrm{SEC}$ AT $26 \mathrm{M}$

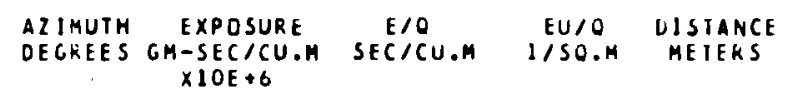

CRISSINU INTEGRATED= $\begin{gathered}1.949 E-04 \\ \text { SEC/SU.M }\end{gathered}$
TEST U6? JUNE 7, 1960 O 0100 TU 0130 PST 2INC SULFIOE RELEASE FRUM ELEVATION OF $26 \mathrm{M}$ $12800 M$ ARC SAMPLER HT $1.5 \mathrm{M} U=8.4 \mathrm{M} / 5 E C$ AT $26 \mathrm{M}$

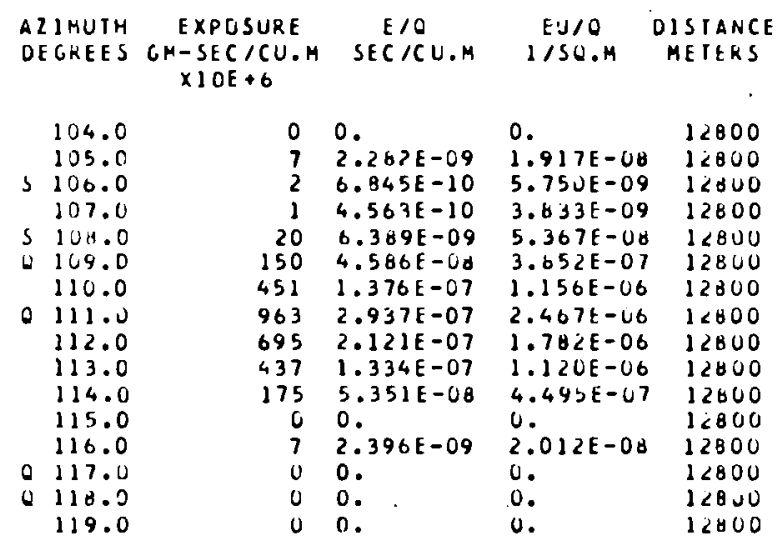

CRUSSHINO INIEGRATEO $0.984 E-04 \quad 1.667 E-03$

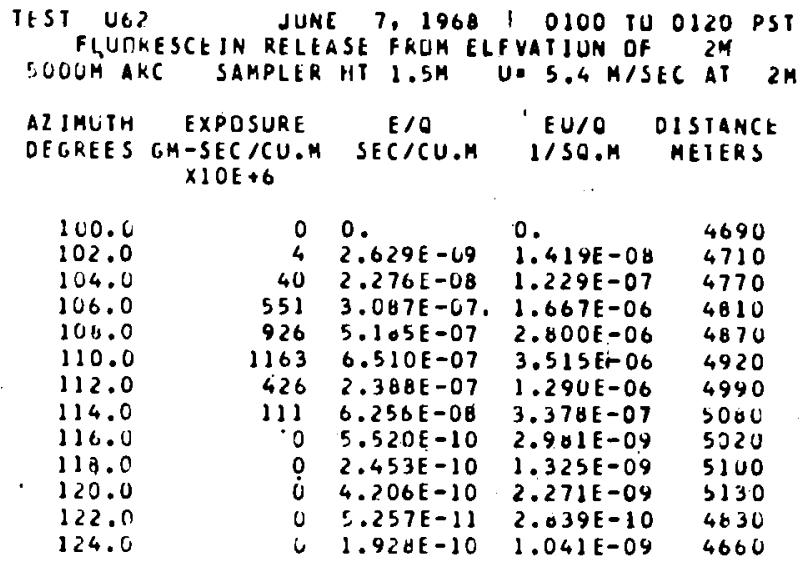

100.0
102.0
104.0
106.0
106.0
110.0
112.0
114.0
116.0
119.0
120.0
122.0
124.0

$\begin{array}{rll}0 & 0 . & 0 . \\ 4 & 2.629 E-19 & 1.419 E-08 \\ 40 & 2.276 E-08 & 1.229 E-07 \\ 551 & 3.087 E-07 . & 1.667 E-06 \\ 926 & 5.105 E-07 & 2.800 E-06 \\ 1163 & 6.510 E-07 & 3.515 E-06 \\ 426 & 2.388 E-07 & 1.290 E-06 \\ 111 & 6.256 E-08 & 3.378 E-07 \\ 0 & 5.520 E-10 & 2.901 E-09 \\ 0 & 2.453 E-10 & 1.325 E-09 \\ \dot{0} & 4.206 E-10 & 2.271 E-09 \\ 0 & 5.257 E-11 & 2.039 E-10 \\ 0 & 1.928 E-10 & 1.041 E-09\end{array}$

4690

4710

4770

4810

4870

4920

4990

5000

5320

5100

3130

4830

4660

\begin{tabular}{|c|c|c|c|c|}
\hline $\begin{array}{l}\text { IEST UEZ } \\
\text { FLUGR } \\
\text { TUDUH AK }\end{array}$ & $\begin{array}{l}\text { JSCEIN RELE } \\
\text { CSCEMLER }\end{array}$ & $\begin{array}{l}\text { E } 7,1908 \\
\text { ASE FRDM E } \\
\text { HT } 1.5 \mathrm{H}\end{array}$ & $\begin{array}{r}0100 \text { IU } \\
\text { LEVATIUN DF } \\
\text { U } 5.4 \text { M/S }\end{array}$ & $\begin{array}{l}01 \angle 0 P S \\
2 M \\
E C A I\end{array}$ \\
\hline $\begin{array}{l}\text { AZIPUIH } \\
\text { DLChEES }\end{array}$ & $\begin{array}{c}\text { EXPDSURE } \\
\text { GH-SEC /CU.H } \\
\times 1 B E+6\end{array}$ & $\begin{array}{c}E / O \\
\text { SEC/CU.M }\end{array}$ & $\begin{array}{c}\text { EU/O } \\
\text { I/SU.M }\end{array}$ & $\begin{array}{l}\text { DISIANCE } \\
\text { MLTERS }\end{array}$ \\
\hline $\begin{array}{l}9 c .0 \\
911.0\end{array}$ & 1 & $H .061 E-10$ & $4.353 E-U 9$ & $\begin{array}{l}6510 \\
65<0\end{array}$ \\
\hline $\begin{array}{r}911.0 \\
100.0\end{array}$ & $\begin{array}{l}\vdots \\
0\end{array}$ & $\begin{array}{l}0 . \\
0 .\end{array}$ & 0. & $\begin{array}{l}65<0 \\
6560\end{array}$ \\
\hline 102.0 & c & 0. & 0. & 0510 \\
\hline $1 \cup 4 . c$ & 2 & $1.542 E-09$ & a. $327 E-09$ & 6650 \\
\hline 4106.0 & 91 & $5.133 \mathrm{E}-68$ & $2.772 \mathrm{E}-07$ & 6720 \\
\hline 4103.0 & 280 & $1.612 E-07$ & $8.705 E-07$ & 6000 \\
\hline 4116.0 & 30 & $2.136 E-08$ & $1.153 E-07$ & 7220 \\
\hline 112.0 & 294 & $1.649 k-07$ & $0.403 E-07$ & 7300 \\
\hline 114.0 & 77 & $4.309 E-08$ & $2.327 E-07$ & 7210 \\
\hline $\begin{array}{l}110.0 \\
11 n .0\end{array}$ & 0 & $3.505 E-10$ & $1.093 E-09$ & 7000 \\
\hline $11 n .0$ & $\iota$ & 0. & u. & 7190 \\
\hline RLISSHINO & INTECRATEDE & $\begin{array}{r}8.728 E-05 \\
\text { SEC/SO.M }\end{array}$ & $\begin{array}{c}4.713 \mathrm{E}-04 \\
1 / \mathrm{M}\end{array}$ & \\
\hline
\end{tabular}

IEST U62 JUNE 7, 1908 O1UO TU 0120 PST H. 5.4 H/5EC AI $2 M$ DLCKEES GH-SECICU.H SECICU.M IJSO.M MLTERS

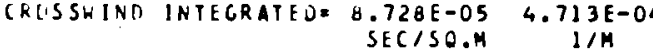

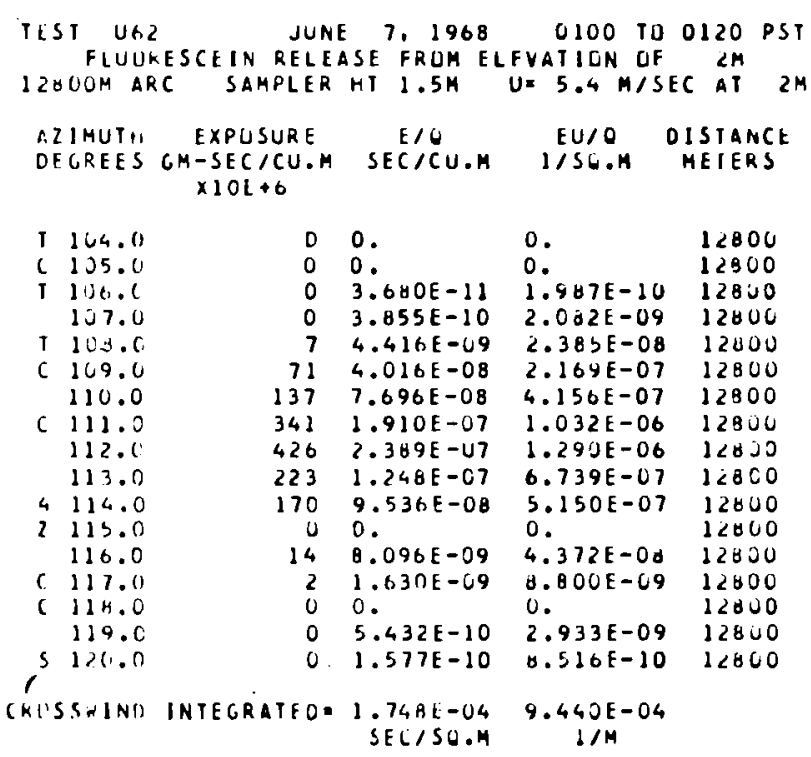


SAMPLINC 4OON TO $12800 \mathrm{M}$, NO TOHER SAMPLING.

ZINC SULFIOE DISPERSED FOR 30 MIN. DISPERSAL DIFFICULIIES RESTRICTED FLUDRESCEIN DISPERSAL ID 25 MIN. 2200M AND 12800 ARCS TRUNCATED ON NDRTHI $12800 M$ DATA EXTRAYQLATED SLIGHTLY

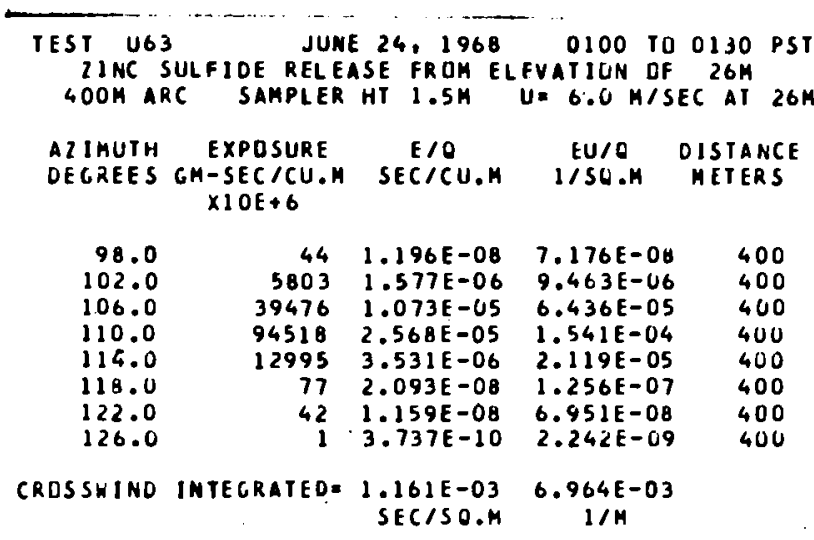

$$
\begin{aligned}
& \text { TEST U63 JUNE } 24,1968 \text { 0105 TO } 0130 \text { PST } \\
& \text { FLULIKESCEIN RELEAST FROM ELFVATION DF } 2 \mathrm{M} \\
& \text { LUOH AKC SAMPLER HT } 1.5 \mathrm{SH} U \text { U } 3.0 \mathrm{M} / \mathrm{SEC} \text { AT } 2 \mathrm{H} \\
& \text { HITHUTH EXPOSURE E/O EUIO DISIANCE } \\
& \text { DEGREES GM-SECICU.M SECICU.H I/SU.M MEIERS } \\
& \times 10 E+6
\end{aligned}
$$

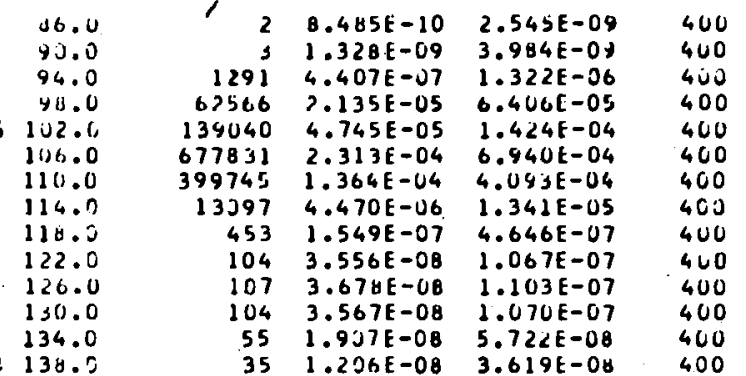

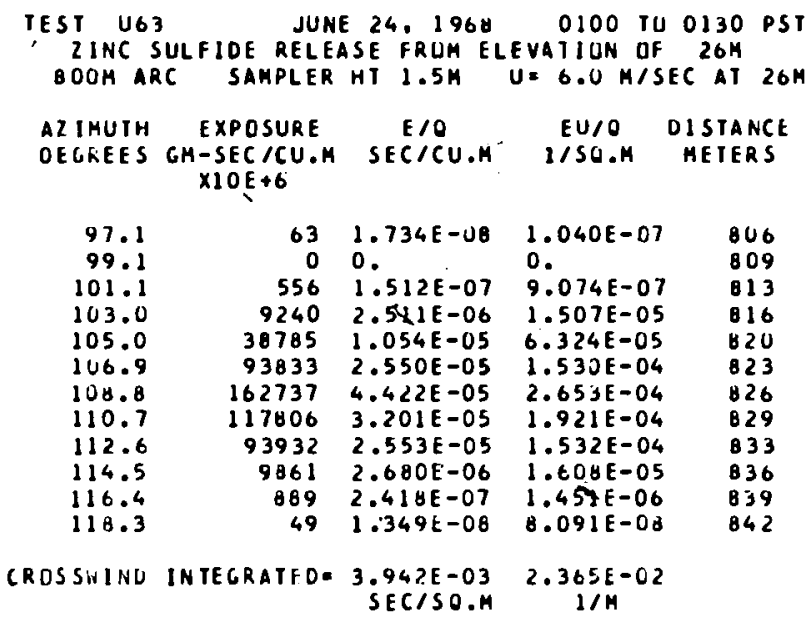

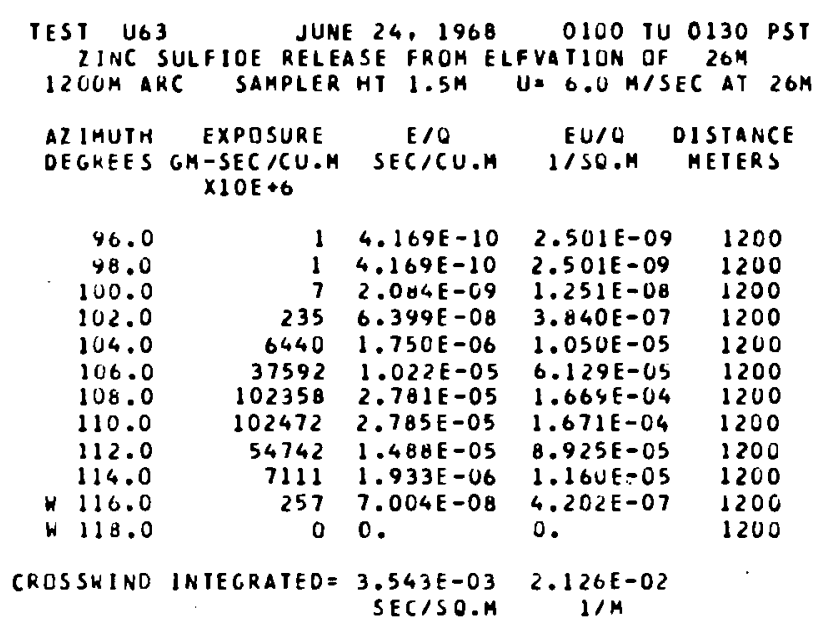

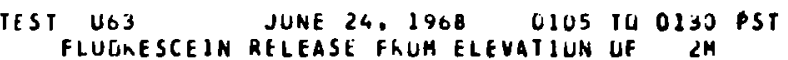
BOUM AKC SAMPLER HI $1.5 \mathrm{H} U=3.0 \mathrm{H} / \mathrm{SEC}$ AT $2 \mathrm{M}$ AZINUTH EXPOSURE $T$ /O EU/O DISTANCE DEGREES GM-SEC/CU.M SFCICU.M I/SO.M MEIERS $X 10 E+6$

$\begin{array}{rrrrr}97.1 & 10 & 3.667 E-09 & 1.100 E-00 & 806 \\ 99.1 & 2240 & 7.646 E-07 & 2.294 E-06 & 809 \\ 101.0 & 20603 & 7.032 E-06 & 2.110 E-05 & 813 \\ 133.0 & 82554 & 2.801 E-05 & 8.402 E-05 & 816 \\ 105.0 & 149007 & 5.086 E-05 & 1.526 E-04 & 820 \\ 106.9 & 331147 & 1.130 E-04 & 3.391 E-04 & 823 \\ 101.0 & 352745 & 1.204 E-04 & 3.612 E-04 & 826 \\ 110.7 & 107972 & 3.665 E-05 & 1.100 E-04 & 829 \\ 112.6 & 19539 & 6.669 E-06 & 2.001 E-05 & 833 \\ 114.5 & 886 & 3.025 E-07 & 9.076 E-07 & 836 \\ 116.4 & 24 & 8.361 E-09 & 2.508 E-00 & 839 \\ 111.3 & 9 & 3.301 E-09 & 9.902 E-04 & 842 \\ 120.2 & 0 & 2.445 E-10 & 7.335 E-10 & 845\end{array}$

CROSSHIND INTECRATED=1.002E-02 3.606E-02

TEST U6J JUNE 24, 1968 OIUS TO 0130 PST FLUURESCEIN RELEASE FRUH ELEVATIUN OF $2 \mathrm{H}$ 120 JM ARC SAMPLER HT $1.5 \mathrm{H} U=3.0 \mathrm{M} / \mathrm{SEC}$ AT $2 \mathrm{H}$ AZI:IUIH EXPUSURE
DEGKEES GR-SECICU.M SECICU.M IISU.M DISTANCE $\times \perp U E+6$

$\begin{array}{rrlll}94.0 & 1 & 5.761 E-10 & 1.720 E-09 & 1200 \\ 96.0 & 0 & 0.0 & 0.0 & 1200 \\ 91.0 & 47 & 1.632 E-06 & 4.895 E-00 & 1260 \\ 100.0 & 141 & 4.841 E-08 & 1.452 E-07 & 1200 \\ 102.0 & 3162 & 1.079 E-06 & 3.230 E-00 & 1200 \\ 104.0 & 31502 & 1.075 E-05 & 3.226 E-05 & 1200 \\ 106.0 & 71490 & 2.440 E-05 & 7.320 E-05 & 1200 \\ 101.0 & 100631 & 3.639 E-05 & 1.092 E-04 & 1200 \\ 110.0 & 61796 & 2.109 E-05 & 6.327 E-05 & 1260 \\ 112.0 & 0352 & 2.051 E-06 & 0.552 E-00 & 1200 \\ 114.0 & 490 & 1.675 E-07 & 5.026 E-07 & 1200 \\ 116.0 & 5 & 2.014 E-09 & 6.043 E-09 & 1200 \\ 113.0 & 2 & 7.613 E-10 & 2.284 E-09 & 1200 \\ 120.0 & 0 & 2.058 E-10 & 6.173 E-1 J & 1200\end{array}$

CROSSWINO INTEGRATED= $4.055 E-03$
SECISO.H 


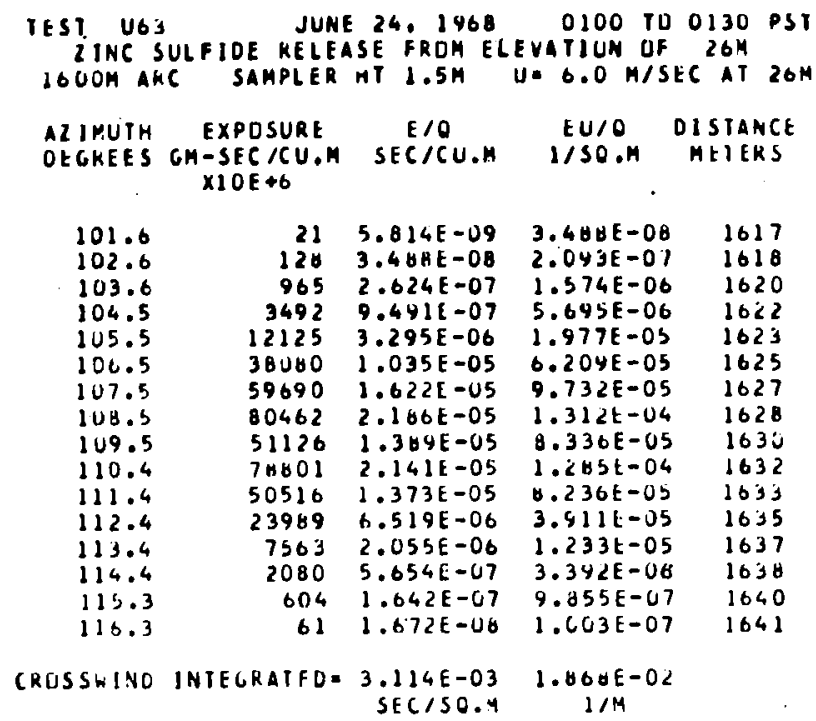

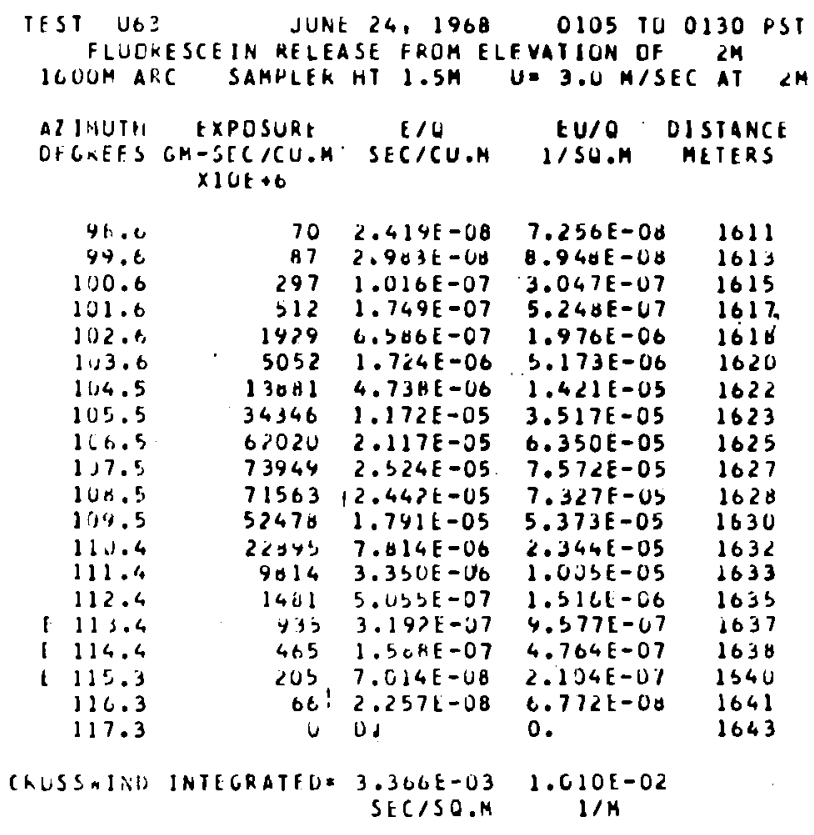

TLST U63 JUNE 24, 1968 C10S TO O1JO PST FLUUKESCLIN RLLEASE FKUM ELFVATILN UF $2 M$ $2 \angle O O M$ AKC SAHPLER HT $1.5 \mathrm{M} U=3 . U \mathrm{M} / S E C$ AT $\angle H$

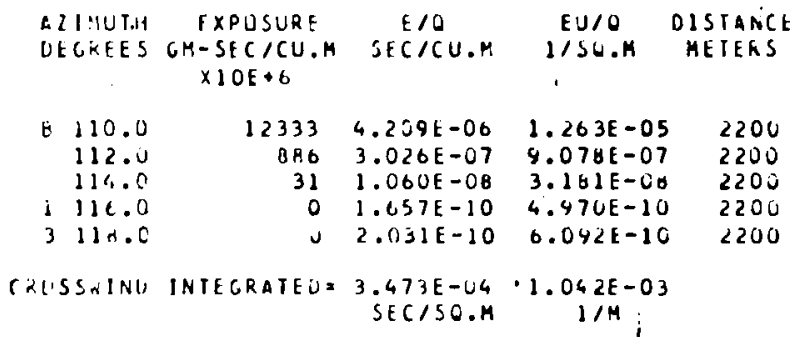

$\begin{array}{lll}114.6 & 72 & 1.921 E=0 B \\ 114.6 & 23 & 6.274 E-09 \\ 110.6 & 0 & 0 .\end{array}$

CKOSSAINU INTEGRATEU: $2.367 E-031.420 F-02$

SEC $/ 52 . M$

$1 / \mathrm{M}$

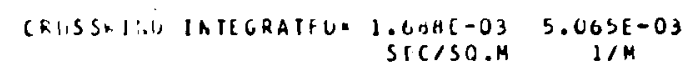




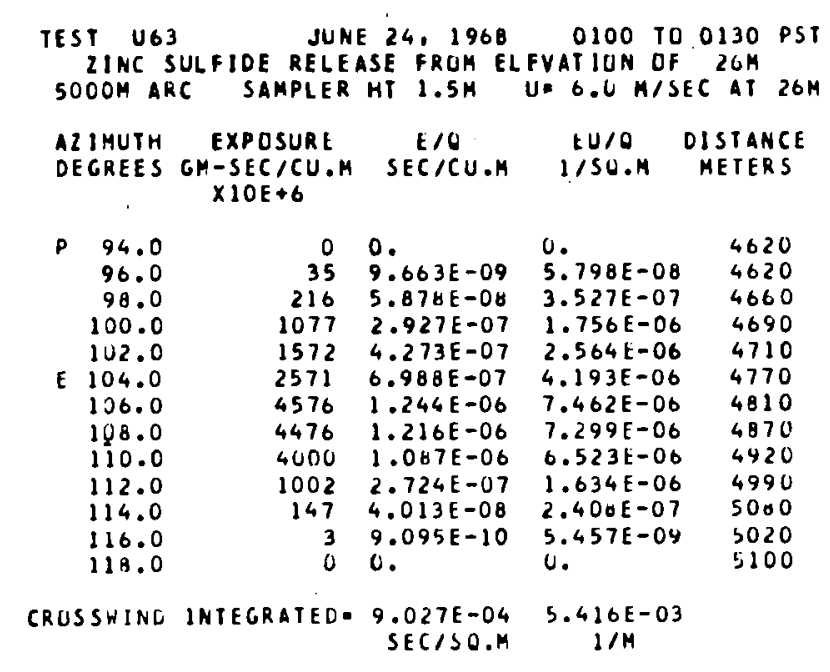

TEST U63 JUNE 24, 1960 OI00 TO 0130 PST ZINC SULFIDE RELEASE FROM ELFVATIUN DF $26 \mathrm{M}$
OOOM ARC SAMPLER HT $1.5 \mathrm{M}$ U. $0.0 \mathrm{M} / \mathrm{SEC}$ AT $26 \mathrm{M}$ $\begin{array}{cccc}\text { AZIMUTH } & \text { EXPOSURE } & \text { EIO } & \text { EU/O DISTANCE } \\ \text { DEGREES GH-SECICU.M SECICU.M I/SO.M METERS } \\ \text { XIOE }+6\end{array}$

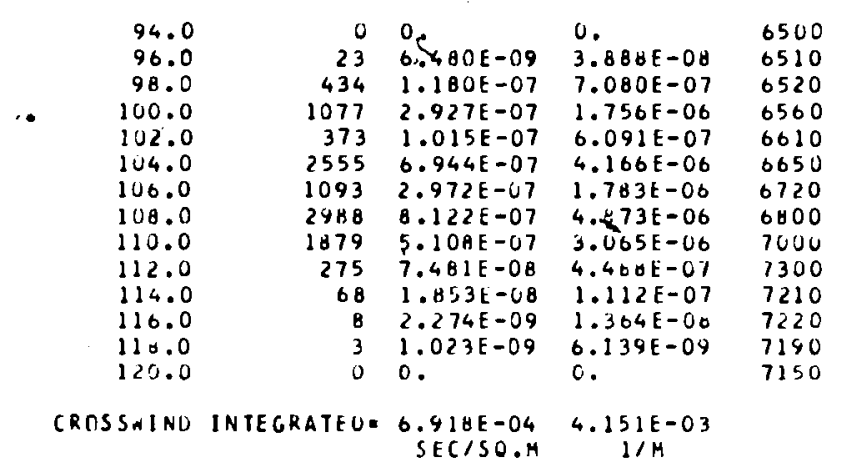

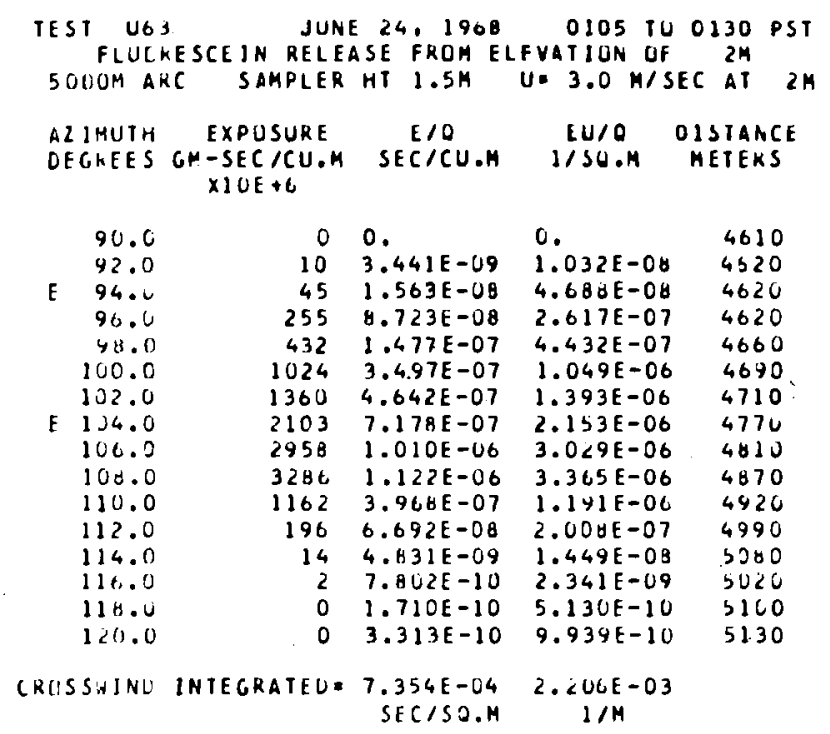

TEST UE3 JUNE 24,1968 C105 TO 0130 PST FLUURESCEIN RFLEASE FROM ELEVATILN DF CM
DOOM ARC SAMPLER HI $1.5 \mathrm{H} \quad U=3.0 \mathrm{H} / \mathrm{SEC}$ AT

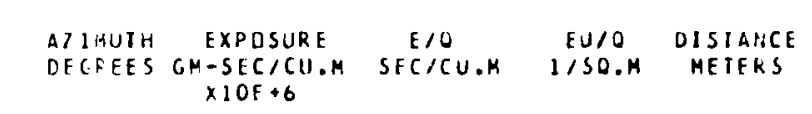

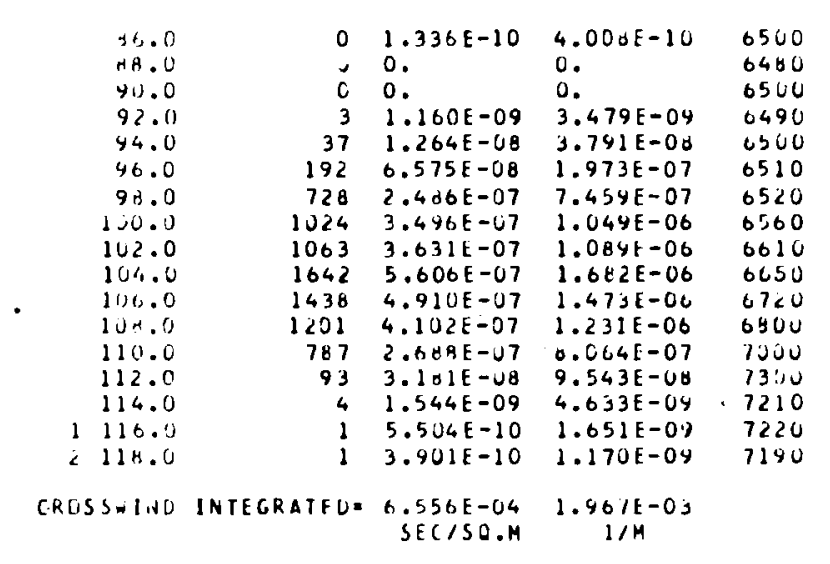




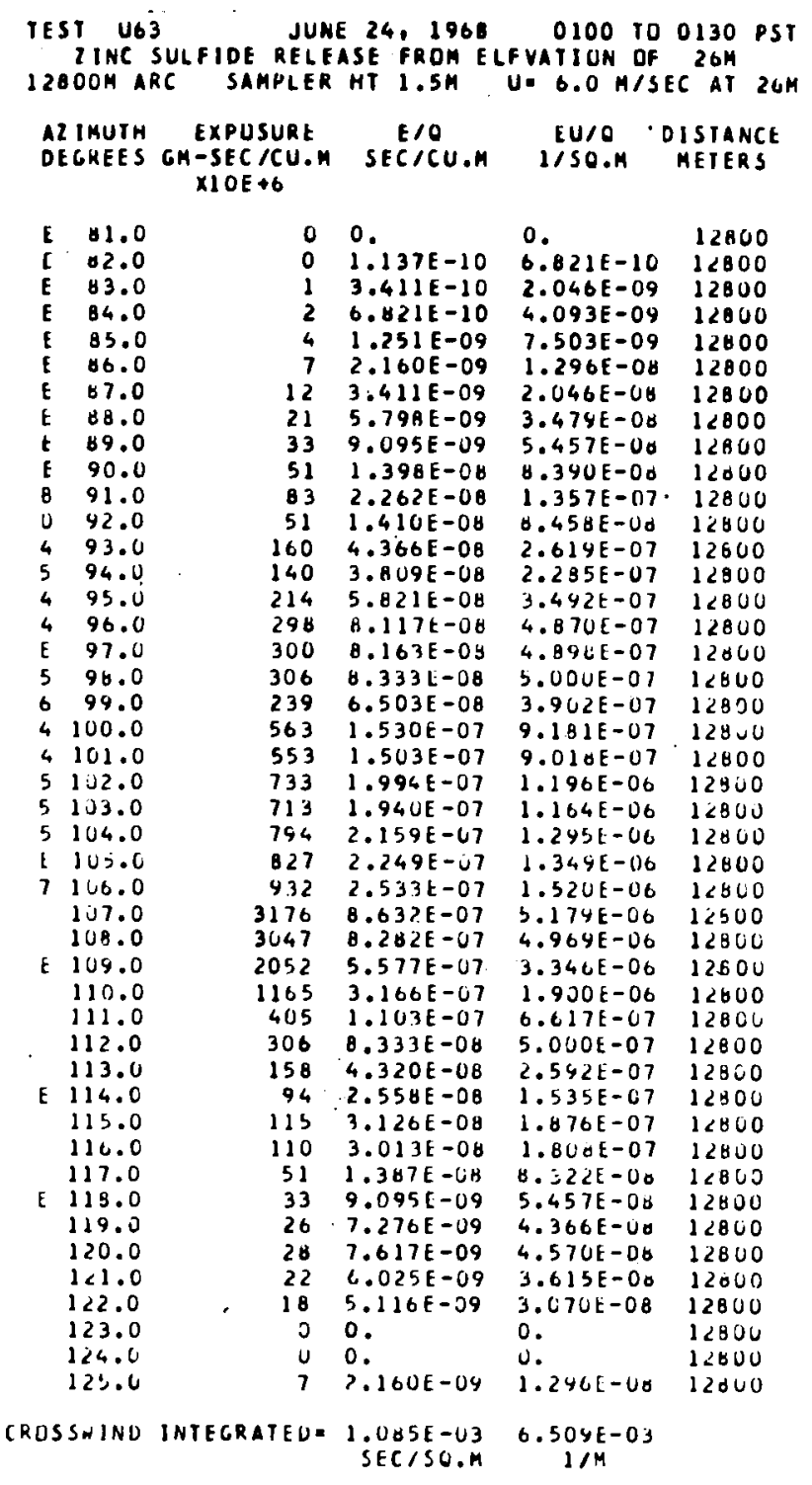

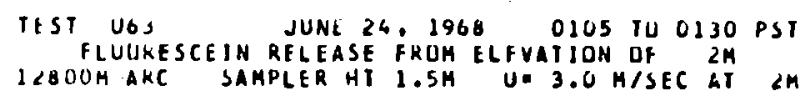

AZIMUTH EXPOSURE
DEGKEES GM-SECICU.M SECICU.M IISO.M OISTANEE
XIOE+6

\section{E 3.0.}

$\begin{array}{ll}E & 03.0 \\ t & 04.0 \\ t & 05.0\end{array}$

$\mathrm{t} 5.0$
$\mathrm{E} \quad 16.0$

F 97.0

F 68.0

F 84.0

$+\quad 30.0$

B 91.0

192.0

493.0

544.0

$4 \quad 45.0$

$4 \quad 96.0$

F 97.0

- 44.0

4 100.

4101.0

5 102.0

5123.0

5124.0

(.) $1 \% .0$

7 1): 1

- 117.0

3103.0

[. 119.1

$1 \mathrm{j} 1 \mathrm{u} .0$

3111.0

1112.0

1113.0

0114.0

$=115.0$

1116.0

2117.0

E 113.0

1114.6

120.0
1121.6

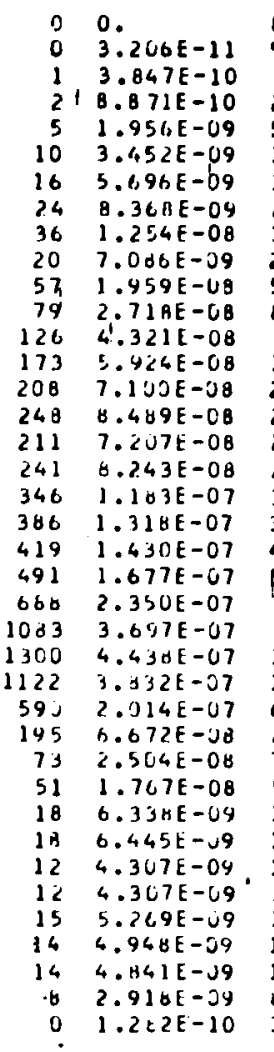

0.

12800

$-11 \quad 12800$

$1.154 E-09 \quad 12800$

$2.661 \mathrm{E}-09$ 1286C

5.867E-09 .12800

$1.036 E-08 \quad 12800$

$1.709 E-O B \quad 12800$

$\begin{array}{ll}1.709 E-08 & 12800 \\ 2.51 U F-08 & 12800\end{array}$

$3.761 \mathrm{E}-0 \mathrm{O} \quad 12800$

$2.126 E-08 \quad 12900$

$5.877 E-08 \quad 12800$

$8.153 E-0812800$

$1.296 \mathrm{E}-07 \quad 12800$

$1.7775-07 \quad 1<B 00$

$2.130 E-07 \quad 12900$

2.547E-07 12800

$2.162 E-0712800$

$2.473 E-07,12800$

$\begin{array}{ll}2.473 E-07 & 12800 \\ 3.55 U E-07 & 1<600\end{array}$

$3.954 E-07 \quad 12300$

$4.241 E-U 7 \quad 12800$

6.032E-07 12800

$\begin{array}{ll}5.032 E-07 & 12800 \\ 7.051 E-07 & 12800\end{array}$

1.1C9E-06 12800

$1.331 E-06 \quad 1 \angle 800$

1:15UE-06 $1 \angle 900$

$.042 \mathrm{E}-27 \quad 12900$

2.002E-07 12860

$7.512 E-06 \quad 12800$

$5.3 U 0 E-0 O \quad 12800$

$1.901 E-001 \angle 6 L U$

$1.933 \mathrm{E}-08 \quad 12830$

$\begin{array}{ll}1.933 E-08 & 12830 \\ 1.292 E-U O & 128 C U\end{array}$

$1.292 \mathrm{E}-0 \mathrm{O} \quad 1 \angle 800$

$1.581 \mathrm{E}-\mathrm{CO} \quad 1<800$

$1.494 E-0 O \quad 1<400$

$1.4525-00$ 1200

$1.452[-08 \quad 12400$

$\begin{array}{ll}8.753 E-04 & 12000 \\ 3.647 E-10 & 12800\end{array}$

CRISSSWIND INIEGRATFUE E.351E-04 1.9J5E-03

$$
\begin{array}{rr}
\text { t.351E-O4 } & 1.935 E-03 \\
\text { SFCISO.M } & 1 / \mathrm{H}
\end{array}
$$


SAHPLING GOOM TO 7OOOH. NO TOWER SAMPLING. ALL ARCS EABKACE CKDSSHINO EXIENI OF TKACER.

IINC SULFIDE DISPERSEO FOR 3U MIN, OISPERSAL UIFFICULIIES RESIRICIEU FLUIKESCEIN DISPERSIL TO 20 MIN.

LAKGE WIND OIRECTION SHEAR BETHEFN TRACER RELEASE LEVELS. TKACER UISIRIGUIIUNS SKEWEO LONG TALL TO THE NURTH.

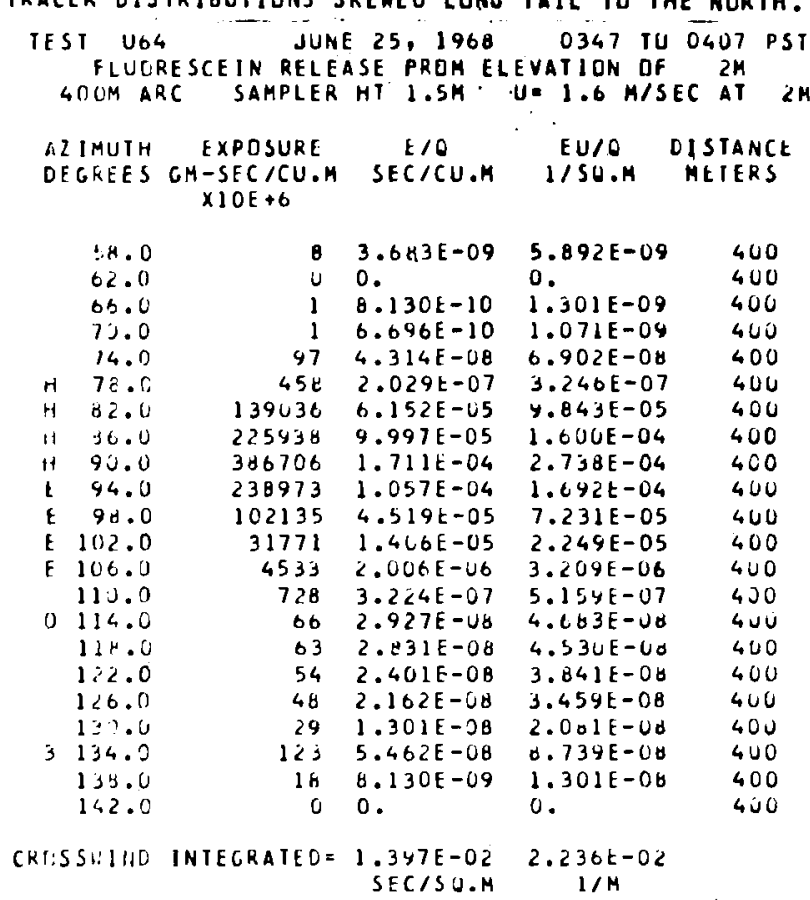

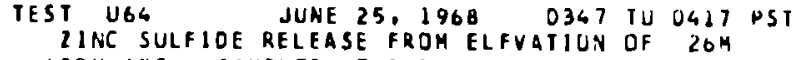
- LDOM ARC SAMPLER HT 1.5M U. $5.3 \mathrm{M} / \mathrm{SEC}$ AT $20 \mathrm{M}$

$\begin{array}{ccccc}\text { ALIMUTH } & \text { EXPOSURE } & \text { E/O } & \text { EJ/O NISIANCE } \\ \text { DFGREES CH-SEC/CU.M } & \text { SECICU.M } & \text { I/SO.M MEIERS } \\ \text { XIOE+6 } & & \end{array}$
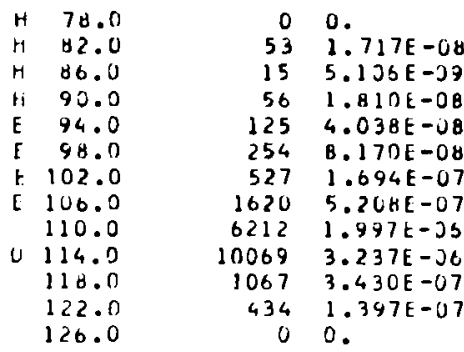

$u$.

$9.1 \cup 3 E-08$

$2.706 E-00$

$9.595 \mathrm{E}-\mathrm{OH}$

C.1 $14 \mathrm{UE}-07$

$4.330 E-07$

$6.9 \forall U E-07$

$2.760 \mathrm{E}-06$

1. $05 \mathrm{~S} \mathrm{E}-25$

1.715E-OS

$1.016 E-36$

$7.4 U S E-07$

0 .

CRUSSHIND INTEGRAIEO= $1.834 E-049.723 E-04$

SFC $/ 50.4$

400
400
400
400
400
400
400
400
400
400
400
400
400

400

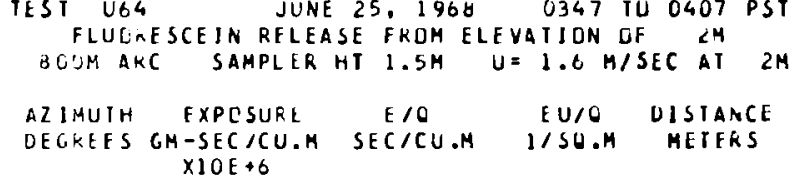

IHST U64 JUNE 25, 1968 O347 I0 0417 PSI 7 INC SULFIDE RELEASE FRIM ELFVATION UF $26 \mathrm{M}$ WOUM ARC SAMPLER HT $1.5 \mathrm{SH} U=5.3 \mathrm{M} / \mathrm{SEC}$ AT 204

AZIHUTH EXPQSURE E/O EU/O UISIANCE OEGREES CM-SECICU.M SECICU.M IISO.M YETERS $\times \perp O E+6$

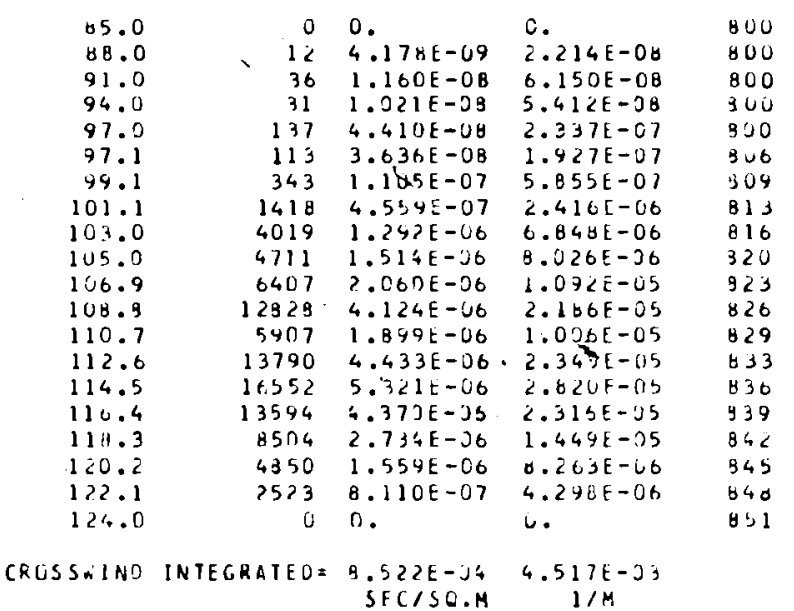

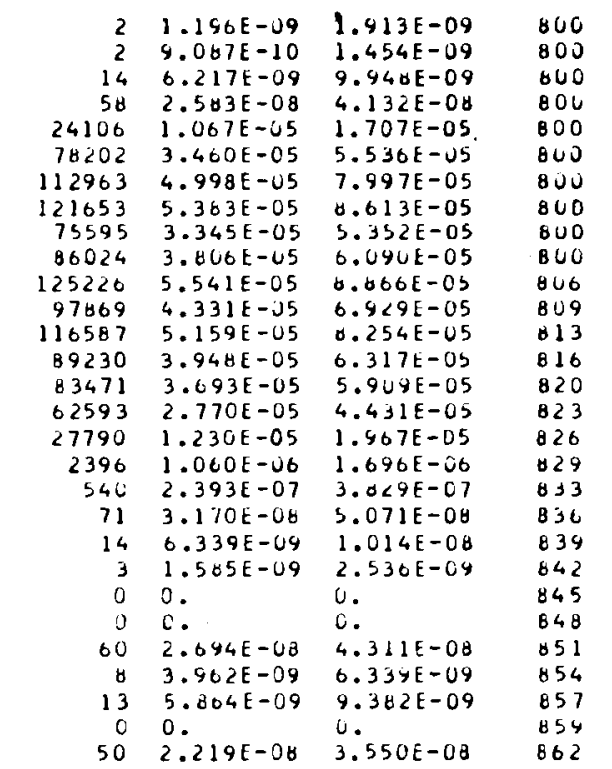

CROSSUIND INIEGRATED = $1.525 E-02 \quad 2.431 E-02$ 


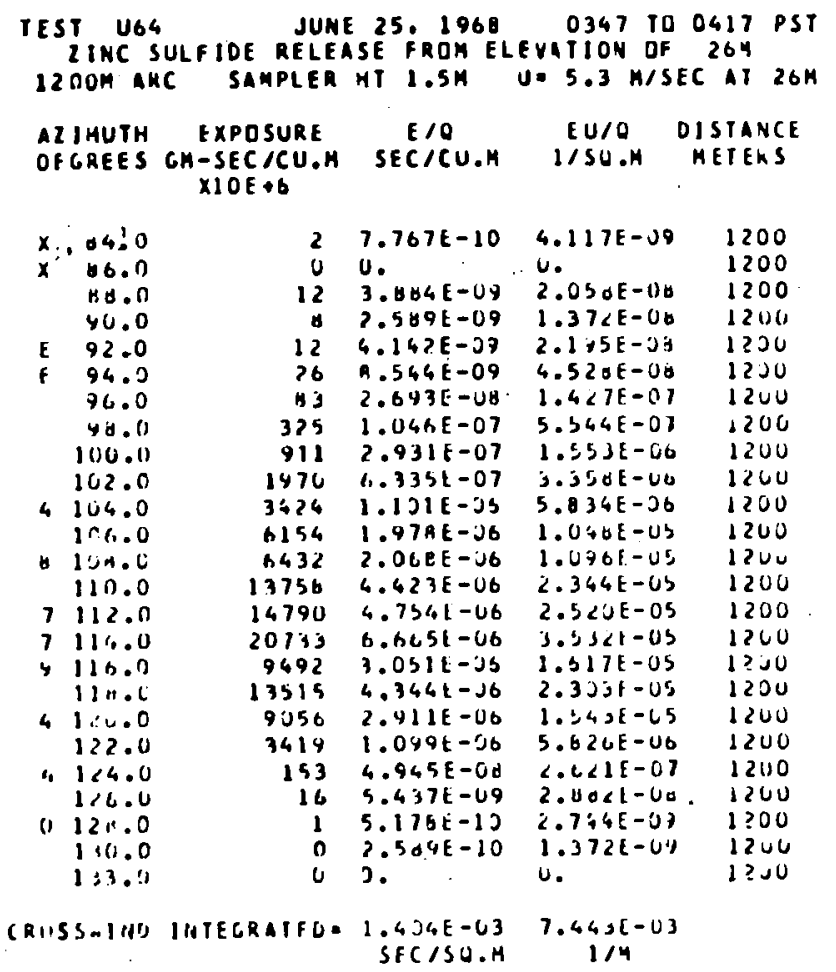

IHSI U64 JUNE 25, 1964 2347 IU 2417 PSI

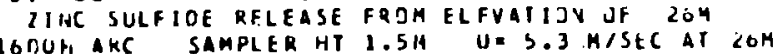
AljYUTH EXPOSURE E/G . TU/O UISTANCE

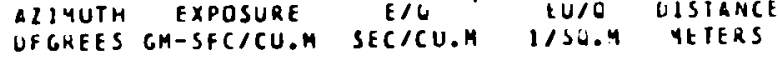
$\times 10 E+6$

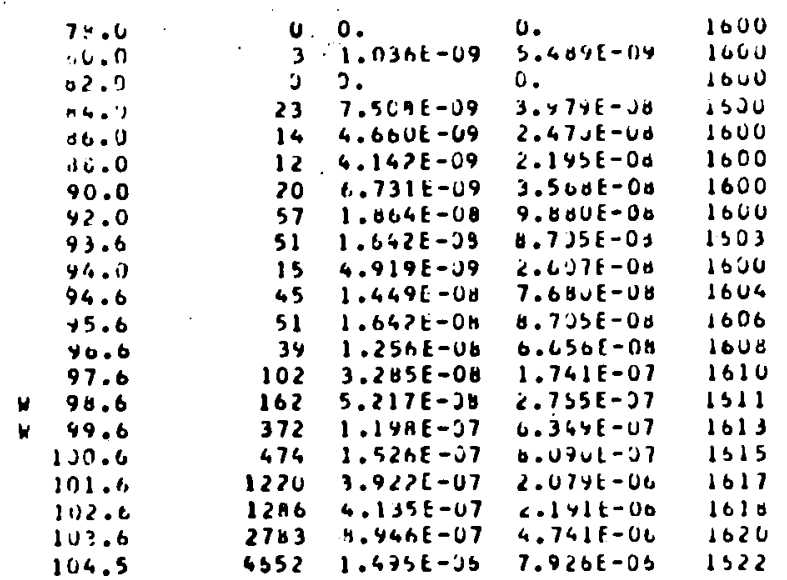

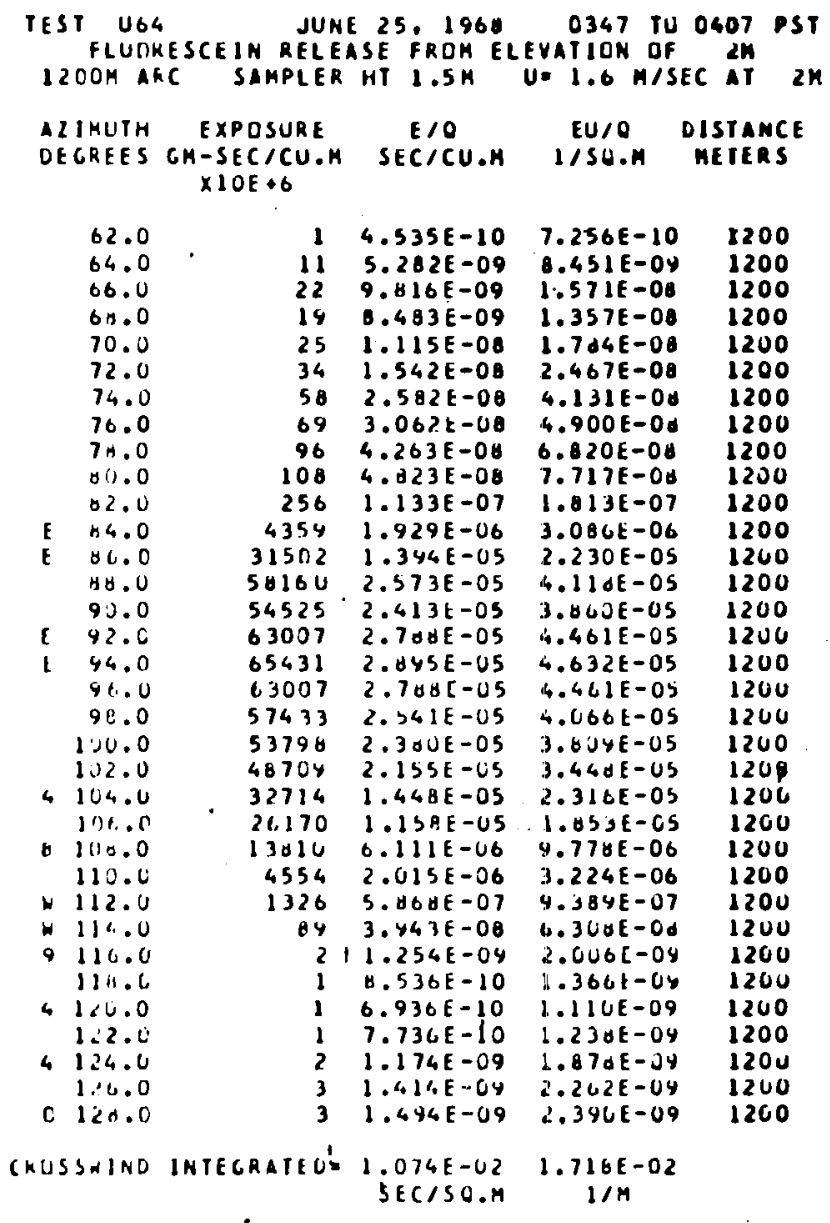

TEST UBS JUAE 25. 1968 0347 TO.0407 PST FLUGRESCEIN RELEASE FKOM ELEVATIUN OF $2 M$
SIIUM AKC SAMPLER HT $1.5 \mathrm{SH}$ VE $1.6 \mathrm{H} / \mathrm{SEC}$ AT $2 \mathrm{H}$

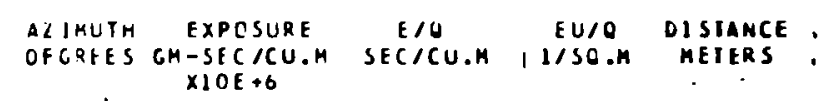

$+06.0$

- 06.0

66.0

70.0

72.0

74.0

76.0

$7 \mathrm{H} .0$

r. U.C

12.0

त4. 0

at. 00

89.0

90.0

92.0

93.6

94.0

44.6

93.6

96.6

97.6

97.6
$4 \quad 49.6$

94.6
1110.6

131.h

102.6

103.6

0
5
5
9
25
44
64
107
144
228
512
1702
8112
17443
41193
49374
24719
59637
47948
42938
39359
33633
30055
22898
34344
31446
24624
24329

2.3

$2.321 t-09$
$2.481 E-09$

$2.441 E-09$
$4.215 E-09$ $1.142 \mathrm{E}-08$

$1.469 E-C 8$

$2.849 E-08$

$4.770 E-00$

$6.397 E-C d$.

$1.013 \mathrm{E}-07$

$2.27 \cup E-07$

$7.535 E-07$

$3.590 E-06$

7.71 OE-06

1. $423 E-05$

$2.185 E-05$

$1.094 \mathrm{E}-05$

$2.639 E-05$

$2.122 \mathrm{E}-05$

$1.900 E-05$

$1.747 E-05$

$1.480 E-05$

1. $336 E-05$

1.U13E-0S

$1.52 J E-05$

1. S'JE-05

$1.267 E-65$

13.

3.71 SE-O9

$3.964 E-U 9$

$6.743 \mathrm{E}-04$

$1.027 E-08$

$3.150 E-08$

$4.550 E-00$

$7.631 E-00$

$1.023 \mathrm{E}-07$

$.621 \mathrm{E}-07$

$1.206 E-06$
.

5.743 E-06

$1.235 E-05$

$2.916 E-05$

$3.496 \mathrm{E}-05$

$1.75 U E-05$

$4.222 \mathrm{E}-05$

3.395 E-OS

3.040 E-05

$2.787 E-0 S$

$2.381 E-05$

C.120E-05

$1.0<1 E-G 3$

$2.432 E-05$

$2.224 E-05$

$2.224 E-05$
$2.026 E-05$

1.732E-0S
1600
1600
1600
1600
1600
1600
1600
1600
1600
1600
1600
1690
1600
1600
1600
1603
1500
1604
1606
1600
1610
1611
1613
1615
1617
1619
1620
1622 


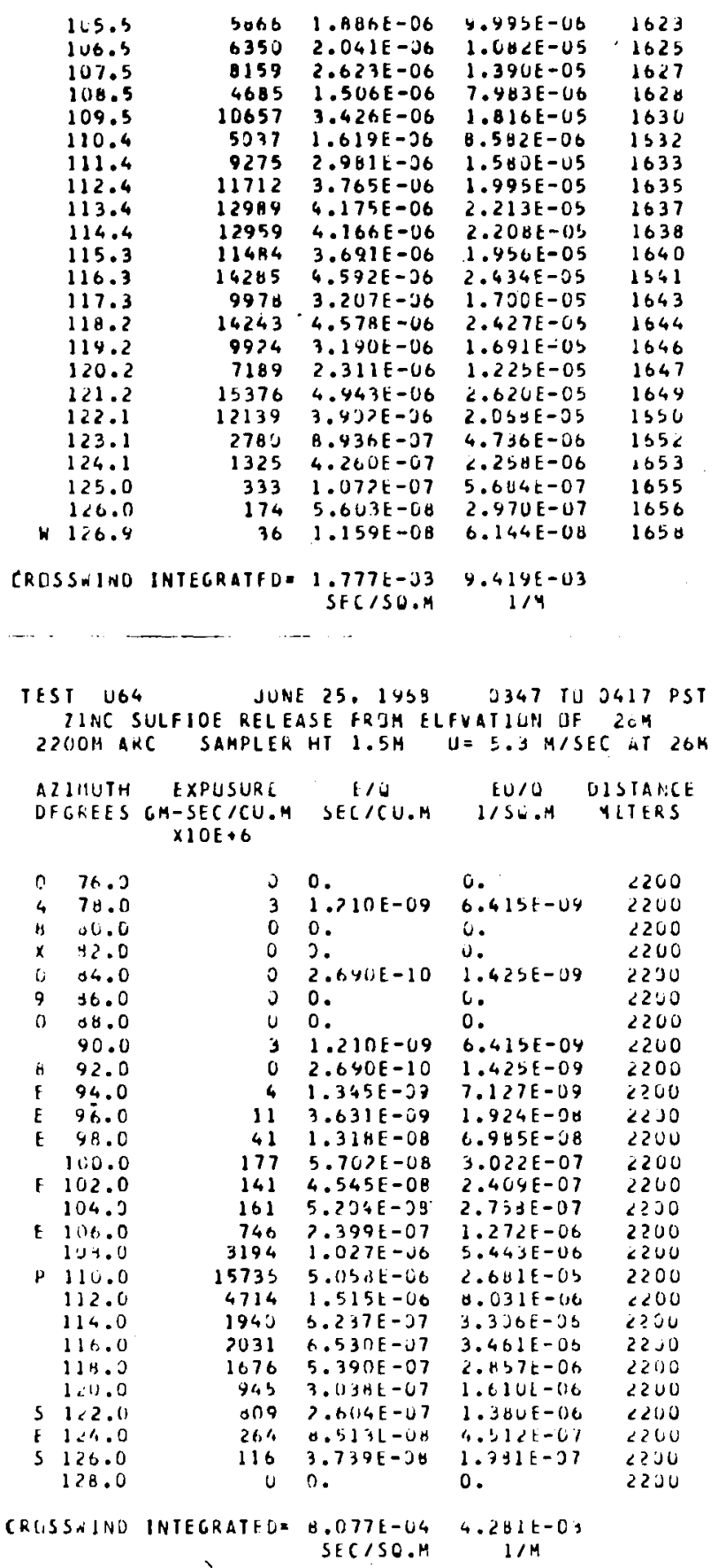

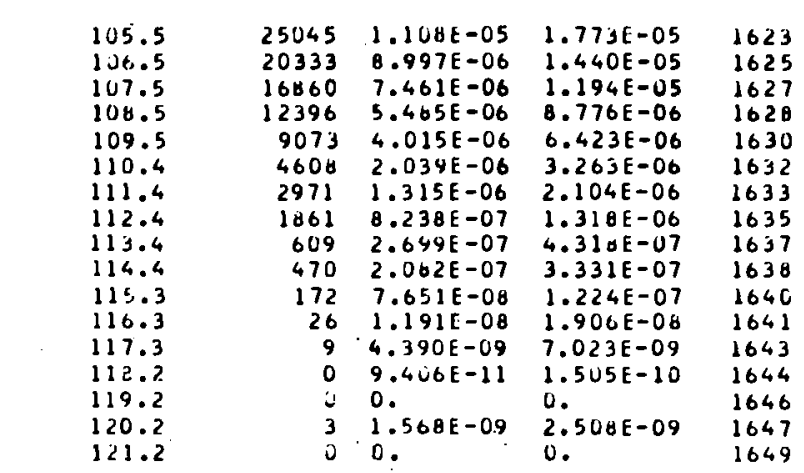

CRUSSWINC INTEGRATED B.298E-U3 $1.328 E-02$
SECISO.M 
TEST U64
ZINC SULFIOE RELEASE FROM ELFVATION OF $250 \mathrm{~N}$ ZINC SULFIOE RFLEASE FROM ELFVATION OF $20 \mathrm{M}$
32 OOM ARC SAMPLER HT $1.5 \mathrm{H}$. U. $5.3 \mathrm{H} / \mathrm{SEC}$ AT $26 \mathrm{H}$

AZIMUTH EXPOSURE EIO
DFCKEES GM-SECICU.M SECICU.M IISU.M OISIANCF
XIOE+G

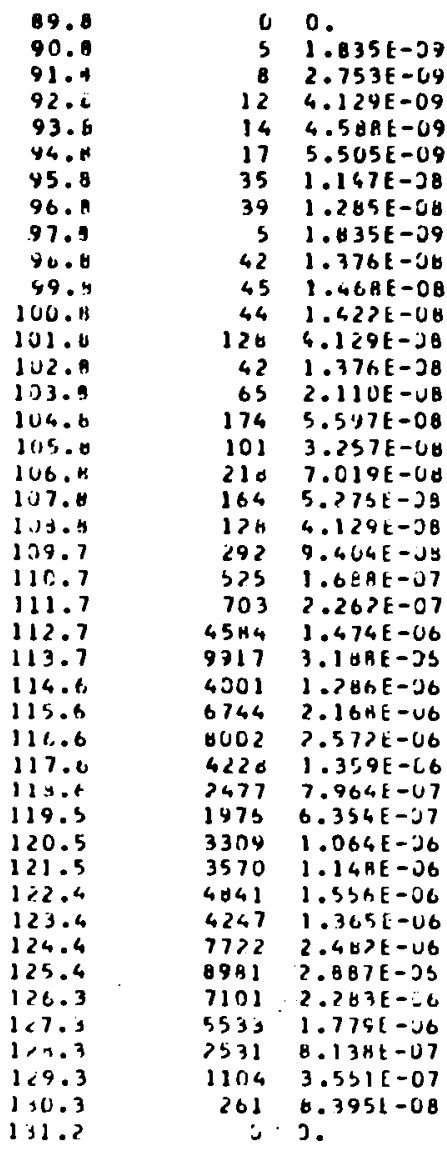

0.

3200

$1.459 \mathrm{E}-0 \mathrm{~B}$ S2J1

$2.1608-083203$

$2.431 E-G d \quad 3205$

C.41 UE-00 1200

$6.070 E-0 Y 3208$

6. $32 B E-O D \quad 3210$

$4.766 E-C 9 \quad 3212$

$7.294[-00 \quad 3213$

7.7 AUE-08 3215

$7.5371-00 \quad 3217$

$2.1945-07 \quad 3 ? 10$

$7.294 E-U d \quad 3220$

1.118E-UT, 222

$2.966 E-07 \cdot 3224$

$2.7<0 E-07 \quad 3225$

$3.7261-07 \quad 3227$

$2.773 E-07$ उ? 29

$2.140[-07 \quad 3230$

$4.436 t-67$ I292

$6.964 \mathrm{f}-07 \quad 3233$

$1.199 \mathrm{E}-00 \quad 3235$

$7.01 \cup E-00 \quad 3237$

$1.67 J E-U 5 \quad 3238$

6.81 aE-US 2240

$1.16 \times E-115 \quad 3261$

$1.363 t-03 \quad 3243$

$7.204 t-36 \quad 3245$

4.<CIE-OC 3240

$3.357 f-36 \quad 3 \geqslant 48$

$5.63 u t-2 b \quad 3249$

b.0स3E-J6 2251

8.267t-ve 1252

$7.23 c t-00 \quad 9253$

1.slet-0, 1215

1.33JE-05 $3 \geqslant 56$

1.21UE-0S 3238

$9.4271-60 \quad 3254$

$4.313 E-66 \quad 3261$

$1.002 t-0 e \quad 3262$

$4.449(-0) \quad 3203$

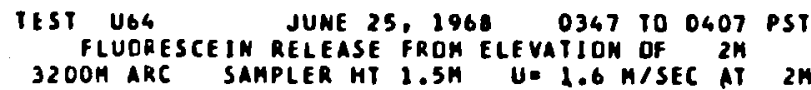

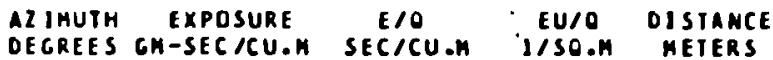

CRISSAIND INIEGRATFD. 1.672E-03 0.004E-CS

SEC 150.4 I T/4 $\times 10 E+6$

\begin{tabular}{|c|c|c|c|c|c|}
\hline & & $\times 1 O E+6$ & - & & \\
\hline & $\begin{array}{l}76.8 \\
77.8 \\
78.8\end{array}$ & $\begin{array}{l}0 \\
2 \\
7\end{array}$ & $\begin{array}{l}0 . \\
1.276 E-09\end{array}$ & 0.042 & $\begin{array}{l}3200 \\
3177 \\
3179\end{array}$ \\
\hline & $\begin{array}{l}79.8 \\
20.8\end{array}$ & $?$ & $\begin{array}{l}4.349 E-09 \\
4.065 E-09\end{array}$ & $\begin{array}{l}6.958 E-09 \\
6.504 E-09\end{array}$ & $\begin{array}{l}3140 \\
3142\end{array}$ \\
\hline & 01.8 & 13 & $6.050 E-09$ & $9.680 E-09$ & 3104 \\
\hline & $8<.0$ & i3 & $0.050 E-09$ & $9.680 E-09$ & 3145 \\
\hline & 83.8 & 15 & $6.759 E-09$ & $1.001 E-00$ & 3187 \\
\hline & $B 4.2$ & 23 & $1.059 E-00$ & $1.694 E-08$ & 3189 \\
\hline & .5 .0 & 29 & $1.245 E-08$ & $2.072 \mathrm{E}-08$ & 3191 \\
\hline & t6.k & 27 & $1.201 E-08$ & $1.921 E-08$ & 3192 \\
\hline & 67.8 & 23 & $1.059 E-08$ & $2.694 \mathrm{E}-00$ & $32 \times 4$ \\
\hline & WH. & 32 & $1.437 \varepsilon-0$. & $2.294 E-08$ & 3140 \\
\hline & 89.8 & 45 & $2.004 E-08$ & $3.207 \mathrm{E}-00$ & 3148 \\
\hline & $9 C . A$ & 64 & $2.055 E-08$ & $4.560 E-08$ & 3199 \\
\hline & 91.0 & 88 & $3.095 E-08$ & $6.232 E-00$ & 320.1 \\
\hline & 42.6 & 61 & $2.713 E-08$ & $4.34 \mathrm{JE}-0 \mathrm{~d}$ & 3203 \\
\hline & Y3.8 & 123 & $5.455 E-08$ & $8.727 E-08$ & 3245 \\
\hline & 94.6 & 126 & $5.546 E-08$ & $8.456 E-00$ & 3206 \\
\hline & 95.6 & 97 & $4.320 E-08$ & $6.412 E-08$ & 3208 \\
\hline & 96.8 & 213 & 9.42 & 1.50 & 3210 \\
\hline & 97.8 & 209 & $9.283 \mathrm{E}-08$ & $1.685 E-07$ & 3212 \\
\hline & 90.8 & $340^{\prime}$ & $1.505 E-07$ & $2.408 E-07$ & 3213 \\
\hline & 99.8 & 476 & $2.107 \mathrm{E}-07$ & $3.371 \mathrm{E}-07$ & 3215 \\
\hline & $200 . \mathrm{A}$ & 048 & $2.871 E-07$ & $4.596 E-07$ & 3217 \\
\hline & 101.9 & 495 & $3.963 \mathrm{E}-07$ & $6.341 E-07$ & 3210 \\
\hline & $1 \cup 2.8$ & 1254 & $5.551 \mathrm{E}$ & $\triangle .88<E-07$ & 3220 \\
\hline & 135.4 & 2084 & $9.226 E-07$ & $1.476 E-00$ & 3222 \\
\hline & $i 04.0$ & 2824 & 1.2 & 2.000 & 3224 \\
\hline & 105.8 & 2357 & $1.041 E-06$ & $1.606 E-06$ & $32<5$ \\
\hline & 106.6 & 3632 & $1, .607 E-06$ & $2.572 \mathrm{E}-06$ & $32<7$ \\
\hline & 207.0 & 4230 & 1.87 & 3.000 & 3224 \\
\hline & l'ie. & 4372 & $1.935 E-06$ & $3.096 E-06$ & 3230 \\
\hline & 130.7 & 5606 & $2.401 E-06$ & $3.964 E-46$ & 3232 \\
\hline 2 & 110.7 & 2386 & 1.0 & $1.64 U E-O C$ & 3233 \\
\hline & iil. 7 & 5180 & $2.292 \mathrm{E}-06$ & $3.667 E-06$. & 3235 \\
\hline & 112.7 & 8971 & $3.97 \cup E-06$ & $6.351 t-66$ & 3237 \\
\hline & 113.7 & 10766 & 4.76 & 7.622 & 3236 \\
\hline & 114.6 & 4911 & $2.173 E-06$ & $3.477 E-06$ & 3240 \\
\hline & 115.6 & 4230 & 1.875 & $3.00 U E-06$ & 3241 \\
\hline & 116.6 & 1254 & $5.551 E-07$ & $6.842 t-07$ & 3243 \\
\hline & $117 . e$ & 425 & & 3.013 & 3245 \\
\hline & $\therefore$ & 240 & 1.0 & 1.7 & 3240 \\
\hline & 114.5 & 219 & $9.704 \mathrm{E}$ & $1.55,36-07$ & 3240 \\
\hline & 126.5 & 416 & $1.036 E-07$ & $2: 437 E-01$ & 3244 \\
\hline & ici.s & 297 & $1.316 E-07$ & $2.105 E-07$ & 3251 \\
\hline & $1<2.4$ & 70 & $3.469 \mathrm{E}$ & $5.551 \mathrm{E}$ & 3252 \\
\hline & 123.4 & 15 & $6.901 E-09$ & $1.104 \mathrm{E}-00$ & 3253 \\
\hline & 124.4 & 16 & 7.18 & 1.150 & 3255 \\
\hline & $1<5.4$ & 15 & $7.043 E-69$ & 1.127 & $3<50$ \\
\hline & $1<6.3$ & 7 & $3.44 \Delta E-j 9$ & $5.540 E-0 Y$ & 3250 \\
\hline & 127.3 & 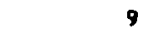 & 4. COSE-09. & $6.504 E-09$ & 3259 \\
\hline & $1 \leq 4$. & 5 & $2.505 E-09$ & $4.008 E-09$ & 3261 \\
\hline
\end{tabular}

LKUSSALINO INTEGRATLO= $\begin{array}{r}1.7 C O E-63 \\ \text { SECISO.M }\end{array}$ 


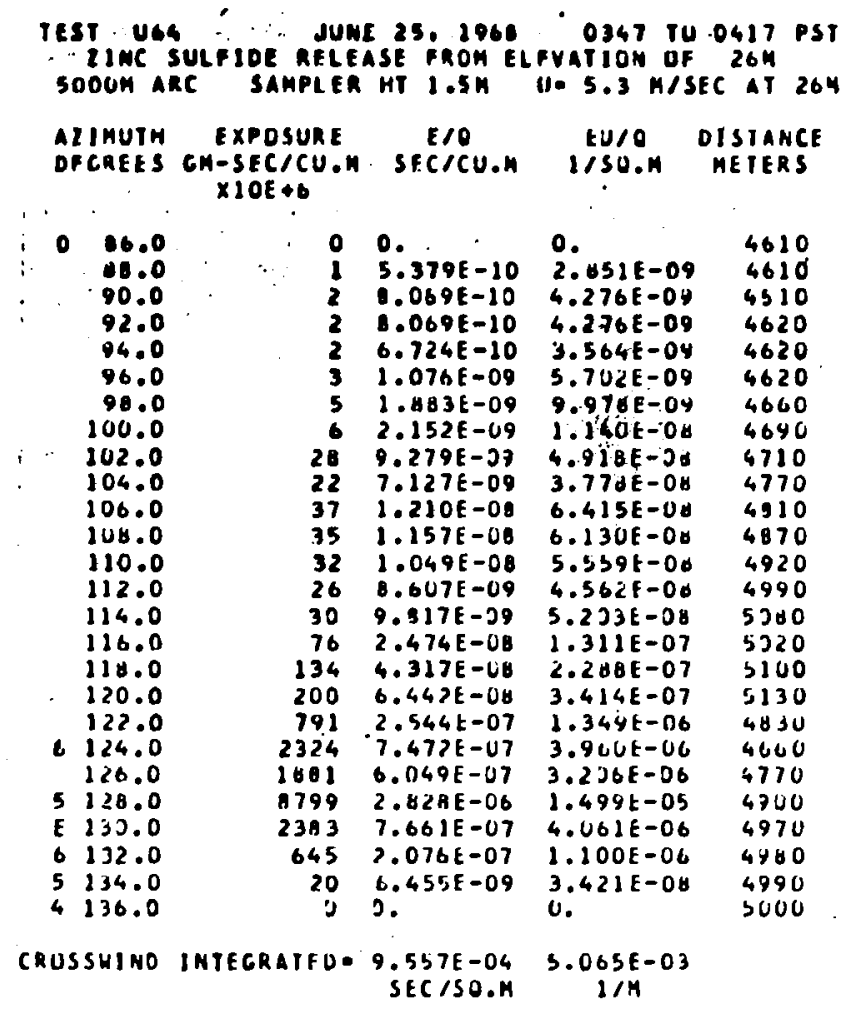

TEST UH4 JUNE 25, 1968 0347 100417 PST IIHC SULFIOE RELFASE FROM ELFVATISN DF 264 70OUM AKC SAMPIER HT $1.5 \mathrm{SM}$ U $5.3 \mathrm{H} / \mathrm{SEC}$ AI $26 \mathrm{M}$ ATIMUTH EXPOSURE
DFGEES GM-SEC/CU.M SEC/CU.M IISO.H UISIANCE $\triangle 1 O E+6$

\begin{tabular}{|c|c|c|c|c|c|}
\hline & $\begin{array}{l}90.0 \\
92.0 \\
94.0 \\
96.0\end{array}$ & $\begin{array}{r}0 \\
1 \\
17 \\
6\end{array}$ & $\begin{array}{l}0 . \\
5.379 E-10 \\
5.51 \div E-09 \\
7.017 E-09\end{array}$ & $\begin{array}{l}0 . \\
2.851 E-09 \\
2.922 t-08 \\
1.039 E-08\end{array}$ & $\begin{array}{l}6500 \\
6490 \\
6500 \\
6510\end{array}$ \\
\hline 4 & $9 A . C$ & u & 0. & u. & 6520 \\
\hline 4 & 100.0 & 0 & 0. & 0. & 0560 \\
\hline & 102.0 & 19 & $6.186 t-09$ & $3.279 E-O B$ & 0610 \\
\hline 5 & 104.0 & 35 & $1.130 E-08$ & $5.987 E-0.0$ & 6650 \\
\hline & 106.0 & 24 & $7.73 \div E-39$ & $\begin{array}{l}4.225 E-03 \\
9.123 E-08\end{array}$ & $\begin{array}{l}5720 \\
6960\end{array}$ \\
\hline h & $\begin{array}{l}1 \text { ud. } 0 \\
110.0\end{array}$ & $\begin{array}{l}53 \\
31\end{array}$ & $\begin{array}{l}1.721 E-08 \\
1.022 E-0 B\end{array}$ & $\begin{array}{l}9.123 E-08 \\
5.417 E-08\end{array}$ & 7000 \\
\hline & 112.0 & 32 & $1.049 E-08$ & $5.554 E-08$ & 7300 \\
\hline 5 & $\begin{array}{l}114.0 \\
116.0\end{array}$ & $\begin{array}{l}35 \\
17\end{array}$ & $\begin{array}{l}1.143 \mathrm{E}-00 \\
6.106 \mathrm{E}-07\end{array}$ & $\begin{array}{l}6.050 E-00 \\
3.279 E-08\end{array}$ & $\begin{array}{l}7210 \\
7220\end{array}$ \\
\hline & 110.0 & 28 & $9.145 E-39$ & $4.047 E-U B$ & 7190 \\
\hline & $\begin{array}{l}120.0 \\
122.0\end{array}$ & $\begin{array}{r}53 \\
186\end{array}$ & $\begin{array}{l}1.721 E-98 \\
5.998 E-08\end{array}$ & $\begin{array}{l}9.123 \mathrm{E}-00 \\
3.17>\mathrm{E}-07\end{array}$ & $\begin{array}{l}7150 \\
7120\end{array}$ \\
\hline & 124.0 & 680 & 2.1 BRE -07 & $1.160 E-06$ & 7100 \\
\hline $\begin{array}{l}4 \\
5\end{array}$ & $\begin{array}{l}126.0 \\
129.3\end{array}$ & $\begin{array}{r}3477 \\
10565\end{array}$ & $\begin{array}{l}1118 E-06 \\
3.396 E-36\end{array}$ & $\begin{array}{l}5.424 E-06 \\
1.0 \supset J E-05\end{array}$ & $\begin{array}{l}7100 \\
7100\end{array}$ \\
\hline & 130.0 & 9550 & $3.070 E-06$ & $1.627 \mathrm{E}-0 \mathrm{~S}$ & 7130 \\
\hline F & 132.0 & 3448 & $1.121 E-06$ & $3.943 \mathrm{E}-06$ & 7150 \\
\hline E & 134.6 & 907 & 2.91 BE -07 & $1.547 E-06$ & 7200 \\
\hline t & 136.0 & 399 & $1.7 \forall 4 E-07$ & $6.8 \cup 7 E-U 7$ & 7250 \\
\hline A & 138.0 & 114 & $3.671 E-3 B$ & $1.9: 5 E-27$ & 7300 \\
\hline 0 & 140.0 & 51 & $1.041 \mathrm{E}-08$ & $8.696 \mathrm{E}-00$ & 7350 \\
\hline 0 & 142.0 & $1 j$ & $4.438 E-09$ & $2.3 b$ दE-Ut & 3250 \\
\hline 0 & 144.0 & $u$ & 0. & & 7110 \\
\hline & 146.0 & 51 & $1.641 E-08$ & $8.696 \mathrm{E}-00$ & 7160 \\
\hline & $5 \times I N D$ & INIEGRATEDE & $\begin{array}{r}2.3 \forall 4 E-03 \\
\text { SFC/SO.M }\end{array}$ & $\begin{array}{c}1.264 \mathrm{E}-32 \\
1 / \mathrm{M}\end{array}$ & \\
\hline
\end{tabular}

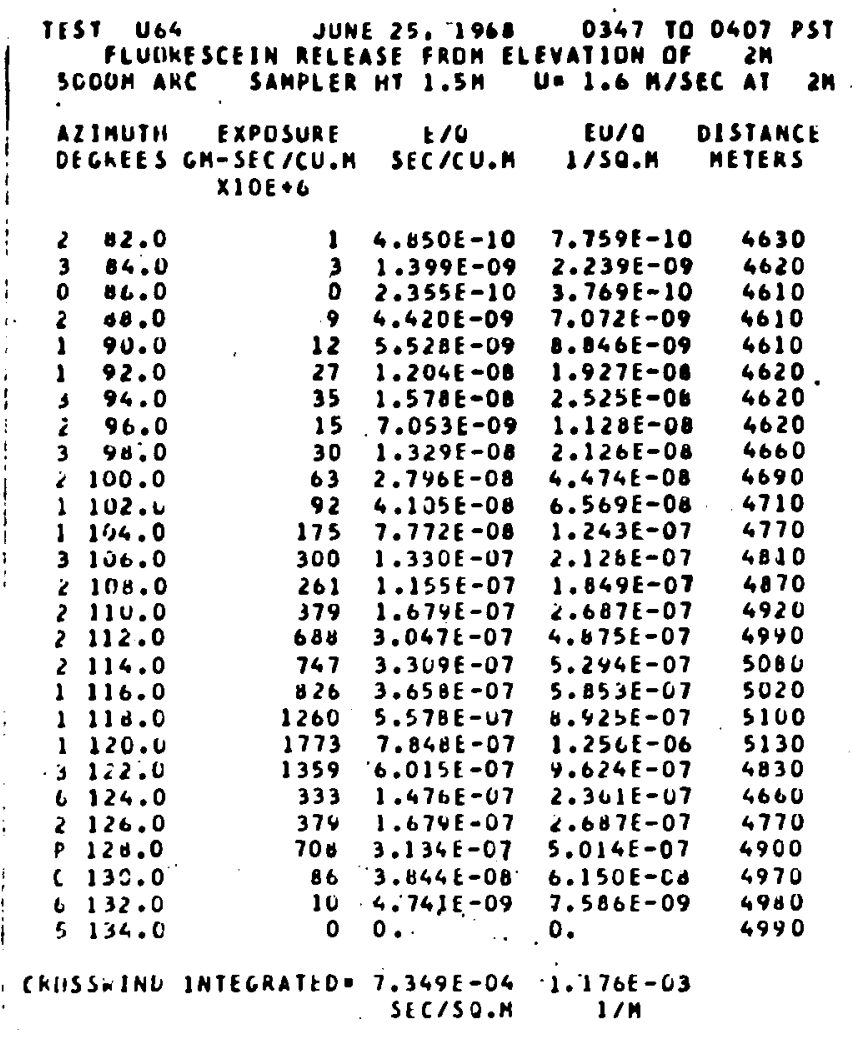

IEST U64 JUNE 25,1968 O347 TO 0407 PST

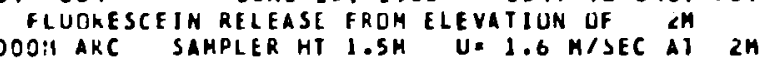

ALIYUIH EXPOSURE

DEGECES GM-SECICU.H SECICU.M H/SO.M METERS $X \perp U E+6$

\begin{tabular}{|c|c|c|c|c|c|}
\hline 1 & $\begin{array}{l}34 . C \\
36.0\end{array}$ & $\begin{array}{l}0 \\
3\end{array}$ & $\begin{array}{l}0 . \\
1.603 E-09\end{array}$ & $\begin{array}{l}0 . \\
2.66 J E-09\end{array}$ & $\begin{array}{l}6500 \\
6540\end{array}$ \\
\hline 5 & is 8.0 & 5 & $2.4111=09$ & $3.657 E-04$ & 6400 \\
\hline 1 & 90.0 & 4 & $2.162 E-09$ & $3.4 B \cup E-09$ & 0500 \\
\hline 4 & 92.0 & 11 & $5.265 \mathrm{E}-09$ & $8.424 \mathrm{E}-09$ & 6490 \\
\hline 1 & Y4.0 & 16 & $7.482 \mathrm{E}-09$ & $1.247 \mathrm{E}-08$ & 0500 \\
\hline 1 & $96 . c$ & 36 & $1.621 \mathrm{E}-08$ & $2.544 E-08$ & 6510 \\
\hline & 90.0 & 50 & $2.245 \mathrm{E}-\mathrm{UB}$ & $91 E-08$ & 6520 \\
\hline 4 & 100.0 & 74 & $3.270 E-\cup 8$ & $5.242 t-00$ & 0500 \\
\hline 2 & 16く.0 & 66 & $2.926 t-08$ & $4.682 t-00$ & 0610 \\
\hline 1 & 104.0 & 70 & $3.161 \mathrm{E}-0 \mathrm{~B}$ & $4.962 E-08$ & 6650 \\
\hline 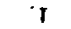 & I verec & 85 & $3.749 \mathrm{E}-5 \mathrm{~d}$ & $6.079 E$ & 6720 \\
\hline s & 135.0 & 125 & $5.545 E-0 d$ & $8.872 \mathrm{E}-0 \mathrm{~B}$ & 6000 \\
\hline 6 & 116.0 & 189 & $0.397 E-08$ & $1.343 E-07$ & 7000 \\
\hline 0 & 112.0 & 129 & $5.720 E-08$ & $9.152 \mathrm{E}-\mathrm{OB}$ & 1300 \\
\hline 5 & iif.c & 222 & $9.852 \mathrm{E}-0 \mathrm{~B}$ & $1.576 E-07$ & 7210 \\
\hline 5 & 116.0 & 380 & $1.603 E-07$. & $2.6945-07$ & 7220 \\
\hline 3 & $11 \% .0$ & 387 & $1.713 E-07$ & $\angle .740 E-07$ & 7190 \\
\hline 6 & $1<0.0$ & 367 & $1.025 E-07$ & $2.000 E-07$ & 7150 \\
\hline i & 122.0 & 047 & $3.749 E-07$ & 5.994 & 7220 \\
\hline 0 & 124.0 & 985 & $4.360 E-07$ & $6.977 E-07$ & 7100 \\
\hline 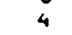 & 126.0 & 2563 & $1.134 E-06$ & 1.815 & 7100 \\
\hline & $1 \neq 0.0$ & 1971 & $8.725 E-07$ & $1.346 E-06$ & 7100 \\
\hline 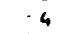 & 131.0 & 1380 & $0.106 E-07$ & $9.770 E-07$ & 7130 \\
\hline 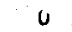 & 132.0 & 43 & $1.937 \mathrm{E}-08$ & $3.099 E-U 8$ & 7150 \\
\hline 6 & 134.0 & 93 & $4.148 E-0 B$ & $6.630 \mathrm{E}-\mathrm{COO}_{0}$ & 7200 \\
\hline$u$ & 130.0 & 11 & $5.265 t-09$ & $0.4 \angle 4 E-U Y$ & 7250 \\
\hline 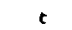 & 130.0 & 17 & $7.621 E-09$ & $1.219 E-0 d$ & 7300 \\
\hline$u$ & 140.0 & 14 & $6.374 E-69$ & $1.020 \mathrm{E}-08$ & 7350 \\
\hline$\iota$ & $14<.0$ & 0 & $2.910 E-09$ & $4.6,0 E-04$ & 7230 \\
\hline$c$ & 144.0 & 0 & $3.603 E-10$ & $5.704 E-10$ & 7110 \\
\hline 4 & 146.0 & 2 & $9.561 E-10$ & $1.530 E-09$ & 7100 \\
\hline & Ac & EGR & $\begin{array}{l}1.114 E-03 \\
S E C / S O . M\end{array}$ & $\begin{array}{c}1.783 E-03 \\
1 / M\end{array}$ & \\
\hline
\end{tabular}


SAMPLING 4OOM TO $12 B 0 J 4$, NO TOHER SAMPLING.

BIMDDAL DISIRIBUTION FOR BOIH TRACERS.

TRACER DISTRIBUTIDNS TRUNCATED ON VDRIH EOGE BEYOND $2200 \mathrm{H}$ ARC.

TRACER OISTR IBUTIDAS TQUNCATED OM VDRTH EDGE EEYOMD 2 ...

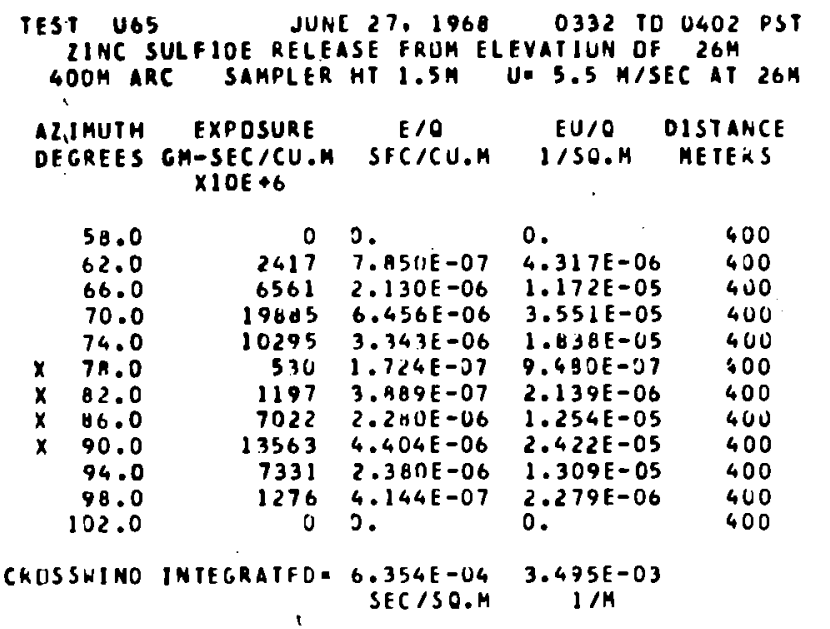

TEST U65 JUNE 27. 1960 D 332 TO 0402 PST 2 INC SULFIOE RELEASE FRJM ELFVATIUN DF 204
POOM ARC SAMPLER MT 1.5 U. UE 5.5 MISEC AT $26 M$
AZIMUTH EXPOSURE TIU EU/O DISTANCE DEGREES CM-SECICU.M SECICU.M I/SO.M METERS $\times 10 \mathrm{O}+6$

52.0
55.0
50.0
61.0
64.0
67.0
70.0
73.0
76.0
79.0
82.0
85.0
88.0
91.0
94.0
97.0
97.1
99.1
101.1
103.0

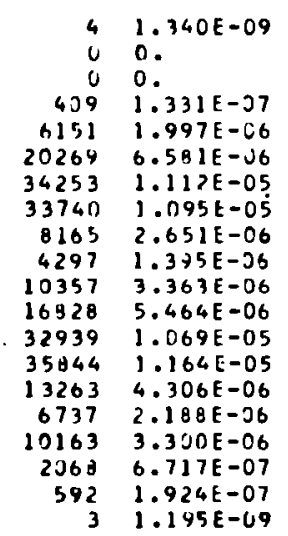

$7.366 E-09$

0.

7.319E-07

$1.09 \rightarrow E-05$

$3.62 J \mathrm{~F}-05$

6. $117 \mathrm{E}-05$

$6.025 \mathrm{~F}-05$

$1.456 \mathrm{~L}-05$

$7.675 E-26$

$1.850 \mathrm{E}-05$

$3.005 E-05$

$5.882 E-05$

$6.401 E-C 5$

$2.36 \forall E-05$

$1.233 E-05$

$1.815 E-05$

$3.694 \mathrm{E}-06$

$1.056 E-06$

800
800
800
300
900
060
800
800
600
300
500
800
800
800
800
900
856
809
813
816

CROSSHIND INTEGRATED= 3.035E-03 1.696E-02
TFSI U65

JUNE ?7, $1968 \quad 0332$ TO 0402 PSI

FLLURESCEIN RELEASE FRUM ELEVATIUN OF 4OOM ARC SAMPLER HT $1.5 \mathrm{M} U=3.0 \mathrm{M} / 5 E C$ AT $2 \mathrm{M}$

AZIMUTH EXPQSURE EIO LIO DISIANCE

OECKEES GM-SEC/CU.M SECICU.M I/SU.M RETERS $\times 10 E+6$

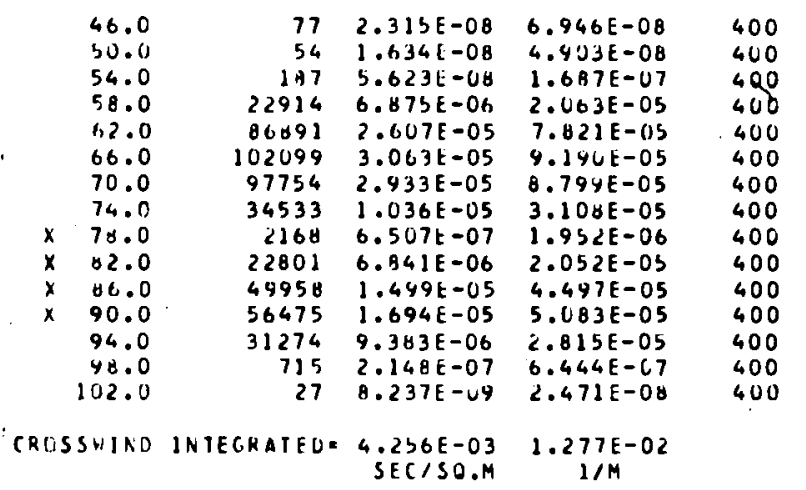

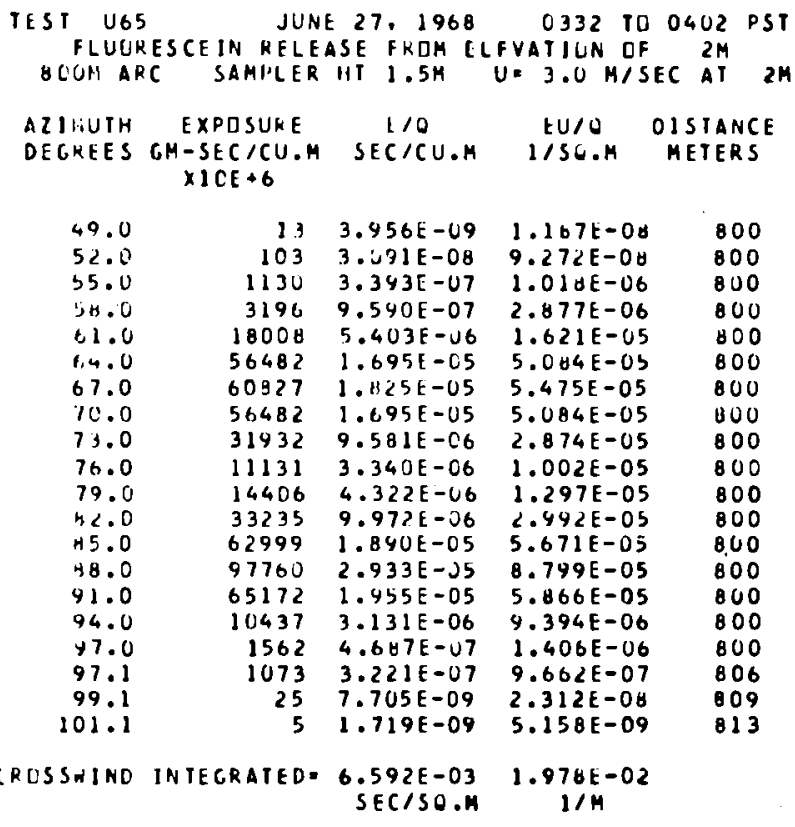




\begin{tabular}{|c|c|c|c|c|}
\hline $\begin{array}{l}\text { TEST U65 } \\
\text { IINC ST } \\
1200 \mathrm{M}\end{array}$ & $\begin{array}{l}\text { ULFIDE RELE } \\
\text { SC SAMPLER }\end{array}$ & $\begin{array}{l}27 \text {. } 1968 \\
\text { SE FROM EL } \\
\text { HT } 1.5 \mathrm{H}\end{array}$ & $\begin{array}{r}0332 \text { TO } \\
\text { EVATIUN OF } \\
\text { J= } 5.5 \mathrm{~m} / \mathrm{S}\end{array}$ & $\begin{array}{l}0402 \text { PSI } \\
=264 \\
S E C A T 26 M\end{array}$ \\
\hline $\begin{array}{l}\text { ALIKUTH } \\
\text { DECREES }\end{array}$ & $\begin{array}{l}\text { EXPOSURE } \\
\text { GH-SEC /CU.H } \\
\text { XIOE+ }\end{array}$ & SEC/CU.H & $\begin{array}{l}\text { Lu/o } \\
\text { lysu.m }\end{array}$ & $\begin{array}{l}\text { DISTANCE } \\
\text { MEIERS }\end{array}$ \\
\hline $\begin{array}{l}40.0 \\
30.0\end{array}$ & $\begin{array}{l}1 \\
1\end{array}$ & $\begin{array}{l}4.9 \text { UIE-10 } \\
4.981 E-10\end{array}$ & $\begin{array}{l}2.740 \mathrm{E}-09 \\
2.740 \mathrm{E}-09\end{array}$ & $\begin{array}{l}1200 \\
1200\end{array}$ \\
\hline $\begin{array}{l}52.0 \\
54.0\end{array}$ & 1 & $\begin{array}{l}4.961 E-10 \\
9.96 \supset E-10\end{array}$ & $\begin{array}{l}2.740 E-09 \\
5.479 E-09\end{array}$ & 1200 \\
\hline 56.0 & 30 & $9.962 \mathrm{E}-09$ & $5.479 E-5 B$ & $\begin{array}{l}1<00 \\
1 ? 00\end{array}$ \\
\hline $\begin{array}{l}58.0 \\
60.0\end{array}$ & $\begin{array}{l}164 \\
704\end{array}$ & $\begin{array}{l}5.330 \mathrm{E}-0 \mathrm{~B} \\
2.289 \mathrm{E}-07\end{array}$ & $\begin{array}{l}2.931 E-07 \\
1.254 E-06\end{array}$ & $\begin{array}{l}1200 \\
1200\end{array}$ \\
\hline 62.0 & 2366 & $7.683 E-07$ & $4.226 E-06$ & 1200 \\
\hline 64.0 & 6076 & $1.973 E-06$ & $1.085 E-05$ & 1200 \\
\hline 66.0 . & $1077 \mathrm{~h}$ & $3.499 \mathrm{E}-06$ & $1.924 E-05$ & 1200 \\
\hline 68.0 & 16468 & $5.347 \mathrm{E}-06$ & $2.941 E-05$ & 1200 \\
\hline 70.0 & IABS5 & $6.125 t-06$ & $3.369 E-05$ & 1200 \\
\hline 72.0 & 18417 & $5.9 B \cup E=06$ & $3.289 \mathrm{E}-05$ & 1200 \\
\hline 74.0 & 9941 & $3.228 E-06$ & $1.775 E-05$ & 1200 \\
\hline 76.0 & 5406 & $1.755 E-06$ & $9.654 E-06$ & 1200 \\
\hline 78.0 & 5193 & $1.606 E-06$ & $9.274 E-06$ & 1200 \\
\hline 80.0 & 3461 & $1.124 \mathrm{E}-06$ & $6.132 \mathrm{E}-06$ & $1 \geqslant 00$ \\
\hline $\begin{array}{l}82.0 \\
84.0\end{array}$ & $49 h^{3}$ & $1.612 \mathrm{E}-06$ & $8.664 E-06$ & 1200 \\
\hline & 6991 & $2.270 E-06$ & $1.248 E-05$ & 1200 \\
\hline$\$ 6.0$ & 16451 & $5.341 \mathrm{E}-06$ & $2.938 E-05$ & 1200 \\
\hline$\$ 8.0$ & 26969 & $8.756 E-06$ & $4.816 \mathrm{E}-05$ & 1200 \\
\hline 90.0 & $2841 d$ & $9.727 \mathrm{E}-06$ & $5.075 E-05$ & 1200 \\
\hline 22.0 & 19250 & $6.250 E-06^{\circ}$ & $3.438 \mathrm{E}-05$ & 1200 \\
\hline 94.0 & 10959 & $3.558 E-06$ & $1.957 \mathrm{E}-05$ & 1200 \\
\hline$\$ 6.0$ & 2512 & $8.159 \mathrm{E}-07$ & $4.487 E-06$ & 1200 \\
\hline 88.0 & 780 & $2.535 \mathrm{E}-07$ & $1.394 \mathrm{~F}-06$ & 1200 \\
\hline 100.0 & 108 & $3.512 \mathrm{E}-08$ & $1.931 E-07$ & 1200 \\
\hline 142.0 & 1 & $4.941 E-10$ & $2.740 \mathrm{E}-09$ & 1200 \\
\hline 194.0 & 0 & כ. & 0 & 1200 \\
\hline CROSSUINO & INTEGRATEDE & $\begin{array}{l}2.92 \mathrm{BE}-03 \\
\text { SEC } / \mathrm{SO} O . \mathrm{M}\end{array}$ & $\begin{array}{c}1.610 E-02 \\
1 / 4\end{array}$ & \\
\hline
\end{tabular}

TEST U65 JULE 27, 1969 0332 TU 0402 PST 2|NC SULFIDE RFLEASE FRJM ELFVATIUN OF 26.4 $16 \mathrm{CPM} A R C$ SAMPLER HT $1.5 \mathrm{M} U=5.5 \mathrm{M} / \mathrm{SEC}$ IT $26 \mathrm{M}$

\begin{tabular}{|c|c|c|c|c|}
\hline $\begin{array}{l}\text { AZIMUTH } \\
\text { DEGREES }\end{array}$ & $\begin{array}{c}\text { EXPOSUKE } \\
\text { GH-SEC/CU.M } \\
\times 1 O E+6 .\end{array}$ & $\begin{array}{c}E / O \\
\text { SFC/CU. Y }\end{array}$ & $\begin{array}{l}\text { EU/O } \\
1 / 50 . M\end{array}$ & $\begin{array}{l}\text { DISIAIICL } \\
\text { METERS }\end{array}$ \\
\hline $\begin{array}{l}50.0 \\
52.0\end{array}$ & $\begin{array}{l}u \\
1\end{array}$ & $\begin{array}{l}0.901 E-10 \\
4.901\end{array}$ & $\begin{array}{l}0 . \\
2.740 E-09\end{array}$ & $\begin{array}{l}1600 \\
1600\end{array}$ \\
\hline 54.0 & 24 & $9.215 E-69$ & $5.064 E-08$ & 1600 \\
\hline 56.0 & 79 & $2.563 E-0 a$ & $1.3 \ngtr 7 E-\cup 7$ & 1500 \\
\hline & 188 & 5.12 & 3.37 & \\
\hline & 560 & 1.75 & 9.65 & 1600 \\
\hline 62.0 & 1214 & $3.960 E-07$ & $2.17 e E-06$ & is 600 \\
\hline 64. & $44 r, 6$ & 1.59 & 0.76 & 1600 \\
\hline 66 . & 4493 & 1.45 & $8.024 \mathrm{E}-06$ & 1600 \\
\hline 68.0 & 9200 & 2.94 & $1.6 \div 3 E-05$ & 1360 \\
\hline 73. & 11633 & 3.77 & 2.07 & 1500 \\
\hline 72. & $5 y 34$ & 1.89 & 1.042 & iscus \\
\hline 74.0 & 6659 & 1.51 & 8.31 & 1660 \\
\hline & & $4.4 \pi$ & 2.426 & 1630 \\
\hline & 1072 & $3.4 \times$ & 1.91 & 1600 \\
\hline$\$ 0.0$ & 476 & 2.45 & 1.53 & isou \\
\hline 82.0 & 1800 & 5.87 & $3.23 \mathrm{~J}$ & 1500 \\
\hline & 2352 & $7 . h$ & 4.2 & itou \\
\hline 80 & $5<15$ & 1.69 & 9.3 & \\
\hline & & & 1.73 & 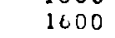 \\
\hline 90 & & 3.1 & 1.72 & 1600 \\
\hline & & 3.5 & 1.958 & $\begin{array}{l}1500 \\
150\end{array}$ \\
\hline 93 & 5974 & 1.9 & 1.06 & 1503 \\
\hline & & & 3.43 & 1500 \\
\hline 94. & 3564 & 1.15 & 6.36 & 1604 \\
\hline & y57 & & $1.710 \mathrm{E}-06$ & \\
\hline & 63 & 2.058 & 1.132 & 1600 \\
\hline & 2 & $5.15 \mathrm{~A}$ & & is $>0$ \\
\hline & 49 & & & $\cdots$ \\
\hline & & $82 \mathrm{E}$ & $9.801 F-09$ & 16 \\
\hline
\end{tabular}

CRLSSHINO INTEGRATEU: $1.642 E-03$ 9.632E-03,

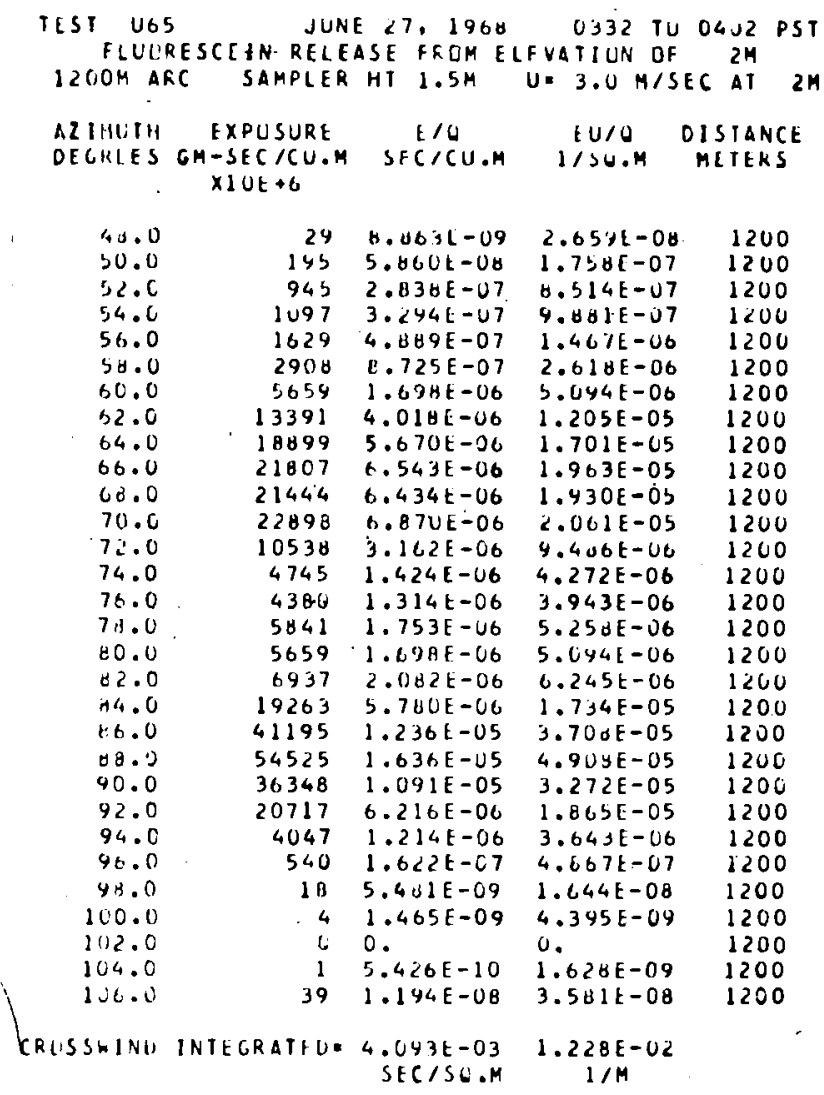

TEST UO5. JUHE 27. $146 \mathrm{~A}$ 0332 TU 0402 PST FLUCKESCEIIN KFLEASE FKIJM ELFVATIUN UF $2 M$ IOCOOK AKC SAMPLER HT $1.5 \mathrm{M} U=3.0 \mathrm{M} / \mathrm{SEC}$ AI $2 \mathrm{M}$ GZIMIITH EXPOSURE K/O EU/O DISTANCE DEGKEES GM-SEC/CU.M SICICU.M IISG.M METERS $\times 10 E+6$

I
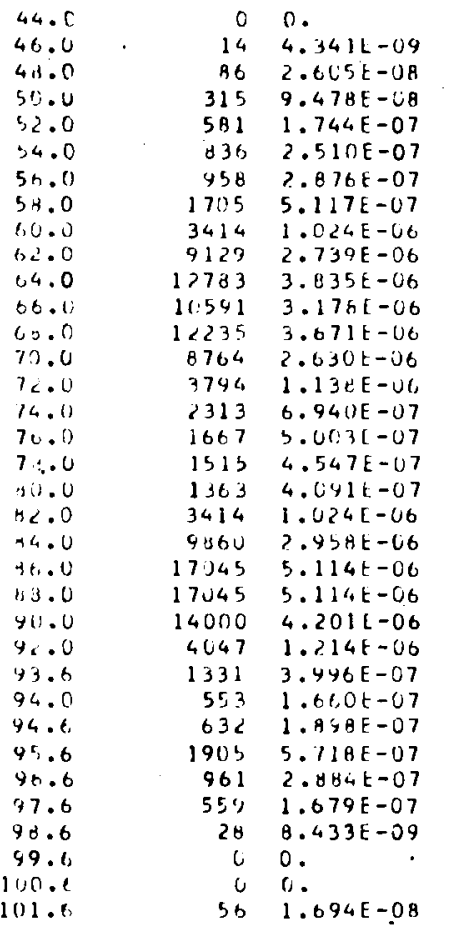

1.302E-00

$1.302 E-00$
$7.814 E-00$

$2.043 \mathrm{E}-07$

$5.231 E-07$

$7.531 \mathrm{E}-07$

$0.628 \mathrm{E}-07$

$1.535 E-06$ $3 . \angle 73 E-60$ $0.218[-06$

$1.151 E-03$

Y. $533 \mathrm{E}-06$

$1.101[-0 b$

7.8YYE-06

$3.415 E-06$.

C.OHCE-UG

$1.501 r-06$

$1.364 \mathrm{E}-0 \mathrm{O}$

$1.227 \mathrm{t}-0 \mathrm{c}$

$3.07 \mathrm{JE}-06$

$8.875 t-06$

$1.594 \mathrm{E}-05$

$1.334 \mathrm{E}-05$

$1.260 E-O 5$

$3.643 \mathrm{E}-06$

1.1 YYE -06

$4.961 \mathrm{E}-07$

$5.644 \mathrm{~F}-07$

$1.716 \mathrm{E}-06$

$4.651 E-07$
$5.037 t-07$

$2.53 U \mathrm{E}-\mathrm{OU}$

0 .

0.

$5.001 t-0 t$

1600

1600

1600

1600

1600

.1600

1500

1600

1600

1600

1600

1600

1600

1600

1600

1600

1600

1600

1600

1600

1600

1603

1600

1604

1606
1608

1610

1611

1613

1615
1617

CRISSSWINU INTEGRATEOE $2.344 t-03 \quad 7.033 E-03$ 


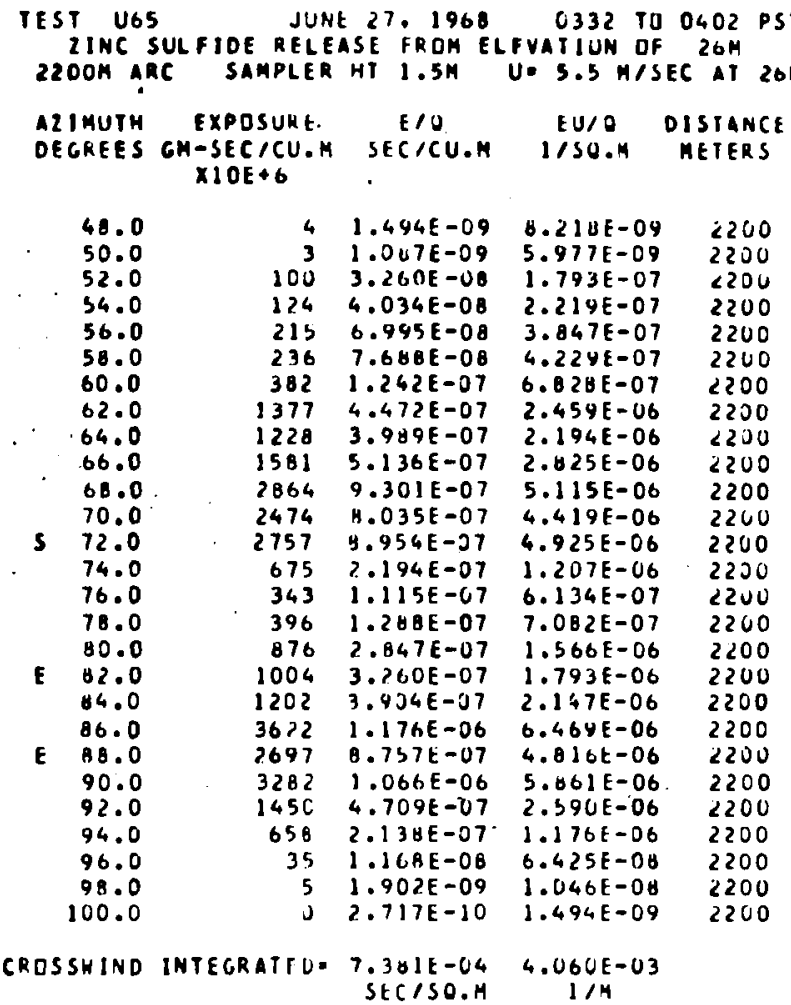

TEST U65 JUNE 27, 19680332 TO 0402 PST IINC SULFIDE RELEASE FROM ELFVATIUV UF $2 L \mathrm{H}$ $3200 M$ ARC SAMPLER HT $1.5 \mathrm{M}$ U. $5.5 \mathrm{M} / \mathrm{SEC}$ AI $26 \mathrm{M}$

AZIMUTH EXPOSURE G E
OEGKEES CM-SECICU.M SFCICU.M IISU.M OISIANCE $\times 10 E+6$

\begin{tabular}{|c|c|c|c|c|}
\hline & $4 A .0$ & 53 & $1.725 E-08$ & $9.4886-08$ \\
\hline & 50.0 & 47 & $1.535 E-08$ & $0.44<E-0 \theta$ \\
\hline & 52.0 & 64 & $2.07>E-C B$ & $1.1 \% 1[-07$ \\
\hline & 54.0 & 140 & $4.3501 E-0 B$ & $2.503 E-07$ \\
\hline & 58.0 & 74 & $2.404 E-0 B$ & $1.322 \mathrm{E}-07$ \\
\hline & 60.0 & 19.2 & $6.252 E-5 B$ & $3.4: 4 E-07$ \\
\hline & 62.0 & $7 ? 3$ & $2.349[-07$ & $1.272 \mathrm{E}-06$ \\
\hline & 64.0 & 769 & $3.167 E-07$ & $1.731 E-06$ \\
\hline & 66.0 & $46 \mathrm{BH}$ & $1.521 E-07$ & $0.367 F-07$ \\
\hline$s$ & $6 \mathrm{~A} .0$ & 1424 & $4.6<46-07$ & $2.543 E-00$ \\
\hline & 70.0 & 2236 & $7.26>E-07$ & $3.994[-66$ \\
\hline & 72.7 & 2396 & $7.78 J E-37$ & $4.27+E-05$ \\
\hline & 73.7 & 2127 & $6.9 \mathrm{CBE}-07$ & $3.779 t-60$ \\
\hline & 74.7 & 513 & 1.66 AE -07 & $9.175 E-07$ \\
\hline & 75.7 & 16.25 & C.629E-J7 & $2.366 E-06$ \\
\hline & 76.8 & 913 & $7.466 E-07$ & $1.631 \mathrm{l}-0 \mathrm{~s}$ \\
\hline & 77.8 & 349 & $1.297 E-07$ & $7.1365-07$ \\
\hline & 79.8 & 777 & $2.524 E-37$ & $1.334[-00$ \\
\hline & 79.8 & 1315 & $4.272 \mathrm{E}-07$ & $2.350 \mathrm{E}-\mathrm{CS}$ \\
\hline & 80.9 & 1467 & $4.7715-07$ & $2.624 E+C 6$ \\
\hline & 31.8 & 3547 & $1.15,7 t-06$ & $6.335 E-C 6$ \\
\hline & 02.8 & 4104 & $1.333 E-06$ & $7.330 \mathrm{E}-6.6$ \\
\hline & 83.8 & 324,9 & $1.055 E-06$ & $5.803 t-00$ \\
\hline & 84.8 & 3172 & $1.035 E-35$ & $5.655 \mathrm{E}-0 \mathrm{D}$ \\
\hline & 85.8 & $318 ?$ & $3.033 E-96$ & $5.65 J E-06$ \\
\hline & 86.9 & 3347 & $1.047 E-06$ & $5.978 \mathrm{E}-06$ \\
\hline & 87.8 & 6417 & $2.214 \mathrm{E}-06$ & $1.217 E-05$ \\
\hline & 48.8 & 6101 & $1.981 E-06$ & $1.000 E-0 S$ \\
\hline & 89.9 & 4036 & $1.311 \mathrm{E}-06^{\prime}$ & $7.209 E-06$ \\
\hline & 90.8 & 1481 & $4.51 J E-37$ & $2.6 \div 5 E-06$ \\
\hline & 91.6 & 1428 & 4.63BE-07 & $2.551 E-06$ \\
\hline & 92.8 & 690 & $2.242 E-07$ & $1.233 E-06$ \\
\hline & 93.8 & $18 B$ & $6.134 E-08$ & $3.374 E-07$ \\
\hline & 94.8 & 69 & $2.251 E-0 B$ & $1.238 E-07$ \\
\hline & 95.0 & 32 & $1.059 E-08$ & $5.825 E-08$ \\
\hline & 96.8 & 80 & $2.600 E-08$ & $1.432 \mathrm{E}-07$ \\
\hline & & & $\begin{array}{l}1.2 \angle 4 E-03 \\
\text { SFC } / S O . M\end{array}$ & $\begin{array}{c}6.841 \mathrm{E}-03 \\
\mathrm{I} / \mathrm{M}\end{array}$ \\
\hline
\end{tabular}

3200

1200

9260

3200

3200

3200

3200

3200

3200
3200

3200

$313 t$

3170

$317 \%$

3173

3173

$3) 77$

3179

3190

1192

3164

3145

3167

3104

3191

3192

3194

3196

3196

3179

3203

3205,

3206

3210

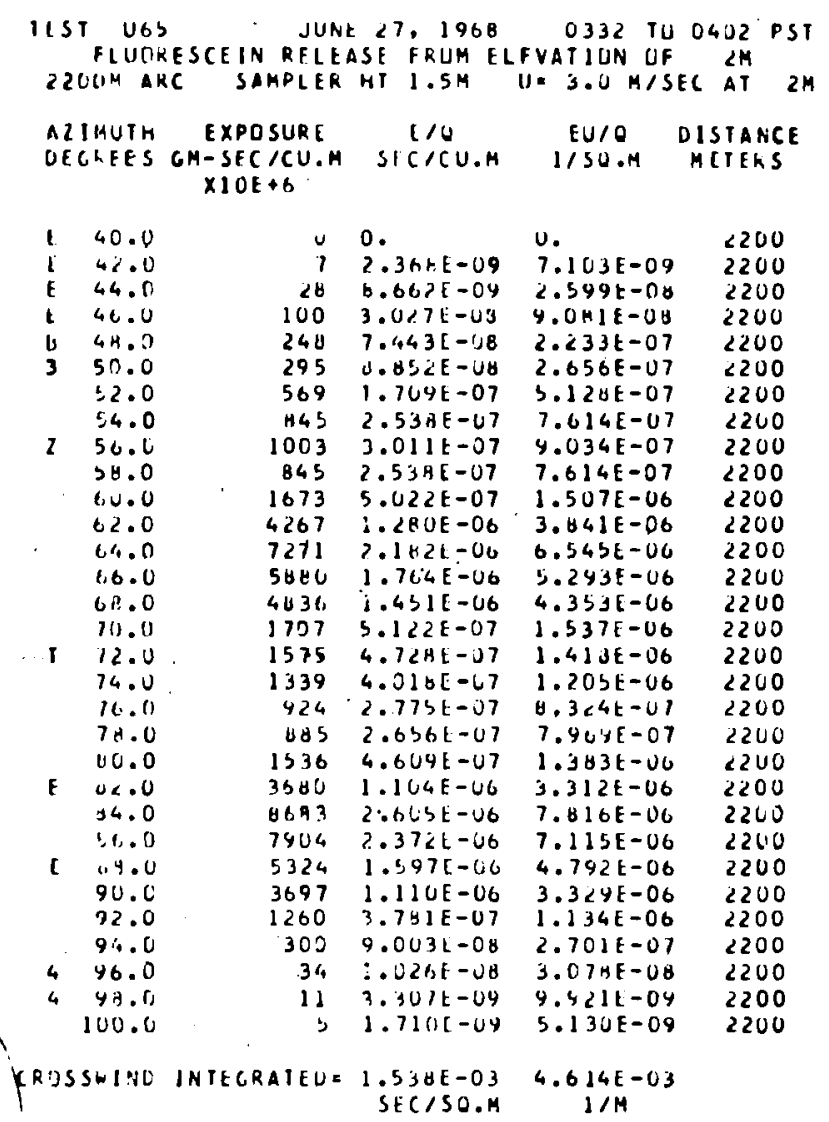

IEST U6S FLULESCEIN FULEASI FH1968 0332 TU 0402 PST

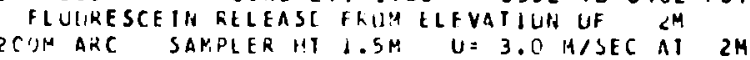

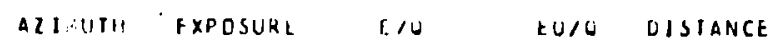

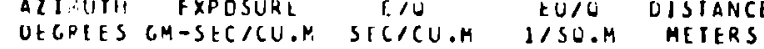 $\times 10 E+6$

\begin{tabular}{|c|c|c|}
\hline $\mathfrak{t}^{\prime}$ & $\Leftrightarrow, 0$ & \\
\hline$T$ & 50.0 & \\
\hline & $\because \sigma_{1}$ & \\
\hline I & 56.0 & \\
\hline & $b=. C$ & \\
\hline & 011.0 & \\
\hline & 2.0 & \\
\hline & $1.4 .1)$ & \\
\hline & 16.0 & \\
\hline$T$ & $B, 4,0$ & \\
\hline 2 & 10.0 & \\
\hline & $7 . .7$ & \\
\hline & 73.7 & \\
\hline & 14.7 & \\
\hline & 75.1 & \\
\hline & 16.8 & \\
\hline & 17.6 & \\
\hline & 74.8 & \\
\hline & 79.4 & \\
\hline & 4.9 & \\
\hline & 1.8 & \\
\hline & 's. & \\
\hline & 83. & \\
\hline & 14.4 & \\
\hline & A3. H & \\
\hline & ut.t & \\
\hline & $\times 7.8$ & \\
\hline & $\because y .8$ & \\
\hline & $89 . h$ & \\
\hline & 90.3 & \\
\hline & 91.8 & \\
\hline & 92.8 & \\
\hline & $93 . \mathrm{H}$ & \\
\hline & 94. A & \\
\hline & 95.8 & \\
\hline & 96.8 & \\
\hline
\end{tabular}
CRUSSWINO INIEGRAIED $\begin{gathered}1.146 E-U 3 \\ \text { SECISO.Y }\end{gathered}$
3200

$10 . .343[-. J 4 \quad 1.813 E-07$

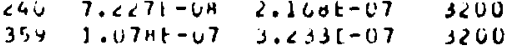
$227 \quad 6.037 \mathrm{E}-08 \quad 2.050 \mathrm{E}-07 \quad 3200$ $201 \quad 6.043 E-U B \quad 1.81 S E-07 \quad 3200$ $280 \quad 0.411 E-08 \quad 2.523 E-U 7 \quad 3200$ OH $5 \quad 2.656 t-07 \quad 7.964 E-07 \quad 3200$ $2243 \quad 6.730 F-07 \quad 2.01 \times E-06 \quad 3200$ $24647.3946-07 \quad 2.2166-06 \quad 3240$ $1160.350 t-07$ l.905t-06 3260 $1420 \quad 4.2636-07 \quad 1.279 t-06 \quad 3200$ $484 \quad 1.433 t-07 \quad 4.35 d-51 \quad 3260$ $11613.404 E-07$ 1.045E-06 3170 $164 \quad 5.0 .906-08$ 1.327L-01 3172 $667 \quad 2.003 t-07 \quad 6.604 k-07 \quad 3173$ OCO 1.AO1E-U7 5.404E-07 $\$ 175$ $45<\cdot 1.357 k-07 \quad 4.0711-07,3177$ $\begin{array}{llll}757 & 2.32725-37 & 6.017 E-J 7 & 3179\end{array}$ $1609 \quad 4.430 \mathrm{~F}-07 \quad 1.449 \mathrm{E}-0 \mathrm{C} \quad 3160$ 24R4 7.45,E-OZ 2.236E-0) 3182 47 US $1.4171-U 6$ 4.23,E-06 3184 $4332 \quad 1.210 F-06 \quad 3.63 U E-06 \quad, 165$ $4503 \quad 1.351 t-06 \quad 4.054 t-00 \quad 3107$ 65100 1.450E-06 5.d51E-0C 3189 $3292 \quad 9.478 E-07 \quad 2.903 E-06 \quad 3191$ $5263 \quad 1.573 t-06 \quad 4.720 t-06 \quad 3192$ $3897 \quad 1.170 \mathrm{t}-06 \quad 3.504 \mathrm{E}-\mathrm{C6} \quad 3194$ $3157 \quad 9.474 E-67 \quad 2.842 t-06 \quad 9190$ $13103.355[-07$ 1.100[-0e 3198 $930 \quad 2.811 L-5 \quad$ U.432E-07 $\quad 1199$ $445 \quad 1.3371-07 \quad 4.01 \cup E-07 \quad 3201$ IHA $5.609 \mathrm{E}-\mathrm{UH} \quad 1.6 \mathrm{H3F}-07 \quad 3203$ $216.410 E-39 \quad 1.923 E-00 \quad 3205$ $13.205 F-109.615 \mathrm{r}-10 \quad 3210$ $1564 \quad 4.6955-.77 \quad 1.404 E-V 6 \quad 3160$ $56 \quad 1.699 E-08 \quad 5.046 E-0 B \quad 3206$ 


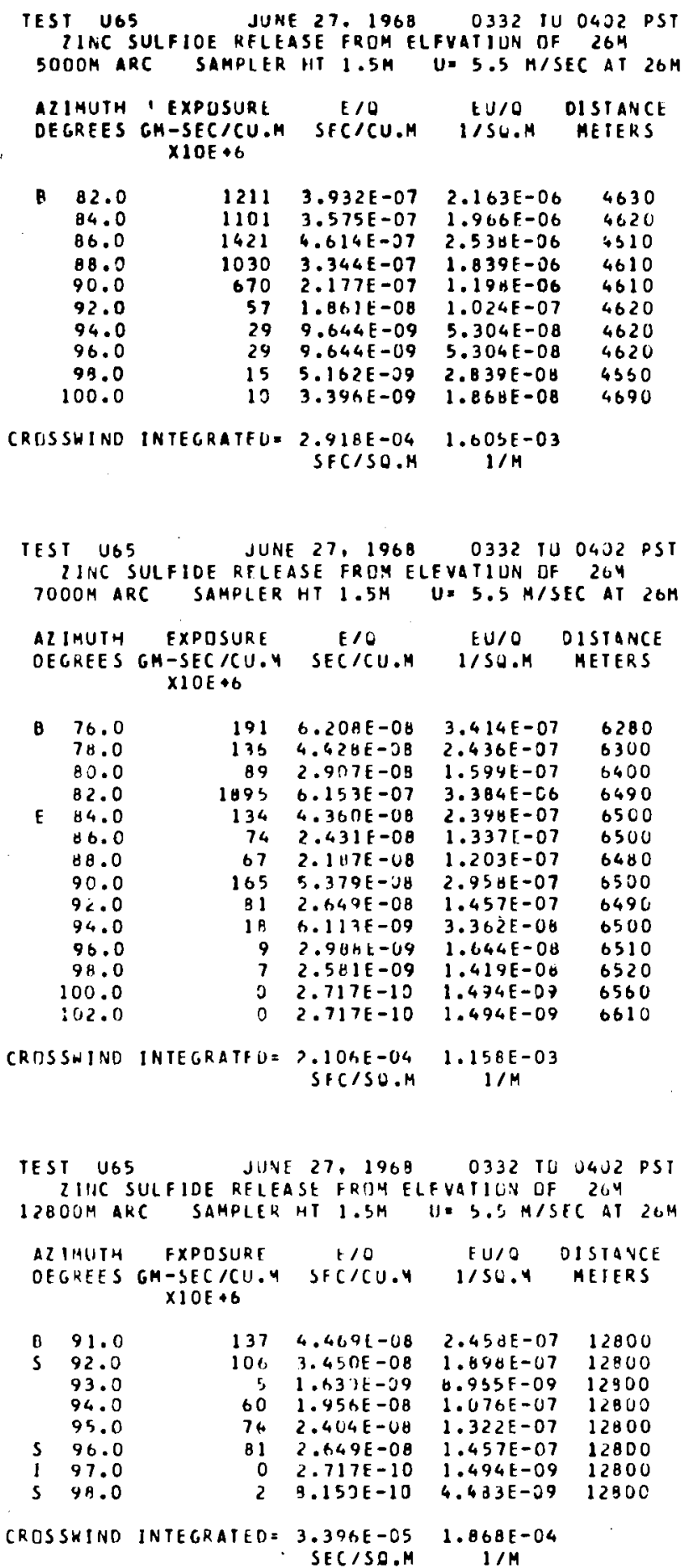

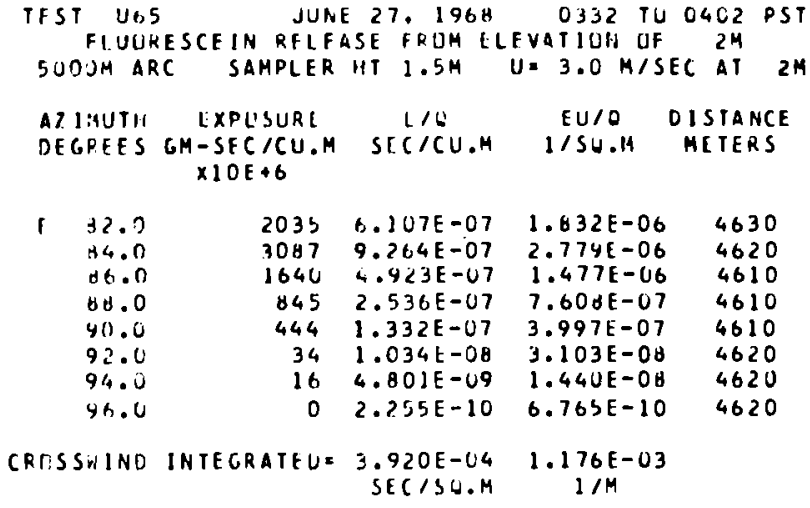




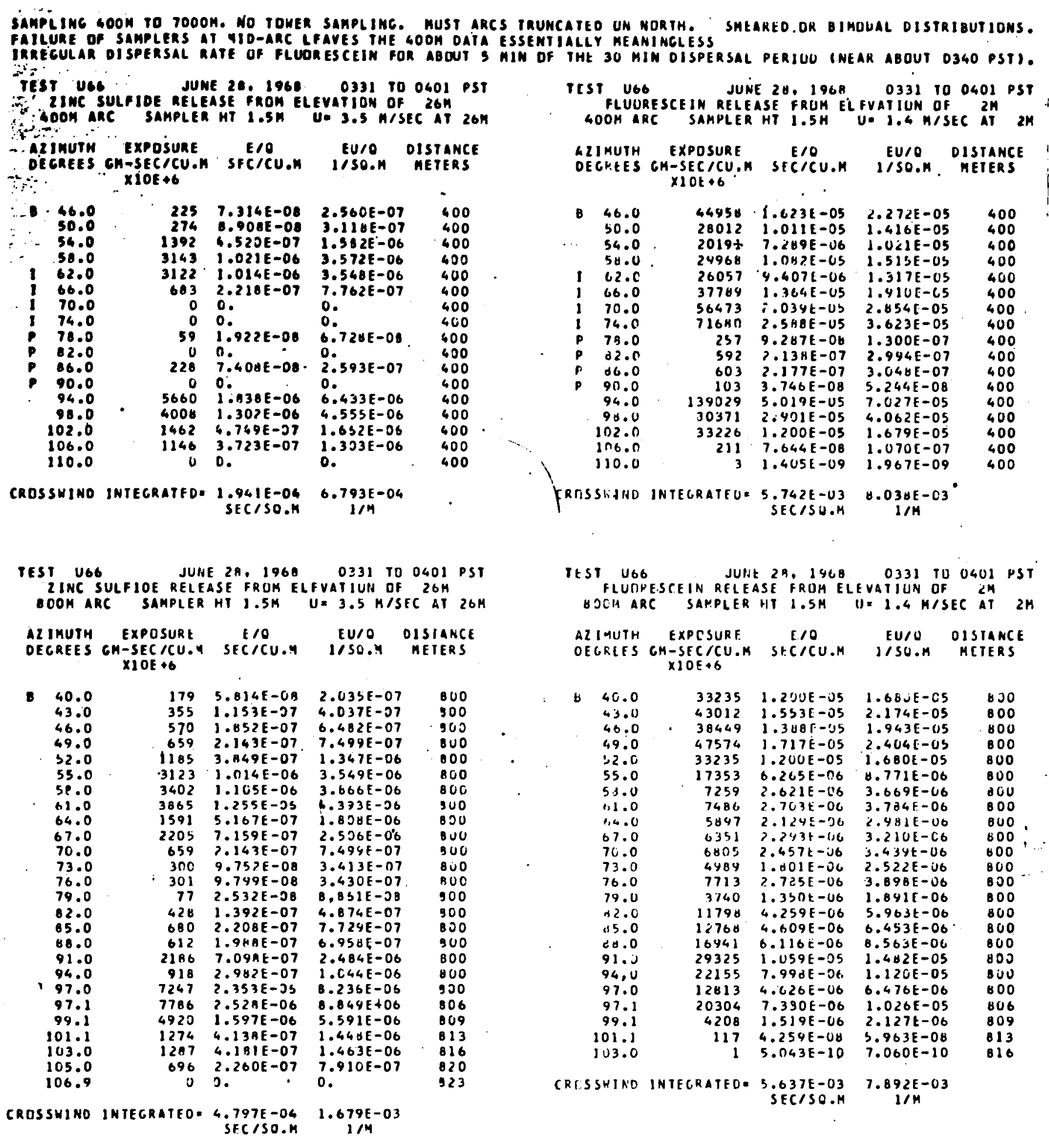




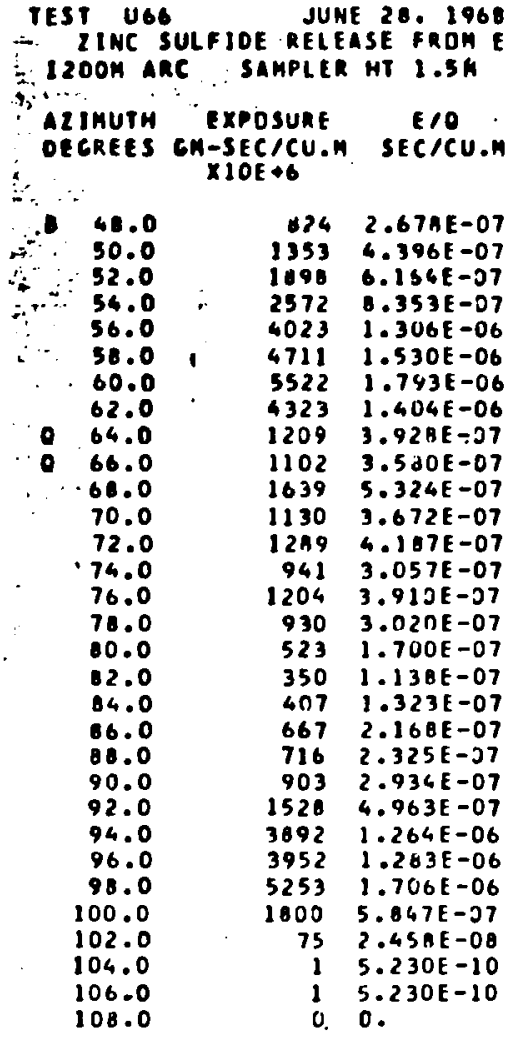

0331 TO 0401 PST U. $3.5^{\circ} \mathrm{M} / \mathrm{SEC}$ AT $26 \mathrm{H}$ EUIO DISTANCE I/SU.M MEIERS

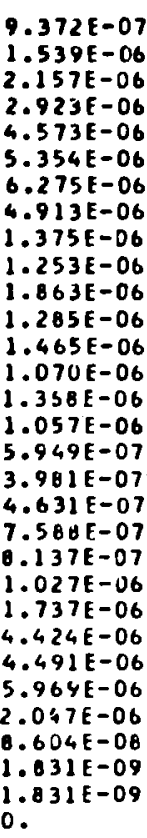
LEVATION OF $26 \mathrm{M}$

TIST U66 FLUURESCEIM RELEASE OHOH ELFYATIDH 100401 PST $1200 \mathrm{M}$ ARC SAMPLER HT $1.5 \mathrm{MH}$ U. $1.4 \mathrm{H} / \mathrm{SEC}$ AT $2 \mathrm{H}$ AZIFUTH EXPOSURE
DECKEES GA-SECKCU.H SECICU.A IISL.M OISIANCE
XIOE+C

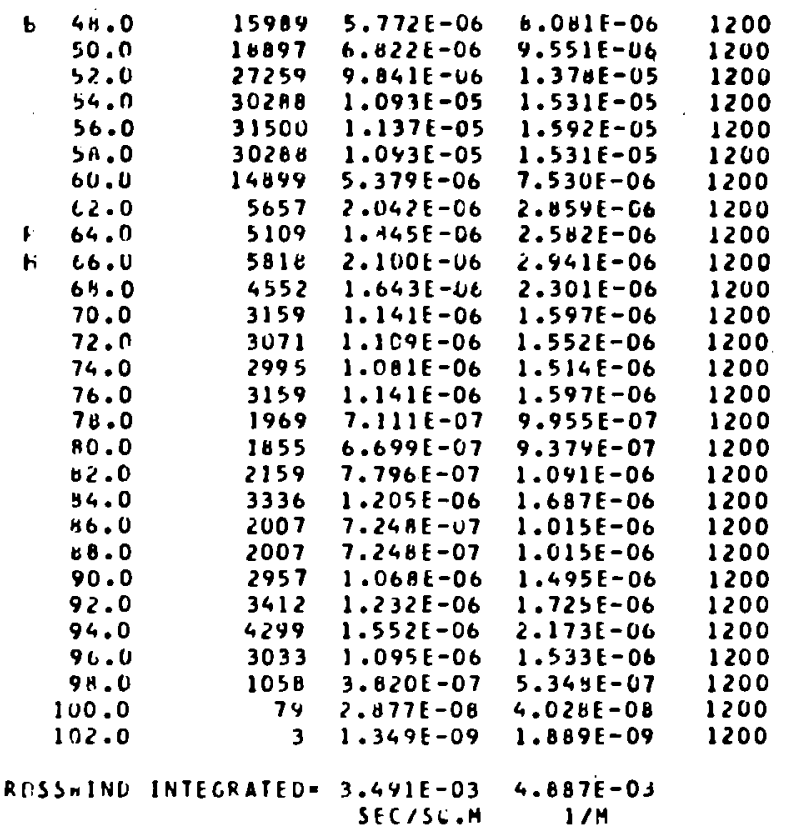

CROSSHIHO INTECRATED= $7.447 E-24 \quad 2.605 E-03$

$1 / \mathrm{M}$

1200
1200
1200
1200
1200
1200
1200
1200
1200
1200
1200
1200
1200
1200
1200
1200
1200
1200
1200
1200
1200
1200
1200
1200
1200
1200

1200

TEST U66 JUNE 28.1968 O331 TU 0401 PST ZINC SULFIDE RELEASE FROH ELEVATIUN OF 264 160OK ARC SAMPLER HT $1.5 \mathrm{M}$ U. 3.5 K/SEC AT $26 \mathrm{H}$ AZIMUTH EXPOSURE
DECREES GH-SEC/CU.M SEC/CU.M I/SO.H MISTANCE $\times 10 E+6$

\begin{tabular}{|c|c|c|}
\hline 48.0 & 539 & $\begin{array}{l}1.752 E-07 \\
1.551 E-07\end{array}$ \\
\hline $\begin{array}{l}50.0 \\
52.0\end{array}$ & $\begin{array}{r}477 \\
1502\end{array}$ & $\begin{array}{l}1.551 E-07 \\
4 . \text { AODE }-07\end{array}$ \\
\hline $\begin{array}{l}32.0 \\
54.0\end{array}$ & 400 & 1.300E-07 \\
\hline 56.0 & 2748 & $0.925 E-07$ \\
\hline $5 n .0$ & 2022 & $6.566 \mathrm{E}-07$ \\
\hline 60.0 & 4163 & $1.352 E-06$ \\
\hline 62.0 & 2833 & $9.200 E-07$ \\
\hline 64.0 & 5704 & $1.852 E-06$ \\
\hline 66.0 & 3063 & 9.44 AE -07 \\
\hline 68.0 & 2532 & B.222E-J7 \\
\hline 70.0 & 493 & $1.603 E-07$ \\
\hline 72.0 & 1640 & $5.327 E-07$ \\
\hline 74.0 & 915 & $2.973 E-07$ \\
\hline 76.0 & 1661 & $5.395 E-D 7$ \\
\hline 78.0 & 459 & $1.491 \mathrm{E}-07$ \\
\hline BO. 0 & 1096 & $3.528 E-j 7$ \\
\hline 82.0 & 276 & ค. $.970 E-0 B$ \\
\hline B4.0 0 & 512 & $1.663 \mathrm{E}-07$ \\
\hline 86.0 & 96 & $3.13 \mathrm{BE}-0 \mathrm{~B}$ \\
\hline$B \theta .0$ & $2 p 6$ & $6.721 E-D 8$ \\
\hline 90.0 & 292 & $9.493 \mathrm{E}-08$ \\
\hline 92.0 & $154 \%$ & $5.000 \mathrm{E}-07$ \\
\hline 94.0 & 11s & $3.760 E-08$ \\
\hline 94.6 & 534 & $1.737 E-07$ \\
\hline 95.6 & 829 & $2.693 \mathrm{E}-07$ \\
\hline 96.6 & 892 & 2.A9AE-07 \\
\hline 97.6 & 2254 & $6.997 E-07$ \\
\hline 98.6 & 1809 & $5.474 E-07$ \\
\hline 99.6 & 1304 & $4.235 E-07$ \\
\hline 100.6 & 1136 & $3.689 E-07$ \\
\hline 101.6 & 411 & $1.337 E-07$ \\
\hline 2.6 & 369 & $1.200 E-07$ \\
\hline$b$ & 0 & 0. \\
\hline
\end{tabular}

CROSSWIND INTEGRATFD= 7.246 E-04 SFC/5O.M $5.420 E-07$ $1.704 E-06$ $4.56 Y E-07$ $3.124 E-06$ 2.24 aE-OB $4.731 E-06$ $3.220 E-06$ $6.483 \mathrm{E}-06$ $3.482 E-06$ $2.978 E-06$ $5.6111 E-07$

$1.864 E-06$ $1.041 \mathrm{E}-06$ 1.88 a -06 $5.217 E-07$ $1.235 \mathrm{E}-06$

3.13 Y -07

$5.821 E-07$

$1.090 \mathrm{E}-07$

$2.352 E-07$

$3.322 E-07$

$1.750 E-36$

$1.309 \mathrm{E}-07$ $6.074 E-07$ $9.426 E-07$ $1.014 E-06$ $2.444 E-06$ 2.056 E-06 $1.482 \mathrm{E}-06$ $1.291 E-06$ $4.201 E-07$ 0 .
$2.535 E-03$

1630
1600
1600
1600
1600
1600
1600
1600
1600
1600
1500
1500
1600
1600
1600
1600
1500
1600
1600
1600
1600
1600
1500
1600
1604
1606
1600
1610
1511
1613
1615
1617
1618
1620

TEST UGG 160014 ARC SAPPIER HT $1.5 \mathrm{M}$ UE $1.4 \mathrm{M} / \mathrm{SEC}$ AT $2 \mathrm{H}$ AZIMUTH EXPOSURE T/O EU/O OISTAMCE OEGRES GH-SECICU.M SFCICU.A I/SU.K MEIERS $\times 10 E+6$
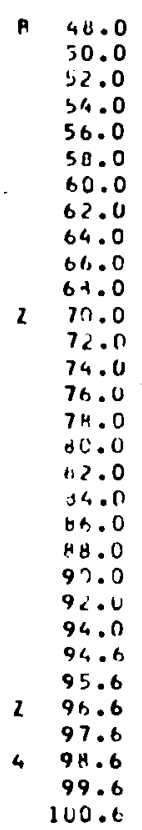

$$
\begin{aligned}
& 11627 \\
& 11627 \\
& 2144 \\
& 15909 \\
& 24714 \\
& 1780 \\
& 13445 \\
& 8945 \\
& 4926 \\
& 341 \\
& 2539 \\
& 2121 \\
& 126 \\
& 958 \\
& 1134 \\
& 860 \\
& 67 \\
& 476 \\
& 284 \\
& 108 \\
& 20 \\
& 32 \\
& 956 \\
& 766 \\
& 79 \\
& 1695 \\
& 421 \\
& 1034 \\
& 335 \\
& 14 \\
&
\end{aligned}
$$

$11627 \quad 4.108 \mathrm{E}-06$ $4.1 \% 8 E-06$
$4.19 H E-06$ $7.74) \mathrm{E}-06$ $5.772 \mathrm{E}-06$ A. $4221-06$ $6.429 E-06$ $6.420 E-06$
$4.854 E-06$ $3.229 E-06$ $1.779 \mathrm{E}-06$ $1.232 \mathrm{E}-06$ $1.232 E-06$
$.167 E-07$ $7.659 \mathrm{E}-07$ $4.506 \mathrm{E}-07$ $3.454 \mathrm{~F}-07$ $4.044 \mathrm{E}-07$

$134 \quad 4.044 \mathrm{E}-07$

$\begin{array}{ll}460 & 3.134 E-07 \\ 67 \mathrm{~B} & 2.444 \mathrm{E}-07\end{array}$

$476 \quad 1.722 \mathrm{E}-07$

$284 \quad 1.026 E-U 7$

$108 \quad 3.922 \mathrm{E}-0 \mathrm{~B}$

$2057.426 t-08$

320 1.156t-07

$956 \quad 3.454 F-07$

$7662.769 E-07$

$7982.842 E-07$

$16956.123 \mathrm{E}-07$

$4212.96 A E-07$

$3.735 \mathrm{E}-07$

$1415.116 E-08$ 0 . $527 \mathrm{E}-11$ $y$

5.877E-06 $5.877 E-06$ $1.084 L-0 S$ $8.081 E-06$ $1.244 \mathrm{E}-0 \mathrm{~S}$ $9.000 E-06$ $6.795 \mathrm{E}-06$ $4.521 E-06$ $2.490 E-06$ $1.725 E-00$ $1.283 \mathrm{E}-06$ $1.072 \mathrm{E}-06$ $6.30 d E-U 7$ $4.836 E-07$ $5.732 E-07$ $4.300 \mathrm{~F}-07$ $3.428 E-07$ $2.411 E-07$ $1.436 E-07$ $5.491 \mathrm{E}-\mathrm{OH}$ $1.040 E-07$ $1.619 \mathrm{E}-07$ 4. $336 E-07$ $3.076 F-07$ $4.035 E-07$ $0.572 E-07$ $4.155 E-07$ $5.229[-07$ $1.695 \mathrm{E}-07$ $7.163 E-04$ 1. $194 E-10$

1600 1600 1600 1600 1600 1690 1600 1600 600 1600 1600 1600 1600 1600 $1600 \cdot 10$ 1600 1600 1600 1600 1600 1600 1600 1604 1606 1608 1610 1613

CRISSIIND INTECRATED. 2.998E-03 4.197E-03

SFC/SO.H $\quad 1 / \mathrm{M}$ 


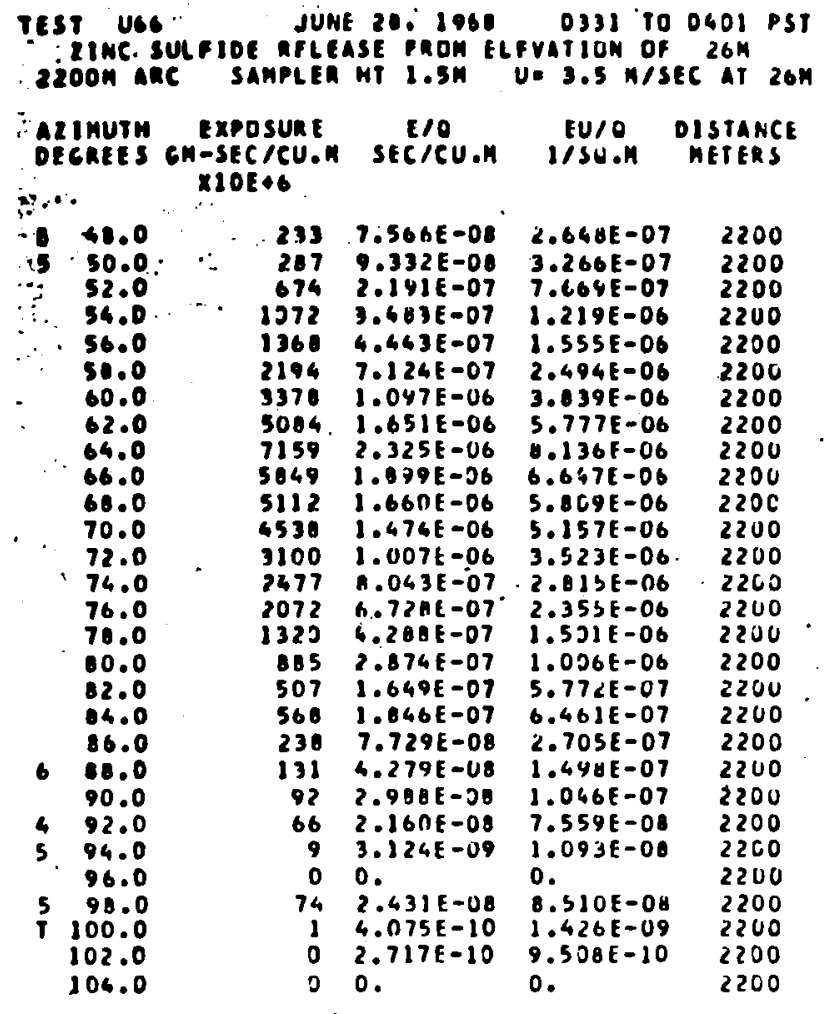

CRDSSHIHD INTECRATED: $1.209 E-03$ 4.232E-03
TFST UAG JUAE 28,1460 0331 TU 0401 PST FLUORESCE IIH RELCASE FKOM ELFVATIUN DF $2 \mathrm{M}$ 220011 ARE SAMPLER HT $1.5 \mathrm{M}$ U. $1.4 \mathrm{M} / \mathrm{SEC}$ AT $2 \mathrm{M}$

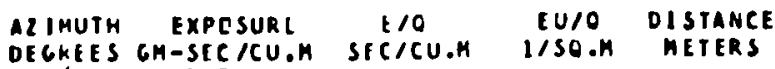
$X 1 \cup E+6$

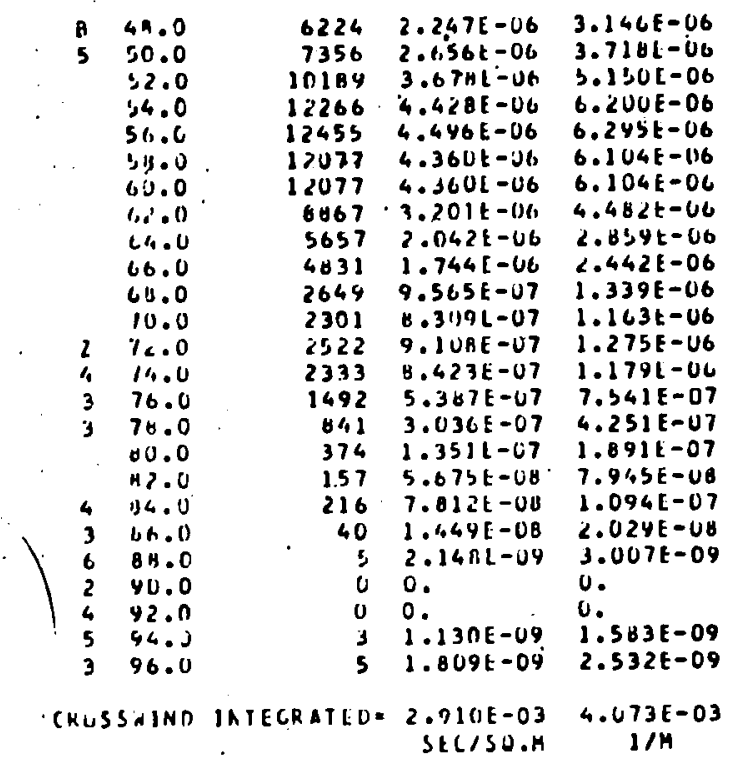

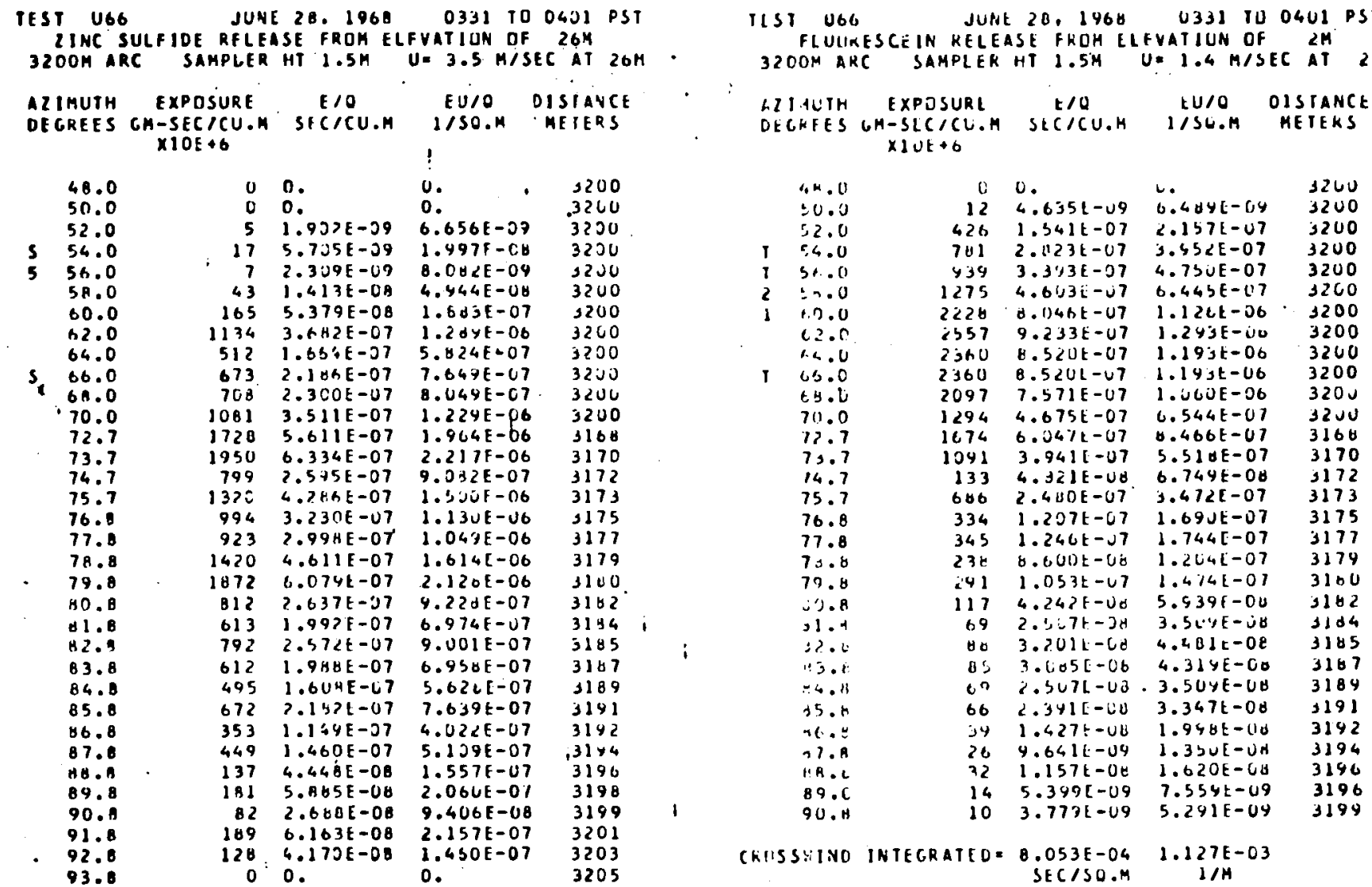




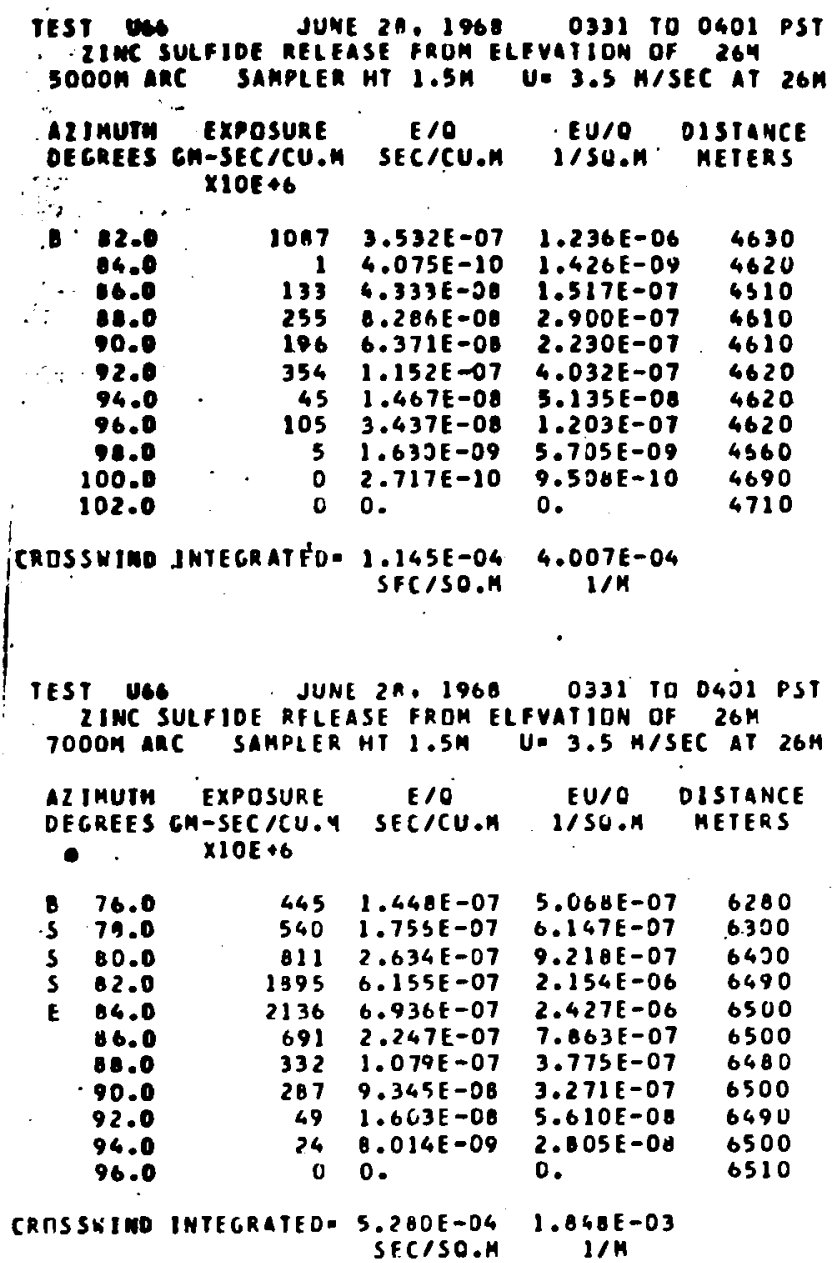

CRISSWIMD INTEGRATED- 5.200E-04 1.048E-03 $1 / \mathrm{M}$ 


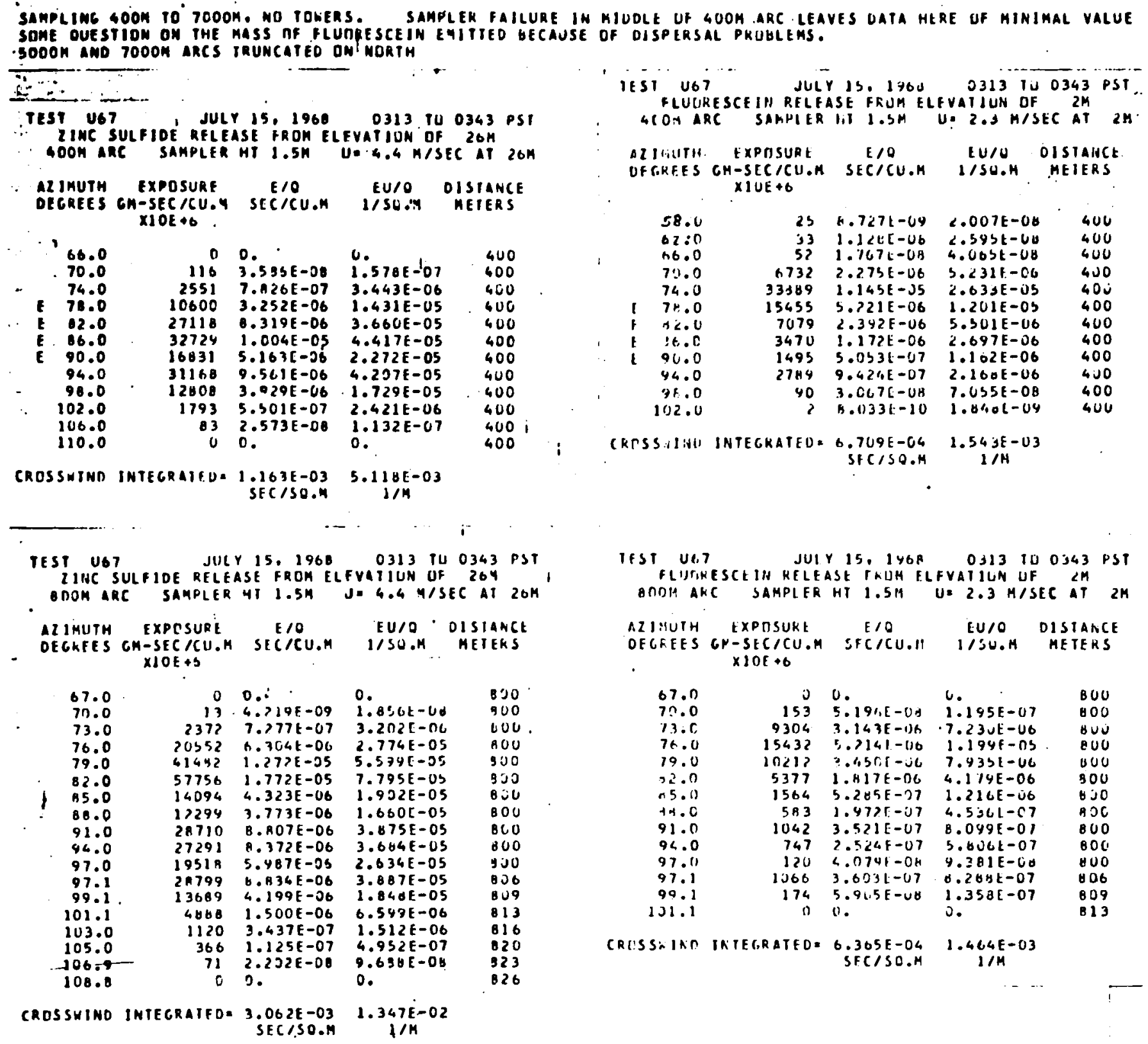




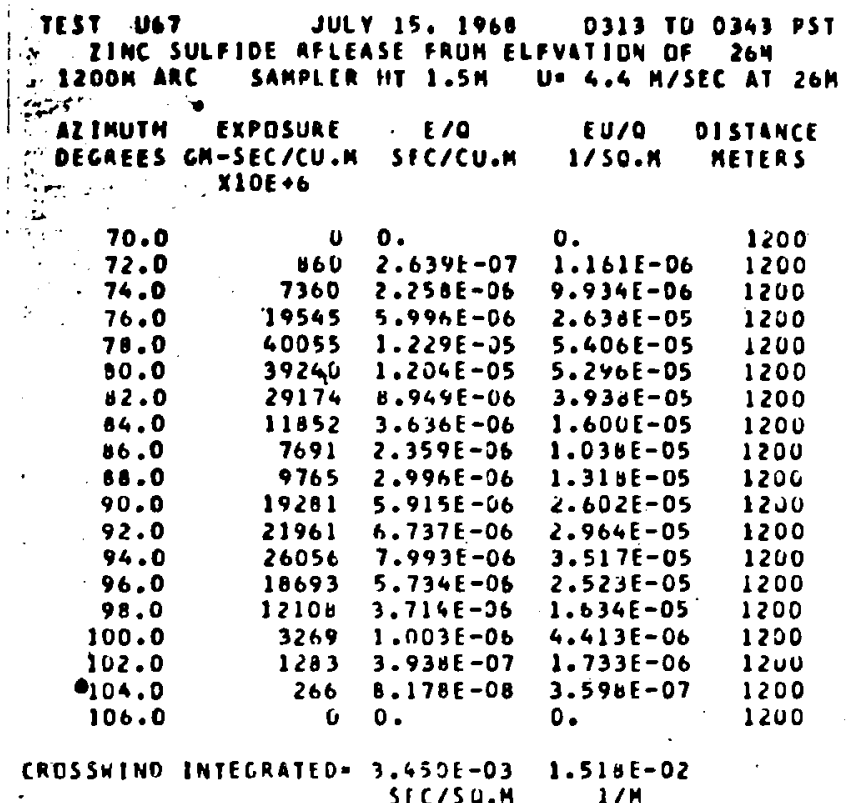

TEST U67 JULY 15, 1969 A 0313 TU 0343 PST
IINC SULFIOE RELEASE FRUM ELEYTIIUV OF 264 IINC SULFIOE RELEASE FRUM ELEVATIUY OF 264
$160 J M$ ARC SAMPLER HT $1.5 \mathrm{H}$ U. 4.4 N/SEC AT $26 \mathrm{M}$

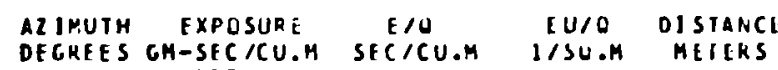
$X 10 \mathrm{E}+6$

\begin{tabular}{|c|c|c|}
\hline 66.0 & i & 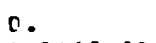 \\
\hline $\begin{array}{l}68.0 \\
70.0\end{array}$ & $\begin{array}{r}16 \\
427\end{array}$ & $\begin{array}{l}4.941 E-6 \\
1.312 E-0\end{array}$ \\
\hline $\begin{array}{l}70.0 \\
72.0\end{array}$ & $\begin{array}{r}427 \\
2219\end{array}$ & $\begin{array}{l}1.312 \mathrm{E}-0 \\
6.109 \mathrm{E}-0\end{array}$ \\
\hline 74.0 & 6407 & $1.97 J E-0$ \\
\hline 76.0 & 16276 & $4.379 E-C$ \\
\hline $7 \pi .0$ & 19353 & $5.937 E-1$ \\
\hline 60.0 & 9316 & $2.85 B E-C$ \\
\hline B 2.0 & 14700 & $4.509 \mathrm{E}-$ \\
\hline 84.0 & .7503 & $1.075 \mathrm{E}-$ \\
\hline 86.0 & 6372 & $1.264 E=5$ \\
\hline $8 B .0$ & 7706. & $8.3311-1$ \\
\hline 90.0 & 5703 & $1.7495-$ \\
\hline 92.0 & 9554 & $2.931 \mathrm{E}-$ \\
\hline 93. & 25369 & $7.702 \mathrm{E}$ \\
\hline 96.0 & 6081 & $1.565 \mathrm{E}-$ \\
\hline 94.6 & 15593 & $6.755 E-$ \\
\hline 95.6 & 13919 & $4.277 \mathrm{E}$ \\
\hline 96.6 & 15119 & $4.63 \mathrm{BE}-$ \\
\hline 97.6 & 14003 & $4.295 E-C$ \\
\hline 98.6 & 9527 & $2.923 \mathrm{E}-\mathrm{C}$ \\
\hline 99.6 & 3890. & $1.1960-C$ \\
\hline 100.6 & $3675^{\circ}$ & $1.127 \mathrm{E}-3$ \\
\hline 101.6 & 1996 & $6.125 \mathrm{E}-$ \\
\hline 102.6 & 1024 & $3.142 \mathrm{E}-\mathrm{C}$ \\
\hline 103.6 & 335 & $1.030 E-$ \\
\hline & 131 & $4.048 E-$ \\
\hline & U & \\
\hline
\end{tabular}

CROSSWINO INTEGRATFU $=2.465 E-03 \quad 1.004 E-02$
TEST U67 JULY 15,1908 O 1913 TU 0343 PST 12 OUM ARE SAMPLER HT $1.5 \mathrm{SM}$ U. $2.3 \mathrm{H}$ HSEC AT $2 \mathrm{H}$

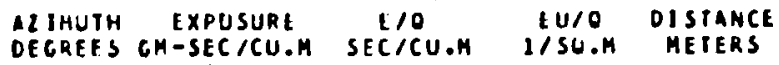
DEGRES GH GEC
XIOE 16

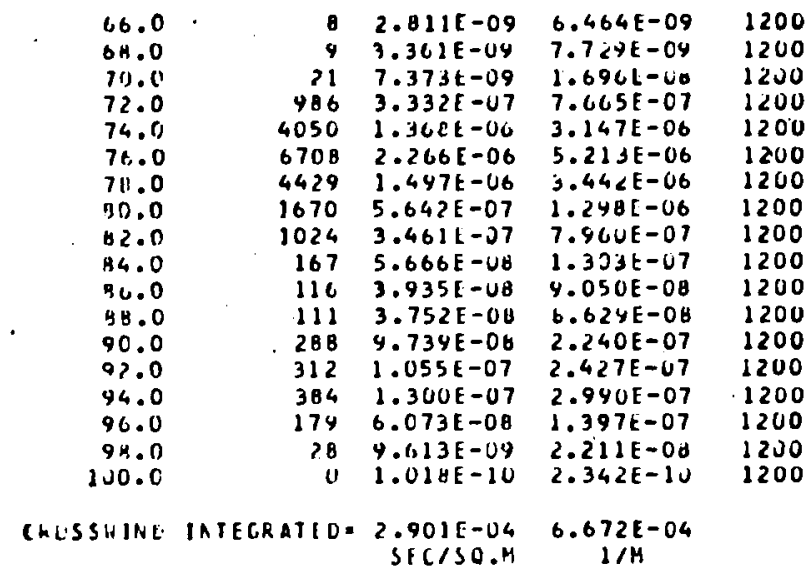

TLST IJ67 JULY 15, 14680313 IL 0343 PST FLWHERSCEIN RILEASE FHUH ELFYATIUN OF IM alliutr exposure lis tulo DISTANCE

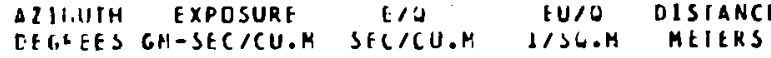
$X \perp C F+C$

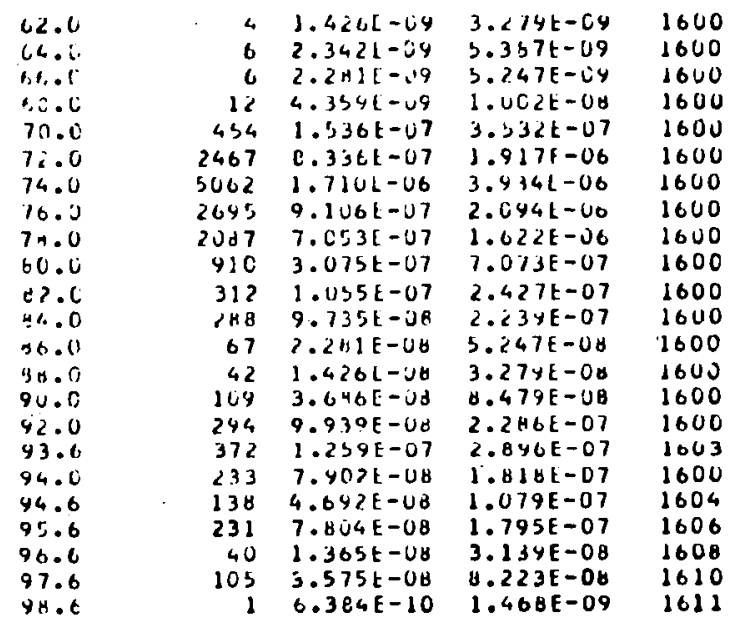

CRUSSHINO INTEGRATEO= $2.084 E-04 \quad 6.633 E-04$ 


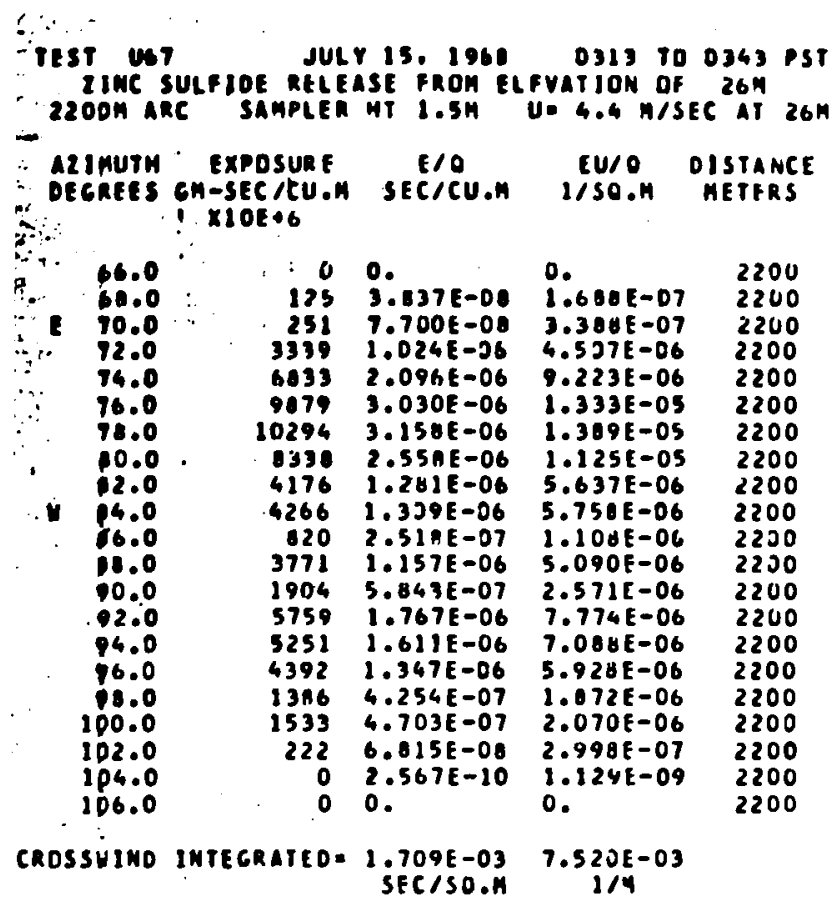

TEST U67 JULY 15, 1938 O313 TO 0343 PST 2 INE SULFIDE RFLEASE FRJM ELEVATION OF $26 \mathrm{M}$ $3206 \mathrm{M}$ ARC SAMPLER HT 1.5M U. 4.4 M/SEC AT $26 \mathrm{M}$ AZIMUTH EXPOSURE E/O EU/O DISTANCE DEGRES CH-SEC/CU.M SECICU.H I/SO.H METERS $\times 10 E \bullet 6$

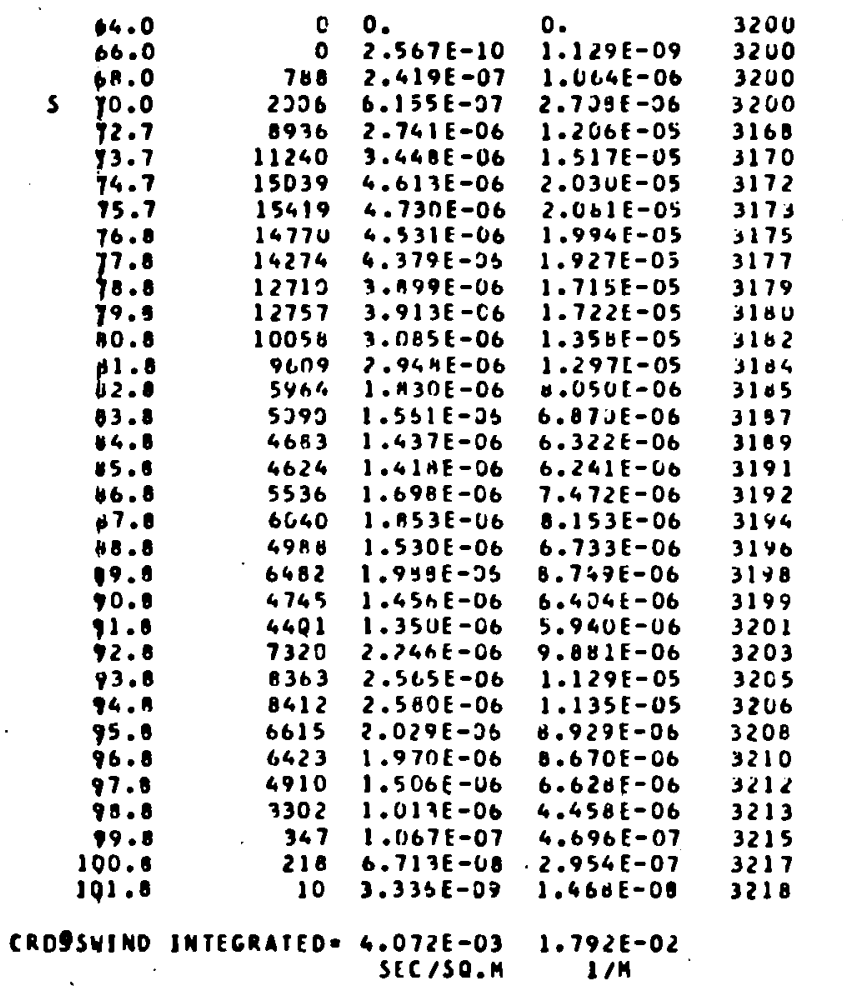

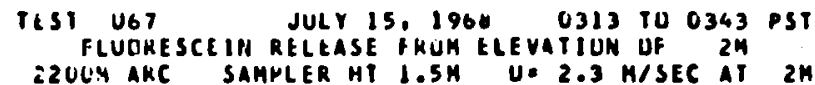
D.LIMUIII EXPQSURE $t / 0$ EU/O OISTAMCE DEGKES GH-SEC/CU.M SEC/CU.M I/SO.M METERS $\times 101+6$

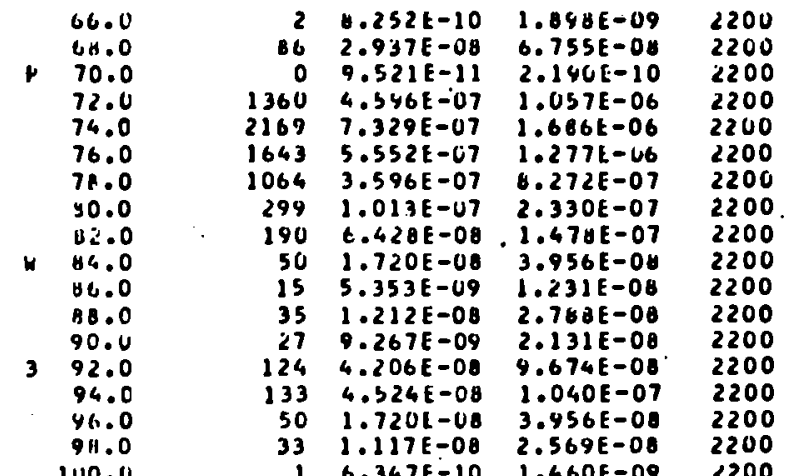

CRDSSHIRO INTECRATEU- $1.097 E-04$ 4.351E-04

SEC/SO.M I/M
TEST U67 JULY 15. 146 A 0313 TU 0343 PST FLUIIKESCEIN KELEASE FRUM ELEYATIUIN OF 32 COP ARC SAMPLER HI $1.3 \mathrm{M}$ U $2.3 \mathrm{H} / \mathrm{SEC}$ AT $2 \mathrm{M}$

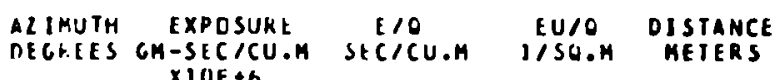
$\times 10 E+6$

\begin{tabular}{|c|c|c|c|c|}
\hline b.o & & $5.395 t-10$ & $1.2=1 E-04$ & 3200 \\
\hline GA. 0 & 224 & $7.5 \cup 5 E-\cup 8$ & $1.745 E-07$ & 3200 \\
\hline 70.0 & 847 & $2.863 E-07$ & $6.584 E-07$ & 3200 \\
\hline 72.7 & $\begin{array}{l}1614 \\
1367\end{array}$ & $\begin{array}{l}5.453 E-U 7 \\
4.620 E-07\end{array}$ & $\begin{array}{l}1.254 E-06 \\
1.063 E-06\end{array}$ & $\begin{array}{l}3168 \\
3170\end{array}$ \\
\hline $\begin{array}{l}73.7 \\
74.7\end{array}$ & $\begin{array}{l}1367 \\
1614\end{array}$ & $\begin{array}{l}4.620 E-07 \\
5.453 E-07\end{array}$ & $\begin{array}{l}1.063 \mathrm{E}-06 \\
1.254 \mathrm{E}-06\end{array}$ & $\begin{array}{l}3170 \\
3172\end{array}$ \\
\hline 75.7 & 1502 & $5.074 t-07$ & $1.167 \mathrm{E}-06$ & 3173 \\
\hline 76.0 & 1035 & $3.447 \mathrm{E}-U 7$ & 8.044E-07 & 3173 \\
\hline 77.0 & 736 & $2.407 t-07$ & $5.720 \mathrm{E}-07$ & 317 \\
\hline $7+.10$ & 501 & $1.6,43 E-07$ & $3.894 \mathrm{E}-07$ & 317 \\
\hline 74.6 & 757 & $2.559 E-07$ & $5.886 E-07$ & 3180 \\
\hline Bu.d & 319 & $1.079 E-07$ & $2.402 \mathrm{E}-07$ & 3182 \\
\hline 01.3 & 130 & $4.406 E-0 B$ & 1.01 & 318 \\
\hline 12.8 & 38 & $1.302 E-08$ & $2.995 E-08$ & 3185 \\
\hline$B 3.6$ & 34 & $1.158 E-0 B$ & $2.663 \mathrm{~F}-00$ & 3187 \\
\hline 84.17 & 37 & $1.266 \mathrm{E}-\mathrm{UH}$ & $2.912 \mathrm{E}-0 \mathrm{~B}$ & 3189 \\
\hline 85.8 & 16 & $5.731 E-09$ & $1.31 \mathrm{BE}-0 \mathrm{~B}$ & 3191 \\
\hline 16.8 & 16 & $5.514 t-09$ & $1.260 E-08$ & 3192 \\
\hline 87.8 & 55 & $1.8 B O E-0 B$ & $4.323 E-08$ & 3194 \\
\hline Y .8 & 10 & $3.457 E-09$ & $7.952 \mathrm{E}-09$ & 3196 \\
\hline 89.8 & 16 & $5.731 E-09$ & $1.310 E-08$ & 3198 \\
\hline 90.4 & 20 & $6.814 E-\cup 9$ & $1.567 E-08$ & 3199 \\
\hline $91 . \mathrm{H}$ & 34 & $1.158 \mathrm{E}-O R$ & $2.663 \mathrm{E}-08$ & 3201 \\
\hline 92.8 & 48 & $1.627 \mathrm{E}-08$ & $3.742 \mathrm{E}-0 \mathrm{O}$ & 3203 \\
\hline 0 & 79 & $2.673 E-0 B$ & $6.149 E-06$ & 3205 \\
\hline 94.8 & 82 & $2.782 E-00$ & $6.398 E-08$ & 3206 \\
\hline 95.8 & 49 & $1.663 \mathrm{E}-08$ & $3.825 E-0 B$ & 3208 \\
\hline & 37 & $1.266 \mathrm{E}-\mathrm{JB}$ & $912 E-08$ & 3210 \\
\hline 97.8 & 9 & $3.241 E-09$ & $7.454 E-09$ & 3212 \\
\hline 99.8 & 1 & $6.424 E-10$ & $1.477 t-09$ & 3213 \\
\hline
\end{tabular}

CRUSSHIND INTECRATED= $2.644 E-04$ 6.002E-04
170

73

3182

105

3187

189

3192

194

3198

3201

3206

3210

3213 


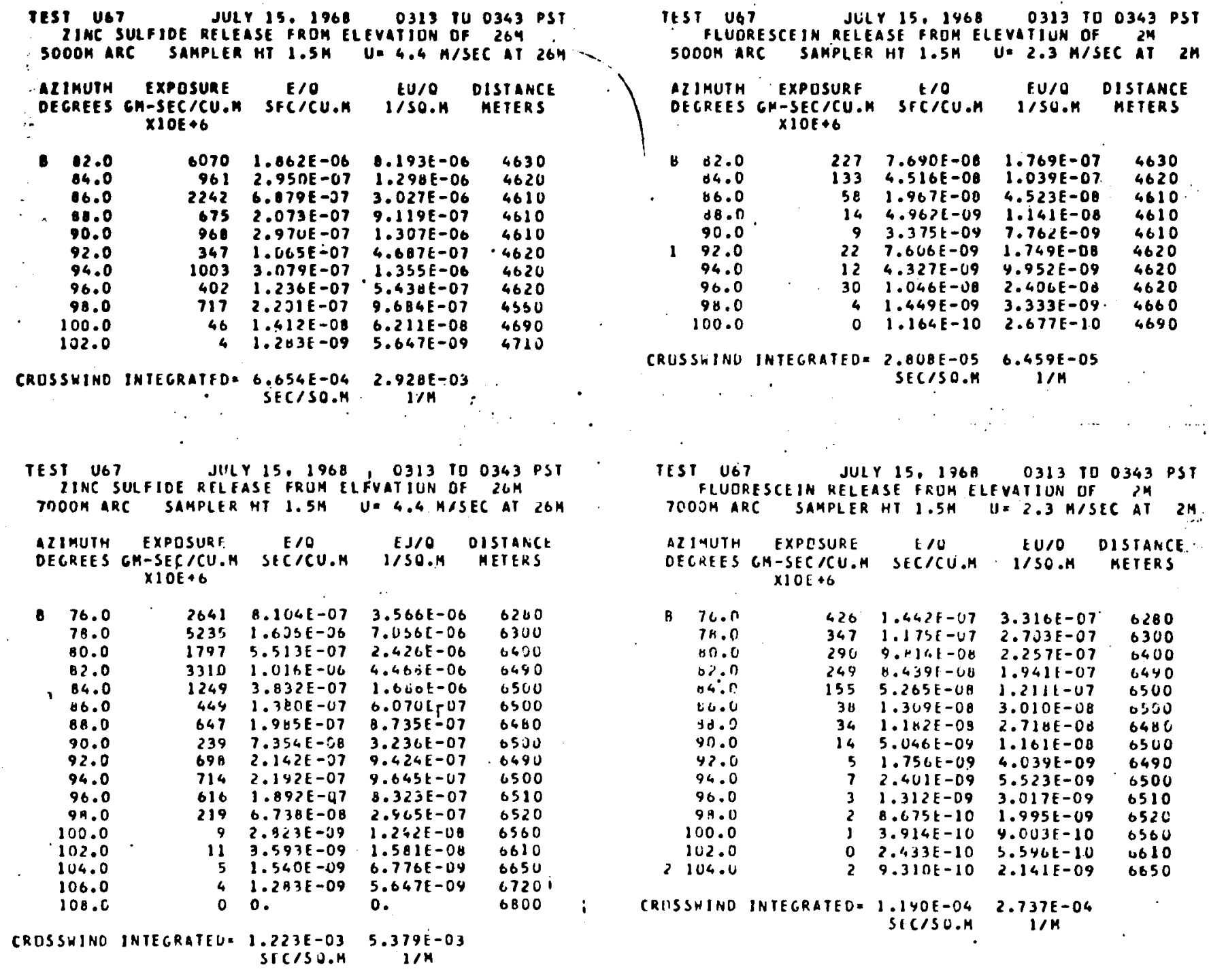


SAMPLINC FRUM $40 O H$ IT IZPOOM. NU TONER SAMPLING. BIMUDAL DISTRIBUTIDNS.

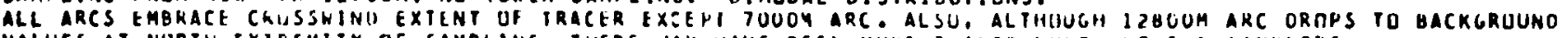
VALUFS AT NURTH FXIREMIIY TF SAMPLING. THERE MAY HAVE BEER MUKE THALER NUKTH UF THE SAHPLERS.

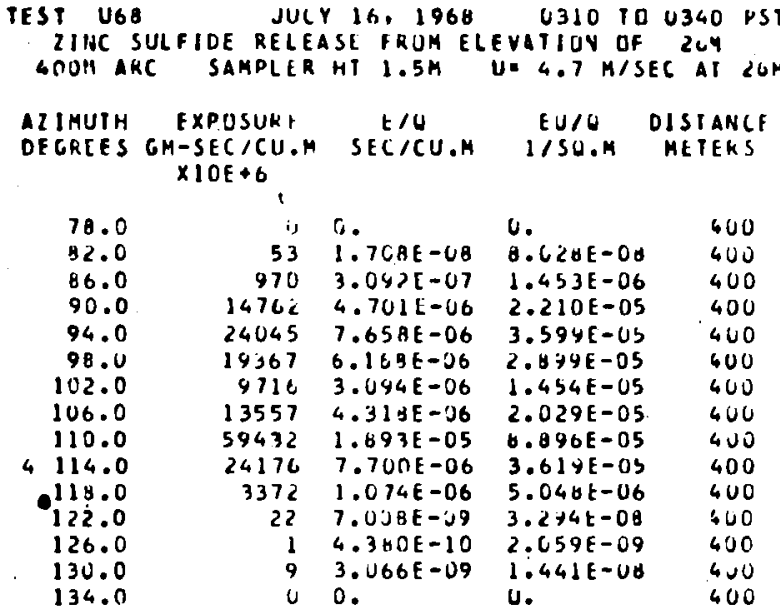

CRIISSHINU INTEGRATTLE J.SUTE-U3 7. UQ4E-03 SECISO.

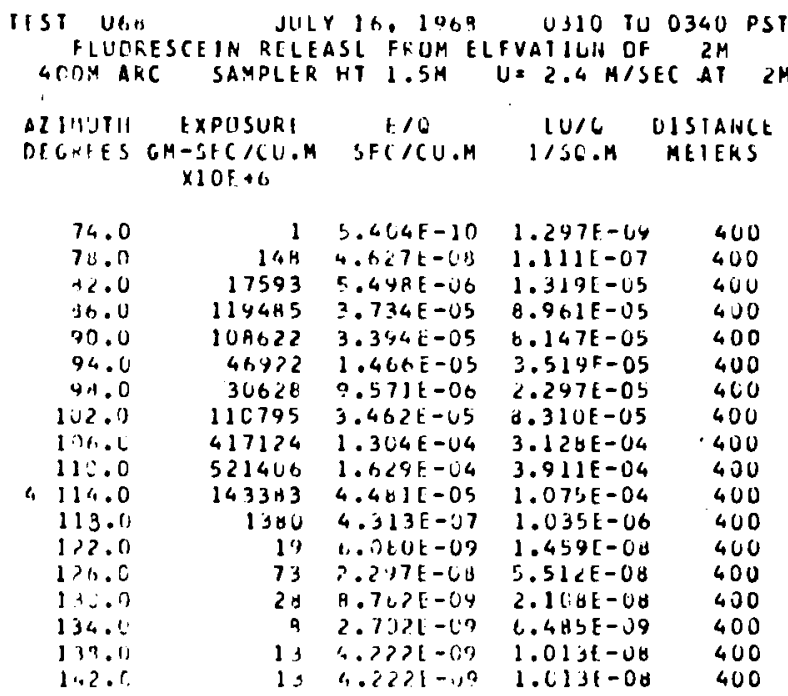

CRISSEIIIL INTEGRATED $=1.324 \mathrm{~F}-02$ 3.17YE-02 SFC/Sid. $1 / \mathrm{H}$

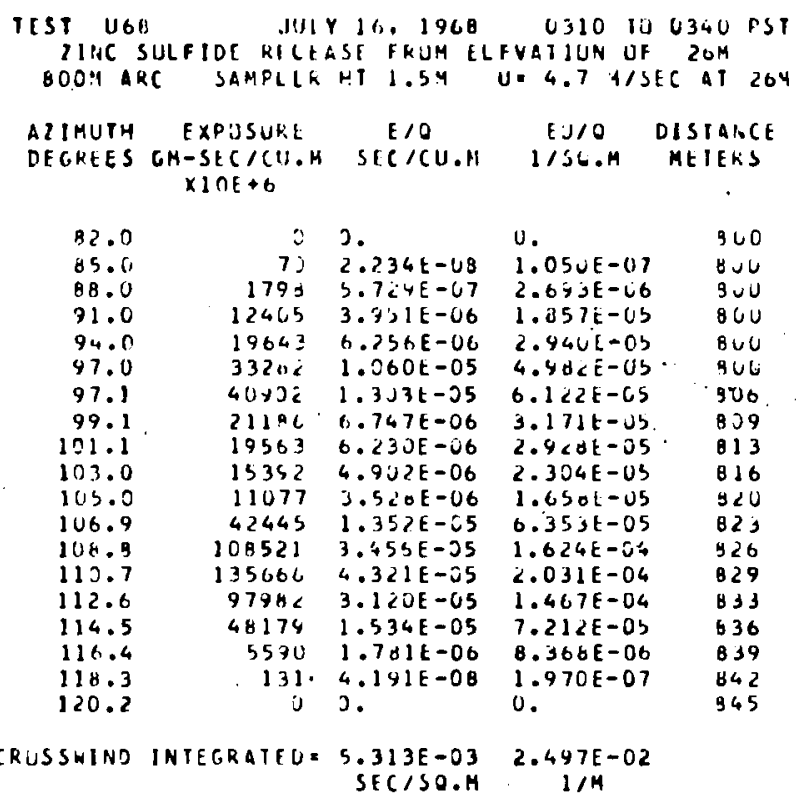

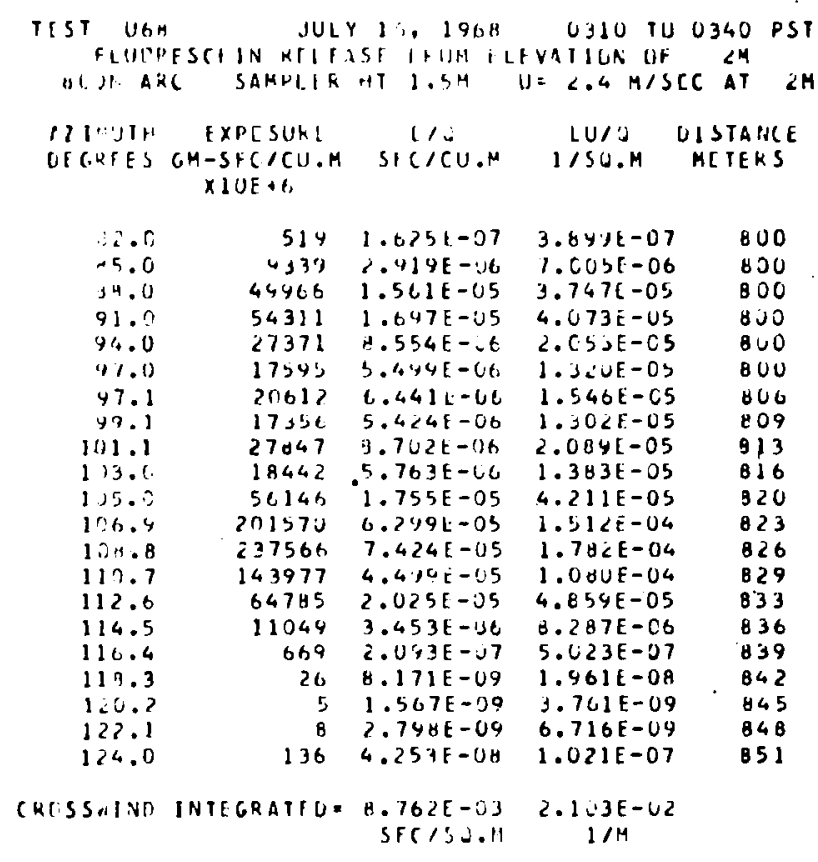




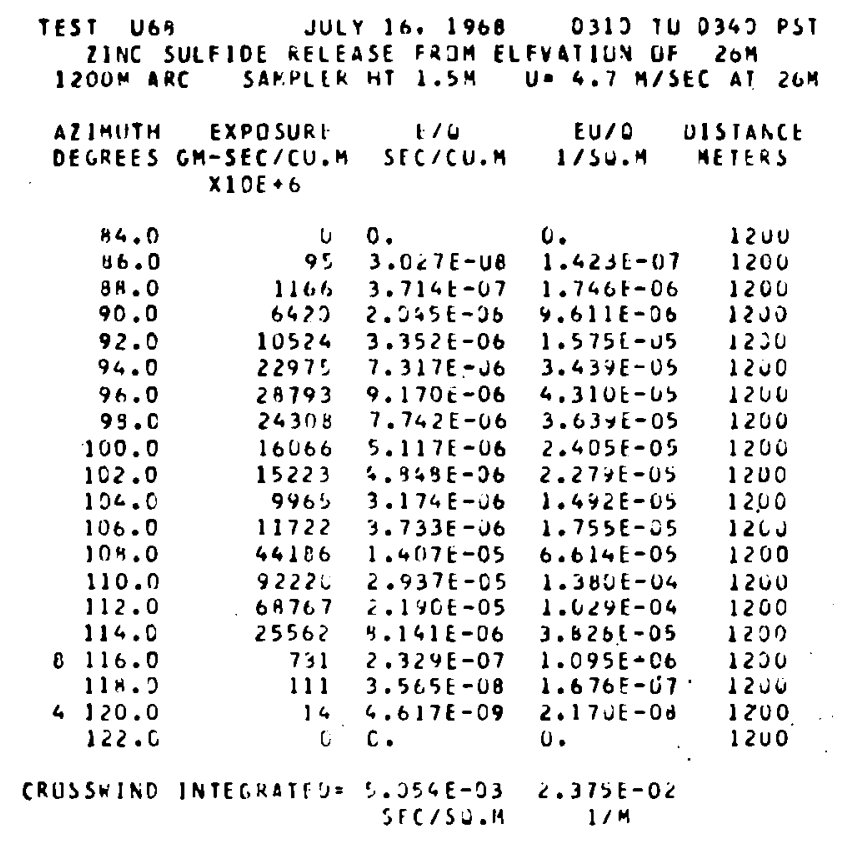

TEST UAH JUIY 16.1968 O 0310 TU 0340 PSI IINC SULFIDE RFLEISS FRLIA ELFVATIUY UF 264 IUCOM ARC SAYPLEK YI $1.5 \mathrm{SM}$ J 4.7 M/SEC AT COH

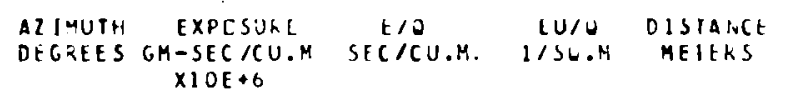

\begin{tabular}{|c|c|c|c|c|}
\hline$B G .0$ & $\because$ & c. & u. & 1500 \\
\hline 16.0 & 18 & $5.960 F-09$ & $2.773 \mathrm{~F}=00$ & 16 u \\
\hline d & 340 & $1.0165 E-07$ & $5.10 u l-07$ & 1600 \\
\hline 90.0 & 2130 & $0.977 E-07$ & $3.27,0-06$ & 1600 \\
\hline 92.0 & 6275 & $1.797 E-56$ & $9.3+4 E-06$ & ISUU \\
\hline 93.6 & $14 y>9$ & $6.733 \mathrm{E}-06$ & $\angle .220 u t-0 s$ & 1503 \\
\hline 96.0 & icsioj & $3.440 E-16$ & $1.617 \mathrm{E}-05$ & $16 \mathrm{Ju}$ \\
\hline 94.6 & 16,499 & $5.754 \dot{E}-06$ & $2.470[-03$ & $150 \%$ \\
\hline 95.6 & $1454 d$ & $4.646, E-06$ & $2.184[-0 s$ & 1606 \\
\hline 96.6 & 18YCE & $5.989 t-06$ & $2.015 \mathrm{E}-0 \mathrm{~S}$ & 1600 \\
\hline+7.6 & 27231 & $7.362 E-j 6$ & $3.325 E-05$ & 1510 \\
\hline $9 \pi .6$ & 10.1013 & $5.2 .27 E-06$ & $2.457 E-0 S$ & 1611 \\
\hline $99 . h$ & 20727 & $6.6 j, E-J E$ & 3.1JSE-US & 2633 \\
\hline $100 . t$ & $16 \% 4+i$ & $\because 395 E-0 c$ & $2.536 \mathrm{~F}-05$ & 1615 \\
\hline 101.6 & $1555 \mathrm{~J}$ & $4.455 E-06$ & $2.32 \times 1-03$ & 1617 \\
\hline 102.6 & $931 R$ & 2. OEFE-UE & $1.395 \mathrm{E}-0 \mathrm{~S}$ & 1618 \\
\hline 103.6 & 9341 & $2.975 \mathrm{~F}-\mathrm{J3}$ & $1.3 \neq 45-05$ & 1520 \\
\hline 104.5 & $70 \%$ & $2.5103 t-20$ & $1.16>15-05$ & 1622 \\
\hline 1113.5 & $b_{2} b_{2}$ & $1.095 \mathrm{E}-06$ & $4.374 E-06$ & $16, j$ \\
\hline $\ln 6.5$ & 12475 & $4.101 \mathrm{E}-\mathrm{OG}$ & $1.927 E-05$ & 162 's \\
\hline 107.5 & 27231 & $4.673 E-06$ & $4.076 \mathrm{~F}-05$ & 1027 \\
\hline 108.5 & 33436 & $1.065 E-05$ & $5.004 \mathrm{E}-0 \mathrm{~b}$ & 1620 \\
\hline 109.5 & 63795 & $2.3 \geq 2 E-35$ & $7.5 \div 7 E-05$ & is $\$ 0$ \\
\hline 119.4 & 6814 त & $2.193 E-05$ & $1.031 \mathrm{E}-64$ & $16 \pm 2$ \\
\hline 111.4 & $592=3$ & $1.03 B E-C 5$ & $8.874 E-\cup 5$ & 1633 \\
\hline 112.4 & 43024 & $1.370 E-05$ & $6.440 E-05$ & 1634 \\
\hline 113.4 & 24204 & $7.703[-06$ & $3.623 E-05$ & 1037 \\
\hline 114.4 & 10935 & $4 . \div 70 t-J 5$ & $1.631 E-35$ & 1534 \\
\hline 115.3 & 4170 & $1.331 E-56$ & $6.2575-06$ & $10+0$ \\
\hline 116.3 & 437 & $1.553 E-07$ & 7.2 YSUE-07 & $15=1$ \\
\hline 117.3 & 31 & $1.005 E-06$ & $4.724 E-O Y$ & 1643 \\
\hline
\end{tabular}

CRISSR.IVU INTEGRATFD= $4.946 E-03 \quad 2.324 E-02$

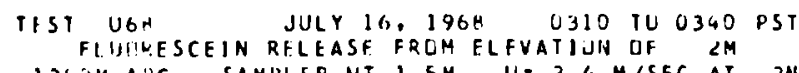
120.NARC SAMPLER HT 1.5M U: $2.4 \mathrm{M} / \mathrm{SEC}$ AT $2 \mathrm{M}$ AZI"UTH EXPUSURF LIZ EU/O UISTANCE DFGLLES GH-SEC/CU.H SECICU.H I/SU.M METERS $X 10 E+6$

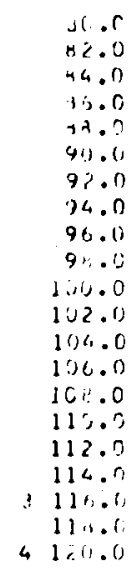

$$
\begin{aligned}
& \text { j } 0 \\
& \begin{array}{rl}
9 & 2.4201-09 \\
90 \% & 2.443 E-07 \\
71 \% 2 & 2.2261-95 \\
22173 & 6.229 E-1.16
\end{array} \\
& 27 \text { CGM } 8.7 \cup G E-J 6 \\
& 21809 \text { a. } 316, \mathrm{t}-6) \\
& 123573.8671-26 .
\end{aligned}
$$

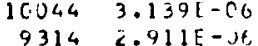

$$
\begin{aligned}
& \begin{array}{l}
9314 \\
9131 \\
2.911 E-J 4 t \\
214006
\end{array} \\
& 11140 \quad 3.461 E-016 \\
& 3.3927 \quad \text { l. OCUE-05 } \\
& 86032 \quad 2.6895-05 \\
& 71491 ; .234 L-05 \\
& 21022 \quad 6.548 \mathrm{E}-06 \\
& 43021.345 E-06 \\
& 207 \quad 0.464 F-1,8 \\
& \begin{array}{ll}
0 & 9.6011-09 \\
2 & 7.7261-10
\end{array}
\end{aligned}
$$

L.

$7.008 \mathrm{E}-\mathrm{Cy}$ $6.822 E-07$ $3.3421-06$ $1.0+3 E-35$ $2.272[-53$ $2.040 \mathrm{E}-015$ $1.6365-05$ Y. $26+F-06$

$7.534 E-U n$ $6.9 B C E-66$ $6.849[-06$ $3.356 E-06$ $2.545 \mathrm{E}-0 \mathrm{~S}$ $6.452 \mathrm{E}-05$ $5.36<\mathrm{E}-\mathrm{US}$

$1.581 \mathrm{E}-\mathrm{C} 5$ $3.221 k-60$

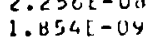

1200

1200

1200

1200
1200

1200

1200

1200

1200

1200

1200

1200

\begin{tabular}{|c|c|c|c|c|}
\hline $32.1=$ & c & $1.1301-10$ & $2.71 \mathrm{sf}-10$ & 1600 \\
\hline$\because 4.0$ & Rl & $2.431-00$ & $0.10401-0)$ & $10 \mathrm{LC}$ \\
\hline 56.0 & $17 \mathrm{MI}$ & $5.567+-27$ & $1.3365-60$ & 1606 \\
\hline 23.1 & 6304 & $2.1+71-1,6$ & b. $17 c t-6 b$ & 1600 \\
\hline $4 \therefore 3$ & 1126,5 & $3.521:-6$ & $8.41 .4 E-U L$ & devo \\
\hline $3 / . n$ & 14537 & $4.5431-46$ & 1.UナUt-Us & 1000 \\
\hline 93.1 & $1: 499$ & $4.00,4 t-0.6$ & $1.1<2[-0 b$ & 1603 \\
\hline 44.0 & 10175 & $3.1 H C t-J t$ & $7.6,1 \mathrm{E}=06$ & 1600 \\
\hline 94.6 & 11692 & $3.6515-06$ & U.7 SCE-UO & 1504 \\
\hline $4 \cdot .6$ & $75 H_{2}$ & $2.36^{2} 1-1-0$ & $5.6: 7 \mathrm{E}-\mathrm{ve}$ & 2656 \\
\hline 96.6 & 9721 & $2.79+6-6$ & $t .671 \mathrm{~F}-26$ & 1008 \\
\hline 47.1 & he:94 & $i \cdot i, t_{t}-j f$ & $3.0111-66$ & $16 i \mathrm{C}$ \\
\hline$y+.6$. & f. 3161 & $2.1,30-60$ & $2 .(4 u t-i) u$ & 1011 \\
\hline 39.6 & 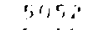 & $1.67 \cdot 1-66$ & $3.7: 3)-60$ & 1013 \\
\hline $100 . t$ & $5,14 \%$ & $1.6 .7) F-i t$ & 4.61 $25-06$ & 1615 \\
\hline 101.0 & $634 ?$ & $1.4 .4 t-6$ & $4.57 \mathrm{LE}-\mathrm{JU}$ & 1617 \\
\hline $122 \cdot 6$ & 4450 & $1.9 y 3 E-10$ & $3 . j+j l-60$ & 1610 \\
\hline $1 \div 3.6$ & 4.56 & $1.1430-06$ & $3.34 \mathrm{st}-06$ & $16<0$ \\
\hline$j$ & 36,7 & $1.6,7 r_{1}-1,1$. & $4.01=1-0 L$ & 16,22 \\
\hline 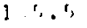 & $76: 3$ & $\therefore 3631-06$ & $3.519+6-6$ & $10<3$ \\
\hline $1 \cdot 4.5$ & 27741 & $7.1141-.20$ & $1.70 \times i-i 0$ & $16<3$ \\
\hline $1,1.5$ & 3444.9 & $1.20>\mathrm{E}-\cup 5$ & $\angle .004 E-6 s$ & 1621 \\
\hline $1 \cdot 5$ & $6<40$ & $2 \cdot C 1>E-5$ & $4.830 t-u b$ & 1628 \\
\hline $1, \therefore$ & 1,201 ? & $1 .{ }^{2} 12 i_{-}-i_{2}$ & $3.106 t-2 b$ & 1630 \\
\hline 1101.4 & $4107 k$ & $1 .: 3>t-u$ & $3.0 \cup \downarrow 1-\cup s$ & 1632 \\
\hline 111.4 & 20820 & 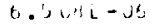 & 1. setid-0b & 1631 \\
\hline 112.4 & 11401 & $3.5631-36$ & $0.5511-60$ & 1635 \\
\hline $11 \div 4$ & $46: 5$ & $1.41+1-16$ & $1.424 t-00$ & 1637 \\
\hline $11 \%$. & $1 \times 77$ & $r_{1} \ldots 6 L L-67$ & $1.4 i J t-06$ & 1636 \\
\hline 111.3 & 276 & $\therefore .4: \Delta 1-u b$ & c. 62 of -07 & 1640 \\
\hline 111.3 & 33 & $1 .(i 5,6)-C)$ & $\therefore 2 s s t-0 c$ & 1041 \\
\hline 117.3 & 5 & $1,\{, y k-04$ & $4.075 \mathrm{E}-0 \%$ & 1643 \\
\hline $11 \times 2$ & $\therefore$ & c. & u. & 1644 \\
\hline
\end{tabular}

1200

1200

1200

1200

1200

1200

1200
(RISS.IIIT INTEGRATEQ $=4.964 E-03 \quad 1.191 E-02$

IEST UE
FLUIRESCEIN RFLEASE FRUY ELFVATIUN UF 16,1908 CII

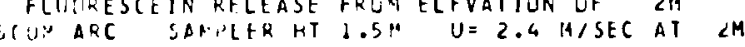

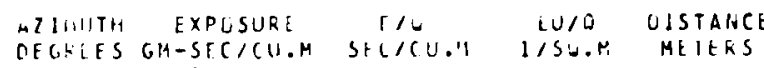
$\times 10 E+6$

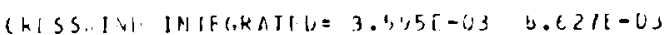


TEST USE

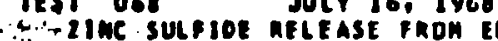

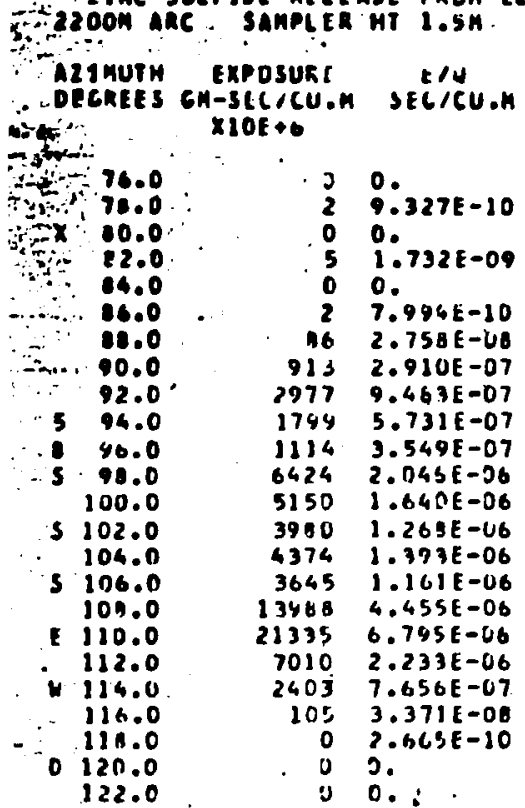

CRUSSHIND INTEGRATFO= 1. RA PE-O3 SICISU.H
O310 I0 0340 kST Fratiun DF $26 \mathrm{~K}$ 4.7.ABSEC AT $26 \mathrm{H}$ EU/O DISIAHCE
IISG OH RETEKS 0. 4.304E-09 0. $0.142 t-09$ 0. $3.757 E-04$ $1.296 E-07$ $1.360 \mathrm{E}-06$ $4.457 E-06$ $2.693 \mathrm{E}-06$ 1.66 dE-06 $9.616 \mathrm{E}-06$ 7.71 UE- 46 $5.950 E-06$ $6.54 \square E-06$ $5.45 \% E-06$ $2.094 E-03$ $3.144 E-25$ $3.590 \mathrm{E}-06$ $1.5+4[-07$ $1.252 \mathrm{~L}-09$ 0. $8.634 E-03$ $1 / \mathrm{H}$

2200
2200
2200
2200
2200
2200
2200
2200
2200
2200
2200
2200
2200
2200
2200
2200
2200
2200
2200
2200
2200
2200
2200
2200

2200
TEST U60 JULY I1". 1968 0310 TU 034J DSI ZIIIC SULFIOE RFLCASE FROM CLFVATIUN GI 264

32004 ARC SAPPLER II $1.5 \mathrm{H}$ U. 4.7 H/SEC AI $26 \mathrm{M}$

$$
\begin{aligned}
& \text { AIJMUTH EXPOSURE T/O EU/O DISTANCE } \\
& \text { DEGREES CM-SFCICU.M SICICU.M IISU.M RETERS } \\
& \times 10 E+6
\end{aligned}
$$

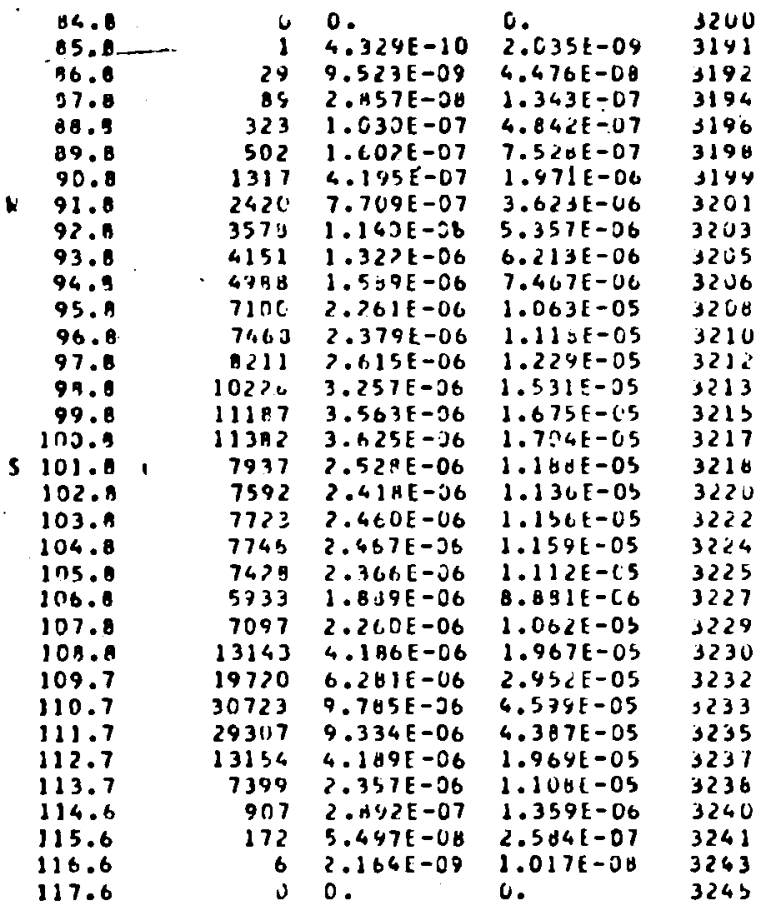

CROSSWIND INTEGRATILE $4.24 \mathrm{BE}-03 \quad 1.996 \mathrm{GE}-02$

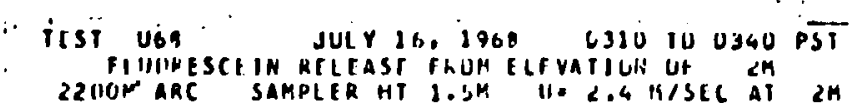

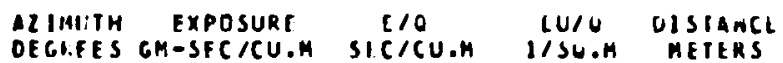
OEGI.FES GM-SFC $/ C O$
$X I O E+H$,

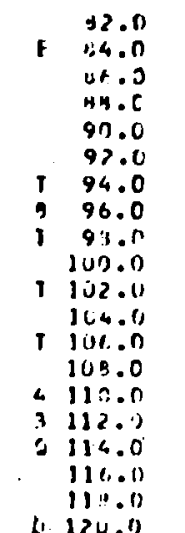

$$
\begin{array}{rl}
6 & 2.055 E-09 \\
55 & 1.742 E-06 \\
531 & 1.605 E-07 \\
3224 & 1.009 L-06 \\
6195 & 2.655 E-06 \\
4873 & 2.773 E-06 \\
6796 & 2.124 E-06 \\
3983 & 1.265 E-06 \\
4552 & 1.4231-06 \\
36 C 3 & 1.126 E-06 \\
3224 & 1.001 E-06 \\
4552 & 1.423 E-06 \\
7362 & 2.3011-06 \\
25175 & 7.867 E-06 \\
15104 & 4.7205-06 \\
4268 & 1.334 E-06 \\
287 & 6.493 E-06 \\
11 & 3.523 E-04 \\
6 & 2.0551-01 \\
2 & 9.1245-10
\end{array}
$$

\section{$4.4921-04$} 4. IGUE-OS $3.935 E-5.7$ $2.414 E-06$ $6.372 \mathrm{E}-06$ C.C55:-06 $5.0471-06$ $2.9481-06$ $3.415 \mathrm{E}-06$ $2.7035-46$ $2.4101-06$ $3.4151-06$ $5.5221-06$ $1.888 \mathrm{E}-\mathrm{CS}$ $1.1\lrcorner 3 L-\cup 5$ $3.2 U 1 E-U 6$ $2.150 \mathrm{E}-07$ $0.455[-c y$ $4.412(-6)$ CRLSSSIND INTEGRATHO. $2.4035-U 3$ 5.766E-03 2200
2200
2200
2200
2200
2200
2260
2200
2200
2200
2200
2200
2200
2200
2200
2200
2200
2200
2200
2200 - SEC/SG.l1

$1 / M$ FLULIRESCE IN RELEASE FKIIM CLPVATILR UF $2 M$ 320OP: ARC SAMPLER IIT $1.5 \mathrm{SM} 11.2 .4$ MISEC AT $2 \mathrm{H}$

\begin{tabular}{|c|c|c|}
\hline ه2. . & 0 & 0 . \\
\hline H. & i) & $1 . n 01 E-16$ \\
\hline A4. B & so & $1.376 E-00$ \\
\hline d5.g & 176 & $5.44 A[-J 8$ \\
\hline 76.3 & 347 & $1.242 E-67$ \\
\hline 87.8 & 1369 & $4.280 E-47$ \\
\hline$\because \ldots$ & $24 A 1$ & $7.758, E-67$ \\
\hline 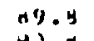 & $\cos 3$ & A. $300,-67$ \\
\hline H.1. & 4367 & $1.427 E-U C$ \\
\hline 91.8 & 5540 & $! .750: t-36$ \\
\hline$y i .4$ & $490 !$ & $1.533 E-U 6$ \\
\hline ys. त & 5024 & $1.820 E-06$ \\
\hline $94 . i$ & 3021 & $1.196 t-06$ \\
\hline 45.11 & 4231 & $1.3<26-66$ \\
\hline 9e. $\mathrm{H}$ & 3356 & $1.044[-06$ \\
\hline 97.8 & 3356 & $1.1149[-v 6$ \\
\hline $4 \cdot .4$ & 4433 & $\therefore 305 t-06$ \\
\hline 49.11 & 3284 & $1.02 \in \varepsilon-06$ \\
\hline 100.0 & 3154 & $4.050[-07$ \\
\hline 101.4 & 2603 & A. SEQtE-U7 \\
\hline lण?.3 & 3191 & $1.091 E-65$ \\
\hline $1,3.4$ & 4366 & $1.364 E-06$ \\
\hline $1 \div 4.5$ & 4633 & $1.3+5 E-: 6$ \\
\hline 1.15 .8 & 4500 & $1.40<E-06$ \\
\hline 1.6 .8 & $t<72$ & $1.960 E-06$ \\
\hline 167.8 & 10025 & $3.133 E-06$ \\
\hline 10.1 .8 & 11967 & $3.740 \mathrm{E}-06$ \\
\hline 140.7 & 17146 & $5.35 \mathrm{Ht}-J 6$ \\
\hline 110.7 & 15527 & 4.852 \\
\hline 111.7 & 8291 & $2.3 y 1 E-0 t$ \\
\hline 112.7 & 2885 & $9.017 E-67$ \\
\hline 113.1 & 860 & $2.7 \cup B\{-07$ \\
\hline 114.6 & 76 & $2.404 E-08$ \\
\hline 115.6 & 16 & $3.074 \mathrm{E}-09$ \\
\hline 110.6 & l & \\
\hline
\end{tabular}
A21411TB EXPOSURL " I/U EU/O DISIARCE DHGEES GM-SEC/CU.M SECICU.M. IISU.M METEKS $X I O E+6$

0. $2.404 r-10 \quad 3187$ $1.300 t-573191$ 2.9U2E-J7 1192 $1.027 E-06 \quad 3194$ $1.8615-06 \quad 3196$ 2.013E-06 3148 $3.420 E=06 \cdot 3199$ $4.22 J E-06 \quad 9201$ $3.67 \mathrm{dt}-36 \quad 3203$ $4.360 E-U 6 \quad 3205$ $3.114 E-06 \quad 3200$ $2.517 E-46 \quad 3210$ 2.517t-LG 3212 3.325t-vo 9213 2.467E-06 3213 $2.300[-06 \quad 3217$ $2.013 t-06$ s210 $2.610[-06 \quad j 220$ $3.275[-06 \quad 3224$ $3.3255-06 \quad 32<4$ $3.375 F-06 \quad 3225$ $4.7051-06 \quad 3227$ 7.31JE-06 3229 $6.976 E-U S \quad 3230$ $1.236 \mathrm{E}-\mathrm{US} \quad 1232$ $1.165 \mathrm{E}-05 \quad 3233$ $6.21 y E-06 \quad 1235$ 2.164t-06 3237 $6.45 Y E-07 \quad 3238$ $5.764 E-08 \quad 3240$ $1.21 \forall \mathrm{E}-08 \quad 3241$ $u$. 3243 


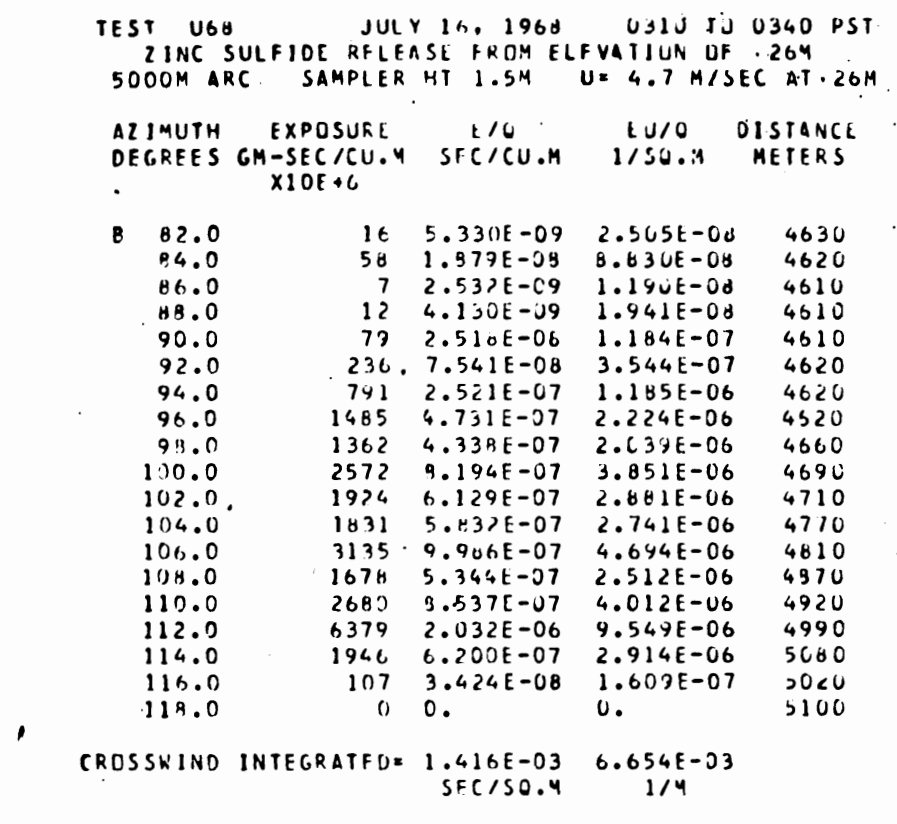

TEST U6B JULY 16.1969 0310 TO 0340 PST 7.INC SULFIDE RFLEASE FROY ELFVATILN LF $26 \mathrm{M}$ $70 O D M A R C$ SAMPLER $4 T 1.5 \mathrm{M} U=4.7 \mathrm{Y} / \mathrm{SEC}$ AT $26 \mathrm{M}$ $\begin{array}{cccc}\text { AZIMUTH EXPESURE } & \text { EIO } & \text { EU/O DISTAIOCE } \\ \text { DEC,FEES GM-SECICU.M SFCICU.M } & \text { I/SJ.M METERS } \\ & \text { XIOE+6 } & & \end{array}$

\begin{tabular}{|c|c|c|c|}
\hline \multirow[t]{17}{*}{ B } & 76.0 & 71 & $2.27 \times E-08$ \\
\hline & 78.0 & 25 & $8.261 \varepsilon-09$ \\
\hline & 80.0 & 56 & $1.749 E-08$ \\
\hline & 82.0 & B9 & $2.525 E-58$ \\
\hline & 84.0 & $4 !$ & $1.49>E-38$ \\
\hline & HA. $O$ & 179 & $5.716 E-0 R$ \\
\hline & \&R. 0 & 190 & $6.076 E-08$ \\
\hline & 90.0 & 283 & $9.034 \mathrm{E}-U \mathrm{~B}$ \\
\hline & 92.0 & 467 & $1.49 B E-07$ \\
\hline & 94.0 & 794 & $2.5>9 E-37$ \\
\hline & 96.0 & 894 & ᄀ. $\mathrm{H} 47 E-07$. \\
\hline & $9 A .0$ & 1714 & $5.461[-07$ \\
\hline & 100.0 & $28 \mathrm{BS}$ & $9.180 E-07$ \\
\hline & 102,0 & 2130 & C.:AGPE-07 \\
\hline & 104.0 & $20 \times 4$ & $0.1135 t-07$ \\
\hline & 106.0 & 1) 35 & $3.615 E-07$ \\
\hline & 160.0 & $130 ?$ & $4.149 E-07$ \\
\hline 5 & 110.0 & 567 & 1. $A C 3 E-47$ \\
\hline & 112.0 & 1077 & $3.431 E-07$ \\
\hline & 114.0 & 1720 & $5.4 .79[-07$ \\
\hline & 116.0 & 530 & $1 . t \cdot 6,4 t-07$ \\
\hline & $11 B .0$ & 21 & $6.7 \div 5 t-07$ \\
\hline & 120.0 & $\therefore$ & I'. \\
\hline
\end{tabular}

CRTISSAIND INTEGRATFD = $\begin{array}{cc}1.427 E-03 & 0.706 E-03 \\ \text { SFC/SU.M } & 1 / M\end{array}$

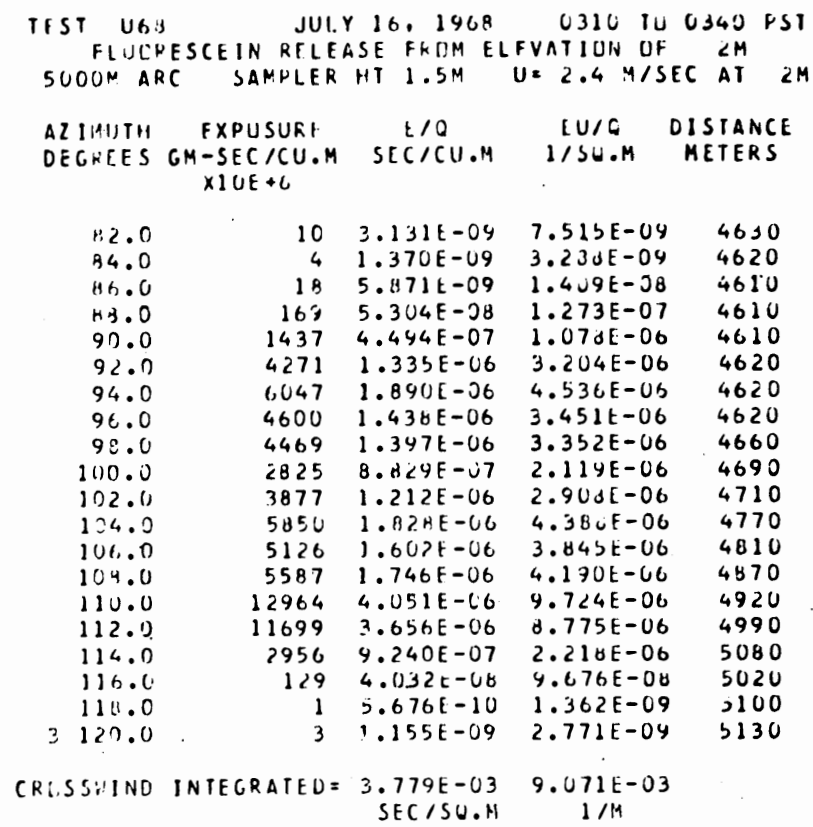

IFST IJGS JUIY 16.1908 CSIO TU 1343 PST
FLUMPESCEIN RELEASE FKLM ELFVATIUN UF $2 M$ FLUTPESCEIN RELEASE FKLM ELFVATIUN UF $2 M$
7CCIIM ARC SAMPIER HIT $1.5 M$ UE $2.4 \mathrm{M} / \mathrm{SEC}$ AT $2 \mathrm{M}$
AZILUTH EXPOSUPCE E/O EU/O DISTAINCE DEGPFES GM-SFC/CU.M SECICU.M IISO.M MEIERS XIOE +6
i. 76.0
$1 . .0$
50.0
यद. 11
$3 \times 4.0$
be. 0
is. 0
90.0
$4 \quad 92.0$
$91 . .1)$
96.1
$9 \times .0$
110.0
$11,2 \cdot 2$
104.0
$417,6.0$
313.0
$b 110.0$
4112.0
11400.
116.0

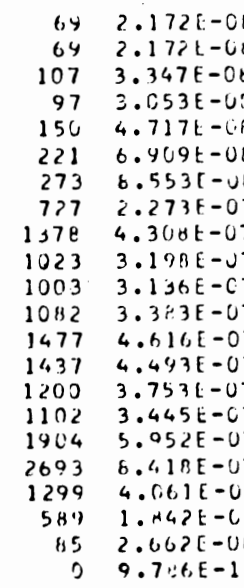

9. $2145-03$

$5.214 E-C H$

H. $03<t-08$

$7.32 \cup E-00$

1. $132 \mathrm{E}-U 7$

1. $050 t-01$

c. $053[-C 7$

\$. $430 L-07$

1. $034 E-06$

$7.675 E-07$

$7.527 t-07$

$8.114 E-07$

$1.108 E-C 6$

1. C 7 U $-0 C$

$9.007 E-07$

8. $2675-07$

1. $4 \angle 8 E-06$

2. $02 U E-C 6$

$2.02 U E-C 6$
$4.747 E-07$

$4.4<61-07$

6.3 BOE -0 d

$2.34+1-11$

CRISSHINO INTEGRATFU = $1.320 E-03 \quad 3.169 E-03$
6280

6300

6460

6490

6500

0500

6480

6500

6490

6500

6510

6520

6560

6610

6650

6720

6800

7000

7300

7210

7190

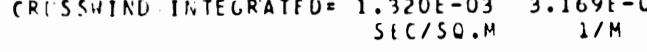




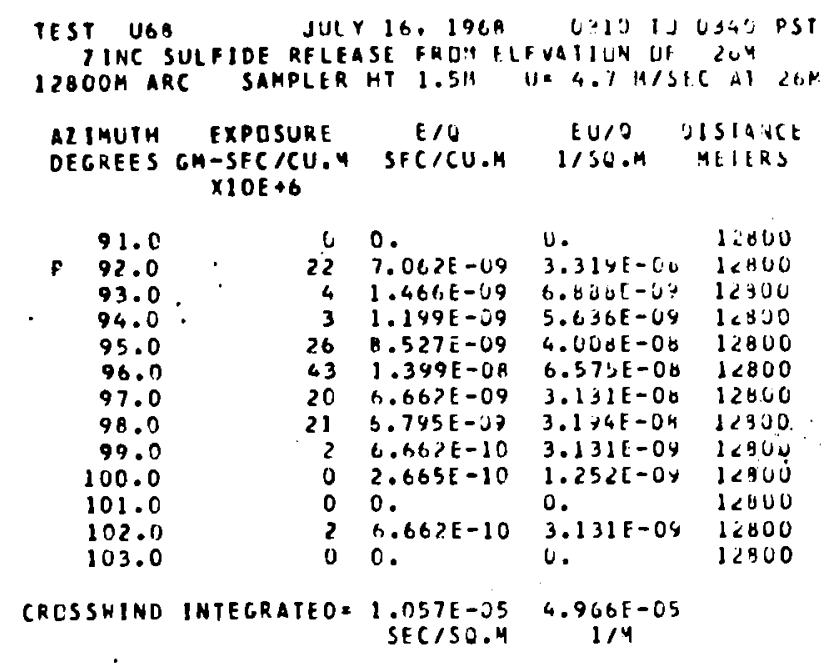

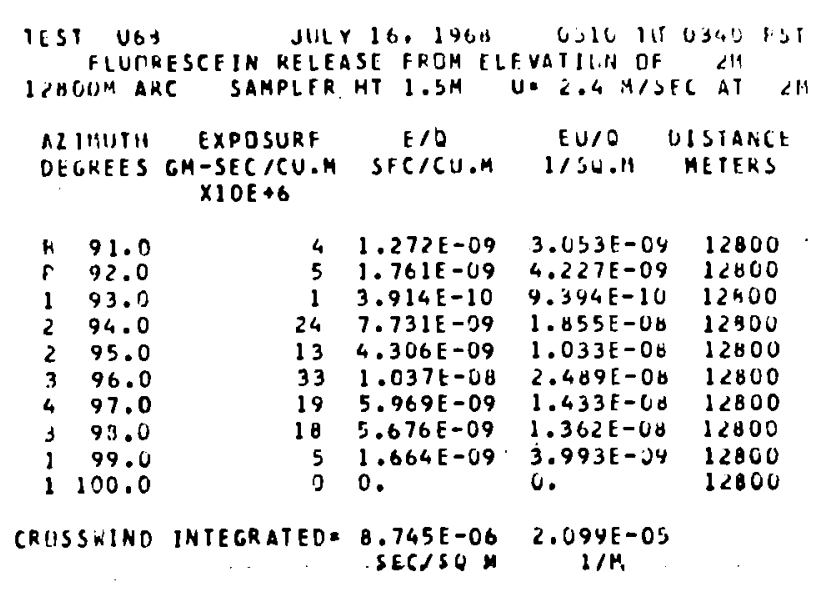


SAHPLING FRTM $400 M$ TO 12 BUOM. NO TOKEK SAKPLING. UNLY ZINC SULFIOE TRACER RELEASEU THIS DATE.

IKACF APPFARS CINTAINFD WITHIN SAYPLERS IO A DISTANCE DF SUUUH, DISTRIBUTIUN SKEHED STRUNGLY TD SDUTH.

TRUIICATED DISTRIHUTIOH GN 7OOJM ARC ANU SIGNIFICANT DUST UN 12 BUOH ARC MAKE IHESE AKC DATA OUESTIONABLE.

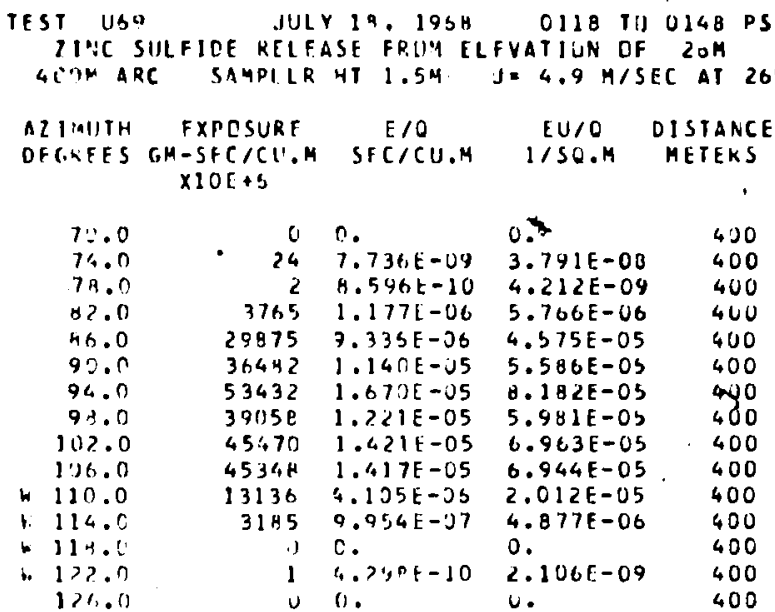

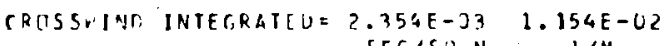

$$
\text { SECISO.: } 1 / 4
$$

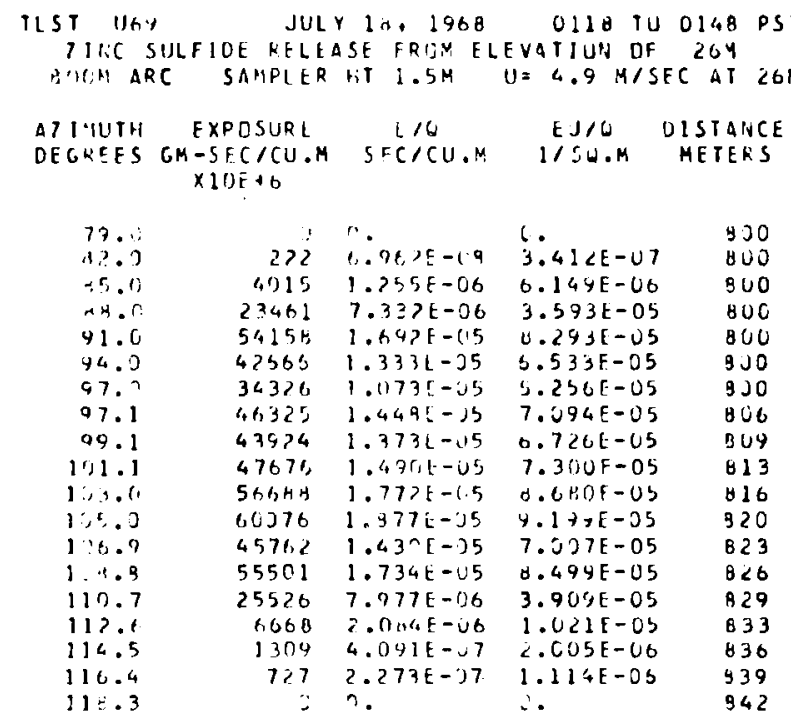

TEISSAINIT IATEGRATFD $=5.115>E-43 \quad 2.475 E-02$

SFCISO. I : $1 / \mathrm{Hi}$. 


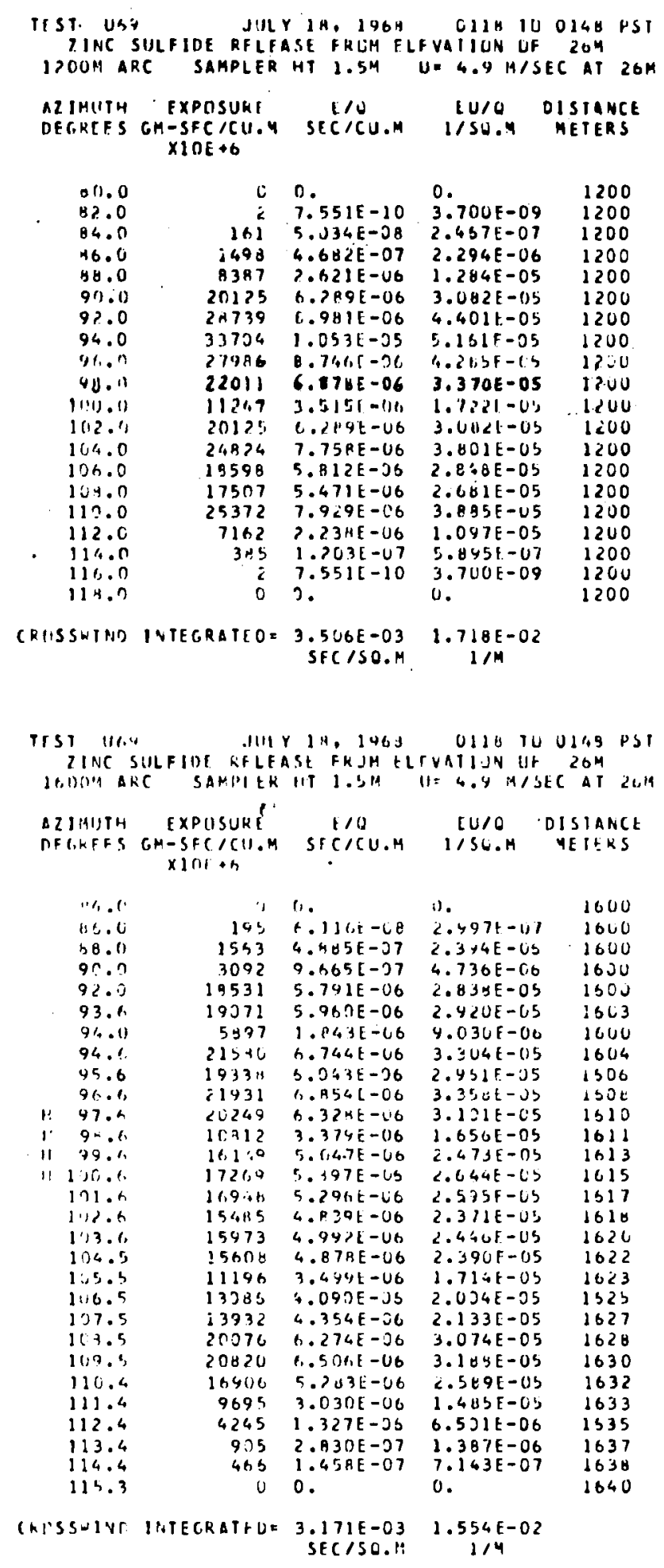




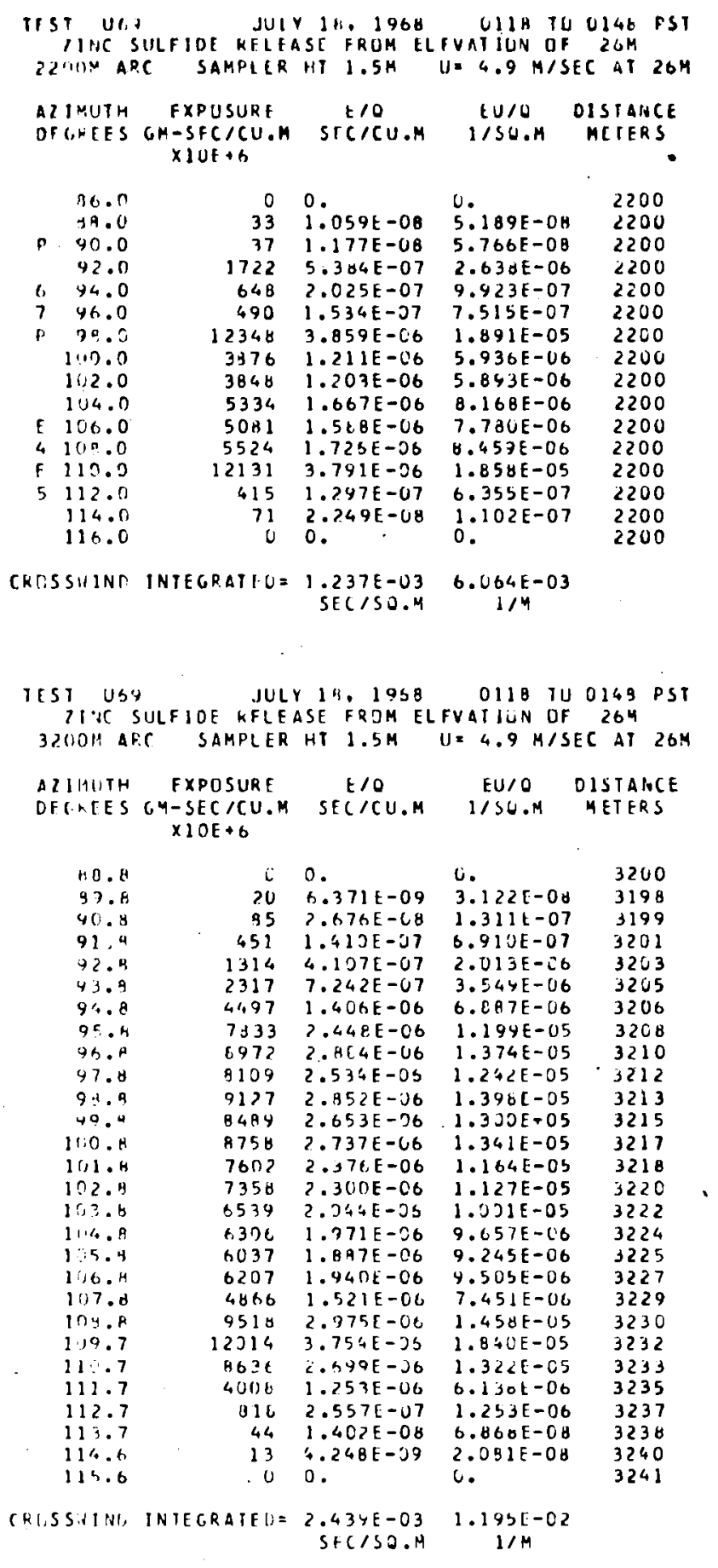




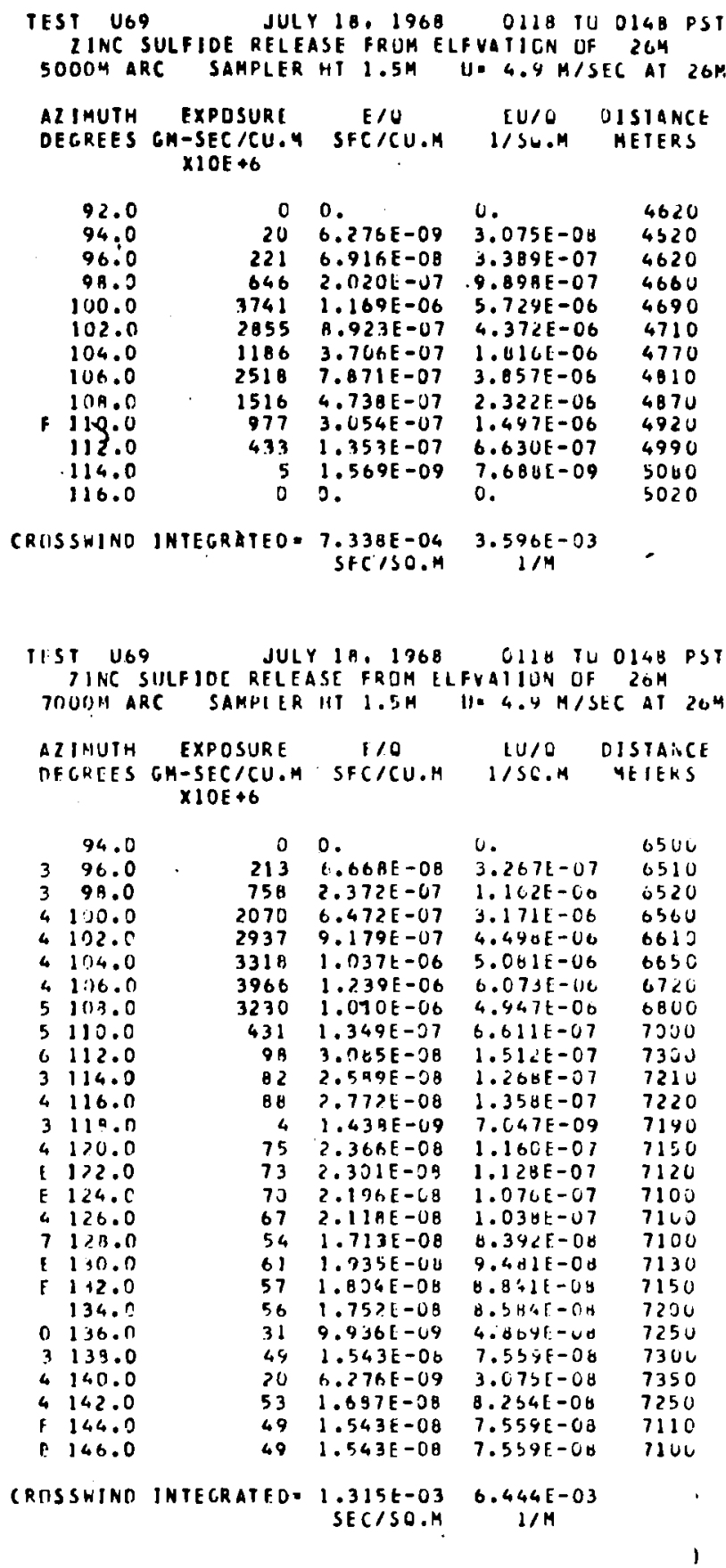




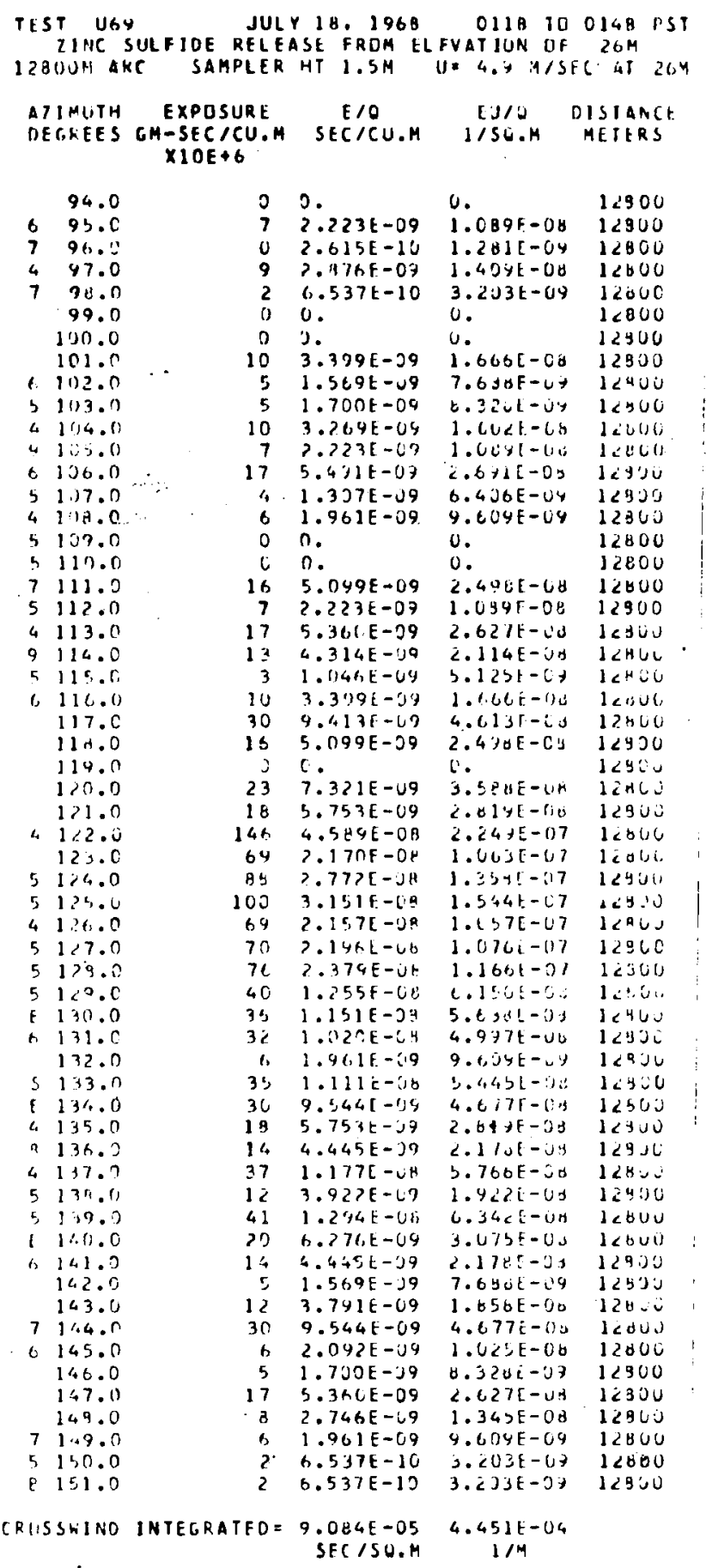


SAMPLINC $400 M$ TO $12000 M$. NO TOHER SAHPLING. DISTRIBUTIDN TENOS TOHARD BIMUDAL. ALL ARCS EXCEPT TOOOM EMRRACE CRESSHIND EXTENT DF BOTH TRACERS. THE SKEHING OF TOUOA DATA TO THE SOUTH IS NOT READILY EXPLAINABLE.

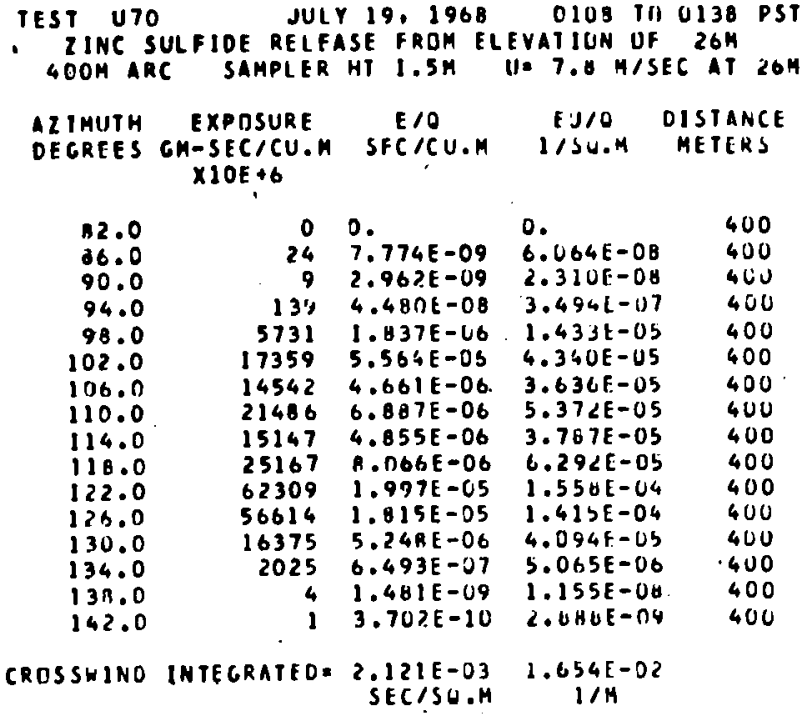

TEST U7O JULY 19, 1968 OTO TO 0138 PST IINC SULFIOE RELEASE FRUM ELEVATIUA DF 264 GOOH ARC SAMPLER HT $1: 5 \%$ UE $7.8 \mathrm{H} / \mathrm{SEC}$ AT $26 \mathrm{M}$

$\begin{array}{cccc}\text { AZIMUTH EXPOSURE } & \text { EIO } & \text { EU/O OISTANCE } \\ \text { DEGKEES GH-SECICU.M SECICU.M IISO.M METERS } \\ \text { XIOE+O }\end{array}$

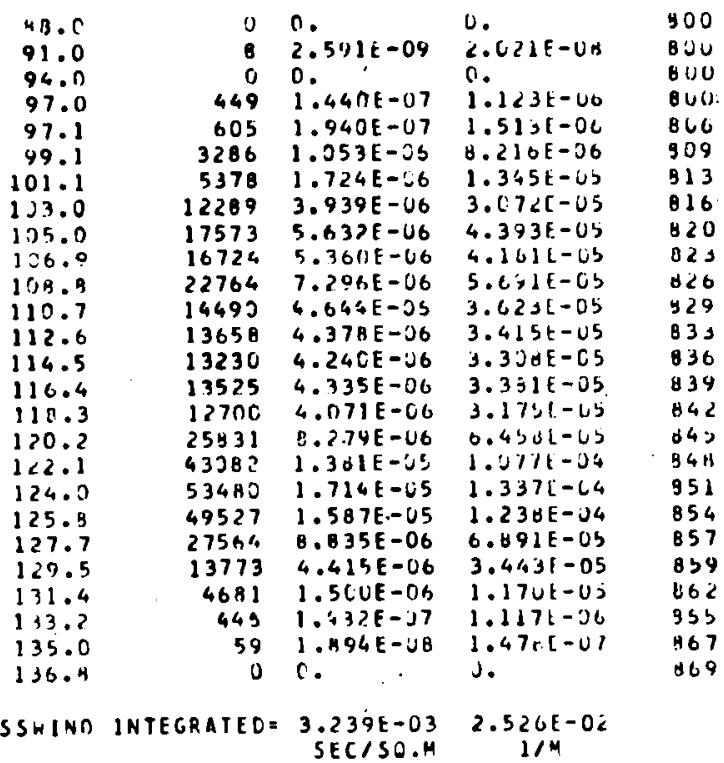

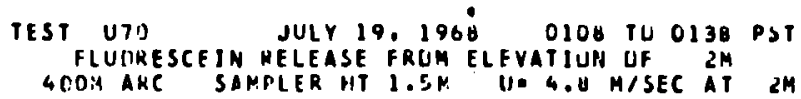
$\times 10 E+6$

90.0
94.0
98.0
102.0
106.0
110.0
114.0
111.0
122.0
126.0
1311.0
134.0
134.0
112.0
146.0
150.0

$\begin{array}{rl}U & 0 . \\ 1044 & 3.964 E-07 \\ 11778 & 4.314 E-06 \\ 39093 & 1.432 E-05 \\ 33228 & 1.217 E-05 \\ 26058 & 9.545 E-06 \\ 41700 & 1.528 E-05 \\ 80372 & 2.944 E-05 \\ 175964 & 6.446 E-05 \\ 156411 & 5.729 E-05 \\ 53432 & 1.957 E-05 \\ 2711 & 9.934 E-07 \\ 62 & 2.296 E-0 B \\ 114 & 5.147 E-09 \\ 16 & 5.939 E-09 \\ 7 & 2.771 E-09\end{array}$

0 .

$1.055 E-06$ $2.071 E-05$ $6.874 \mathrm{E}-05$

$5.842 E-05$ $4.582 E-05$ $7.332 E-05$ $1.413 E-04$ $3.094 \mathrm{E}-04$ $2.750 t-04$ $9.395 E-O S$ 4. $768 \mathrm{E}=06$ $4.768 E-06$
$1.102 E-07$ $2.471 E-0$ 2. $851 E-08$

$1.330 E-03$

$$
\begin{aligned}
& 400 \\
& 400 \\
& 400 \\
& 400 \\
& 400 \\
& 400 \\
& 400 \\
& 400 \\
& 400 \\
& 400 \\
& 400 \\
& 400 \\
& 400 \\
& 400 \\
& 400 \\
& 400
\end{aligned}
$$

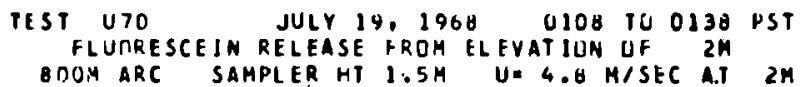

AZJIUTH EXPQSURE E/O EU/O DISTANCE OECPEES CH-SECICU.M SECICU.H IISU.H. METERS $\times 10 \mathrm{E}+\mathrm{C}$

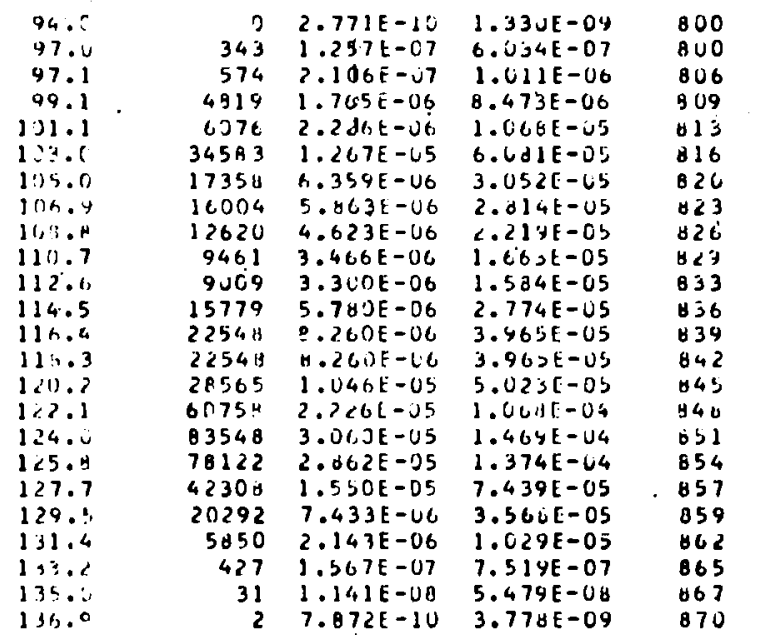

CRUSSAINL JNTEGRATED $4.989 E-03$ 2.395E-02 


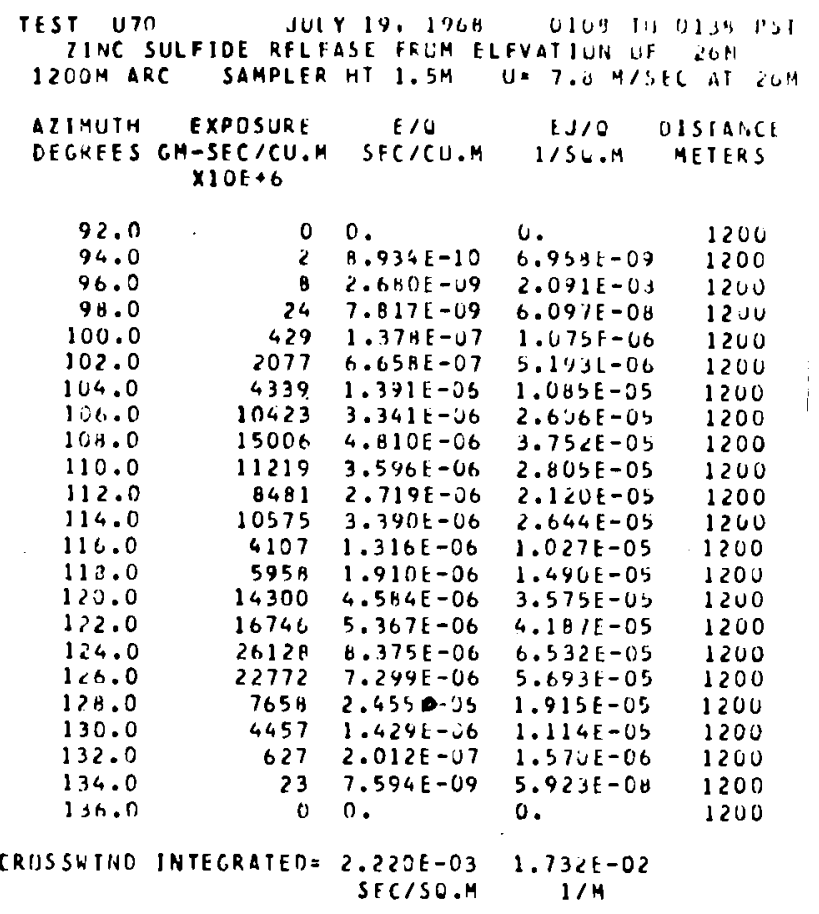
IFSI 117 17) JULY $19,196 \mathrm{H}$ FLUUINESCFIN RELEASL FRDM CLFVATIUN TU 0138 Hb $1200 M$ ARC SAHPLER HT $1.5 M$ UE 4.6 M/SEC AT $2 M$

\begin{tabular}{|c|c|c|c|c|}
\hline $\begin{array}{l}\text { AZ JWUUTH } \\
\text { DEGKEES }\end{array}$ & $\begin{array}{c}\text { EXPQSURE } \\
\text { CH-SEC /CU.H } \\
\times 1 O E+6\end{array}$ & $\begin{array}{c}E / O \\
S E C / C U . H\end{array}$ & $\begin{array}{l}\text { CU/O } \\
1 / S O E . H\end{array}$ & $\begin{array}{c}\text { DISIANCE } \\
\text { NETERS }\end{array}$ \\
\hline
\end{tabular}

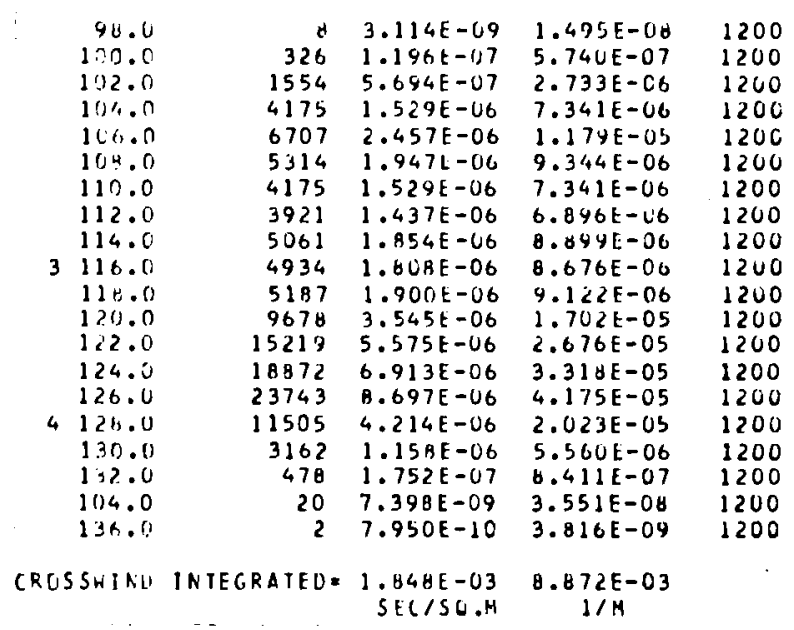

IEST U70 JULY 19, 1968 O1U8 TU 0136 PST 2INC SULFIDE RELEASE FROM ELFVATILN OF 264 $160 J M$ ARC SAMPLER HI 1.5M W. $7.6 \mathrm{M} / \mathrm{SEC}$ AT $26 \mathrm{M}$ AZIMITH EXPOSURT E/Q LJIO DISTANCL DEGREES GH-SCC/CU,H SECICU,H I/SE.M MEILKS X]OE +6

\begin{tabular}{|c|c|c|c|c|}
\hline $\begin{array}{l}92.0 \\
93.5\end{array}$ & $\begin{array}{l}1 \\
3\end{array}$ & $\begin{array}{l}4.467 E-10 \\
1.21 \mathrm{BE}-09\end{array}$ & $\begin{array}{l}3.454 t-09 \\
9.502 F-C 9\end{array}$ & $\begin{array}{l}1600 \\
1603\end{array}$ \\
\hline $9 \cdot .0$ & 10 & $3.350[-03$ & $2.013 t-03$ & 1600 \\
\hline 94.6 & $3 y$ & $1.218 \mathrm{E}-0 \mathrm{~B}$ & $4.5 \cup 2 L-01$ & 1604 \\
\hline 95.6 & $u$ & 0. & 0. & 1606 \\
\hline 46.6 & 0 & 0. & u. & $1 S 68$ \\
\hline 97.6 & 45 & $1.462 E-08$ & $1.14 \cup E-0 \%$ & 1610 \\
\hline 98.6 & 0 & 0. & c. & 1611 \\
\hline 99.6 & 0 & 0. & 0 . & 1613 \\
\hline 100.6 & • & 0 & 0. & 1615 \\
\hline 101.6 & 93 & $2.985 E-08$ & $2.320 \mathrm{t}-07$ & 1617 \\
\hline 102.6 & 269 & 3.6 & -07 & 1510 \\
\hline $1 \sim 3.6$ & 271 & $8.710 t-30$ & $6.7 \%+1-\cup 7$ & 1520 \\
\hline 194.5 & 1347 & 4.31 HE -07 & $3.360 t-06$ & 1622 \\
\hline 1115.5 & 2392 & $7.66 \mathrm{HL}-07$ & $5.981[-00$ & 1623 \\
\hline $\begin{array}{l}106.5 \\
107.5\end{array}$ & $\begin{array}{l}5441 \\
8061\end{array}$ & $\begin{array}{l}1.872 E-06 \\
2.340 \mathrm{E}-06\end{array}$ & $\begin{array}{l}1.4601-05 \\
2.213 t-05\end{array}$ & $\begin{array}{l}1625 \\
1627\end{array}$ \\
\hline 100.5 & 12122 & $3.805 E-06$ & $3.031 t-05$ & 1528 \\
\hline 134.5 & 7947 & $2.547 \mathrm{~L}-06$ & $1.487 \mathrm{~F}-U \mathrm{~b}$ & 1530 \\
\hline 110.4 & $55 A 3$ & $1.79 J \mathrm{E}-06$ & $1.390 E-05$ & 1632 \\
\hline 111.4 & 8274 & 2.6328 & 2.00 & 1633 \\
\hline 112.4 & 9423 & $3.1 \times 1[-46$ & $2.4815-03$ & 1615 \\
\hline $1 \cap 3.4$ & 7604 & $2.5 C 2 E-06$ & $1.951 k-u s$ & 1637 \\
\hline 114.4 & 4311 & $1.35 ?[-35$ & 1.07 SE -05 & 1538 \\
\hline 115.3 & 3707 & $1.1 \mathrm{~b}$ il $-0 \mathrm{O}$ & $4.20 \times 1-06$ & 1640 \\
\hline 116.3 & 3610 & 1.157 & $9.021 E-U U$ & 1641 \\
\hline 117.3 & 4511 & $1.44 h E-06$ & $1.1205-05$ & 1643 \\
\hline 116.2 & 5837 & $1.871 E-06$ & 1.43 y -03 & 1644 \\
\hline 119.2 & 6316 & $2.025 t-66$ & $1.57 \times E-05$ & 1646 \\
\hline $1<0.2$ & 7723 & $2.475 E-33$ & $1.931 t-35$ & 1347 \\
\hline 121.2 & B884 & $2 . B 4 A E-16$ & $2.221 E-05$ & 1349 \\
\hline $1<2.1$ & 11712 & $3.754 E-06$ & $2.9 \angle 0 E-05$ & 1650 \\
\hline 123.1 & 13089 & $4.195 E-06$ & $3.272 \mathrm{E}-0 \mathrm{~s}$ & 1652 \\
\hline 124.1 & 12916 & $4.141 E-06$ & $3.23 \cup E-05$ & 1653 \\
\hline 125.0 & 8511 & $2.72 \mathrm{BE}-06$ & $2.12 \mathrm{UE}-05$ & 1655 \\
\hline 126.0 & 12818 & $4.10 B E-36$ & $3.2 J 5 E-05$ & 1556 \\
\hline 126.9 & 13764 & $4.412 E-06$ & $3.441 \mathrm{E}-05$ & 1658 \\
\hline $1<7.9$ & 9593 & $3.075 E-06$ & $2.39 \square E-05$ & 1659 \\
\hline $\begin{array}{l}128.6 \\
179.7\end{array}$ & $\begin{array}{l}3833 \\
1940\end{array}$ & $\begin{array}{l}1.229 E-06 \\
6.219 E-07\end{array}$ & $\begin{array}{l}4.593 E-06 \\
4.851 E-06\end{array}$ & $\begin{array}{l}1600 \\
1562\end{array}$ \\
\hline
\end{tabular}

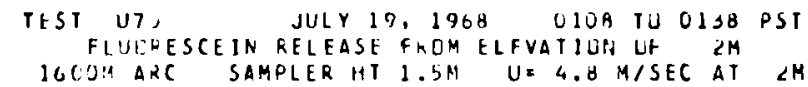

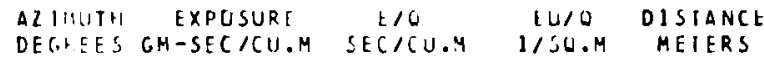
$\times 10 \mathrm{OE}+6$

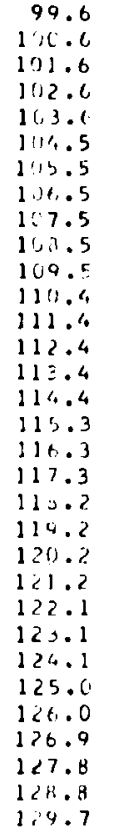

0.

$\begin{array}{ll}0 . & 1600 \\ 1.7 .03 E-08 & 1615 \\ 2.454 E-07 & 1617 \\ 6.85 U E-07 & 1618 \\ 0.103 E-07 & 1620 \\ 3.477 E-00 & 1622 \\ 3.413 L-06 & 1623 \\ 1.045 E-U S & 1625 \\ 1.072 E-0 S & 1647 \\ 1.307 E-05 & 1626 \\ 6.093 E-06 & 1630 \\ 4.523 E-0 C & 1632 \\ 6.442 E-06 & 1639 \\ 7.837 E-06 & 1635 \\ 6.7911-06 & 1637 \\ 3.913 E-06 & 1638 \\ 4.610 E-06 & 1640 \\ 6.267 E-06 & 1641 \\ 8.361 E-06 & 1543 \\ 7.576 E-06 & 1644 \\ 6.361 E-06 & 1646 \\ 7.314 E-06 & 1647 \\ 7.052 E-06 & 1644 \\ 1.404 E-05 & 1650 \\ 1.359 E-05 & 1652 \\ 1.673 E-05 & 1653 \\ 1.124 E-05 & 1655 \\ 2.274 E-05 & 1656 \\ 2.093 E-05 & 1658 \\ 1.072 E-05 & 1659 \\ 3.913 E-06 & 1660 \\ 2.527 E-06 & 1662\end{array}$




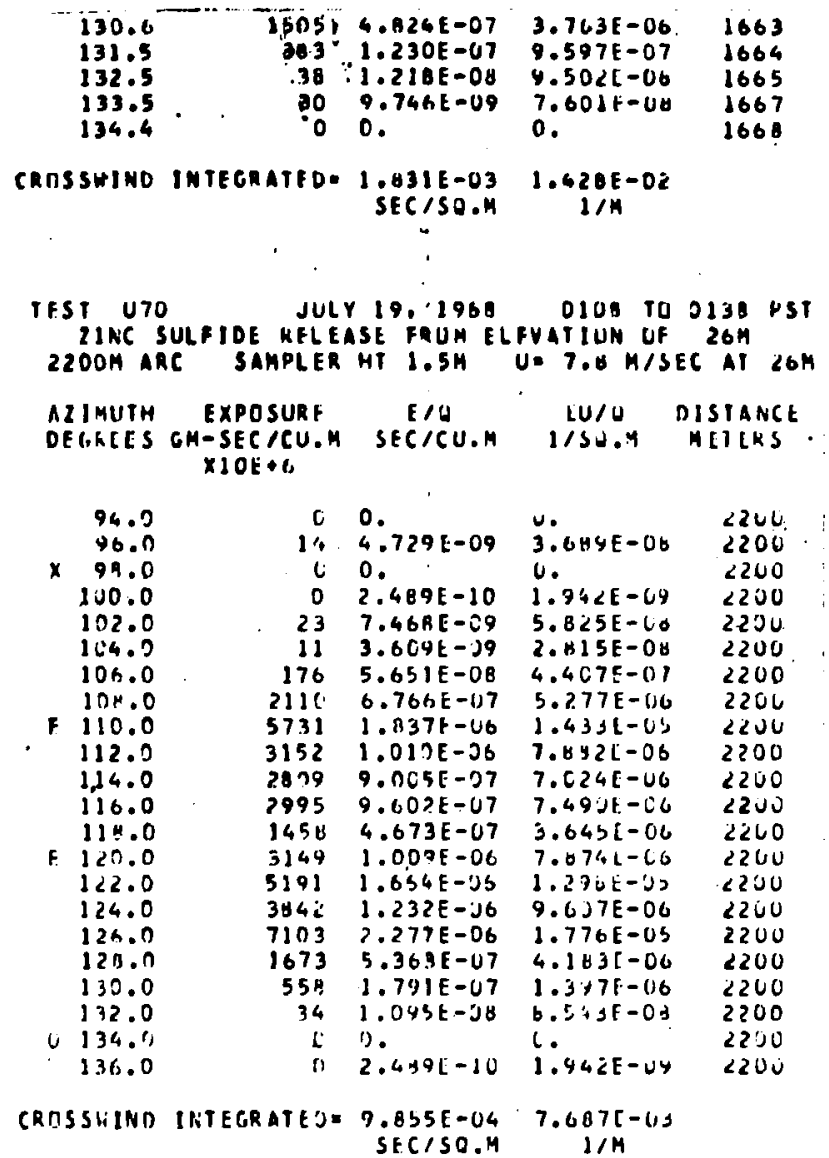

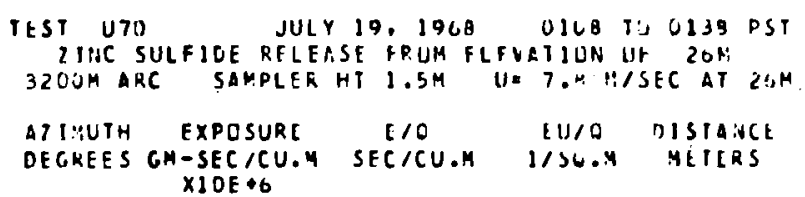

(RISS.IINR INTEGRATFU) $=1.737 t-U 3 \quad 1.3 Y J F-02$

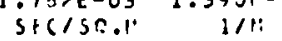

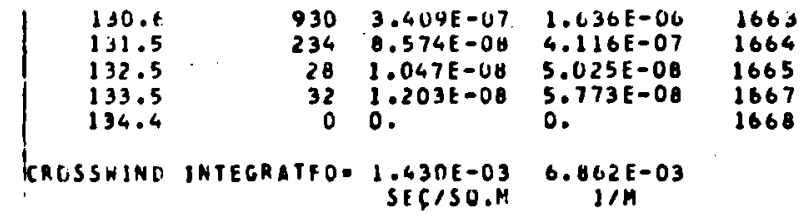

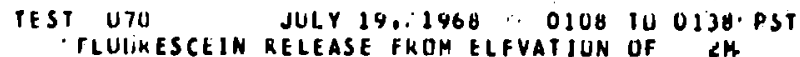

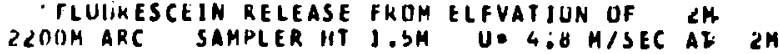

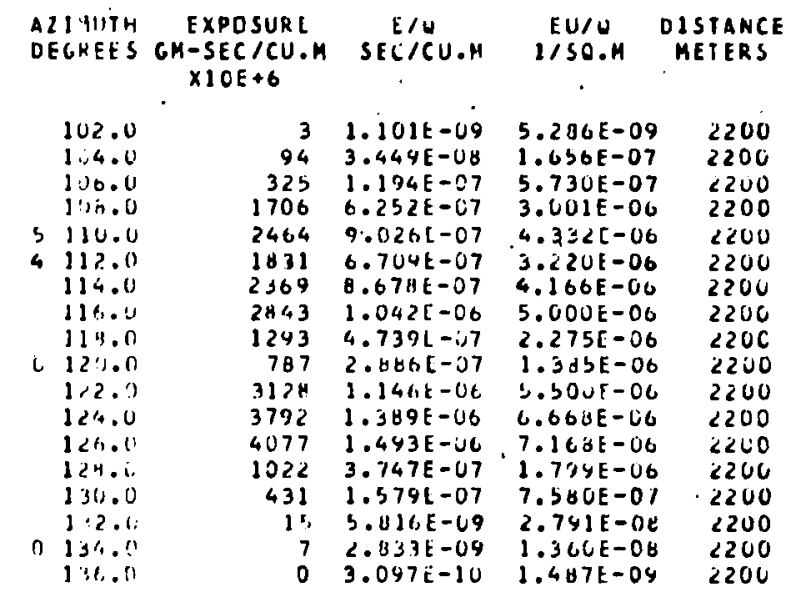

CRLISSRIVI: INTEGRATEUE $7.369 t-043.537 t-03$ IEST U70) JULY 19,1908 CIUO TO 0138 PST
FLULAESCEIN RELEASE FRUM ELFVATION OF $2 \mathrm{M}$ $321, \mathcal{}$ " ARC SAMPLER HT $1.3 \mathrm{BH} U=4.8 \mathrm{M} / \mathrm{SEC}$ AT $2 \mathrm{M}$

A7.IEUTH EXPOSURF E/U EU/O OISTANCE DEG,EES GM-SECICU.M SECICU.M IISA.M METEKS $\times 10 E+6$

\begin{tabular}{|c|c|c|c|c|}
\hline 13.9 & 0 & 0. & u. & 3200 \\
\hline $1,4.6$ & 0 & 0. & u. & \\
\hline $1:$ : & 3 & $1.6148-09$ & $6.70<t-09$ & 3225 \\
\hline $1.16 .:$ & 1 & $4.695 E-10$ & $2.254 E-09$ & 3227 \\
\hline 17.8 & 254 & $9.313 E-68$ & $4.476 E=07$ & 3229 \\
\hline $1 \therefore . n$ & 906 & $3.322 E-67$ & $1.595 E-06$ & 3230 \\
\hline $1,0.1$ & 1064 & $0.116 L-37$ & $2.936 E-06$ & $3<32$ \\
\hline 113.7 & 2477 & $9.074 E-07$ & $4.356 E-06$ & 1233 \\
\hline 111.7 & 3150 & $1.154 k-06$ & $5.53 \times E-60$ & 3235 \\
\hline 112.7 & 3890 & $1.425 \mathrm{E}-06$ & $6.840 E=06$ & 1237 \\
\hline 113.7 & 3957 & $1.450 \mathrm{E}-06$ & $6.954 E-06$ & 3238 \\
\hline 114.1 & 4832 & $1.770 E-06$ & $8.497 E-06$ & 3240 \\
\hline 115.6 & 3015 & $1.1050-00$ & $5.302 t-00$ & 3241 \\
\hline $11 \cdot .6$ & 1471 & $6.655[-07$ & $3.281 E-06$ & 3243 \\
\hline 117.6 & 1288 & $4.719 E-07$ & $2.265 E-06$ & $\$ 245$ \\
\hline $11+\ldots$ & 1131 & $4.144[-07$ & $1.989 E-06$ & 3246 \\
\hline 119.5 & 1736 & $6.36 ? E-C 7$ & $3.054 E-06$ & 3246 \\
\hline 12.1 .5 & 2544 & $9.321 E-67$ & $4.474 E=06$ & 2249 \\
\hline 121.9 & 3352. & $1.22 \mathrm{HE}-06$ & $5.894 E-06$ & 3251 \\
\hline $1<2.4$ & 3234 & $1.203 E-06$ & $3.775 \mathrm{E}-06$ & 3252 \\
\hline $12 \div .4$ & 4159 & $1.524 E-16$ & $7.314 \mathrm{~F}-06$ & 3253 \\
\hline $1,4.4$ & 3621 & $1.326 E-06$ & $0.367[-06$ & 1255 \\
\hline $1.1, .4$ & 3023 & $1.4 \cup 0 E-36$ & $6.722 E-06$ & 3256 \\
\hline 124.3 & 2544 & $9.321 E-07$ & $4.474 E-06$ & 3258 \\
\hline 1.1 .3 & 1041 & 3.A15E-U7 & $1.831 E-06$ & 3259 \\
\hline $1<e^{3} 3$ & 339 & $1.244 E-07$ & $5.973 E-07$ & 3261 \\
\hline $1 / 0.3$ & 73 & $2.700 F-08$ & $1.296 E-07$ & 3262 \\
\hline $1 \% 1.3$ & 14 & $5.478 t-09$ & $2.624 E-08$ & 3263 \\
\hline 1.1 .2 & 1 & $7.043 \mathrm{E}-10$ & $3.381 E-04$ & 3264 \\
\hline 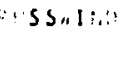 & & $\begin{array}{r}1.113 E-03 \\
\text { SEC/SO.M }\end{array}$ & $\begin{array}{c}5.341 E-03 \\
1 / M\end{array}$ & \\
\hline
\end{tabular}




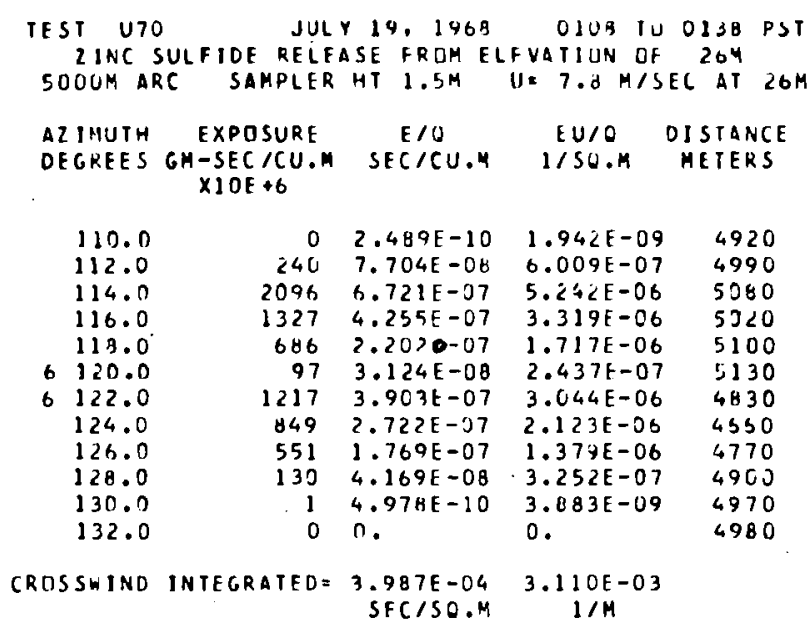

TEST U7O JULY 19, 1968 OLOB TU 0138 PST 2 INC SULFIDE RFLEASE FROM ELEVATION LF 264 $7000 M$ ARC SAMPLER HT $1.5 M \quad U=7$. U M/SEC AT $26 \mathrm{~m}$ ATIMUTH EXPOSURE E/O LU/G DISIATCE DEGKFES GM-SEC/CU.M SECICU.M WSAM METERS $\times 1 O E+S$

\begin{tabular}{|c|c|c|c|c|c|}
\hline & A 6.0 & $\therefore$ & 0. & u. & .500 \\
\hline 4 & 38.0 & e & $2.314 E=09$ & $2.015[-.0 \mathrm{~d}$ & 6400 \\
\hline 4 & 90.0 & 13 & $4.232 \mathrm{E}-09$ & $3.301 \mathrm{~F}-00$ & osuo \\
\hline 4 & 92.0 & 50 & $1.61+\mathrm{E}-U \mathrm{~B}$ & $1.2<<t-07$ & 6640 \\
\hline 4 & 94.0 & 21 & $3.975 E-09$ & $5.436 E-39$ & 5500 \\
\hline 4 & 96.0 & 32 & $4.721 E-09$ & $5.24 \angle E-E D$ & 6510 \\
\hline 4 & 99.0 & 22 & $7.343[-09$ & $5.72 \times F=0 \mathrm{t}$ & 6520 \\
\hline , & 100.0 & H & $7.73 \mathrm{HE}-69$ & $2.13 \omega F-0 t$ & 65011 \\
\hline 4 & 102.0 & 20 & $0.472 E-\cup 9$ & s.c4ul-0o & 6610 \\
\hline 4 & 104.0 & 24 & $7.7171-09$ & $0.014[-00$ & 6650 \\
\hline 4 & 106.0 & ค & $7.353 F-j 9$ & $2.233[-00$ & 6720 \\
\hline [ & $1,4,0$ & 2.2 & $7.343 t-199$ & $5.7<0[-00$ & 6900 \\
\hline $\mathrm{E}$ & 110.0 & 55 & 1.7MUE-UB & 1. उEHE-U7 & 7000 \\
\hline 5 & 112.0 & 10 & $\supset .+3 A E-U \theta$ & $2.213 E-07$ & 1300 \\
\hline 4 & 114.0 & 546 & $1.752 \mathrm{E}-\mathrm{U} 7$ & $1.367 t-c 6$ & 1210 \\
\hline 4 & 116.0 & 1774 & $5.688 E-07$ & $4.437 E-06$ & 7220 \\
\hline & $1]+.0$ & 3256 & $1.044 t-36$ & $8.14]\left[-C_{6}\right.$ & 7190 \\
\hline & $1<0.0$ & 1593 & $5.1 \cup 7 E-\cup 7$ & $3.5 \% J E-C_{0}$ & 7150 \\
\hline 7 & 122.0 & 594 & $1.919 E=07$ & $1.497 \mathrm{E}=00$ & 7120 \\
\hline r & 124.0 & 1251 & $4.0118-67$ & $3.1<y[-06$ & $71 \cup 0$ \\
\hline 5 & $1<0.0$ & 770 & $2.40+1-67$ & $1.9235-0 c$ & 7100 \\
\hline 4 & 129.0 & 151 & $4.956 E-0 A$ & 3.7 y $6 E-07$ & 7100 \\
\hline & 130.0 & 100 & $3.211 t-6 H$ & $2.535 t-67$ & 7130 \\
\hline r & $1 ; 2.0$ & 100 & $3.236 E-06$ & $2.524 E-07$ & 7150 \\
\hline 4 & 134.0 & 105 & $3.373 t-08$ & $2.031 t-07$ & 7200 \\
\hline 4 & 136.0 & he & D.DISE-UE & $1.720 \mathrm{E}-07$ & 7250 \\
\hline & 1 s. 0 & 73 & $2.358 \mathrm{~L}-U \theta$ & $1.83 b 5=07$ & 1300 \\
\hline $\mathrm{E}$ & 360.0 & 72 & $2.327 \mathrm{E}-\mathrm{OH}_{4}$ & $1.015[-07$ & 7350 \\
\hline 4 & 142.0 & 71 & $2.303 E=O A$ & $1.796, F-07$ & 7250 \\
\hline 7 & 1.4 .0 & 44 & $1.431 E=08$ & $1.11 \cup E-07$ & 7110 \\
\hline $\mathrm{P}$ & $14 b . c$ & 100 & $3.236 E-08$ & $2.324 E-07$ & 7100 \\
\hline & INO & NTEGRATEO= & $\begin{array}{r}9.966 E-04 \\
\text { SEC/SO.M }\end{array}$ & $\begin{array}{c}t .9 \mathrm{~J} j[-03 \\
1 / M\end{array}$ & \\
\hline
\end{tabular}

TEST U7U JULY 19, 1968 O 108 TU 0138 PST FLUURESCEIN RELEASE FRDM ELEVATIUN OH $2 M$
SAOM ARC SAMPLER HT $1.5 \mathrm{M}$ U

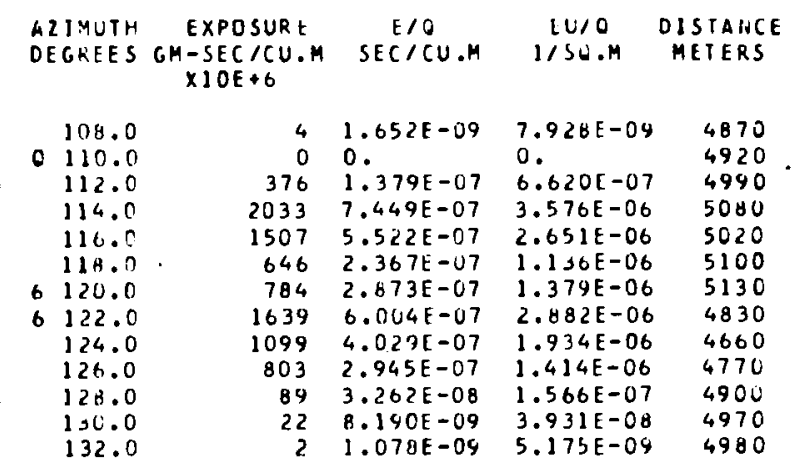

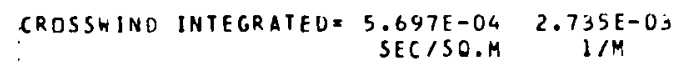

TEST UTO JULY 19: 1968 OLOB TU 0138 PST FLURRESCEIN RELEASE FRUM FLFVATIUN DF LM
SAMPLER HT $1.5 M$ UE 4.6 M/SEC AT $2 M$

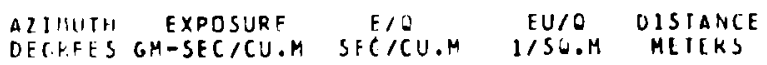
$\times 10 E+6$

490.0

492.5

494.1

446.0

$19 \% .0$

4100.0

$41 \% 2.0$

$4 \quad 1: 4.0$

41.6 .0

E 134.0

9110.0

5112.0

4114.0

4116.0

$411 \% .0$

5120.0

7122.0

T. 124.0

5126.0

4129.1

5130.0

f 132.0

4134.0

4136.0

$413, i^{2}$

F 140.0

4112.0

7144.0

A 146.0

00.

00.0.

0500

$\begin{array}{rlll}4 & 1.721 E-09 & 8.259 E-09 & 6440 \\ 72 & 2.63 A E-08 & 1.260 E-07 & 650 C\end{array}$

$4 \quad 1.721 \mathrm{t}-09 \quad 6.254 \mathrm{E}-09 \quad 6510$

$23 \quad 3.603 E-09 \quad 4.129 E-00 \quad 6520$

$\begin{array}{llll}23 & 3.603 E-09 & 4.129 E-00 & 6520 \\ 29 & 1.067 E-00 & 5.120 E-08 & 6560\end{array}$

$176.538 E-09$ 3.13EE-0B 6310

$15 \quad 5.5 \% 6-09 \quad 2.643 E-60 \quad 6650$

i 0.00606

$31.376 E-09 \quad 6.6071-09$ tEUC

$4 \quad 1.721 t-09 \quad 0.259 E-09 \quad 7000$

$\begin{array}{rlll}B 4 & 3.047 E-0 B & 1.487 E-07 & 7300\end{array}$

$\begin{array}{rlll}353 & 1.294 \mathrm{E}-\mathrm{C} 7 & 6.211 \mathrm{E}-07 & 7210\end{array}$

$\begin{array}{llll}1135 & 4.160 E-C 7 & 1.997 E-06 & 7220 \\ 1392 & 5.100 E-07 & 2.448 E-0 C & 7190\end{array}$

$\begin{array}{rlll}1392 & 5.100 E-07 & 2.44 B E-06 & 7190 \\ 721 & 2.643 E-07 & 1.269 E-06 & 7150\end{array}$

$93 \mathrm{H} \quad 3.438 \mathrm{E}-07 \quad 1.650 \mathrm{E}-06 \quad 7120$

$977 \quad 3.5 \mathrm{H} 2 \mathrm{E}-07 \quad 1.714 \mathrm{E}-06 \quad 7100$

$757 \quad 2.774 E-07 \quad 1.331 E=06 \quad 7136$

$103 \quad 3.765 E-08$ 1.817E-U7 7100

$\begin{array}{rlll}103 & 3.765 E-O B & 1.817 E-U 7 & 710 U \\ 42 & 1.544 E-0 U & 7.433 F-O B & 7130\end{array}$

3) $1.308 \mathrm{E}-08 \quad 6.277 \mathrm{E}-00 \quad 7150$

$279.979 \mathrm{E}-\mathrm{C9} \quad 4.75 U E-00 \quad 7200$

$33 \quad 1.23$ Y F-OB $5.446 E-00 \quad 7250$

28 1.U32E-00 $4.955 E-00 \quad 73 U E$

$27 \quad 9.979 E-09 \quad 4.700 E-00 \quad 7350$

$\begin{array}{llll}27 & 9.979 E-09 & 4.790 E-08 & 7350 \\ 27 & 9.979 E-09 & 4.79 U[-0 \mathrm{i} & 7250\end{array}$

$24 \quad 8.947 \mathrm{E}-69 \quad 4.295 \mathrm{E}-0 \mathrm{O} \quad 7110$

CRISSSHINO INIEGRATED $=6.306 E-04 \quad 3.027 E-03$ 


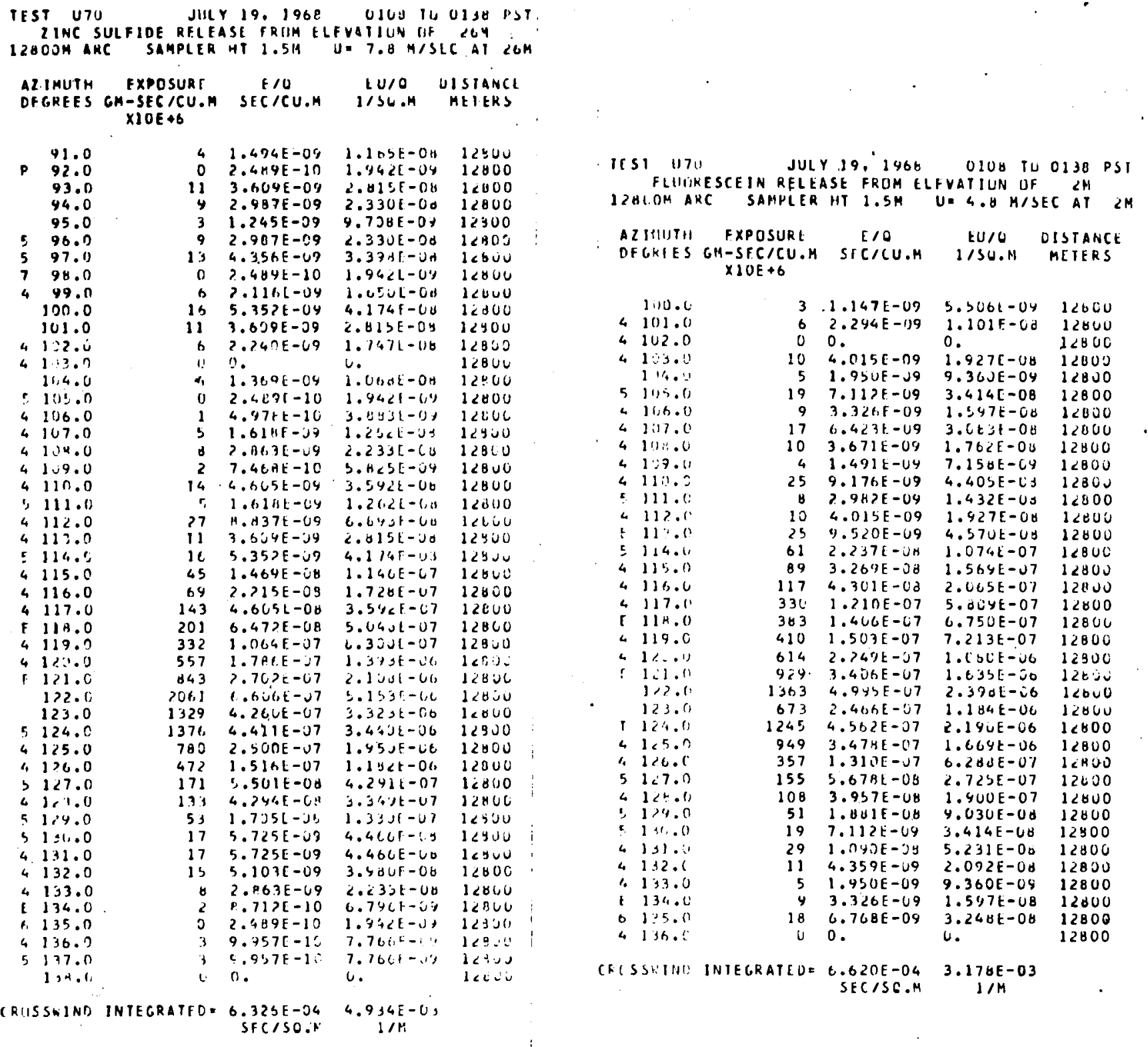




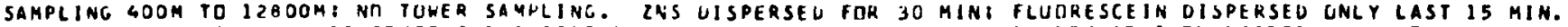
ARCS DUT TO $3200 M$ EMBKACF CRUSSHIND EXTENT UF TRACER, 5OOOM, TOUUM ANU 128OOM ARCS TRUNCAIED UN HURTH.

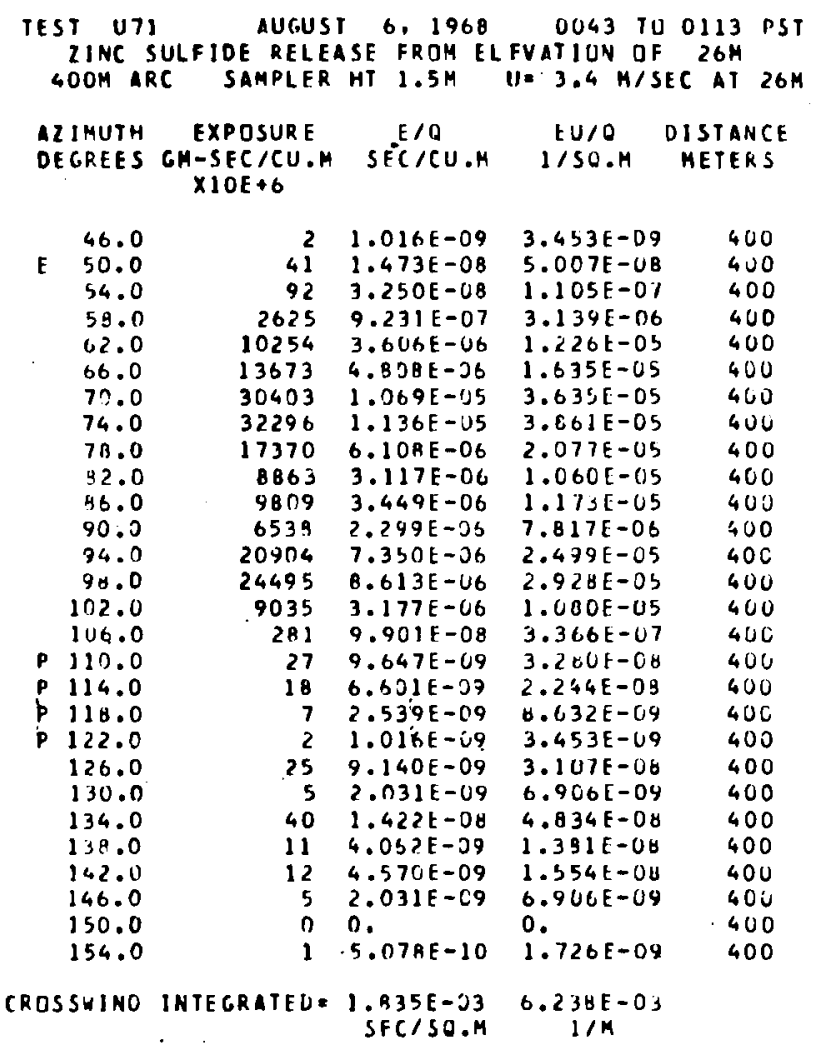

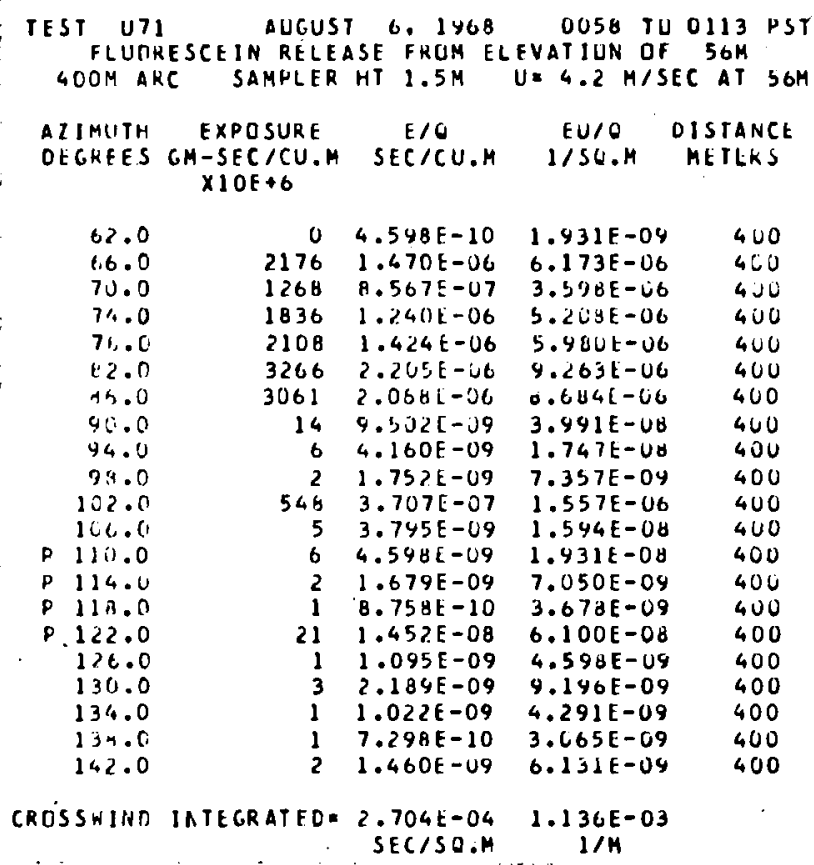




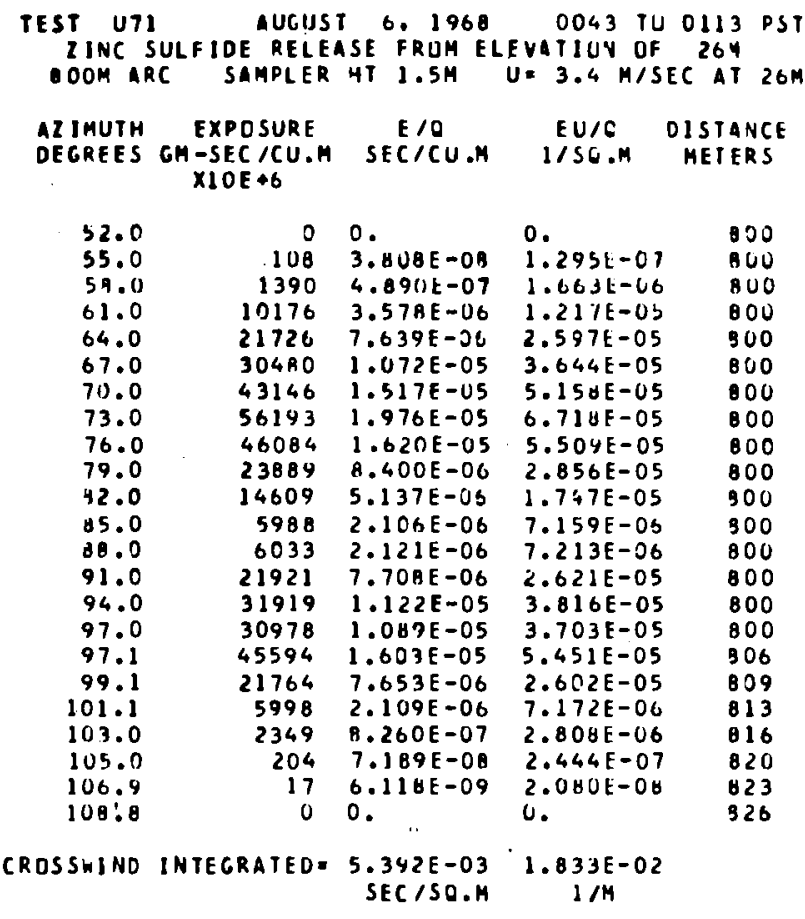

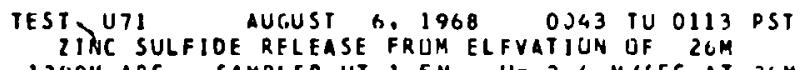
$12110 M$-ARC SAMPLER HT $1.5 \mathrm{M}$ U. $3.4 \mathrm{M} / \mathrm{SEC}$ AT $26 \mathrm{M}$ AZIMUTH EXPQSURE
DEGREES GM-SECVCU.M SEC/CU.M IIS/O DISTANCE
XIOE+6

\begin{tabular}{|c|c|c|c|c|c|}
\hline & $\begin{array}{l}54.0 \\
56.0\end{array}$ & $\begin{array}{ll}1 \\
81\end{array}$ & $\begin{array}{l}5.666 E-10 \\
2.86 \cap E-00\end{array}$ & $\begin{array}{l}1.926 E-09 \\
9.725 E-00\end{array}$ & $\begin{array}{l}1200 \\
1200\end{array}$ \\
\hline & 38.0 & 711 & $2.501 E-07$ & $0.502 E-07$ & 1200 \\
\hline & 66.0 & 1540 & $5.415 \mathrm{E}-07$ & $1.841 \mathrm{E}-06$ & 1200 \\
\hline & 62.0 & 5428 & $1.909 \mathrm{~F}-06$ & $6.49 \cup \mathrm{E}-06$ & 1200 \\
\hline & 64.0 & 17362 & $6.231 E-06$ & $2.135 \mathrm{E}-0 \mathrm{~S}$ & 1200 \\
\hline & 36.0 & 32186 & $1.132 \mathrm{E}-05$ & $3.848 E-05$ & 1200 \\
\hline & 68.0 & 30093 & $1.05 B E-05$ & 3.59 \& -05 & 1200 \\
\hline & 70.0 & 29591 & $1.041 \mathrm{E}-25$ & $3.534 E-05$ & 1200 \\
\hline & $72 . \mathrm{C}$ & 32334 & $1.137 \mathrm{E}-05$ & $3.866[-\cup 5$ & 1200 \\
\hline & 74.0 & 40597 & $1.427 E-05$ & $4.853 \mathrm{~F}-05$ & 1200 \\
\hline & 16.0 & 41734 & $1.407 \mathrm{E}-05$ & $4.944 E-0.5$ & 1200 \\
\hline & 78.0 & 44079 & $1.550 E-05$ & $5.27 \cup E-05$ & 1200 \\
\hline & 20.0 & 38587 & $1.357 \mathrm{E}-05$ & $4.6136-05$ & 1200 \\
\hline & $\$ 2.0$ & 21772 & $7.65 \mathrm{SE}-0 \mathrm{~S}$ & 2.60 IE -05 & 1200 \\
\hline & N4.0 & 12234 & $4.302 \mathrm{E}-\mathrm{i} 6$ & $1.463 E-J 5$ & 1200 \\
\hline & 16.0 & 7102 & $2.407 E-06$ & $8.491 \mathrm{~F}-\mathrm{co}$ & 1200 \\
\hline & 58.0 & 6816 & $2.397 \mathrm{E}-06$ & U.1 4 YE-06 & 1200 \\
\hline & 90.0 & 13377 & $4.704 \mathrm{E}-06$ & $1.599 \mathrm{E}-05$ & 1200 \\
\hline & 92.0 & 14212 & $4.997 t-06$ & $1.694 F-05$ & 1200 \\
\hline 4 & 94.0 & 13477 & $4.799 t-06$ & $1.611 \mathrm{E}-\mathrm{JS}$ & 1200 \\
\hline 6 & 96.0 & 11815 & $4.154 E-66$ & $1.412 \mathrm{E}-0 \mathrm{OS}$ & 1200 \\
\hline & 94 . & 0217 & $2.804 E$ & $9.824 \mathrm{E}-06$ & 1200 \\
\hline & $1 \cup 0.0$ & 1719 & 6. $00,6 E-07$ & $2.050 E-0 Q$ & 1200 \\
\hline & 102.0 & 826 & $2.906[-07$ & $9.679 E-07$ & 1200 \\
\hline 0 & 104.0 & 3 & $1.133 \mathrm{E}-09$ & $3.852 E-09$ & 1200 \\
\hline & 106.0 & 0 & $2.832 \mathrm{E}-10$ & $9.624 E-10$ & 1200 \\
\hline 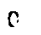 & 108.0 & 13 & $4.815 E-09$ & $1.637 \mathrm{E}-08$ & 1200 \\
\hline & 110.0 & 10 & $3.682 E-09$ & $1.252 \mathrm{E}-08$ & 1200 \\
\hline & & 0 & & 0. & 1200 \\
\hline & & $A T E D$ & $\begin{array}{r}6.281 E-03 \\
S F C / S O . M\end{array}$ & $\begin{array}{c}2.135 E-02 \\
1 / M\end{array}$ & \\
\hline
\end{tabular}

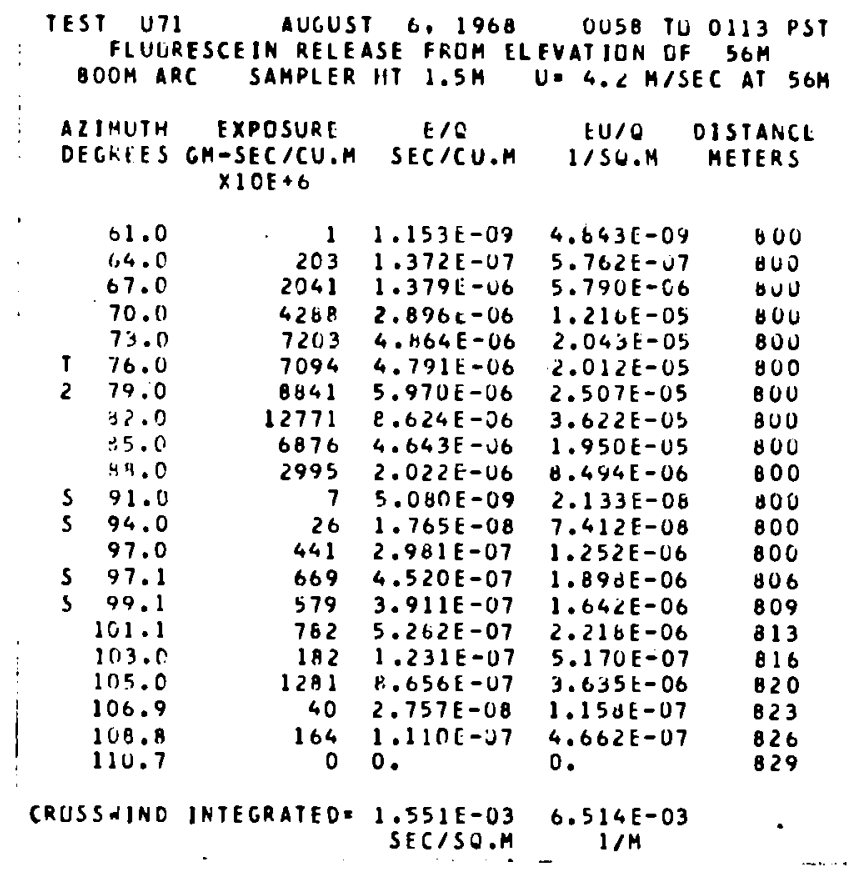

TEST U71 AUCUST 6, 1968 OUSQ TU 0113 PST FLUORESCE IN RELEASE - FROM ELEVATIUN OF SOM $120 O M$ ARC SAMPLER HT $1.5 \mathrm{M} U=4.2 \mathrm{M} / \mathrm{SEC}$ 4T $56 \mathrm{M}$ CRISSHINO INTEGRATED: $\begin{gathered}2.244 E-03 \\ \text { SECISO.14 }\end{gathered}$ 
TEST U71 AUGUST 6. 1968 O043 TU 0113 PST ZINC SULFIDE RELEASE FRUM ELEYATIUN OF 264 160OM ARC SAMPLER HI $1.5 \mathrm{M} U=3.4 \mathrm{M} / \mathrm{SEC}$ AT $26 \mathrm{M}$

\begin{tabular}{|c|c|c|c|c|}
\hline $\begin{array}{l}\text { AZ JMUTH } \\
\text { DECREES }\end{array}$ & $\begin{array}{c}\text { EXPOSURE } \\
\text { GM-SEC/CU.M } \\
\times 1 O E+6\end{array}$ & $\begin{array}{c}E / O \\
\text { SEC/CU.M }\end{array}$ & $\begin{array}{c}E J / O \\
1 / S O . M\end{array}$ & $\begin{array}{l}\text { DISIANCE } \\
\text { MEIEKS }\end{array}$ \\
\hline
\end{tabular}

\begin{tabular}{|c|c|c|c|c|c|}
\hline & 56.0 & 0 & 0 . & 0. & 1500 \\
\hline & 54.0 & 28 & $9.912 E-09$ & $3.370 E-68$ & 1600 \\
\hline & 10,0 & 325 & $1.144 E-07$ & $3.890 E-07$ & 1600 \\
\hline & $\$ 2.0$ & 1387 & $4.880 E-07$ & 1.65 yE -06 & 1600 \\
\hline & 64.0 & 5715 & $2.010 E-06$ & $.0 .033 \mathrm{E}-06$ & 1600 \\
\hline & 66.0 & 12307 & $4.222 \mathrm{E}-06$ & $1.435 E-05$ & 1500 \\
\hline & 68.0 & 18590 & 6. $537 E-06$ & $2.222 E-0,5$ & 1600 \\
\hline & 70.0 & 16849 & $5.924 E-06$ & $2.014 E-05$ & 1600 \\
\hline & 72.0 & 1432.5 & $3.037 k-06$ & $1.7131-05$ & 1600 \\
\hline & 74.0 & 13043 & $4.586 E-06$ & $1.559 E-105$ & 1600 \\
\hline & 76.0 & 33845 & $1.190 E-05$ & $4.046 t-05$ & 1600 \\
\hline & 78.0 & 37677 & $1.325 \mathrm{E}-05$ & $4.534 E-05$ & 1500 \\
\hline & 80.0 & 23828 & $3.379[-06$ & $2.849 E-05$ & 1600 \\
\hline & 82.0 & 16781 & $5.901 E-06$ & $2.006 E-U S$ & 1600 \\
\hline & $B 4.0$ & 14254 & $5.012 E-06$ & $1.704 E-05$ & 1600 \\
\hline & 96.0 & 10755 & $3.782 E-06$ & $1.236 \mathrm{E}-05$ & 1600 \\
\hline & $B 8.0$ & 4603 & $1.619 \mathrm{E}-06$ & $5.504 E-06$ & 1600 \\
\hline & 90.0 & 5889 & $2.071 E-05$ & $7.041 E-06$ & 1500 \\
\hline & 92.0 & 17186 & $6.043 E-06$ & $2.055 E-05$ & 1600 \\
\hline & 93.6 & 26815 & $9.429 E-66$ & $3.206 E-05$ & 1603 \\
\hline & 94.0 & 17354 & $6.102 \mathrm{E}-06$ & $2.075 E-05$ & 1600 \\
\hline & 94.6 & 25348 & $8.913 E-06$ & $3.030 E-05$ & 1604 \\
\hline & 95.6 & 16650 & $5.855 E-06$ & $1.491 \mathrm{E}-05$ & 1606 \\
\hline & 96.6 & 13098 & $4.606 E-06$ & $1.536 E-05$ & 1508 \\
\hline & 97.6 & 10131 & $3.562 \mathrm{E}-06$ & $1.211 E-05$ & 1610 \\
\hline 7 & 99.6 & 3260 & $1.147 E-06$ & $3.894 \mathrm{E}-06$ & 1611 \\
\hline & 99.6 & 4751 & $1.671 E-06$ & $5.601 E-06$ & 1613 \\
\hline & 100.6 & 2333 & $3.222 t-07$ & $2.7+5 t-06$ & 1515 \\
\hline & 101.6 & 1256 & $4.417 E-07$ & $1.50<E-O C$ & 1617 \\
\hline $\mathrm{c}$ & 102.6 & 643 & $2.262 \mathrm{E}-07$ & $7.684 E-\cup 7$ & 1610 \\
\hline & 103.6 & 270 & $9.511 \mathrm{E}-08$ & $3.234 E-07$ & 1620 \\
\hline & 104.5 & 63 & $2.219 \mathrm{E}-08$ & $7.546 E-08$ & 1622 \\
\hline & 105.5 & 33 & $1.162 \mathrm{E}-08$ & $3.952 t-08$ & 1623 \\
\hline & 106.5 & 78 & $2.748 E-08$ & $9.3 \div 2 E-08$ & 1525 \\
\hline & 107.5 & 0 & 0. & 0. & 1627 \\
\hline & IN & $A T E D=$ & $\begin{array}{r}5.886 E-03 \\
\text { SEC/SO.M }\end{array}$ & $\begin{array}{c}2.001 E-02 \\
1 / M\end{array}$ & \\
\hline
\end{tabular}

TEST U71 AUGUST 6. 1968 O 0043 TO 0113 PST ZINC SULFIDE RELEASE FROM ELFVATIUN LIF 264 $2200 M$ ARC SAMPLER HT $1.5 \mathrm{M} U=3.4 \mathrm{M} / \mathrm{SEC}$ AT $26 \mathrm{M}$ AZIIIUTH EXPOSURE E/O EU/O DISTANCE DEGREES GM-SEC/CU.M SECICU.M I/SE.M METERS $X 10 E+6$

\begin{tabular}{|c|c|c|c|c|c|}
\hline & 56.0 & 0 & 0. & o. & 2200 \\
\hline \multirow[t]{6}{*}{$x$} & $o() .0$ & c & o. & l. & $\angle 200$ \\
\hline & $t 2.0$ & 170 & $5.9 B .7 E-J B$ & $2.036 E-07$ & 2200 \\
\hline & 64.0 & 376 & $1.324 E-07$ & $4.501 E-67$ & J220 \\
\hline & 6.6 .0 & 1253 & $4.406 E-07$ & $1.490 E-06$ & 2200 \\
\hline & $\operatorname{ts} .0$ & $120: 9$ & $4.254 E-07$ & $1.446 f-66$ & 2200 \\
\hline & 701.0 & 7026 & $2.471 \mathrm{E}-06$ & $8.40 O E-U G$ & 2200 \\
\hline \multirow[t]{3}{*}{$E$} & 72.0 & 4712 & $1.657 \mathrm{E}-\mathrm{J6}$ & $5.634 F-06$ & 2200 \\
\hline & 74.0 & 2163 & $7.607 E-07$ & $2.586 E-06$ & 2200 \\
\hline & 76.0 & 4214 & $1.482 \mathrm{E}-6 \mathrm{~B}$ & $5.030 \mathrm{E}-06$ & 2200 \\
\hline \multirow[t]{3}{*}{$E$} & 79.0 & 3440 & $1.210 E-06$ & $4.113 \mathrm{E}-06$ & $<200$ \\
\hline & 80.0 & 2434 & $8.562 E-07$ & $2.911 E-06$ & 2200 \\
\hline & 82.0 & 4115 & $1.447 E-06$ & $4.920 \mathrm{E}-06$ & 2200 \\
\hline 5 & $94 . ?$ & 1112 & $3.912 \mathrm{E}-07$ & $1.33 \cup E-06$ & 2200 \\
\hline \multirow[t]{5}{*}{$s$} & $B 6.0$ & 1465. & $5.155 E-07$ & $1.753 E-06$ & 2200 \\
\hline & 88.0 & 2556 & $8.940 E-07$ & $3.05 C E-U 6$ & .2200 \\
\hline & 90.0 & 3513 & $1.235 E-06$ & $4.2100 t-00$ & 2200 \\
\hline & 92.0 & 1669 & $5.870 E-07$ & $1.996 \mathrm{t}-06$ & 2200 \\
\hline & 94.0 & 13150 & $4.624 E-J 5$ & $1.512 \mathrm{E}-0 \mathrm{~S}$ & 2200 \\
\hline 4 & 90.0 & 802 & $2.423 E-07$ & $9.54 \mathrm{BE}-6.7$ & $\angle 2.50$ \\
\hline 0 & 98.0 & 253 & $8.900 E-\cup B$ & $3.026 E-67$ & 2200 \\
\hline & 100.0 & 987 & $3.472 \mathrm{E}-07$ & $1.180 E-06$ & 2200 \\
\hline$s$ & 1.02 .0 & 66 & $2.324 E-08$ & $7.903 E-08$ & 2200 \\
\hline s & 104.0 & 12 & $4.413 E-09$ & $1.500 E-00$ & 2200 \\
\hline & 106.0 & 0 & $1.471 \mathrm{E}-10$ & $5.002 E-10$ & 2200 \\
\hline & SWIND & ATED & $\begin{array}{r}1.531 E-03 \\
S F C / S O . M\end{array}$ & $\begin{array}{c}5.206 E-03 \\
1 / M\end{array}$ & \\
\hline
\end{tabular}

\begin{tabular}{|c|c|c|c|c|c|}
\hline TES & $\begin{array}{l}5 T \text { UT1 } \\
F L U I J R \\
600 M A R\end{array}$ & $\begin{array}{l}\text { AUCUS } \\
\text { RESCEIN RELE } \\
\text { KC SAMFLER }\end{array}$ & $\begin{array}{c}\text { T } 6,1968 \\
\text { ASE FRDM E } \\
\text { HT } 1.5 M\end{array}$ & $\begin{array}{r}0058 \mathrm{TL} \\
\text { FVATIUN Ut } \\
U=4.2 \mathrm{M} / \mathrm{s}\end{array}$ & $\begin{array}{r}0113 \text { PST } \\
\text { S6M } \\
S E C A T \text { SGM }\end{array}$ \\
\hline & $\begin{array}{l}2 \text { IIIUTH } \\
\text { EGREES }\end{array}$ & $\begin{array}{c}\text { EXPOSURL } \\
\text { GM-SEC /CU.M } \\
\times 1 O E+6\end{array}$ & $\begin{array}{c}1 / 2 \\
\text { SECC/CU.M }\end{array}$ & $\begin{array}{l}E U / O \\
1 / S O . M\end{array}$ & $\begin{array}{l}\text { DISTANCE } \\
\text { METEKS }\end{array}$ \\
\hline & 02.0 & 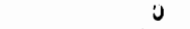 & $2.442 E-10$ & $1.026 E-C y$ & 1600 \\
\hline & 64.0 & 14 & $1.010 E-O B$ & $4.240 \mathrm{E}-00$ & 1600 \\
\hline & 66.0 & 67 & $4.527 E-08$ & $1.901 E-07$ & 1600 \\
\hline & h3.0 & 555 & $3.752 E-07$ & 1.576 & 1600 \\
\hline & $70 \cdot 0$ & 872 & $5.889 E-07$ & $2.474 E-06$ & 1600 \\
\hline & 72.0 & 1859 & $1.256 E-06$ & $5.274 E-06$ & 1600 \\
\hline & 74.0 & 1935 & 1.307 & 5.48 & 1600 \\
\hline & 76.0 & 6392 & $4.316 E-06$ & $1.813 E-05$ & 1600 \\
\hline & 78.0 & 8584 & $5.796 E-06$ & $2.434 E-05$ & 1600 \\
\hline & 80.0 & 5113 & $3.453 E-06$ & $1.450 E-05$ & 1600 \\
\hline & $ن<.0$ & 3773 & $2.548 E-06$ & $1.07 \cup E-05$ & 1600 \\
\hline & $\$ 4.0$ & $85 B 4$ & $5.746 E-06$ & $2.434 E-U 5$ & 1600 \\
\hline & 86.0 & 8949 & $6.043 E-06$ & $2.538 \mathrm{E}-0 \mathrm{~S}$ & 1600 \\
\hline & त. . 0 & 4139 & $2.795 E-06$ & $1.174 E-05$ & 1600 \\
\hline & 90.0 & 4049 & $2.735 E-06$ & $1.149 \mathrm{E}-0 \mathrm{~S}$ & 1600 \\
\hline & 92.0 & 2011 & $1.358 t-06$ & $5.70 S E-06$ & 1600 \\
\hline & 93.6 & 3764 & $2.542 E-1.6$ & F-US & 1603 \\
\hline & 44.6 & 1100 & $7.42 B E-J 7$ & $3.220 E-06$ & 1600 \\
\hline & 94.6 & 2524 & $1.705 E-06$ & $7.160 E-06$ & $160 \%$ \\
\hline & 95.6 & 1532 & $1.035 E-06$ & $4.346 E-06$ & 1606 \\
\hline & 96.6 & 2574 & $1.738 t-06$ & $7.300 E-06$ & 1608 \\
\hline & 97.6 & 3318 & $2.241 t-06$ & $9.41 \cup E-06$ & 1610 \\
\hline 7 & 98.0 & 2921 & $1.973 E-v 6$ & $8.285 E-06$ & 1611 \\
\hline & 09 & 3814 & $2.575 E-46$ & $1.0 H \angle E-05$ & 1613 \\
\hline & 100.6 & 1978 & $1.336 E-06$ & $5.612 E-00$ & 1615 \\
\hline & 101.6 & & 1.872 & $7.863 E-06$ & 1617. \\
\hline .2. & $102 \cdot 6$ & 476 & $3.219 t-07$ & $1.35<E-06$ & 1618 \\
\hline 2. & $10,3.6$ & 554 & $3.745 E-07$ & $1.573 E-06$ & 1620 \\
\hline & $1 \cap 4.5$ & 58 & $3.955 E-08$ & $1.661 E-07$ & 1622 \\
\hline & 105.5 & 9 & $6.534 E-09$ & $2.746 E-08$ & 2623 \\
\hline & 106.5 & 1034 & $6.983 E-07$ & $2.733 \mathrm{E}-06$ & 1625 \\
\hline & 107.5 & 0 & 0. & 0 . & 1627 \\
\hline & $1 \cup B .5$ & 7 & $4.944 E-09$ & $2.077 E-00$ & 1628 \\
\hline & 109.5 & 0 & 0. & & 1630 \\
\hline & 110.4 & 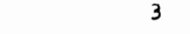 & $2.233 F-09$ & $9.378 E-09$ & 1632 \\
\hline & Dי & 11 & $\begin{array}{r}2.65 \mathrm{BE}-03 \\
\text { SEC/SO.M }\end{array}$ & $\begin{array}{c}1.116 \mathrm{E}-02 \\
1 / \mathrm{M}\end{array}$ & \\
\hline
\end{tabular}

TEST U71 AUC,UST 6. 1968 OCS8 TU 0113 PST FLUORESCEIN RELEASE FRUM ELFVATIUN DF $56 M$ 2200 M ARC SAM.PLER. HT $1.5 \mathrm{M} U=4.2 \mathrm{M} / \mathrm{SEC}$ AT $56 \mathrm{M}$ AZIMUIH EXPOSURE
DIGIES GM-SECICU.M SECICU.M IISO.M DISIANCE XIOE+6

\begin{tabular}{|c|c|c|c|c|c|}
\hline & $\angle 2.0$ & 1 & $1.264 E-09$ & $5.3 \angle 8 F-0 Y$ & 2200 \\
\hline & 14.0 & 1 & $1.275 E-09$ & $5.355 E-09$ & 2200 \\
\hline & 66.0 & 1 & 1.0U9E-09 & $4.236 E-09$ & 2200 \\
\hline & $6 \cdot .0$ & 16 & $1.091 \mathrm{t}-08$ & $4.582 E-08$ & 2200 \\
\hline & 70.0 & 222 & $1.503 E-07$ & $6.314 t-07$ & 2200 \\
\hline 8 & 72.0 & 360 & $2.436 k-07$ & $1.023 \mathrm{E}-06$ & 2200 \\
\hline & 14.0 & 452 & $3.057 E-07$ & $1.284 E-06$ & 2200 \\
\hline & 75.0 & 1064 & $7.187 E-07$ & $3.010 E-06$ & 2200 \\
\hline E & 74.0 & 1445 & $9.762 \mathrm{E}-07$ & $4.100 E-60$ & 2200 \\
\hline & 00.0 & 1548 & $1.046 E-06$ & $4.392 E-06$ & 2200 \\
\hline & 32.0 & 2339 & $1.500 E-06$ & $6.635 E-06$ & 2200 \\
\hline 5 & 34.0 & 3414 & $2.3 \cup 6 E-06$ & $9.684 E-06$ & 2200 \\
\hline$s$ & 30.0 & 1163 & $7.853[-07$ & $3.290 t-06$ & 2200 \\
\hline & \pm 2.0 & 2037 & $1.376 E-06$ & $5.779 E-06$ & 2200 \\
\hline & 90.0 & 3130 & $2.114 \mathrm{E}-06$ & $8.877 E-06$ & 2200 \\
\hline & $92 . c$ & 525 & $3.546 E-07$ & $1.489 E-06$ & 2200 \\
\hline & 94.0 & 1580 & $1.067 t-06$ & $4.48<E-06$ & 2200 \\
\hline 9 & 46.0 & 275 & $1.859 E-07$ & $7.806 E-07$ & 2200 \\
\hline & 98.0 & 472 & $3.191 E-07$ & $1.340 E-06$ & 2200 \\
\hline & 100.0 & 867 & $5.855 E-07$ & $2.459 E-06$ & 2200 \\
\hline T & 102.0 & 275 & $1.859 E-07$ & $7.800 \mathrm{E}-07$ & 2200 \\
\hline T & 104.0 & 176 & $1.193 E-07$ & $5.009 E-07$ & 2200 \\
\hline 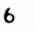 & $10 t .0$ & 347 & $2.347 t-07$ & $9.857 E-07$ & 2200 \\
\hline & 108.0 & 48 & $3.298 E-08$ & $1.385 E-07$ & 2200 \\
\hline 0 & 110.0 & 23 & $1.586 \mathrm{E}-08$ & $6.6600-08$ & 2200 \\
\hline 5 & 112.0 & 2 & $1.396 E-09$ & $5.861 \mathrm{E}-09$ & 2200 \\
\hline 4 & 114.0 & 1 & $1.142 E-09$ & $4.795 E-09$ & 2200 \\
\hline & 116.0 & 1 & $6.978 E-10$ & $2.931 E-09$ & .2200 \\
\hline & WINO & JEG & $\begin{array}{l}1.130 E-03 \\
\text { SEC/SO.M }\end{array}$ & $\begin{array}{c}4.747 \mathrm{E}-03 \\
\mathrm{I} / \mathrm{M}\end{array}$ & $\because$ \\
\hline
\end{tabular}




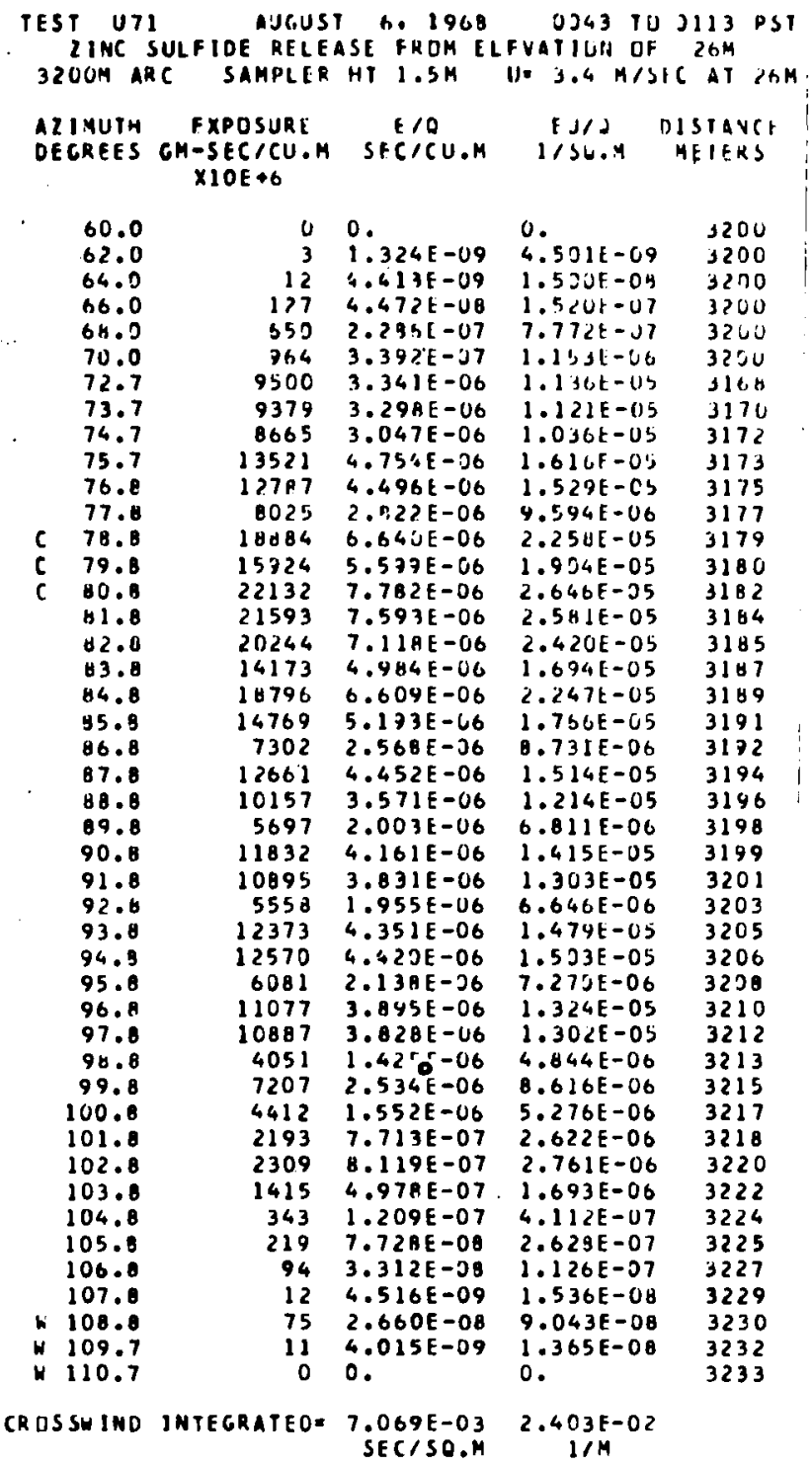

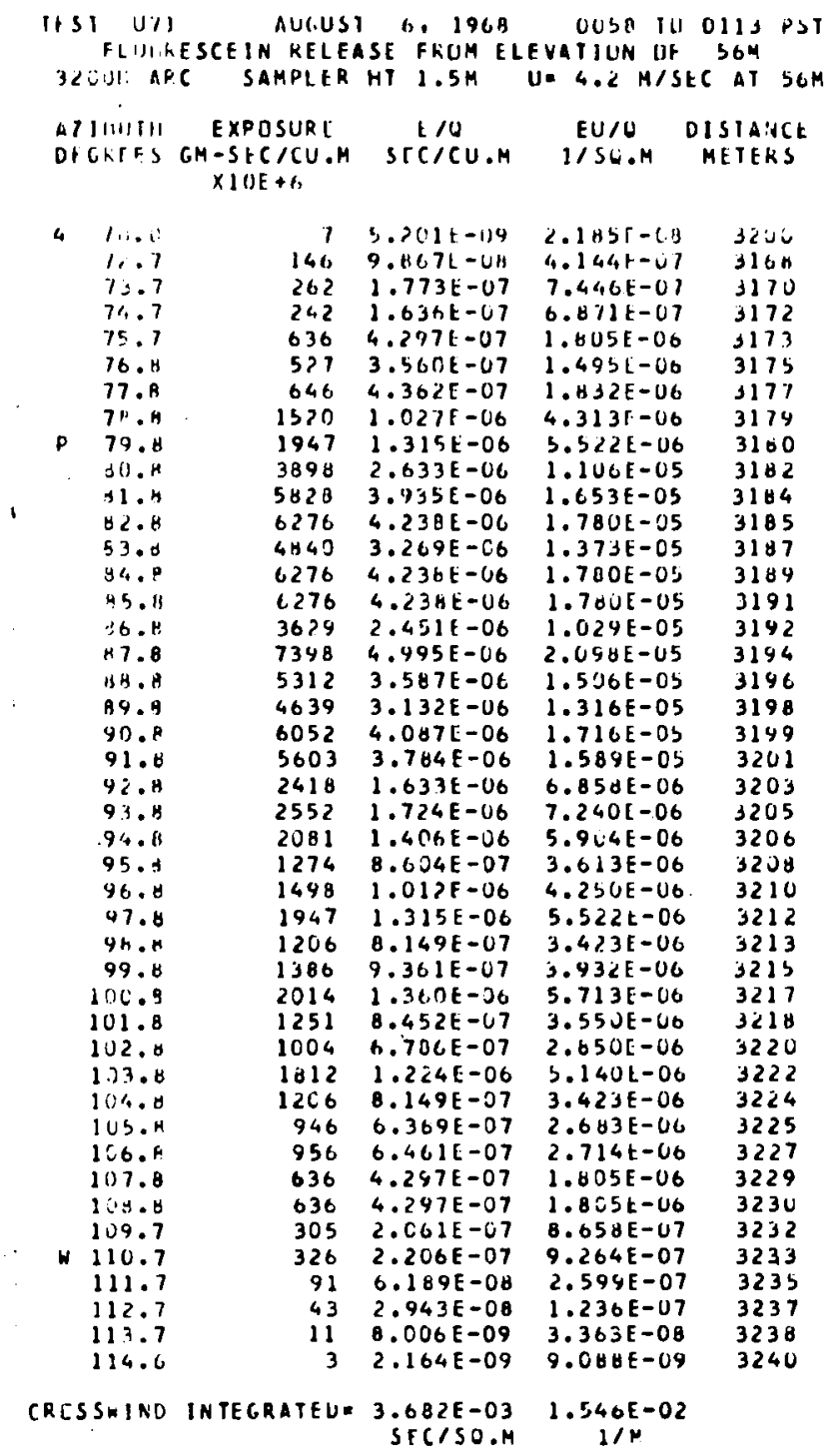




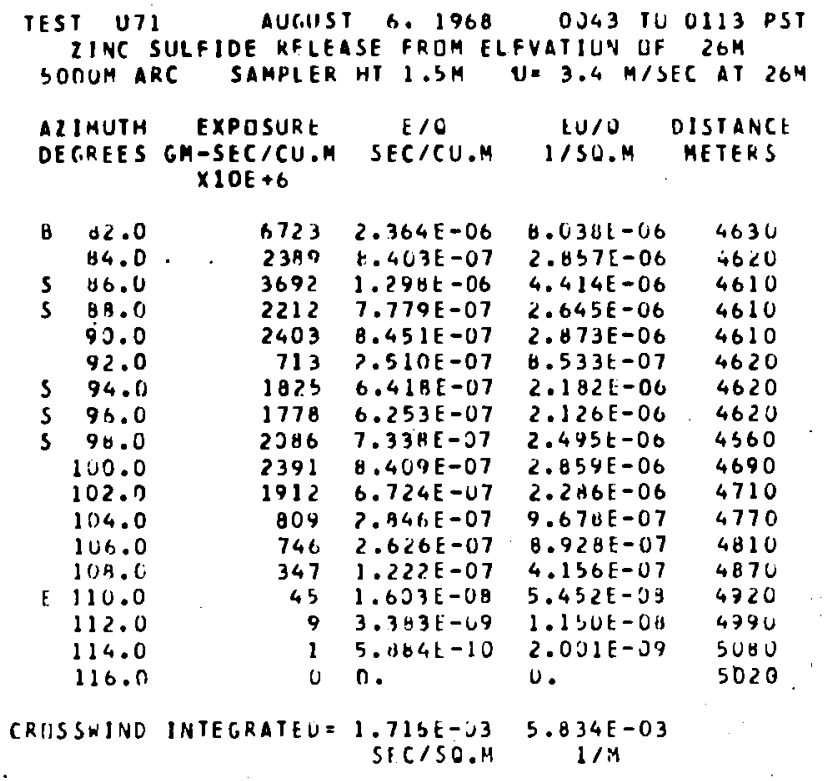

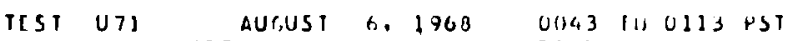
IINC SULFIDE RELIASE FRJIA ELFVATILIS OF $20 \mathrm{M}$

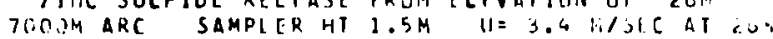

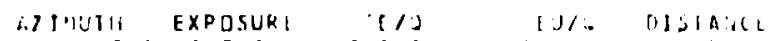

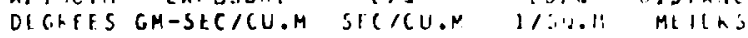
$\times 10 E+C$

\begin{tabular}{|c|c|c|c|c|c|}
\hline $\mathrm{F}$ & 76.0 & 612 & $2.154[-07$ & $7.3<21-07$ & $6 \geq 06$ \\
\hline . & $7+0$ & 701 & $2.7+21-07$ & $4.1,5,3 t-07$ & 6320 \\
\hline \% & $(10.1)$ & 11711 & $4.1161-c 7$ & $103+4+60$ & 6460 \\
\hline 4 & $\therefore 0$ & 1493 & $5 . .9001-07$ & $1.7 a:(-1) t$ & 0403 \\
\hline F. & 14.0 & 2001, & $1.47>1-67$ & 1. $.4,1-100$ & ๘) \\
\hline 6 & $\Delta 6.0$ & 727 & $2.4281-37$ & $0.43: !-27$ & vive \\
\hline 4 & $\mathrm{AH} .0$ & $1 \cup A S_{3}$ & $3.416 .1-07$ & 1. $.2+7 E-i$, & 0450 \\
\hline 4 & $3 n .0$ & 1176 & $4.144 t-67$ & $1.4018 t-60$ & us Uu. \\
\hline & $7 ? .0$ & $147 i$ & $5.171 E-07$ & $1.750[-110$ & 6490 \\
\hline 5 & 34.0 & 1091 & $3.5 \mathrm{~g}+1-67$ & $1.3 c>1-0 t$, & $0 \$ 1,0$ \\
\hline 4 & 96.9 & 1590 & $5.34,1 t-37$ & 2.0?]1-0. & 6310 \\
\hline 1 & 25.0 & 1901 & $6.44 R[-07$ & C.j7yL-Ub & 0520 \\
\hline 4 & $1: 11.0$ & $251 \%$ & 8. 93วE -.7 & $3.11<\mathrm{i}-106$ & 6500 \\
\hline 4 & 102.0 & 3116 & $1.046 t-06$ & $3.726 i-26$ & 0510 \\
\hline 4 & $204.0)$ & 2553 & 4.47 & 3.6 & 6050 \\
\hline 4 & 106.0 & 1655 & $5.42 J E-O 7$ & $1.97 \times E-O 0$ & 6720 \\
\hline 4 & 179.0 & 686 & $2.414 E-07$ & 0.2 auf -67 & 6020 \\
\hline 3 & 11100 & כ & $1.471 \mathrm{E}-10$ & $5.03<\mathrm{F}-1 \mathrm{v}$ & 7000 \\
\hline
\end{tabular}

CFIISS:IND INTFCRATPDE $1.949 E-U 3$ G.740t-lis

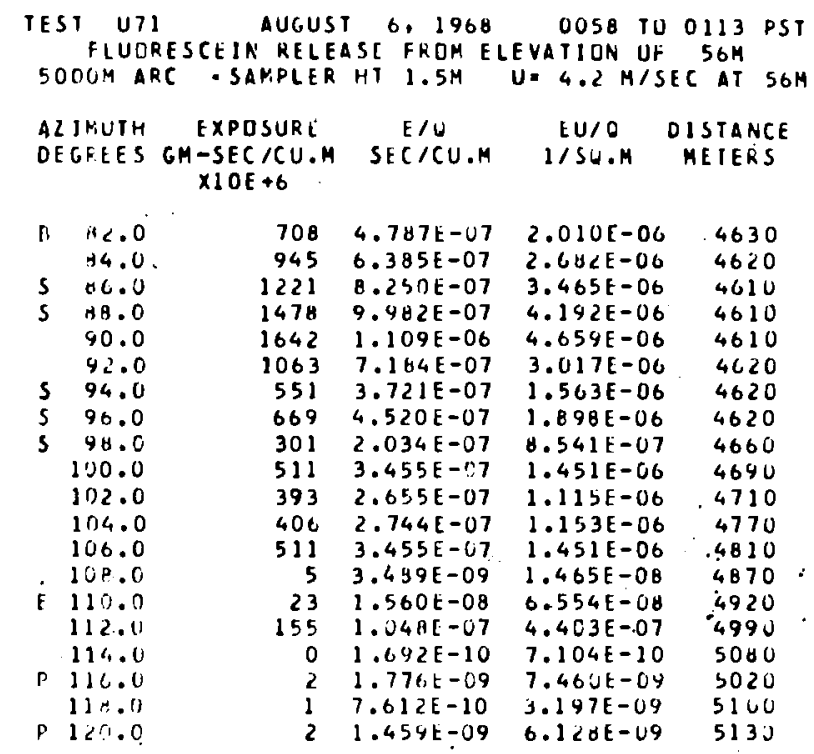

(RISSHINO INTEGRATFD $=1.160 E-U 3$ 4.673E-03

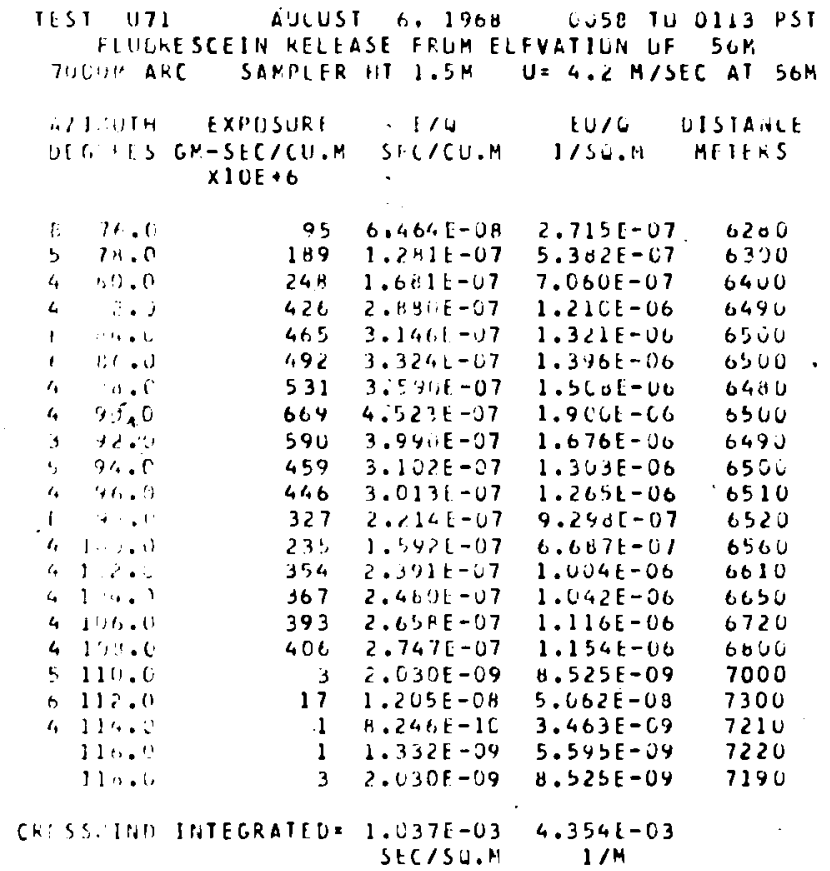




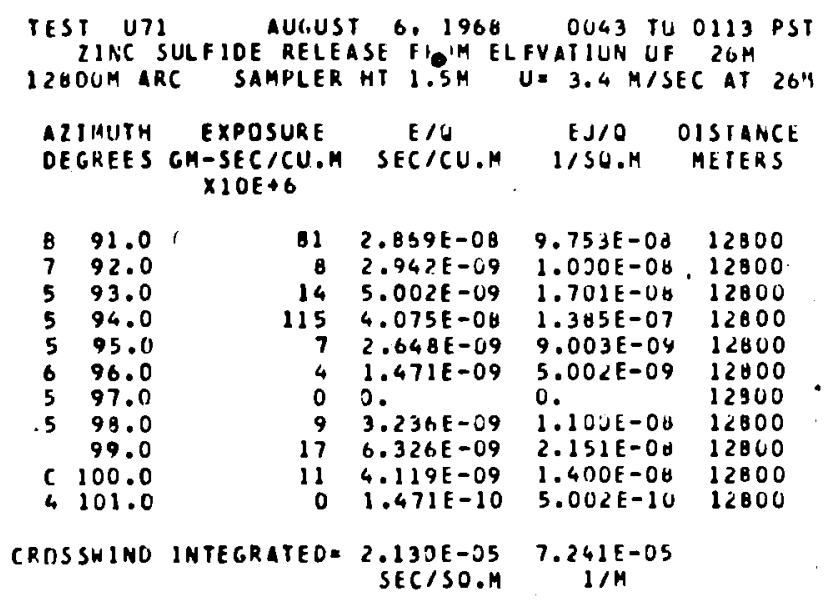

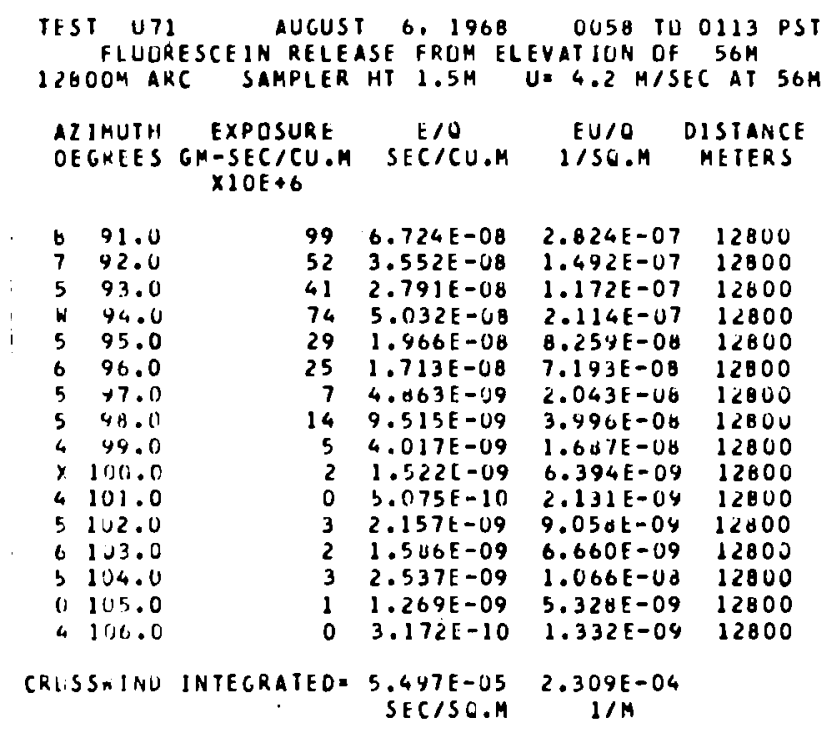


SAMPLING 4OOM TD 700OA: NU TUWER SAMPLING. LNS UISPERSAL FOK SU MINI FLUOKESCEIN UISPERSED ONLY LAST 2O MIN. OISTRIBUTIONS ARE BIMLDAL DK MULTIYUUAL.

ALL ARCS EMBRACE CRUSSHINU EXIENT DF IRALER.

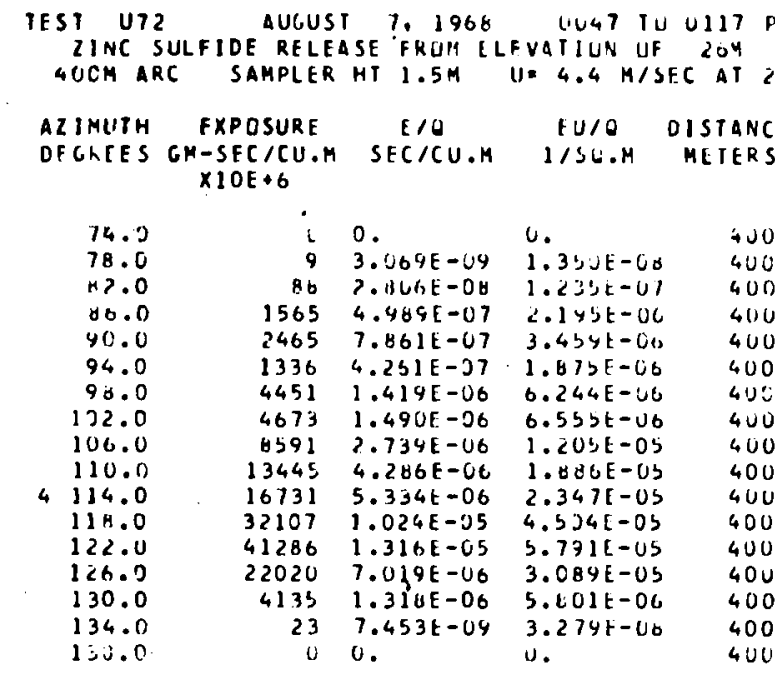

(RIISSI.IND INTEGRATFU $=1.361 E-03$ S.S. SUE $-U 3$

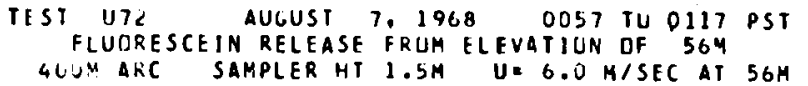

$\begin{array}{cccc}\text { ATIMUTP EXPCSURE } & \text { E/O } & \text { EU/O OISTANCE } \\ \text { DEGHES GM-SECICU.M SECICU.M IISO.M RETERS } \\ \text { XIOE }\end{array}$

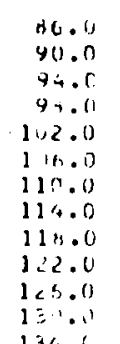

$\begin{array}{rl}0 & 0 . \\ 16 & 7.662 E-09 \\ 0 & 1.505 E-10 \\ 6 & 2.996 E-09 \\ 6 & 3.146 L-09 \\ 15 & 7.211 E-09 \\ 5 & 2.344 F-119 \\ 2926 & 1.359 E-06 \\ 7 & 3.553 E-09 \\ 41 & 1.915 E-08 \\ 4 & 2.293 E-09 \\ 0 & 2.559 E-10\end{array}$

0.

$4.597 \mathrm{E}-00$

400

$4.03 \angle E-10$

$1.797 \mathrm{E}-0 \mathrm{~S}$

$1.8+18 \mathrm{E}-08$

$4.326 E-08$

$1.436 E-U d$

B. $152 \mathrm{~L}-0 \mathrm{G}$

$2.132 \mathrm{E}-\mathrm{OB}$

$1.149 E-07$

$1.376 E-00$

$1.535 E-0 Y$

0.

400
400
400
400
400
400
400
400
400
400
400
400

CRISSH.IA. INTECRATHD $=3.430 E-05.2 .350 E-04$ SECISO.H $\quad 1 / \mathrm{M}$

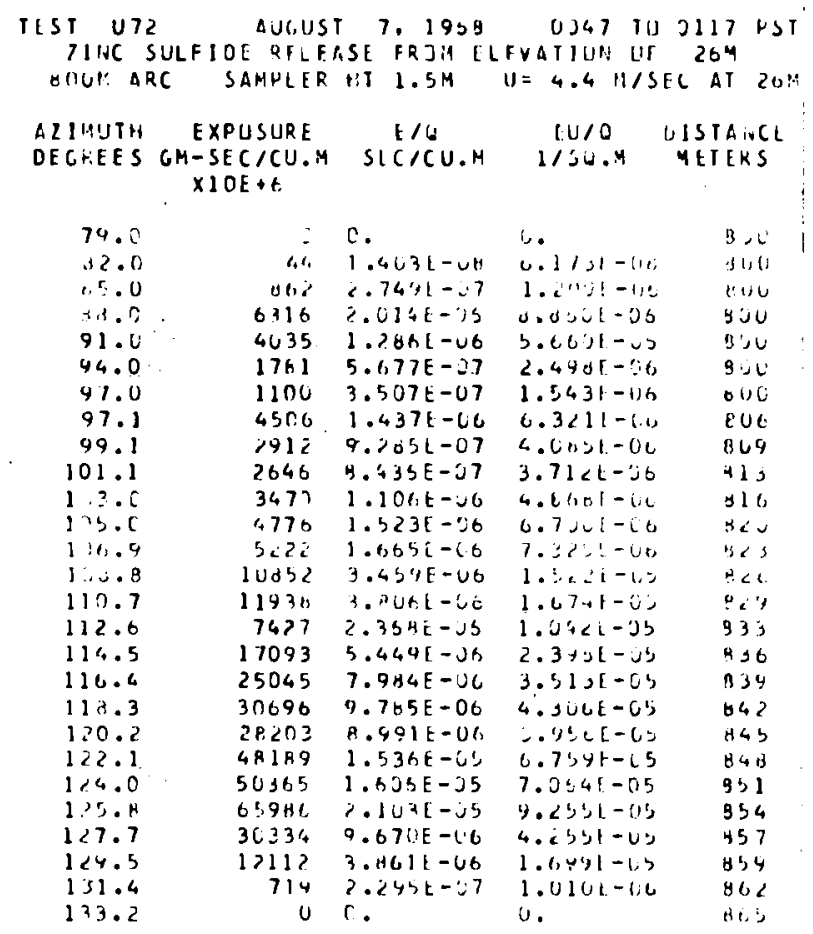

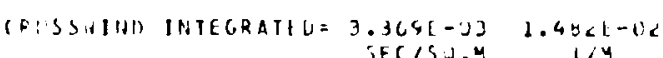

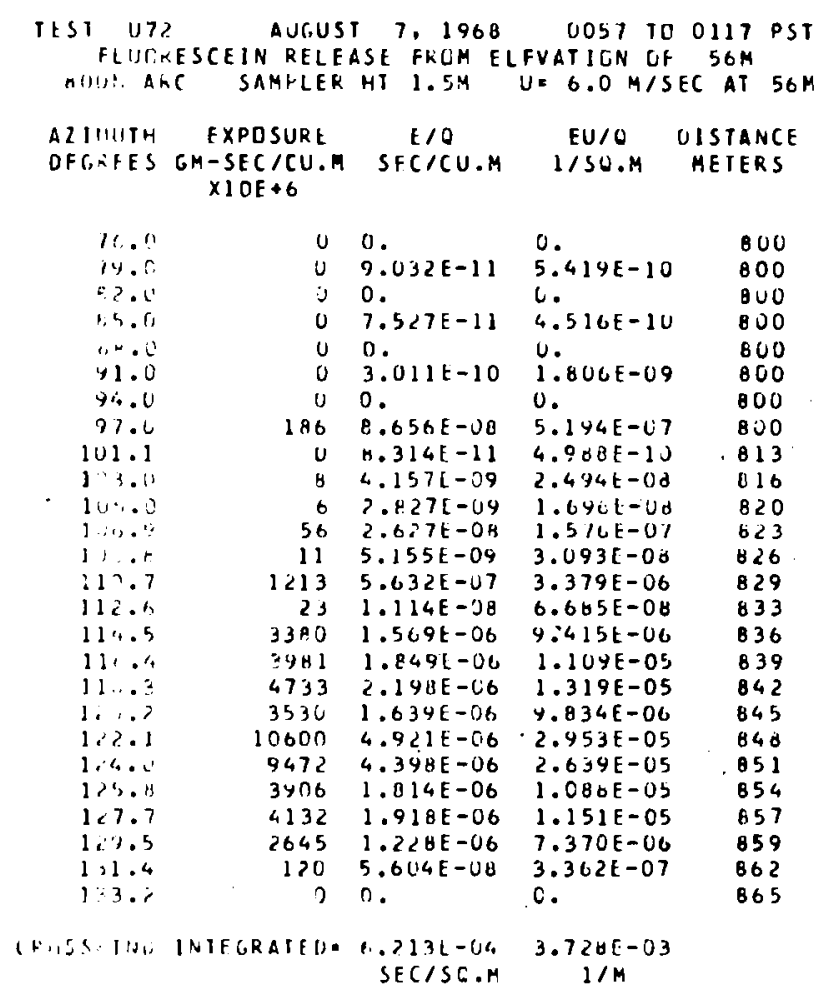




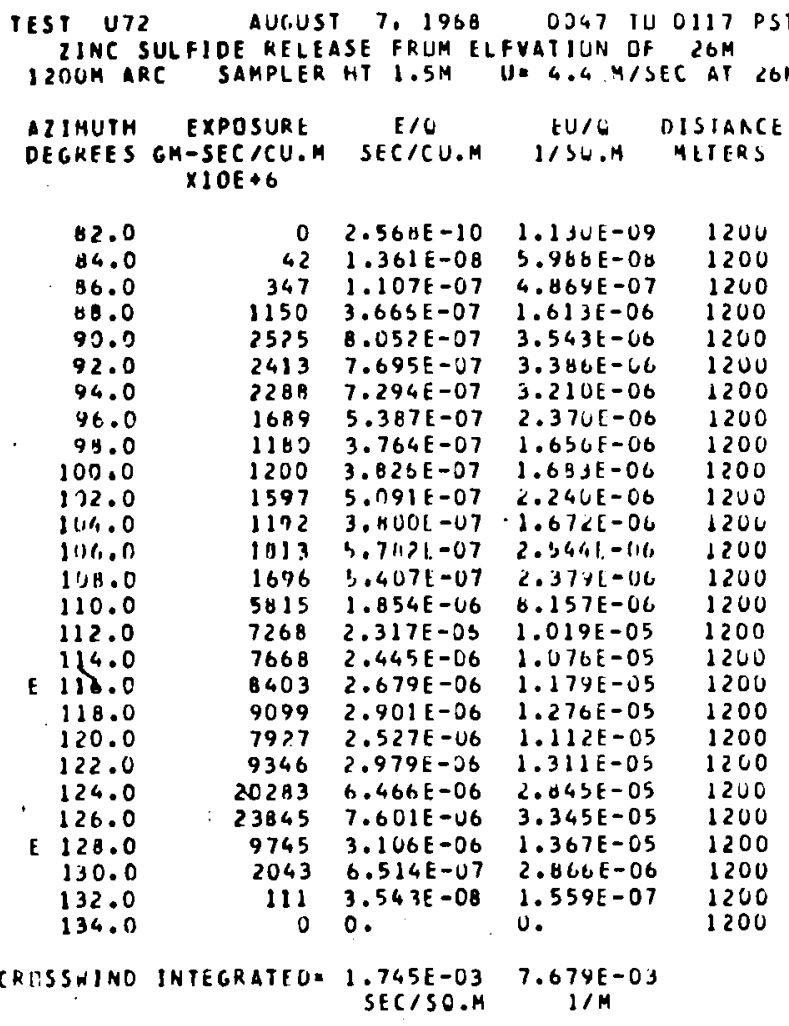

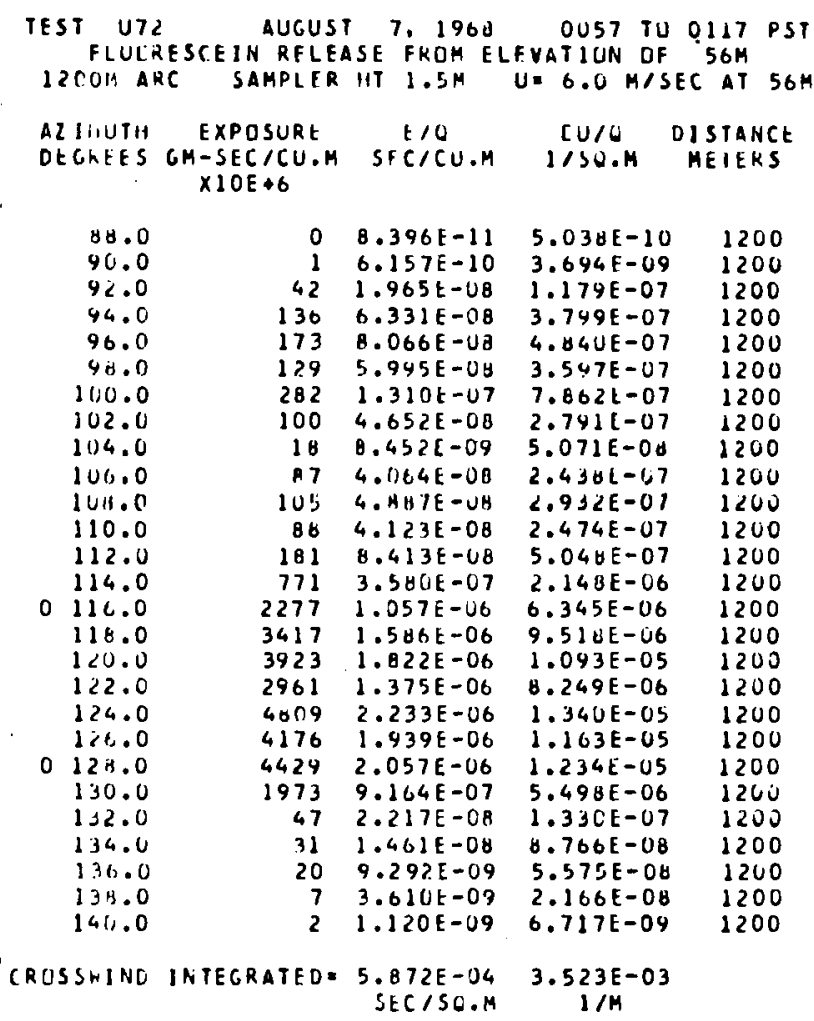




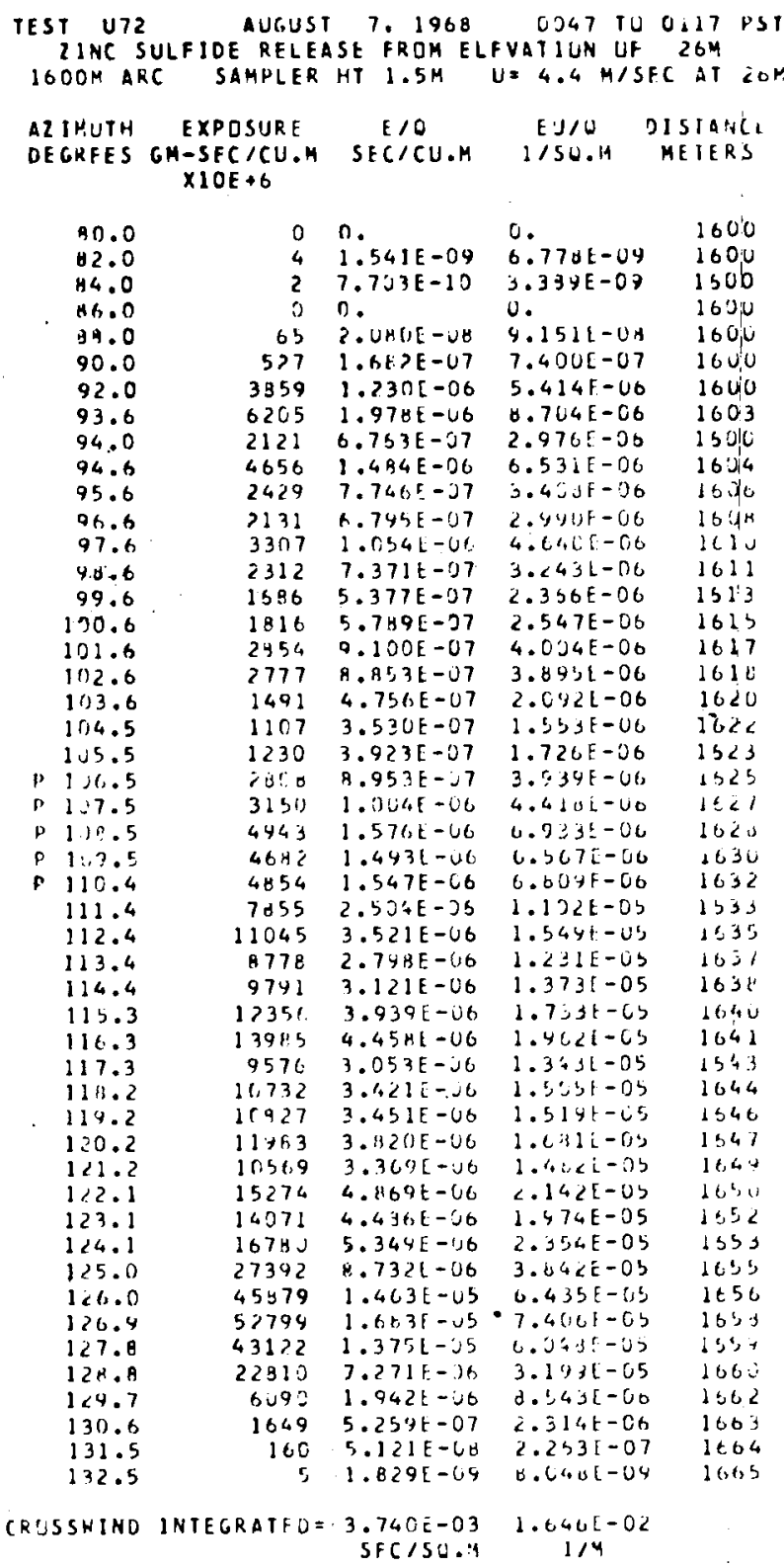

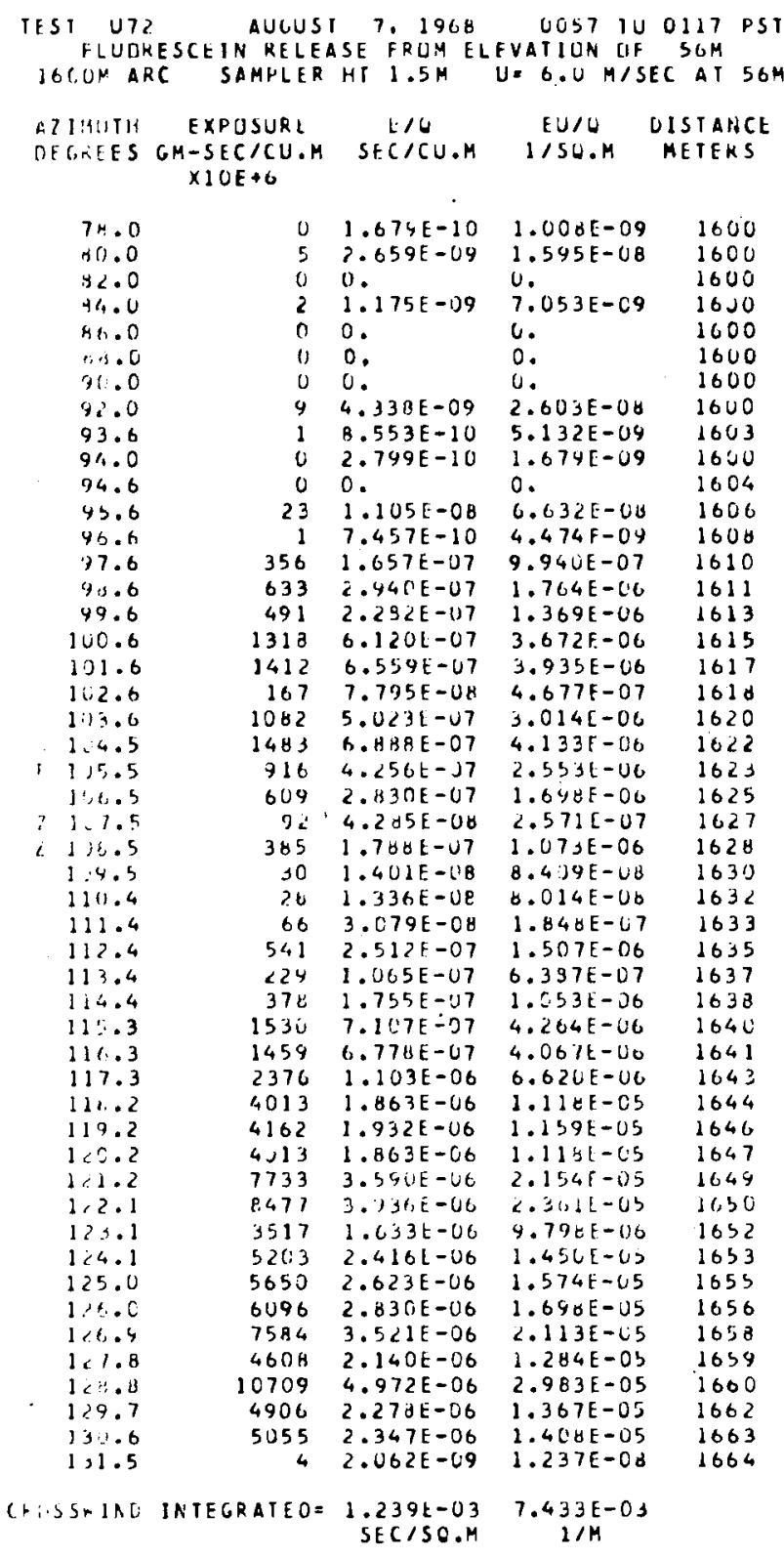


TEST UT2 AUCUST 7. 1968 OJ47 TO 0117 PST
IINC SULFIDE RFLEASE FQDM ELFVATION DF $26 \mathrm{M}$ IINC SULFIDE RFLEASE FQDM ELFVATIUN DF $26 \mathrm{M}$
$2200 \mathrm{M}$ ARC SAMPLER HT $1.5 \mathrm{SM}$ U. 4.4 MISEC AI $26 \mathrm{M}$

\section{EXPOSURE E/O IU/O DISIANCE DECREES GM-SECICU.M SECICU.M IISU.H METERS $\times 10 \mathrm{~A}+6$}

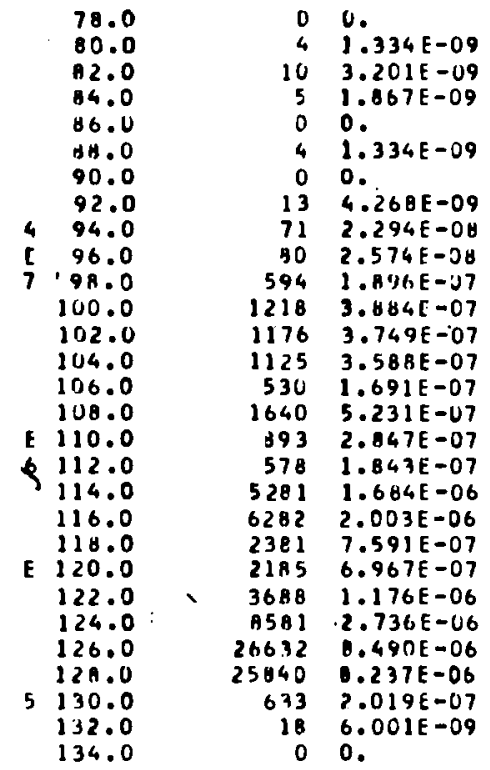

$\begin{array}{ll}U . & 2200 \\ 5.66 U E-09 & 2200 \\ 1.406 E-08 & 2200 \\ 0.215 E-09 & 2260 \\ 0 . & 2200 \\ 5.868 E-09 & 2200 \\ 0 . & 2200 \\ 1.878 E-08 & 2200 \\ 1.009 E-07 & 2200 \\ 1.135 E-07 & 2200 \\ 8.344 E-07 & 2200 \\ 1.7 U 9 E-06 & 2260 \\ 1.650 E-06 & 2200 \\ 1.574 E-06 & 2200 \\ 7.441 E-07 & 2200 \\ 2.301 E-06 & 2200 \\ 1.253 E-06 & 2200 \\ 8.110 E-07 & 2200 \\ 7.408 E-06 & 2200 \\ 8.812 E-06 & 2200 \\ 3.340 E-06 & 2200 \\ 3.065 E-06 & 2200 \\ 5.173 E-06 & 2200 \\ 1.204 E-05 & 2200 \\ 3.7361-05 & 2200 \\ 3.624 E-05 & 2200 \\ 8.864 E-07 & 2200 \\ 2.641 E-08 & 2200 \\ 0 . & 2200\end{array}$

CRUSSWINO INTEGRATEO=12.190E-03 9.637E-03 SFC/SO.M $1 / \mathrm{M}$

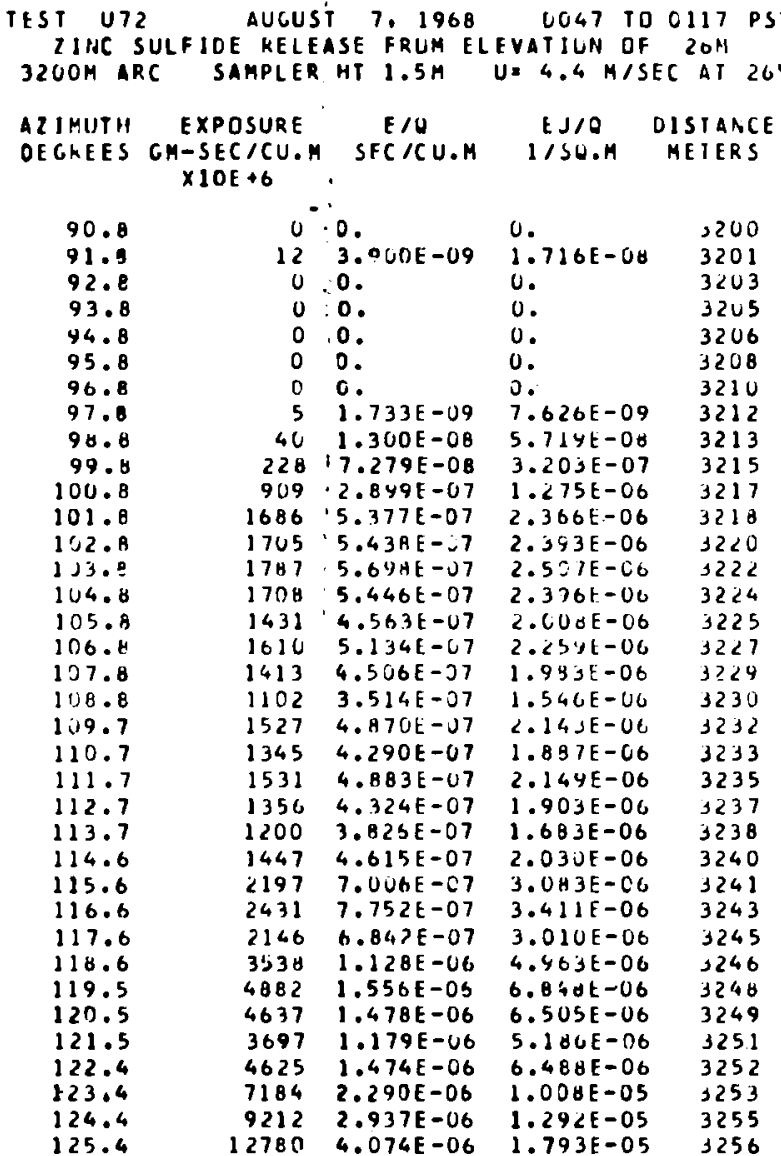

\begin{abstract}
IEST U72 AUGUST 7,1968 0057 TU 0117 PST FLUUKESCEIN RELEASE FRUM ELFVATION OF $56 \mathrm{M}$ 22 OUM ARC SAMPLER HT $1.5 \mathrm{M}$ U $6.0 \mathrm{M} / S E C$ AT $56 \mathrm{M}$
\end{abstract}

ALIMUTH EXPQSURE
DECHEES GM-SECICU.M SFCICU.M IISO.M METERS
XIOE

494.0
496.0
791.0
100.0
102.0
104.0
4106.0
104.0
0110.0
6112.0
114.0
116.0
2118.0
0120.0
122.0
124.0
126.0
121.0
130.0
132.0
0
134.0
136.0
134.0
140.0

$\begin{array}{rl}2 & 1.192 E-09 \\ 0 & 3.198 E-11 \\ 43 & 2.021 E-08 \\ 03 & 3.846 E-0 U \\ 143 & 6.656 E-08 \\ 246 & 1.146 E-07 \\ 165 & 7.676 E-08 \\ 152 & 7.094 E-08 \\ 123 & 5.741 E-08 \\ 68 & 3.176 E-08 \\ 222 & 1.035 E-07 \\ 439 & 2.043 E-07 \\ 670 & 3.111 E-07 \\ 1321 & 6.133 E-07 \\ 3035 & 1.409 E-06 \\ 1991 & 9.247 E-07 \\ 3889 & 1.806 E-06 \\ 3604 & 1.674 E-06 \\ 2235 & 1.038 E-06 \\ 1 J & 4.747 E-09 \\ 5 & 2.58 B E-09 \\ 2 & 1.279 E-09 \\ 3 & 1.410 E-09 \\ 0 & 4.507 E-10\end{array}$

$\begin{array}{ll}7.153 E-04 & 2200 \\ 1.914 E-10 & 2200 \\ 1.212 E-07 & 2200 \\ 2.338 E-07 & 2200 \\ 3.945 E-U 7 & 2200 \\ 6.073 E-07 & 2200 \\ 4.606 E-07 & 2200 \\ 4.257 E-07 & 2200 \\ 3.445 E-07 & 2200 \\ 1.906 E-07 & 2200 \\ 6.211 E-U 7 & 2200 \\ 1.226 E-06 & 2200 \\ 1.867 E-06 & 2200 \\ 3.68 C E-06 & 2200 \\ 8.456 E-06 & 2200 \\ 5.548 E-06 & 2200 \\ 1.083 E-05 & 2200 \\ 1.004 E-0 S & 2200 \\ 6.226 E-06 & 2200 \\ 2.670 E-U J & 2200 \\ 1.553 E-U 8 & 2200 \\ 7.676 E-09 & 2200 \\ 8.461 E-U 9 & 2200 \\ 2.704 E-09 & 2200\end{array}$

CRI'SSHINU INTEGRATEDE 6.583 E-04 3.950E-03

$$
\text { SECISO.M } \quad 1 / M
$$

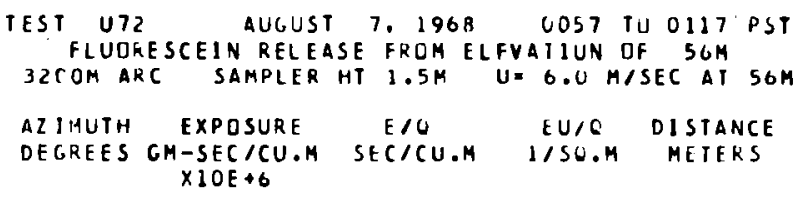




\begin{tabular}{|c|c|c|c|c|c|}
\hline & $\begin{array}{l}126.3 \\
127.3 \\
125.3 \\
129.3 \\
130.3 \\
131.2 \\
132.2\end{array}$ & $\begin{array}{r}16547 \\
18392 \\
9441 \\
2867 \\
341 \\
47 \\
0\end{array}$ & $\begin{array}{l}5.275 E-06 \\
5.663 E-06 \\
3.010 E-06 \\
9.142 E-07 \\
1.08 A E-07 \\
1.517 E-04 \\
0 .\end{array}$ & $\begin{array}{l}2.3211-05 \\
6.3401-05 \\
1.3241-05 \\
4.0235-06 \\
4.7855-07 \\
6.673 E-08 \\
0 .\end{array}$ & $\begin{array}{l}3256 \\
3254 \\
3261 \\
3262 \\
3263 \\
3264 \\
3266\end{array}$ \\
\hline \multicolumn{2}{|c|}{ RIISSAIND } & INTEGRATFD= & $\begin{array}{r}2.250 E-03 \\
S F C / 50 . M\end{array}$ & \multicolumn{2}{|l|}{$\begin{array}{c}9.401 \mathrm{E}-03 \\
1 / 4\end{array}$} \\
\hline & $\begin{array}{l}S T \text { T } 72 \\
2 I N C S \\
O N J H A R\end{array}$ & $\begin{array}{l}\text { AULUST } \\
\text { RELEAS }\end{array}$ & 7,1960 & $\begin{array}{l}\text { OUL7 TII } \\
\text { VATIUN IIF }\end{array}$ & $\begin{array}{r}0117 \\
26 M\end{array}$ \\
\hline & $\begin{array}{l}\text { I IAUTH } \\
\text { CEEEES }\end{array}$ & $\begin{array}{c}\text { EXPUSUR } \\
\text { CM- } 5[C / C H . M \\
\times 1 O F+6\end{array}$ & $\begin{array}{c}E / 0 \\
\text { SrClCU.M }\end{array}$ & $\begin{array}{l}1 \mathrm{~J} / 6 \\
1 / 36 . M\end{array}$ & $\begin{array}{l}\text { IS I I } \\
\text { MF It }\end{array}$ \\
\hline & 90.0 & 0 & 0. & 0. & 4530 \\
\hline & $\begin{array}{l}100.0 \\
102.0\end{array}$ & $\begin{array}{l}5 \\
3\end{array}$ & $\begin{array}{l}1.734 \mathrm{E}-09 \\
1.067 \mathrm{E}-09\end{array}$ & & \\
\hline & 104.0 & 0 & 0. & i. & 47711 \\
\hline & 106.0 & 37 & $1.1 \otimes 7 E-\cup A$ & 5.22 & 4610 \\
\hline & 104.0 & 136 & $4.34 \mathrm{HL}-D \mathrm{P}$ & 1.91 & .970 \\
\hline & 110.0 & 395 & 1.271 & 3.57 & $1+\angle C$ \\
\hline & 112.0 & 410 & $E-U 7$ & 5.84 & $4: 41$ \\
\hline & 114.0 & 192 & $-O H$ & 2.05 & $311 \times 0$ \\
\hline & 110.0 & 235 & 7.51 & 3.31 & 3020 \\
\hline & $11 \mathrm{H} . \mathrm{O}$ & 197 & 1.2 & 3.5 & $1(21)$ \\
\hline & 120.0 & 1196 & 3.4 & $1.6,7$ & 190 \\
\hline & $122 \cdot 1$ & 1156 & 3.011 RE -07 & $1.6,2$ & 4951 \\
\hline & $1<4.9$ & 1100 & 3.51 & 1.54 & .66, \\
\hline & $\begin{array}{l}1.6 .0 \\
1 \therefore 4.0\end{array}$ & $\begin{array}{l}3 \div 5 \% \\
2120\end{array}$ & $\begin{array}{l}1.1365-66 \\
6.776,5-67\end{array}$ & $\begin{array}{l}0.4 \times 11=06 \\
2.4 \times 11-06\end{array}$ & $\begin{array}{l}.770 \\
.9110\end{array}$ \\
\hline i: & 1.111 .0 & 147 & $5.4 \mathrm{BH}-1) \mathrm{H}$ & $\therefore 1.125-07$ & .076 \\
\hline h & $1+2.0$ & 2 & (4.0.0) -10 & 3.5211 .07 & \\
\hline & 134.0 & $y$ & 1). & $\therefore$ & 4313 \\
\hline & (I) ND & $\Delta T H=$ & $\begin{array}{l}1.0421-114 \\
5+C 130 . N\end{array}$ & $\begin{array}{c}2+1,0,1-v s \\
1 / 1\end{array}$ & \\
\hline
\end{tabular}

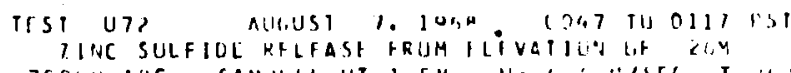

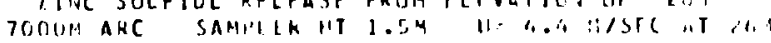

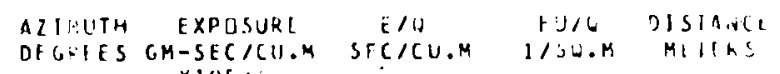
$X 10 \mathrm{E} \rightarrow 6$

\begin{tabular}{|c|c|c|c|}
\hline & $\mathrm{H}_{4} . \mathrm{O}$ & 11 & 0 . \\
\hline & 44.0 & 0 & $n$. \\
\hline & 1114.0 & 9 & כ. \\
\hline & $1: c \cdot \pi$ & ; & ). \\
\hline & $1 \mathrm{H} . \mathrm{J}$ & $\therefore$ & $n$. \\
\hline 5 & 112.0 & 3 & $2.134 E-09$ \\
\hline 4 & 114.11 & $2+3$ & F. $.41 S F-C H$ \\
\hline & 116.0 & $10 \%$ & $? .23,01-27$ \\
\hline & $11 \mathrm{H} .0$ & 473 & $3.13+1-17$ \\
\hline & $1<U .0$ & $7 \times 7$ & $2.511 !-: 77$ \\
\hline & 122.0 & 750 & $2.341 E-J 7$ \\
\hline 0 & $\begin{array}{l}124.0 \\
1=6.0\end{array}$ & $\begin{array}{r}647 \\
2705\end{array}$ & $\begin{array}{l}7.063 E-L 7 \\
\text { A. } 6<5 E-07\end{array}$ \\
\hline$x$ & 120.0 & 1698 & $5.415 E-07$ \\
\hline E & 130.0 & 1277 & $4.07 \geqslant E-07$ \\
\hline 0 & 132.0 & 1130 & $3.607 E-07$ \\
\hline 4 & 134.0 & 105 & $3.374 \mathrm{E}-0 \mathrm{~B}$ \\
\hline 5 & 136.0 & 0 & 0 . \\
\hline
\end{tabular}

$$
\text { c. } \quad(1,0,0)
$$$$
\text { a. }
$$$$
\text { - }
$$$$
\text { - t, t.2. }
$$$$
\text { .3495-0. } 7300
$$$$
\therefore 7031-07 \quad 721,
$$$$
\text { 4. 1.1.1-0y leci }
$$$$
\text { 1.51,1-96 7140 }
$$$$
1.1: 3 i-60 \quad 1150
$$

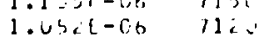$$
\text { 4.07 UE-U7 I]00 }
$$$$
\text { 3.743t-06 71i0 }
$$$$
\text { 2. } 382+-66 \quad 7100
$$$$
1.792 \mathrm{E}-06 \quad 7130
$$$$
\text { 1.5A5E-06 } 7150
$$$$
1.465 F-07 \quad 7200
$$$$
U \text {. }
$$

1250

$$
\begin{aligned}
& 121.3 \\
& 127.3 \\
& 1211.3 \\
& 129.3 \\
& 130.3 \\
& 131.2 \\
& 132.2 \\
& 133.2 \\
& 134.2
\end{aligned}
$$

$\begin{array}{rr}7850 & 3.644 E-06 \\ 10542 & 4.814 E-06 \\ 5831 & 2.767 E-06 \\ 4373 & 2.030 t-06 \\ 649 & 3.014 t-07 \\ 33 & 1.577 E-08 \\ 15 & 7.141 E-09 \\ 45 & 2.123 E-08 \\ 0 & 1.488 E-10\end{array}$

$2.107 E-O S$ $2.937 E-05$
$1.624 E-05$ $1.624 E-05$ $1.21 \mathrm{HE}-05$ 1. A JYE-V6 $9.462 E-08$ $4.285 E-08$ $4.285 E-08$
$1.274 E-07$ 3266

$\begin{array}{llll}45 & 2.123 E-08 & 1.274 E-07 & 3267 \\ 0 & 1.488 E-10 & 8.927 E-10 & 3268\end{array}$

\begin{tabular}{|c|c|c|c|c|}
\hline Tf 5 T & $\triangle \cup G U S T$ & 1968 & 0557 & \multirow{2}{*}{$\begin{array}{r}0117 \\
5011\end{array}$} \\
\hline FLUTY & ESCE IN & $2 D M E L$ & FVATIUN UF & \\
\hline (U, Ji: $A R^{2}$ & SAMPLER & H:T 1.54 & $U=0 . U \mathrm{~N} / \mathrm{SE}$ & EC AT $50: 1$ \\
\hline $\begin{array}{l}\therefore Z 1 \because: 1 T H \\
\angle H 6, E E S\end{array}$ & $\begin{array}{c}\text { EXPDSURE } \\
\text { GM-SEC /CU.M } \\
\times 1 O E+6\end{array}$ & $\begin{array}{c}\mathrm{L} / \mathrm{U} \\
S H C H . M\end{array}$ & $\begin{array}{c}E U / 2 \\
1 / S 2 . H\end{array}$ & $\begin{array}{l}\text { DISTANCE } \\
\text { METEKS }\end{array}$ \\
\hline ¿. 112.0 & $u$ & $3.344 t-10$ & $2.0 C 6 E-U Y$ & 7360 \\
\hline$\Leftrightarrow 11 \% .0$ & 11 & $1, .231, E-09$ & $3.2461-00$ & $7<10$ \\
\hline $11 \% .0$ & 94 & $4.6 \angle 7 L-\angle B$ & $2.7101-07$ & 1220 \\
\hline 113.0 & $24 t$ & $1.1 S 6 E-07$ & $0.935 F-117$ & 1190 \\
\hline $1 \therefore \ldots$ & 97 & $4,3,36 t-00$ & $2.72 d E-01$ & 7150 \\
\hline$=1=2.5$ & ou & $2.796 E-08$ & $1.677 \mathrm{E}-\mathrm{L} 7$ & 1120 \\
\hline$\therefore 124.0$ & 196 & $9.115 \mathrm{E}-U \mathrm{~B}$ & $5.46 Y E-U 7$ & 7100 \\
\hline$=1>0.0$ & 2169 & $1.007 E-06$ & $6.042[-00$ & $71 \cup 0$ \\
\hline $21 \div 6.0$ & 1202 & $5.5 \div 2 t-07$ & $3.34+t-06$ & 7100 \\
\hline $11=0.0$ & 1084 & $5.033 \mathrm{E}-07$ & $3.020 E-06$ & 7130 \\
\hline $61 \$ 2.0$ & 1084 & $5.033 \varepsilon-07$ & $3.020 E-06$ & 7150 \\
\hline 6134.0 & 39 & $1.832 E-J \theta$ & $1.099 E-07$ & 7200 \\
\hline 5136.0 & 0 & $7.269 E-11$ & $4.361 E-10$ & 7250 \\
\hline CKlis Sin IND & INTECRATEO = & $\begin{array}{l}7.264 E-04 \\
S E C / S O . M\end{array}$ & $\begin{array}{c}4.358 E-03 \\
1 / M\end{array}$ & \\
\hline
\end{tabular}

(KL:SSAINO) INTEGRATEOE 1.2H3E-03 7.7UUE-03 SECISO.H $\quad 1 / M$

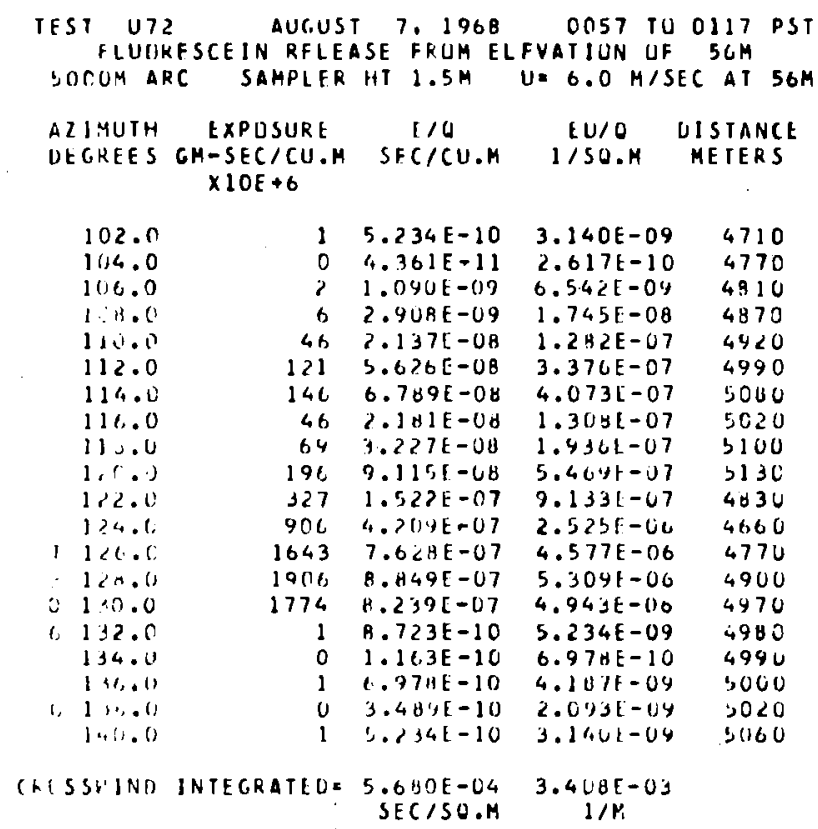


SAMPLING $400 M$ TO 220004 NO TUHER SAYPLING. ALL ARCS EMGRACE CRUSSHINO EXTENT DF TRACER OISTRIBUTIDN.

2NS DISPERSAL FOR 30 CONTINUOUS MIN. FLUURESCEIN DN FIRST 5 HIN. DFF IO MIN, THEN UN IS MIN. IFLUURESCEIN OM AT OLLOPST. OFF AT OLISPST. ON AT OISSPST AND DFF AGAIN AT O2IOPSI.I FLUDRESCEIN DISPERSAL DEVICE PRUBLEMS.

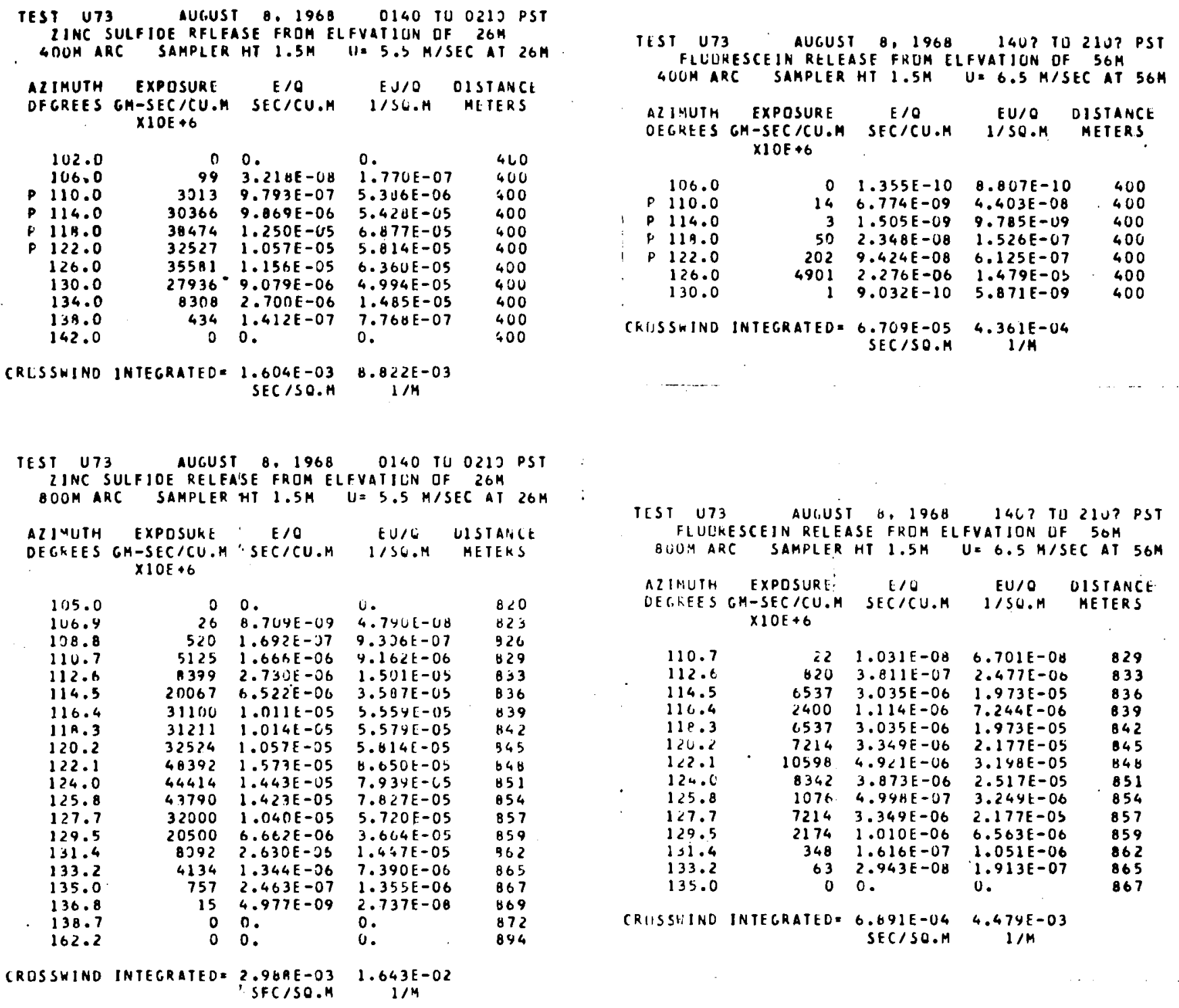




\begin{tabular}{|c|c|c|c|c|}
\hline \multirow{2}{*}{$\begin{array}{l}\text { TEST UT3 } \\
\text { ZINC SU } \\
120 O M \text { ARC } \\
\text { AZIMUTH } \\
\text { DEGKEES O }\end{array}$} & \multicolumn{2}{|c|}{$\begin{array}{l}\text { AUS,UST BO } 195 \mathrm{H} \\
\text { LFIUE RFLEASF FRUM }\end{array}$} & \multicolumn{2}{|c|}{ 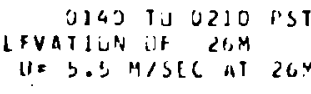 } \\
\hline & $\begin{array}{l}\text { EXPOSURE } \\
\text { GH-SEC/CU.M } \\
\times 1 O E+6\end{array}$ & $\begin{array}{c}\text { E/Q } \\
\text { SEC/CU.Y. }\end{array}$ & $\begin{array}{l}\operatorname{lu} / C \\
1 / S U .4\end{array}$ & $\begin{array}{l}\text { DISTANGL } \\
\text { METHRS }\end{array}$ \\
\hline 1 บ9.? & 1 & c. & 0. & 1200 \\
\hline 110.0 & 175 & $\because$ TCSE-0O & $3.1+18-U 1$ & 1200 \\
\hline 112.0 & 303 & 9.86 HE -60 & $3.4 a^{3}+7-61$ & $\$ 200$ \\
\hline 114.0 & 3557 & $9.935 t-37$ & $5.43,5 t-06$ & 1200 \\
\hline 116.0 & 3480 & $1.1311-26$ & $6.2611-00$ & 1236 \\
\hline $11 \mathrm{~B} .0$ & 10915 & $3.547 E-66$ & $1.4511-61$ & $12 v 0$ \\
\hline 120.0 & 12547 & $9.07 A E-06$ & $2.2431-63$ & 1200 \\
\hline 1.2 .0 & 9971 & $3.241 \mathrm{t}-06$ & $1.7 a c t-0 s$ & J 240 \\
\hline 124.0 & $9 \div 85$ & $3.713 t-06$ & $1.70 / \mathrm{L}-0 \mathrm{3}$ & 1260 \\
\hline$i, t .0$ & 11494 & 3. HS?E-J6 & $2.124 t-05$ & 1200 \\
\hline $11<n .3$ & 2137 & $6.9478-07$ & $3.6211-0 b$ & 1200 \\
\hline 139.2 & 1449 & $4.7 \because h E-U 7$ & C.DAQE-UG & 1200 \\
\hline 132.0 & 343 & $1.11+[-0]$ & $0.2411-07$ & 1200 \\
\hline 134.0 & y & $3.1411-04$ & $1.7200-00$ & 1200 \\
\hline 136.0 & 'j & o. & u. & $1<00$ \\
\hline CKIISSHIND & INIEGRATFD $=$ & $\begin{array}{l}9.007 \mathrm{~L}-04 \\
\text { stcksw.ti }\end{array}$ & $\begin{array}{l}4.91,45-0,3 \\
1 / M\end{array}$ & \\
\hline IEST 073 & aurius & 10.190 & $0140 \quad 111$ & 0212 \\
\hline ZIMC 5 & F. KFLF & ASE FRUM FE & LFVATIU: UF & 20.4 \\
\hline IACOP AR & SAMPIFR & $H T 1.5 \mathrm{H}$ & $\|=S . ל M / J F$ & $C$ C स T $20 \%$ \\
\hline AZ THUTH & EXPOSURI & t/u & $17 / 12$ & UISTARE \\
\hline DFCKEES & $\begin{array}{c}G M-S F C / C U \cdot M \\
\quad \times 1 U F+Q\end{array}$ & $S F C / C U . M$ & $1 / 520 \%$ & MrTEXS \\
\hline 104.3 & $\because$ & c. & u. & 1523 \\
\hline 109.5 & 120 & 4.107 & $2 .<3 \in[-0)]$ & itsol \\
\hline 110.4 & 120 & $3.924 E$ & $2.1545-67$ & is se \\
\hline 111.4 & 172 & $5.610 \mathrm{E}-0 \mathrm{~B}$ & $3.0 * 6 t-67$ & 1633 \\
\hline 112. & 367 & 2.81HE -07 & $1 . S 5 \cup E-0 G$ & $i \in 35$ \\
\hline 113.4 & 1756 & 5.70 de -67 & $3.13 \%-06$ & 1637 \\
\hline 314.4 & 3729 & $1.212 E-06$ & $6.6 D E E-06$ & 1630 \\
\hline 115.3 & 4691 & $1.521 E-00$ & $0.360 k-00$ & 1040 \\
\hline 116.3 & 12765 & $4.149 \mathrm{E}-J \mathrm{~S}$ & $2.2+2 E-05$ & 1341 \\
\hline 117.3 & 13956 & $4.536 E-i b$ & $.2 .475 F-05$ & 1643 \\
\hline 114.2 & 229 & $7.476, E-66^{\circ}$ & - 4. JuOE-US & 1644 \\
\hline 319.2 & 226 & 7.424 & $4.0 B 3 E-65$ & 1646 \\
\hline 120.2 & 27718 & $C .0 \cup 9 E-06$ & $4.9558-035$ & 1647 \\
\hline 121.2 & 3.3349 & $1.044 E-05$ & $5.401 F-05$ & 1649 \\
\hline 122 & 30433 & $1.002 t-35$ & $5.511 E-05$ & 1550 \\
\hline 123.1 & 21577 & $7.012 \mathrm{E}-\mathrm{Cb}$ & $3.057 \mathrm{~F}-05$ & 1552 \\
\hline 124.1 & & $8.185 E-06$ & $4.5 \partial 2 E-05$ & 1653 \\
\hline & 432 & 1.40 & $7.726 \mathrm{E}-05$ & 1655 \\
\hline & & 7.47 & 4 ic & \\
\hline & & 4.7 & 2.0 & \\
\hline 12 & 14 & 437 & $\begin{array}{l}x-0, \\
t-0,\end{array}$ & $\begin{array}{l}1030 \\
1359\end{array}$ \\
\hline 120.8 & $391 \mathrm{~A}$ & $1.274 E-06$ & $7.004 t-06$ & 1600 \\
\hline $1<9.7$ & 2211 & 7.18 & $3.953 \mathrm{E}-06$ & 1662 \\
\hline 130.6 & 740 & $2.40 H E-07$ & $1.324 \mathrm{E}-06$ & 1663 \\
\hline 6131.5 & 540 & $1.757 \mathrm{E}-07$ & $9.663 t-07$ & 1664 \\
\hline 14132.5 & 21 & $7.135 E-09$ & $3.924 F-08$ & 1665 \\
\hline 133. & 0 & & 0 . & 1367 \\
\hline CROSSWINO & INTEGRATEOE. & $\begin{array}{l}2.900 E-03 \\
\text { SEC/SQ.M }\end{array}$ & $\begin{array}{c}1.595 \mathrm{E}-02 \\
1 / M\end{array}$ & \\
\hline
\end{tabular}

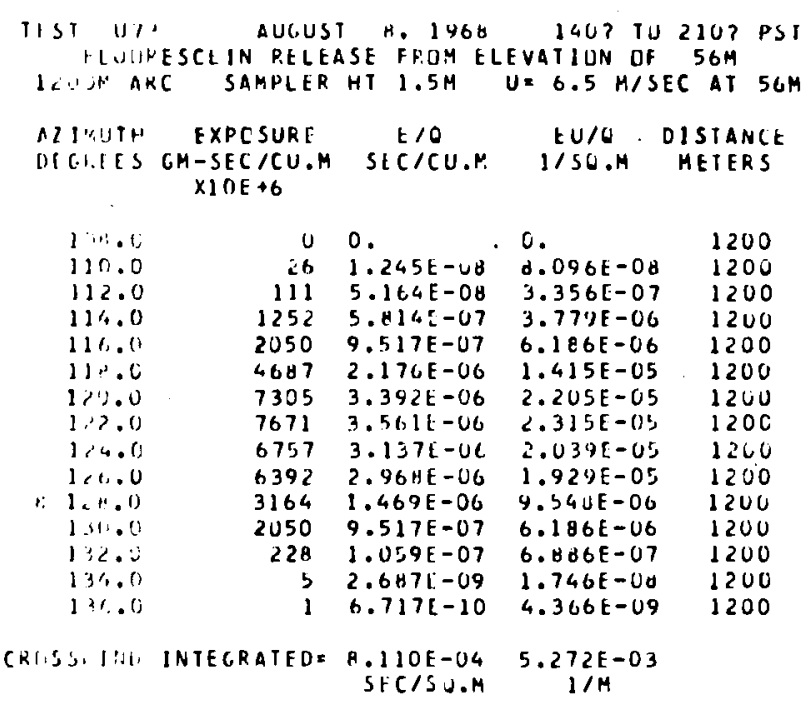
TEST UT: AULUST B, 1968 140? TU $210 ?$ PST TI. ULIKESCE IN RELEASE FRDM ELEVATIUN DF $56 \mathrm{M}$ 160 ARC SARPLER HT $1.5 \mathrm{M} U=6.5 \mathrm{M} / \mathrm{SEC}$ AT 5614

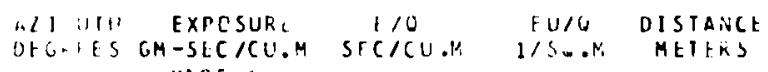 $\times 10 E+6$

\begin{tabular}{|c|c|c|c|}
\hline & ל. & 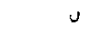 & c. \\
\hline & $1] 1.4$ & 7 & $3: 5,17 \bar{c}-19$ \\
\hline & 111.4 & 123 & $5.724 t-08$ \\
\hline & 112.4 & 633 & $2.441 E-07$ \\
\hline & 111.4 & 822 & $3.412 t-07$ \\
\hline & 114.4 & 769 & $3.573 t-07$ \\
\hline & 112.3 & 2823 & $1.311 E-06$ \\
\hline & 116.3 & 3219 & $1.445 E-06$ \\
\hline & 117.3 & 4311 & $2.002 E-06$ \\
\hline & $11 \pm .2$ & 6692 & $3.167 \mathrm{f}-06$ \\
\hline & 119.2 & 11305 & $5.249 E-06$ \\
\hline & $1<0.2$ & 8329 & $3.867 E-06$ \\
\hline • & $1 \therefore 1.2$ & 8180 & $3.748 E-06$ \\
\hline & 122.1 & 18349 & ค. $519 E-06$ \\
\hline & 123.1 & 13388 & $6.216 E-66$ \\
\hline & 124.1 & 14876 & $6.907 E-06$ \\
\hline & $1<5.0$ & 11900 & $5.525 \mathrm{E}-06$ \\
\hline & 126.0 & 7436 & $3.452 \mathrm{E}-06$ \\
\hline & $1<6.9$ & 4757 & $2.209 E-46$ \\
\hline & 127.8 & 6096 & $2.831 \mathrm{E}-06$ \\
\hline & 128.0 & 6692 & $3.107 E-06$ \\
\hline & 129.7 & 1156 & $5.369 E-07$ \\
\hline & 130.6 & 704 & $3.270 E-07$ \\
\hline E & 151.5 & 704 & $3.270 E-07$ \\
\hline$z$ & 132.5 & 0 & $4.3 d 6 E-10$ \\
\hline & 133.5 & 0 & 0. \\
\hline
\end{tabular}

J.

2. $201 E-30$ 1633 1635 $2.462 \mathrm{E}-06 \quad 1637$ $2.322 \mathrm{E}-00 \quad 1638$ H.519E-66 $164 \mathrm{~J}$ $9.717 \mathrm{E}-06 \quad 1641$ $1.301 E-05 \quad 1643$ $2.019 E-05 \quad 1644$ $3.412 \mathrm{E}-05 \quad 1646$ $2.513 E-05 \quad 1647$ $2.469 E-05 \quad 1649$ $5.537 E-05 \quad 1650$ 4. $040 E-05 \quad 1652$ $4.489 \mathrm{E}-05 \quad 1653$ $3.591 E-05 \quad 1655$ $2.244 E-05 \quad 1656$ $1.436 E-05 \quad 1658$ $1.840 E-U 5 \quad 1659$ $2.019 E-05 \quad 1660$ $3.490 E-06 \quad 1662$ $2.126 E=06 \quad 1663$ $2.126 E-06 \quad 1664$ $2.851 E-09 \quad 1665$

CRIISS:HIND INTEGRATED= 1.715E-03 1.11SE-02 1667 


\begin{tabular}{|c|c|c|c|c|}
\hline $\begin{array}{l}\text { PESI U7? } \\
\text { XNAC } \\
22004 \text { Af }\end{array}$ & $\begin{array}{l}3 \\
\text { SULF IDE RUES } \\
\text { RC SAMPLER }\end{array}$ & $\begin{array}{l}\text { T A } 1968 \\
\text { ASE FROM EL } \\
\text { HI } 1.5 \mathrm{M}\end{array}$ & $\begin{array}{r}0140 \text { TU } \\
\text { FVATIUN UF } \\
U=3.5 \mathrm{~m} / \mathrm{s}\end{array}$ & 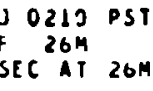 \\
\hline $\begin{array}{l}\text { AZIMUTH } \\
\text { DEGREES }\end{array}$ & $\begin{array}{c}\text { EXPOSURE } \\
\text { CH }-5 E C / C U, H \\
\times 1 O E+6\end{array}$ & $\begin{array}{c}\text { K/O } \\
\text { SEC/CU.M }\end{array}$ & $\begin{array}{c}E U / O \\
1 / S O . M\end{array}$ & $\begin{array}{l}\text { DISTANCE } \\
\text { METERS }\end{array}$ \\
\hline $\begin{array}{r}110.0 \\
7112.0 \\
114.0 \\
116.0 \\
118.0 \\
F \quad 120.0 \\
122.0 \\
124.0 \\
126.0 \\
128.0 \\
130.0 \\
132.0 \\
0 \\
134.0\end{array}$ & $\begin{array}{r}0 \\
4 \\
367 \\
447 \\
2621 \\
7111 \\
7210 \\
2805 \\
2930 \\
971 \\
65 \\
15 \\
0\end{array}$ & $\begin{array}{l}0 . \\
1.360 E-09 \\
1.194 E-07 \\
1.453 E-07 \\
8.518 E-07 \\
2.311 E-06 \\
2.343 E-06 \\
9.119 E-07 \\
9.523 E-07 \\
3.156 E-07 \\
2.121 E-0 B \\
5.167 E-09 \\
0 .\end{array}$ & $\begin{array}{l}0 . \\
7.478 E-09 \\
6.566 E-07 \\
7.994 E-07 \\
4.695 E-06 \\
1.271 E-05 \\
1.209 E-05 \\
5.016 E-06 \\
5.230 E-06 \\
1.736 E-06 \\
1.167 E-07 \\
2.842 E-00 \\
0 .\end{array}$ & $\begin{array}{l}2200 \\
2200 \\
2200 \\
2200 \\
2200 \\
2200 \\
2200 \\
2200 \\
2200 \\
2200 \\
2200 \\
2200 \\
2200\end{array}$ \\
\hline CRUSSHINO & INTEGRATFO & $\begin{array}{l}6.127 E-04 \\
\text { SEC /SO.M }\end{array}$ & $\begin{array}{c}3.370 E-03 \\
1 / M\end{array}$ & \\
\hline
\end{tabular}

TEST U73 AURUST B. 1968 O14J TU 0210 PST ZINC SULFIDE RELEASE FRDM ELFVATION OF $26 \mathrm{M}$ $3200 M$ ARC SAMPLER HT $1.5 \mathrm{M} U=5.5 \mathrm{M} / \mathrm{SEC}$ AT $26 \mathrm{M}$ AZIMUTH EXPOSURE E/O EU/O DISTANCE DEGKFES GH-SEC/CU.M SEC/CU.M I/SG.M METERS $\times 10 E+6$

\begin{tabular}{|c|c|c|}
\hline 6.8 & $u$ & 0. \\
\hline 111.7 & $u$ & 0. \\
\hline 112.7 & 35 & $1.144 t-0$ \\
\hline 113.7 & BO & 2.6 USE-Jo \\
\hline 114.6 & $53 \%$ & 1.P.86, -67 \\
\hline 115.6 & $12 \mathrm{hS}$ & $4.113 t-07$ \\
\hline 113.6 & 2119 & $6.487 E-07$ \\
\hline 117.6 & 3616 & $1.175 t-06$ \\
\hline 114.6 & 7114 & $2.312 t-00$ \\
\hline 119.5 & 10332 & $3.358 \mathrm{E}-3 \mathrm{~S}$ \\
\hline 120.5 & 13710 & $4.45 \mathrm{RE}-\mathrm{UB}$ \\
\hline 121.5 & 12552 & $4.079 E-06$ \\
\hline 122.4 & 12151 & $3.949 E-06$ \\
\hline 123.4 & 9518 & $3.093 E-06$ \\
\hline 124.4 & 6442 & $2.224 \mathrm{E}-\mathrm{UC}$ \\
\hline 125.4 & 3871 & 1.25 AE-0S \\
\hline 126.3 & 2615 & $8.499 E-07$ \\
\hline $1<7.3$ & 505 & $1.643 E-07$ \\
\hline 128.3 & 110 & $3.143 E-08$ \\
\hline 129.3 & 5 & $1.767 E-09$ \\
\hline 130.3 & 0 & 0. \\
\hline & & \\
\hline
\end{tabular}

3227

c. 3235

$6.3171-08 \quad 3237$

$1.4331-07$ 3238

$1.0311-6.63240$

$2.26<E-06 \quad 3241$

$3.746 E-06 \quad 3243$

e. $465 \mathrm{~F}-06 \quad 3245$

$1.272 \mathrm{E}-\mathrm{CS} \quad 3246$

$1.647 E-05 \quad 3246$

$2.4521-0\rangle \quad 3249$

$2.244 E-05 \quad 3251$

$2.172 E-05 \quad 3252$

$1.7 U 1 E-05 \quad 3253$

$1.223 \mathrm{E}=05 \quad 3255$

$6.919 E-06 \quad 3256$

$4.674 E-06 \quad 3258$

$4.038 \mathrm{E}-07 \quad 3259$

$2.114 \mathrm{E}-07 \quad 3261$

$9.714 E-0 Y \quad 3262$

0.3278

CRLSSHIND INTEGRATEUE $1.559 E-03$ 8.376E-U.3

SEC/SU.M IIM

TEST U73 AUCUST B. 1960 O140 TU 0210 PST IINC SULFIDE RFLEASE FRUM ELFVATIUN UF $26 \mathrm{M}$ $5000 M$ ARC SAMPLER HT $1.5 \mathrm{M}$ U $3.5 \mathrm{M} / \mathrm{SEC}$ AT $26 \mathrm{M}$

AZIMUTH EXPOSURE L/U EU/O DISIANCE DECREES GA-SEC/CW.M SECICU.M I/SJ.A METERS $\times 10 E+6$

\begin{tabular}{|c|c|c|c|c|}
\hline 112.0 & 0 & 0 . & c. & 4990 \\
\hline 114.0 & 4 & $1.4 \times(1,1-1) 4$ & $0.2261-0 y$ & , 080 \\
\hline 116.0 & 51 & $1.659 \mathrm{E}-64$ & $4.123[-11 d$ & $20<0$ \\
\hline $11 \mathrm{H} .0$ & 596 & $1.93+t-37$ & $1.60,6 t-60$ & 5100 \\
\hline 120.0 & 612 & $1.991 E-\cup 7$ & $1 .(1+2)-116)$ & 2130 \\
\hline 122.0 & 2700 & H. 77AE- 07 & $4.82 d L-06$ & 4630 \\
\hline & $36 ?$ & $1.177 \mathrm{E}-07$ & $6.4761-07$ & 4660 \\
\hline 0 & 238 & $7.736 \mathrm{E}-\mathrm{UH}$ & $4.2595-07$ & 477 \\
\hline 128.0 & 17 & $5.867 E-07$ & $3.216 E-00$ & 4700 \\
\hline 30 & 0 & $2.719 E-10$ & OY & 497 \\
\hline
\end{tabular}

CRISSAIND INTECRATEUE=2.544K-U4 1.3991-03

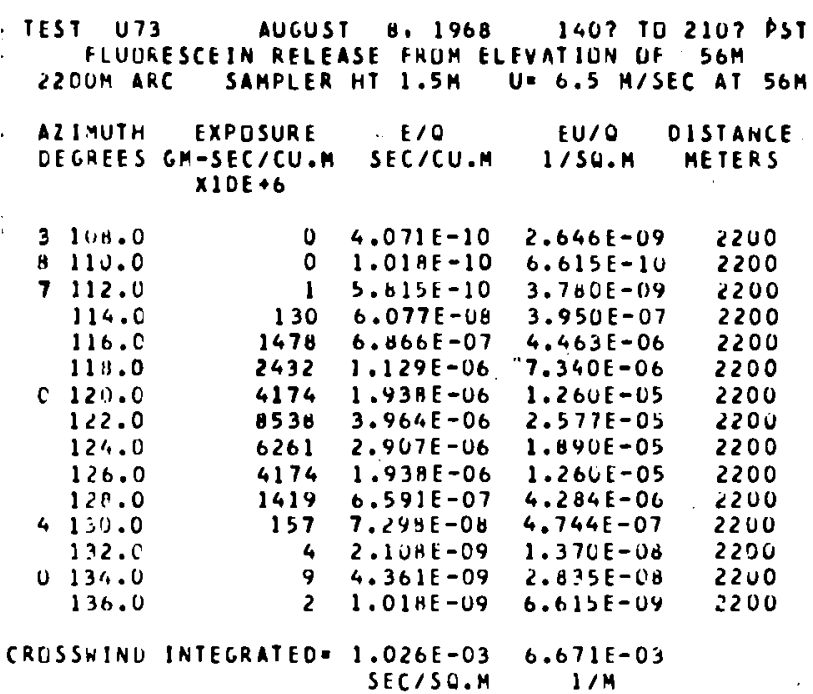

TEST U7S AUGUST 8,1968 140? TU 210 ? PST FLULIRESCE IN RELEASE FROM ELEYATION OF $56 \mathrm{M}$ $320.3 M$ ARC SAMPLER HT $1.5 M$ U 6.5 M/SEC AT $56 M$ AZIMUTH EXPOSURE
DEGREES GM-SEC/CU.M SEC/CU.M IJSO.M OISTARCE $\times 1 O E+6$

\begin{tabular}{|c|c|c|c|c|}
\hline 112.7 & 5 & $2: 72 \mathrm{LL}-U \mathrm{Y}$ & $1.7715-08$ & 3237 \\
\hline 113.7 & $\theta$ & 3:91AE -04 & $2.547 \mathrm{~F}-\mathrm{Cd}$ & 3238 \\
\hline 114.6 & 230 & $1: 106 E-67$ & $7.180[-07$ & 3240 \\
\hline 115.6 & 8119 & $3: 759 E-U 7$ & $2.443 t-06$ & 3241 \\
\hline 116.6 & 1455 & $6: 759 E-67$ & $4.394 E-00$ & 3243 \\
\hline 117.6 & 3093 & $1.436 \mathrm{E}-06$ & $9.335 E-06$ & 3245 \\
\hline $11^{n} \cdot 6$ & 2891 & $1.342 \mathrm{E}-0 \mathrm{t}$ & $8.726 E-06$ & 3246 \\
\hline 110.5 & 4170 & $1: 936 E-06$ & $1.250 E-05$ & 3240 \\
\hline 120.5 & 5179 & $2.405 E-06$ & $1.563 \mathrm{E}-0 \mathrm{~S}$ & 324.9 \\
\hline 121.5 & 7400 & $3: 436 E-06$ & $2.233 \mathrm{E}-0 \mathrm{~S}$ & 3251 \\
\hline 122.4 & 6727 & $3.123 E-06$ & $2.03 \cup E-05$ & 3252 \\
\hline 123.4 & 5830 & $2.707 E-06$ & $1.759 \mathrm{E}-0 \mathrm{~S}$ & 3253 \\
\hline 124.4 & 8970 & $4.165 E-06$ & $2.707 t-05$ & 3255 \\
\hline 125.4 & 6279 & $2.915 E-06$ & $1.095 E-05$ & 3256 \\
\hline 126.3 & 2555 & $1.186 t-06$ & $7.711 E-06$ & 3258 \\
\hline 1.27 .3 & 962 & $4.40 \mathrm{HE}-07$ & $2.904 E-06$ & 3259 \\
\hline 124.3 & 103 & $4.810[-08$ & $3.127 E-07$ & 3261 \\
\hline $1<0.3$ & 3 & $1.736 E-09$ & $1.12 \forall E-08$ & 3262 \\
\hline C.SSWINO & INTEGRATED= & $454 \mathrm{E}-03$ & -03 & \\
\hline
\end{tabular}

TEST U73 AUGUST 8,1968 140? TU 210? PST FLUIIRESCEIN RELEASE FRUM ELFVATIUN UF 5 LM SNOJM AKC SAMPLER HT $1.5 \mathrm{M} U=6.5 \mathrm{M} / \mathrm{SEC}$ AT $56 \mathrm{M}$ $\begin{array}{lcc}\text { AZIHUTH EXPOSURE } & \text { E/O } & \text { EU/O DISTANCE } \\ \text { DEGHEES GM-SEC/CU.H SECICU.M } & \text { I/SU.M METERS }\end{array}$ $\times 10 E+6$

\begin{tabular}{|c|c|c|c|c|}
\hline 110.0 & 0 & $2.908[-1]$ & $1.89 \mathrm{JE}-1 \mathrm{U}$ & $49<0$ \\
\hline 112.0 & 6 & $3.053 \mathrm{E}-\mathrm{CQ}$ & $1.984 \mathrm{E}-00$ & 4990 \\
\hline 114.0 & 0 & $3.198 E-10$ & $2.07 Y E-0 ?$ & 5080 \\
\hline $11 \%: 0$ & 31 & $1,48 \triangle E-O B$ & $Y .63 Y E-U U$ & .5020 \\
\hline $11 . .0$ & 827 & $3: 842 \mathrm{E}-07$ & $2.49 \forall t-06$ & .5100 \\
\hline 17.0 & 2109 & $1.007 E-06$ & $6.546 \mathrm{E}-06$ & 5130 \\
\hline $1<2.6$ & 2432 & $1.129 E-06$ & $7.339 E-66$ & 4830 \\
\hline 164.01 & 1971 & $9.154 E-07$ & $5.950 \mathrm{E}-06$ & 4060 \\
\hline $1,6.0$ & 1643 & $7.62 B E-07$ & $4.930[-06$ & 4770 \\
\hline $1, \cdots 0$ & 216 & $1.003 E=07$ & $6.520 E-07$ & 4900 \\
\hline $1+1.0$ & 7 & $3.634 t-09$ & $2.362 \mathrm{E}-08$ & 4970 \\
\hline $1=2.0$ & 1 & $42052 E-10$ & $3.024 E-09$ & 4980 \\
\hline $13 \pi . n$ & 0 & $4.071 E-10$ & $2.640 E-09$ & 4990 \\
\hline & & $\begin{array}{l}7.362 E-04 \\
\text { SECISO.M }\end{array}$ & $\begin{array}{c}4.785 t-03 \\
1 / M\end{array}$ & \\
\hline
\end{tabular}




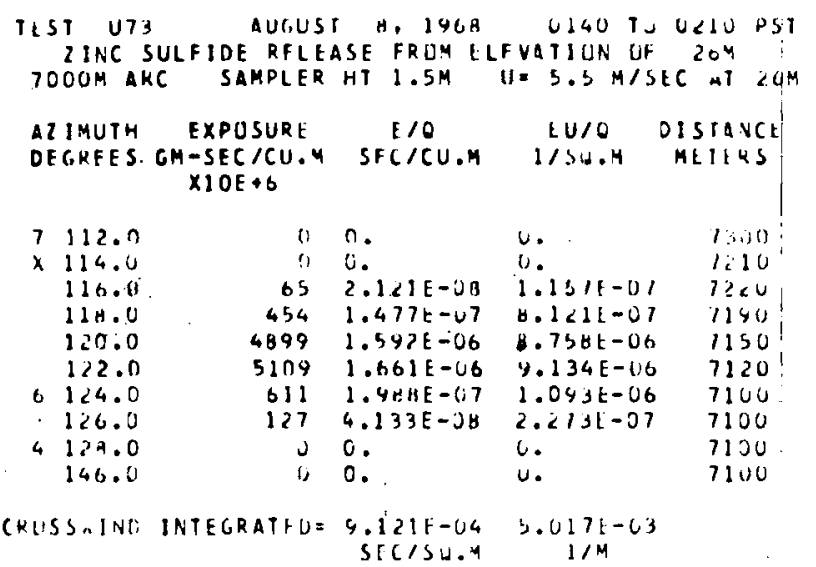

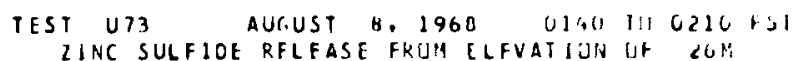

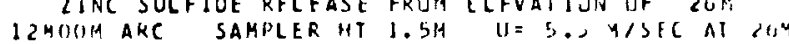
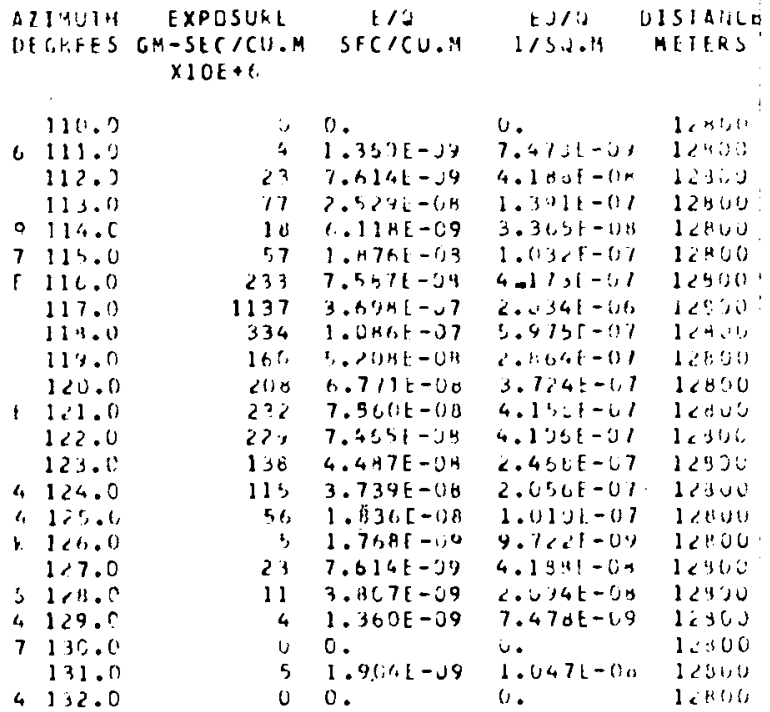

0.

U. $1, x+1,3$

1.301t-01 12H:10

3. $3(., 5 t-1) B \quad 1 \angle H_{U}$

1.03, $5-07$ 12400

$4-1,1-0,1<500$.

$2.4341-40 \quad 1<0)$

$8,475[-117$ 1<4,

$\therefore 1: 040-01$ dates:

$3.7 \div 4 t-61 \quad 1<850$

4.11,i-el ledu'

$4.1030-01$ 1 3030

$2.456 E-27 \quad 12930$

2.05GE-01 12300

$1.0131-07 \quad 1<140$

9.7<21-0\% 1<म00

$4.1941=0 \times 1<360$

$20.04 t-6 \theta \quad 12930$

$7.47 d \mathrm{t}-69$ lis 40

1.04 $71-0,300$

i.

$1<310$

CROSSHIND INIEGRATFD= 2.?35E-114 $\quad 1.224 E-13$

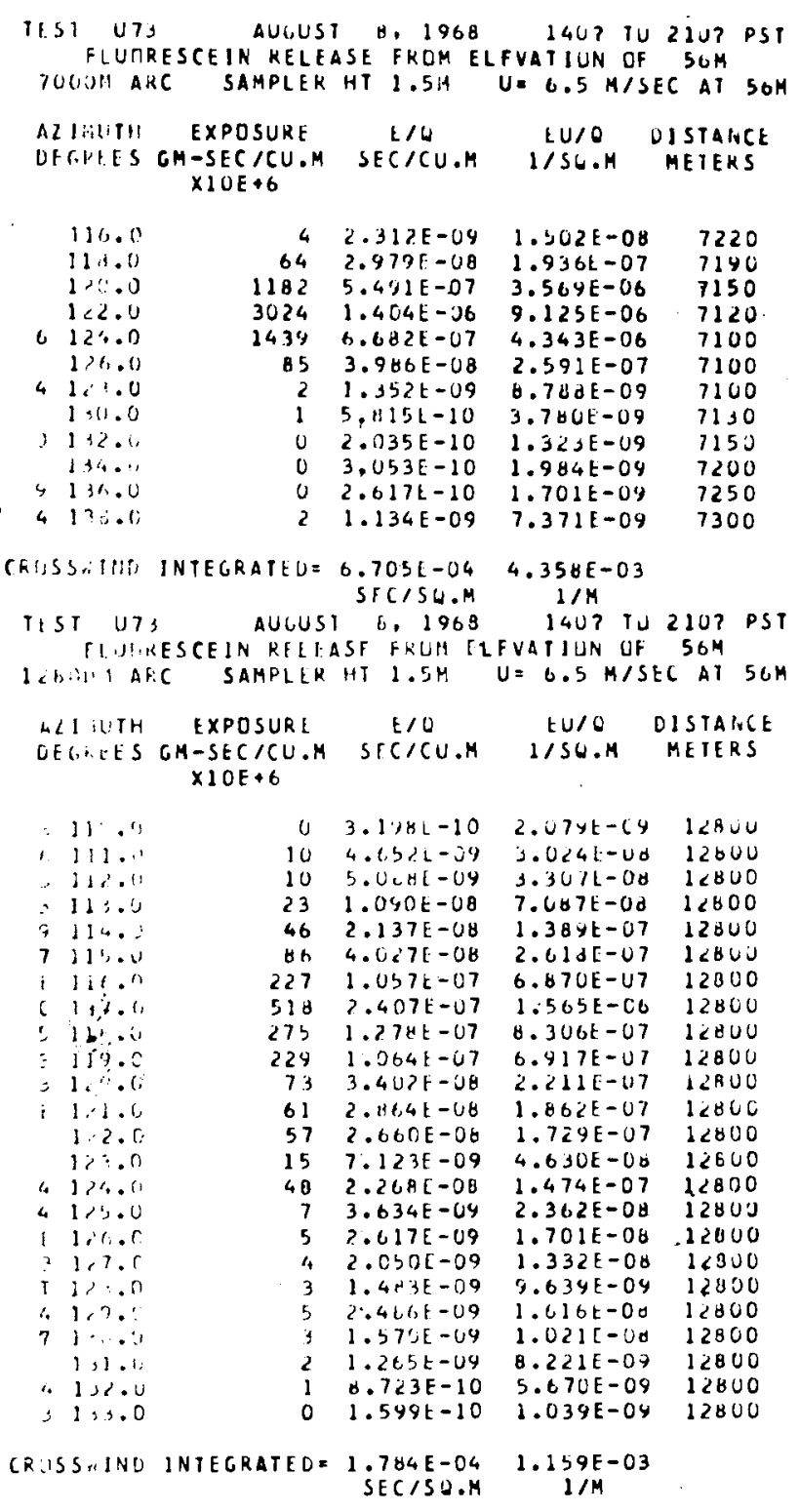


SAMPLING $400 M$ TO 7HOOY. NO TOWER SAMPLING. TRACEK SKEWEU TO NOKTH.

ALL AKCS EMBRACE CROSSHINO EXTLNT. OF TRACER.

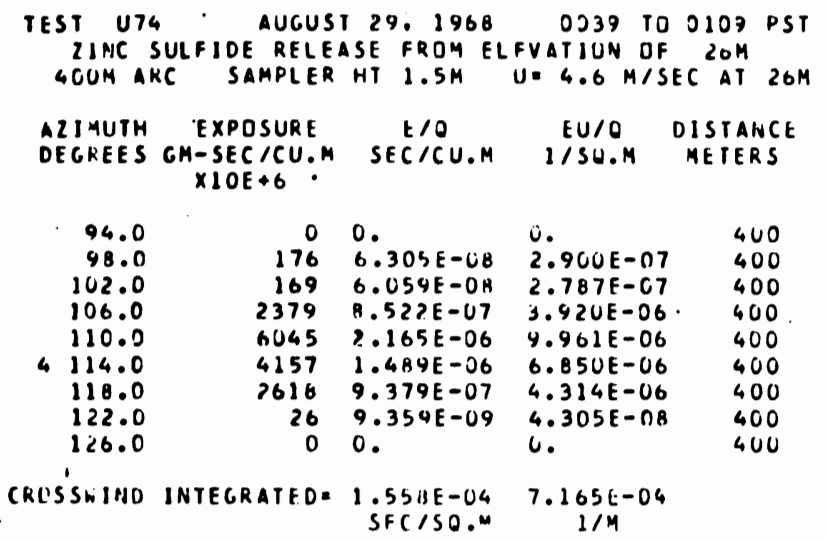

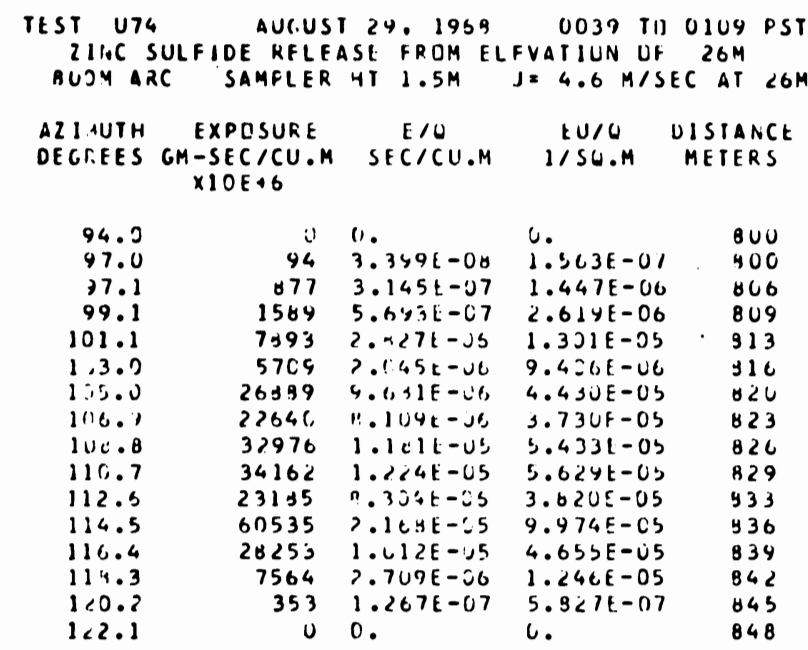

CRISSAIND INTEGRATFU = ?.4\%BE- 33 1.14YE-02

SFC/SO.4 $1 / 4$

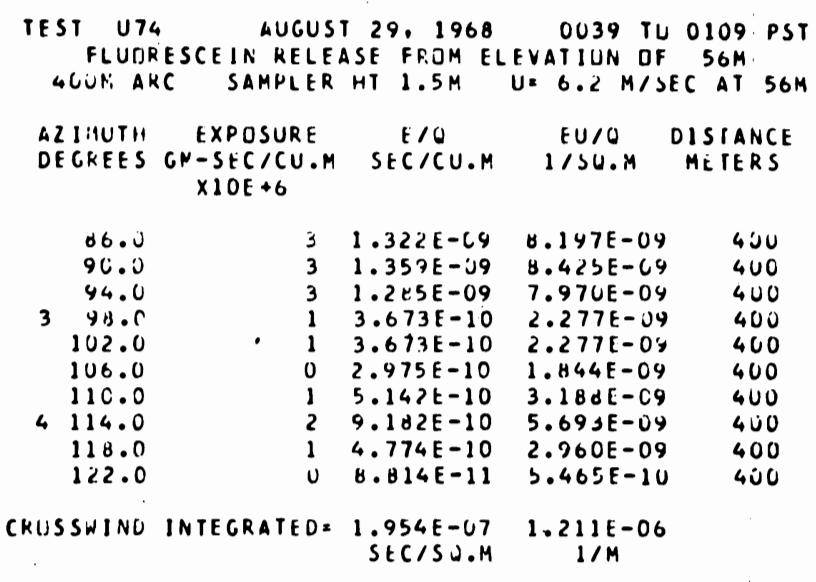

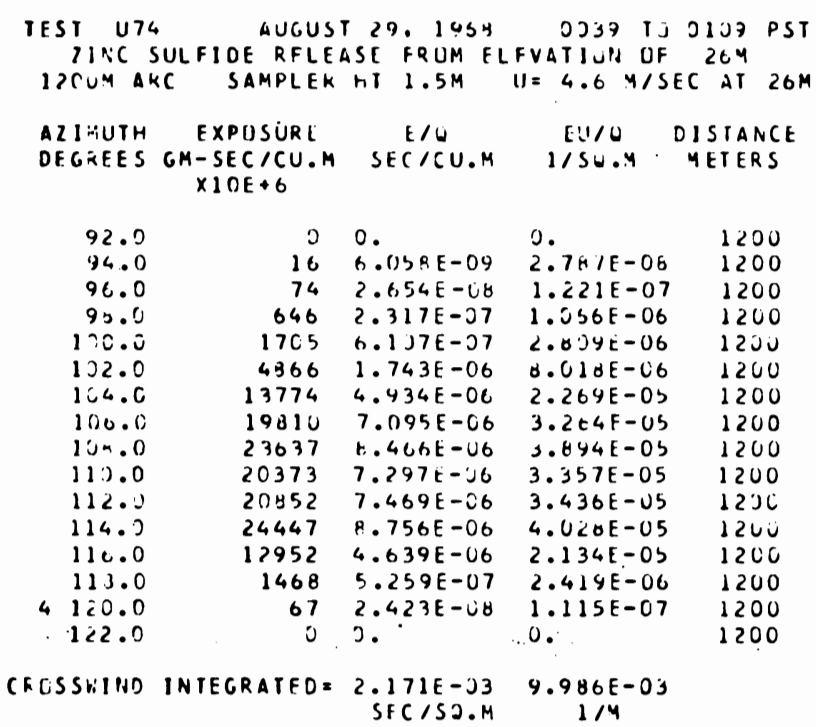

TEST U74 AUCOSST 29.1368 UO39 TJ OLOS PST FLIIIIRESCEIN RELEASE FRUM ELEVATIUN OF $56 \mathrm{M}$ GUUH AKC SAMPLER HT $1.54 \quad U=6.2$ M/SEC AT $56 M$ ALIAUTH EXPCSURI. FIU
UFCKEES GM-SECICU.M SHLICU.M IUIO DISTANCE
XIUE TO

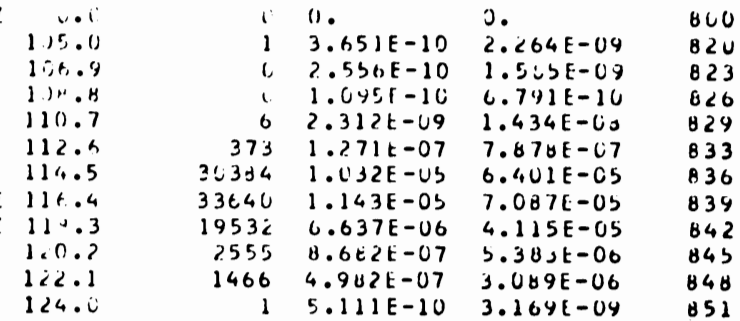
CRISSSIINU INTEGRATLU= B.310E-O4 5.157E-03 SEC/SG.M $1 / M$

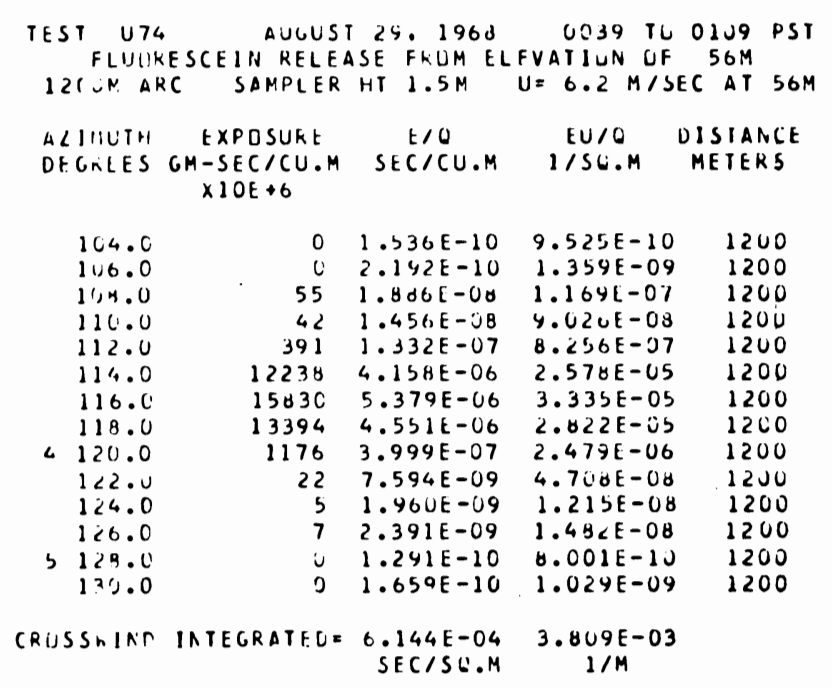




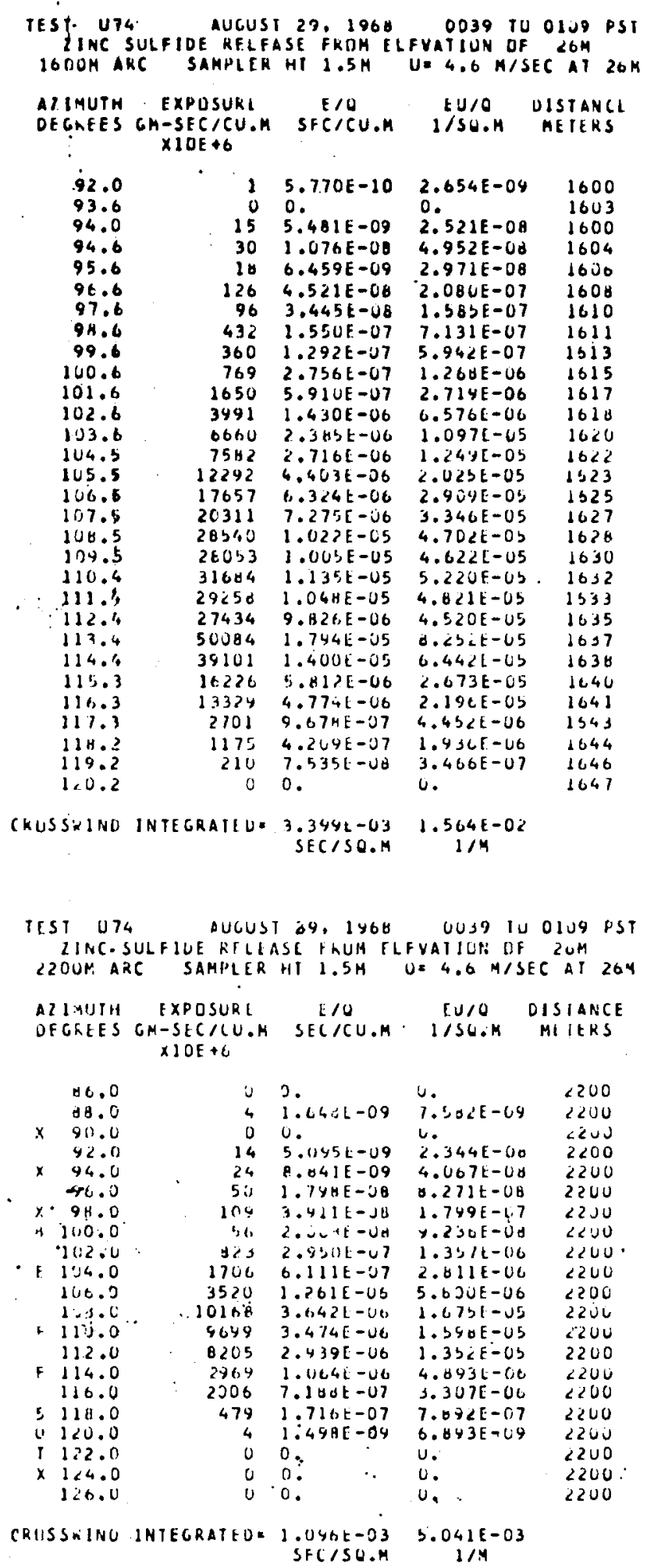

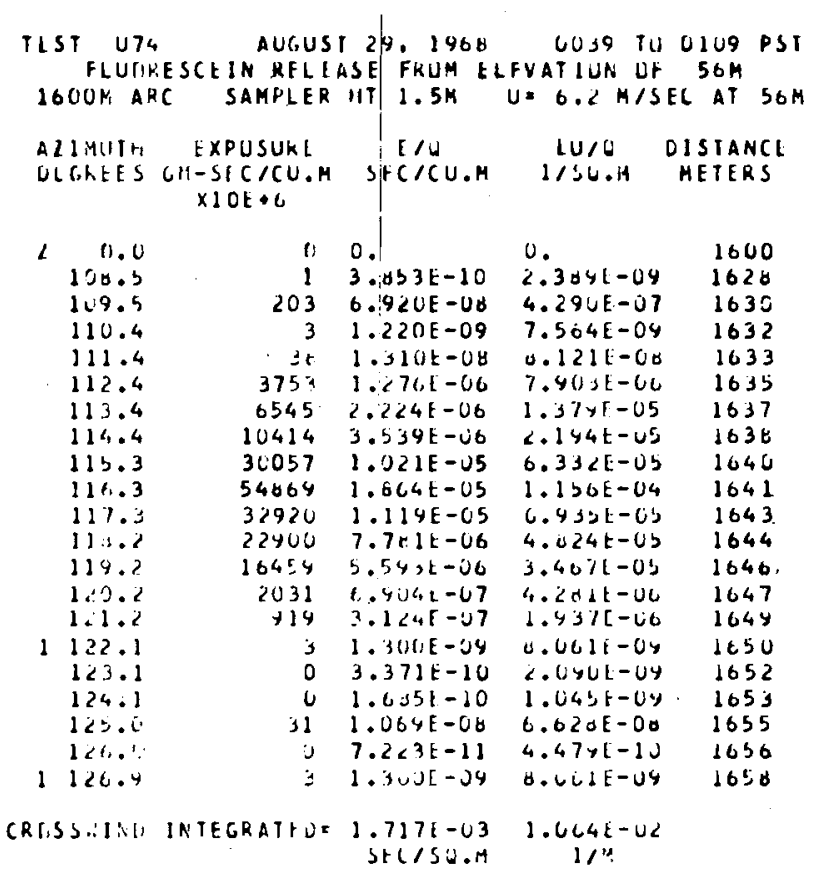

CRLSSHIAE INTEGRATED= 6.789E-04 4.209E-03

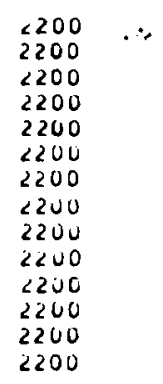




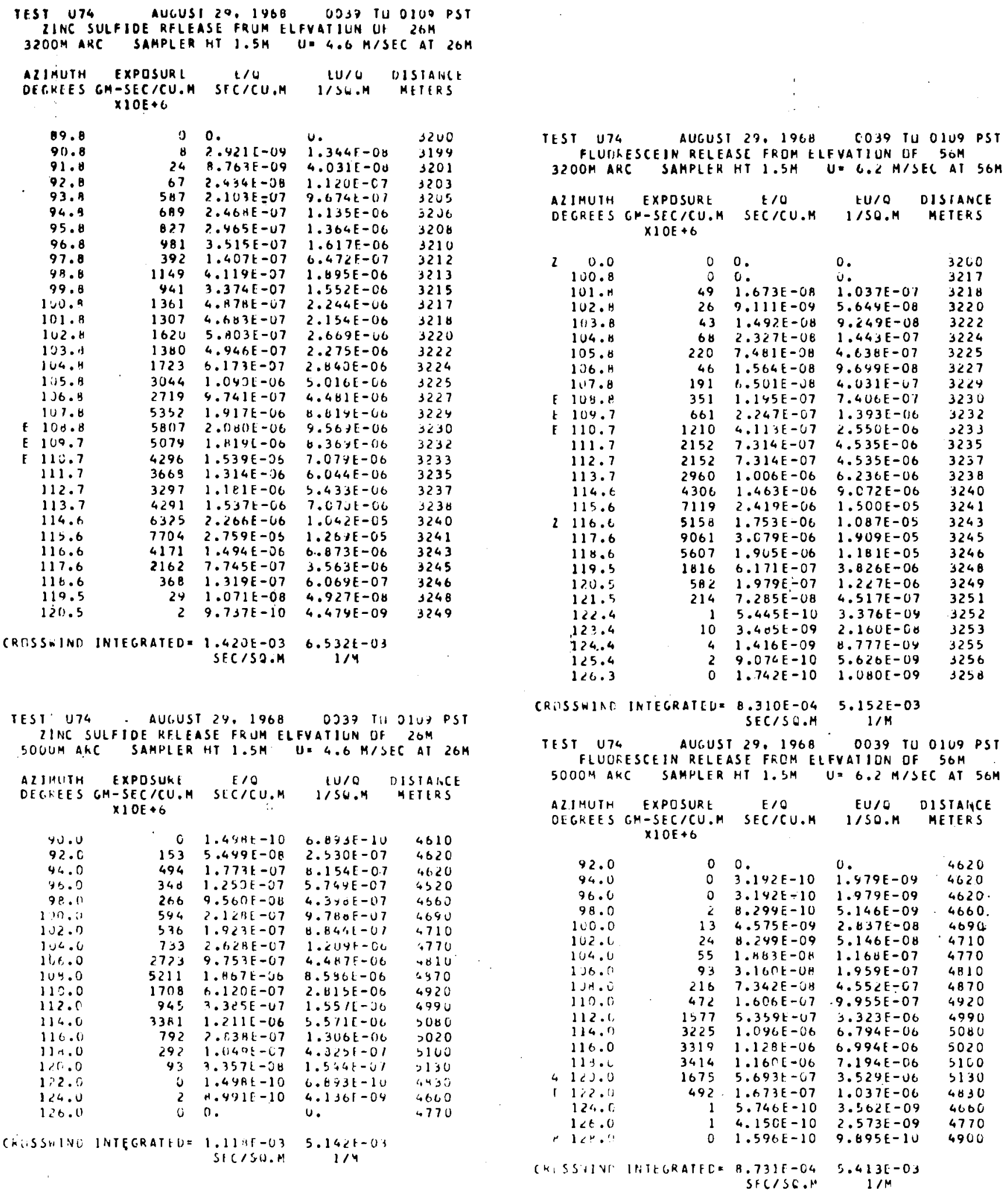




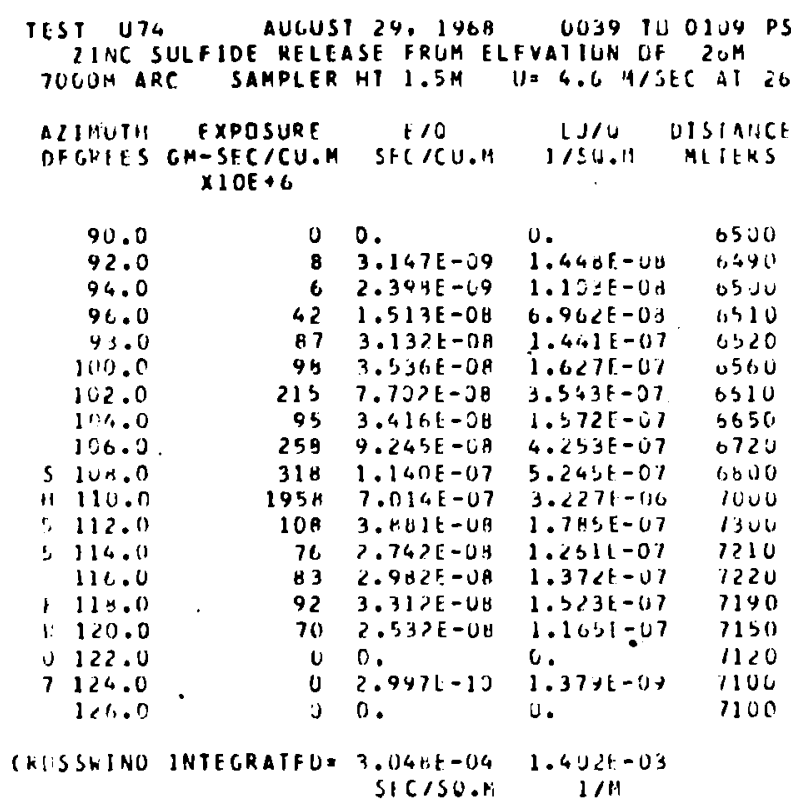

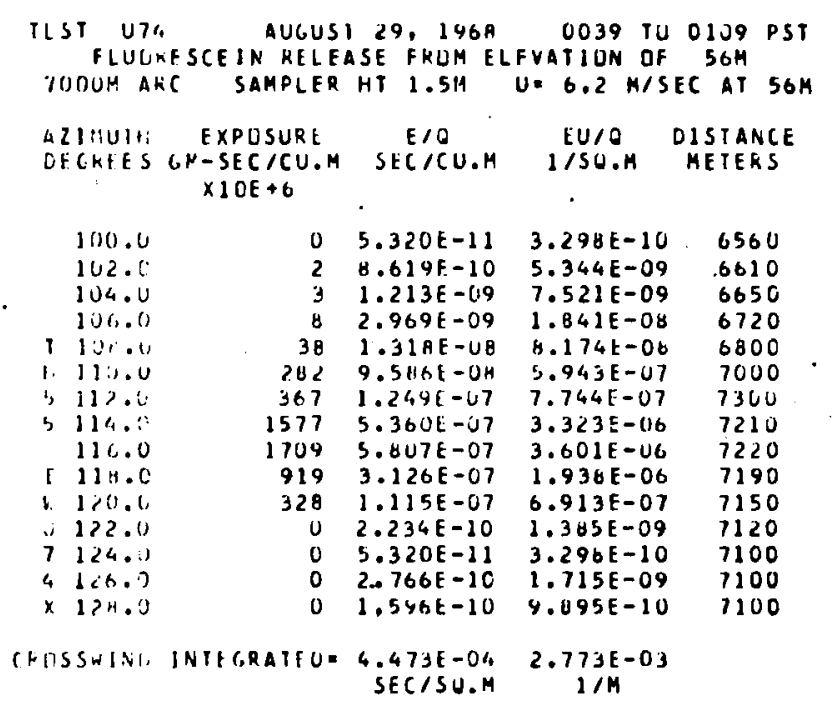


SAMPLINC 4OOM TO $12800 M$, BUT NO TRACLR OBSERVED AT $12000 \mathrm{M}$. NU TLEER SAMPLINE.

500OM AND 700OM ARCS TRUNCATED ON NORTH. NINU SPEED MEASUREU AT 12 GOUM ARC AVERAGEU ABUUT O.L M/SEC AT I. SM ELEVATION. RELATIVELY LARGE DIRECTIUN SHEAR HITH ELEVATION (AGUUT 20 OEGREES FRUM ISM TU GIM).

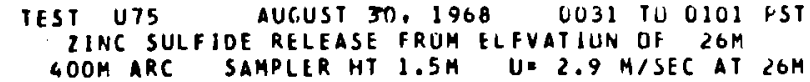

\begin{tabular}{|c|c|c|c|c|}
\hline $\begin{array}{l}\text { AZIMUTH } \\
\text { DFGREES }\end{array}$ & $\begin{array}{l}\text { EXPDSURE } \\
\text { CM-SEC /CU.M } \\
\times 1 O E+6\end{array}$ & $\begin{array}{c}E / O \\
S E C / C U . M\end{array}$ & $\begin{array}{l}E U / O \\
1 / S O \cdot M\end{array}$ & $\begin{array}{l}\text { DISTANCE } \\
\text { METERS }\end{array}$ \\
\hline $\begin{array}{l}62.0 \\
66.0 \\
70.0 \\
74.0 \\
78.0 \\
32.0 \\
56.0 \\
90.0 \\
94.0\end{array}$ & $\begin{array}{r}5 \\
86 \\
594 \\
13389 \\
2980 \\
1273 \\
3083 \\
460 \\
0\end{array}$ & $\begin{array}{l}2.052 E-09 \\
3.078 E-08 \\
2.114 E-07 \\
4.756 E-06 \\
1.059 E-06 \\
4.525 E-07 \\
11.095 E-06 \\
3.057 E-07 \\
0 .\end{array}$ & $\begin{array}{l}5.951 E-09 \\
8.926 E-0 B \\
6.129 E-07 \\
1.379 E-05 \\
3.071 E-06 \\
1.312 E-06 \\
3.176 E-06 \\
8.867 E-07 \\
0 .\end{array}$ & $\begin{array}{l}400 \\
400 \\
400 \\
400 \\
400 \\
400 \\
400 \\
400 \\
400\end{array}$ \\
\hline JSSHINO & INTEGRATED & $\begin{array}{l}2.210 E-O 4 \\
\text { SEC/SO.M }\end{array}$ & $\begin{array}{c}6.40 \mathrm{UE}=04 \\
1 / \mathrm{M}\end{array}$ & \\
\hline
\end{tabular}

TEST UT5 AUCUST 30,1968 OOSOI TO 0101 PST

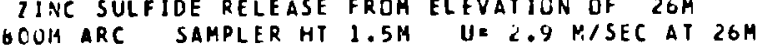
$\begin{array}{llll}\text { LIIHUTH EXPOSURE } & : F / O & \text { EU/O DISIARCE } \\ \text { DEGKEES GP-SECICU.M SEC/CU.H IISO.M HETERS }\end{array}$ $\times 10 E+6$

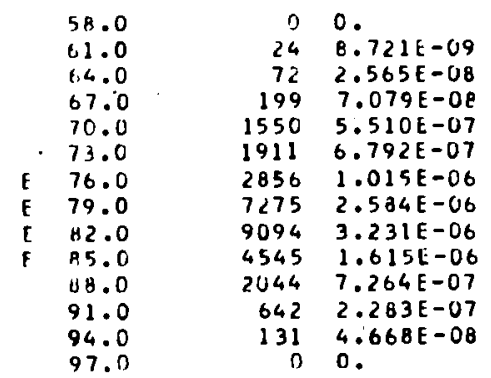

CROSSIIND INTEGRATED $=4.516 E-04$ 1.31UE-03

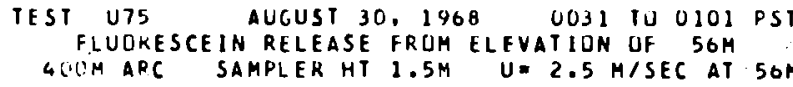

AZIMUTH EXPCSURL E/O CU/O DISTANCE

DEGKEES GM-SEC/CU.M SEC/CU.M I/SO.M METERS $\times 10 E+6$

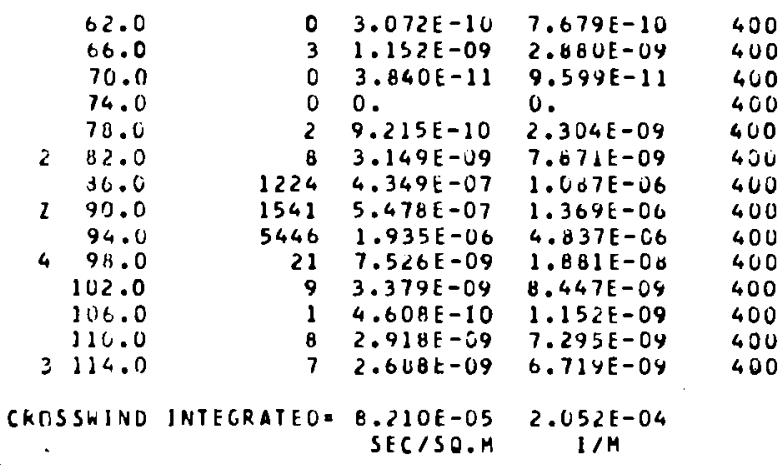

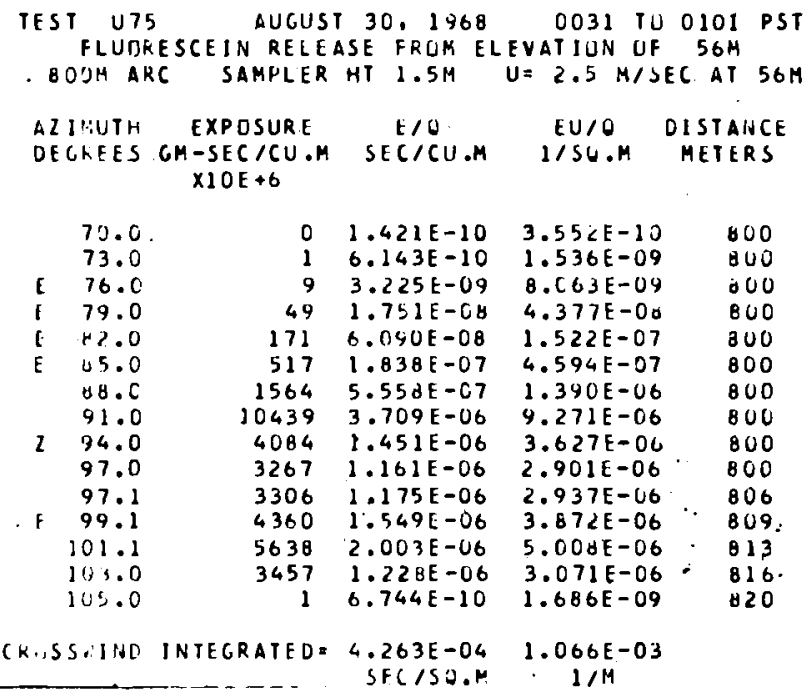




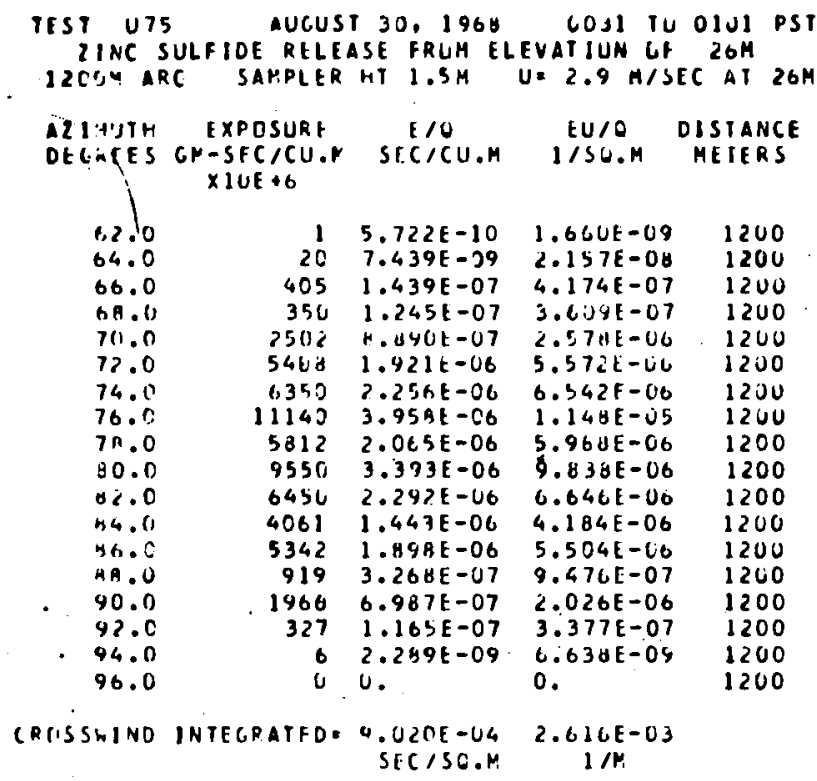

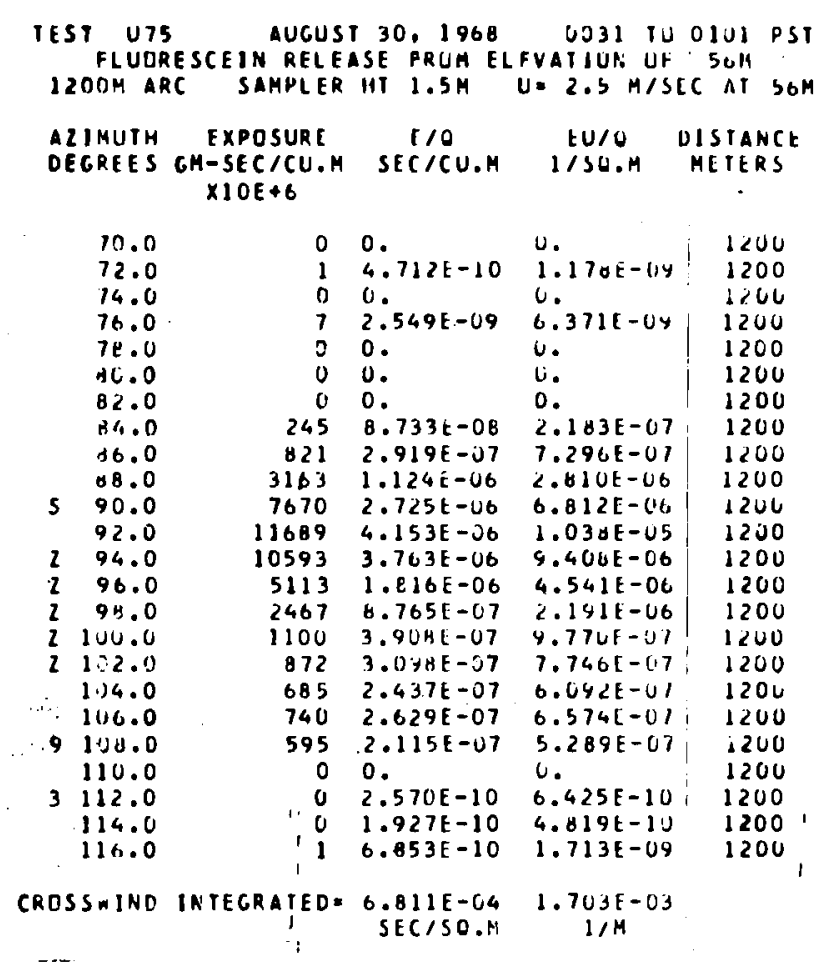

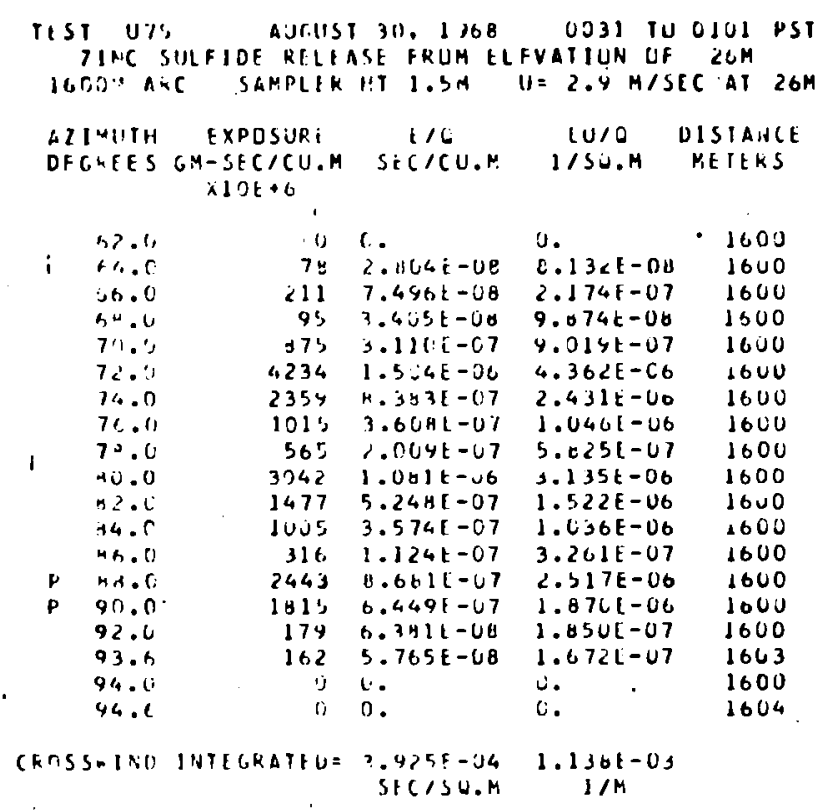

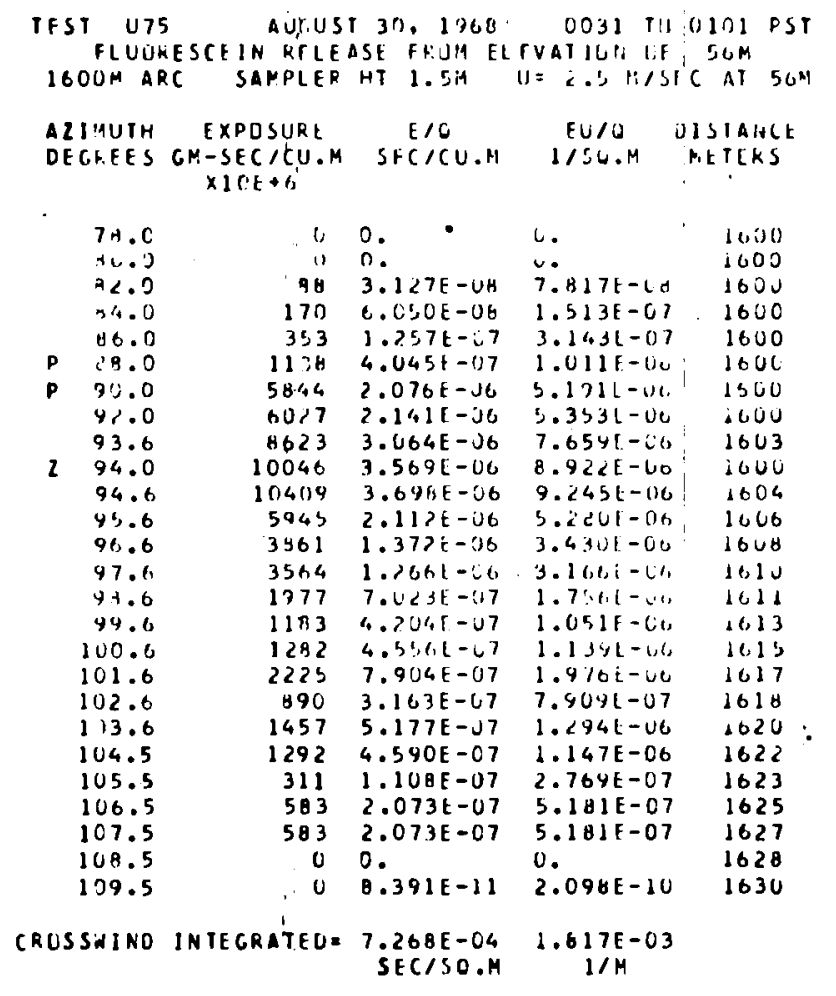




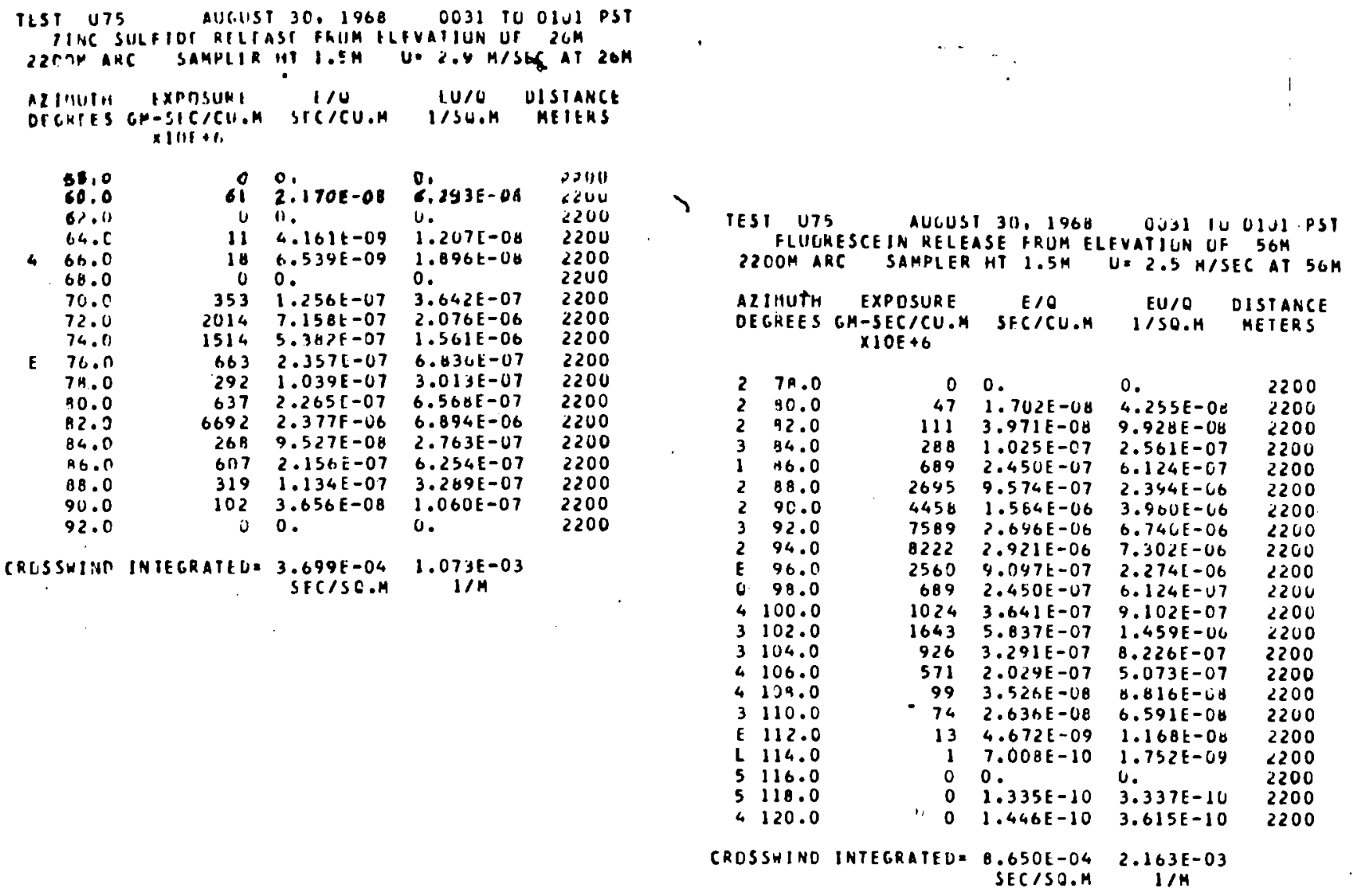

TEST U75 AUCUST 30, 1968 OO31 IU OIOI PST

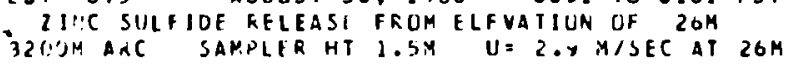

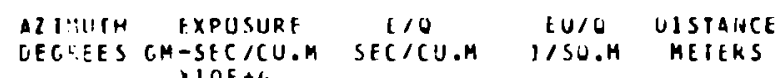
$1.1 \mathrm{OE}+6$

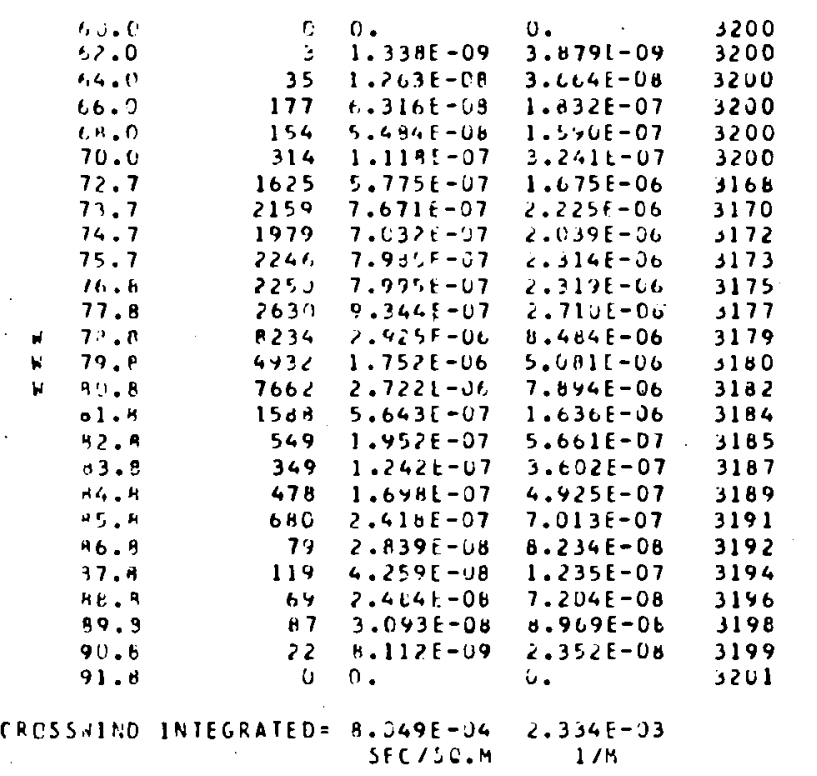

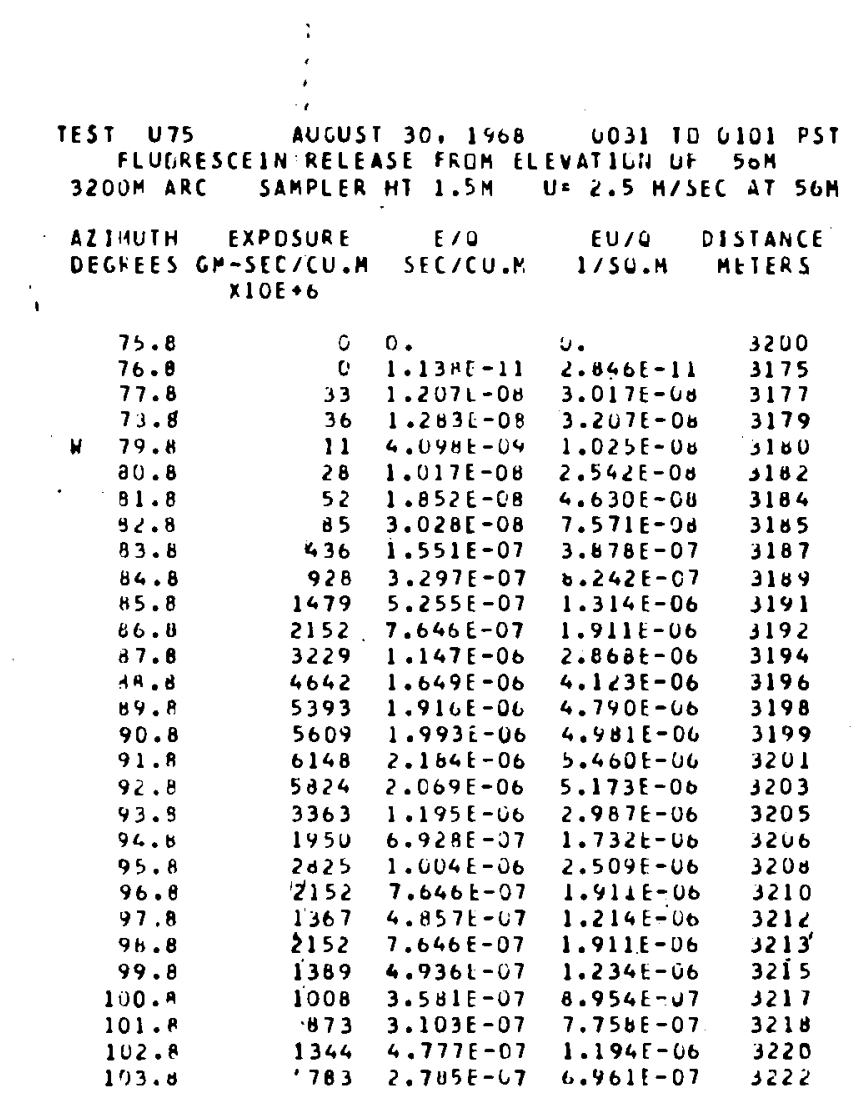




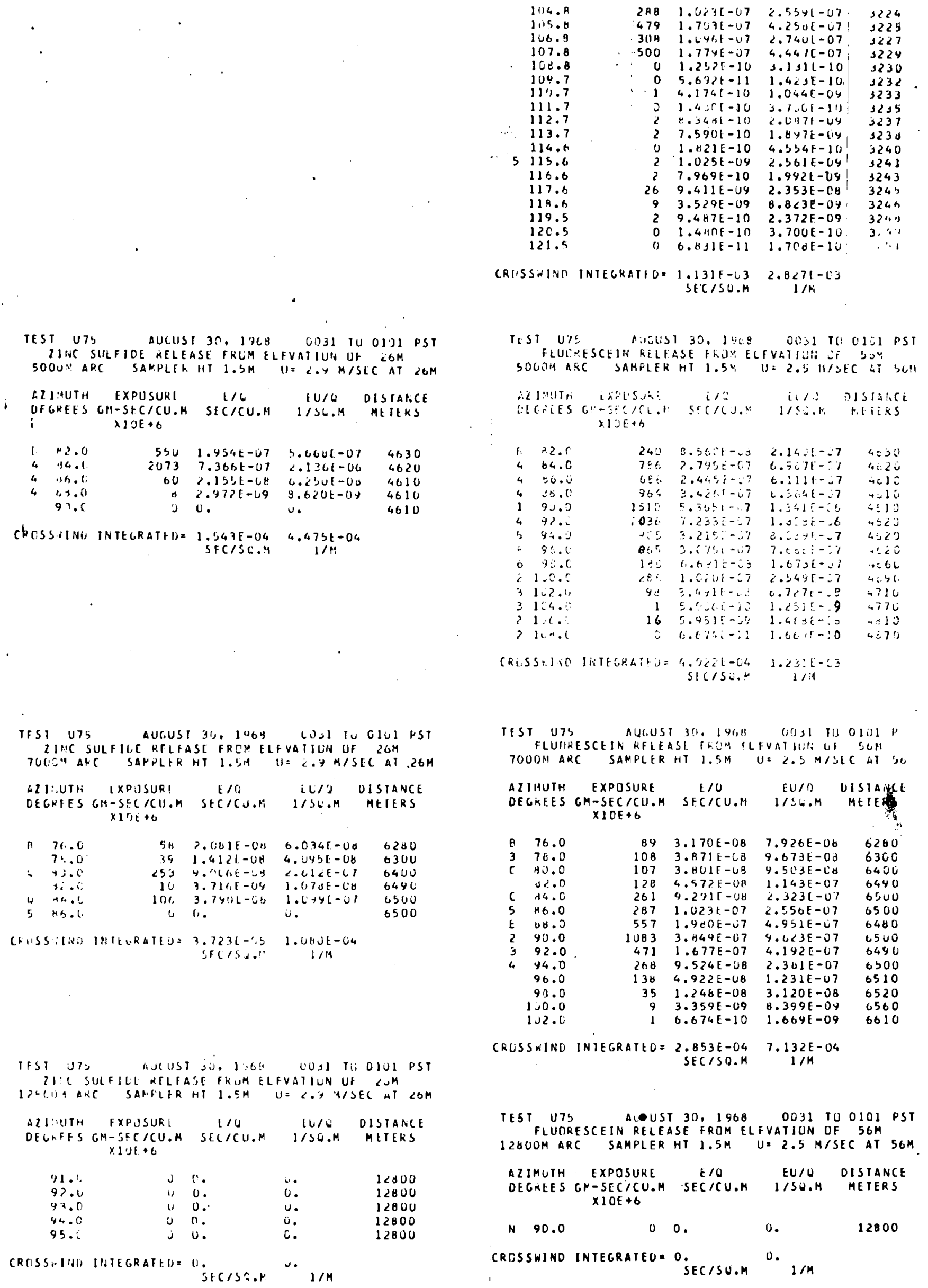


SAMPLIHC $400 M$ IO 7OOOM. WII TOHEK SAHPLING.

50OOM AHU 7CCOM ARCS TRUNCATEU DN NORIK.

TEST U76 SEPTEMBEK 16. 1468 U206 TU 0236 PST IINC SULFIUE RELEASE FRUH ELFVATIUN DF $26 \mathrm{M}$ 4COM AKC SARPLER MT $1.5 \mathrm{SH}$ U. 6.5 H/SEC AT $26 \mathrm{H}$

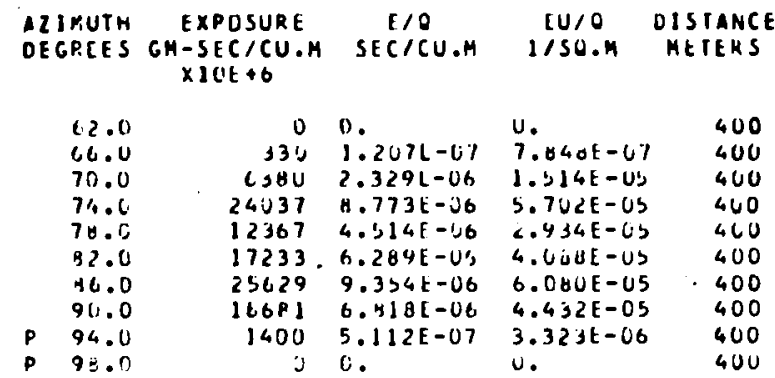

CKOSSRINU INIEGRATFL= 1.CJ1E-U3 7.026E-U3

TEST U76 SEPTEMGER 16.1960 02J6 TJ 0236 PST 2 INC SULFIDE HELEASE FRDM ELFVAIIUN UF $26 \mathrm{~K}$ OODM ARC SAMPLIR IIT $1.5 \mathrm{M} U=0.5 \mathrm{M} / \mathrm{SEC}$ AT $26 \mathrm{M}$

AZIMUTH EXPUSURE E/U EU/O OISTANCE OECNEES CM-SECICU.H SECICU.M I/SU.M METERS $\times 1$ it +6

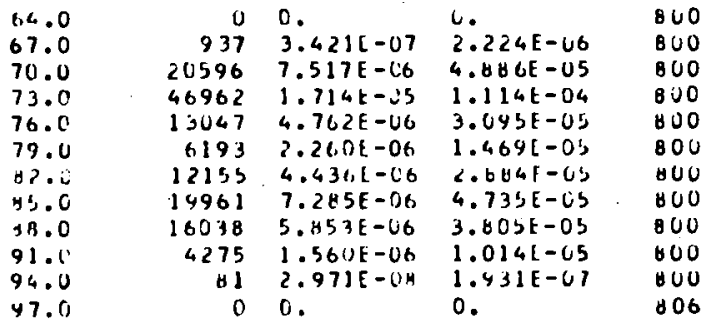

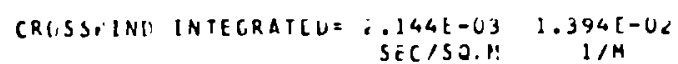

IFST U76 SHPTCMEEK $16,196 \mathrm{H}$, 200 TU 0236 PST IINC SULFIUE RILEASE FRUM FLFVATILIN UT 2014 $1203 M$ ARC SAMPLEK HT $1.5 \mathrm{M} \quad 1=6.5$ M/SLC AT $26 M$ AZINIIII EXPUSUKL $t / 0$ EU/O OISIAIYCE DECFEES GM-SHCICU.M SEC/CU.M I/SU.M MEIERS $\times 10 \mathrm{O}+6$

\begin{tabular}{|c|c|c|c|c|c|}
\hline & 64.2 & $\therefore$ & 1. & . & 1200 \\
\hline & 64.0 & 57 & C. IUOE-UB & $1.365 f-67$ & 1200 \\
\hline & so.0 & 3184 & $1.1 .62 \mathrm{E}-06$ & $1.55 e t-66$ & 1200 \\
\hline & $7 ! .0$ & 20338 & $7.4 \angle 3 \mathrm{E}-\mathrm{CG}$ & $4 . \forall \angle \supset F-0 j$ & $1<00$ \\
\hline & 72.0 & 32943 & $1.204 \mathrm{E}-015$ & $7.6<4 t-01$ & 1200 \\
\hline & 74.0 & 20520 & $7.489 t-26$ & 4. $8601-03$ & $12 \cup 0$ \\
\hline & 76.0 & 4759 & $1.737 \mathrm{E}-36$ & $1.124 \mathrm{E}-\mathrm{C} 5$ & $12 \cup 0$ \\
\hline & 73.0 & 4134 & $1.509 \bar{E}-66$ & $3.610 \mathrm{E}-06$ & $12 Q 0$ \\
\hline & 37.6 & 5756 & $=.101 t-06$ & $1.36, E-U S$ & 1200 \\
\hline & 4.2 .0 & 6,236 & $2.2761-006$ & $1.47 \cdot 56-03$ & 1200 \\
\hline & 54.0 & 19964 & $3.272 E-C 6$ & $2.127 \mathrm{t}-0 \mathrm{~b}$ & 1200 \\
\hline & EC.C & 11174 & $4.07 \mathrm{BE}-\mathrm{C} 6$ & $2.651 \mathrm{t}-\mathrm{cs}$ & 1200 \\
\hline & $\mathrm{EH} . \mathrm{C}$ & 4224 & $3.36 .7 \bar{E}-06$ & 2.1 nof $-U S$ & 1200 \\
\hline & $91 . .7$ & 3270 & $1.2035-1.0$ & $7.0<11-00$ & 1200 \\
\hline & 97.0 & 345 & $1.200[-67$ & $6.18 \cdot 31-11]$ & 1200 \\
\hline & 44.0 & 0 & 0. & u. & 1200 \\
\hline
\end{tabular}

CRIISSNIND INTEGRATEUE 2.002L-03 1.JJCL-0L SEC/SO.1:

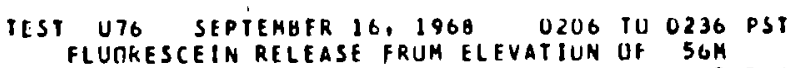
GOUM ARC SAMPLER HT $1.5 \mathrm{M}$ U. $7.1 \mathrm{M} / \mathrm{SEC}$ AT $56 \mathrm{M}$

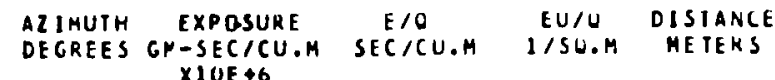

62.0
66.0
70.0
74.0
76.0
62.0
86.0
90.0
94.0
91.0
102.0
126.0
110.0
114.0
118.0
122.0

$\begin{array}{rl}0 & 0 . \\ 0 & 2.730 E-10 \\ 3 & 1.237 E-09 \\ 5 & 2.160 E-09 \\ 0 & 1.526 E-10 \\ 69 & 2.581 E-08 \\ 765 & 2.844 E-07 \\ 9 & 3.525 E-09 \\ 5 & 2.080 E-09 \\ 4 & 1.598 E-09 \\ 5 & 2.080 E-09 \\ 9 & 3.425 E-09 \\ 38 & 1.417 E-08 \\ 5 & 2.200 E-09 \\ 2 & 0.753 E-10 \\ 5 & 1.959 L-09\end{array}$

$1.93 \forall E-09$ $8.780 E-0 Y$ $1.534 \mathrm{E}-08$ $1.063 \mathrm{E}-04$ $1.832 \mathrm{E}-07$ 2.019E-06 $2.019 E-06$
$2.5 U 3 E-C B$ 1. $477 \mathrm{E}-08$ $1.135 E-06$ $1.477 \mathrm{E}-08$ $2.417 E-08$ 1. $006 E-07$ $1.562 E-06$ $6.215 E-09$ 400 400 400
400 400 400 400 400
400 400 400 400 400 400
400 400

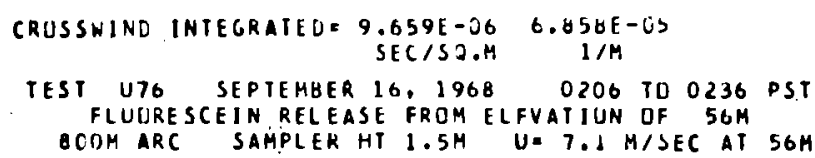
$\times 10 E \div 6$

$\begin{array}{lrrrr}67.0 & 0 & 2.011 \mathrm{~F}-11 & 1.996 \mathrm{E}-10 & 800 \\ 70.0 & 725 & 2.695 \mathrm{E}-07 & 1.913 \mathrm{E}-06 & 800 \\ 73.0 & 2654 & 9.862 \mathrm{E}-07 & 7.002 \mathrm{E}-00 & 800 \\ 76.0 & 6658 & 2.4731-06 & 1.756 \mathrm{E}-05 & 800 \\ 79.0 & 1519 & 5.646 \mathrm{E}-07 & 4.006 \mathrm{E}-06 & 800 \\ 02.0 & 997 & 3.706 \mathrm{E}-07 & 2.632 \mathrm{E}-06 & 800 \\ B 5.0 & 5239 & 1.916 \mathrm{E}-06 & 1.362 \mathrm{E}-0 \mathrm{~S} & 600 \\ 86.0 & 9824 & 3.649 \mathrm{E}-06 & 2.591 \mathrm{E}-05 & 800 \\ 91.0 & 1837 & 6.826 \mathrm{E}-07 & 4.847 \mathrm{E}-06 & 800 \\ 94.0 & 99 & 3.638 \mathrm{E}-0 \mathrm{OB} & 2.626 \mathrm{E}-07 & 800 \\ 97.0 & 2 & 7.669 \mathrm{E}-10 & 5.445 \mathrm{E}-09 & 800 \\ 97.1 & 0 & 2.262 \mathrm{E}-10 & 1.606 \mathrm{E}-09 & 806 \\ 99.1 & 0 & 1.863 \mathrm{E}-10 & 1.323 \mathrm{E}-09 & 809\end{array}$

CRCSSHIVO INIEGRATEO=4.599E-04 3.265E-03 $S F C / 50 . M \quad 1 / 4$

IEST U76 SEPIEMBER 16, 1964 O206 TO 0236 PST FLUURESCE IN RELEASE FFUM ELFVATIUN UF $56 \mathrm{M}$ $1200 M$ ARC SAMPLER HT $1.5 \mathrm{M} U=7.1 \mathrm{M} / \mathrm{SEC}$ AI SGM

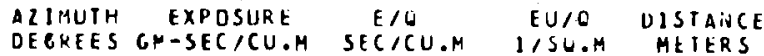
$X 10 E+6$

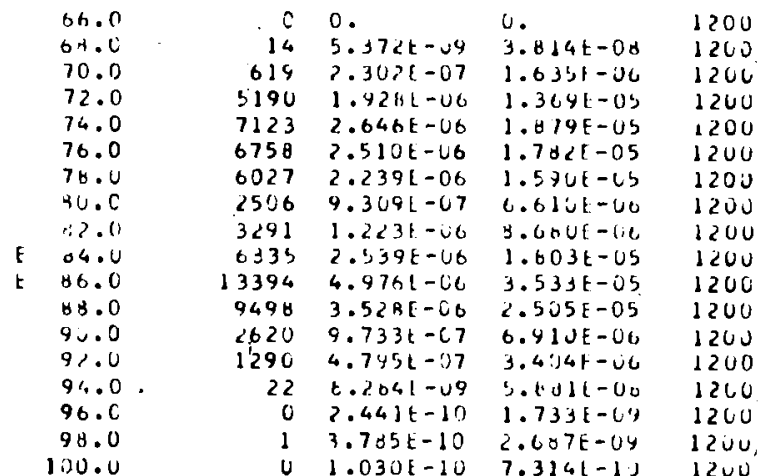

CRUSSWINO INIEGRATEU. $1.014 t-03 \quad 7.202 E-03$ 


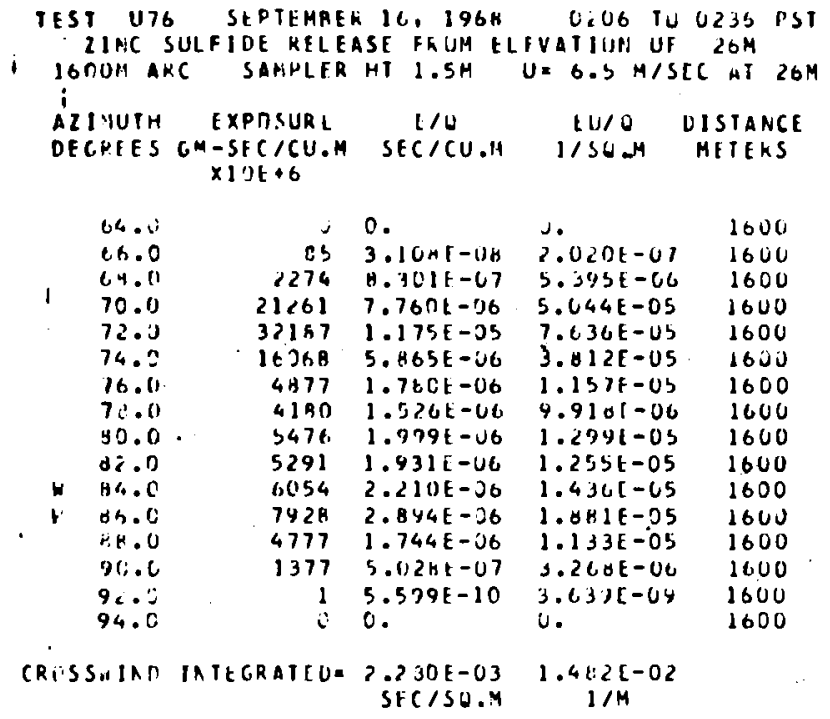

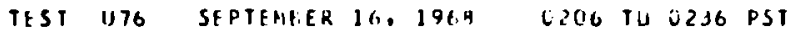
2 II:C SULFIOE RFLEASE FRUM LLFVATILR: OF 2 OM 2200 AKC SAPIPLER III $1.5 \mathrm{M} U=6.5 \mathrm{M} / \mathrm{SEC}$ AT $26 \mathrm{M}$

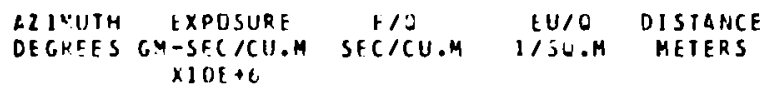

\begin{tabular}{|c|c|c|c|c|c|}
\hline & $\begin{array}{l}1.2 .0 \\
64.0\end{array}$ & $\begin{array}{l}\dot{U} \\
3\end{array}$ & $\begin{array}{l}0 . \\
1.374 t-03\end{array}$ & $\begin{array}{l}U . \\
8.93 \angle E-U 9\end{array}$ & $\begin{array}{l}2200 \\
2200\end{array}$ \\
\hline & $\begin{array}{l}1.6 .0 \\
59.0 \\
70.0\end{array}$ & $\begin{array}{r}126 \\
1245 \\
11437\end{array}$ & $\begin{array}{l}4.626 E=09 \\
4.527 E=07 \\
4.174 E-06\end{array}$ & $\begin{array}{l}3.007 E-07 \\
2.943 E-06 \\
2.713 E-05\end{array}$ & $\begin{array}{l}2200 \\
2200 \\
2200\end{array}$ \\
\hline & $\begin{array}{l}72.0 \\
74.0\end{array}$ & $\begin{array}{r}14006 \\
2714\end{array}$ & $\begin{array}{l}5.134 E-06 \\
9.96 \text { A }-07\end{array}$ & $\begin{array}{l}3.237 E-05 \\
e .440 E-06\end{array}$ & $\begin{array}{l}2200 \\
2200\end{array}$ \\
\hline & 76.0 & 3269 & $1.143 \mathrm{E}-06$ & $7.755[-06$ & 2200 \\
\hline & $7 \varepsilon .0$ & 1415 & $3.167 E-67$ & $3.359 \varepsilon=06$ & 2200 \\
\hline & 010.0 & 949 & $3.4 C 5 E-1.7$ & $2.232 E-06$ & 2200 \\
\hline & $\$ 2.0$ & 963 & $3.515 E-07$ & $2.2 \times 5 L=06$ & 2200 \\
\hline & 04.0 & 1002 & $3.6605-67$ & 2.37 YE-06 & 2200 \\
\hline & 86.0 & 1219 & $4.452 \mathrm{k}-07$ & $\angle .6 \times 4 E-06$ & 2200 \\
\hline 2 & $d \pm . C$ & 49 & $1.917 E-08$ & $1.181 E-07$ & 2200 \\
\hline & 90.0 & 6 & $2.290 E-04$ & $1.489 E-08$ & 2200 \\
\hline & 92.0 & 0 & 0. & 0 & 2200 \\
\hline
\end{tabular}

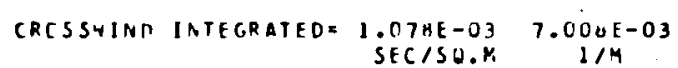

TEST U76 SFPTEMBIR 16, 196 H UCU6 TU OO236 PST FLUURESCEIA RELFASE TKIIM LLFVATIUN UR SUH $160 O M$ ARC SAMPLER HT $1.5 \mathrm{M} U=7.1$ Y/SLC $11 \mathrm{JGM}$

\begin{tabular}{|c|c|c|c|c|c|}
\hline \multicolumn{2}{|c|}{$\begin{array}{l}\text { A.z InUTH } \\
\text { OEGHEES }\end{array}$} & $\begin{array}{c}\text { EXPOSURE } \\
\text { GM-SEC ICU.M } \\
\times 1 O E+C\end{array}$ & $\begin{array}{c}t / C \\
S E C / C U . M\end{array}$ & $\begin{array}{l}1 U / 0 \\
1 / S W . M\end{array}$ & $\begin{array}{l}\text { GISIANCL } \\
\text { MEIERS }\end{array}$ \\
\hline & $\begin{array}{l}6 h .0 \\
70.0\end{array}$ & $\begin{array}{r}0 \\
403\end{array}$ & $\begin{array}{l}1.7031-11 \\
1.4481-07\end{array}$ & $\begin{array}{l}3.3341-10 \\
1.0641-06\end{array}$ & $\begin{array}{l}1600 \\
1600\end{array}$ \\
\hline 2 & 72.0 & 3924 & $1.65 H L-U 0$ & $1.035 \mathrm{~L}-\mathrm{U}$ & bovis \\
\hline$L$ & $\begin{array}{l}74.0 \\
16.0\end{array}$ & $\begin{array}{r}5696 \\
11324\end{array}$ & $\begin{array}{l}2.116 E-06 \\
4.207 E-06\end{array}$ & $\begin{array}{l}1.30<\mathrm{U}-05 \\
2.96 .7 E-05\end{array}$ & $\begin{array}{l}1600 \\
1000\end{array}$ \\
\hline & $\begin{array}{r}7 \mathrm{H} .0 \\
00.0\end{array}$ & $\begin{array}{l}566 ? \\
4050\end{array}$ & $\begin{array}{l}2.1031-06 \\
1.3 \cup 51-06\end{array}$ & $\begin{array}{l}1.1098-u s \\
1.009 t-v b\end{array}$ & $\begin{array}{l}1600 \\
1600\end{array}$ \\
\hline 2 & $8<.0$ & 3670 & $1.364 E-06$ & $9.60<E-06$ & 1600 \\
\hline 4 & 54.8 & 5063 & $1.901 E-06$ & $1.335 E-10 S$ & dive \\
\hline$W$ & $\begin{array}{l}d 6.0 \\
68.0\end{array}$ & $\begin{array}{l}8950 \\
7089\end{array}$ & $\begin{array}{l}3.325 t-06 \\
2.633[-06\end{array}$ & $\begin{array}{l}2.361 \mathrm{E}-\mathrm{US} \\
1.870 \mathrm{t}-0 \mathrm{~S}\end{array}$ & $\begin{array}{l}1600 \\
1600\end{array}$ \\
\hline & 90.0 & 2506 & $9.309[-07$ & $6.010 E=00$ & 1600 \\
\hline $\mathbf{E}$ & 93.6 & 4 & $1.755 \mathrm{t}-$ U9 & $1.246 \mathrm{E}-00$ & $160:$ \\
\hline 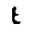 & 94.0 & 1 & $5.173 t-10$ & צy & 1600 \\
\hline & D. & EC & $\begin{array}{l}1.211[-03 \\
\text { SEC } 150.11\end{array}$ & $\begin{array}{c}4.39 \Delta \overline{\mathrm{E}}-0 \mathrm{~J} \\
1 / 11\end{array}$ & \\
\hline
\end{tabular}

IEST U76 SEPTEMBER 16, 196A 0206 TU 0236 PST FLURRESCE IN RELEASE FRUIA ELFVATIUN DF 5 GM $22 \mathrm{COM} A R C$ SAMPIER HIT $1.5 \mathrm{M} U=7.1 \mathrm{M} / \mathrm{SEC}$ AT $56 \mathrm{M}$

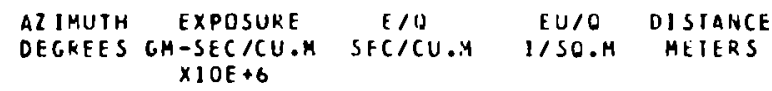

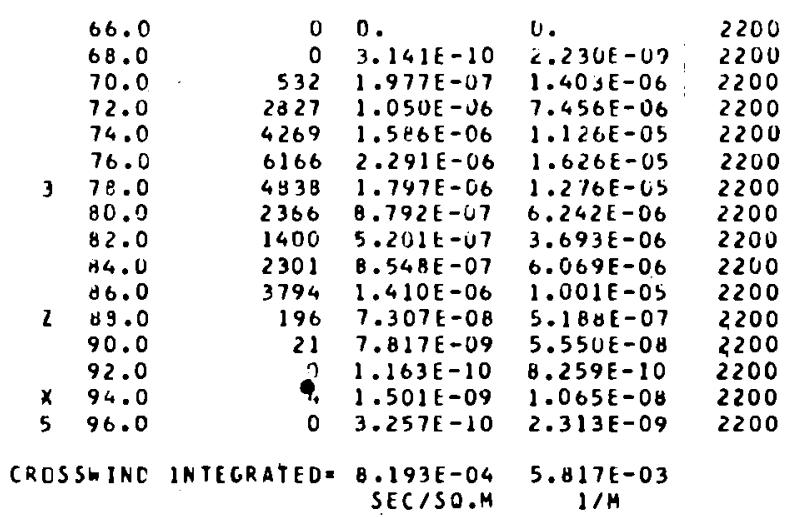


TEST U76 SFPTEMAEK 16, 1960 0206 TU 0236 PST IINC SULFIDE RELEASE FROM ILFVATIUN OF $26 \mathrm{H}$ 32 OCH ARC SAMPLER HT $1.5 \mathrm{M} U=6.5 \mathrm{M} / \mathrm{SEC}$ AT $26 \mathrm{M}$

AIIMLITH EXPQSURE T/U EU/O DISTANCE DEGRFES GR-SEC/CU.H SEC/CU.M I/SU.M METERS $\times 10 E+6$

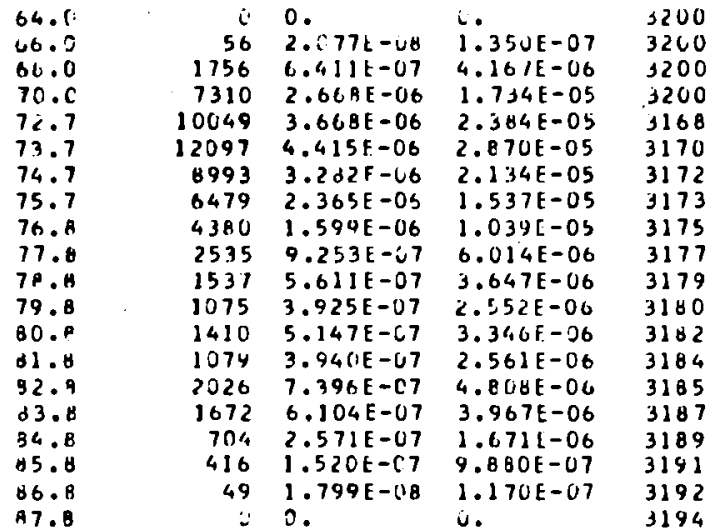
CRIISSHINA IATEGRATEO= $1.709 E-03$ 1.111E-OL

TEST UT6 SEPTEMAEK 16, 1968 O2 0206 TU 0236 PST 2IARC SULFIDE RELEASE FROM ELFVATIUN OF $26 \mathrm{M}$ SOOOM ARC SAMPLER HT $1.5 M$ U = 6.5 MIJEC AT $26 \mathrm{M}$ AZITUTH EXPOSURE E/O EU/O DISTANCE DFGEES CH-SECICU.M SECICU.M I/SC.H MEIERS $\times 10 \mathrm{O}+6$

\begin{tabular}{|c|c|c|c|c|}
\hline$t 2.0$ & 366 & $1.338 t-07$ & $8.694 E-07$ & 46 \\
\hline A4.0 & 107 & $3.939 f-U 8$ & $2.561 \mathrm{E}-07$ & 462 \\
\hline 86.0 & 5 & $1.905 E-64$ & $1.290 \mathrm{E}-00$ & \\
\hline$\$ 4.0$ & 5 & $2.13 \times 1-09$ & $1.38 y[-0 y$ & 461 \\
\hline 90.0 & 12 & $4.42 \cup 8-09$ & $C .87 \cup P=08$ & 461 \\
\hline 92.0 & 3 & $1.374 t-09$ & $0.93<E-69$ & 462 \\
\hline 94.0 & 3 & $1.222 \mathrm{E}-09$ & $7.94 \mathrm{JE}-0 \mathrm{Y}$ & 462 \\
\hline 96.0 & 2 & $1.06,4 \mathrm{E}-09$ & $6.44 / E-U Y$ & 462 \\
\hline $\begin{array}{r}90.0 \\
100.0\end{array}$ & .3 & $\begin{array}{l}1.374 E-04 \\
1.985 E-09\end{array}$ & $\begin{array}{l}0.432[-04 \\
1.290[-00\end{array}$ & \\
\hline 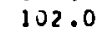 & & & & \\
\hline
\end{tabular}

CR1.SSMINC INTEGRATED= $3.049[-U 5$ '1.992E-U4 SECISO.M TIIM
TEST U76 SEPTEMBER 16,1968 02U6 TU U236 PST FLUORESCEIN RRELEASE FRDM ELEVATIUN UF 5CM $3200 M$ ARC SAMPLER HT $1.5 \mathrm{M} U=7.1 \mathrm{M} / \mathrm{SEC}$ AT $56 \mathrm{M}$ AZIMUTH EXPOSURE
DEGRES GM-SEC/CU.M SEC/CU.M I/OUOO IISTANCE $\times 1 O E+6$

66.0
68.0
76.0
72.7
73.7
74.7
75.7
76.8
77.8
79.8
79.8
80.8
81.8
82.8
63.8
84.8
85.8
86.8
87.8
88.8
89.8
96.8

$\begin{array}{rl}0 & 0 . \\ 40 & 1.487 E-08 \\ 473 & 1.757 E-07 \\ 3027 & 1.125 E-06 \\ 5382 & 2.000 E-06 \\ 6504 & 2.416 E-06 \\ 6728 & 2.500 E-06 \\ 7401 & 2.750 E-06 \\ 6055 & 2.250 E-06 \\ 6055 & 2.250 E-06 \\ 2421 & 8.996 E-07 \\ 2018 & 7.496 E-07 \\ 2354 & 6.746 E-07 \\ 2892 & 1.075 E-06 \\ 4104 & 1.525 E-06 \\ 2960 & 1.100 E-06 \\ 1524 & 5.663 E-07 \\ 640 & 2.378 E-07 \\ 95 & 3.538 E-08 \\ 1 & 6.984 E-10 \\ 2 & 9.762 E-10 \\ 0 & 0 .\end{array}$

0. $1.248 E-06 \quad 3200$ $7.985 E-06 \quad 3168$ $1.420 E-05 \quad 3170$ $1.716 E-05 \quad 3172$ $1.775 E-05 \quad 3173$ $1.952 \mathrm{E}-05 \quad 3175$ $1.597 E-0 S \quad 3177$ $1.597 E-05 \quad 3179$ $1.597 E-05$
$6.387 E-06$
5.3180 $3.322 E-06 \quad 3182$ $0.210 E-60 \quad 3184$ $7.630 E-06 \quad 9165$ $1.022 E-05 \quad 3187$ $1.022 E-05$
$7.007 E-06$
$4.02189-06$ $4.021 \mathrm{E}-06 \quad 3191$ $1.688 \mathrm{E}-06 \quad 3192$ $2.512 E-07 \quad 3194$ 4.95YE-OY 3196 6.931 E-09 3198 0 .

3199

CRUSSHIMD INTEGRATED $1.332 E-03 \quad 9.456 E-03$ SEC/SO.M $1 / \mathrm{M}$

TEST U76 SEPTEMBER 16, 1968 0206 TU 0236 PST FLUGRESCEIN RELEASE FRDH ELFVATIUN UF $50 \mathrm{M}$ SOOOM ARC SAMPLER HT $1.5 \mathrm{M}$ U. $7.1 \mathrm{M} / \mathrm{SEC}$ AT $56 \mathrm{M}$ AZIMUTH EXPOSURE E/O EU/O DISTANCE DEGREES GH-SECICU.M SECICU.M I/SO.M METERS $\times 10 E+6$

$B$ a

$\begin{array}{rrrrr}22.0 & 1577 & 5.860 \mathrm{E}-07 & 4.161 \mathrm{E}-06 & 4630 \\ \mathrm{H4.0} & 159 & 5.912 \mathrm{E}-08 & 4.197 \mathrm{E}-07 & 4620 \\ 86.0 & 5 & 2.024 \mathrm{E}-09 & 1.437 \mathrm{E}-0 \mathrm{~B} & 4610 \\ 88.0 & 0 & 8.143 \mathrm{E}-11 & 5.781 \mathrm{E}-10 & 4610 \\ 90.0 & 0 & 1.512 \mathrm{E}-10 & 1.074 \mathrm{E}-09 & 4610\end{array}$

CRLSSHIND INTEGRATED= $1.046 E-04 \quad 7.427[-64$ SECISU.M TIM

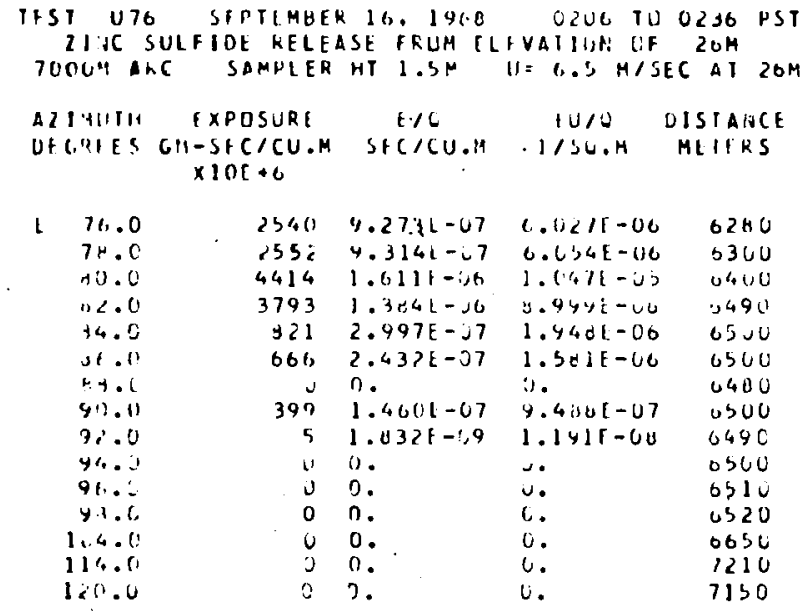

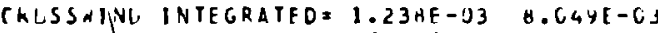

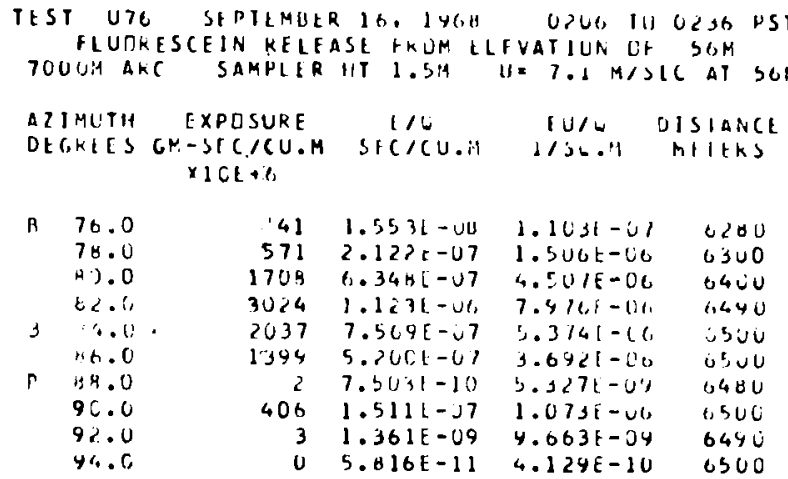

CRUSSHIND INTEGRATED $=7.708 E-045.473 E-03$ 
SARPLING 4OOM ID 12 GOUR. NU IUHIK SAHPLINC. IXTREME SIIFI IN PLUKE CLNTERLINE LUCATIUN SIARIING AGLUT IGOUM.

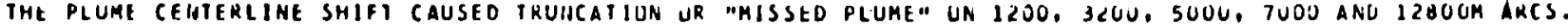
FULURESCFIN WAS MUT ASSAYER FAR FNIUGH NUKIH UN THE IBOOA AKC TU BE SURE UF TRUNCATILA.

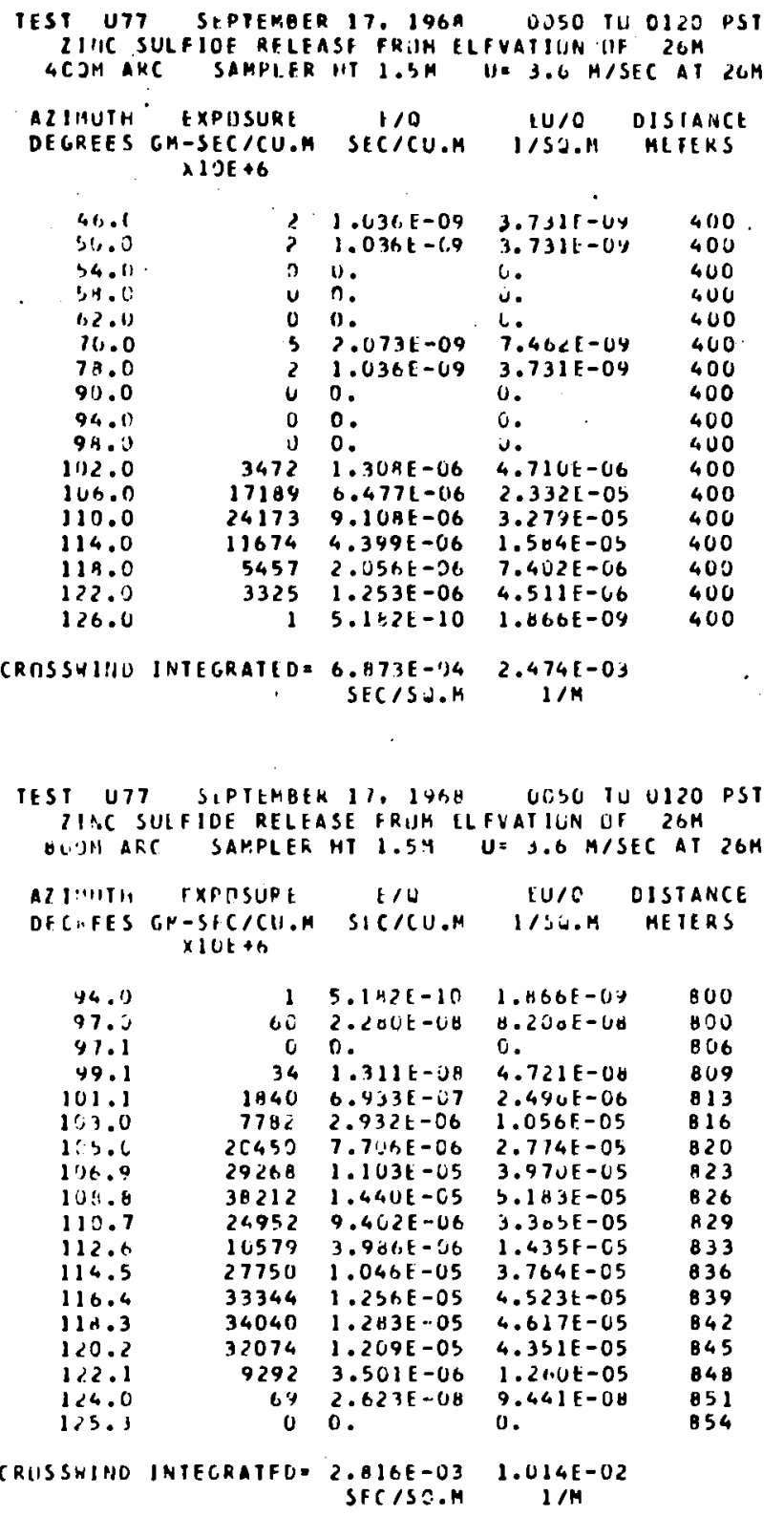

\begin{tabular}{|c|c|c|c|c|}
\hline $\begin{array}{r}\text { TEST U77 } \\
\text { FLUUR } \\
40,1) M \quad A R\end{array}$ & $\begin{array}{l}\text { SFPTEMBE } \\
\text { SESCE IN RELE } \\
\text { SC SAMPIER }\end{array}$ & $\begin{array}{l}\text { K } 17,1960 \\
\text { ASE FROM EL } \\
\text { HT } 1.5 M\end{array}$ & $\begin{array}{r}\text { CJSC TU } \\
\text { LFVAIIUN UF } \\
\text { II. } 3.7 \mathrm{M} / \mathrm{S}\end{array}$ & $\begin{array}{l}0120 \text { PSI } \\
\text { SUM } \\
\text { SL AI SGM }\end{array}$ \\
\hline $\begin{array}{l}\text { ALIMUTH } \\
\text { DECKEES }\end{array}$ & $\begin{array}{c}\text { EXPIISURE } \\
\text { GM-SEC/CU.H } \\
\times 1 O E+6\end{array}$ & $\begin{array}{c}n / U \\
S+C / C U . H\end{array}$ & $\begin{array}{l}\text { IU/O } \\
\text { l SU.M }\end{array}$ & $\begin{array}{l}\text { IDISIALCE } \\
\text { ME TERS }\end{array}$ \\
\hline $\begin{array}{r}86.0 \\
90.0 \\
94.0 \\
96.0 \\
102.0 \\
106.0 \\
110.0 \\
114.0 \\
118.0 \\
122.0\end{array}$ & $\begin{array}{r}3 \\
6 \\
0 \\
12 \\
0 \\
2040 \\
1019 \\
3811 \\
2108 \\
0\end{array}$ & $\begin{array}{l}1.227 \mathrm{E}-04 \\
2.296 \mathrm{E}-04 \\
0 . \\
4.551 \mathrm{E}-09 \\
3.166 \mathrm{E}-1 \mathrm{C} \\
7.472 \mathrm{E}-07 \\
3.732 \mathrm{E}-07 \\
1.396 \mathrm{E}-06 \\
7.722 \mathrm{E}-07 \\
0 .\end{array}$ & $\begin{array}{l}4.54 U E-04 \\
6.443 E-07 \\
0 . \\
1.684 E-08 \\
1.172 E-08 \\
2.765 E-06 \\
1.381 E-06 \\
5.163 E-06 \\
2.857 E-06 \\
0 .\end{array}$ & $\begin{array}{l}400 \\
400 \\
400 \\
400 \\
400 \\
400 \\
400 \\
400 \\
400 \\
400\end{array}$ \\
\hline CROSSINIAD & INTEGRATED= & $\begin{array}{r}9.2 O B E-U S \\
\text { SECISO.M }\end{array}$ & $\begin{array}{c}3.406 E-04 \\
1 / H\end{array}$ & \\
\hline
\end{tabular}

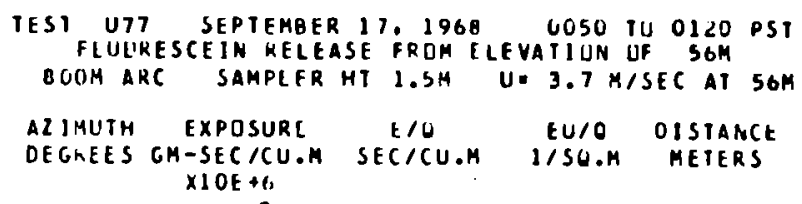




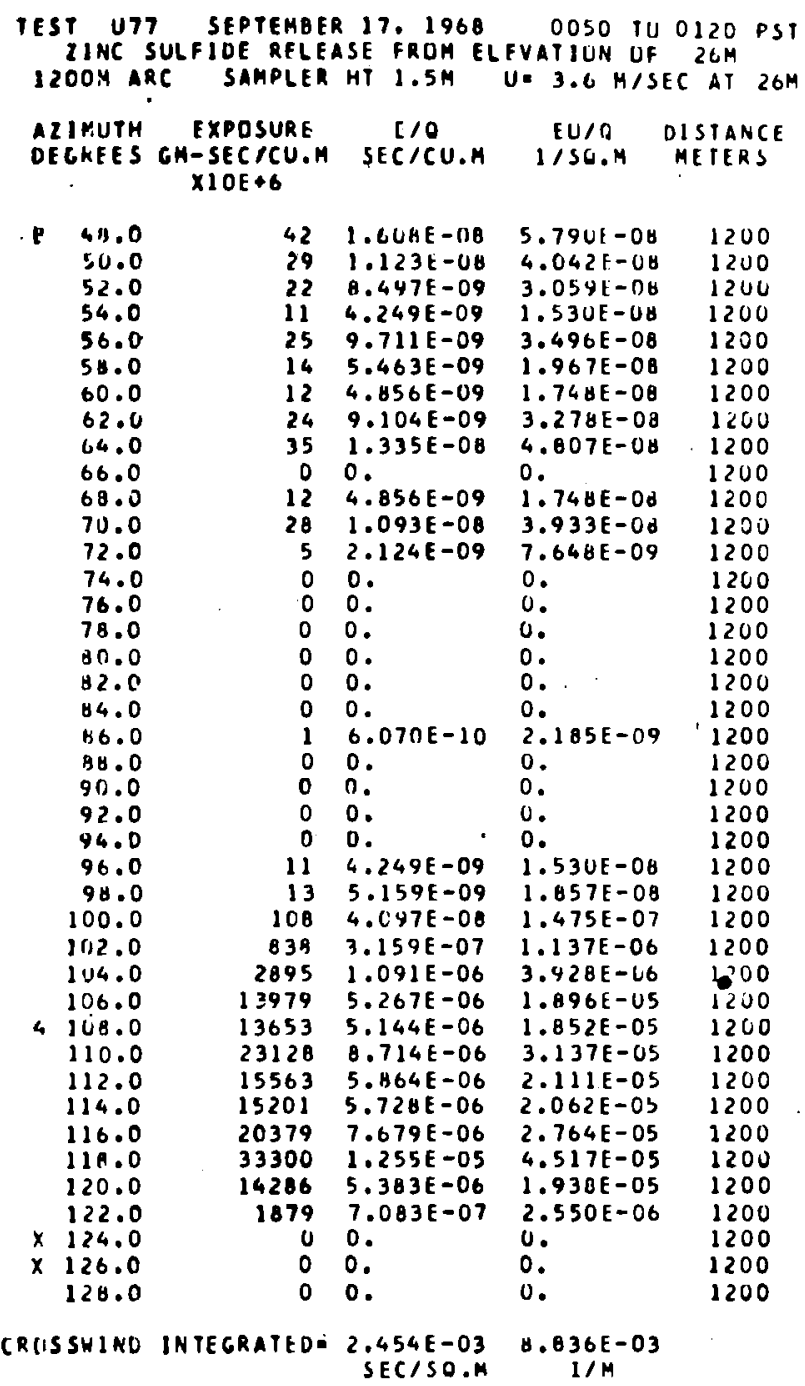

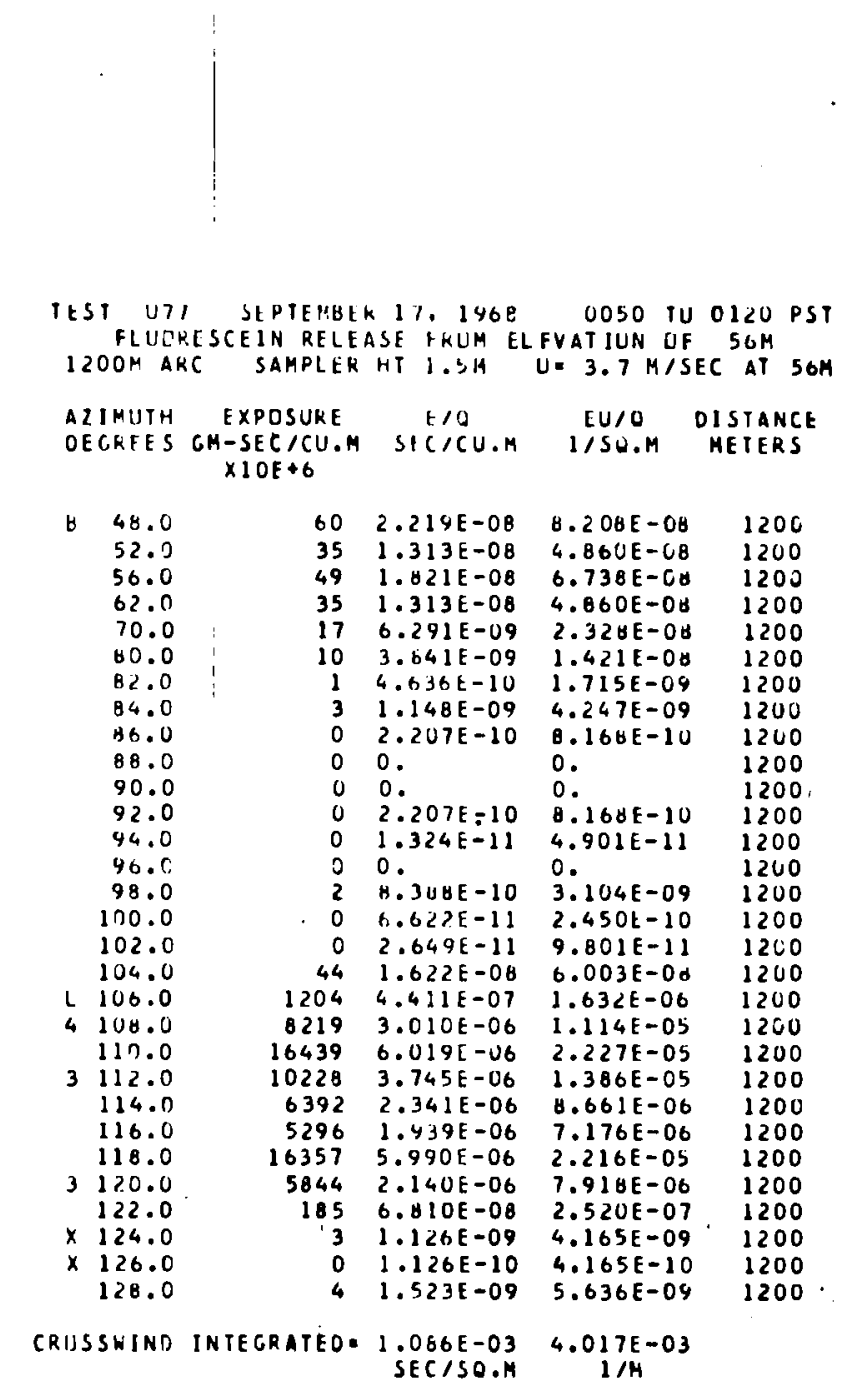




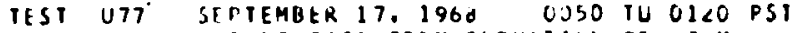
2IIIC SULFIUE RFLEASE FRCM ELFVAIILN OF $26 \mathrm{M}$ $16 \mathrm{com}$ AKC SAMPLER HI $1.5 \mathrm{M} \mathrm{U}=3.6 \mathrm{M} / \mathrm{SEC}$ AT $26 \mathrm{M}$

\begin{tabular}{|c|c|c|c|c|}
\hline $\begin{array}{l}\text { AZIYLITH } \\
\text { UE CREES }\end{array}$ & $\begin{array}{l}\text { EXPQSURE } \\
\text { GM-SEC } / C U . H \\
\text { XIOE } 46\end{array}$ & $\begin{array}{c}E / O \\
\text { SFC/CU.M }\end{array}$ & $\begin{array}{l}\text { Eu/o } \\
1 / 50.11\end{array}$ & $\begin{array}{l}\text { DI SI IANCE } \\
\text { MEIEKS }\end{array}$ \\
\hline ate. & 0 & 0. & $u$. & 1600 \\
\hline 57 & 0 & 0. & u. & 16 \\
\hline 56.6 & 0 & $n$. & u. & 1500 \\
\hline Buect & 0 & 0. & & 1600 \\
\hline $6,4.1$ & 1 & $6.270 E-10$ & $2.185 \mathrm{E}-04$ & 1600 \\
\hline 64.0 & 0 & 0. & u. & 1600 \\
\hline 72. & 0 & 0. & u. & 1600 \\
\hline 76.0 & 0 & $3.035 E-10$ & $1.093 E-09$ & 160 \\
\hline 80.0 & 3 & 1.210 & 4.37 & 1600 \\
\hline 34. & ci & i. & 0 . & 1600 \\
\hline$H B .0$ & $3 y$ & $1.487 E-C B$ & $5.353 E-00$ & 1600 \\
\hline 38. & 33 & 1.27 & 6.31 & 1600 \\
\hline 90 & 95 & 3.50 & 1.28 & 1600 \\
\hline 92.0 & 31 & $1.1 B 4 E-0 B$ & $4.261 E-00$ & 1600 \\
\hline 93.6 & 150 & 5.60 & 2.03 & 1603 \\
\hline 94.1 & 37 & 1.39 & 5.02 & 1600 \\
\hline 44.1 & 264 & 9.96 & 3.50 & 1604 \\
\hline $9 ! .6$ & 147 & 5.5 & 1.9 & 1606 \\
\hline 96.6 & 108 & 4.07 & 1.46 & 1608 \\
\hline & 61 & & 1.1 & 1610 \\
\hline $93 . t$ & 280 & 1.08 & 3.91 & 1611 \\
\hline 99.6 & 159 & 6.00 & $2.161 E-07$ & 1613 \\
\hline 2100 & 399 & 1.50 & 5.422 & 1615 \\
\hline $101 . t$ & 162 & 6.1 & 2.20 it -07 & 1617 \\
\hline $1 \div 2$ & 444 & 1.0 & 6.0 & 161 \\
\hline & 510 & 1.5 & 6.9 & 1620 \\
\hline נ & 937 & 3.53 & 1.27 & 1622 \\
\hline & 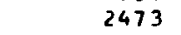 & 9.3 & 3.3 & 16 \\
\hline & 7 & 2.3 & 8.4 & 1625 \\
\hline 107.5 & 9216 & 3.6 & 1.33 & 162 \\
\hline $1<3$ & & & 2.4 & 1620 \\
\hline 109.5 & & 8.8 & & 1630 \\
\hline $\begin{array}{l}113.4 \\
111.4\end{array}$ & $\begin{array}{l}30235 \\
23016\end{array}$ & $\begin{array}{l}1.139 \\
8.672\end{array}$ & $\begin{array}{l}4.1 \\
3.1\end{array}$ & $\begin{array}{l}1632 \\
1633\end{array}$ \\
\hline 112.4 & 25617 & 9.7 & 3.50 & 163 \\
\hline & & & & \\
\hline 114.4 & 26166 & 9.45 & 3.54 & 16 \\
\hline 115.3 & 373 & & 5.06 & \\
\hline 116.3 & 40069 & $1.516 \mathrm{E}$ & $5.43 \mathrm{SE}-05$ & 364 \\
\hline 117 & 44842 & 1.690 & $6.0 \mathrm{dJE}$ & 16 \\
\hline 114.2 & 34268 & 1.29 & 4.646 & 164 \\
\hline 119 & 148 & $5.506 E$ & 2.011 & 16 \\
\hline 120. & 5169 & $1.94 \mathrm{BE}-06$ & $7.012 \mathrm{E}-06$ & 164 \\
\hline 1 & 1927 & $6.885 \varepsilon-07$ & $2.479 E-06$ & \\
\hline & $1 \div 8$ & $5.204 \mathrm{f}-0 \mathrm{~B}$ & $1.875 E-07$ & 165 \\
\hline 121 & u & & & 1552 \\
\hline
\end{tabular}

CRLSSDINA: INIEGRATED= $3.902 E-03 \quad 1.405 E-02$

\begin{tabular}{|c|c|c|c|c|}
\hline $\begin{array}{l}\text { EST U77 } \\
\text { FLUUK } \\
1600 \text { AK }\end{array}$ & $\begin{array}{r}\text { SEPTEMEE } \\
\text { SSCE IN RELE } \\
\text { IC SAMPLER }\end{array}$ & $\begin{array}{l}17,1968 \\
\text { RSE FRUM EL } \\
\text { HI } 1.5 \mathrm{HA}\end{array}$ & $\begin{array}{r}0050 \mathrm{TO} \\
\text { LFVATIUN UF } \\
U=3.7 \mathrm{M} / \mathrm{S}\end{array}$ & $\begin{array}{l}0120 \\
\begin{aligned} 0 \\
\text { SOH }\end{aligned} \\
S E C \text { AT }\end{array}$ \\
\hline $\begin{array}{l}\text { ALIMUIH } \\
\text { DEGKEES }\end{array}$ & $\begin{array}{c}\text { EXPOSURE } \\
\text { GH-SEC/CU.M } \\
\times 1 O E+6\end{array}$ & $\begin{array}{c}E / D \\
\text { SEC/CU.M }\end{array}$ & $\begin{array}{c}\text { EU/O } \\
1 / 50.4\end{array}$ & $\begin{array}{l}\text { DISTANC } \\
\text { METERS }\end{array}$ \\
\hline 100.6 & 0 & 0 . & c. & 161 \\
\hline 101.6 & $u$ & 0 . & 0. & \\
\hline 102.6 & 217 & $7.957 E-08$ & $2.944 E-07$ & 1618 \\
\hline 103.6 & 82 & $3.027 E-08$ & $1.120 \mathrm{E}-07$ & 1620 \\
\hline 134.5 & 0 & $1.73 \cup E-11$ & $6.400 E-11$ & 1622 \\
\hline 105.5 & 0 & $9.514 \mathrm{E}-11$ & $3.520 \mathrm{E}-10$ & 2623 \\
\hline 106.5 & & $1.730 E-07$ & $6.400 E-07$ & 1625 \\
\hline 107.5 & 9.4 & $1.133 \mathrm{E}-07$ & $4.19<E-07$ & 1627 \\
\hline $10 * .5$ & 1237 & $4.532 E-07$ & $1.677 \mathrm{E}-06$ & 1628 \\
\hline 109.5 & 4015 & $1.470 E-06$ & $5.440 \mathrm{E}-06$ & 1630 \\
\hline 2110.4 & 14382 & $5.266 E-06$ & $1.944 \mathrm{E}-05$ & 1632 \\
\hline 111.4 & 15374 & $5.630 E-06$ & $2.083 E-0 S$ & 1633 \\
\hline 112.4 & 22319 & $8.173 E-06$ & $3.024 E-03$ & 1635 \\
\hline 113.4 & 14382 & $5.266 E-06$ & $1.949 E-05$ & 1637 \\
\hline 114.4 & 7438 & $2.724 \mathrm{E}-06$ & $1.0085-05$ & 1638 \\
\hline 115.3 & 11406 & $4.177 \mathrm{E}-06$ & $1.545[-05$ & 1640 \\
\hline 116.3 & 19321 & $7.075 E-06$ & $2.618 E-05$ & 1641 \\
\hline 117.3 & 9540 & $3.493 E-06$ & $1.293 E-05$ & 1643 \\
\hline 118.2 & 20753 & $7.599 E-06$ & $2.812 \mathrm{E}-05$ & 1644 \\
\hline 119.2 & 15981 & $5.852 E-06$ & $2.165 \mathrm{E}-03$ & 1646 \\
\hline 120.2 & 5057 & $1.852 t-06$ & $6.852 \mathrm{E}-06$ & 1647 \\
\hline 121.2 & 1188 & $4.350 t-07$ & $1.610 E-0 B$ & 1649 \\
\hline 122.1 & 136 & $5.016 \mathrm{E}-08$ & $1.836 t-07$ & 1650 \\
\hline 123.1 & 400 & $1.496 E-07$ & $5.536 \mathrm{E}-07$ & 1652 \\
\hline 124.1 & 63 & $2.335 E-08$ & $8.640 \mathrm{E}-0 \mathrm{~V}$ & 1653 \\
\hline 123.0 & 15 & $5.622 \mathrm{E}-09$ & $2.000 t-00$ & 1655 \\
\hline $12 t .0$ & 188 & $6.914 E-08$ & $2.560 E-07$ & 1656 \\
\hline 126.9 & 146 & $5.362 E-08$ & $1.984[-07$ & 1658 \\
\hline 127.8 & & 0 . & 0 . & 1659 \\
\hline
\end{tabular}

CRUSSHIND INIEGRATEO= $1.646 E-03 \quad 6.234 E-C_{3}$

SEC/SO.M $1 / 11$
TEST U77 SEPTCMUER 11, 1968 GSSO TU $01 \angle 0$ PST 2IIGC SULFIOE RELEASE FKCIM FLEVATIUN UF ZGM T2UUIP $\triangle$ RC SAMPLER HI $1.3 \mathrm{H}$ HE 3.6 H/SEC AT $26 \mathrm{M}$

QZIIUTH EXPOSURL E/O EU/O DISTANCE DFGFES GHISHC/CII.H SFCICU.M I/SU.M MEIERS $x \quad 1 \cup E \rightarrow 6$

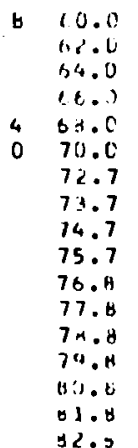

\begin{tabular}{|c|c|}
\hline & ر \\
\hline & $3.70 \mathrm{HE}-U \mathrm{t}$ \\
\hline 29 & $1.1119-08$ \\
\hline $11 \mathrm{~s}$ & $4.335 t-00$ \\
\hline & $3.783[-08$ \\
\hline 23 & H.670E-09 \\
\hline 305 & $1.152 \mathrm{E}-07$ \\
\hline 275 & $1.040 \mathrm{E}-07$ \\
\hline 530 & $1.947 E-07$ \\
\hline 411 & $1.552 E-07$ \\
\hline 262 & 9. $+345-00$ \\
\hline 505 & $1.405 E-07$ \\
\hline 774 & $2.919 E-07$ \\
\hline 301 & $1.137 \mathrm{E}-07$ \\
\hline 63 & $2.402 \mathrm{t}-07$ \\
\hline 49 & $1.859 \mathrm{E} \cdot 07$ \\
\hline & $3.754 \mathrm{E}-\mathrm{j} 7$ \\
\hline
\end{tabular}

$1.334 \mathrm{E}-07$ $1.356[-01$ $4.0248-08$ $1.301 \mathrm{E}-\mathrm{Cr}$ $1.362 \mathrm{E}-\mathrm{U}$ ग.1 $21 E-08$ $4.146 E-07$ $3.743 \mathrm{E}-07$ $7.190 t-07$ $5.500 E-07$ $3.55 \mathrm{dE}-07$ $0.654 \mathrm{E}-07$ $1.031 \mathrm{E}-06$ $4.093 E-67$ $8.647 \mathrm{E}-07$ $6.693[-07$

$1.353 E-06$

$x$

TEST U77 SEPTEMGER 17,1968 OUSO IU 0120 PST
FLUCRESCEIN RELEASF FROM ELFVATIUN OF SOM 32 COM ARC SAMPLER HT $1.5 \mathrm{~N} U=3.7 \mathrm{M} / \mathrm{SEC}$ AT $56 \mathrm{M}$

AZIMUTH EXPOSURE E/G EU/O DISTANCE DEGKES CM-SECICU.M SECICU.M I/SO.M METERS $X 10 E+6$

$B$

$$
\begin{aligned}
& 60.0 \\
& 62.0 \\
& 64.0 \\
& 66.0 \\
& 61.0 \\
& 70.0 \\
& 72.7 \\
& 73.7 \\
& 74.7 \\
& 75.7 \\
& 76.8 \\
& 77.8 \\
& 78.8 \\
& 79.8 \\
& 80.8 \\
& 81.8 \\
& 12.8
\end{aligned}
$$$$
4 \quad 6 \mathrm{H.0}
$$$$
\text { o } 70.0
$$

$\begin{array}{llll}.224 & 8.221 E-C B & 3.042 E-07 & 3200 \\ 211 & 7.763 E-08 & 2.872 E-07 & 3200 \\ 155 & 5.699 E-08 & 2.109 E-07 & 3200 \\ 177 & 6.501 E-0 B & 2.406 E-07 & 3200 \\ 211 & 7.763 E-08 & 2.872 E-07 & 3200 \\ 202 & 7.419 E-08 & 2.745 E-07 & 3200 \\ 141 & 5.183 E-08 & 1.918 E-07 & 3168 \\ 360 & 1.320 E-07 & 4.884 E-07 & 3170 \\ 144 & 5.300 E-08 & 1.961 E-07 & 3172 \\ 275 & 1.007 E-07 & 3.727 E-07 & 3173 \\ 221 & 8.116 E-08 & 3.003 E-07 & 3175 \\ 119 & 4.361 E-08 & 1.614 E-07 & 3177 \\ 189 & 6.943 E-08 & 2.569 E-07 & 3179 \\ 68 & 2.523 E-08 & 9.335 E-0 B & 3180 \\ 328 & 1.203 E-07 & 4.450 E-07 & 3182 \\ 520 & 1.907 E-07 & 7.055 E-07 & 3184 \\ 851 & 3.119 E-07 & 1.154 E-06 & 3185\end{array}$




\begin{tabular}{|c|c|c|}
\hline.$\mu$ & 163 & $7.6781-07$ \\
\hline $\mathrm{H}_{4}, \mathrm{H}$ & 022 & 3.0 मा -O \\
\hline ns.p & 490 & 1.N44 $1-07$ \\
\hline 46.4 & 600 & $2.566 E-07$ \\
\hline$\$ 7.0$ & 974 & $3.672 \mathrm{E}-07$ \\
\hline AK. A & Yos, & $3.411 t-1.7$ \\
\hline dy.t & 1451 & $3.4708-07$ \\
\hline$a r ., n$ & $5,5,4$ & $3.0 \times 01-07$ \\
\hline 91.4 & 1314 & $4.4791-67$ \\
\hline 92.8 & 1766 & $0.65 \cup[-07$ \\
\hline 73.2 & 1394 & $5.275 E-U 7$ \\
\hline 44.6 & 1867 & $1.037 t-67$ \\
\hline 95.4 & 1428 & $0.068[-07$ \\
\hline 46.5 & $105 j$ & $6.906 E=07$ \\
\hline 77.8 & 2.15: & $0.1 \cup 2 \downarrow-67$ \\
\hline $9 \times$ & 1669 & h. $\angle \forall Y L-U 7$ \\
\hline 99.8 & 2003 & $7.3496-07$ \\
\hline 10jor & 1427 & $3.3771-07$ \\
\hline 101.0 & TAB & $\because 9901-67$ \\
\hline $1: 12.4$ & 467 & $1.76>1-67$ \\
\hline 3.6 & $40 ;$ & $1.521 t-67$ \\
\hline i., $x_{n}, \mathrm{~h}$ & $4 \% 1$ & $1.0646-37$ \\
\hline 1135.11 & 192 & $2.2336-67$ \\
\hline $100 . \mathrm{H}$ & 399 & $1.5065-177$ \\
\hline 167.8 & 247 & $9.321 L-i, 8$ \\
\hline 1.17 .0 & 429 & $3.124 t-07$ \\
\hline 1.9 .7 & 834 & $3.15 n[-07$ \\
\hline 110.7 & 2176 & B.194:-C7 \\
\hline 111.7 & 3401 & $1.432 \mathrm{E}-06$ \\
\hline 132.7 & 4034 & 1.42?L-Ua \\
\hline 113.7 & 1066 & $1.5331-06$ \\
\hline 114.6 & $5 \times 63$ & $1.904 E-06$ \\
\hline 115.6 & 3411 & $1.2 \cup 5 E-\cup 6$ \\
\hline 116.6 & 3461 & $1.304 \mathrm{k}=06$ \\
\hline 117.6 & 151 & $3.7360-08$ \\
\hline & 14 & $5.1<18-1) 4$ \\
\hline 114.5 & & \\
\hline
\end{tabular}
Sicisu.p.
CPISSHIVO INIFGRATIDE 1.31ME-1.3
1.0901 .06 $1.113 t-06$
$6.0561-67$ $9.2371-07$ $1.3221-06$ $1.22 d E-06$ $7.32<E-07$ $1.7 \% 1-06$ C.3"16-00 $1.6495-06$ 2. $3315-06$ $2.472 t-66$ $2.315 E-46$ $2.917 t-86$
$6.204 t-56$ 2. $71 \mathrm{EE-06}$ $1.936 t-106$ $1.009[-66$ C. $3+2 \mathrm{E}-\mathrm{U}$ ? अ. $401-01$ $5.5421-07$ S. $421 E-07$ $3.421 E-07$
$3.356[-07$ $1.12 S E-46$ $1.134 \mathrm{E}-66$ $2.95 \angle E-06$ 2. $1578-66$ u. S) U1-L1 $7.1435-06$ 4. 6 COLl-co $4.676 E-66$ 1.0440 .01 0 .

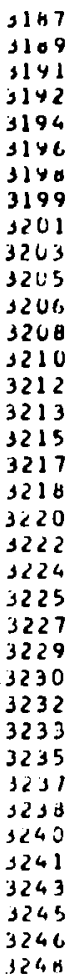
$4.747 t-03$ $1 / M$ $1.46,4 E-66$ $2.016,35-07$ 1

TEST U77 STPTEMELR 27, 19LB GUBO TU OLLO PST 21I.C SULFIDE KELEASE FKGM [LFVATILN UF 20 M SOOU. ARC SAPHLER WT 1.5M H- 3.6 M/SEC AI $26 \mathrm{M}$

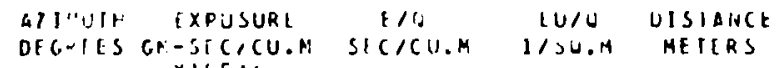
$x \perp 6 \varepsilon+6$

\begin{tabular}{|c|c|c|c|c|c|}
\hline y & 2.0 & 944 & $3.578 t-07$ & $1.2 \mathrm{n} b \mathrm{~F}-06$ & 4630 \\
\hline & 04.0 & 2053 & $3.971 \mathrm{E}-07$ & $1.430 \mathrm{t}-06$ & 4620 \\
\hline & $=6.0$ & 4505 & $1.69 \mathrm{~Hz}-26$ & $6.11][-06$ & 4510 \\
\hline & 9.9 & 954 & $3,46,1 B E-1,7$ & $1.2276-06$ & 4610 \\
\hline & $9 \cdot \% .6$ & $\$ 22$ & 3. JY4t - U7 & $1.116 E-46$ & 4610 \\
\hline & 42.0 & $29 A$ & $1.124 \mathrm{E}-07$ & $4.046 E-01$ & 4620 \\
\hline & 94.0 & 292 & $1.142 t-07$ & $3.967 l-07$ & 4620 \\
\hline & to. 0 & 541 & $2.040 E-07$ & $7.34 \mathrm{SE}-07$ & 4620 \\
\hline & 90.0 & 174 & $6.763 \mathrm{E}-U B$ & $2.435 F-07$ & $456 \mathrm{U}$ \\
\hline & 1.3 .3 & $28 \%$ & $1 . \operatorname{cst} E-6]$ & $3.8<2[-07$ & 4690 \\
\hline 4 & $1,2.6$ & 45 & $1.71 A t-U 9$ & G.1AßE-U & $671 \mathrm{U}$ \\
\hline 4 & $1,4.14$ & 97 & $3.677 E-65$ & $1.3<2\{-0\}$ & $477 \mathrm{C}$ \\
\hline & $I \leqslant, 0.0$ & 204 & $7.693 E-O H$ & $2.76,9 \mathrm{E}-07$ & 4810 \\
\hline & $1: A, C$ & 164 & $6.195 E-j 8$ & $2.23 \cup E-07$ & 4870 \\
\hline & i1). & 243 & $9.150 \mathrm{E}-0 \mathrm{~B}$ & $3.247 \mathrm{E}-07$ & 4720 \\
\hline & 112.11 & $10 \%$ & $4.114 t-28$ & $1.4 E 1 E-0 \%$ & 4490 \\
\hline l & 114.6 & (1) & 0. & u. & b000 \\
\hline 6 & $i 1 . . \therefore$ & is & 0. & u. & 5020 \\
\hline & $11 * .0$ & 12 & $4.571 \mathrm{~F}-1,9$ & $1.6468-08$ & 3100 \\
\hline 6 & $1<0.0$ & $u$ & c. & u. & 5136 \\
\hline$\because$ & $1<2.0$ & v & 0. & u. & 4330 \\
\hline$x$ & $1<6.0$ & 11 & 0. & u. & $466 i$ \\
\hline & $1,6$. & 4 & $1.734 E-\cup 4$ & $0.24<E-09$ & 4770 \\
\hline & $1<0.4$ & 12 & $4.7205-159$ & $1.70<t-0 \mathrm{H}$ & 4900 \\
\hline 5 & 100.0 & 2 & $7.082 E-10$ & $2.097[-69$ & 4970 \\
\hline & 132.6 & 3 & $2.207 E-09$ & $7.945 f-09$ & 4900 \\
\hline & 134.0 & $i$ & $0.305 t-10$ & $2.27 U E-O Y$ & 4990 \\
\hline & $1 \mathrm{~s}, \mathrm{r}$ & 3 & 0 . & c. & 5000 \\
\hline
\end{tabular}

CRESSMIND INIEGRATEU $=0.547 E-04 \quad 2.357 E-03$

\begin{tabular}{|c|c|c|c|c|c|}
\hline & 73.6 & 139 & $1.2421-07$ & $4.5935-07$ & 3167 \\
\hline & $U 4,0$ & 9 & $3.423 t-6 H$ & $1.2(1,5-1) t$ & 5104 \\
\hline & 45.8 & 160 & $5 . \mathrm{H} 671-04$ & $2.17 \mathrm{HE}-0 ?$ & 1191 \\
\hline & $\begin{array}{l}36.8 \\
17.8\end{array}$ & $\begin{array}{l}456 \\
307\end{array}$ & $\begin{array}{l}1.672 \mathrm{E}-07 \\
1.125 \mathrm{E}-07\end{array}$ & $\begin{array}{l}6.1018-01 \\
4.1611 \mathrm{~F}-6\end{array}$ & $\begin{array}{l}3192 \\
3194\end{array}$ \\
\hline & 84.8 & 296 & 1.04ist-07 & $4.0161-01$ & 3176 \\
\hline & 09.0 & 467 & $1.7111-107$ & $0.3325-07$ & 1198 \\
\hline & 40.6 & 24.3 & B. $8491-018$ & $3.2481-01$ & 1149 \\
\hline & 41.6 & 862 & $3.1541-07$ & $1 . j e ; i-u d$ & 3201 \\
\hline & $\begin{array}{l}92.5 \\
93.6\end{array}$ & $\begin{array}{l}1926 \\
476\end{array}$ & $\begin{array}{l}4.13372-17 \\
1.7561-27\end{array}$ & $\begin{array}{l}1.73=1-00 \\
6.4761-27\end{array}$ & $\begin{array}{l}3203 \\
3265\end{array}$ \\
\hline & 34.8 & 63' & $2.41, t-07$ & $8.9378-01$ & $32 \mathrm{lb}$ \\
\hline & 95.8 & bl. & $2.220 \mathrm{t}-\mathrm{J} 7$ & $0.213[-0]$ & 3208 \\
\hline & $96 . A$ & 531 & $1.9461-67$ & $7.200 \mathrm{E}-0 ?$ & 3210 \\
\hline & 97.8 & $79 \mathrm{H}$ & $2.024 t-07$ & $1.002 E-00$ & 3212 \\
\hline & 96.8 & 451 & $3.119 E-117$ & $1.154 \mathrm{E}-06$ & 3213 \\
\hline & 99.6 & 043 & $3.237 t-07$ & $1.193 F-06$ & 3215 \\
\hline & 100.8 & 595 & $2.1+1 t-07$ & $11.060[-07$ & 3217 \\
\hline & 101.8 & 289 & $1.046 F-07$ & $3.871[-07$ & 3218 \\
\hline & 132.8 & 451 & $1.672 t-1.57$ & $0.187[-.17$ & $12<0$ \\
\hline & 103.8 & 243 & H.AYQE - UH & $3.29 a^{1}[07$ & $\$ 22 i$ \\
\hline & 1014.8 & 43 & $1.3 N+1-0 H$ & $3, \theta \operatorname{col}-\cos$ & $\$ 224$ \\
\hline & 105.8 & 109 & $4.004 t-0 y$ & $1.483 t-07$ & $322 \%$ \\
\hline & 106.8 & 60 & $2.445 E-0 B$ & $9.0428-010$ & 3227 \\
\hline 3 & 107.8 & 35 & $1.62 \forall E-O B$ & $5.2021-00$ & 3224 \\
\hline & $100 . A$ & 189 & $6.943 \mathrm{E}-\mathrm{Ob}$ & $2.569 i-07$ & 3230 \\
\hline & 154.7 & 176 & $6.447 \mathrm{E}-0 \mathrm{H}$ & $2.3451:-01$ & 1232 \\
\hline & 110.7 & 713 & $2.6116-07$ & $4.66 \mathrm{CF}-111$ & 3233 \\
\hline & 111.7 & 1200 & $4.42015-07$ & $1.630 t-06$ & 3234 \\
\hline & 112.7 & 1314 & $0.6,461-07$ & $2.4501-06$ & 3237 \\
\hline & 113.7 & 5311 & $1.47138-06$ & $7.2311-00$ & 3238 \\
\hline & 114.6 & H97:1 & $3.76,1-06$ & $1.21 \mathrm{st}$ & 3240 \\
\hline & 115.6 & 126211 & $4.6,21+-i, 6$ & $1.71 \cup F-\cup S$ & $324 !$ \\
\hline 2 & 116.6 & 7046 & $2.4748-06$ & $1.0081-0 ;$ & 3243 \\
\hline & 111.6 & 1500 & $5.4361-67$ & coussi-iots &, 245 \\
\hline & $11^{4} .6$ & 731 & $2.7011-07$ & $4.4 \times 31-111$ & 5246 \\
\hline & 119.5 & 347 & $1.1401-67$ & 3.252 & 3240 \\
\hline & 120.5 & 349 & $1 .\left\langle t^{\prime} 1 t-U\right\rangle$ & $4.74 \dot{U} l-U l$ & 1249 \\
\hline & $1<1.5$ & 9 & $3.64][-64$ & $1.280 \mathrm{~L}-0 d$ & 3251 \\
\hline & 122.4 & 1 & $1.114 b L-10$ & $\angle .60 ; 0 t-09$ & 3252 \\
\hline & 12. 1.4 & (1 & $1.17+1-10$ & $4.34,1-14$ & 3213 \\
\hline & & $\triangle 1 F D=$ & 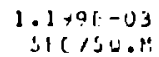 & $\begin{array}{c}4.437 E-U 1 \\
1 / 4\end{array}$ & \\
\hline
\end{tabular}

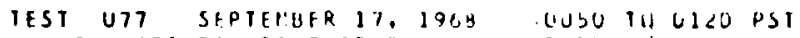
FLUUKESCEIN RFLFASE FHUM ELFVATIUN UIF SOK SCOUI: ARC SAMPLIR IIT $1.5 \mathrm{FH} U=3.7 \mathrm{M} / \mathrm{SIC} \triangle 1$ 5GM

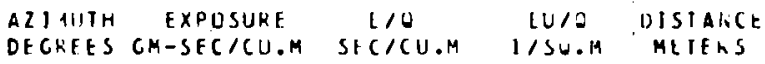
DECREES GM-SECICU.M SHCICU.M 1/SW.A MLIELS
XIUE +6

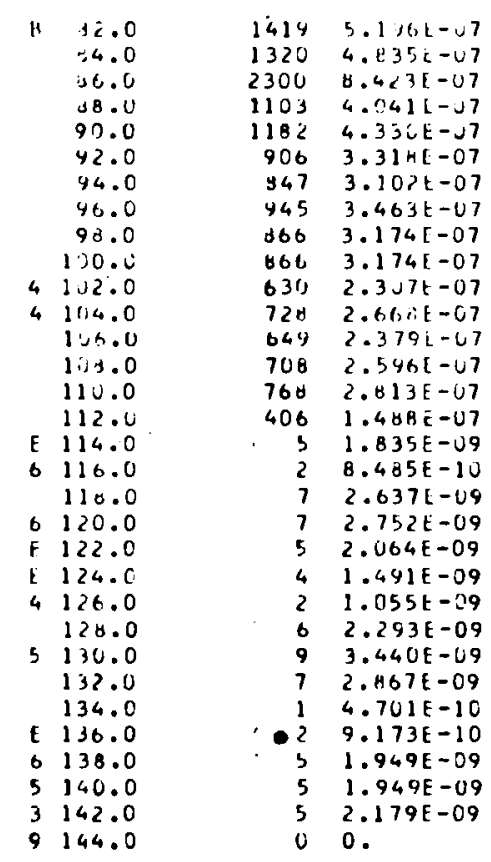

CROSSHIND INTEGRATEO=9.408E-04 SEC/5O.M
$1.4231-06 \quad 4010$ $1.7341-00 \quad 4620$ $3.117 E-06 \quad 4310$ $1.4956-00 \quad 4616$ $1.0021-00 \quad 4610$ $1.2281-06 \quad 4620$ $1.146 \mathrm{~F}-06 \quad 4620$ $1.201 E-06 \quad 4620$ $1.174 \mathrm{E}-06 \quad 4660$ $1.174 \mathrm{E}-06 \quad 469 \mathrm{U}$ Q.536E-07 4710 $4.0721-07 \quad 4770$ 6. BU3E-07 4810 $4.605 E-07 \quad 4870$ $1.041 E-00 \quad 47<0$ $5.507 E-07 \quad 4990$ $6.780 \mathrm{E}-095000$ $3.139 E-0 Y \quad 5020$ $9.758 t-09 \quad 5100$ $1.010 E-08 \quad 5130$ $7.637 E-09 \quad 4830$

$5.515 E-09 \quad 4660$

$3.903 E-09 \quad 4770$ B.485E-OY 4900 $1.273 E-08 \quad 4970$ $1.061 E-O B \quad 4980$ $1.739 E-09 \quad 4990$ $3.394 \mathrm{E}-09 \quad 500 \mathrm{C}$ $7.212 \mathrm{E}-09 \quad 5020$ $7.212 E-09 \quad 5060$ $8.061 E-09 \quad 5090$ 0 . 5130 


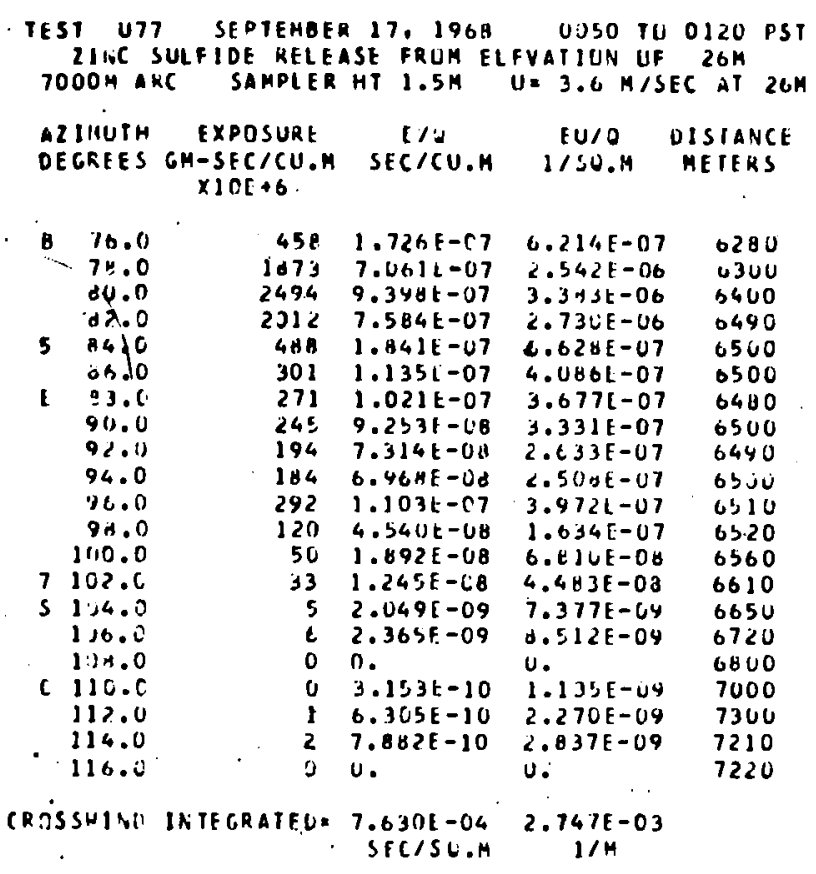

TFST U77 SEPTEMEEK 17, 196A UU5O TU U1<O PST 7 IIIC SULFIDE KELEASE TRUM ELFVATIUN OF $2 G M$

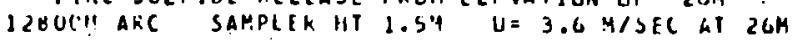

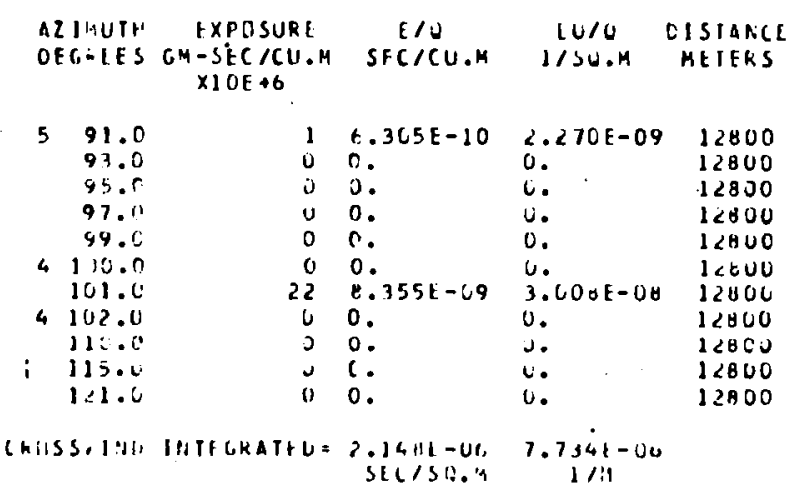

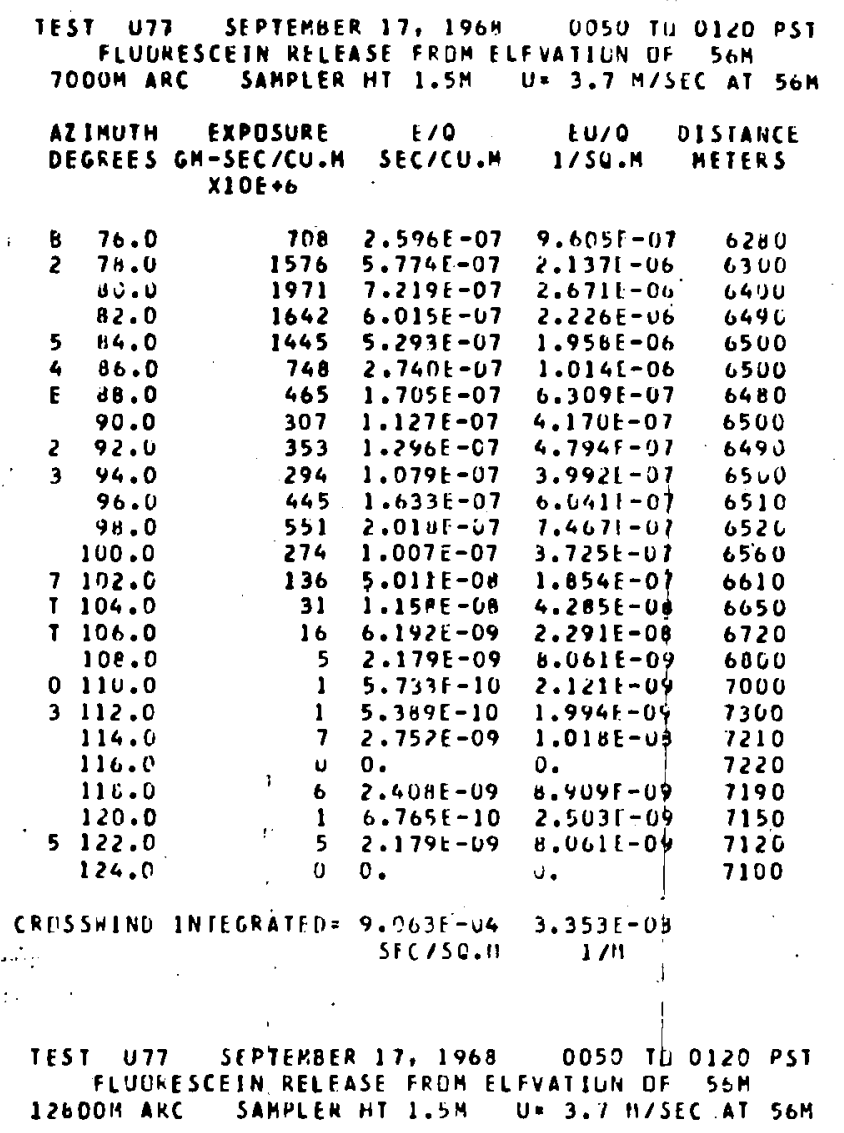

\begin{tabular}{|c|c|c|c|c|c|}
\hline \multicolumn{2}{|c|}{$\begin{array}{l}\text { AZIRUTH } \\
\text { DECIAEES }\end{array}$} & $\begin{array}{c}\text { EXPUSURE } \\
\text { GM-SEC } / C U . M \\
\times 1 O E+6\end{array}$ & \multirow{2}{*}{$\begin{array}{c}t / 0 \\
\operatorname{sic} / C U .4 \\
2.893 E-09\end{array}$} & \multirow{2}{*}{$\begin{array}{c}1 U / 0 \\
1 / S O . M \\
0.4 E S E-C Y\end{array}$} & $\begin{array}{l}\text { OISTENCE } \\
\text { METEKS }\end{array}$ \\
\hline p & 91.0 & & & & 12800 \\
\hline & Y 2.0 & 2 & $7.457 t-10$ & $2.7581-04$ & 12800 \\
\hline & 93.0 & & $2.293[-10$ & $0.4851-10$ & $1<B<0$ \\
\hline 5 & 44.0 & $u$ & $2.293 \mathrm{E}-10$ & B.4 SSL-10 & $1<\varepsilon \cup 6$ \\
\hline 5 & 95.0 & 2 & $9.1731-10$ & $3.3940-07$ & 12000 \\
\hline 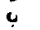 & $9 h .0$ & 3 & $1.142 t-09$ & $4.41<[-14 y$ & $12 a \cup b$ \\
\hline 9 & 97.0 & 1 & $4.3571-10$ & $1.0121-0 y$ & 12 120 \\
\hline & $4 B .0$ & $i$ & 4.1 & 1.4 & \\
\hline & 99.0 & i & $5.364[-10$ & $1.994 \mathrm{~F}-04$ & $1<\theta 00$ \\
\hline & 130.0 & 0 & $3.440 \mathrm{E}-11$ & 1.27 ,E-10 & $1<800$ \\
\hline & 101.0 & 1 & $4.357 t-10$ & $1.61 / F-04$ & $128 \cup$ \\
\hline & 102. & & $1.949 E-10$ & $1.21 \mathrm{e}:-10$ & $1<H \cup 0$ \\
\hline & i) 3.0 & 0 & $9.173 f-11$ & $\because .3 \forall n 1-10$ & $1<b \cup c$ \\
\hline & 104.0 & 0 & 1.005 & $3.940 !-10$ & $1: 000$ \\
\hline & jus.0 & 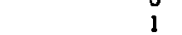 & $4.3571-10$ & $1.61<1-04$ & $1 \angle B \cup C$ \\
\hline 7 & $1 \cup 0.0$ & 0 & $2.064 \mathrm{E}-10$ & $7.637 \mathrm{E}-1 \mathrm{C}$ & 12900 \\
\hline & $1=7 . c$ & ) & 2.941 & $1.103 \mathrm{f}-0 \mathrm{G}$ & $1280 \mathrm{C}$ \\
\hline & iUM.O & $c$ & $1.37 t_{2} E$ & $5 .(14) \&-10$ & 12000 \\
\hline & 104.0 & 1 & $3.389 t-10$ & $1.94 \div 1-(1)$ & $1<8 \cup 0$ \\
\hline & 110. & 0 & $2.9 \mathrm{t}$ & $1.1031-6,4$ & 12800 \\
\hline & & 0 & 0. & u. & $1 \angle B O O$ \\
\hline & & & $\begin{array}{l}2.1431- \\
\text { Sec/so }\end{array}$ & $\theta .113[-1\} G$ & \\
\hline
\end{tabular}


SAMPLING 4OOM TO 7OSOM. NO TUWER SAHPLING. ALL ARCS EMBRAGE CRUSSWIND EXTENT OF TRACER.

TRACE OF MAIN FELL UURING IRACER RELEASE. UP TO 0.03 IHCHES UF RAIH FELL DURING PICKUP OF SAMPLESI ALTHOUGH

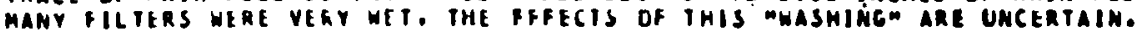

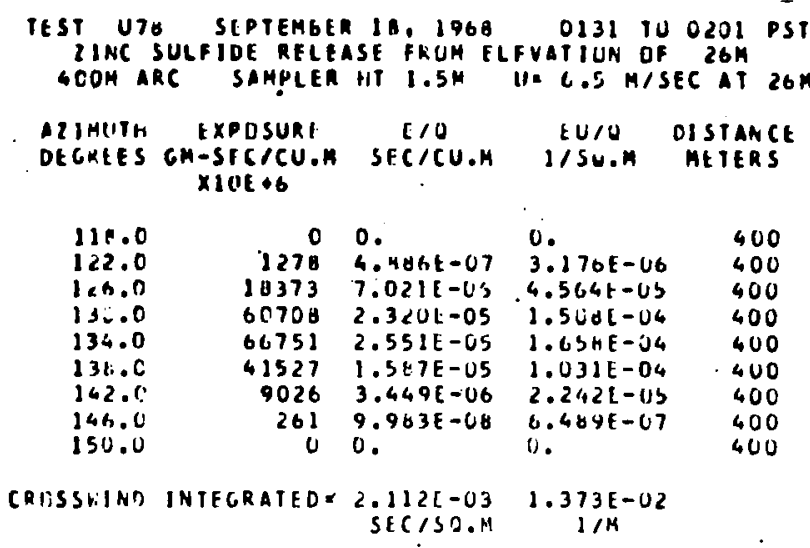

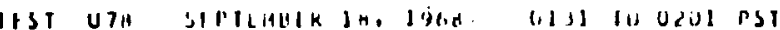
IINC SULFIOE HILIASI FKUM ILFVAIIIIN UI CUM ELCA ARC SAMULER HT I.SM U EIMITH FXPUSURE DFL.XEES GM-SECICU.M SICICU.M I/SG.M MEIERS $x \perp 0 t+6$

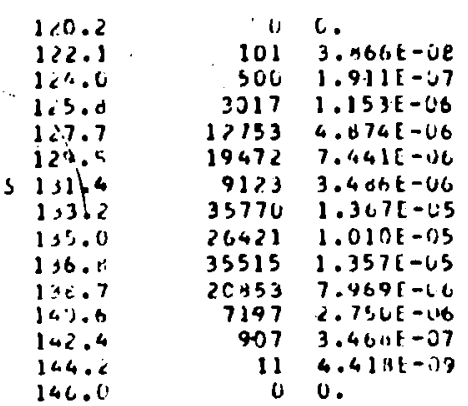

CRUSSAINO INIELRATLO= 1.UCLE-C3 $1.104 E-02$

ᄂ.

$2.313 E-67$ $1.24<t-00$ $7.456 E-V 6$ $3.160 \mathrm{E}-\mathrm{N}$ 1.. 0 sot-us $2.2005-05$ $\forall .0 \forall 4 E-O S$ $6.562 t-05$ $0.021 E-0 S$ 5.1 dut -05 1. 7 \& $\&$ E-OS $2.25,6-06$ $2.0725-40$ u. 643
648
651
854
857
859
802
665
667
069
072
074
176
878
080

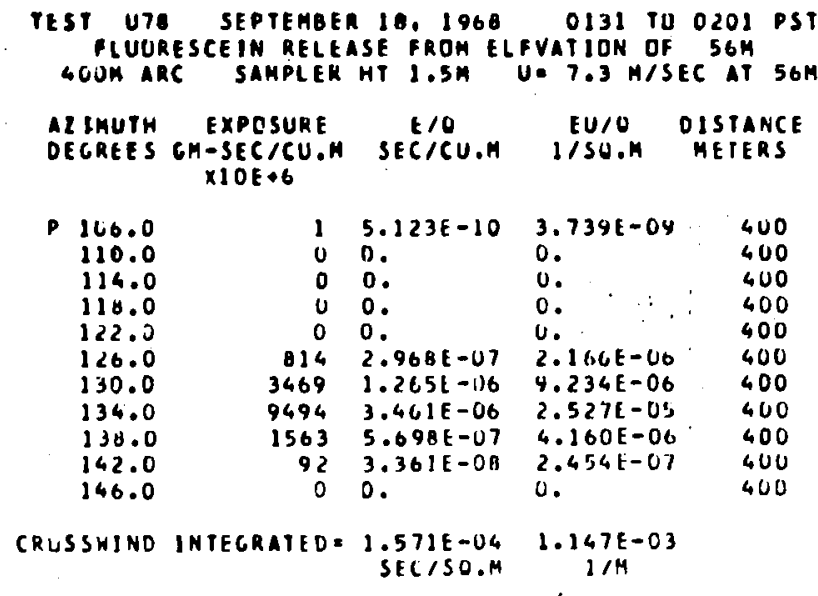

\footnotetext{
i
}

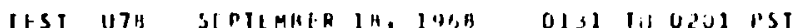 FLUIKESCEIII KELFASE FKIIY LLFVATIJN UI SUM BODH ARC SAHPLER HI $1.5 \%$ U $=7.3 \mathrm{M} / 5 \mathrm{SC}$ AI $50 \mathrm{M}$ AZIMIJIH FXPUSURL LO HUIO UISTANCE DEChFES CH-SEC/CU.M SHCICII.H I/SO.M MEIERS $\times 10 E+6$
122.1
$12+.0$
1.5 .6
127.7
129.3
1131.4
133.2
134.9
1136.9
133.7
143.6
1142.4
144.2
5146.0

I 147.8

$\begin{array}{rl}C & 1.175 E-10 \\ 20 B & 7.544 E-0 A \\ 1250 & 4.559 E-67 \\ 9694 & 3.536 E-06 \\ 379 A 9 & 1.345 E-05 \\ 44692 & 1.022 E-05 \\ 71623 & 2.611 E-C 5 \\ 24818 & 9.04 B E-06 \\ 11730 & 4.277 E-06 \\ 665 H & 2.42 H E-U 6 \\ 1250 & 4.559 t-07 \\ 75 & 2.763 E-08 \\ 7 & 2.020 E-69 \\ 7 & 2.690 E-09 \\ 18 & 6.738 E-09\end{array}$

A. $\$ 79 \mathrm{E}-1$

$5.344 t-47$

$3.3201-00$ $2.551 E-05$

$1.011 \mathrm{~L}-\mathrm{J4}$

1. $104 \mathrm{E}-04$

$1.900 t-04$

$6.6055-05$

$3.122 \mathrm{E}-0$

$3.122 \mathrm{E}-0$
$1.772 \mathrm{E}-0$

$1.772 t-05$
$3.3211-06$

$2.0175-07$

$2.034 t-02$

1.964 E-Cd

$4.919 E-00$

848
051
854
857
859
862
665
867
870
472
874
876
878
880
862

CRUSSHIND INIEGRATFD-?.097E-03 1.531F-02 
TEST U70 SEPTEMEER 18. 1968. 0131 TO 0201 PST ZINC SULFIDE RELEASE FROM ELEVATIOH DF $26 \mathrm{H}$ 1200M ARC SAKPLER HT 1.5H U. Q.5 M/SEC AT $26 \mathrm{~K}$

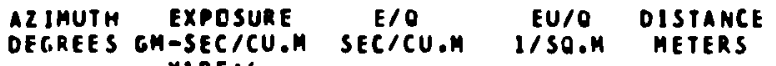
$\times 10 E+6$

$\begin{array}{rrrrr}\times 122.0 & 0 & 0 . & 0 . & 1200 \\ 124.0 & 73 & 2.748 E-08 & 1.019 E-07 & 1200 \\ 126.0 & 1695 & 6.477 E-07 & 4.210 E-06 & 1200 \\ 128.0 & 3732 & 1.426 E-06 & 9.270 E-06 & 1200 \\ 5130.0 & 0903 & 3.402 E-06 & 2.211 E-05 & 1200 \\ 132.0 & 12354 & 4.7211 E-06 & 3.069 E-05 & 1200 \\ 134.0 & 14764 & 5.642 E-06 & 3.667 E-05 & 1200 \\ S 136.0 & 17032 & 6.509 E-06 & 4.231 E-05 & 1200 \\ 134.0 & 4412 & 1.686 E-06 & 1.096 E-05 & 1200 \\ 140.0 & 2209 & 0.441 E-07 & 5.467 E-06 & 1200 \\ 142.0 & 287 & 1.100 E-07 & 7.147 E-07 & 1200 \\ 144.0 & 6 & 2.518 E-09 & 1.637 E-08 & 1200 \\ 146.0 & 0 & 2.798 E-10 & 1.819 E-09 & 1200 \\ & & & & \\ \text { CROSSHIND IMTECRATED } & 1.048 E-03 & 6.812 E-03 & \end{array}$

TEST U78 SEPTEMBER 18, 1968 O 0131 TU 0201 PST 21NC SULFIDE RELEASE FROM ELFVATION OF $26 \mathrm{H}$

AZIMUTH EXPOSURE E/O EU/O DISTANCE DEGREES GM-SECICU.M SECICU.M I/SO.M METERS $X 10 E+6$

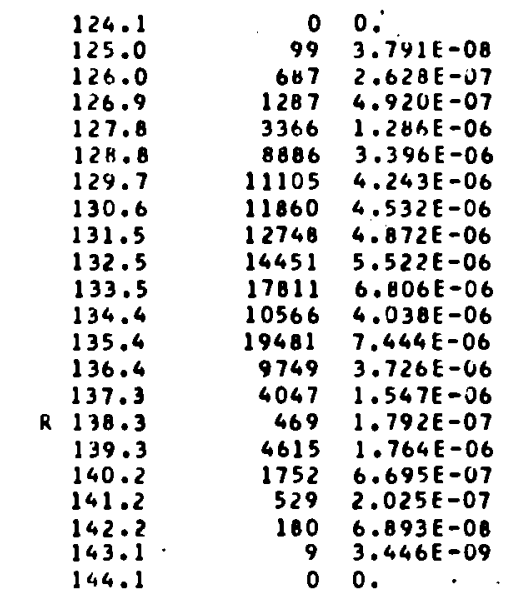

CRUSSNIND INTEGRATED= $1,417 E-03 \quad 9.213 E-03$ $1600 M$ ARC SAMPLER.HT $1.5 \mathrm{M}$ U. $6.5 \mathrm{H} / \mathrm{SEC}$ AT $26 \mathrm{M}$

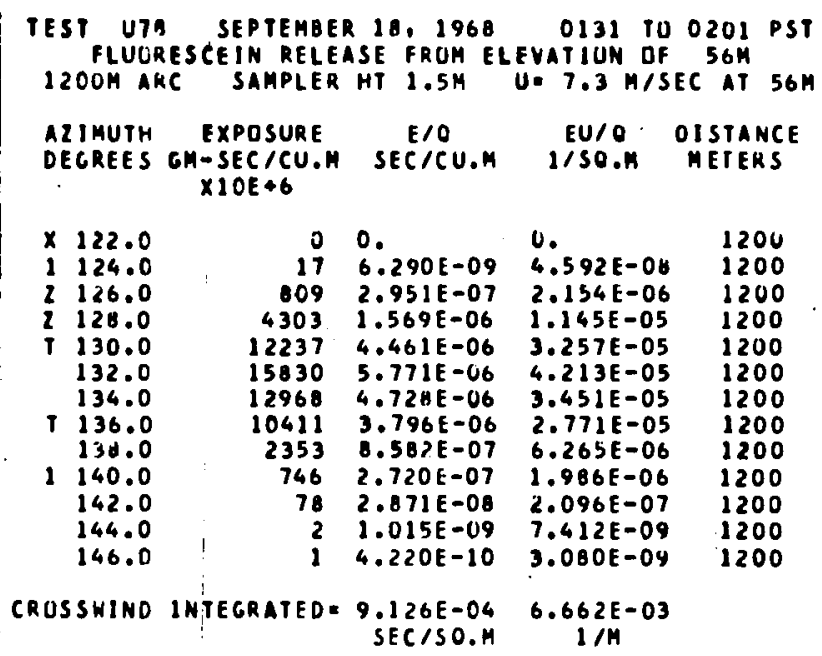

TEST UTA SEPTEMBER 18, 1968 O 0131 TU 0201 P.ST FLUORES CE IN RELEASE FROM ELFVATION OF $56 \mathrm{M}$ $1600 \mathrm{M}$ ARC SAMPLER HT $1.5 \mathrm{M} U=7.3 \mathrm{M} / \mathrm{SEC}$ AT $56 \mathrm{M}$

AZIMUTH EXPOSURE EIO EU/O DISTANCE DECREES CH-SEC/CU.M SECICU.M I/SO.M METERS $\times 10 E+6$

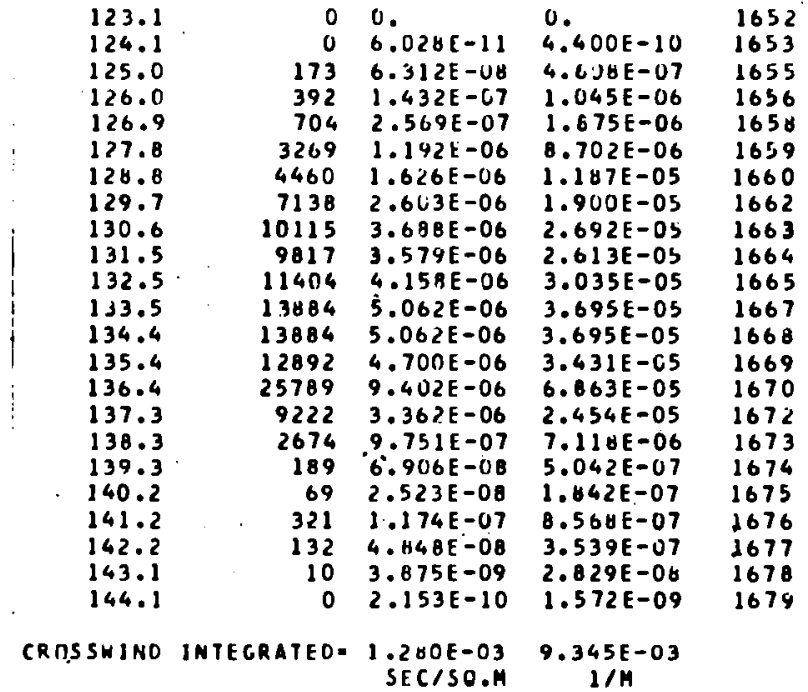

TEST U7B SEPTEMBER 18, 1968 OT31 TO 0201 PST ZINC SULFIOE RELEASE FROM ELFVATION OF $26 \mathrm{H}$

$2200 \mathrm{M}$ ARC SAHPLER HT $1.5 \mathrm{M} U=6.5 \mathrm{M} / \mathrm{SEC}$ AT $26 \mathrm{M}$

ALIMUTH EXPOSURE E/O EU/O DISTANCE DECKFES GM-SEC/CU.M SEC/CU.M I/SO.M HETERS $\times 10 E+6$

$\begin{array}{rrlll}122.0 & 0 & 0 . & & \\ \times 124.0 & 23 & 8.831 E-09 & 5.74 C E-0 B & 2200 \\ 126.0 & 206 & 7.902 E-08 & 5.136 E-07 & 2200 \\ 12 A .0 & 1094 & 4.184 E-07 & 2.720 E-06 & 2200 \\ 130.0 & 4412 & 1.686 E-06 & 1.096 E-05 & 2200 \\ 132.0 & 3110 & 1.184 E-06 & 7.725 E-06 & 2200 \\ E 134.0 & 3108 & 1.188 E-06 & 7.721 E-06 & 2200 \\ 136.0 & 3081 & 1.178 E-06 & 7.655 E-06 & 2200 \\ 7138.0 & 718 & 2.747 E-07 & 1.745 E-06 & 2200 \\ 140.0 & 591 & 2.2611 E-07 & 1.470 E-06 & 2200 \\ 142.0 & 60 & 2.314 E-08 & 1.504 E-07 & 2200 \\ 144.0 & 0 & 0.0 . & 0 . & 2200 \\ \text { CRIISSHIND INTEGRATED } & 4.815 E-04 & 3.130 E-03 & \end{array}$

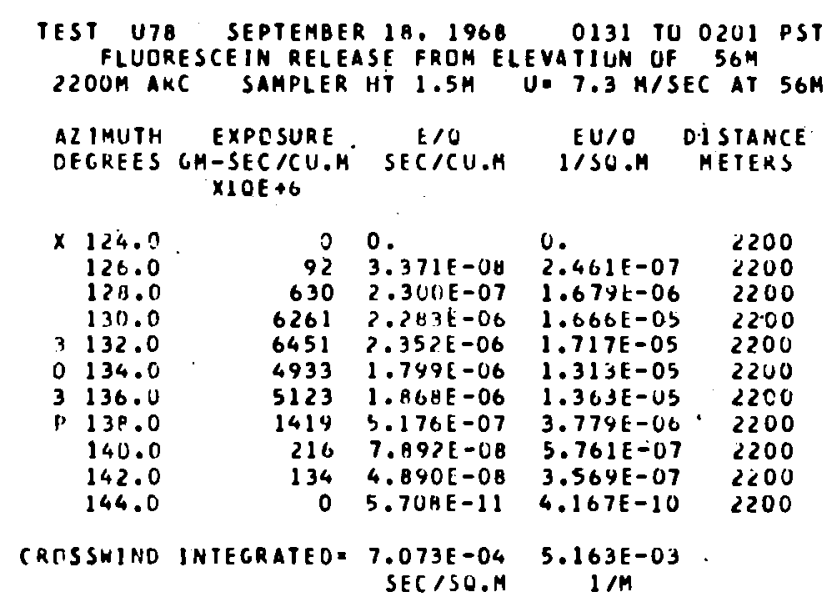




\begin{tabular}{|c|c|c|c|c|}
\hline $\begin{array}{l}\text { TFST UTE } \\
\text { IINCS S } \\
32 \text { UOM AR }\end{array}$ & $\begin{array}{l}\text { SEPTEMBE } \\
\text { ULFIDE RELE } \\
\text { IC SAMPLER }\end{array}$ & 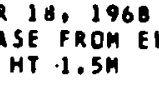 & $\begin{array}{r}0131 \text { TU } \\
\text { LFVATIUN OF } \\
\text { U. } 6.5 \mathrm{~m} / \mathrm{s}\end{array}$ & $\begin{array}{l}0201 \text { PST } \\
264 \\
\text { EC AT } 26 \%\end{array}$ \\
\hline $\begin{array}{l}\text { AL IMUTH } \\
\text { DEGREES }\end{array}$ & $\begin{array}{l}\text { EXPOSURE } \\
\text { GM-SEC /CU.H } \\
X \perp O E+6\end{array}$ & $\begin{array}{c}E / O \\
\text { SEC/CU.H }\end{array}$ & $\begin{array}{c}E U / 0 \\
1 / S Q . M\end{array}$ & $\begin{array}{l}\text { DISTANCE } \\
\text { METERS }\end{array}$ \\
\hline $\begin{array}{l}125.4 \\
126.3 \\
127.3 \\
129.3 \\
129.3 \\
130.3 \\
131.2 \\
132.2 \\
133.2 \\
134.2 \\
135.2 \\
136.2 \\
137.1 \\
138.1 \\
139.1 \\
140.1 \\
141.1 \\
142.0\end{array}$ & $\begin{array}{r}14 \\
14 \\
54 \\
496 \\
705 \\
2028 \\
4060 \\
4142 \\
4349 \\
4022 \\
3732 \\
3173 \\
2446 \\
2056 \\
451 \\
277 \\
64 \\
0\end{array}$ & $\begin{array}{l}0 . \\
5.454 E-09 \\
2.082 E-08 \\
1.099 E-07 \\
2.697 E-07 \\
1.081 E-06 \\
1.552 E-06 \\
1.583 E-06 \\
1.662 E-06 \\
1.043 E-06 \\
1.426 E-06 \\
1.213 E-06 \\
9.350 E-07 \\
7.858 E-07 \\
3.252 E-07 \\
1.061 E-07 \\
2.474 E-00 \\
0.0\end{array}$ & $\begin{array}{l}0 . \\
3.545 E-08 \\
1.353 E-07 \\
1.234 E-06 \\
1.753 E-06 \\
7.023 E-06 \\
1.009 E-05 \\
1.029 E-05 \\
1.080 E-05 \\
1.198 E-05 \\
9.271 E-06 \\
7.882 E-06 \\
6.078 E-06 \\
5.108 E-06 \\
2.114 E-06 \\
6.896 E-07 \\
1.611 E-07 \\
0 .\end{array}$ & $\begin{array}{l}3256 \\
3258 \\
3259 \\
3261 \\
3262 \\
3263 \\
3264 \\
3266 \\
3267 \\
3268 \\
3270 \\
3271 \\
3272 \\
3273 \\
3274 \\
3276 \\
3277 \\
3270\end{array}$ \\
\hline CROSSWIND & INIEGRATEDE & $\begin{array}{r}7.290 E-04 \\
\text { SEC/SO.H }\end{array}$ & $\begin{array}{c}4.738 E-03 \\
\mathrm{I} / \mathrm{M}\end{array}$ & \\
\hline
\end{tabular}

TEST UTY SEPTEMBER 18.1960 0131 TU 0201 PST IINC SULFIOE RELEASE FROA ELEVATION OF $26 \mathrm{M}$ SUOOM ARC SAMPLER HT 1.5M U 6.5 M/SEC AT $26 \mathrm{M}$

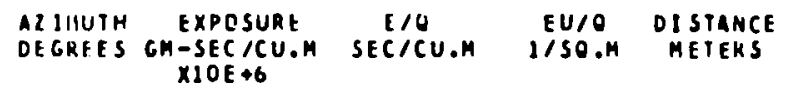
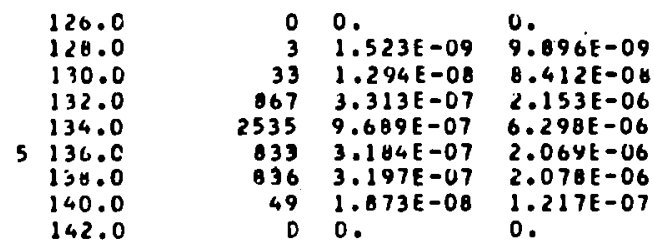

4770
4900
4970
4900
4990
5000
5020
5060
5090

CRMSSWIND INTECRATEU: $3.438 E-04 \quad 2.235 E-03$ SEC/SO.H

$1 / M$

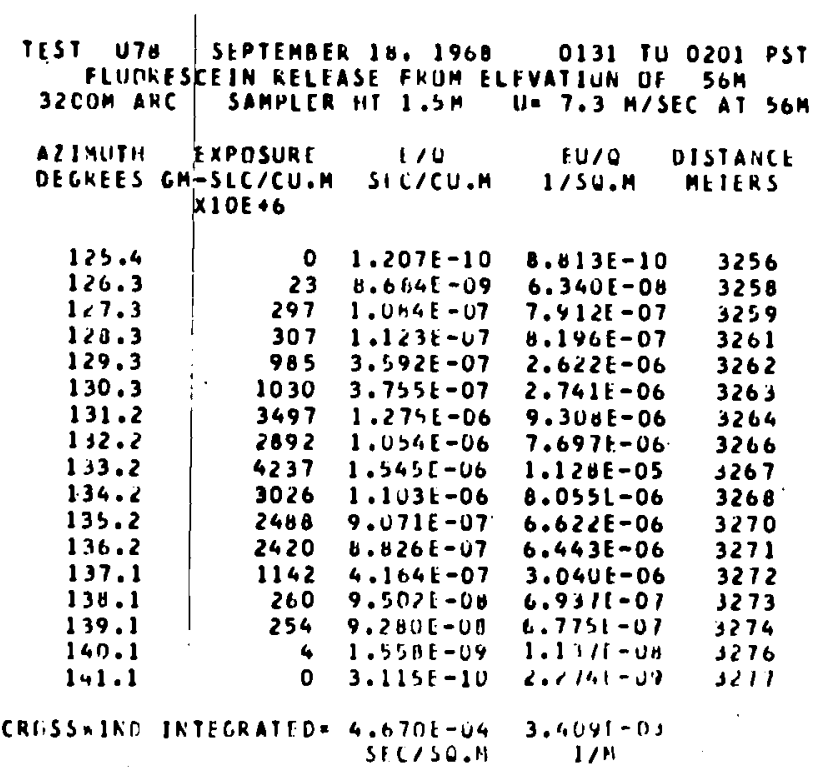

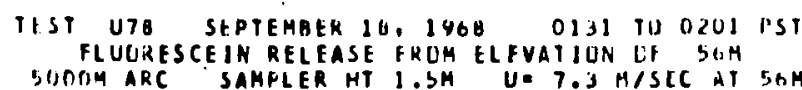
MIIYUTH EXPOSURE C/O EU/O DISIANCL DICKFES GH-SECICU.M SICICU.M IISO.M MEIERS $\times 10 E+6$

$\begin{array}{lrrrr}126.0 & 0 & 6.030 E-11 & 3.000 E-10 & 4770 \\ 121.0 & 0 & 1.027 E-10 & 7.50 U E-10 & 4900 \\ 130.0 & 0 & 2.991 E-09 & 2.183 E-00 & 4970 \\ 132.0 & 601 & 2.514 E-07 & 1.835 E-06 & 4980 \\ 134.0 & 1511 & 5.511 E-07 & 4.023 E-06 & 4990 \\ 5136.0 & 1511 & 5.511 E-07 & 4.023 E-06 & 5000 \\ 138.0 & 229 & 8.359 E-08 & 6.102 E-07 & 5020 \\ 140.0 & 4 & 1.694 E-09 & 1.237 E-08 & 5060 \\ 142.0 & 0 & 1.142 E-11 & 8.334 E-11 & 1090\end{array}$

CRUSSHIND IATEGRATED- $2.514 E-04 \quad 1.835 E-03$ 
SAMPLINC 4OOM TO 12 GOOM. NO TOHER SAMPLING. SAMPLING ARC AT $2200 \mathrm{H}$ WAS NOT ACTIVATEO.

ALL ARCS EMBKACE CRIISSHINO EXTENT OF TRACER.

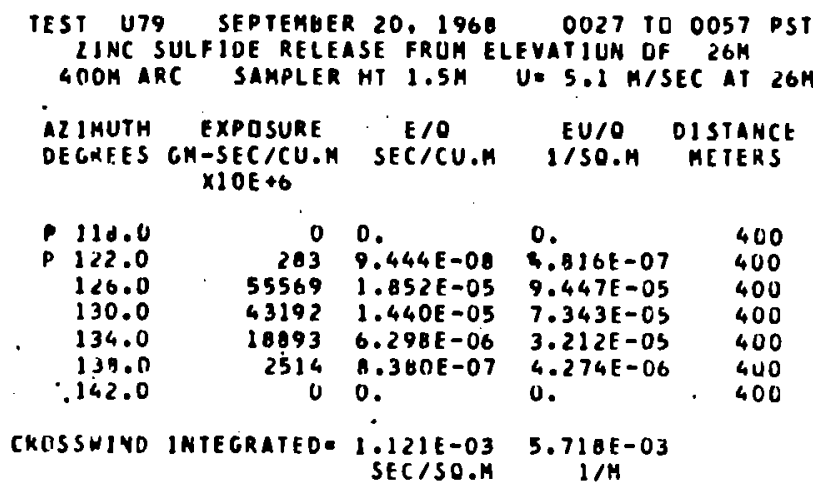

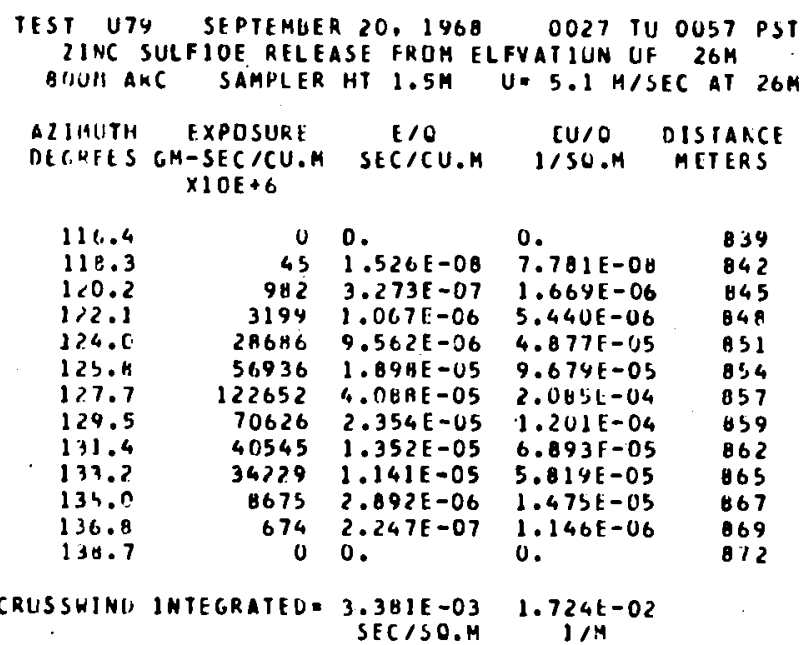

IFST U79 SEPTEMBER 20, 1968 OOL7 TL DUS7 PST IINC SULFIOE RFLFASF FRUM FLFVATIUN IJF $26 \mathrm{M}$ 120UH AKC SAMPLER HT $1.5 \mathrm{M} U=3 . J$ MISEC AT $26 \mathrm{~A}$ AZIYUTH EXPDSURE EIO EU/O OISIANCE DEGLEES. CM-SEC/CU.H SEC.ICU.M IISU.M MEIERS $\times 10 \mathrm{OE}+\mathrm{C}$

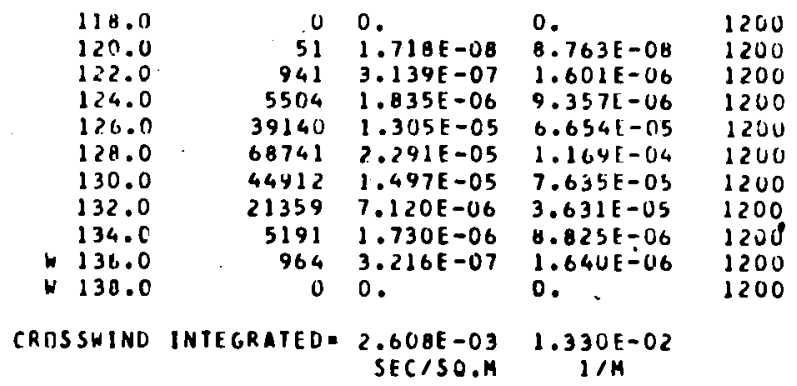

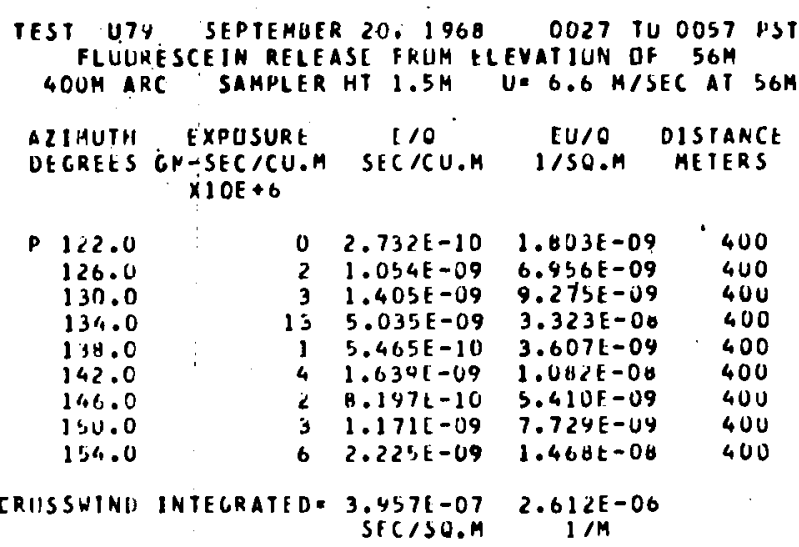

ILST U7Y SEPTEMBER 20, 1960 OOO27 TU 00S7 PST FLUIIIESCEIN RELIASF FHDM ELFVATIUH UF $56 \mathrm{H}$ HOUIA ARC SAMPLER IIT $1.5 \mathrm{M}$ U. $6.6 \mathrm{M} / \mathrm{SEC}$ AT $50 \mathrm{M}$

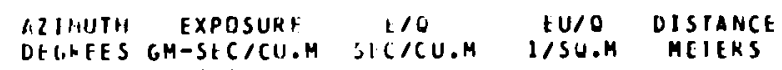
$\times 10 \mathrm{E}+6$

3.701F-03

112.6
114.5
116.4
118.3
110.2
112.1
124.0
125.4
127.7
129.5
131.4
133.8
134.9
136.9
134.9
140.6

$$
\begin{aligned}
& \text { is } u \text {. } \\
& 29.90: 1 E-10 \\
& \text { 1) } 3 . \cos 3[-09 \\
& 51 \text { 1. } 017 \mathrm{t}-0 \mathrm{~B} \\
& \begin{array}{ll}
105 & 3.012 L-08 \\
236 & 0.59 B t-08
\end{array} \\
& \begin{array}{r}
236 \\
46 \quad 1.698 t-04 \\
623 t-0 B
\end{array} \\
& 6232.253 \mathrm{~F}-07 \\
& 10000 \text { 3.9U1E-OB } \\
& 19325 \quad 7.0515-06 \\
& 14462 \quad 3.367 \text { E-06 } \\
& 49331.742 \mathrm{E}-06 \\
& 49331.762 \mathrm{E}-106 \\
& 4 \quad 1.772 \mathrm{E}-09 \\
& 16.11745-10 \\
& 00 \text {. }
\end{aligned}
$$

$6.574 \mathrm{~L}-09$ $3.731 \mathrm{E}-08$

$1.233 t-07$

2. $516 t-07$

$5.675+-07$

$3.675 t-07$
$1.100[-07$

$1.4 U 7 F-06$

$2.574 \mathrm{E}-0 \mathrm{~S}$

$4.634[-05$

$3.342 E-05$

$1.176 \mathrm{~F}-0 \mathrm{~S}$

$1.176 \mathrm{E}-05$

1.1 1 UE $-0 \mathrm{~B}$

$4.01<E-04$

0 .

CRIISSWINO IHTEGRATEDE S. GOEE-UG

$1 / \mathrm{M}$

800
636
839
842
845
848
851
634
857
859
862
065
867
870
672
674
6

TIST U7, SIPTENBER 20, 190 FLUIJRESCEIN RELEASE FRUM ELFYAIIUN UF $50 \mathrm{M}$ I POOM ARCI SAMPLER HT $1.5 \mathrm{M}$ U. $6.6 \mathrm{M} / \mathrm{SEC}$ AT SGH

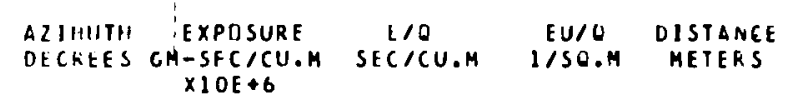

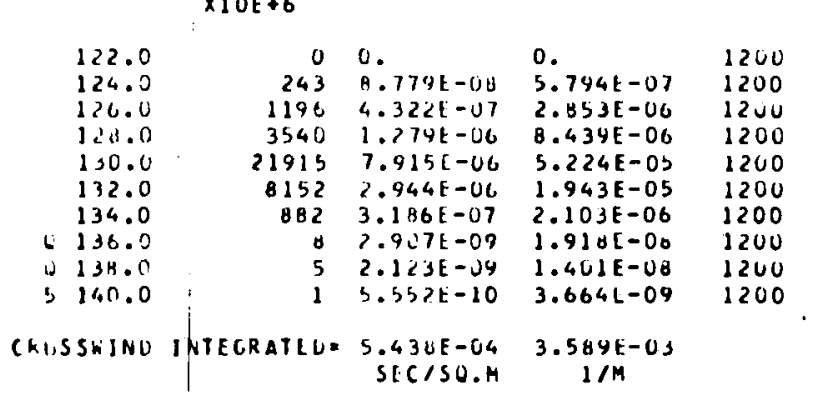




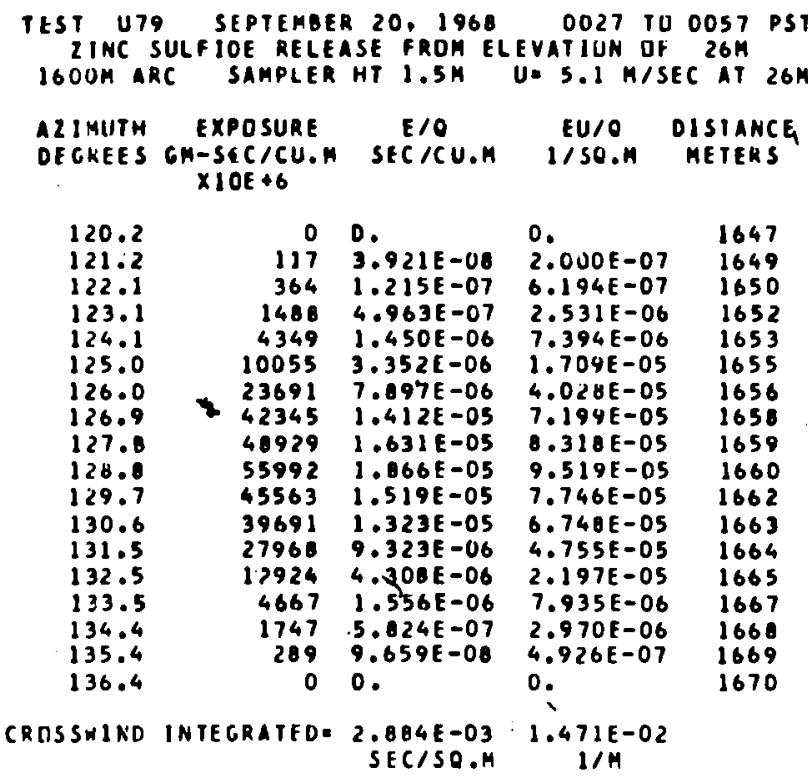

TEST U79 SEPTEHEER 20, 1968 OO27 TU 0057 PST 2 INC SULFIDE RELEASE FROM ELEVATIDN DF $26 \mathrm{M}$ $3200 M$ ARC SAMPLER HT $1.5 \mathrm{SM} U=5.1 \mathrm{M} / \mathrm{SEC}$ AT $26 \mathrm{H}$

A2IAUTH EXPOSURE EIO EU/O DISTANCE DEGREES CM-SECICU.M SECICU.H I/SO.H METERS $\times 10 E+6$

$\begin{array}{lrl}117.6 & 0 & 0.1 \\ 118.6 & 33 & 1.133 E-0 B \\ 119.5 & 232 & 7.748 E-08 \\ 120.5 & 1168 & 3.896 E-07 \\ 121.5 & 4210 & 1.404 E-06 \\ 122.4 & 4462 & 1.487 E-06 \\ 123.4 & 4417 & 1.472 E-06 \\ 124.4 & 7379 & 2.460 E-06 \\ 125.4 & 11363 & 3.794 E-06 \\ 126.3 & 17922 & 5.974 E-06 \\ 127.3 & 17209 & 5.736 E-06 \\ 128.3 & 8100 & 2.700 E-06 \\ 129.3 & 4908 & 1.636 E-06 \\ 130.3 & 2252 & 7.507 E-07 \\ 131.2 & 1205 & 4.019 E-07 \\ 132.2 & 221 & 7.385 E-08 \\ 133.2 & 0.385 & 0.06\end{array}$

0.

$5.777 E-08$

$3.951 E-07$

$1.987 E-06$

$7.158 E-06$

$7.586 E-06$

$7.510 E-06$ - 3253

$1.254 E-05 \quad 3255$

$1.935 E-05 \quad 3256$

$3.047 E-05 \quad 325$

$2.926 E-05 \quad 3259$

$1.377 \mathrm{E}-05 \quad 326$

1.377E-05 3261

3.829E-06 3263

$2.050 E-06 \quad 3264$

$3.766 E-07 \quad 3266$

0 .

3267

CRISSHINO INTECRATED= $1.573 E-03$ B.024E-03 $S E C / 50.4$

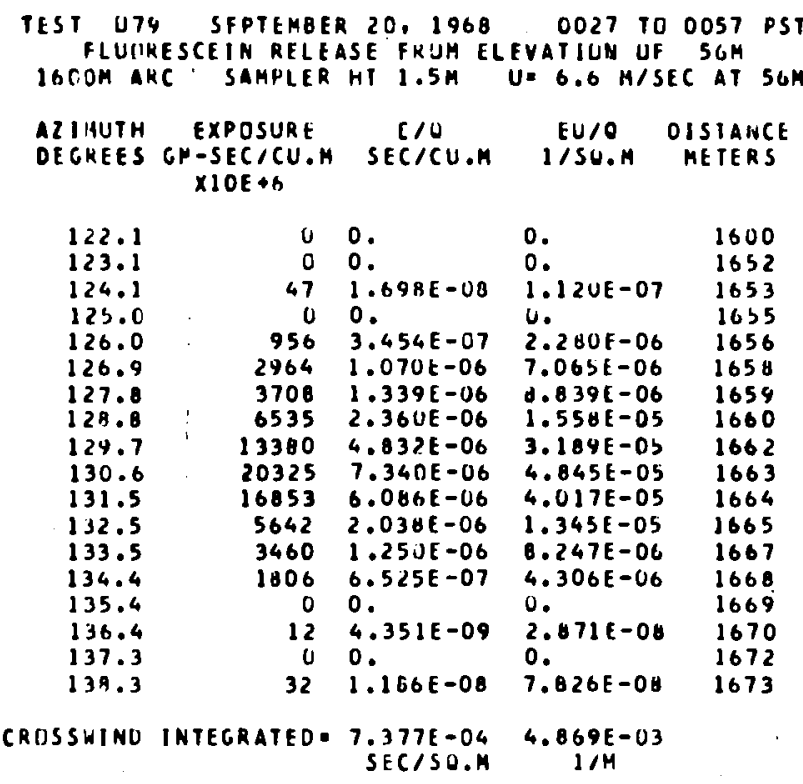

TEST U79 SEPTEHEER 20, 1968 0027 TU 0057 PST FLUOKESCEIN RELEASE FRDM ELFVATIUN DF $56 \mathrm{M}$ 32 COM ARC SAMPLER HT $1.5 \mathrm{H} U=6.6$ H/SEC AT $56 \mathrm{H}$

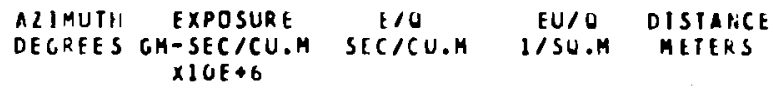

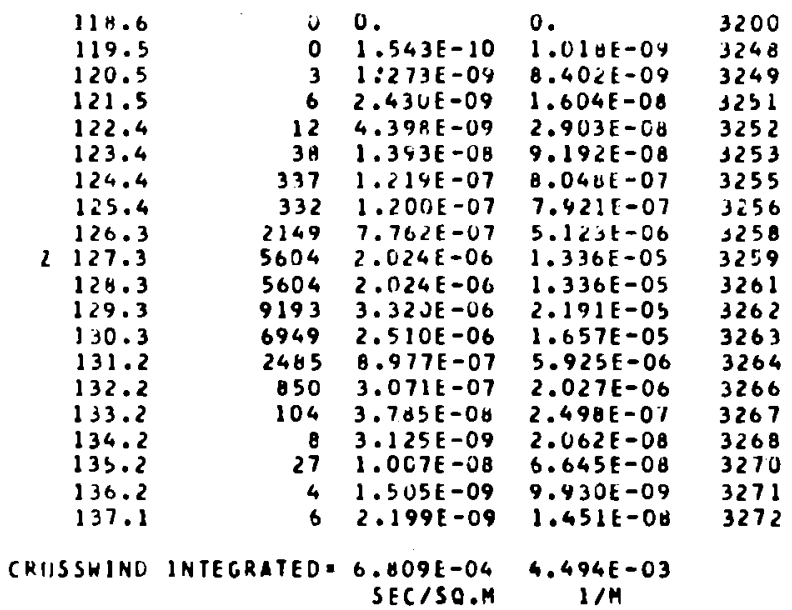




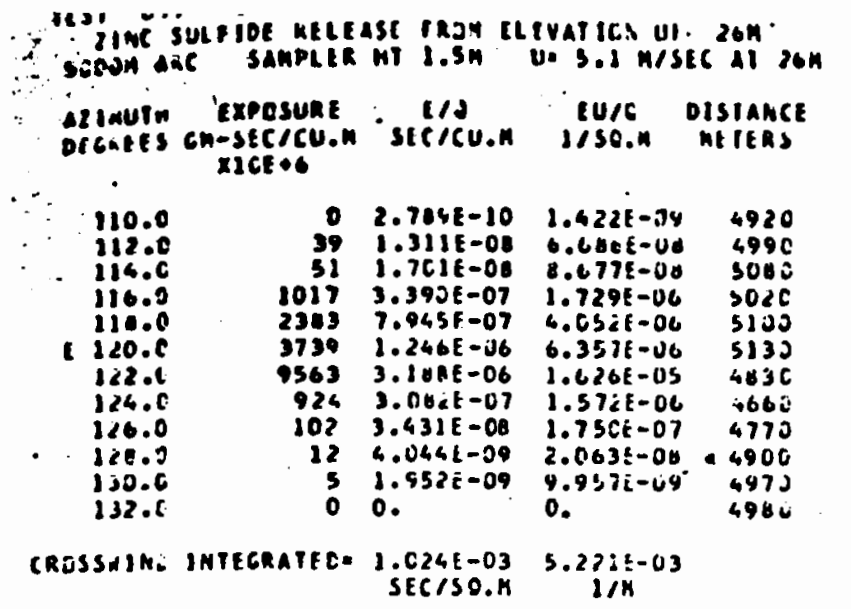

IISI UT: SEPIEPEFA 20.1768 CUA: IL ESST IST

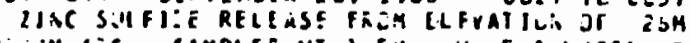

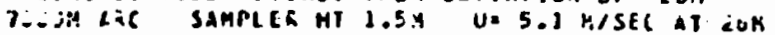

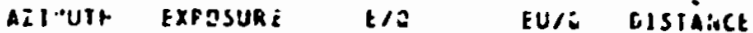
$U: C, F E S$ GP-SEG/CU.P SECICU.A I/SE.S METERS $x] \leq \leqslant+6$

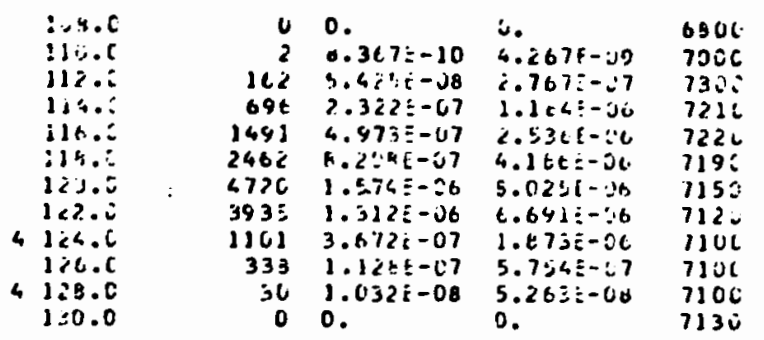

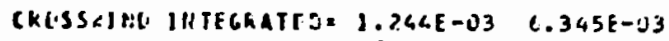

$$
\text { SECASE.A TH }
$$

IEST UT, SEPIEREEK 20, 1968 OOZ7 IO 2057 VST ClIIC SULF:CE RELEASE F

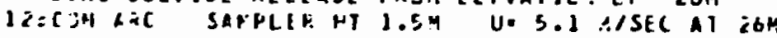

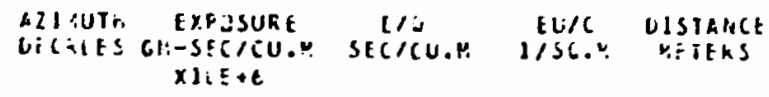

\begin{tabular}{|c|c|c|c|c|c|}
\hline & 1.00 & C & $2.7 \dot{4} \leq t-10$ & $1.422 \mathrm{i}-04$ & 12800 \\
\hline & $\begin{array}{l}111.2 \\
102.2\end{array}$ & 3 & $\begin{array}{l}2.650-09 \\
0 .\end{array}$ & $\therefore .351 \div-00$ & $\begin{array}{l}1<802 \\
1<806\end{array}$ \\
\hline & $1: 3.8$ & 6 & $2.731 i-39$ & $1.13=i-i \theta \theta$ & $i<800$ \\
\hline & $1: 4.6$ & 61 & $2.6365-3 d$ & $1.53=5-27$ & 12800 \\
\hline & 1.25 .1 & $z$ & $8.367 \bar{E}-10$ & $4.2615-57$ & ICBUC \\
\hline 7 & $\begin{array}{l}19.6 \\
3: 7.6 \\
3) .6\end{array}$ & $\begin{array}{l}45 \\
192 \\
150\end{array}$ & $\begin{array}{l}1.50 t-68 \\
6.4-1-08 \\
5.08 i-06\end{array}$ & $\begin{array}{l}7.601=-00 \\
3.2705-50 \\
2.360=-27\end{array}$ & $\begin{array}{l}12800 \\
18560 \\
12600\end{array}$ \\
\hline$t$ & $\begin{array}{l}19.5 \\
119.0\end{array}$ & $\begin{array}{l}212 \\
303\end{array}$ & $\begin{array}{l}7.07 c 1-39 \\
1.0128-07\end{array}$ & $\begin{array}{l}3.60 e-27 \\
3.1045-21\end{array}$ & $\begin{array}{l}\text { i } \angle 806 \\
i<3 c .\end{array}$ \\
\hline & $1 ! 1.1$ & $7:$ & $8.3 .11-i b$ & $1.216:-07$ & 12506 \\
\hline & $\begin{array}{l}1: 2.6 \\
11 \div .0\end{array}$ & $\begin{array}{l}3 \equiv t \\
28 \vdots\end{array}$ & $\begin{array}{l}1.1<::-c 7 \\
9.5<\div:-j t\end{array}$ & $\begin{array}{l}5.6 z c t-0.7 \\
4.69<-i 37\end{array}$ & $\begin{array}{l}1 \text { lac } \\
\text { lenui }\end{array}$ \\
\hline t & $\begin{array}{l}114.5 \\
119.5\end{array}$ & $\begin{array}{l}223 \\
203\end{array}$ & $\begin{array}{l}7.4471-00 \\
6.74<1-04\end{array}$ & $\begin{array}{l}3.7+05-27 \\
3.40<=-37\end{array}$ & i<bou \\
\hline & $11 n . i$ & 25: & F. $8,47 \div-08$ & $\begin{array}{l}3.40 .4=-57 \\
4.3 .3=5-07\end{array}$ & $\begin{array}{l}1<360 \\
12606\end{array}$ \\
\hline & $117 \cdot c$ & 241 & $3.0478-08$ & $4.104:-07$ & 12300 \\
\hline & $11 P . c$ & 34 & $1.1576-06$ & $3.90 \div:-0 b$ & .2960 \\
\hline & $114 . \%$ & 18 & $6.136,[-24$ & $3.12 y=-20$ & $1<50 i$ \\
\hline & $1 / 2.6$ & 10 & $3.4 \leq 0, t-39$ & $1.770 t-00$ & $1<80$. \\
\hline & 1.1.1. & 5 & $1.6131-69$ & $3.24 c:=-4,4$ & 12600 \\
\hline & $1,2.6$ & 6 & $1.3 \% 5 \div-10$ & $7.11<:-10$ & $1 / 800$ \\
\hline
\end{tabular}

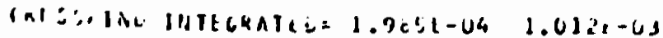

$$
\text { Silligen }
$$

\begin{tabular}{|c|c|c|c|c|}
\hline $\begin{array}{l}\text { Allavith } \\
\text { olgates }\end{array}$ & $\begin{array}{c}\text { Fxprswke } \\
\text { Gn-sis/cu.n } \\
x)=600\end{array}$ & $\begin{array}{c}\cos ^{\prime 0} \\
\text { setcou.t. }\end{array}$ & $\begin{array}{l}14 / 0 \\
1 / 5 E .4\end{array}$ & $\begin{array}{l}\text { oisianci: } \\
\text { mitiks }\end{array}$ \\
\hline $\begin{array}{l}112.0 \\
114.0 \\
116.0 \\
111.0 \\
0120.0 \\
122.0 \\
124.0 \\
126.0 \\
120.0 \\
139.0 \\
132.0 \\
134.0\end{array}$ & $\begin{array}{r}2 \\
2 \\
76 \\
193 \\
379 \\
1436 \\
1306 \\
2625 \\
146 \\
264 \\
11 \\
0\end{array}$ & $\begin{array}{l}0.029 E-10 \\
7.331 E-10 \\
2.767 E-00 \\
7.059 E-08 \\
1.371 E-J 7 \\
5.196 E-C 7 \\
3.627 E-07 \\
1.021 E-U 6 \\
3.057 E-07 \\
9.347 E-C H \\
4.044 E-O Y \\
0 .\end{array}$ & $\begin{array}{l}5.2498-34 \\
4.052 E-09 \\
1.0231-07 \\
4.6351-57 \\
9.0435-07 \\
3.4268-36 \\
2.394 E-06 \\
0.736 E-06 \\
2.011 E-26 \\
6.301 E-37 \\
2.742 E-30 \\
0 .\end{array}$ & $\begin{array}{l}4940 \\
3000 \\
5620 \\
5100 \\
3130 \\
4530 \\
4660 \\
4770 \\
4900 \\
4970 \\
4980 \\
4490\end{array}$ \\
\hline KESS:IND & INIEGRATEO & $\begin{array}{l}\text { 4. ZYSEE-04 } \\
\text { SEC SSO. }\end{array}$ & $\begin{array}{c}2.027 t-c 3 \\
1 / 14\end{array}$ & \\
\hline
\end{tabular}

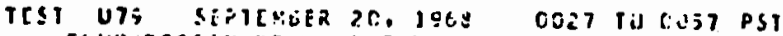
FLULB.ESCIIS RELEASI FISY FLFATIOS OF BOA

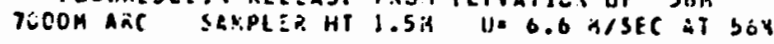

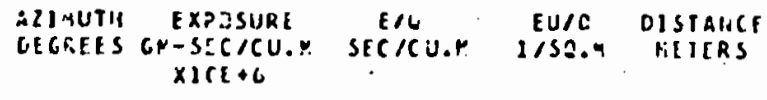

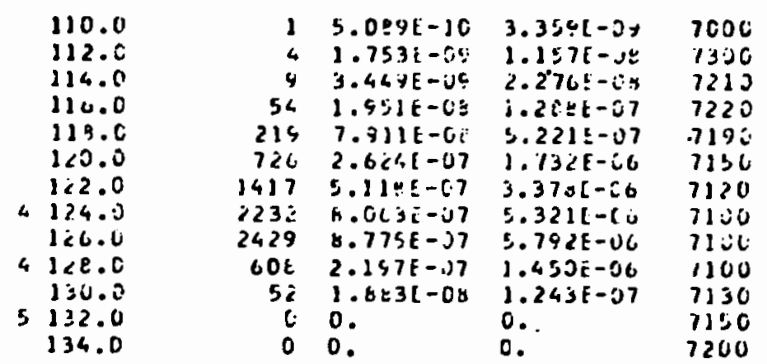

CRCSSAIND IHTEGRETFC. 6.553E-04 4.584E-IJ SEC/SO.N II:

IEST U7Y SEPIEMJEK 20, 196: 0027 TU COST PST

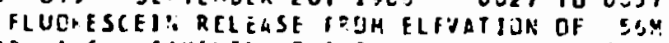

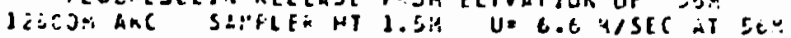

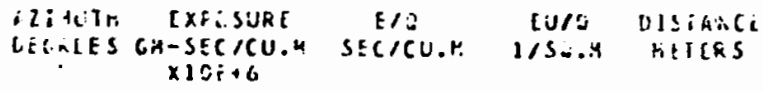

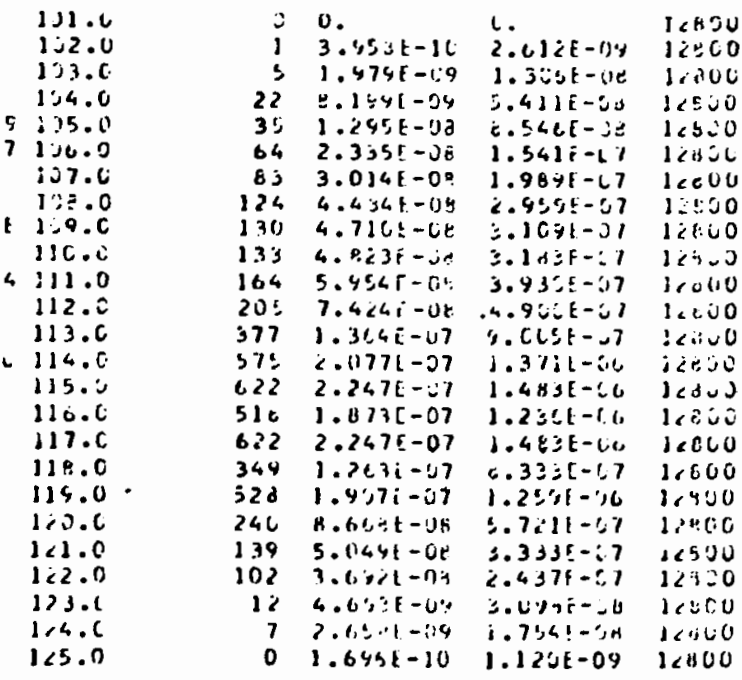

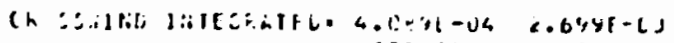

srejo.k. 114 
SAMPLING 4OOM TO 320OM. NO TOWER SAMHLING. ALL AKCS TRUNCATED FUK ZNS RELEASE FROM ZGH. SOME ARCS TRUNCATED IUN BLUOKESCEIN KELEASE FETM 56M. TRACER "SHEARED" HIUELY IN CKOSSHIND UIREGTIUN. IHERMALLY VERY STABLE ATHUSPHERE. EUUIPAENT DIFFICULTIES DURING FLUQRESCEIN ASSAY.

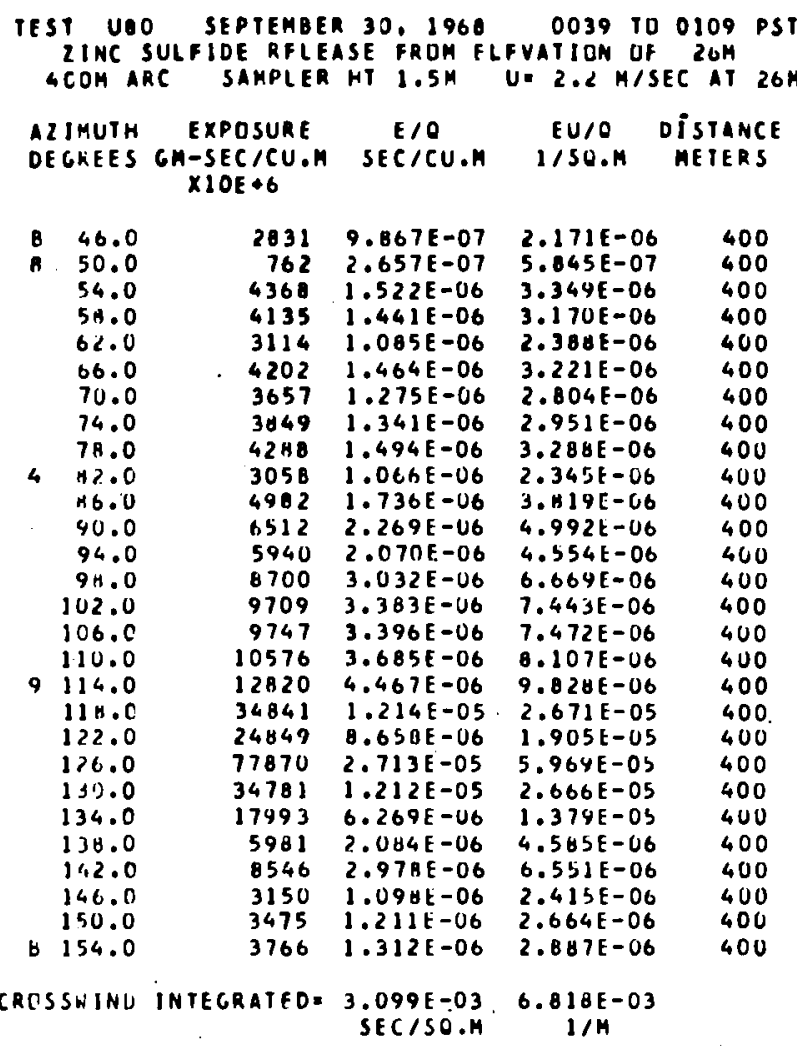

IEST U8O SEPTEMBER 30. 1968 TOO TU 0109 PST 2 INC SULFIDE RELEASE FROM ELEVATIUN OF $26 \mathrm{M}$ OCJM ARC SAMPLER HT $1.5 \mathrm{M} U=2.2 \mathrm{MISEC}$ AT $26 \mathrm{M}$

\begin{tabular}{|c|c|c|c|c|}
\hline $\begin{array}{l}\text { AL IMUTH } \\
\text { DEGKEES }\end{array}$ & $\begin{array}{c}\text { EXPCSURE } \\
\text { GH-SEC /CU.M } \\
\times 1 O E+6\end{array}$ & $\begin{array}{c}E / 0 \\
\text { SEC/CU, }\end{array}$ & $\begin{array}{l}E U / O \\
1 / S O \cdot M\end{array}$ & $\begin{array}{l}\text { DISTANCE } \\
\text { METERS }\end{array}$ \\
\hline
\end{tabular}

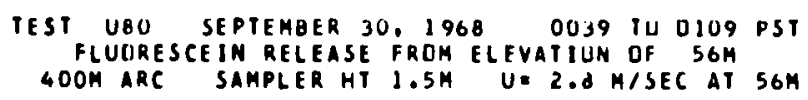

AZIMUTH EXPCSURE
DEGRES GH-SECICU.M SECICU.M
XIOE*6

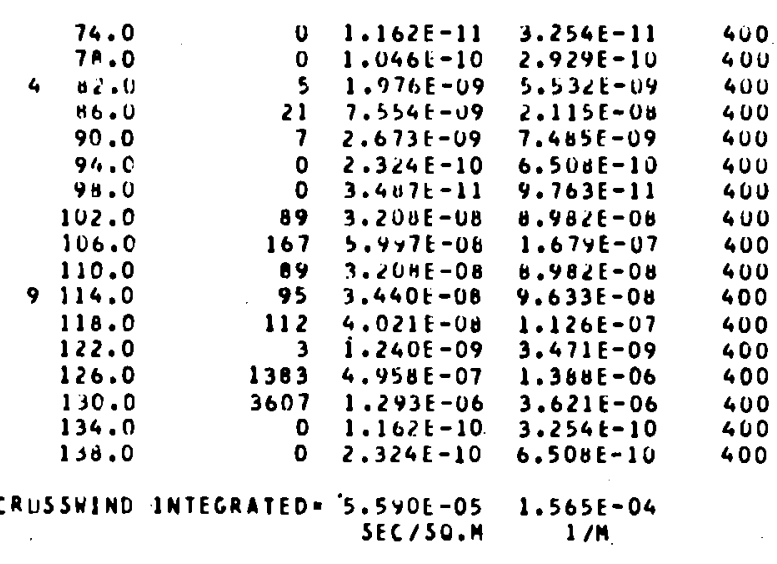

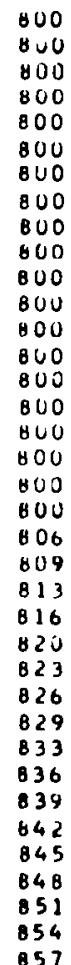

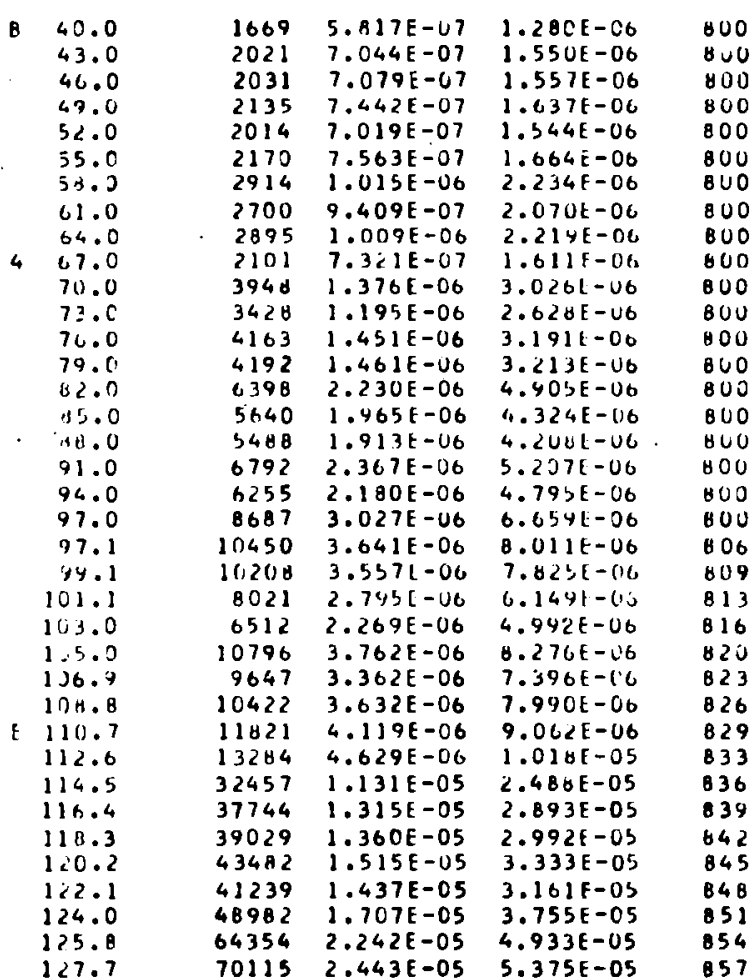

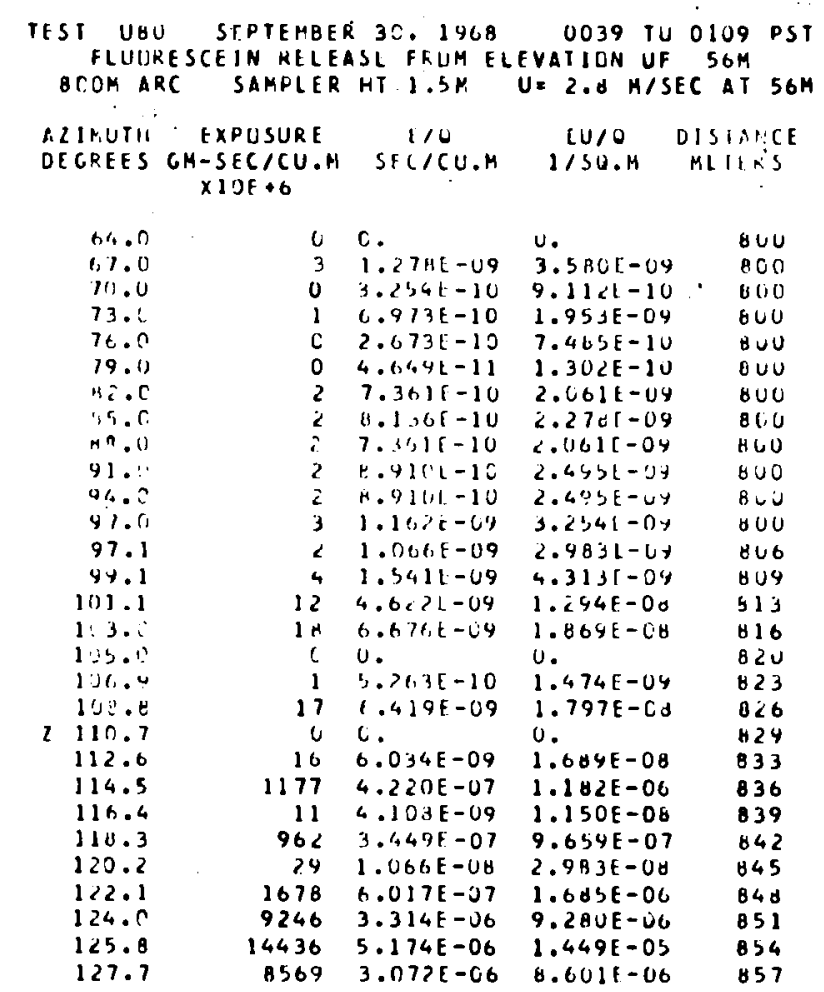




\begin{tabular}{|c|c|c|c|c|c|}
\hline & 120.5 & 110111 & $4.15 C E-U S$ & $4.1316-\mathrm{Cs}$ & ASY \\
\hline & 111.4 & 49935 & $1.740 \mathrm{E}-05$ & $3.820 \mathrm{~L}-0 \mathrm{~b}$ & 402 \\
\hline & 133.2 & 67294 & $2.171 E-05$ & 4.77 b-US & 065 \\
\hline & 135.0 & 59970 & $2.040 E-65$ & $4.597 E-05$ & 867 \\
\hline I & 136.0 & 54515 & $1.849 E-05$ & $4.17 \times[-05$ & 869 \\
\hline $\mathbf{E}$ & 134.7 & 43609 & $1.520 E-05$ & $3.343 t-05$ & 072 \\
\hline E & 140.6 & $327 c 3$ & $1.140 E-05$ & $2.507 E-05$ & 874 \\
\hline 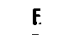 & 142.4 & 27745 & $9.667 E-06$ & $2.127 \mathrm{E}-0 \mathrm{~S}$ & 876 \\
\hline E & 144.2 & 26369 & $9.18 \mathrm{HE}-06$ & $2.021 E-0 S$ & 878 \\
\hline & 146.0 & 21975 & $7.657 \mathrm{E}-66$ & $1.685 \mathrm{SE}-\mathrm{US}$ & $\triangle 40$ \\
\hline & 147.8 & 15481 & $5.394 E-06$ & $1.187 E-05$ & $8 B 2$ \\
\hline & 149.6 & 17878 & $6.229 E-06$ & $1.370 E-05$ & 884 \\
\hline & 151.4 & 15012 & $5.231 \mathrm{E}-06$ & $1.151 E-05$ & 806 \\
\hline & 153.2 & 13084 & $4.559 t-06$ & $1.003 E-05$ & 487 \\
\hline & 155.0 & 10071 & $3.504 E-06$ & $7.721 t-06$ & B89 \\
\hline & $156 . \mathrm{A}$ & 7018 & $2.445 E-06$ & $5.380 E-06$ & $\theta 90$ \\
\hline & $13 A .6$ & 5851 & $2.039 E-06$ & $4.486 \mathrm{E}-06$ & 892 \\
\hline & 160.4 & 5541 & $1.931 E-06$ & $4.248 E-06$ & 893 \\
\hline B & 162.2 & 5787 & $2.017 E-06$ & $4.437 E-06$ & 894 \\
\hline CRO & INU & VTEGRATEDE & $\begin{array}{r}1.130 E-02 \\
S E C / 50 . H\end{array}$ & $\begin{array}{c}2.487 \mathrm{E}-02 \\
1 / \mathrm{M}\end{array}$ & \\
\hline
\end{tabular}

TEST UOO SEPTEMBER 30, 1968 0039 TO 0109 PST

ZINC SULFIUE RFLEASE FRUM ELEVATION OF $26 \mathrm{M}$ $120 O M$ ARC SAMPLER HT $1.5 \mathrm{M} U=2.2 \mathrm{M} / \mathrm{SEC}$ AT $26 \mathrm{M}$

CRUSSMING INTEGRATEO= B.679E-03 1.909E-02

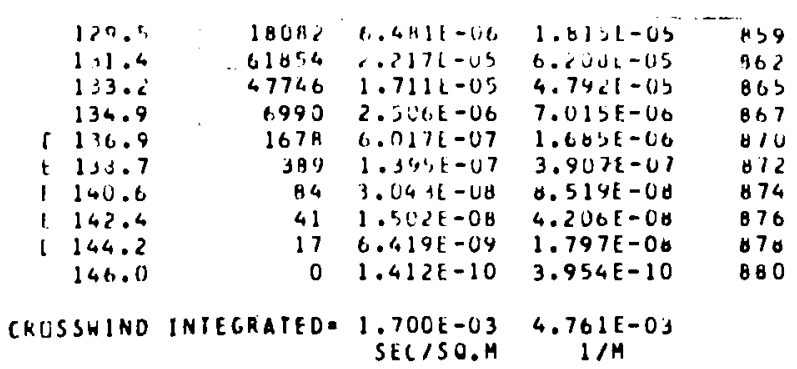

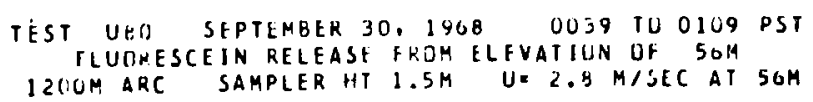

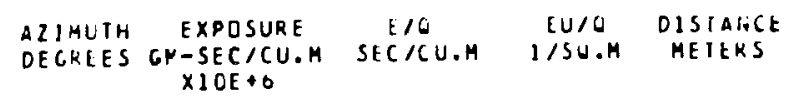

92.0
94.0
96.0
98.0
100.0
102.0
104.0
116.0
118.0
110.0
112.0
114.0
116.0
119.0
120.0
122.0
124.0
126.0
124.0
130.0
112.0
134.0
136.0
134.0
140.0
142.0
144.0
146.0
148.0
150.0
152.0
154.0
156.0

\begin{tabular}{|c|c|}
\hline U & 0 . \\
\hline & 0. \\
\hline 43 & $1.556 L-08$ \\
\hline 88 & $3.176 t-08$ \\
\hline 140 & $5.337 E-08$ \\
\hline 160 & $5.769 t-08$ \\
\hline 420 & $1.566 E-07$ \\
\hline 438 & $.1 .571 E-07$ \\
\hline 245 & $8.794 E-08$ \\
\hline 377 & $1.355 \mathrm{E}-07$ \\
\hline 390 & $1.400 E-07$ \\
\hline 1935 & $6.936 E-07$ \\
\hline 1099 & $3.941 \mathrm{E}-07$ \\
\hline 1441 & $5.166 E-07$ \\
\hline 1327 & $4.758 \mathrm{E}-0.7$ \\
\hline 719 & $2.5 \mathrm{HOE}-67$ \\
\hline 517 & $1.854[-07$ \\
\hline 1251 & $4.486[-07$ \\
\hline 6581 & $2.359 E-06$ \\
\hline 8583 & $3.077 t-06$ \\
\hline 23627 & $8.469 t-06$ \\
\hline 31503 & $1.129[-05$ \\
\hline 2656 & $9.523 E-07$ \\
\hline 466 & $1.672 E-07$ \\
\hline 947 & $3.397 E-07$ \\
\hline 812 & $2.911 E-07$ \\
\hline 335 & $1.204 E-07$ \\
\hline 649 & $2.327 E-07$ \\
\hline 209 & $7.498 \mathrm{E}-08$ \\
\hline 27 & $9.940[-09$ \\
\hline 41 & $1.441 \mathrm{E}-08$ \\
\hline & $3.436[-09$ \\
\hline 11 & $4.106 E-09$ \\
\hline
\end{tabular}

i. $\quad 1200$

0 .

$4.350 E-U E-1200$ $4.844 t-00 \quad 1200$ $1.494 E-07 \quad 1200$ $1.615 E-01 \quad 1200$ $4.217 E-07 \quad 1200$ $4.399 \mathrm{E}-07 \quad 1200$ $2.462 E-07 \quad 1200$ $3.743 \mathrm{E}-07 \quad 1200$ $3.921 E-07 \quad 1200$ $1.942 E-06 \quad 1200$ $1.104 E-06 \quad 1200$ $1.447 \mathrm{E}-06 \quad 12 \mathrm{~J} 0$ $1.332 \mathrm{E}-06 \quad 1200$ $7.224 \mathrm{E}-07 \quad 1200$ b. $191 \mathrm{E}-07 \quad 1200$ $1.256 \mathrm{k}-06 \quad 1200$ $6.60 S E-00 \quad 1200$ $8.614 E-06 \quad 1200$ $2.371 E-05 \quad 1200$ $3.162 E-05 \quad 1200$ $2.666 \mathrm{E}-06 \quad 1200$ $2.666 \mathrm{E}-06$
$4.633 \mathrm{E}-07 \quad 1200$ $9.511 E-07 \quad 1200$ H.15UE-07 1200 $3.37 \cup E-07 \quad 1200$ $6.516 E-07 \quad 1200$ $2.099 E-07 \quad 1200$ $2.743 E-08 \quad 1200$ $4.175 E-00 \quad 1200$ $9.620 E-04 \quad 1200$ CRUSSHINO INTEGRATED $=1.307 E-03 \quad 3.66 U E-03$ SECISO.H I/M 
TEST UAR SEPTEMBER 30. 1960 O0039 TO 0109 PST 2INC SULFIDE RELEASE FROM ELEVATIUN UF $26 \mathrm{H}$

$1600 M$ ARC SAMPLER HT $1.5 \mathrm{M} U=2.2 \mathrm{M} / \mathrm{SEC}$ AT $26 \mathrm{M}$

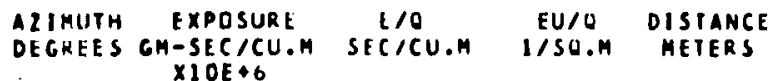

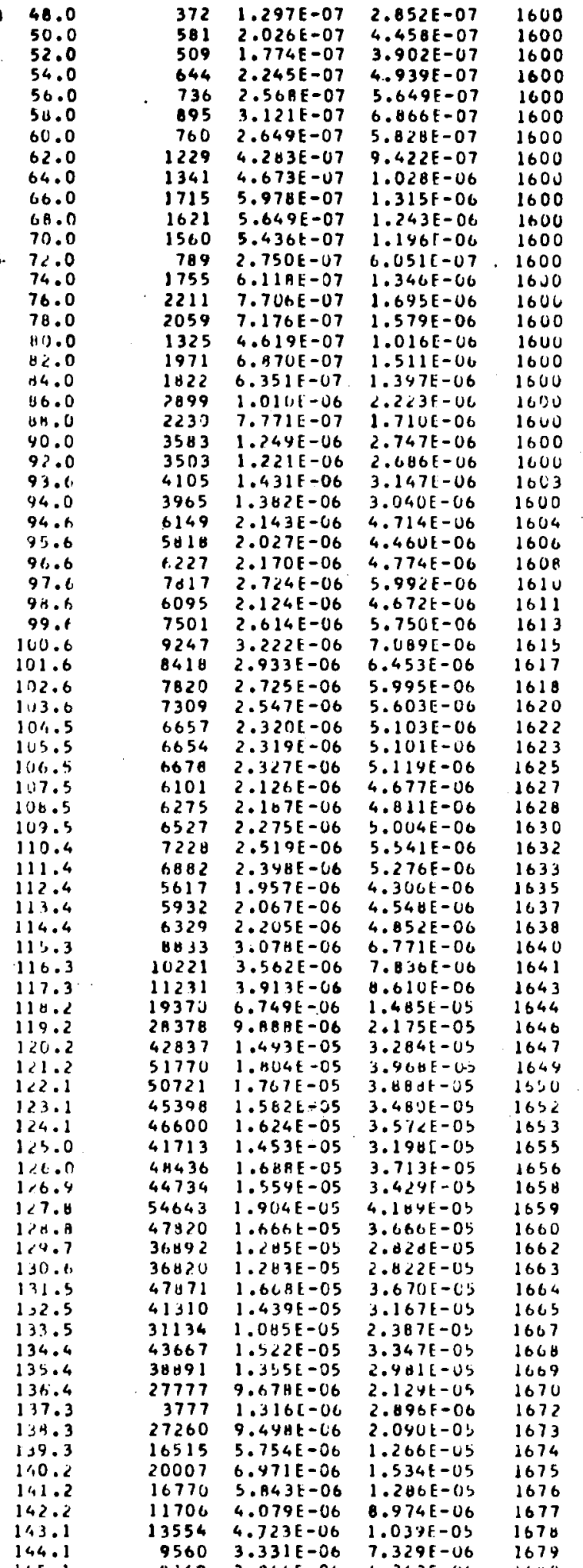
TEST UTU SIPTEMBIR 311. 196A 0039 TU OLU PST FIUITESCFIN RELIASE FHUY ILEVAIIUN UF $56 \mathrm{M}$ IBCUM ARC SAMPLER HT $1.54 \mathrm{U}=\therefore$ LO HISEC AT SGM
AZIMIITH EXPOSURE IIU EU/O DISIANICE DEGREES GH-SEC/CU.H SEC/CU.M I/SO.M METERS $\times 10 E+6$

\begin{tabular}{|c|c|c|c|c|}
\hline 96.6 & $\iota$ & u. & $\because$ & 1600 \\
\hline 99.1 & 11 & $2.340 t-10$ & $7.112 \mathrm{E}-10$ & 1013 \\
\hline 160.6 & 16 & $3.743 t-64$ & $1.607 E-00$ & 1615 \\
\hline 101.6 & 36 & $1.3111-08$ & $3.670 t-U d$ & 1617 \\
\hline 1.12 .6 & $1 \theta$ & $0.50>b-09$ & $1 . \forall<1 E-00$ & 1618 \\
\hline $1<3.6$ & 20 & 7.31 HE-O9 & $2.105 E-0 d$ & 1620 \\
\hline 164.5 & 20 & $7.518 \mathrm{t}-09$ & $2.105 E-0 d$ & 1622 \\
\hline 109.5 & 1112 & $3.673 \mathrm{E}-08$ & $1.020 \mathrm{t}-07$ & 1023 \\
\hline $1 \cdot 20.5$ & 1295 & $a, 6436-1) 7$ & $1.300 t-06$ & 1625 \\
\hline 1.7 .5 & 400 & 0. & & 1627 \\
\hline 111.t.5 & 400 & $1.434 t-07$ & $4.015 E-07$ & 1628 \\
\hline 104.3 & $\begin{array}{l}140 \\
563\end{array}$ & $5.627 \ddot{0}-08$ & $1.40 b t-07$ & 1630 \\
\hline $\begin{array}{l}110.4 \\
111.4\end{array}$ & $\begin{array}{l}563 \\
215\end{array}$ & $2.018[-07$ & $5.631 E-07$ & 1632 \\
\hline 111.4 & $\begin{array}{r}215 \\
67\end{array}$ & $7.7361-08$ & $2.166 \mathrm{~F}-07$ & 1633 \\
\hline 112.4 & $\begin{array}{r}67 \\
563\end{array}$ & $2.703 E-6 \theta$ & $6.728 t-08$ & 163 \\
\hline 113.4 & 563 & $2.01 B[-07$ & $5.651 t-07$ & 1637 \\
\hline $114 \cdot 4$ & 350 & $1.256 E-07$ & $3.517 E-07$ & 1638 \\
\hline 111.3 & $9 \% 1$ & $3.373 t-67$ & $9.4441-07$ & 1640 \\
\hline 116.3 & 1054 & $3.746,[-07$ & $1.00 j E-06$ & 1641 \\
\hline 117.3 & 773 & $2.7 A O E=07$ & $7.784 F-07$ & 1643 \\
\hline 114.2 & 12 & $4.471) t=09$ & $1.252 E-U B$ & 1644 \\
\hline 119.2 & 1035 & $3.7118-47$ & $1.039 E-66$ & 1646 \\
\hline 1.0 .2 & 1602 & $5.743 t-07$ & $1.6013[-06$ & 1647 \\
\hline $1: 1.2$ & 2754 & 9. मY>t-J7 & $2.770[-06$ & 1649 \\
\hline $1: 2 \cdot 1$ & 81,6 & $3.034 L-67$ & $0.496 \mathrm{E}-07$ & 1650 \\
\hline $1<\ldots .1$ & $1<71$ & $4.530 E-\cup 7$ & $1.276 E-06$ & 1052 \\
\hline $1 ? 4.1$ & 610 & $2.1671-07$ & $0.12 \mathrm{SE}-07$ & 1653 \\
\hline $1 \% 0$ & 752 & $2.695 E-07$ & $7.547 \mathrm{E}-07$ & 1655 \\
\hline $1: 0.0$ & 563 & $2.01 \mathrm{HE}-07$ & $3.631 E-07$ & 1656 \\
\hline $1<6.4$ & 470 & $1.6011 \mathrm{E}-07$ & $4.726 E-07$ & 1658 \\
\hline $1<7.8$ & 704 & $2.5261-07$ & $7.0735-07$ & 1659 \\
\hline 124.0 & 531 & $1.9 .0 B 1-07$ & $5.338 t-07$ & 1660 \\
\hline $12 \% .7$ & $16 \mathrm{H}^{2}$ & $6.011 L-07$ & $1.664 f-66$ & 1602 \\
\hline 130.1 & 970 & $3.107 E-07$ & $4.010 t-01$ & 1661 \\
\hline $1,1.5$ & 8031 & $2.0976-66$ & $8.061 E-06$ & 1664 \\
\hline $1,2.3$ & 19837 & $7.1101-00$ & $1.941 t-05$ & 1665 \\
\hline 193.5 & $22 \mathrm{HOH}$ & $6.2076-06$ & $2.294 \mathrm{E}-\mathrm{US}$ & 1667 \\
\hline 134.4 & 21467 & $7.644 t-06$ & $2.154 \mathrm{E}-05$ & 1668 \\
\hline 1512.4 & 16004 & 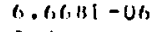 & $1.66,7 \mathrm{k}-0 \mathrm{~s}$ & 1669 \\
\hline $1=6.0$ & H77, & $3.140 L-146$ & $0.0671-66$ & 1670 \\
\hline 121.3 & 9013 & $3.240101-07$ & $9.01<t-07$ & 1672 \\
\hline $1+4+3$ & 5204 & $1 . H C b 6-06$ & $3.223[-06$ & 1673 \\
\hline 157.1 & $252 s$ & & & 1674 \\
\hline 140.7 & $3<70$ & $1.172 t-06$ & $3.282 t-06$ & 1675 \\
\hline 141.2 & 2674 & $9.587 k-07$ & $2.634 E-06$ & 1676 \\
\hline 2.2 & 1335 & $4.787 L-\cup 7$ & $1.340 t-v_{0}$ & 1677 \\
\hline 143.1 & 539 & $1.434 E-07$ & 3.414 & \\
\hline $1,4.1$ & 704 & 07 & $273 E$ & 16 \\
\hline $14 ! .1$ & $2 A G$ & $1.0,2 B l-47$ & $2,077 t-07$ & 1600 \\
\hline
\end{tabular}




$\begin{array}{rrr}146.0 & \$ 752 & 3.050 E-06 \\ 146.9 & 3722 & 1.994 E-06 \\ 147.9 & 3979 & 1.307 E-06 \\ 148.8 & 1941 & 6.765 E-07 \\ 149.7 & 814 & 2.038 E-07 \\ 150.7 & 393 & 1.372 E-07 \\ 151.6 & 69 & 2.409 E-08 \\ 152.6 & 69 & 2.409 E-08 \\ 153.5 & 57 & 1.990 E-08 \\ 154.5 & 0 & 0 . \\ 155.4 & 0 & 0 .\end{array}$

TRCSSHINO INTEGRATFD $1.186 E-02$ 2.60HE-02

$\begin{array}{ll}6.709 E-06 & 1641 \\ 4.387 E-06 & 1642 \\ 3.050 E-06 & 1643 \\ 1.480 E-06 & 1684 \\ 6.244 E-07 & 1685 \\ 3.010 E-07 & 1686 \\ 5.299 E-06 & 1687 \\ 5.299 E-08 & 1680 \\ 4.377 E-08 & 1688 \\ 0 . & 1689 \\ 0 . & 1690\end{array}$

SEC/SO.M

1/M

TEST UBO SEPTEMBER 30, 1960 O0039 TU 0109 PST 2 INC SULFIDE RELEASE FROM ELEVATIUN DF $26 \mathrm{H}$ $2200 \mathrm{H}$ ARC SAMPLER HT $1.5 \mathrm{H} \mathrm{U}=2.2 \mathrm{M} / \mathrm{SEC}$ AT $26 \mathrm{M}$ ALINUTH EXPOSURE EIO
DEGREES GH-SEC/CU.M SEC/CU.M I/SO.M METENS XIOE 6
XIOH SEC/CU.M I/SO.M METEIIS

B $\quad 4.0$

650.0

452.0

554.0

556.0

558.0

500.0

562.0

5. 64.0

536.0

50.0

470.0

472.0

374.0

E 76.0

78.0

00.0

$0<.0$

84.0

95.0

4 dB. 0

90.0

92.0

96.0

E
E 98.0

6100.0

4102.0

3164.0

E 106.0

5108.0

7110.0

5112.0

6114.0

7116.0

1119.0

$+120.0$

4122.0

7124.0

126.0

5128.0

7130.0

7132.0

134.0

6136.0

138.0

140.0

7142.0

4144.0

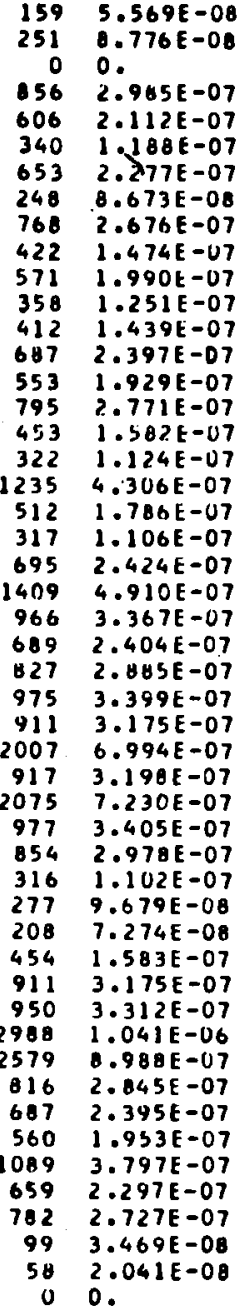

00 .

$.976 E-04$ SECISO.H
1.225E-07 $1.931 \mathrm{C}-07$

$6.568 E-07$

$4.647 E-07$

$2.614 E-07$

5.009E-07

1.908E-07

$5.884 E-07$

$3.242 E-07$

$4+378 E-07$

2. $752 E-07$

$3.165 E-07$

$5.272 E-07$

$4.243 E-C 7$

$6.097 E-07$

3.4 日UE-U7

$2.473 \mathrm{E}-07$

$9.4731-07$

$2.434 E-07$

$5.333 E-07$

$1.080 E-06$

$7.408 E-07$

5.206E-07

$6.347 E-07$

$7.479 E-07$

$6.985 \mathrm{E}-07$

$1.534 \mathrm{E}-06$

$7.036 \mathrm{E}-07$

$7.492 \mathrm{E}-07-2200$
$6.552 \mathrm{E}-07$

$6.552 \mathrm{E}-07 \quad 2200$

$2.424 \mathrm{E}-07 \quad 2200$

$2.129 \mathrm{E}-07 \quad 2200$

$1.600 \mathrm{E}-07 \quad 2200$

$3.463 E-07 \quad 2200$

$6.985 E-07 \quad 2200$

$7.286 \mathrm{E}-07 \quad 2200$

$2.291 E-06 \quad 2200$

$1.977 \mathrm{E}-\mathrm{U} 6 \quad 2200$

$6.260 \mathrm{E}-07 \quad 2200$

$5.269 E-07 \quad 2200$

4.297E-07 2200

8.354E-07 2200

$5.054 \mathrm{E}-07 \quad 2200$

$6.000 E-07 \quad 2200$

$7.633 \mathrm{E}-08 \quad 2200$

$4.490 \mathrm{E}-0 \mathrm{~B}$

0.

2200

$.195 E-03$

\begin{tabular}{|c|c|c|c|c|c|}
\hline 2 & $\begin{array}{l}146.0 \\
146.9 \\
147.9 \\
148.9 \\
149.7 \\
150.7\end{array}$ & $\begin{array}{r}515 \\
201 \\
67 \\
59 \\
43 \\
5\end{array}$ & $\begin{array}{l}1.049 E-07 \\
7.228 E-08 \\
2.487 E-08 \\
2.149 E-08 \\
1.556 E-08 \\
2.100 E-09\end{array}$ & $\begin{array}{l}6.177 t-01 \\
2.024 E-07 \\
6.965 E-00 \\
6.016 E-0 d \\
4.351 E-08 \\
5.874 E-04\end{array}$ & $\begin{array}{l}1681 \\
1682 \\
1683 \\
1684 \\
1685 \\
1686\end{array}$ \\
\hline CR & 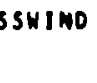 & IHTEGRATED. & $\begin{array}{r}1.396 E-03 \\
\text { SEC/SO.M }\end{array}$ & $\begin{array}{c}3.409 E-03 \\
1 / \mathrm{H}\end{array}$ & , \\
\hline
\end{tabular}

TEST UBU SEPTEMBER 30,1968

0039 TU 0109 PST FLUURESCE IN RELEASE FROM ELEVATIUN DF 56M 2200 ARC SAMPLER HI $1.5 \mathrm{M} \mathrm{U}=2.8 \mathrm{H} / \mathrm{SEC}$ AI $36 \mathrm{H}$

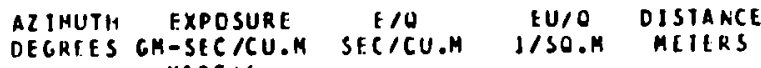
$\times 10 E+6$

5.00

13.0

54.0

50.0

488.0

690.0

92.0

44.0

96.0

94.0

6100.6

4102.0

104.0

9106.0

51118.0

7110.0

5112.0

114.0

116.0

$11 \% .0$
$0 \quad 120.0$

4122.0

7124.0

5126.0

5128.0

7130.0

7132.0

0134.0

6136.0

$913 \mathrm{H} .0$

6140.0

7142.0

4144.0

3146.0

5148.0

5150.0

4152.0

154.0

150.0

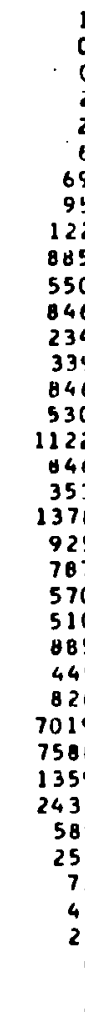

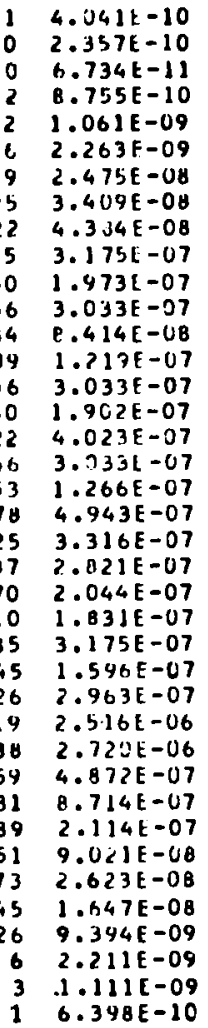

$1.131 \mathrm{E}-0 ?$

6. $600 E-10$

$1.886 E-10$

$2.451 E-09$

$2.97 \dot{U E}-09$

$6.336 E-09$

$6.93 \mathrm{JE}-\mathrm{OE}$

$9.544 \mathrm{E}-0 \mathrm{~B}$

$1.22 \forall \mathrm{E}-07$

6. $890 E-07$

$5.524 \mathrm{E}-07$

$0.494 E-07$

$2.3 S C E-07$

$3.412[-07$

B. 494 E- 07

$5.326 E-07$

$1.127 \mathrm{E}-06$

8. $494 E-07$

$3.544 E-07$

$1.304 E-06$

$9.280 E-07$

7.900 E-0?

$5.722 \mathrm{E}-07$

$5.120 \mathrm{E}-07$

$8.890 E-01$

$4.468 E-07$

$0.296 E-07$

7. $044 \mathrm{E}-06$

$7.616 E-06$

$1.364 E-06$

$2.440 E-06$

$5.920 \mathrm{E}-07$

$2.326 E-07$

$7.344 E-00$

$4.610 \mathrm{E}-0 \mathrm{O}$

$2.630 \mathrm{E}-\mathrm{OB}$

$6.191 E-04$

$3.111 \mathrm{E}-0 \mathrm{Y}$

$1.791 E-09$

CROSSHINO INTEGRATED= $0.968 \mathrm{E}-04$ 2.511E-03 SEC / 50.14
$22 \cup 0$

2200

2200

2200

2200

2200

2200

$\angle 200$

$\angle 200$

2200

2200

2200

2200

2200

2200

2200

2200

2200

2200

2200
2200

2200

$\angle 200$

2200

2200

2200

2200

2200

2200
2200

2200

2200

$\angle 200$

2200
2200 
TEST UBO SEPTEMUER 30, 1968 0034 TU 0109 PST ZINC SULFIDE RELEASE FROM ELEVATION UF $20 \mathrm{PH}$ $3200 \mathrm{M}$ ARC SAMPLER HT $1.5 \mathrm{M}$ U. $2.2 \mathrm{M} / \mathrm{SEC}$ AT $26 \mathrm{M}$

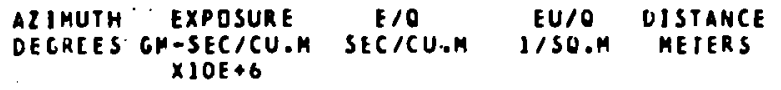

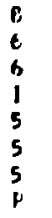

48.0
$t \quad 50.0$
6

$6 \quad 50.0$

154.0

556.0

558.0

50.0

62.0

464.0

466.0

60.0

70.0

72.7

74.7

15,7

76.8

77.8

H 74.8

H 70.8

P. 1.8

42.8

d $3 . \mathrm{H}$

A4. 8

BS.A

Ho.

17.8

4.8

89.8

40.8

91.8

93.8

94.8

95.8

96.8

$9 \mathrm{~J} .8$

$9 \% .8$.

100.0

101.8

11)2.

107.8

$1 \mathrm{C} 4 . \mathrm{H}$

125.8

$106 . \mathrm{B}$

105.0

104.7

110.7

111.7

112.7

113.7

114.6

115.6

115.6
117.6

$11 \mathrm{H} .6$

119.5

120.5

121.5

o 122.4

123.4

$2 \quad 7.289 \mathrm{E}-10 \quad 1.604 \mathrm{E}=0 \mathrm{O}$

$2.415 E-10 \quad 6.414 E-10$

0 0.

. 0 . 0

$3.015 E-08$

$2.373 E-08$

$2.086 E-04$

$3.335 E-08$

0.

$431.516 E-00$

0

$1.385 E-08$

$3.047 E-08$

$2.213 F-08$

$1.006 E-00$

-09
0

$\begin{array}{rl}23 & 0.163 \mathrm{E}-09 \\ 111 & 3.079 \mathrm{E}-08\end{array}$

$2057.161 \mathrm{t}-08$

$232 \quad 0.106 \mathrm{E}-08$

$\begin{array}{ll}301 & 1.049 E-07 \\ 306 & 1.069 \mathrm{E}-07\end{array}$

$\begin{array}{ll}306 & 1.069 E-07 \\ 246 & 0.603 E-08\end{array}$

$790 \quad 2.755 E-07$

$943 \quad 3.287 \mathrm{E}-07$
$2224 \quad 7.753 \mathrm{E}-07$

$\begin{array}{ll}2224 & 7.753 E-07 \\ 1527 & 5.3211 E-07\end{array}$

$11443.98 B E-07$

$\begin{array}{ll}1762 & 6.1 .41 E-57 \\ 3065 & 1.060 E-06\end{array}$

30653
1803
1375

1375
1084
$10.5348-07$

$\begin{array}{ll}1884 & 6.584 E-07 \\ 1953 & 6.80 H E-07\end{array}$

25A1 $8.996 \mathrm{BE}-07$

$2137 \quad 7.449 E-07$

$1377 \quad 4.799 E-07$

$\begin{array}{ll}1261 & 4.396 E-07 \\ 2333 & 4.130 E-07\end{array}$

$1084 \quad 3.779 E-07$

$1030 \quad 3.540 E-07$

$10703.730 \mathrm{E}-07$

$913 \quad 3.283 \mathrm{E}-07$

$896 \quad 3.123 E-07$

$\begin{array}{ll}543 & 1.895 E-07 \\ 636 & 2.21 B E-07\end{array}$

$\begin{array}{ll}636 & 2.21 B E-07 \\ 558 & 1.944 E-U 7\end{array}$

558
311
$4.944 E-07$

$479 \quad 1.671 E-07$

$\begin{array}{ll}562 & 1.954 \mathrm{E}-07 \\ 34 \mathrm{~S} & 1.203 \mathrm{E}-07\end{array}$

$34 \mathrm{~S}$
$21.203 E-07$
$7.6 U A E-08$

$\begin{array}{ll}21 \mathrm{H} & 7.6 \mathrm{BAE}-08 \\ 553 & 1.929 \mathrm{E}-07 \\ 583 & 2.034 \mathrm{E}-07\end{array}$

$5832.034 E-07$

231 A. $056 E-08$

$2659.249 E-U 8$

$2699.399 E-08$

$1194.177 \mathrm{E}-08$

82 2. $084 E-08$

$\begin{gathered}101 \\ 19\end{gathered} 6.531 \mathrm{E}-08$

$\begin{array}{ll}19 & 6.962 E-09 \\ 36 & 1.343 E-08\end{array}$

$\begin{array}{ll}38 & 1.343 E-08 \\ 74 & 2.586 E-08\end{array}$

$672.337 E-08$

$622.180 E-08$

$49 \quad 1.740 E-08$

227.956 E-09

$4.973 E-09$

$611 F-09$

$1.796 \mathrm{E}-0.6$
$.533 \mathrm{E}-0 \mathrm{O}$
$1.575 E-07$

$1.533 \mathrm{E}-0 \mathrm{~B}$
$1.573 \mathrm{E}-07$

$1.783 \mathrm{E}-07$

2.30 U[ -07

$2.352 E-07$
$1.893 E-07$

$6.061 E-07$

$7.231 E-07$

$1.706 \mathrm{E}-06$

$1.171 E-06$
$0.774 E-07$

1.351E-06

$2.3508-06$

$1.444 \mathrm{E}-06$

$1.055 E-06$
$1.448 E-06$

$1.494 \mathrm{E}-06$

1.97 YE- 06

$1.634 \mathrm{E}-06$

$1.056 E-66$

$9.6718-07$

$1.789 E-06$

$7.094 E-07$

0.2 USE -07

$7.002 E-07$

$6.870 E-07$

4.16 yE -07

$4.879 E-U 7$
$4.278 E-U 7$

$2.385 E-07$

$3.676 \mathrm{E}-07$

$4.31 \cup t-07$

2.6471 .07

$1.674 E-U 7$
$4.245 E-U 7$

$4.474 t-07$

$1.772 \mathrm{E}-07$

$2.746 E-07$

$2.033 E-07$
$2.060 E-07$

$9.190 E-08$

$6.345 E-08$

$7.767 E-08$

$1.532 \mathrm{E}-08$
$2.954 \mathrm{E}-\mathrm{JB}$

$5.689 \mathrm{E}-00$

$5.142 E-08$

$4.014 E-08$

$3.829 \mathrm{E}-0 \mathrm{O}$

$1.750 \mathrm{E}-08$

$1.094 \mathrm{E}-08$
$2.188 \mathrm{E}-09$

CRUSSHIND INTEGRATED= $\begin{gathered}.120 E-04 \\ \text { SEC/SO.M }\end{gathered}$

$1 / M$
$\$ 200$

$\$ 200$

3200

3200

3200

3200

3200

3200

3200

3200

3160

3170

172

3173
1175

3177

3174

3180

3102

3185

1107

3169

3191

3192

3196

3198

3199

3201

3205

3206

3298

3210

3213

3215

3217

3220

3222

3224

3227

$\$ 229$

3230

3232

3233

3235

3238

3240

3241

3243

3246

3248

3249

3251

3253

TIST UGO I SEPTLMAER 30, 1308 OU99 TU 0109 PST FLUIIRESICE IN RELLAST FRUM ELEVATIUN UF S6M $3200 \mathrm{M}$ ARC SARPLER HI $1.5 \mathrm{M}$ U= 2.8 H/SEC AT SGM

\begin{tabular}{|c|c|c|c|}
\hline $\begin{array}{l}\text { ALIHU } \\
\text { DEGKE }\end{array}$ & $\begin{array}{l}\text { EXPOSURE } \\
\text { GK-SEC/CU.H }\end{array}$ & $\begin{array}{l}\text { E/O } \\
\text { SEC/CU.H }\end{array}$ & $\begin{array}{l}\text { EU/O } \\
\text { I/SU.M }\end{array}$ \\
\hline
\end{tabular}

DEGREES GK-SEC/CU.H SECICU.A I/SU.M MEIERS

$X 10 E+$

7
6
6
5
5
5
5
5
4
4
7
5

$$
\begin{aligned}
& 5 \\
& 5 \\
& 5 \\
& 5 \\
& 6 \\
& 6 \\
& 6 \\
& 6 \\
& 6 \\
& 7 \\
& 7 \\
& 7
\end{aligned}
$$

4.00
52.0

52.0

56.0

38.0

60.0

62.0

66.0

6,00

70.6

73.7

14.7

75.7

76.11

77.6

76.0

70.8

(1). 0

H. 1.

42.0

1) 1.0

$14 . \mathrm{A}$

45.4
16.0

16.6
07.6

115.

69.

9:1.

प1.

92.4

$93 . t$

$94 . \mathrm{H}$

$95 . \mathrm{H}$

96.11

97.8

.93 .6

प9.1

100.7

IU1. 8

162.6

11) 4.4

1114.8

1. . . .

116.1

4117.8

1128.4

109.7

110.7

2111.7

112.7

113.7

114.6

115.6

116.6

117.6

$11 \mathrm{H} . \mathrm{h}$

119.5

120.5

121.5

- 122.4

123.4

124.4

125.4 


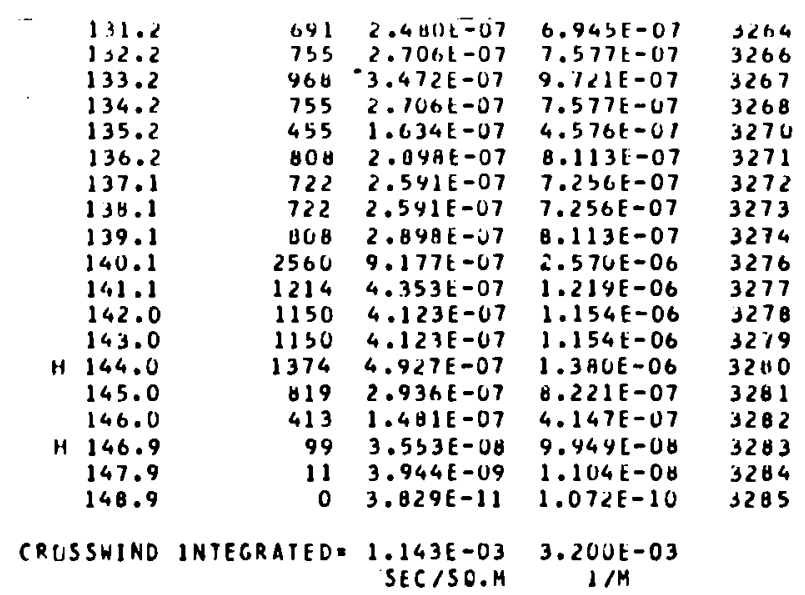




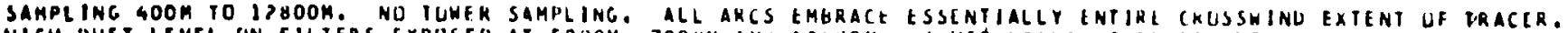

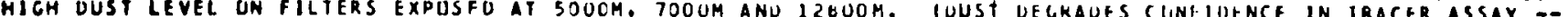
ESPECIALLY ZNS.I LARGE HINO DIRECTIUN VAKIANCE FUR RELATIVELY HIGH WIND SPLEU.

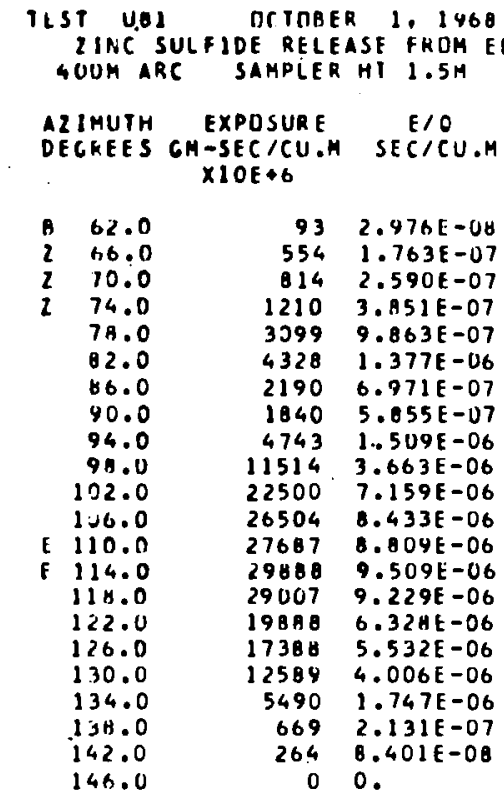

CRESSHIND INTEGRATEO= $\begin{gathered}1.975 E-03 \\ \text { SECISC.M }\end{gathered}$

CRLSSHIND INTEGRATEU: $\begin{array}{rl}1.975 E-03 & 1.126 E-02 \\ \text { SEC/SG.M } & 1 / M\end{array}$

0111 TU U141 HST UETIUN UF 20 M $U=5.7 \mathrm{M} / \mathrm{SEC} A 1 \mathrm{COM}$

EU/O OISTARCE ISSU.H MEIFRS

$\begin{array}{ll}1.646[-U 1 & 400 \\ 1.005 E-06 & 400 \\ 1.477 E-06 & 400 \\ 2.195 E-06 & 400 \\ 5.622 E-06 & 400 \\ 7.849 E-C 6 & 400 \\ 3.973 E-06 & 400 \\ 3.337 E-06 & 400 \\ 8.602 E-06 & 400 \\ 2.080 E-05 & 400 \\ 4.081 E-05 & 400 \\ 4.807 E-05 & 400 \\ 5.021 E-05 & 400 \\ 5.420 E-05 & 400 \\ 5.261 E-05 & 400 \\ 3.607 E-05 & 400 \\ 3.153 E-05 & 400 \\ 2.283 E-05 & 400 \\ 9.957 E-06 & 400 \\ 1.215 E-06 & 400 \\ 4.789 E-07 & 400 \\ 0 . & 400\end{array}$

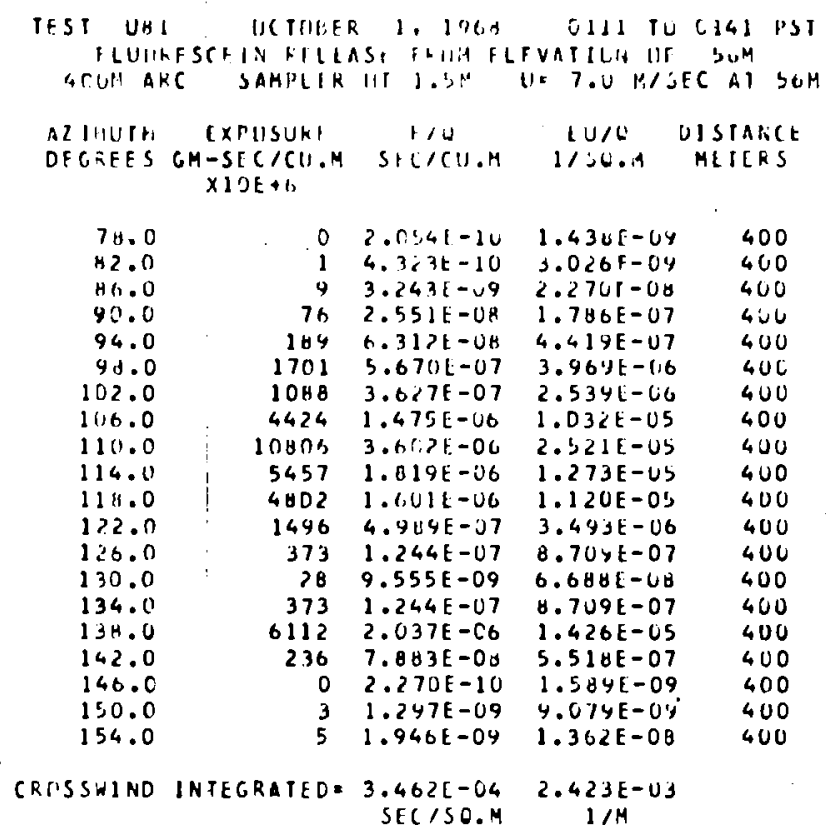

0111100141 PST ZIHC SULFIOE RELEASE FROM ELFVATIUN UF $20 \mathrm{M}$
8 OOH ARC SAMPLER HT $1.5 \mathrm{MH}$ U: $5.7 \mathrm{M} / \mathrm{SEC}$ AT $26 \mathrm{M}$ 7 IIIC SULFIOE RELEASE FROM ELFVATIUN UF $20 \mathrm{M}$
8 OOH ARC SAMPLER HT $1.5 \mathrm{M}$ U: $5.7 \mathrm{H} / \mathrm{SEC}$ AT $26 \mathrm{M}$ AZ JiAUTH EXPOSURE
DEGRES GM-SEC/CU.M SEC/CU.H I/SU.M OISTANCE $\times 10 E+6$ MUT.M HETERS

\begin{tabular}{|c|c|c|c|}
\hline & 70.0 & 0 & \\
\hline & 73.0 & 19 & $6.126 E-09$ \\
\hline & 76.0 & 72 & $2.319 E-U B$ \\
\hline & 79.0 & 1151 & $3.663 E-07$ \\
\hline & 02.0 & 416 & $1.326 E-07$ \\
\hline & 05.0 & 844 & $2.6 \forall 7 E-07$ \\
\hline & 30.0 & 2802 & $5.990 E-07$ \\
\hline & 91.0 & 1467 & $4.669 \mathrm{E}-07$ \\
\hline & $\$ 4.0$ & 1453 & $4.625 E-07$ \\
\hline & 97.0 & 1686 & $5.365 E-07$ \\
\hline & 97.1 & 1766 & $5.622 \mathrm{E}-07$ \\
\hline & 99.1 & 1920 & $6.137 E-07$ \\
\hline & 101.1 & 2274 & $7.236 \mathrm{E}-07$ \\
\hline & 103.0 & 3692 & $1.175 \mathrm{E}-06$ \\
\hline & $1<5.0$ & 8352 & $2.657 \mathrm{E}-06$ \\
\hline & 106.9 & 14304 & $4.551 \mathrm{E}-06$ \\
\hline & 108.8 & 17145 & $5.455 E-06$ \\
\hline & 110.7 & 21220 & $6.752 \mathrm{E}-0 \mathrm{~S}$ \\
\hline & 112.6 & 22553 & $7.176 E-06$ \\
\hline & 114.5 & 31409 & $9.994 \mathrm{E}-06$ \\
\hline & 116.4 & 44164 & $1.405 \mathrm{E}-05$ \\
\hline & $11 \mathrm{H} \cdot 3$ & 53340 & $1.697 E-05$ \\
\hline & 120.2 & 24817 & $7.896 E-06$ \\
\hline & $1<2.1$ & 21743 & $6.918 E-06$ \\
\hline & 124.0 & 21456 & $6.827 E-06$ \\
\hline & 125.8 & 15898 & $5.05 \mathrm{BE}-06$ \\
\hline & $1<7.7$ & 9493 & $3.020 E-06$ \\
\hline & 129.5 & 11285 & $3.591 E-06$ \\
\hline & 131.4 & 2580 & $8.210 E-07$ \\
\hline & 133.2 & 253 & $0, U \forall 1 E-0 B$ \\
\hline & 135.0 & 0 & \\
\hline
\end{tabular}

0.

$3.49 \angle \mathrm{E}-0 \mathrm{Y}$ $1.322 \mathrm{E}-07$ 2. 000 E-06 $7.557 E-07$ $1.531 \mathrm{E}-06$ $3.415 \mathrm{E}-06$ $2.661 \mathrm{E}-06$ $2.636 t-06$ 3.05 E E-J6 $3.204 t-06$ $3.498 E-06$ $4.126 E-06$ $0.696 E-06$ $1.515 \mathrm{E}-0 \mathrm{~S}$ $2.594 E-05$ $3.110 \mathrm{E}-05$ $3.644 E-05$ $4.090 E-05$ $5.696 \mathrm{E}-05$ $8.004 E-U 5$ $9.674 E-U 5$ $4.501 E-05$ $3.943 \mathrm{E}-\mathrm{US}$ $2.883 \mathrm{E}-05$ $1.722 \mathrm{E}-0 \mathrm{~S}$ $2.047 E-05$ $4.680 \mathrm{E}-06$ 4.606 E- 07 0 .

CKIJSSHIND INTEGRATEU. $3.012 E-03 \quad 1.717$ E-02

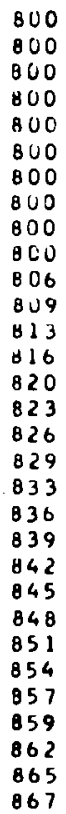

867

\begin{tabular}{|c|c|c|c|c|}
\hline $\begin{array}{r}\text { TEST URI } \\
\text { FLUINR } \\
\text { GOOMAR }\end{array}$ & $\begin{array}{l}\text { OCIDBE } \\
\text { RESICIN RELE } \\
\text { RC SAMPLER }\end{array}$ & $\begin{array}{l}R \quad 1,1968 \\
\text { ASE FRUM E } \\
\text { HI } 1 . S M\end{array}$ & $\begin{array}{r}0111 \text { TU } \\
\text { LEVATIUN UF } \\
U=7 . U \mathrm{H} / \mathrm{SE}\end{array}$ & $\begin{array}{l}0141 \text { PST } \\
=5 Q M \\
S E C A T \text { SOM }\end{array}$ \\
\hline $\begin{array}{l}\text { ALIMESTH } \\
\text { DE G, EEES }\end{array}$ & $\begin{array}{c}\text { EXPOSURL } \\
\text { CM-SEC /CU.H } \\
\times 1 O E+6\end{array}$ & $\begin{array}{c}\text { LAU } \\
\text { SEC/CU.M }\end{array}$ & $\begin{array}{l}\text { EU/O } \\
\text { I/SG.M }\end{array}$ & $\begin{array}{l}\text { UISIAINCE } \\
\text { METERS }\end{array}$ \\
\hline $\begin{array}{l}08.0 \\
91.0\end{array}$ & 4 & $0.1147 t-48$ & $\begin{array}{l}\text { U. } \\
1.0135-07\end{array}$ & $\begin{array}{l}8 \mathrm{JU} \\
600\end{array}$ \\
\hline $\begin{array}{l}91.0 \\
94.0\end{array}$ & $\begin{array}{r}43 \\
1\end{array}$ & $\begin{array}{l}1.06 t-68 \\
6.017 t-10\end{array}$ & $\begin{array}{l}1.013 t-0 \\
4.21<k-0,\end{array}$ & 800 \\
\hline 97.0 & 79 & $2.6305-u s$ & $1.845 \mathrm{t}-07$ & $\forall \cup 0$ \\
\hline 97.1 & "u & $1.361 t-05$ & $9.5<7 t-0 B$ & 806 \\
\hline 00.1 & 50.4 & d.e. & $1.316 E-06$ & $B \cup 4$ \\
\hline 101.1 & 1427 & $4.7511 E-07$ & $3.3306-06$ & 613 \\
\hline $11,3.6$ & 3220 & $1.0765-00$ & $7.5341-06$ & 036 \\
\hline 105.0 & 4281 & $1.427 E-00$ & $9.991 \mathrm{E}-60$ & 820 \\
\hline 106.9 & 6949 & 2.3 SCE-UO & $1.631 \mathrm{E}-\mathrm{US}$ & 823 \\
\hline 103.8 & 14887 & $4.962 t-06$ & $3.474 E-U 5$ & 926 \\
\hline 110.7 & 10600 & $3.533 E-06$ & $2.473 t-65$ & 829 \\
\hline 112.0 & 17294 & $5.765 \mathrm{E}-0 \mathrm{C}$ & 4.0 S SE-US & 833 \\
\hline $11 \% .5$ & 19550 & $6.517 t-06$ & $4.5626-05$ & 836 \\
\hline 116.4 & 9697 & $3.232 \mathrm{E}-06$ & $2.263[-65$ & 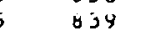 \\
\hline 118.3 & 11502 & $3.834 \mathrm{E}-06$ & $2.684 E-U S$ & 842 \\
\hline 120.2 & 7092 & $2.631 E-U e$ & $1.042 i-U S$ & 81.5 \\
\hline 122.1 & 2251 & $7.504 E-07$ & $5.253 E-06$ & 840 \\
\hline 124.0 & 1619 & $5.398 E-67$ & $3.77 \forall E-06$ & 451 \\
\hline $125 . \mathrm{A}$ & 3481 & $1.327 t-06$ & $9.2 B Y E-\cup 6$ & A 54 \\
\hline 127.7 & 1032 & $3.442 t-07$ & $2.40 Y E-06$ & 857 \\
\hline 129.5 & 1950 & $0.501 E=07$ & $4.551 \mathrm{E}-\mathrm{US}$ & $4 b 9$ \\
\hline 131.4 & 1176 & $3.922[-07$ & $2.745[-06$ & 802 \\
\hline 133.2 & 402 & $1.343 E-C 7$ & $9.402 E-07$ & 805 \\
\hline 135.0 & 7 & $2.308 E-49$ & $1.671 E-08$ & 867 \\
\hline 136.9 & 3 & $1.313 L-09$ & $9.193[-0 x$ & 870 \\
\hline 138.7 & 5 & $1.671 \mathrm{E}-09$ & $1.170 \mathrm{E}-00$ & 872 \\
\hline 140.6 & 6 & 0 & 0. & 874 \\
\hline 142.4 & 0 & 0. & 0 . & $B 76$ \\
\hline SHIND & NTEGRATED & $\begin{array}{r}1.112 E-03 \\
\text { SEC/SD.M }\end{array}$ & $\begin{array}{c}7.782 E-03 \\
1 / M\end{array}$ & \\
\hline
\end{tabular}




\begin{tabular}{|c|c|c|c|}
\hline \multicolumn{2}{|c|}{$\begin{array}{l}\text { AZIMUTH } \\
\text { DECKEES }\end{array}$} & $\begin{array}{c}\text { EXPOSURE } \\
\text { GM-SEC/CU.H } \\
\text { XIOE }+6\end{array}$ & $\begin{array}{l}\text { E/O } \\
\text { SEC/CU }\end{array}$ \\
\hline & 40.0 & & 0. \\
\hline & 42. & 2 & 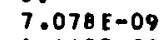 \\
\hline & 84. & 52 & $1.6660 \mathrm{E}$ \\
\hline & 36.0 & 113 & 3.6 \\
\hline & 89. & & \\
\hline & 90. & 751 & $\begin{array}{l}1.118 \\
7.078\end{array}$ \\
\hline & 92.8 & $\begin{array}{l}228 \\
335\end{array}$ & \\
\hline & $9 n$. & 602 & 2.1 \\
\hline & YH. O & 396 & 1.2 \\
\hline & 100.0 & 663 & 2.1 \\
\hline & 10 & 1338 & \\
\hline & 104. & 1227 & $3.905 E-0$ \\
\hline & 10 & & $8.240 E$ \\
\hline & 108. & 3006 & $9.565 E-07$ \\
\hline & 11 & 4055 & 1.29 \\
\hline & 11 & 5983 & 1.9 \\
\hline & 114.0 & 11536 & 3.67 \\
\hline & 116.1 & 22243 & 7.0 \\
\hline & 118. & 20673 & 6.5 \\
\hline & 120. & 10789 & 3.4 \\
\hline & 12 & $\begin{array}{l}9073 \\
7538\end{array}$ & $\begin{array}{l}2.88 \\
2.39\end{array}$ \\
\hline & 126.0 & 3540 & $1.126 E-06$ \\
\hline & $1<B$ & 470 & $2.499 E-0$ \\
\hline & 15 & 19 & 6.102 \\
\hline & 13 & 1 & $\begin{array}{l}4.081 E-10 \\
1.464 E-09\end{array}$ \\
\hline & & $\begin{array}{l}4 \\
0\end{array}$ & $\begin{array}{l}1.464 t-09 \\
0 .\end{array}$ \\
\hline & & 0 & \\
\hline
\end{tabular}

CRUSSHIND INIEGRATEU $1.431 E-03$ SEC/SO.M
0111 TU 0141 PST LEATIUN OF 264 . 5.7 H/SEC AT $26 \mathrm{M}$ EU/O DISTANCE
I/SO.M METLRS 0. $4.034 \mathrm{E}-0 \mathrm{OH} \quad 1200$ $9.460 E-0 \mathrm{O} \quad 1200$ $2.059 E-07 \quad 1200$ $4.215 \mathrm{E}-07 \quad 1200$ $6.371 E-07 \quad 1200$ $4.034 E-07 \quad 1200$ 6.07 YE-07 1200 $1.600 \mathrm{E}-06 \quad 1200$

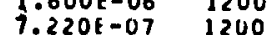
$\begin{array}{ll}1.220 E-07 & 1200 \\ 1.203 E-06 & 1200\end{array}$ $2.42 \forall E-06 \quad 1200$ $2.2261-06 \quad 1200$ $4.697 \mathrm{E}-06.1200$ $5.452 \mathrm{E}-06 \quad 1200$ $7.355 E-06 \quad 1200$ $1.085 E-05 \quad 1200$ $2.092 t-05 \quad 1200$ $4.034[-05 \cdot 1200$ $3.749 \mathrm{E}-6 \mathrm{~S} \quad 1200$ $1.957 E-05 \quad 1200$
$1.645 E-05$
1.3200 $1.367 E-05 \quad 1200$ $6.420 E-06 \quad 1200$ $0.542 E-07.1200$ $3.47 U E-0 d \quad 1200$ $2.702 \mathrm{E}-09 \quad 1200$ $8.347 f-0 y \quad 1200$ 0 .

1200

$0.156 E-03$ $1 / \mathrm{M}$

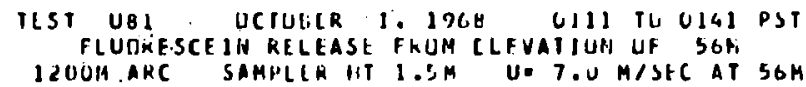

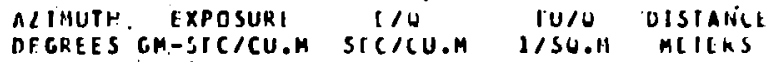
xiof +6

\begin{tabular}{|c|c|c|c|c|}
\hline 174,0 & 11 & 0. & 0. & 1200 \\
\hline $96 . .0$ & $i$ & $3.1101-1,4$ & $1.5 .14 t-00$ & $1<00$ \\
\hline $4 \mathrm{H.0}$ & 13 & $4.4<11-1,9$ & $3.04 \mathrm{st}-0_{6}$ & $1<00$ \\
\hline 100.0 & his & $2.1100-00$ & $1.4771 .-21$ & 1200 \\
\hline joz.r & 112 & $3.73+1-U t$ & $2.01 \cup[-\pi 1$ & dásu \\
\hline $\left.1: i_{6}, 0\right)$ & 574 & 1. $\mid(x, 1)-i) 7$ & $1.2 \circlearrowleft C F-0 Q$ & 1200 \\
\hline J.66.1 & 14,79 & $4.9311-117$ & $3.46,1-116$ & 1200 \\
\hline 1011.0 & 1137 & $3.7 y, t-07$ & $2.65,1-1) 6$ & live \\
\hline 110.0 & $46 H ?$ & $1.5016-00$ & $1.0 \times 1[-0 ;$ & 1200 \\
\hline 112.6 & $6, y 6,1$ & $\therefore .300 t-06$ & $\left.1.6<4 l-v^{\prime}\right)$ & 1.jo \\
\hline $11 / 40$ & 10754 & 3.5utit-ib & $2.11<E-\cup S$ & 1200 \\
\hline 111,0 & (1.334t & $2.2786 L-06$ & 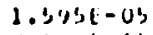 & 1200 \\
\hline $\begin{array}{l}1111.0 \\
1.0 .0\end{array}$ & $\begin{array}{r}17744 \\
6574\end{array}$ & $\begin{array}{l}1.2611-66 \\
2.1011-06\end{array}$ & $\begin{array}{l}2.4031-6 \\
1.534 t-0:\end{array}$ & $\begin{array}{l}1200 \\
1200\end{array}$ \\
\hline $1 \times 2.0$ & 2125 & $7.0 \times 4 F-07$ & $4.957 E-J 6$ & 1200 \\
\hline 124.0 & 1365 & $4, \supset J 2 t-67$ & $3.1 \mathrm{JCL}-0 t$ & 1200 \\
\hline $1<6.0$ & 2044 & G.A $90 \mathrm{OF}-07$ & $4.731 k-06$ & $1<00$ \\
\hline $1<R, 0$ & 2049 & C.H3)(-כ) & $4.78][-00$ & 1200 \\
\hline 130.0 & yoy & $3.23 \% 2-47$ & $2.123 L-i c$ & 1200 \\
\hline 132.0 & 2 & $9.1,66 t-10$ & $6.7521-09$ & 1200 \\
\hline 131.0 & $i$ & $4.4 a 1 F-10$ & $3.025 E-0$. & 1200 \\
\hline 136.0 & 3 & $1.14,1-69$ & 6.01 (1-U? & 1200 \\
\hline
\end{tabular}

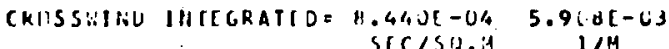

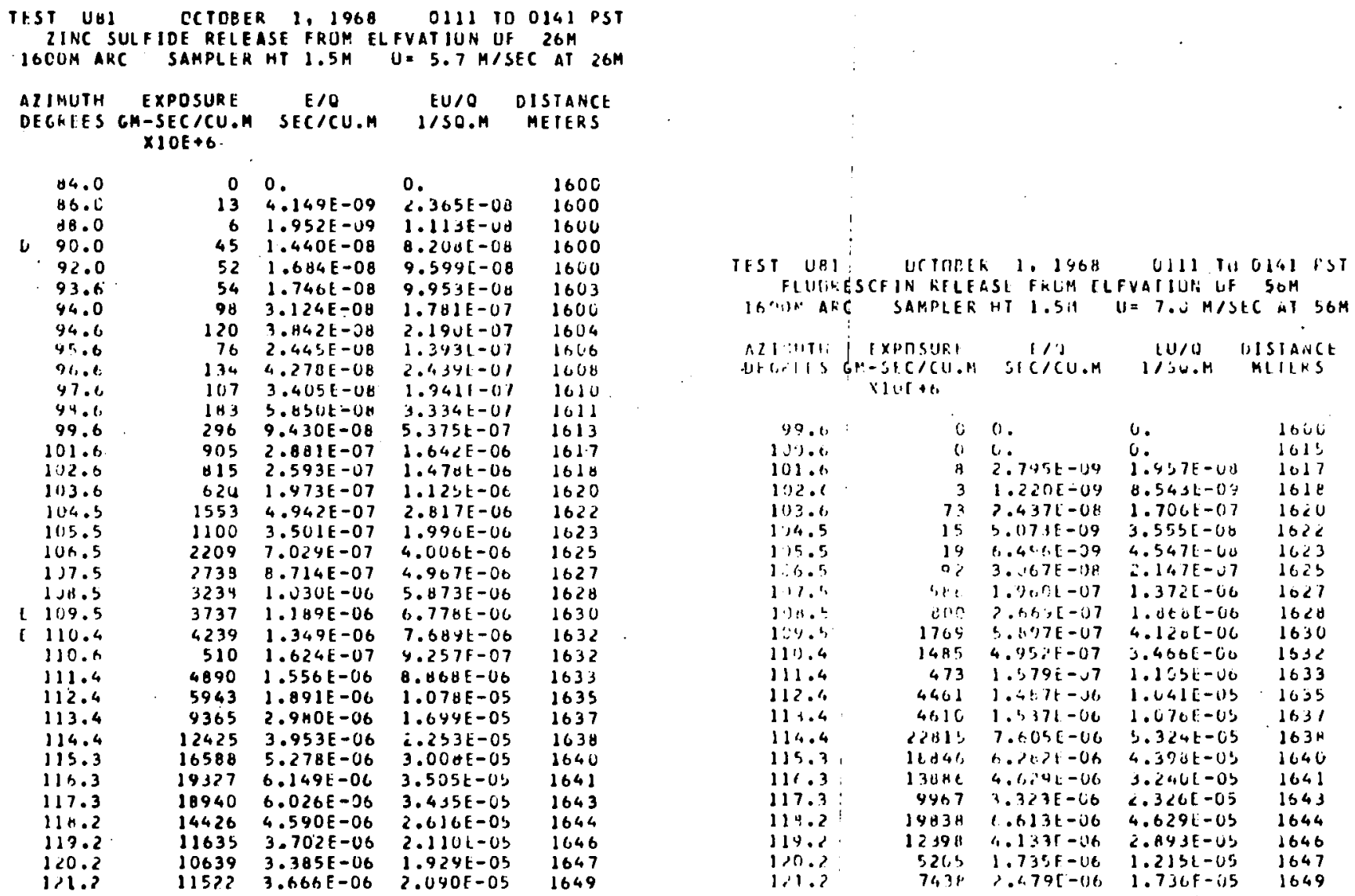




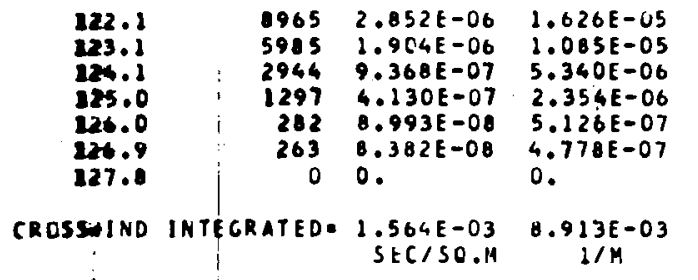

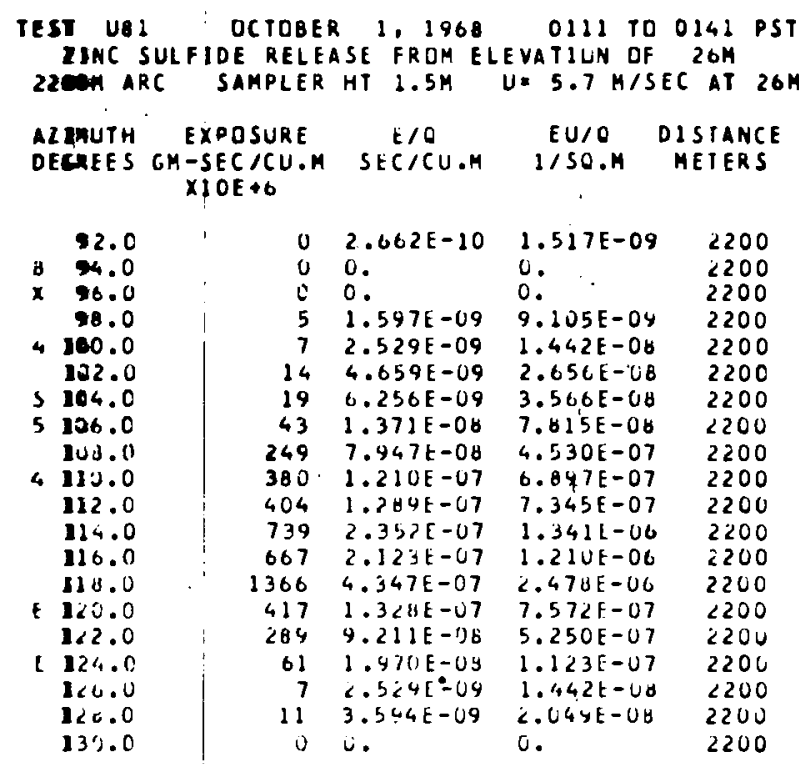

CRISS. IHU INTEGRATEU: $1.145 E-U 4 \quad 6.5 \angle 8 E-04$

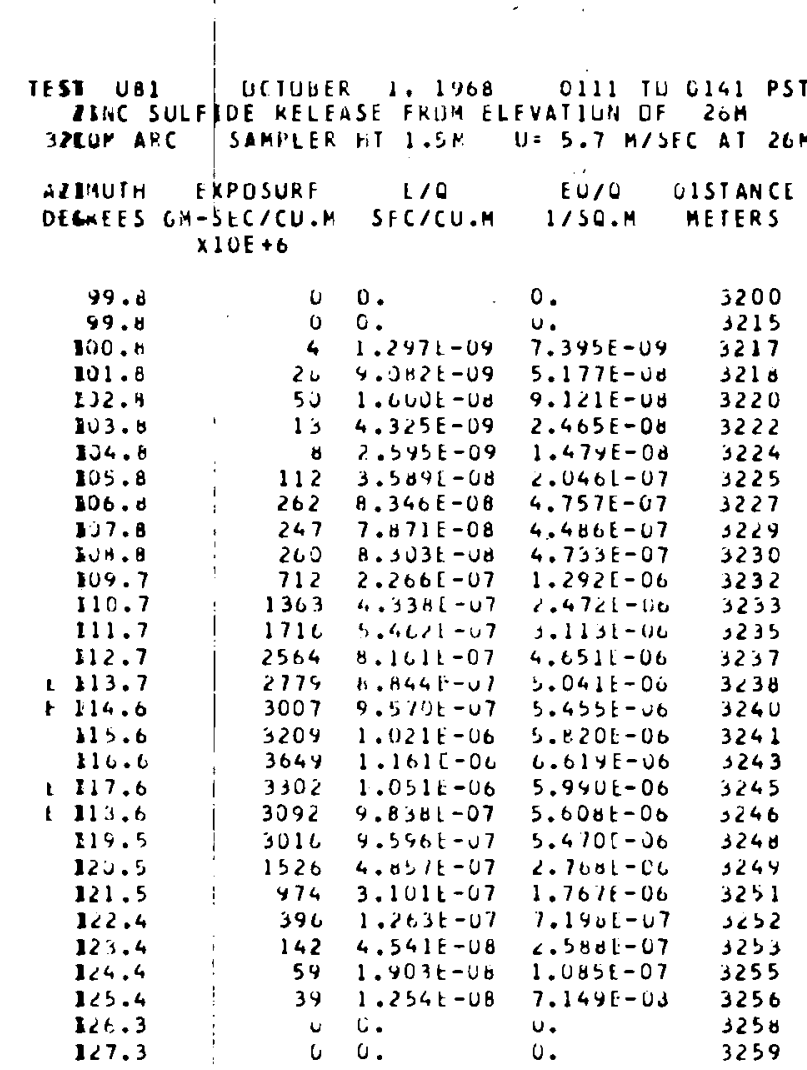

CKLSSWIND INTEGRATEDE $5.730 E-04.3 .266 E-03$

$$
\begin{array}{cc}
5.730 E-04 \\
\text { SECISG.M } 3.26 C E-O B
\end{array}
$$

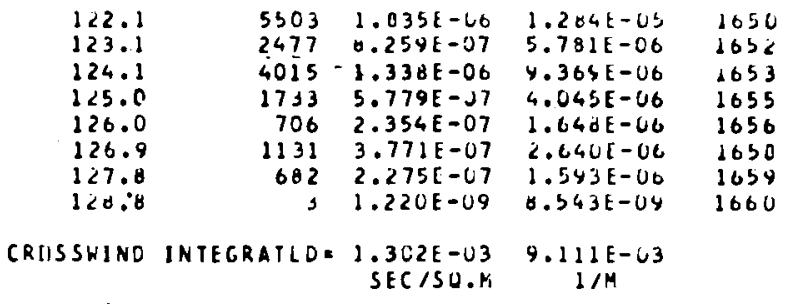

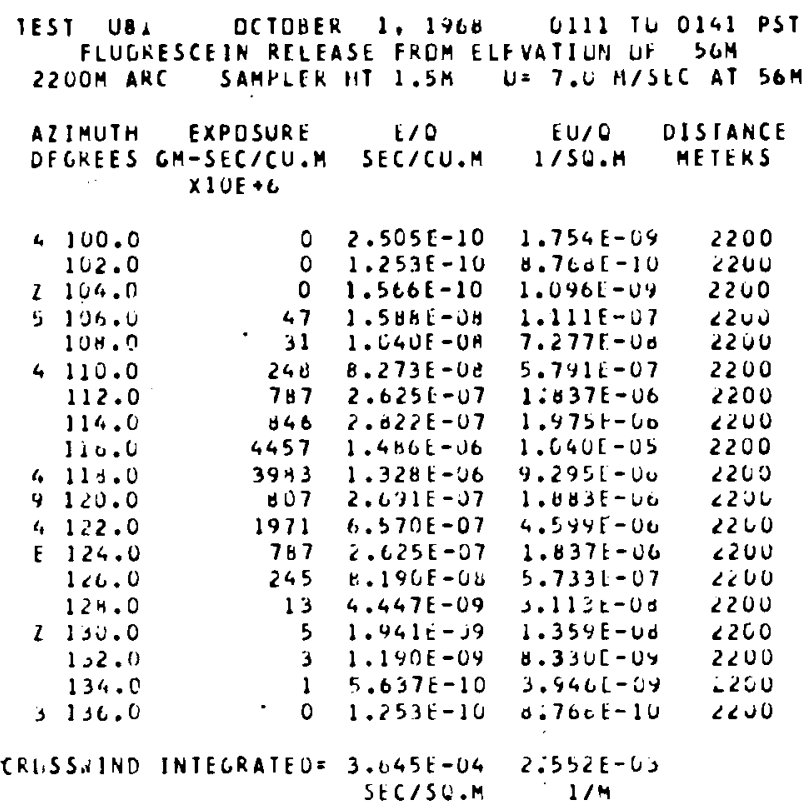

TESI UEI OCTMEER 1, 1968 U111 TU 0241 PST FLUOKESCEIN RELEASE FROM ELFVAIIUIN UT $56 \mathrm{M}$ $3200 M$ ARC SAMPLER HT $1.5 \mathrm{M} U=7.2 \mathrm{M} / \mathrm{SEC}$ AT $56 \mathrm{M}$

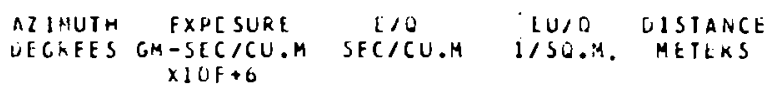

\begin{tabular}{|c|c|c|}
\hline 103.4 & j & 0. \\
\hline 104.8 & c & 0. \\
\hline 105.8 & 9 & $3.094 \mathrm{k}-09$ \\
\hline 166.8 & 87 & $2.9 J t, E=0 \theta$ \\
\hline 107.8 & 1's & $4.467 t-04$ \\
\hline $1,8.8$ & 293 & $9.77 B E-0 B$ \\
\hline 1014.7 & 314 & $1.049 E-107$ \\
\hline 110.7 & 30 & $1.7018-07$ \\
\hline 111.7 & 684 & $2.249 \mathrm{~L}-07$ \\
\hline 112.7 & 958 & $3.1961-07$ \\
\hline 111.7 & 1676 & $5.3 \pm 3 E-\cup 7$ \\
\hline 114.6 & 3493 & $1.165[-60$ \\
\hline 115.6 & 3493 & $1.165 E-66$ \\
\hline 116.0 & 60,1 & $2.017 i=06$ \\
\hline 117.6 & 3430 & $1.277 \mathrm{E}-06$ \\
\hline 110.6 & 2080 & $6.935 E-07$ \\
\hline 119.5 & 2753 & $0.17 n[-07]$ \\
\hline 121.3 & 2733 & $9.17+16-c 7$ \\
\hline $1<1.5$ & 2090 & $6.33+E-\cup 7$ \\
\hline 122.4 & 29141 & $7.0 \leq 7-07$. \\
\hline 123.4 & 1604 & $5.365 t-07$ \\
\hline $12 \frac{1}{.4}$ & 1532 & 5.10 is -67 \\
\hline 125.4 & 1003 & $3.613 E-37$ \\
\hline 126.3 & 261 & $0.710 E-U A$ \\
\hline $1<7.3$ & 194 & $6.644 E-U N$ \\
\hline $12 M \cdot 3$ & 142 & $0.431 k-08$ \\
\hline 129.3 & 1 & $4.4 \cup 9 t-10$ \\
\hline 130.3 & 3 & $1.2025-64$ \\
\hline $1,1.2$ & s & $1.816 t-29$ \\
\hline
\end{tabular}
$0 . \quad 3200$ 3224 $.1667-60 \quad 3225$ $2.034 L-01 \quad 32<7$ $3.1411-0 \% \quad 32<9$ $6.0445-67$ S250 $7.34 \mathrm{JF}-67 \quad 3232$ $1.1 \%$ OU 3233 1.CU. 2.2371-06 9297 $3.41<\mathrm{L}-06 \quad 9230$ d. $1525-60 \quad 3240$ B.13टk-Ue 3241 $1.41<E-U S .3243$ D. $337 E-06 \quad 3245$ 4. $254-110 \quad 324 C$ $6.42 b 1-40 \quad 3249$
$4.854-v 0$ $4.034 E-00 \quad 3201$

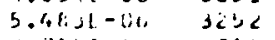
3. $75 \pm=60$ 125 $3.570 E-U L \quad 3255$ $2.5241-00 \quad 3250$ 6. $197 \mathrm{~L}-17 \quad 3258$ 4. $651 E-17 \quad 3254$ $4.5 \cup 2 E-07 \quad 3261$ 4.487 [-CY 202 b.973E-US $326 \mathrm{~s}$ 1.271t-UD $12 \$ 4$

CRLSSHIND INIEGRATED = 7.UO2E-U4 4.95OE-UI ifit 


\begin{tabular}{|c|c|c|c|c|}
\hline $\begin{array}{l}\text { EST UEI } \\
21 N C \text { SI } \\
\text { SOOOH AR }\end{array}$ & $\begin{array}{l}\text { DCIOEE } \\
\text { FIOERELE } \\
\text { SAMPLER }\end{array}$ & $\begin{array}{l}R \\
R \text { ASE } \\
\text { A FRUM EL } 1968 \\
\text { HT } 1.5 \mathrm{SH}\end{array}$ & $\begin{array}{r}0111 \text { IL } \\
\text { LFVATIUN of } \\
U=5.7 \mathrm{HIS}\end{array}$ & $\begin{array}{lll}264 \\
C 12\end{array}$ \\
\hline $\begin{array}{l}\text { AZIMUTH } \\
\text { DECKEES }\end{array}$ & $\begin{array}{c}\text { EXPOSURE } \\
\text { GP-SEC/CU.A } \\
\text { XIOE +6 }\end{array}$ & $\begin{array}{l}\text { SECO } \\
\text { SEC/CU.M }\end{array}$ & $\begin{array}{l}\text { EU/O } \\
1 / 50 . \mathrm{H}\end{array}$ & $\begin{array}{c}\text { OISTANCE } \\
\text { HETERS }\end{array}$ \\
\hline $\begin{array}{l}4106.0 \\
4108.0 \\
4110.0 \\
4112.0 \\
4114.0 \\
4116.0 \\
4118.0 \\
4120.0 \\
4122.0 \\
5124.0 \\
5126.0 \\
7128.0 \\
130.0\end{array}$ & $\begin{array}{r}0 \\
6 \\
25 \\
77 \\
204 \\
167 \\
100 \\
38 \\
15 \\
0 \\
0 \\
0 \\
0\end{array}$ & $\begin{array}{l}0 . \\
1.997 E-09 \\
B .120 E-09 \\
2.476 E-08 \\
6.496 E-08 \\
5.324 E-08 \\
5.990 E-0 B \\
1.225 E-08 \\
5.058 E-04 \\
2.662 E-10 \\
0 . \\
0 . \\
0 .\end{array}$ & $\begin{array}{l}\text { U. } \\
1.136 E-06 \\
4.628 E-0 . \\
1.411 E-07 \\
3.703 E-07 \\
3.035 E-07 \\
3.414 E-07 \\
6.980 E-08 \\
2.083 E-08 \\
1.517 E-08 \\
6 . \\
0 . \\
0 .\end{array}$ & $\begin{array}{l}4810 \\
4870 \\
4920 \\
4990 \\
5080 \\
5020 \\
5100 \\
5130 \\
4830 \\
4660 \\
4770 \\
4900 \\
4970\end{array}$ \\
\hline SW & & & $\begin{array}{r}2.317 \mathrm{E} \\
1 / \mathrm{M}\end{array}$ & \\
\hline
\end{tabular}

TEST UOI OCTOBER 1, 1968 O 0111 TO 0141 PST ZINC SULFIDE RELEASE FRUA ELFVATION OF .26H TOCOM ARC SAMPLER HT $1.5 \mathrm{M}$ UE $5.7 \mathrm{M} / \mathrm{SEC}^{2}$ AT $26 \mathrm{M}$

AZIMUTH EXPOSURE
DEGRES EH-SECICU.M SEC/CU.M I ISO.M UISIANCE
XIOE+6

$\begin{array}{ll}6 & 100.0 \\ 5 & 102.0 \\ 6 & 104.0 \\ 6 & 136.0 \\ 6 & 108.0 \\ 6 & 110.0 \\ 6 & 112.0 \\ 7 & 114.0 \\ 6 & 116.0 \\ 6 & 118.0 \\ 5 & 120.0 \\ 7 & 122.0 \\ 6 & 124.0 \\ 7 & 126.0 \\ 6 & 128.0\end{array}$

$\begin{array}{rl}0 & 0 . \\ 1 & 5.324 E-10 \\ 6 & 1.947 E-09 \\ 66 & 2.103 E-09 \\ 166 & 5.29 d E-09 \\ 228 & 7.268 E-08 \\ 431 & 1.372 E-07 \\ 636 & 2.025 E-07 \\ 670 & 2.134 E-07 \\ 391 & 1.246 E-07 \\ 227 & 7.241 E-0 \theta \\ 117 & 3.740 E-08 \\ 8 & 2.795 E-09 \\ 14 & 4.526 E-09 \\ 0 & 0 .\end{array}$

4.

$3.0358-04$

$1.13 \forall E-0 B$

$1.194 E=07$

3. $020 E-07$

$4.143 E-07$

$7.8<3 E-07$

$1.154 \mathrm{E}-06$

$1.216 t-06$

$7.102 E-07$

$4.12 \forall[-07$

$2.132 E-07$

$1.393 E-08$

$2.500 E-0 \mathrm{~B}$

6560

6610

6650

6720

6800

7000

7300

7220

7190

7150

7120

7100

1100 CRTSSININD INTEGRATED= $2.360 E-04 \quad 1.345 E-03$

$1 / \mathrm{H}$

TEST U81 DCTDEER 1, 1468 0111 IU 0141 PST IINC SULFIDE RELEASE FRUM ELFVATIUN UF $20 \mathrm{M}$ $12800 \mathrm{H}$ ARC SAMPLER HT $1.5 \mathrm{M} U \mathrm{U}$ 5.7 M/SEC AT $26 \mathrm{M}$

AIIMUTH EXPOSURE E/O EU/O DISTANCE DEGREES CH-SEC/CU.M SECICU.M I/SO.M DETERS $\times 10 E+6$

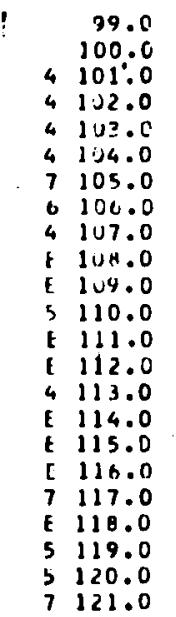

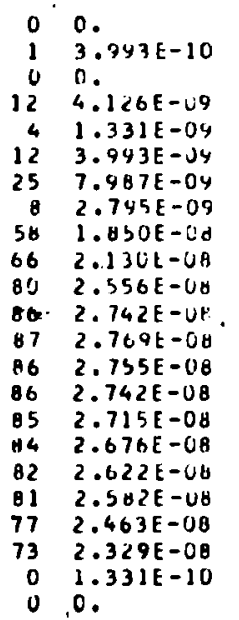

0 .

12000

$2.276 t-04 \quad 12800$

0.

$2.35<\mathrm{E}-00$

$7.587 E-04 \quad 12800$

$2.276[-C B \quad 12800$

$4.552 E-08 \quad 12860$

$1.543 \mathrm{E}-00 \mathrm{O} 12000$

$1.055 \mathrm{E}-07 \quad 12800$

$1.214 E-07 \quad 12600$

$1.4576-07 \quad 12800$

$1.563 \mathrm{E}-07 \quad 12800$

$1.570 E-07 \quad 12000$

$1.571 t-01 \quad 1<800$

$1.50 \mathrm{EE}-07 \quad 22800$

$1.548[-07 \quad 12800$

$1.525 E-07 \quad 22800$

$1.495 \mathrm{E}-07 \quad 12800$

$1.4721-07 \quad 12800$

$1.404 E-07 \quad 12800$

$1.328 E-07 \quad 12800$

$7.5871-10 \quad 12800$

CRUSSWIND INTEGRATLOO= $7.821 E-05 \quad 4.45 U E-04$ SEC/SU.M TIIM

TEST UBS OCTUBER 1,1968 O111 TU 0141 PST FLUOKESCEIN RELEASE FROM ELFYATILN UF $56 \mathrm{~B}$ SOOOM ARC SAMPLER HI $1.5 \mathrm{M} U=7.0 \mathrm{M} / \mathrm{SEC}$ AT $56 \mathrm{M}$ ALIMUTH EXPOSURE GIO
DECREES CM-SECICU.M SECICU.M IISC.M DISIIANCE $X 10 E+6$

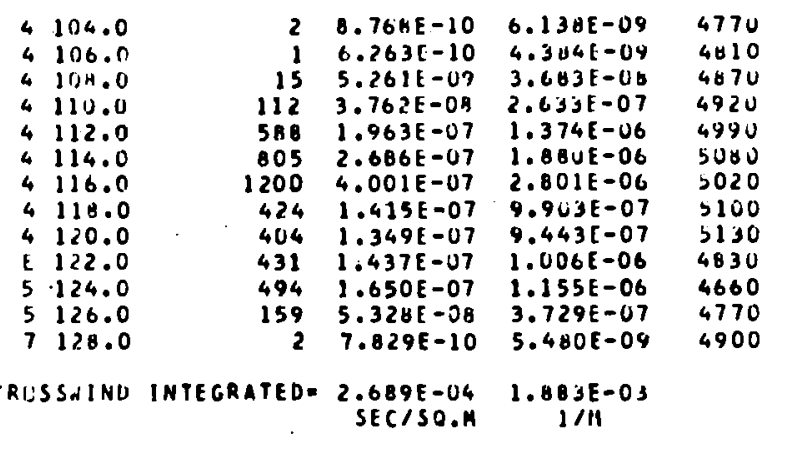

TEST UO1 DCTOBER 1, 1968 O111 10 0141 PST FLUDRESCE IN RELEASE FRUM. ELEVATION UF bOM TDUDM ARC SAKPLER HI $1.5 \mathrm{M}$ U. $7.0 \mathrm{M} / \mathrm{SEC}$ AI $56 \mathrm{M}$

ALJIIUTH FXPOSURE L/O EU/O DISIANCE DFGHES GP-SEC/CU.M SECICU.M I/SU.H MLIEKS $\times 10 E+6$
5132.0
e 1.04 .0
"106.0
6108.0
A 110.0
b 112.0
7114.0
h 116.0
6118.0
7122.0
B 124.0
7126.0
i $1<H . C$
p $1,2.0$

$\begin{array}{rl}0 & 0 . \\ 11 & 3.904 E-09 \\ 150 & 3.029 E-0 U \\ 207 & 6.914 E-08 \\ 746 & 2.489 E-07 \\ 1082 & 3.607 E-07 \\ 1141 & 3.804 E-C 7 \\ 1259 & 4.199 E-07 \\ 1509 & 5.032 E-07 \\ 726 & 2.423 E-07 \\ 687 & 2.292 E-07 \\ 142 & 4.766 E-08 \\ 286 & 9.545 E-08 \\ 20 & 6.827 E-09 \\ 1 & 1.323 E-10 \\ 4 & 1.399 E-U 4 \\ 0 & 0.3\end{array}$

0.

$2.733 E-0 \theta$ $3.5 \angle U E-07$ $4.040 E-07$

$1.74<E-06$

$2.525 E-06$

$2.663 \mathrm{E}-66$

$2.439 E-06$

$3.522 E-06$

$1.646[-06$

$1.604 t-06$

$3.3361-07$

$6.601 t-07$

$4.77 Y E-00$

$3.7201-1) 4$

$9.7911-09$

0 .

0610

6650

6720

7000

7300

7216

7220

7150

7126

7100
1100

IIśd

1130

$71 b 0$

1200

CRLSSNIND INIEGRATED= 6.647E-U4 4.653E-U3

SEC/SO.M I/M

IEST UA1 TRTOEER 111968 O 111 TU 0161 PST FLUINESCEIN RELEASE FRUM ELFVATIIUN UF SOM

12ADOH AKC SAMPLER HT 1.SM U: 7.0 MISEC.AT BQM

AZIMUTH EXPOSURE E/: EU/O DISIAIOLE DEGREES GM-SECICU.M SEG/CU.M I/SE.M METERS $X I I E+6$.

1. 99.0

4 J 10.0

4.101 .6

$41: 2.0$

4103.0

1104.0

7105.0

$t$ lise.0

4107.0

5 I. $A .11$

4. 1119.0

5110.0

i. $111 . \mathrm{C}$

b 112.0

4113.0

(1) 114.0

5113.0

H. 116.0

7117.0

$911 \% .0$

5119.0

51211.0

7121.0

3122.0

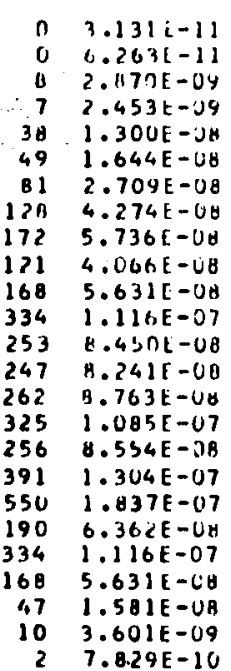

$2.192[-10 \quad 12600$ $4.3341-101+1300$ $2.00 ?[-4012400$ 1:717L-JU 128J0 $9.097 E-l a$ 12dUU $1.1515-0712000$ $1.890 \mathrm{t}-0712800$ $4.442 F-07 \quad 12400$ $4.015 \mathrm{E}-0112800$ $2.8401-57 \quad 12800$ $3.94<E-07 \quad 1<060$ 7.614t-07 12600 5.9]b[-07 12800 j.7uti-ur 1atuo $0.134[-0.712840$ $7.345 E-07$ 1<BUL $5.988 t-U 7$ 128UU $9.130 \mathrm{E}-0112800$ $1.280 t-06$ 12800 $4.453 E-07 \quad 12000$ $7.814 E-07 \quad 12800$

$3.94<t-07 \quad 1<H O 0^{\circ}$

1.107E-07 12600

$2.521 E-0012800$ 3.480t-us 12000 


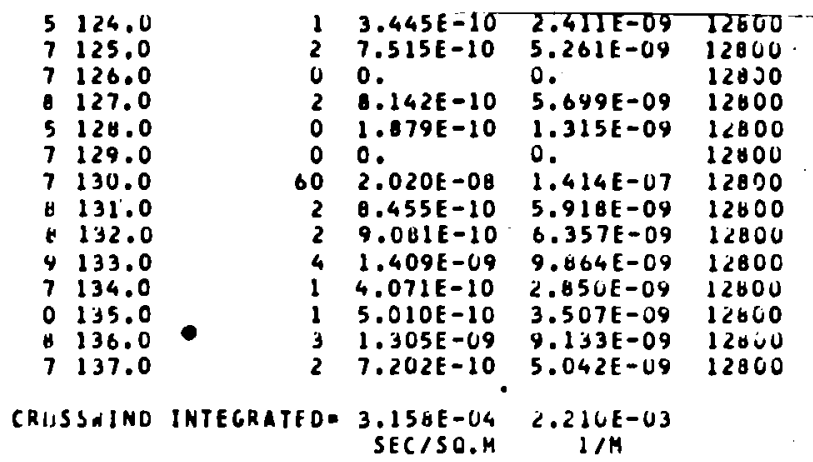


SAMPLINC 400M IO $12800 M$. NU TOHFK SAMPLING. ALL ARCS EMBKACE CRUSSHINO EXTENT OF IRACER. NU FLUDKESCEIN DETFCIEU AT 4OOM ARC. HIGH DUST LEVEL UN FILIERS DN 5OUO, 700O AND 12 UOOM ARCS. IDUST DEGRAUES CONFIDENCE IN TRACER ASSAY -- PARTICULAKLY LNS.) LARGE UIRECIIUN SHEAR. PLUME CENTERLINE-SHIFIS LUCATIGN STARTING AT BZOOM.

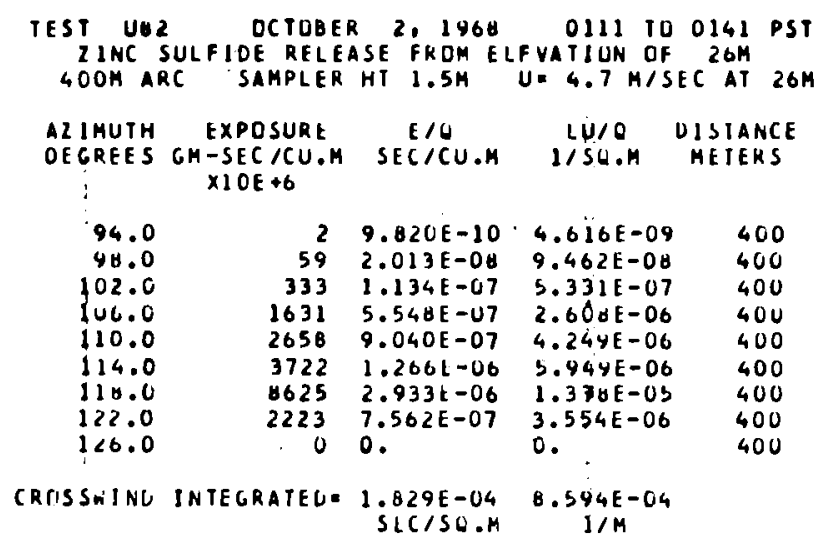

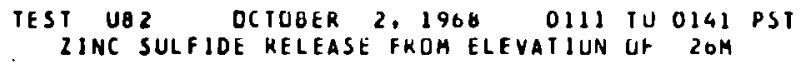
OOOM ARC : SAMPLER HT $1.5 \mathrm{KM}$ U $=4.7 \mathrm{M} / \mathrm{SEC}$ AT $26 \mathrm{M}$ ALIMUTH EXPUSURE LIO EU/O OISTARICE OEGKEES CH-SEC/CU.M SEC/CU.M I/SG.M MEIERS $X \perp O E+6$

\begin{tabular}{|c|c|c|c|c|}
\hline $\begin{array}{l}97.0 \\
97.1\end{array}$ & 100 & $\begin{array}{l}0 . \\
3.4 U Y E-08\end{array}$ & $\begin{array}{l}0.602 E-07 \\
1.602\end{array}$ & $\begin{array}{l}800 \\
806\end{array}$ \\
\hline 99.1 & 542 & $1.844 \mathrm{E}-07$ & $8.667 \mathrm{E}-07$ & 809 \\
\hline 101.1 & 1458 & $4.959 \mathrm{E}-07$ & $2.331 \mathrm{E}-06$ & 813 \\
\hline 103.0 & 1449 & $4.928 E-07$ & $2.316 E-06$ & B16 \\
\hline 105.0 & 2214 & $7.531 E-07$ & $3.340 E-06$ & 820 \\
\hline 106.9 & 4534 & $1.542 E-06$ & $7.247 E-06$ & 823 \\
\hline 108.0 & 7847 & $2.06 \theta E-06$ & $1.254 E-05$ & 826 \\
\hline 110.7 & 15713 & $5.343 E-06$ & $2.511 E-05$ & 829 \\
\hline 112.6 & 22281 & $7.576 E-06$ & $3.501 E-05$ & 833 \\
\hline 114.5 & 35864 & $1.220 \mathrm{E}-05$ & $5.735 E-05$ & 836 \\
\hline 116.4 & 31327 & $1.003 E-05$ & 5.coget -05 & 839 \\
\hline 110.3 & 40114 & $1.364 E-05$ & $6.411 \mathrm{E}-0 \mathrm{~b}$ & 842 \\
\hline $1<0.2$ & 20945 & $7.1<2 E-06$ & $3.347 E-05$ & 845 \\
\hline $1<2.1$ & 11352 & $3.860 E-06$ & $1.014 E-05$ & 640 \\
\hline 124.0 & 5409 & $1.839 E-66$ & $8.645 E-00$ & 851 \\
\hline 125.8 & 91 & $3.099 \mathrm{E}-\mathrm{U} 8 \mathrm{E}$ & $1.457 \mathrm{E}-07$ & 854 \\
\hline 127.7 & 0 & 0 . & 0 . & 857 \\
\hline נs & & $\begin{array}{l}1.960 E-U 3 \\
\text { SEC/SO.M }\end{array}$ & $\begin{array}{c}0.932 \mathrm{E}-\mathrm{US} \\
1 / \mathrm{M}\end{array}$ & \\
\hline
\end{tabular}

IEST UQZ OCTOEEK 2,1968 O111 TO 0141 PST ZIHC SULFIDE KILEASE FKUM ELEVATIUN UE $26 M$
ZUOA AKC SAMPLER HT 1.5 .4 U $4.7 \mathrm{M} / S E C$ AT $20 \mathrm{M}$ AZIMUTH EXPOSURE EIU EUIU DISTANCE DEGRES GM-SEC/CU.M SEC/CU.M I/SH.M MEIERS $\times 10 t+6$

\begin{tabular}{|c|c|c|c|c|c|c|}
\hline & 00.0 & & 33 & $1.123 t-c 8$ & $5.277[-08$ & 1200 \\
\hline & 90.0 & & 50 & $1.725 \mathrm{E}-\mathrm{JH}$ & $\Delta .1 \cup y E-C \theta$ & 1200 \\
\hline & 42.0 & & 40 & $1.5005-08$ & $7.466 \mathrm{E}-00$ & 1200 \\
\hline & $4 x_{1} .0$ & & 105 & 3.SOHE-OU & 1.CEOE-U7 & 1200 \\
\hline & 96.0 & & 214 & $7.449 L=08$ & $3.501 E-07$ & 1200 \\
\hline & y. & & 413 & 1.4 uest-67 & $0.6101-07$ & 1200 \\
\hline & $1,1.11$ & & 654 & (.) $1 / t-U 7$ & 1.0'st-Uo & 1200 \\
\hline & $1,2$. & & 1534 & 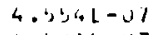 & $2.14 \sqrt{ } \mathrm{l}-00$ & 1200 \\
\hline & $1 \therefore 0^{\circ}$ & & dyss & C.todit-u7 & $J . U Y J E-U C$ & 1200 \\
\hline & lise..e & & 5283 & $1.7 \% / c-66$ & $8.444 t-66$ & 1200 \\
\hline & Iuseo: & & $71 \mathrm{AC}$ & $2.444 L-6 C$ & I.1AGE-U! & 1200 \\
\hline & 116.2 & & $153 \times 0$ & $5.23^{\prime}, k-60$ & $2.4 c] t-u s$ & 1200 \\
\hline$\downarrow$ & 112.0 & & 12144 & $4.104 t-v e$ & $1.441 t-U 4$ & 1200 \\
\hline & $11<$. & $\vdots$ & 2745, & $\because \theta, \Delta, t-v e$ & $4.4601-U S$ & 1200 \\
\hline $\mathrm{t}$ & $11 . .0$ & . & 25,247 & $t \therefore a b+1-v_{0}$ & GOUYSE-US & 1260 \\
\hline & $11: 0$ & & 217116 & $1.3011-16$ & $3.46 y E-035$ & 1200 \\
\hline : & $1 . \cup \cdot v$ & & 766. & $2.548,8-66$ & $1.213 t-64$ & $12 \pi$ \\
\hline & $1 \therefore 0$ & 1 & $361: 5$ & $1 . \angle C A B-v 0$ & $3.703 t-60$ & 1200 \\
\hline s & $12 c_{0} . .6$ & & 314 & $1.071 L-\cup 7$ & $5.033 t-67$ & 1200 \\
\hline & $1: 0.0$ & & 37 & $1.472 t-v 8$ & G. $\angle C B t-V_{0}$ & 1200 \\
\hline & 1.0 .11 & & c & 0. & u. & 1200 \\
\hline & & & alli & $\begin{array}{l}1,4706-03 \\
316 / 51.001\end{array}$ & $\begin{array}{c}0.74 \cup \Gamma-0 ; \\
1 / M\end{array}$ & \\
\hline
\end{tabular}

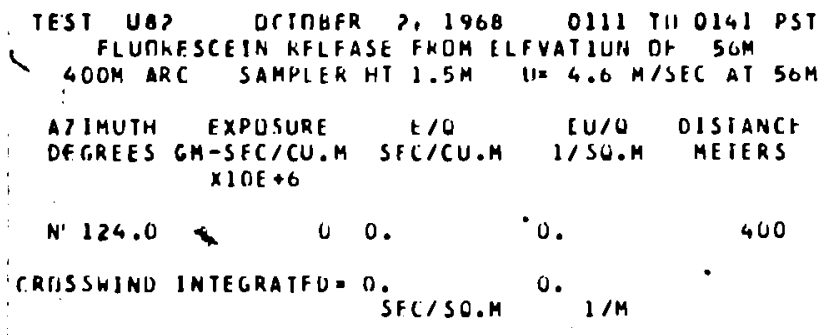




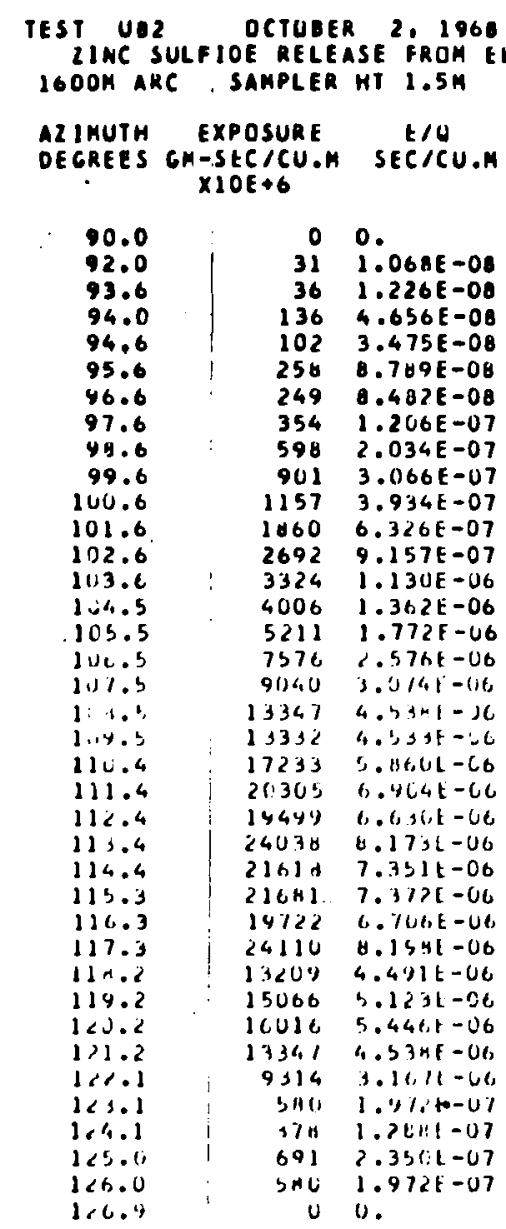

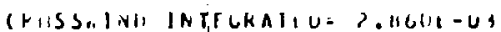

引I $1, \lambda, M$

\begin{abstract}
1. $344 t-02$
$1 / \mathrm{M}$
\end{abstract}

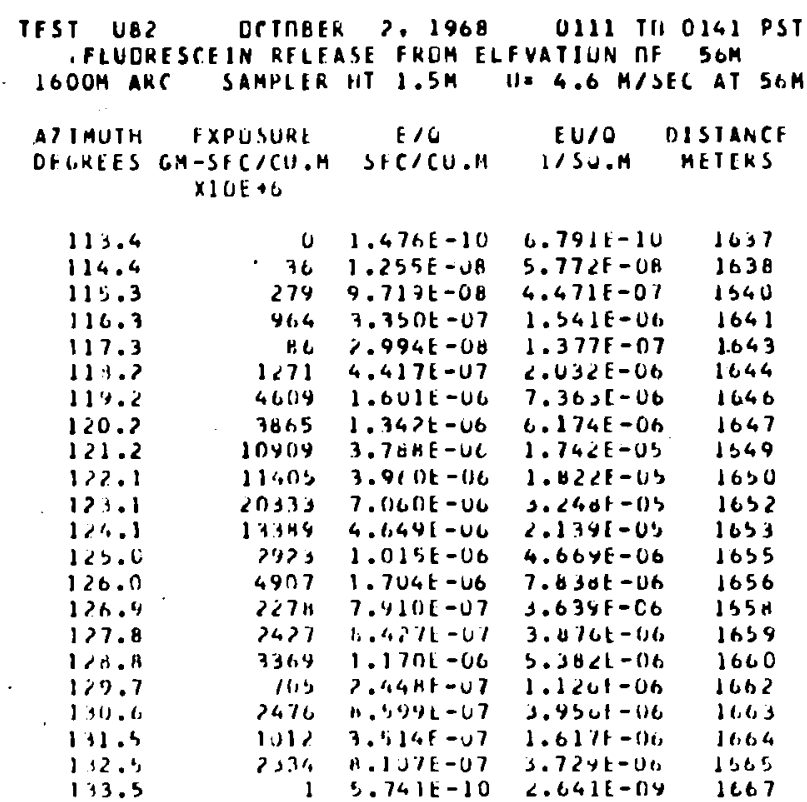

(RIISSWINO IATECRATIDE $10.656 E-04$ 3.4ACF-0) SFCISO.R IIM 


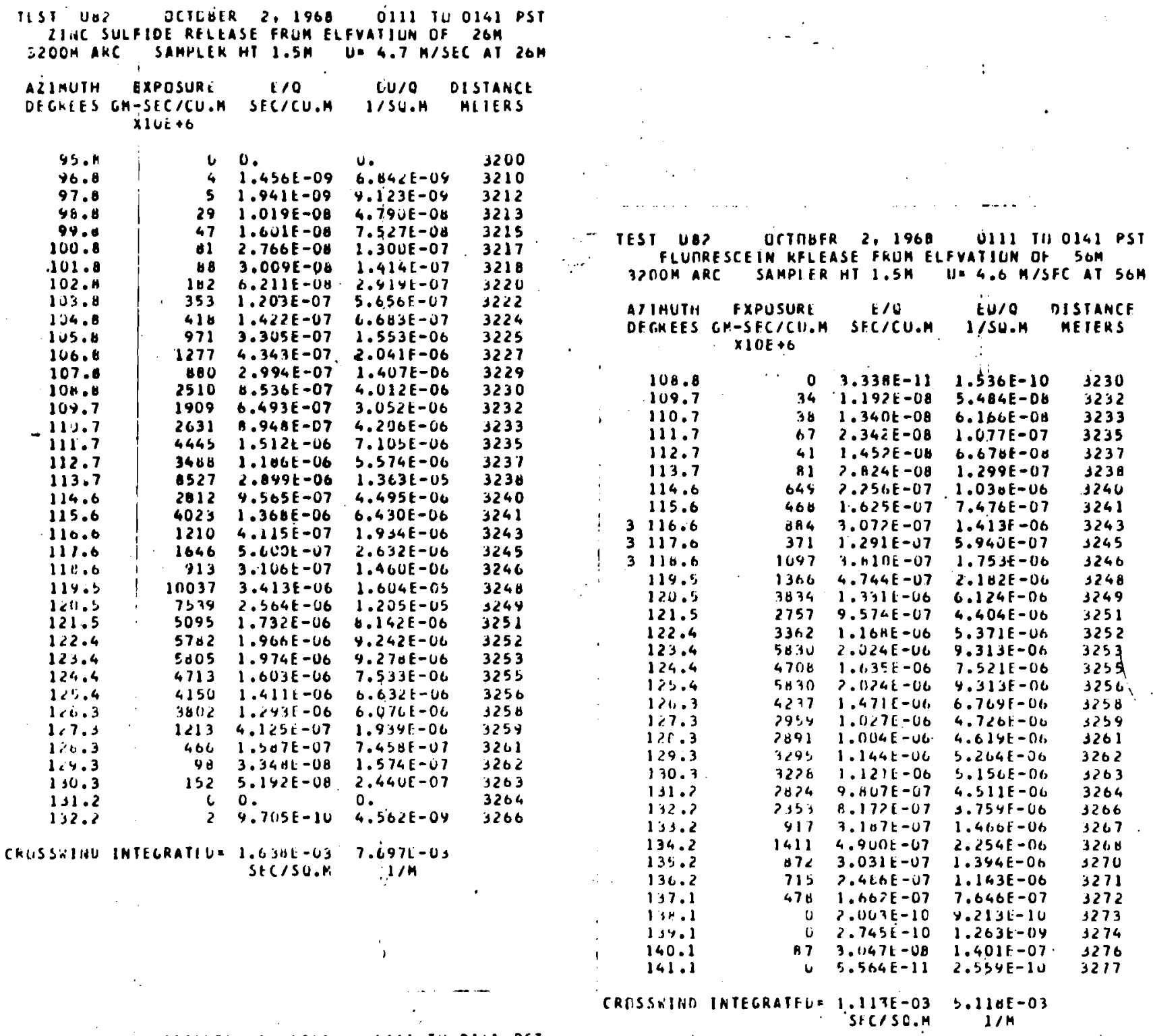

IEST U82 DCTOBER 2, 1968 O111 TU 0141 PSI IINC SULFIOE RELEASE FRUM ELEVATIUN OF $26 \mathrm{M}$ OUM $26 \mathrm{M}$

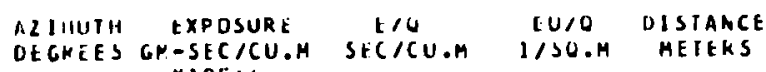
$X \perp O E+6$

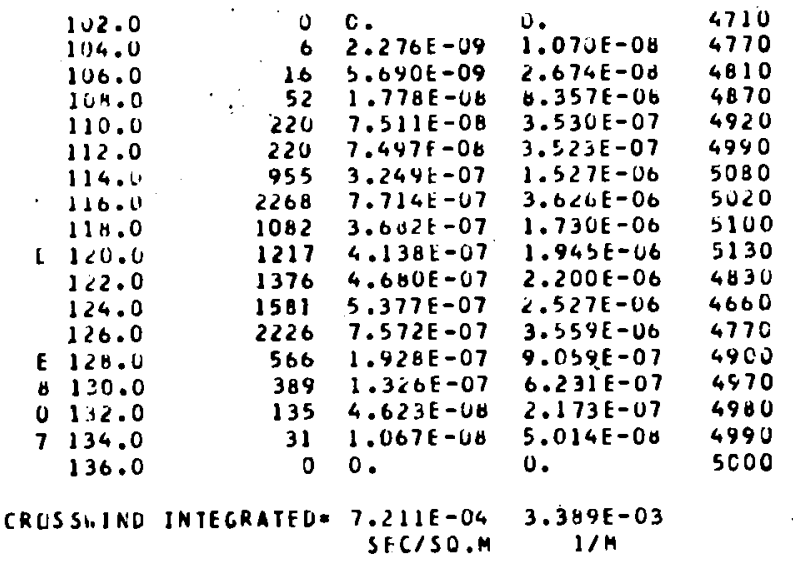

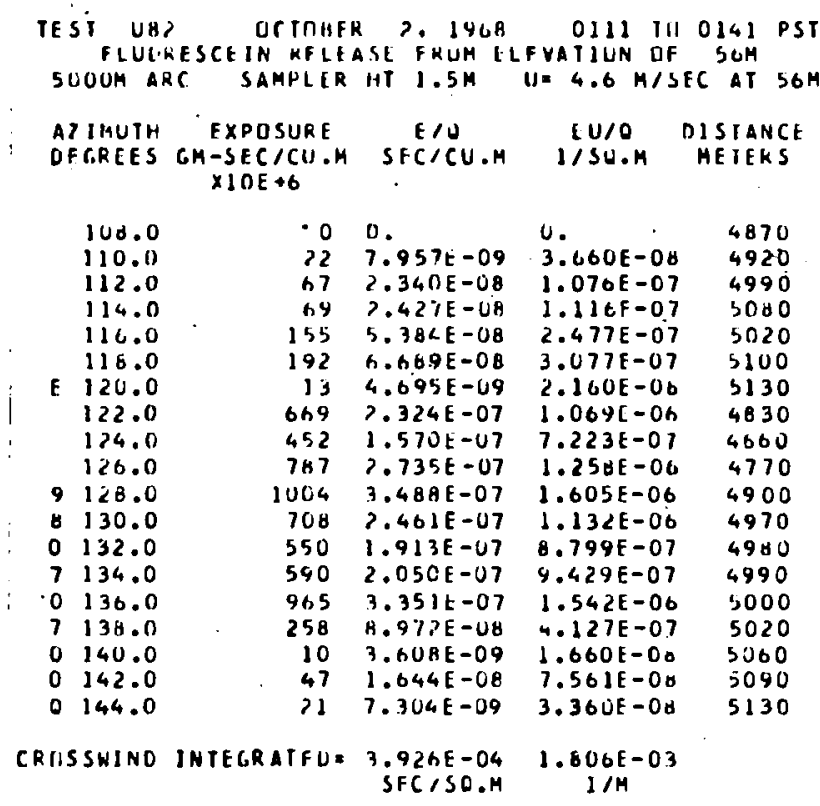




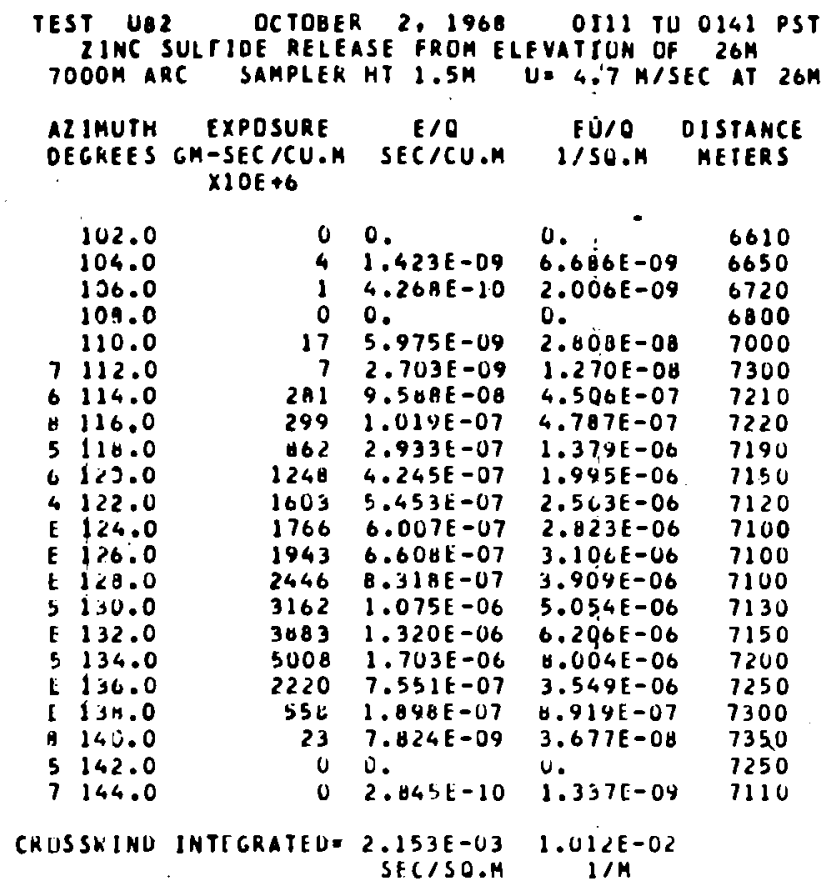

TEST UO2 DCTUBER 2, 1968 O111 TU 0141 PST ZINC SULFIOE RELEASE FRDM ELEVATIUN DF $26 \mathrm{M}$ $1280, M$ ARC SAMPLER HI $1.5 \mathrm{M}$.U. $4.7 \mathrm{M} / S E C$ AT $26 \mathrm{M}$ AZIMUTH EXPCSURE E/O EU/O OISIANCE DEGREES GM-SEC/CU.M SECICU.M I/SQ.M METERS $X 10 E+6$

\begin{tabular}{|c|c|c|c|}
\hline & 119.0 & 0 & 0. \\
\hline & $1<0.0$ & 2 & $7.113 E-10$ \\
\hline $\mathbf{P}$ & 121.0 & B & $2.845 t-09$ \\
\hline & 122.0 & 30 & $1.038 E-08$ \\
\hline 0 & 123.0 & 0 & 0. \\
\hline 8 & 124.0 & 5 & $1.849 E-09$ \\
\hline & 125.0 & 41 & $4 \mathrm{LdE}-0 \mathrm{O}$ \\
\hline $\mathbf{t}$ & $1<6.0$ & 140 & $4.766 F-68$ \\
\hline t & 127.0 & 351 & $1.145 E-07$ \\
\hline 5 & 128.0 & 758 & $2.574 E-07$ \\
\hline 5 & 129.0 & 1130 & $3.044 \mathrm{E}-07$ \\
\hline s & 130.0 & 1341 & $4.562 E-U 7$ \\
\hline 4 & 131.0 & 1234 & $4.212 t-07$ \\
\hline I & 132.0 & 1717 & $041 E-07$ \\
\hline 4 & $1 \$ 3.0$ & 2660 & $9.073 t-07$ \\
\hline & 134.0 & ค 8 & $3.002 \bar{\varepsilon}-0 b$ \\
\hline 4 & 135.0 & 79 & $2.717 t-60$ \\
\hline 8 & 136.0 & 28 & $9.531 \mathrm{E}-09$ \\
\hline 6 & 137.0 & 10 & $3.414 E-09$ \\
\hline 7 & 139.0 & 13 & $4.644 E-09$ \\
\hline & 132.0 & 16 & $5.548 E-09$ \\
\hline & 140 & 0 & \\
\hline
\end{tabular}

CRISSNIND INTEGRATFDE $7.347 E-04 \quad 3.453 E-U S$
0.

$0 . \quad 12800$

$3.343 E-09 \quad 1 \angle 0 L U$

$1.337 \mathrm{E}-0 \mathrm{~B} \quad 12800$

$4.801 E=08 \quad 12800$

U. 12800

$8.692 \mathrm{E}-0 \mathrm{Y} \quad 12800$

$6.61 Y E-08 \quad 12000$

$2.240 E-07 \quad 12800$

$5.616 E-07 \quad 12600$

$1.212 \mathrm{E}-06 \quad 1 \angle 000$

I. 007 E-00 12000

$\begin{array}{ll}1.807 E-06 & 12800 \\ 2.144 E-06 & 12800\end{array}$

$2.745 E-06 \quad 12800$

$4.264 E-06 \quad 12800$

$1.411 E-07 \quad 12800$

$1.277 E-07 \quad 12600$

$4.480 E-001 \angle 800$

$1.605 E-00 \quad 12800$

$2.206 E-00 \quad 1<800$

$\begin{array}{ll}2.60 J E-0 B & 12800 \\ 0 . & 12800\end{array}$
1. $980 E=06 \quad 12800$

$1 / M$

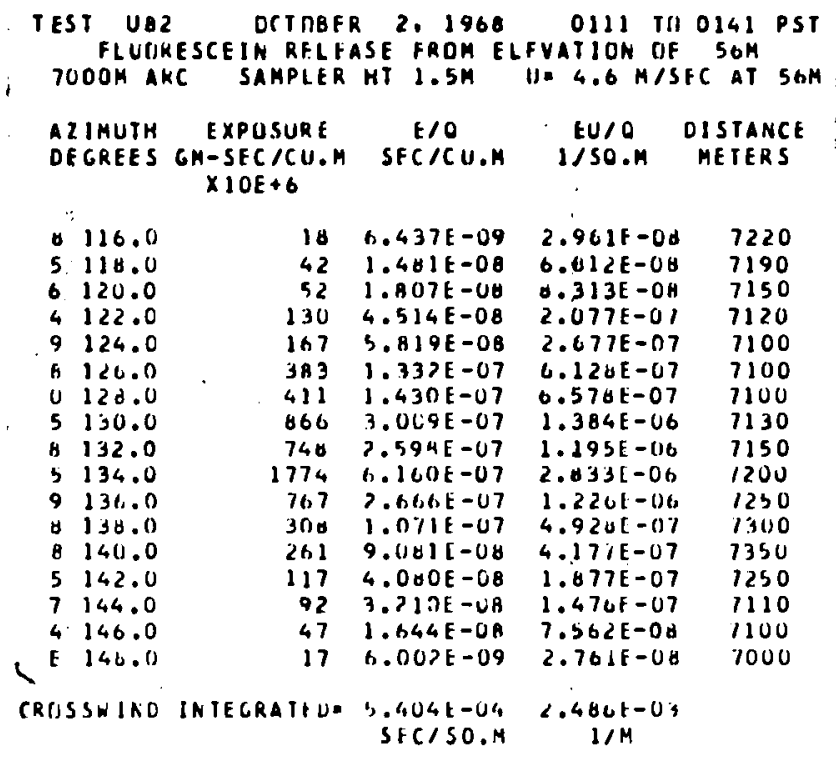

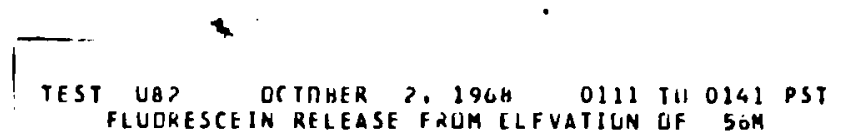
$1280 O M$ ARC SAMPLER HT $1.5 \mathrm{M} U=4.6 \mathrm{M} / \mathrm{SEC}$ AT $56 \mathrm{~A}$
AIIMUTH FXPUSURE E/O EU/O OISTANCE DFGREES CIM-SFC/CU.M SFCICU.M IISU.M MEIERS $X] \cap E+6$

b 124.0

1125.0

9126.0

9127.0

5128,0

5129.0

5130.0

4131.0

E 132.0

4133.0

E 134.0

4135.0

* 136.0

6137.0

3139.0

3140.0

+ 141.0

3142.0

3143.0

514.4 .0

1145.0

3146.0

4147.0

146.0

144.11

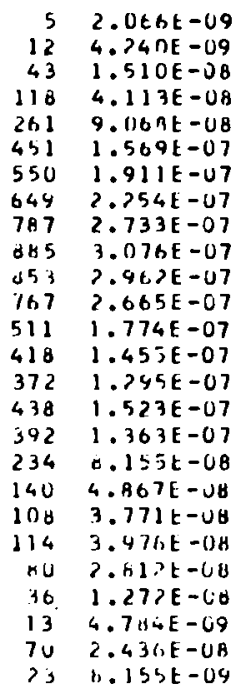

9.50;E-09

$1.451 \mathrm{E}-08$
$6.947 \mathrm{E}-08$

$6.947 E-08$

1. $892 \mathrm{E}-07$

7. $217[-U ?$

.. 79 st -07

d. $793 \mathrm{E}-07$
$1.037 \mathrm{E}-06$

$1.257 \mathrm{E}-06$

1. $415 E-06$

1. $226 \mathrm{E}-06$

$1.226 E-06$
$6.163 E-07$

$6.092 \mathrm{E}-07$

$5.957 E-07$

$7.007 E-07$

$0.27<E-07$

3. $75 \perp F-07$

2. $239 E-07$

$1.735 E-07$

$1.829 \mathrm{E}-117$

1. $243 \mathrm{t}-\mathrm{U} 7$

$5.852 \mathrm{E}-00$

$2.201 \mathrm{E}-\mathrm{OA}$

$1.12 \cup E-07$

3. $731 \mathrm{t}-0 \mathrm{~d}$

12800

12800

12800

12400

12400

$1<B \cup 0$

12800

12900

12600

12000

12800

12900

12800

12800

12400

12800

12840

12800

12800

$1 \angle B \cup 0$

$12 B 00$

$1 \angle 900$
12000

CKUSSBINL INTEGRATIU = 6.47CR-U4 $2.977 F-0$ S

SFCISO.N 
SAMPLING $400 M$ TO 7UOOH. NII TUWER SAMPLING. ALL ARCS EMBRACE CRUSSHIND EXTENT OF RRACER. CENTERLINES UF FLUIJESSLIN AND ZINC SULFIDE PLUMES AT SIGNIFICANTLY DIFFERENT OIRECTIUNS. THERMALLY VERY SIABLE ATMUSPHERE. LAKGE OIKECTIEN SHEAR HITH HEIGHT.

\begin{tabular}{|c|c|c|c|c|c|}
\hline $\begin{array}{l}\text { TEST UEI } \\
\text { 2INC } \\
400 M A B\end{array}$ & $\begin{array}{l}3 \text { OCTUEER } \\
\text { SULF IOE RELE } \\
\text { RC SAMPLER }\end{array}$ & $\begin{array}{l}\text { R } 3.1968 \\
\text { ASE FRDM E } \\
\text { HT } 1.5 \mathrm{M}\end{array}$ & $\begin{array}{l}0112 \text { TU } \\
\text { ELFVATIUN DF } \\
\text { UE } 3.2 \mathrm{H} / \mathrm{S}\end{array}$ & $\begin{array}{r}0142 \\
F \quad 26 M \\
\text { SEC AT }\end{array}$ & $\begin{array}{l}\text { PS1 } \\
26 H\end{array}$ \\
\hline $\begin{array}{l}\text { AIIMUTH } \\
\text { OECREES }\end{array}$ & $\begin{array}{c}\text { EXPOSURE } \\
\text { GM-SEC /CU.H } \\
\times 1 O E+6\end{array}$ & $\begin{array}{c}L / 0 \\
\text { SEC/CU.H }\end{array}$ & $\begin{array}{c}C U / O \\
1 / 50 . M\end{array}$ & $\begin{array}{l}\text { DISTA } \\
\text { METE }\end{array}$ & $\begin{array}{l}\text { NCt } \\
\text { RS. }\end{array}$ \\
\hline $\begin{array}{l}102.0 \\
106.0 \\
110.0 \\
114.0 \\
118.0 \\
122.0\end{array}$ & $\begin{array}{r}0 \\
527 \\
7026 \\
14767 \\
496 \\
0\end{array}$ & $\begin{array}{l}0 . \\
1.796 E-07 \\
2.397 E-06 \\
5.038 E-06 \\
1.695 E-07 \\
0 .\end{array}$ & $\begin{array}{l}0 . \\
9.351 E-07 \\
1.247 E-05 \\
2.620 E-05 \\
8.813 E-07 \\
0 .\end{array}$ & & $\begin{array}{l}00 \\
00 \\
00 \\
50 \\
00 \\
00\end{array}$ \\
\hline CRESSHIND & INTEGRATF O= & $\begin{array}{r}2.174 E-04 \\
\text { SECISO.M }\end{array}$ & $\begin{array}{c}1.130 E=03 \\
1 / K\end{array}$ & & \\
\hline $\begin{array}{c}\text { TESI UB3 } \\
2 \text { IIIC } \\
\text { BCO4 AR }\end{array}$ & $\begin{array}{l}3 \text { UC TOBER } \\
\text { SULF JOE RELEA } \\
\text { RC SAMPLER }\end{array}$ & $\begin{array}{l}\text { R } 3,1968 \\
\text { ASE FRUM E } \\
\text { HT } 1.5 \mathrm{M} .\end{array}$ & $\begin{array}{r}0112 \mathrm{TU} \\
\text { ELFVATION OF } \\
\text { UE } 5.2 \mathrm{~m} / \mathrm{S}\end{array}$ & $\begin{array}{r}0142 \\
\text { SEC } 2 \text { AT }\end{array}$ & $\begin{array}{l}\text { PST } \\
26 \%\end{array}$ \\
\hline $\begin{array}{l}\text { ALIMUTH } \\
\text { OECREES }\end{array}$ & $\begin{array}{c}\text { EXPUSURE } \\
\text { GH-SEC /CU.M } \\
\times 1 O E+6\end{array}$ & $\begin{array}{c}E / O \\
S E C / C U . M\end{array}$ & $\begin{array}{l}\text { EU/O } \\
\text { I/SO.M }\end{array}$ & $\begin{array}{l}\text { OLSIA } \\
\text { MLIEA }\end{array}$ & $\begin{array}{l}\text { NCE } \\
\text { RS }\end{array}$ \\
\hline $\begin{array}{l}99.1 \\
101.1 \\
1.33 .0 \\
105.0 \\
106.9 \\
108.8 \\
110.7 \\
112.6 \\
114.5 \\
116.4 \\
118.3 \\
120.2\end{array}$ & $\begin{array}{r}8 \\
30 \\
474 \\
2675 \\
5041 \\
12349 \\
15343 \\
16530 \\
20028 \\
10645 \\
1017 \\
8\end{array}$ & $\begin{array}{l}2.463 E-09 \\
1.034 E-0 B \\
1.614 E-07 \\
0.128 E-07 \\
1.720 E-06 \\
4.229 E-116 \\
5.137 E-06 \\
5.641 E-06 \\
6.633 E-06 \\
3.632 E-06 \\
3.473 E-07 \\
2.468 E-09\end{array}$ & $\begin{array}{l}1.544 E-08 \\
5.403 E-0 \theta \\
8.413 E-07 \\
4.747 E-06 \\
8.943 E-06 \\
2.144 E-0 b \\
2.669 E-05 \\
2.933 E-0 S \\
3.553 E-05 \\
1.089 E-05 \\
1.806 E-06 \\
1.544 E-08\end{array}$ & $\begin{array}{l}8 \\
0 \\
6 \\
8 \\
8 \\
6 \\
1 \\
8 \\
1 \\
8 \\
8 \\
B\end{array}$ & $\begin{array}{l}09 \\
13 \\
16 \\
20 \\
23 \\
26 \\
24 \\
33 \\
36 \\
39 \\
42 \\
45\end{array}$ \\
\hline $\begin{array}{c}\text { RUSSININD } \\
.\end{array}$ & INTEGRATFDE & $\begin{array}{r}7.903 E-04 \\
\text { SEC/SO.M }\end{array}$ & $\begin{array}{c}4.10 Y E-U 3 \\
1 / M\end{array}$ & & \\
\hline
\end{tabular}
TEST UA3 OCTUBER 3, 1968 $400 M$ ARC SAMPLER HT $1.5 \mathrm{M} U=6.6 \mathrm{H} / \mathrm{SEC}$ AT $56 \mathrm{M}$

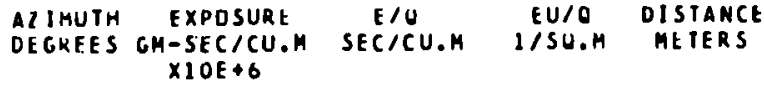

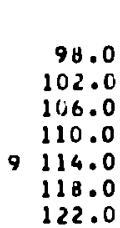

CROSSHIND INTEGRATED: $1.691 E-06$ S.116E-OS
TEST UB3 UCTRBER 3,1968 0112 TU 0112 PST FLUDRESCE IN RELEASE FROM ELFVATION DF S6H WUDH ARC SAMPLER HT $1.5 \mathrm{M} U=6.6 \mathrm{M} / \mathrm{SEC}$ AT $56 \mathrm{M}$

AZIIUTH EXPOSURE T/O EU/Q UISTANCE DECKEES GM-SECICU.M SECICU.M I/SO.M METEKS $\times 10 E+6$

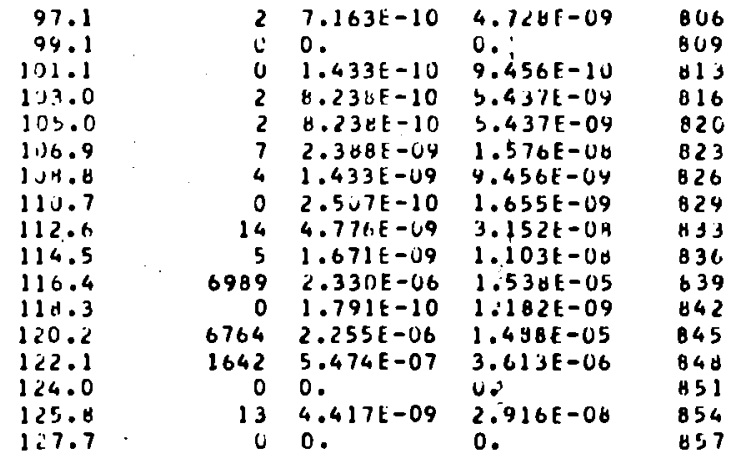

CRIJSSHIND INTEGRATEDE $1.439 E-04$ 9.496E-04

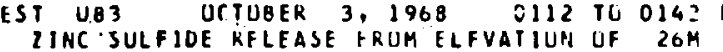
$1200 M$ AKC SAMPLER HT 1.SM UE 5.2 MISEC AT $26 M$

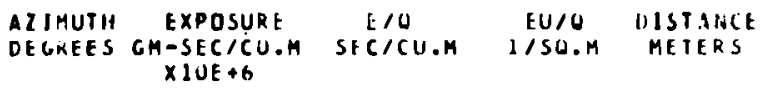

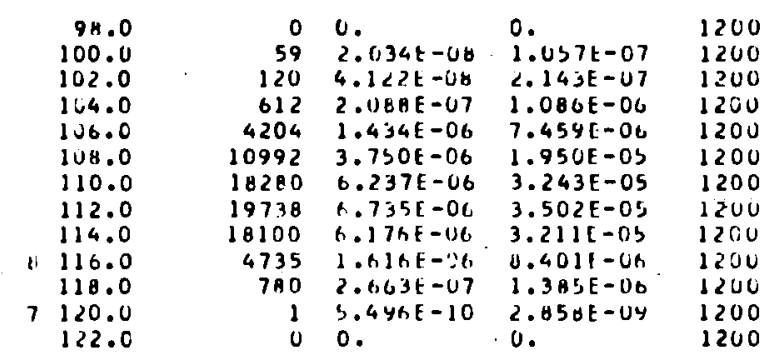

CRIISSHIND INIEGRATEO= $1.109 E-03 \quad 5.764 E-03$ SEC/SU.M I/M
TEST UAS. IICTMEER 3.1968 U112 TU O142 RST FIUURESCE IN RELEASE FRUM tLFVAT ILN UF SGM 12 IIJH ARC SAMPLER HT $1.5 \mathrm{M} U=6.6 \mathrm{M} / \mathrm{SEC}$ AT $50 \mathrm{H}$

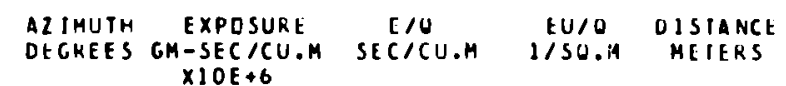

\begin{tabular}{|c|c|c|c|c|c|}
\hline & $134 . c$ & 1 & $6.631 E-10$ & $4.377 E-09$ & $120 \mathrm{~J}$ \\
\hline & 106.0 & 3 & $1.266 t-09$ & $0.356 F-09$ & 1200 \\
\hline & 104.0 & 4 & $07 E-09$ & $7 E-09$ & 1200 \\
\hline & 110.0 & 2 & $9.043 \mathrm{E}-10$ & $5.966 E-09$ & 1200 \\
\hline & 112.0 & 9 & $3.255 E-00$ & $2.149 E-00$ & 1200 \\
\hline & $11 \ldots$ & 1326 & $4.42 .7 t-07$ & $\| \times k-60$ & $1<00$ \\
\hline & 116.0 & 2541 & $3.47+[-07$ & $3.69<1-016$ & 1230 \\
\hline 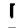 & 114.0 & $12 \mathrm{HH}$ & 4.DU5E -07 & 2.6310 & 1200 \\
\hline & 120.0 & 1668 & $3.5611-07$ & $3.6111-6,6$ & 1200 \\
\hline & 122.0 & 1098 & $3.662 E-07$ & $2.417 \mathrm{~F}-06$ & 1200 \\
\hline & 124.0 & 15 & $5.299 E-U 9$ & $3.4 \times 7 E-0$. & 1200 \\
\hline & 120.0 & 339 & $1.130 E-07$ & $7.400[-07$ & 1200 \\
\hline & $1<B .0$ & 9 & $E-09$ & $O \angle Y E-U E$ & 1200 \\
\hline & 130.0 & 64 & $2.140 E-00$ & $1.413 t-01$ & 1200 \\
\hline & 1.12 .0 & 6 & $2.291 \mathrm{f}-109$ & $1.512 \mathrm{E}-0 \mathrm{U}$ & 1200 \\
\hline & 134.6 & 36 & $1.216[-100$ & $0.024 t-0$, & 1200 \\
\hline & 136.0 & 51 & $1.718 t-00$ & $1.134 \mathrm{f}-07$ & 1200 \\
\hline & 154.0 & 3 & $1.3<6 E-09$ & $8.734 E-09$ & 1200 \\
\hline 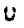 & 140.0 & 2 & H. $.449 E-10$ & $5.57 \cup E-04$ & 1200 \\
\hline
\end{tabular}

CEIJSSHINL INTEGRATTD- 1.JULE-O4 7.8ICE-04 


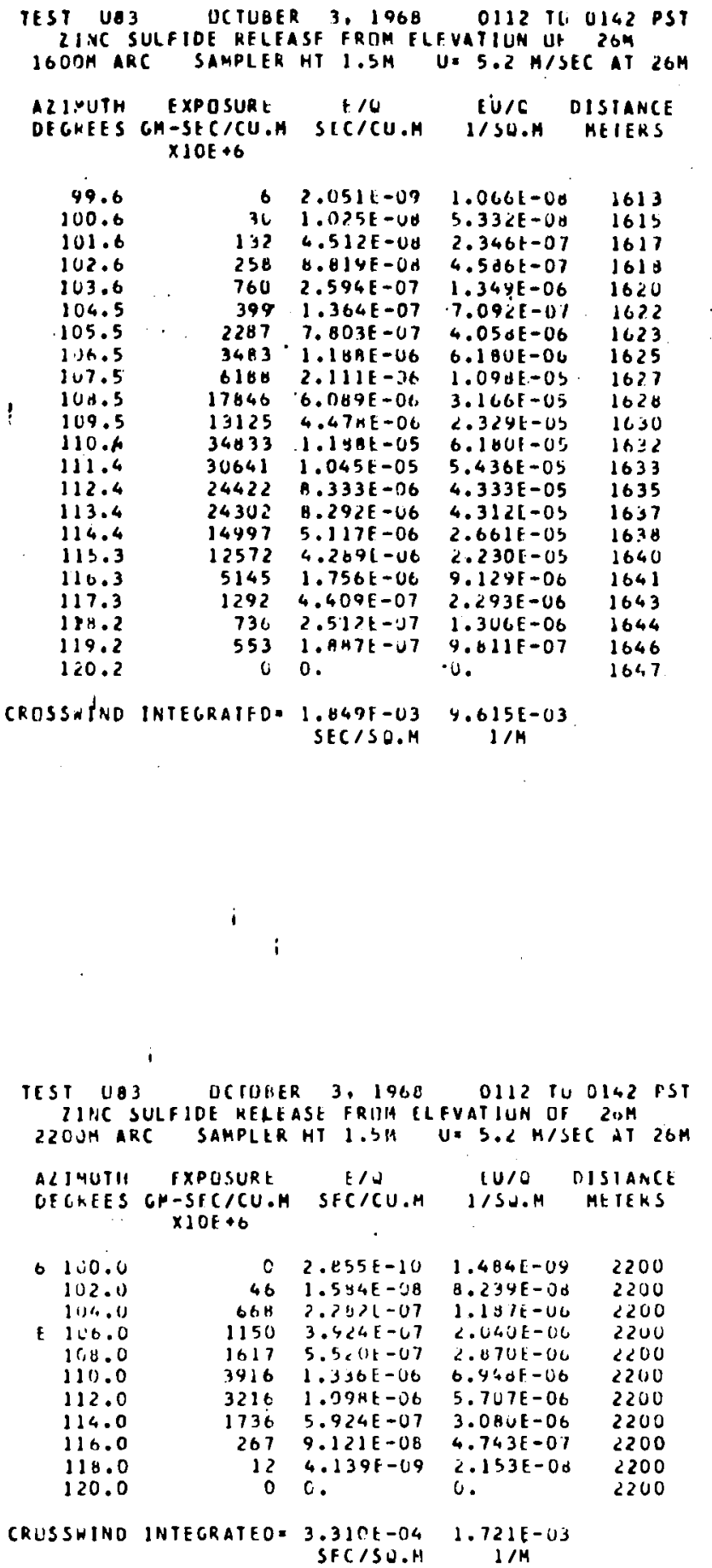

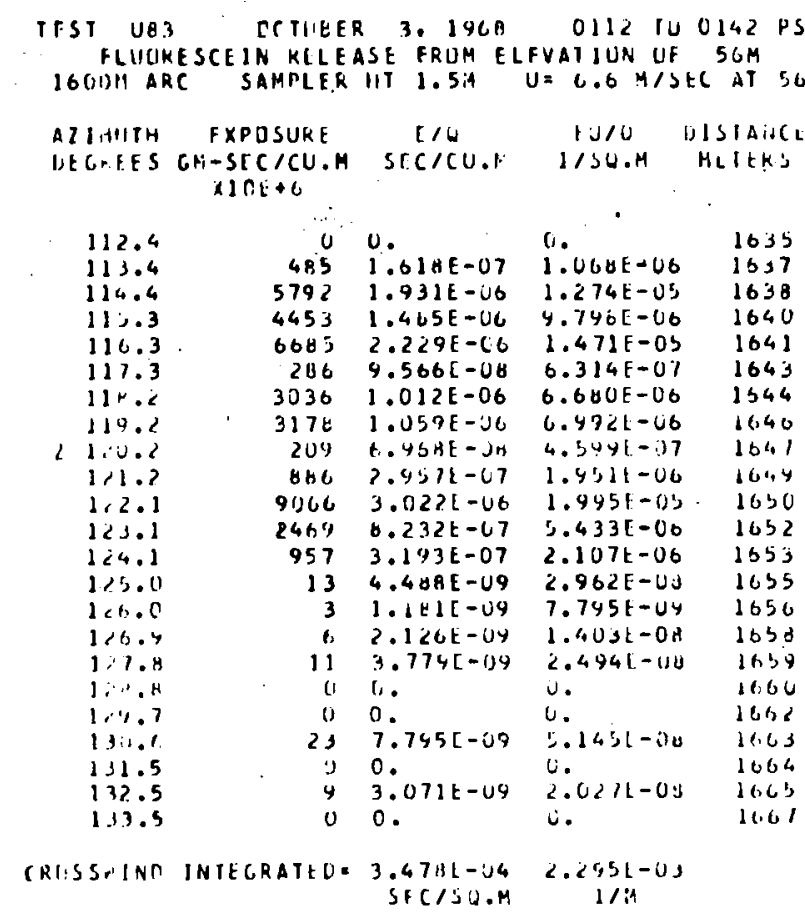

TESI UQ3 UCILHEK 3, IYUE U112 1J 61.2 DST

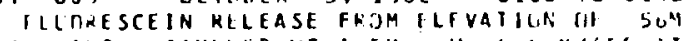

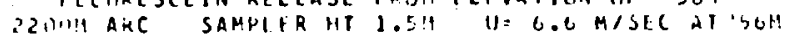

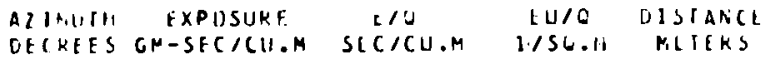
$X 10 \mathrm{O}+6$

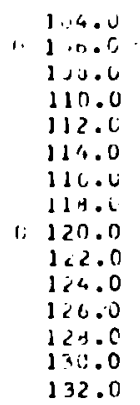

CRUSSHIND INTEGRAIED=2.543E-U4 SECISO.N

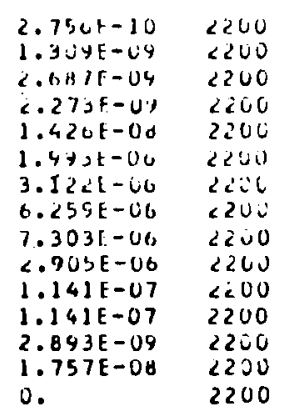

0 .

$1.678 E-03$ $1 / \mathrm{M}$ 


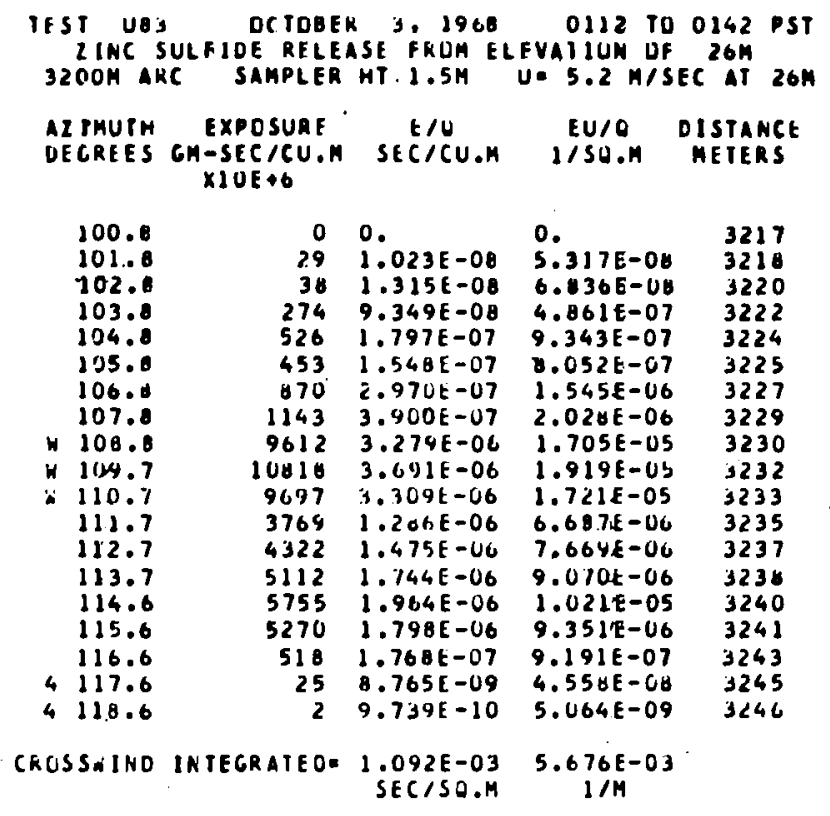

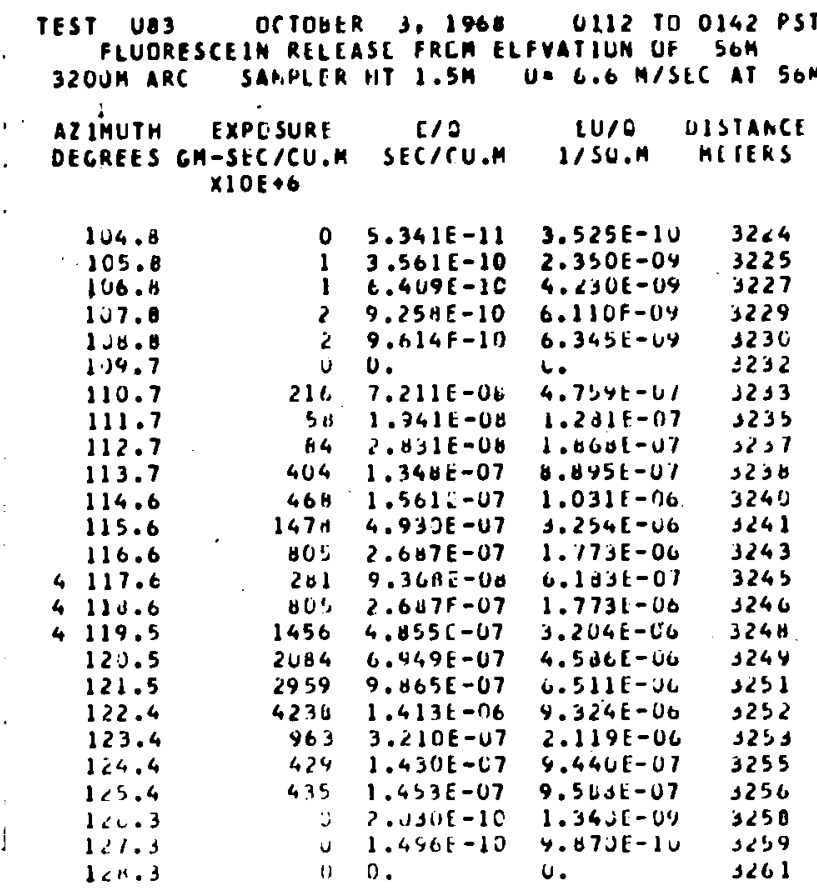

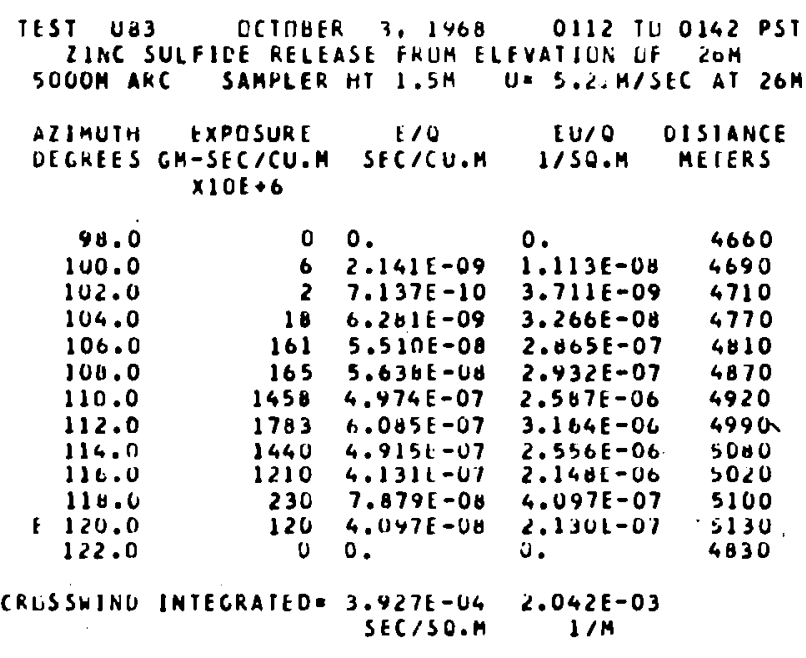

CRUSSIINO INIEGRATIC = $3.145 E-04$
SECISO.14

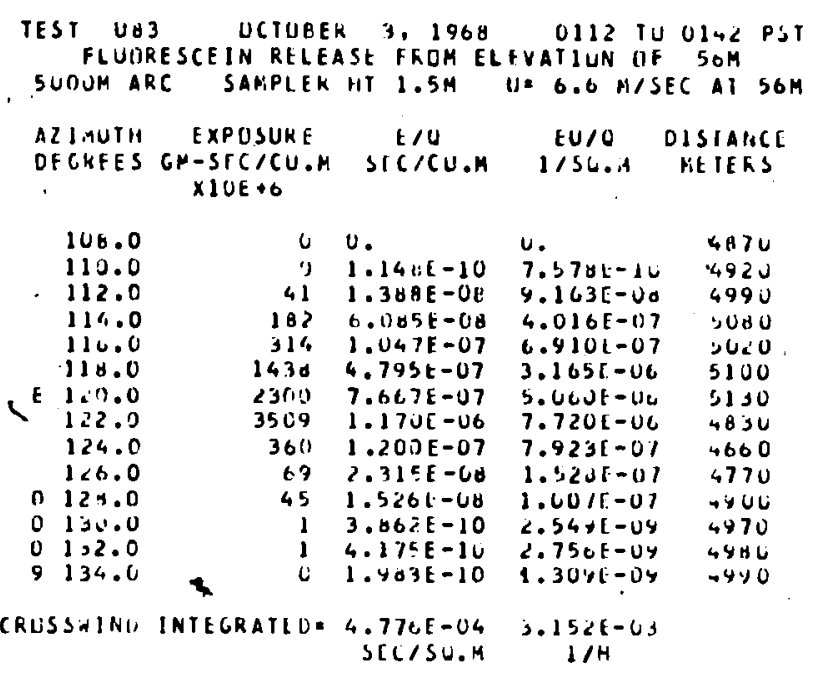




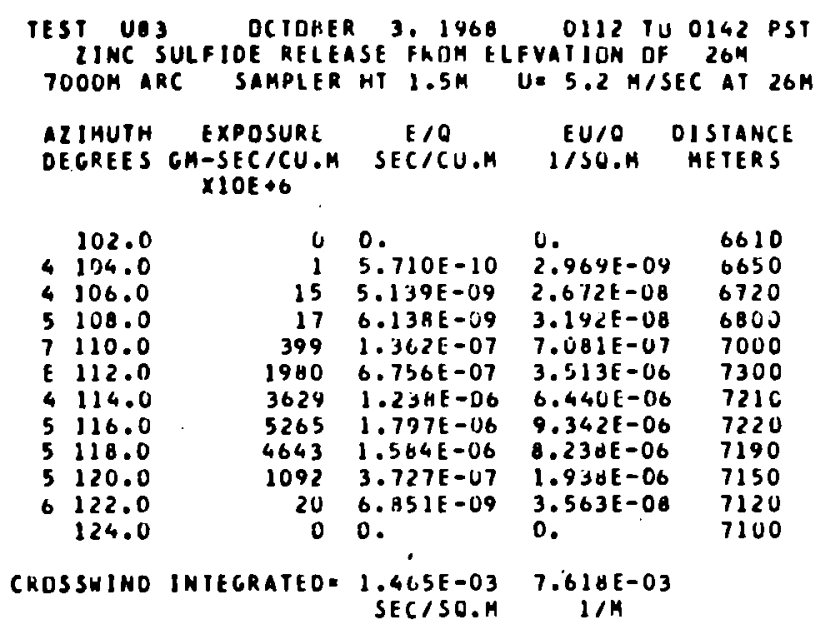

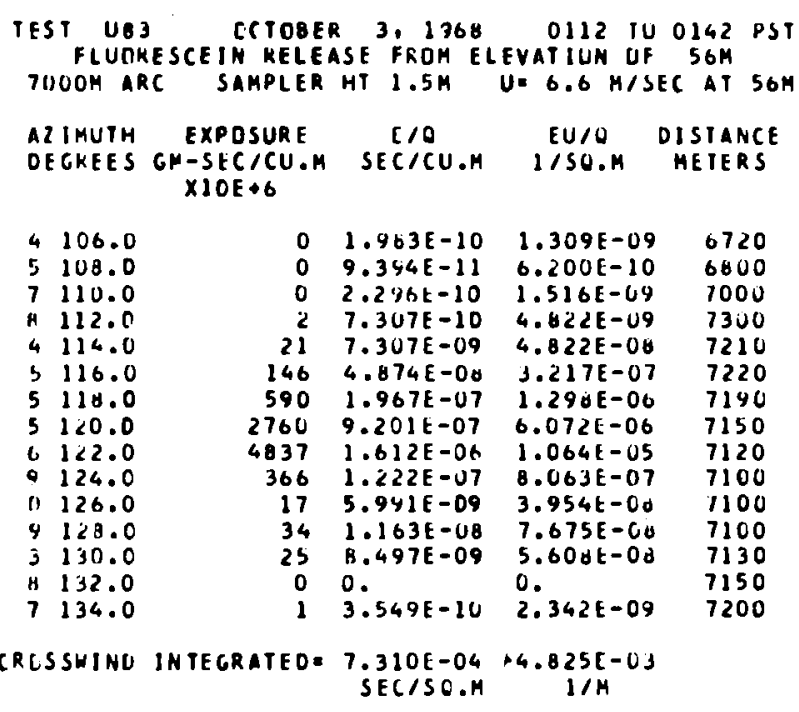


SAMPLINL 4OOM TO 12 UOOMI NO TOWER SAMPLING. ONLY ZNS TRACER WAS UISPERSED. NO TRACER IIASERVED DN GOOA DR GUOM ARCS. WIJH THE EXCEPIIUN UF IHE 12 OUJA AKC. ALL ARCS EXPUSEO TU TRACER EMBRACED THE ENTIRE CRUSSHINO OISIRILUTIUN.

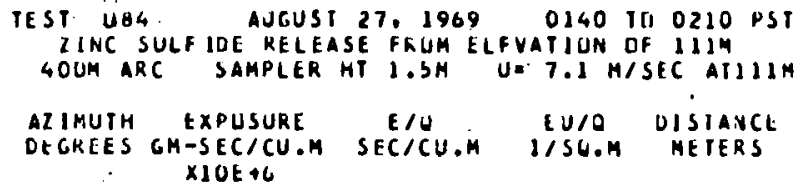

N 79.0 00.0 .400

CRUSSHINU INIECKATFOA U. SEC/SU.M U. I/M

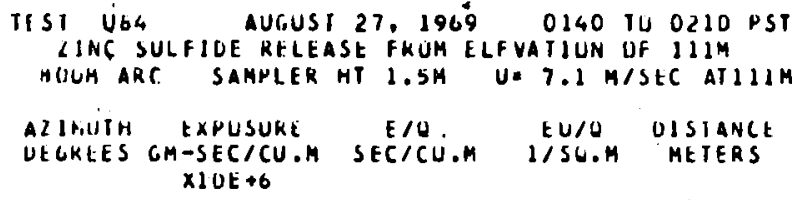




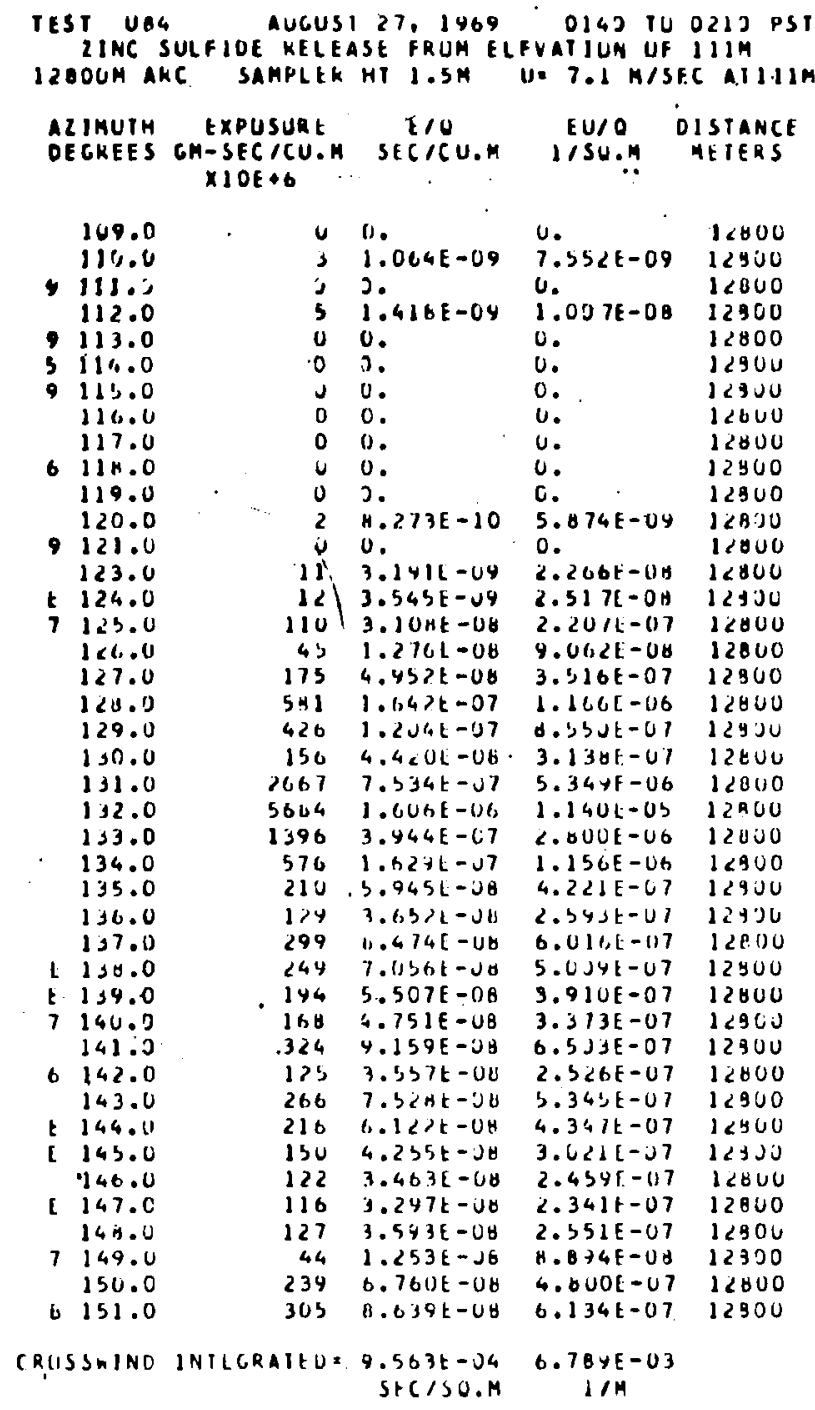




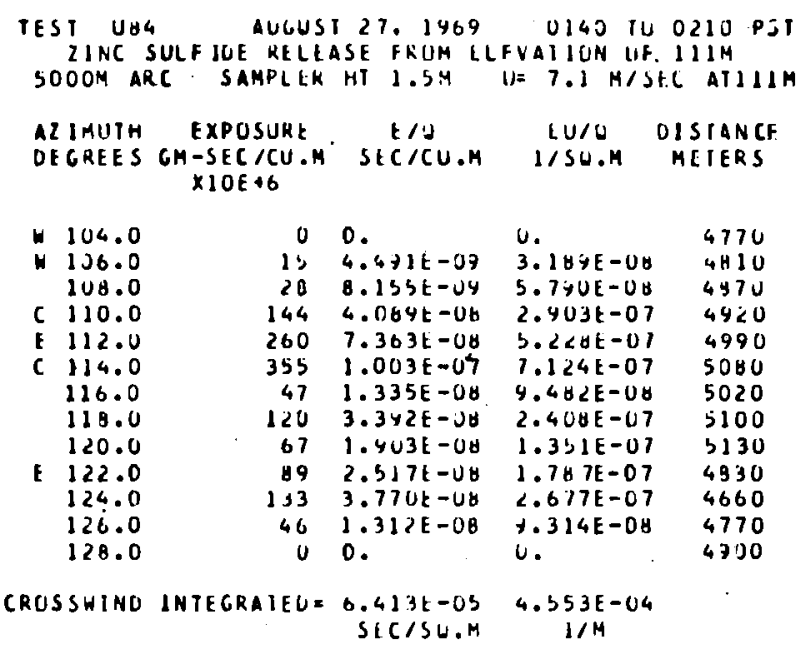

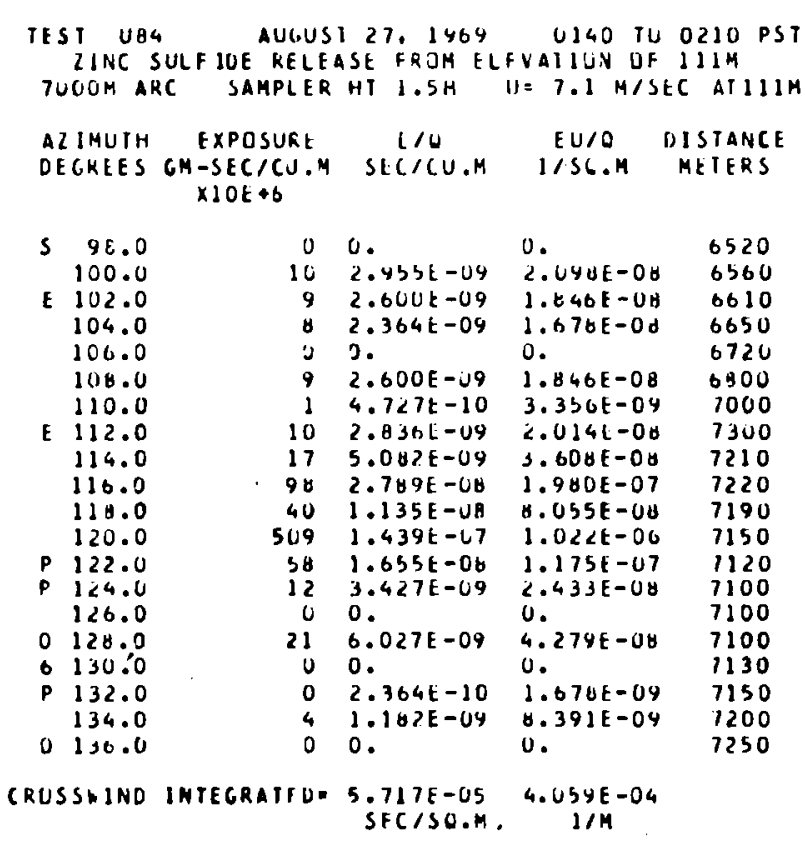


SAHPL ING 400 TO $12000 M$ N NO TOWER SAMPLING.

ALL ARCS EMBRACE CRUSSWINC EXIENT OF IRACER UISTRIBUTIONS. NO 2MS TRACER UBSERVEU UN 4 UOM ARC. SPURAOIC FLUIIRESCEIN DISPERSAL UIIRINE FIKSI IU \#IN OF TESTI DK UURING LAST 20 MIN.

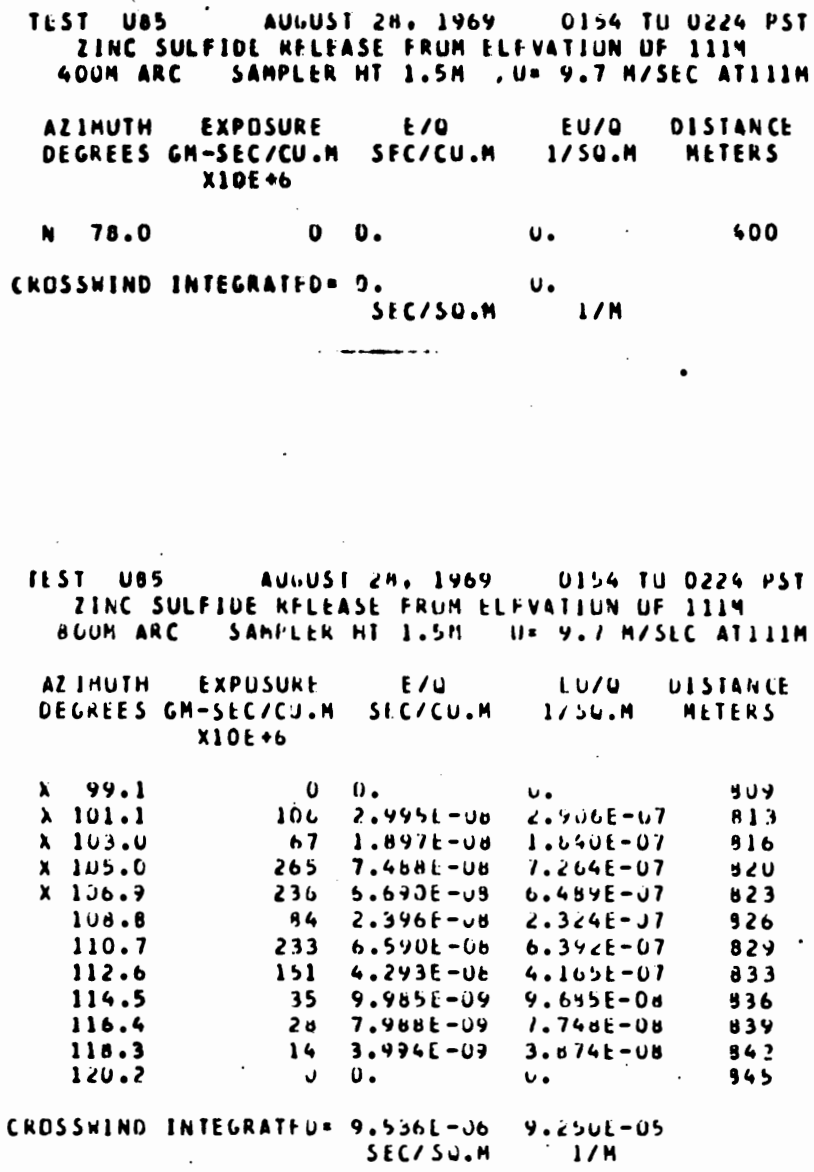

CROSSHIND INTEGRATHU: 9.SSGL-JO Y.ZSUE-US

$$
\text { SECISU.M TMULE }
$$




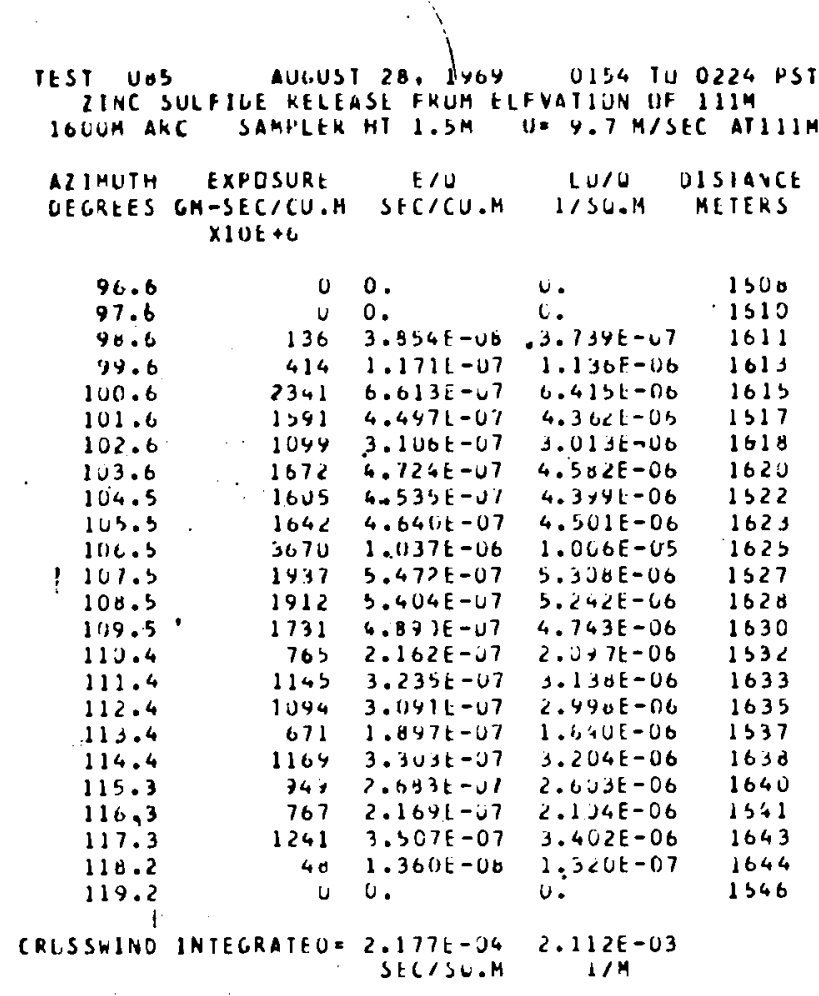

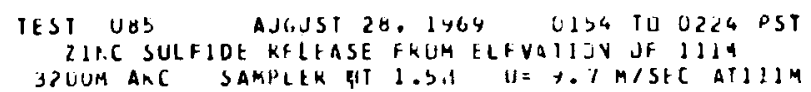

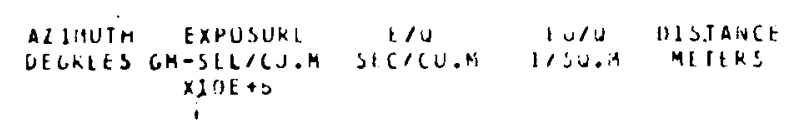

$+41.0 \quad 1$

\begin{tabular}{|c|c|c|}
\hline 41.0 & $\mathrm{u}$ & u. \\
\hline $9 y, 8$ & 13 & $9,5,41-1,4$ \\
\hline 44,8 & bo & 1 1. B sit $1-U B$ \\
\hline 160.6 & 330 & $y .3 j \cup \dot{E}-j \forall$ \\
\hline 101.9 & dB 7 & $2.5 v 2 t-v 7$ \\
\hline $1 \mathrm{~J} 2 \cdot 0$ & 1367 & $3.42 J E-J 7$ \\
\hline 103.0 & לנ & $3.117 \mathrm{t}-07$ \\
\hline 134.8 & $1<7 y$ & $3 . t .13 t-J 7$ \\
\hline lus.to & $\Delta U>Y$ & $5.7,31-07$ \\
\hline 106.8 & 2866 & 3. U पतt $-U 7$ \\
\hline $101 \cdot 3$ & $22 \pi 4$ & $5.3+7 E-\cup 7$ \\
\hline$\angle \operatorname{lin} \theta, \theta$ & $\angle U \backsim R$ & $5.7 B b L-67$ \\
\hline$l 1 \cup 9.7$ & $\therefore<40$ & $0 .$. oul $-\cup l$ \\
\hline 2110.7 & I†G & $5.0,57 \mathrm{~L}-\mathrm{Jl}$ \\
\hline 111.7 &.$<23$ & $t,<d<t-0 t$ \\
\hline 112.7 & 1804 & $3 . / 74 t-07$ \\
\hline 113.7 & $15 H B$ & $\because \because \forall \forall+E-U 7$ \\
\hline 114.6 & $97 \mathrm{~J}$ & $2.741 t-37$ \\
\hline 115.6 & 150 & $4.762 \mathrm{E}-\mathrm{UH}$ \\
\hline 116.6 & so & $1.6511-06$ \\
\hline 127.6 & 2 & $7.6740-10$ \\
\hline 115.6 & 0 & 0 . \\
\hline
\end{tabular}

CRDSSAIND INTEGRATLL: $4.0241-043.409 \mathrm{H}$-US

$\because 31120$ 4. $3111-0, \quad 3213$ 1215 Y.ubGt-U7 $\$ 217$ $2.432 \mathrm{E}-\mathrm{UE} \quad 3210$ 3. $J 3 E-J B \quad 3220$ 3. A. U Dt-116 3222 $3.3615-00 \quad 3225$ 1. $835=00 \quad 3227$ D. CUSE-IH 3229 $\therefore 613 t-06 \$ 230$ c.. (16, - 116 323 ? $3.46 / t-1,03233$ $0.0+31-06 \quad 3 \geqslant 35$ S. $1<$ it-UG 3231 4. $354 E-U 6 \quad 3230$ 2.1 $34 \mathrm{E}-U 7$ ? 124 1. 6 Cict-07 $324 j$ 7.444E-U 3245 $\begin{array}{ll}7.4445-27 & 3245 \\ 6 . & 3246\end{array}$

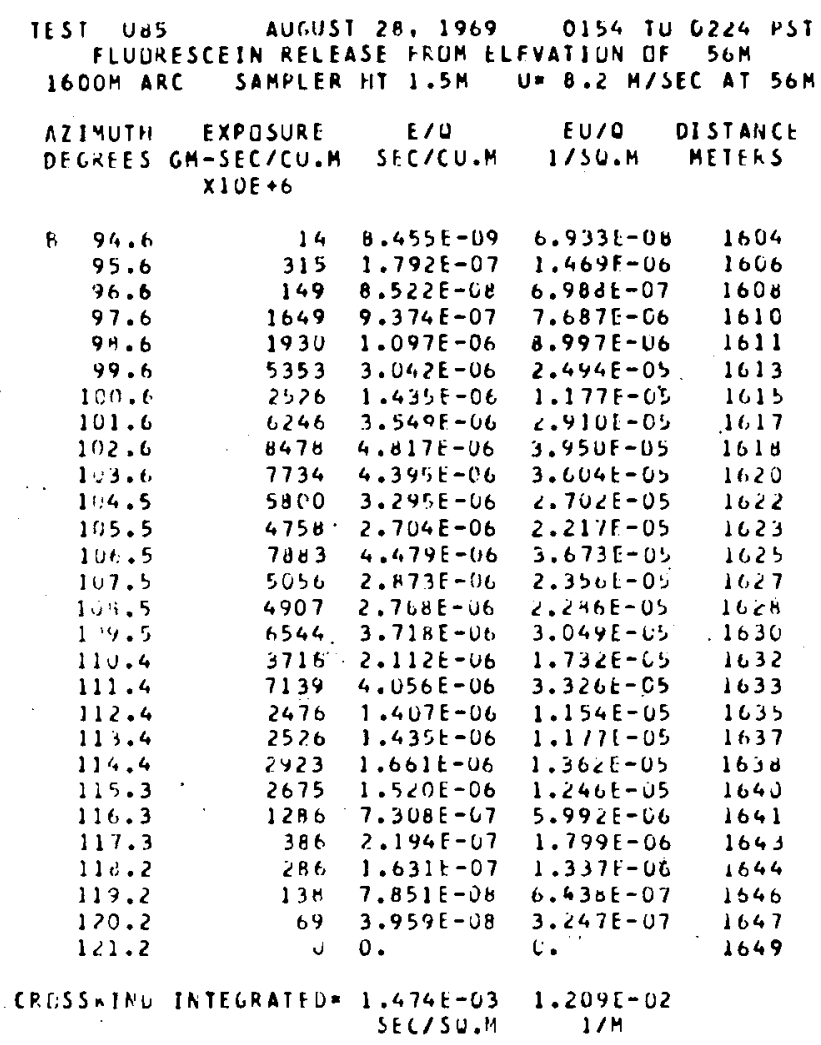

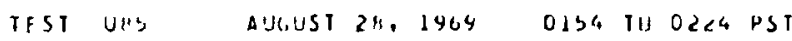

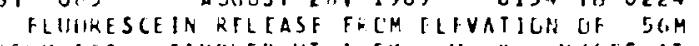
IOROM ARC SAMPLER HT I.SM UIS H.C MISLC AT DOM

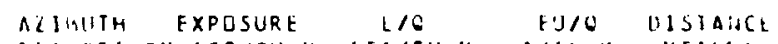
DHCKEES GM-SICICU.M SECICU.M I/SL.M MEILKS $X \perp C E+G$ SFC/SU.M I/Y

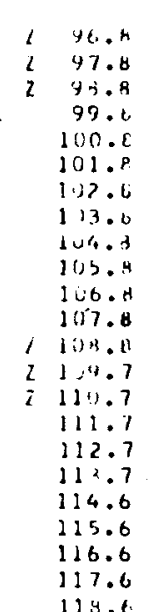

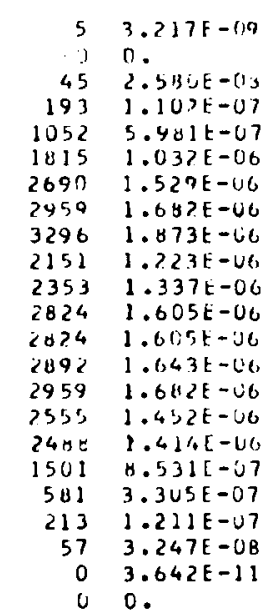

$2.6 ; 1+1,00$

2.11 1 SE- 7

2.113E-0.7

$4.90 S E-C E$ H. TS SUE-00 1. $253 \mathrm{E}-0 \mathrm{~S}$

1.37\%-13

1. $530 \mathrm{~F}-\mathrm{u}$

1.0U31-08

1. 0 y $7:-0$,

1. $316 E-0$

$1.3161-0,5$

$1.3401-2$,

1.37ye-us

1. $141 t-01$

1. $12,40,-03$

$6.4931-06$

c. $710 t-06$

$9.924 E-31$

$2.663 E-C 7$

$2.986 E-10$

0 .

3216

3: 12

513

$321 ?$
+217

करा

3720

$3<2<$

3224

ic 23

$12<7$

3224

1231

3251

3235

3235
3291

$1<34$

2240

3241

3243

3245

CKUSSWIND INTEGRATEDE 1.123E-03 4.207E-03

\author{
$M$
}

T 


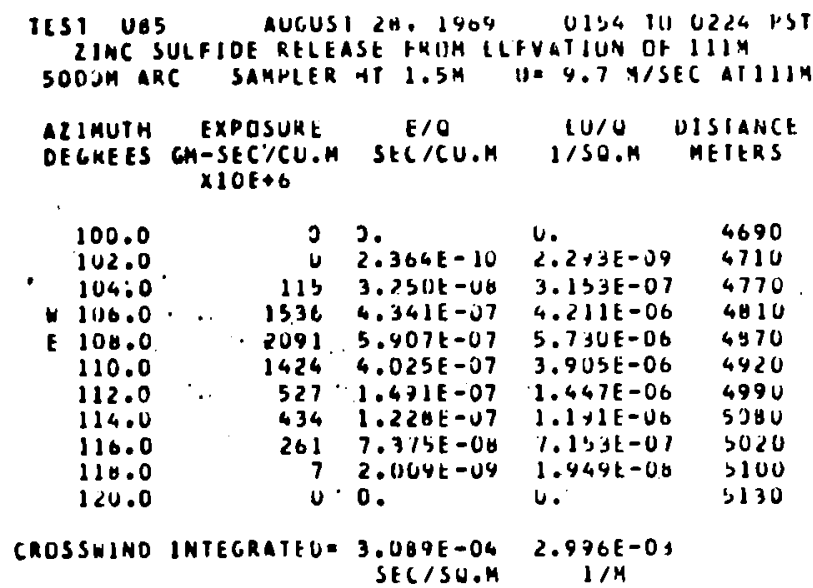

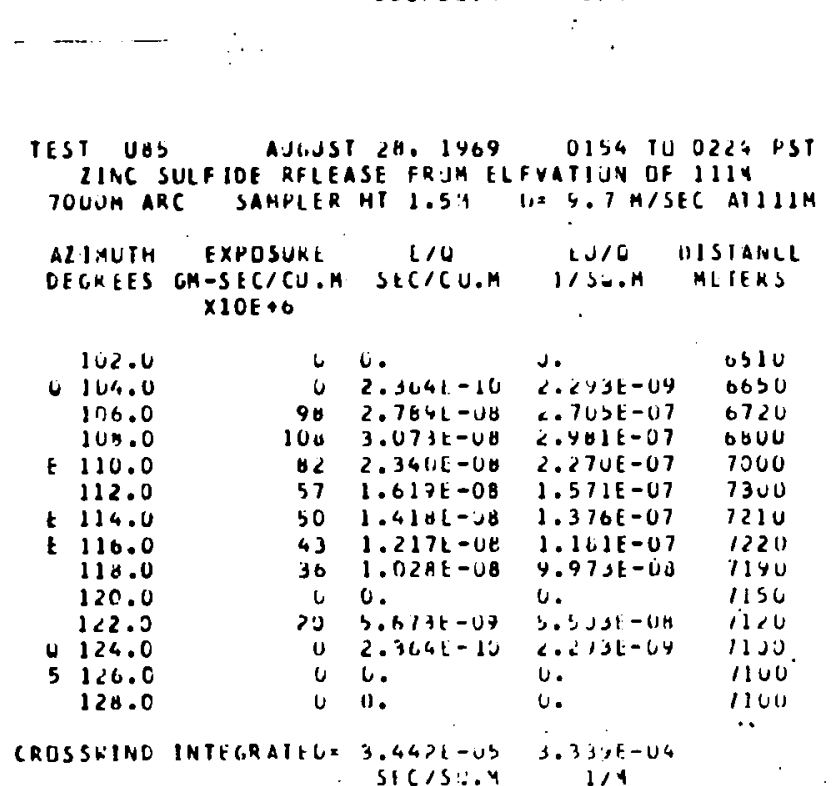

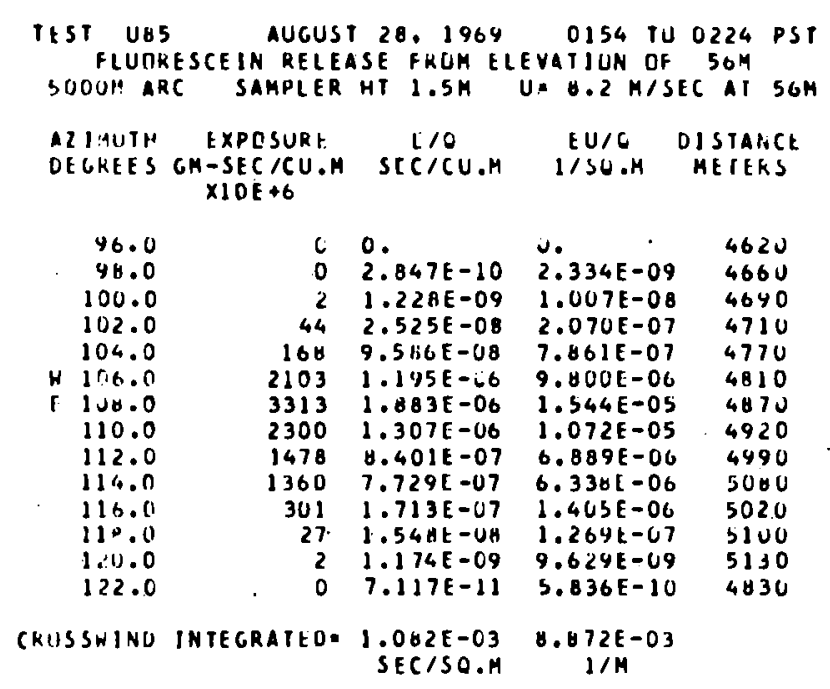

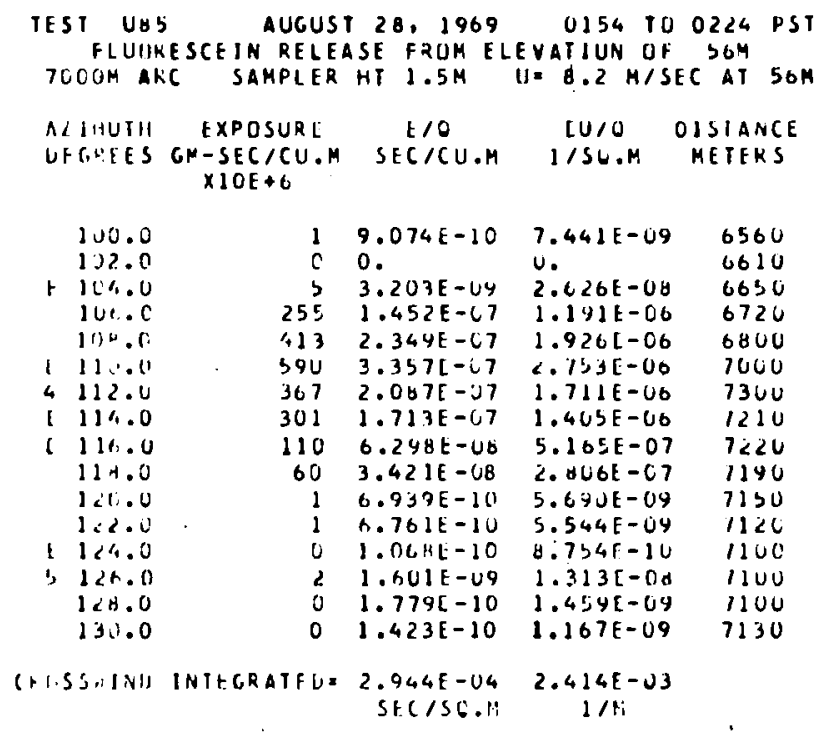

ISST UHS AUSUST 2 A. 1969 UI54 TU O2L4 PST

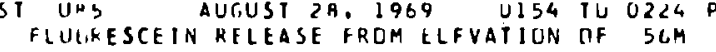
IBACOII ARC SAMFIER HT $1.5 \mathrm{M} U=8.2 \mathrm{M} / \mathrm{SEC}$ AT $56 \mathrm{M}$ AIIIUTH EXPOSURE E/O EU/O OISTAILE DEGRFES GM-SFC/CU.M SECICU.M 1/SO.M MEIETS $X \perp 0 E+6$

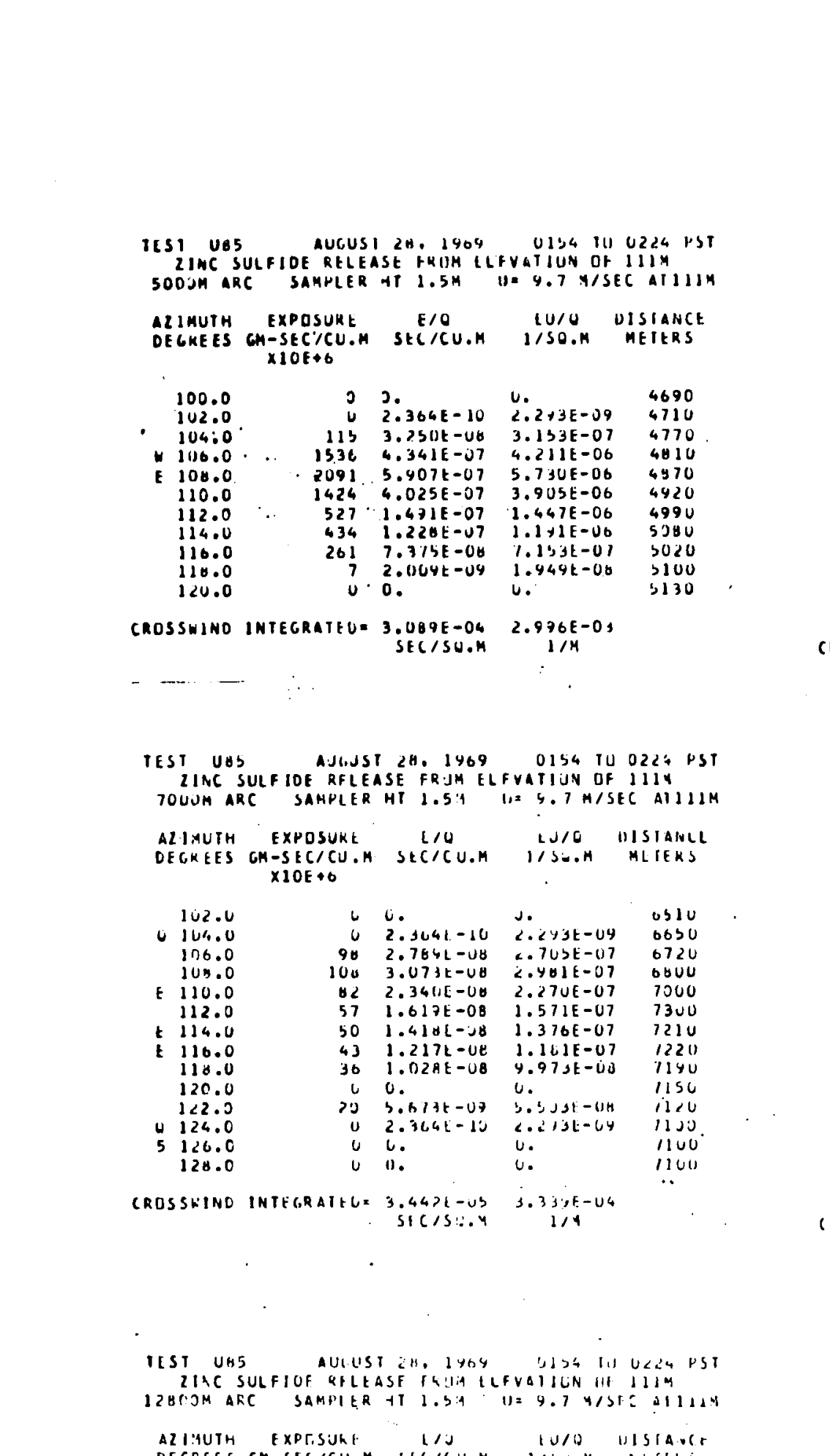

$\begin{array}{lllll}107.0 & 0 & 1.779 \mathrm{E}-11 & 1.459 \mathrm{E}-10 & 12800 \\ 101.0 & 0 & 4.804 \mathrm{E}-09 & 3.939 \mathrm{E}-06 & 12800\end{array}$

$1 J 4.0 \quad 7 \quad 4.270 t-093.502 \mathrm{t}-0 \mathrm{~B} \quad 12800$

$110.0 \quad 392.242 E-08 \quad 1 . E 3 H E-07126.10$

$111.0 \quad 114$ 6.7UHE-64 S.5UJE-67 1<甘G

$112.0 \quad 492 \quad 7.777 \mathrm{E}-07 \quad 2.213 \mathrm{E}-06 \quad 12600$

$113.0 \quad 275 \quad 1.564 \mathrm{E}-07 \quad 1.20<\mathrm{E}-100 \quad 12600$

$7114.0 \quad 518 \quad 7.946 E-67 \quad 2.416 \mathrm{~F}-60 \quad 12000$

$4+15.0 \quad 380 \quad 2.162 \mathrm{E}-07 \quad 1.773 \mathrm{E}-00 \quad 12000$

$\begin{array}{lllll}115.0 & 380 & 2.162 \mathrm{E}-07 & 1.773 \mathrm{E}-00 & 12000 \\ 110.0 & 367 & 2.087 \mathrm{E}-07 & 1.711 \mathrm{E}-00 & 12800\end{array}$

$117.0 \quad 143$ A.129E-08 6.666E-C.7 $12 \mathrm{H} U \mathrm{O}$

$H 11 H .0 \quad B 6 \quad 4.928 E-00 \quad 4.0416-07 \quad 12800$

$4119.0 \quad 7 \quad 4.440 \mathrm{E}-09 \quad 3.647 \mathrm{~L}-0 \mathrm{U} \quad 1<\mathrm{HOO}$

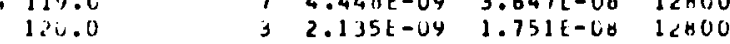

$4121.0 \quad 0 \quad 2.669 \mathrm{E}-102.180 \mathrm{E}-04 \quad 1280 \mathrm{U}$

$122.0 \quad 0 \quad 2.847 \mathrm{E}-10 \quad 2.334 \mathrm{E}-\mathrm{U} 4 \mathrm{Y} 12800$

$123.0 \quad 0 \quad 1.06+10-100.754 F-1 \mathrm{~J} 12800$

CRLSSHIND INTEGRATFO. 3.110E-04 2.55UE-03 


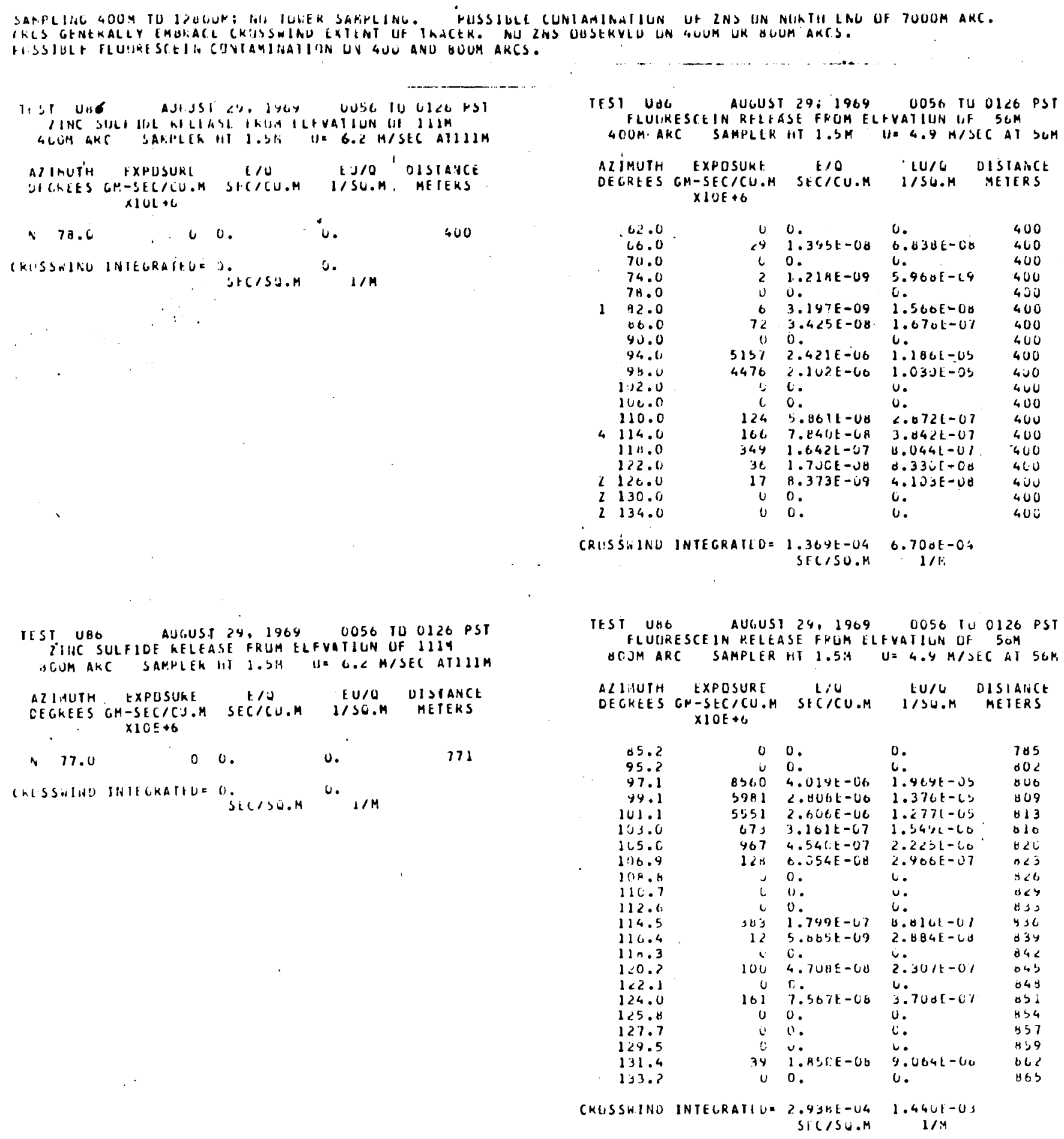




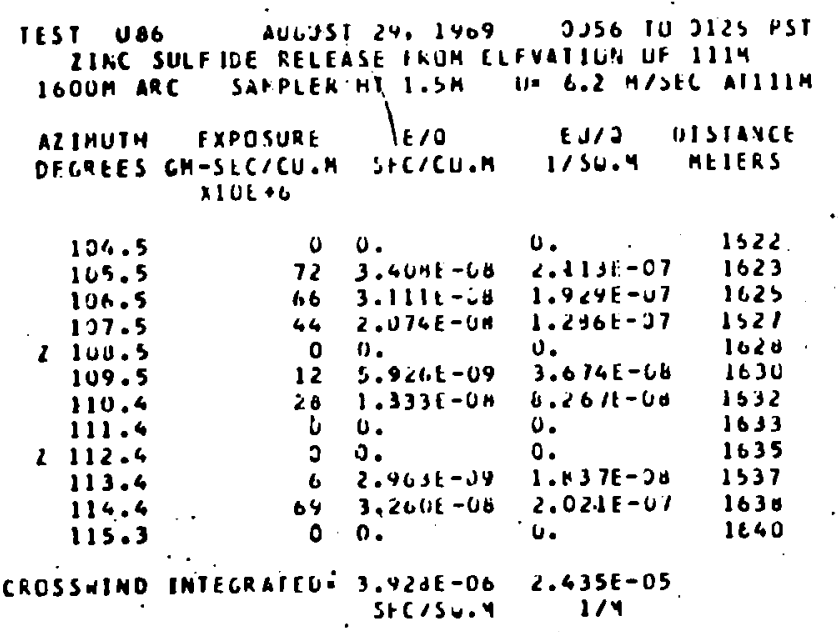

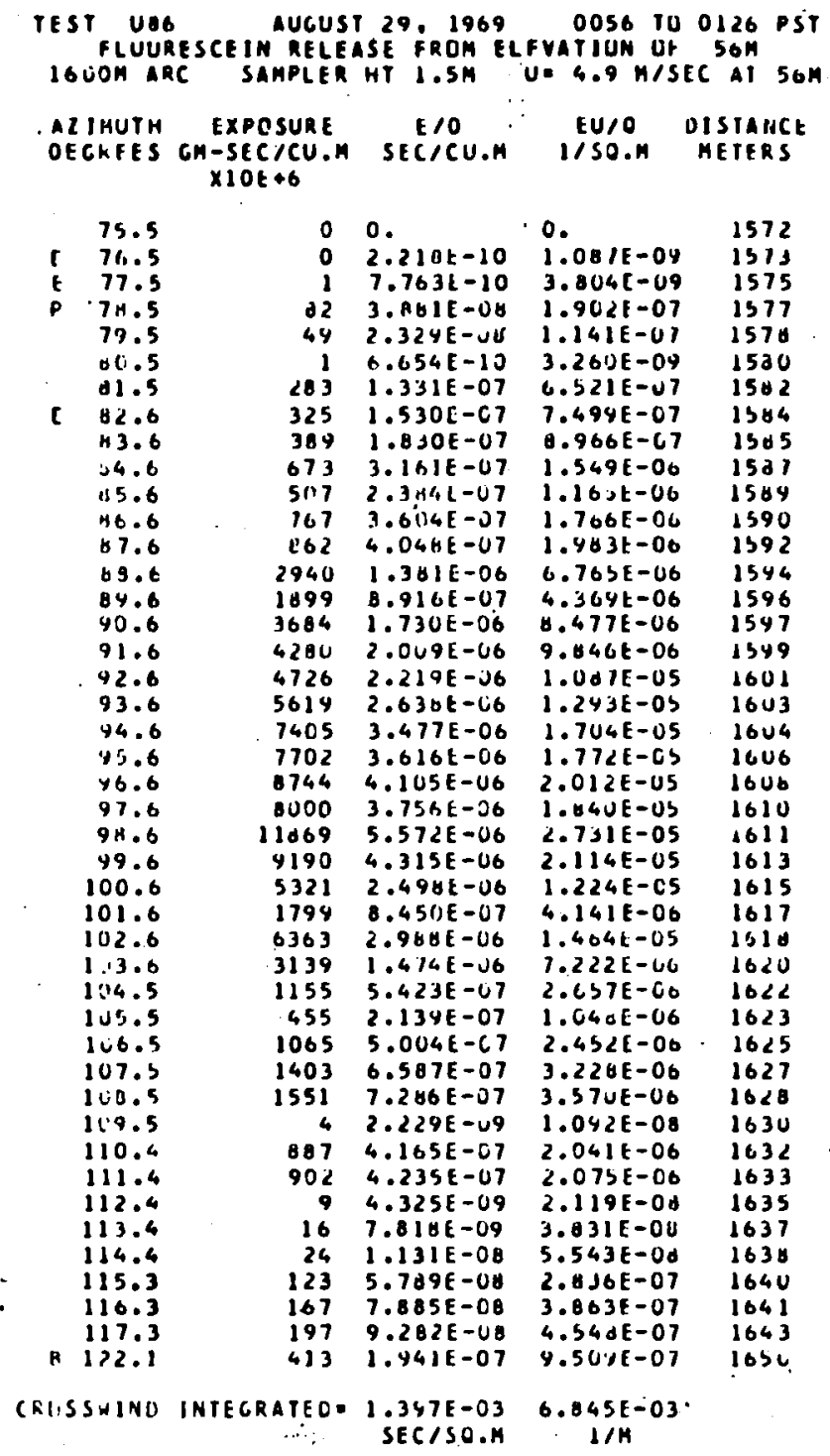




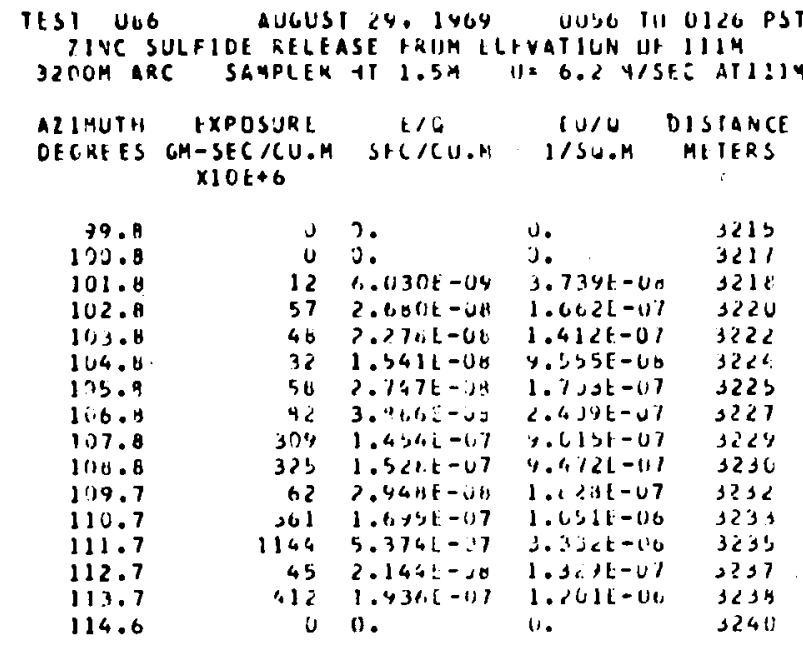

CROSSNINO INTEGRATILE 7.7,OT-JE 4.7IGL-U4

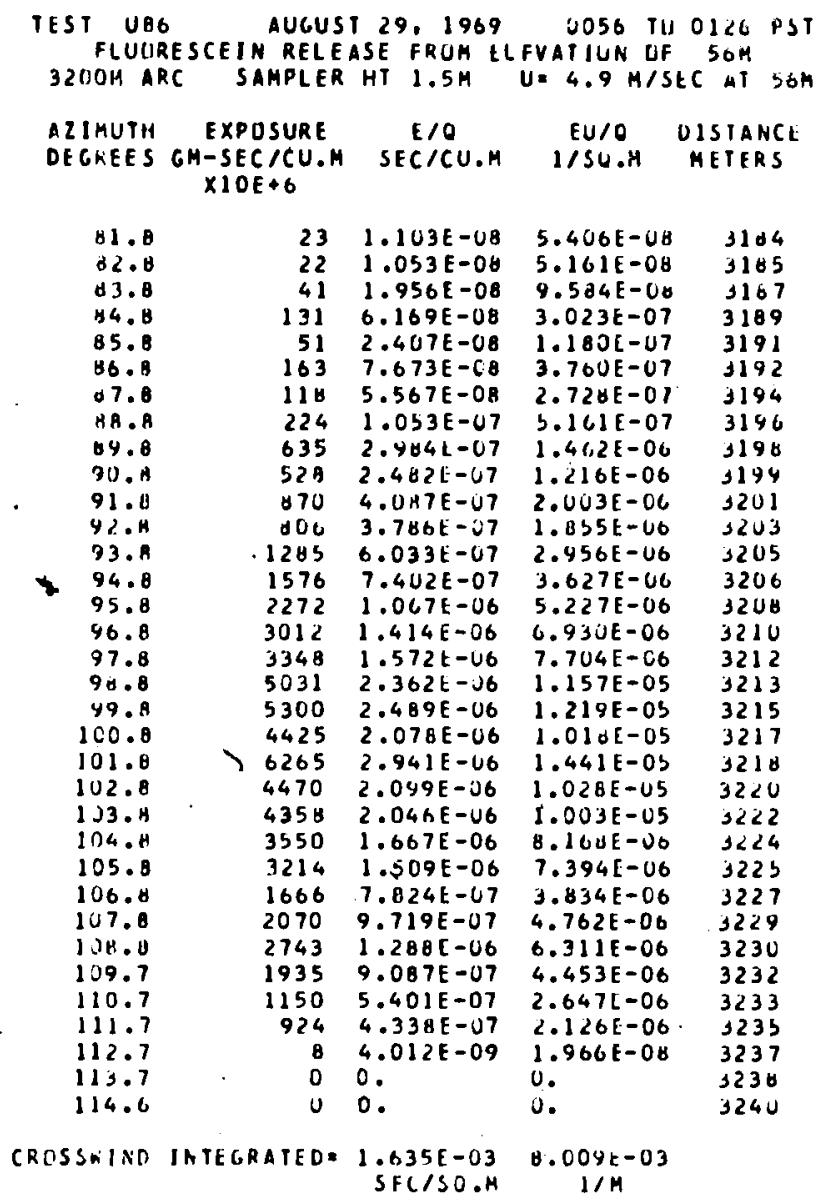

TEST UB6 AUGUST 29.1969 0056 TU 01 16 PST FLUIIRESCEIN RELEASE FROM ELFVATIUN UF 564 5 UOU. AKC SAMPLER HT $1.5 \mathrm{H}$ UE $4.9 \mathrm{M} / \mathrm{SEC}$ AT $56 \mathrm{H}$

AZIMUTH EXPOSURL E/W - EU/O UISIAISCL DECKEES CM-SEC/CU.M SEC/CU.M J/SU.M MEILKS $X 10 E+6$

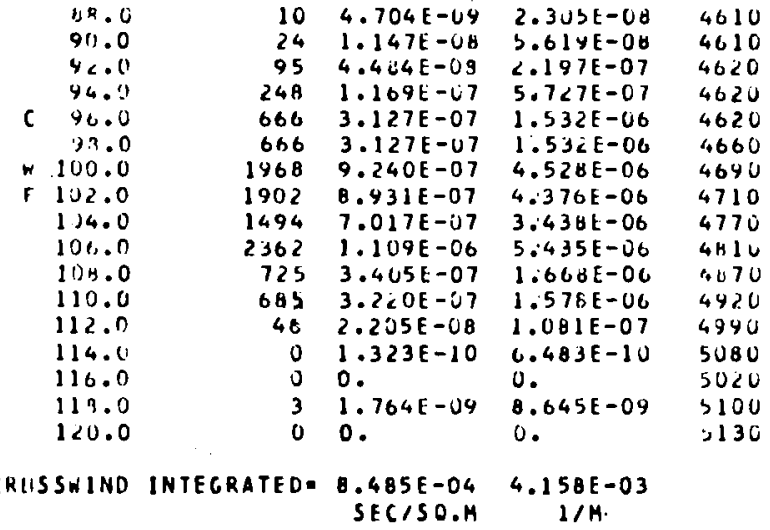

CRUSSWIND INTEGRAIIUE, ?.90\%t-US, $1.770 t-04$

$$
\begin{aligned}
& 450 \\
& +2<11 \\
& \text { is } \\
& \begin{array}{l}
4060 \\
4590
\end{array} \\
& 4710 \\
& 477 \\
& 4+10 \\
& 4010 \\
& 4+21 \\
& 4495 \\
& 201 \\
& \text { Jid } \\
& \text { गu } \\
& \text { it } 30
\end{aligned}
$$


TEST U66 AUCUST 29.1969 0056 TU OLS DST ZINC SULFIDE RELEASE FRON ELFVATIUN OF IIIM 70.0OM ARC SAMPLER HT $1.3 \mathrm{SH} 11=6.2$ H/SEC ATI11A AZINUTH FXPOSURE
DEGRESS CH-SECICU.M SECICU.M IISO.M METERS $\times 1 O E \bullet 6$

76.0
71.0
80.0
82.0
86.0
16.0
48.0
90.0
92.0
94.0
96.0
98.0
100.0
102.0
104.0
106.0
104.0
110.0
112.0
114.0
116.10
118.0
120.0
122.0
124.0
126.0

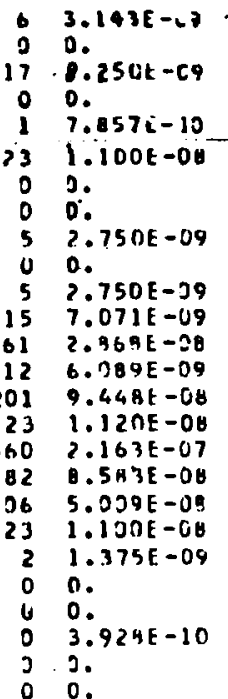

$871 \mathrm{E}-89$

$0.0201-00$ 0.

1. 7U5E-08

1.705E-0. 4. $384 \mathrm{E}-0 \mathrm{~B}$ 1.77 yE -07 $3.775 E-39$ 5. BS dE- 07 $6.9411-06$ $1.341 \mathrm{E}-06$ $5.32<E=07$ $3.1055-07$ 6. $52 U E-O D$ 8. $525 E-09$

$2.436 E-09$ 0.

s.

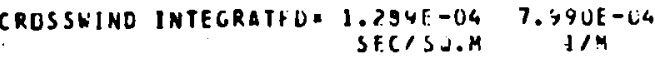

TEST UEG AUTUSI 24, 1464 C056 T1, 0126 PST 7 INC SULFIDE KILLASE FKIIM LLIVATIUI IJF IIIM $1 \angle B O O H$ ARC SAMPLIR III $1 .: M$ U. 6.2 MISIC AIIIIH

AZIHUTK EXPUSULE E/S LU/O UISTIVCL DEGREES GM-SFCICU.M SICICU.H I/SO.H HETEKS $\times 10[46$

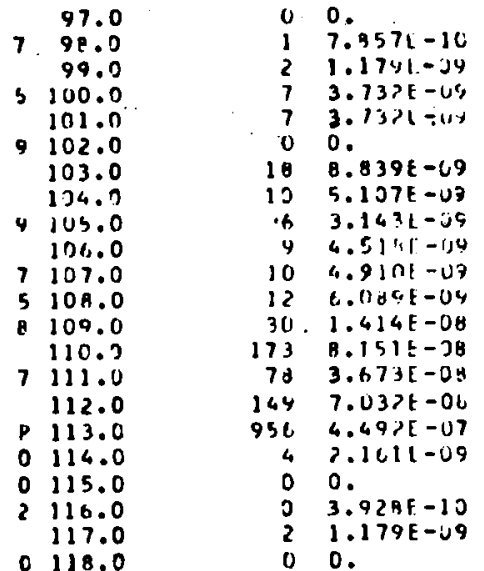

12900

4.8710.09 128001

$2.314 E-08 \quad 12860$

$\therefore .316 E-11312800$

$5.4 B U E-0 B \quad 12900$

$3,1,6 E-C D 12820$

$1.9180-4812420$

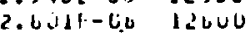

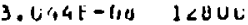

$3.775 E-U B$ l $\angle 30 \mathrm{O}$

$8.768 E-06 \quad 1<300$

$5.484 E-U 7 \quad 12300$

$2.277 \mathrm{E}-J 7$ 125 JU

$4.260 E-071<800$

$2.785 E-60 \quad 1 \angle 400$

$1.3401-001290 \mathrm{C}$

$2.436 E-19 \quad 12900$

$7.357 E-J>129 J C$

0 .

12800

CROSSWINA INTEGRATIO= $1.553 t-069.663 E-04$

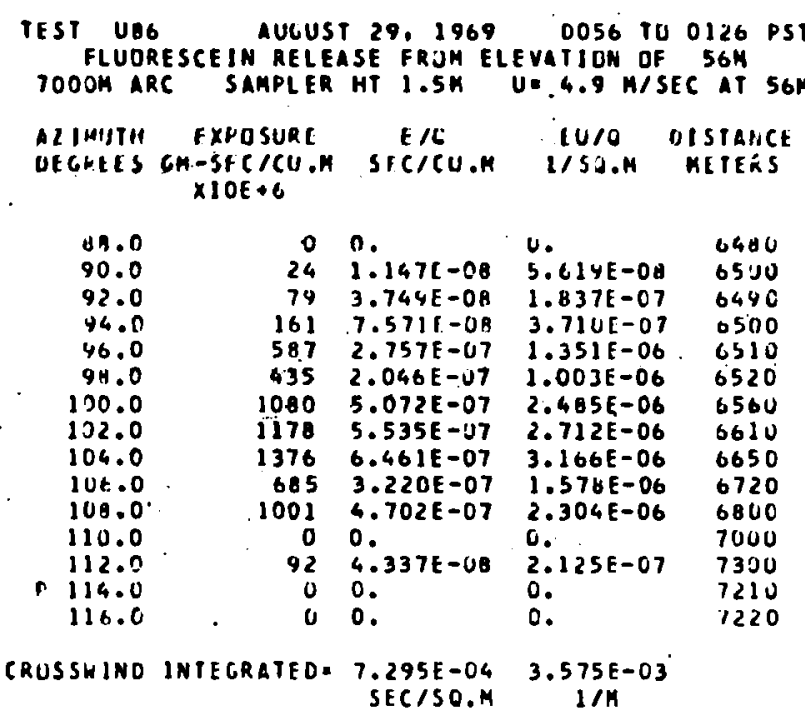

IEST UEG. AUGUST 29,1909 UOSB TO 0126 PST FLUDKESCEIN RELHASE FRUM ELEVATIUN OF S6M

12QCIA ARC SAMPLER MT $1.5 \mathrm{M} \quad 11=4.9 \mathrm{HISEC}$ AI $56 \mathrm{H}$ DEGKEES GH-SECICU.H SECICU.M I/SOAR METERS $\times 10 E+6$

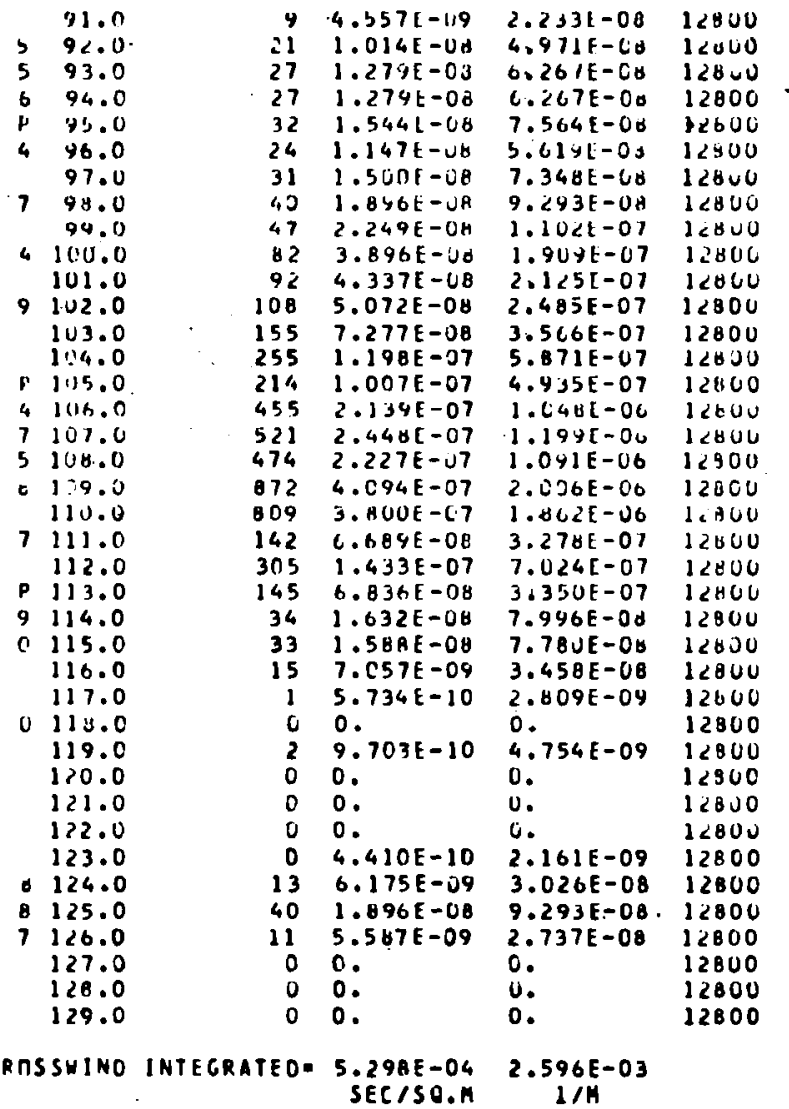




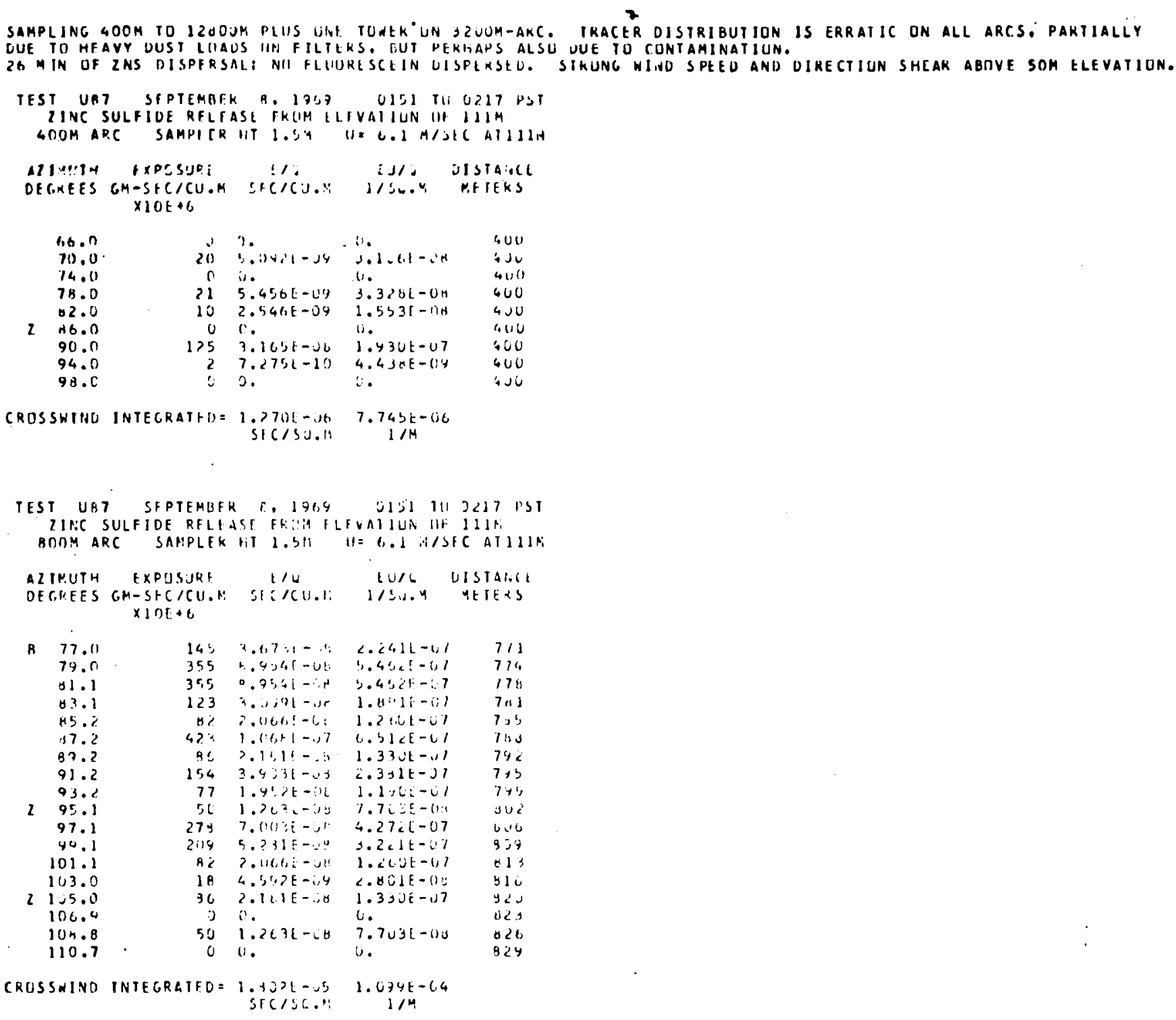




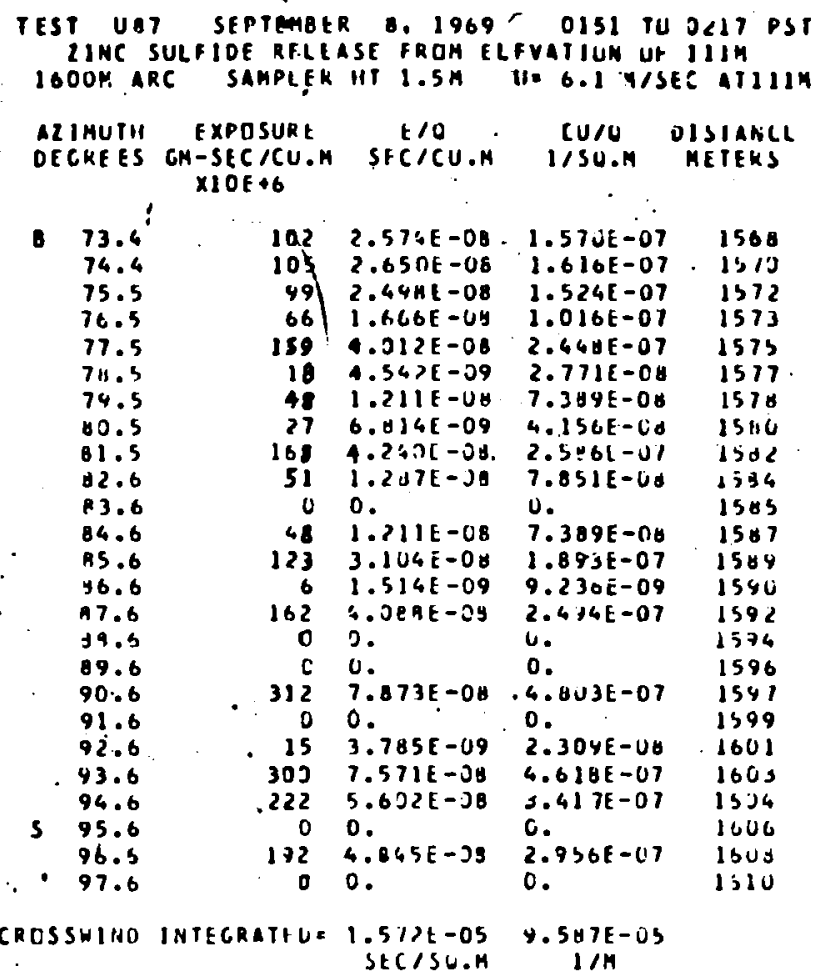

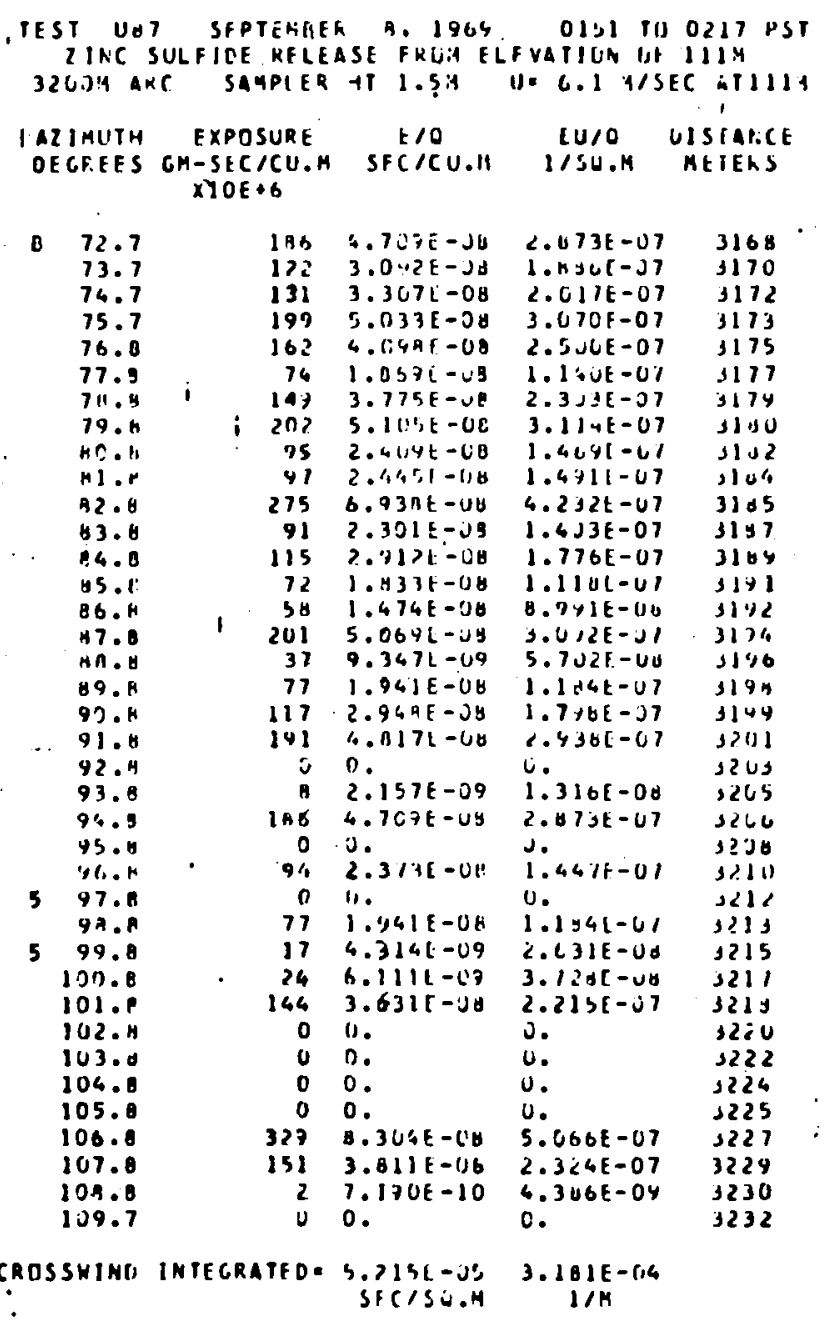




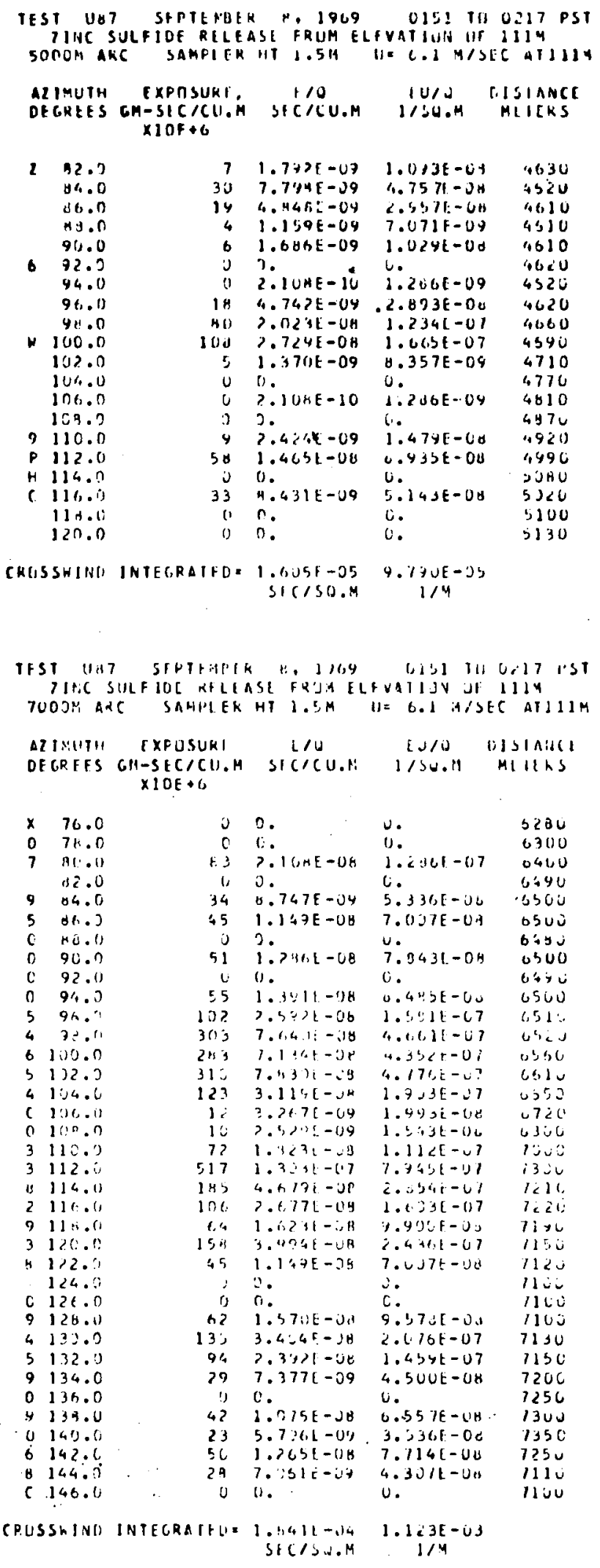




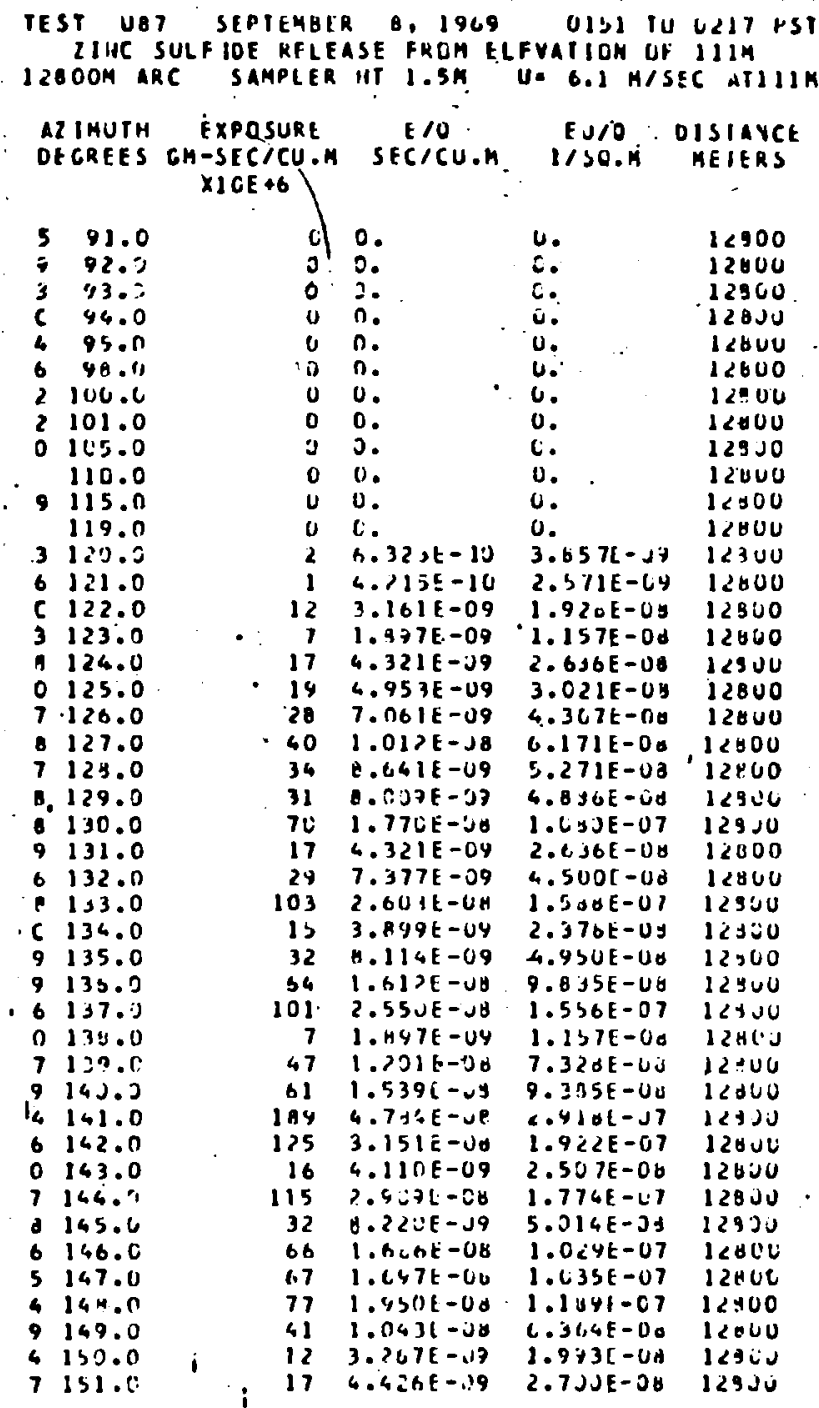

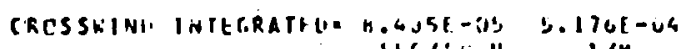

StC $131 ; .11 \quad 1 / \mathrm{M}$

\footnotetext{
TOHER CATA FOLLON....
TEST UBT SAPTEHOTK $A, 1469$ OIDI TU $0<17$ UST -2INC SULFIOE RELEASE TKOTY CLFVATIUH III IIIM 3200N ARC! Je C.I M/SIC AtIIII
}

AZIFHTH HE ICHIT EXPOSURF F/O EU/U JISPAHCL

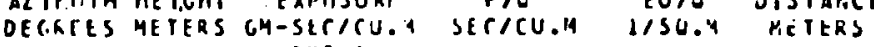
alUE +h

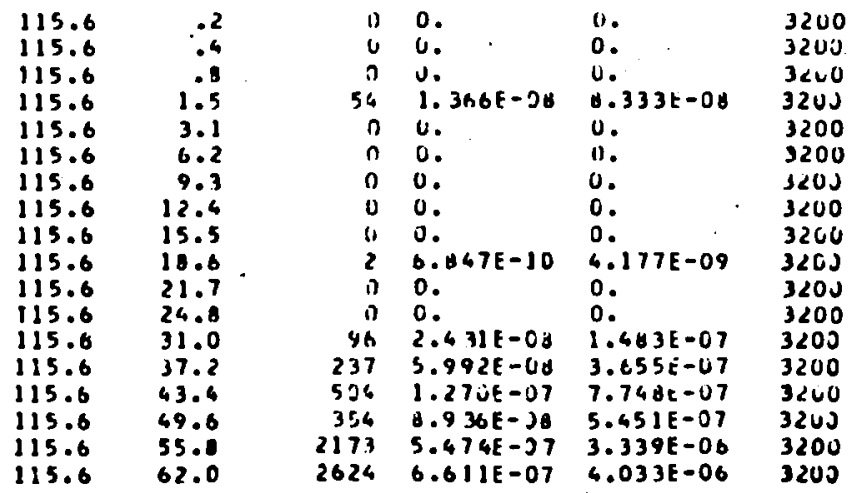




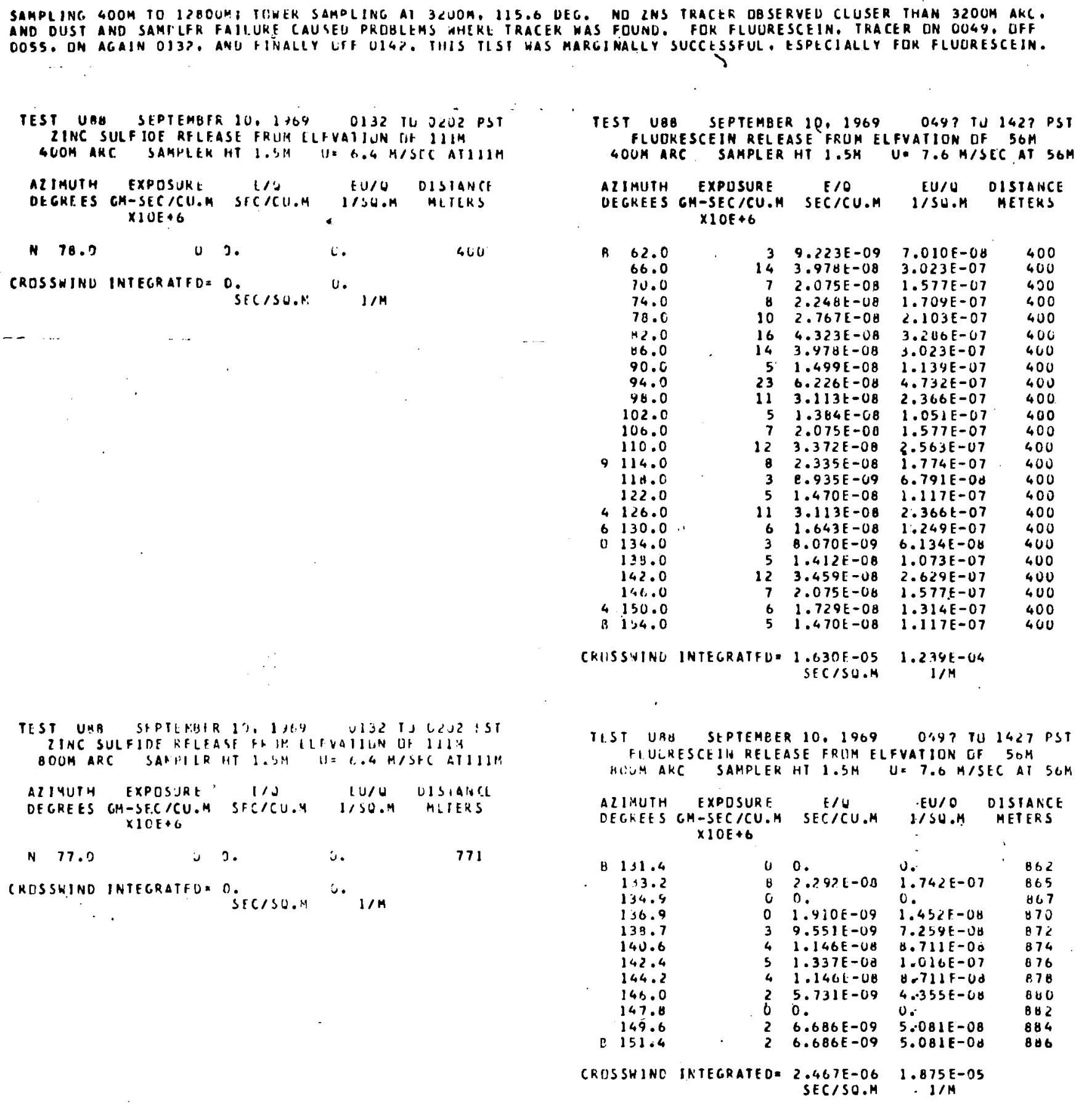




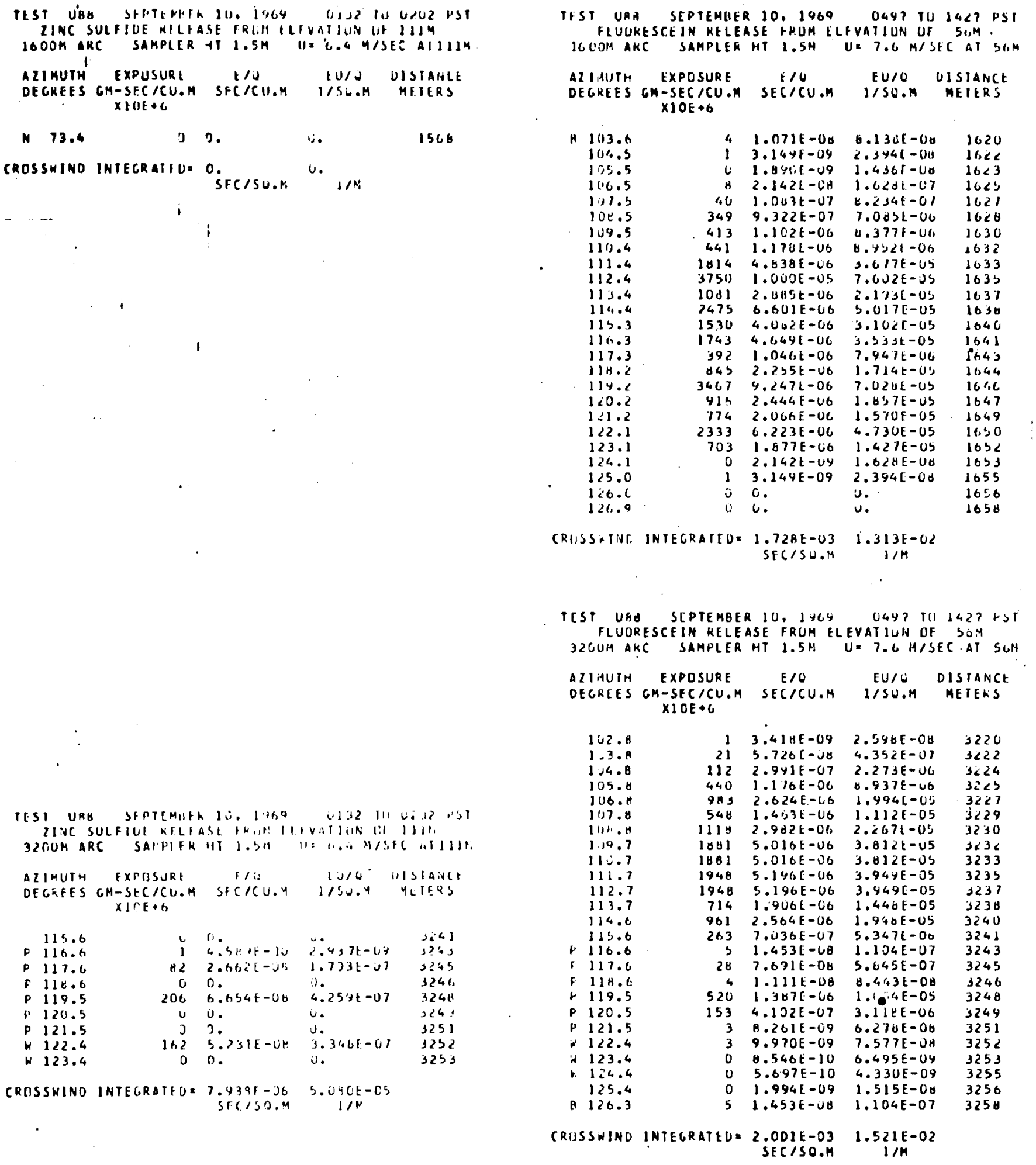




\begin{tabular}{|c|c|c|c|c|}
\hline $\begin{array}{l}\text { ALIMUTH } \\
\text { OE GREES }\end{array}$ & $\begin{array}{l}\text { EXPOSURE } \\
\text { GP-SEC/CU.M } \\
\text { XIOE }+6\end{array}$ & $\begin{array}{c}E / O \\
S E C / C U . M\end{array}$ & $\begin{array}{c}E U / O \\
1 / S O . K\end{array}$ & $\begin{array}{r}\text { OISTANC } \\
\text { METERS }\end{array}$ \\
\hline $\begin{array}{l}118.0 \\
120.0 \\
122.0 \\
\\
124.0 \\
4126.0 \\
7128.0 \\
1130.0\end{array}$ & $\begin{array}{r}0 \\
125 \\
26 \\
0 \\
46 \\
0 \\
0\end{array}$ & $\begin{array}{l}0 . \\
4.022 E-08 \\
8.610 E-09 \\
0 . \\
1.493 E-08 \\
0 . \\
0 .\end{array}$ & $\begin{array}{l}0 . \\
2.574 E-07 \\
5.510 E-06 \\
0.5 \\
4.557 E-08 \\
0 . \\
0 .\end{array}$ & $\begin{array}{l}5100 \\
5130 \\
4830 \\
4660 \\
4770 \\
4900 \\
4970\end{array}$ \\
\hline
\end{tabular}

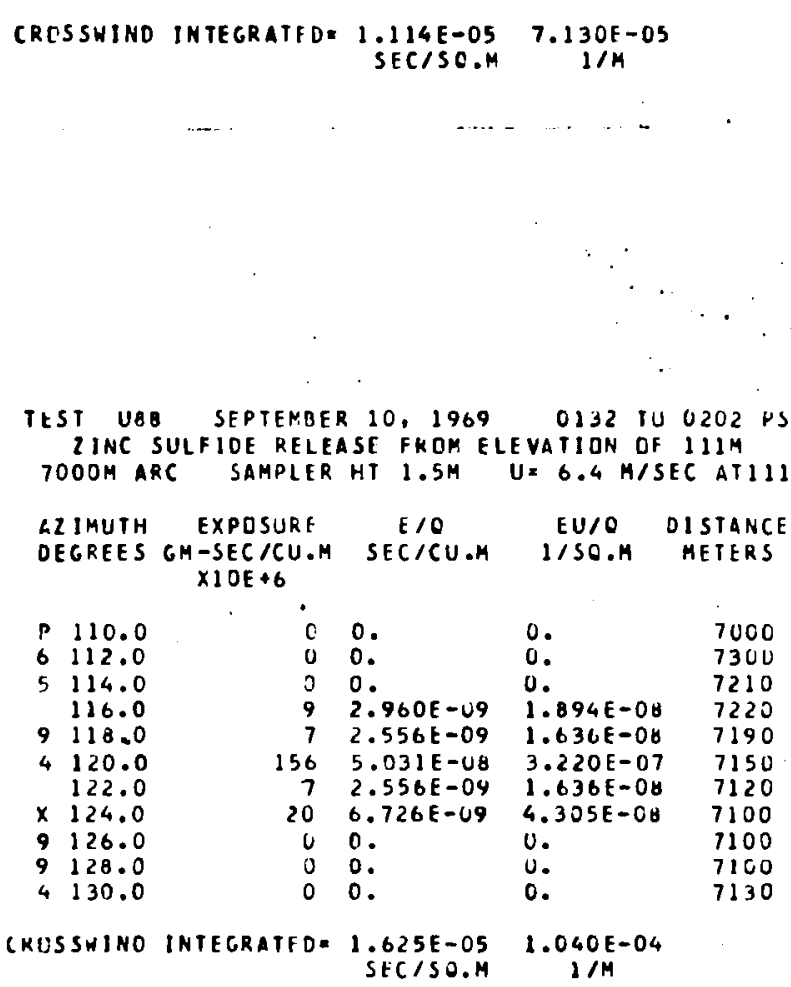

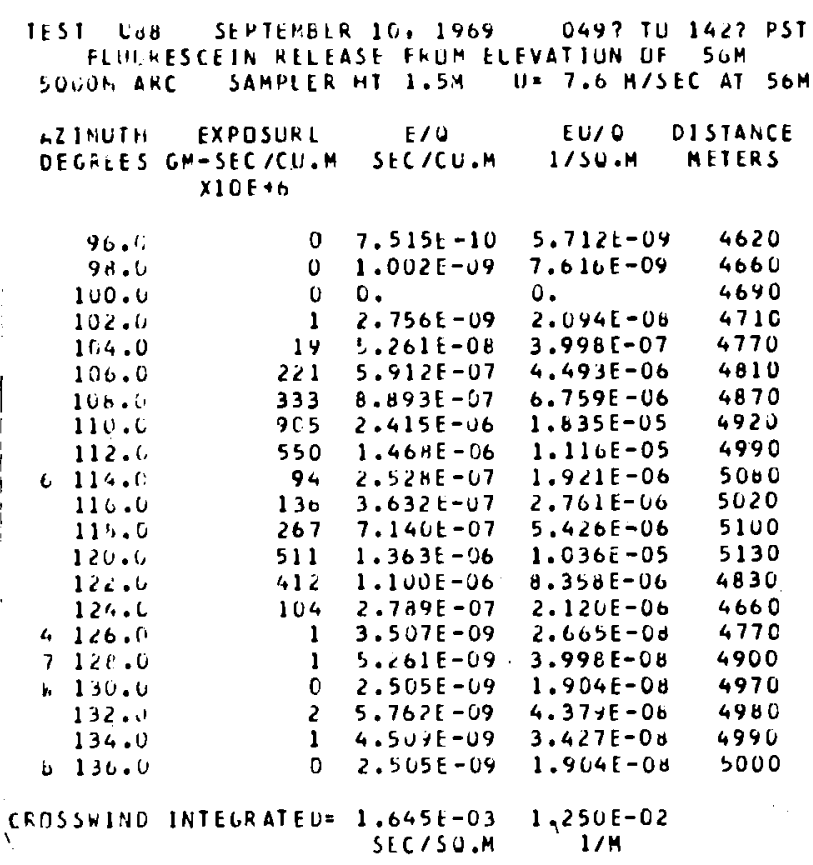
ITST LAB SEPTENBER 10, 1969 G4Y? TU 142? PST FLUIJRESCEJiN KELEASE FKOM ELEVATIUN UF $50 M$ 7 JUUH ARC SAPIPIER HT $1.5 \mathrm{M} U=7.6 \mathrm{M} / \mathrm{SEC}$ AT $50 \mathrm{M}$
AZLIKTH EXPUSUKE EIO EUID UISTANCE DEGREE CM-SECICU.M SECICU.M IISO.M MEIERS $\times 10 \mathrm{O}+6$

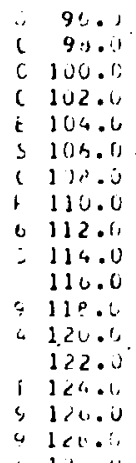

(.) 130.1
CPISSIINE, INTECRATID=9.957F-U4 $5+C 156.14$

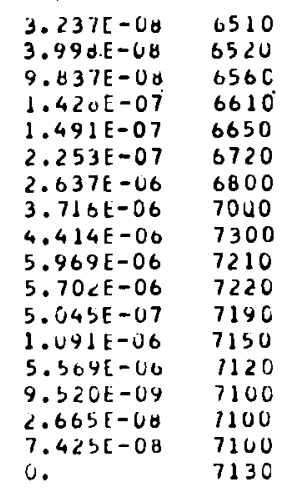

0.

$7.5605-03$

$1 / 4$ 


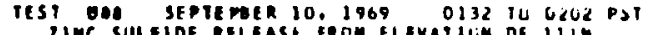

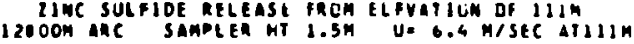

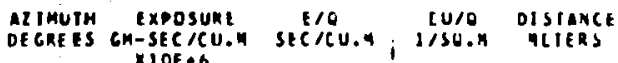
$\times 1$ OE $\rightarrow 6$
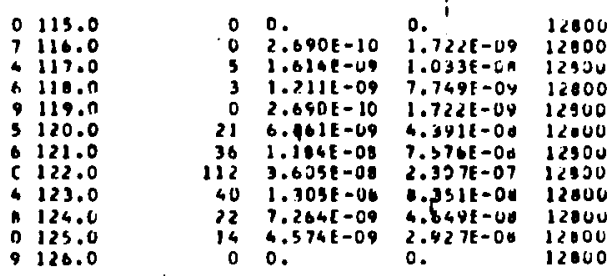

CRUSSHIMO IMTEGRATEO 1.A54E-05 1.197E-06 :

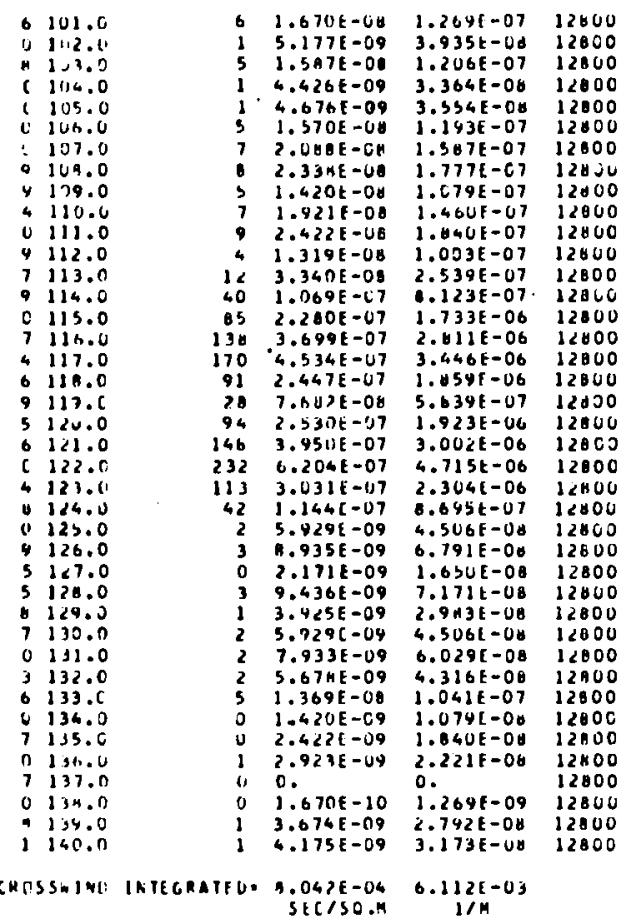

TOWER DATA FELLEW....

TEST UEA SEPTEMBER 10, 1969 O49? TU 142? PST FLULDESCEIN RELEASE FROY ELFVATIUN UF $50 \mathrm{BH}$

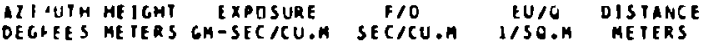
$x \quad x=A$

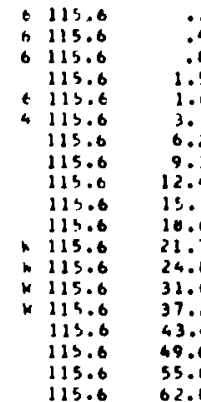

115.6
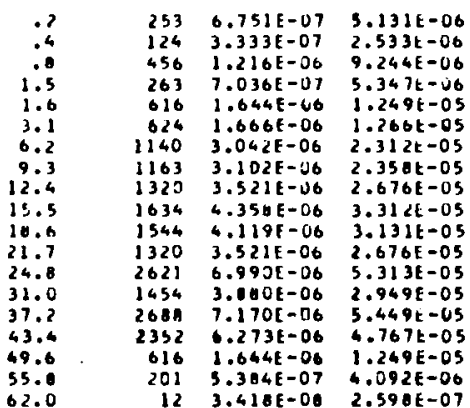

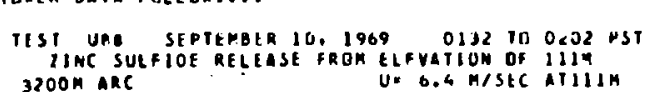

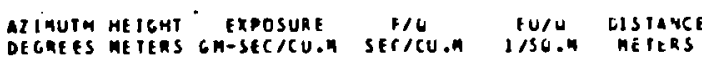
$15 E \circ 6$

6113.6
6115.5
6115.6
6113.6
4115.6
115.5
115.6
115.6
115.6
115.6
115.6
119.6
115.6
113.6
113.6
115.6
115.6
115.6

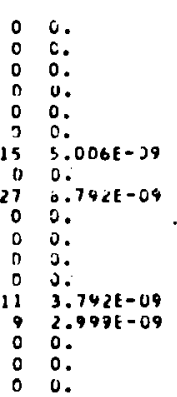

$n$.
i.
i.
i.

3<2 $3<0$

1.. 3000

$3.2345-30$

$3<i j$

S.edit-ly $3<00$

0 .

$3.427 t-c \theta$

$1.919 t-6 t$

6.

0. 


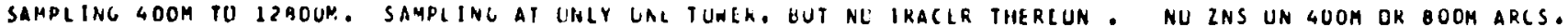
ZIKC SULFIDE TRACER EAHRACIU BY CKIISSWINU EXTENT IIF AKCS. FLUUKESCEIN CRUSSTINU UISIKIBUTIUNS SEEM IU EXTEND CGF EUGES OF ARCS FREGUENIIY. HUT CRNTAMIHATIUN IS POSSIBILITY. HEITHER IRACER OBSERVED AT ILBOOA ARC.

\begin{tabular}{|c|c|c|c|c|}
\hline $\begin{array}{l}\text { TEST UU9 } \\
\text { IINC SL } \\
\text { ANOH ARC }\end{array}$ & $\begin{array}{l}\text { SEPTEMUER } \\
\text { SULF IDE RELEA } \\
\text { C SAMPI.ER }\end{array}$ & $\begin{array}{l}11,1069 \\
\text { ISE FRUM IL } \\
\text { HI } 1.3 \mathrm{HA}\end{array}$ & $\begin{array}{l}0110 \\
\text { FVATIUN } \\
\text { lI= 3.6 H }\end{array}$ & $\begin{array}{l}\text { TS C148 PST } \\
\text { AF } 1114 \\
\text { ISF } 4 T 111 \mathrm{M}\end{array}$ \\
\hline $\begin{array}{l}\text { AlIMUTH } \\
\text { DEGRES }\end{array}$ & $\begin{array}{c}\text { EXPOSURE } \\
\text { CM-SFC CU.M } \\
\times 10 F+6\end{array}$ & $\begin{array}{c}{[/ U} \\
\text { SHC/CU.A: }\end{array}$ & $\begin{array}{l}\operatorname{LU/0} \\
\text { l/SG.M }\end{array}$ & $\begin{array}{l}\text { U) SIANCE } \\
\text { MEIERS }\end{array}$ \\
\hline 78.0 & 0 & 0. & 0. & 400 \\
\hline
\end{tabular}

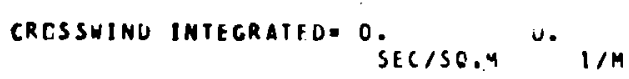

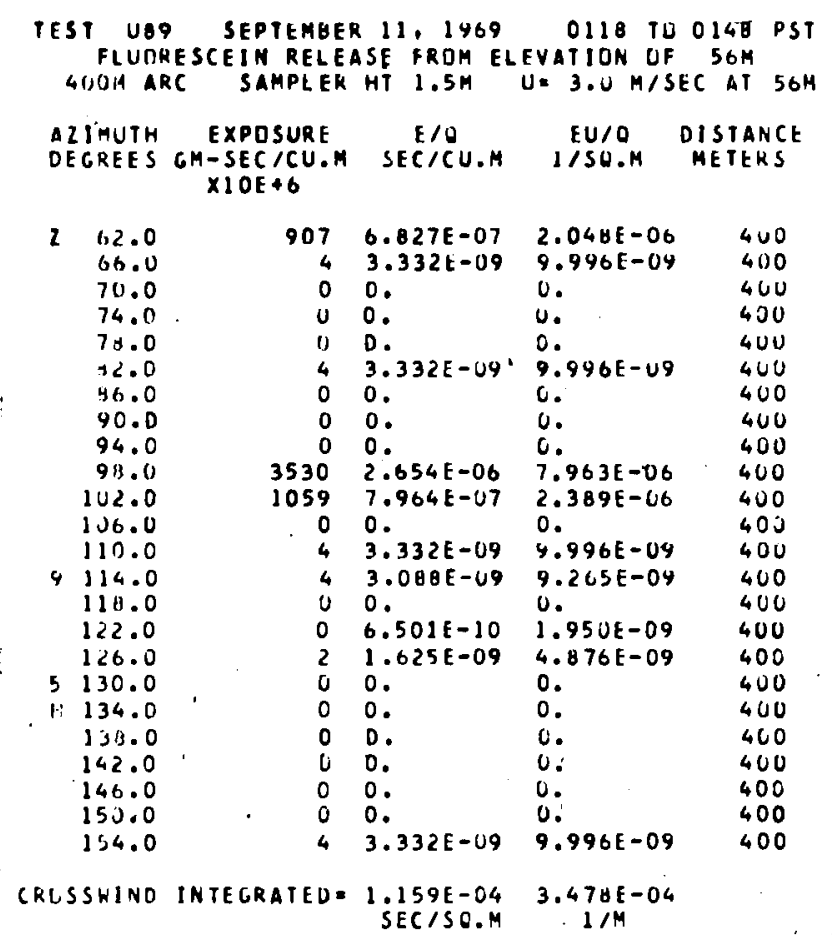

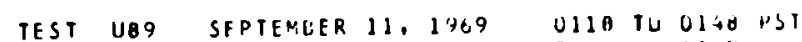
ZIHC SULFIUE RELEASE FRLM FLFVGTIUN UT 11 IM

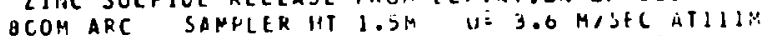

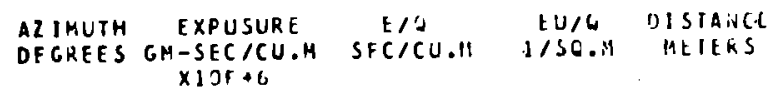

N $77.0 \quad 0 \quad 0.0 \quad 771$

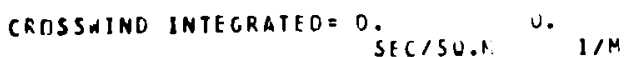

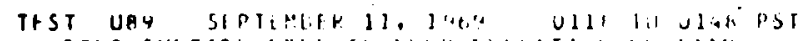

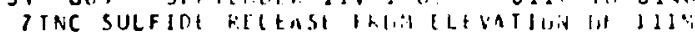

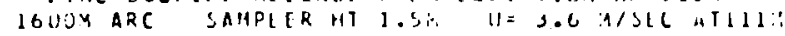

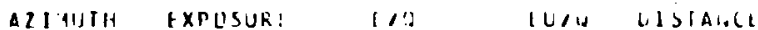

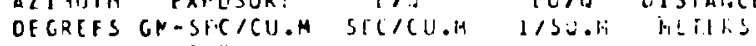
$x \geq 10+1$,

\begin{tabular}{|c|c|c|c|c|}
\hline $\begin{array}{l}91.6 \\
92.6\end{array}$ & 15 & $\because \therefore k 1 c-\Omega ?$ & 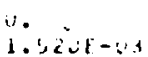 & $\begin{array}{l}15: 4 \\
16,1\end{array}$ \\
\hline 73.6 & 0 & i. & i. & dous \\
\hline 94.6 & 150 & $4.340 !-3 !$ & $1.5: 0[-67$ & 1604 \\
\hline 95.6 & 324 & $9.110 t-6$ & $3 .<t 2 f-0)$ & 1066 \\
\hline 96.6 & 760 & $2.1160-07$ & $1.69 y 1-(1)$ & 1660 \\
\hline 97.6 & 1573 & $3.95+1-, 7$ & $1.38+[-10$ & 1616 \\
\hline 93.6 & 67.1 & $1 . x+31-11$ & $0.77 \times\{-j 7$ & $16: 1$ \\
\hline 06 & v) & 0. & i. & in 19 \\
\hline
\end{tabular}

CRIISSHIND INTEGRATIO= $2.607 E-65$ Y.372E-L.S

\begin{tabular}{|c|c|c|c|c|}
\hline $\begin{array}{r}\text { THST UBY } \\
\text { FLUUK } \\
\text { BUUA ARE }\end{array}$ & $\begin{array}{l}\text { SEPTEMGED } \\
\text { XESCEIN RELE } \\
\text { C SAMPLER }\end{array}$ & $\begin{array}{l}\text { R } 11,1969 \\
\text { ASE FRDM EL } \\
\text { HT } 1.5 \mathrm{SM}\end{array}$ & $\begin{array}{r}\text { OIIB TU } \\
\text { LFVATION OF } \\
\text { UE } 3.0 \mathrm{~m} / \mathrm{S}\end{array}$ & $\begin{array}{l}U \text { O148 PST } \\
F \text { SGM } \\
\text { SEC AT } 56 M\end{array}$ \\
\hline $\begin{array}{l}\text { AZIMUTH } \\
\text { DEGKEES }\end{array}$ & $\begin{array}{c}\text { EXPUSUKE } \\
\text { GH-SEC } / C U . M \\
\times 1 C E+6\end{array}$ & $\begin{array}{c}E / O \\
S E C / C U . M\end{array}$ & $\begin{array}{c}E U / 0 \\
1 / S Q . M\end{array}$ & $\begin{array}{l}\text { UISIANCL } \\
\text { MEIEKS }\end{array}$ \\
\hline 41.2 & 0 & 0. & 0. & 795 \\
\hline 93.2 & 540 & $4.064 E-07$ & $1.219 E-06$ & 79.9 \\
\hline .93 .2 & 712 & $5.356 E-07$ & $1.607 E-06$ & 602 \\
\hline 97.1 & 0 & 0 & 0. & 800 \\
\hline 99.1 & $675 !$ & $5.076 t-00$ & $1.523 E-05$ & 809 \\
\hline 101.1 & 3291 & $2.475 f-06$ & $7.424 t-00$ & 413 \\
\hline 103.0 & 816 & $6.140 \mathrm{E}-07$ & $1.842 \mathrm{E}-06$ & 010 \\
\hline $1: 5.0$ & 0 & 0. & 0 . & $8<0$ \\
\hline 136.9 & 712 & 5.356 & 1.60 & $82 ;$ \\
\hline $108 . \theta$ & 1163 & $8.750 E-07$ & $2.625 \mathrm{E}-06$ & 826 \\
\hline 110.7 & 0 & 0 . & ü. & 629 \\
\hline 112.6 & 0 & 0. & u. & bat \\
\hline ISSHINO & INTEGRATEU= & $\begin{array}{l}2.936 E-04 \\
\text { SEC/SO.M }\end{array}$ & $\begin{array}{c}8.002 E-06 \\
1 / M\end{array}$ & \\
\hline
\end{tabular}

TEST IJEY SEPTEMUER 11. IYG9 CIIO IU CIAO PSI FLUIIRESCEIN RFLFASE FRUM ELIVATIUN UF $5 U H$ IOUUM ARC SAMPLER HT $1.5 \mathrm{TH} U=3.0 \mathrm{M} / \mathrm{SEC}$ AI $50 \mathrm{M}$

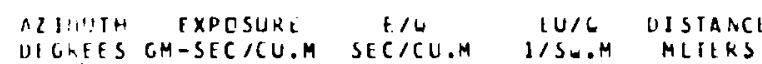
$X 1 O E+6$

\begin{tabular}{|c|c|c|c|c|}
\hline $4+6$ & 0 & 0. & u. & $15 \times 4$ \\
\hline 89.6 & 747 & $5.621 E-U 7$ & $1.6 B G E-06$ & 1596 \\
\hline 90.2 & 381 & $2.868[-0]$ & d. $G$ LSE-OI & $13 \% 7$ \\
\hline 91.1 & 460 & $3.5 C d t-07$ & 1. $05 \angle E-0 t$ & 154 \\
\hline 92.6 & 76 & $5.772 \mathrm{E}-0 \mathrm{~B}$ & $1.732 \mathrm{E}-07$ & 160 \\
\hline 93.6 & $u$ & c. & ᄂ. & 160 \\
\hline 94.6 & 168 & $1.270 E-07$ & $3.81 \cup E-07$ & $16 \mathrm{~J}$ \\
\hline 95.6 & 395 & $2.975 E-07$ & $6.924 E-01$ & 160 \\
\hline 96.6 & 2273 & $1.709 t-06$ & $4.126 \mathrm{t}-00$ & 160 \\
\hline 97.6 & 2868 & $2.157 \mathrm{E}-0 \mathrm{t}$ & $6.471 E-06$ & 161 \\
\hline yit. 6 & 3060 & $2.903 \mathrm{k}-06$ & $8.709 \mathrm{E}-06$ & 1511 \\
\hline 99.1 & 2860 & $2.157 E-06$ & $6.471 t-06$ & 161 \\
\hline 160.6 & 4158 & $3.127 E-06$ & $9.380 \mathrm{E}-06$ & 161 \\
\hline 101.6 & 1621 & $1.214 \mathrm{E}-06$ & $3.656 E=06$ & 161 \\
\hline $102 \cdot t$ & 388 & $2.922 \mathrm{E}-07$ & $8.765 E-07$ & 161 \\
\hline
\end{tabular}




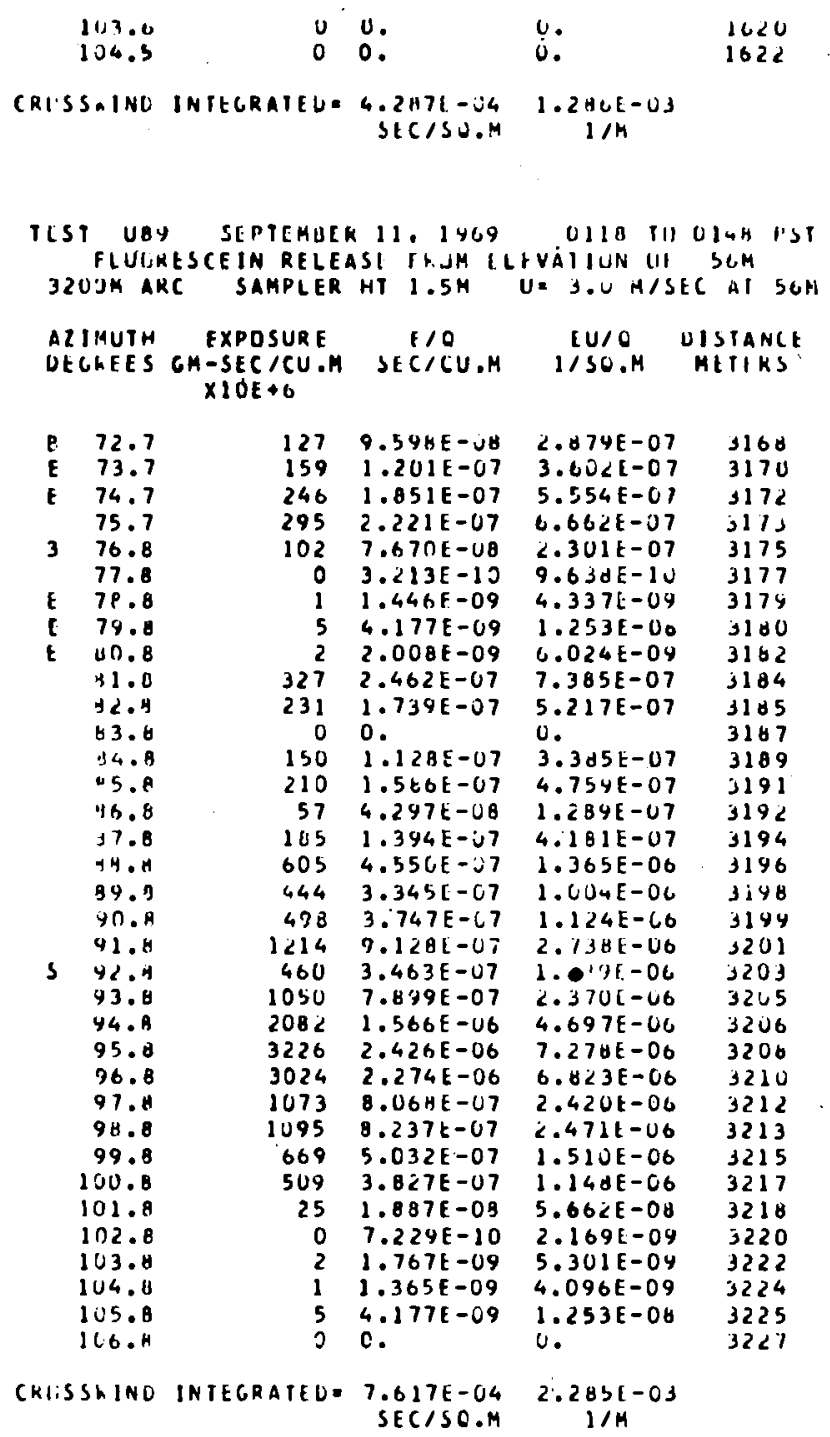

TEST UA9 SEPTEABER 11 . 1969 UIIS TU ULA PSI

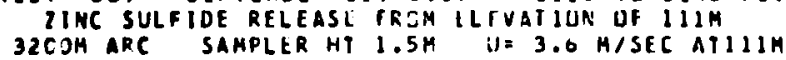

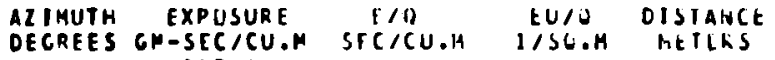
$\times 10 E+6$

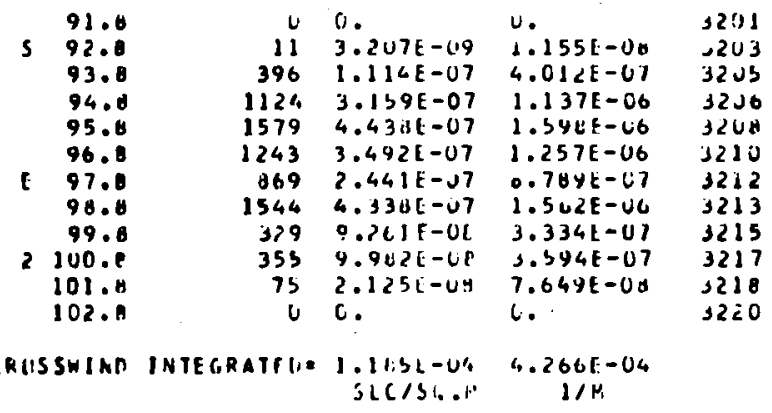

TEST UBQ SFPIEMRER 11, 1\%6,9 UIIH IU UI4O PST ZINC SULFIDE RELEASF FPOA LLIUTIUN UI $111 \mathrm{H}$ 5OOOM AKC SAMPLER HT $1.5 \mathrm{MA} \quad 11=3.0$ HISEC ATIIIM A2IMUTH EXPUSURE IIU TU/L OISTAINCE DECPEES GM-SFC/CU.M SFCICU.M HSG.M MEITKS $x \perp D E+6$

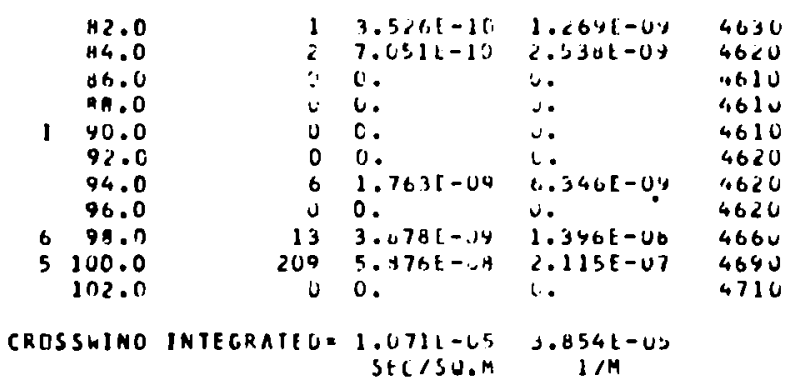

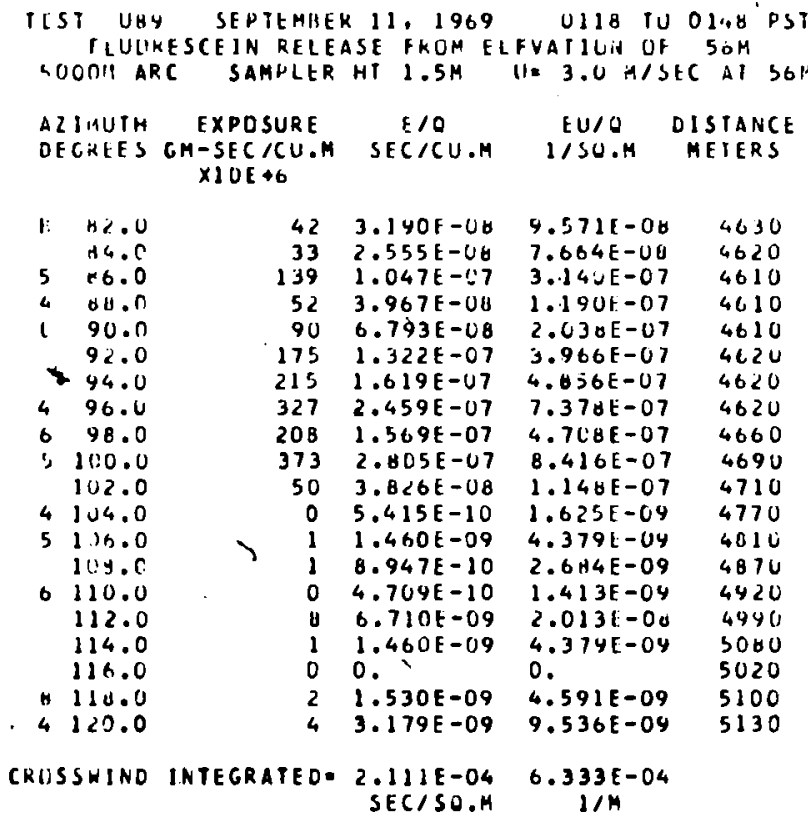




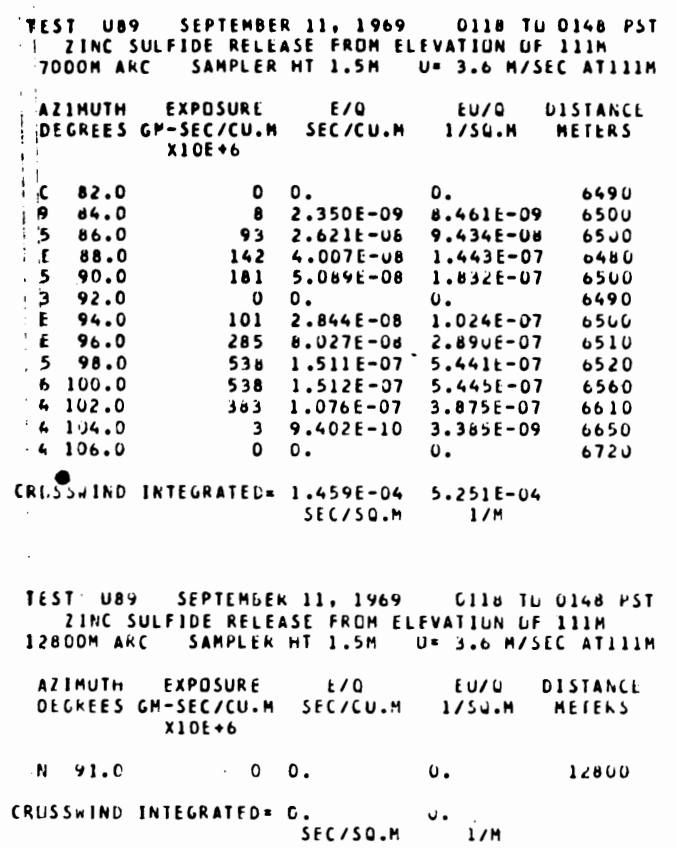

TLIMEF UATA FOLLLU...

TEST UBY SEPTEPDEF 11 , 29E9 $011 E$ TU 0148 PST IIIIC SULFIDE KELEASE FKUM ELFVATILN OF IIIM
$3200 M$ ART
$U=3.6$ MISEC ATIIIH

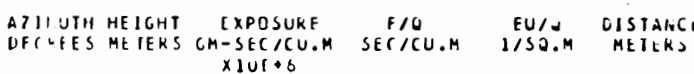
1: 115.6

.2
u 0.
0 .

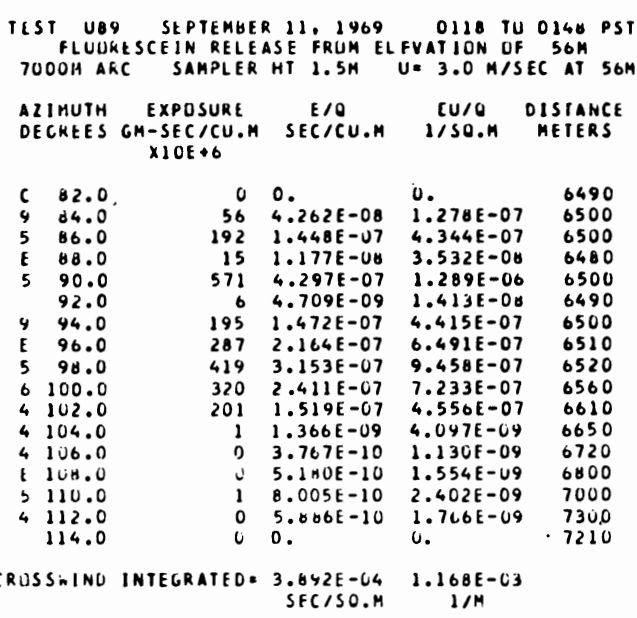

TEST UO9 SEPIEABER 11,1909 O118 TU 0145 PST FLUUTKESCEIN KELEASE FKOM ELFVATION OF 5GM $126 C O P A K C$ SAMPLER HT $1.5 \mathrm{SH} U=3.0 \mathrm{M} / \mathrm{SEC}$ AT $56 \mathrm{H}$ AZIRUTH EXPOSURL
DECKEES GN-SECICU.M SECICU.M I IOSO.M MISTANCE N $95.0 \quad 0 \quad 0$. CKIISSIIND INTEGRATHD= $0_{\text {SHCISO.M U. I/M }}$

TOHEK UATA FIJLLOW....

TEST UES SEPTEMBER 11. 1969 O118 TU 0148 PST FLUORESCE IN RELEASE FROM ELFVATIUN OF $56 \mathrm{M}$
U. $3.0 \mathrm{M} / \mathrm{SEC}$ AT $56 \mathrm{M}$

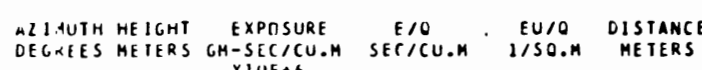
$\begin{array}{llllll}11 & 114.6 & .2 & 0 & 0 . & 0 .\end{array}$ 


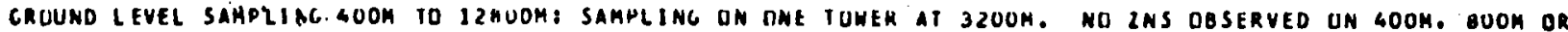

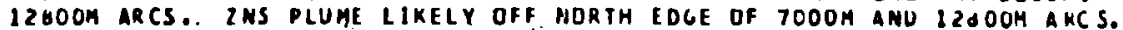

FLUURESCEIN DISPFRSAL FOR DNLY 4 MIN. IHUS. SISNIFICANCE CF FLUURESCEIN MEASUREMENTS IS MINIMAL.

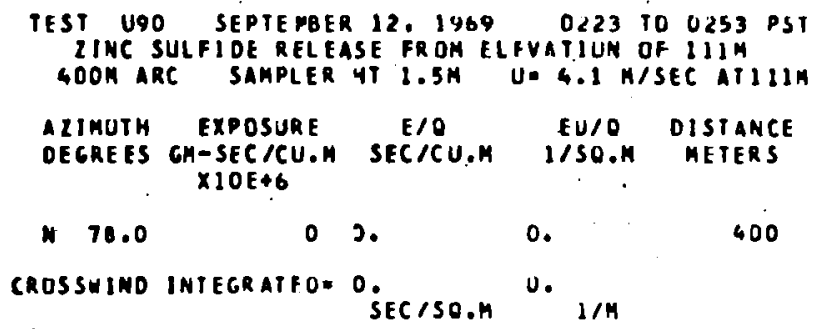

TEST U9O SEPTEMBER 12,1969 O 0223 TU 0253 PST IINC SULFIDE RELEASE FRLH ELFVATIUN UF $111 \mathrm{M}$

DOOM ARE SAMPLER HT $1.5 \mathrm{SH}$ U 4.1 M/SEC ATIIIH

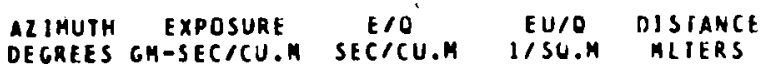
$\times 10 E+6$

N $77.0 \quad 00.00171$

CROSSHINO INTECRATED: O SFC/SU.M O. $1 / 4$

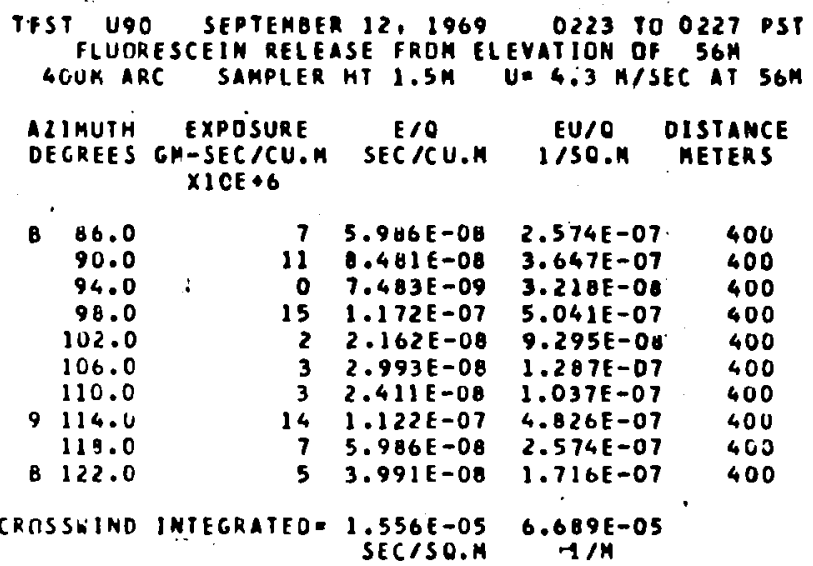

ItST U9O SHPTEHGER 12, $1969 \quad 0223$ TU 0227 PST FLUDRESCEIN RELEASE FRDH ELEVATIUN DF $56 \mathrm{M}$

BOUM ARC, SAMPLER HT $1.5 \mathrm{H}$ U. 4.3 H/SEC AT $56 \mathrm{H}$

ALIMUTH EXPOSURE E/O EU/O DISTANCE OECKLES CH-SECICU.M SECICU.H I/SO.H METEKS $X \perp 0 E+6$

97.1
99.1
101.1
103.0
103.0
106.9
120.8
110.7
112.6
114.5
116.4
111.3
120.2
122.1
124.0

$$
\begin{array}{r}
-5 \\
0 \\
0 \\
2 \\
8 \\
27 \\
0 \\
0 \\
7 \\
22 \\
4 \\
0 \\
13 \\
1
\end{array}
$$$$
\begin{aligned}
& 3.657 E-00 \\
& 0 . \\
& 0 . \\
& 2.204 E-08 \\
& 6.612 E-08 \\
& 2.144 E-07 \\
& 0.0 \\
& 5.510 E-09 \\
& 5.510 E-08 \\
& 1.736 E-07 \\
& 3.306 E-08 \\
& 0.3057 E-08 \\
& 3.057 E-07 \\
& 1.075 E-07 \\
& 1.107 E-08
\end{aligned}
$$$$
\text { (1) }
$$$$
1.659 \mathrm{E}-07
$$$$
0 .
$$$$
0 .
$$$$
9.478[-08
$$$$
2.043 E-C 7
$$$$
9.241 E-07
$$$$
0 \text {. }
$$$$
\text { 5. 3स } 3 \text { प } 0 \text { - }
$$$$
2.364 E-07
$$$$
7.464 \mathrm{E}-07
$$$$
1.422 E-07
$$$$
0 .
$$

1. $059 \mathrm{E}-07$

4. $620 E-07$

$4.739 E-O B$

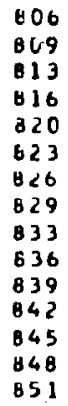

CRIISSWIND INTEGRATEO= $2.123 E-059.127 E-05$

$$
\text { SECISO.H } \quad 1 / \mathrm{H}
$$

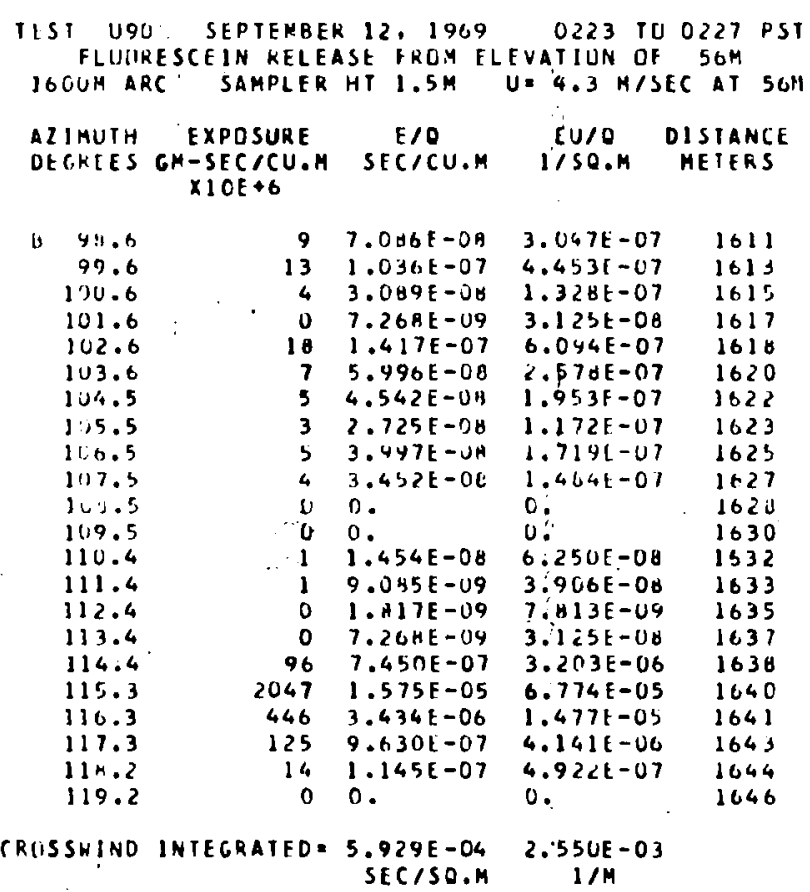

\begin{tabular}{|c|c|c|c|c|}
\hline $\begin{array}{l}\text { AZIMUTH } \\
\text { DEGEEES }\end{array}$ & $\begin{array}{c}\text { EXPOSURF } \\
\text { GM-SECICU.A } \\
\times 1 O E+G\end{array}$ & $\begin{array}{c}\text { rou } \\
\text { SHC/CU.M }\end{array}$ & $\begin{array}{l}\text { EU/O } \\
\text { J/SU.M }\end{array}$ & $\begin{array}{l}\text { OISTANCE } \\
\text { METIRS }\end{array}$ \\
\hline 97.6 & (. & 0. & U. & 1510 \\
\hline $4 p .6$ & j & $4.1231-10$ & $3.3346-09$ & 1611 \\
\hline 97.6 & 18 & $4.6746-09$ & $1.490 \mathrm{E}-00$ & 1013 \\
\hline 100. & 0 & 0. & $i$. & isl', \\
\hline 101.6 & 0 & 0 . & u. & ie \\
\hline 102.5 & 212 & $5 . B+B L-158$ & $2.3316-07$ & 1610 \\
\hline 103.6 & u & 0. & u. & 1520 \\
\hline 104.5 & 748 & $7.0231-07$ & $d .2 \times 3 t-v 1$ & $16<2$ \\
\hline 105.5 & $7 y$ & $2.112 \mathrm{i}-0 \mathrm{~B}$ & $3 .(5,4 f-6)$ & $1<>3$ \\
\hline 106.5 & 456 & $1.235 t-07$ & $\therefore .00<1-07$ & is 5 \\
\hline 107.3. & 222 & 6.0111 & $2.41, b 2-01$ & 16,27 \\
\hline 100.5 & 474 & 1.2036 & $3 .<B<E-U 1$ & 1526 \\
\hline 109.5 & 315 & $9.529[-0 \mathrm{~B}$ & $3.497 E-07$ & 1630 \\
\hline 110.4 & 916 & $2 .+7+[-0]$ & $1.016 E-1) 6$ & 1632 \\
\hline iil. & 556 & $1.303 E-07$ & $0.161 \mathrm{E}-01$ & 1333 \\
\hline 112.4 & 237 & $6.617[-0 H$ & $200916-0)$ & 1635 \\
\hline 113.4 & c & 0 . & & 16.37 \\
\hline
\end{tabular}

CRUSSHIND INTECRATFC: $3.179 F-95 \quad 1.303 E-J 4$

$$
\text { SICISO.M }
$$


TEST U9O SEPTEMEER 12, 1969 0223 TO 0253 PST ZINC SULF IDE RELEASE FROM ELFVATIUN COF $111 \mathrm{M}$ $3200 M$ ARC SAMPLER HT $1.5 \mathrm{M} U=4.1 \mathrm{M} / \mathrm{SEC}$ ATIIIM

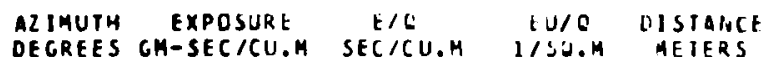
$\times 10 E \$ 6$

\begin{tabular}{|c|c|c|c|c|c|}
\hline & 89.0 & 0 & 0. & U. & 3196 \\
\hline & 90.8 & 0 & 0. & 6. & $\$ 199$ \\
\hline & 91.3 & 81 & $2.199 \mathrm{I} .08$ & $9.014[-00$ & 1201 \\
\hline & 92.8 & 221 & $5.479[-11 \mathrm{~A}$ & $2.451 t-01$ & 3203 \\
\hline & 93.10 & 42 & $1.157 \mathrm{E}-0 \mathrm{~B}$ & $\because 144 E-O H$ & 3205 \\
\hline & 94.8 & 179 & $3.510 L-30$ & $1.494 E-01$ & $3<06$ \\
\hline & 95.8 & c & 0. & u. & 3208 \\
\hline H & 96.3 & 204 & $5.679 E-08$ & $2.3 \angle 5 E-07$ & 3210 \\
\hline H & 97.8 & 256 & $6.943 E-06$ & $2.047 E-07$ & 3212 \\
\hline & 98.8 & 130 & $3.747 \mathrm{E}-0 \mathrm{H}$ & $1.534 \mathrm{E}-01$ & $3 \div 13$ \\
\hline & 99.8 & 124 & $3.355 E-39$ & $1.376 \mathrm{E}-07$ & 3215 \\
\hline & 100.8 & 346 & $9.373 \mathrm{E}-\mathrm{L}$ & $3.843 E-j 7$ & 3217 \\
\hline $\mathbf{E}$ & 101.8 & 368 & $9.9 b>E-08$ & $4 . \cup 8 \cup E-07$ & 3218 \\
\hline & 102.8 & 406 & $1.099 \mathrm{E}-07$ & $4.507 E-67$ & $3 \geqslant 20$ \\
\hline & 103.8 & 498 & $1.345 E-07$ & $5.514 E-07$ & 3222 \\
\hline & 104.8 & 402 & $1.08 \mathrm{HE}-07$ & $4.400 E-07$ & 3224 \\
\hline & 105.0 & 1805 & $4.879 E-07$ & $2.00: 1 E-06$ & 3225 \\
\hline & 106.8 & 1391 & $3.761 E-07$ & $1.34<E-06$ & 3227 \\
\hline & 107.0 & 1600 & $4.347 \mathrm{E}-07$ & $1.7 A 2 E-06$ & 3229 \\
\hline 3 & 108.8 & 204 & $5.5 \ln E-: 19$ & $2.262 \mathrm{E}-117$ & 3230 \\
\hline & 109.7 & 2022 & $5.466[-j]$ & $2.241 \mathrm{E}-06$ & $\$ 232$ \\
\hline & 110.7 & 2675 & $7.237 \mathrm{k}-\mathrm{j} 7$ & $\angle .905 E-00$ & 3233 \\
\hline & 111.7 & 2564 & $6.931 E-\cup 7$ & $2.1 .42 t-06$ & 3235 \\
\hline & 112.7 & 1073 & $7.901 k-077$ & $1.16,4 E-06$ & 3237 \\
\hline & 113.7 & 154 & $4.166[-U B$ & $1.70 B E-07$ & $\$ 230$ \\
\hline & 114.6 & 214 & $5.78 \% t-U 4$ & $2.37<E-07$ & 3240 \\
\hline 2 & 115.6 & 134 & $3.626 E-U A$ & $1.447 E-07$ & $3 x^{2}+1$ \\
\hline & 116.6 & 15 & $4.743 t-099$ & $1.7 \% U E-O B$ & 3243 \\
\hline & 117.6 & (1) & 0. & U. & 3245 \\
\hline
\end{tabular}

CRUSSHIND INTECRATIU: $2.5 H 3 L-U 4$, T.6SYE-US ¿lc/so.m

TEST U9O STPIEPAER 12, 1969 10223 TI) ULSO PST IINC SULFIOE RELEASE FRUA ILFVATIUN UH III: $500 J M$ IRC SAMPLER HT $1.54 \quad 11=4.1$ M/DEC AIIIIM A2IMUIH EXPOSURE IIU IJ/O WISIAINCL DEGRES GK-SECLCU.M SICICU.M IISU.M METERS $X 1 J E+6$

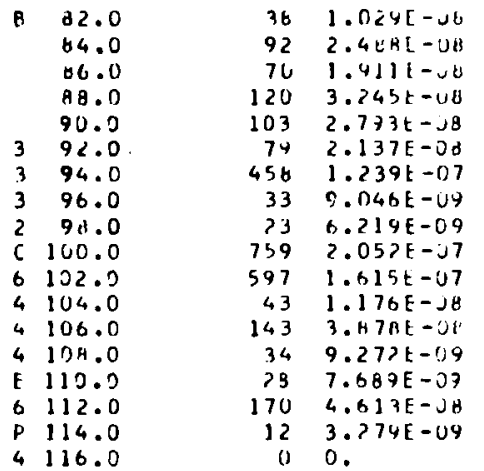

$4.214 E-0 B \quad 4630$ 1. $U 2 U E-07 \quad 4620$ $7.835 t-0 y \quad 4510$ 1.S31f-01 4010 $1.165 E-07$ 4510

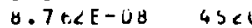
$5.081 E-07 \quad 4620$ 3. $7 U 9 E-00 \quad 4620$ $2.550 E-00 \quad 4530$ $0.414[-07 \quad 4690$ $6.6<(1 E-0) \quad 4710$ 4. $A 211 E-08-4770$ $1.3,46 E-01 \quad 4410$ $3.8 \mathrm{CLE}-0 \mathrm{O} \quad 4 \mathrm{E} 70$ $3.1 \mathrm{BCE}-0 \mathrm{OH} \quad 4920$ 1. $\rightarrow \rightarrow 1 \mathrm{E}-\mathrm{U} 7 \quad 477 \mathrm{~J}$ $1.344 \mathrm{E}-08 \quad 3000$

CRUSSAIND INIEGRATED $=1.245 E-134 \quad 5.104 E-014$ SEC/SU.K $1 / M$

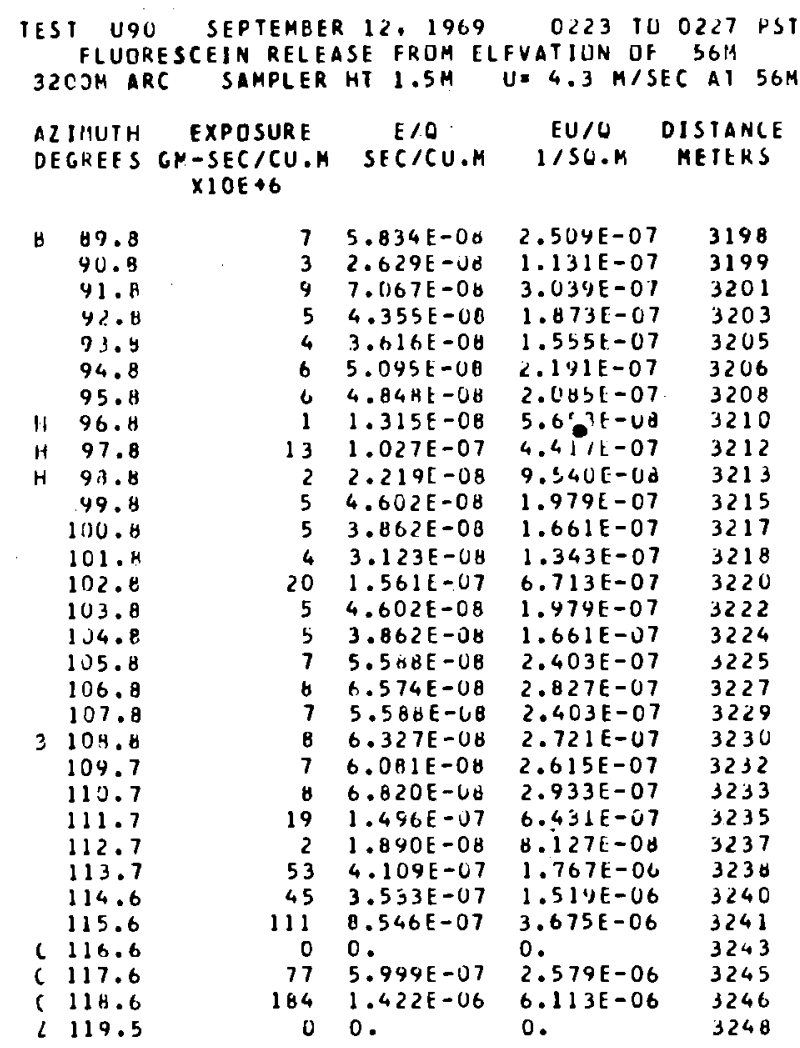

CRISSSHINU INIEGRATED $2.763 E-04 \quad 1.188 E-03$

IEST U90 SEPTEMBER 12, 1969 O223 TU 0227 PST FLUIIRESCEIN RELEASE IRDM ELEVATIUN OF $50 \mathrm{M}$ SOUUM ARC SAMPLER HT $1.5 \mathrm{M} U=4.3 \mathrm{M} / S E C$ AT $5 O M$

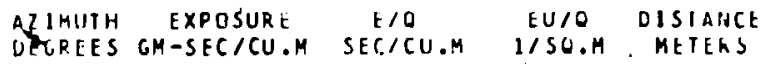
$X 10 E+6$
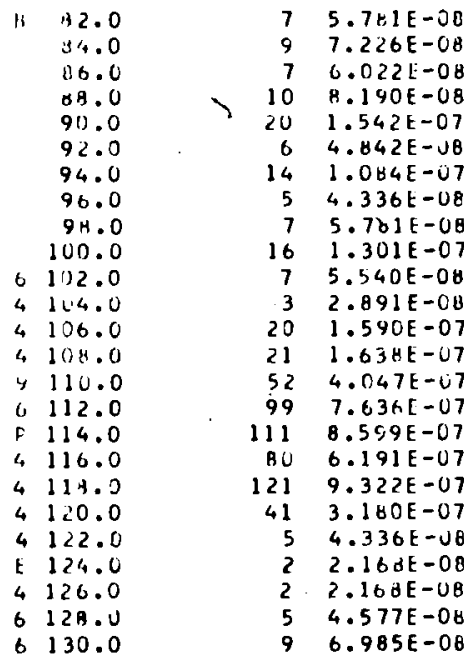

$2.480 \mathrm{E}-07$ $3.107 E-07$ $2.589 \mathrm{E}-07$ $3.522 \mathrm{E}-07$ $6.629 E-07$ $2.032 \mathrm{E}-07$ $4.661 \mathrm{E}-07$

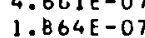
$1.864 E-07$ $2.486 E-07$ $5.593 \mathrm{E}-07$ $2.382 \mathrm{E}-07$ 1. $243 \mathrm{E}-07$ $6.836 \mathrm{E}-07$ $7.0431-07$ $1.740 E-06$ $3.283 E-06$ $3.69 d \mathrm{dE}-06$ $2.662 \mathrm{E}-06$ $4.008 \mathrm{E}-06$ $1.367 E-06$

$1.864 \mathrm{E}-07$

$9.322 \mathrm{E}-0 \mathrm{~B}$

$9.322 \mathrm{E}-0 \mathrm{~B}$

$1.968 E-C 7$

3.004E-07

4630

4620

4010

4610

4610

4620

4620

4620

4600

4640

4710

4770

4810

4870

4920

4990

5000

5020

5100

3130

4630

4680

4770

4900
4970

CRUSSHIND INTEGRATED $=9.207 E-04 \quad 3.959 E-03$ SEC/SO.M 


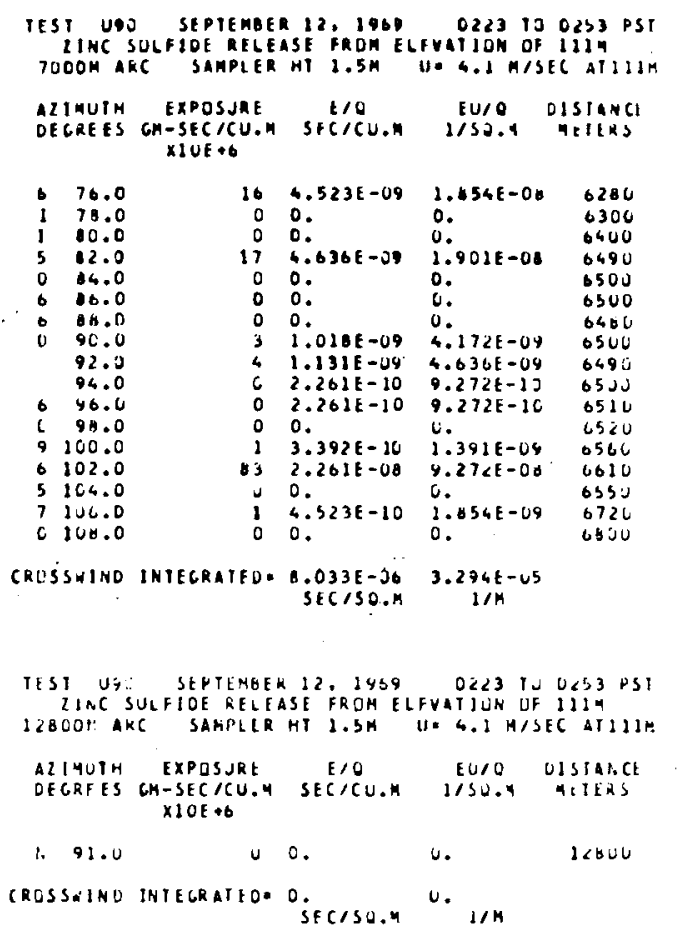

TULER UATA FULLOH....

TEST U9J SEPIEKBFA 12,2969 O 0223 IJ 0253 PSI Z1NC SULF DOE RELEASE FRUM ELFYA]ION OF $111 \mathrm{~K}$
$U=4.1$ H/SEC MTIIM

ALIAUTH HEIGHT EXPOSUEE FIO EU/O DASTANCE OEGREES METERS GP-SLCICU.M SETICU.M IISO.M METESS

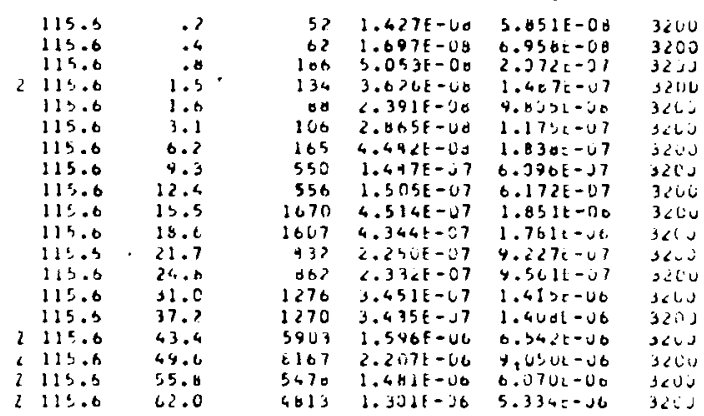

IEST UYO SEPTEMBER 12, 2469 O223 TO U227 PST

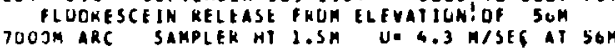

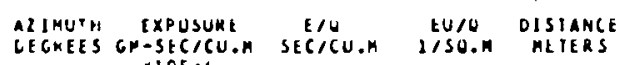
AIOE +6

\begin{tabular}{|c|c|c|c|c|c|}
\hline h & 76.0 & 3 & $2.674 E-08$ & $1.150 E-07$ & 6280 \\
\hline$t$ & 74.0 & 1 & $1.645 E-08$ & C.2ISE-OD & 0300 \\
\hline & 60.0 & 2 & $1.734 t=06$ & $7.450 E-08$ & 6400 \\
\hline b & 32.6 & 3 & $2.674 \mathrm{E}-0 \mathrm{~b}$ & $1.15 \cup E-07$ & 6490 \\
\hline 0 & 44.0 & 2 & $2.096 E-00$ & $9.011 \mathrm{f}-00$ & 6500 \\
\hline 6 & 86.0 & 6 & $0.263 \mathrm{E}=0 \mathrm{~B}$ & $2.693 E-07$ & 6300 \\
\hline & B8.C & 0 & $7.226 E-49$ & $3.107 E-08$ & 6480 \\
\hline & 9.0 & 3 & $2.312 E-48$ & $9.943 \mathrm{E}=0 \mathrm{~d}$ & 0500 \\
\hline & 42.0 & 5 & 4.DYSE-OU & $1.761 E-07$ & 0440 \\
\hline & $94 \cdot C$ & 1 & $7.9496-09$ & $3.61 \cup[-08$ & 6500 \\
\hline$c$ & $\$ 6.0$ & 4 & $3.469 E-68$ & $1.69<6-07$ & 6310 \\
\hline & $\$ 0.0$ & 1 & $1.510 \mathrm{E}-0 \mathrm{t}$ & $6.525 E=0$ & 0520 \\
\hline 4 & 112.0 & 4 & $3.107 \mathrm{E}-48$ & $1.336 E-07$ & 6560 \\
\hline 6 & $1 / 2.0$ & 3 & $2.529[-08$ & $1.08 \& E-U 7$ & 6010 \\
\hline r & 134.0 & 3 & $3.902 E-U \theta$ & $3.676 t-07$ & 6630 \\
\hline 7 & $10 \% .0$ & 1 & $3.061 E-U 6$ & $1.11+[-07$ & 6720 \\
\hline i, & 12.3 .0 & 2 & $1.607 E-60$ & $7.7601-00$ & 6800 \\
\hline & 110.0 & 4 & $3.396 E-48$ & $1.4600-07$ & 7000 \\
\hline & & & $\begin{array}{r}1.0775-04 \\
\text { SHC150.4 }\end{array}$ & $\begin{array}{c}4.631 E-14 \\
11 \mathrm{M}\end{array}$ & \\
\hline
\end{tabular}

IESI U9. SFPTEMBER 12, 1964 C223 TU 0227 PST HLULAESCEIN RELEASE FROM ELEVATIUN OE SOH I COLOM ARC SAMPLER HT D.SH U

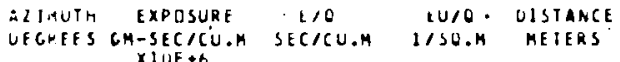

\begin{tabular}{|c|c|c|c|c|c|}
\hline b & 91.0 & [1] & $8.6>P E-u b$ & $3.724 \mathrm{E}-07$ & $128 \cup 0$ \\
\hline 5 & $9<.6$ & 3 & $2,066 E-60$ & $1.253 E-07$ & $1<600$ \\
\hline & $y \therefore u$ & 12 & $5.344 \mathrm{E}-08$ & $4.04 \cup E-07$ & $1<800$ \\
\hline & $94 . i$ & 16 & $1.301 t-07$ & $5.5+3 E-27$ & 12000 \\
\hline & 33.6 & 10 & 7.7 तE -GD & $3.314 \mathrm{E}-07$ & 12800 \\
\hline & ×u. & 0 & C. $H$ HEE-Ob & $2.0726-07$ & 12000 \\
\hline & 47.6 & 4 & 3.131106 & $1.347 f-07$ & $1<000$ \\
\hline & 90.6 & 1. & $1.1328-68$ & $4.0 c a t-v a$ & $1<b<0$ \\
\hline & & 1 teskn & $\begin{array}{l}1.133 t-04 \\
\text { sictsiti }\end{array}$ & $\begin{array}{c}4.07 \pm E-04 \\
1114\end{array}$ & \\
\hline
\end{tabular}

HH.FH LITA FILLUH....

IIST UQU SEHIEMaER 12, 1464 U 223 TU O227 PST Fi IITEFSCEIN RELEASE FRUH ELEVAT IUN DF. SOM

ALI SUTH HEICHT EXPOSUKE FIU EUTO UISTANCE

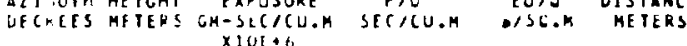

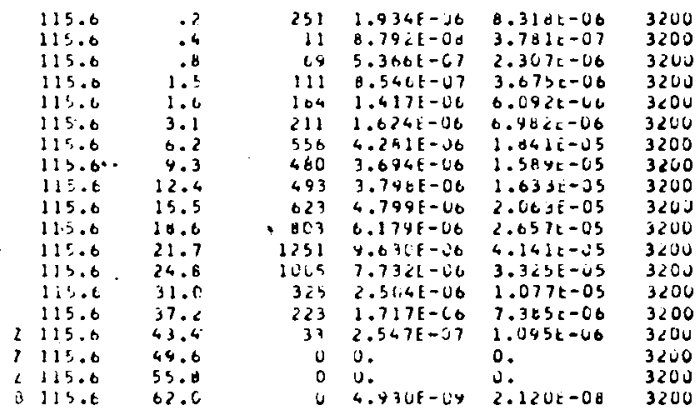




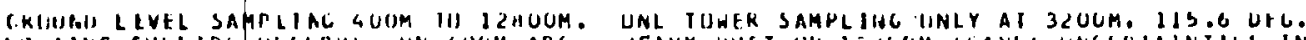

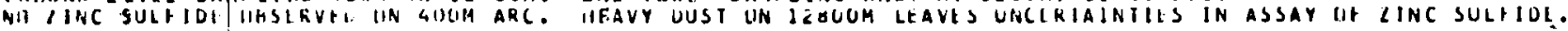

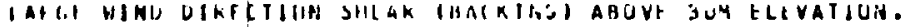

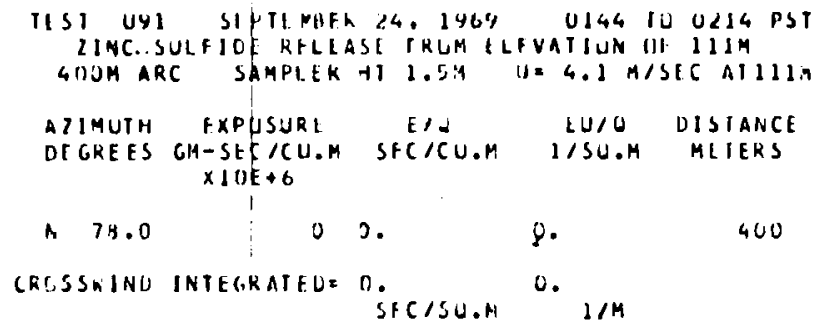

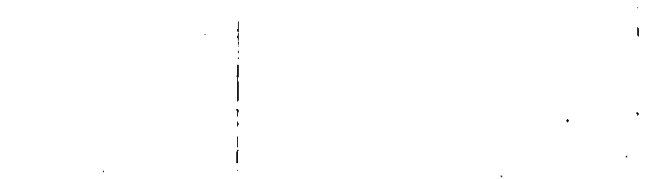

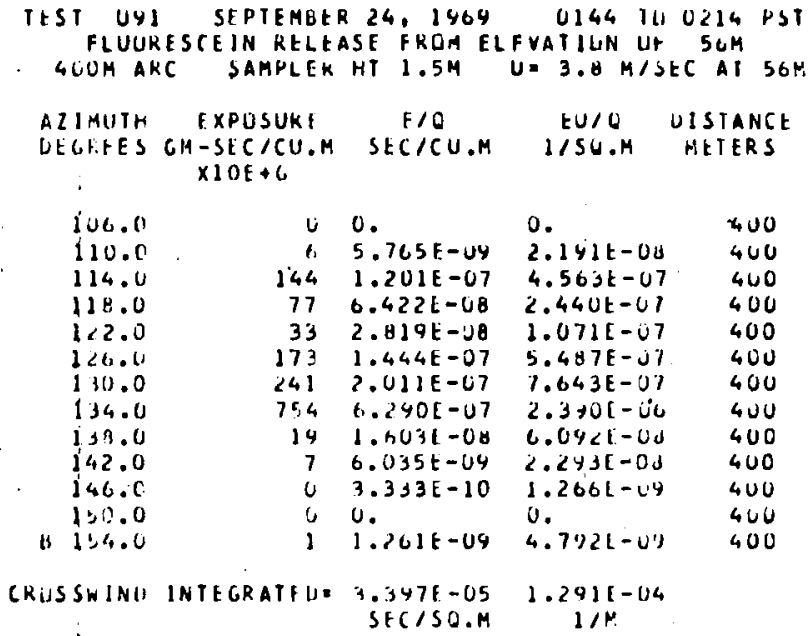

TEST U91 SEPTEMUER 24, 1909.0144 TU 0214 PST FLUORESCEIN RLLFASE FROM ELEVATIUN IIF SEM. WOUM AKC SAMPLIR HT 1.5M IJE 3.6 M/JLC AI SOM

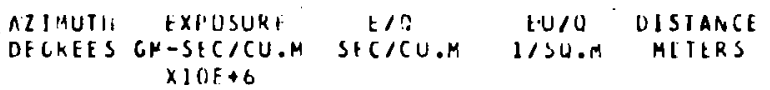

\begin{tabular}{|c|c|c|c|c|}
\hline 110.7 & J & 0. & c. & 829 \\
\hline 112.6 & 's & $2.945 E-11$. & $1.1 S+E-10$ & 33 \\
\hline 114.5 & 190 & $1.591 E-07$ & $0.04,[-07$ & 030 \\
\hline 116.4 & 412 & $3.441 \mathrm{f}-67$ & $1.300 \mathrm{~F}-00$ & 834 \\
\hline$\$ 113.3$ & 996 & $8.3 \cup 7 t-07$ & $3.150 \mathrm{t}-60$ & 61,2 \\
\hline $1<0.2$ & 503 & $4.695 t-67$ & $1.734 \bar{E}-00$ & 845 \\
\hline 12122.1 & 423 & $3.531 \mathrm{E}-07$ & $1.34<[-.6$ & 440 \\
\hline $1<4.2$ & 552 & $40005 \mathrm{E}-37$ & $1.75 \cup E-J 6$ & 851 \\
\hline 125.0 & 996 & $H .307 E-07$ & $3.15 c t-j c$ & 854 \\
\hline 127.7 & 1462 & $1.2145-00$ & $4.031 \mathrm{E}-00$ & 457 \\
\hline 129.5 & 1175 & $9.744 E-07$ & $3.724 t-06$ & 859 \\
\hline $1: 1.4$ & 1462 & $1.219 E-06$ & $4.6311-00$ & 067 \\
\hline 133.2 & 1392 & $1.161 \mathrm{E}-J 6$ & $4.411 E-00$ & 365 \\
\hline 134.9 & $\angle C 24$ & $1.607 E-60$ & $0.41<E-J 0$ & 867 \\
\hline 136.4 & 2701 & $2.251 \mathrm{E}-06$ & O.S5OE-UC & $47 \mathrm{~L}$ \\
\hline 138.7 & 2024 & $1.667 \mathrm{~L}-06$ & $0.4121-00$ & 872 \\
\hline 140.6 & 3679 & $3.0<6 \mathrm{E}-0 \mathrm{e}$ & $1.10 \% 1-05$ & $874_{0}$ \\
\hline 142.4 & 3152 & $2.627 \mathrm{E}-06$ & $9.584 t-00$ & 876 \\
\hline 144.2 & 1740 & $1.457 \mathrm{E}-06$ & $5.530 t-00$ & 870 \\
\hline $1 \div 6.0$ & 2071 & $1.726 E-U C$ & $6.5090-66$ & 000 \\
\hline 16.7 .8 & 203 & $5.8545-07$ & $2.2201-06$ & $80<$ \\
\hline 149.6 & 380 & $3.173 E-07$ & $1.2 \cup C E-\cup U$ & 804 \\
\hline 151.4 & 455 & $3.800 t-07$ & $1.444 \mathrm{E}-6 \mathrm{G}$ & $H \otimes 6$ \\
\hline 153.3 & 423 & $3.5316-37$ & I.34aE-UE & you \\
\hline $1=5.6$ & 31 & $1.6 .27 \mathrm{E}-\mathrm{Cl}$ & $9.981 \mathrm{~F}-\mathrm{Co}$ & 809 \\
\hline 156.0 & 11 & $E .0560-09$ & $3 . \angle A Y E-U D$ & equ \\
\hline 130.6 & 4.4 & $3.701 \mathrm{E}-49$ & 1. 4 UeE-U, & 892 \\
\hline 100.4 & 16 & $1.552\left[-0_{0}\right.$ & $5.496 E-0 B$ & 693 \\
\hline 162.2 & 0 & $3.000 E-10$ & $1.474 E-0 Y$ & $8 \times 4$ \\
\hline UIN & IE & $\begin{array}{l}.0 .760 E-U 4 \\
\text { SEC/SU.M }\end{array}$ & $\begin{array}{c}2.569 E-U S \\
1 / 14\end{array}$ & \\
\hline
\end{tabular}




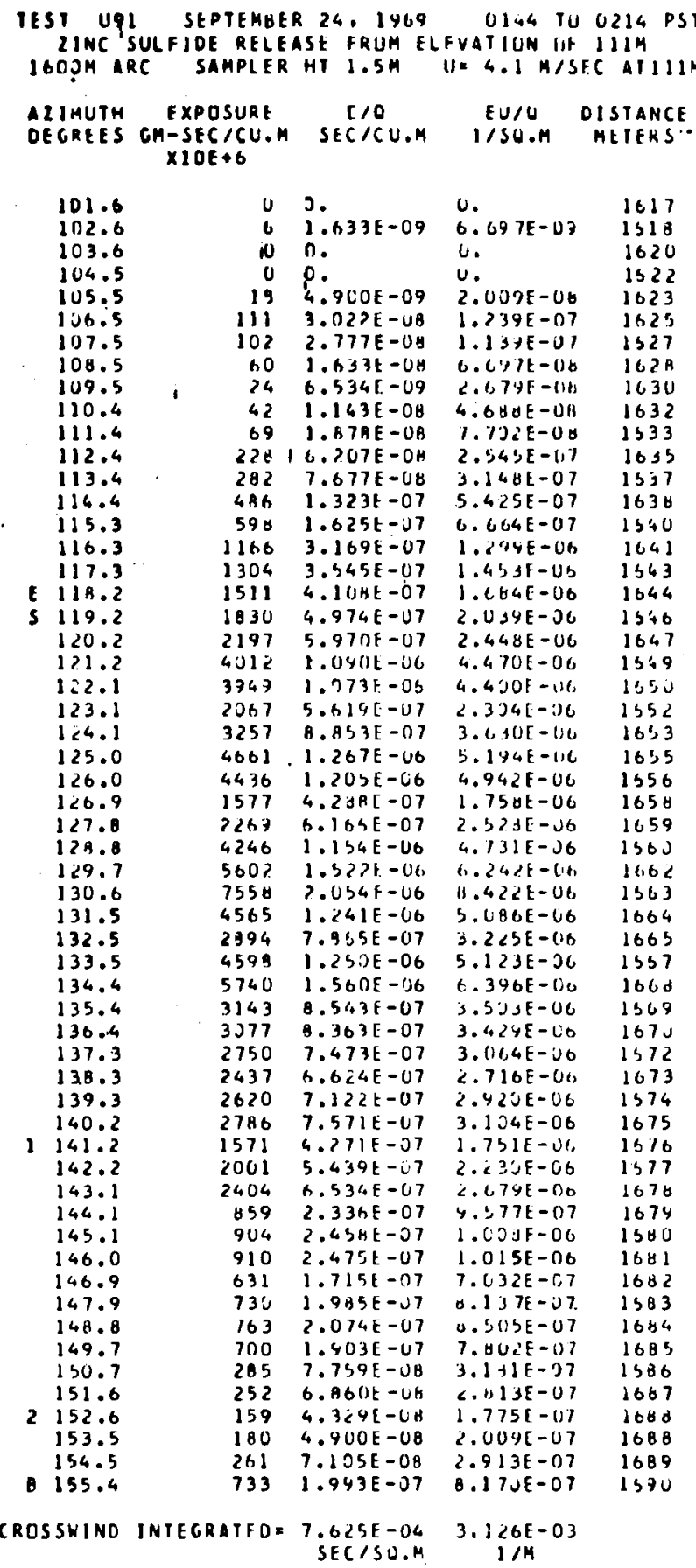

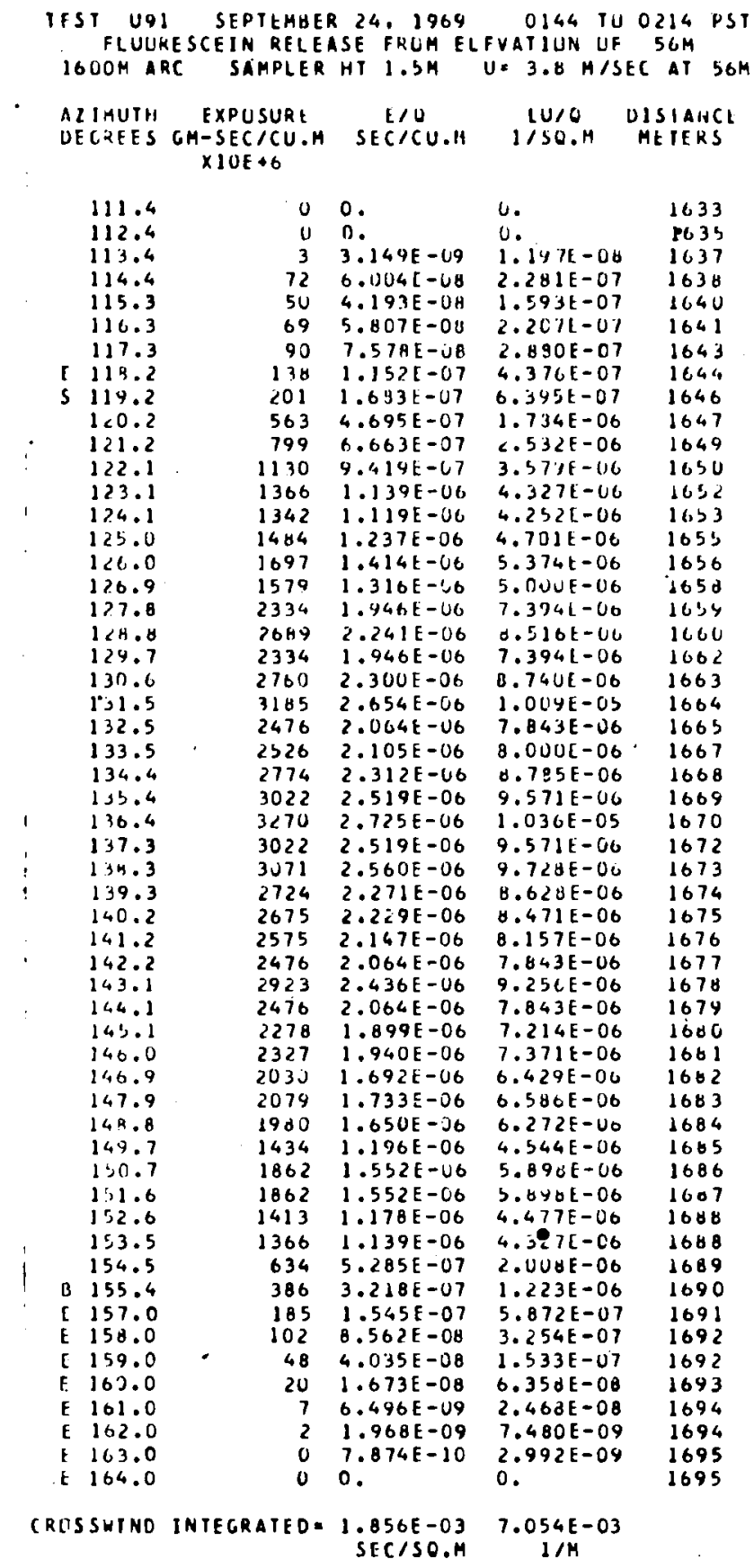




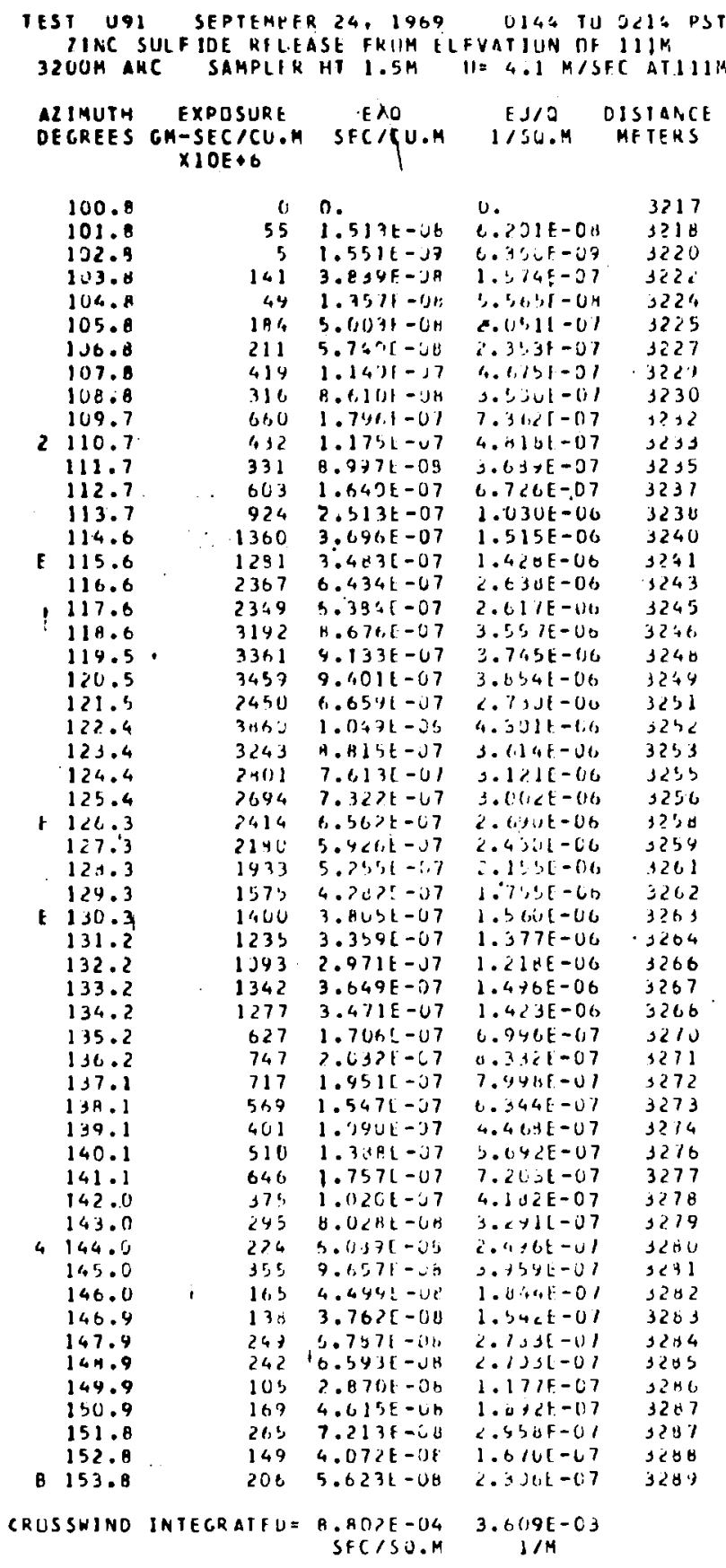

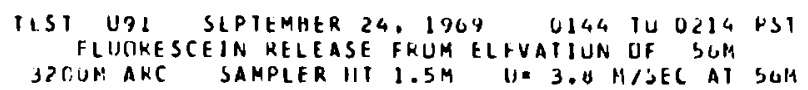

ALIMUTH EXPOSURE
DEGHES CM-SECICU,M SECICU.M IISOAM MEIERS
XIOE \&

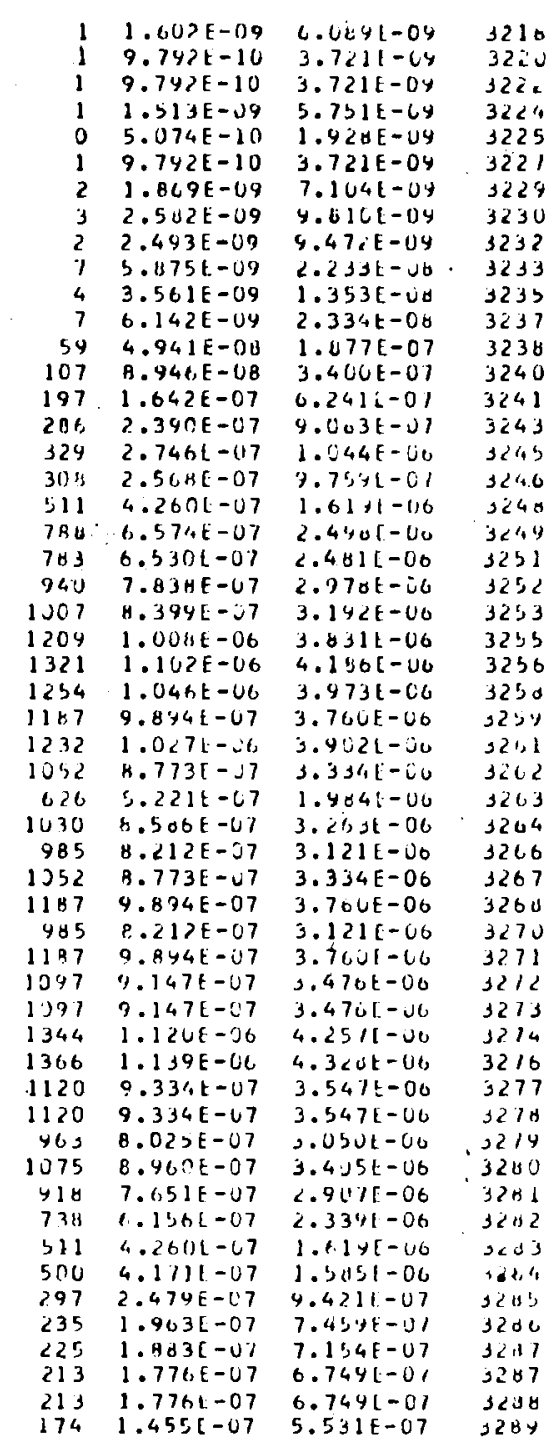

CRI.SSHIND INTEGRATED= 1.531E-03 5.8IUE-03

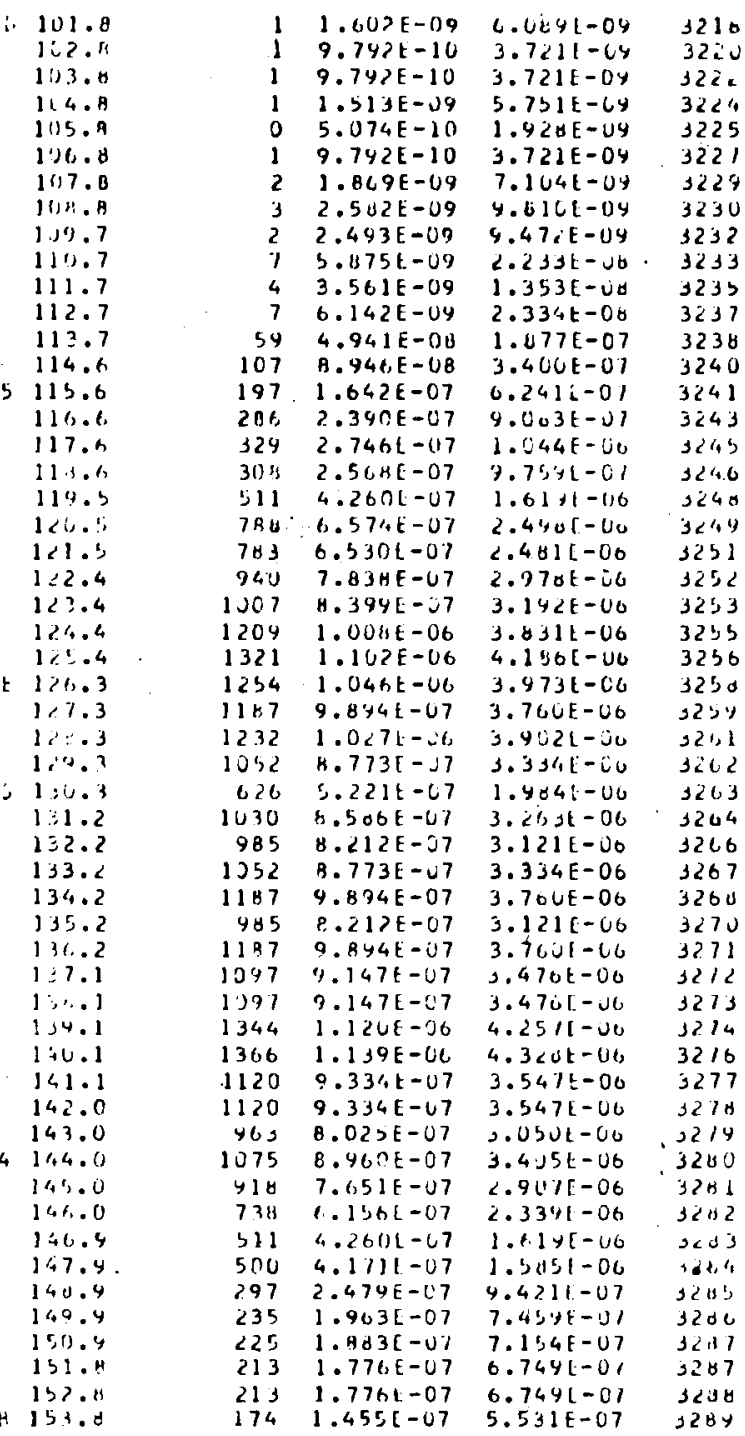
SEC/SO.M $1 / 14$ 
TEST U91 SEPTEABFR 24, 1969 ŨI44 TU O214 FST ZINC SULF IDE RELEASE FRUM ELFVATIUH UF IIIM SOOOM ARC SAMPLER HT $1.5 \mathrm{H}$ H. 4.1 M/SEC ATIIIM

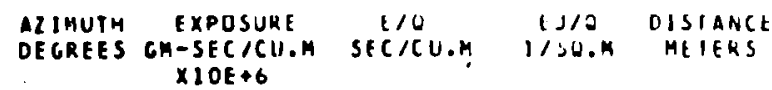

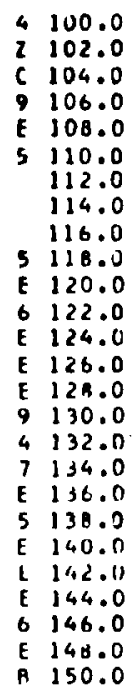

CRUSSHIND INTECRATHU: $2.2001-04 \quad 9.20,1 E-014$ siciso. 14

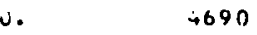

0.

$1.212 \mathrm{E}-0 \mathrm{~B}$

$7.17 \mathrm{dE}-08$

$1.109 E-07$
$1.012 t-07$

$3.012 t-07$

0.76 YE -1$) 7$

$5.551 \mathrm{E}-07$

$0.440 E-07$

$7.429 E-07$

$3.347 \mathrm{E}-07$

$1.700 \mathrm{E}-07$

$1.617 t-07$

2.CS $1 E-07$

$1.3565-07$

$4.035 E-08$

4. $6,1 \mathrm{E}-\mathrm{D}$

".) $112 l-0$ -

$3.26 .31-04$

$\therefore, 317 t-1,8$

$2.937 \mathrm{E}-0 \mathrm{~B}$

$3.330 t-0 y$
4690
4710
4770
4910
4870
4920
4990
5080
5020
5100
5130
4830
4660
4770
4700
4970
4980
4940
5000
5020
5360
5090
5190
3190
5240
5300
IEST UY1 SEPTEMAER 24, 1409 U10,4 111 O214 PST ZINC SULFIDE RTLEASE FRUM LLFVAIIUN UI IIIY TOOUM ARC SAMPLER HT. $1.5 .4 \quad U=1.1 \mathrm{M} / \mathrm{STC}$ ATI11M

AZIMUTH EXPOSURE EIN IU/O DISIATCE OEGREES GM-SEC/CJ.M SILCU.P I/SW.M MLIEKS $\times 10 E+0$

$\begin{array}{ll}7 & 100.0 \\ 6 & 132.0 \\ 9 & 104.0 \\ 7 & 106.0 \\ F & 108.0 \\ 9 & 110.0 \\ 8 & 112.0 \\ 0 & 114.0 \\ 0 & 116.0 \\ 7 & 114.0 \\ E & 120.0 \\ & 122.0 \\ t & 124.0 \\ E & 126.0 \\ E & 128.0 \\ E & 130.0 \\ 7 & 112.0 \\ E & 134.0 \\ E & 136.0 \\ 7 & 134.0 \\ 0 & 140.0 \\ 5 & 142.0 \\ 0 & 144.0 \\ 7 & 146.0\end{array}$

0.

4. $3<2 t-10$ 1.372E-Jy $1.277 E-07$ $1.3946-07$ $1.4445+07$ $2.2054-07$ $1.235[-0]$ 3. (6) E.E-0 $7.3435-07$ 1. C. $4<8-06$ 1.4 J 1 - 06 $9.4<5 E-01$ 4. SISE-D) $2.823[-0)$ 1. $\times 44 k-07$ $1.205[-07$ 1. $0.53[-07$

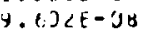
$7.424 \mathrm{E}-0 \mathrm{O}$ $2.004 \mathrm{~F}-0 \mathrm{~B}$ 1. 1 eise-UE $9.322 E-10$ $1.674 \mathrm{~F}-38$ $1 / 14$
6560

6610

3550

$672 \mathrm{~J}$

t,duO

$1 \mathrm{~J} \mathrm{~J}$

1900

7210

12213

7150

150

1160

7100

1100

7110

7150

7200

7250

1300

(350

7110

$710 \mathrm{~J}$
CKOSSHIND INTEGRATED= $4.073 E-04$ 1.670E-U3
SEC/SO.H

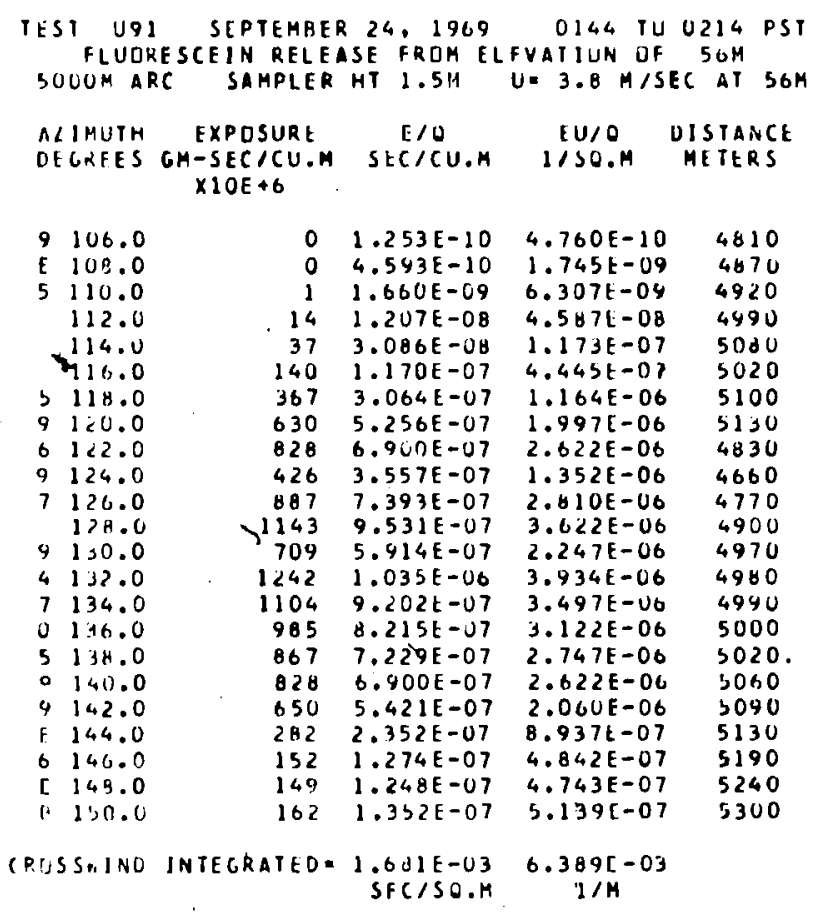

I ST UYI SEPTEMBER 24, 1969 O144 IU 0214 PST FLLORESCEIN RELEASE FHOH ELEVAIIUN UF $56 \mathrm{M}$

TUD.M ARC SAMPLER HT $1.5 \mathrm{M} U=3.8 \mathrm{M} / \mathrm{SEC}$ AT $56 \mathrm{M}$
AZ IUUTH EXPCSURE
OECREES GM-SEC/CU.M SECICU.M I/SO.M M MEIERS $\times 10 E+6$

71 1 4.0

6102.0

4104.0

$71: 6.0$

t 1114.0

4110.0

1112.0

D $11 \% .0$

(1) 116.0

7114.0

3120.0

1.2 .0

1124.0

$t 1<0.0$

012.00

1130.0

7132.0

134.0

I 146.0

$1+3.0$

146.0

5142.0

U 144.0

2

$\begin{array}{rl}O & 3.288 E-10 \\ 0 & 4.854 E-10 \\ 0 & 4.593 E-10 \\ 0 & 3.810 E-10 \\ 1 & 9.290 E-10 \\ 10 & 8.940 E-09 \\ 9 & 7.896 E-09 \\ 19 & 1.549 E-08 \\ 48 & 4.025 E-08 \\ 228 & 1.900 E-07 \\ 156 & 1.300 E-07 \\ 675 & 5.632 E-07 \\ 051 & 8.763 E-07 \\ 854 & 7.119 E-07 \\ 453 & 3.777 E-07 \\ 236 & 1.968 E-07 \\ 135 & 1.130 E-07 \\ 255 & 2.133 E-07 \\ 492 & 4.105 E-07 \\ 611 & 5.092 E-07 \\ 223 & 1.859 E-07 \\ 67 & 5.591 E-08 \\ 5 & 4.556 E-09 \\ 41 & 3.477 E-08\end{array}$

$1.249 E-09$
$1.644 E-04$

1. $745 \mathrm{E}-0$

$1.44 \times E-09$

$3.530 E-64$

3. $397 \mathrm{E}-08$

3. $001 \mathrm{E}-\mathrm{JU}$

$6.075 \mathrm{E}-0 \mathrm{~d}$

$1.5,90 \mathrm{E}-07$

$7.222 E-07$

$4.941 E-07$

$2.140 E-U Q$

3. 33 UE -06

<. $705 \mathrm{E}-06$

$1.435 E-06$

$7.4179 E-07$

$4.293 \mathrm{E}-07$

E. $104 \mathrm{E}-07$

$1.560 \mathrm{t}-0 \mathrm{C}$

$1.935 E-06$

$7.063 E-07$

2. $125 \mathrm{E}-07$

1. $731 \mathrm{E}-0 \mathrm{0}$

1. $321 \mathrm{E}-07$

6500

6610

6650
6720

6800

7000

7300

7210

7190

7150

7120

7100

7100

7130

7153

1200
1250

7300

7350

7253

7110

CKIISSHIND INTEGRATED $=1.162 E-03 \quad 4.417 \mathrm{E}-03$ 


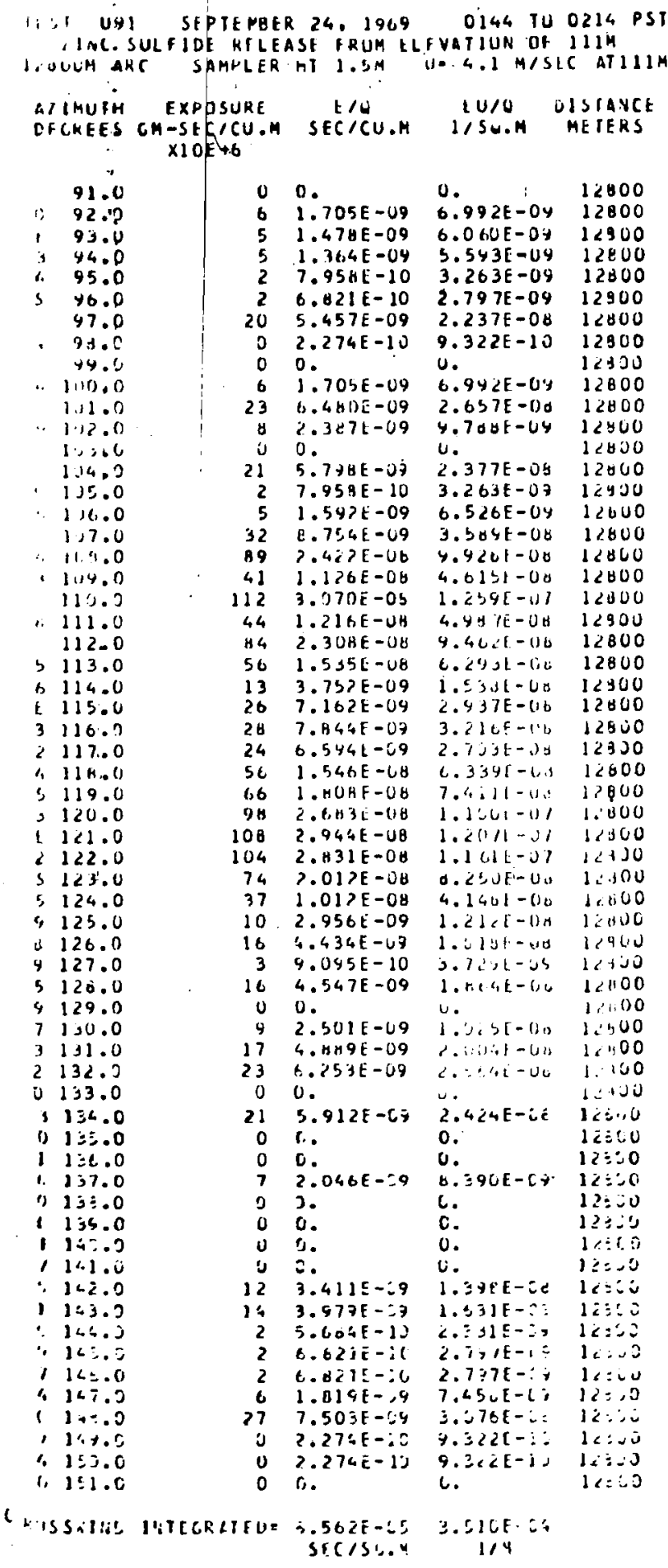

TEST. U91 SEPTEMUEK 24, 19690144 IL U214 PST FLUUKESCEIN NELLASE FRUM ELFVATIUT: IIF JUK. IZOUJM AKC SAMPLIR III I.S:I UE 3.0 MISFE AI SGM

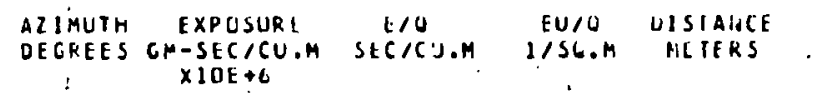

$\begin{array}{rl} & 91.0 \\ 0 . & 92.0 \\ 1 & 93.0 \\ & 94.0 \\ 4 & 95.0 \\ 5 & 96.0 \\ & 97.0 \\ 4 & 95.0 \\ & 99.0\end{array}$

4100.0

4101.0

9102.0

4103.0

104.0

105.0

5106.0

107.0

4113.0

61119.0

110.0

$t 111.0$

4112.0

5113.6

6114.0

C 115.0

116.0

117.0

$411 \mathrm{d.0}$

5119.0

123.0

L 121.0

122.0

5123.0

5124.0

$\therefore 125.0$

$+126.0$

9127.0

$5128: 0$

$y 129.0$

7130.0

131.0

132.0

$0 \quad 133.0$

134.0

0 195.0

1136.0

- 137.0

0134.0

c 139.6

l. 140.0

7141.0

5142.6

1143.6

0 144.0

9145.0

7146.0

4147.0

(1 149.1

4149.0

A 151.0

c. 133.0

E 135.0

\section{.}

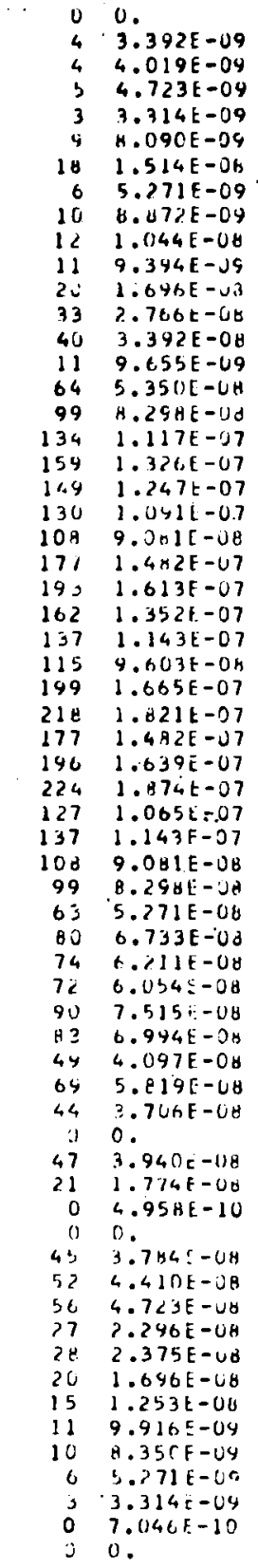

0.

$1.2896-00012000$

$1.527 E-00 \quad 12400$

$1.7455-4012800$

$1.25 \times 5-0012800$

$3.074 E-0812800$

$5.751 E-08 \quad 126 J U$

2.003 lic 12800

$3.3725-0512000$

$9.4601-1) B 12800$

$3.5740-0012800$

G.44EE-CA 12800

1.031E-07 12800

$1.285[-0712000$

$3.60 y E-c 0 \quad 1<060$

$2.03 \div[-07$ 1 2060

$3.13 j \mathrm{j}-6$ 12 $120 \mathrm{k}$

$4.244 t-v 7$ 1.28JJ

2.037t-01 12JC0

$4.7401-57 \quad 1<860$

$4.1451-07 \quad 12800$

$3.451 t-0712600$

$5.632 \mathrm{~F}-\mathrm{U} 7 \mathrm{1} 26 \mathrm{JJ}$

$0.120[-0712400$

$3.1376-01 \quad 12000$

$4.34 \mathrm{jF}-\mathrm{CP} 1<800$

$3.6445-07 \quad 12800$

$6.3271-0712000$

$6.922 \mathrm{E}-07 \quad 1280 \mathrm{U}$

$5.632 \mathrm{E}-07$ 12RUO

$\begin{array}{ll}5.632 \mathrm{E}-07 & 12800 \\ 0.223 \mathrm{~F}-01 & 12000\end{array}$

$7.120 E-07 \quad 12800$

$4.0461-07 \quad 12800$

$4.343 \mathrm{E}-07 \quad 1<800$

$3.451 E-07 \quad 12800$

$3.153 E-67 \quad 1<800$

$2.003 E-07 \quad 12600$

$2.5585-07 \quad 12800$

$2.360 f-07 \quad 1<800$

$2: 3011-07 \quad 12800$

$2.856[-07 \quad 12600$

2.CSOE-CI 12RUO

$1.557 \mathrm{E}-07 \quad 12800$

$3.2115-07$.12800

$1.400 E-07,12000$

$\because$

$1.497[-117 \quad 12800$

$6.7431-08 \quad 12000$

$1.0846-0 y \quad 12300$

1.

$1.43 M I-07 \quad 1<800$

$1.676 F=07 \quad 12800$

$1.7435-0112000$

$0.720 E-60 \quad 1<860$

$9.0241-c 012000$

$6.446 E-68 \quad 12400$

$4.7601-08 \quad 1<800$

$3.7601-j 81<800$

$3.17 j E-0012000$

c.voji-va lavou

$1.2541-6012800$

$2.677[-09 \quad 12600$

u. 12000

CRUSSWINO INTEGRAILU $=8.000 \mathrm{E}-04 \quad 3.040 \mathrm{~L}-09$ 


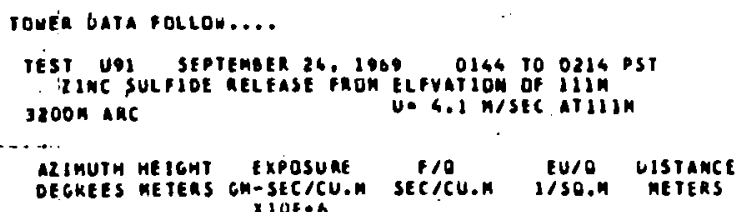

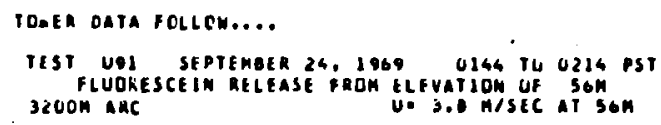

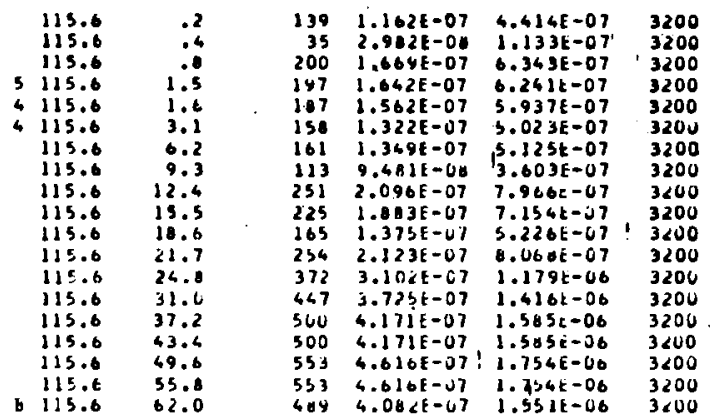


CROUND LEVEL SAMPLING GOUY TO 12 SULM: HJTH PUSSIULE EXCEPTJON OF FLUDRESCEIN AT $12 B O O M$. ALL ARCS EMBRACE THE CROSSHINO EXTENT OF IHE TKACEK. TKACER UISTRIUUTIONS EXIEND ABOVF TUP UF THE SINGLE SAMPLING TOHER. MO LIMC SULFIOE lIGSERYLL UN GUUM. GUJH UK IGUUM AKCS

TEST U92 SEPTEMSEK 26 , 1960 CU4H TII OJIV PSI

ZINC SULF IOE RFLEASE FROM ELFVAIIUY OF III

4 OOM ARC SAMPLER HT $1.5 \mathrm{SM} U=5.4$ M/SEC ATIIIM

ALIMUTH EXPOSURE
DEGREES CH-SECICU.M SECICU.M IIU/O OISTANCE
XIOE+G $\mathrm{X} 10 \mathrm{E}+6$

$146.0 \quad 00.00460$

CRUSSHINO INTECRATED. O. SEC/SO.ti $* 1 / \mathrm{H}$

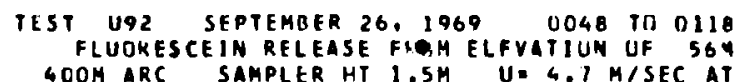

TEST U92 SEPTEMBER 26, 1969 GOOMORESCEIN RELEASE FRAM ELFVATIUN UF 564

AZIMUTH EXPOSURE E/Q EUIO DISTANCE DEGREES GH-SECICU.M SECICU.H IISO.M METERS $\mathrm{XIOE}+6$
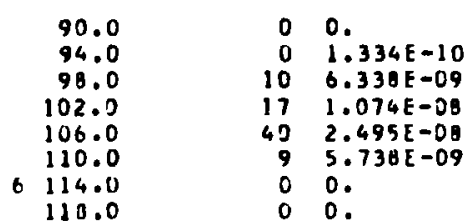

$6.272 E-10$ $2.979 E-00$ $5.049 E-08$

$1.173 E-07$

$2.697 \mathrm{E}-\mathrm{OH}$ 0.

CRUSSHINO INTEGRATED= $1.33 H E-06 \quad 6.287 E-06$ SFC/SO.M O. $1 / M$
TEST U92 SEPTEHBER 26.1969 CO4B TU 0119 PST IINC SULFIDE RELEASE FROM ELFVATIUN UF IIIM GOOM ARC SAMPLEK HT $1.5 \mathrm{M} U=5.4 \mathrm{M} / \mathrm{SEC}$ ATI11M $\begin{array}{lccc}\text { AZIRUTH EXPOSURE } & E / O & \text { EU/O UISTALCE } \\ \text { OEGREES CM-SECICU.M SECICU.M } & 1 / \text { SU.Y MLIERS }\end{array}$ $\times 10 \mathrm{E}+6$

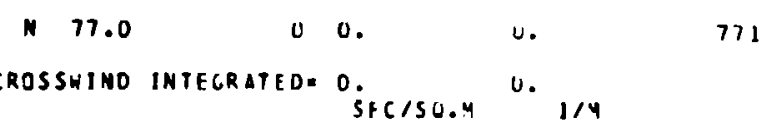

TEST U92 SEPTLMBEK 26, 1969 LO48 JU DI1Y HST ZINC SULFIOE RELEASE FRUM CLFVAIIUN LIT IIIM $1600 M$ ARC SAMPLER HT $1.5 \mathrm{M} U=5.4 \mathrm{M} / \mathrm{SEC}$ AIIIIM $\begin{array}{lccc}\text { AZIMUTH EXPOSURE } & \text { E/O } & \text { EJ/O OISTANCE } \\ \text { DEGREES CH-SECICU,M SFC/CU.H IISU.M MITLES }\end{array}$ XJ OE +6

N 73.4 J 3.4 v. 1568

CROSSHIND INTEGRATEOE O. SECISU.R. U. IIH

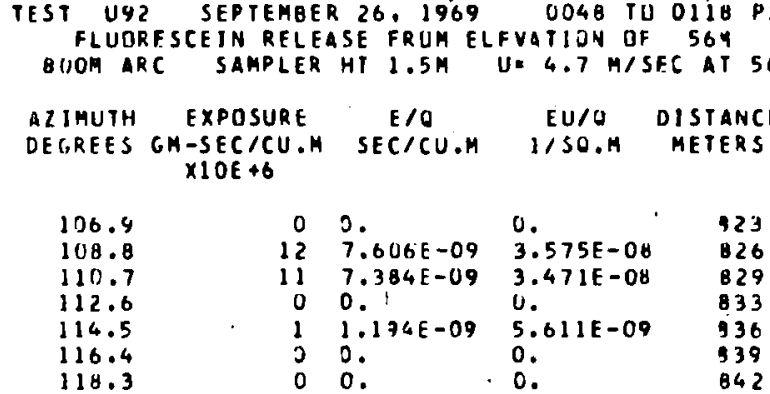

CRESSHIND INTEGRATED= $4.444 E-07 \quad 2.089 E-06$ SECISO.M $\quad 1 / \mathrm{M}$

TEST U92 SEPTEMEER 26, 1969 OO4B TO 0118 PST FLUTRESCEIN RELEASE ERUM ELFVATIUN OF $56 \mathrm{M}$ I6OJM ARC SAMPLER MT $1.5 M \quad U=4.7$ MIJEC AI $56 \mathrm{M}$

AZIMUTH EXPDेSURE E/Q EU/O OISTANCE DEGREES GM-SECICU,M SECICU.M IISU.M METERS $X 10 E+6$

\begin{tabular}{l}
99.0 \\
$E \quad 90.0$ \\
$E \quad 91.0$ \\
\hline 92.0 \\
\hline 93.0 \\
\hline 93.6 \\
94.6 \\
95.6 \\
96.6 \\
47.6 \\
91.6 \\
99.6 \\
100.6 \\
101.6 \\
102.6 \\
103.6 \\
104.5 \\
115.5 \\
116.5 \\
107.5 \\
108.5 \\
109.5 \\
110.4 \\
111.4 \\
112.4 \\
113.4 \\
114.4 \\
115.3
\end{tabular}

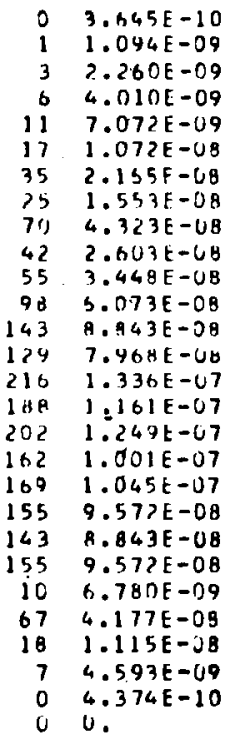

$1.713 E-09$ $5.140 E-O S$ $1.062 \mathrm{E}-\mathrm{OH}$ $1.885 \mathrm{E}-08$

$3.324 \mathrm{E}-\mathrm{CO}$

$5.037 \mathrm{E}-08$

1.01 UE -07

$1.5945-39$

$2.032 t-117$

$1.22 j[-07$

1.621t-01

$2.654 \mathrm{~F}-07$

4. $156 E-07$

$3.745 \mathrm{E}-07$

$6.261 t-67$

$5.450 F-07$

5. $27 \cup E-07$

$4.7 J 5 E-07$

$4.91 J E-U 7$

$4.444 \mathrm{E}-07$

4. $136 E-07$

$4.499 \mathrm{E}-07$

3.1 $17 \mathrm{E}-08$

$1.753 E-07$

$5.242 E-3 B$

$2.159 E=08$

$2.056 E-09$

0 .

1595

1596

1598

1600

1602

1503

1504

1506
$160 \%$

1510

1611

1513

is 15

1617

$15 \times 0$

1622

1523

1325

1620

1630

1632

1633

1637

1638

CRIISSHIND INTEGRATFD: 3.572E-U5 1.726E-04 
TEST U92 SEPTEMBER 26. 1969 TO48 TU O118 PST IINC SULFIOE RELEASE FKUM ELFVAT SUN OF IIIM 3200 ARC SAMPLER HT $1.5 \mathrm{M}$ U. $5.4 \mathrm{M} / \mathrm{SEC}$ ATIIIH

A2IYUTH EXPOSJRE

DECREES GH-SECICU.M SFCICU.Y USU.M MLIERS

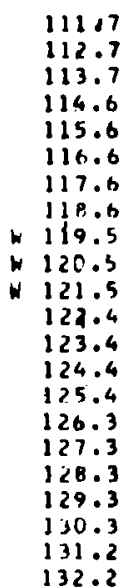

$$
\begin{aligned}
& 246 \\
& 458 \quad 1.290 E-07 \\
& 3209.169 \mathrm{E}-J \mathrm{~S} \\
& 3419.326 E-U O \\
& 3339 \\
& 00 . \\
& 7202.101 \mathrm{t}-\mathrm{U} \\
& 1634.14 \\
& 00 . \\
& \text { O } 0.145 E-U A C \\
& 143.987 t-69 \\
& 2 . \\
& 0 . \\
& 7.973 E-18 \\
& 3.104 t-09 \\
& \text { a.7711 -0y } \\
& i^{12} 3 .
\end{aligned}
$$$$
143.907 t-04 \quad 2.1531-110
$$$$
27.973 E-10 \quad 4.305 t-09
$$$$
\text { - } 2.392 \mathrm{E}-04 \quad 1.292 \mathrm{E}-00
$$

$2.153 t-08$

U.

$\because 30, E-1,4$

1. $722 \mathrm{E}=0 \mathrm{OL}$

4. I SCE-U

$1.437 t=0$

u.

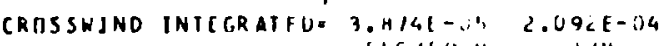

$$
\text { SAC SH.H }
$$

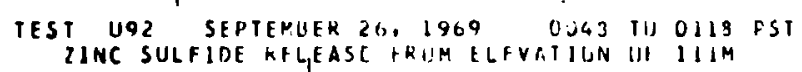
SUOUM ARC SAMPIER HI L.SM $U=S . M$ MISIC ATIIIH:

ALIMUTH EXPOSJRF I/6 IU/W UISIANLL DEGKEES CH-SFCICU.H UL/CU.M IISU.M YEIRKS $\times 101+6$

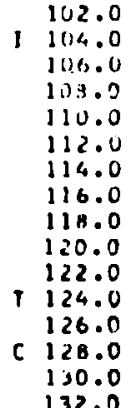

$$
\begin{aligned}
& 00 . \\
& \text {. } \\
& \text { i) } 0 \\
& .317+10 \\
& 2 . \\
& 4.6 .19,1-101 \\
& \text { 41. } 1.2471-02 \\
& \begin{array}{l}
130 \\
96.00115-U A \\
2.742 E-6 E
\end{array}
\end{aligned}
$$

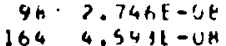

$$
\begin{aligned}
& 164 \quad 4.5411=U H \\
& 235 \quad 3.579 \mathrm{t}-0 \mathrm{E} \\
& 00 . \\
& 0 \quad 2.337 t-10 \\
& 0 \text {. } \\
& 1.169 \mathrm{E}-\mathrm{U9}
\end{aligned}
$$

ᄂ.

-

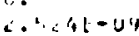

1. तU, OU - OU

¿. OSSt-01

$1.443 \mathrm{E}=07$

C. 4 OUE -07 $3.533 \mathrm{E}-\mathrm{UT}$

$6.342 E-07$

$1.202 E-0 S$ .

$6.311 E-0 S$

U.

CRDSSHIND INTECRATFD= $5.407 E-0 S$ 2.920E-04
TEST U92 SEPTEMAER 26, 1469 FLUDRESCEIN RELEASE FROM ELFVATIJY OF 564 $3200 M$ ARC SAMPLER HT $1.5 \mathrm{M} U=4.7 \mathrm{H} / \mathrm{SEC}$ AI $56 \mathrm{M}$

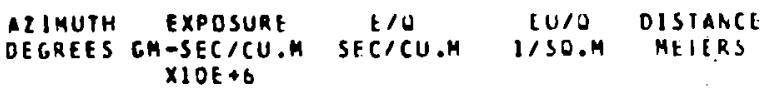

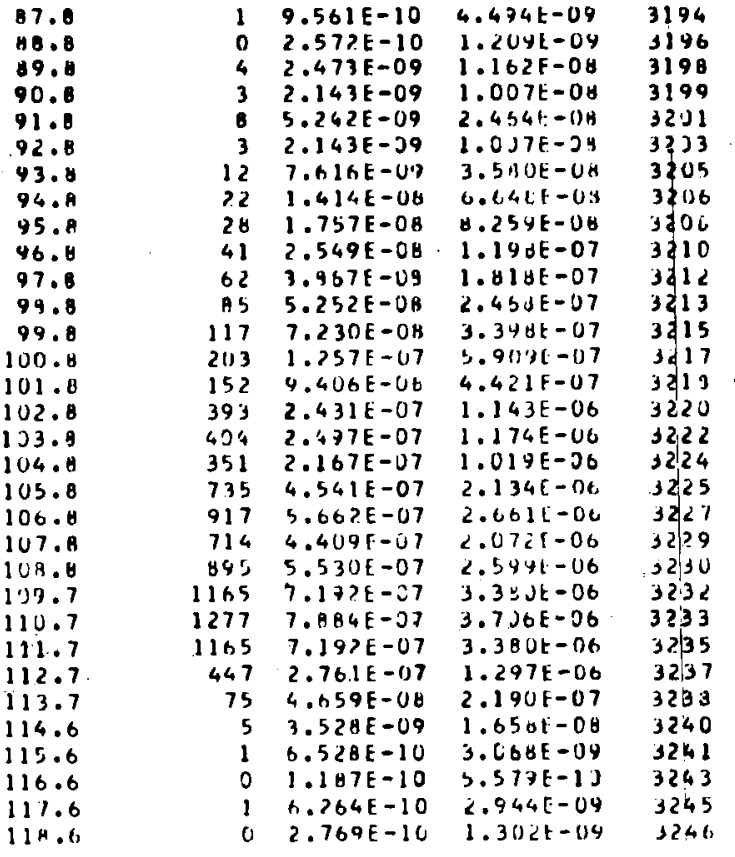

(KIISSWINU INTEGRATED $=3.147 E-04 \quad 1.51)$ IL-OS SFC/SO.M 1/4 
TEST UQ2 SEPTEMEEK 26. 1964 O248 TU 0119 PST ZINC SULF IDE RELEASE FRUM ELFVATIUN OF IIIH TOOOM ARC SAMPLER HT $1.5 \mathrm{M}$ U1:5.4 M/SEC AT1114 AZIMUTH EXPOSURE FIU DJIO DISTANCE

DEGRES GM-SEC/CU.H SEG/CU.M I/SH.M METERS $\times 10 \mathrm{O}+\mathrm{C}$
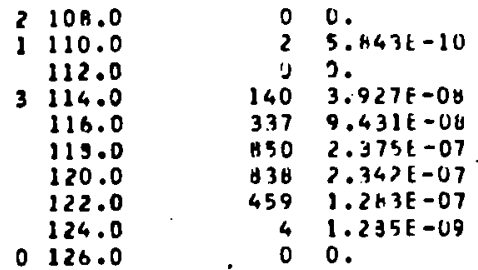

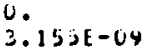

$2.12 U E-07$

$3.093 E-07$

$1.20<E-00$

$1.20 S E-06$

$6.929 E-07$

$6.942 E-09$

0 .

0126.0

CROSSHIND INTECRATED $1.841 \mathrm{E}-04 \quad 9.9396-04$ SEC/SO.M $\quad 1 / M$

6000
7000
7300
7210
1220
7190
7150
7120
7100
7100

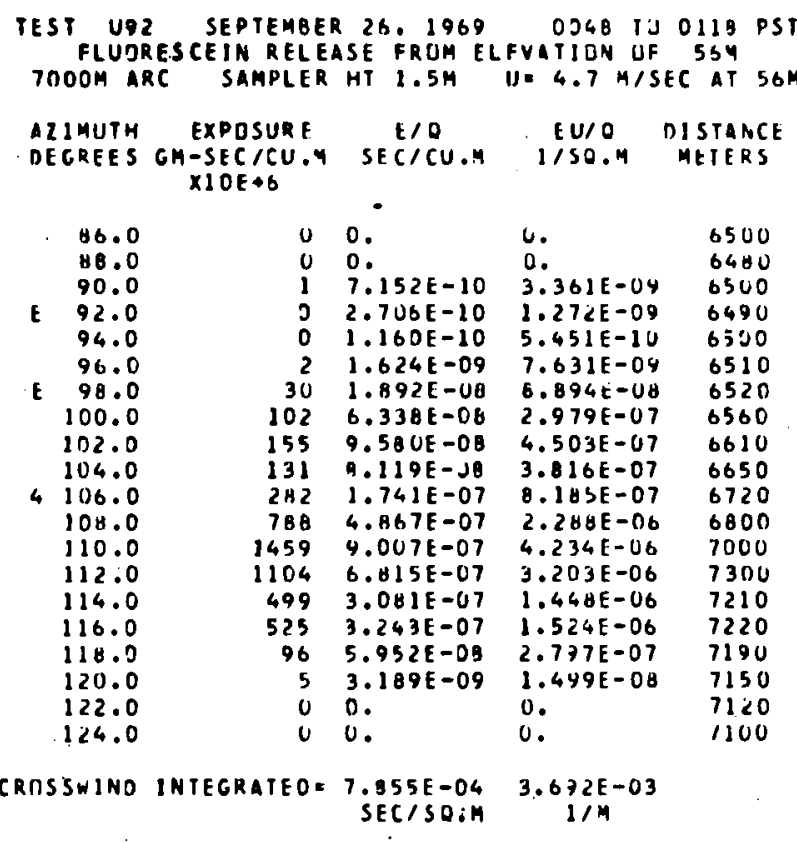

TEST U92 SEPTEMBER 26, 1969 UO4H IIJ O11B PST FLUDRESCEIN RELEASE FRUM ELFVAIIJV DF 564

$1280 O M$ ARC SAMPLER HT $1.5 \mathrm{H} U=4.7 \mathrm{M} / \mathrm{SEC}$ AT $56 \mathrm{H}$

$\begin{array}{lcc}\text { ALIMUTH EXPOSURE } & \text { E/O } & \text { EU/O UISIANCE } \\ \text { DECKEES CM-SECICU.M SEC/CU.H I/SH.M MEIEKS }\end{array}$ $\times 10 E \bullet 6$

TEST U92 SEPTEMBEK 26. 1969 C346 IU J119 PST ZINE SULF IOE RELEASE FKUM ELFVATIUN UF $111 \mathrm{M}$ $12800 M$ ARC SAMPLER HT $1.5 \mathrm{M} U=5.4 \mathrm{M} / \mathrm{SEC}$ MTIIIM

\begin{tabular}{|c|c|c|c|c|c|}
\hline \multicolumn{2}{|c|}{$\begin{array}{l}\text { AZ IMUTH } \\
\text { OECEEES }\end{array}$} & $\begin{array}{c}\text { EXPDSURE } \\
G H-S E C / C U . M \\
\times 1 O E+6\end{array}$ & $\begin{array}{c}\mathrm{L} / 0 \\
\text { SECICU.M }\end{array}$ & $\begin{array}{c}t J / 2 \\
1 \% \leq \cup . M\end{array}$ & $\begin{array}{l}\text { DISIAYCL } \\
\text { MEIERS }\end{array}$ \\
\hline 3 & $\begin{array}{l}106.0 \\
107.0\end{array}$ & $\mathfrak{l}$ & $\begin{array}{l}0 . \\
3.5 i / 6 t-10\end{array}$ & $\begin{array}{l}\text { l. I S } 3 t-U y \\
\text { l. }\end{array}$ & $\begin{array}{l}1<\theta<0 \\
1<d \dot{U}\end{array}$ \\
\hline & $1 \cup B .0$ & 27 & $7.3531=(19$ & (1. 1) $2<E-(1)$ & 12460 \\
\hline 7 & 109.0 & 15 & $4.3 i 41-i 4$ & C. ISbt-lin & $12 y 20$ \\
\hline 3 & 110.0 & 5 & $1.1141-69$ & b. $2040 t-114$ & 12400 \\
\hline 5 & 111.0 & 23 & $0.661 \mathrm{E}-00$ & $3.597 \mathrm{E}-00$ & $12 H O U$ \\
\hline & 112.0 & 23 & $1.427[-09$ & $3.471 \mathrm{E}-0 \mathrm{~d}$ & 12500 \\
\hline$s$ & 113.0 & 27 & $7.596 E-69$ & $4.10 \mathrm{CE}-00$ & 12800 \\
\hline 3 & 114.0 & 30 & $8.6485-07$ & $4.67 \cup E-0$. & $1 \angle 960$ \\
\hline E & 115.0 & 25 & $7.012 E-09$ & $3.750 E-U H$ & $1<930$ \\
\hline 2 & 116.0 & 22 & $0.31][-04$ & $3.400 \mathrm{E}-00$ & 12800 \\
\hline 3 & 117.0 & 6 & $1.67111-c 7$ & $1.010 t-00$ & 12005 \\
\hline 2 & 110.0 & 10 & $2.927 t-64$ & $1.17 u k-48$ & 12160 \\
\hline 6 & 119.0 & 6 & $1.8731-09$ & $1.010 E-130$ & 128.10. \\
\hline 1 & 120.0 & 1 & $3.506 E-10$ & $1.8+3 E-04$ & $1<y .00$ \\
\hline I & 121.0 & 0 & & & $1<000$ \\
\hline & 122.0 & 0 & $1.164 \mathrm{E}-10$ & $6.311 E-10$ & $1<8(10)$ \\
\hline & 3.0 & 0 & 0 . & L. & 12800 \\
\hline 8 & 124.0 & 5 & $1.519 E-69$ & $8.204 E-03$ & 12800 \\
\hline 9 & .0 & $u$ & 0. & i. & 12900 \\
\hline
\end{tabular}

CRUSSWIND INTEGRATED= $1.454 E-657.853 E-0 S$ SECISU.H
(2)

b 91.0

4.92 .0
E 93.0

43.0
494.0

495.0

496.0

97.0

$798 \%$

99.0

6100.0

4102.0

113.0

104.0

$t 105.0$

106.0

107.0

IกA. 0

7109.0

110.0

5111.0

113.0

114.0

9115.0

115.0
116.0

117.0

$11 \mathrm{u} . \mathrm{C}$

() 119.0

120.3

E 121.0

122.0

123.0

4124.0

$+125.0$

$-126.0$

- 127.0

912.4 .0

B 124.0

7330.0

$1 \$ 1.0$

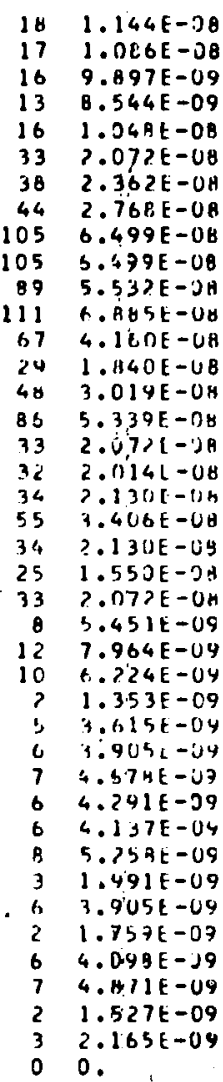

5.37 UE-38

$5.106 E-0 B \quad 1 \angle 800$

$4.652 E-0812800$

$4.016 E-08 \quad 12800$

$4.924 E-08 \quad 12900$

$9.739 \mathrm{E}-0 \mathrm{E} \quad 12500$

$1.110 E-07 \quad 12800$

$1.361 E-07 \quad 12800$

$3.054 \mathrm{E}-07 \quad 12800$

$3.054 \mathrm{E}-0712900$

$2.6525-07 \quad 12350$

$3.236 t-01 \quad 12800$

$1.95 b 5-07 \quad 12800$

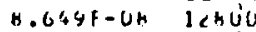

$1.41 \% \mathrm{~F}-07$ 12BUU

$2.5 J 4 E-117 \quad 12900$

$9.7 \pm$ yf -0 A 12930

$4.4671-0135$ 12400

1.0011-01 12800

$1.001 t-0712800$

1.0J1E-U7 12900

$7.236 \mathrm{E}-2 \mathrm{H} \quad 12320$

$9.734 E-0 S 12800$

$2.562 t-00 \quad 1<H 00$

$3.743 \mathrm{~F}-0 \mathrm{~d} 12400$

$2.423 F-11$ H 12 AJO

6.36Ut-liy 12b110

$1.6+95=041>400$

1. Cis?-01, 1>AUO

$2.1+7 t-J e \quad 1<46$

c.01/F-0. 1230

$1.944[-U B \quad 1 \angle A O O$

$2.4711-100 \quad 12000$

9.35 ती $09 \quad 1 \angle 500$

1. Hist-0n 12HUO

b.2S7E-UY 12500

$1.926[-3] 12930$

$2.26 y t-0412800$

$7.1771-0 y \quad 12800$

$1.010 \mathrm{E}-0 \mathrm{O} \quad 12800$

0 .

12800 


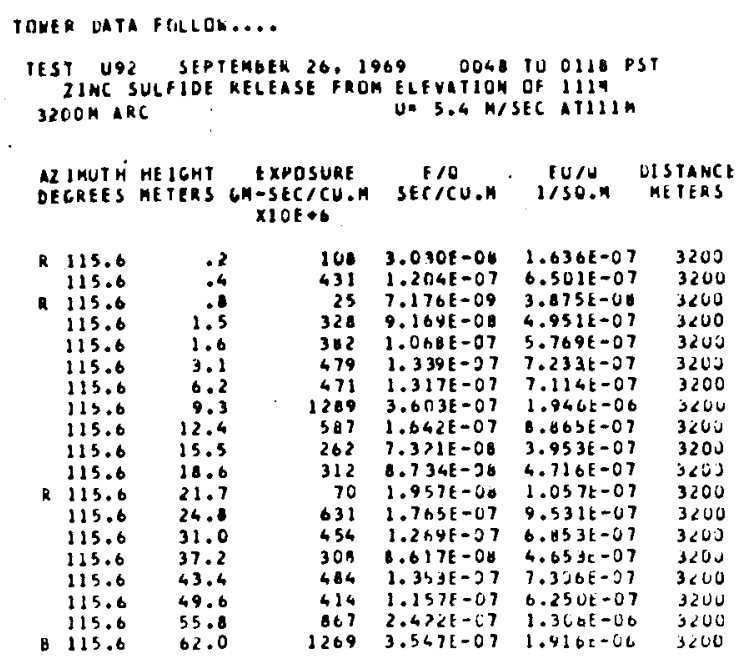

IDAF DATA FOLLOW....

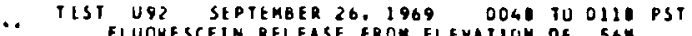
FLUOKESCEIN RELEASE FRON ELFYATIOH OF 564
3200 URC

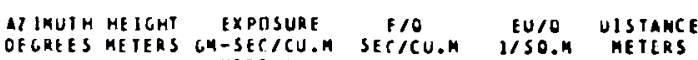
$X 10 E \div 6$

\begin{tabular}{|c|c|c|c|c|c|}
\hline $\begin{array}{l}115.6 \\
115.6 \\
115.6\end{array}$ & .2 & $\begin{array}{l}0 \\
4 \\
1\end{array}$ & $\begin{array}{l}0 . \\
2.60 B 5 E-00 \\
1.022 E-00\end{array}$ & $\begin{array}{l}0 . \\
1.224 E-08 \\
4.104 t-09\end{array}$ & $\begin{array}{l}3200 \\
3200 \\
3200\end{array}$ \\
\hline 115.6 & 1.5 & $i$ & $6.5>8 E-10$ & $3.060 t-09$ & 3200 \\
\hline 125.6 & 1. A & 7 & $5.63 B E-09$ & $2.650 E=08$ & 3200 \\
\hline 115.6 & 3.1 & 6 & $4.055 E-09$ & $1.996 \mathrm{E}-90$ & 3200 \\
\hline 113.6 & 6.2 & 104 & $6.439 E-08$ & $3.026 t-07$ & 3200 \\
\hline $\begin{array}{l}115.6 \\
113.6\end{array}$ & $\begin{array}{r}9.3 \\
12.4\end{array}$ & $\begin{array}{l}308 \\
895\end{array}$ & $\begin{array}{l}1.9 n 3 E-07 \\
5.53 \cup E=07\end{array}$ & $\begin{array}{l}8.946 E-07 \\
2.599[-06\end{array}$ & $\begin{array}{l}3200 \\
3200\end{array}$ \\
\hline 115.3 & 15.5 & 1691 & $1.03 B E-06$ & $4.377 \mathrm{t}-06$ & 3200 \\
\hline 115.6 & 10.6 & 2555 & $1.57 B E-26$ & $1.625 E-26$ & $3<02$ \\
\hline 115.6 & 21.7 & 4036 & $2.492 E-06$ & $1.171 E-05$ & 3200 \\
\hline & 24.8 & 5932 & $3.6+2 E-06$ & $1.721 E-05$ & 3200 \\
\hline 115.6 & 31.0 & 7766 & $4.794 E-0 B$ & $2.253 E-0.5$ & 3200 \\
\hline 115.3 & 37.2 & 12278 & $7.591 E-06$ & $3.5681-05$ & 3200 \\
\hline 5.6 & 43.6 & 11650 & $7.17 \angle E-26$ & $3.380 E-05$ & 3200 \\
\hline 5.6 & 49.6 & 10679 & $6.542 E=36$ & $3.278 E-D 5$ & 3202 \\
\hline 115.0 & 58 & 10679 & $0.59<t-66$ & $3.098 E-05$ & 3200 \\
\hline .6 & 62.0 & 10356 & $0.393 E-06$ & $3.005 t-05$ & $3<00$ \\
\hline
\end{tabular}


GRIJUHG LEVEL AND TUWEK SAMPLING 200M TO 3200M. ALL ARCS ERURACE CKUSSHINU EXTENT UF IRACER DISTRIGUTIUNS AL THOUGH IRACERS TRUNCATEU AT TOPS UF TOHERS. EXTRAPOLATIOA IS OISTIMCT PUSSIBILIIY UA IHIS IEST RELATIVELY SMPLL AMDUNT JF RHUDAMINE DISPERSLU LEAVES CONSIUERABLE UNCERTAINTIESIN INJIVIDUAL EXPUSURE VALUES.

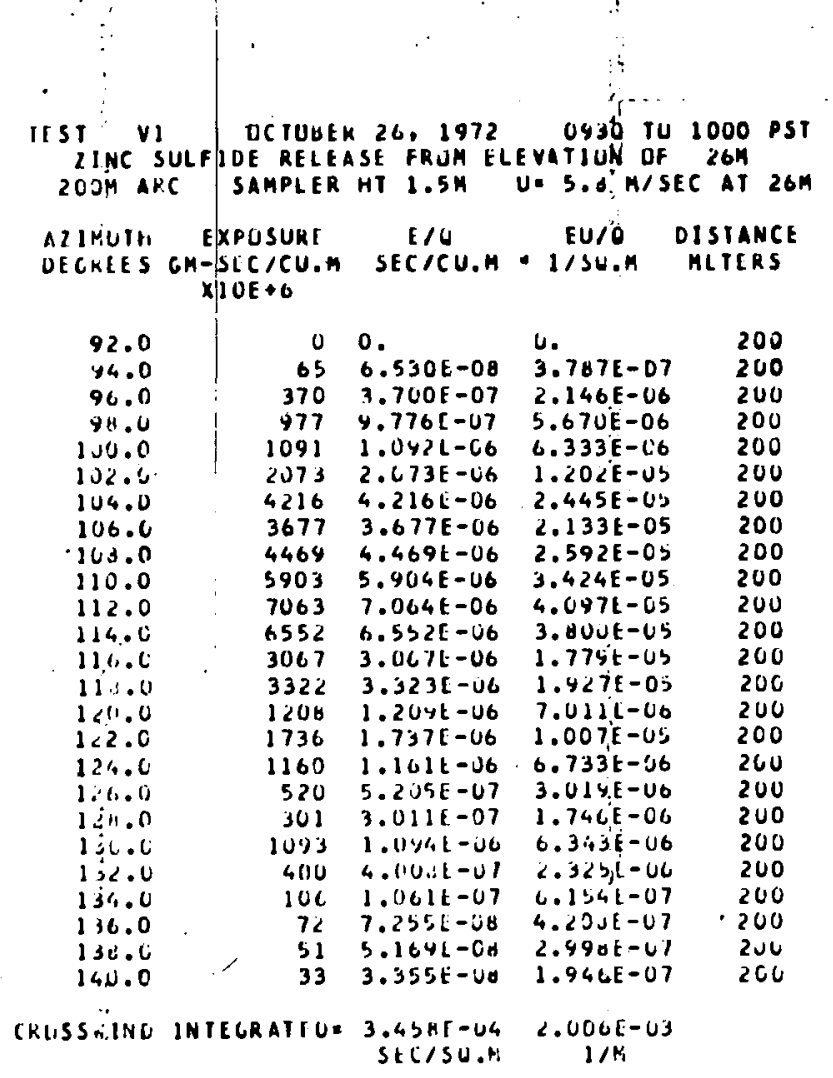

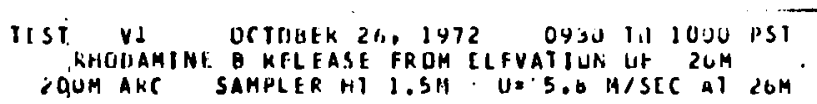

TLSI VI CCIDEER 26. 1972 0930 TU 1000 PST ZINC SULFIDE RELCASE FKUM ELFYATIUN OF $26 \mathrm{M}$ GOUI AKC SAMPLER IIT $1.5 \mathrm{M}$ U. 5 . A M/SEC AT $26 \mathrm{M}$ AIJAUIH EXPUSURE EIU EU/O OISIANCL LIGKEES CM-SECICU.M SECICU.M I/SO.M METEKS $X 10 \mathrm{E}+\mathrm{C}$

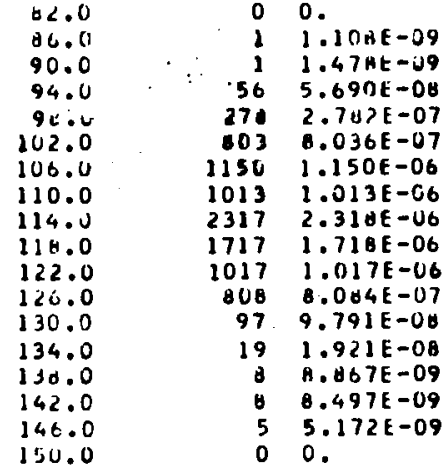

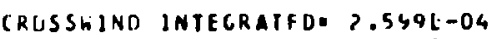
SEL/SO.M
0.

$6.428 E-04$ $0.571 E-09$ 3. $300 E-07$ $1.614 E-06$ $4.661 t-06$ $6.671 F-00$ 5.67 UE-06 1.344E- 05 $9.962 \mathrm{E}-06$ $5.899 E-06$ $4.682 E-06$ $5.678 . E-07$ $1.114 E-07$ $5.143 E-06$ $4.929 \mathrm{E}-0 \mathrm{E}$ $3.000 E-O D$ 0. 1. $507 E-03$

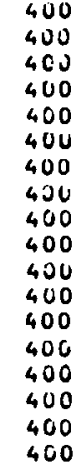

400

400

400

400

400

400

400

400

400

400

400

400
TEST VI UCTUHER KS, $147 \%$ USSO IU IOUO PSI RIIUIIAMLIHE O RFLFASL FKUH, ELFVAIIUI L 264 41.UM AKC SAMPLIK HI $1.5 \mathrm{H}$ HE S.D M/SEC.AT LCM

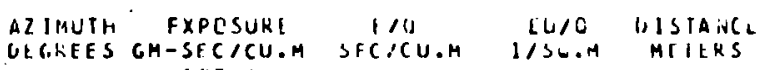
$X 10 E+6$
390.6 94.10
94.10
102.0
$10 \mathrm{c} \cdot \mathrm{C}$
110.0
114.0
116.0
122.0
$1<6.0$
4130.5
4134.0

$\begin{array}{rl}1 & 1.143 E-06 \\ 5 & 5.36: E-06 \\ 95 & 9.511 E-07 \\ 236 & 2.524 E-06 \\ 320 & 3.207 E-06 \\ 196 & 1.959 E-06 \\ 362 & 3.627 E-06 \\ 343 & 3.434 E-06 \\ 320 & 3.207 E-06 \\ 34 & 5.457 E-07 \\ 19 & 1.923 E-07 \\ 5 & 5.925 E-08\end{array}$

$6.63<t-04$ $3.075 \mathrm{E}-\mathrm{U} 7$ $5.516 E-C t$ $1.498 \mathrm{t}-\mathrm{C}$ ', 1. $B 6 C E-O S$ $1.136 E-05$ 2. $101 \mathrm{E}-05$ 2. $202 E-155$ $1.860 \mathrm{t}-0 \mathrm{~b}$ $3.105 E-06$

1. $115 \mathrm{E}-06$ $3.43 \cup E-07$

400 460 400 4रJ 400 400 400 400 400
400 400

CROSSHIND INTEGRATLO= $3.676 E-U 4$ S. $292 E-0$; 
IEST VI OCTOBER 26. 19720330 TU 1000 PST 2INC SULFIDE RELEASE FRUM ELFVATIUN DF $20 \mathrm{M}$ DOUH ARC SAMPLER HT $1.5 \mathrm{M}$ U $=3.0 \mathrm{M} / \mathrm{SEC}$ aT $20 \mathrm{M}$

\begin{tabular}{|c|c|c|c|c|}
\hline $\begin{array}{l}\text { AZI I HUTH } \\
\text { DEGREES }\end{array}$ & $\begin{array}{l}\text { EXPOSURE } \\
\text { CH-SEC/CU.H } \\
\times 1 O E+6\end{array}$ & $\begin{array}{c}E / U \\
\text { SEC/CU.M }\end{array}$ & $\begin{array}{c}\text { EU/O } \\
1 / S O . M\end{array}$ & $\begin{array}{l}\text { DISIANCE } \\
\text { METEKS }\end{array}$ \\
\hline
\end{tabular}

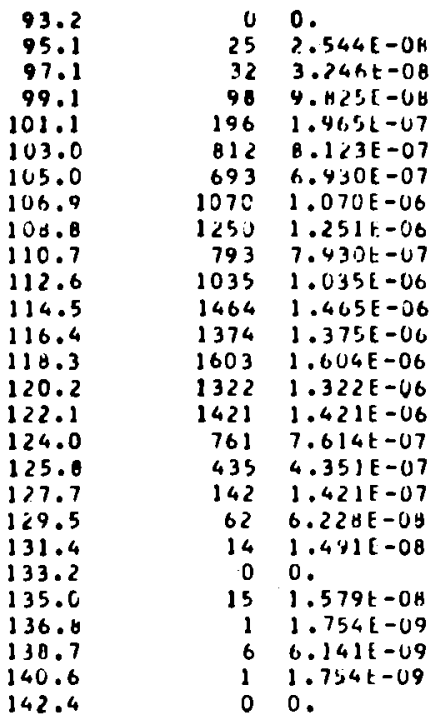

l.

l. $476 t-07$

$1.083[-01$

$3.649 E-07$

$1.140 \mathrm{E}-0 \mathrm{C}$

$4.712 \mathrm{E}-06$

$4.020 F-06$

$6.207 t-06$

$7.256 t-i 6$

$4.600 E-16$

$6.604 t-06$

$8.4975-06$

$7.973 \mathrm{E}-06$

$4.3011-06$

$7.660 \mathrm{E}-\mathrm{CO}$

$0.243 \mathrm{E}-06$

$4.416 E-0 C$

$2.324 \mathrm{E}-110$

$0.243[-07$

$3.61<E-07$

B.6 SUE-Od

U.

$4.158 F-00$

$1.01 d E-0 d$

$3.5621-08$

$1.014 \mathrm{t}=0 \mathrm{~d}$

c.

799
802
806
809
813
816
820
963
826
829
833
836
839
842
845
848
851
854
657
859
862
865
867
869
672
874
876

CRUSSHINO INIEGRATFU: $4.059[-04 \quad 2.354$ F-03 SLC/SU.M

$1 / \mathrm{M}$

TEST VI OCIMOER 26. 1972

OY30 TO 1000 PST ZINC SULFIOF RELEASE FRUM ELFVATIUN UF $26 \mathrm{M}$ $1200 \mathrm{M}$ AKC: SAMPLER HT $1.5 \mathrm{M} U=5.0 \mathrm{M} / \mathrm{SEC}$ AT $26 \mathrm{M}$

\begin{tabular}{|c|c|c|c|c|}
\hline $\begin{array}{l}\text { A } 11 \mathrm{HA} \\
\text { DECKE }\end{array}$ & $\begin{array}{c}\text { EXPOSURF } \\
\text { GH-SEC/CU.M }\end{array}$ & shécu. & $\begin{array}{l}\operatorname{los} 10 \\
1 / 50.4\end{array}$ & $\begin{array}{l}\text { UISIAIICE } \\
\text { MEIFRS }\end{array}$ \\
\hline
\end{tabular}
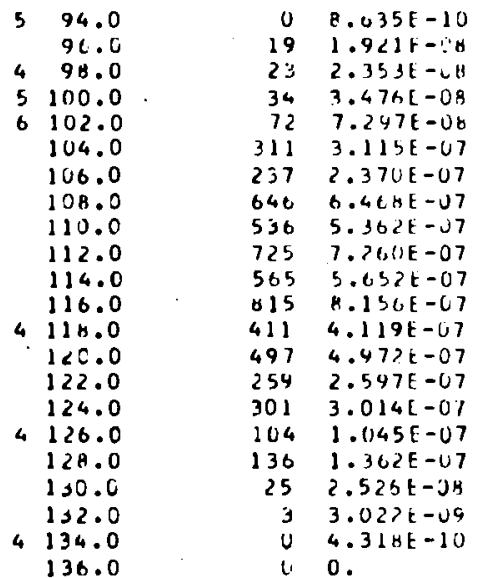

3. vunt-uy

$1.114 t-0 \%$

$1.30, b E-C$.

$2.0161-01$

$1.23<8-07$

$1.007[-46$

$1.375 E-06$

$3.7511-06$

3.11UE-UO

$4.211 E-06$

$3.27 U E-06$

$4.7, O E-C 6$

$2.3895-66$

$2.384 F-06$

$1.306 E-U 6$

$1.740 E-0 C$

$6.060 E-0$

$7.401[-0]$

$1.455 E-U 7$

$1.753 E-L 6$

$2.5346-09$

.

1200

1200

$1<00$

1200

1200

1200

1700

1260

$1: 00$

1200

1200

1200

1200

1200

1200

1200

1200

1 c00 $\begin{array}{llllll}\text { TEST VI OCTDBER } 26.1972 & 0930 & \text { TU } & 1000 & \text { PST } \\ \text { RHIDDAMINE B RELEASE FROM ELFVATIUN OF } 26 \mathrm{M} & \end{array}$ BCOM ARC SAMPLER HT $1.5 \mathrm{M}$ U= $5.8 \mathrm{M} /$ SEC AT $26 \mathrm{M}$

\begin{tabular}{|c|c|}
\hline DEGRLE & $\begin{array}{c}\text { EXPUSURE } \\
\text { GP-SEC/CU. }\end{array}$ \\
\hline
\end{tabular}

\begin{tabular}{|c|c|c|}
\hline 45.1 & 3 & $3.789 \mathrm{E}-\mathrm{OH}$ \\
\hline 47.1 & 2 & $2.067 E-08$ \\
\hline 94.1 & 6 & 6. $.200 E-08$ \\
\hline 101.1 & 17 & $1.737 E-07$ \\
\hline 2103.0 & 87 & ค. $7838-07$ \\
\hline 105.0 & 80 & $8.044 E-07$ \\
\hline $1: 6.9$ & 94 & $9.477 \mathrm{E}-07$ \\
\hline ICH.h & $10 \mathrm{~A}$ & 1.0 KE - U6 \\
\hline 110.7 & 9 & $4.300 E-U E$ \\
\hline $112 . t$ & 84 & $0.439 E-07$ \\
\hline 114.5 & 115 & $1.154 E-06$ \\
\hline 116.4 & 91 & $9.12+E-67$ \\
\hline 110.3 & 80 & $8.094 E-07$ \\
\hline 120.2 & 63 & $6.372 E-07$ \\
\hline 122.1 & 77 & $7.750 \mathrm{E}-07$ \\
\hline $1<4.0$ & 39 & $3.961 E-07$ \\
\hline 125.6 & 24 & $2.4 B D E-07$ \\
\hline 127.7 & 13 & $1.343 \mathrm{E}-07$ \\
\hline 129.5 & 3 & $3.100 E-08$ \\
\hline 131.4 & 0 & 0 . \\
\hline 133.2 & 0 & c. \\
\hline & & \\
\hline
\end{tabular}

$\begin{array}{ll}2.198 E-07 & 802 \\ 1.199 E-07 & 806 \\ 3.596 E-01 & 809 \\ 1.014 E-0 C & 813 \\ 5.044 E-06 & 816 \\ 4.695 E-06 & 820 \\ 5.494 F-06 & 823 \\ 6.243 E-00 & 826 \\ 5.344 E-07 & 829 \\ 4.895 E-06 & 833 \\ 6.693 E-06 & 836 \\ 5.294 E-06 & 839 \\ 4.695 E-06 & 842 \\ 3.696 E-06 & 845 \\ 4.495 E-06 & 848 \\ 2.297 E-06 & 851 \\ 1.438 E-06 & 854 \\ 7.791 E-07 & 857 \\ 1.748 E-07 & 859 \\ 0.79 & 862 \\ 0.9 & 865 \\ 0.1 & 967\end{array}$

CRISSWIND INTEGRATHOE 2 TO6E-04 1.616 WE-03

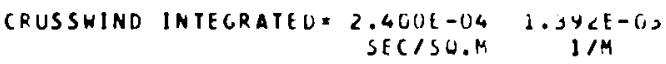


TEST V1 DCTOBER 26. 1972 0930 TU 1000 PST 2INC SULFIDE RELEASE FRUM ILIVATIIUN UT ZUM $1600 M$ ARC SAMPLER HT 1.514 W. 5.O M/SEC AT. 264

\begin{tabular}{|c|c|c|c|c|}
\hline $\begin{array}{l}\text { ALIIIUTH } \\
\text { DECKEES }\end{array}$ & $\begin{array}{c}\text { EXPOSURE } \\
\text { EM-SEC /CU.H } \\
\times 10 E+6\end{array}$ & $\begin{array}{c}E / O \\
\text { StC } / C U . M\end{array}$ & $\begin{array}{l}\text { IU/O } \\
\text { I/SO.M }\end{array}$ & $\begin{array}{l}\text { DISTANCE } \\
\text { MEIEKS }\end{array}$ \\
\hline $\begin{array}{l}91.6 \\
98.6\end{array}$ & $\begin{array}{l}6 \\
2\end{array}$ & $\begin{array}{l}6.049[-09 \\
2.746 t-09\end{array}$ & $\begin{array}{l}3.532 E-0 \mathrm{~B} \\
1.570 E-00\end{array}$ & $\begin{array}{l}1610 \\
1611 \\
1613\end{array}$ \\
\hline $\begin{array}{r}99.6 \\
100.6\end{array}$ & $\begin{array}{l}37 \\
53\end{array}$ & $\begin{array}{l}3.7 N 4 E-08 \\
5.345 E-08\end{array}$ & $\begin{array}{l}2.140 E-07 \\
3.100 E-07\end{array}$ & $\begin{array}{l}1613 \\
1615\end{array}$ \\
\hline 101.6 & .79 & $7.944 \mathrm{E}-00$ & $.4 .631 E-07$ & 1617 \\
\hline $\begin{array}{l}102.6 \\
103.6\end{array}$ & $\begin{array}{c}100 \\
89\end{array}$ & $\begin{array}{l}1.06 \text { Q } \\
0.931 E-07\end{array}$ & $\begin{array}{l}5.847 E-07 \\
5.180 E-07\end{array}$ & $i 610$ \\
\hline 104.5 & 144 & $1 . .448 E-07$ & $0.390 E-07$ & 1622 \\
\hline 105.5 & 129 & $1.249 \mathrm{E}-07$ & $7.535 E-07$ & 1623 \\
\hline 106.5 & 221 & $2.21 / 2 \mathrm{E}-07$ & $1.283 E-06$ & 1625 \\
\hline 107.5 & 98 & 9. $\mathrm{H} 7 \mathrm{AE}-\mathrm{OH}$ & $5.729 E-07$ & 1627 \\
\hline $\begin{array}{l}100.5 \\
109.5\end{array}$ & $\begin{array}{l}231 \\
410\end{array}$ & $\begin{array}{l}2.314 t-07 \\
4.107 E-07\end{array}$ & $\begin{array}{l}1.342 \mathrm{E}-0 \mathrm{O} \\
2.3132 \mathrm{E}-\mathrm{C.6}\end{array}$ & $\begin{array}{l}1626 \\
1630\end{array}$ \\
\hline 110.4 & 205 & $2.050 \mathrm{E}-07$ & $1.189 E-06$ & $1<32$ \\
\hline 111.4 & $14 \mathrm{H}$ & $1.409 t-07$ & $0.633 t-01$ & 1633 \\
\hline 112.4 & 334 & $3.349 t-47$ & $1.443 E-06$ & 1633 \\
\hline 113 & 250 & $2.5036-07$ & $1.4521-06$ & $16: 37$ \\
\hline 114.4 & 230 & $2.307 E-67$ & $1.334 t-66$ & 1638 \\
\hline 115.3 & 221 & $2.2121-07$ & $1.293 t-06$ & 1640 \\
\hline 116.3 & 401 & $4.0191-07$ & $2.331 E-16$ & 1641 \\
\hline 117.3 & 338 & $3.340 E-07$ & $1.966 E-06$ & $1 \in 43$ \\
\hline 116.2 & 252 & $2.524 E-07$ & $1.404 E-06$ & 1644 \\
\hline 119.2 & 320 & $3.261 \mathrm{f}-07$ & $1.842 \mathrm{E}-0 \mathrm{U}$ & 1646 \\
\hline 120.2 & 284 & $2.14 ; E-07$ & $1.646 E-06$ & 1647 \\
\hline 121.2 & $125^{\circ}$ & $1.23 d t-07$ & $7.299 \mathrm{E}-07$ & 1649 \\
\hline 122.1 & 196 & $1.969 \mathrm{E}-67$ & $1.142 \mathrm{E}-06$ & 1650 \\
\hline 123.1 & 105 & $1.056 E-07$ & $6.122 E-07$ & 1652 \\
\hline 124.1 & 272 & $2.727 E-107$ & $1.581 E-06$ & 1653 \\
\hline 125.0 & 135 & $1.360 E-07$ & $7.86 d E-07$ & 1655 \\
\hline 126.0 & 106 & $1.069 t-07$ & $6.2005-07$ & $1+36$ \\
\hline 126.0 & 71 & $7.104 E-0 B$ & $4.121 \mathrm{E}-1.77$ & 1658 \\
\hline 127.8 & 37 & $3.709 E-08$ & $2.198 \mathrm{E}-07$ & $165 y$ \\
\hline 124.8 & 24 & $2.436 t-00$ & $1.413[-07$ & 1660 \\
\hline 129.7 & 44 & $4.939 E-06$ & $2.865 E-07$ & 1662 \\
\hline & 9 & 9.4 & $5.494 E-08$ & 1663 \\
\hline 131.5 & 0 & & & 1664 \\
\hline
\end{tabular}

CRESSHIND INTEGRATFD $=1.597 E-U 4 \quad 4.263 E-04$ SEC/SC.M

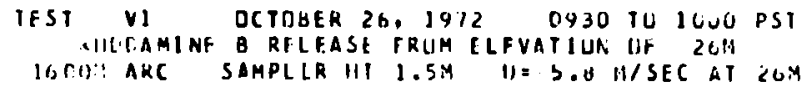

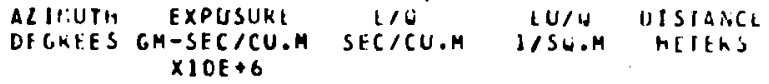

\begin{tabular}{|c|c|c|c|c|}
\hline 9.6 & 10 & $1.022 \mathrm{E}-07$ & $5.929 E-07$ & 1611 \\
\hline 94.6 & 12 & $1.295 E-07$ & $7.51 \cup E-U 7$ & 1013 \\
\hline 100.5 & 17 & $1.772[-07$ & $1.020 E-06$ & 1615 \\
\hline 101.6 & 0 & 0. & 0. & 16,17 \\
\hline 172.1 & 13 & $1.363 E-07$ & $7.405 E-07$ & 1510 \\
\hline 113.6 & 1 & $1.117 E-68$ & $1.054 E-67$ & 1020 \\
\hline 194.5 & 10 & $1.027 E-07$ & $5.929 E-07$ & 1022 \\
\hline 104.3 & 18 & $1.227 E-07$ & $7.114 \mathrm{E}-07$ & 1623 \\
\hline 100.5 & 14 & $1.431 E-07$ & $\triangle .300 E-07$ & 1625 \\
\hline 107.5 & 18 & $1.840 \mathrm{E}-07$ & $1.067 \mathrm{E}-06$ & 1527 \\
\hline 139.5 & 15 & $1.567 E-07$ & $9.091 E-07$ & 1628 \\
\hline 149.5 & 10 & $1.022 E-47$ & $5.9<9 E-07$ & 1630 \\
\hline 110.4 & is & $1.567 E-07$ & $9.091 \mathrm{E}-07$ & 1632 \\
\hline .111 .4 & 12 & $1.205 E-07$ & $7.51 \cup E-07$ & 1633 \\
\hline 112.4 & 17 & $1.772 F=07$ & $1.620 t-06$ & ios5 \\
\hline 113.4 & 16 & $1.636 E-07$ & $9.456 E-07$ & 1537 \\
\hline 114.4 & 19 & 1.9 UHE -67 & $1.1 \cup 7 E-06$ & 1638 \\
\hline 115.3 & 20 & $2.044 E-07$ & $1.186 E-0 B$ & 1040 \\
\hline $11 t \cdot .3$ & $y$ & 6.178E-OA & $4.743 E-01$ & 1641 \\
\hline 117.3 & 12 & $1.227[-67$ & $7.1145-07$ & 1643 \\
\hline $11 \cdot . ?$ & 15 & $1.567 E-.17$ & $4.0415-67$ & i 8,44 \\
\hline 119.2 & 16 & $1.636 \mathrm{E}-\mathrm{C} 7$ & $9.4 \mathrm{HCE}-0.7$ & 1546 \\
\hline $1: 3 . ?$ & 17 & $1.704 E-U 7$ & $3.601 f-01$ & $16: 7$ \\
\hline $1 \because 1.2$ & 22 & $2.249 \mathrm{E}-. .27$ & $1.1304 \mathrm{E}-06$ & 1644 \\
\hline $1 \div 2.1$ & 35 & $3.521 \mathrm{E}-07$ & $2.0428-06$ & 1650 \\
\hline 123.1 & 16 & $1.336 E-07$ & $9.406,1-67$ & 1692 \\
\hline 124.1 & 13 & $1.363 E-27$ & $7.905 t-07$ & 1653 \\
\hline $1<5.0$ & 1 & $1 . R] 7 E-U B$ & $1.054 \mathrm{~F}-07$ & 1655 \\
\hline $12^{\prime}, \ldots .11$ & 11 & $1.15 \mathrm{kF}-\mathrm{C} 7$ & $6.7191-07$ & 1650 \\
\hline $1 ? 6.9$ & h & $6.315 \mathrm{~F}-4$ & $3.9531-07$ & $\mid c ; 5,0$ \\
\hline 1.7 .8 & y & $6.859 \mathrm{E}-66$ & $5.1 \mathrm{~J} J \mathrm{f}-\mathrm{C} 7$ & $165 y$ \\
\hline $1<6 \cdot \theta$ & B. & $0.17 \mathrm{BE}-08$ & $4.74 J E-07$ & 1660 \\
\hline $1,4.7$ & 6 & $6.133 \mathrm{E}-08$ & $3.557 E=07$ & 1662 \\
\hline $130 . t$ & 6 & $6.133 E-i \theta$ & $3.557 E-07$ & 1663 \\
\hline
\end{tabular}

(KISSAINU) INTEGRATFD $=1.241 E-04$ 7.24dF-U4 SFC/S2.M $1 / \mathrm{M}$
TLST VI HCTIIPER 26, 1917 OY30 1111060 HSI

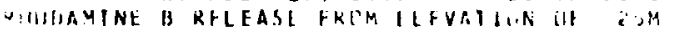

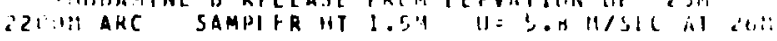

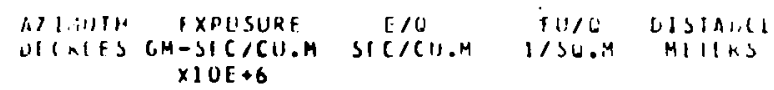
$\times 10 E+6$

\begin{tabular}{|c|c|c|c|c|}
\hline 134.0 & A & $4.0 .241-34$ & $5,1, \cdots+1-1,7$ & $22 \omega)$ \\
\hline 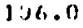 & 11 & 1.1 HE -07 & $0,0 \div 21-1,1$ & $\therefore$ iv \\
\hline$(\ldots, \ldots)$ & 13 & $1.347[-07$ & $\forall . C \alpha_{1} \alpha_{1}[-0]$ & .2(1) \\
\hline 116.0 & 12 & $1.2 \mathrm{~d}+[-67$ & $7.44+1-0.1$ & $\therefore 00$ \\
\hline 112.0 & 14 & $1.490 t-077$ & U. $64 \mathrm{UE}-U 7$ & ¿2: ن \\
\hline 114.11 & 11 & $1.1 \| 1 \mathrm{~F}-: 17$ & (.. Ah) E $=57$ & $?<0 U$ \\
\hline 116.2 & 20 & $2.0031-27$ & $1 . d i x t=6$ & $\therefore 1$, \\
\hline 114.0 & 27 & $2.1271-017$ & $1.5741-1) 6$ & 2200 \\
\hline $1 . n .0$ & 16 & $1,635,[-1 ; 7$ & $3.01118-07$ & 2260 \\
\hline 1.7 .0 & 34 & $1.4901-07$ & $0.64 \cup \mathrm{E}-\mathrm{U}$ & $c<00$ \\
\hline 124.0 & 12 & $1.234 E-07$ & $7.4485-01$ & $\angle 200$ \\
\hline $1.1 \%, 0$ & 5 & $5.547 \mathrm{E}-\mathrm{UE}$ & $3.217 t-u 1$ & 2200 \\
\hline $1 / 3.6$ & 5 & $5.239 E-U d$ & $3 . \cup 39 E-U 7$ & 2200 \\
\hline $1+10.0$ & 0 & 0. & c. & $\angle 206$ \\
\hline
\end{tabular}

(KI SSHINI) INTEGRATIOE $1.35 \mathrm{BL}-\mathrm{U} 47.366 \mathrm{~K}-04$ SECISW.11 


\begin{tabular}{|c|c|c|}
\hline \multicolumn{3}{|c|}{$\begin{array}{l}\text { TEST VI DCTOBER } 26,1972 \\
\text { ZINC SULFIOE RELEASE FRUM EL } \\
32 \text { OOH ARC SAMPLER HT } 1.5 \mathrm{SM}\end{array}$} \\
\hline $\begin{array}{l}\text { AZI I MUTH } \\
\text { DE GREES }\end{array}$ & $\begin{array}{l}\text { EXPDSURE } \\
\text { CM-SEC /CU.H } \\
\text { XIOE } \$ 6\end{array}$ & $\begin{array}{c}E / U \\
\text { SEC/CU.M }\end{array}$ \\
\hline 97.0 & 1 & $1 . N 26 t=09$ \\
\hline $9 A . n$ & 3 & $3.2+3 L=09$ \\
\hline 99.8 & 32 & $2.227 \mathrm{t}-\mathrm{UB}$ \\
\hline 100.8 & 17 & $1.7165-08$ \\
\hline 101.0 & io & $1.05 \% 1-0 \theta$ \\
\hline 102.6 & it & $1.8<6 L-U B$ \\
\hline 103.0 & 33 & $3.323 L-00$ \\
\hline 104.0 & 22 & $2.264 F-38$ \\
\hline 105.8 & 57 & $5.7335-60$ \\
\hline 106.8 & 75 & 7.33 HE $-O B$ \\
\hline 107.0 & 78 & $7.014 t-04$ \\
\hline 108.8 & 110 & $1.183 E-07$ \\
\hline 109.7 & 129 & $1.296 E-07$ \\
\hline 110.7 & 104 & $1.072 \mathrm{E}-07$ \\
\hline 111.7 & 44 & $9.443 \mathrm{E}-06$ \\
\hline 112.7 & 102 & $1.027[-07$ \\
\hline 113.7 & 143 & $1.435 \mathrm{k}-07$ \\
\hline 114.6 & 120 & $1.201 \mathrm{E}-07$ \\
\hline 115.6 & 104 & $1.041 \mathrm{E}-07$ \\
\hline 116.6 & 86 & O.B96E-OB \\
\hline 117.6 & 123 & $1.23 \mathrm{HE}-07$ \\
\hline 110.6 & 112 & $1.121 \mathrm{E}-07$ \\
\hline 119.5 & 58 & $5.806 E-0 B$ \\
\hline 120.5 & 104 & $1.04 \mathrm{HE}-07$ \\
\hline 121.5 & 63 & $6.353 E-08$ \\
\hline 122.4 & 59 & $5.915 \mathrm{E}-6 \mathrm{~B}$ \\
\hline 123.4 & 98 & $9.859 E-08$ \\
\hline 124.4 & 23 & $2.373 E-08$ \\
\hline 125.4 & 86 & $8.690 \mathrm{E}-0 \mathrm{~B}$ \\
\hline 126.3 & 20 & $2.00 \mathrm{BE}-0 \mathrm{~S}$ \\
\hline 127.3 & 7 & $7.668 E-09$ \\
\hline $1<0.3$ & 0 & 0 \\
\hline
\end{tabular}

CRUSSHIND INTEGRATED $=1.167 E-04 \quad 6.766 E-C 4$
0930 TU 1000 PST GVATIUN UF $20 \mathrm{M}$

EU/O DISTANCE 1/S2.M METERS

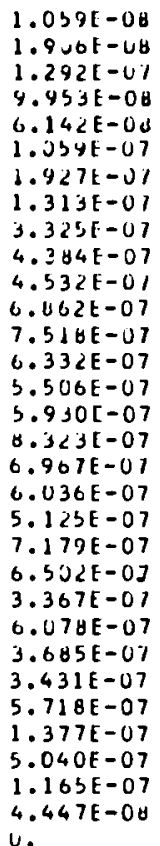

3212

$32: 3$

3215

3217

$321 \mathrm{H}$

3222

3224

$3<25$

3227

3229

3230

3232

3233

3235

3237

3230

324,0

3241

3243

3245

3246

3248

249

3251

3252

3253

3255

$32 \% 6$

3250

3259

3261

$$
\text { SEC/SO.M } \quad 1 / \mathrm{M}
$$
$U=5.0 \mathrm{H} / \mathrm{SEC}$ AT $26 \mathrm{H}$

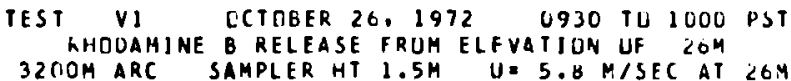

AZIMUTH EXPOSURE E/O EU/O DISIANCE DECREES GM-SEC/CU.H SEC/CU.M IISO.H METERS $\times 10 E+6$

\begin{tabular}{|c|c|c|c|c|c|}
\hline $\begin{array}{l}91.6 \\
99.0\end{array}$ & & $\begin{array}{l}0 \\
1\end{array}$ & $\begin{array}{l}0 . \\
1.335 E-08\end{array}$ & $\begin{array}{l}0.746 t-08 \\
7.70\end{array}$ & $\begin{array}{l}2213 \\
3213\end{array}$ \\
\hline $166 . B$ & & 0 & 0. & i. & Jili \\
\hline 101.6 & & & 0. & 0. & 3218 \\
\hline $1 \cup 2.8$ & & 0 & $n$. & 0. & 3220 \\
\hline 103.0 & & i. & 0. & u. & 3222 \\
\hline $10 \% .8$ & & 0 & $4.109 E-U Y$ & $2.383 E-0 v$ & 3224 \\
\hline $16,5, H$ & & 1 & $1.43 \mathrm{HE}-11)$ & $0.3+21-06$ & 3223 \\
\hline 106.16 & & 1 & $1.0276-60$ & s.jou-cou & side \\
\hline (1) 7.8 & & 1 & $1.233 \mathrm{E}-13 \mathrm{H}$ & 7.1 jul-Ub & $32<4$ \\
\hline I..$A$ & & i & $1.349 E-U H$ & $1.072 \mathrm{~F}-07$ & 3230 \\
\hline 109.7 & & 1 & $1.746 E-0 B$ & $1.013 \mathrm{E}=07$ & 3232 \\
\hline 110.7 & & 2 & $2.774 E-08$ & $1.60 Y E-07$ & 3231 \\
\hline 111.7 & & 6 & $0.164 E-08$ & $3.575 E-07$ & 3235 \\
\hline 112.7 & & 2 & $2.465 E-38$ & $1.43 \cup E-07$ & 3237 \\
\hline 113.7 & & 2 & $2.465 E-08$ & $1.430 t-01$ & 3236 \\
\hline 114.6 & & 3 & 3.34 & 1.90 & 3240 \\
\hline 113.6 & & 2 & $2.465 \mathrm{E}-\mathrm{UB}$ & $1.430[-07$ & 3241 \\
\hline $11+.6$ & & 2 & $2.774 E-28$ & $1.609 E-07$ & 3243 \\
\hline 117.6 & & 1 & $1 . \operatorname{An} 9 E-O H$ & $1.072 E-07$ & 3245 \\
\hline 111.6 & & 2 & $2.774 E-08$ & 1.6 USE -07 & $\$ 246$ \\
\hline 119.5 & & 5 & $5.547 \mathrm{E}-0 \mathrm{~B}$ & $3.217 \mathrm{E}-07$ & 3248 \\
\hline 120.5 & & 2 & $2.774 E-U B$ & 1.604 & 3249 \\
\hline 121.5 & & 2 & $2.157 \mathrm{E}-08$ & $1.251 \mathrm{E}-07$ & 3251 \\
\hline 122.4 & & 5 & $5.234 E-08$ & $3.034 E-07$ & 3252 \\
\hline 123.4 & & 1 & $1.027 \mathrm{E}-00$ & $5.910 \mathrm{E}-\mathrm{OH}$ & 3253 \\
\hline 124.4 & & 5 & $5.2396-08$ & $3.039[-07$ & 3255 \\
\hline $1<5.4$ & & 2 & $2.157 E-08$ & $1.251 \mathrm{E}-07$ & 3256 \\
\hline $1 / 2.3$ & & 4 & 4.DOKE-OB & $2.324 \mathrm{E}$ & 32 \\
\hline 127.3 & & 1 & $1.644 \mathrm{E}$ & 9.53 & 3259 \\
\hline 16.3 & & 3 & $3.638 E-06$ & $2.145 E-07$ & 3261 \\
\hline $1<9.3$ & & 2 & $2.465 \mathrm{E}-\mathrm{OA}$ & 1.43 & 3262 \\
\hline 130.3 & & 2 & $2.157 E-08$ & $1.251 E-07$ & 3263 \\
\hline $58-i d u$ & & & & -04 & \\
\hline
\end{tabular}

TUEFR DATA FCLLOH....
TFST VI CCTUBER 26, 1972 U930 TU 1000 H I KHIDUAMINE B RELEASE FRUM ELFVATILIN IJF Li,M ZOCA ARC U $=3,0$ MISEC AI ZUNi

\begin{abstract}
ZINC SULFIDE RELEASE FRUM ELEVATIUN OF $20 M$
2OOM ARC
TEST VI UCTUHER 20,1972 20930 TUL LUUO PST
\end{abstract}

\begin{tabular}{|c|c|c|c|c|c|}
\hline $\begin{array}{l}\text { AL JMUTH } \\
\text { DE CREES }\end{array}$ & $\begin{array}{l}\text { HE IGHT } \\
\text { ME TERS }\end{array}$ & $\begin{array}{c}\text { EXPOSURE } \\
\text { GM-SEC/CU.M } \\
\times 1 O E+6\end{array}$ & $\begin{array}{c}F / O \\
\text { SET/CU.M }\end{array}$ & $\begin{array}{l}F U A \\
\text { BSL.M }\end{array}$ & $\begin{array}{l}\text { WISTANCE } \\
\text { ME TLRSS }\end{array}$ \\
\hline 102. & .4 & 2311 & 2.3111 .06 & $1.34 \cup t-05$ & $\therefore 70$ \\
\hline 102. &.$b$ & 2131 & $2.132 t-06$ & $1.2301=03$ & 20.5 \\
\hline 102 & 1.5 & $\angle 073$ & 2.07 & $1.20<1-05$ & $i 00$ \\
\hline 102 & 2.5 & & $2 \cdot 1 h$ & $1.251 t-05$ & \\
\hline 102.0 & 4.6 & 2114 & 2.11 yt -00 & $1.229 E-05$ & -200 \\
\hline 102.0 & 5.5 & 2561 & $2.5 h+t-06$ & $1.485 t-25$ & 200 \\
\hline 10 & 7.0 & 2001 & $\angle \cdot t \cap \angle t-06$ & $1.504 k-05$ & 200 \\
\hline 2.0 & 8.5 & 36,50 & $3.6501-06$ & $2.117 t-0 b$ & 200 \\
\hline 102.1 & 10.0 & $6 H$ & 6.85 & 3.9 & 200 \\
\hline 10 & & 61 & 6.13 & $3.560 t-u b$ & 200 \\
\hline 102. & 16.0 & 77 & $7.7 \mathrm{~B}$ & $4.517 E-05$ & 200 \\
\hline 102. & 19.0 & 11732 & 1.1 & $6.405 L-05$ & 200 \\
\hline 102. & 22.0 & 1317 & $1.310 \mathrm{~F}$ & $7.643 k-05$ & 200 \\
\hline 102.0 & 25.0 & 12106 & 1.2111 .05 & $7.022 t-05$ & 200 \\
\hline 102. & 28.0 & 8014 & $8.0141-06$ & $4.648 t-05$ & $\angle 60$ \\
\hline & 31.0 & 7262 & 7.2 tict -06 & $4.21<6-05$ & 200 \\
\hline 102.5 & 32.8 & 6920 & $6.921 E-36$ & $4.014 t-0.5$ & 260 \\
\hline
\end{tabular}

ALIOUTH HEIGHT EXPTSURE F/O TU/J UISTANCE OELKEES METERS GM-SEC/RU.M SEC/CU.M I/SG.M MLTERS $\times 10 F+b$

$\begin{array}{lr}112.0 & .4 \\ 102.0 & .0 \\ 102.0 & 1.5 \\ 102.0 & 2.5 \\ 132.0 & 4.0 \\ 132.0 & 5.5 \\ 102.0 & 7.0 \\ 1112.0 & 8.5 \\ 162.0 & 10.0 \\ 102.0 & 13.0 \\ 102.0 & 16.0 \\ 102.0 & 19.0 \\ 102.0 & 22.0 \\ 132.0 & 25.0 \\ 1112.0 & 28.0 \\ 102.0 & 31.0 \\ 1112.0 & 32.0\end{array}$




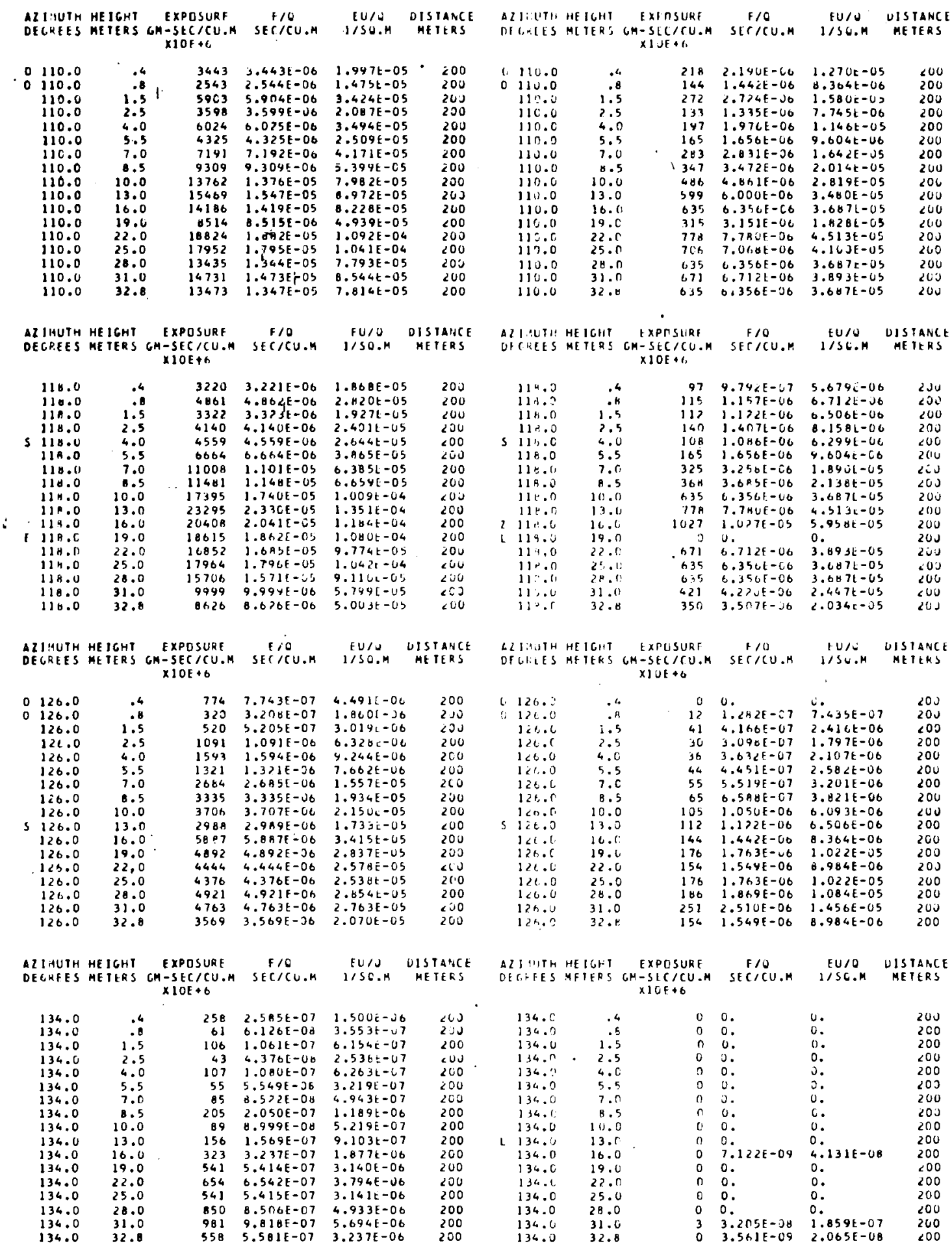




\begin{tabular}{|c|c|c|c|c|c|c|c|c|c|c|c|}
\hline 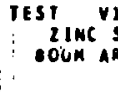 & & $\begin{array}{l}\text { ER 26, } \\
\text { EASE F }\end{array}$ & $\begin{array}{l}72 \quad 0930 \\
\text { ELFYAIIOK } \\
\text { U. S.8 KI }\end{array}$ & $\begin{array}{l}\text { TU. } 1000 \\
\text { NOF } 264 \\
\text { SEC AT } 26 \%\end{array}$ & PSI & $\begin{array}{r}\text { IEST } \\
\text { PHOOL } \\
\text { OOM } A R\end{array}$ & IME & $\begin{array}{l}\text { JUBFK } 26,197 \\
\text { RELEASE FROA }\end{array}$ & 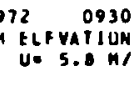 & $\begin{array}{l}\text { IU } 1000 \text { PS } \\
\text { OF } 26 \mathrm{H} \\
\text { SEC } 21 \mathrm{~T} 26 \mathrm{M}\end{array}$ & PSI \\
\hline $\begin{array}{l}\text { LZINUTH } \\
\text { DE GREES }\end{array}$ & $\begin{array}{l}\text { HE IGHT } \\
\text { ME TERS }\end{array}$ & $\begin{array}{l}\text { EXPOSURE } \\
\text { GH-SEC/CU.H } \\
\text { XIOE+6 }\end{array}$ & $\begin{array}{c}E / 0 \\
\text { SEC/CU.N }\end{array}$ & $\begin{array}{l}\text { EU/O } \\
1 / 50.4\end{array}$ & $\begin{array}{c}\text { OIS IANCE } \\
\text { METERS }\end{array}$ & $\begin{array}{l}\text { AZI I HUIH } \\
\text { OEGREES }\end{array}$ & $\begin{array}{l}\text { ME ICHI } \\
\text { ME TERS }\end{array}$ & $\begin{array}{c}\text { EXPOSURE } \\
\text { CH-SEC/CU.H } \\
\times 1 O E+6\end{array}$ & $\begin{array}{c}F / 0 \\
\text { SEC/CU.n }\end{array}$ & $\begin{array}{c}60 / 0 \\
2 / 50.4 \\
.\end{array}$ & \\
\hline $\begin{array}{l}105.0 \\
105.0 \\
105.0 \\
105.0 \\
105.0 \\
105.0 \\
105.0 \\
105.0 \\
105.0 \\
105.0 \\
105.0 \\
105.0 \\
105.0 \\
105.0 \\
105.0 \\
105.0 \\
105.0 \\
105.0\end{array}$ & $\begin{array}{l}.3 \\
1.5 \\
1.5 \\
2.1 \\
4.2 \\
6.3 \\
1.4 \\
10.5 \\
12.6 \\
14.7 \\
16.8 \\
21.0 \\
25.8 \\
29.4 \\
33.6 \\
37.8 \\
42.0\end{array}$ & $\begin{array}{r}1997 \\
1435 \\
1603 \\
693 \\
1935 \\
1305 \\
1270 \\
1336 \\
1238 \\
974 \\
1031 \\
996 \\
1094 \\
827 \\
890 \\
807 \\
725 \\
496\end{array}$ & $\begin{array}{l}1.997 E-06 \\
1.436 E-06 \\
1.603 E-06 \\
6.930 E-07 \\
1.936 E-06 \\
1.305 E-06 \\
1.270 E-06 \\
1.337 E-06 \\
1.239 E-06 \\
9.766 E-07 \\
1.032 E-06 \\
9.960 E-07 \\
1.094 E-06 \\
.271 E-07 \\
1.907 E-07 \\
1.077 E-07 \\
7.251 E-07 \\
4.965 E-07\end{array}$ & $\begin{array}{l}1.150 E-05 \\
6.327 E-06 \\
9.300 E-06 \\
4.020 E-06 \\
1.123 E-05 \\
7.5772-06 \\
7.367 E-06 \\
7.7531-06 \\
7.105 E-06 \\
5.653 E-06 \\
3.983 E-06 \\
5.7777-06 \\
6.365 E-06 \\
4.797 E-06 \\
3.166 E-06 \\
4.684 E-06 \\
4.2061-06 \\
2.800 E-06\end{array}$ & $\begin{array}{l}120 \\
020 \\
020 \\
120 \\
620 \\
620 \\
020 \\
020 \\
122 \\
420 \\
420 \\
020 \\
020 \\
120 \\
020 \\
020 \\
120 \\
.20\end{array}$ & $\begin{array}{l}105.0 \\
105.0 \\
105.0 \\
105.0 \\
105.0 \\
105.0 \\
105.0 \\
305.0 \\
1115.0 \\
165.0 \\
105.0 \\
105.0 \\
105.0 \\
105.0 \\
105.0 \\
105.0 \\
105.0 \\
105.0\end{array}$ & $\begin{array}{r}.3 \\
.5 \\
1.1 \\
1.5 \\
2.1 \\
4.2 \\
6.3 \\
1.4 \\
10.5 \\
12.6 \\
14.8 \\
16.8 \\
21.0 \\
25.2 \\
24.4 \\
33.6 \\
37.8 \\
42.0\end{array}$ & $\begin{array}{l}63 \\
43 \\
53 \\
60 \\
56 \\
56 \\
53 \\
53 \\
56 \\
46 \\
41 \\
43 \\
22 \\
29 \\
22 \\
10 \\
22 \\
22 \\
25 \\
25\end{array}$ & $\begin{array}{l}6.372 E-07 \\
4.306 E-07 \\
5.339 E-07 \\
8.094 E-07 \\
5.613 E-07 \\
5.319 E-07 \\
5.339 E-07 \\
5.613 E-07 \\
4.650 E-L 7 \\
4.654 E-67 \\
4.306 E-07 \\
2.234 E-07 \\
2.976 E-07 \\
2.234 E-07 \\
1.894 E-07 \\
2.234 E-07 \\
2.234 E-07 \\
2.5 B 3 E-07\end{array}$ & $\begin{array}{l}3.096 t-06 \\
2.697 E-06 \\
3.097 E-06 \\
4.695 E-06 \\
3.296 E-06 \\
3.047 t-06 \\
3.097 t-06 \\
3.296 E-06 \\
2.697 t-06 \\
2.697 t-06 \\
2.497 t-06 \\
1.249 E-06 \\
1.698 t-06 \\
1.244 E-06 \\
1.099 E-06 \\
1.299 t-06 \\
1.299 E-06 \\
1.498 E-06\end{array}$ & $\begin{array}{l}.20 \\
620 \\
620 \\
620 \\
020 \\
020 \\
020 \\
020 \\
020 \\
020 \\
620 \\
020 \\
020 \\
020 \\
020 \\
020 \\
020 \\
020\end{array}$ \\
\hline $\begin{array}{l}\text { AZ IMUTH } \\
\text { OECREES }\end{array}$ & $\begin{array}{l}\text { ME IGHT } \\
\text { MEIERS }\end{array}$ & $\begin{array}{c}\text { EXPOSUKE } \\
\text { GH-SEC/CU.A } \\
\text { XIOE } \bullet\end{array}$ & $\begin{array}{c}\text { F/O } \\
\text { SE C/CU.H }\end{array}$ & $\begin{array}{l}\text { EU/U } \\
1 / 50.1\end{array}$ & DIS & $\begin{array}{l}\text { AlWUTH } \\
\text { UECXESS }\end{array}$ & $\begin{array}{l}\text { HEICH? } \\
\text { WE TERS }\end{array}$ & $\begin{array}{c}\text { EXPOSUKF } \\
\text { GM-StCGICU.M } \\
\text { AIUE }\end{array}$ & SESICU.M & $\begin{array}{c}\text { IU/O } \\
\text { I/S6.M }\end{array}$ & $\begin{array}{l}\text { ISTANCE } \\
\text { MEIEKS }\end{array}$ \\
\hline $\begin{array}{r}112.6 \\
112.6 \\
112.6 \\
112.6 \\
112.6 \\
112.6 \\
112.6 \\
112.6 \\
112.6 \\
112.6 \\
1112.6 \\
112.6 \\
1112.6 \\
112.6 \\
112.6 \\
112.6 \\
112.6 \\
112.6\end{array}$ & $\begin{array}{r}.3 \\
.5 \\
1.1 \\
1.5 \\
2.1 \\
4.2 \\
6.3 \\
6.4 \\
10.5 \\
12.6 \\
14.7 \\
16.8 \\
21.0 \\
25.2 \\
29.4 \\
33.6 \\
37.8 \\
42.0\end{array}$ & $\begin{array}{l}1935 \\
1607 \\
1549 \\
1035 \\
1227 \\
1621 \\
1191 \\
1360 \\
1661 \\
1623 \\
1431 \\
1478 \\
1361 \\
1291 \\
1120 \\
1402 \\
1219 \\
1203\end{array}$ & $\begin{array}{l}1.935 E-06 \\
1.600 E-06 \\
1.550 E-06 \\
1.035 E-06 \\
1.227 E-06 \\
1.621 E-06 \\
1.191 E-06 \\
1.360 E-06 \\
1.661 E-06 \\
1.424 E-06 \\
1.432 E-06 \\
1.476 E-06 \\
1.362 E-06 \\
1.292 E-06 \\
1.124 E-06 \\
1.403 E-06 \\
1.219 E-06 \\
1.203 E-06\end{array}$ & $\begin{array}{l}1.122 t-05 \\
9.325 E-06 \\
6.484 E-06 \\
6.004 t-06 \\
7.117 E-06 \\
9.404 E-06 \\
6.910 E-06 \\
7.409 E-06 \\
9.635 E-06 \\
8.256 t-66 \\
8.304 E-06 \\
8.575 E-06 \\
7.897 E-06 \\
7.493 t-06 \\
6.548 E-06 \\
0.136 E-06 \\
7.073 E-06 \\
6.970 E-06\end{array}$ & $\begin{array}{l}033 \\
633 \\
633 \\
833 \\
633 \\
633 \\
633 \\
833 \\
833 \\
033 \\
033 \\
633 \\
633 \\
033 \\
033 \\
033 \\
833 \\
033 \\
033 \\
633\end{array}$ & $\begin{array}{l}112.6 \\
112.6 \\
112.6 \\
112.6 \\
112.6 \\
112.6 \\
112.6 \\
112.6 \\
112.6 \\
112.6 \\
1112.6 \\
112.6 \\
1112.6 \\
112.6 \\
112.6 \\
112.6 \\
112.6 \\
112.6\end{array}$ & $\begin{array}{r}.3 \\
.5 \\
1.1 \\
1.5 \\
2.1 \\
4.2 \\
6.3 \\
8.4 \\
10.3 \\
16.8 \\
14.7 \\
16.0 \\
21.8 \\
25.2 \\
29.6 \\
33.6 \\
37.8 \\
42.0\end{array}$ & $\begin{array}{l}67 \\
23 \\
53 \\
04 \\
60 \\
76 \\
67 \\
70 \\
74 \\
67 \\
6 \\
74 \\
6 \\
711 \\
611 \\
10 \\
49 \\
46\end{array}$ & $\begin{array}{l}6.717 E-07 \\
6.372 E-67 \\
5.334 E-07 \\
9.434 E-07 \\
6.075 E-07 \\
7.406 E-07 \\
6.717 E-07 \\
7.061 E-07 \\
7.406 E-07 \\
6.717 t-67 \\
0.406 E-07 \\
7.406 E-07 \\
6.544 E-08 \\
7.0615-C 7 \\
6.076 E-07 \\
6.07 C E-07 \\
4.454 E-07 \\
4.6561-07\end{array}$ & 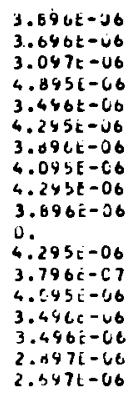 & 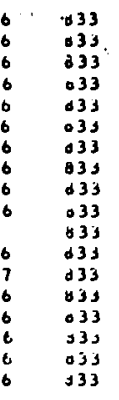 \\
\hline $\begin{array}{l}\text { ALIMUIH } \\
\text { OEGREES }\end{array}$ & $\begin{array}{l}\text { IGHT } \\
\text { TERS }\end{array}$ & $\begin{array}{r}\text { EXPOS } \\
\text { GN-SECA } \\
\text { XIOE. }\end{array}$ & $\begin{array}{l}\text { E/O } \\
\text { SET/CU.H }\end{array}$ & $\begin{array}{c}E U / 0 \\
1 / 50.4 \\
.\end{array}$ & $\begin{array}{l}\text { UISIANCE } \\
\text { ME IERS }\end{array}$ & $\begin{array}{l}\text { AZISUIT } \\
\text { OFi-AESS }\end{array}$ & $\begin{array}{l}\text { HEIGHT } \\
\text { METERS }\end{array}$ & $\begin{array}{c}\text { EXYDSURF } \\
\text { CM-SICICU.M } \\
\text { MIUH+t }\end{array}$ & $\begin{array}{l}F / S \\
\text { SET/CU.A }\end{array}$ & $\begin{array}{l}\text { EUA } \\
\text { d/SE.h }\end{array}$ & $\begin{array}{l}\text { WISTANCE } \\
\text { ME TEKS }\end{array}$ \\
\hline $\begin{array}{l}120.2 \\
120.2 \\
120.2 \\
120.2 \\
120.2 \\
120.2 \\
120.2 \\
120.2 \\
120.2 \\
120.2 \\
5120.2 \\
120.2 \\
120.2 \\
120.2 \\
120.2 \\
120.2 \\
120.2 \\
120.2\end{array}$ & $\begin{array}{r}.3 \\
.5 \\
1.1 \\
1.5 \\
2.1 \\
4.2 \\
6.3 \\
8.4 \\
10.5 \\
12.6 \\
14.7 \\
16.8 \\
21.0 \\
25.2 \\
29.4 \\
33.6 \\
37.8 \\
42.0\end{array}$ & $\begin{array}{l}1699 \\
1308 \\
1544 \\
1322 \\
1054 \\
1089 \\
1196 \\
1192 \\
1219 \\
1110 \\
760 \\
1396 \\
1262 \\
1367 \\
849 \\
1304 \\
1356 \\
1225\end{array}$ & $\begin{array}{l}1.700 E-06 \\
1.300 E-06 \\
1.565 E-06 \\
1.322 E-06 \\
1.054 E-06 . \\
1.089 E-06 \\
1.196 E-06 \\
1.192 E-06 \\
1.220 E-06 \\
1.118 E-06 \\
7.663 E-07 \\
1.396 E-C 6 \\
1.282 E-06 \\
1.360 E-06 \\
1.494 E-07 \\
1.304 E-06 \\
1.356 E-06 \\
1.225 E-06\end{array}$ & $\begin{array}{l}9.057 t-06 \\
7.587 C-06 \\
0.960 E-06 \\
7.660 E-06 \\
6.1161-06 \\
6.316 E-06 \\
6.927 t-06 \\
6.915 E-06 \\
7.075 E-06 \\
6.486 E-06 \\
4.444 E-06 \\
.098 E-06 \\
7.637 E-06 . \\
7.932 E-06 \\
4.926 E-06 \\
7.565 E-06 \\
7.860 E-06 \\
7.106 E-06\end{array}$ & $\begin{array}{l}045 \\
045 \\
045 \\
045 \\
845 \\
045 \\
045 \\
845 \\
045 \\
045 \\
845 \\
045 \\
045 \\
045 \\
145 \\
045 \\
045\end{array}$ & $\begin{array}{l}1<0.2 \\
1<0.2 \\
120.2 \\
120.2 \\
1>0.2 \\
120.2 \\
120.2 \\
120.2 \\
120.2 \\
120.2 \\
120.2 \\
110.2 \\
120.2 \\
120.2 \\
120.2 \\
120.2 \\
120.2 \\
120.2\end{array}$ & $\begin{array}{r}.3 \\
1.5 \\
1.1 \\
1.5 \\
2.1 \\
4.2 \\
6.3 \\
8.4 \\
10.5 \\
12: 6 \\
14.7 \\
16.6 \\
21.0 \\
25.2 \\
29.4 \\
33.6 \\
37.8 \\
42.0\end{array}$ & $\begin{array}{l}46 \\
49 \\
53 \\
63 \\
53 \\
53 \\
46 \\
53 \\
53 \\
53 \\
10 \\
56 \\
56 \\
56 \\
36 \\
53 \\
56 \\
56\end{array}$ & $\begin{array}{l}4.64 U E-U 7 \\
4.994 E-07 \\
3.334 E-C 7 \\
6.376 E-07 \\
5.33+E-07 \\
5.334 E-07 \\
4.650 E-07 \\
5.3391-U 7 \\
5.334 E-U 7 \\
3.334 E-07 \\
1.094 E-57 \\
5.663 E-07 \\
5.643 E-07 \\
5.643 E-07 \\
3.617 E-07 \\
5.334 E-U 7 \\
5.643 E-U 7 \\
5.643 E-07\end{array}$ & 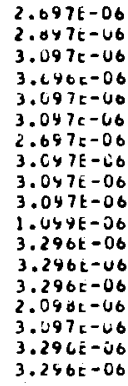 & $\begin{array}{l}045 \\
045 \\
045 \\
045 \\
045 \\
045 \\
045 \\
045 \\
045 \\
045 \\
045 \\
045 \\
045 \\
045 \\
045 \\
345 \\
045 \\
045\end{array}$ \\
\hline
\end{tabular}




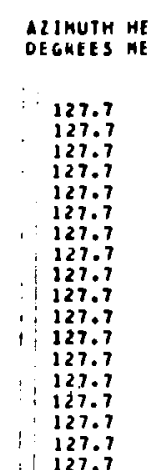

(a)

EXPOSUR $x 106 \oplus 6$
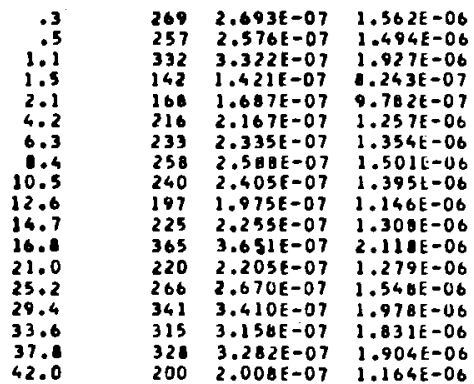

DISTANCE
METERS

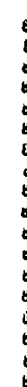

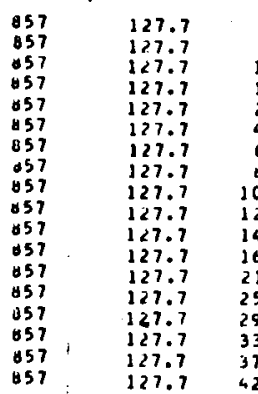

.3
1.5
1.1
2.5
4.2
6.3
8.4
10.5
12.6
14.7
16.8
21.0
25.2
29.4
33.4
37.8
42.0
ESICICU. xioteo
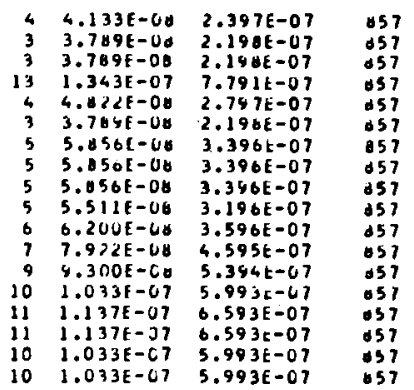

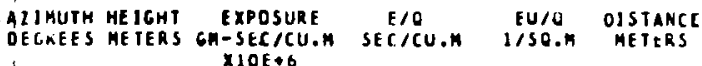

$\begin{array}{rrrrrr}135.0 & .3 & 33 & 3.367 E-08 & 1.953 E-07 & 867 \\ 135.0 & .5 & 25 & 2.576 E-00 & 1.494 E-07 & 06 \\ 135.0 & 1.1 & 0 & 0.0 & 0.0 & 867 \\ 135.0 & 1.5 & 15 & 1.579 E-08 & 9.130 E-08 & 867\end{array}$

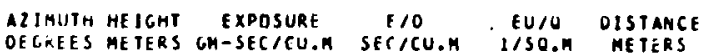
XIOE +6

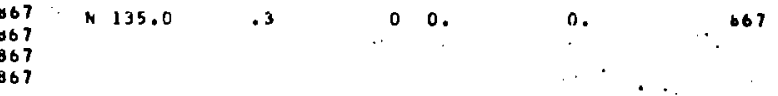

TOHE R OATA FOLLOH....

TEST VI OCTOEER 26. 1972 0830 TO 1000 PST IINC SULFIOE RELEASE FROM ELEVATION OF $26 \mathrm{M}$
1OOOM ARC

AZIMUTH HEIGHT EXPQSURE F/O EU/U OISTANCE DECKEES MEIERS GM-SEC/CU.M SEC/CU.H I/SO.M METERS
XIOE

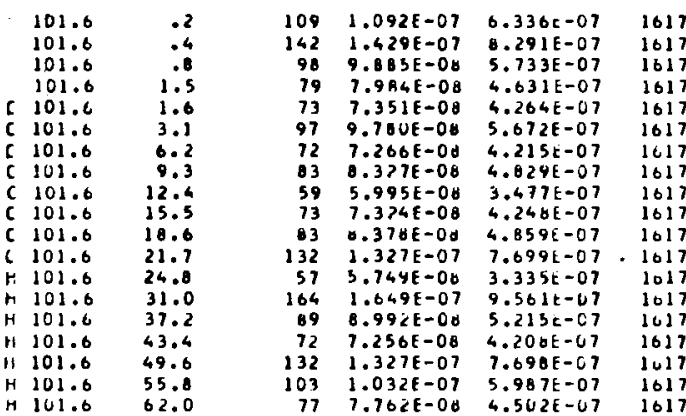

THLER UATA FMLLOW...

IEST VI OCIOEER 26, 19720930 TO 1000 OSI

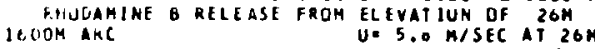

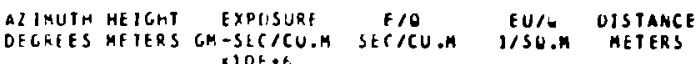

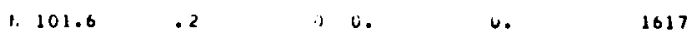

ALIMUTH HEIGHT EXPOSURE F/O EU/O OISTANCE DECHEES METERS GH-SEC/CU.A SEC/CU.M I/SO.H REIERS

ALIMUIH HEIGHT EXPCSURE F/O EU/O OIS.TANCE UECKEES METERS GM-SIC/CU.M SEC/CU.H I/SO.M METERS

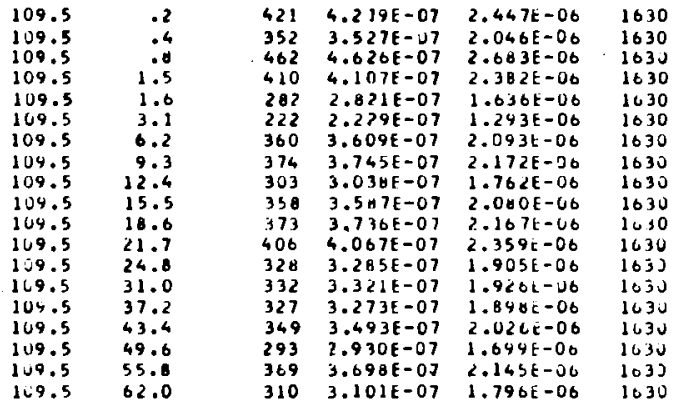

\begin{tabular}{|c|c|}
\hline $\begin{array}{l}119.5 \\
164.5\end{array}$ & .2 \\
\hline 109.5 & .6 \\
\hline $\begin{array}{l}109.5 \\
109.5\end{array}$ & $\begin{array}{l}1.5 \\
1.6\end{array}$ \\
\hline $\begin{array}{r}149.5 \\
109.5\end{array}$ & $\begin{array}{l}3.1 \\
6.2\end{array}$ \\
\hline 109.5 & 9.3 \\
\hline 160,5 & 12.4 \\
\hline 169.5 & 13.5 \\
\hline 177.5 & 18.6 \\
\hline 1.4 .5 & 21.7 \\
\hline 109.5 & 24.8 \\
\hline 104.5 & 31.0 \\
\hline 109.5 & 37.2 \\
\hline 10.5 & 43.4 \\
\hline iju.s & \\
\hline $3=4.5$ & it, \\
\hline
\end{tabular}

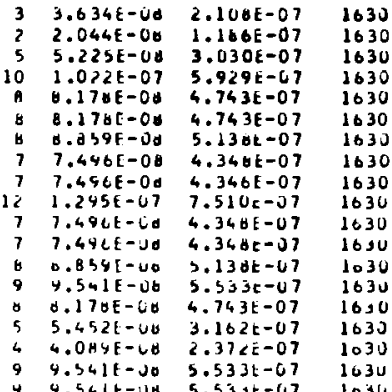




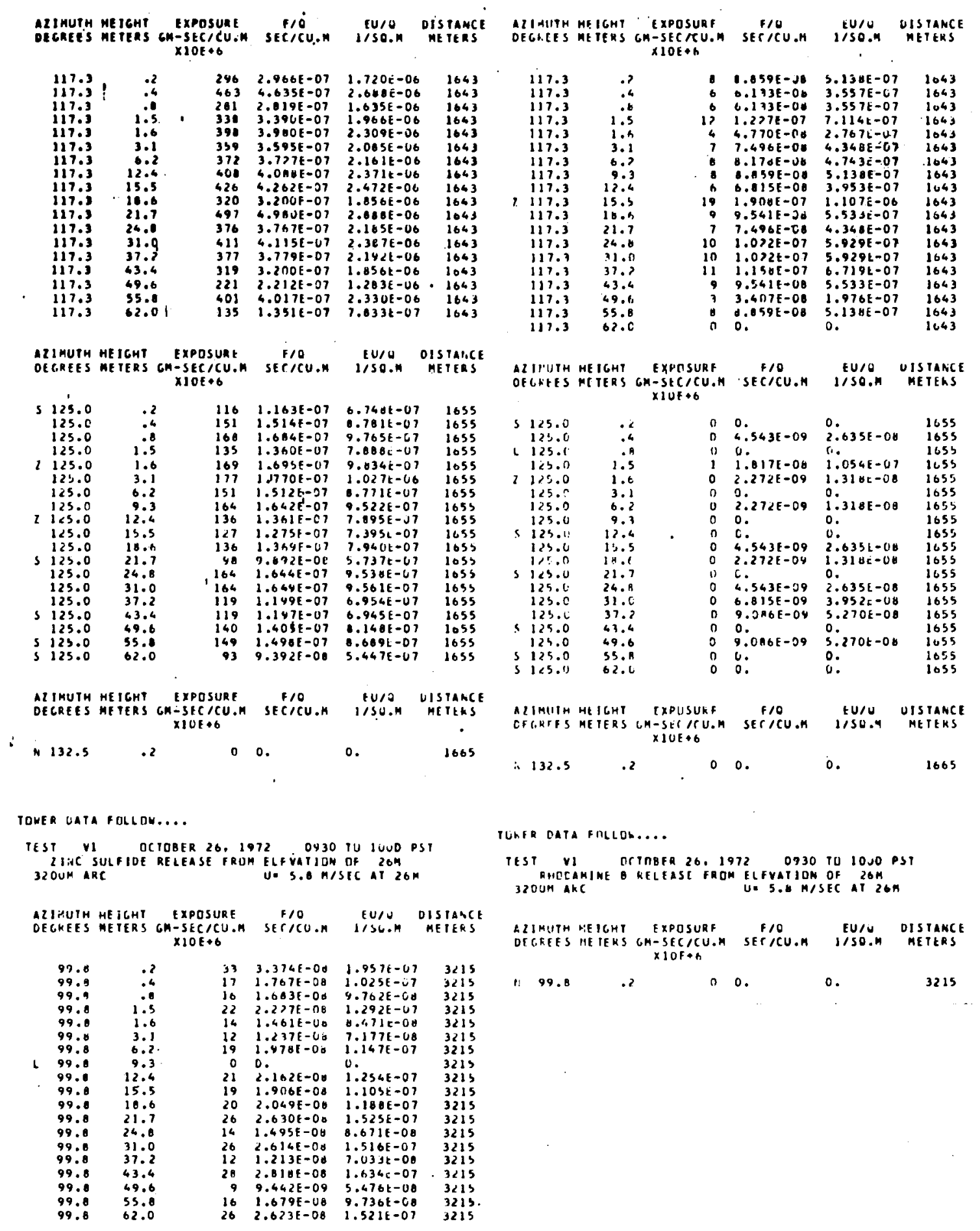




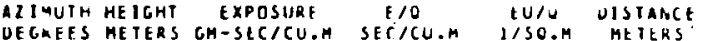
$\times 106+6$

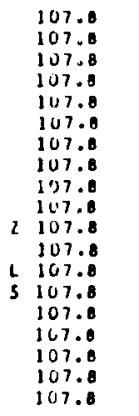

.2
.4
.8
1.5
1.6
3.1
6.2
9.3
12.4
15.5
16.0
21.7
24.8
31.0
37.2
43.4
49.6
55.8
62.0
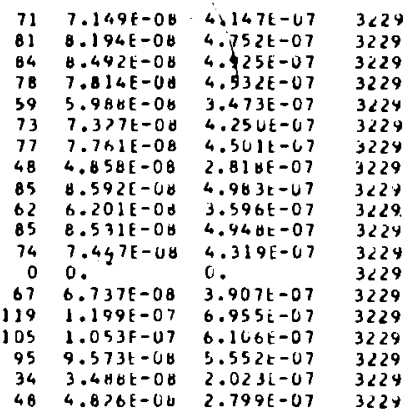

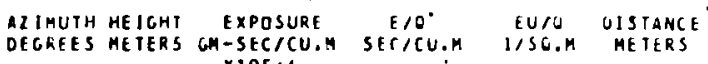
$\times 10 E \bullet 6$
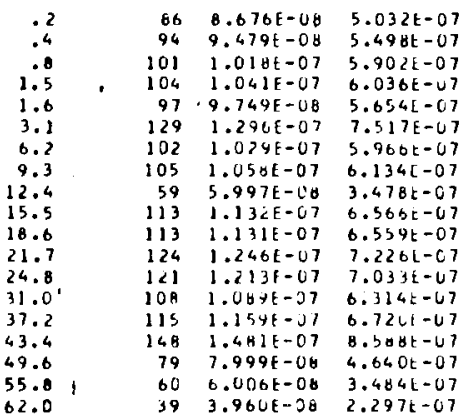

3241

$3<44$

3241

3241

3241

3241

3241

$3<41$

3241

$3<41$

$3<41$

3241

3241

3241

3113.6

115.6

62.0

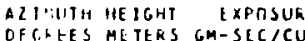
$x \quad \times E+6$

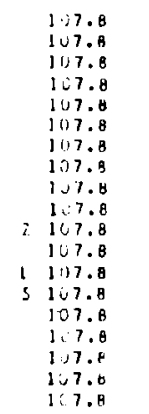

.2
.4
.8
1.5
1.6
3.1
6.2
9.3
12.4
19.5
14.6
21.7
24.8
31.0
37.2
43.4
49.6
55.4
62.1

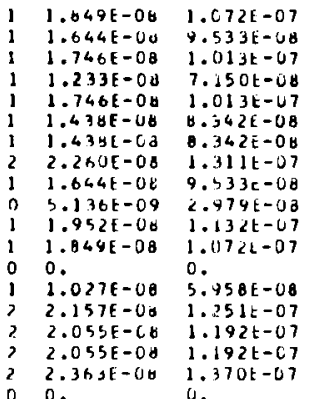

DISTANCE

DIIUUTH HEIGH EXPOSURF F/O TU/U UISIANCE $.362 \mathrm{E}-0 \mathrm{O} \quad 1.370 \mathrm{E}-07 \quad 3224$ $\times 10 t+h$

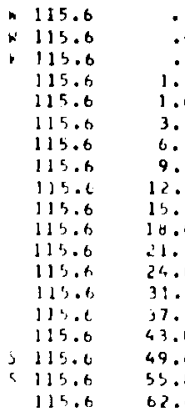

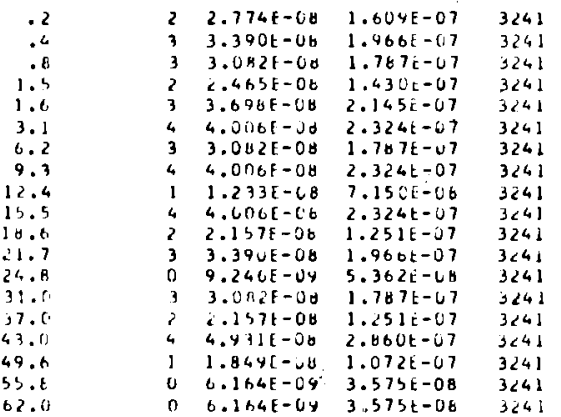

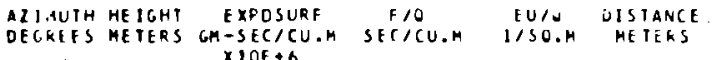

D2 1MUTH HEIGHT EXPOSURF

6.1B4E-6Y 3.575t-08

3241

DERES ME IELS GM-SECMU.H

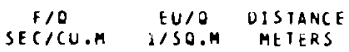

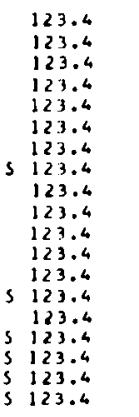

.2
.4
.8
1.5
1.6
3.1
6.2
9.3
12.4
15.5
18.6
21.7
24.8
31.0
37.2
43.4
49.6
55.8
62.0

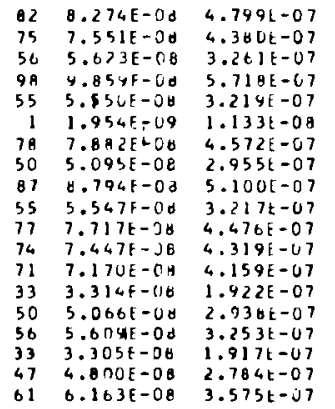

$3<53$
$3<53$
3253
$3<53$
3253
3253
3253
3253
3253
3259
3259
3253
3253
3253
3253
3253
3253
3253
3253

ALIHUIH HEIGHT TKPOSURE DEGRES METERS GM-SECICU.H $\times 10 E \bullet B$
$\begin{array}{llll}N & 131.2 & .2 & 0\end{array}$

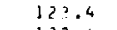

123.4

123.4
123.4

123.4
123.4

13

123.4

123.4

$1<3.4$

123.4

123.4

123.4
123.4

5123.4

12
5 123.4

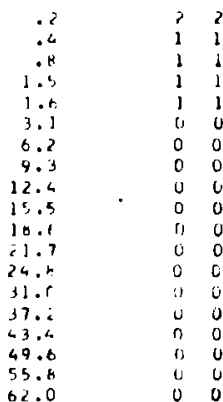

\begin{tabular}{|c|c|c|}
\hline $2.1571-00$ & $1.251 \mathrm{E}-07$ & $3<53$ \\
\hline $1.869 \mathrm{E}-00$ & $1.072 E-67$ & 3251 \\
\hline $1.541 \mathrm{E}-0 \mathrm{~B}$ & $8.937 E-00$ & 3253 \\
\hline $1.027 \mathrm{E}-\mathrm{OH}$ & $5.958 t-08$ & $3<33$ \\
\hline $1.049 E-08$ & $1.07 \approx E-67$ & 3253 \\
\hline 0 . & 0. & 3253 \\
\hline 0. & 0. & 3253 \\
\hline 0 . & 0. & 3251 \\
\hline u. & 0. & $3<b 3$ \\
\hline 0. & o. & 3253 \\
\hline 0. & 0. & 3253 \\
\hline 0 . & 0. & $325]$ \\
\hline 0. & 0 . & $3<53$ \\
\hline i. & c. & 3253 \\
\hline 0. & 0. & $3<53$ \\
\hline 0. & 0 . & $3<53$ \\
\hline 0. & 0. & 3253 \\
\hline$u$. & 0. & $3<53$ \\
\hline 0. & 0. & 3253 \\
\hline
\end{tabular}

A2IMUTH HEIGHT EXPTSURF F/O EU/J UISTANCE DFCKEES HETFRS GH-SEC/CU. $X] 6 F \cdot 6$

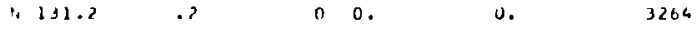


GROUND LEVEL AND TOHER SAMPLING 2UUM TO 32UOM. FULLOAING SUME EXTRAPOLATIDN AT SUUTH EDGE. ALL ARCS EMBRACE THE CROSSHIND EXTENT DF TRACEK UISIRIBUTIUNS. 13 OF ZO TOYERS "HIT" UY TRACERS ALL VEKTICAL. DISIRIBUTIONS TRUNCATED AT TOP. ALTHO NI RAIN UURING THACER RELEASE. PRIUR RAIH LEFT HILTERS WET: EFFECT. IF AHY, UHKNUWA.

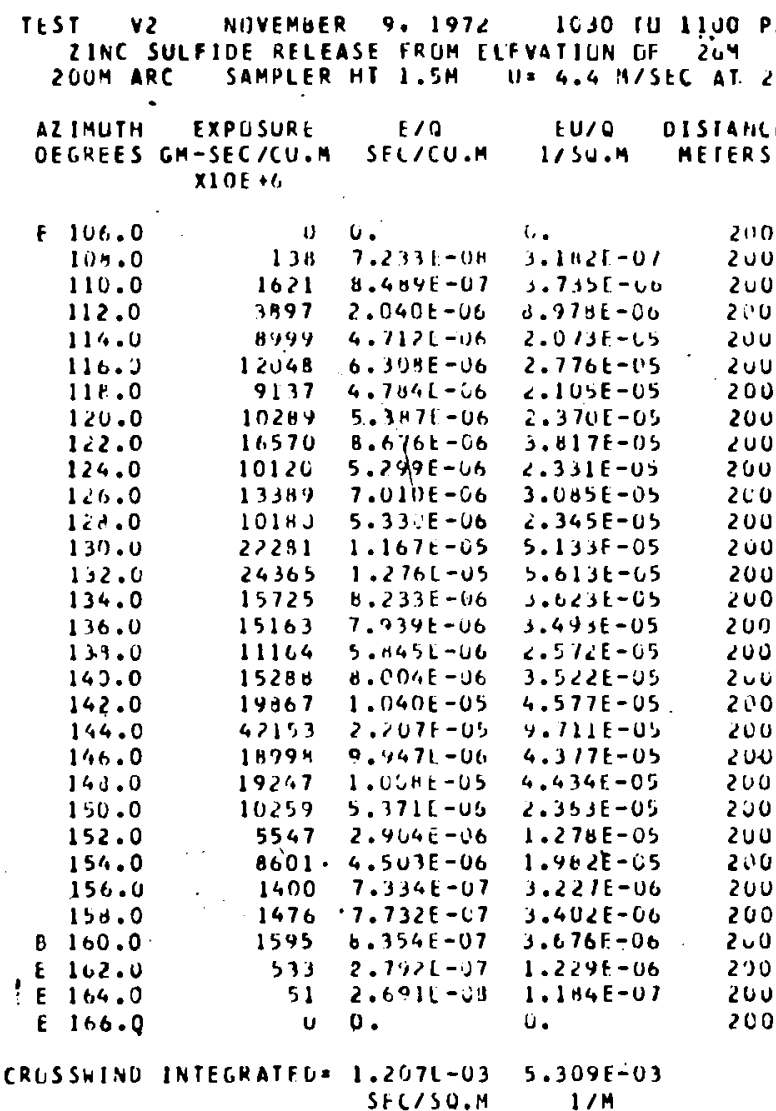

TEST V2 NUVEMHER 9.1972 1030 TU 1100 FST IINC SULFIOE KELEASE FRLIM ELEVATIUN OF 264

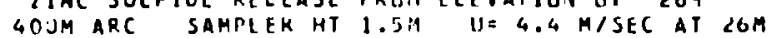
AZIMUIH EXPOSURE FIO IU/O DISIAIICE UFGKEES GP-SEC/CU.M SFCICU.M I/SO.M MEIEKS $\times 10 E+C$

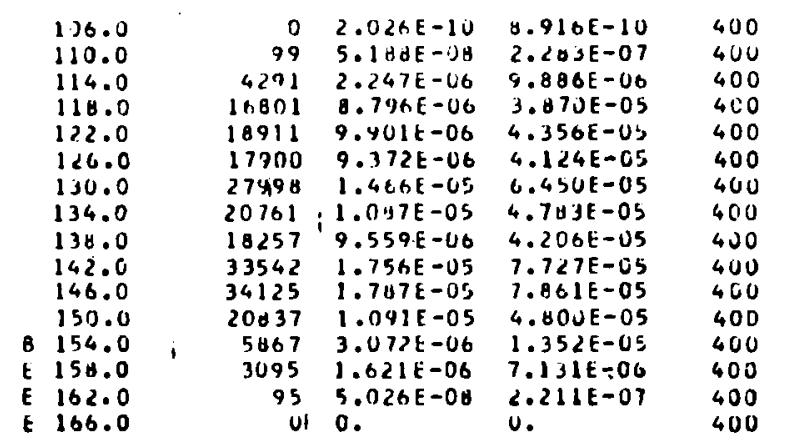

CROSSHIND INTEGRATED= $3.254 E-03 \cdot 1.432 E-02$ $\begin{array}{rr}.254 E-03 & 1.432 E \\ S E C / S O . M & 1 / H\end{array}$
IEST V2 NIIVEABER 9,1972 COO: AKC SAMPLEK HT $1.5 \mathrm{M}$ II: $4.4 \mathrm{H} / \mathrm{SEC} 4 \mathrm{~T} 26.4$

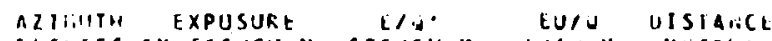 DECHEES CM-SECICU.H SECICU.H WSU.A HETEKS $x \in[0+6$

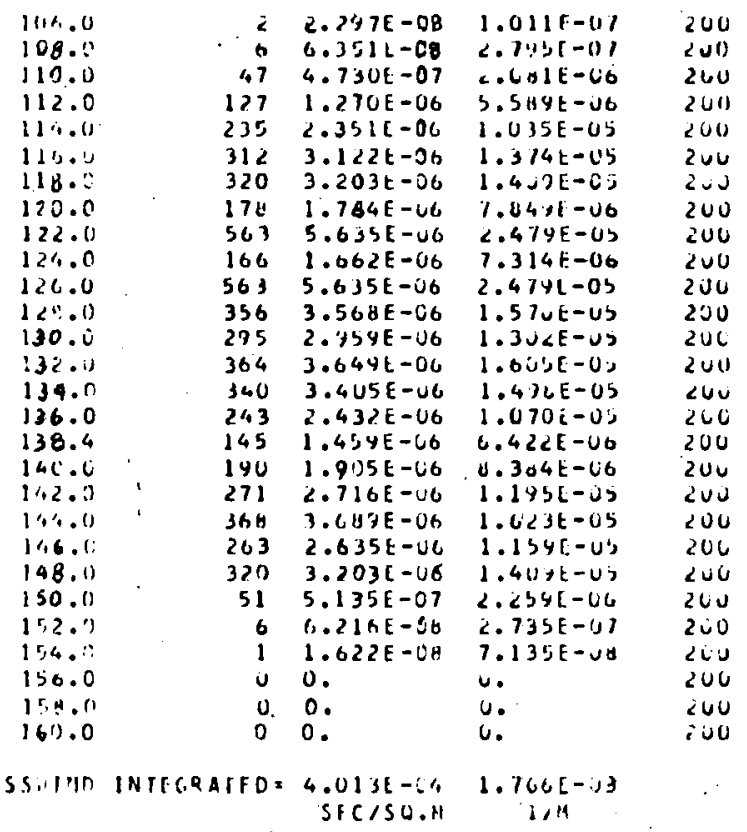


TEST V2 NCVEMBER 4. $1972 \quad 1030$ TO 1100 PST ZINC SULFIDE RELEASF FRUA ELFVATIUN UF $26 \mathrm{M}$ DOOH ARC SAMPLER HT $1.5 \mathrm{M}$ U = $4.4 \mathrm{M} / \mathrm{SEC}$ AT $2 \mathrm{GM}$

AZIMUTH EXPOSURE
DEGKEES GH-SEC/CU.M SFC/CU.M
XIOE $\$ 6$

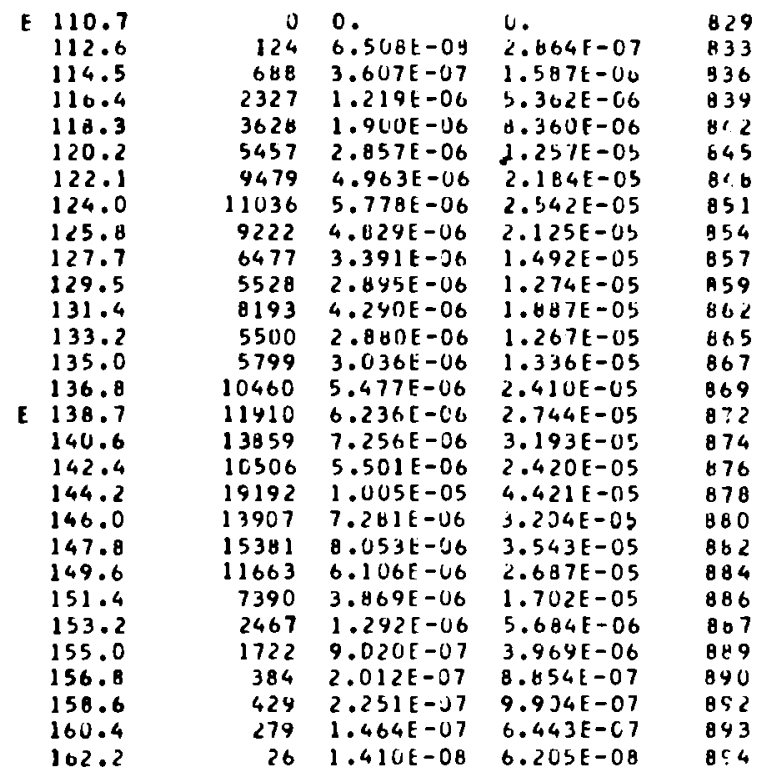

CRUSSWIND INTEGRATED $=2.811 \mathrm{E}-03 \quad 1.237 \mathrm{E}-02$

$$
\text { SFC15O.M } 11 \mathrm{M}
$$

IEST V2 NOVEMBER 9, 1972 1630 TU 11 LO PST 2INC SULFITE RELEASE FRUM FLEVATIUN UF $26 M$ 12004 ARC SAMPLER HT $1.5 \mathrm{M} \quad \|=4.4$ M/SEC AT $20 \mathrm{M}$

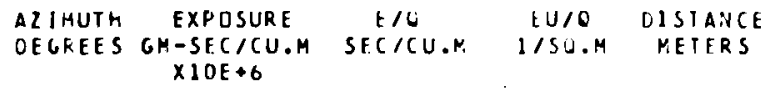

\begin{tabular}{|c|c|c|c|c|c|}
\hline & 110.0 & v & 0. & & 1200 \\
\hline & & 9 & 4. HOUE -O9 & $\therefore .13 \forall E-\cup J$ & 1200 \\
\hline & 114.0 & $\begin{array}{r}25 \\
524\end{array}$ & $\begin{array}{l}1.35 h E-08 \\
2.744 E-07\end{array}$ & $\begin{array}{l}5.96 B E-08 \\
1.207 E-06\end{array}$ & $\begin{array}{l}1260 \\
1200\end{array}$ \\
\hline & 118.0 & 1368 & $7.167 \mathrm{E}-07$ & $3.151 \mathrm{f}-00$ & 1200 \\
\hline & 120.0 & 1934 & $1.0136-06$ & $4.457 E-06$ & 1260 \\
\hline & 122.6 & 2906 & $1.522 E-06$ & C.695E-UG & 1200 \\
\hline & 124.0 & 3192 & $1.672 E-U 6$ & $7.355 \mathrm{E}-0 \mathrm{O}$ & 1200 \\
\hline & 126.0 & 6196 & 3. $244 E-06$ & $1.427 E-0 b$ & 1200 \\
\hline & $12 t .0$ & 4356 & $2.281 \mathrm{E}-06$ & $1.004 E-03$ & 1200 \\
\hline & 130.0 & 6165 & $3.22 \mathrm{PE}-06$ & $1.4 \angle 0 E-05$ & 1200 \\
\hline & 132.0 & 3120 & $1.634 E-06$ & $7.184 F-06$ & 1200 \\
\hline & 134.0 & 4063 & $2.127 F-06$ & $9.3 B \cup E-\cup 6$ & 1266 \\
\hline & 136.0 & 5615 & $2.9100 E-06$ & $1.294 E-05$ & 1200 \\
\hline & $13 H .0$ & $\cos 3$ & $3.1447-06$ & $1.517 \mathrm{~F}-05$ & 1200 \\
\hline & 140.0 & 3501 & $1.033 E-06$ & $6.067 E-106$ & 1260 \\
\hline & 142.0 & 5639 & $2.953 E-36$ & $1.294 \mathrm{E}-\mathrm{Cb}$ & 1200 \\
\hline & 144.0 & 6012 & $4.195 E-U 6$ & $1.346 E-05$ & 1200 \\
\hline & 146.0 & 5701 & $2.4 u 51-00$ & $1.313 E-U 5$ & 1200 \\
\hline & 149.0 & 3920 & $2.0531-06$ & $4.033 E-06$ & 1200 \\
\hline & 150.0 & $3+75$ & $2.0011-06$ & $y .15,3 t-06$ & 1200 \\
\hline & 152.0 & 2152 & $1.127 E-06$ & $4.43 \cup E-06$ & 1206 \\
\hline & 154.0 & $1<32$ & $6.454 E-67$ & $2.840 E-0 G$ & 1260 \\
\hline$B$ & 156.0 & 297 & $1.557 E-07$ & $6.85 j E-07$ & 1200 \\
\hline & 158.0 & 323 & $1.6931-67$ & $7.4 \mathrm{SOE}-67$ & 1200 \\
\hline & 160.0 & 64 & $3.36 \mathrm{BE}-0 \mathrm{~A}$ & $1.482 E-07$ & 1200 \\
\hline & 162.0 & 3 & $2.034 t-04$ & $8.95<E-09$ & 1240 \\
\hline & 164.0 & $u$ & $0_{0}$ & u. & 1200 \\
\hline
\end{tabular}

CROSSHIND INTEGRATED $=1.774 \mathrm{~F}-03 \quad 7.805 E-03$ SECISO.M T.8 $1 / M$

TEST V2 NOVEHAER 9. 1372 1030 IU 1100 PST RHIUDAMINE B RELEASE FROM ELEVATIUN OF $20 \mathrm{H}$ BCOM ARC SAMPLER HT $1.5 \mathrm{M} U$ U $4.4 \mathrm{M} / \mathrm{SEC}$ AT $26 \mathrm{M}$

ILIHUTH FXPOSURE F/O TU/O DISIANCE DEGREES GM-SEC/CU.M SEC/CU.H I/SU.M METEKS $X 1 O E+6$

110.7
112.8
114.5
116.4
115.3
120.2
132.1
124.0
125.8
127.7
129.5
111.4
133.2
135.0
136.8
138.7
147.6
142.4
114.2
146.0
147.9
149.6
151.4
153.2
155.0
156.8
159.6
160.4
162.2

$\begin{array}{rl}0 & 0 . \\ 6 & 6.536 E-00 \\ 42 & 4.248 E-07 \\ 140 & 1.408 E-06 \\ 234 & 2.366 E-06 \\ 384 & 3.850 E-06 \\ 676 & 6.761 E-06 \\ 728 & 7.262 E-06 \\ 65 \theta & 6.567 E-06 \\ 641 & 6.413 E-06 \\ 463 & 4.634 E-06 \\ 390 & 3.902 E-06 \\ 536 & 5.370 E-06 \\ 306 & 3.065 E-06 \\ 536 & 5.370 E-06 \\ 571 & 5.718 E-06 \\ 584 & 5.892 E-06 \\ 405 & 4.053 E-06 \\ 1006 & 1.006 E-05 \\ 763 & 7.630 E-06 \\ 554 & 5.544 E-06 \\ 416 & 4.163 E-06 \\ 238 & 2.386 E-06 \\ 81 & 8.131 E-07 \\ 64 & 6.431 E-07 \\ 23 & 2.366 E-07 \\ 28 & 2.889 E-07 \\ 3 & 3.791 E-08 \\ 2 & 2.614 E-08\end{array}$

0.

$2.676 t-u$ $1.6691-06$ $6.195[-06$ $1.03 \cup[-0 S$ $1.694 \mathrm{E}-\mathrm{US}$ 2.97 b -05 $3.2 \cup 4 E-U !$ $2.840 E-05$ $2.822 \mathrm{E}-0 \mathrm{~S}$ $2.034 t-05$ $1.717 E-05$ $2.363 F-U S$ $1.349 \mathrm{E}-0 \mathrm{~S}$ $2.363 E-05$ 2. $516 E-05$ $2.592 \mathrm{E}-05$ $1.706 E-05$ $4.420 F-05$ $3.357 t-03$ $2.439 \mathrm{E}-0 \mathrm{~S}$ $1.832 \mathrm{~F}-05$ $1.050 E-05$ $3.570 E-06$

$2.830 E-06$ $1.041 \mathrm{E}-06$ $1.271 \mathrm{E}-06$ $1.660 \mathrm{E}-07$ des H3 3 036 039 442 045 048 $\forall$ 054 पड 1 059 062 dos 867 669 872 874 676
678 800 862 844 886 867 $\forall \forall 9$ 890 892 393

CKISSWIR:D INTEGRATFD=2.918E-03 1.284E-02 SEC/SO.M $\quad 1 / \mathrm{M}$

IEST V2 NOVEMGER 9,1972 ID 10 TU 1100 PST IHICLAMINE B RELEASE FKDM ELFVATILN DF $26 \mathrm{M}$ $1200 M A R C$ SAMPLER $1.5 \mathrm{M}$ U. $4.4 \mathrm{M} / \mathrm{SEC}$ AI $26 \mathrm{H}$

ALIMIITH EXPOSURE EIO EU/O DISIANCE DEGAES GH-SEC/CU.M SEC/CU.M IISJ.M MEIERS $\times 10 \mathrm{E}+6$

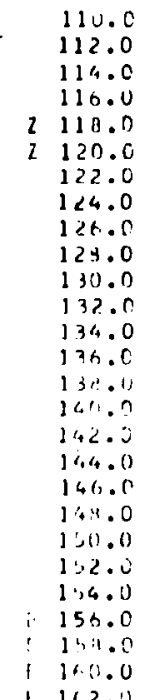

(FUSSAINU INTEGRATEU: $1.762 E-03$
U.

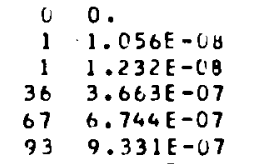

$93 \quad 9.331 \mathrm{E}-07$

$\begin{array}{ll}122 & 1.226 \mathrm{E}-06 \\ 213 & 2.133 \mathrm{E}-06\end{array}$

$\begin{array}{ll}213 & 2.133 E-06 \\ 274 & 2.747 E-06\end{array}$

$345 \quad 3.869 E-06$

$3.429 \mathrm{E}-06$

$1.782 \mathrm{E}-06$

$1.343 E-06$

$2.601 \mathrm{E}-166$

$2.221 E-46$

2. $2517 E-U 6$

$3.253 t-06$

$3.429 E-U 6$

$3.693 \mathrm{E}-\mathrm{V} 6$

$1.723 E-V 6$

$1.284 E-\cup 6$

$5.336 E-U 7$

$2.455 E-07$

$6.601 E-00$

$1.320 E-00$

0 .

SEC/SO.M
$647 \mathrm{E}-08$

$5.421 E-08$

$1.612 \mathrm{E}-06$

$2.967 t-60$

$.100 \mathrm{E}-06$

$5.393 t-06$

. 3 + $5 E-06$

1. ¿UYE-US

$1.702 \mathrm{E}-05$

$1.509 E-U S$

$7.040 \mathrm{t}-0 \mathrm{U}$

$5.9 \cup 4 F-00$

$1.144 \mathrm{E}-05$

$9.7715-06$

$9.771 \mathrm{E}-06$

$9.9 \cup u t-00$

$1.431 E-05$

$1.5 u \rightarrow 1-04$

$1.625 \mathrm{t}-05$

$7.56<t-60$

$5.651 \mathrm{t}-U \mathrm{G}$

$2.340 E-26$

1. CBOt-06

$2.934 \mathrm{~L}-017$

0 .

$7.752 E-03$
1200

1200

1200

1200

1200

1200

12.00

$1<00$

1200

1200

1200

1200

1200

1200

1200
1200

I 2 (wi)

$120 \mathrm{~J}$

120

1200

1200

1200 


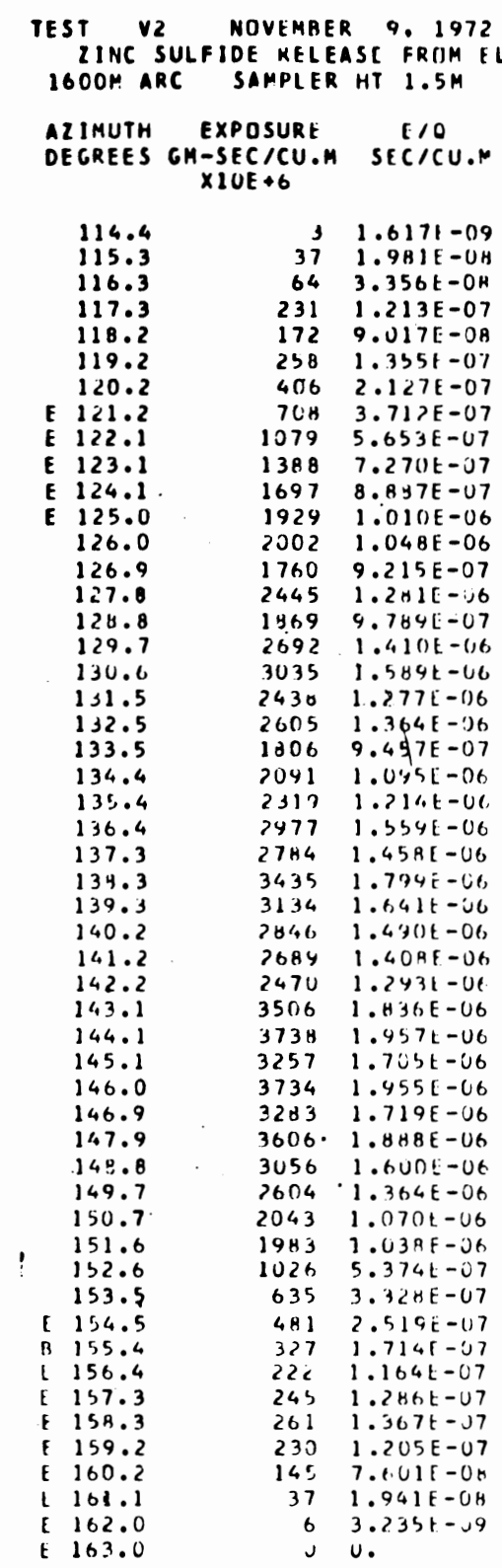

IUJC TU 11 OJT PST

FVATIUN DF $26 M$ MISEC AT 26 : EUIO OISIANCE
I/SO.M METERS

7.116E-UY $0.717 F-U S$ $1.477 E-07$ $5.337 \mathrm{E}-07$ $3.967 \mathrm{E}-\mathrm{C} 7$ $5.96 U F-01$ y. $35 B E-07$ $1.633 \mathrm{E}-06$ $2.4 R 7 E-06$ $3.199 \mathrm{E}-\mathrm{LG}$ $3.910 E-06$ $4.444 E-06$ $4.613 E-06$ $4.055 t-06$ $5.634 E-46$ 4. $307 E-06$ C. $2021-06$ C. $494 \mathrm{E}-0 \mathrm{~B}$ $5.61 \mathrm{JE}-06$ 1.. $U(3)-116$ 4. $161 \mathrm{E}-06$ 4. $E J \square E-O D$ ,. $343 t-06$ $6.8581-06$ $6.1414 E-00$ $7.9135-O C$ $7.221 \mathrm{E}-20$ C. SSOE-06 6.1951-06 $5.641 \mathrm{E}-6 \mathrm{t}$ b. $074 \mathrm{E}-0 \mathrm{C}$ Q. $612 \mathrm{E}-\mathrm{CO}$ $7.504 E-06$ b. 6 ن $4 E-1 J 6$ 7. $565 \mathrm{E}-\mathrm{U}$ 8. $306 \mathrm{E}-\mathrm{VE}$ $7.042 \mathrm{~F}-\mathrm{Cb}$ $6.001 \mathrm{E}-06$ 4. 707 E-06 4. $569 \mathrm{E}-06$ 2. $31.4 \mathrm{~F}-06$ $1.4645-00$ $1.108 \mathrm{E}-06$ $7.543 \mathrm{E}-U 7$ 5.124E-07 $5.657 \mathrm{E}-0$ 6. Clat $-U$ 1. $3 \cup 2 E-07$ $3.343 E-07$ 8. $540 E-O B$ $1.423 \mathrm{E}-\mathrm{UO}$ i.

1694

1695

CRUSSHIND INTEGRATFD= $1.277 t-03 \quad 3.618 \mathrm{E}-0 \mathrm{3}$

TEST VL NOVEMGER 9. 1972 1030 TU ILUJ PST . HHIUAHINE B RELEASF FKUM ELEVATIUN UF $26 M$ IF RI;A AKC SAMPIER IIT $1.5 \mathrm{M} \quad H=-4.4 \mathrm{M} / 3 L C$ AI 26.1

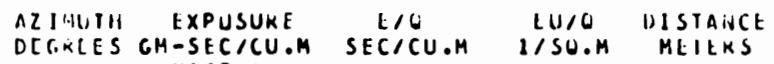
$X \perp O E+6$

$1.13: 4$

111.4
113.3

116.3

111.3

114.2

$11 \%$

1., 1.8

11.1 .2

11.2 .1

F 123.1

i $14 . .1$

$1 \quad 125.0$

$1: 6.0$

$1 \div 9.9$

$1<1.0$

$1 \cdot 1 \cdot 11$

144.7

$1 \% 1.1$

$1,1.2$

$1 \therefore 2.5$

13.5

$131 . .4$

1,0

$1: 7.3$

$1 \therefore 3$

$1 \% 0.0$

$1 \cdot 1.2$

$1 \div 2.2$

$1,3.1$

$1,4.1$

$14 \div .2$

146.0

$1 \% .9$

19.7 .4

11.8 .6

14.4 .7

19.87

$1,1 \cdot 6$

$1,2 \cdot 1$
12.1

$1, \therefore .5$

1,54

1. 156.4

1.7.1

$11,2.3$

$11,4.3$

.

$\begin{array}{ll}0 & 0 \\ 0 & 8\end{array}$

0.

E.C.11-10

H.621t-10

$1.7<4 t-08$

$7.414 t-U i$

5.000t-UO

9. $3371-013$

b. $336 t-6 \%$

4. $3: 335-.37$

H. 3 HBL-UT

B. 3BRE-CI

A. 3 U B E - O ?

B. $3 \triangle 8 E-U 7$

$7.94 \cap E-U 7$

1. $263 t-46$

1. $.063[-06$

1. $339 t-66$

1. $431,1-116$

1. 26, 3E-06

1. $297 \mathrm{~F}-\mathrm{vb}$

1. $306 t-v 6$
$1.016 t-06$

1. 2 Y $7 \mathrm{t}-4 \mathrm{C}$

$1.2631-06$

$1.5 .4[-36$

1.534t-00

1. 53 ? 1 -6u

1. 00 :i-ice

$1.4 \% 01-06$

$1.435 E-i b$

1. $0.4 t-J 6$

2. $504 t-66$

2. $5 \mathrm{C} 4 \mathrm{t}-\mathrm{Ut}$

1. $115 E-(t)$

1. $349 E-$ UO

2. $050(-1) 6$

1. $953 E-60$

1. 7i, 1)E- U6

$1.470 \mathrm{~L}-1) 6$

$1.007 t-06$

7.94i)t-i7

7 \&. $7,7 t-7$

4. $217 i-j$

$7.629[-6)$

$141.374 t-47$
$111.121 t-07$

4 5.)UOE-U

$2.759 t-20$

(i) c.

$3.7438-0.4$

3. 7 y 1 - Uy

1.5 $5 U E-U U$

$3: 202[-0]$

2.2 UUL -07

$4.1 .14 \mathrm{t}-07$

3. ज3 उद - 00

$3.6 \% 11-v 1$.

.6\%11-U0

$3.691 t-v c$

$3.6+1 t-06$

$3.691 \mathrm{E}-00$

$3.493 E-C 6$

$5.557 \mathrm{E}-\mathrm{vo}$

$4.67, L-00$

6. $1711-40$

c.jlut-vo

๖.১り أ - UC

$5.7 J 4 E-J U$

C. $C 1<1-0 Q$

4.4 UUL-UO

5: 7)yl-Ue.

๖.๖๖7:-06

c.t1st-ic

1.. $1111-1) 6$,

$6.7111-0 t$

$7.011 .1-1 / 0$

$6.467 E-06$

6. $310 L-U O$

$0.136 E-v 0$

$1: 1$ UCt-Us

$1: 10 i t-u b$

7 पyen1-60

$0.1 \mathrm{sel}-0 \mathrm{c}$

$4.047 t-U C$

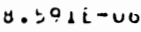

7.es $3 t-0 c$

$1.40 / L-v 6$

$4.4 j J l-v e$

$3.4,5 !-0 j$

2.4.11-vis

1. $16,1-2 e$

1.15/t-1;0

$0.000 t-41$

$4.431 i-67$

¿.2uvt-u7

1.C 4 t $-v 7$

u.

(1). SS IH: IHTLCRATEL $=1.370 E-U$ S G.UBUE-US
1631

$16 \mathrm{sli}$

1640

1641

$16+4$

1646

1641

16 穴

1612

1613

165'

$16 b 6$

1654

1639

1660

$160<$

1301

1004

1605

1001

1668

$16,6 y$

$16,1 \mathrm{~J}$

1612

101.3

1014.

1676

1617

1670

1619

1006

1501

1602

1603

1604

16 k6

$120 \%$

16.91

$16: 3$

$15 \rightarrow 1$

1691

1692

1643

$$
\text { SEC/SU.M } 1 / M
$$


IEST V2 NOVEABER Y, $19 / 2$ 1030 IO 1100 PST IINC SULFIDE RELEASH FRCM LLFVATIUN DF LLM $2200 \mathrm{M}$ ARC SAMPLER HT $1.5 \mathrm{M}$ U. $4.4 \mathrm{M} / \mathrm{HEC}$ AT $26 \mathrm{M}$

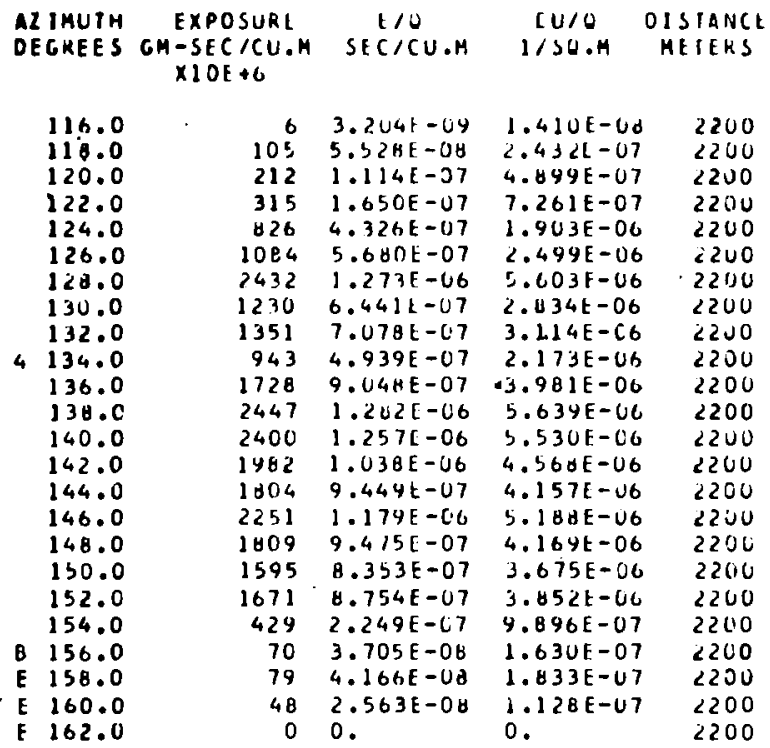

CRISSHIND INIEGRATED=1.079E-U3 4.746E-03

SECISU.M $\quad 1 / M$

IEST V2 NOVEMBER 4,1972 1030 TO 1100 HST IINC SULFIDE RFLEASE FRUM ILFVATIUN UF ZUM $3200 M$ ARC SAMPLER HI $1.5 \mathrm{M} U=4.4 \mathrm{M} / \mathrm{SEC}$ AI $26 \mathrm{M}$

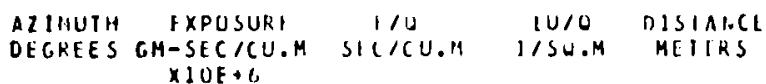

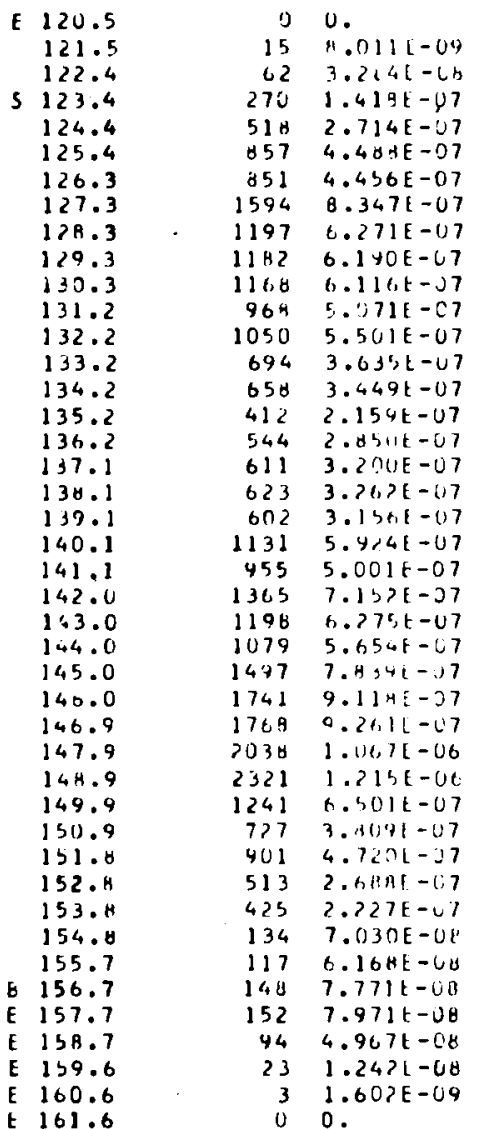

$\therefore \quad 3249$

3251

$-67 \quad 3252$

$6.23 y t-01 \quad 3233$

$1.144-66 \quad 325$

$1.975 E-06 \quad 3256$

$1.901 \mathrm{t}-06 \quad 3258$

$3.6731 .06 \quad 3259$

$2.759 E-00$,261

$2.724 \mathrm{E}-06 \quad 3262$

$2.091 \mathrm{t}-50 \quad 3263$

$2.2311-06 \quad 326.4$

$2.421 F-C 6 \quad 3266$

$1.59+1-06 \quad 3267$

$1.5111-06 \quad 3260$

$9.449 \mathrm{E}-07 \quad 321 \mathrm{U}$

$1.254 \mathrm{E}-06 \quad 3211$

$1.400 E-0 B \quad 3272$

$1.434 \mathrm{~F}=\mathrm{CQ} \quad \$ 273$

$1.38 \%-06 \quad 3274$

$2.0078-06 \quad 3270$

$2.200 E-06 \quad \$ 277$

$3.147 \mathrm{t}-0 \mathrm{O} 3278$

$2.761 E=06 \quad 3274$

$2.4 \mathrm{BOE}-\mathrm{UC} \quad 1280$

$3.4 .91-06 \quad 3281$

4. U1<F-CO 3262

$4.075 E-06$ J2H3

$4.043 E-06 \quad 3284$

$2.347 \mathrm{E}-\mathrm{Ct} \quad 328 \mathrm{~S}$

$2.10 \mathrm{OU}-U C$
1.060

1. $77 \mathrm{E}-\mathrm{OU} \quad 3247$

1. $1 \mathrm{HBF}-\mathrm{CE} \quad 3203$

$9.799 \mathrm{E}-07 \quad 3209$

$3.093 \mathrm{E}-07 \quad 3290$

$2.714 \mathrm{~F}-07 \quad 3240$

$3.414 t-07 \quad 1291$

$3.507 E-07 \quad 3292$

C.IYSE-U7 3292

$3.463 \mathrm{E}-08 \quad 3293$

7. USUE-04

3294

0 .

CRUSSHINO INTEGRAIED=9.621E-04 4.321E-U3

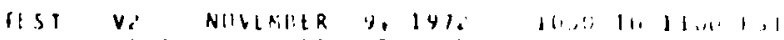

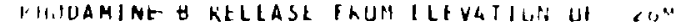
Z2TRA ARC SAMPLLR HI $1.5 \mathrm{M} U=4.4 \mathrm{M} / \mathrm{JEL}$ AI CUM

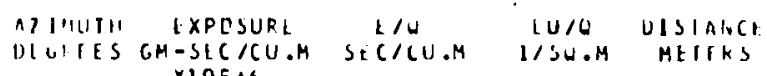
$\times 10 E+6$

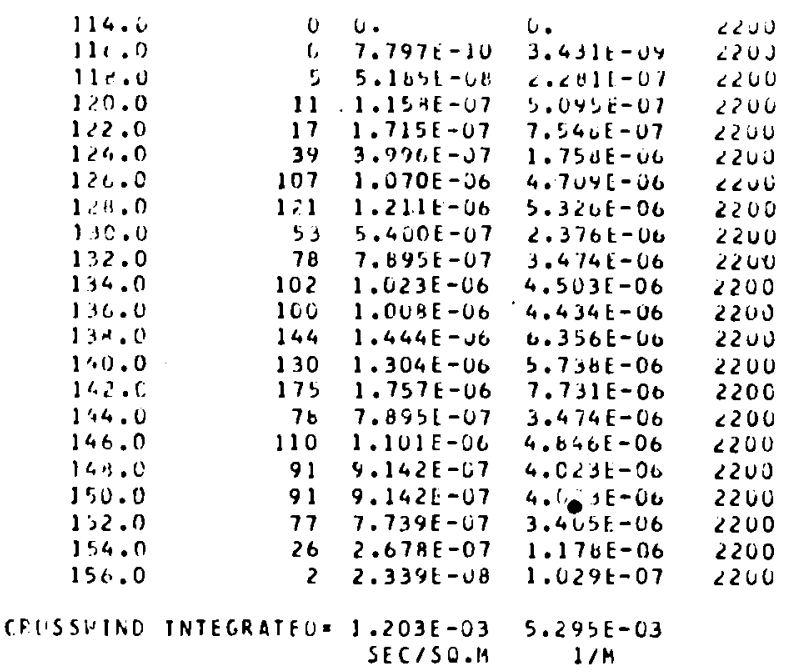

ITST V2 NUVEMUER $\%, 1472$ 1030 TL 1100 PST WHIIDAMINE B RELEASE FKOJM ELFVATIUN UF $2 J M$ 32NIM ARC SAMPLER HT $1.54 \quad U=.6 . .4$ M/SEC AT $26 M$

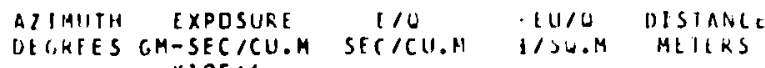
$x 10 F+6$

\begin{tabular}{|c|c|c|c|c|}
\hline 120.5 & 0 & $1.940 \mathrm{E}-3 \circ$ & $i .577 \mathrm{E}-\cup 9$ & 3249 \\
\hline 121.5 & 1 & $1.404 \mathrm{E}-\mathrm{OH}$ & $0.1755-0 b$ & 221 \\
\hline $1 ; 2.4$ & 3 & 3. $\{1,1[-01\}$ & $1.3341-67$ & 9238 \\
\hline 123.4 & 1.3 & $1.345 \mathrm{E}-6.4$ & $5.41\}[-07$ & 3233 \\
\hline 124.4 & 21 & $2.172 \mathrm{E}-07$ & $9.5556-07$ & sLb \\
\hline 125.4 & 33 & $3.398 \mathrm{E}-U \%$ & $1.441 E-06$ & 3256 \\
\hline 128.3 & 29 & $2.932[-0]$ & $1 . \angle \% O E-06$ & 258 \\
\hline 127.3 & 63 & $6.335 E-07$ & $2.70 \mathrm{JE}-016$ & 3257 \\
\hline $1<2.3$ & 52 & $5.244 E-07$ & $2.307 t-06$ & 3261 \\
\hline $1<9.3$ & 63 & $6.335[-07$ & $2.7 \unlhd \forall[-06$ & $\$ 262$ \\
\hline 130.3 & 50 & 5. Oesest-jo & $2: 23 y E-06$ & 3263 \\
\hline $1+1.2$ & 52 & $5.244 E-97$ & $2: 307 E-00$ & 3264 \\
\hline $1: 2.2$ & 52 & $5.244 E-07$ & $2.307 E-00$ & 3260 \\
\hline 133.2 & 35 & $3.591 E-67$ & $1.3 d u t-06$ & 3267 \\
\hline 134.2 & $2 B$ & $2.930 E-07$ & $1.2+5 k-06$ & 3268 \\
\hline 191.2 & 23 & $2.374 E-07$ & $1.045 E-06$ & 3270 \\
\hline 136.2 & 25 & $2.5 i 6 t-01$ & $112 \mathrm{E}-0 \mathrm{~b}$ & 271 \\
\hline 137.1 & 30 & $3.033 i-i 7$ & $1.35 \Delta t-v b$ & 3212 \\
\hline $1=1.1$ & 30 & $3.033 E=07$ & $1.335[-06$ & 9213 \\
\hline $1,4.1$ & 3b & $3.540 E-07$ & $1.5 b a[-6 b$ & 3214 \\
\hline $1+v \cdot 1$ & 60 & $6.023 E-07$ & $2.65 U E-06$ & 9276 \\
\hline 141.1 & 64 & $6.491 E-07$ & $2.0>6 t-06$ & 3277 \\
\hline $1+2.0$ & 68 & $6.8033 E-07$ & $2.493 \mathrm{t}-60$ & $327 \mathrm{~B}$ \\
\hline 143.0 & 63 & $6.135[-07$ & $2.750 \mathrm{t}-10$ & 3.79 \\
\hline 144.0 & 61 & $6.174 t-07$ & $2.71+6-66$ & 3280 \\
\hline 145.0 & 80 & 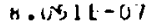 & $3.5+2 \mathrm{t}-06$ & 3201 \\
\hline $1+60$ & $O H$ & $0.050 E-07$ & $3.605 t-06$ & 3212 \\
\hline 146.0 & 68 & $8.830 E-07$ & $3.885 E-06$ & 3203 \\
\hline 147. & 100 & $1.056 E-06$ & $4.717 E-06$ & 3204 \\
\hline 1.4 .9 .9 & 110 & $1.101 E-06$ & $4.840 E-66$ & 3205 \\
\hline $11 \% \cdot 1$ & 52 & $5.244 E-07$ & $2: 307 t-16$ & 3200 \\
\hline ] $\because i .2$ & 30 & $3.033[-07$ & $1.3136-06$ & $3\langle 8\rangle$ \\
\hline $151 . \%$ & 31 & $3.135 t-67$ & $1.379 t-06$ & 3287 \\
\hline 152.0 & 21 & $2.121 \mathrm{E}-67$ & $9: 332 L-07$ & 3288 \\
\hline 151.8 & 18 & $1.817 t-07$ & $7.944 E-C 7$ & 3289 \\
\hline $154 . H$ & 3 & $3.321 \mathrm{E}-0 \mathrm{~B}$ & $1.601 E-07$ & 3290 \\
\hline 135.7 & b & $5.497 E-08$ & $2: 41$ YE -07 & 3240 \\
\hline 156.7 & 2 & $2.534 \varepsilon-08$ & $1.115 \mathrm{E}-07$ & 2201 \\
\hline
\end{tabular}

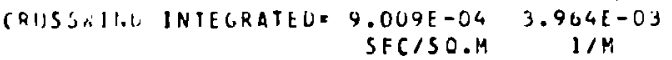


TOMER DATA FOLLOW...

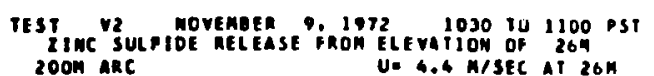

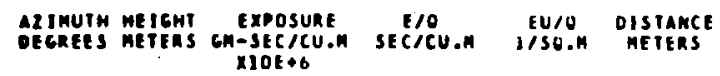

102.0 . 140 .

0 .

200

AZINUTH HEIGHT EXPOSURE E/O EUNO UISIANCE DECRES HETERS GM-SECKCU.A SEC/CU.M l/SO.M METERS

$\begin{array}{rr}110.0 & .4 \\ 110.0 & .0 \\ 110.0 & 1.5 \\ 110.0 & 2.5 \\ 110.0 & 4.0 \\ 110.0 & 3.5 \\ 110.0 & 7.0 \\ 110.0 & 1.5 \\ 110.0 & 10.0 \\ 110.0 & 13.0 \\ 110.0 & 16.0 \\ 110.0 & 19.0 \\ 110.0 & 22.0 \\ 110.0 & 25.0 \\ 110.0 & 28.0 \\ 110.0 & 31.0 \\ 110.0 & 32.8\end{array}$

$\begin{array}{rll}742 & 3.085 E-07 & 1.710 E-06 \\ 1017 & 5.330 E-07 & 2.345 E-06 \\ 1621 & 0.484 E-07 & 3.735 E-C 6 \\ 2341 & 1.226 E-06 & 5.394 E-06 \\ 1573 & 0.238 E-07 & 3.625 E-06 \\ 1348 & 7.060 E-07 & 3.106 E-06 \\ 2156 & 1.179 E-06 & 4.960 E-06 \\ 2308 & 1.200 E-06 & 5.317 E-06 \\ 2142 & 1.122 E-06 & 4.935 E-06 \\ 1900 & 9.951 E-07 & 4.379 E-06 \\ 2071 & 1.005 E-06 & 4.772 E-06 \\ 256 & 1.343 E-07 & 5.911 E-07 \\ 376 & 1.971 E-07 & 8.673 E-07 \\ 458 & 2.400 E-07 & 1.056 E-06 \\ 168 & 1.823 E-08 & 3.082 E-07 \\ 253 & 1.376 E-07 & 5.833 E-07 \\ 152 & 7.977 E-08 & 3.510 E-07\end{array}$

200
200
200
200
200
200
200
200
200
200
200
200
200
200
200
200
200

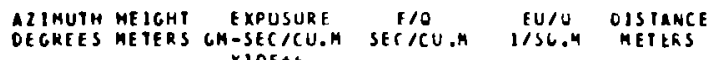
$\times 10 E+b$

$\begin{array}{rr}9110.0 & .4 \\ 9118.0 & .0 \\ 118.0 & 1.5 \\ 118.0 & 2.5 \\ 118.0 & 4.0 \\ 110.0 & 5.5 \\ 110.0 & 7.0 \\ 119.0 & 9.5 \\ 118.0 & 10.0 \\ 118.0 & 13.0 \\ 118.0 & 16.0 \\ 118.0 & 19.0 \\ 118.0 & 22.0 \\ 119.0 & 25.0 \\ 110.0 & 28.0 \\ 110.0 & 31.0 \\ 110.0 & 32.0\end{array}$

\begin{tabular}{|c|c|c|}
\hline $4 n 60$ & 2.56 & \\
\hline 6573 & $3.442 \mathrm{E}-00$ & $1.514 E-05$ \\
\hline 9137 & $4.784 \mathrm{~F}-6 \mathrm{~B}$ & c.105k-05 \\
\hline 10601 & $5.5511-00$ & $2.442 t-U 5$ \\
\hline 8080 & $4.230 E-36$ & $1 . B B I K-U S$ \\
\hline 23712 & $1.241 \mathrm{E}-05$ & $5.46<\varepsilon-05$ \\
\hline 94? & 1.9 >YL -05 & $8.0441-05$ \\
\hline 36509 & $1.911 \mathrm{E}-\mathrm{OS}$ & $8.411 t-05$ \\
\hline 35314 & $8498-05$ & $0.135 E-05$ \\
\hline 790 & $1.979 E-J 5$ & $0.700 \mathrm{E}-05$ \\
\hline 43237 & $2.2648-0 b$ & $9.9611-05$ \\
\hline songe & $2.673 E-05$ & 1).154t-04 \\
\hline & & $7.005 \mathrm{E}-05$ \\
\hline 40520 & $2.122 \mathrm{E}-35$ & $9.335 \mathrm{i}-05$ \\
\hline 50752 & $2.657 t=05$ & $1.169 \mathrm{c}=06$ \\
\hline & 05 & $1 E-\cup 5$ \\
\hline 22185 & $1.16,2 E-05$ & $5.1111=05$ \\
\hline
\end{tabular}

200
200
200
200
200
200
200
200
200
200
200
200
200
200
200
200
200

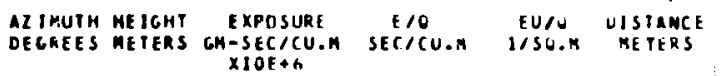

$\begin{array}{ll}0 & 126.0 \\ 0 & 126.0\end{array}$

126.0

126.0

326.0

126.0

1.5

$126.0 \quad 10.0$

13.0

126.0

126.0 !

126.0

126.0

126.0

.4
1.8
2.5
6.0
5.5
7.0
.5
0.0
3.0
6.0
9.0
2.0
5.0
28.0
31.0
32.8

$2842 \quad 1.486 \mathrm{EF}-06 \quad 6.54 \mathrm{dt}-06$ $6613 \quad 2.415 \mathrm{t}-06 \quad 1.063 t-0$ $1913 \mathrm{H}$ 22536 I. $140 E-05$ $5.191 E-U 5$

$27061 \quad 1.459 E-05 \quad 0.410 E-65$

35944 I.SBjE-05 O.28UE-OS

49148 2.573E-05 $1.132 E-64$

$430562.254 E-05 \quad 9.918 E-05$

$71749 \quad 3.757 E-05 \quad 1.653 E-04$

$65567 \quad 3.433 \mathrm{E}-0 \mathrm{~S} \quad 1.510 \mathrm{E}-04$

$71054 \quad 3.720 \mathrm{~F}-05 \quad 1.637 \mathrm{E}-04$

$28569 \quad 1.496 E-05 \quad 6.581 E-05$

$72990 \quad 3.822 \mathrm{E}-05 \quad 1.682 \mathrm{E}-04$

$\begin{array}{lll}37914 & 3.032 E-05 & 1.334 E-04 \\ 33551 & 1.757 E-05 & 7.729 E-05 \\ 39425 & 2.064 E-05 & 9.002 E-05\end{array}$

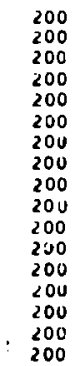

200
200
200
200
200
200
200
200
200
200
200
200
200
200
200
200
200 rowt datA FnLLOW...

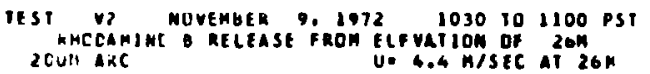

AZI:UYH HEICHT EXPOSURE E/O EU/O DISTANCE

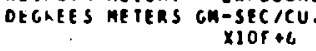

$\begin{array}{llllll}402.0 & .4 & 0 & 0 . & 0 . & 200\end{array}$

AZIMUTH HEIGHT EXPOSURE F/O EU/O OISTANCE PEC.HEES METERS GM-SEC/CU.H SECICU.M I/SO.M METERS

$\begin{array}{lr}110.0 & .4 \\ 110.0 & .8 \\ 110.0 & 1.5 \\ 110.0 & 2.5 \\ 110.0 & 4.0 \\ 110.0 & 5.5 \\ 1110.0 & 7.0 \\ 110.0 & 10.5 \\ 110.0 & 10.0 \\ 110.0 & 13.0 \\ 110.0 & 16.0 \\ 110.0 & 19.0 \\ 110.0 & 22.0 \\ 110.0 & 25.0 \\ 11000 & 29.0 \\ 110.0 & 31.0 \\ 110.0 & 32.8\end{array}$

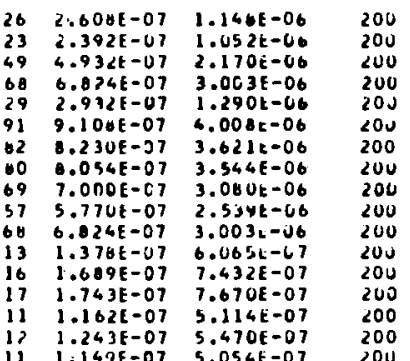

4IDHTH HE IGHT EXDOSURF F/O EU/U UISTANCE DFCLEES METERS $\mathrm{CM}-\mathrm{SEC} / \mathrm{CU}$

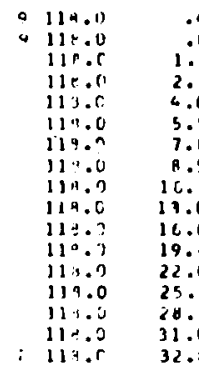

.4
1.8
2.5
2.5
5.0
7.5
7.5
10.0
19.0
16.0
19.0
22.0
25.0
28.0
31.0
32.8

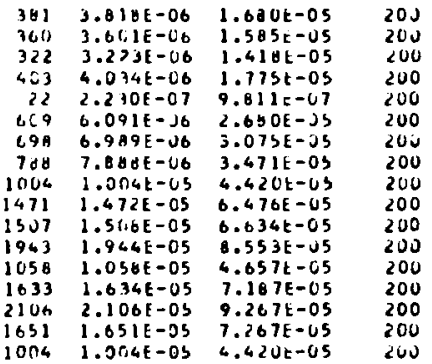

ATIYCIT HEIGH EXPLSLAR F/O EU/O OISTANCE LETEYS METER: GM-SETICO

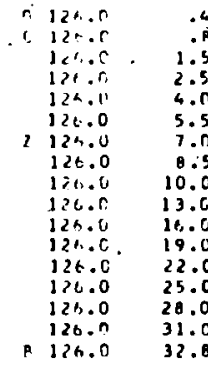

.4
1.5
2.5
4.0
5.5
7.0
8.5
10.0
13.0
16.0
19.0
22.0
25.0
28.0
31.0
32.0




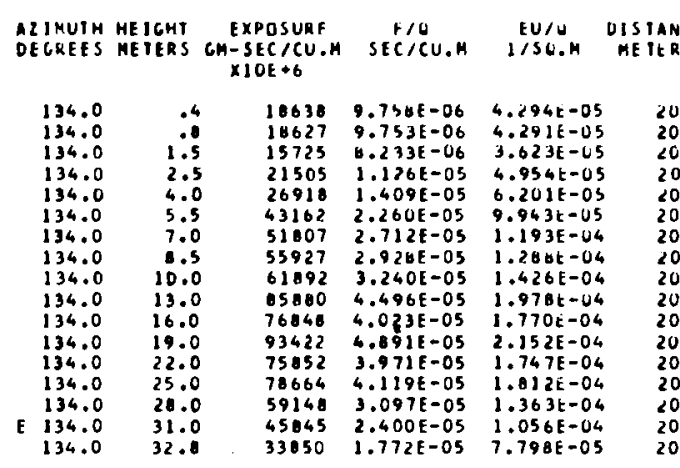

TUHER DATA FOLLLW...

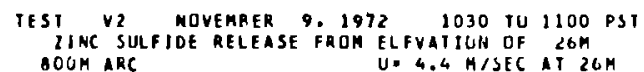

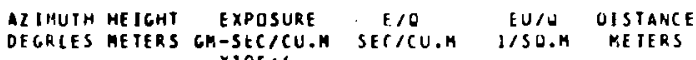
$\times 10 E+6$

0 .

$* 2 i$

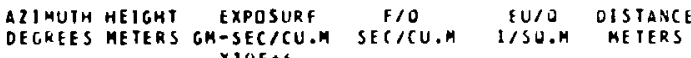
$\times 10 \mathrm{E}+6$

112.6
112.6
112.6
112.6
112.6
112.6
112.6
112.6
112.6
112.6
112.6
112.6
112.6
112.6
112.6
112.6
1112.6
112.6

.3
1.5
1.5
2.5
4.2
6.3
8.4
10.5
12.6
14.7
16.8
21.0
25.2
29.4
33.6
37.8
42.0

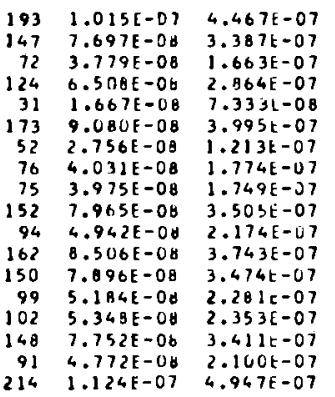

633
833
833
233
833
033
033
833
833
833
033
633
833
033
033
833
833
833
833

DIIMUTH HEIGHT EXPOSURF

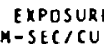
$\begin{array}{ll}x & 10 \mathrm{O} \rightarrow \mathrm{C}\end{array}$

\begin{tabular}{|c|c|c|c|c|}
\hline $\begin{array}{l}120.2 \\
120.2 \\
120.2\end{array}$ & $\begin{array}{r}.3 \\
: 5 \\
1.1\end{array}$ & $\begin{array}{l}5541 \\
5353 \\
6421\end{array}$ & $\begin{array}{l}2.901 E-06 \\
2.803 E-06 \\
3.362 E-06\end{array}$ & $\begin{array}{l}1.277 t-05 \\
1.233 t-05 \\
1.479 t-05\end{array}$ \\
\hline $\begin{array}{l}120.2 \\
120.2\end{array}$ & $\begin{array}{l}1.5 \\
2.1\end{array}$ & $\begin{array}{l}5457 \\
5745\end{array}$ & $\begin{array}{l}2.857 E-06 \\
3.000 E-06\end{array}$ & $\begin{array}{l}1.257 L-05 \\
1.324 E-05\end{array}$ \\
\hline 120.2 & 4.2 & 5400 & $2.027 E-06$ & $1.244 E-05$ \\
\hline $12 \theta$ & 6.3 & $\$ 602$ & $2.9331-06$ & $1.291 E-U 5$ \\
\hline 120 & 0.4 & 5392 & $2.823 E-06$ & $1.24 \angle E-05$ \\
\hline 120 & 10.5 & 5153 & $2.694 \mathrm{~F}-06$ & $1.187 t-05$ \\
\hline 120. & 12.6 & 5087. & $2.664 E-0 C$ & $1.172 \mathrm{E}-05$ \\
\hline 120.2 & 14.7 & $\begin{array}{l}4153 \\
5383\end{array}$ & $\begin{array}{l}2.175 E-D O \\
2.818 E-06\end{array}$ & $\begin{array}{l}9.569 k-06 \\
1.240 t-05\end{array}$ \\
\hline is & 21.0 & 4932 & 2.5 B $3-06$ & $\begin{array}{l}1.2400-05 \\
1.136 t-05\end{array}$ \\
\hline & 25.2 & 5154 & $2.699 \mathrm{E}-06$ & i.100t-os \\
\hline 120 & 29.4 & 3299 & $1.728 \mathrm{E}-06$ & $7.601 E-06$ \\
\hline & & 2151 & $1.127 t-06$ & $4.957 E-06$ \\
\hline & & 3740 & $1.958 \mathrm{E}-08$ & $8.617 \mathrm{E}-06$ \\
\hline 120.2 & 42,0 & 3745 & $1.961 \mathrm{E}-06$ & $6.627 \mathrm{E}-06$ \\
\hline
\end{tabular}

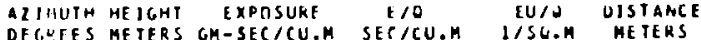
$x 20 E+6$
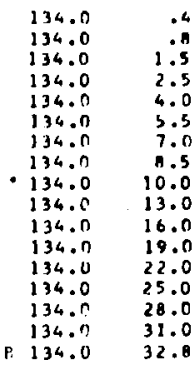

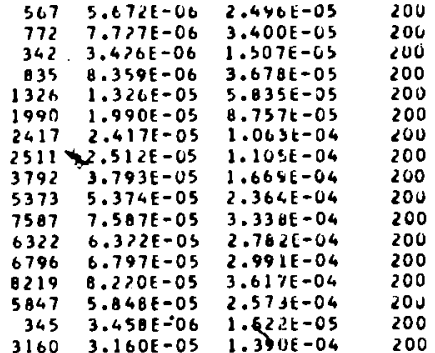

TOMER DATA FULLOW...

TEST V2 NCVEMGER $9,1972 \quad 1030101100$ PST RIIUDAMINE B RELEASE FROM ELFVATIUN OF $26 \mathrm{M}$
UDOM ARC

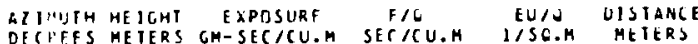
$\times 106+6$

1. $1.15 .0 \quad 0 \quad 00.00 \quad 020$

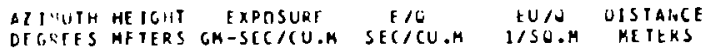
$\times 10 E+6$

\begin{tabular}{|c|c|c|c|c|c|}
\hline 112.5 & .3 & 16 & $1.6 \mathrm{RGE}-07$ & $7.420 E-07$ & d 33 \\
\hline 112.6 & 3 & 14 & $1.451 E-07$ & $6.384 E-L 7$ & B34 \\
\hline $112 . t$ & 1.1 & 12 & $1.216 E-07$ & $5.349 E-07$ & 833 \\
\hline 312.6 & 1.5 & 6 & $0.536 \mathrm{E}-0 \mathrm{~d}$ & $2.87 b t-07$ & 833 \\
\hline 112.6 & 2.1 & 13 & $1.333 \mathrm{E}-07$ & $5.867 t-07$ & 033 \\
\hline 112.6 & 4.2 & 12 & $1.216 E-07$ & $5.369[-57$ & d33 \\
\hline 112.6 & h. 3 & $\theta$ & $0.627 \mathrm{E}-0 \mathrm{\theta}$ & $3.756 E-07$ & 433 \\
\hline 112.6 & 8.4 & 12 & $1.21 \in E-07$ & $5.349 E-07$ & 833 \\
\hline $112 . t$ & 10.5 & 13 & $1.333 \mathrm{E}-07$ & $5.867 E-67$ & 833 \\
\hline 112.6 & 12.6 & 22 & $2.275 E-117$ & $1.001 E-06$ & d33 \\
\hline 112.6 & 14.7 & 12 & $1.216 E-07$ & $5.349 k-07$ & 833 \\
\hline 112.0 & 16.8 & 19 & $1.922 E-07$ & $8.455 f-67$ & d33 \\
\hline 112.6 & 21.0 & 26 & $2.027 E-.7$ & $1.156 E-06$ & 833 \\
\hline $112 . t$ & 25.7 & 25 & $2,5100-07$ & $1.104 E-06$ & 833 \\
\hline 112.1 & 29.4 & 30 & $3.090 E-07$ & $1.363 \mathrm{E}-06$ & B33 \\
\hline 112.6 & 33.8 & 23 & $2.392 t-07$ & $1.053 \mathrm{E}-06$ & d3s \\
\hline 112.6 & 37.6 & 5 & $5.098 E-0 B$ & $2.243 E-07$ & 433 \\
\hline 112.6 & 42.0 & 27 & $2.745 \mathrm{E}-07$ & $1.208 \mathrm{t}-0 \mathrm{~b}$ & 633 \\
\hline
\end{tabular}

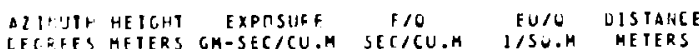
LECOFES METERS GM-SEC/CU
XIOE+G

\begin{tabular}{|c|c|c|c|c|c|}
\hline 179.2 & .3 & 561 & $5.017 E-56$ & $2.471 E-C b$ & 045 \\
\hline 120.7 & .5 & 596 & $5.96 .26-06$ & $2.623 E-05$ & 045 \\
\hline 120.2 & 1.1 & 642 & $6.4>2 E-06$ & $2.8<B E-05$ & 845 \\
\hline 120.2 & 1.5 & 334 & $3.85 \cup E-06$ & $1.694 E-65$ & 845 \\
\hline 120.2 & 2.1 & 619 & $0.192 \mathrm{E}=06$ & $2.725 E-05$ & 345 \\
\hline 120.7 & 4.2 & 711 & $7.112 E-06$ & $3.129 \varepsilon-35$ & 845 \\
\hline 126.2 & b. 3 & 607 & $0.077 \mathrm{E}-06$ & $2.674 E-05$ & 145 \\
\hline$i 2 n, ?$ & B. 4 & 665 & $0.652 \mathrm{~F}-06$ & $2.927 k-05$ & 345 \\
\hline $1<0.2$ & 10.5 & 458 & $4.562 t-06$ & $2.016 \mathrm{t}-0 \mathrm{~s}$ & 645 \\
\hline 120.2 & 12.6 & 711 & $7.112 f-06$ & $3.129 t-05$ & 045 \\
\hline 120.2 & 14.7 & 699 & $0.997 E-0 B$ & $3.079 t-65$ & 045 \\
\hline 120.2 & 16.8 & 745 & $7.458 E-06$ & $3.281 t-05$ & 845 \\
\hline 120.2 & 21.0 & 680 & 6.คA $2 E-06$ & $3.028 E-05$ & 345 \\
\hline 120.2 & 25.2 & 619 & $6.192 E-06$ & $2.725 t-05$ & 045 \\
\hline 120.2 & 29.4 & 538 & $5.387 E-06$ & $2.370 t-05$ & 845 \\
\hline 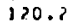 & 33.6 & 262 & $2.620 E-00$ & $1.156 E-05$ & $\checkmark 45$ \\
\hline 120.2 & & 492 & $4.927 E-06$ & $2.168 E-05$ & 045 \\
\hline 120.7 & 42.0 & 487 & $4.875 E-26$ & $2.145 t-05$ & 845 \\
\hline
\end{tabular}




\begin{tabular}{|c|c|c|c|c|c|c|c|c|c|c|c|}
\hline $\begin{array}{l}\text { AZI I AUTH } \\
\text { DEGREES }\end{array}$ & $\begin{array}{l}\text { HE ICHT } \\
\text { ME TERS }\end{array}$ & $\begin{array}{c}\text { EXPOSURE } \\
\text { CN-SEC/CU.N } \\
\times 1 O E+6\end{array}$ & $\begin{array}{l}\text { F/O } \\
\text { SEC/CU.M }\end{array}$ & $\begin{array}{l}\text { EU/J } \\
\text { I/SO.M }\end{array}$ & $\begin{array}{c}\text { DISIANCE } \\
\text { METERS }\end{array}$ & $\begin{array}{l}\text { ALYUUTH } \\
\text { DE G, IES }\end{array}$ & $\begin{array}{l}\text { HE IGHT } \\
\text { METERS }\end{array}$ & $\begin{array}{c}\text { EXPQSURF } \\
\text { GM-SECICU.M } \\
\times] O E+6\end{array}$ & $\begin{array}{c}\text { F/O } \\
\text { SEC/CU.M }\end{array}$ & $\begin{array}{l}\text { EU/u } \\
\text { I/SO.A }\end{array}$ & $\begin{array}{l}\text { DISTANCE } \\
\text { METERS }\end{array}$ \\
\hline $\begin{array}{l}127.7 \\
127.7 \\
127.7 \\
127.7 \\
127.7 \\
127.7 \\
127.7 \\
127.7 \\
127.7 \\
127.7 \\
127.7 \\
127.7 \\
127.7 \\
127.7 \\
127.7 \\
127.7 \\
127.7 \\
127.7\end{array}$ & $\begin{array}{r}.3 \\
1.5 \\
1.1 \\
1.5 \\
2.1 \\
4.2 \\
6.3 \\
10.4 \\
10.5 \\
12.6 \\
14.7 \\
16.0 \\
21.0 \\
25.2 \\
29.4 \\
33.6 \\
37.8 \\
42.0\end{array}$ & $\begin{array}{l}10809 \\
10689 \\
9251 \\
6477 \\
10189 \\
0364 \\
0976 \\
0486 \\
7513 \\
7209 \\
6298 \\
0649 \\
1575 \\
5939 \\
4343 \\
3267 \\
836 \\
2309\end{array}$ & $\begin{array}{l}5.660 E-06 \\
5.597 E-06 \\
5.150 E-06 \\
3.391 E-06 \\
5.335 E-06 \\
4.379 E-06 \\
4.700 E-06 \\
4.443 E-06 \\
3.934 E-06 \\
3.775 E-06 \\
3.298 E-06 \\
4.528 E-06 \\
1.251 E-07 \\
3.110 E-06 \\
2.274 E-06 \\
1.711 E-06 \\
4.389 E-07 \\
1.209 E-06\end{array}$ & $\begin{array}{l}2.490 E-05 \\
2.462 E-05 \\
2.269 E-05 \\
1.492 E-05 \\
2.347 E-05 \\
1.927 E-05 \\
2.060 E-05 \\
1.955 E-05 \\
1.731 E-05 \\
1.661 E-05 \\
1.451 E-05 \\
1.993 E-05 \\
3.630 E-06 \\
1.368 E-05 \\
1.001 E-05 \\
7.527 E-06 \\
1.931 E-06 \\
5.320 E-06\end{array}$ & $\begin{array}{l}857 \\
857 \\
857 \\
857 \\
857 \\
657 \\
857 \\
057 \\
857 \\
857 \\
057 \\
857 \\
857 \\
057 \\
857 \\
857 \\
857 \\
057 \\
057\end{array}$ & $\begin{array}{l}127.7 \\
127.7 \\
127.7 \\
127.7 \\
127.7 \\
127.7 \\
127.7 \\
177.7 \\
127.7 \\
127.7 \\
127.7 \\
127.7 \\
1127.7 \\
127.7 \\
127.7 \\
127.7 \\
E \\
B \\
B \\
127.7 \\
127.7\end{array}$ & $\begin{array}{r}.3 \\
.5 \\
1.1 \\
1.5 \\
2.1 \\
4.2 \\
6.3 \\
8.4 \\
10.5 \\
12.6 \\
14.7 \\
16.8 \\
21.0 \\
25.2 \\
29.4 \\
33.6 \\
37.8 \\
42.0\end{array}$ & $\begin{array}{r}961 \\
1190 \\
869 \\
641 \\
1099 \\
1007 \\
778 \\
1007 \\
1099 \\
1053 \\
961 \\
854 \\
920 \\
1007 \\
839 \\
716 \\
655 \\
609\end{array}$ & $\begin{array}{l}9.616 E-06 \\
1.191 E-05 \\
6.698 E-06 \\
6.413 E-06 \\
1.099 E-05 \\
1.007 E-05 \\
7.7 A C E-06 \\
1.007 E-05 \\
1.099 E-05 \\
1.053 E-05 \\
9.616 E-06 \\
8.545 E-06 \\
9.204 E-06 \\
1.007 E-05 \\
8.392 E-06 \\
7.169 E-06 \\
6.557 E-06 \\
6.098 E-06\end{array}$ & $\begin{array}{l}4.232 E-05 \\
5.240 E-05 \\
3.827 E-05 \\
2.822 E-05 \\
4.837 t-05 \\
4.433 E-05 \\
3.423 E-05 \\
4.433 E-05 \\
4.837 E-05 \\
4.635 E-05 \\
4.231 t-05 \\
3.760 E-05 \\
4.050 E-05 \\
4.433 t-05 \\
3.693 E-05 \\
3.154 E-05 \\
2.885 E-05 \\
2.683 E-05\end{array}$ & $\begin{array}{l}857 \\
857 \\
857 \\
857 \\
657 \\
657 \\
857 \\
457 \\
857 \\
857 \\
457 \\
057 \\
857 \\
857 \\
057 \\
857 \\
057 \\
857\end{array}$ \\
\hline
\end{tabular}

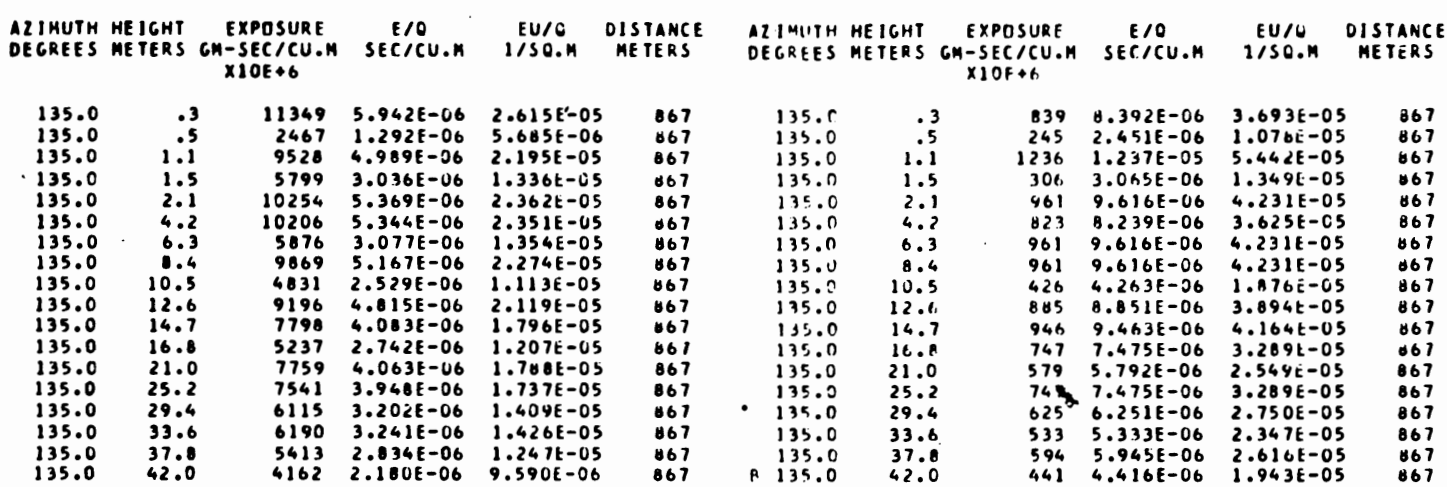

TOHER DATA FOLLCW....

TEST V. NUVERBER 9.19721030 TO 1100 PST IIMC SULFIOE RELEASE FROM ELFVATION OF $26 \mathrm{~K}$
$1600 \mathrm{M}$ ARC
UE $4.4 \mathrm{M} / \mathrm{SEC}$ AT $26 \mathrm{M}$

AZIMUTH HEIGHT EXPOSURE E/O EU/J OISTANCE

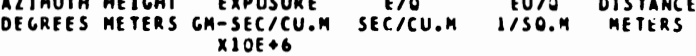
$x 10 E+6$

N $101.6 \quad .2$

0. 1617

TMUER MATA FRLLOW....

$>$

IEST V2 NOVEMBER 9, $1972 \quad 1030$ TU 1100 PST KHIDOAMINE B RFLEASE FROM ELEVATIUN OF $26 \mathrm{M}$
$1600 M$ URC

WZIYUTH HEIGHT EXPOSURF F/O EU/O DISTANCE DEGFES METERS GM-SECICU.H SECICU.M I/SO.M METERS
$N 101.6$
.2
o 0 .
0.

1617

AZ IMUTH HE IGHT EXPOSURE ME/O M EU/O OISTANCE
DEGREES METERS GH-SECICU.M SEC/CU.M I/SO.M METERS

AZIMUTH HEIGHT EXPOSURE F/O EU/O DISTANCE

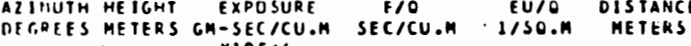
$x 105 * 6$

$\begin{array}{llllllllllllllll}N & 109.5 & .2 & 0 & 0 . & 0 . & 1630 & \text { N } & 109.5 & .2 & 0 & 0 . & 0 . & 1630\end{array}$ 


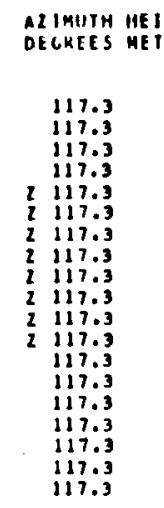

Exposure CSECICU

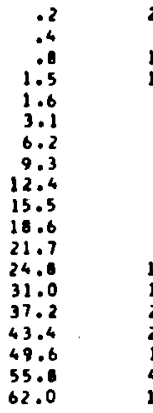

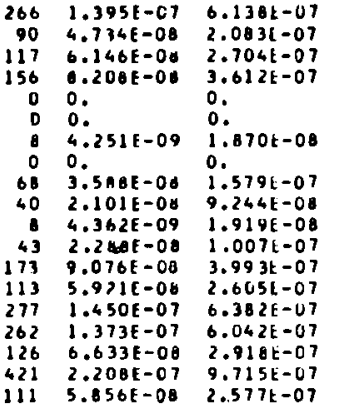

1643

1643

1643
1643

1043

1643

64

1643

1643

1643
1643
1645

1643

1643
1643
1643
1643

1643

1643

1643

AZIMUTH HEIGH

EXPOSURF ExpOSURE
MUT IU

111.11
12.00
123.0
125.0
125.0
125.0
125.0
125.0
125.0
125.0
125.0
125.0
125.0
125.0
125.0
125.0
125.0
125.0

1.4
.8
1.5
1.6
3.1
6.2
9.3
12.4
15.5
11.6
21.7
24.8
31.0
37.2
43.4
49.6
55.8
62.0
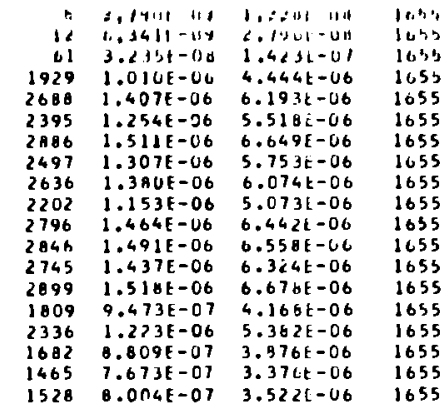

AZIMUTH HEICHT

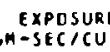

F/O EU/A OISTANCE

132.5

.2
.4
.8
1.5
1.6
3.1
6.2
9.3
12.4
15.5
10.6
21.7
24.8
31.0
37.2
43.4
49.6
55.8
62.0

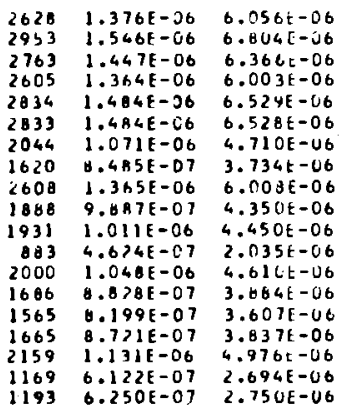

1665

1665
1065

1665
1665
1665
1665
1665

1665

1665

132.5

62.0

$\begin{array}{lll}1169 & 6.122 E-07 & 2.694 E-06 \\ 1193 & 6.250 E-07 & 2.750 E-06\end{array}$

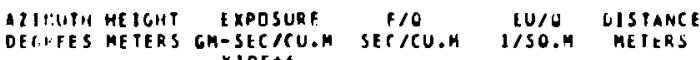

$\begin{array}{lr}117.3 & .2 \\ 117.3 & : 4 \\ 117.3 & .8 \\ 117.3 & 1.5 \\ 117.3 & 1.6 \\ 1117.3 & 3.1 \\ 1117.3 & 6.2 \\ 1117.3 & 9.3 \\ 1117.3 & 12.4 \\ 1117.3 & 15.5 \\ 1117.3 & 11.0 \\ 7117.3 & 21.8 \\ 1117.3 & 24.8 \\ 117.3 & 31.8 \\ 117.3 & 37.2 \\ 117.3 & 43.4 \\ 117.3 & 49.6 \\ 117.3 & 55.8 \\ 117.3 & 62.0\end{array}$

1643

.2
.4
1.5
1.5
3.1
6.2
9.3
2.4
5.5
1.8
1.7
4.8
7.0
3.2
9.6
5.8
2.0

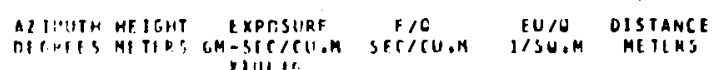

1.1 .1
113.01
112.01
125.0
125.0
125.0
125.0
125.0
125.0
125.0
125.0
125.0
125.0
125.0
125.0
125.0
125.0
125.0
125.0

1.1
1.5
1.5
1.6
3.1
6.7
9.3
12.4
15.5
19.6
21.7
24.0
31.0
37.2
43.4
49.6
55.8
62.0

\begin{tabular}{|c|c|c|}
\hline & is, & \\
\hline & u. & b. \\
\hline & J. & 0. \\
\hline & $0.836 E-07$ & 3.46 \\
\hline & 1.ABAE-06 & $\theta .26 b c-0$ \\
\hline & $2.491 E-06$ & $1.096 t-05$ \\
\hline & $2.339 E-06$ & $1.029 E-05$ \\
\hline & $2.566 t-06$ & $1.124 E-0$ \\
\hline & $2.491 E-06$ & $1.096 \mathrm{k}-0 \mathrm{~s}$ \\
\hline & $3.173 E-06$ & $1.3966=05$ \\
\hline & $3.022 \mathrm{E}-06$ & $1.329 t=05$ \\
\hline & $2.41 b E-06$ & $1.062 t-05$ \\
\hline & $2.870 E-06$ & $1.263 \mathrm{E}-05$ \\
\hline & $2.339 E-06$ & $1.029 E-05$ \\
\hline & $2.566 E-06$ & $1.129 \mathrm{E}-05$ \\
\hline & $\begin{array}{l}2.642 \mathrm{~F}-06 \\
2.491 \mathrm{E}-06\end{array}$ & $\begin{array}{l}1.163 E-05 \\
1.096 E-05\end{array}$ \\
\hline & 2.11 it-06 & $9.209 E=06$ \\
\hline & & 7054 \\
\hline
\end{tabular}

labs iobs

1635

1655

1055
1655

1655
1655

1655
1655 1655
1655 1655
1655 1655
1655 1655
1655 1655 1635

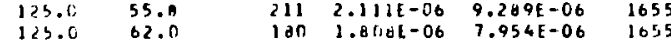

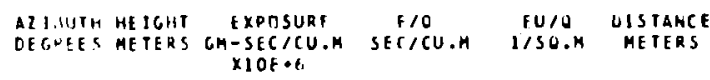

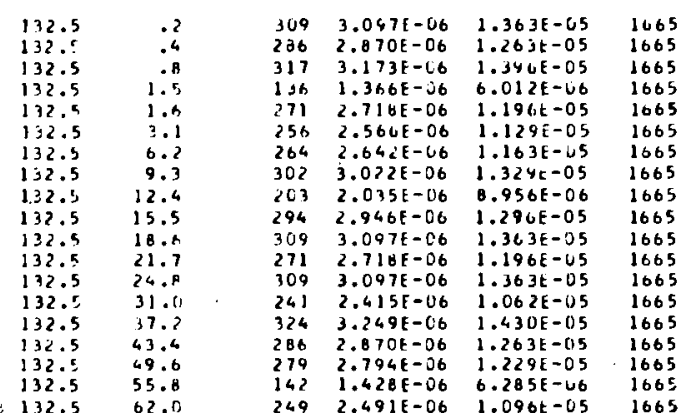


TOWER DATA FOLLOY....

TEST V2 NOVERGER 919721030 TU 1100 PST $21 M C$ SULFIDE RELEASE FROM ELEVATION OF 2OH
$3200 \mathrm{U}$ ARC

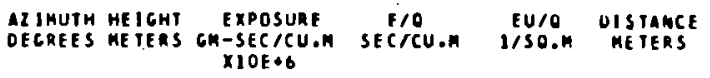

TORE DATA FILLCOH....

IFST V2 MOVEABE $9,1972 \quad 1030$ IU 1100 PSI KHODANINE ORELASE FROM ELFVATION OF $26 \mathrm{M}$
320 MERE
UE $4.4 \mathrm{M} / \mathrm{SEC}$ AT $26 \mathrm{M}$

ADIHUTH REICHT EXPTSURE F/O EU/O DISTANCE

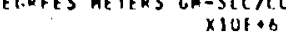

$99.8 \quad .2$

$0 . \quad 3215$

AzIMUTA HEICHT EXPOSURE F/O EU/O DISTANCE DEGREES METERS GH-SEC/CU.M SEC/CU.M I/SO.M METERS $\times 10 t=6$

o.

3224

AIIMUTH HEIGHT EXPOSURE F/O EU/O UISTALICE DEGRES ME TERS GH-SEC/CU.M SET/CU.M I/SO.M METERS $x 10 E+6$

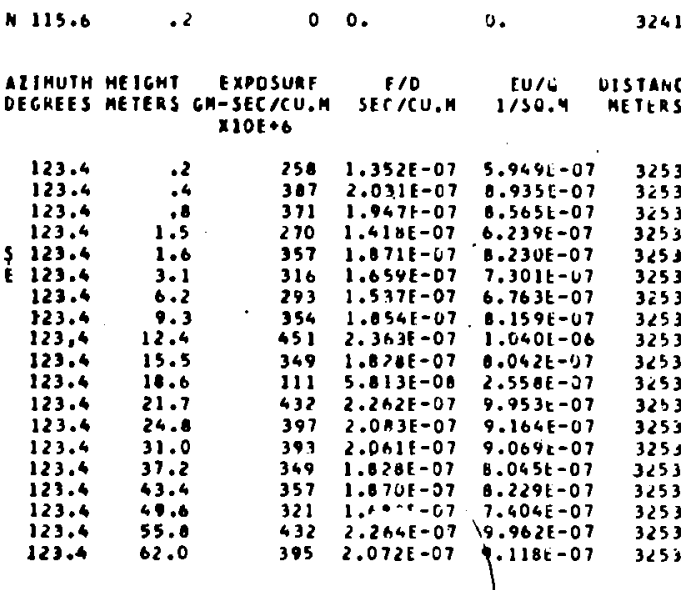
AZIHUTH HEIGHT EXPOSURE GF
OEGRES METERS GM-SEC/CU.M SEC/CU.M DU/A DISTANCE

$\begin{array}{rr}131.2 & .2 \\ 131.2 & : 4 \\ 131.2 & .0 \\ 131.2 & 1.5 \\ 131.2 & 1.6 \\ 131.2 & 3.1 \\ 131.2 & 6.2 \\ 131.2 & 9.3 \\ 131.2 & 12.4 \\ 131.2 & 15.5 \\ 131.2 & 19.6 \\ 131.2 & 21.7 \\ 131.2 & 24.8 \\ 131.2 & 31.0 \\ 131.2 & 37.2 \\ 131.2 & 43.4 \\ 131.2 & 49.6 \\ 131.2 & 55.6 \\ 131.21 & 62.0\end{array}$
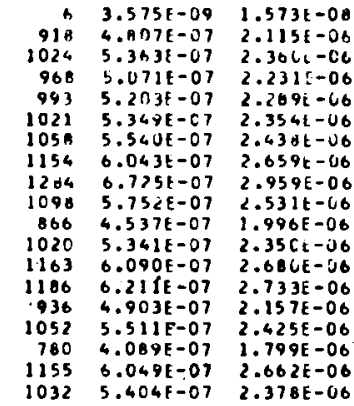

$3<64$

$3<64$

3264

3264

3264

3264

3264

3264

3264

3264

3264

3264

3264

3264

3264 $\begin{array}{rrrrrr}1.8 & .7 & 0 & 0 . & 0 . & 3215\end{array}$

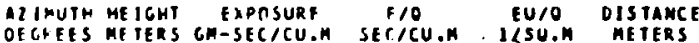
IUf 16

$\begin{array}{llllll}N 117.0 & .2 & 0 & 0 . & 0 . & 3229\end{array}$

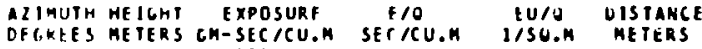
$\times 10 t+6$

$\begin{array}{lllll}N 115.6 & .2 & 0 & 0 . & 0\end{array}$

0.324

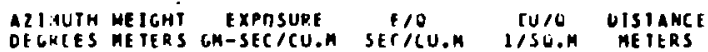
$\times 1 \cup F+6$

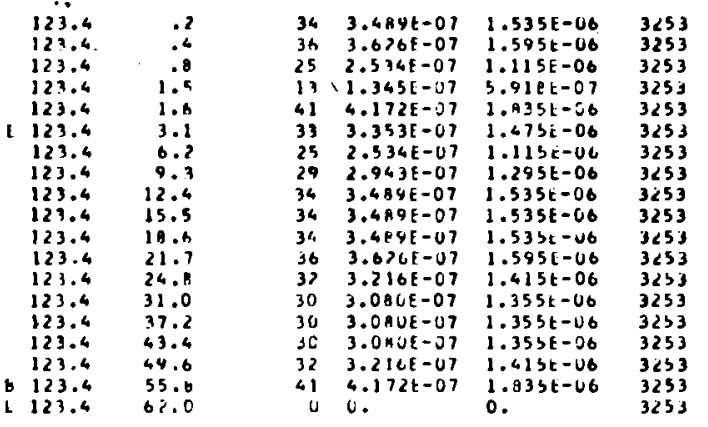

DzIIIUTH MEIGHT EXPOSURE F/O EU/J DISTANCE DFCUES METERS CR-SEC/CU.H SETICU.M I/SO.M METERS x)

\begin{tabular}{|c|c|c|c|c|c|c|}
\hline & 1.2 & .2 & 0 & $0.187 \mathrm{~F}-\mathrm{CH}$ & $3.60<t-00$ & 3264 \\
\hline & 131. & & 95 & $9.54 d t-07$ & $4.201 k-06$ & 32664 \\
\hline & 131.2 & .6 & 112 & $1.1>6 F-06$ & $4.956 \mathrm{E}-06$ & 3264 \\
\hline & 131.2 & 1.5 & 52 & $3.244 E-07$ & $2.307 E-06$ & $3<04$ \\
\hline E & 131.2 & 1.6 & 119 & 1.1 YSE-C6 & $5.230 \mathrm{~L}-06$ & $3<64$ \\
\hline & 131.2 & 3.1 & $12 \mathrm{~B}$ & $1.264 E-06$ & $5.560 t-08$ & 3264 \\
\hline $\mathbf{F}$ & 131.2 & 6.2 & 133 & $1.332 \mathrm{E}-08$ & $5.862 E-06$ & 3264 \\
\hline & 131.2. & 9.3 & 140 & $1.401 t-06$ & $0.1636-06$ & 3264 \\
\hline & 131.2 & 12.6 & 126 & $1.264 E-66$ & $5.560 t-06$ & 3264 \\
\hline & 131.2 & 15.3 & 122 & $1.27 \times E=06$ & $5.4 C Q E=06$ & 3264 \\
\hline & 191.2 & 19.6 & 85 & $0.519 f-07$ & $3.746 t=06$ & $3<64$ \\
\hline & $13: .2$ & 21.7 & 98 & $9.891 E-07$ & $4.352 E-00$ & 3264 \\
\hline & 131.2 & 24.8 & 119 & $1.195 \mathrm{E}-06$ & $5.250 E-06$ & 3264 \\
\hline & 131.2 & 31.0 & 122 & $1.279 \mathrm{E}-06$ & $5.409 \bar{c}-06$ & 3264 \\
\hline & 131.2 & 37.2 & 102 & $1.023 t-06$ & $4.563 t-06$ & 3264 \\
\hline 0 & 131.7 & 43.4 & B] & B.175E-07 & $3.597 E-06$ & 3264 \\
\hline & 131.2 & 49.6 & 61 & $0.117 \mathrm{E}-07$ & $2.6911=06$ & 3264 \\
\hline c & 131.2 & & 133 & $1.332 E-06$ & $5.062 t=06$ & 3264 \\
\hline 6 & 31 & 2.5 & 129 & $1.296 t-06$ & $5.711 E=06$ & 326 \\
\hline
\end{tabular}




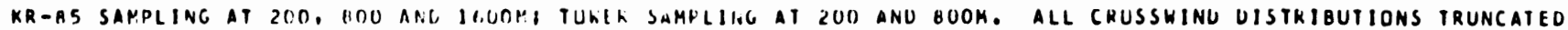
ON STUTH. SIX UF TEN TIIWLRS "HIT". BUT UISTRIGUIIIINS IRUNCAIED AT TUP:-MAY HAVE BEEN TRACER UN TUHER AT HOOM. 112.6 DEG. BUT VERY NEAK BACKGRDUND. LUW VULIAGES UN MANY GUOM SENSIJKS MAY HAVELEAU IO UNDERESTIMATES OF XIO.

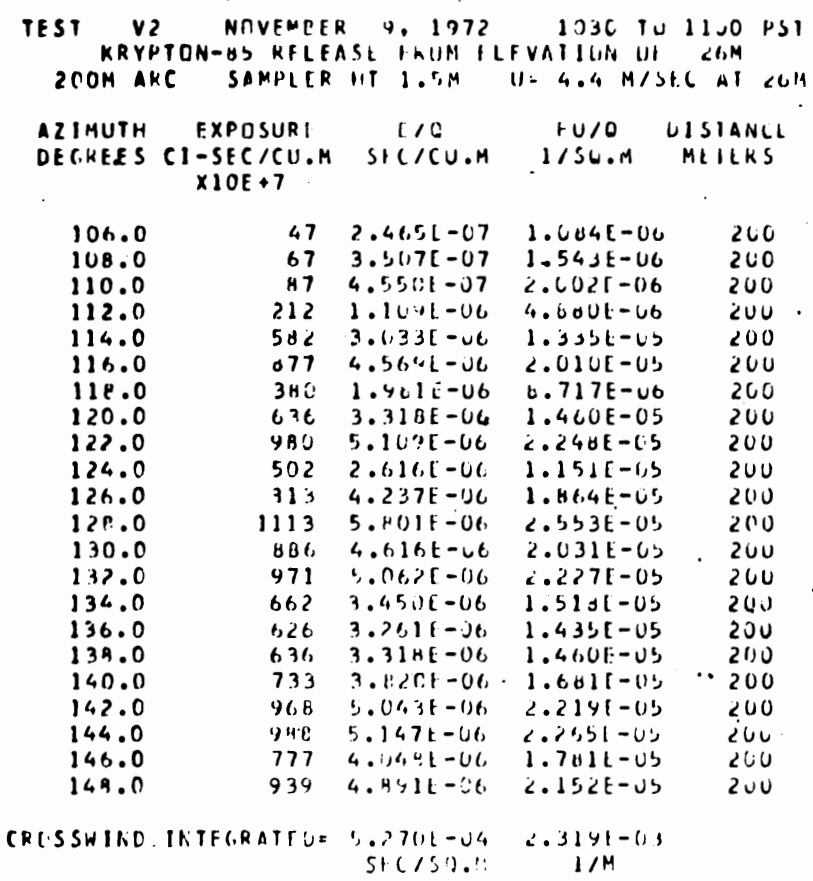

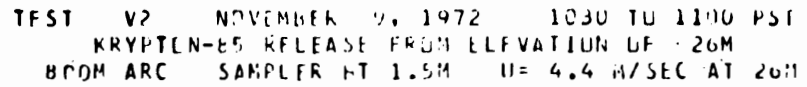

Q.ZIMUTH EXPCSUKL HIS LU/G DISTAIVCE DECKEES CI-SECICU.M SH ICU.M I/SH.H METEKS $\times 10 \mathrm{E}+7$

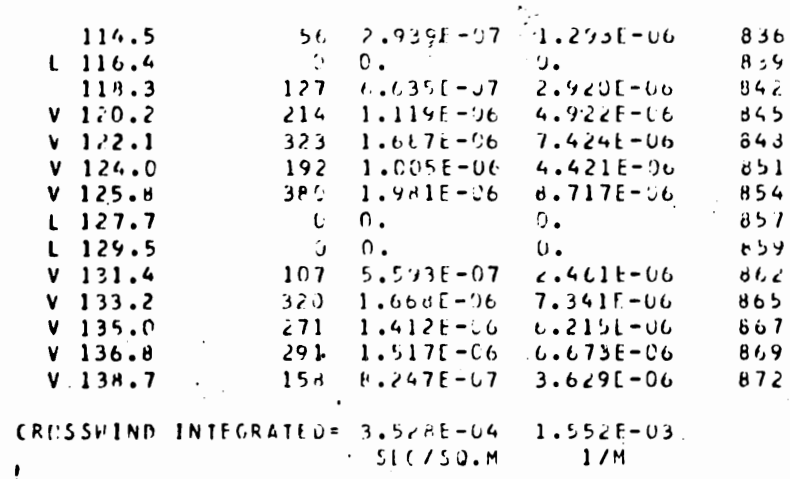

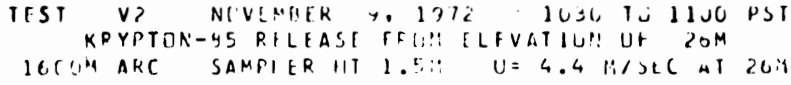


TOHER DATA FOLUU....

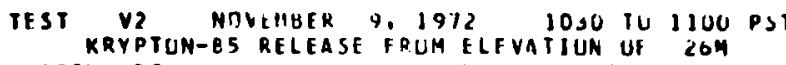
zOOH ARC $U=4.4 \mathrm{M} / \mathrm{SEC} A \mathrm{I} 2 \mathrm{M}$

\begin{tabular}{|c|c|c|c|c|c|}
\hline $\begin{array}{l}\text { AZIMUTH } \\
\text { DEGREES }\end{array}$ & $\begin{array}{l}\text { HE ICHT } \\
\text { METERS }\end{array}$ & $\begin{array}{c}\text { EXPUISURF } \\
\text { CI-SECICU.H } \\
\times 1 \cup[+7\end{array}$ & $\begin{array}{c}F / O \\
\text { SEC } / \text { CU.H }\end{array}$ & $\begin{array}{l}\text { EU/U } \\
\text { I/SO.n }\end{array}$ & $\begin{array}{l}\text { DISIANCE } \\
\text { METEKS }\end{array}$ \\
\hline
\end{tabular}

$\begin{array}{llllll}A & 192.0 & 1.5 & 0 . & 200\end{array}$

\begin{tabular}{|c|c|c|c|c|c|c|}
\hline $\begin{array}{l}\text { AZI I MUTH } \\
\text { DE GREES }\end{array}$ & $\begin{array}{l}\text { HEICHT } \\
\text { HETERS }\end{array}$ & $\begin{array}{r}E x \\
C I-5 \\
x 10\end{array}$ & $\begin{array}{l}\text { Jht } \\
0.14\end{array}$ & $\begin{array}{c}\text { I/U } \\
\text { SEC/CU.H }\end{array}$ & $\begin{array}{c}1 U / U \\
1 / S O . M\end{array}$ & $\begin{array}{c}\text { DISIANCE } \\
\text { METEKS }\end{array}$ \\
\hline $\begin{array}{l}110.0 \\
110.0 \\
110.0 \\
110.0 \\
110.0 \\
110.0 \\
1110.0\end{array}$ & $\begin{array}{r}1.5 \\
4.0 \\
7.0 \\
13.0 \\
19.0 \\
25.11 \\
32.0\end{array}$ & 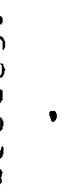 & $\begin{array}{r}87 \\
1 \\
47 \\
227 \\
107 \\
50 \\
7\end{array}$ & $\begin{array}{l}4.55 \cup F-U 7 \\
0 . \\
2.4635-07 \\
1.145 E-06 \\
5.593 E-07 \\
2.644 E-07 \\
0 .\end{array}$ & $\begin{array}{l}2.002 E-06 \\
0 . \\
1.064 \mathrm{t}-06 \\
5.214 \mathrm{E}-06 \\
2.461 \mathrm{t}-.6 \\
1.16 \mathrm{EE}-06\end{array}$ & $\begin{array}{l}200 \\
200 \\
200 \\
200 \\
200 \\
200 \\
200\end{array}$ \\
\hline
\end{tabular}

$\begin{array}{ccclll}110.0 & 25.0 & 50 & 2.644 E-07 & 1.164 E-06 & 200 \\ 110.0 & 32.8 & n & 0 . & 0 . & 200\end{array}$

AZIAUTH HEIGHT EXPIISURE DELREES METERS CI-SFC/CII.H, SER/CU.M IISG.M METERS XIUE +7

\begin{tabular}{|c|c|c|c|c|}
\hline $\begin{array}{l}118.0 \\
118.0 \\
118.0 \\
118.0 \\
119.0 \\
118.0 \\
119.0\end{array}$ & $\begin{array}{r}1.5 \\
4.0 \\
7.0 \\
13.0 \\
19.0 \\
25.0 \\
37.0\end{array}$ & $\begin{array}{r}300 \\
680 \\
2346 \\
2449 \\
3720 \\
3461 \\
3414\end{array}$ & $\begin{array}{l}1.381 E-06 \\
3.345 F-06 \\
1.243 E-05 \\
1.276 E-05 \\
1.420 E-05 \\
1.8131-05 \\
2.02+1-05\end{array}$ & $\begin{array}{l}1.717 t-60 \\
1.560 t-05 \\
5.466 t-65 \\
3.614 t-05 \\
0.525 t-J 5 \\
7.933 t-05 \\
0.926 t-0 S\end{array}$ \\
\hline
\end{tabular}
ALIMUITH HEIGHT EXPISUIRF
DEGPEES METERS CI-SETICU.M SECICU.M I/SO.M METERS $\times 10 \%+7$

\begin{tabular}{|c|c|c|c|c|}
\hline $\begin{array}{l}126.0 \\
126.0 \\
126.0 \\
126.0 \\
126.0 \\
126.0 \\
120.0\end{array}$ & $\begin{array}{r}1.5 \\
4.0 \\
7.0 \\
13.0 \\
19.0 \\
25.0 \\
32.0\end{array}$ & $\begin{array}{r}611 \\
1547 \\
2462 \\
5045 \\
4437 \\
5962 \\
11\end{array}$ & $\begin{array}{l}4.237 E-06 \\
3.266 E-06 \\
1.283 E-0 S \\
2.626 F-0 S \\
2.311 E-0 S \\
3.074 F-05 \\
0 .\end{array}$ & $\begin{array}{l}1.654 E-05 \\
3.637 L-U 5 \\
5.643 E-U 5 \\
1.156 E-U 4 \\
1.017 E-L 4 \\
1.353 L-U 4 \\
U .3\end{array}$ \\
\hline
\end{tabular}

\begin{tabular}{|c|c|c|c|c|c|}
\hline $\begin{array}{l}\text { ALINIITH } \\
\text { DEGREES }\end{array}$ & $\begin{array}{l}\text { HE JGHT } \\
\text { METERS }\end{array}$ & $\begin{array}{c}\text { EXPASGRF } \\
\text { CI-SECICU.A } \\
\times 1 U F+7\end{array}$ & $\begin{array}{c}F / 0 \\
\text { sected. }\end{array}$ & $\begin{array}{c}\text { IU/U } \\
\text { ISSU.H }\end{array}$ & $\begin{array}{l}\text { USI Aiale } \\
\text { METikS }\end{array}$ \\
\hline $\begin{array}{l}134.0 \\
134.0 \\
134.0 \\
134.0 \\
134.0 \\
134.0 \\
134.0\end{array}$ & $\begin{array}{l}1.5 \\
4.0 \\
7.0 \\
13.0 \\
19.0 \\
25.0 \\
32.6\end{array}$ & $\begin{array}{l}667 \\
1011 \\
2191 \\
3447 \\
510.6 \\
5737 \\
2694\end{array}$ & $\begin{array}{l}3.430[-06 \\
5.27 \mathrm{~J}-26 \\
1.141 \mathrm{~L}-05 \\
2.004 \mathrm{E}-05 \\
2.641 \mathrm{~L}-03 \\
2.986[-05 \\
1.301 \mathrm{E}-05\end{array}$ & $\begin{array}{l}1.510 \mathrm{c}-0 \mathrm{~b} \\
2.314 \mathrm{~L}--5 \\
5.02<\mathrm{L}-05 \\
8.817 \mathrm{~L}-05 \\
1.164 \mathrm{~L}-04 \\
1.314 \mathrm{t}-6,4 \\
5.727 \mathrm{~L}-65\end{array}$ & $\begin{array}{l}200 \\
200 \\
200 \\
200 \\
200 \\
200 \\
200\end{array}$ \\
\hline
\end{tabular}




\begin{tabular}{|c|c|c|c|c|c|c|}
\hline 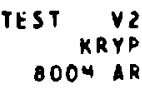 & $\begin{array}{l}\text { NOV } \\
\text { PTON-BS } \\
\text { RT }\end{array}$ & $\begin{array}{l}\text { IEHBER } \\
\text { RFLEAS }\end{array}$ & $\begin{array}{l}9,19 \\
\text { E FROM }\end{array}$ & $\begin{array}{l}972 \quad 1030 \\
\text { LLFVAI IUN } \\
\text { UE } 4.4 \mathrm{HI}\end{array}$ & $\begin{array}{l}T 111100 \mathrm{P} \\
\text { OF } 20 \mathrm{M} \\
\text { SEC AT } 26 \mathrm{M}\end{array}$ & \\
\hline $\begin{array}{l}\text { ALIMUTH } \\
\text { DEGREES }\end{array}$ & $\begin{array}{l}\text { HE IGHT } \\
\text { ME TERS }\end{array}$ & \multicolumn{2}{|c|}{$\begin{array}{c}\text { EXPOSUR.F } \\
\text { CI-SEC } / C U . M \\
\times 10 E+7\end{array}$} & $\begin{array}{c}1 / 0 \\
S E C / C U . H\end{array}$ & $\begin{array}{l}10 / 0 \\
1 / 50 . M\end{array}$ & $\begin{array}{l}\text { WISTAHGE } \\
\text { NEIEKS } \\
\text {. }\end{array}$ \\
\hline$N 105.0$ & 1.5 & & 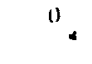 & 0. & 0 & 820 \\
\hline $\begin{array}{l}\text { AZIMUTH } \\
\text { OEGKEES }\end{array}$ & $\begin{array}{l}\text { HE IGHT } \\
\text { METERS }\end{array}$ & $\begin{array}{r}\text { EXPl } \\
C I-S E C \\
\times 10 \%\end{array}$ & $\begin{array}{l}\text { SIIKF } \\
\text { /C.1)..4 } \\
+7\end{array}$ & $\begin{array}{c}\text { I/O } \\
\text { SEC/CU.H }\end{array}$ & $\begin{array}{l}1010 \\
1 / 50.4\end{array}$ & $\begin{array}{l}\text { UIS I ANCE } \\
\text { Hit ILKS }\end{array}$ \\
\hline N 112.6 & 1.5 & & 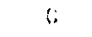 & 0. & u. & 033 \\
\hline $\begin{array}{l}\text { AZIHUTH } \\
\text { DECREES }\end{array}$ & $\begin{array}{l}\text { HE IGHT } \\
\text { METERS }\end{array}$ & $\begin{array}{r}\text { Expin } \\
\text { C1-Stc } \\
\times 10 t\end{array}$ & $\begin{array}{l}\text { SUKt } \\
\text { ICU.H } \\
+1\end{array}$ & $\begin{array}{c}1 / 0 \\
\text { SI } / \mathrm{CO} . \mathrm{H}\end{array}$ & $\begin{array}{l}1 U / W \\
1 / S C . H\end{array}$ & $\begin{array}{l}\text { UISIAREt } \\
\text { H.LIIES } \\
\text {. }\end{array}$ \\
\hline $\begin{array}{ll}v & 120.2 \\
v & 120.2 \\
v & 120.2 \\
v & 120.2 \\
& 120.2 \\
& 120.2 \\
& 120.2\end{array}$ & $\begin{array}{l}1.5 \\
5.0 \\
12.0 \\
19.0 \\
26.0 \\
34.0 \\
1.2 . ?\end{array}$ & & $\begin{array}{l}214 \\
343 \\
243 \\
367 \\
505 \\
316 \\
384\end{array}$ & $\begin{array}{l}1.1111-J 6 \\
1.792 E-06 \\
1.270 E-06 \\
1.0166-06 \\
2.6311-06 \\
1.6491-36 \\
1.0711-06\end{array}$ & $\begin{array}{l}4.92<t-16 \\
7.083 E-06 \\
3.504 t-06 \\
8.200 t-56 \\
1.159 E-05 \\
7.257 E-06 \\
8.926 t-26\end{array}$ & $\begin{array}{l}343 \\
845 \\
345 \\
343 \\
345 \\
345 \\
343\end{array}$ \\
\hline $\begin{array}{l}\text { AL IHUTH } \\
\text { DECRIES }\end{array}$ & $\begin{array}{l}\text { HE ICHT } \\
\text { ME TEKS }\end{array}$ & $\begin{array}{r}\text { Fypo } \\
\text { 1-StC } \\
\times 101\end{array}$ & $\begin{array}{l}5(15: 1 \\
1 C \cup, 11 \\
+7\end{array}$ & $\begin{array}{c}1 / 0 \\
3[C / C U . r\end{array}$ & $\begin{array}{c}1 \mathrm{U} / \mathrm{J} \\
1 / 56.11\end{array}$ & $\begin{array}{l}\text { UIS I ALCE } \\
\text { :ItLES }\end{array}$ \\
\hline $\begin{array}{r}127.7 \\
127.7 \\
127.7 \\
177.7 \\
127.7 \\
1127.7 \\
127.7\end{array}$ & $\begin{array}{l}1.5 \\
3.11 \\
12.0 \\
19.0 \\
26.0 \\
34.0 \\
42.0\end{array}$ & 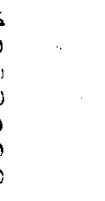 & $\begin{array}{r}0 \\
453 \\
567 \\
576 \\
424 \\
1 . \\
545\end{array}$ & 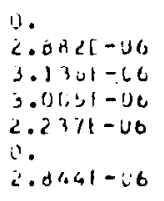 & $\begin{array}{l}0.36 H t-35 \\
1.26 H \\
1.3611-05 \\
1.322 \mathrm{~L}-35 \\
4.4432-136 \\
\because .2511-05\end{array}$ & $\begin{array}{l}351 \\
1551 \\
151 \\
127 \\
457 \\
151 \\
157\end{array}$ \\
\hline $\begin{array}{l}\text { AZIAUIH } \\
\text { DESREES }\end{array}$ & $\begin{array}{l}\text { HE I GHT } \\
\text { METERS }\end{array}$ & $\begin{array}{r}\text { ExPLi } \\
\text { CI-SE } \\
\times 10 F\end{array}$ & $\begin{array}{l}S, P, F \\
\text { IrU. } \\
1\end{array}$ & $\begin{array}{c}1 / 0 \\
\operatorname{sic} / 60 .\end{array}$ & $\begin{array}{l}1, U / 4 \\
1 / S O . N\end{array}$ & $\begin{array}{l}\text { US TAiCE } \\
\text { "itiks }\end{array}$ \\
\hline $\begin{array}{r}135.0 \\
\mathrm{l} 135.0 \\
135.0 \\
135.0 \\
135.0 \\
135.0 \\
135.0\end{array}$ & $\begin{array}{r}1.5 \\
5.0 \\
12.0 \\
14.11 \\
26.1 \\
34.1 \\
42.0\end{array}$ & & $\begin{array}{l}271 \\
69 \\
694 \\
484 \\
393 \\
3145 \\
454\end{array}$ & 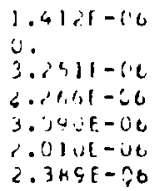 & 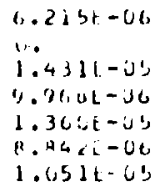 & 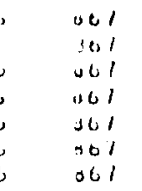 \\
\hline
\end{tabular}


GRUUND LEVEL AND TOHER SAMPLING TCOM TO 3200 M. MUST ARCS TRUACATED ON SOUTH END. BUT CENTERLINE ALWAYS CBSERVED. MOST VERTICAL DISTRIHUTIONS TRUNCATED AT TOPS DF TUWERS.

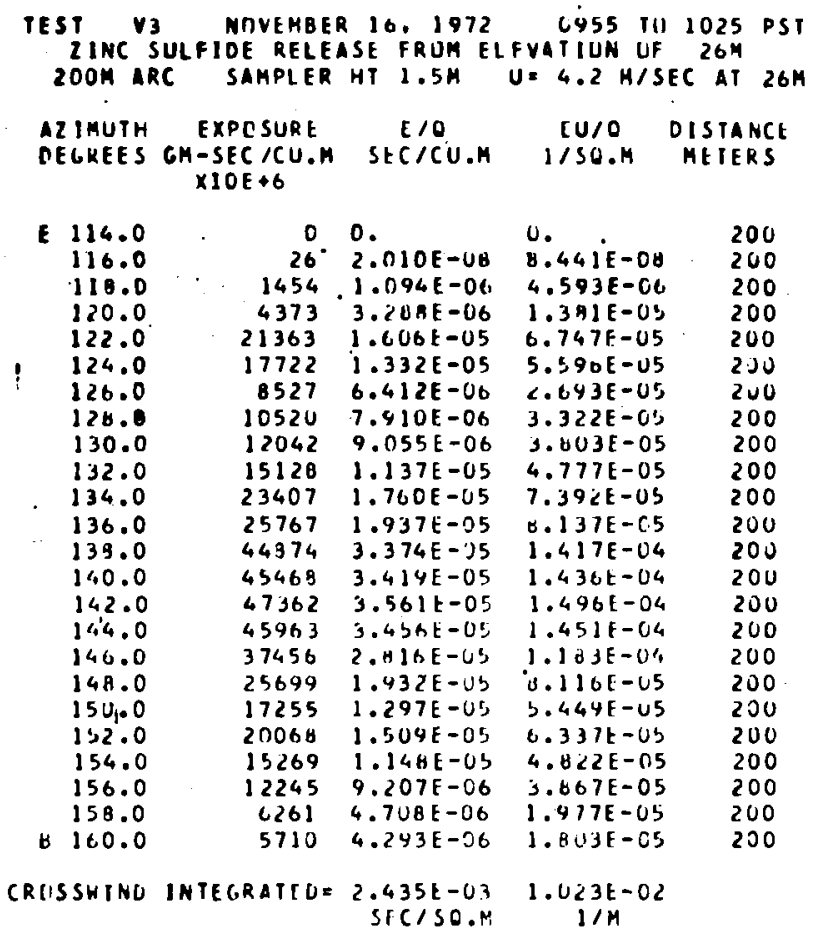

TEST V3 NIJVEMBÉR 16.1972 O955 TU 1025 PST IINC SULFIDE RFLEASE FRUM ILFVAIILN UH $26 \mathrm{M}$ 4 COM ARC SAMTLER IIT $1.5 \mathrm{SM} \mathrm{H}=4.2 \mathrm{M} / \mathrm{SLC}$ AT $26 \mathrm{M}$

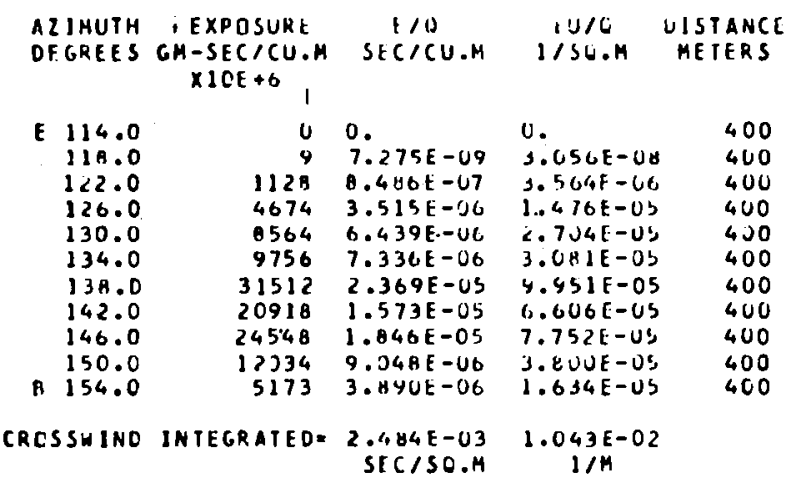

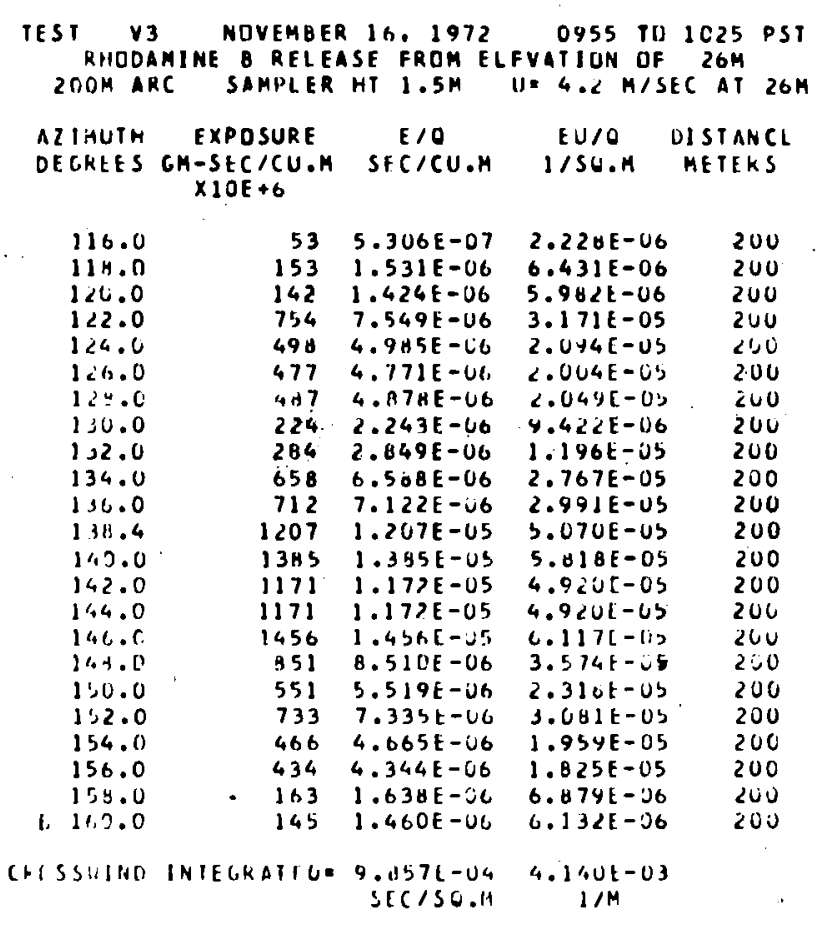

TESI VS NEVEMEER 16, 1972 0955 IL IU2S I'SI IIIIOAIINF B RELEASF FKOM ILFVATIUN UI CGM

WIOH AKC SAMPIER FI $1.5 \mathrm{M} \quad \mathrm{H}=4.2 \mathrm{M} / \mathrm{SEC}$ AT $20 \mathrm{M}$

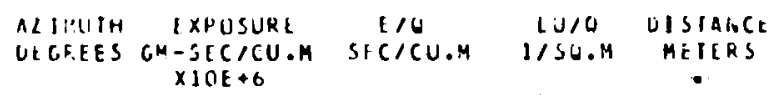

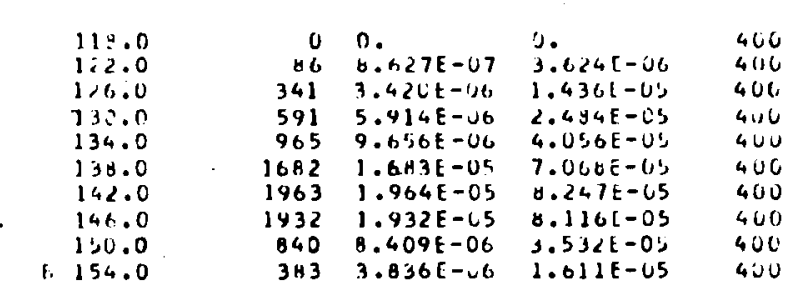

CRTSSHINOD INTEGRATEDE $2.454 E-03$ 1.031E-02

$1 / M$ 


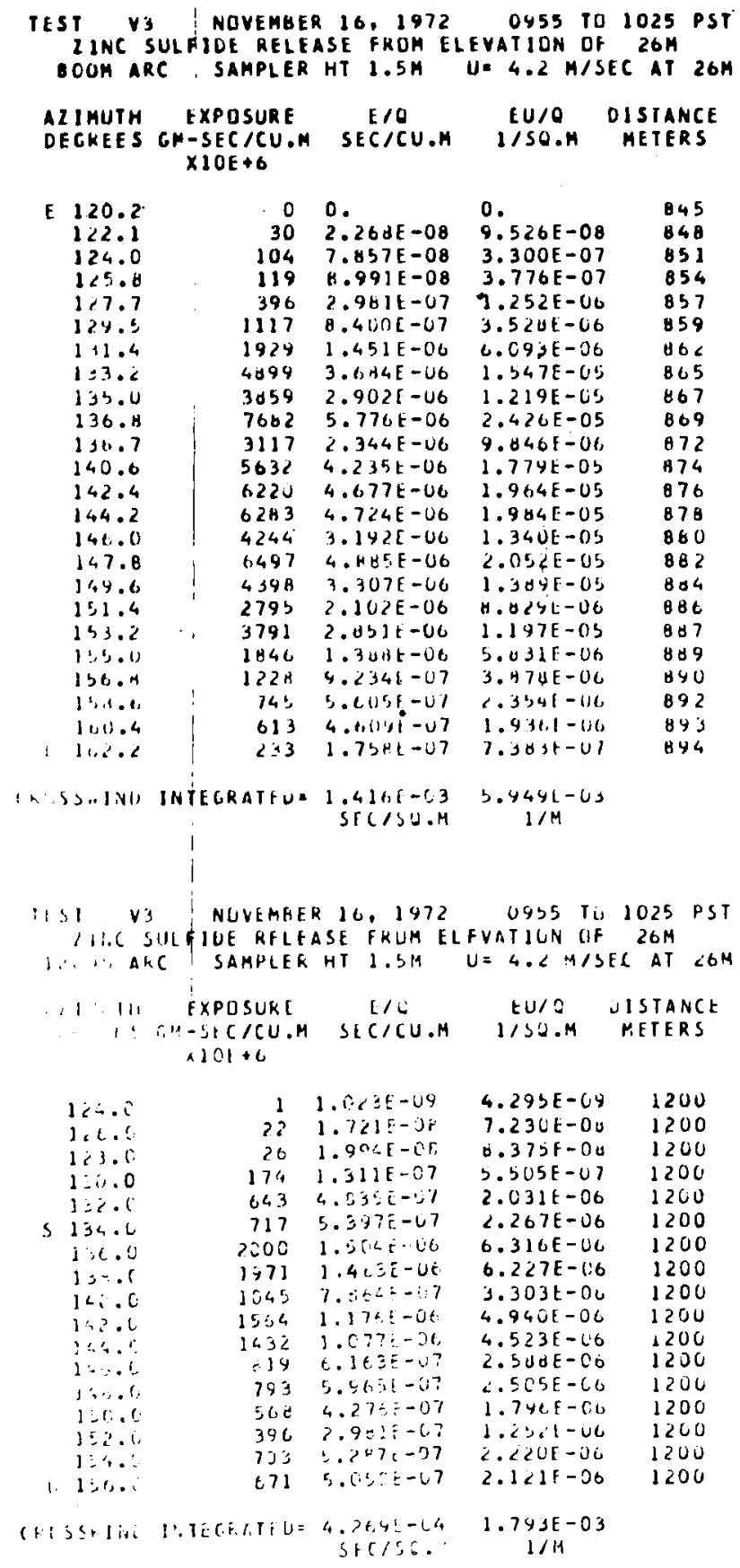

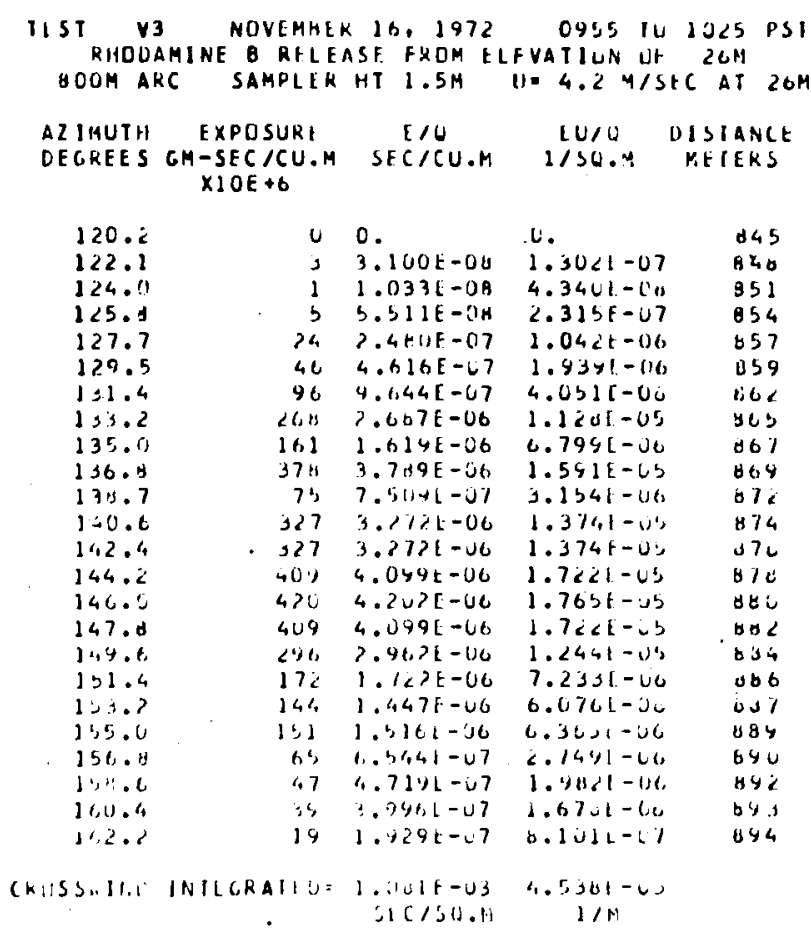

IEST VI NIJVERFFK 16, 1972 LS55 IU IU25 HST

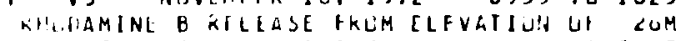

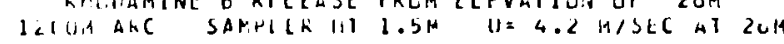

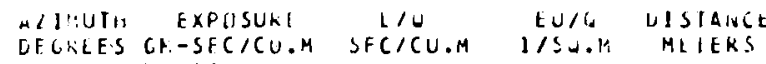
$\times 10 E+0$

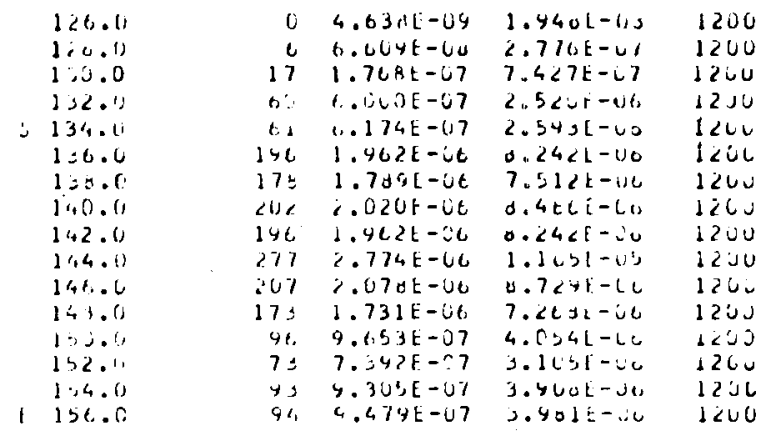

(RISSSINO INTECRAIIUE SFCISO.M $\quad 1 / 4$ 


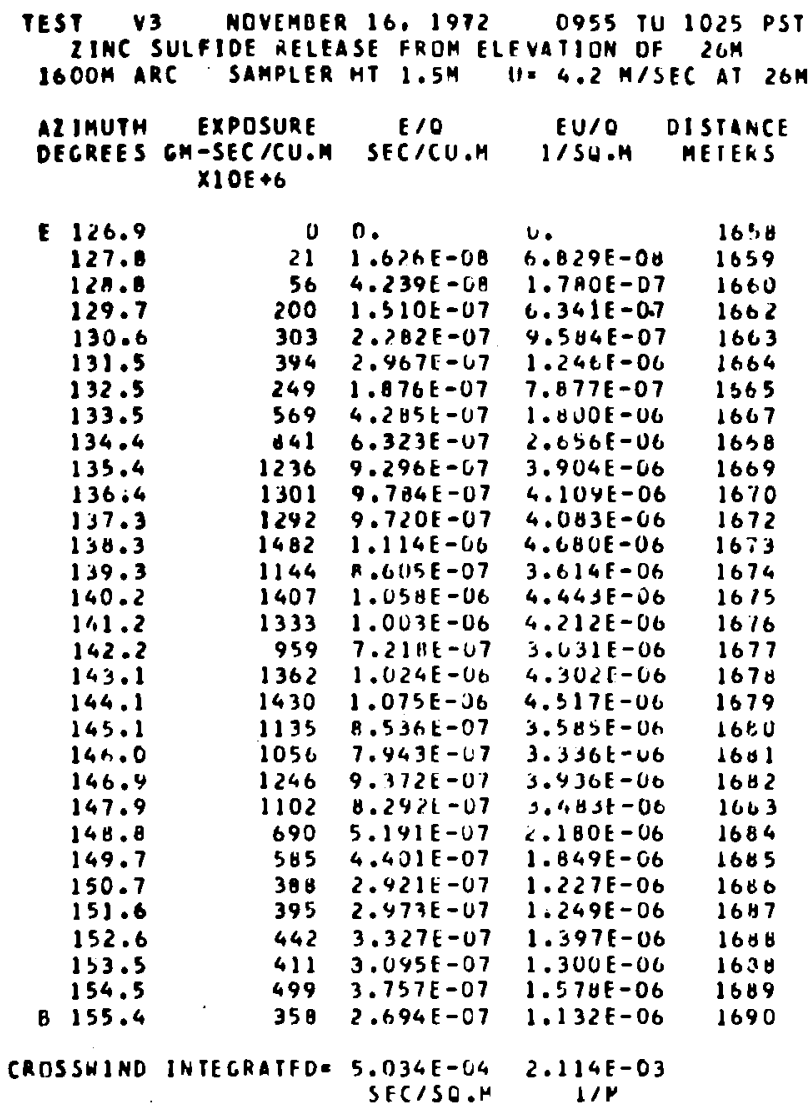

TEST V3 NUVEROER 16. 1972 0955 IU 1025 PST ZINC SULFIDE RELEASE FRDM [LFVATIUN UF $20 \mathrm{OM}$ $2200 M$ ARC SAMPLER HT 1.5H U $=4.2 \mathrm{M} / \mathrm{SEC}$ TI $26 \mathrm{M}$

\begin{tabular}{|c|c|c|c|c|c|}
\hline \multicolumn{2}{|c|}{$\begin{array}{l}\text { ALIMUTH } \\
\text { OEGREES }\end{array}$} & $\begin{array}{c}\text { EXPUSURE } \\
\text { GM-SEC /CU.A } \\
\text { XIOE } \$ 6\end{array}$ & $\begin{array}{c}H / U \\
\text { SEC }\end{array}$ & $\begin{array}{l}i U / 0 \\
i / S O . M\end{array}$ & $\begin{array}{l}\text { DISIAHCE } \\
\text { MEIEKS }\end{array}$ \\
\hline $\mathbf{E}$ & $\begin{array}{l}126.0 \\
1<8.0\end{array}$ & $\begin{array}{r}0 \\
10\end{array}$ & $\begin{array}{l}0 . \\
\text { E.053E-U9 }\end{array}$ & $\begin{array}{l}C .30 \angle E-08 \\
3.30 \angle E\end{array}$ & $\begin{array}{l}2200 \\
2200\end{array}$ \\
\hline & 130.0 & 28 & $2.1 \angle H E-\angle B$ & $0.939 E-v 8$ & 2200 \\
\hline & 132 & 64 & $4.461 E-U H$ & $2.141 E-07$ & $223 v$ \\
\hline & 134.0 & 91 & $6.043 E-08$ & $\angle .875 E-07$ & 2200 \\
\hline & $\begin{array}{l}136.0 \\
133.0\end{array}$ & $\begin{array}{l}310 \\
450\end{array}$ & $\begin{array}{l}2.333 E-1) 7 \\
3.385 E-07\end{array}$ & $\begin{array}{l}1.7 \% 7 \mathrm{E}-07 \\
1.422 \mathrm{t}-06\end{array}$ & $\begin{array}{l}2200 \\
\angle 200\end{array}$ \\
\hline & 140.0 & 486 & $3.656 E-j 7$ & $1.53 \zeta E-06$ & 2200 \\
\hline & $\begin{array}{l}142.0 \\
144.0\end{array}$ & $\begin{array}{l}423 \\
300\end{array}$ & $\begin{array}{l}3.106 F-67 \\
2.709 E-C 7\end{array}$ & $\begin{array}{l}1.537 E-v 6 \\
1.134 E-V 6\end{array}$ & $\begin{array}{l}22 \mathrm{JU} \\
2200\end{array}$ \\
\hline & $\begin{array}{l}146.0 \\
14 H .0\end{array}$ & $\begin{array}{l}407 \\
248\end{array}$ & $\begin{array}{l}3.060 E-07 \\
1.067 E-07\end{array}$ & $\begin{array}{l}1.2 \mathrm{HSE}=06 \\
7.040 \mathrm{E}-07\end{array}$ & $\begin{array}{l}2200 \\
2200\end{array}$ \\
\hline & 150.0 & 159 & $1.199 t-07$ & $3.037 t-07$ & 2200 \\
\hline & 152.0 & 110 & $8.312 t-08$ & $9.491 E-07$ & 2200 \\
\hline & 154.0 & 169 & $1.274 E-07$ & $5.351 E-07$ & 2200 \\
\hline 8 & 156.0 & 219 & $1.648 E-07$ & $6.922 \mathrm{E}-\mathrm{U} 7$ & 2200 \\
\hline
\end{tabular}

CRUSSHIND INTECRATED= $2.043 E-0 \% \quad 8.583+-04$
TEST Y3 NOVEMOER 16.1972 0955 TU 1025 PST RIIODAMINE $B$ RELEASE FRDM ELFYATIUN OF $26 \mathrm{M}$ $1600 M$ ARC SAMPLER HT $1.5 \mathrm{M}$ U $=4.2 \mathrm{H} / \mathrm{SEC}$ AT $26 \mathrm{M}$

AZIMUTH EXPOSURE
OECREES EM-SECICU.M SEC/CU.M IISO.H MISTANCE
XIOE+6

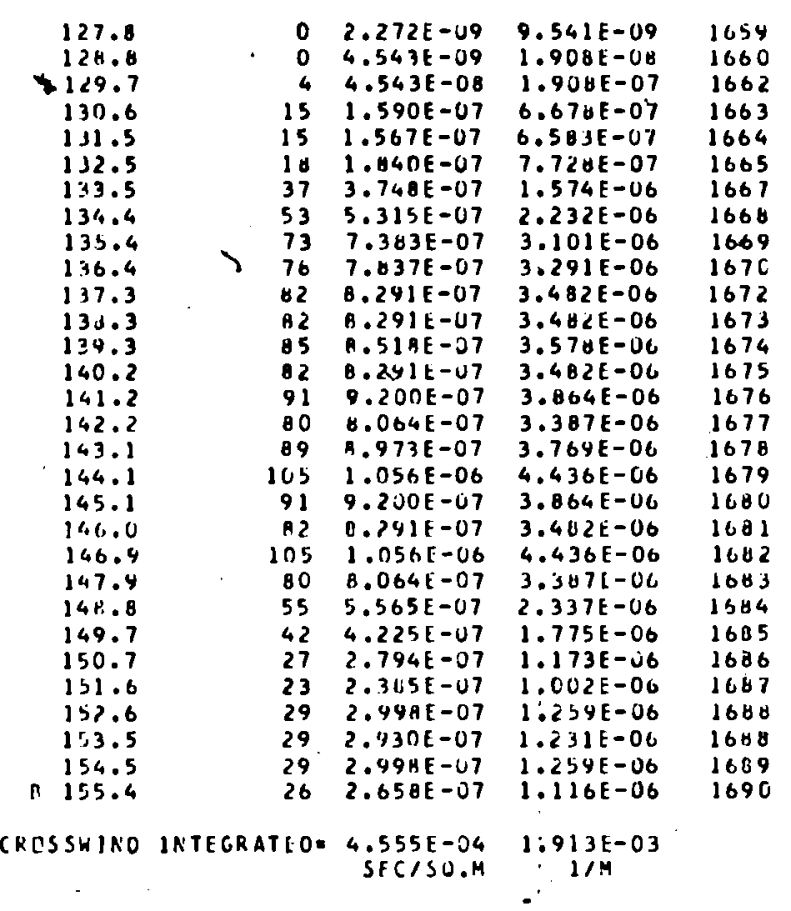

TEST V3 NOVEMBER 1h, 1972 .0955 TL 1025 PST KMIICAMINE B RELEASE FRUM ELFVATIUN OF $26 \mathrm{M}$ I 2 COM ARC SAMPLER HT 1.5 .4 U 4.2 M/SEC $4126 \mathrm{M}$ $\begin{array}{ccc}\text { AZIMUTH EXPOSURE } & \text { E/U } & \text { EU/O DISTANCE } \\ \text { CFGEEES CM-SECICU.M SECICU.M IJSA.M METERS } \\ \text { XIOE } 46\end{array}$

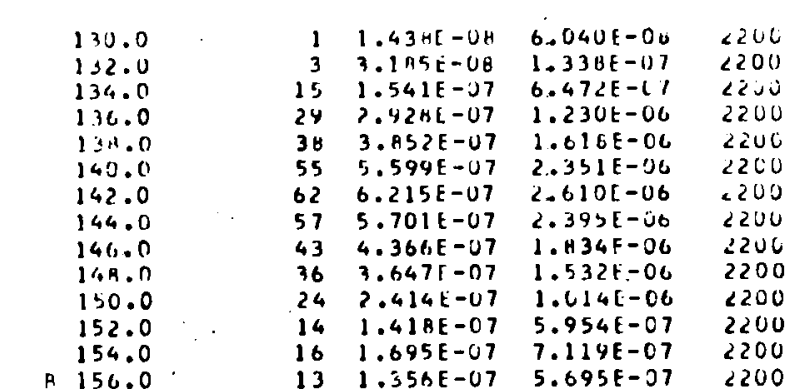

CRIISSHIND INTEGRATED= 3.163E-U4 $1.329 E-03$ 


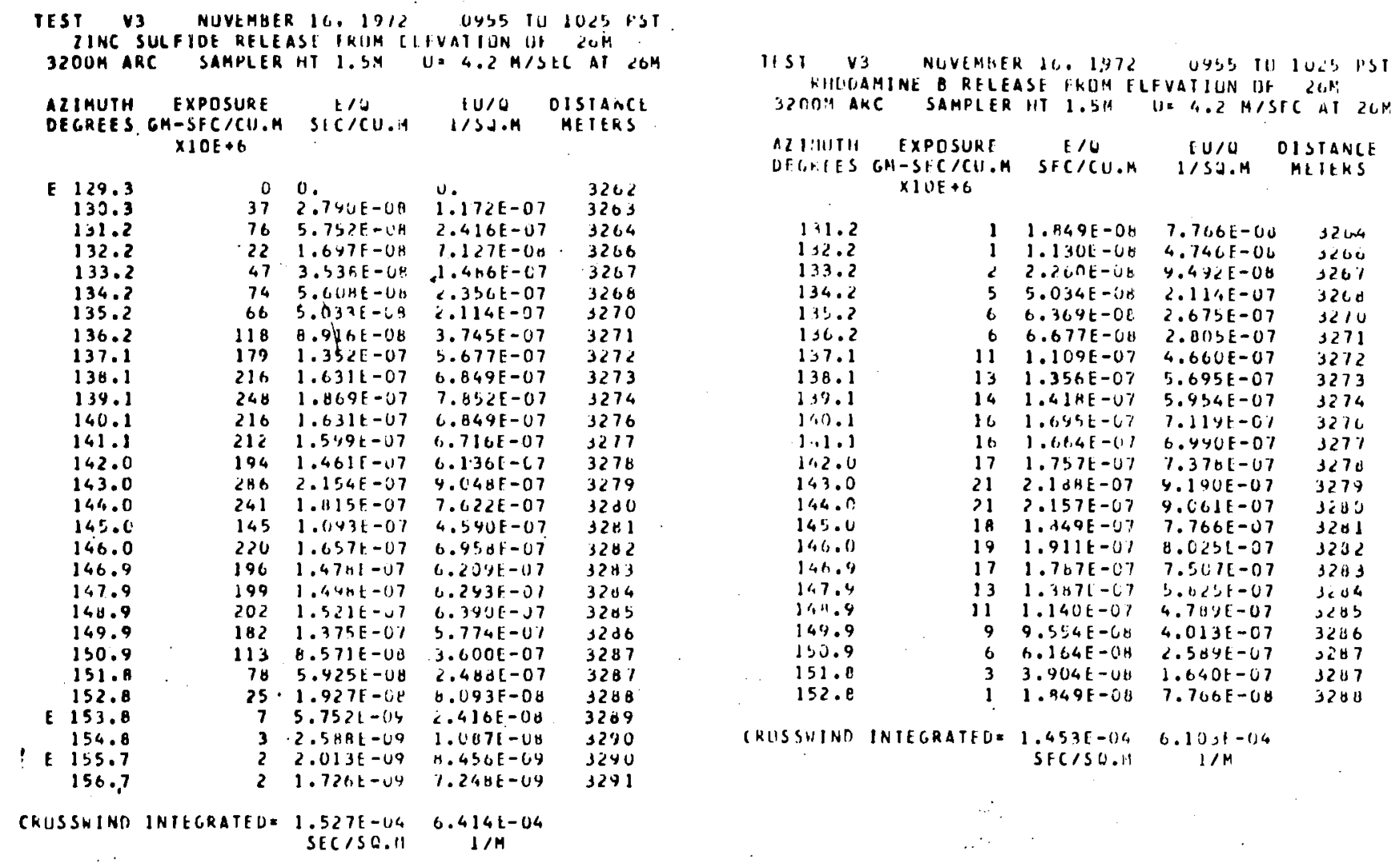


TOWER DATA FOLLOM....

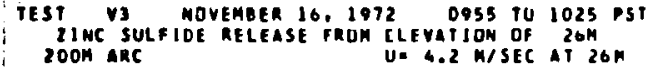

AZIMUTH HE IGHT EXPOSURE
DEGRES HETERS CH-SEC/CU.M SEC/CU.M I/SO.M DISTAMCE XIOE +6

$\begin{array}{llllll}102.0 & .4 & 0 & 0 . & 0 . & 200\end{array}$

AZIMUTH HEICLT EXPOSURF FIO FU/O UISTANCE DEGREES METERS GM-SEC/CU.N SEC/CU.H I/SO.M METEKS
XIOEOG

$\omega 110.0 \quad .4 \quad \ldots 00 . \quad \ldots \quad 0.200$.

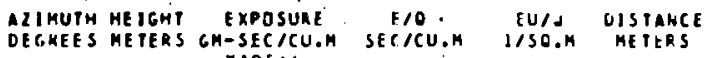
$0 \mathrm{t}+6$

\begin{tabular}{|c|c|c|c|c|c|}
\hline $\begin{array}{l}11 t .0 \\
12 R .0\end{array}$ & 5.5 & 1061 & $\begin{array}{r}0.041 E-07 \\
7.945 E-07\end{array}$ & $\begin{array}{l}3.377 t-66 \\
3.354 t-66\end{array}$ & 160 \\
\hline $\begin{array}{l}116.01 \\
113.0\end{array}$ & $\begin{array}{l}7.0 \\
0.5\end{array}$ & $\begin{array}{l}3000 \\
3033\end{array}$ & $\begin{array}{l}2.3101-06 \\
2.2811-06\end{array}$ & $\begin{array}{l}9.727 t-06 \\
4.57 b E-U 6\end{array}$ & 200 \\
\hline 118.0 & 10.0 & 3870 & $2.9101-06$ & $1.222 t-05$ & 200 \\
\hline $11 \% .0$ & 13.0 & 6643 & $3.1471-66$ & $2.162 t-05$ & <UJ \\
\hline & 16.0 & $755 \%$ & $5.6 \mathrm{nO}$ & $-0 s$ & \\
\hline 130.0 & $19.0^{\prime}$ & 20654 & $0.011 E-06$ & $3.365 L-05$ & 200 \\
\hline & & 10520 & $7.910 E-06$ & $3.322 \mathrm{t}-05$ & 2 \\
\hline 118.0 & 25.0 & 6470 & $4.8 B S E-a_{b}$ & $2.043 L-05$ & 203 \\
\hline 110.0 & 20.0 & 5828 & $4.3 \mathrm{n} 2 \mathrm{E}-06$ & $1.041 t-03$ & 260 \\
\hline & & 5010 & $3.767 E-06$ & $1.582 i-05$ & 200 \\
\hline & & 175 & $.091 E-06$ & $1.634 t-05$ & \\
\hline
\end{tabular}

AZIMUTH HETGHT EXPOSURF F/O EU/J DISTANGE DEGEES METERS CH-SEC/CU.H SEC/CU.M I/SG.M METEKS

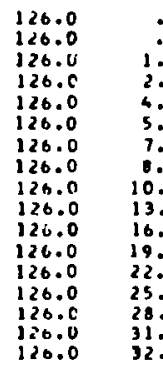

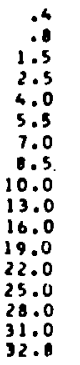

$1610 \quad 1.211 t-06 \quad 5.087 t-06$ $2895 \quad 2.177 E-06 \quad 9.144 t-06$ $0527 \quad 6.412 t-06 \quad 2.643 t-U S$ 13.25 1.006L-0.

$13325 \quad 1.002 t-034.208 t-05$

$12843 \quad 9.6945-06 \quad 4.072 t-05$

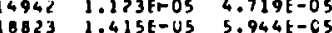

11314 8.507E-06 3.573E-0S

$4662 \quad 3.506 E-06 \quad 1.472 E-U 5$

10750 O.OB,E-06 3.395E-US

$98537.404 E-06$ 3.112t-US

$1920 \quad 5.955 E-46 \quad 2.501 \mathrm{c}-05$

14250 1.072E-0S 4.5U3E-05

$12754 \quad 4.5906-06 \quad 4.02 d t-J 5$

$\begin{array}{lll}13703 & 1.074 t-05 & 4.3281-05 \\ 15573 & 1.171 \mathrm{t}-05 & 4.910 \mathrm{t}-05\end{array}$

200
200
200
200
200
200
200
200
200
200
200
200
201
200
200
200
200

AZIMUTH HEIGHT EXPOSUEE E/O EU/O WISTANCE OEGRES HETERS GM-SEC/CU.N SEC/CU.A WISU.N METEKS xiot 40

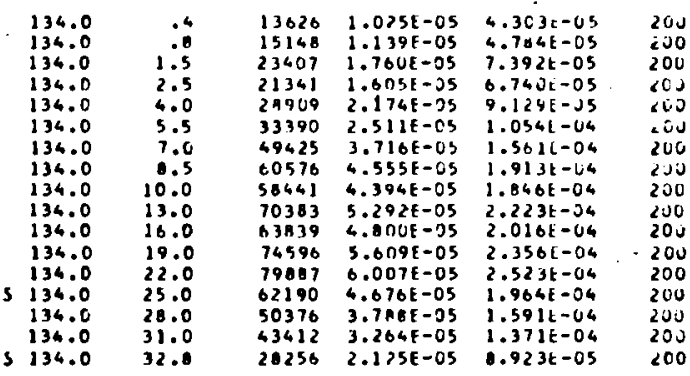

TURER DATA FGLLOW....
IEST VJ MOYEMHER $1 \mathrm{~h}, 1972$ 095S TU 1025 PST RHDDAMIME B RELEASE FROM ELLYATIOA OF $26 \mathrm{M}$

AIIMUTM HEIGHT EXPQSURE F/O EU/O DISTANCE DEGRESS METEMS GH-SEC/CU

$\begin{array}{llllll}\text { N } 102.0 & .4 & 0 & 0 . & 0 . & 200\end{array}$

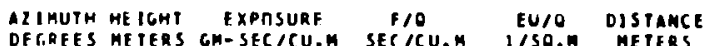
DFTREES METERS GM-SEC/CU.A SET/CU.A I/SO.N METERS
XIOE+6

$\begin{array}{llllll}110.0 & .4 & 0 & 0 . & 0 . & 200\end{array}$

ALIHUTH HEICHT EXPOSUAF F/O EUAO DISTANCE

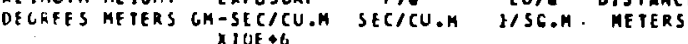

\begin{tabular}{|c|c|c|c|c|c|}
\hline $\begin{array}{l}11 H .0 \\
11 n .0\end{array}$ & .4 & $\begin{array}{l}33 \\
35\end{array}$ & $\begin{array}{l}5.306 t=07 \\
3.514 t=07\end{array}$ & $\begin{array}{l}2.2280-00 \\
2.318 E-0 B\end{array}$ & $\begin{array}{l}200 \\
<00\end{array}$ \\
\hline 116.0 & 1.5 & 153 & $1.531 \varepsilon-06$ & $6.431 t=06$ & 200 \\
\hline 118.0 & & 32 & $3.276 E-07$ & $1.370 \mathrm{E}-06$ & \\
\hline 110.0 & 4.0 & 16 & $1.674 \mathrm{E}-07$ & $7.029 t-07$ & \\
\hline $11 n .0$ & 3.5 & 25 & $2,5>0[-07$ & $1.062 \mathrm{E}=06$ & 200 \\
\hline $11+.6$ & 7.0 & 43 & $4.344 E-07$ & $1.025 E-06$ & 200 \\
\hline iil. & 0.5 & 34 & $3.490 f-07$ & $1.466 \mathrm{t}=00$ & 20 \\
\hline 114.0 & 10.0 & 47 & $9.25 \forall \varepsilon-07$ & $3.08 B \mathrm{t}=00$ & 20 \\
\hline $11 \mathrm{H}, 1$ & 13.0 & 147 & $1.4748-.06$ & $5.442 t-06$ & 200 \\
\hline 1110 & 16.0 & .27 & $2.2791-06$ & $9.571 t-06$ & 20 \\
\hline 114.0 & 19.0 & $33 n$ & $3.3 \times 36-06$ & $1.4211-05$ & $<00$ \\
\hline $11+.2$ & 22.0 & 313 & $3.1 S Q E-U 6$ & $1.331 \mathrm{t}-05$ & \\
\hline $11 \% .0$ & 25.0 & 210 & $2.101 E-06$ & $8.024 \mathrm{t}-00$ & $<0$ \\
\hline 116.0 & 28.0 & 170 & $1.7 D+E-66$ & $7.179 t=06$ & \\
\hline $11^{r} .0$ & 31.0 & 156 & $1.567 t-06$ & $6.580 t-06$ & \\
\hline 16.1 & & 174 & $1.745 E-06$ & $400-0$ & \\
\hline
\end{tabular}

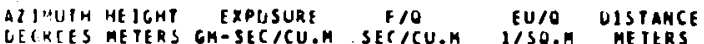
AIOE +6

123.0

6126.0

126.0

$1<t \cdot 0$

2126.0

$1<6.0$

$1<6.0$

128.0

126.0

126.0

126.

$126.0 \quad 25.0$

136.0

126.6

.4
1.5
2.5
4.0
5.5
7.0
10.5
10.0
13.0
16.0
19.0
22.0
25.0
21.00
31.0
32.6

$96 \quad 9.614 E-07 \quad 4.036 E-06 \quad 200$

$477 \quad 4.771 E-6 e$ 2.004t-05 200

252 2.57OE-G6 $1.042 E-G S$

$3613.81 C F-16$ 1.600E-US 200

$348 \quad 3.490 E-66$ 1.66EE-J5 200

in 3.169F-06 $1.331 \mathrm{E}-65$ 20J

$2742.7426-06 \quad 1.1326-65 \quad 200$

$2870.87 B E-072.0496-06$

$227 \quad 2.2791-36$ 9.571E-06

$192 \quad 1.973 E-06 \quad 8.0762-06 \quad 200$

435 $4.550 t-06 \quad 1.914 t-65 \quad 200$

$346 \quad 3.490 E-06 \quad 1.466 t-05$ CUJ

$\begin{array}{llll}370 & \$ .7695-06 & 1.555 E-U S & 200 \\ 645 & 4.451 E-06 & 1.0645-05 & 200\end{array}$

A2I TUTH MEIGHT EXPOSURF T10 EUNA WISIANCE MIUE
MEO

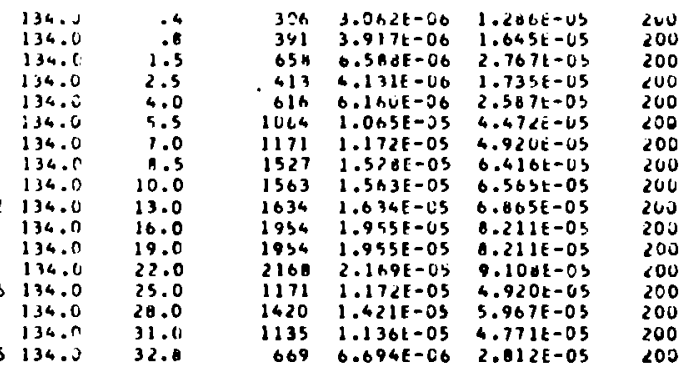


rowe nata FOLLOW....

TES! V3 NOVEHOER 10.1972 OQS5 TO 1025 PST DOOHE SULFIOE RFLEASE FROH ELFYATIOH OF $20 \mathrm{H}$

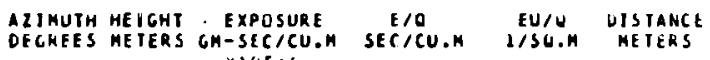
$\times 10 \mathrm{E}+6$
TUEER DAIA FOLLOK....

IEST V3 NOVEHBER 16. 1972 U955 TO 1025 PSI AHODAMINE B RELEASE FRUM ELIVATION OF $26 \mathrm{H}$
DCUM AKC

AZIMUTH HEICMT EXPUSURF F/O EU/O DISTANCE OECREES METERS GM-SECICU.M SET/CU.M H SO.M METERS

i. $145.0 \quad .3 \quad 0$

o.

d 20

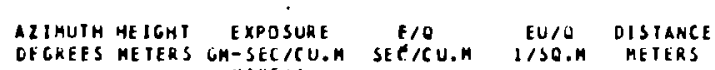

ALIMUTH HEIGHT EXFIISUKF F/O EUIJ DISTANCE

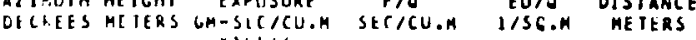
$x$ IOE +6

v.

1. $112.6 \quad .3$ I 6 .

o.

d 33

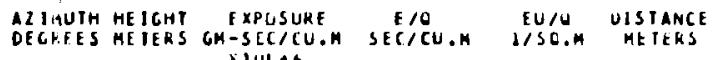

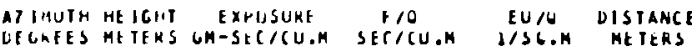
AlUL $+b$

c 0.0

0. $d 4 s$

1. $120.2 \quad .3 \quad$ J 0.

0 .

45

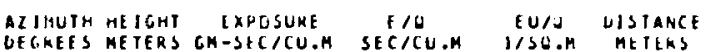

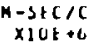

AZIKUIH HEICHT EXPLISIJRF

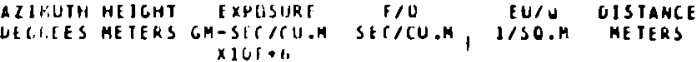

057
057
051
057
057
057
057
057
057
057
057
057
057
-557
057
457
051
057

057
057
051
057
457
057
057
057
657
057
051
057
057
357
057
457
051
057

$\begin{array}{rr}127.7 & .3 \\ 127.7 & .3 \\ 127.7 & 1.1 \\ 127.7 & 1.5 \\ 127.7 & 2.1 \\ 127.7 & 4.2 \\ 127.7 & 0.3 \\ 127.7 & 8.4 \\ 127.7 & 10.5 \\ 127.7 & 12.0 \\ 127.7 & 14.7 \\ 127.7 & 10.8 \\ 127.7 & 42.0 \\ 127.7 & 25.2 \\ 127.7 & 29.4 \\ 127.7 & 33.6 \\ 127.7 & 37.8 \\ 5127.7 & 42.0\end{array}$
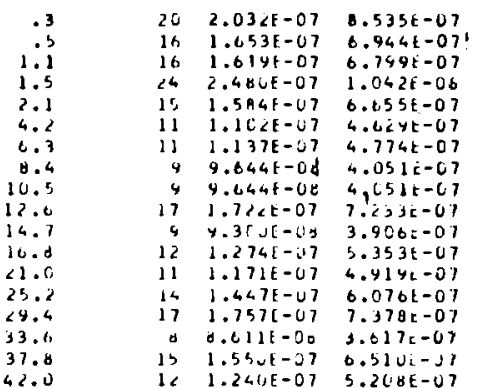

d5?

057

as

ist

d 57

457

d57

us 7

057

057
057

457
057

as

057

ALITUIH HEIGHT EXPCSUKF T/O LU/U WISTANGE DECKES METERS ON-SEC/CU.M SEC/CU.M L/SG.M METCKS

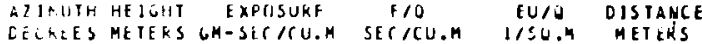
$x \mid J E+e$

\begin{tabular}{|c|c|c|c|c|}
\hline 133.0 & 3 & 5476 & $4.419 E-J 6$ & $1.056 c-05$ \\
\hline 135.0 & .5 & 1826 & $1.373 t-06$ & $5.7671-60$ \\
\hline 135.6 & 1.1 & 7103 & $5.3 \mathrm{ABE}-0 \mathrm{G}$ & $2.202 \mathrm{~L}-0 \mathrm{~b}$ \\
\hline 135.0 & 1.5 & 3859 & $\angle .90<t-66$ & $1.2145-05$ \\
\hline 135.0 & & 6060 & $5.104 E-06$ & $2.16 y_{C}=05$ \\
\hline 135.8 & 4.2 & $5 \mathrm{HAS}_{4}$ & $4.394 E-06$ & $1.846 c-05$ \\
\hline 155.0 & 6.3 & 6015 & $4.974 E-66$ & C.Joyt-us \\
\hline 135.6 & $6 \cdot 4$ & 4831 & $3.633 E-06$ & $1.32 C L-0 b$ \\
\hline 135.0 & 10.5 & 5370 & $4.030 E-06$ & $1.090 i-15$ \\
\hline 135.0 & $1<.6$ & 5184 & $3.85 C E-36$ & $1.097 t-43$ \\
\hline 135.0 & 14.7 & $53 \div 4$ & $4.03: 5-60$ & $1.644 t-03$ \\
\hline 19300 & 16.0 & 5797 & $4.350[-U c$ & $1.829 t-u b$ \\
\hline 135. & 21.0 & 5474 & $4.110 E-=6$ & $1.729 E-j 5$ \\
\hline 135.0 & 25.2 & 5241 & $3.470 E-C_{0}$ & $1.67 d t-03$ \\
\hline 135.6 & 29.4 & 5345 & $4.019 L-60$ & $1.60 b c-0)$ \\
\hline$: 33.0$ & 33.6 & 4315 & $3.267 \mathrm{t}-0 \mathrm{O}$ & $1.304 L=U S$ \\
\hline 135.0 & 37.8 & 2403 & $1.007 E-20$ & $7.54 v c-40$ \\
\hline 135.0 & 42.0 & 4313 & $3.243 E-00$ & $1.36<t-3)$ \\
\hline
\end{tabular}

\begin{tabular}{|c|c|c|}
\hline del & & $13 \% .6$ \\
\hline 067 & & 1950 \\
\hline 067 & & 135.0 \\
\hline 107 & & 135,0 \\
\hline 867 & & i35.J \\
\hline 167 & & 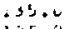 \\
\hline 067 & & 1.35 .0 \\
\hline$\infty 01$ & 2 & $13: 0$ \\
\hline 167 & & 135.0 \\
\hline $0 t>7$ & & 134.0 \\
\hline 467 & & $235 . \mathrm{C}$ \\
\hline$=07$ & & $1=5.0$ \\
\hline acl & & $1+5,6$ \\
\hline set & 1 & $1.2 \% 0$ \\
\hline 007 & & 155.0 \\
\hline 367 & & $1=5.0$ \\
\hline$\alpha 61$ & 5 & 135.0 \\
\hline & & 135.0 \\
\hline
\end{tabular}

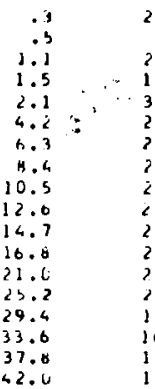

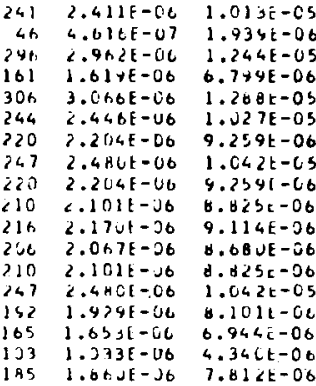

367

867

367
667

867

067
067

067
$0: 7$

467

067

.067

067
067
067

067
067

467

d 67

067
567
067 


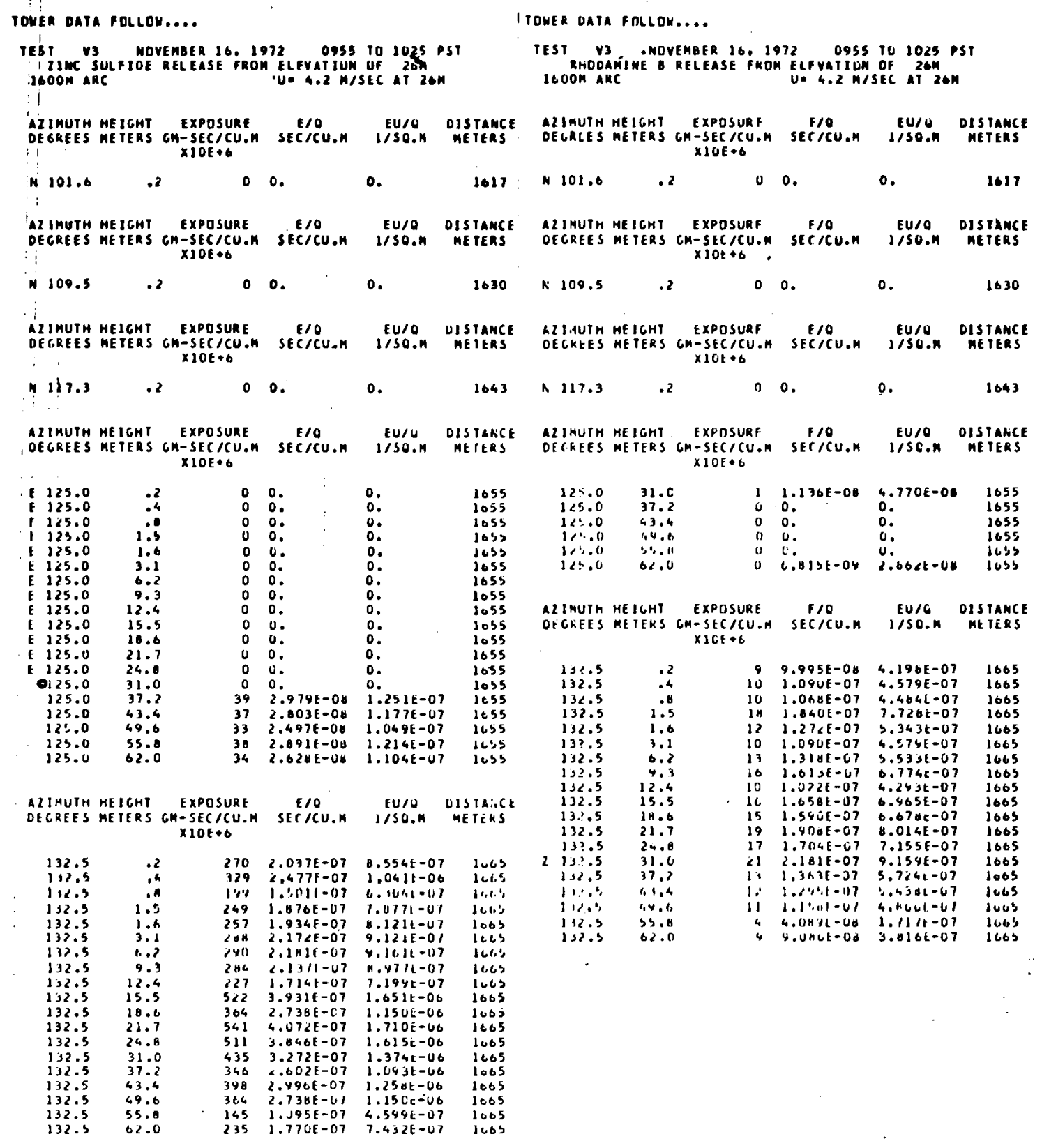


TORER DATA FOLLOW....

TEST: V3 NOVEABER 16,1972 0955 TO 1025 PSI 21MC SULFIOE RELEASE FROM ELEVATIUN OF $26 \mathrm{M}$

ATIMUIM HEILHT EXPOSURE F/O DU EUIO OASTANCE DECKEES METERS GR-SEC/CU.H SEC/CU.M I/SG.H METERS XIOE+6

99.8

00 .

0.

3215

A2IMUTH HEICHT EXPOSURE E/O EULU. DISTANCE $\times 10 E+6$

$107.8 \quad .2$

00.

0.

3224

AZIMUTH HE IGHT

A2 IRUTH HETGHT EXPGSURE E/O E
$\times 10 \mathrm{SE}+6$

N $315.6 \quad .2$

00.

OISTANCE

AZIMUTH HEIGHT EXPQSURE E/O

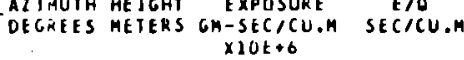

$\begin{array}{lllll}M & 123.4 & 0 & 0 & 0 .\end{array}$

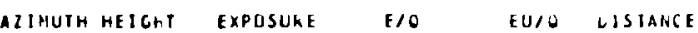
OEGKEES HETERS GH-SEC/CU.H SEC/CU.M liso.M METERS $\times 10 F+b$

$\begin{array}{rr}1131.2 & . \\ 131.2 & \\ 131.2 & . \\ 131.2 & 1 . \\ 131.2 & 1 . \\ 131.2 & 3 . \\ 131.2 & 6.2 \\ 131.2 & 9 . \\ 131.2 & 12 . \\ 131.2 & 15 . \\ 131.2 & 18 . \\ 131.2 & 21 . \\ 131.2 & 24.0 \\ 131.2 & 31.0 \\ 131.2 & 37 . \\ 131.2 & 43.4 \\ 131.2 & 49.6 \\ 131.2 & 55.0 \\ 13122 & 62 .\end{array}$

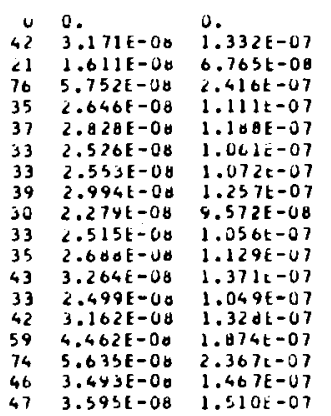

$3<64$
3264
3264
3264
3204
3264
3264
3264
3264
3264
$3<64$
$1<64$
3264
3264
3264
3264
3264
3264
3264

GE LATA FILLOOH.....

TEST V3 NUVEMBEK 16.1972 U9BS TU 1025 PST KRIULAHINE B RELEASE PROM ELFYAT IUM OF $26 \mathrm{~K}$
SZUOM G.2 M/SEC AT $26 \mathrm{~K}$

ALIMUIH HEIGHT EXPOSURE F/O EU/U UISTANCE DEGREES METERS GM-SEC/CU.M SEC/CU.M W/SU.M METERS

$\times 99.9 \quad .2$ บ 0.

0.

3215

AZIMUTH HEIGHT EXPUSURE F/C EU/O DISTAMCE OECKEES HETERS GH-SEC/CU.M SET/CU.M D.SO.M METEKS

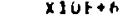

$167.8-20 \quad 0 \quad 0$.

$0 . \quad 3229$

ALIMUTH HEIGHT EXPOSURF EM EUIU DISTANCE DECREES METERS GM-SEC/CU.H SEC/CU.H THSO.H. METERS IOE +6

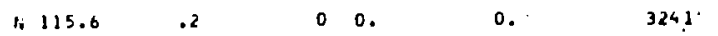

AZIMUTH HEIGHT EXPQSURE E/O EU/O DISTANCE DEGREES ME TERS CH-SEC/CU
XIOETh

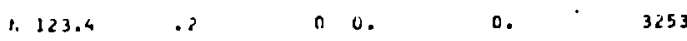

DIIIUTH HEIGHT EXPUSURE F/O EU/O OISTANCE DEGREES METERS GM-SEC/CU.A SEC/CU.A LISO.H METERS $\times 30 \mathrm{E}+6$

\begin{tabular}{|c|c|c|c|c|c|}
\hline 191.2 & .7 & 0 & 0. & 0. & $\begin{array}{l}3264 \\
3264\end{array}$ \\
\hline 131.2 & .4 & 1 & $\begin{array}{l}1.335 E-0 A \\
9.24 C E-O Q\end{array}$ & $\begin{array}{l}5.609 t-D \theta \\
3.803 t=08\end{array}$ & $\begin{array}{l}3264 \\
3264\end{array}$ \\
\hline $\begin{array}{l}131.2 \\
131.2\end{array}$ & $\begin{array}{l}.0 \\
1.5\end{array}$ & 1 & $1.0<9 F-00$ & $7.706 \mathrm{E}-00$ & 3264 \\
\hline 131.2 & 1.6 & 0 & C.1BGE-04 & $2.509 k-00$ & 32 \\
\hline 131.2 & 3.1 & 0 & $2.055 E-0 Y$ & $0.629 t-09$ & 326 \\
\hline $1,1.2$ & 6.2 & 0 & $3.08<F-09$ & $1.294 E-00$ & 326 \\
\hline 131.2 & 9.3 & 0 & $5.136 E-04$ & $2.1578-08$ & 326 \\
\hline 131.2 & 12.4 & 1 & $1.434 E-08$ & $6.040 E-0 B$ & 3204 \\
\hline 111.2 & 15.5 & 0 & $4.109 \mathrm{E}-09$ & $1.726 \mathrm{E}-0 \mathrm{~B}$ & 3264 \\
\hline 131.2 & $18 . \mathrm{h}$ & 0 & $3.136 E-09$ & $2.157 c-08$ & 326 \\
\hline 191.2 & 21.7 & 0 & $5,13 \in[-\cup 4$ & $2.157 E-08$ & 3264 \\
\hline 131.2 & 24.8 & 0 & $5.1368-04$ & $2.157 c-08$ & 320 \\
\hline 131.2 & 31.0 & 0 & $0.164 E-34$ & $2.589 \div-08$ & $3<6$ \\
\hline 131.2 & 37.2 & 0 & $0.210 f-24$ & $3.432 t-00$ & 3264 \\
\hline 131.2 & 43.4 & 0 & $7.141 E-J 4$ & $3.02 \cup E-00$ & 320 \\
\hline 131.2 & 49.6 & 0 & $1.191 E-09$ & $3.02 \mathrm{UL}-0 \mathrm{O}$ & \\
\hline 131.2 & 55.8 & 1 & $1.4 \div 0 E-00$ & $6.040 E-68$ & $3<0$ \\
\hline 13 & 02.0 & $\dot{0}$ & $5.13 u t-0 y$ & $2.157 t-06$ & \\
\hline
\end{tabular}


GROUAD LEVEL AND TRHER SAMPL JNG TCOM TU IGOOM - WITH MODITIONAL THO TUHERS SAMPLING AT $3200 M$.

ALL ARCS EMBRACE CRUSSMIND EXTENT UF TRACER. TUWER SAMPLES TKUNCATED AT TOP. ABDUT 9 CM SNOH DEPTH ON GRUUAO DURING EXPERIMENT. THERMALLY STABLE II LULEST GM. BUT NEUTRAL ABOVE.

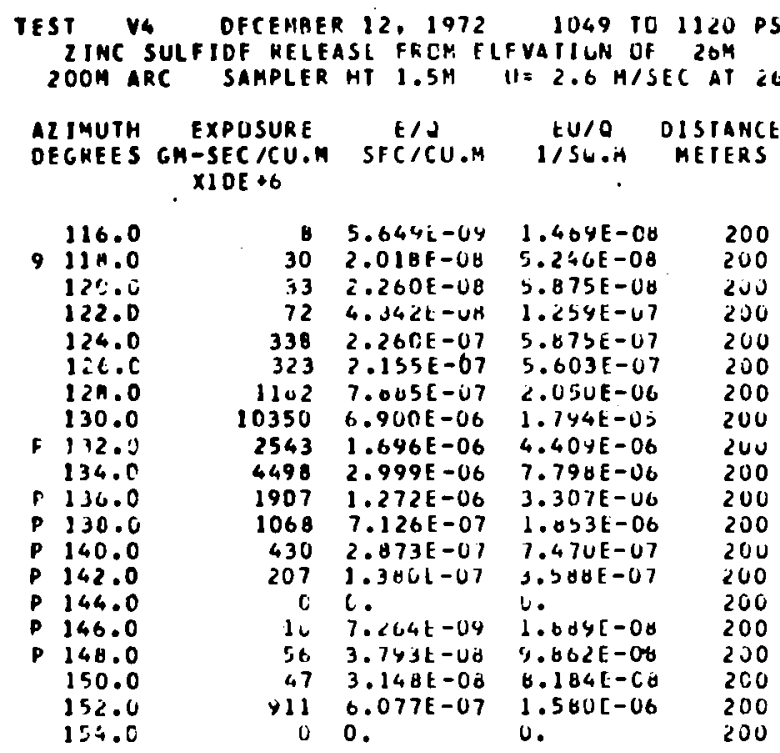

CKIISSHIHO INTEGRATFD= $1.119[-04 \quad 2.9 C 7 F-04$

TEST V4 DECE:IUEK 12, 1972 1049 TU 1120 NST IINC SULFIUE RELEASE FRUA TLFVATION DF $26 M$ BOUR AKC SAPPILR HI I.SM $U=2.6 \mathrm{HISEC} \mathrm{AT} \mathrm{LGM}$

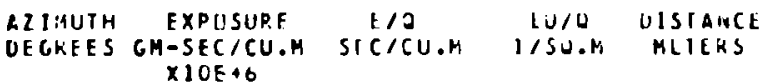

\begin{tabular}{|c|c|c|c|}
\hline 114.3 & 2 & 1. SuJE - y & 4. UGUE-OY \\
\hline $\begin{array}{l}120.2 \\
122.1\end{array}$ & $\begin{array}{l}174 \\
917\end{array}$ & $\begin{array}{l}1.163 t-07 \\
5.44 \% E-07\end{array}$ & $\begin{array}{l}3.0 \angle 4 E-U 7 \\
1.417 E-06\end{array}$ \\
\hline 122.1 & 3058 & $2.034 E-06$ & $3.302 E-06$ \\
\hline 125.8 & 12766 & $0.471 E-06$ & 2.2 \\
\hline 127.7 & 18046 & $1.203 E-05$ & 3.1206 \\
\hline .129 .5 & $1025 \mathrm{t}$ & $1.217 \mathrm{E}-0 \mathrm{~s}$ & 3.165 \\
\hline 131.4 & 14426 & $9.617 \mathrm{E}-6 \mathrm{t}$ & C.SO1E \\
\hline 133.2 & 9021 & $6.014 \mathrm{E}-06$ & $1.564 \mathrm{E}$ \\
\hline . C & 4309 & $2.673 E-06$ & $7.470 \mathrm{E}$ \\
\hline 136.8 & 13895 & $9.264 E-06$ & 2.40 \\
\hline 130.7 & 29100 & $1.441 t-65$ & 3.045 \\
\hline 140.6 & 16357 & $1.090 t-65$ & 2.635 \\
\hline 142.4 & 6067 & $4.045 t-66$ & 1.0 \\
\hline 144.2 & 11814 & $7.676 E-06$ & $2.046 \mathrm{E}-0$ \\
\hline 168.0 & 7526 & $5.217 E-06$ & $1.357 \mathrm{E}-\mathrm{t}$ \\
\hline 147.8 & 16778 & $1.112 E-05$ & $2.90 \mathrm{DE}=$ \\
\hline 149.6 & 10972 & $7.315 i-06$ & $1.902 \mathrm{E}-$ \\
\hline 151.4 & 1607 & $1.072 E-26$ & .7871 \\
\hline & 236 & $1.577 t-07$ & 4.100 \\
\hline & 2 & $1.561 \mathrm{E}-04$ & 4. U6UE - \\
\hline
\end{tabular}

8,2

645

848

851

254

854

859

362

865

407

864

872

874

876

876

840

$80<$

604

806

807 RHUDARINE B RELEASE FROH ELFVATILN UF $26 \mathrm{H}$

$200 M$ ARC SAMPLER HT $1.5 \mathrm{M} U=2.6 \mathrm{M} / \mathrm{SEC}$ AI $26 \mathrm{M}$

AZIMUTH EXPOSURE $2 / 0$ EU/O UISIANCE

DECREES GH-SEC/CU.M SEC/CU.M I/SO.H MEIEKS $\times 10 E+6$
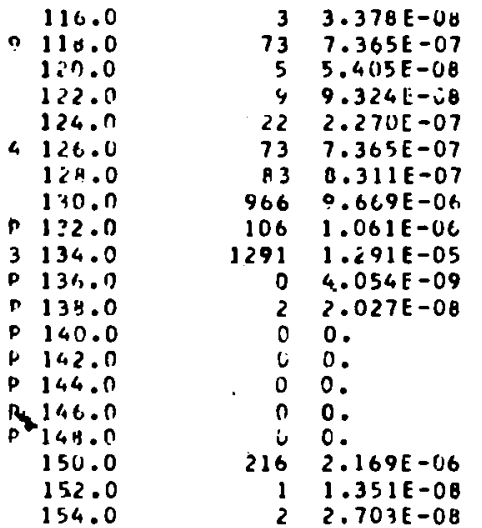

$\begin{array}{ll}8.764 E-00 & 200 \\ 1.915 E-00 & 200 \\ 1.405 E-07 & 200 \\ 2.424 E-07 & 200 \\ 5.903 E-07 & 200 \\ 1.915 E-06 & 200 \\ 2.161 E-06 & 200 \\ 2.514 E-05 & 200 \\ 2.758 E-06 & 200 \\ 3.357 E-05 & 200 \\ 1.054 E-08 & 200 \\ 5.270 E-00 & 200 \\ 0 . & 200 \\ 0 . & 200 \\ 0 . & 200 \\ 0 . & 200 \\ 0.0 & 200 \\ 5.639 E-06 & 200 \\ 3.514 E-00 & 200 \\ 7.627 E-00 & 200\end{array}$

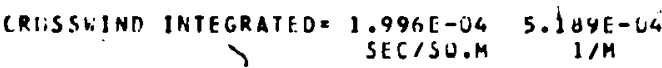

IIST V4 OECEMUER.12: 1972 1049 TU1118 PST RHIDAHINE B RELEASF FRUM ELFVATIUN UF $26 \mathrm{M}$ aCOM ARC SAMPLER HT $1.5 \mathrm{M} U=2.6 \mathrm{M} / \mathrm{SEC}$ AT $20 \mathrm{M}$

AZIYLTH EXPOSURE LIO EU/O DISTANCE

DEGFIES GM-SECICU.M SEEICU.M I/SO.M MEIERS $X \perp 0 E+6$

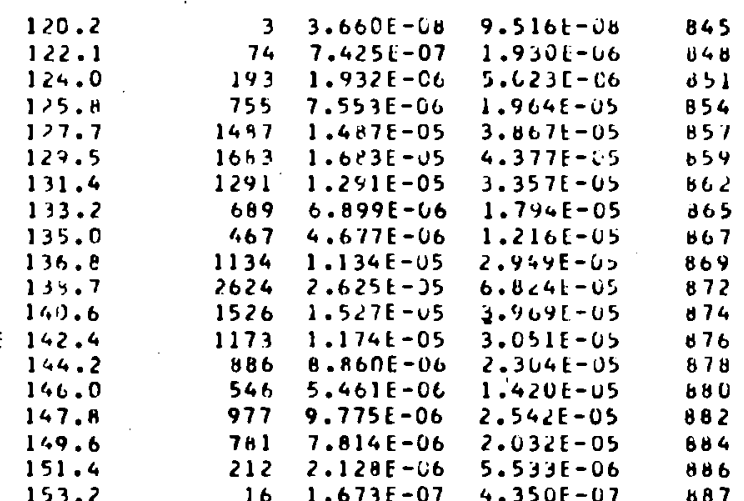

G D.SSIIND INTEGRATEU $4.614 \mathrm{E}-\mathrm{CH} \quad 1.200 \mathrm{H}-02$
CRUSSHINO INTEGRATED $=3.037[-03 \quad 9.457 E-03$ 


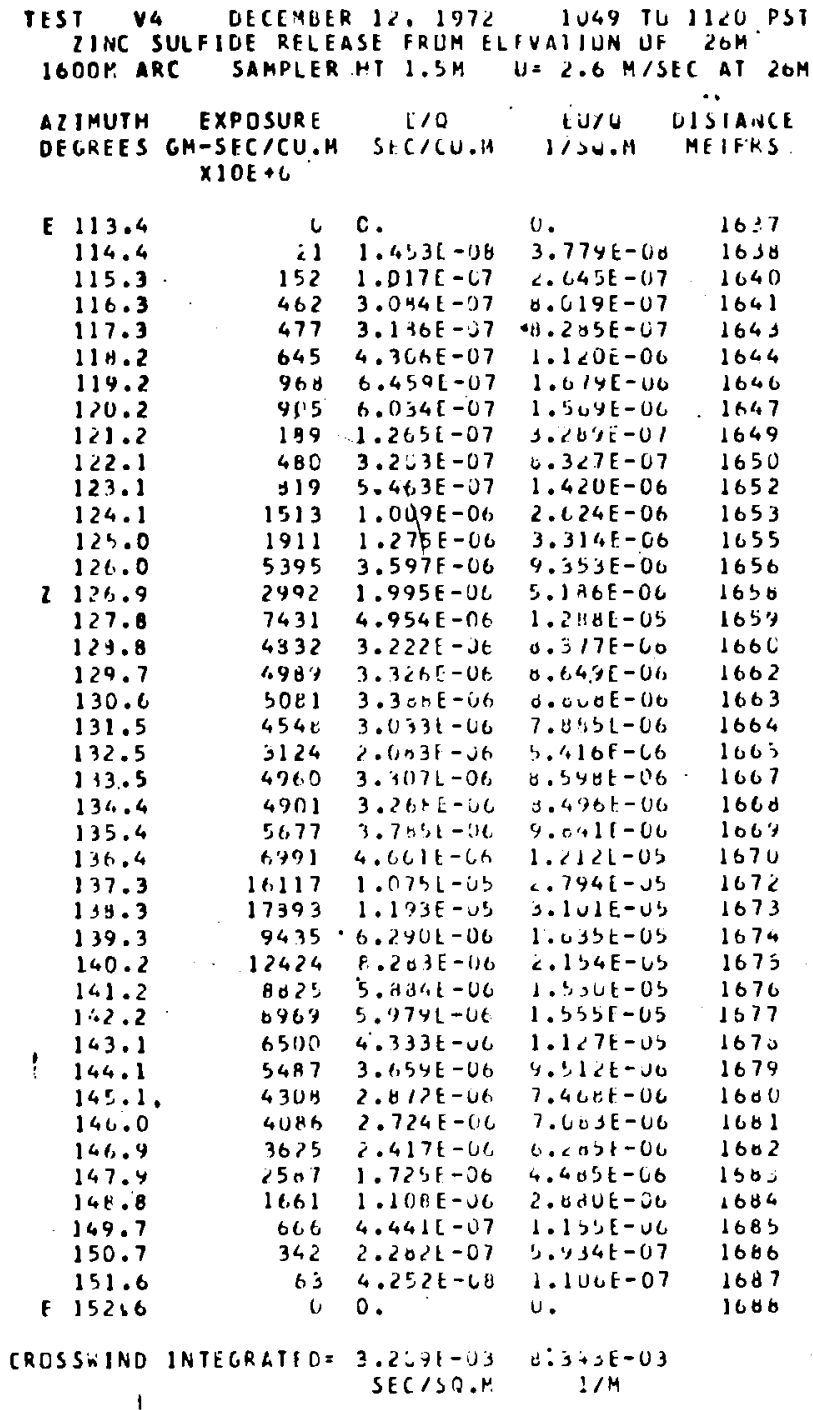
IEST V4 DECEMBER 12, 1972 1049 IU 1118 PST RIIJOAMINE B RELEASE FRUH ELEVAIIUN UF ZUM IHCA ARC SAMPLER HT $1.5 \mathrm{M} \quad 1=2.0 \mathrm{M} / \mathrm{SEC}$ HI $26 \mathrm{H}$

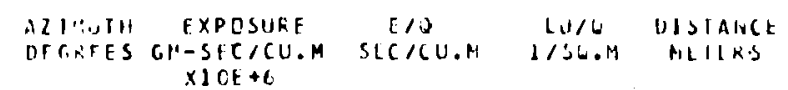

111.3
110.3
117.3
118.2
119.2
119.2
1.1 .2
122.1
127.1

$0.021 E-609$

1.

$9.14 \hat{E}-100 \quad 1641$

$\begin{array}{llll}5 & 5.431 E-06 & 1.41 C E-67 & 1643 \\ 6 & 1.466 \mathrm{E}-0 \mathrm{~b} & 1.631 \mathrm{E}-67 & 1644\end{array}$

$12 \quad 1.243 \mathrm{E}-67 \quad 3.362 \mathrm{E}-67 \quad 10.4$

$353.517 \mathrm{E}-670.145 \mathrm{E}-07$ 154

$14 \quad 1.422 \mathrm{E}-07 \quad 3.69 \mathrm{JE}-07 \quad 1049$

$\begin{array}{llll}14 & 1.422 \mathrm{E}-07 & 3.69 j \mathrm{E}-\mathrm{C} 7 & 1649 \\ 92 & 9.207 \mathrm{E}-07 & 2.394 \mathrm{E}-\mathrm{C} 6 & 165 \mathrm{~L}\end{array}$

$123.1 \quad 86$ B.6\%OE-07 $2.259 E-66$ 1652

$124.1 \quad 143 \quad 1.434 E-06 \quad 3.737 E-06 \quad 1033$

$125.0 \quad 185 \quad 1.852 E-06 \quad 4.814 E-06 \quad 1635$

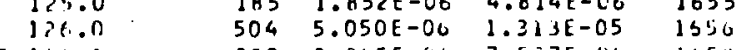

$1>7.8$

$1,1 . .4$

$1,9.1$

131,6

$1 ; 1.5$

$1 ? 2.5$

133.5

$13 \times .4$

$11, .6$

$1,7.3$

$1 \% \cdot 3$

1.9 .3

$140 . ?$

141.2

$1+2.7$

143.1

$1 \therefore 4.1$

161.1

146.0

1.6 .9

147.4

143.4

34.3. 7

$15,0.7$

151.6
$1: 2.8$

$$
\begin{aligned}
& 280 \\
& 542
\end{aligned}
$$$$
542
$$$$
392
$$

4.6
375

375
272

323
238

238

306
350
556

1134

1315
617

617

$9.7 \quad 9.171 E-06$

5 ค $5.13265-60$

$453 \quad 4.533 t-06$

436

$341: 3.4121-U 6$

$3+343.843 t-U 6$

$3413.412 k-C 6$

$315 \quad 3.153 \mathrm{E}-\mathrm{iG}$

$20 \mathrm{H} 2.004 \mathrm{E}-6 \mathrm{C}$

$7.655 \mathrm{E}-07$

$4.724 \mathrm{E}-07$

1. $31,5 E-07$

0 .

CKISSAII, INTEGRATEOE 3,6,51E-63

SEC/SO.
$7.527 \mathrm{E}-06 \quad 1653$

$1.5+3 t-05+64$

$1.022 \mathrm{E}-\mathrm{US} \quad 16.60$

y. $760 \mathrm{E}-00 \quad 1603$

$7.070 \mathrm{E}-00 \quad 1664$

$8.423 E-06 \quad 1605$

$7.751 E-00 \quad 1607$

$7.975 t-60 \quad 1660$

$4.3201-06 \quad 166$

$1.4411-05 \quad 1610$

$2.944^{2}=-65$

3.4Cut-C5 1619

1.6.4E-0S 1674

$2.411 \mathrm{t}-0 \mathrm{~S} \quad 1612$

1.t<7[-us 1070

1.51)1-0: 167

1.17yi-v夕 1510

1.134E-is 1614

6.t11t-lio 164

$4.492 \mathrm{~F}-\mathrm{Uh} \quad 1601$

6.8711-06 160

$6.199 \mathrm{E}-00 \quad 1605$

$3.4200-06 \quad 1604$

$1.490 E-06 \quad 1605$

$1.220 E-00 \quad 1606$

$3.497 \mathrm{~L}-07 \quad 168 \%$

v.

Jó。
$1.134 \mathrm{E}-0 \mathrm{~S} \quad 16.62$ 
TOWER DATS FOLLOM....

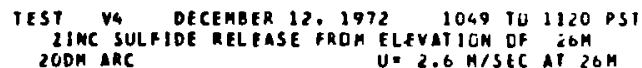

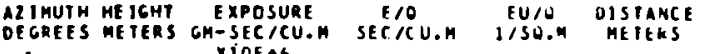
$X I O E \bullet 6$
TOEER CATA F RLCOK,...

ItSI V4 OECEMBER 12, 1972 1049 IU 1118 PST

KHUTIAHIAE D RELEASE FRUM LLFVATIUA UF $20 \mathrm{M}$

AZIMUTH HEIGHT EXPOSURF F/O EU/O UISTANCE $\times 102+6$

\begin{tabular}{|c|c|c|c|c|c|}
\hline 102.0 & .4 & 0 & 0. & 0. & \\
\hline $\begin{array}{l}\text { AL IMUTH } \\
\text { DEGREES. }\end{array}$ & $\begin{array}{l}\text { HE IGH } \\
\text { ME TERS }\end{array}$ & $\begin{array}{c}\text { EXPOSURE } \\
\text { GH }-5 E C / C U, H \\
\times 1 O E \bullet 6\end{array}$ & $\begin{array}{c}\text { F'U } \\
\text { SEC'CU.M }\end{array}$ & $\begin{array}{l}E U / \sigma \\
1 / S C . H\end{array}$ & $\begin{array}{l}\text { UIST } \\
\text { MET }\end{array}$ \\
\hline 110.0 & .4 & L & 0 . & & \\
\hline $\begin{array}{l}\text { AZIMUUTH } \\
\text { DEGREES }\end{array}$ & $\begin{array}{l}\text { HE ICHI } \\
\text { HE TERS }\end{array}$ & $\begin{array}{c}\text { EXPDSURE } \\
\text { CN-SEC/CU.A } \\
\times 10 E+6\end{array}$ & SEC/CU.M & $\left.\right|_{1 / 50.4}$ & UIS \\
\hline 110. & .4 & 73 & 4.894 & 1.2 & \\
\hline 1.18 & .8 & 0 & 0 . & 0. & \\
\hline $\begin{array}{l}118.0 \\
118.0\end{array}$ & $\begin{array}{l}1.5 \\
2.5\end{array}$ & $\begin{array}{l}30 \\
81\end{array}$ & $\begin{array}{l}2.016 \\
5.407\end{array}$ & $\begin{array}{l}3.246 E-C Q \\
1.406 E-07\end{array}$ & \\
\hline 118.0 & 4.0 & 156 & 1.0 & 2.30 & \\
\hline $\begin{array}{l}118.0 \\
118.0\end{array}$ & $\begin{array}{l}5.5 \\
7.0\end{array}$ & $\begin{array}{l}347 \\
466\end{array}$ & $\begin{array}{l}2.31 \\
3.10\end{array}$ & $\begin{array}{l}0.02 \\
0.07\end{array}$ & \\
\hline $11 A .0$ & & 510 & 3.40 & $0.6486-U 7$ & \\
\hline $11 \mathrm{~d} .0$ & 10.0 & 371 & 2.47 & 6.43 & \\
\hline $\begin{array}{l}11 \mathrm{~A} .0 \\
118.0\end{array}$ & $\begin{array}{l}13.0 \\
16.0\end{array}$ & $\begin{array}{l}769 \\
519\end{array}$ & $\begin{array}{l}5.126 E-07 \\
3.4 \text { HOE }-07\end{array}$ & $\begin{array}{l}1.333 L-U 6 \\
8.94 C L-07\end{array}$ & \\
\hline 118.0 & 19.0 & 3105 & 2.07 & $5.3821-66$ & \\
\hline 110.0 & 22.0 & 3145 & $2.097 t$ & $5.43<6-06$ & \\
\hline 11 & 25.0 & 4775 & $3.184 E=06$ & $3.27 \varepsilon t-116$ & \\
\hline 118.0 & 28.0 & 5001 & $3.8 \mathrm{BHE}-$ & $1.0061-05$ & \\
\hline & 7 & & $1.4135-06$ & $3.855 t-06$ & \\
\hline 11 & 32.0 & 3402 & .2 to[ -96 & $5.897 \mathrm{E}-06$. & \\
\hline
\end{tabular}

AZIMUIH HEIGHE EXPOSURE W/O EUA UISIAIAC DECFEES!MEIERS GH-SEC/CU.H SEC/CU.H LISU.P HETERS

\begin{tabular}{|c|c|c|c|c|}
\hline 0126.0 & .4 & 43 & z. BAYE-CD & $7.459 t-58$ \\
\hline$=126.0$ & . & 170 & $1.134 \mathrm{E}-01$ & $2.96<t-17$ \\
\hline 126.0 & 1.5 & 323 & $2.153 \mathrm{E}-07$ & $5.6011-1,7$ \\
\hline 126.01 & 2.5 & 1376 & $9.176[-07$ & $2.306 t-06$ \\
\hline$i<6.0$ & 4.0 & $3 \times 32$ & $2.5539-00$ & h. $443 t-J 6$ \\
\hline 126.0 & 5.5 & 4269 & $2.8476-10$ & $7.401 \mathrm{t}-\mathrm{Jt}$ \\
\hline 126.0 & 3.0 & 5746 & $3.811 t-66$ & $9.4611-46$ \\
\hline 126.0 & 0.5 & 7445 & $4.9471-1,6$ & $1.244 \mathrm{~L}-\mathrm{US}$ \\
\hline 120.0 & 10.0 & 11504 & $7.71 \mathrm{JF}-0 \iota$ & 3.1, $34=03$ \\
\hline $12 t . r$ & 13.6 & ZJЗ ЗА & 1.jayf-us & $30342-05$ \\
\hline 126.0 & 16.0 & 35176 & $2.345 \mathrm{~F}-035$ & $6 . J 4 / t-3$, \\
\hline 128.0 & 19.0 & 59127 & $3.941 \mathrm{E}-\mathrm{US}$ & $1.42 b_{2}-v_{4}=$ \\
\hline 126.0 & 22.0 & 82749 & $5.517 E-05$ & $1.434 c-64$ \\
\hline 126.0 & $25.0 !$ & 76511 & $5.101 E-05$ & $1.326 t-64$ \\
\hline 126.0 & 20.0 & 72334 & $4.8228-25$ & $1.254 \mathrm{E}-04$ \\
\hline $12 \pi \cdot c$ & 31.0 & 42587 & $2.839 E-05$ & $7.381 t-05$ \\
\hline 126.0 & & 27326 & $1.02<E-05$ & $4.736 t-65$ \\
\hline
\end{tabular}

\begin{tabular}{|c|c|}
\hline Lo. & $12 \mathrm{k} . \mathrm{c}$ \\
\hline 200 & $412 b .0$ \\
\hline$<00$ & $412 \mathrm{~h} .0$ \\
\hline 200 & 131.1 \\
\hline 200 & 126.6 \\
\hline $20 \%$ & 120.0 \\
\hline 200 & $12 A . \therefore$ \\
\hline 200 & $1 \geq 6.6$ \\
\hline 2000 & $1>n, n$ \\
\hline 6110 & jer. \\
\hline 2נJ & 121.0 \\
\hline 200 & 123.6 \\
\hline 200 & $12 \cos$ \\
\hline$\angle 00$ & $12 t .0$ \\
\hline 200 & $1<t .0$ \\
\hline 200 & $1 \% 0$ \\
\hline
\end{tabular}

t. $142.0 \quad .4 \quad 000$

o.

200

AIINUTH HEIGHT EXPOSURE F/O EU/A UISTANCE DIGRES MFTERS GH-SEC/CU,H

$\therefore 1111.0 \quad .4 \quad 000.0$

0. 200

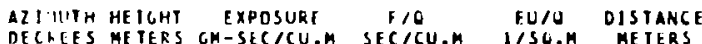

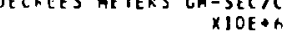

\begin{tabular}{|c|c|c|c|c|c|}
\hline (I) 118.0 & .4 & 147 & $1.4 H O E+06$ & $3.867 t-06$ & 200 \\
\hline C' 114.11 & .8 & 91 & $\forall .1<<E-0>$ & $2.372 E-06$ & 200 \\
\hline $411,1.0$ & 1.5 & 73 & $7.345 t-07$ & $1.915 t-06$ & 200 \\
\hline 114.6 & 2.3 & $i$ & $1.672 t-04$ & $4.216 E-U 8$ & 200 \\
\hline 119.0 & 4.0 & 27 & $2.716 E-07$ & $7.062 t-07$ & 200 \\
\hline 114.0 & 5.5 & 3 & $3.37 \cup[-O B$ & $8.704 t-08$ & 200 \\
\hline $11 \therefore .1$ & 7.6 & B & $8.514 \mathrm{E}-48$ & $2.214 t-07$ & 200 \\
\hline 112.0 & d. 5 & 7 & $7.703 \mathrm{E}-0 \mathrm{~B}$ & $2.003 \mathrm{E}-\mathrm{U} 7$ & 200 \\
\hline $11 . .0$ & 10.0 & 0 & 0 . & u. & 200 \\
\hline $11 p \cdot c$ & 13.0 & 0 & 0. & 0. & 200 \\
\hline $11 \div 0$ & 16.2 & 0 & 0. & 0. & $\angle 00$ \\
\hline $11 n .0$ & 14.0 & 1 & $1.757[-08$ & $4.56 \mathrm{dE}=0 \mathrm{U}$ & 200 \\
\hline 111.8 & 22.0 & 4 & $4.73 \cup[-] 6$ & $1.230 t-07$ & 200 \\
\hline 1$] H .0$ & 25.0 & 27 & $2.716 E-\cup 7$ & $7.062 t=07$ & 200 \\
\hline $11 \% .0$ & 28.0 & 57 & $5.743 E-07$ & $1.493 \mathrm{E}-06$ & 200 \\
\hline 1$)^{\mu} .0$ & 31.0 & 38 & $3.451 E-C 7$ & $1.001 t-06$ & iou \\
\hline 110.0 & 32.8 & 61 & $0.149 E-07$ & $1.599 k-06$ & 200 \\
\hline
\end{tabular}

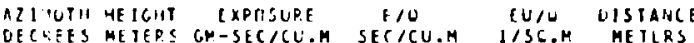
OECLEES METERS CP-SEC/CL.M SETICU.M I/SG.M METLRS
XIUPOE

$12 \mathrm{i}$.

.4
. .5
1.5
2.5
4.0
5.5
7.0
10.5
17.0
13.0
16.0
19.0
22.0
23.0
29.0
31.0
32.8

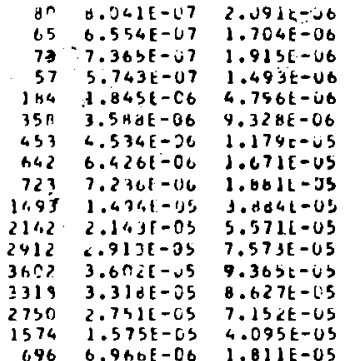

200
200
200
200
200
200
200
200
200
200
200
200
200
200
200
200
200

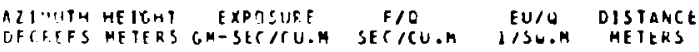

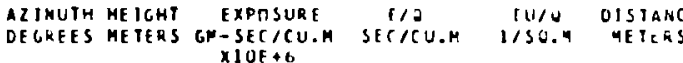

\begin{tabular}{|c|c|c|c|c|}
\hline 134.0 & .4 & 7603 & $4.936 E$ & $1.203 L-U 5$ \\
\hline 134.0 & .8 & 3057 & $2.0345-06$ & $5.360 t-60$ \\
\hline 134.0 & 1.5 & 4574 & $310506-06$ & $7.4305-06$ \\
\hline 134.0 & 2.5 & 6013 & $4.3 \cap y-0 b$ & $1.04<t-$ - 5 \\
\hline & 4.0 & $1171 \mathrm{~h}$ & $7.011 k-i 0$ & $2.0 \Delta I E-C+5$ \\
\hline 134.0 & 5.5 & 21230 & $1.415 t-05$ & $3.6 H U E-O S$ \\
\hline 134.0 & 7.1 & $290<4$ & $1.930 t-c 5$ & $5.038 \mathrm{t}-05$ \\
\hline 134.0 & 0.5 & 44941 & $2.494 t-03$ & $7.797:-05$ \\
\hline 134.0 & 10.0 & 54724 & $3.648 t-05$ & $9.6001-65$ \\
\hline 134.0 & 13.0 & 79650 & $5.31 \cup E-35$ & $1.381 t-64$ \\
\hline 134.0 & 16.0 & 121053 & $a .07 \cup[-0\}$ & $2.09 \mathrm{tE}-04$ \\
\hline 134.0 & 19.0 & 50246 & $3.3501-63$ & $8.749[-03$ \\
\hline 134.0 & 22.0 & 218714 & $1.458 t-04$ & $3.791 \mathrm{t}-04$ \\
\hline 134.0 & 25.0 & 314494 & $2.096 E-04$ & $3.451 E-04$ \\
\hline 134.0 & 20.0 & 176285 & $1.175 E-i 6$ & $3.056 t-04$ \\
\hline 134.0 & 31.0 & 183837 & $1.276 t-04$ & $3.163 t-j 4$ \\
\hline 134.0 & 32.8 & 201905 & $1.346 E-U 4$ & $3.500 \mathrm{E}-04$ \\
\hline
\end{tabular}

200
200
200
200
200
200
260
200
200
2100
200
200
200
200
200
200
200
134.5
114.0
134.0
134.6
134.0
134.0
134.1
134.0
134.0
134.1
134.0
134.0
136.0
134.0
134.0
134.0
134.0

$134 . \mathrm{C}$

.3
.8
1.5
2.5
4.0
5.5
7.9
9.5
13.0
13.0
16.0
19.0
22.0
25.0
28.0
31.0
32.0

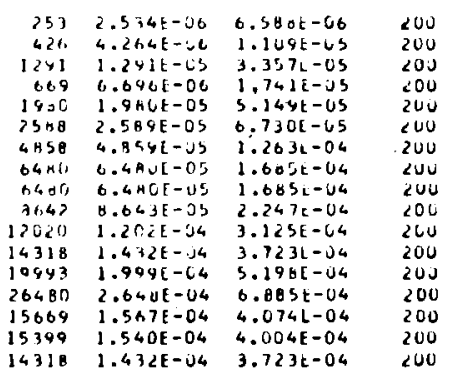


TOWER DATA F DLLOW....

IEST VG DECEMBER 12, 1972 1069 1O 1120 OST DOOM SULFIDE RELEASE FROM ELFVATIUN OF $26 \mathrm{G}$

ALIMUIH HEJGHT EXPOSURE T/O EU/O DISTANCE DEGRES METERS CN-SEC/CU.M SEC/CU.A IISO.M METERS

$\begin{array}{llllll}N 105.0 & .3 & 0 & 0 . & 0 . & 020\end{array}$

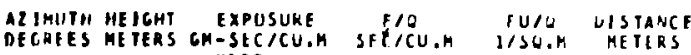
$\times 10 \mathrm{E}+6$

$\begin{array}{llllll}112.6 & .3 & 0 & 0 . & 033\end{array}$

AZIMUTH HEJGHT EXPOSURF FIO EU/L DISTANCE DECKEES METERS GA-SEC/CU.M SEC/CU.H ISU.M METERS

\begin{tabular}{|c|c|c|c|c|}
\hline $\begin{array}{l}120.2 \\
120.2\end{array}$ & .3 & $\begin{array}{l}218 \\
133\end{array}$ & $\begin{array}{l}1.454 E-07 \\
0.896 E-08\end{array}$ & $\begin{array}{l}3.7 \forall \angle E-C 7 \\
2.313 E=07\end{array}$ \\
\hline 120.2 & 1.1 & 139 & $9.2901-0 \mathrm{E}$ & $2.415 t-07$ \\
\hline 120.2 & 1.5 & 174 & $1.163 \mathrm{E}-07$ & $3.024 t-07$ \\
\hline 120.7 & 2.1 & 213 & $1.471 \mathrm{E}-07$ & $3.694 E-07$ \\
\hline 120.2 & 4.2 & 229 & $1.53 C E-07$ & $3.978 t-J 7$ \\
\hline 120.2 & 6.3 & 277 & $1.65 C E-07$ & $4.811 \mathrm{t}=07$ \\
\hline 120.2 & B. 4 & 158 & $1.053 E-07$ & $2.743 \mathrm{E}-07$ \\
\hline 120.2 & 10.5 & 181 & $1.212 \mathrm{E}-07$ & $3.151 t-67$ \\
\hline 120.2 & 12.6 & 70 & $4.713 E-08$ & $1.225 \mathrm{t}-67$ \\
\hline $1>0.2$ & 14.7 & 129 & $8.6 \cap 4 E-08$ & $2.237 \mathrm{E}-07$ \\
\hline 120.2 & 16.0 & 106 & $7.111 E-C \theta$ & $1.049 \mathrm{E}-07$ \\
\hline 120.2 & 21.0 & 105 & $7.034 \mathrm{E}-0 \mathrm{~B}$ & $1.830 E-07$ \\
\hline 120.2 & 25.2 & 86 & $5.766 E-0 B$ & $1.444 \mathrm{E}-07$ \\
\hline 120.2 & 29.6 & 32 & $2.155 \mathrm{E}-0 \mathrm{~d}$ & $5.602 t-08$ \\
\hline 120.2 & 33.6 & 44 & $\angle .970 E-0 B$ & $7.721 k-08$ \\
\hline & 37.8 & 16 & $1.1055-08$ & $2.873 E-08$ \\
\hline 1 & 42.0 & & 9 & $82 t=$ \\
\hline
\end{tabular}

$$
\begin{aligned}
& 845 \\
& 845 \\
& 045 \\
& 045 \\
& 845 \\
& 845 \\
& 045 \\
& 045 \\
& 045 \\
& 845 \\
& 045 \\
& 145 \\
& 645 \\
& 845 \\
& 045 \\
& 845 \\
& 845 \\
& 145
\end{aligned}
$$

AIIHUTIS HEJGH

EXPOSURE

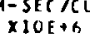

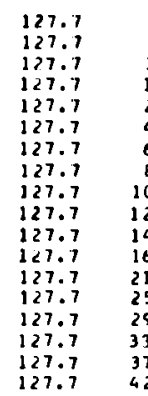

.3
1.5
1.5
2.1
4.2
6.3
8.4
10.5
12.6
14.7
16.8
21.0
25.2
29.4
33.8
37.8
42.0
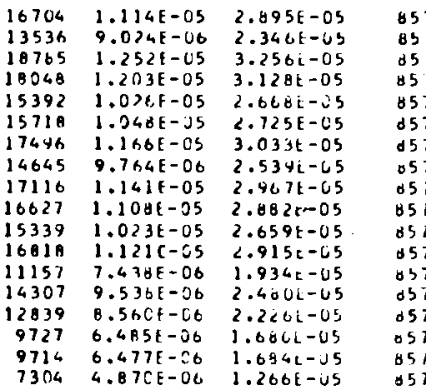

857
851
857
857
857
057
057
857
857
851
857
857
857
857
057
057
851
857

AI MUTA HEICHT

7304 $4.87 C E=06 \quad 1.266 L_{-}-25$
TOWE R DATA FIILLOW....

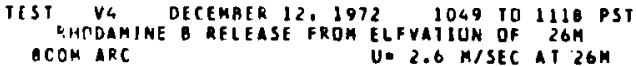

A2 JMUTH HEICHT EXPTSURE E/O EU/O DISIANCE DECPEES METERS GM-SECICU.A SEC/CU.M IISU.M METEKS
XIUFOG

N $105.0 \quad .3 \quad 000$.

$0 . \quad 020$

ALIMUTH HFIGHT EXPUSURE F/O EU/O UISTANCE OECIIES METERS GM-SEC/CU.M SEC/CU.M I/SO.M NETERS

N 112.6 $.3 \quad 00$.

0.

433

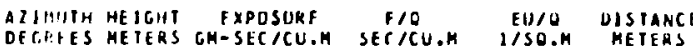
$X 1 O H+6$
$X$

120.2
120.2
120.2
120.2
120.2
120.2
120.2
120.2
120.2
120.2
120.2
$1<0.2$
120.2
120.2
122.2
120.2
120.2
120.2

.3
.5
1.1
1.5
2.1
4.2
6.3
8.5
10.5
12.6
14.7
16.8
21.0
25.2
29.4
33.6
37.8
42.0

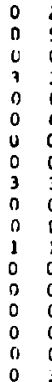

$2.614 E-09$
$5.274 E-09$
0.
$3.660 E-00$
0.5
$6.536 E-09$
0.
0.
$3.399 E-08$
0.
0.
$1.046 E-00$
0.
0.
0.
0.
0.

$6.797 t-09$
$1.359 t-08$

0 .

$9.516 E-00$

$1.699 \mathrm{E}-0 \mathrm{O}$

0

B.837E-00

0.

$2.719 E-08$

0 .

0 .

0.

$1.0201-08$

AIIMUTH HEIGHT EXPOSURF F/O EUIO OISTANCE DFCKEES METERS CH-SEC C
X10E+b

\begin{tabular}{|c|c|c|c|c|}
\hline $1<7.7$ & .3 & 1029 & $1.030 \mathrm{t}-05$ & $2.677 k-0.5$ \\
\hline 127.7 & 1.5 & 1487 & $1.487 E-05$ & $3.867 t-05$ \\
\hline 127.7 & .5 & 1199 & 1.2 ULE-05 & $3.114 i-05$ \\
\hline 127.7 & 1.1 & 1565 & $1.566 \mathrm{E}-05$ & $4.071 \mathrm{E}-05$ \\
\hline 127.7 & & 1369 & $1.37 \cup E-05$ & $3.561 \mathrm{k}=0 \mathrm{~b}$ \\
\hline 127.7 & 4.2 & 1252 & $1.252 \mathrm{E}-05$ & $3.255 E-05$ \\
\hline 127.7 & 6.3 & 1291 & $1.291 \mathrm{E}=05$ & $3.357 t-05$ \\
\hline $1 \geqslant 7.7$ & 8.4 & 1016 & $1.017 E-J 5$ & $2.644 E-05$ \\
\hline 127.7 & 10.5 & 1147 & $1.147 \mathrm{E}-\mathrm{CS}$ & $2.983 E-05$ \\
\hline 127.7 & 12.6 & 1082 & 1.0 2E -05 & $2.813 t-05$ \\
\hline 127.7 & 14.7 & 1042 & $1.0 \mathrm{HE}-0 \mathrm{~S}$ & $2.813 E-05$ \\
\hline 127.7 & 16.8 & 964 & $4.644 E-06$ & $2.506 \mathrm{k}-05$ \\
\hline 127.7 & 21.0 & 624 & $6.246 E-06$ & $1.624 \mathrm{E}-05$ \\
\hline $1 ? 7.7$ & 25.7 & 742 & $1.472 \mathrm{E}-06$ & $1.930 E-05$ \\
\hline 127.7 & 29.4 & 585 & $5.856 E-J 6$ & $1.52<E-05$ \\
\hline $1 \geqslant 7.7$ & $33, t$ & 369 & $3.697 t-06$ & .06 \\
\hline 127.7 & 37.1 & 350 & $3.501 t-60$ & $9.102 z-06$ \\
\hline & & 4 & $2.050 E-06$ & \\
\hline
\end{tabular}

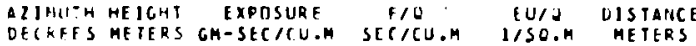

$\begin{array}{ll}135.0 & .3 \\ 135.0 & .5 \\ 135.0 & 1.1 \\ 135.0 & 1.5 \\ 135.0 & 2.1 \\ 135.0 & 4.2 \\ 135.0 & 6.3 \\ 135.0 & 8.4 \\ 135.01 & 10.5 \\ 135.0 & 12.6 \\ 135.0 & 14.7 \\ 133.0 & 16.0 \\ 135.0 & 21.0 \\ 135.0 & 25.2 \\ 135.0 & 29.0 \\ 135.0 & 33.6 \\ 135.0 & 37.0 \\ 135.0 & 42.0\end{array}$

.3
1.5
1.5
2.1
4.2
6.3
10.4
10.5
12.6
14.7
16.8
21.0
23.2
29.4
33.6
37.9
42.0
$x 10 E+1$

\begin{tabular}{|c|c|c|c|c|}
\hline 9.0 & .3 & 4359 & $2.90 \mathrm{BE}-06$ & $6[-96$ \\
\hline & .5 & 629 & $4.195[-07$ & $i-0 e$ \\
\hline 135.0 & 1.1 & 4322 & $2.0 H 2[-06$ & $7.44<0-36$ \\
\hline 135.0 & 1.5 & 4309 & $2.079 E-06$ & $7.470 t-06$ \\
\hline 135.0 & 2.1 & 5126 & $3.41+1-80$ & a.88GL-U6 \\
\hline & 4. & 033 & $4.022 \mathrm{E}-06$ & 1.U4GE-US \\
\hline 135.0 & 6.3 & 7073 & $4.715 E-00$ & $1.226 L-05$ \\
\hline 135. & & $719 \mathrm{~A}$ & $4.799 t-66$ & $1-05$ \\
\hline 135.0 & 10.5 & 6979 & $4.653 \mathrm{E}-08$ & $1.210 k-05$ \\
\hline 135.0 & 12.6 & 9686 & $6.457 E-06$ & $1.679 \mathrm{~b}-05$ \\
\hline 135.0 & 14.7 & 8535 & $5.691 \mathrm{E}-00$ & $2.480 E-05$ \\
\hline 135.0 & 26.8 & 9160 & $6.107 \mathrm{E}-06$ & $1.58 \Delta t-05$ \\
\hline 135.0 & 21.0 & 10270 & $6.847 E-06$ & $1.780 t-05$ \\
\hline 135.0 & 25.2 & 8599 & $5.733 E-06$ & $1.491 \bar{c}=05$ \\
\hline 135.0 & 29.4 & 7636 & $5.0911-00$ & $1.324 t-65$ \\
\hline 135.0 & & A285 & $5.524 E-06$ & $1.4361-05$ \\
\hline 35.0 & 37.8 & 9177 & e.21 UE-06 & $1.591 \mathrm{c}-0 \mathrm{~s}$ \\
\hline 35 & 43 & 7797 & $5.190 E-06$ & $1.352 \mathrm{E}-05$ \\
\hline
\end{tabular}

667
867
067
867
067
067
867
067
867
667
867
067
867
067
067
867
067
067

135.0 
TQWER QATA FULLUH....

TEST V4 DECEHGER 12, $1972^{\circ} 1049$ 1U 1120 PST ZIINC SULFIOE RELEASE FRDM ELFYATIUN OF $26 \mathrm{M}$ $1600 M$ ARC U. $2.6 \mathrm{H} / \mathrm{SEC}$ AT $26 \mathrm{~N}$

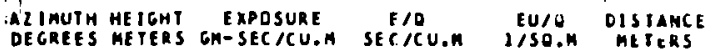
$\times 10 E+6$

* $101.6 \quad .210001617$

AZIMUTH HEIGKT EXPOSURE E/O EU/A DISTANCE DECKEES METERS GH-SEC/CU.M SEC/CU.M IISO.M METEKS

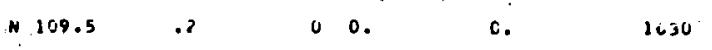

AZIMUTH HEIGHT EXPOSURE H/O EU/O OISIANCE DEGKES METERS CM-SEC/CU.N SEC/CU.M I/SO.K NETEKS

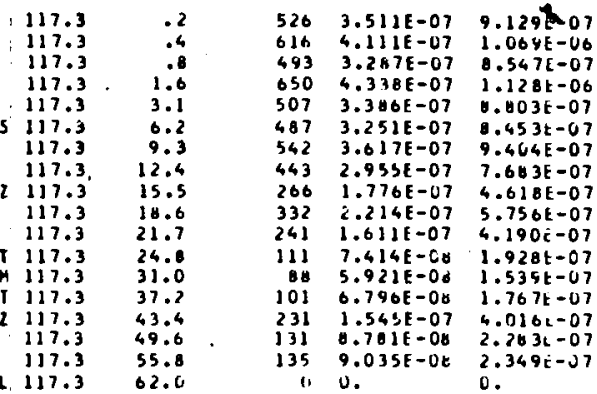

1643

1643

104

1643

164

1043

1043

1043
1043
1643

1043
1643
1043

1443

1043

1043

1043

AZIMUIM HEIGHT EXPQSURE F/O EU/U UISTANCE OEGHEES METERS GM-SECICU.M SEC/CU.M I/SG.M METCRS

\begin{tabular}{|c|}
\hline $\begin{array}{l}125.0 \\
125.0 \\
125.0 \\
125.0 \\
125.0 \\
125.0 \\
125.0 \\
125.0 \\
125.0 \\
125.0 \\
125.0 \\
125.0 \\
125.0 \\
125.0 \\
125.0 \\
125.0 \\
125.0 \\
1.5 .0\end{array}$ \\
\hline
\end{tabular}
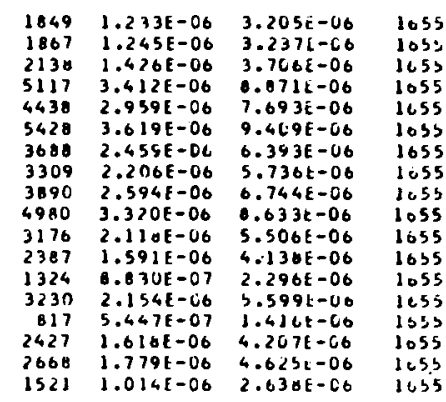

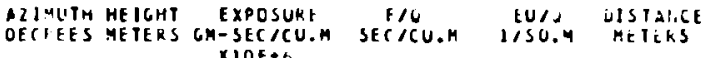

\begin{tabular}{|c|c|c|c|c|c|}
\hline 132.5 & .8 & $\begin{array}{l}3416 \\
3622\end{array}$ & $\begin{array}{l}2.544 F-06 \\
2.615 E-06\end{array}$ & $\begin{array}{l}6.615 \mathrm{~b}=06 \\
6.27 \mathrm{~g}_{\mathrm{r}}=06\end{array}$ & $\begin{array}{l}1,05 \\
1665\end{array}$ \\
\hline 132.5 & .0 & 4708 & $3.192 \mathrm{E}-06$ & $\begin{array}{l}0.200=06 \\
8.300 \bar{c}-60\end{array}$ & $\begin{array}{l}1005 \\
10<5\end{array}$ \\
\hline 132.5 & 1.6 & 4561 & $3.041 E-06$ & $7.957 \mathrm{c}-0 \mathrm{D}$ & joes \\
\hline 132.5 & 3.1 & 3677 & $2.585 E-0 b$ & $0.72 E E-06$ & 1005 \\
\hline 132.5 & 0.2 & 3770 & $2.516 \mathrm{f}-0 \mathrm{t}$ & $0.535 E-06$ & 1065 \\
\hline $\begin{array}{l}132.5 \\
132.5\end{array}$ & $\begin{array}{r}9.3 \\
12.4\end{array}$ & $\begin{array}{l}2670 \\
4077\end{array}$ & $\begin{array}{l}1.7616-06 \\
2.71 b E-06\end{array}$ & $\begin{array}{l}4.624 E-C D \\
7.067 E-06\end{array}$ & $\begin{array}{l}1005 \\
1005\end{array}$ \\
\hline 192.5 & 15.5 & 2984 & $1.989 \mathrm{E}-06$ & $5.172 \mathrm{E}-6 \mathrm{G}$ & loos \\
\hline 132.5 & 10.0 & 2543 & $1.696 t-06$ & $4.404 t-v e$ & 1065 \\
\hline 132.5 & 21.7 & 3280 & $2.1 A 7 t=06$ & $5.606 t=06$ & docs \\
\hline 132.5 & 24.8 & $37 \mathrm{Cb}$ & $2.471 E-06$ & $0.424 E-60$ & 1005 \\
\hline 132.5 & 31.0 & 1463 & $9.756 f-67$ & $2.537 t-06$ & 1665 \\
\hline 132.5 & 37.2 & 2414 & $1.612 E-06$ & $4.191 t-06$ & icos \\
\hline 132.5 & .4 & 2297 & $1.53<t-66$ & $3.9 d<i-06$ & lues \\
\hline 132.5 & & 1826 & $1.210 t-100$ & $3.166:-0 t$ & lues \\
\hline $\begin{array}{l}132.5 \\
132.5\end{array}$ & $\begin{array}{l}55.8 \\
62.0\end{array}$ & 995 & $\begin{array}{l}0.636 L-07 \\
0.7395-07\end{array}$ & $1.725 t-06$ & Wo's \\
\hline
\end{tabular}

Inito LATA FOLLOW....

TEST VU DECEMES 12,1972 1044 TU 2118 PST 1600 TRC $U$. $6.6 \mathrm{~K} / \mathrm{SEC}$ AT $26 \mathrm{~A}$

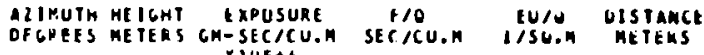

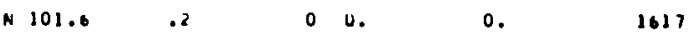

AZIMUTH HEIGHT EXPOSUAF E/O FU/U OISIANCE

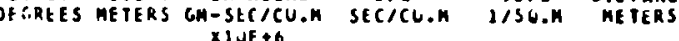

$\begin{array}{llll}N 109.3 & .2 & 0 & 0\end{array}$

o.

1630

ALIAUTH AEIGHT EXPOSURE T/O EUAN UISTANCE DE ENEES ME IERS GH-SEC/CU.N SEC/CU.N IJSO.K METERS

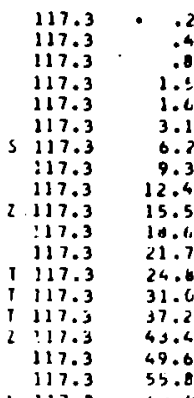

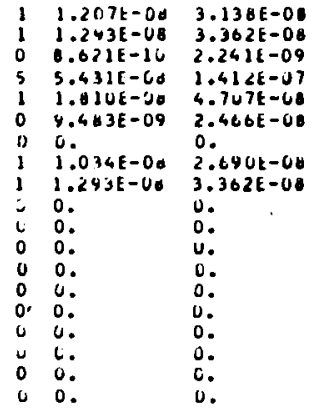

2643

1643

1643

1643

1043

1043

1643

1643

1043

64

1040

1643

1043

1043
1643

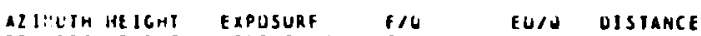

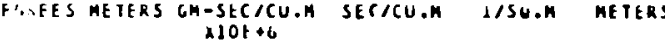

\begin{tabular}{|c|c|c|c|c|c|}
\hline $\begin{array}{l}125.0 \\
1.5 .0\end{array}$ & $\begin{array}{l}.2 \\
.4\end{array}$ & $\begin{array}{l}140 \\
145\end{array}$ & $\begin{array}{l}1.901 E-06 \\
1.955 t=06\end{array}$ & $\begin{array}{l}4.949 k-06 \\
5.003 k-06\end{array}$ & \\
\hline iasov & . A & 192 & $1.924 E-00$ & $5.016 E-06$ & \\
\hline 125.2 & 1.5 & 165 & $1.652 \mathrm{E}-06$ & $4.014 i-06$ & \\
\hline 125.0 & 1. & 479 & $4.7916-06$ & $1.246 E-05$ & \\
\hline $1<5.6$ & 3.1 & 461 & 4.CTYE-C6 & $1.201 E-05$ & \\
\hline 225.0 & $c .2$ & $4<7$ & $4.274 E-66$ & $1.1111-05$ & \\
\hline 123.0 & 9.3 & 289 & $2.095 t-i 6$ & $7.5 \div 7 c-06$ & \\
\hline $1 / 5.6$ & 12.4 & 255 & $2.55 U E-36$ & $c .630 t-06$ & \\
\hline $1<5.0$ & 15.5 & 3114 & $3.843 E-30$ & $9.49<E-00$ & \\
\hline 123.6 & 18.6 & 336 & $3.5 \times 4 E-06$ & $4.320 \mathrm{~L}=06$ & \\
\hline 1. 205.0 & 21.7 & 272 & $6.72<t-46$ & $7.07 \& t-06$ & \\
\hline 124.0 & 24.0 & 179 & $1.000 E-00$ & $4.600 t-06$ & \\
\hline 125.0 & 31.0 & 48 & $4.810 t-07$ & $1.251 t-66$ & \\
\hline 123.0 & 37.2 & 296 & $2.9 A+1-36$ & $7.75 \mathrm{BE}-20$ & \\
\hline 125.0 & 49.4 & 35 & $3.517 E-07$ & $9.145 c-07$ & \\
\hline 3.0 & & 187 & $1.87 \pm[-06$ & $4.402 t=00$ & \\
\hline $1<3.0$ & $55 . \mathrm{H}$ & 172 & $1.72<1-46$ & $4.47 \Delta t=06$ & \\
\hline 1.5 .0 & $62 \cdot 6$ & $1<1$ & $1.231 t-46$ & $3.201 t-06$ & \\
\hline
\end{tabular}

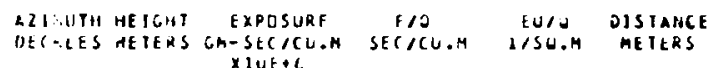

\begin{tabular}{|c|c|c|c|c|c|}
\hline 132.5 & .2 & 242 & $a .421 t-0 b$ & $6.244 \mathrm{E}-06$ & 1665 \\
\hline 132.5 & .4 & 255 & $2.5 \mathrm{~s}, \mathrm{t}-\mathrm{Ct}$ & $0.63 j t-16$ & 1665 \\
\hline+32.5 & .8 & 236 & 2.3este-jo & $0.154 E-0 C$ & 1605 \\
\hline $\begin{array}{l}132.5 \\
132.5\end{array}$ & $\begin{array}{l}1.5 \\
1.0\end{array}$ & $\begin{array}{l}323 \\
289\end{array}$ & $\begin{array}{l}3.24 C E-06 \\
2.0 \times 5 E-j 6\end{array}$ & $\begin{array}{l}6.463 c-06 \\
7.527 k-06\end{array}$ & $\begin{array}{l}1665 \\
1665\end{array}$ \\
\hline$: 32.5$ & 3.1 & 263 & $2.63 C E-16$ & $6.0341=06$ & 1065 \\
\hline $1: 2$ & a.e. & 255 & $2.55 \mathrm{U} E-J 6$ & $6.62 \mathrm{Jt}-06$ & 1665 \\
\hline 132.5 & 9.3 & 190 & $1.91) 1 i-v e$ & $4.749 t-66$ & 1065 \\
\hline 132.5 & 12.4 & $1 \times 5$ & $1.91,51-60$ & $5 . c_{0} 3 t-06$ & 1005 \\
\hline 132.5 & 15.5 & 192 & $1.4>4 E-06$ & $5.016 \mathrm{E}-60$ & 1065 \\
\hline 132.5 & 10.6 & 164 & $1.645 t-06$ & $4.277 t-06$ & 1665 \\
\hline 132.5 & 21.7 & 234 & $2.343 E-56$ & $0.052 t-46$ & 1005 \\
\hline & 24.8 & 234 & $2.343 E-00$ & $0.04 \angle E=06$ & 1065 \\
\hline 132.8 & 31.0 & 01 & $0.17<t-111$ & $2.1<3 t-60$ & 1665 \\
\hline $13<.3$ & 37.6 & 164 & $1.04 \div 1-00$ & 4.257L-Ue & $106 \%$ \\
\hline $13 / .3$ & 43.4 & 174 & $1.740 t=00$ & $4.546 k=06$ & $16 \mathrm{~F}$ \\
\hline 137.5 & 49.6 & 141 & $1.41<E-60$ & $3.671 \mathrm{k}-06$ & 1608 \\
\hline & & 79 & $7.914 t-47$ & $2.058 t-00$ & 1005 \\
\hline & & 7 & & & \\
\hline
\end{tabular}


TUMER DATA FOLLOW....

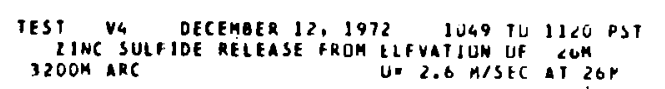

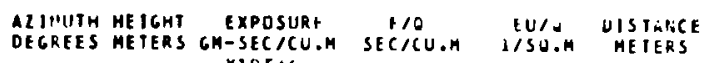

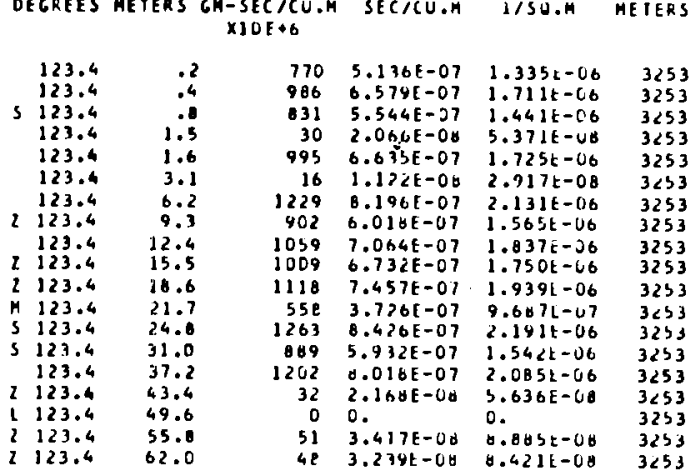

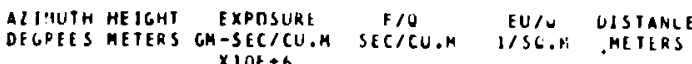

$\begin{array}{rrr}\text { E } 131.2 & .2 \\ 131.2 & .4 \\ 131.2 & .0 \\ 131.2 & 1.6 \\ 131.2 & 3.1 \\ 1131.2 & 0.2 \\ & 131.2 & 9.3 \\ L & 131.2 & 12.4 \\ & 131.2 & 15.5 \\ 2 & 131.2 & 18.6 \\ 2 & 131.2 & 21.7 \\ 2 & 131.2 & 24.8 \\ 2 & 131.2 & 31.0 \\ 7 & 131.2 & 37.2 \\ 2 & 131.2 & 43.4 \\ 2 & 131.2 & 49.6 \\ 2 & 131.2 & 55.8 \\ 2 & 131.2 & 62.0\end{array}$

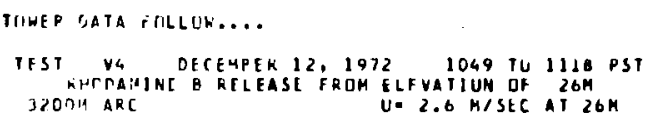

TIHEP EATA TILLLUK...

TFST V4 DECEMPEK 12,1972 1049 TU 1110 PST

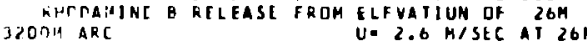

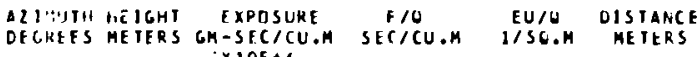

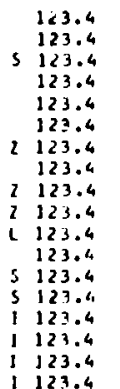

.2
.4
.8
1.6
1.1
6.2
9.3
12.4
13.5
10.6
21.7
24.8
31.0
37.7
43.4
49.6
55.8
62.0

$$
\begin{array}{r}
100 \\
94 \\
76 \\
104 \\
9 \\
97 \\
60 \\
86 \\
75 \\
100 \\
84 \\
61 \\
83 \\
0 \\
0 \\
1 \\
0
\end{array}
$$$$
\begin{array}{rll}
100 & 1.001 E-06 & 2.603 \mathrm{E}-06 \\
94 & 9.427 \mathrm{E}-07 & 2.451 \mathrm{E}-06 \\
76 & 7.673 \mathrm{E}-07 & 1.995 \mathrm{E}-06 \\
06 & 1.046 \mathrm{E}-06 & 2.725 \mathrm{E}-06 \\
0 & 0.0 & 0 . \\
97 & 9.778 \mathrm{E}-07 & 2.542 \mathrm{E}-06 \\
60 & 6.035 \mathrm{E}-07 & 1.569 \mathrm{E}-06 \\
86 & 8.608 \mathrm{E}-07 & 2.238 \mathrm{E}-06 \\
75 & 7.556 \mathrm{E}-07 & 1.964 \mathrm{E}-06 \\
00 & 1.001 \mathrm{E}-06 & 2.603 \mathrm{E}-06 \\
0 & 0 . & 0 . \\
84 & 0.491 \mathrm{E}-07 & 2.208 \mathrm{E}-06 \\
61 & 6.152 \mathrm{E}-07 & 1.600 \mathrm{E}-06 \\
83 & 0.374 \mathrm{E}-07 & 2.177 \mathrm{E}-06 \\
0 & 0.0 & 0 . \\
0 & 0 . & 0 . \\
1 & 0 . & 0 . \\
0 & 0 . & 0 .
\end{array}
$$

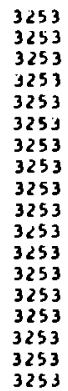

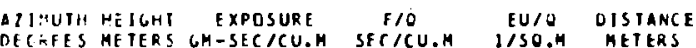

$$
\text { EXPOSURE FIO }
$$$$
\text { Evera }
$$

3253 $-S E C / C U$
$X 1 O F+6$

111.2
131.2
131.2
131.2
131.2
131.2
131.2
131.2
131.2
131.2
131.2
1131.2
1131.2
1131.2
1131.2
1131.2
1311.2
1313.2

.2
.1 .8
1.8
3.1
6.2
9.3
12.4
15.5
18.6
21.7
24.8
31.0
37.2
43.4
49.6
55.8
62.0$$
00 .
$$$$
0.0
$$$$
0 .
$$$$
\text { 4. } 136 t-07 \quad 3<64
$$$$
\text { l. } 1.591 t-07 \quad 4.136 t-07 \quad 3264
$$$$
130 \quad 1.309 E-06 \quad 3.404 E=06 \quad 3264
$$$$
127 \quad 1.270 E-06 \quad 3.3025-06 \quad 3264
$$$$
119 \quad 1.192 E-06 \quad 3.100 E-06 \quad 3264
$$$$
\text { 14? } 1.476 \text { E-06 } 3.708 E-06 \quad 3264
$$$$
0 \text { 0. } 0.004
$$$$
123 \quad 1.231 E-06 \quad 3.201 E-66 \quad 3264
$$$$
130 \quad 1.309 \mathrm{E}-06 \quad 3.404 \mathrm{E}-06 \quad 3264
$$$$
00 \text {. }
$$$$
\begin{array}{ll}
0 & 0 . \\
0 & 0 .
\end{array}
$$$$
00 .
$$$$
2 \quad 2.690 \mathrm{E}-08 \quad 6.994 E-00
$$$$
\text { Q. } 9.357 E-09
$$$$
0 \text { : } 0 \text { : }
$$

3204

3264

3264 3264 3264 
GROUND LEVEL AND TOWER SAMPLINC PGOM IO 3ZUUM. ALL ALCS EMUKACE CRUSSHIND EXIENT DF UISTRIBUTION.

16 TOMERS "HIT" BY TRACER: ALTHUUEH DISIRIGUIJUNS TRUNCATEU AT TOPS UF TOHERS. EXTRAPOLATIUN HOULO BE

REASDHABLE. VERTICAL CROSS SESTILNS IIIROUGH TOWER AKCS SHUWS SIGNIFICANT OIRECTION SHEAR UF TRACERS WITH HIT.

TEST VS SEPTENBER 5, 1973 USOI TU 0531 PST

ZINC SULFIDE RELEAST FRDM CLFVATIUN DF $26 \mathrm{M}$

$200 M$ ARC SAMPLER HT $1.514 \quad 11=4.3$ HISHC AT $26 M$

AZIMUTH EXPOSURE E/U EJ/O UISTANCE

DEGREES CM-SEC/CU.M SFC/CU.H DISU.M MEIEKS $X \perp O E+6$

\begin{tabular}{|c|c|c|c|c|c|}
\hline & 16.0 & c & 0. & 6. & ¿Uu \\
\hline & $\begin{array}{l}06.0 \\
38.0\end{array}$ & $\begin{array}{l}124 \\
296\end{array}$ & $\begin{array}{l}6.959 t-v t \\
1.515,5-v 7\end{array}$ & $\begin{array}{l}\text { C. } \$>t E-07 \\
6.513 E-07\end{array}$ & 200 \\
\hline & 90.0 & 615 & $3.316 E-67$ & $1.4 \angle 6 E-60$ & <Ju \\
\hline & $\begin{array}{l}92.0 \\
94.0 \\
96.0\end{array}$ & $\begin{array}{l}1270 \\
1543 \\
2648\end{array}$ & $\begin{array}{l}6.850 t-07 \\
0.331 t-07 \\
1.4265-06\end{array}$ & $\begin{array}{l}2.940 E-00 \\
3.342 E-06 \\
0.140 E-06\end{array}$ & $\begin{array}{l}200 \\
200 \\
200\end{array}$ \\
\hline & $\begin{array}{r}98.0 \\
100.0\end{array}$ & $\begin{array}{l}1187 \\
2489\end{array}$ & $\begin{array}{l}6.4301-i 7 \\
1.342 \mathrm{E}-36\end{array}$ & $\begin{array}{l}2.71,2 E-U C \\
5.77 \& E-06\end{array}$ & $\begin{array}{l}200 \\
200\end{array}$ \\
\hline & 102.0 & 1468 & $1.061 t-U_{6}$ & $4.562 E-06$ & 200 \\
\hline & 104.0 & 3750 & $2.0265-06$ & $0.71 \triangle E-06$ & 200 \\
\hline & 106.0 & 3542 & $1.410 F-66$ & $3.212 E-U 6$ & $<\cup 0$ \\
\hline & $10 H .0$ & 1946 & $1.049[-06$ & $4.512 \mathrm{~L}-06$ & 200 \\
\hline & $\begin{array}{l}110.0 \\
112.0\end{array}$ & $\begin{array}{l}2128 \\
1247\end{array}$ & $\begin{array}{l}1.149 E-66 \\
6.727 E-67\end{array}$ & $\begin{array}{l}4.935 E-\cup 6 \\
2.093 E-06\end{array}$ & $\begin{array}{l}200 \\
200\end{array}$ \\
\hline & 114.0 & 636 & $3.437 t-07$ & $1.476 E-C 6$ & 200 \\
\hline $\mathbf{E}$ & 116.0 & 122 & $6.618 E-C 8$ & $2.846 \mathrm{t}-07$ & 200 \\
\hline & $11 \% .0$ & 43 & $2.320 E-0 B$ & $9.97 \pm[-60$ & 200 \\
\hline & 120.0 & 127 & $6.841 t-08$ & $\therefore 903 E-07$ & 200 \\
\hline & $\begin{array}{l}122.0 \\
124.0\end{array}$ & $\begin{array}{r}124 \\
11\end{array}$ & $\begin{array}{l}6.954 t-10 d \\
6.141 t-49\end{array}$ & $\begin{array}{l}2.992 E-07 \\
2.6 .40 E-08\end{array}$ & $\begin{array}{l}200 \\
200\end{array}$ \\
\hline H & 126.0 & ) & 0. & u. & 200 \\
\hline
\end{tabular}

CRISSSWINO INTEGPATIDE 9.7L1E-US 4.1 HUL-04

$$
\text { SrCISO. A }
$$

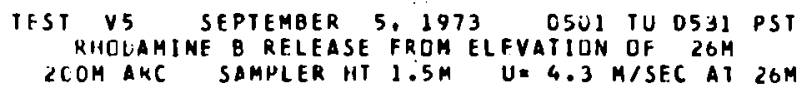
$\times 10 E+6$

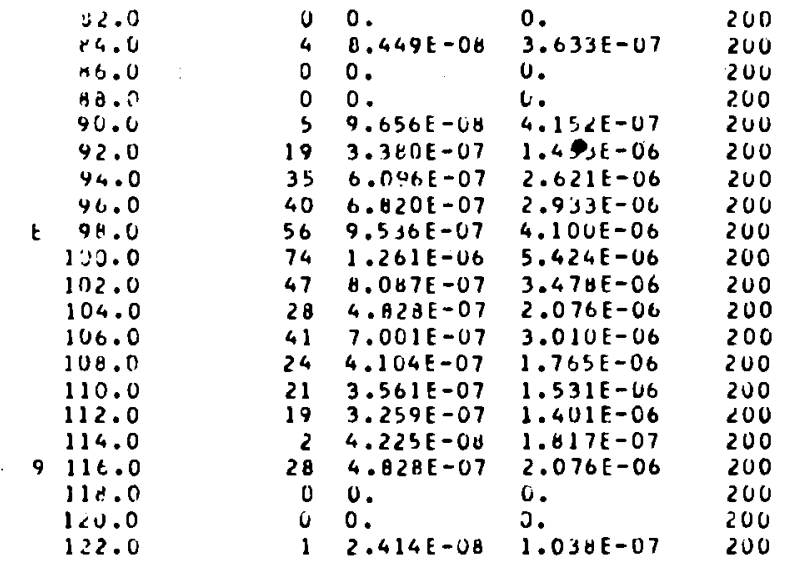

CRISSHIND INTECRATED $=5.347 E-05 \quad 2.294 E-04$

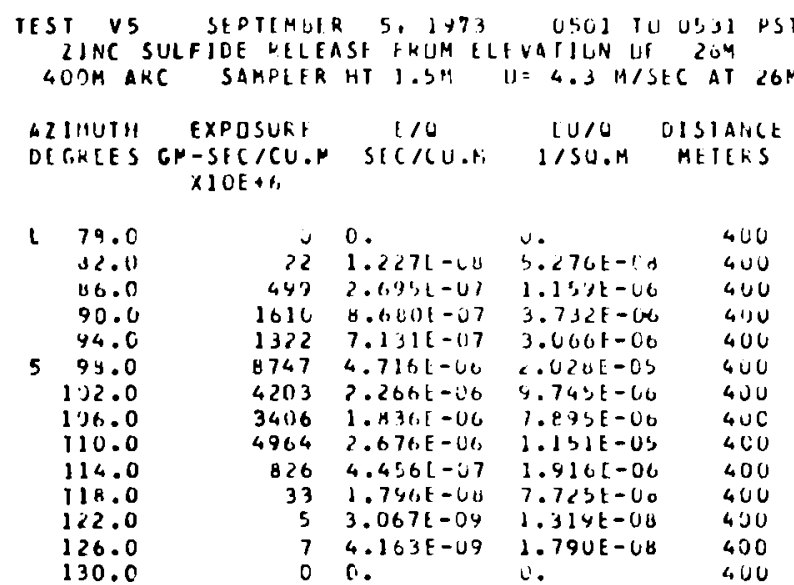

CROSSWIAD INTEGRATEO=3.801t-OG 1.66JE-03
TIST VS SEPTEMRER 5, 1973 OSUI TL 0S9I PST KIICDAMINE $O$ RELEASE FRUM LLFVATIUN UF COM $40 O M$ ARC SAMPLER HT $1.5 \mathrm{M} \quad \mathrm{U}=4.3 \mathrm{M} / \mathrm{SEC}$ AT $26 \mathrm{M}$

ALIIUTH EXPOSURE ETO LUIO DISTANCE DEGREES GM-SEC/CU.M SFCICU.M I/SU.M METERS $X 10 E+6$

L $\quad 7.0$

02.0

46.0

90.0

94.0

5.98 .0

102.0

106.0

110.0

114.0

116.0

$1<2.0$

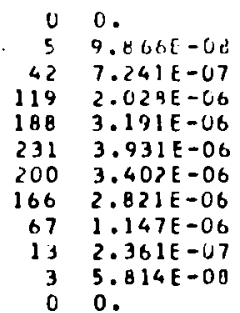

$4.2+2 t-07$

$3.114 \mathrm{E}-06$

$0.72 U E-06$

1. $372 E-03$

$1.690 \mathrm{E}-05$

$1.463 \mathrm{E}-05$

1. $213 E-05$

$4.932 \mathrm{E}-06$

$1.015 \mathrm{E}-\mathrm{U}$

2. $500 \mathrm{E}-0$

0 .

400

400

400

400

400
400

400

400

400

400

400

(ROSSHIND INTEGRATED $=4.925 E-04 \quad 2.116 E-03$ 


\begin{tabular}{|c|c|c|c|c|}
\hline $\begin{array}{l}\text { TEST VS } \\
\text { ZINC SI } \\
\text { OOH AR }\end{array}$ & $\begin{array}{l}\text { SFPTEMUE } \\
\text { SULFIDE RELE } \\
\text { RC SAMPLER }\end{array}$ & $\begin{array}{l}\text { SE } 1973 \\
\text { ASE FRUM E } \\
\text { HT } 1.34\end{array}$ & $\begin{array}{l}\text { CSOL TU } \\
\text { LFVATIUN HF } \\
U=4.3 \mathrm{~m} / \mathrm{SE}\end{array}$ & $\begin{array}{rl}\text { OS33 } & \text { PST } \\
264 & \\
S E C A T & 26 M\end{array}$ \\
\hline $\begin{array}{l}\text { AL IHUTH } \\
\text { DEGREES }\end{array}$ & $\begin{array}{c}\text { EXPOSURL } \\
\text { CM-SEC } / C U+H \\
\times 1 O E+6 .\end{array}$ & $\begin{array}{c}\text { L/L } \\
\text { SEC/CU.M }\end{array}$ & $\begin{array}{c}t \cup / 0 \\
1 / 56.4\end{array}$ & $\begin{array}{l}\text { DISTANCE } \\
\text { HEIEKS }\end{array}$ \\
\hline 77.0 & $\begin{array}{l}0 \\
0\end{array}$ & 0. & i. & 771 \\
\hline$\$ 1.1$ & 42 & $2.310 L-O B$ & $9.933 E-O B$ & 778 \\
\hline 03.1 & 274 & $1.47 \mathrm{HE}-07$ & $6.357 E-07$ & 701 \\
\hline 45.2 & 782 & $4.2176-07$ & $1.013[-00$ & 705 \\
\hline 47.2 & 1710 & $9.220 \mathrm{E}-07$ & $9.90\}[-06$ & 768 \\
\hline 49.2 & 3167 & $1.707 E-06$ & $7.3426-06$ & 792 \\
\hline 91.2 & 5477 & $2.453 t-66$ & $1.21 U E-U 5$ & 745 \\
\hline 93.2 & $6024^{\circ}$ & $3.250 \mathrm{t}-06$ & $1.3905-U b$ & 799 \\
\hline 95.1 & 4780 & $2.577 E-U E$ & $1.100 E-05$ & 802 \\
\hline 97.1 & 4776 & $5.771[-00$ & $2.2 \cot -05$ & 806 \\
\hline 99.1 & 12328 & $6.646 \mathrm{t}-0 \mathrm{t}$ & $2.050 \mathrm{~L}-1 \mathrm{~s}$ & 804 \\
\hline 101.1 & $1390 \mathrm{~K}$ & $7.498 t-66$ & $3.224 E-03$ & 613 \\
\hline 103.0 & 17601 & $9.4 \mathrm{HQE}-0 \mathrm{~B}$ & ¿.USUE-OS & 816 \\
\hline E 125.0 & 15911 & $0.57 \mathrm{HL}-46$ & $3 . C H O E-\angle 5$ & 820 \\
\hline 106.9 & 14911 & $8.034 E-06$ & $3.457 E-05$ & 823 \\
\hline 108.8 & 11156 & $6.014 E-0 B$ & $2.586 E-45$ & 826 \\
\hline 110.7 & 3030 & 1.63 HE- 66 & $7.044 \mathrm{E}-06$ & 829 \\
\hline 112.6 & 1630 & $0.831 t-07$ & $3.797 E-0 b$ & 8.33 \\
\hline 114.5 & 437 & $4.314 L-07$ & $1.011 \mathrm{f}-\cup 6$ & $8: 6$ \\
\hline 116.4 & 100 & $5.41>t-58$ & $2.327 \mathrm{E}-07$ & 834 \\
\hline 118.3 & 1 & B.evUt -10 & $2.09 \forall E-64$ & 1442 \\
\hline CROSSHINR & IATEGRATFU & $\begin{array}{r}1.0446-03 \\
\text { sicisto. }\end{array}$ & $\begin{array}{c}7.920 \mathrm{E}-\mathrm{US} \\
\mathrm{I} / \mathrm{M}\end{array}$ & \\
\hline
\end{tabular}

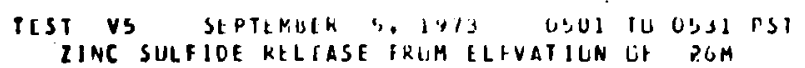

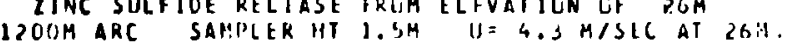

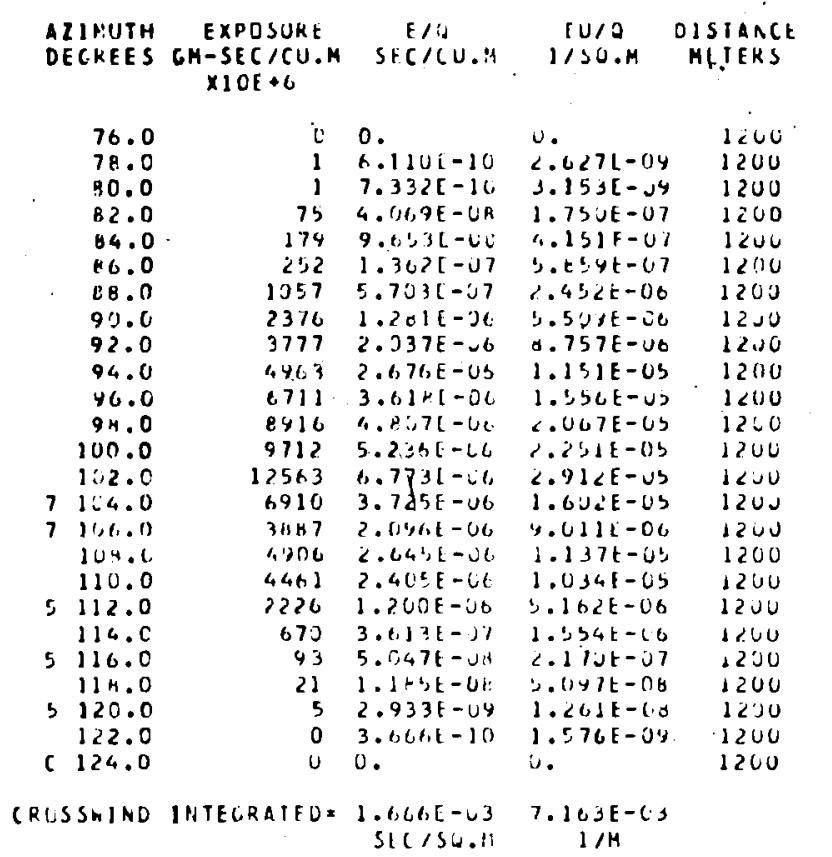

SICISU.ll I/M

TEST V5 SLPIEMBER 5. 1973 USU1 TU U531 PST ZINC SULFIUE RELEASE FKUM ELFVATIUN IJF $26 \mathrm{M}$ I BOOOH ARC SAMPLER HT $1.5 \%$ " $1=4.3 \mathrm{H} / \mathrm{SEL}$ AI $26 \%$

\begin{tabular}{|c|c|c|c|c|}
\hline $\begin{array}{l}\text { ALIMUTH } \\
\text { OFGREES }\end{array}$ & $\begin{array}{c}\text { EXPOSURE } \\
\text { CH-SEC/CU.H } \\
\times 1 O L+C\end{array}$ & $\begin{array}{c}1 / x \\
\text { sic/cu.t }\end{array}$ & $\begin{array}{l}1 \mathrm{U} / 0 \\
1 / 56 . \mathrm{H}\end{array}$ & $\begin{array}{l}\text { DISIAIJCL } \\
\text { MEIERS }\end{array}$ \\
\hline <o & $u$ & 0. & 1.. & 0 \\
\hline$\forall 1$. & ${ }_{16}^{2}$ & $\begin{array}{l}1.36+1-09 \\
9.119[-09\end{array}$ & $\begin{array}{l}3 . \forall 0<E-0 y \\
3, y<1 E-0 .\end{array}$ & $\begin{array}{l}1502 \\
1504\end{array}$ \\
\hline
\end{tabular}

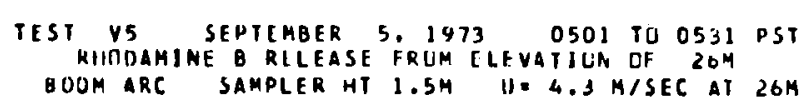

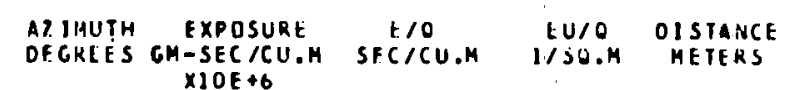

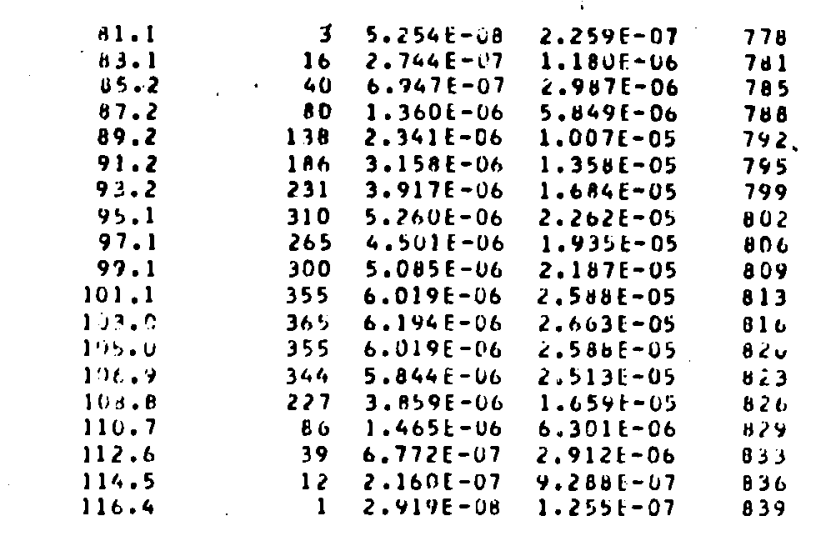

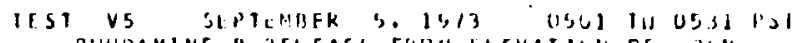
FHUDAMINE B RLLEASE FKUN KLFVATIUN DF ZUM 1200.4 ARC SAMPLER HT $1.5 \mathrm{SH} U=4.3 \mathrm{M} / S E C$ AT $20 \mathrm{M}$

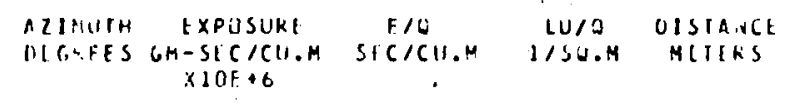

\begin{tabular}{|c|c|c|c|c|c|}
\hline & $\begin{array}{l}+2.0 \\
14.0\end{array}$ & 13 & $\begin{array}{l}3.503 E-08 \\
1.436 E--7\end{array}$ & $\begin{array}{l}2.360 E-1.7 \\
\text { d. } 324 E-i 7\end{array}$ & $\begin{array}{l}1200 \\
1200\end{array}$ \\
\hline & H(..0) & 13 & $2.260 \mathrm{k}-07$ & $9.7101-07$ & $12 \mathrm{~L}$ \\
\hline & $\therefore A .0$ & 40 & $7.87\} E-07$ & $3.365 E-6.6$ & 1200 \\
\hline & $y: 0$ & 57 & $1.4: 15 t-06$ & $0.305 t-06$ & 1206 \\
\hline & 92.0 & 113 & $1.927 \mathrm{E}-06$ & $0.266[-106$ & 1200 \\
\hline & N. . & 138 & $2.340 \mathrm{E}-46$ & $1.006 E-05$ & 12011 \\
\hline & 90.0 & 150 & $2.643 \mathrm{E}-\mathrm{C6}$ & $1.1 \operatorname{sis}[-35$ & 1200 \\
\hline & $2+.0$ & 140 & $3.724 E-16$ & $1.310 \mathrm{t}-0 \mathrm{~S}$ & 1200 \\
\hline & 1.10 .0 & 230 & $3.9121-106$ & J.CE2T-OSS & $i<00$ \\
\hline & 102.0 & 216 & $4.0101-06$ & $1.7 c^{4} 1-0 s$ & 1260 \\
\hline & 134.0 & 172 & $7.924 E-06$ & $1.2601-05$ & 1200 \\
\hline E & 130.0 & 132 & $2.241 \mathrm{E}-\mathrm{CO}$ & $9.6305-06$ & 1200 \\
\hline 7 & ח & $\begin{array}{r}106 \\
58\end{array}$ & $\begin{array}{l}\text { 1. } B \cup 9 E-\cup 6 \\
9.836 E-07\end{array}$ & $\begin{array}{l}7.77 \cup E-U C \\
4.230 E-O C\end{array}$ & $\begin{array}{l}12 \cup 0 \\
1200\end{array}$ \\
\hline ; & 112.6 & 36 & $6.2 \cup B E-C 7$ & $2.006 t-00$ & 1200 \\
\hline & $11 \cdot .11$ & 11 & $1.4361-i 17$ & $0.324 t-07$ & 1210 \\
\hline 5 & 116.6 & 6 & $1.1605-07$ & b. $(2 \cup t-0)$ & l¿Ju \\
\hline & $11+0$ & 1 & $2.06,4 E-08$ & $0.073 E-00$ & İue \\
\hline$C+: ?$ & $\ln D$ & R:T[CRATFD $=$ & $\begin{array}{r}1.247 \mathrm{E}-03 \\
\mathrm{SFC} / \mathrm{SO} 0 . \mathrm{M}\end{array}$ & $\begin{array}{c}5.362 \mathrm{E}=0 \mathrm{~S} \\
1 / \mathrm{M}\end{array}$ & \\
\hline
\end{tabular}

THST VS SEPTEMUFR 5, 1973 DSUI TII U5SI HSI

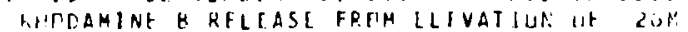

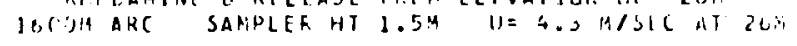
ALIIUTH EXPUSUPE EIO TU/O UISIARCE OEGREES GM-SECICU.H SECICU.M IISO.M HEIERS $X] \cup E+6$

112.6 .00$.

1564 


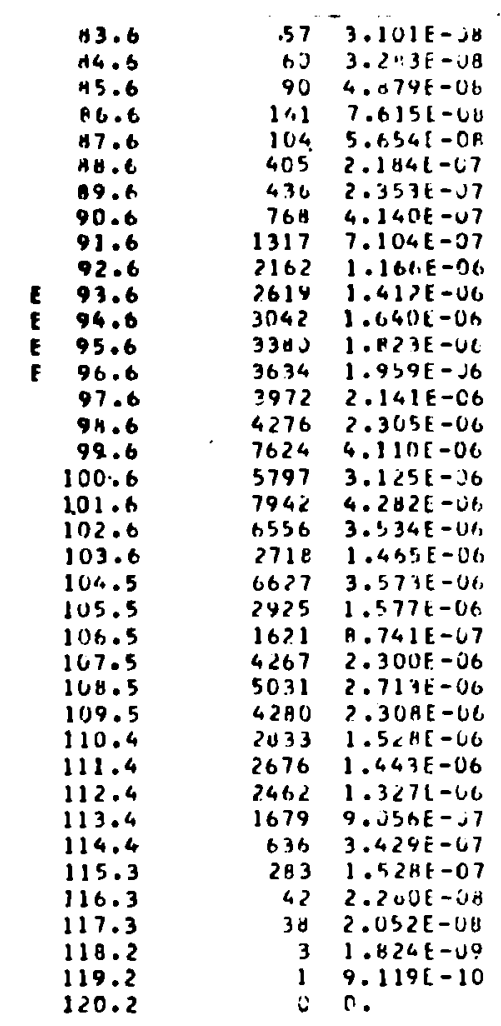

CROSSHIND INIEGRATIDE $1.395 E-3 . ?$

SFC/SL.N

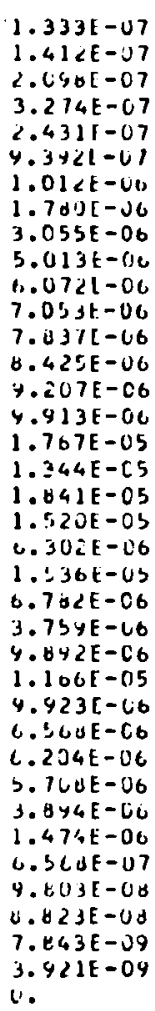

1565

1587

1584

1540

1542

1594

1346

1549

1601

1603

love

$16 \cup 8$

1610

1611

1613

1615

1617

$16: 0$

1620

$16<2$

1623

1625

1628

1630

1632

1533

1635

1637

1630

$16<.0$

1641

1643

1644

1646
1647

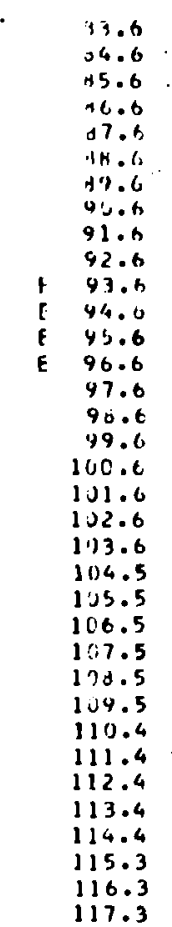

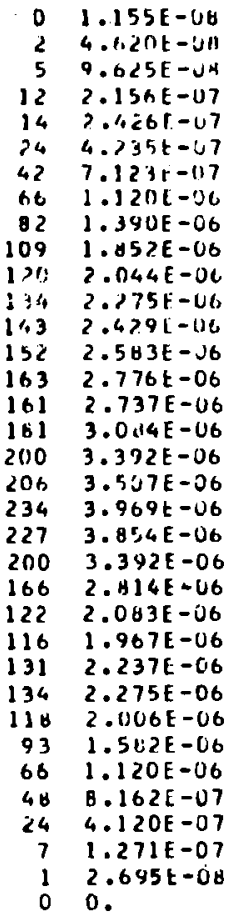

4. $4671-04$

$1.967[-01$

4. $134 E-07$

$9.271 L-01$

$1.04+1-06$

1. $1<11-06$.

3.vist-tib

4. 81 a L-U

$5.976 t-06$

$7.963 t-U_{6}$

$0.731 \mathrm{r}-00$

4.7141-06

$1.0456-05$

$1.111 \mathrm{E}-0 \mathrm{~S}$

1. $194 E-0 S$

1.177E-US

$1.326 \mathrm{E}-0 \mathrm{~S}$

$1.459 \mathrm{E}-0 \mathrm{~S}$

1. SLUE-US

$1.707 E-J S$
$1.657 E-05$

$1.454 E-05$

1. $210 \mathrm{E}-05$

$6.956 E-06$

8. 4 CUE-06

$9.614 \mathrm{E}-06$

$9.784 \mathrm{E}-06$

$8.62 S E-06$

6. $604 E-06$

4.816 E-06

3. $510 \mathrm{U}-06$

$1.771 \mathrm{E}-06$

$5.463 \mathrm{~F}-07$

1. 1 प $45-07$

0 .

1565

1501

1584

1406
11,42

11,44

is

1541
1594

1601

1603

1604

1606
1608

1610

1611

1515

1617

1010

1622

1625

1625

1627

1630

1632

1633

1635
1637

1638

1641

1643

$1 / \mathrm{H}$

TEST VS SEPTEHEER 5, 1973 ZJUI TU 0531 PST 2 INC SULFIDE RELEASE FRIM ELFVAIIUN OF $2 O M$ $3200 \mathrm{H}$ ARC SAHPLER HT $1.5 \mathrm{MM} U=4.3 \mathrm{M} / \mathrm{SEC}$ AT $26 \mathrm{M}$

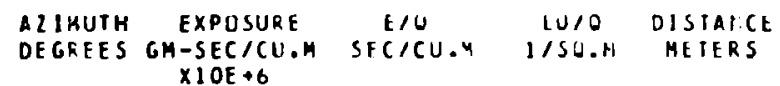

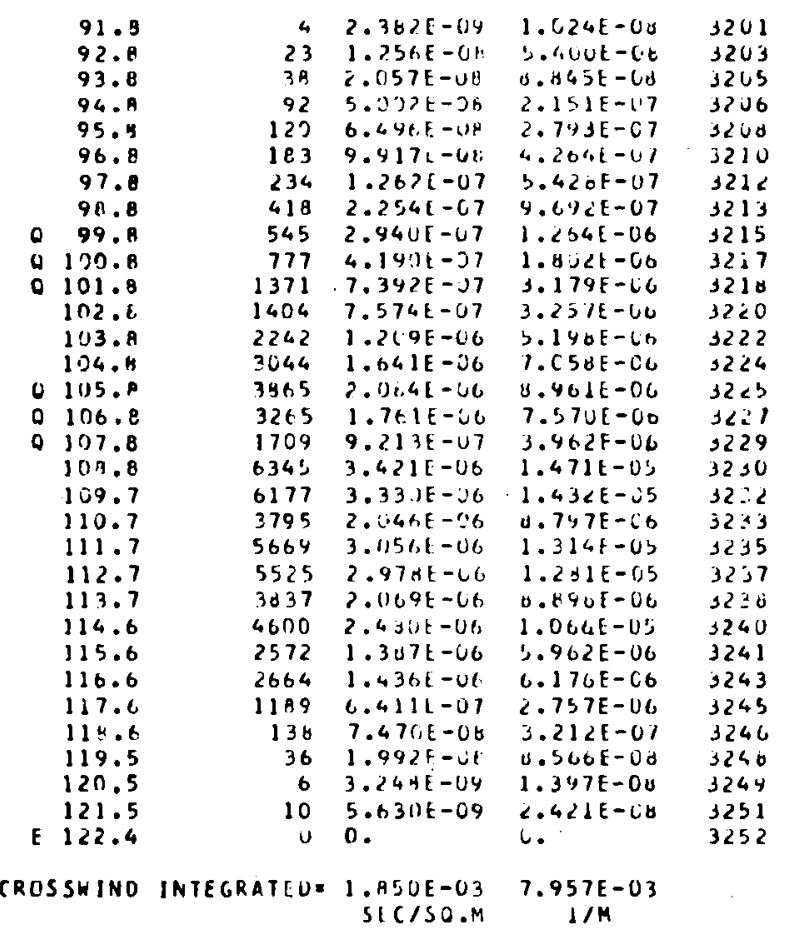

TEST VS SEPTEMBER 5,1973 0SO1 TU 0531 PST RHUOAMINE B RELEASE FROM ELFVATILN DF C6N $3200+1$ ARC SAMPLER HI $1.5 \mathrm{M} U=4.3 \mathrm{M} / \mathrm{SEC}$ AT $26 \mathrm{M}$

ALIMUTH EXPOSURE
DEGPEES CH-SECICU.M SFCICU.M
XIOE +6

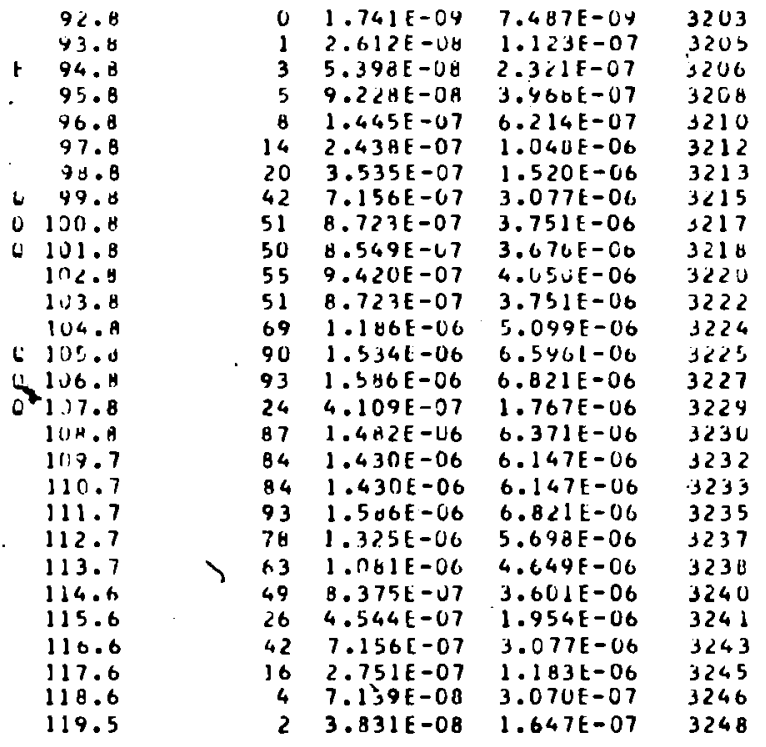

CRISSHINO INTEGRATEDE $1.146 E-03 \quad 4.936 E-03$
206

208

212

615

218

224

3225

3229

233

237

230

3241

245

3248 
TOLER DATA FOLLOW....

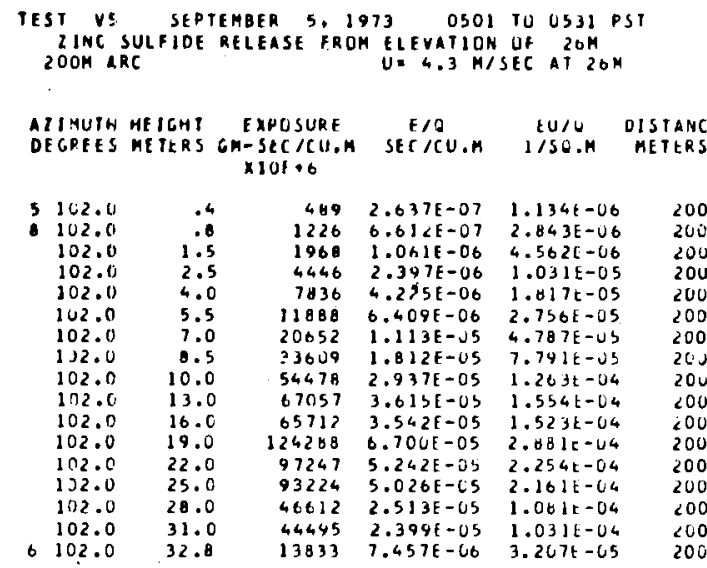

AIIMUTH HEIGHT EXPOSURF E/U EU/J LISTARCE DECPEES METERS GH-SEC/CU.M SEC/CU.H IISG.M MEILRS

\begin{tabular}{|c|c|c|c|c|}
\hline 110.0 & .4 & 1161 & $6.261 E-07$ & $2.64<1-60$ \\
\hline 110.0 & .8 & 1547 & $0.613 \mathrm{E}-\mathrm{J} 7$ & $3.704 t-J 6$ \\
\hline 110.0 & 1.5 & 2128 & $1.148 \mathrm{E}-06$ & $4.935 t-06$ \\
\hline 110.0 & 2.5 & 1186 & $6.39 \in E-07$ & $2.73 \cup x-00$ \\
\hline 110.0 & 4.0 & 43,8 & $2.33 j E-06$ & $1.00 \mathrm{gt}-\mathrm{us}$ \\
\hline 110.0 & 5.5 & 5069 & $3.164 E-06$ & $1.361 \mathrm{c}-4 \mathrm{~S}$ \\
\hline 110.0 & 7.0 & 11052 & $5.956 E-106$ & $2.562 t-65$ \\
\hline 110.0 & 0.5 & 22737 & $1.226 \mathrm{~F}-\mathrm{JS}$ & $5.271 k-65$ \\
\hline 110.0 & 10.0 & 34852 & $1.0796-05$ & $8.074 E-65$ \\
\hline 110.0 & 13.0 & 60067 & $3.56 c 1-05$ & $1.5311-04$ \\
\hline 110.0 & 16.0 & 110887 & $5.978 t-05$ & $2.570 t-34$ \\
\hline 110.0 & 19.0 & 157124 & B.67UK-JS & $3.64<t-64$ \\
\hline 110.0 & 22.0 & 229131 & $1.233 E-04$ & $5.311 k-04$ \\
\hline 110.0 & 25.0 & 231105 & $1.246 \mathrm{E}-04$ & $5.357 \mathrm{t}-04$ \\
\hline 110.0 & 28.0 & 180628 & $9.737 \mathrm{E}-\mathrm{OS}$ & $4.1871-64$ \\
\hline 110.0 & 31.0 & 204245 & $1.101 \mathrm{E}-04$ & $4.735 t-14$ \\
\hline 110.0 & 32 & 101262 & $5.459 \mathrm{E}-0 \mathrm{~S}$ & $2.347 t-04$ \\
\hline
\end{tabular}

AZIMUTH HEIGHI EXPOSURE FIU EL/O OISIANCE $x$ TLE

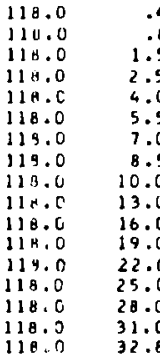

TONER DATA FCLLUW.... TEST VS SEPTEMBER 5. 1973 OSOI TO C.531 PST
RHODAMINE BRELEASE FROM ELFVATIUN OF $26 \mathrm{MH}$
ZUUM ARC
U. 4.3 H/SEC A.T 264

AIIPUTH HEIGHT EXPOSURE F/O EUIU DISTANCE DEGREES MEIERS GM-SEC/CU.M SEC/CU.M I/SO.M METERS

3102.0
102.0
102.0
102.0
102.0
102.0
102.0
102.0
102.0
112.0
112.0
132.0
102.0
102.0
102.0
112.0
112.0
$x i 0 F+6$

.4
.8
1.5
2.5
4.0
5.5
7.0
8.5

$\begin{array}{rlll}55 & 9.355 E-07 & 4.023 E-06 & 200 \\ 71 & 1.207 E-06 & 3.190 E-06 & 200 \\ 47 & 8.0 B 7 E-07 & 3.478 E-06 & 200 \\ 21 & 3.561 E-07 & 1.531 E-06 & 200 \\ 72 & 1.225 E-06 & 5.268 E-06 & 200 \\ 132 & 2.239 E-06 & 9.628 B-06 & 200 \\ 185 & 3.144 E-06 & 1.352 E-05 & 200 \\ 345 & 5.860 E-06 & 2.520 E-05 & 200 \\ 441 & 7.490 E-06 & 3.221 E-05 & 200 \\ 805 & 1.365 E-05 & 5.868 E-05 & 200 \\ 719 & 1.270 E-05 & 5.245 E-05 & 200 \\ 1595 & 2.704 E-05 & 1.153 E-04 & 200 \\ 1702 & 2.645 E-05 & 1.241 E-04 & 200 \\ 1453 & 2.463 E-05 & 1.059 E-04 & 200 \\ 730 & 1.238 E-05 & 5.323 E-05 & 200 \\ 516 & 8.757 E-06 & 3.766 E-05 & 200 \\ 399 & 6.766 E-06 & 2.909 E-05 & 200\end{array}$

AII IHJTH HEIGHT EXPOSIJAF F/O EU/O DISTANCE DECLES METEKS GH-SEC/CU.M SEC/CU.H I/SO.M METERS $\times 10 \mathrm{E}+6$

3110.0
110.0
110.0
110.0
110.0
112.0
110.0
110.0
110.0
110.0
110.0
110.0
110.0
110.0
110.0
110.0
110.0
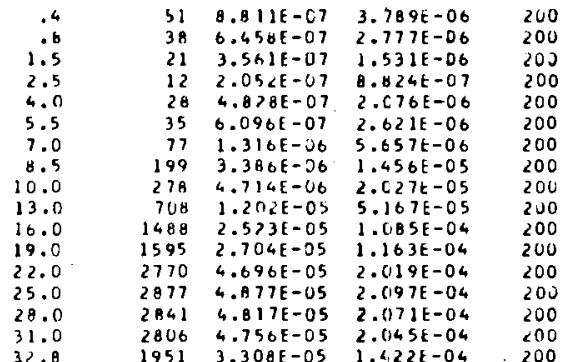

AII"UTH HEIGHT EXPTSURF F/O IU/J DISIANCE DECHES AETERS CM-SEC/CU $\times 10 \mathrm{E}+6$

\begin{tabular}{|c|c|c|c|c|c|}
\hline 113.0 & .4 & 0 & & & 200 \\
\hline 119.0 &.$B$ & 2 & $3.621 \mathrm{E}-0 \mathrm{D}$ & $1.557 \mathrm{E}=07$ & 200 \\
\hline 113.6 & 1.5 & 0 & 0. & 0. & 200 \\
\hline 112.0 & 2.5 & 0 & 0 . & 0. & 200 \\
\hline 119.0 & 4.0 & 0 & 0. & 0 . & 200 \\
\hline 11.4 .0 & 5.5 & 0 & 0. & 0. & 200 \\
\hline 116.0 & 7.0 & 1 & $3.01 \Delta t-U \theta$ & $1.298 \mathrm{E}-07$ & 200 \\
\hline 119.0 & 8.5 & 2 & $4.820 E-0 B$ & $2.070 E-07$ & $2<0$ \\
\hline 11 n. & 10.0 & 11 & $1.931 E-67$ & $0.305 t=07$ & 200 \\
\hline $11 \mathrm{H} .6$ & 13.0 & 32 & $5.556 \mathrm{E}-07$ & $2.308 \mathrm{E}-06$ & 200 \\
\hline $11 H .6$ & 16.0 & 73 & $1.243 E-06$ & $5.34 \mathrm{bt}-06$ & 200 \\
\hline 112. & 14,0 & 135 & $2.299 E-06$ & $9 . A B B E-U C$ & 200 \\
\hline 110.0 & 22.0 & 167 & $2.043 E-06$ & $1.222 \mathrm{E}-05$ & 200 \\
\hline $11 \% .0$ & 25.0 & $23 \mathrm{H}$ & $4.050 E-06$ & $1.741 \mathrm{k}-05$ & 200 \\
\hline $11 \mathrm{H} \cdot \mathrm{C}$ & 28.0 & 345 & 5.8 SOE -06 & $2.520 \mathrm{E}-05$ & $\angle 00$ \\
\hline 114.0 & 31.0 & 292 & $4.955 E-06$ & $2.1316-05$ & 200 \\
\hline 1 1甘.0 & 32 & 249 & $4.231 \mathrm{E}-06$ & $1.819 E-0 S$ & \\
\hline
\end{tabular}




\begin{tabular}{|c|c|c|c|c|c|c|c|c|c|c|c|}
\hline $\begin{array}{l}\text { A2IMUTH } \\
\text { OE CREES }\end{array}$ & $\begin{array}{l}\text { HE IGHT } \\
\text { METERS }\end{array}$ & $\begin{array}{c}\text { EXPOSURE } \\
\text { GH-SEC/CU.H } \\
\times \perp U E+6\end{array}$ & $\begin{array}{l}\text { E/O } \\
\text { SEC/CU.M }\end{array}$ & $\begin{array}{c}E U / O \\
1 / 50 . M\end{array}$ & $\begin{array}{l}\text { DISTANCE } \\
\text { ME TERS }\end{array}$ & $\begin{array}{l}\text { AZIMUTH } \\
\text { OEGREES }\end{array}$ & $\begin{array}{l}\text { HEIGHT } \\
\text { METERS }\end{array}$ & $\begin{array}{c}\text { EXPOSURE } \\
\text { GH-SEC/CU.M } \\
\times 10 E+6\end{array}$ & $\begin{array}{c}\text { E/O } \\
\text { SEC/CU.N }\end{array}$ & $\begin{array}{l}\text { EU/O } \\
1 / S O . N\end{array}$ & $\begin{array}{c}\text { DISTANCE } \\
\text { ME TERS }\end{array}$ \\
\hline $\begin{array}{ll}0 & 126.0 \\
0 & 126.0 \\
8 & 126.0 \\
126.0 \\
126.0 \\
126.0 \\
126.0 \\
126.0 \\
126.0 \\
126.0 \\
126.0 \\
126.0 \\
126.0 \\
126.0 \\
126.0 \\
126.0 \\
126.0 \\
126.0\end{array}$ & $\begin{array}{r}.4 \\
1.0 \\
1.5 \\
2.5 \\
4.0 \\
5.05 \\
7.0 \\
8.5 \\
10.0 \\
13.0 \\
16.0 \\
19.0 \\
22.0 \\
25.0 \\
28.0 \\
31.0 \\
32.0\end{array}$ & $\begin{array}{r}2 \\
0 \\
0 \\
145 \\
48 \\
56 \\
50 \\
24 \\
20 \\
23 \\
40 \\
17 \\
10 \\
12 \\
0 \\
21 \\
15\end{array}$ & $\begin{array}{l}1.430 E-09 \\
0 . \\
0 . \\
7.831 E-08 \\
2.627 E-08 \\
3.065 E-08 \\
2.702 E-08 \\
1.328 E-08 \\
1.097 E-08 \\
1.265 E-08 \\
2.193 E-08 \\
9.285 E-09 \\
5.605 E-09 \\
6.970 E-09 \\
3.894 E-10 \\
1.155 E-06 \\
8.110 E-04\end{array}$ & $\begin{array}{l}6.147 E-09 \\
0 . \\
0 . \\
3.367 E-07 \\
1.130 E-07 \\
1.318 E-07 \\
1.162 E-07 \\
5.710 E-08 \\
4.717 E-00 \\
5.440 E-00 \\
9.430 E-00 \\
3.992 E-08 \\
2.410 E-08 \\
2.997 E-00 \\
1.677 E-09 \\
4.465 E-00 \\
3.487 E-00\end{array}$ & $\begin{array}{l}200 \\
200 \\
200 \\
200 \\
200 \\
200 \\
200 \\
200 \\
200 \\
200 \\
200 \\
200 \\
200 \\
200 \\
200 \\
200 \\
200\end{array}$ & ii 126.0 & . & 0 & 0. & 0. & $\begin{array}{r}200 \\
-\ldots\end{array}$ \\
\hline
\end{tabular}


TDHER DATA FIILLOH....

TEST VS SEPTEABER 5.1973 0SO1 TU 0531 PST IINC SULFIOE RELEASE FROM ELEVATIUN OF 264 BOOM ARC U. $4.3 \mathrm{M} / \mathrm{SEC}$ AT $26 \mathrm{M}$
TOHER IHATA FIILLLIH....

TEST VS SEPTEMBER S, 1973 USUI TU OS31 PST RHDDAMINE H RELEASE FROM ELFVATION OF $26 \mathrm{H}$

BUOH ARC UE $4.3 \mathrm{H} / \mathrm{SEC}$ AT $26 \mathrm{H}$

\begin{tabular}{|c|c|c|c|c|c|c|c|c|c|c|c|}
\hline $\begin{array}{l}2 \text { IMUTH } \\
\text { ECREES }\end{array}$ & $\begin{array}{l}\text { HE IGHT } \\
\text { ME TERS }\end{array}$ & $\begin{array}{c}\text { EXPUSURE } \\
\text { GM-SEC/CU.H } \\
\times 1 O E+6\end{array}$ & $\begin{array}{c}\text { F/O } \\
\text { SEC/CU.M }\end{array}$ & $\begin{array}{l}E \cup / U \\
1 / S O . M\end{array}$ & $\begin{array}{l}\text { DISTANCE } \\
\text { ME TtRS }\end{array}$ & $\begin{array}{l}\text { A2 JPHUTH } \\
\text { DEGREES }\end{array}$ & $\begin{array}{l}\text { HE IGHT } \\
\text { ME IERS }\end{array}$ & $\begin{array}{l}\text { IEXPOSURE } \\
\text { CM-SLC/CU.M } \\
\text { XIUE+G }\end{array}$ & $\begin{array}{c}\text { F/O } \\
\text { SES /CU.M }\end{array}$ & $\begin{array}{c}E U / U \\
1 / S U . M\end{array}$ & $\begin{array}{l}\text { DISTANCE } \\
\text { METERS }\end{array}$ \\
\hline $\begin{array}{l}105.0 \\
105.0 \\
105.0 \\
105.0 \\
105.0 \\
105.0 \\
105.0 \\
105.0 \\
105.0 \\
105.0 \\
105.0 \\
105.0 \\
105.0 \\
10.5 .0 \\
105.0 \\
105.0 \\
105.0 \\
105.0\end{array}$ & $\begin{array}{l}.3 \\
.5 \\
1.1 \\
1.5 \\
2.1 \\
4.2 \\
6.3 \\
8.4 \\
10.5 \\
12.6 \\
14.7 \\
16.8 \\
21.0 \\
25.2 \\
29.4 \\
33.6 \\
37.8 \\
42.0\end{array}$ & $\begin{array}{l}4161 \\
15676 \\
11858 \\
15911 \\
19797 \\
25932 \\
25307 \\
31347 \\
31166 \\
42903 \\
29562 \\
47453 \\
41784 \\
31437 \\
23052 \\
8810 \\
4527 \\
2782\end{array}$ & $\begin{array}{l}2.243 E-06 \\
8.451 E-06 \\
6.393 E-06 \\
8.578 E-06 \\
1.067 E-05 \\
1.398 E-05 \\
1.344 E-05 \\
1.690 E-05 \\
1.680 E-05 \\
2.313 E-05 \\
1.594 E-05 \\
2.558 E-05 \\
2.253 E-05 \\
1.695 E-05 \\
1.243 E-05 \\
4.750 E-06 \\
2.441 E-06 \\
1.500 E-06\end{array}$ & $\begin{array}{l}9.646 E-06 \\
3.634 E-05 \\
2.749 E-05 \\
3.688 E-05 \\
4.589 E-05 \\
6.011 E-05 \\
5.866 E-05 \\
7.267 E-05 \\
7.225 E-05 \\
9.945 E-05 \\
6.853 t-05 \\
1.100 E-04 \\
9.686 E-05 \\
7.267 E-05 \\
5.344 E-05 \\
2.042 E-05 \\
1.050 E-05 \\
6.450 E-06\end{array}$ & $\begin{array}{l}\forall 20 \\
\forall 20 \\
820 \\
020 \\
620 \\
620 \\
820 \\
820 \\
020 \\
820 \\
920 \\
820 \\
820 \\
820 \\
620 \\
620 \\
820 \\
820\end{array}$ & $\begin{array}{l}105.0 \\
105.0 \\
105.0 \\
105.0 \\
105.0 \\
105.0 \\
105.0 \\
105.0 \\
105.0 \\
105.0 \\
105.0 \\
105.0 \\
105.0 \\
105.0 \\
105.0 \\
105.0 \\
105.0 \\
105.0\end{array}$ & $\begin{array}{r}.3 \\
1.5 \\
1.1 \\
2.5 \\
4.1 \\
6.3 \\
8.4 \\
10.5 \\
12.6 \\
14.7 \\
16.8 \\
21.0 \\
25.2 \\
29.4 \\
33.6 \\
37.8 \\
42.0\end{array}$ & $\begin{array}{r}71 \\
355 \\
313 \\
355 \\
396 \\
479 \\
530 \\
654 \\
799 \\
842 \\
861 \\
851 \\
992 \\
854 \\
551 \\
344 \\
176 \\
93\end{array}$ & $\begin{array}{l}1.270 E-06 \\
6.019 E-06 \\
5.314 E-06 \\
6.014 E-06 \\
6.720 E-06 \\
8.171 E-06 \\
8.996 E-06 \\
1.110 E-05 \\
1.355 E-05 \\
1.513 E-05 \\
1.460 E-05 \\
1.443 E-05 \\
1.6 B 2 E-05 \\
1.443 E-05 \\
9.347 E-06 \\
5.844 E-06 \\
2.943 E-06 \\
1.582 E-06\end{array}$ & $\begin{array}{l}5.247 E-06 \\
2.588 E-05 \\
2.287 E-05 \\
2.548 E-05 \\
2.889 E-05 \\
3.492 E-05 \\
3.868 E-05 \\
4.772 E-05 \\
5.827 E-05 \\
6.504 E-05 \\
6.278 E-05 \\
6.203 E-05 \\
7.232 E-05 \\
6.228 E-05 \\
4.019 E-05 \\
2.513 E-05 \\
1.283 E-05 \\
6.003 t-06\end{array}$ & $\begin{array}{l}820 \\
420 \\
820 \\
620 \\
820 \\
820 \\
620 \\
620 \\
820 \\
820 \\
020 \\
820 \\
820 \\
620 \\
820 \\
420 \\
820 \\
820\end{array}$ \\
\hline
\end{tabular}

\begin{tabular}{|c|c|c|c|c|c|c|c|c|c|c|c|}
\hline $\begin{array}{l}2 \text { I I HUTH } \\
\text { DECREES }\end{array}$ & $\begin{array}{l}\text { HE IGHT } \\
\text { ME IERS }\end{array}$ & $\begin{array}{c}\text { EXPOSUKF } \\
\text { GH-SECICU.H } \\
\times 10 E+6\end{array}$ & $\begin{array}{c}\text { E /O } \\
\text { SEC/CU.M }\end{array}$ & $\begin{array}{l}E U / 0 \\
1 / S O . H\end{array}$ & $\begin{array}{l}\text { DISTANCE } \\
\text { METERS }\end{array}$ & $\begin{array}{l}\text { AZIMUTH } \\
\text { UE CREES }\end{array}$ & $\begin{array}{l}\text { HE IGHT } \\
\text { ME TERS }\end{array}$ & $\begin{array}{c}\text { EXPDSURF } \\
\text { CH-SECICU.M } \\
\times 1 O E+6\end{array}$ & $\begin{array}{c}\text { F/O } \\
\text { SEC /CU.M }\end{array}$ & $\begin{array}{l}E U / 0 \\
1 / S Q . M\end{array}$ & $\begin{array}{l}\text { DISTANCE } \\
\text { METERS }\end{array}$ \\
\hline $\begin{array}{l}112.6 \\
112.6 \\
112.6 \\
112.6 \\
112.6 \\
112.6 \\
112.6 \\
112.6 \\
112.6 \\
112.6 \\
112.6 \\
112.6 \\
112.6 \\
1112.6 \\
112.6 \\
112.6 \\
112.6 \\
112.6\end{array}$ & $\begin{array}{l}.3 \\
1.5 \\
1.1 \\
1.5 \\
2.1 \\
4.2 \\
6.3 \\
1.4 \\
10.5 \\
12.6 \\
14.7 \\
16.8 \\
21.0 \\
25.2 \\
29.4 \\
33.6 \\
37.8 \\
42.0\end{array}$ & $\begin{array}{l}1793 \\
1826 \\
1714 \\
1638 \\
2736 \\
3907 \\
6015 \\
12599 \\
15881 \\
15430 \\
19177 \\
26719 \\
34717 \\
47313 \\
42070 \\
39350 \\
1367 \\
2070\end{array}$ & $\begin{array}{l}9.670 E-07 \\
9.846 E-07 \\
9.244 E-07 \\
8.831 E-07 \\
1.475 E-06 \\
2.107 E-06 \\
3.243 E-06 \\
6.792 E-06 \\
0.562 E-06 \\
0.310 E-06 \\
1.034 E-05 \\
1.440 E-05 \\
1.872 E-05 \\
2.551 E-05 \\
2.260 E-05 \\
2.121 E-05 \\
7.371 E-07 \\
1.116 E-06\end{array}$ & $\begin{array}{l}4.158 E-06 \\
4.234 E-06 \\
3.975 E-06 \\
3.797 E-06 \\
6.343 E-06 \\
9.05 \theta E-06 \\
1.395 E-05 \\
2.921 E-05 \\
3.662 E-05 \\
3.577 E-05 \\
4.446 E-05 \\
6.194 E-05 \\
6.048 E-05 \\
1.097 E-04 \\
9.752 E-05 \\
9.122 E-05 \\
3.170 E-06 \\
4.800 E-06\end{array}$ & $\begin{array}{l}833 \\
033 \\
833 \\
833 \\
833 \\
833 \\
833 \\
033 \\
833 \\
833 \\
833 \\
833 \\
833 \\
833 \\
033 \\
433 \\
833 \\
433 \\
833\end{array}$ & $\begin{array}{l}112.6 \\
112.6 \\
112.6 \\
112.6 \\
112.6 \\
112.6 \\
1112.6 \\
112.6 \\
1112.6 \\
112.6 \\
112.6 \\
112.6 \\
112.6 \\
112.6 \\
112.6 \\
112.6 \\
112.6 \\
112.6\end{array}$ & $\begin{array}{l}.3 \\
1.3 \\
1.1 \\
1.5 \\
2.1 \\
4.7 \\
6.3 \\
8.4 \\
10.5 \\
12.6 \\
14.7 \\
16.6 \\
21.0 \\
23.2 \\
29.4 \\
33.6 \\
37.8 \\
42.0\end{array}$ & $\begin{array}{r}28 \\
32 \\
28 \\
39 \\
35 \\
67 \\
107 \\
148 \\
241 \\
303 \\
396 \\
0 \\
644 \\
747 \\
784 \\
789 \\
0 \\
520\end{array}$ & $\begin{array}{l}4.846 E-07 \\
5.546 E-07 \\
4.846 E-07 \\
6.772 E-07 \\
6.072 E-07 \\
1.063 E-06 \\
1.313 E-06 \\
2.516 E-06 \\
4.092 E-06 \\
5.143 E-06 \\
6.770 E-06 \\
0.1 \\
1.092 E-05 \\
1.267 E-05 \\
1.337 E-05 \\
1.337 E-05 \\
0.3 \\
8.821 E-06\end{array}$ & $\begin{array}{l}2.084 E-06 \\
2.385 E-06 \\
2.084 E-06 \\
2.912 E-06 \\
2.611 E-06 \\
4.569 E-06 \\
7.807 E-06 \\
1.082 E-05 \\
1.760 E-05 \\
2.212 E-05 \\
2.889 E-05 \\
0.0 \\
4.697 E-05 \\
5.450 E-05 \\
5.751 E-05 \\
5.751 E-05 \\
0.7 \\
3.793 E-05\end{array}$ & $\begin{array}{l}833 \\
833 \\
833 \\
833 \\
833 \\
833 \\
833 \\
833 \\
833 \\
833 \\
833 \\
833 \\
833 \\
633 \\
833 \\
833 \\
833 \\
833\end{array}$ \\
\hline
\end{tabular}

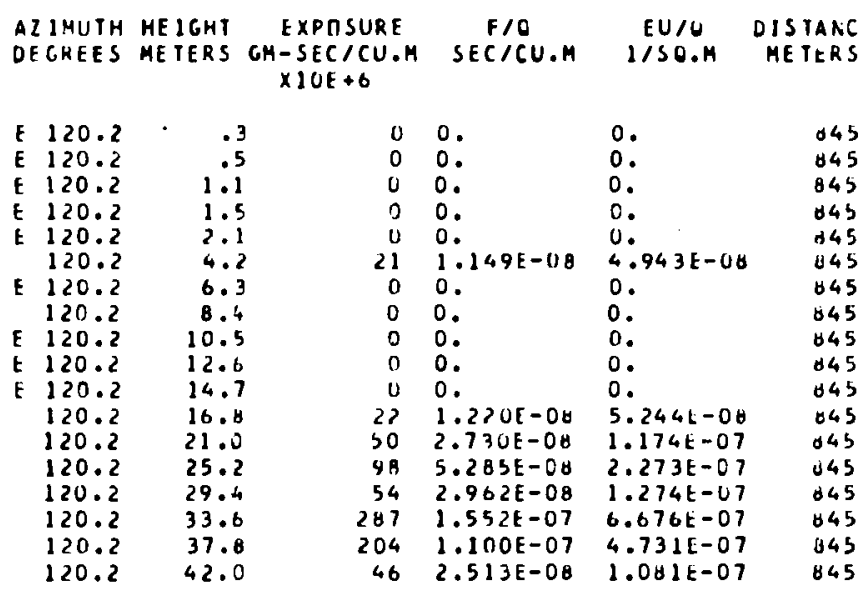

AZTMUTH HEIGHT EXPUSURF F/O EU/O UISTANCE DEGKEES MEIERS GM-SECICU.H SECICU.H I/SO.M HETERS

\section{$\times 10 E+6$}

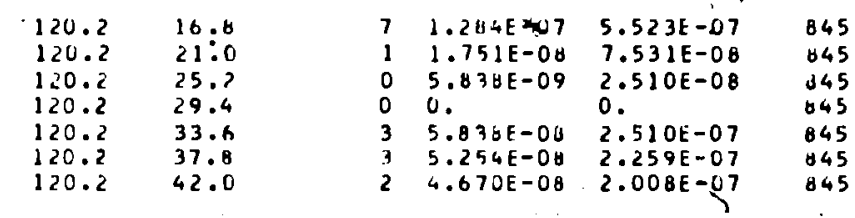

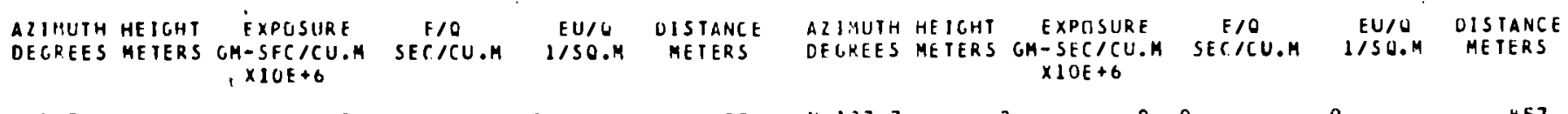
$\begin{array}{lllll}N 127.7 & .3 & 0 & 0 & 0\end{array}$
$857 \quad N 127.7 \quad .3 \quad 0 \quad 0$.
0.

657

AZIMUTH HEIGHT EXPOSURE F/O EU/O DISTANCE ALIHUTH HEIGHT EXPOSURE OTE EIO DECREES METERS GM-SECICU.M SECICU.M I/SO.M METERS OEGREES HETERS GM-SECICU.M SECICU.M IISU.H METERS $\times 10 E+6$
N 135.0
.3
00.
0.
267
$\begin{array}{llll}N & 135.0 & 0 & 0\end{array}$
0.

467 
TONER DATA FOLLCW....

IEST VS SEPTEMBER 5, 1973 0501 TU OSII PST ZINC SULFIDE KELEASE FRDM ELFYATION OF $26 \mathrm{M}$ $1600 \mathrm{M}$ ARC $U=4.3 . M / S E C$ AI $26 \mathrm{M}$
TUAER DATA FILLDH....

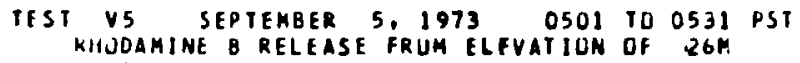
GIIUM ARC U. $4.3 \mathrm{~K} / \mathrm{SEC}$ AI $26 \mathrm{M}$

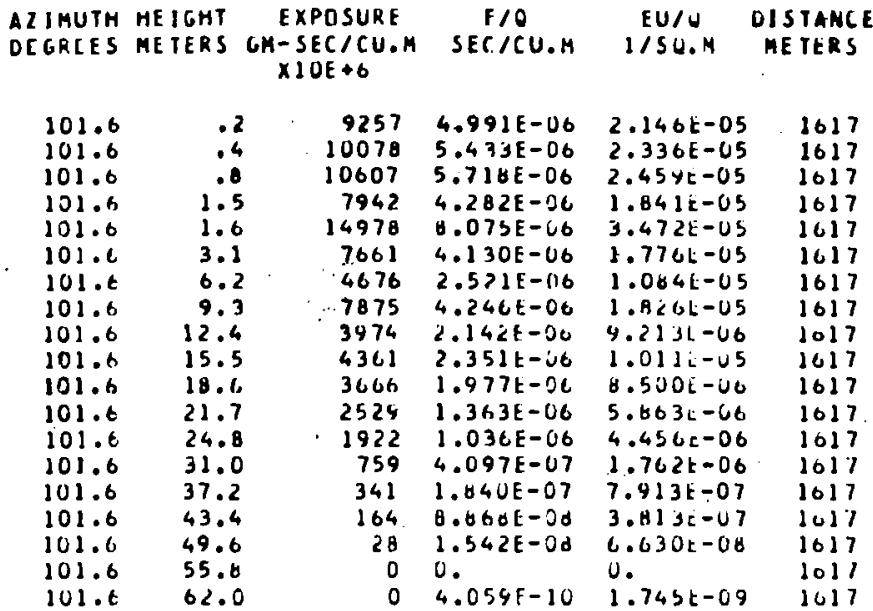

A2JHUTH HEIGHT EXPUSURE F/O LU/J OISIAINCE DEGMEES METERS GM-SECICU.M SECICU.M I/SU.M METLKS XIOE $\rightarrow 6$

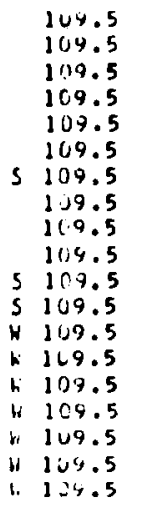

.2
.4
.0
1.5
1.6
3.1
6.2
9.3
12.4
15.5
18.6
21.7
24.6
31.0
37.2
43.4
49.6
55.8
62.0

1030
1030
1030
1030
1630
1630
1630
1630
1030
1030
1030
1030
1030
1030
1630
1630
1030
1630
1030

A2 ISUTH
DEGRESS
101.6
101.6
101.6
101.6
101.6
101.6
101.6
101.6
101.1
101.6
101.6
101.6
101.6
101.6
101.6
101.6
101.6
101.6
101.6

HE I
HE IGH
ETERS GH

EXPOSURE $\times 10 \mathrm{P} \rightarrow 6$

.2
.4
.8
1.5
1.6
3.1
6.2
9.3
12.4
15.5
18.6
21.7
24.8
31.0
37.2
43.4
49.6
55.8
62.1

EU/G UISIANCE I/SO.H MEIERS

\author{
1/
}

$.142 E-06$

1017

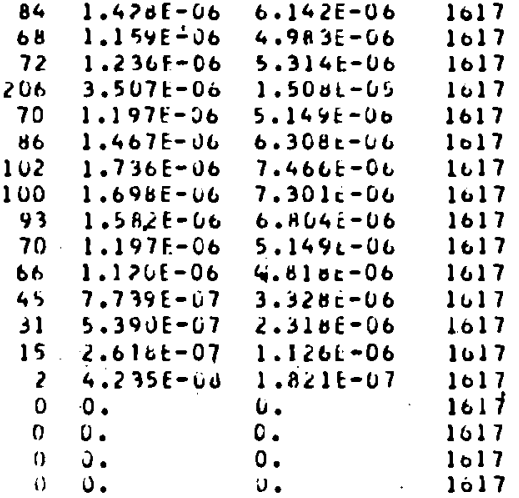

WIIIUTH HEIGHT EXPUSURE F/O EU/U UISTANCE DECHIS MEIERS GM-SECICU.M SERICU.M IISO.M MEIEKS $x \mid 01+1$.

$\begin{array}{ll}104.5 & .7 \\ 119.5 & .4 \\ 1104.5 & .8 \\ 109.5 & 1.5 \\ 109.5 & 1.6 \\ 1119.5 & 3.11 \\ 119.5 & 6.2 \\ 1199.5 & 9.3 \\ 107.5 & 12.4 \\ 169.5 & 15.5 \\ 109.5 & 10.6 \\ 124.5 & 21.7 \\ 11.9 .5 & 24.6 \\ 1119.5 & 31.11 \\ 1114.5 & 37.2 \\ 109.5 & 43.4 \\ 1139.5 & 49.6 \\ 104.5 & 55.8 \\ 104.5 & 62.0\end{array}$

$\begin{array}{rl}36 & 6.199 E-07 \\ 31 & 5.390 E-07 \\ 31 & 5.390 E-07 \\ 134 & 2.275 E-06 \\ 33 & 5.671 E-07 \\ 43 & 7.354 E-07 \\ 72 & 1.236 E-06 \\ 91 & 1.544 E-06 \\ 100 & 1.690 E-00 \\ 130 & 2.545 E-06 \\ 101 & 3.084 E-06 \\ 179 & 3.045 E-06 \\ 177 & 2.430 E-06 \\ 16 H & 2.053 E-06 \\ 343 & 5.817 E-06 \\ 63 & 1.082 E-06 \\ 20 & 3.542 E-07 \\ 0 & 1.425 E-07 \\ 3 & 5.390 E-08\end{array}$

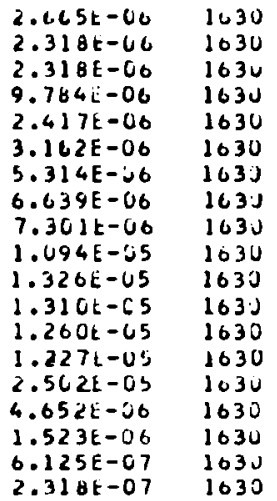

1030
1030
1630
1630
1630
1630
1630
1631
1630
1630
1630
1630
1630
1630
1030
1630
1630
1630
1030
AZIMUTH HEJGHT EXPUSURE F/U EU/J UISIATHE DECREES METERS GM-SEC/CU.M SEC/CU.M IISU.M METCKS $X$
$X 10 E+C$

$\begin{array}{rr}117.3 & .2 \\ 117.3 & .4 \\ 117.3 & .8 \\ 117.3 & 1.5 \\ 117.3 & 1.6 \\ 117.3 & 3.1 \\ 117.3 & 6.2 \\ 117.3 & 9.3 \\ 5117.3 & 12.4 \\ 117.3 & 15.5 \\ 117.3 & 18.6 \\ 117.3 & 21.7 \\ 117.3 & 24.8 \\ 117.3 & .31 .0 \\ 117.3 & 37.2 \\ 117.3 & 43.4 \\ 117.3 & 49.6 \\ 117.3 & 55.4 \\ 117.3 & 62.0\end{array}$

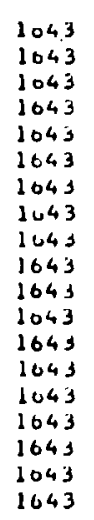

AZ IISUTH HEIGHT EXPOSURE $-S E C / C U O M$
$X \perp O E+0$

$\begin{array}{lr}117.3 & .2 \\ 117.3 & .4 \\ 117.3 & .6 \\ 117.3 & 1.3 \\ 117.3 & 1.0 \\ 117.3 & 3.1 \\ 117.3 & 6.2 \\ 117.3 & 9.3 \\ 5117.3 & 12.4 \\ 117.3 & 15.5 \\ 117.3 & 18.6 \\ 117.3 & 21.7 \\ 117.3 & 24.4 \\ 117.3 & 51.4 \\ 117.3 & 37.7 \\ 117.3 & 49.4 \\ 117.3 & 49.6 \\ 117.3 & 55.8 \\ 117.3 & 62.0\end{array}$

AZIMUTH HEIGHT EXPOSURE F/O EU/W ULSTANCE

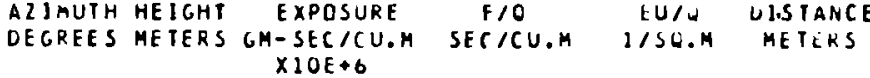
A2 JALTH HEIGHT EXPOSURF F/O EU/O DISTANCE

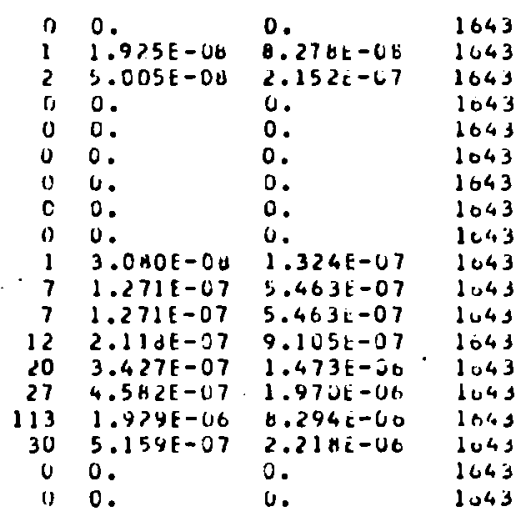
DECKLE METERS CH-SEC/CU.H
XIOE+6
N 125.0

.2
u.
1653

$1: 125.0 \quad .2 \quad 13$. u.

10s 


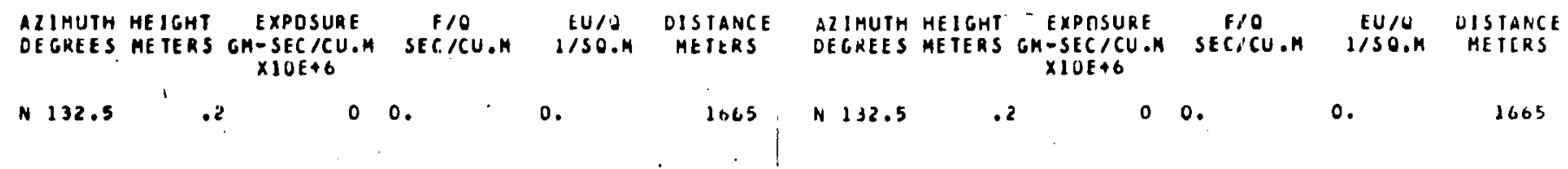

TOHER OATA FDLLOW...

:TOHER DATA FQLLDH....

TEST Y5 SEPYEMBER 5, 1973 0502 TO 0331 PST ZINC SULFIDE RELEASE FROH ELFVATION OF $26 \mathrm{~K}$ $3200 \mathrm{ARC} U=4.3 \mathrm{M} / \mathrm{SEC}$ AT $26 \mathrm{M}$
TEST V5 SEPTEMBER. 5, 1973 0501 TU 0531 PST KHUDAHINE B RELEASE FROH ELEVATION OF $26 \mathrm{M}$
$3200 M$ ARC U. $4.3 \mathrm{M} / \mathrm{SEC}$ AT $26 \mathrm{~F}$

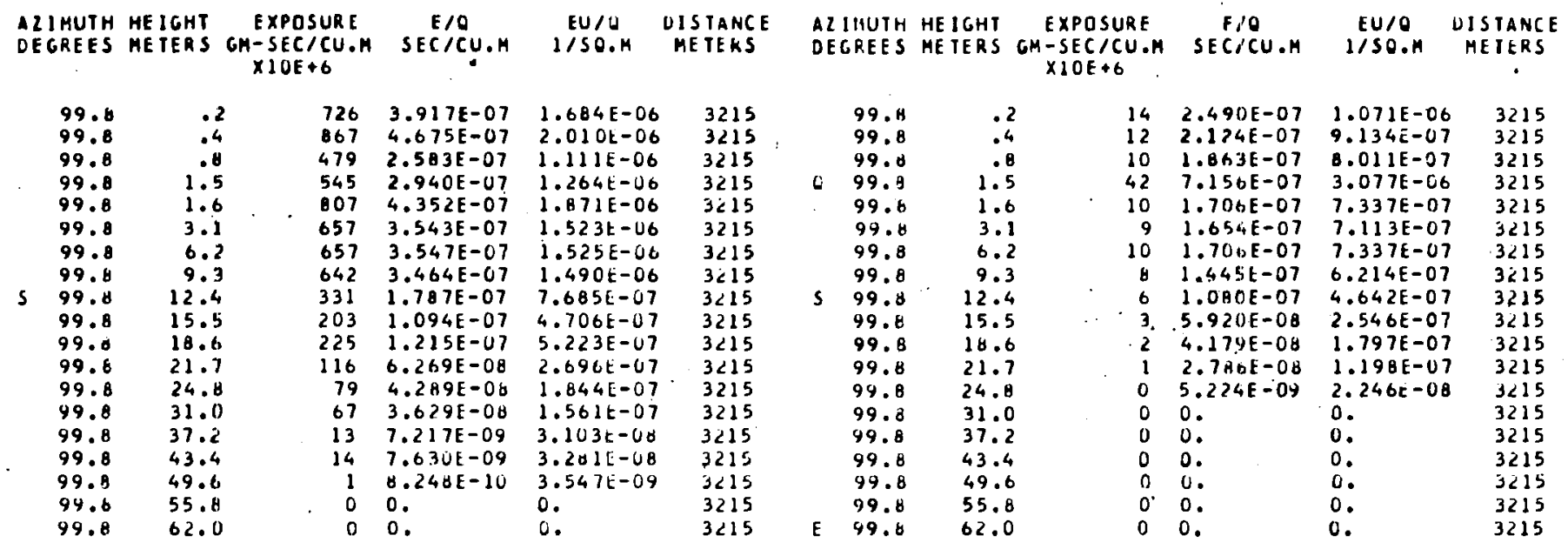

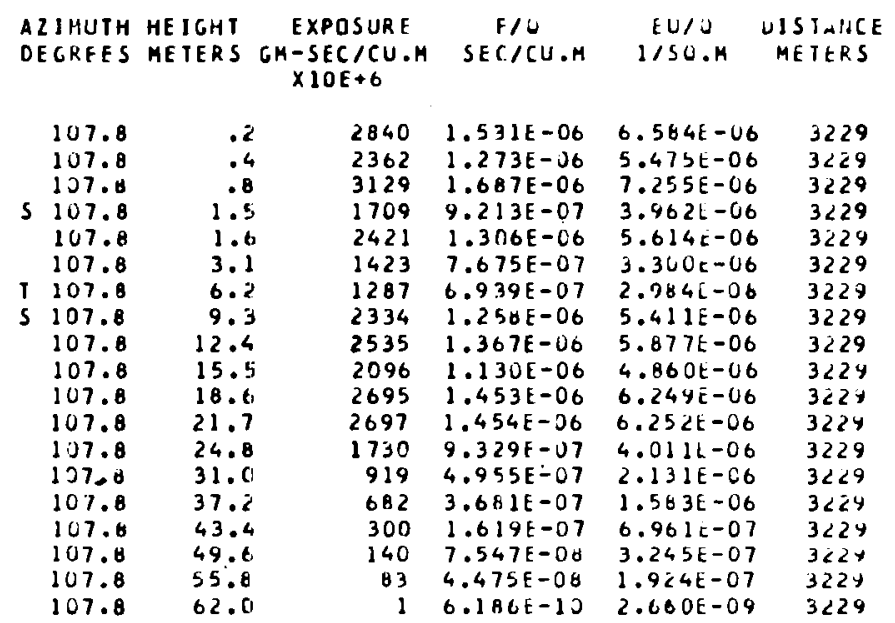

AZIMUTH HEICHT EXPOSURF FIO EUIJ OISTANCE DEGKEES METERS GK-SEC/CU.M SECICU.M I/SO.M METERS

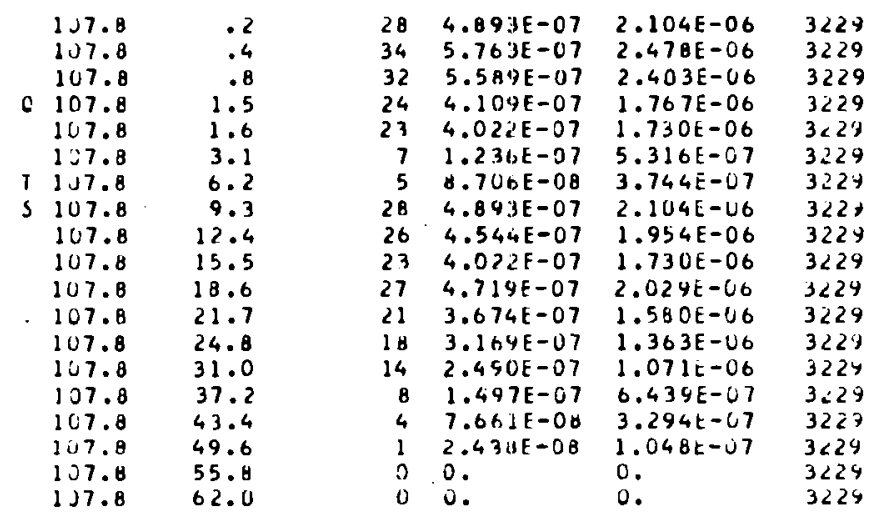

ALIMUTH HEIGHT EXPOSURF F/O EUIJ DISTANCE DEGREES HETERS GM-SEC/CU.M SEC/CU.M I/SU.M HETEKS

AZIIAUTH HEIGHT EXPOSURF E/O EU/O UISTANCE OEGREES METERS GH-SEC/CU.M SECICU.M. I/SO.M METERS

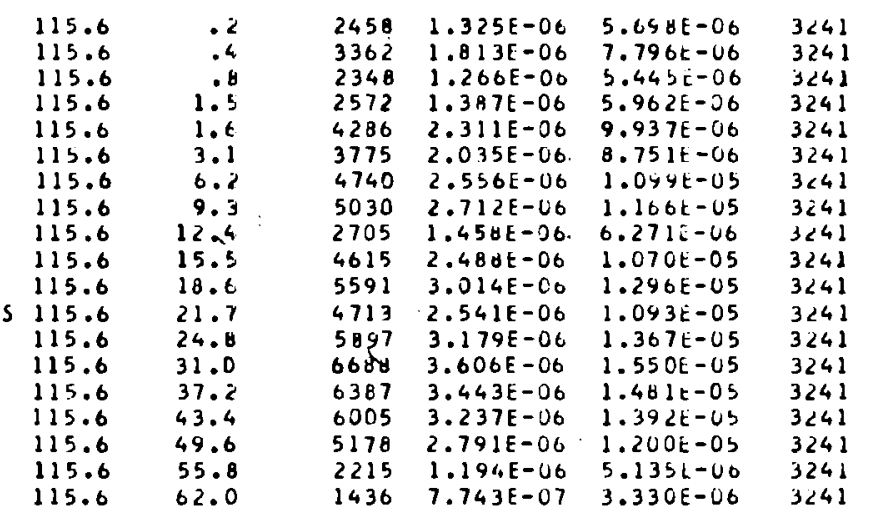

$\begin{array}{lr}115.6 & .2 \\ 115.6 & .4 \\ 115.6 & .8 \\ 115.6 & 1.5 \\ 115.6 & 1.6 \\ 115.6 & 3.1 \\ 115.6 & 6.2 \\ 115.6 & 9.3 \\ 115.6 & 12.4 \\ 115.6 & 15.5 \\ 115.6 & 18.6 \\ 115.6 & 21.7 \\ 115.6 & 24.8 \\ 115.6 & 31.0 \\ 115.6 & 37.0 \\ 115.6 & 43.0 \\ 115.6 & 49.6 \\ 115.6 & 55.8 \\ 115.6 & 62.0\end{array}$

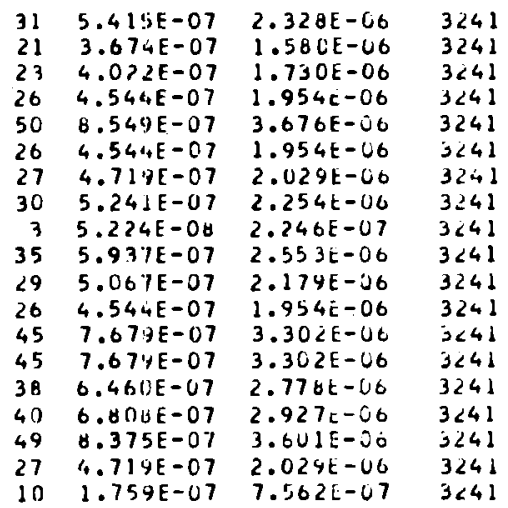




\begin{tabular}{|c|c|c|c|c|c|c|c|c|c|c|c|}
\hline $\begin{array}{l}\text { CZIMUTH } \\
\text { DECREES }\end{array}$ & $\begin{array}{l}\text { ME ICHT } \\
\text { ME TERS }\end{array}$ & $\begin{array}{c}\text { EXPGSURE } \\
\text { GM-SEC/CU. } \\
\times 10 E+6\end{array}$ & $\begin{array}{c}F / \dot{Z} \\
\text { SEC/EU.K }\end{array}$ & $\begin{array}{l}i v / 6 \\
1 / 52.4\end{array}$ & $\begin{array}{l}\text { WISTAhCe } \\
\text { Mi TCKS }\end{array}$ & $\begin{array}{l}\text { AZ IMUTH } \\
\text { DEGREES }\end{array}$ & $\begin{array}{l}\text { HEICHT } \\
\text { HE TERS }\end{array}$ & $\begin{array}{c}\text { EXPOSURE } \\
\text { CH-SEC/CU.A } \\
\times 1 O E+6\end{array}$ & $\begin{array}{c}E / O \\
\text { SEC/CU.H }\end{array}$ & $\begin{array}{l}\text { EU/O } \\
\text { I/SO.H }\end{array}$ & $\begin{array}{l}\text { DISTANCE } \\
\text { METERS }\end{array}$ \\
\hline $\begin{array}{l}123.4 \\
123.4 \\
123.4 \\
123.4 \\
123.4 \\
123.4 \\
123.4 \\
123.4 \\
123.4 \\
123.4 \\
123.4 \\
123.4\end{array}$ & $\begin{array}{l}.2 \\
3.1 \\
12.4 \\
15.5 \\
10.8 \\
21.8 \\
31.8 \\
37.2 \\
43.8 \\
49.6 \\
55.8 \\
62.0\end{array}$ & $\begin{array}{l}2 \\
11 \\
16 \\
1 \\
0 \\
4 \\
4 \\
11 \\
4 \\
20 \\
48 \\
16\end{array}$ & 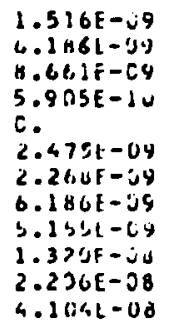 & 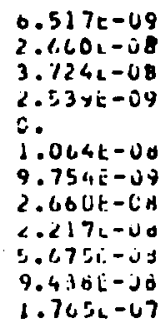 & $\begin{array}{l}3253 \\
3233 \\
3253 \\
3253 \\
3253 \\
3253 \\
3253 \\
3253 \\
3253 \\
3253 \\
3253 \\
3<53\end{array}$ & N 123.4 &.$\quad{ }^{-2}$ & 0 & 0. & 0. & $\begin{array}{l}3253 \\
\ldots\end{array}$ \\
\hline $\begin{array}{l}\text { A I IMUTII } \\
\text { DECREES }\end{array}$ & $\begin{array}{l}\text { HE ICHI } \\
\text { HE TERS }\end{array}$ & $\begin{array}{c}\text { EXPOSUKR } \\
\text { GH-SEC/CU.A } \\
\times 1 \cup E+0\end{array}$ & $\begin{array}{c}F / 0 \\
S E C /: U . M\end{array}$ & $\begin{array}{l}10 / 0 \\
1 / 56.4\end{array}$ & $\begin{array}{l}\text { UISTALICE } \\
\text { METERS }\end{array}$ & $\begin{array}{l}\text { AITMUTH } \\
\text { DECREES }\end{array}$ & $\begin{array}{l}\text { HE ICIST } \\
\text { ME TERS }\end{array}$ & $\begin{array}{c}\text { EXPQSURE } \\
\text { CH-SEC/CU.H } \\
\times 1 O E+6\end{array}$ & $\begin{array}{c}\text { E'O } \\
\text { SEC/CU.H }\end{array}$ & $\begin{array}{l}\text { ru/u } \\
\text { I/SO.H }\end{array}$ & $\begin{array}{l}\text { DISTANCE } \\
\text { METERS }\end{array}$ \\
\hline
\end{tabular}




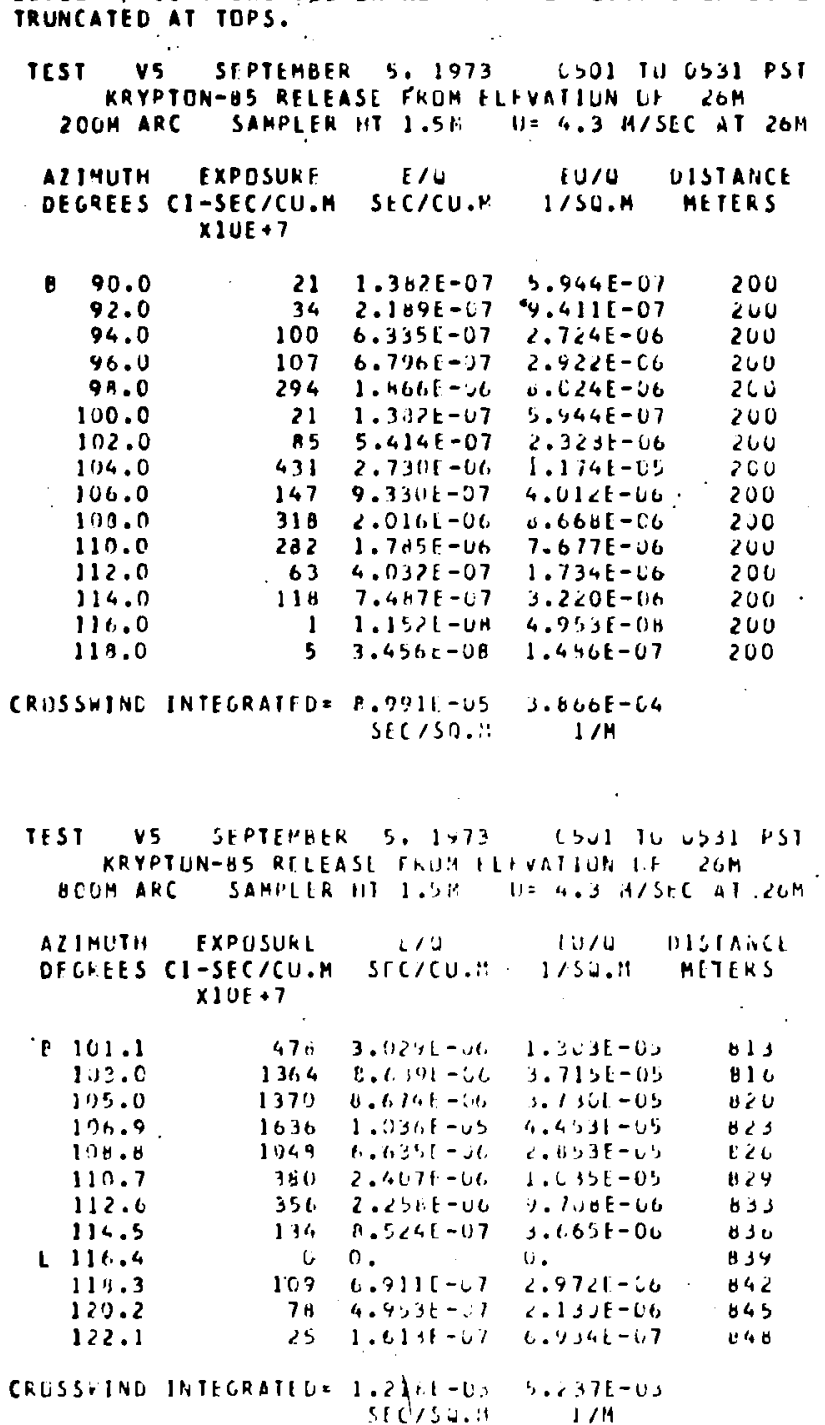


TOWER UATA FIJLLUW....

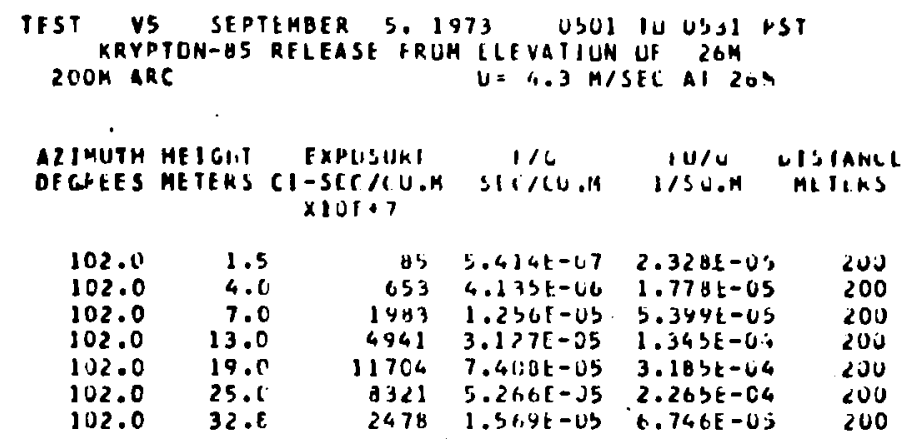

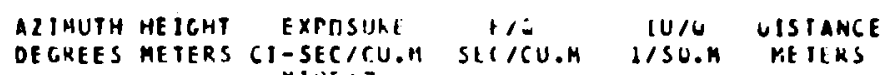
$\times \mathfrak{L O E}+7$

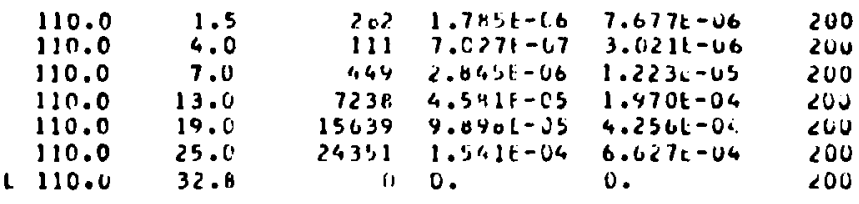

\begin{tabular}{|c|c|c|c|c|c|}
\hline $\begin{array}{l}2 \text { IMUTH } \\
\text { EGREES }\end{array}$ & $\begin{array}{l}\text { HE IGHT } \\
\text { METERS }\end{array}$ & $\begin{array}{c}\text { EXPOSIIRF } \\
C I-S E C / C U . M \\
\times 10 t+7\end{array}$ & $\begin{array}{c}f / C \\
\text { SEC / }\end{array}$ & $\begin{array}{l}r U / 0 \\
\text { I/SU.H }\end{array}$ & $\begin{array}{l}\text { UISTANCE } \\
\text { METEKS }\end{array}$ \\
\hline $\begin{array}{l}118.0 \\
114.0 \\
114.0 \\
110.0 \\
118.0 \\
118.0 \\
119.0\end{array}$ & $\begin{array}{r}1.5 \\
4.0 \\
7.0 \\
13.0 \\
19.0 \\
25.0 \\
32.0\end{array}$ & $\begin{array}{r}5 \\
74 \\
154 \\
114 \\
1654 \\
2644 \\
262 ?\end{array}$ & $\begin{array}{l}3.456 t-00 \\
4.773 t-07 \\
4.7911-07 \\
1.6261-06 \\
1.017 t-05 \\
1.67 .6 t-05 \\
1.600 t-05\end{array}$ & $\begin{array}{l}1.4 t 6 t-07 \\
2.031 E-06 \\
4.21 U E-06 \\
1.129 t-05 \\
4.5 C 2 t-05 \\
7.147 t-05 \\
7.138 t-05\end{array}$ & $\begin{array}{l}200 \\
200 \\
200 \\
200 \\
200 \\
200 \\
200\end{array}$ \\
\hline
\end{tabular}

MZIHUTH HEIGHT EXPISIIKF I/O LU/J UISIANCE DECREES METERS CI-STC/CU.M SECICU.M I/SH.M HEILRS $\times j 01+7$
N 126.0
1.5
$v \quad 0$
0.
200

AZIMUTH HEIGHT EXPQSURE H/U CL/J UISTANCE DECFEES METERS CI-SECICU.M SLIICU.M IISC.N. METERS $\times 1 \mathrm{OE}+7$
$N 134.0$
1.5
i) 0.
0.
200 


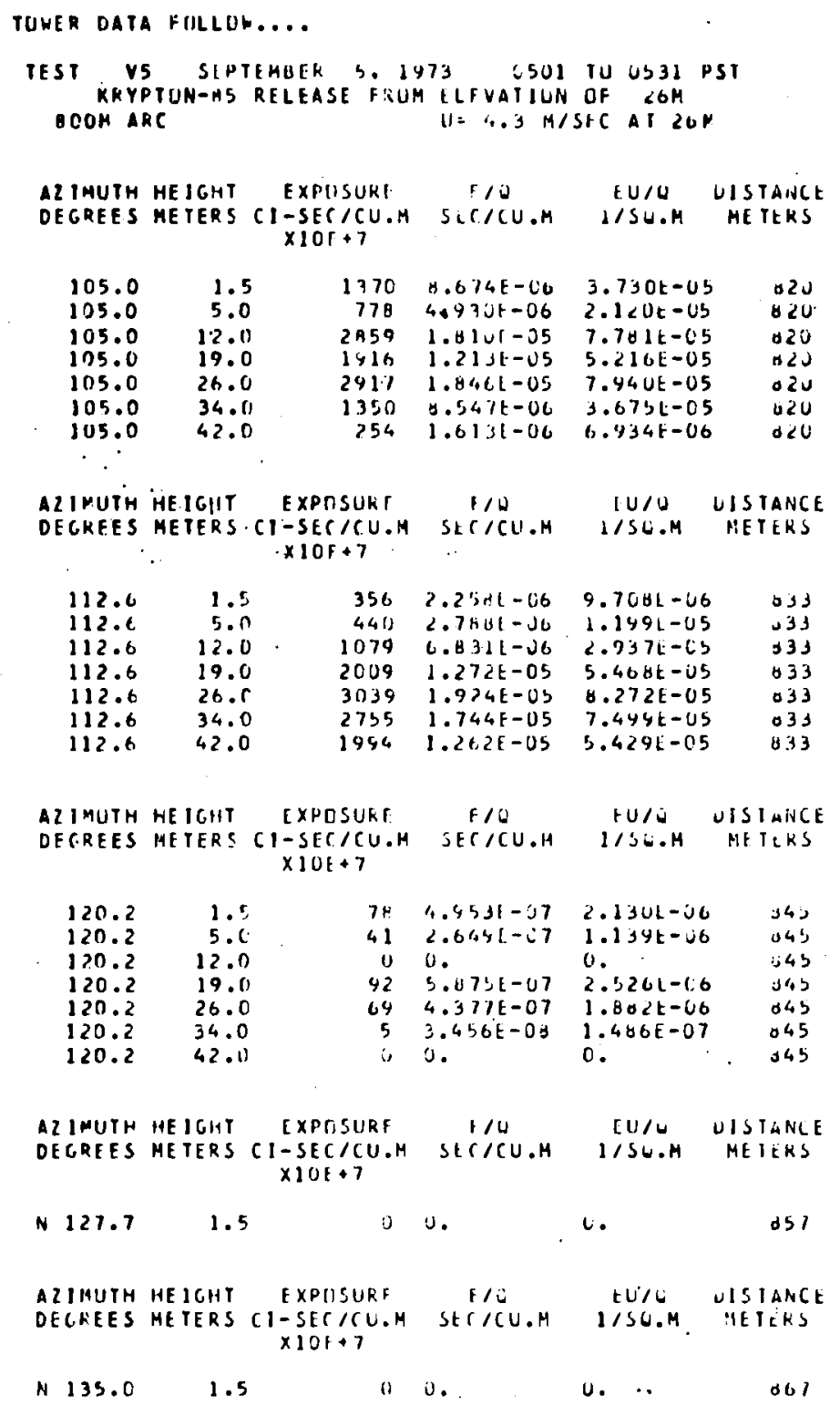




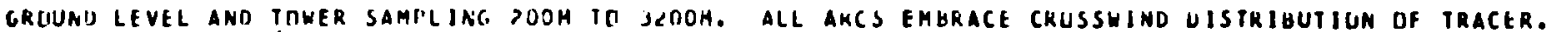
TOHERS TRUNCATF TUP OF VIRTICAL IISTRIRUTIUN. BUT IXTKAPULAIJUN WLULD NDI UE UNREASUNABLE.

\begin{tabular}{|c|c|c|}
\hline $\begin{array}{l}\text { EST } \\
\text { Z INC } \\
2 \text { OOM AI }\end{array}$ & $\begin{array}{l}\text { 6 SFPTLABE } \\
\text { SULFIUE RFEE } \\
\text { RC SAMPLER }\end{array}$ & $\begin{array}{l}\text { A } 13.1973 \\
\text { ASt FRUM IL } \\
\text { HT } 1.5 \mathrm{SM}\end{array}$ \\
\hline $\begin{array}{l}\text { ALIMUTH } \\
\text { DEGREES }\end{array}$ & $\begin{array}{c}\text { EXPOSURE } \\
\text { GH-SEC } / C U . M \\
\times I O E+6\end{array}$ & $\begin{array}{c}\text { E } / U \\
S E C / C U . M\end{array}$ \\
\hline $\begin{array}{l}102.0 \\
104.0 \\
106.0 \\
100.0 \\
110.0 \\
112.0 \\
114.0 \\
116.0 \\
118.0 \\
120.0 \\
122.0 \\
124.0 \\
126.0 \\
129.0 \\
130.0 \\
132.0 \\
134.0\end{array}$ & $\begin{array}{r}0 \\
22 \\
12 \\
229 \\
2357 \\
8220 \\
15076 \\
14767 \\
12933 \\
9623 \\
10499 \\
7446 \\
3301 \\
750 \\
229 \\
5 \\
0\end{array}$ & $\begin{array}{l}0 . \\
9.779 E-199 \\
5.334 E-J 9 \\
9.912 E-C 4 \\
1.016 E-06 \\
3.543 E-06 \\
6.494 E-06 \\
6.365 E-06 \\
5.575 E-06 \\
4.234 E-06 \\
4.648 E-06 \\
3.382 E-06 \\
1.423 E-U 6 \\
3.236 E-U 7 \\
9.912 E-C 8 \\
2.223 E-03 \\
0 .\end{array}$ \\
\hline
\end{tabular}

CRUSSHIND INTEGRATED= 2.602 E-04

\footnotetext{
CRUSSHINO INTEGRATEOE 2 SECISH.M
}

OS16 III 0546 PST FVATIUN LF 2GM $U=6.4 \mathrm{H} / \mathrm{SEC}$ AI $26 \mathrm{M}$ EUIO DISTANCE IISJ.M MEIEKS

U. $259 E-68$

$3.414 \mathrm{E}-\mathrm{OH}$ $6.344 E-07$

6. $503 E-06$ $2.260 E-03$ $4.159 t-U 5$ 4.074E-05 $3.560 E-25$ 2.710E-05 $3.007 E-O S$ 2. $165 \mathrm{E}-0 \mathrm{~S}$ $9.136 E-06$ $2.071 E-06$ $0.344 E-07$ $1.422 E-06$ 0 .

$1.665 E-03$ $1 / \mathrm{H}$
200

200

200

2110

200

200

200

200

200

200

200

200

200

200

200

TEST VO SEPIEMBER 13, 1973 US16 1U C546 PST IIHC SULFIDE RELEASE PRUM LLEVATIUN OF 264 4OOM ARC SAMPLER HI $1.511 \quad U=6.4 \mathrm{M} / \mathrm{SEC}$ AI $2 G \mathrm{M}$

\begin{tabular}{|c|c|c|c|c|}
\hline $\begin{array}{l}\text { ALI:AUTH } \\
\text { OEGREES }\end{array}$ & $\begin{array}{l}\text { EXPOSUKE } \\
\text { GN-SEC/CU.H } \\
\times 1 O E+6\end{array}$ & $\begin{array}{c}E / O \\
S E C / C U . M\end{array}$ & $\begin{array}{l}\text { LU/O } \\
\text { I/SU.H }\end{array}$ & $\begin{array}{l}\text { DISIANCE } \\
\text { MEIERS }\end{array}$ \\
\hline $\begin{array}{l}86.0 \\
90.0\end{array}$ & $\begin{array}{l}0 \\
1\end{array}$ & $\begin{array}{l}1.66 .1 \bar{E}-10 \\
6.673 \mathrm{E}-10\end{array}$ & $\begin{array}{l}1.16 \cup E-09 \\
4.271 E-J 4\end{array}$ & $\begin{array}{l}400 \\
400\end{array}$ \\
\hline 94. & 3 & $1.501 E-39$ & $9.6 i 9 E-C 9$ & 400 \\
\hline 98.0 & 30 & $1.335 t-08$ & $t .542 t-08$ & 400 \\
\hline$* 102.0$ & 0 & $3.337 E-1 C$ & $2.135 E-09$ & 400 \\
\hline E 106.0 & 45 & $1.952 \mathrm{t}-0.4$ & $1.2 .9 E-07$ & 400 \\
\hline $\begin{array}{l}110.0 \\
114.0\end{array}$ & $\begin{array}{r}907 \\
12710\end{array}$ & $\begin{array}{l}3.912 E-57 \\
5.4 \times 1 E-36\end{array}$ & $\begin{array}{l}1.504 E-06 \\
3.538 E-C 5\end{array}$ & $\begin{array}{l}400 \\
400\end{array}$ \\
\hline 118.0 & 6268 & $2.702 E-J t$ & $1.724 E-05$ & 402 \\
\hline 122.0 & 10416 & $4.491[-06$ & $\angle . B 74 E-U S$ & 400 \\
\hline 126.0 & 4190 & $1.306 E-U C$ & $1.156 E-05$ & 400 \\
\hline 130.0 & 168 & $7.257 \mathrm{E}-08$ & $4.645 E-07$ & 400 \\
\hline 134.0 & 27 & $1.201 E-08$ & $\begin{array}{r}7.680 E-08 \\
3.030 E-C 8\end{array}$ & $\begin{array}{l}400 \\
400\end{array}$ \\
\hline 142 & 6 & $2.664 E-00$ & $1.7 \cup B E-O B$ & 400 \\
\hline 146.0 & 4 & $1.035 \mathrm{E}-04$ & $1.174 E-0 d$ & 400. \\
\hline
\end{tabular}

CROSSHIND INTEGRATED= $4.189 E-04 \quad 2.681 E-03$

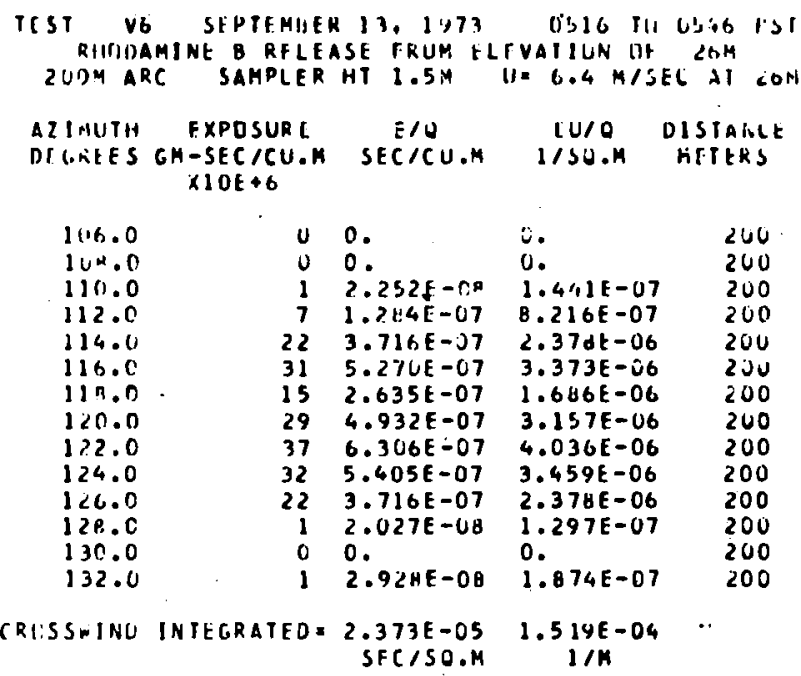

RHODAMINE B

10OM ARC DATA

MISSING 


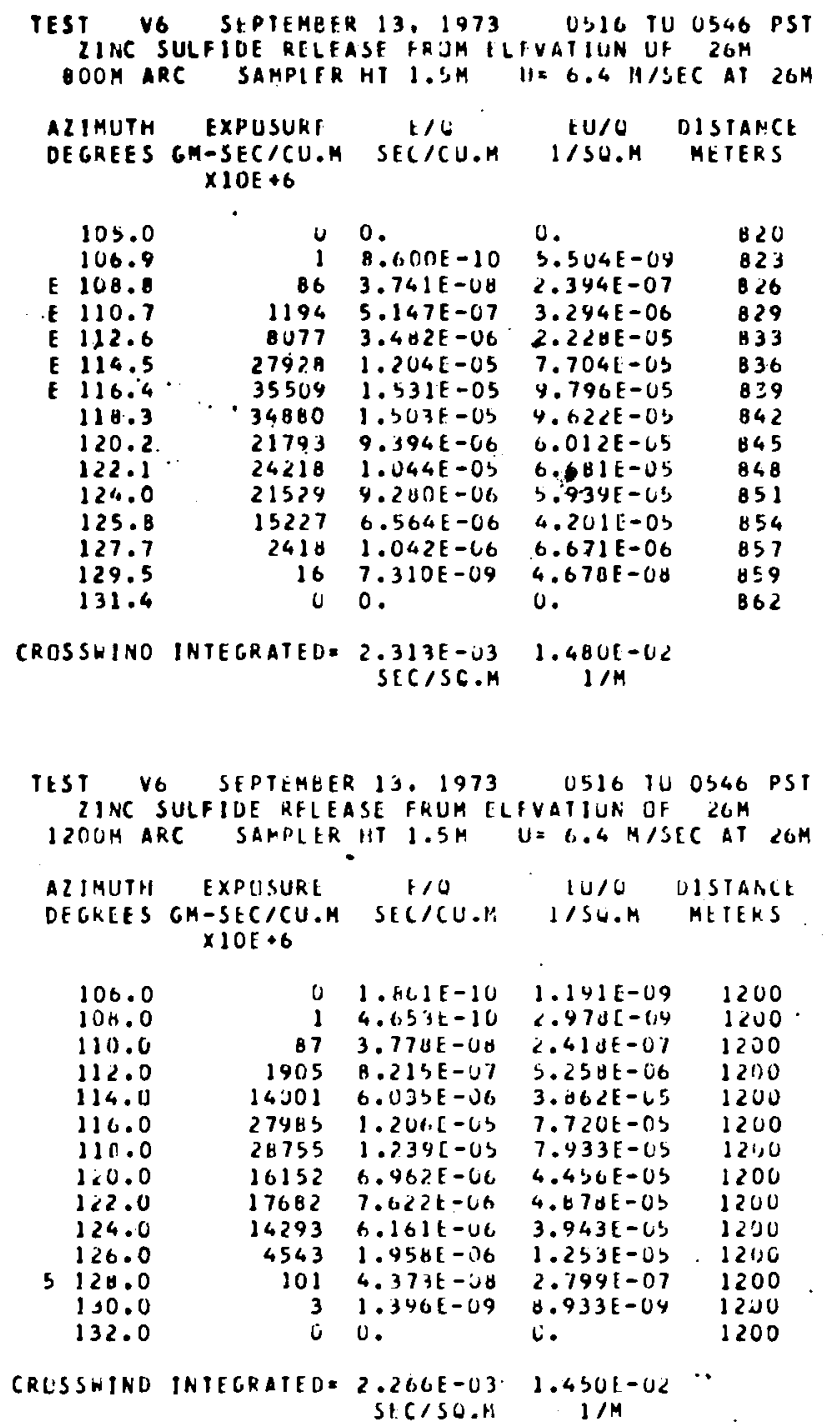
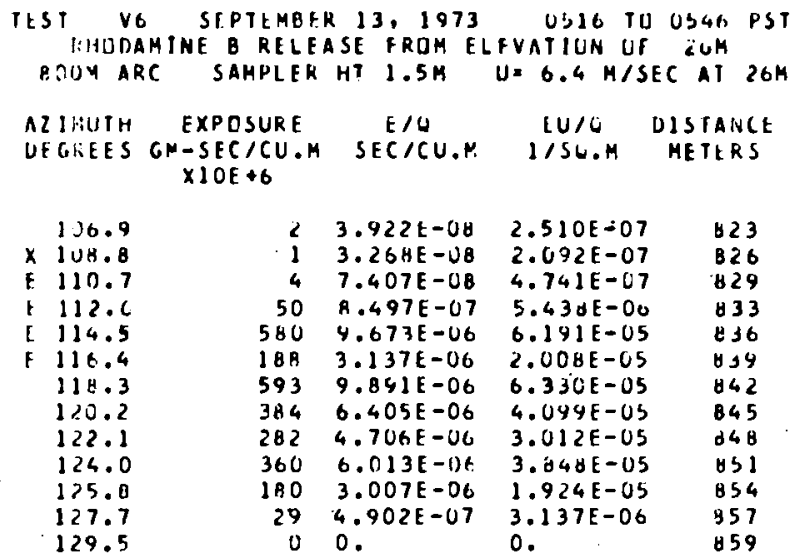

CRESSHINO INTEGRATED $=1.233 E-03$ SEC/SO.H

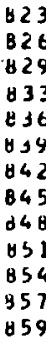

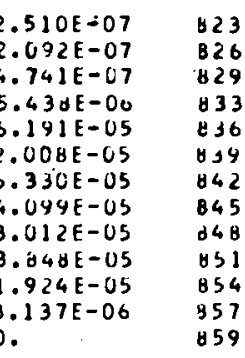

$1 / M$
$7.869 \mathrm{E}-03$

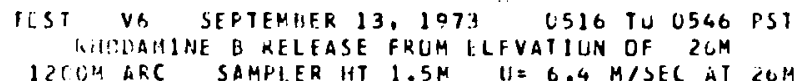
ALIUITH EXPOSURE EISTANCE DEGRES CM-SECICU.M SECICU.M IISO.M MEILKS $\times 10 E+6$

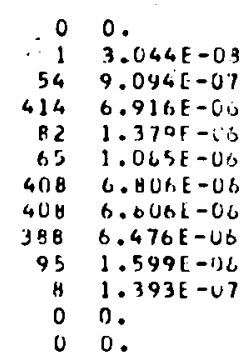
$S F(150.4$

CRISSSINO INTEGRATEO= 1.347 E-03 0.618E-03

0 .

1200

$1.96 \mathrm{EE}-07$ 1200 $5.420 \mathrm{~F}-06 \quad 1200$ $4.426 \mathrm{E}-115 \quad 12 \div 0$ A. $124 \mathrm{E}-\mathrm{OC} \quad \mathrm{L}, \mathrm{UU}$ $6.9411-06 \quad 1200$ $4.15 t, t-C b \quad 1200$ $4.356 E-05 \quad 1200$ $\begin{array}{ll}4.356 E-05 & 1200 \\ 4.165 E-05 & 1200\end{array}$ 1. $0<3[-05$ 1. $91 \mathrm{~d}[-07$ 0.1260 0.1200 $1 / M$ 
TEST V6 SEPIEABER 13, 1973 0516 TO 0546 PST 2 INC SULFIOE RELEASE FRUM ELFYATIUN OF $26 \mathrm{M}$ 1600M ARC SAMPLER MT $1.5 \mathrm{M}$ U. $6.4 \mathrm{M} / \mathrm{SEC}$ AT $26 \mathrm{M}$

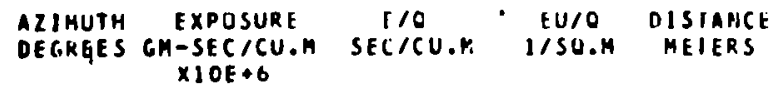

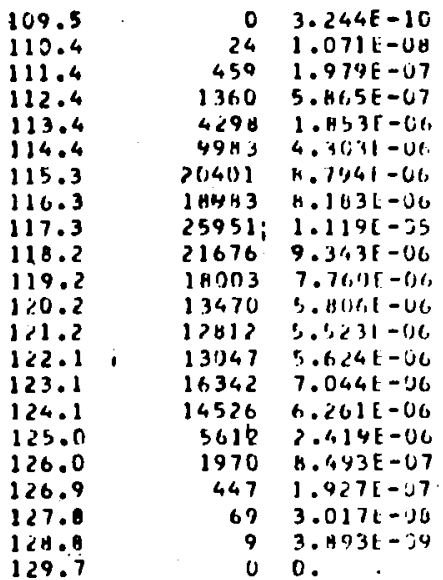

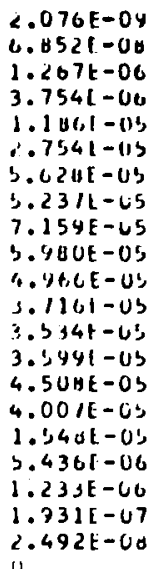

1630

1632

1633

1635

1631

1630

1640

1641

1643

1614

1646

1641

1349

1630

1632

1653

1635

1656

1658

16,9

1660

1662

CROSSININU INTEGRATEDE $2.390 E-U 3$ 1.S3UE-0.

$$
\text { SFCISO.M } 1 / M
$$

IEST VO SIPTLMLEK $13,1 \$ 73$ US1L TL U:46 NST ZINC SULFIDE PHLEASF FRIIH LLFVATIUR UF $26 \mathrm{M}$ 32 UOM ARC SAMPIER HT $1.5 \mathrm{H} \quad \|=6.4 \mathrm{M} / \mathrm{SEC}$ AT $26 \mathrm{M}$

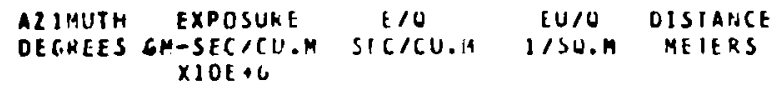

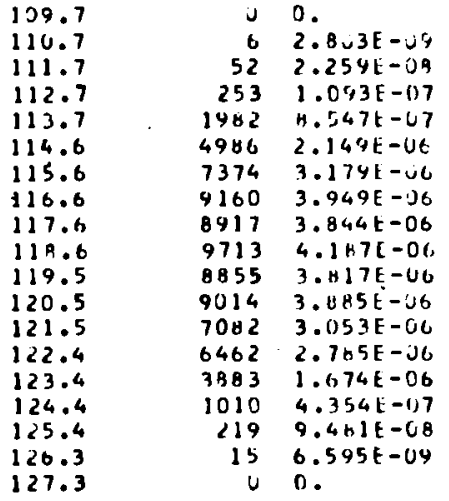

L. 3232

$1.794 E-C B \quad 3233$ $1.44 \mathrm{CL}-07 \quad 3235$ $6.496 \mathrm{E}-07 \quad 3237$ $5.4701-06 \quad 3230$ $1.3751-05 \quad .3240$ $2.034 \mathrm{E}-\mathrm{LS} \quad 3241$ $2.527 \mathrm{E}-U \mathrm{~S} \quad 3243$ $\begin{array}{ll}2.5 \angle 7 E-U S & 3243 \\ 2.46 U E-C 5 & 3245\end{array}$ 2. 6 HUE- 135 3246 $2.4431-03$ 3260 $2.487 E-05$ 3249 1.954 E-US 3251 $1.763 \mathrm{E}-\mathrm{U}$ S $32 \% 2$ $1.071 E-05 \quad 3253$ C. $787 E-06 \quad 3255$ 6. $668 E-67 \quad 3236$ 4.221E-C 3250 u.

3250

CROSSHINO INTEGRATED= $1.001 E-U 3 \quad 1.204 E-02$
IEST VG SFPTEMBER 13, 1973 0516 TU 0546 PST RHLDAMINE B RELEASE FROM ELEVATIUN OF 264 $160 O M$ ARC SAMPIER HT $1.5 \mathrm{M} U=6.4 \mathrm{M} / \mathrm{SEC}$ A 2 ON XPOSURE
AI IiNUTII UEGREES GR-SEC/CU. $\times 20 E+6$

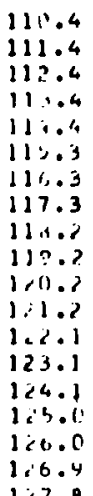

$1 \cdot 7 \cdot 8$

(KUSSAIHI) INTERRATEU= 2.07SE-OS
SFC 10

EUIU DISIAHCE I/SO.H MEILRS

v.

$$
\begin{array}{rl}
J & 0 \\
6 & 1.063 t-07 \\
23 & 10.3101-07 \\
115 & 1.2631-06
\end{array}
$$

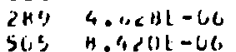

SC5 $5.1201-06$

$531 \quad 8.051 \mathrm{~L}-06$

$4 A 7 \quad 4.132 E-06$

29 B $4.971 t-46$

$2.43 .136 \mathrm{~L}-110$

$2713.673 \mathrm{E}-\mathrm{U} 6$

$37 \mathrm{I} \quad 6.264 \mathrm{t}-26$

211] $4.634[-1) / 3$

$1432.399 E-06$

$477.9021-07$

$1.365[-1] 7$

$1>.074 \mathrm{t}-10$

$6.6055-07$ 2.7byt-UG

$1.2601-1) b$

$3.0401-0$

$5.3191-01$

3.04t1-US

$5.664 \mathrm{E}-\mathrm{UD}$

$3.2 \mathrm{SSF}-\mathrm{U}$

$3.9176-1)$

$3.11121-05$

c.34ir-0s

$4.0 C y[-35$

$2.998 E-03$

$1.396 t-0$

$5.057 \mathrm{t}-0 \mathrm{c}$

$0.7361-07$

$1.834 \mathrm{E}-131$

1632

1633

1635

16,37

1630

1040

1641

1643

1644

$16,1,0$

1647

1647

16,00

1652

16,3

1650

1558

16,59
2. $363 \mathrm{t}-\mathrm{Cl}$

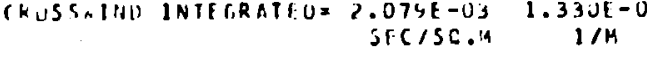

ILST VG SHPTEHBER 13,1413 OS16 TL CS46 PST HIIJUAPINE B RELEASE FRLIH ELFVATIUH UF ZIOR: 320JiA ARC. SAMPIER HT $1.5 \mathrm{Y}$ $\mathrm{I}=6.4 \mathrm{H} / \mathrm{SEC}$ AI 26.4

$$
\text { ATIIITH }
$$

$t / 0$ DFREES GA-SEC/CI $\times 10 E+6$

110.7
111.7
117.7
113.7
114.6
115.6
116.6
117.6
113.6
119.5
123.5
121.5
122.4
123.4
124.4
123.4
123.3

$\begin{array}{rl}0 & 0.0 \\ 1 & 1.749 E-U 0 \\ 10 & 1.819 E-07 \\ 59 & 9.877 E-07 \\ 195 & 3.093 E-J 6 \\ 275 & 4.587 E-06 \\ 295 & 4.977 E-06 \\ 283 & 4.717 E-66 \\ 263 & 4.392 E-06 \\ 240 & 4.003 E-06 \\ 193 & 3.223 E-06 \\ 177 & 2.963 E-06 \\ 119 & 1.488 E-06 \\ 69 & 1.163 E-06 \\ 20 & 3.379 E-117 \\ 4 & 7.147 E-08 \\ 0 & 7.797 E-09\end{array}$

0.

LU/O DISIANCL $1 / 56 . H$ MLItRS

$1.164 E-06 \quad 3237$

$6.321 E-116.1236$

$1.974 \mathrm{E}-05 \quad 3240$

$2.9361-45 \quad 3<41$

3.1 $85 E-03 \quad 3243$

$3.014 E-05 \quad 9245$

$2.4111-053240$

$2.502 E-05 \quad 3240$

$2.063 E-0 S \quad 3249$

$2.063 E-05$
$1.696 E-05$
1.275251

$1.273 E-C 5 \quad 3252$

$7.444 E-06 \quad 3253$

$2.162 E-06 \quad 3255$

$4.574 \mathrm{E}-07 \quad 3256$

$4.990 \mathrm{E}-08 \quad 3256$

CKUSSWINA IATEGRATFDE $2.030 E-03$ 1.24YE-02 
IOWER CATA FILLOH....

\section{TESI V6 SEPTEMBER 13, 1973 O516 IJ OS46 PST ZINC SULFIDE RELEASE FROH ELEVATION OF $26 \mathrm{M}$ 2OOM ARC U. $6.4 \mathrm{H} / \mathrm{SEC}$ AT $26 \mathrm{M}$}

\begin{tabular}{|c|c|c|c|c|c|}
\hline $\begin{array}{l}\text { ALIMUTH } \\
\text { DEGKEES }\end{array}$ & $\begin{array}{l}\text { HE IGHT } \\
\text { ME TERS }\end{array}$ & $\begin{array}{l}\text { EXP.OSURE } \\
\text { GH-SEC / CU.H } \\
\times 1 O E+6\end{array}$ & $\begin{array}{c}E / O \\
S E C / C U . M\end{array}$ & $\begin{array}{l}E U / U \\
I / S C . H\end{array}$ & $\begin{array}{l}\text { UISTANCE } \\
\text { ME TERS }\end{array}$ \\
\hline $\begin{array}{l}102.0 \\
102.0 \\
102.0\end{array}$ & $\because$ & $\begin{array}{l}6 \\
4 \\
0\end{array}$ & $\begin{array}{l}3.000 E-09 \\
1.920 E-09 \\
0 .\end{array}$ & $\begin{array}{l}1.920 E-00 \\
1.229 E-10 \\
0 .\end{array}$ & $\begin{array}{l}200 \\
200 \\
200\end{array}$ \\
\hline
\end{tabular}

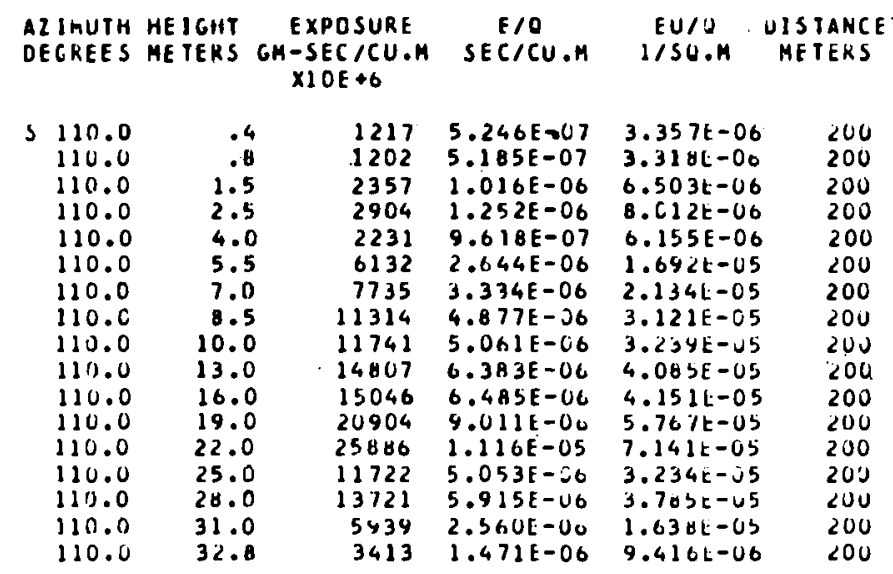

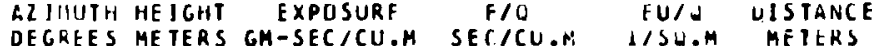
$X I O E+6$

$\begin{array}{lr}118.0 & .4 \\ 110.0 & .8 \\ 118.0 & 1.5 \\ 118.0 & 2.5 \\ 118.0 & 4.0 \\ 118.0 & 5.5 \\ 116.0 & 7.0 \\ 118.0 & 8.5 \\ 118.0 & 10.0 \\ 110.0 & 13.0 \\ 118.0 & 16.0 \\ 110.0 & 19.0 \\ 118.0 & 22.0 \\ 118.0 & 25.0 \\ 110.0 & 28.0 \\ 118.0 & 31.0 \\ 111.0 & 32.0 \\ \end{array}$

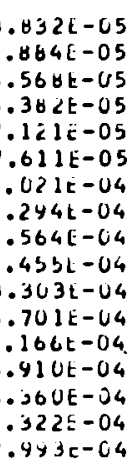

TULEF CATA FILLLLW....

TEST VO SEPTEMBER 13,1973 OS16 TO 0546 PST KHOOAHINE B RELEASE FROM ELFYATICN DF $26 \mathrm{M}$

2OOH AKC

$U=6.4 \mathrm{M} / \mathrm{SEC}$ A $26 \mathrm{M}$
AZIHIJTH HEIGHT EXPOSURE : F/O EU/U UISTANCE DECP.EES METERS GM-SEC/CU.M. SEC/CU.M I/SO.M MEIERS $\times 10 F+6$

N IU2.C .4 00 .

0.

200

A2IMUIH HEIGHT EXPIISURE IIO EUIO UISTANCE DEGKEES METERS CM-SECICU.H SEI/CU.H 1/SO.M METERS
XIUF+6

$\begin{array}{rr}5110.0 & .4 \\ 110.0 & .0 \\ 110.0 & 1.5 \\ 110.0 & 2.5 \\ 110.0 & 4.0 \\ 110.0 & 5.5 \\ 110.0 & 7.0 \\ 110.0 & 8.5 \\ 110.0 & 10.0 \\ 11100 & 13.0 \\ 110.0 & 16.0 \\ 110.0 & 14.0 \\ 110.0 & 22.0 \\ 110.0 & 25.0 \\ 110.0 & 28.0 \\ 110.0 & 31.0 \\ 110.0 & 32.0\end{array}$

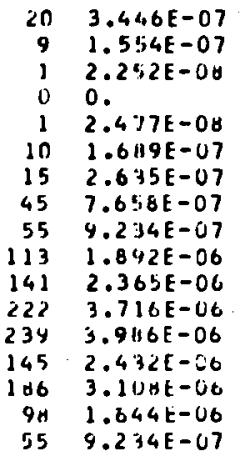

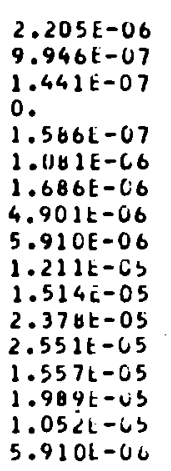

200
200
200
200
200
200
200
200
200
200
200
200
200
200
200
200
200

AZI:IUIH HEIGHT DITIUTH HEIGHI EXPOSURE EXPQSURE F/O $\times 10 \mathrm{~F}+6$

\begin{tabular}{|c|c|}
\hline 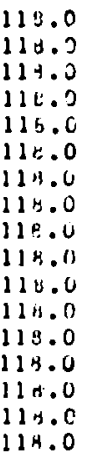 & $\begin{array}{r}.4 \\
1.0 \\
1.5 \\
2.5 \\
4.0 \\
5.5 \\
7.0 \\
1.5 \\
15.0 \\
13.0 \\
16.0 \\
14.0 \\
22.0 \\
25.0 \\
28.0 \\
31.0 \\
32.0\end{array}$ \\
\hline
\end{tabular}

EUIU UISTANCE ISW.M METEKS

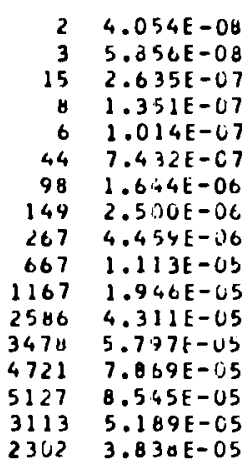

200
200
200
200
200
200
200
200
200
200
200
200
200
200
200
200
200

AZIMUIH THEICHT DECHEES METERS GM-SEC/CU X
$\times 1 O E+C$

$\begin{array}{ll}0 & 126.0 \\ 0 & 126.0 \\ 120.0 \\ 126.0 \\ 126.0 \\ 126.0 \\ 126.0 \\ 126.0 \\ 126.0 \\ 126.0 \\ 126.0 \\ 126.0 \\ 126.0 \\ 126.0 \\ 126.0 \\ 126.0 \\ 126.0\end{array}$

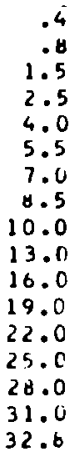

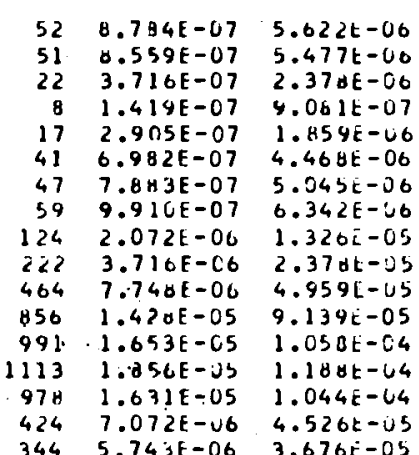

$3.676 \bar{E}-05$
EU/O UISTANCE I/SO.M METERS OEGREES METERS GM-SEC /CU
$\times 1 O E+6$

$\begin{array}{llr}9126.0 & .4 \\ 9126.0 & .8 \\ 126.0 & 1.5 \\ 126.0 & 2.5 \\ 126.0 & 4.0 \\ 126.0 & 5.5 \\ 126.0 & 7.0 \\ 126.0 & 8.5 \\ 126.0 & 10.0 \\ 126.0 & 13.0 \\ 126.0 & 16.0 \\ 126.0 & 19.0 \\ 126.0 & 22.0 \\ 126.0 & 23.0 \\ 126.0 & 28.0 \\ 126.0 & 31.0 \\ 120.0 & 32.8\end{array}$

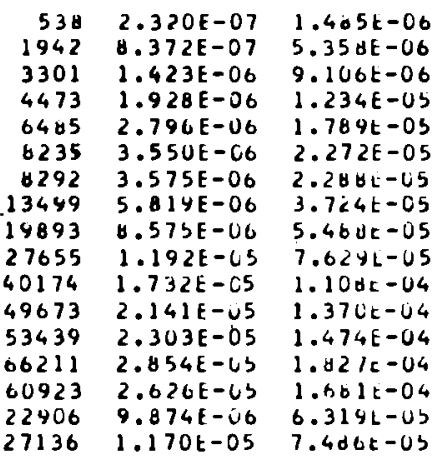

200
200
200
200
200
200
200
200
200
200
200
200
200
200
200
200
200

Q2 IIIUTH HE IGHT OEGREES METERS GHOSEC SURE EXPOSURE
$X I O E+6$
F 10

EU/N UISTANCE I/SU.M METERS
A I IIUTH HE IGH DFCKCES METERS GM-SEC/CU.A SEC/CU.M $x \perp \cup E+C$
$\begin{array}{ll}.920 L-O R & 200 \\ . & 20 U \\ & 200\end{array}$
$\begin{array}{r}134.0 \\ \hline \quad 134.0\end{array}$
.4
.6
1.5
$6 \quad 3.000 E-09$
0 .
N. 134.0
.4
i) 0 .
134.0
0 0.
. 0

I/SC.M MEIERS 
TOWER DATA PLLOW....

TEST Yo SEPTEMBER 13:1973 0516 TO 0546 PST ZINC SULFIDE RELEASE FRON ELEVATIUN OF $26 \mathrm{H}$ BOOM ARE
THEE DATA FOLLOW....

ILST V6 SEPTEMBER 13, 1973 O516 TO 0546 PST EIIUDAMINE RELEASE FROM ELFYATION OF $26 \mathrm{~A}$

DOUK AKC UE 6.4 MISEC AT $26 \mathrm{M}$

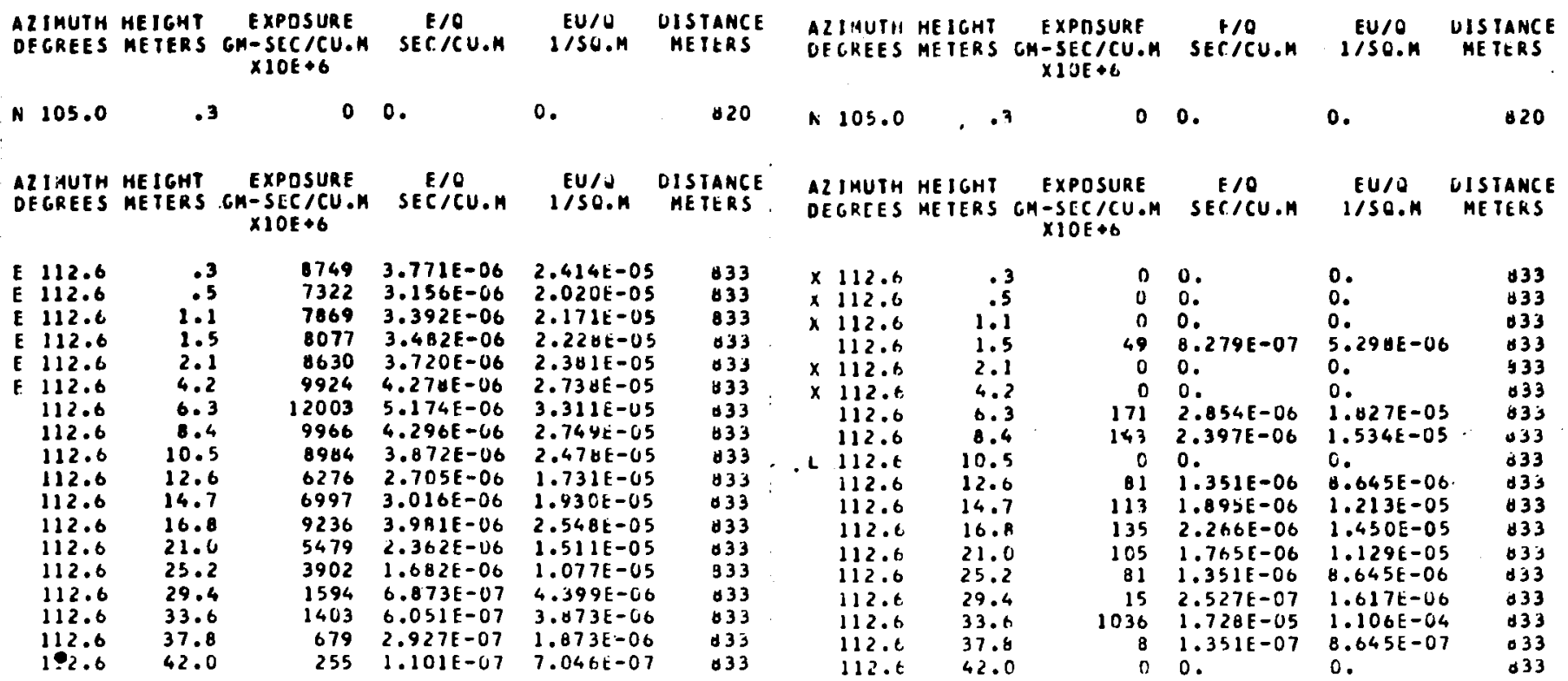
FIO DISTANCE $\times 1 O E+6$

$\begin{array}{ll}120.2 & .3 \\ 120.2 & .5 \\ 120.2 & 1.1 \\ 120.2 & 1.5 \\ 120.2 & 2.1 \\ 120.2 & 4.2 \\ 120.2 & 6.3 \\ 120.2 & 8.4 \\ 120.2 & 10.5 \\ 120.2 & 12.6 \\ 120.2 & 14.7 \\ 120.2 & 16.8 \\ 120.2 & 21.0 \\ 120.2 & 25.2 \\ 120.2 & 29.4 \\ 120.2 & 33.6 \\ 120.2 & 37.8 \\ 120.2 & 42.0\end{array}$

$\begin{array}{lll}20477 & 8.027 E-06 & 5.649 E-05 \\ 21107 & 9.098 E-06 & 5.823 E-05 \\ 18638 & 8.034 E-06 & 5.142 E-05 \\ 21793 & 9.394 E-06 & 6.012 E-05 \\ 26795 & 1.155 E-05 & 7.392 E-05 \\ 25960 & 1.119 E-05 & 7.162 E-05 \\ 28769 & 1.240 E-05 & 07.937 L-U 5 \\ 5856 & 2.524 E-06 & 1.615 E-05 \\ 23733 & 1.023 E-05 & 6.547 E-05 \\ 19608 & 8.452 E-06 & 5.409 E-05 \\ 15917 & 6.861 E-06 & 4.391 E-05 \\ 21766 & 9.382 E-06 & 6.0 C 5 E-05 \\ 20132 & 8.676 E-06 & 5.554 E-05 \\ 17545 & 7.563 E-06 & 4.840 E-05 \\ 14893 & 6.420 E-06 & 4.109 t-05 \\ 19814 & 0.541 E-00 & 5.466 E-05 \\ 12443 & 5.364 E-00 & 3.433 t-05 \\ 7760 & 3.340 E-06 & 2.143 L-05\end{array}$

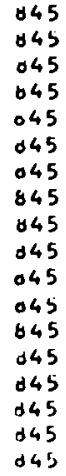

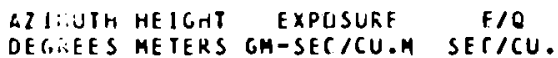
DECLICU.M SET/CU.M $X \perp 0 E+C$

$\begin{array}{ll}120.2 & .3 \\ 120.2 & .5 \\ 120.2 & 1.1 \\ 120.2 & 1.5 \\ 120.2 & 2.1 \\ 120.2 & 4.2 \\ 120.2 & 6.3 \\ 120.2 & 8.4 \\ 120.2 & 10.5 \\ 120.2 & 12.6 \\ 120.2 & 14.7 \\ 120.2 & 16.8 \\ 120.2 & 21.0 \\ 120.2 & 25.2 \\ 129.2 & 29.4 \\ 120.2 & 33.6 \\ 120.2 & 17.8 \\ 120.2 & 42.0\end{array}$

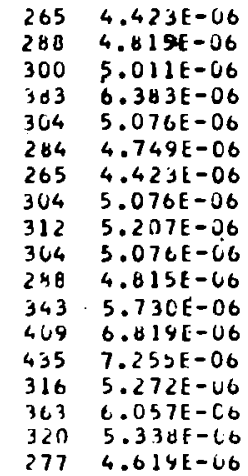

EU/O OISTANCE I/SO.M HETERS

$.431 \mathrm{k}-05$

$3.081 t-05$

$3.207 E-05$

$4.045 E-U 5$

$3.040 c-05$

$3.040 c-05$
$2.831 t-05$

$2.831 t-05$
$3.249 E-U 5$

$3.33<E-25$

$3.249 E-05$

$3.081 E-05$

$3.667 t-05$

$4.364 E-05$

$4.643 \mathrm{i}-05$

$3.374 E-U 5$

$3.8765-45$

$3.416 t-05$

$2.956 c-05$

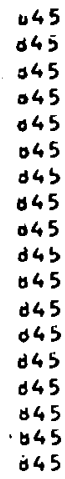

AZINUTH HEICHT WECLEES METEKS GHEXPIISUR

EXPIISUR
EXC/CU. $\times 10 r+6$

$\begin{array}{lr}127.7 & .3 \\ 127.7 & .5 \\ 127.7 & 1.1 \\ 127.7 & 1.5 \\ 127.7 & 2.1 \\ 127.7 & 4.2 \\ 127.7 & 6.3 \\ 127.7 & 8.4 \\ 127.7 & 10.9 \\ 127.7 & 12.6 \\ 127.7 & 14.7 \\ 127.7 & 16.4 \\ 127.7 & 21.0 \\ 127.7 & 23.2 \\ 127.7 & 24.4 \\ 127.7 & 33.6 \\ 127.7 & 37.8 \\ 127.7 & 42.0\end{array}$

\begin{tabular}{|c|c|c|}
\hline & & \\
\hline & & \\
\hline & & \\
\hline & & \\
\hline 16 & & $\theta$. \\
\hline & & \\
\hline 72 & & \\
\hline & & \\
\hline & & \\
\hline & 7 & .06 \\
\hline $\begin{array}{l}2405 \\
2013\end{array}$ & & 6. \\
\hline 20 & & \\
\hline $1 / 1$ & & - Oc \\
\hline 86 & & \\
\hline & 2 & 06 \\
\hline & & \\
\hline & & \\
\hline
\end{tabular}

AZ1:11STH HEIGHT DFGEEES METEKS CM-SEC/CU.H SETICU.M $\times 10 \mathrm{E}+6$

$\begin{array}{ll}857 & 127.7 \\ 057 & 137.7 \\ 857 & 177.7 \\ 057 & 127.7 \\ 857 & 127.7 \\ 057 & 127.7 \\ 057 & 127.7 \\ 057 & 127.7 \\ 057 & 127.7 \\ 051 & 127.7 \\ 857 & 127.7 \\ 057 & 137.7 \\ 051 & 127.7 \\ 057 & 127.7 \\ 057 & 127.7 \\ 057 & 127.7 \\ 057 & 127.7 \\ 057 & 127.7\end{array}$

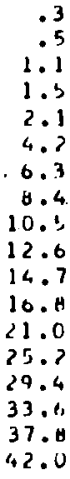

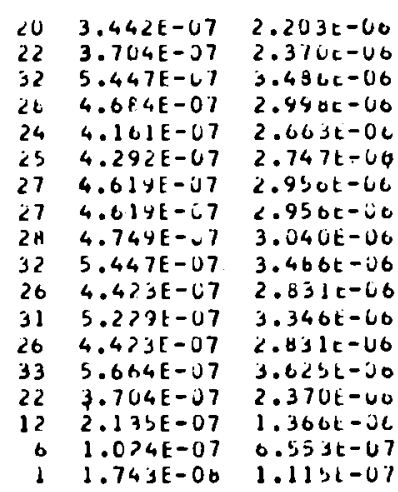

o5 5 d) 7 057 ab ab 7 057 357 057 d 57 051 os 7 657 057 557 نS 7 व 7 os 7 657
ALIILITI HEIGHT EXPOSURE H/U IU/L OISTANCE DEGKEES METERS GM-SECICU.M SECICU.M LISU.M METEKS $\times 101+1$
N 135.0

00.

6.

867

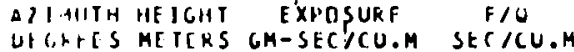
$\times 10 E+6$ 
TOHER DATA FOLLOW....

TEST VO SEPTEHEER 13. 1973 U316 TU 0546 PST IINC SULFIOE KELEASE FROM ELPYATIUN DF $26 \mathrm{G}$ $1600 \mathrm{M} A R C$ U 6.4 M/SEC AT $26 \mathrm{M}$

\begin{tabular}{|c|c|c|c|c|c|}
\hline $\begin{array}{l}\text { AZIMUTH } \\
\text { DE GREES }\end{array}$ & $\begin{array}{l}\text { HEICH T } \\
\text { ME TERS }\end{array}$ & $\begin{array}{c}\text { EXPOSURE } \\
\text { GM-SEC/CU.H } \\
\times 1 O E+6\end{array}$ & $\begin{array}{l}\text { E/O } \\
\text { SECC/CU.H }\end{array}$ & $\begin{array}{l}\text { EU/O } \\
\text { IESO.n }\end{array}$ & $\begin{array}{l}\text { DISTANCE } \\
\text { METERS }\end{array}$ \\
\hline 101.6 & .2 & D & 0. & 0 : & 1617 \\
\hline $\begin{array}{l}\text { AZIHUTH } \\
\text { DEREES }\end{array}$ & $\begin{array}{l}\text { HE ICHT } \\
\text { METERS }\end{array}$ & $\begin{array}{c}\text { EXPQSURE } \\
\text { EM-SEC/CU.H } \\
\text { XIOE+6 }\end{array}$ & $\begin{array}{c}E / O \\
S E C / C U . M\end{array}$ & $\begin{array}{l}\text { EU/O } \\
\text { I/SU.M }\end{array}$ & $\begin{array}{l}\text { DISTANCE } \\
\text { METERS }\end{array}$ \\
\hline $\begin{array}{l}109.5 \\
109.5 \\
109.5 \\
109.5 \\
109.5 \\
109.5 \\
109.5\end{array}$ & $\begin{array}{l}.0 \\
1.5 \\
1.6 \\
3.1 \\
6.2\end{array}$ & $\begin{array}{r}12 \\
122 \\
16 \\
0 \\
24 \\
21 \\
1\end{array}$ & $\begin{array}{l}3.828 E-09 \\
5.290 E-08 \\
7.323 E=09 \\
3.244 E-10 \\
1.071 E-08 \\
9.236 E-09 \\
7.696 E-10\end{array}$ & $\begin{array}{l}2.450 \mathrm{E}-08 \\
3.385 \mathrm{E}-07 \\
4.687 \mathrm{E}-08 \\
2.076 \mathrm{E}-09 \\
6.852 \mathrm{E}-08 \\
5.911 \mathrm{E}-08 \\
4.925 \mathrm{E}-09\end{array}$ & $\begin{array}{l}1630 \\
1630 \\
1630 \\
1630 \\
1630 \\
1630 \\
1630\end{array}$ \\
\hline
\end{tabular}

IEST VG SLPTEMBER 13, $1+73$ US16 TU 0S46 PST HIISTAIINE B R[LEASE FRUM LLFVATION UF 2OM 160011 ARS U

AZIYUTH HEIGHT EXPOSURF E/O EUIG DISTANCE UFCRLES METERS GM-SECICU.M SEC./CU.M J/SO.M METEKS $\times 10 E+6$

1. 101.6 . 0 0. 0 .

1617

AZJIUTH HEIGHT EXYOSURE EIO EU/O DISTANCE DEGREES METERS GM-SECICU.M SETICU.H I/SO.H METERS $\times 10 E+6$

$\begin{array}{lr}109.5 & .2 \\ 109.5 & .4 \\ 109.5 & .8 \\ 109.5 & 1.6 \\ 109.5 & 3.1 \\ 104.5 & 6.2 \\ 109.5 & 9.3 \\ 109.5 & 12.4 \\ 109.5 & 15.5 \\ 109.5 & 18.6 \\ 109.5 & 21.7 \\ 109.5 & 24.8 \\ 109.5 & 31.0 \\ 109.5 & 37.2 \\ 109.5 & 43.4 \\ 104.5 & 49.6 \\ 109.5 & 55.8 \\ 119.5 & 62.0\end{array}$

$\begin{array}{ll}5 & 9.339 E-08 \\ 7 & 1.322 E-07 \\ 0 & 0.0 \\ 3 & 6.170 E-08 \\ 1 & 0.1 \\ 4 & 7.759 E-08 \\ 0 & 0 . \\ 0 & 0 . \\ 2 & 4.310 E-08 \\ 6 & 0 . \\ 3 & 5.747 E-08 \\ 0 & 1.006 E-08 \\ 5 & 4.334 E-08 \\ 3 & 5.460 E-08 \\ 2 & 4.310 E-08 \\ 0 & 1.006 E-08 \\ 3 & 5.891 E-08 \\ 1 & 2.874 E-08\end{array}$

$\begin{array}{ll}5.977 E-07 & 1630 \\ 8.460 E-07 & 1030 \\ 0.9 & 1630 \\ 3.954 t-07 & 1030 \\ 0 . & 1630 \\ 4.966 t-07 & 1630 \\ 0 . & 1630 \\ 0 . & 1630 \\ 2.759 E-07 & 1630 \\ 0 . & 1630 \\ 3.678 E-07 & 1630 \\ 6.437 E-08 & 1630 \\ 5.977 t-07 & 1630 \\ 3.494 t-07 & 1630 \\ 2.759 E-07 & 1630 \\ 6.437 t-08 & 1630 \\ 3.770 E-07 & 1630 \\ 1.839 E-07 & 1630\end{array}$

AZ IRUTH HEIGHT
DEGREES METERS

EXPUSURF

F/O

EUIU DISTAT.CE $\times 10 E+6$

117.3
117.3
117.3
117.3
117.3
117.3
117.3
117.3
117.3
117.3
117.3
117.3
117.3
117.3
117.3
117.3
117.3
117.3
117.3

.2
.4
1.8
1.8
3.1
6.2
9.3
12.4
15.5
18.6
21.7
24.8
31.0
37.2
43.4
49.6
55.8
62.0

\begin{tabular}{|c|c|c|}
\hline $\begin{array}{l}26164 \\
19908 \\
26520 \\
25951 \\
27272 \\
23493 \\
24668 \\
27656 \\
25431 \\
23395 \\
25124 \\
23359 \\
17679 \\
17432 \\
14607 \\
6914 \\
1525 \\
375 \\
7\end{array}$ & $\begin{array}{l}1.128 E-05 \\
8.581 E-06 \\
1.143 E-05 \\
1.119 E-05 \\
1.176 E-05 \\
1.142 E-05 \\
1.063 E-05 \\
1.192 E-05 \\
1.096 E-0 S \\
1.004 E-05 \\
1.063 E-05 \\
1.007 E-U 5 \\
7.6>0 E-06 \\
7.514 E-06 \\
6.296 E-06 \\
2.980 E-06 \\
6.574 E-07 \\
1.619 E-07 \\
3.056 E-04\end{array}$ & $\begin{array}{l}7.218 E-05 \\
5.492 t-05 \\
7.316 E-05 \\
7.159 E-05 \\
7.523 t-05 \\
7.300 E-05 \\
6.805 t-05 \\
7.629 t-05 \\
7.016 E-05 \\
6.454 t-0 S \\
6.931 E-05 \\
6.444 t-05 \\
4.877 t-05 \\
4.809 E-05 \\
4.030 t-05 \\
1.908 E-05 \\
4.208 E-06 \\
1.036 E-06 \\
1.966 t-08\end{array}$ \\
\hline
\end{tabular}

1643

1043

1643

$164 \mathrm{~s}$

1643

1643

1643

1643

1643

1043

1643

1643

1043

1643

1643

1649

1043

1643
AIIIIUTH HEIGHT

DECAEES METERS GH SESSUR

\section{3}

117.3

117.3

117.3

117.3

117.3

117.3

$117: 3$

E 117.3

117.3

117.3

117.3

117.3

117.3

117.3

117.3

117.3

117.3

.117 .3

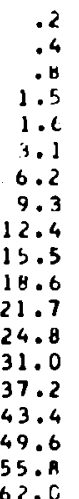

.2
.4
.8
1.5
1.6
3.1
6.2
9.3
12.4
15.5
18.6
21.7
24.8
31.0
37.2
43.4
49.6
55.9
62.0

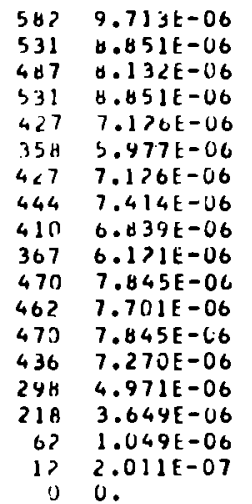

$6.216 E-05$

$5.664 E-05$

$5.205 E-05$

$5.664 E-05$

$4.561 \mathrm{E}-05$

3. $125 \mathrm{E}-05$

$4.561 \mathrm{E}-0 \mathrm{~S}$

$4.745 E-05$

$4.377 E-05$

$3.917 E-05$

$5.021 t-05$

$4.929 E-05$

$5.021 E-05$

$4.653 E-05$

$3.162 t-05$

$2.336 E-05$

$6.713 \mathrm{E}-06$

$1.2 \forall 7 \mathrm{E}-06$

STANCE

1643

1643

1643
1643

1643

1643

.1643

1643
1643

1643

1643

1643

1643

1643

1643

1643

1643

1043
EU/O UISTANCE 1/SO.M HETERS

A2 IMUTH HEIGH

EXPTSURE

E/O - SECTCL

$\begin{array}{lr}125.0 & .7 \\ 125.0 & .4 \\ 125.0 & .8 \\ 125.0 & 1.5 \\ 125.0 & 1.0 \\ 125.0 & 3.1 \\ 125.0 & 6.2 \\ 125.0 & 9.3 \\ 125.0 & 12.4 \\ 125.0 & 15.5 \\ 125.0 & 18.0 \\ 125.0 & 21.7 \\ 125.0 & 24.0 \\ 121.0 & 31.0 \\ 125.0 & 37.2 \\ 125.0 & 43.4 \\ 125.0 & 49.6 \\ 125.0 & 55.8 \\ 125.0 & 62.0\end{array}$

$\begin{array}{ll}6056 & 2.611 E-06 \\ 5313 & 2.290 E-06 \\ 7107 & 3.063 E-06 \\ 5612 & 2.419 E-06 \\ 5666 & 2.443 E-00 \\ 3457 & 2.352 E-06 \\ 4997 & 2.154 E-06 \\ 4787 & 2.061 E-06 \\ 4440 & 1.914 E-06 \\ 4617 & 1.990 E-06 \\ 7467 & 3.219 E-06 \\ 5535 & 2.366 E-06 \\ 6716 & 2.674 E-06 \\ 3122 & 1.346 E-06 \\ 3442 & 1.444 E-06 \\ 3377 & 1.456 E-06 \\ 1293 & 5.577 E-07 \\ 902 & 3.892 E-07 \\ 13 B & 5.983 E-06\end{array}$

$1.671 t-u s$

$1.466 t-05$
$1.961 t-05$

$1.548 \mathrm{~L}-05$

1. 56 st -05

$1.5 C 5 t-u 5$

$1.379 t-0 s$

$1.319 L-05$

1. $225 E-01$

2. $060 t-0 S$

$1.527 \mathrm{E}-0 \mathrm{~S}$

1.715E-US

$8.614 t-06$

$9.318 \mathrm{r}-06$

$3.569 \mathrm{E}-06$

$3.569 E-06$
$2.491 E-06$

$3.824 t-07$

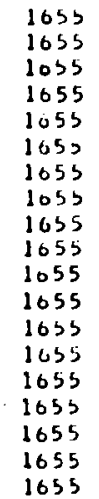

1655
1655
1055
1655
1055
1655
1655
1055
1655
1655
1055
1055
1655
1655
1655
1655
1655
1655
1655

AZ JIUTH HEIGH

AZIMUTH HEIGHT EXPTSURE - E/O EU/J UISTANCE DEGRES HETERS GH-SEC/CU.H SET/CU.M I/SO.M METERS
XIUE+O

$\begin{array}{lr}125.0 & .2 \\ 125.0 & .4 \\ 125.0 & .8 \\ 125.0 & 1.5 \\ 125.0 & 1.6 \\ 125.0 & 3.1 \\ 125.0 & 6.2 \\ 125.0 & 9.3 \\ 125.0 & 12.4 \\ 125.0 & 13.5 \\ 125.0 & 13.6 \\ 125.0 & 21.7 \\ 121.0 & 24.0 \\ 123.0 & 31.0 \\ 125.0 & 37.2 \\ 125.0 & 43.4 \\ 125.0 & 49.6 \\ 1<5.0 & 55.8 \\ 125.0 & 62.0\end{array}$

EXPUSUR

RE $\times 10 \mathrm{~F}+6$

EUTU DISTANCE

62.0

MIIIUIH HEIGHT EXPOSURF

SS $9.195 \mathrm{t}-\mathrm{C}$

. $805 E-06$

55 4.19CE-07 5.AB5E-06

$427.040 E-07 \quad 4.5 L 6 E-J 6$

$143 \quad 2.394 \mathrm{E}-06 \quad 1.530 \mathrm{E}-05$

4, 7.C 7 SE -07

$2003.340[-06$

$37 \quad 6.17 a 1=07$

उH $\quad 0.466 E-07$

$43.7 .376 \mathrm{O}-07$

4) $7.040 E-U 7$

46. $7.754 \mathrm{E}-07$

$416.897 t-07$

41) $6.753 E-U 7$

$37 \quad 0.322 E-U 7$

27
19
$19.2 \% U E-07$

$9 \quad 1.667 \mathrm{E}-07$

$4.074 t-06$

$2.1431-05$

$3.954 \mathrm{~L}-06$

4.13dE-06

$4.690 c-00$

$.506 t-66$

$4.966 E-06$

$4.414 \mathrm{E}-06$

$4.322 L-06$

$4.046 t-06$

$2.943 \mathrm{E}-06$

$2.06 \cup k-06$

$1.067 \mathrm{E}-6 \mathrm{~B}$

$1.931 \mathrm{E}-07$

1635

1655

1655
1655

1655

1655

1655

1055

1655
1655

1655

1655

1655

1655
1655

1655

1055

1055

1655

o.

1665
EU/U DISTANCE I/SO.M METERS 
TOLER DATA FOLLOH....

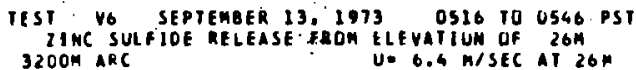

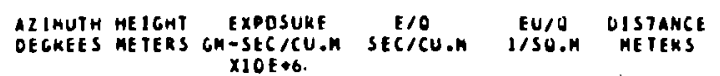

o.

3215

AZJHUIN HEICHI EXPOSURE IF EU/O DISTANCE DECKEES METERS GM-SEC/CWD

0.

3224

ALIMUIH HEIGIHT EXPOSURE F/O EU/U DISTARICE DECREES METERS GM-SEC/CU.M SECICU.M ISSH.M METERS
XIOE+6

C 115.6

c 115.6

C 115.6

115.6

115.6
115.6

115.6

115.6 6.

$115.6 \quad 12.4$

$115.6 \quad 15.5$

\begin{tabular}{ll}
115.6 & 10.6 \\
\hline & 115.6
\end{tabular}

$115.6 \quad 24.6$

115.6 . 31.0

$115.6 \quad 43.4$

$\begin{array}{ll}115.6 & 49.6 \\ 115.6 & 55.0\end{array}$

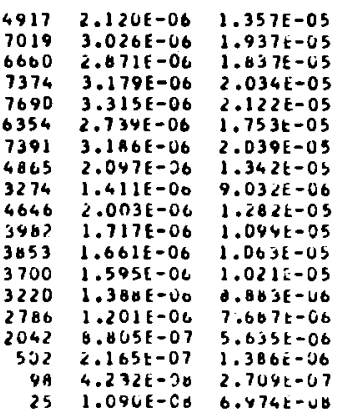

3241

$3<41$

3241

3241

3241

3241

$3 \times 41$

3241

$3<41$

$3<41$

$3<41$

3241

$3<41$

3241

3241

3241

AZIYUTH HEIGHT EXPOSURE F/O EU/O OISIANCE

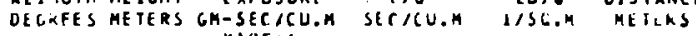

$\begin{array}{lr}123.4 & .2 \\ 123.4 & .4 \\ 123.4 & .8 \\ 123.4 & 1.5 \\ 123.4 & 1.6 \\ 123.4 & 3.1 \\ 123.4 & 6.2 \\ 123.4 & 9.3 \\ 123.4 & 12.4 \\ 123.4 & 15.5 \\ 123.4 & 18.6 \\ 123.4 & 21.7 \\ 123.4 & 24.6 \\ 123.4 & 31.0 \\ 123.4 & 37.2 \\ 123.4 & 43.4 \\ 123.4 & 44.6 \\ 123.4 & 55.8 \\ 123.4 & 62.0\end{array}$

\begin{tabular}{|c|c|}
\hline $\begin{array}{l}2471 \\
3314\end{array}$ & $\begin{array}{l}1.062 E-00 \\
1.424 E-06\end{array}$ \\
\hline 4426 & $1.900 E-06$ \\
\hline 3803 & $1.674 \mathrm{E}-06$ \\
\hline $\begin{array}{l}1810 \\
2530\end{array}$ & $\begin{array}{l}7.837 \mathrm{E}-07 \\
1.094 \mathrm{E}-0 \mathrm{E}\end{array}$ \\
\hline 2970 & $1.200 E-06$ \\
\hline 2450 & $1.056 E-06$ \\
\hline 4453 & $20 E=0 t$ \\
\hline 4699 & $2.026 E-06$ \\
\hline 3728 & $1.6075-06$ \\
\hline 4002 & $1.725 E-06$ \\
\hline $40<4$ & $1.735 E-66$ \\
\hline 2062 & $1.234 E-06$ \\
\hline 3260 & $1.405 E-6.6$ \\
\hline 2274 & $9.805 F-07$ \\
\hline 678 & $0.424 E-0 ?$ \\
\hline & $E=07$ \\
\hline 243 & $1.051 \in-07$ \\
\hline
\end{tabular}

ALITUTH HEIGHT EXPOSURE F/O EU/J UISIAINE OECKEES METERS GM-SECKCU.M SETICU.M ISSU.M HEIIKS
IOLFP DATA FOLLUW....

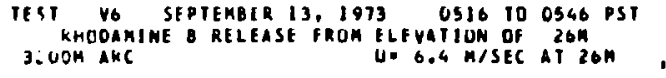

AZIMUTH HEIGHT EXPOSURE F/O EU/O DISTARCE $\times 10 E+6$

1. 99.8 .2 0 $0.0 . \quad 3215$

ATIMUTH HEIGHT EXPOSURF F/O EU/Y DISTANCE DECWES METERS GM- SLCTCLOH
$\times 1 O E+6$.

$\begin{array}{llllll}A & 107.8 & 0 & 0 & 3229\end{array}$

A)IMUTH HFIGH EXPOSURF F/O EU/O DISTANCE DEGRES HETERS CM-SECICU.M SEC/CU.M I/SO.K HETERS

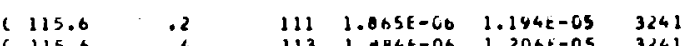

$\begin{array}{llllll}115.6 & .2 & 113 & 1.0446 t-06 & 1.206 t-05 & 3241\end{array}$

$\begin{array}{llrlll}115.6 & .6 & 90 & 1.514[-06 & 5.669 t-06 & 324] \\ 115.6 & 1.5 & 275 & 4.587 t-06 & 2.436 t-05 & 324]\end{array}$

$\begin{array}{llllll}113.6 & 1.5 & 275 & 4.587 E-06 & 2.936 E-05 & 3241 \\ 115.6 & 1.6 & 123 & 2.0531-j 6 & 1.314 E-J 5 & 3241\end{array}$

$115.6 \quad 3.1 \quad 115 \quad 1.023 E-06 \quad 1.231 E-05 \quad 3241$

$115.6 \quad 6.2 \quad 1232.053 \mathrm{E}-06 \quad 1.314 \mathrm{E}-05 \quad 3241$

$115.6 \quad 9.3 \quad 38 \quad 1.675 \mathrm{E}-06 \quad 9.440 \mathrm{E}-06$

$\begin{array}{llllll}115.6 & 12.4 & 42 & 7.147 E-37 & 4.574 E-66 & 3241\end{array}$

$315.5 \quad 1071.7976-061.144 E-05 \quad 3241$

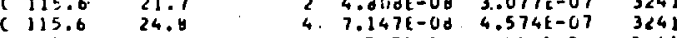

$1315.6 \quad 31.0 \quad 0 \quad 9.747 E-040.233 E-00 \quad 3<4$

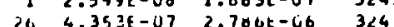

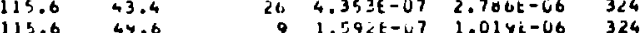

$\begin{array}{llllll}115.6 & 44.6 & 1.59 \angle E-U 7 & 1.014 i-06 & 3241 \\ 111.6 & 55.8 & 1 & 2.4645-C 6 & 1.562 t-07 & 3241\end{array}$

$115.6 \quad 62.0 \quad 010.041$

ALT.UTH HEIGHT EXrOSURA F/O EU/O DISTANCE

XIUE $C L$.

\begin{tabular}{|c|c|c|c|c|c|}
\hline 123.4 & .2 & 21 & $3.574 E-07$ & $2.207 t-66$ & 3253 \\
\hline 12344 & .4 & ld & $3.114 E-J 7$ & $1.99 C E-J 6$ & 3253 \\
\hline 123.4 &. $\mathrm{H}$ & 19 & 3.1 AूR $-\cup 7$ & $2.03 d t-06$ & 3253 \\
\hline 123.4 & 1.5 & ta & $1.163 E-06$ & $7.444 E-06$ & 3253 \\
\hline 123.4 & 1.8 & 21 & $3.6391-07$ & $2.329 E-06$ & 3253 \\
\hline 123.4 & 3.1 & 17 & $2.054 \mathrm{E}-07$ & $1.0305-06$ & 3253 \\
\hline 123.4 & 6.2 & 27 & $4.54 E F-07$ & $2.411 E=06$ & 3253 \\
\hline 123.4 & 9.3 & 20 & $3.374 E-J 7$ & $2.10<E-06$ & 3253 \\
\hline 123.4 & 12.4 & 27 & $4.613 E-07$ & $2.453 k-06$ & 3253 \\
\hline 123.4 & 13.5 & 24 & $4.094 E-67$ & $2 .+B 20 L=C 6$ & 3253 \\
\hline 123.4 & 18.6 & 26 & $4.4 \mathrm{H} 3 \mathrm{~F}=07$ & $2.009 E-06$ & 3253 \\
\hline 123.4 & 21.7 & 30 & S. UBOE-J7 & $3.244 t-06$ & $3<53$ \\
\hline $1<3.4$ & 24.8 & 32 & $5.450 t-67$ & $3.443 E-16$ & 3253 \\
\hline 123.4 & $\$ 1.0$ & 30 & $5.133 E-07$ & $3.205 t-06$ & 3253 \\
\hline 123.4 & 37.0 & 29 & $4.93=t-67$ & $3.16 C E-0 B$ & 3253 \\
\hline 123.4 & 43.0 & 23 & $3.894 t-67$ & $0.454 t-06$ & 3253 \\
\hline $179.4^{\circ}$ & 44.6 & 20 & $3.444 E-07$ & $2.204 E-06$ & 3253 \\
\hline 123.6 & $55 . \mathrm{n}$ & 11 & $1.8841-07$ & $1.260 E-06$ & 3253 \\
\hline $1<3.4$ & 62.8 & b & 1. Unet -07 & $0 . d 20 r-07$ & 3253 \\
\hline
\end{tabular}

AZI.IUTH HEIGHT EXPUSURT F/O EUIO DISTANCE

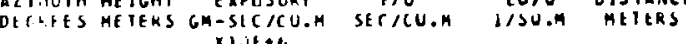

$\begin{array}{llllll}11 & 131.2 & .2 & 0 . & 0 . & 3264\end{array}$

$131.2 \quad .2-$ i 0.

$u$

$3<04$ 


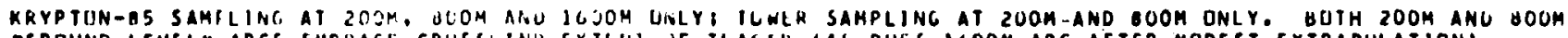
-C.ROUND-LEVEL" ARCS EMERACI CRUSSIIIHU EXILINI JF IRACER IAS DUES IGOOH ARC AFTER MODEST EXTRAPULATIONI.

THREE MIDOLE TOHFR AT ECTH 2UOM ANL GOOM INICKCEPT IRACEK PEAK CONCENTRATIEM SAMPLED, BUT TRUNCATION AT TOP.

TEST V6 SEPTEMBTR 13,1273 US16 TU 0346 PST

KRYPTON-HS RFLEASE TRIIM ILIVAIIUN UF $26 \mathrm{M}$

$20: M$ ARC SAMPLER HIT $1.5 \mathrm{M} \quad \mathrm{H}=6.4 \mathrm{H} / \mathrm{SEC}$ AI $26 \mathrm{H}$

AZSHUTH EXPOSURF $t / 6$ CU/G OISIAKC

DECREES CI-SECICU.M SECICU.M I/SA.H HEIEKS $\times 1 O E+7$

\begin{tabular}{|c|c|c|c|}
\hline $\begin{array}{l}104.0 \\
106.0 \\
104.0 \\
110.0 \\
112.0 \\
114.0 \\
116.0 \\
119.0 \\
120.0 \\
122.0 \\
124.0 \\
126.0 \\
128.0 \\
110.0\end{array}$ & $\begin{array}{r}67 \\
69 \\
44 \\
251 \\
360 \\
420 \\
425 \\
293 \\
387 \\
416 \\
214 \\
83 \\
178 \\
29\end{array}$ & 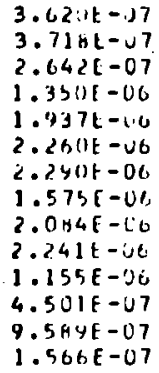 & 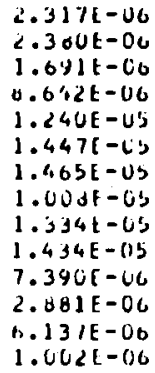 \\
\hline SSNINO & NIECRATEDE & $\begin{array}{l}1.214 E-04 \\
5 E C / 50 . \text { it }\end{array}$ & $\begin{array}{c}7.800 E-04 \\
1 / M\end{array}$ \\
\hline
\end{tabular}

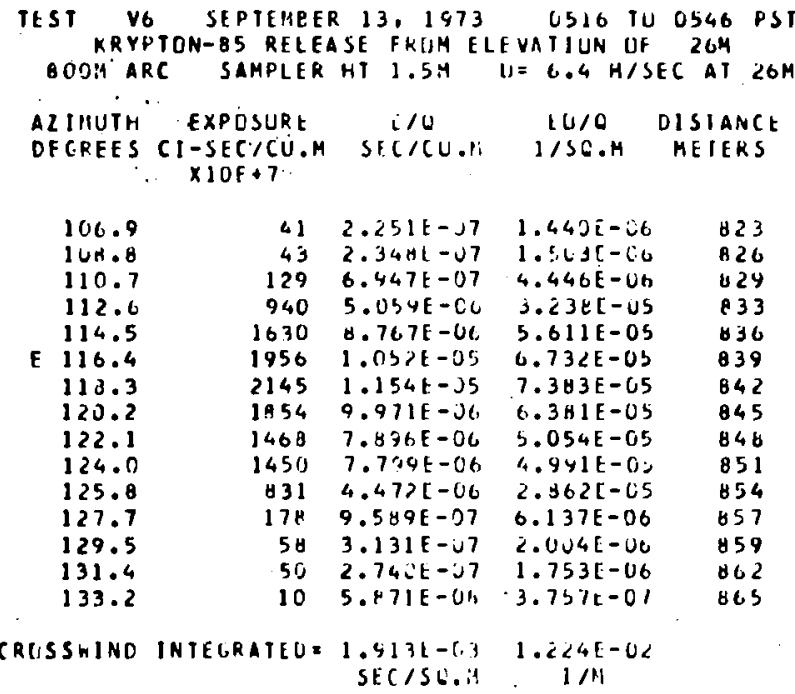

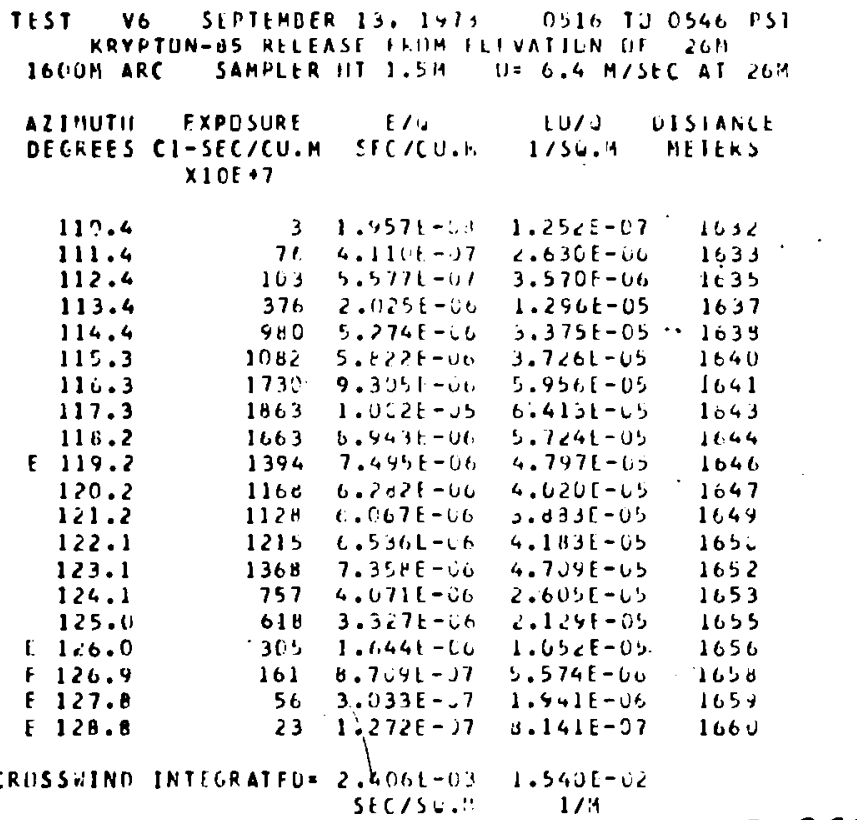




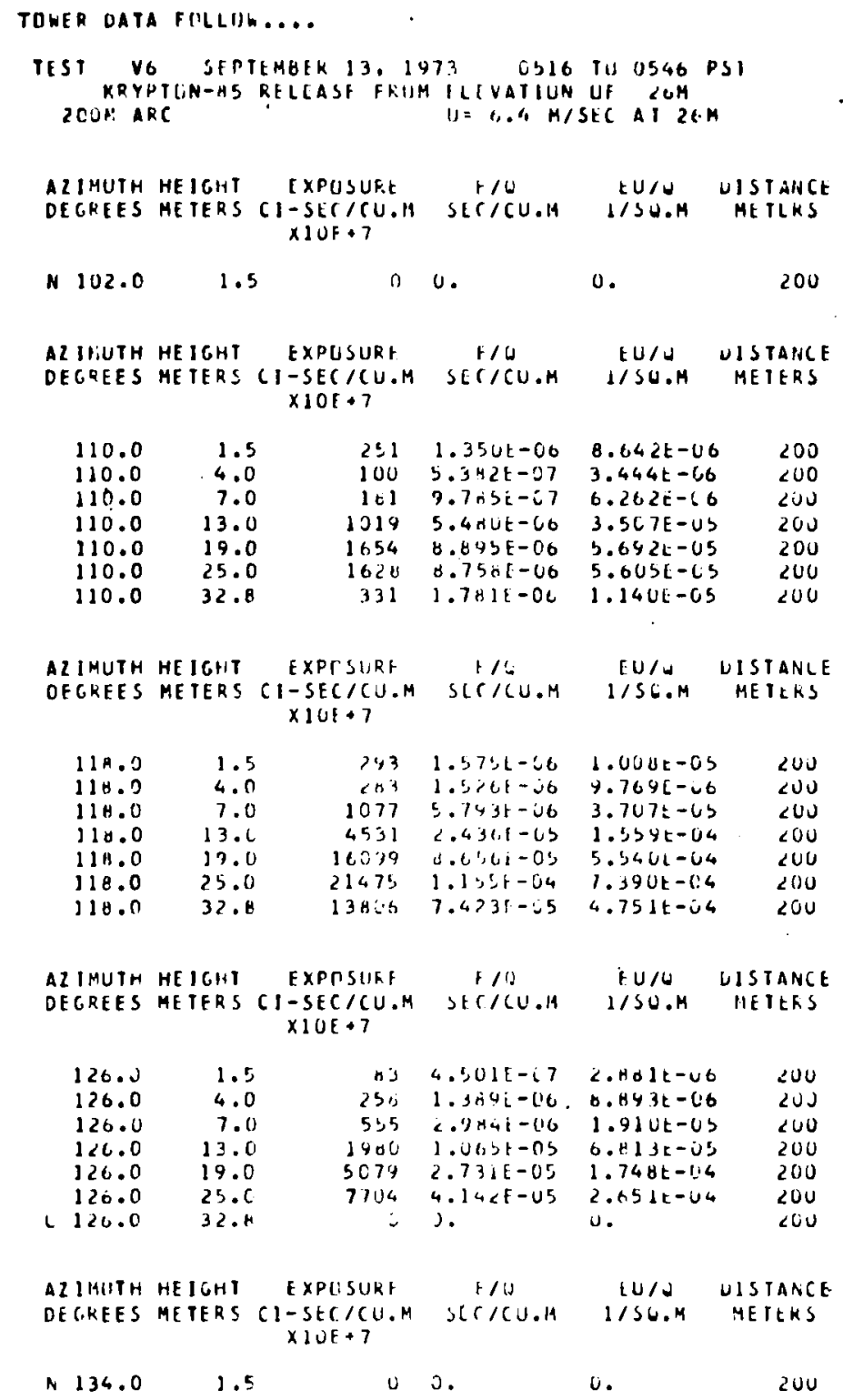


TOHER DATA FLLLUH....

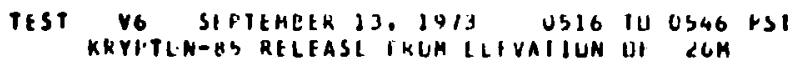
HOOM ARC UE O.4 MISEC AI LON

\begin{tabular}{|c|c|c|c|c|c|}
\hline $\begin{array}{l}\text { AZ INUTH } \\
\text { DECREES }\end{array}$ & $\begin{array}{c}\text { HE IGITT } \\
\text { ME IERS } \\
\text {. }\end{array}$ & $\begin{array}{c}\text { EXPOSUKE } \\
\text { CI-SEC/CU.H } \\
\text { XIUE +7 }\end{array}$ & $\begin{array}{c}F / U \\
\text { str./CU.H }\end{array}$ & $\begin{array}{c}E U / J \\
1 / S N \cdot K\end{array}$ & $\begin{array}{l}\text { UI SI IANCE } \\
\text { HE IEKS }\end{array}$ \\
\hline N 105.0 & 1.5 & $\begin{array}{l}0 \\
.\end{array}$ & 0 & 0. & 820 \\
\hline $\begin{array}{l}\text { AL JUUTH } \\
\text { DECKEES }\end{array}$ & $\begin{array}{l}\text { HE IGHT } \\
\text { ME TERS } \\
\therefore . .\end{array}$ & $\begin{array}{l}\text { EXPUSURE } \\
C 1-S E C / C U . M \\
\times 1 \cup t+7\end{array}$ & $\begin{array}{c}F / d \\
\sec / C U, H\end{array}$ & $\begin{array}{l}E U / U \\
1 / S O . H\end{array}$ & $\begin{array}{l}\text { DISTANCE } \\
\text { METLKS }\end{array}$ \\
\hline $\begin{array}{l}112.6 . \\
112.6 \\
112.6 \\
112.6 \\
112.6 \\
112.6 \\
112.6\end{array}$ & $\begin{array}{l}1.5 \\
5.0 \\
12.0 \\
19.0 \\
26.0 \\
34.0 \\
42.0\end{array}$ & $\begin{array}{r}440 \\
6311 \\
677 \\
411 \\
376 \\
123 \\
72\end{array}$ & $\begin{array}{l}3.05+t-66 \\
3.43 \pm t-16 \\
3.640 t-06 \\
2.211 t-06 \\
2.025 t-06 \\
6.654 E-07 \\
3.914 E-07\end{array}$ & $\begin{array}{l}3.236 t-U S \\
2.19 \forall t-U S \\
2.33 U t-U S \\
1.41 b t-U S \\
1.24 G E-U S \\
4.25 \Delta t-U 6 \\
2.50 b t-U 6\end{array}$ & $\begin{array}{l}435 \\
033 \\
633 \\
033 \\
033 \\
033 \\
033\end{array}$ \\
\hline $\begin{array}{l}\text { AI I AUTH } \\
\text { DE GREES }\end{array}$ & $\begin{array}{l}\text { HE JGHT } \\
\text { METERS }\end{array}$ & $\begin{array}{c}\text { EXPOSURF } \\
\text { CI-SEC/CU.H } \\
\times 10 \mathrm{E}+7\end{array}$ & $\begin{array}{c}F / U \\
S E C / C U . H\end{array}$ & $\begin{array}{c}\text { EU/U } \\
\text { H/SL.M.M }\end{array}$ & $\begin{array}{l}\text { UISTANCE } \\
\text { METLKS }\end{array}$ \\
\hline $\begin{array}{l}120.2 \\
120.2 \\
120.2 \\
120.2 \\
120.2 \\
120.2 \\
120.2\end{array}$ & $\begin{array}{l}1.5 \\
5.0 \\
12.0 \\
19.0 \\
26.0 \\
34.0 \\
42.0\end{array}$ & $\begin{array}{l}1854 \\
1412 \\
1845 \\
1949 \\
2154 \\
1679 \\
1334\end{array}$ & $\begin{array}{l}9.971 E-06 \\
7.593 E-06 \\
9.972 E-66 \\
1.0461-05 \\
1.159 E-05 \\
9.032 E-06 \\
7.172 E-06\end{array}$ & $\begin{array}{l}6.301 E-U S \\
4.460 t-U S \\
6.350 E-U S \\
6.761 t-U S \\
7.415 E-05 \\
5.760 t-U S \\
4.59 J E-U S\end{array}$ & $\begin{array}{l}045 \\
042 \\
045 \\
014 \\
145 \\
045 \\
045\end{array}$ \\
\hline
\end{tabular}

AZIHUTH HEIGHT EXPISUEF FIO EU/U VISIANCE DETRFES METERS CI-SECICU.H SECICU.H IISU.A ME IERS $\times 10 \mathrm{~F}+7$

\begin{tabular}{|c|c|c|c|c|c|}
\hline $\begin{array}{l}127.7 \\
1227.7 \\
1227.7 \\
127.7 \\
127.7 \\
127.7 \\
127.7\end{array}$ & $\begin{array}{l}1.5 \\
5.0 \\
12.0 \\
19.0 \\
26.0 \\
34.0 \\
42.0\end{array}$ & $\begin{aligned} 110 \\
1011 \\
211 \\
171 \\
191 \\
125 \\
7 \%\end{aligned}$ & $\begin{array}{l}9.5 B 41-07 \\
4.04<E-07 \\
1.135 E-00 \\
1.1+3 E-07 \\
9.7+51-07 \\
6.7 b<E-07 \\
4.01<1-07\end{array}$ & $\begin{array}{l}6.137 c-06 \\
3.131 L-06 \\
7.204 t-06 \\
5.087 t-06 \\
6.262 t-06 \\
4.321 t-06 \\
2.560 t-06\end{array}$ & $\begin{array}{l}051 \\
051 \\
451 \\
057 \\
057 \\
551 \\
057\end{array}$ \\
\hline $\begin{array}{l}2 \text { JMUTH } \\
\text { E GREES }\end{array}$ & $\begin{array}{l}\text { HE IGHT } \\
\text { ME TFRS }\end{array}$ & $\begin{array}{l}\text { ExROSURI } \\
\text { CI-SEC } / C U \text {. F } \\
\times 1 \cup F+7\end{array}$ & $\begin{array}{c}1 / C \\
\text { SEC/CU. }\end{array}$ & $\begin{array}{c}E U / U \\
1 / S O . M\end{array}$ & $\begin{array}{l}\text { UISI ANLE } \\
\text { ME ILKS }\end{array}$ \\
\hline 135.0 & 1.5 & 9 & - & 0. & 061 \\
\hline
\end{tabular}




\section{IXC SUTIDS}

GRUUHU LEVEL ANO IOHER SAMPLING POOH TO 3200M. AFTER MDDEST EXTRAPOLATION UF ZNS TU SOUTH. ALL ARCS EMBRACE THE CRDSSHINC EXTENT UF THIS TRACER. EICHTEEN OF TWENTY TOHERS MHITN IN THIS TEST LUKINC UNSTABLE CDNOITIONS. BUT ALL DISTRIBUTIUNS TRUNCATED AT TOPS DF TOHERS. MUST UNSTABLE CONOITIUNS DF IHIS FIELO SEKIES.

\section{roreros-es}

ALTHOUGH SAMPLING AT 20C. BOO AND 1 GOUM. DIFFUSIOH HAS SO GKEAT IHAT KRYPTUN DETECTEO HITH CUNFIOENCE OMLY AT 200M. ALL 5 TOHERS AT 20 OM HIT AY TRACER BUT TRUIHCATEO BAOLY AT IUP IN ALL CASES. GROUNO LEVEL SAMPLIMG ALSO TRUNCATED AT SEUTH. ESTIMATE AT 122 DEG BASEU ON SIMILARETY BETWEEH ZNS ANO KRYPION CRUSSWINU OISTRIGUTIUNS.

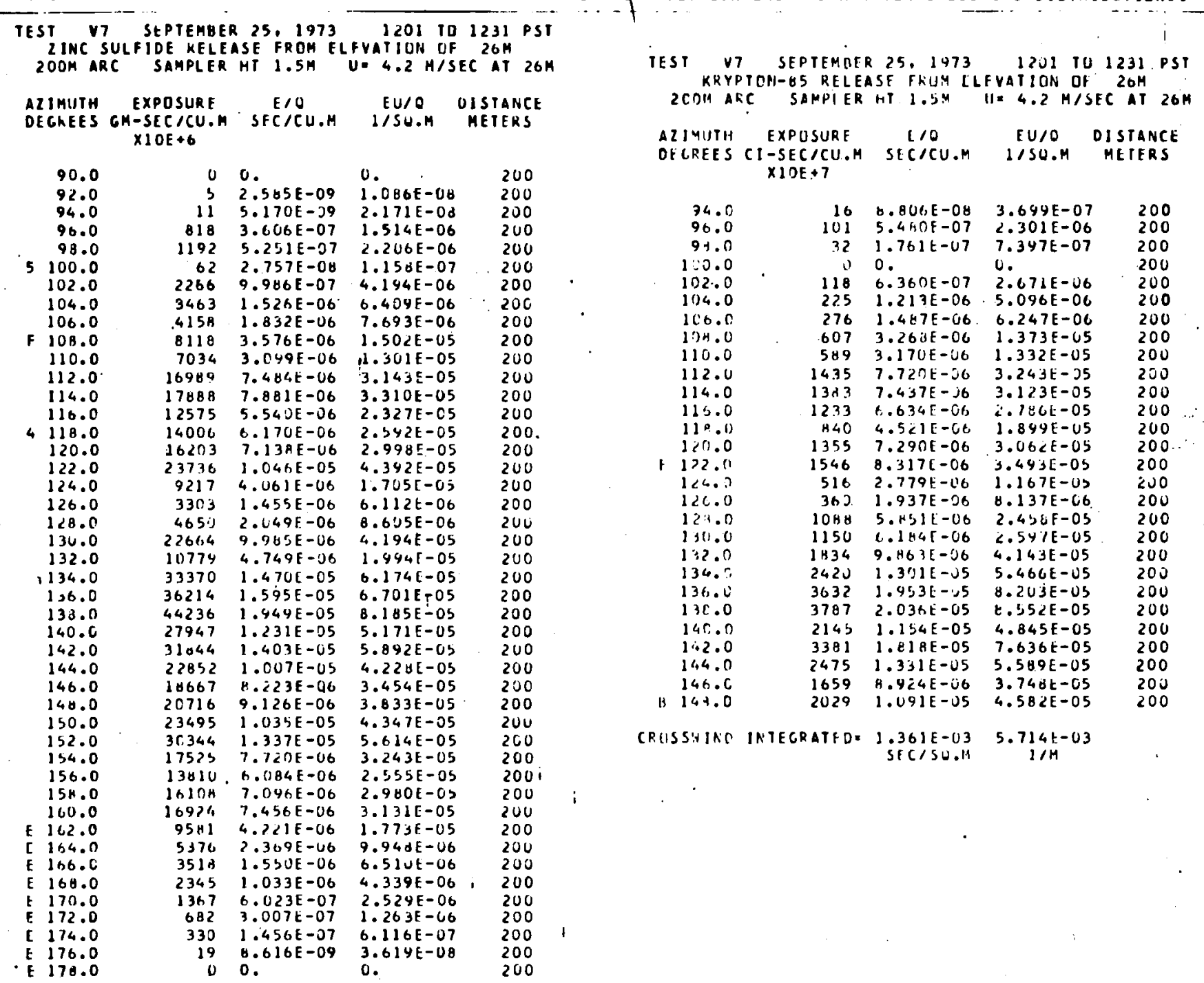

CRISSHINO INTEGRATEO= 1.711E-03 7.287E-03 


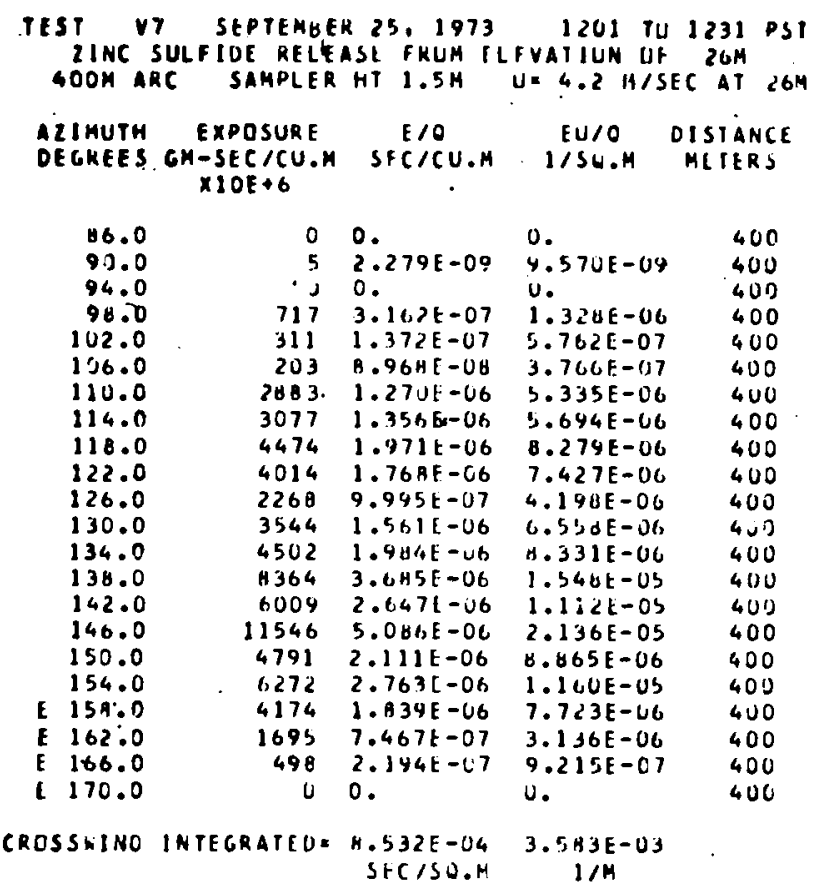

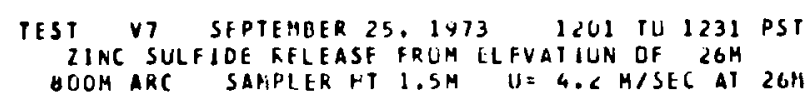

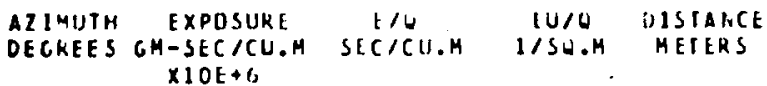

95.1

97.1

101.1

133.0

155.0

106.9

108.8

110.7

112.6

114.5

116.4

110.3

$1<0.2$

122.1

124.0

125.1

127.7

129.5

131.4

133.

135.0

$13 \mathrm{~B} . \mathrm{H}$

133.7

140.6

142.4

144.2

146.0

147.8

149.6

151.4

153.2

155.0

156.8

150.6

160.4

162.2

E 164.0

E 166.0

CRUSSHIND INTECRATED= 4.135E-04.

CRUSSHIND INTECRATED
$4.135 E-04$
SECISO.M

$\checkmark$.

$4.167 \mathrm{t}-09 \quad 1.750 \mathrm{E}-0 \mathrm{E}$ 2. $145 E-0$ 1.20dE-0 5.042E-UA $2.116[-07$ $1.250 E-37 \quad 5.2511-07$

$1.367 F-07$ C.S61F-17

$2.717 \mathrm{t}-07$ ग.731E-0\%

$2.371[-07 \quad 4.9598-07$

$2.8131-07 \quad 1.1 \mathrm{~d} 1=00$

$5.547 \mathrm{E}-07 \quad 2.33 \cup \mathrm{E}-06$

$4.111 \mathrm{t}-07 \quad 1.731 \mathrm{t}-\mathrm{U} 6$

$2.379 E-07 \quad 9.994 t-67$

$3.653 E-06$

$2.982 E-06$

$1.423 E-06$

6. $231 E-07$

Q. $834 \mathrm{E}-\mathrm{C}$.

Y. $609 \mathrm{E}-07$

$1.8501-06$

1.t. $12[-00$

C. $0351-00$

$2.737 \mathrm{E}-06$

$2.956 t-06$

C. C 1 OE-DO

$2.962 E-40$

$3.310 E-06$

$3.7445-06$

$1.671 \mathrm{E}-06$

$2.862 E-06$

$4.314 E-U 6$

3.34 uf -06

C. $303 \mathrm{E}-106$

$1.731 \mathrm{~F}-0 \mathrm{C}$

$1.582 \mathrm{k}-06$

$1.083 E-06$

$2.975 \mathrm{E}-\mathrm{OH}$

$2.450 E-06$

1. $750 E-O B$

0 .

$1.737 \mathrm{E}-03$

CRUSSHIND INTECRATED
$4.135 E-04$
SECISO.M

$$
\begin{aligned}
& 802 \\
& 806 \\
& 809 \\
& 813 \\
& 816 \\
& 820 \\
& 823 \\
& 826 \\
& 809 \\
& 833 \\
& 836 \\
& 839 \\
& 842 \\
& 845 \\
& 848 \\
& 851 \\
& 854 \\
& 457 \\
& 859 \\
& 862 \\
& 865 \\
& 867 \\
& 809 \\
& 872 \\
& 874 \\
& 876 \\
& 878 \\
& 800 \\
& 832 \\
& 884 \\
& 886 \\
& 887 \\
& 889 \\
& 890 \\
& 892 \\
& 843 \\
& 894 \\
& 895 \\
& 896
\end{aligned}
$$




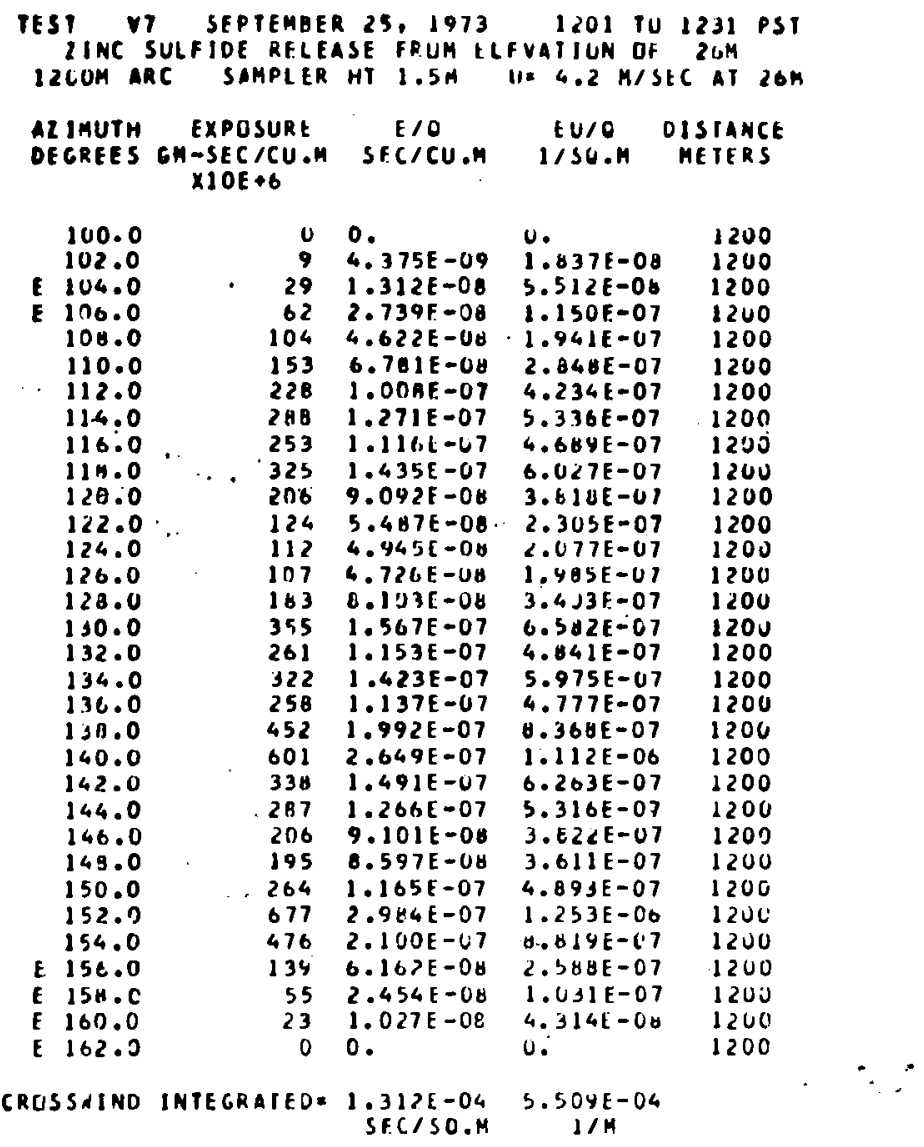

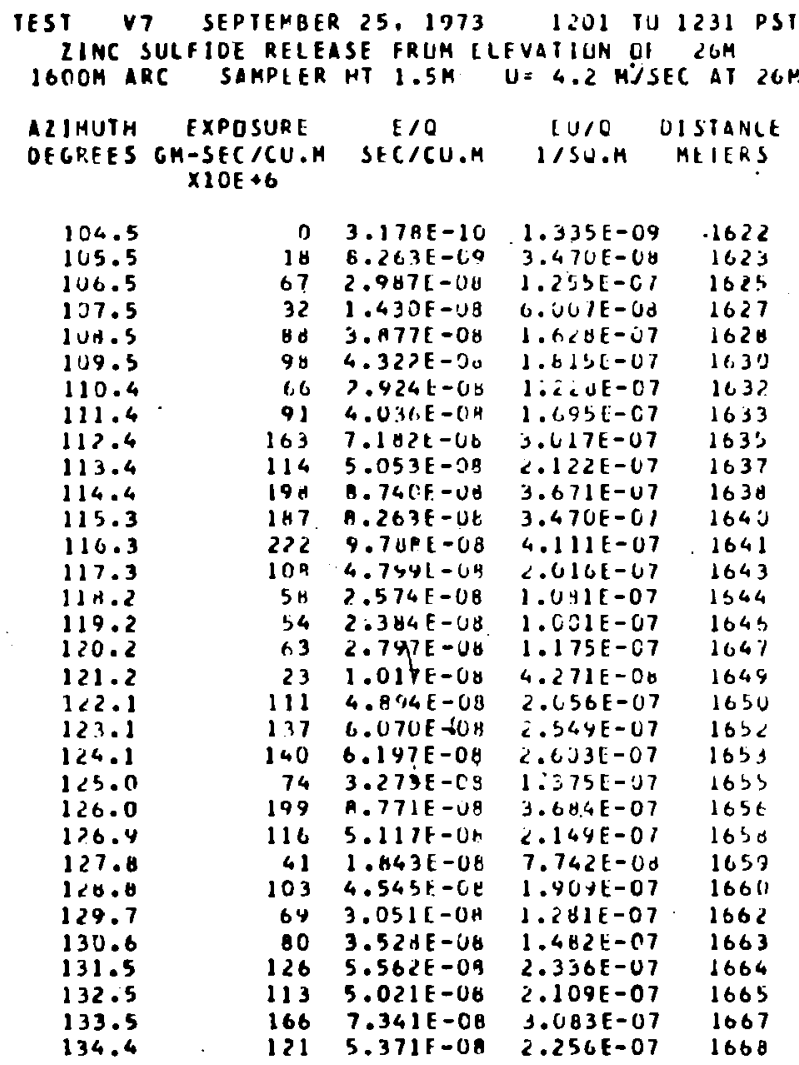




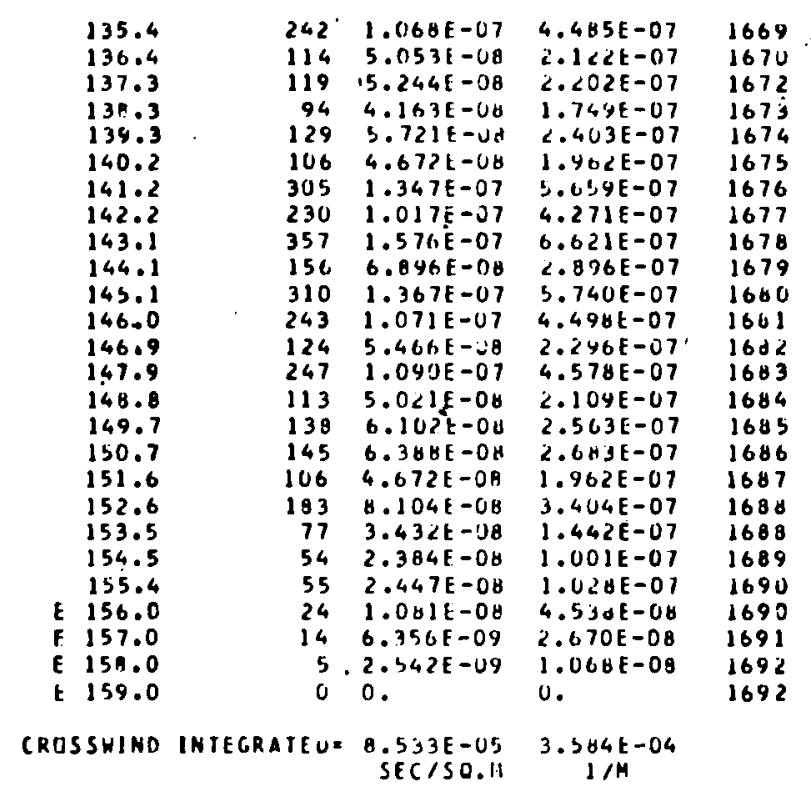

TEST V7 SEPTEPBER 2\%, $1973 \quad 1201$ IU 1231 PST IINC SULFIDE RELEASE FKIJM ILFVATIUN DF $20 \mathrm{M}$
$3200 \mathrm{H}$ ARC SAMPLER HT $1.5 \mathrm{M}$ II= $4.2 \mathrm{M} / \mathrm{JEC}$ AT $26 \mathrm{M}$ AZIMUTH EXPOSURE
OEGRES GP-SECICU.M SIC/CU.M I ISO.M METERS
XIOE +6

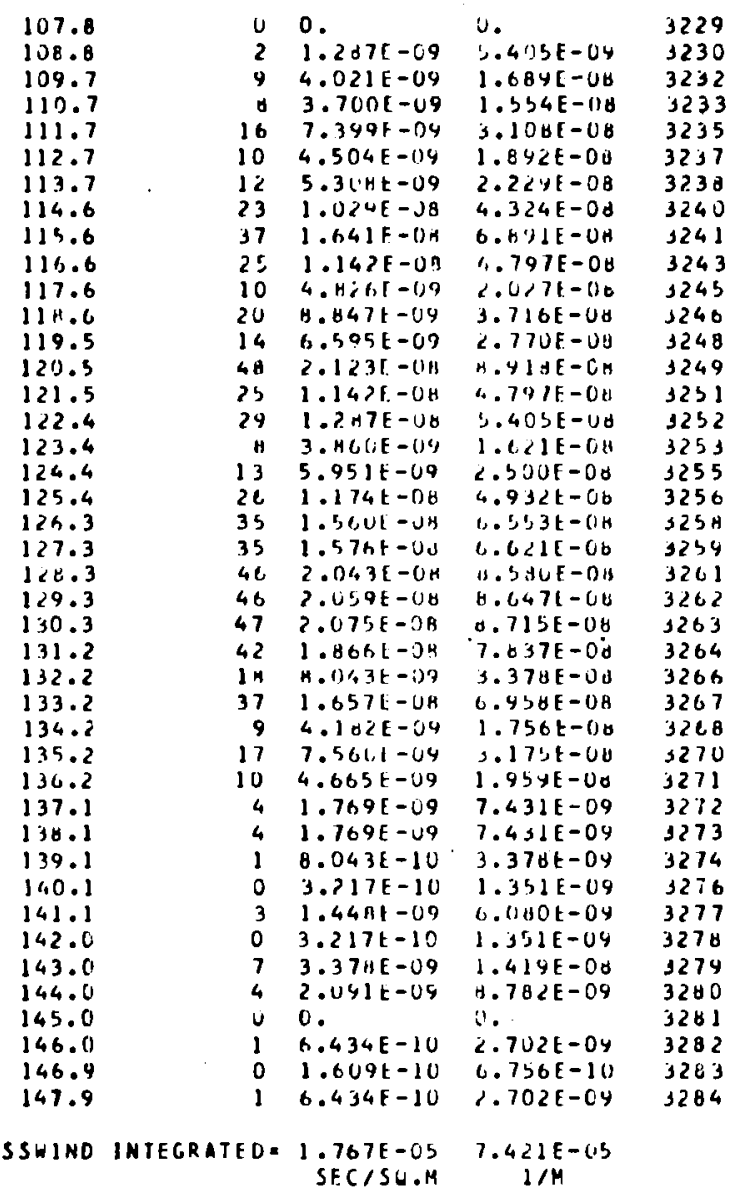




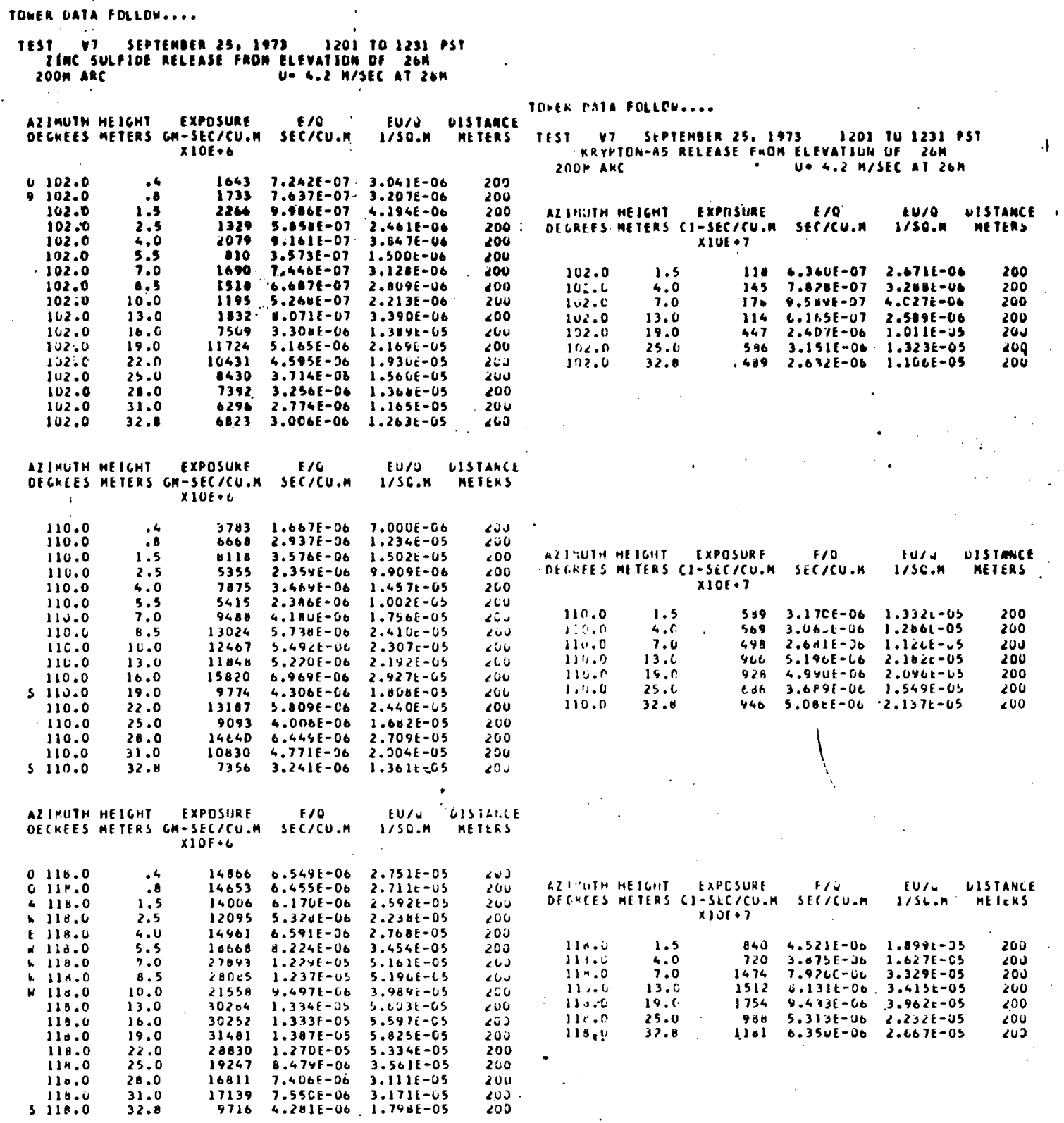




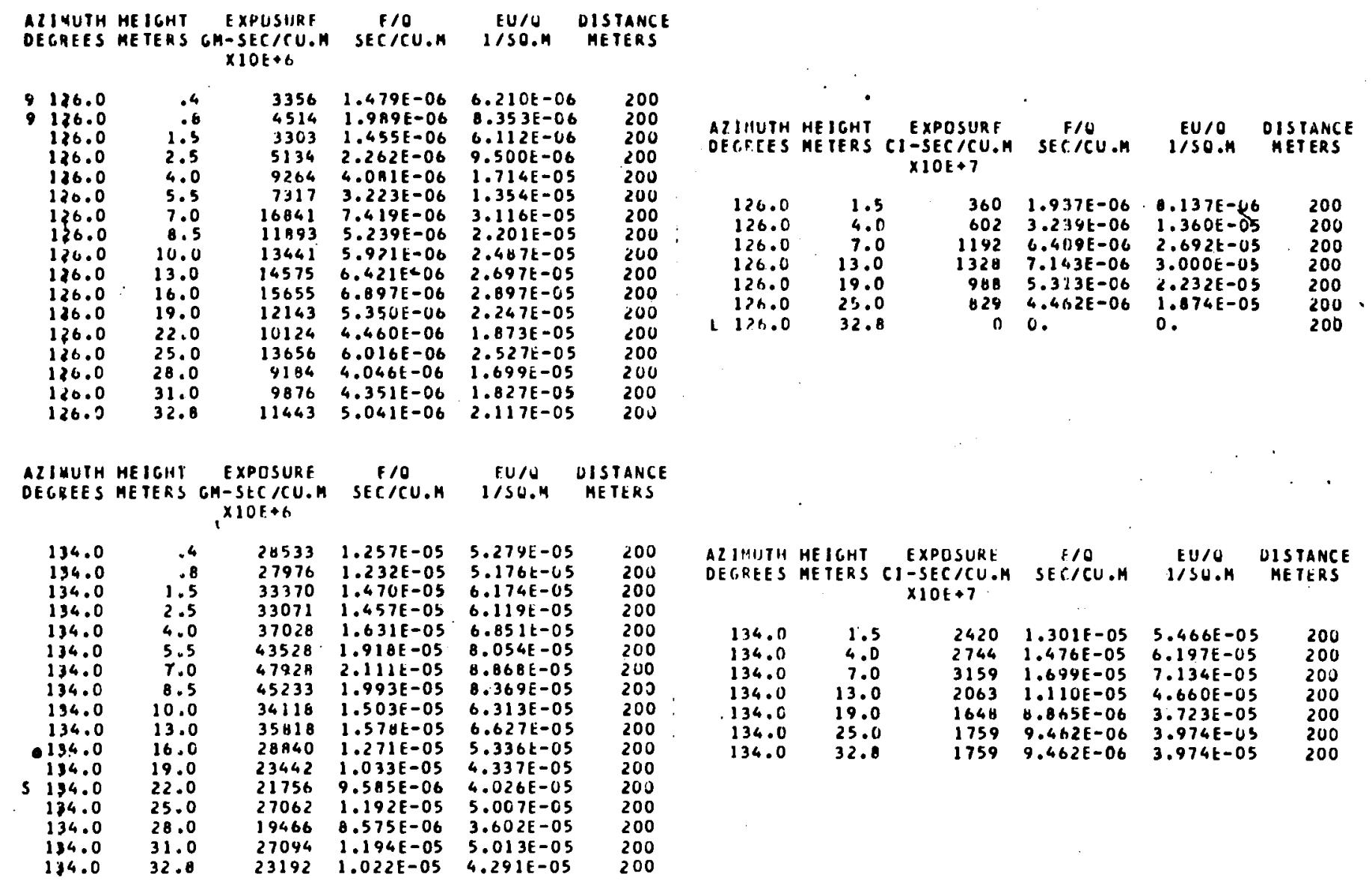

TOWER DATA FCLLEW....

\begin{tabular}{|c|c|c|c|c|c|c|}
\hline $\begin{array}{l}\text { EST V } \\
\text { ZINC } \\
\text { BOOM AI }\end{array}$ & $\begin{array}{l}7 \text { SEP } \\
\text { SULFIUE } \\
\text { RC }\end{array}$ & $\begin{array}{l}\text { TEMBER } 2 \\
\text { RELEASE }\end{array}$ & $\begin{array}{l}25,1 \\
\text { E FRDI }\end{array}$ & $\begin{array}{l}13 \quad 1201 \\
\text { ELFVATION } \\
U=4.2 \mathrm{M} /\end{array}$ & $\begin{array}{l}\text { TO } 1231 \text { P } \\
\text { DF } 26 \mathrm{M} \\
\text { SEC AT } 26 \mathrm{M}\end{array}$ & PST \\
\hline $\begin{array}{l}\text { ALIMUTH } \\
\text { DECEEES }\end{array}$ & $\begin{array}{l}\text { HE IGHT } \\
\text { METERS }\end{array}$ & $\begin{array}{r}\text { EXPOS } \\
\text { GH-SECS } \\
\times 1 O F\end{array}$ & $\begin{array}{l}\text { SURE } \\
\text { ICU.M } \\
\text { } 6\end{array}$ & $\begin{array}{c}F / O \\
\text { SEC/CU.M }\end{array}$ & $\begin{array}{l}E \cup / 0 \\
1 / 50.1\end{array}$ & $\begin{array}{c}\text { DISTANCE } \\
\text { METERS }\end{array}$ \\
\hline $\begin{array}{l}105.0 \\
105.0 \\
105.0 \\
105.0 \\
105.0 \\
105.0 \\
105.0 \\
105.0 \\
105.0 \\
105.0 \\
105.0 \\
105.0 \\
105.0 \\
105.0 \\
105.0 \\
105.0 \\
105.0 \\
105.0\end{array}$ & $\begin{array}{l}.3 \\
1.5 \\
1.1 \\
2.1 \\
4.2 \\
6.3 \\
6.4 \\
10.5 \\
12.6 \\
14.7 \\
16.8 \\
21.0 \\
25.2 \\
29.4 \\
33.6 \\
37.8 \\
42.0\end{array}$ & $\begin{array}{l}3 \\
5 \\
1 \\
5 \\
1 \\
2 \\
3 \\
6 \\
5 \\
6 \\
7 \\
4 \\
5 \\
2 \\
4 \\
6 \\
8 \\
0\end{array}$ & $\begin{array}{l}455 \\
239 \\
258 \\
355 \\
285 \\
284 \\
413 \\
378 \\
212 \\
354 \\
468 \\
314 \\
301 \\
206 \\
146 \\
281 \\
177 \\
191\end{array}$ & $\begin{array}{l}2.005 E-07 \\
1.054 E-07 \\
1.139 E-07 \\
1.567 E-07 \\
1.256 E-07 \\
1.255 E-07 \\
1.873 E-07 \\
1.666 E-07 \\
9.366 E-08 \\
1.562 E-07 \\
2.062 E-07 \\
1.384 E-07 \\
1.329 E-07 \\
9.107 E-08 \\
6.450 E-08 \\
1.239 E-07 \\
7.798 E-08 \\
1.445 E-08\end{array}$ & $\begin{array}{l}8.422 E-07 \\
4.428 E-07 \\
4.785 E-07 \\
6.581 E-07 \\
5.274 t-07 \\
5.271 E-07 \\
7.656 t-07 \\
6.994 E-07 \\
3.934 E-07 \\
6.561 E-07 \\
8.660 t-07 \\
5.812 E-07 \\
5.504 E-07 \\
3.825 E-07 \\
2.709 E-07 \\
5.204 E-07 \\
3.275 E-07 \\
3.547 t-07\end{array}$ & $\begin{array}{l}620 \\
820 \\
820 \\
020 \\
620 \\
020 \\
620 \\
020 \\
820 \\
020 \\
020 \\
020 \\
020 \\
020 \\
020 \\
020 \\
820 \\
020\end{array}$ \\
\hline
\end{tabular}




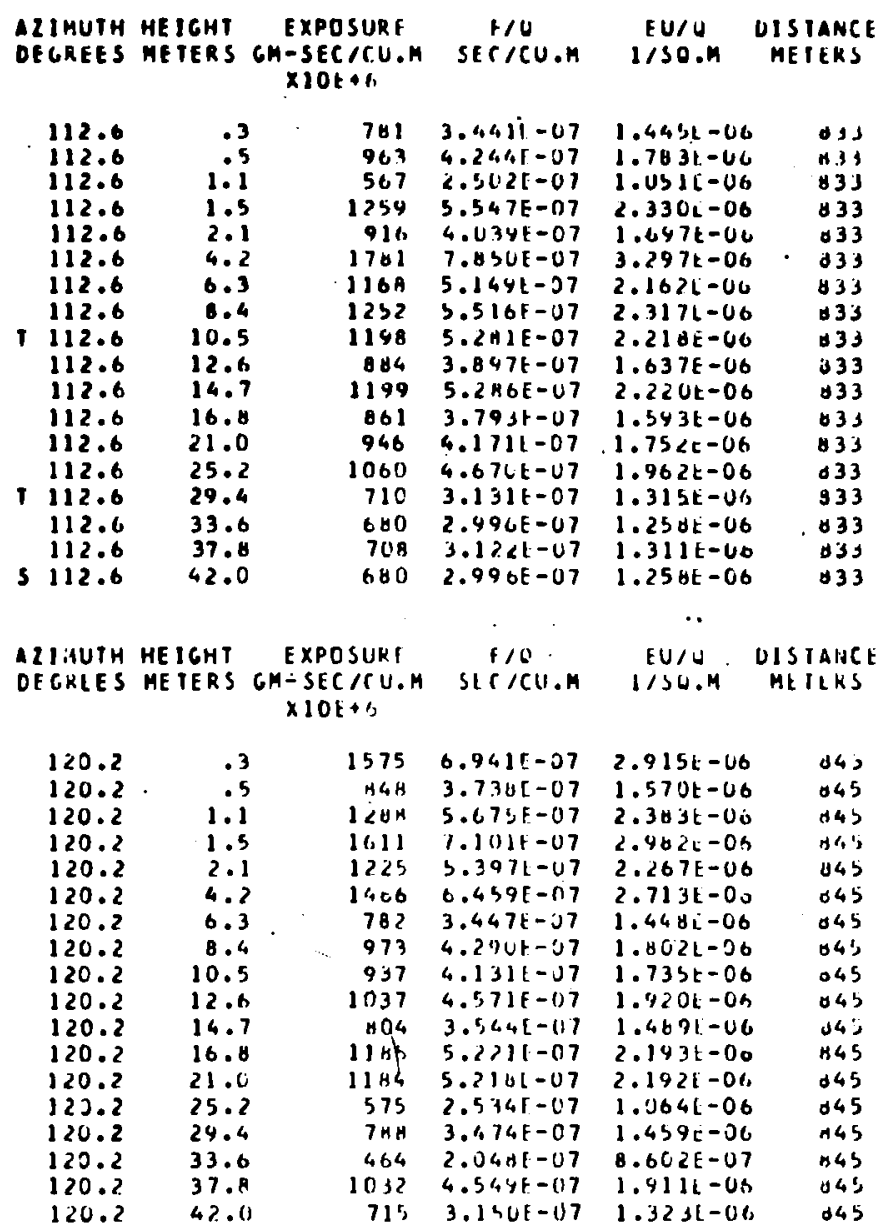

AZIMUTH HEICHT EXPUSUKL 10 EU/U DISTANCE DEGREES METERS GM-SEC/CU.M SETICU.M IISU.M MEIERS $\times 10 E+6$

\begin{tabular}{|c|c|c|c|c|c|}
\hline 127.7 & 3 & $\begin{array}{l}474 \\
427\end{array}$ & $\begin{array}{l}2.11<1-07 \\
1.8 \times 5-27\end{array}$ & $\begin{array}{r}4.070 t-07 \\
7.40-07\end{array}$ & $\begin{array}{l}057 \\
057\end{array}$ \\
\hline 127.7 & 1.1 & 587 & $2.5460-07^{\circ}$ & 1. $008 t-00$ & 057 \\
\hline 127.7 & 1.5 & 519 & $2.2 H \otimes E-\cup 7$ & $9.6051-07$ & 057 \\
\hline 127.7 & 2.1 & $64 ?$ & $2.47+5 .-07$ & $1.100 \mathrm{t}-06$ & ob 7 \\
\hline 127.7 & 4.2 & 626 & $2.7501-67$ & $1.159 i=00$ & 057 \\
\hline 127.7 & 6. 3 & 669 & $2.347[-67$ & $1.23 B t-O t$ & 851 \\
\hline 127.7 & B .4 & 635 & 2.74 צ[ $[-07$ & $1.176 t-06$ & ubl \\
\hline 127.7 & 10.5 & 429 & $1.891,[-67$ & $7.9506-07$ & 0 o \\
\hline 127.7 & 12.0 & 152 & $2.43<1-07$ & $1.02<t-06$ & $\$ 1$ \\
\hline 127.7 & 14.7 & $n 03$ & $2.656 \mathrm{~B}-07$ & $1.117 t-00$ & 051 \\
\hline 127.7 & 16.9 & 745 & $3.2 H\langle E-U 7$ & $1.373 i-06$ & 051 \\
\hline 127.7 & 21.0 & 6.62 & 2.91 เ1-017 & $1.220 L=06$ & 457 \\
\hline 127.7 & 25.2 & 622 & $2.74<t-107$ & $1.132 t=06$ & 057 \\
\hline 127.7 & 29.4 & 250 & $1.105 f-07$ & $4.642 t-07$ & 057 \\
\hline 127.7 & 33.6 & 1.44 & $2.17 \mathrm{st}-07$ & $9.140 t-07$ & 457 \\
\hline 127.7 & 37.8 & $31 A$ & $1.402 E-07$ & $5.1490 t-07$ & 057 \\
\hline 127.7 & 42.0 & 249 & $1.0476-07$ & $4.608 E-07$ & as 1 \\
\hline
\end{tabular}




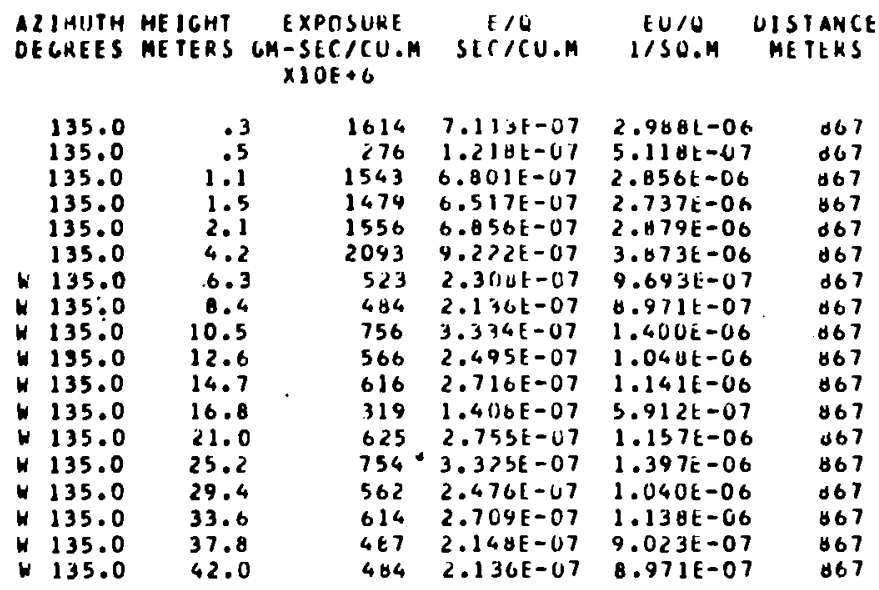

TOHER DATA FIILLOH....

TEST VI SEPTEMBER 25,1973 1201 TO 1231 PST
2INC SULFIOE RELEASE FROM FLFVAIIUN UF $26 \mathrm{M}$

$2 I N C$ SULFIOE RELEASE FROM FLFVAIIUN UF $26 \mathrm{M}$
$1600 M$ ARC
U. 4.2 M/SEC AT $26 \mathrm{M}$

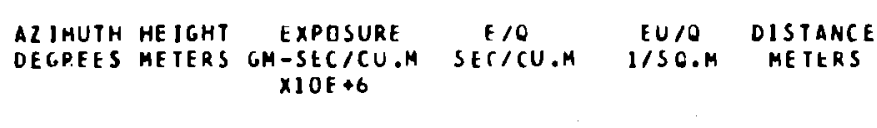

16 $101.8 \quad 110.20 .1617$

ALIMUTH HEIGHT EXPOSURE F/O EU/U OISTANCE

DEGREES MEIERS GM-SECICU.M SECICU.M I/SO.M METLRS $\times 10 E+6$

\begin{tabular}{|c|c|c|c|c|c|}
\hline 109.5 & .2 & 54 & $2.347 \mathrm{E}-20$ & $1.002 E-07$ & 1630 \\
\hline 109.5 & .4 & $7 \mathrm{H}$ & $3.430 k-00$ & $1.443 t=67$ & 1630 \\
\hline 109.5 & .8 & 39 & $1.757[-08$ & $7.381 \mathrm{c}-08$ & 1630 \\
\hline 109.5 & 1.5 & 90 & $4.3>2 \mathrm{t}-00$ & $1.815 t-67$ & 1630 \\
\hline 109.5 & 1.6 & 44 & $1.97 \cup E-00$ & d. $276 \mathrm{c}-0 \mathrm{~b}$ & 1630 \\
\hline $1 ; 9.5$ & 3.1 & 121 & $5.35 \times 1-: 08$ & $2.251 t-07$ & 1630 \\
\hline 109.5 & 6.2 & 117 & $5.16,3 t-U 0$ & $2.171 t-07$ & $163 \mathrm{~J}$ \\
\hline 109.5 & 9.3 & 112 & $4.4760-08$ & $2.0901-07$ & 1030 \\
\hline 109.5 & 12.4 & 32 & $2.320 t-60$ & $9.769 \mathrm{~L}-0 \mathrm{U}$ & 1030 \\
\hline 109.5 & 15.5 & 119 & $5.250 t-0 B$ & $2.205 t-07$ & 1630 \\
\hline 109.5 & 18.6 & 76 & 3.34 of -00 & $1.406=-07$ & 1630 \\
\hline 109.5 & 21.7 & 99 & $4.370 E-J 6$ & $1.839 t-i 7$ & 1030 \\
\hline 109.5 & 24.8 & 63 & $3.646 t-0 \mathrm{~J}$ & $1.552 t-07$ & 1630 \\
\hline 109.5 & 31.0 & 60 & $2.663 \mathrm{E}-00$ & $1.118 i-07$ & 1630 \\
\hline 109.5 & 37.2 & $9 \theta$ & $4.35<8-4 d$ & $1.828 L-07$ & 1030 \\
\hline 109.5 & 43.4 & 79 & $3.516 E-00$ & $1.477 t-07$ & 1630 \\
\hline 1.29 .5 & 49.6 & 40 & $1.7 H I F-j 8$ & $7.481 \mathrm{E}-0.08$ & 1630 \\
\hline 1119.5 & 55.6 & 31 & $2.26,1 \mathrm{l}-\mathrm{U}$ & $4.499 t-U_{d}$ & $163 \mathrm{~J}$ \\
\hline 109.5 & 62.0 & 17 & $7.773 t=09$ & $3.265 t-00$ & 1630 \\
\hline
\end{tabular}

AZIKUTH HEIGHT EXPOSURE F/O EU/U UISIANCE DEGPEES METERS GH-SECICU.M SETICU.M IISU.M MEIERS $\times 10 t+6$

\begin{tabular}{|c|c|c|c|c|c|}
\hline 117.3 & 4 & 6 & $2.934 \mathrm{~F}-08$ & $1.3 .32 t-07$ & 1643 \\
\hline 117.3 & .4 & 0 & $2.960 t=08$ & $1.243 t-07$ & 1643 \\
\hline 117.3 & .6 & 70 & $3.117[-0.0$ & $1.3041-07$ & 1643 \\
\hline 117.3 & 1.5 & $10 \mathrm{H}$ & 4.7 Y Y & $2.016 E-07$ & 1643 \\
\hline 117.3 & 1.0 & 126 & $5.54 \mathrm{st}-0 \mathrm{U}$ & $2.345 \mathrm{i}-07$ & 1643 \\
\hline 117.3 & 2.1 & 156 & $6.912[-\cup \theta$ & $2.903 t-07$ & 1643 \\
\hline 117.3 & 6.2 & 49 & $4.36 y 1=118$ & $1.83 \mathrm{si}-07$ & 1643 \\
\hline 117.3 & 9.3 & 102 & $4.50111-00$ & 1. HYUL -07 & 1645 \\
\hline 117.3 & 12.4 & $10 \%$ & $4.674 E-0.8$ & $1.942 t-07$ & 1643 \\
\hline 117.3 & 15.5 & 91 & $4.013 k-6 \theta$ & $1.607 \mathrm{E}-U 7$ & 1643 \\
\hline 117.3 & 18.6 & 176 & $7.794 \mathrm{E}=00$ & $3.274 t-07$ & 1043 \\
\hline 117.3 & 21.7 & 65 & $2.8+46-00$ & $1.211 \mathrm{E}-07$ & 1643 \\
\hline .3 & 24.8 & 137 & $6.057 E-06$ & $2.544 t-07$ & 1643 \\
\hline .3 & 31.0 & 66 & $3.793 E-U B$ & $1.593 \mathrm{E}-07$ & 1643 \\
\hline & & $11 \mathrm{~B}$ & $5.213 E-08$ & $2.189 \mathrm{t}-07$ & 1643 \\
\hline & 43.4 & 163 & $7.205 E-08$ & $3.026 E-07$ & 1643 \\
\hline 11 & & 94 & $4.164 t-08$ & $1.749 E-07$ & 1643 \\
\hline & & 143 & $6.33 \mathrm{dE}-0 \mathrm{~d}$ & $2.662 \mathrm{t}-07$ & 1643 \\
\hline & 62 & 71 & $3.133 E-08$ & $1.316 t-07$ & 1643 \\
\hline
\end{tabular}




\begin{tabular}{|c|c|c|c|c|c|c|}
\hline & $\begin{array}{l}\text { MUTH } \\
\text { REES }\end{array}$ & $\begin{array}{l}\text { HE ICHT } \\
\text { ME TERS }\end{array}$ & $\begin{array}{l}\text { EXPDSURE } \\
\text { CH-SEC/CU.M } \\
\times 1 O E+6\end{array}$ & $\begin{array}{c}F / O \\
\text { SEC/CU.H }\end{array}$ & $\begin{array}{l}E \cup / 2 \\
1 / 56.4\end{array}$ & $\begin{array}{l}\text { UIS I ARCE } \\
\text { HETERS }\end{array}$ \\
\hline & $\begin{array}{l}125.0 \\
125.0 \\
125.0 \\
125.0 \\
125.0 \\
125.0 \\
125.0 \\
125.0 \\
125.0 \\
125.0 \\
125.0 \\
125.0 \\
125.0 \\
125.0 \\
125.0 \\
125.0 \\
125.0 \\
125.0 \\
125.0\end{array}$ & $\begin{array}{l}.2 \\
.4 \\
1.8 \\
1.5 \\
3.1 \\
6.2 \\
9.3 \\
12.4 \\
15.5 \\
18.6 \\
21.7 \\
24.0 \\
31.0 \\
37.2 \\
43.4 \\
49.6 \\
55.6 \\
62.0\end{array}$ & $\begin{array}{r}91 \\
45 \\
118 \\
74 \\
69 \\
131 \\
71 \\
126 \\
144 \\
36 \\
148 \\
117 \\
64 \\
48 \\
138 \\
60 \\
16 \\
97 \\
74\end{array}$ & $\begin{array}{l}4.03 U E-J 8 \\
2.007 E-08 \\
5.206 E-08 \\
3.273 E-08 \\
3.941 E-08 \\
5.716 E-08 \\
3.170 E-08 \\
5.591 E-08 \\
6.567 E-08 \\
1.607 E-08 \\
6.515 E-08 \\
5.191 E-08 \\
3.421 E-06 \\
2.135 E-08 \\
5.656 E-08 \\
2.656 E-08 \\
7.221 E-04 \\
4.2116 E-08 \\
3.296 E-08\end{array}$ & $\begin{array}{l}2.693 E-07 \\
8.430 E-08 \\
2.106 E-07 \\
1.375 E-07 \\
1.655 E-07 \\
2.430 E-07 \\
1.331 E-07 \\
2.340 E-07 \\
2.758 E-07 \\
6.750 E-06 \\
2.745 E-07 \\
2.100 E-07 \\
1.644 E-07 \\
6.968 E-08 \\
2.375 E-07 \\
1.115 E-07 \\
3.033 E-08 \\
1.301 E-07 \\
1.305 E-07\end{array}$ & $\begin{array}{l}1655 \\
1655 \\
1655 \\
1655 \\
1655 \\
1655 \\
1655 \\
1655 \\
1655 \\
1655 \\
1655 \\
1655 \\
1655 \\
1655 \\
1055 \\
1655 \\
1655 \\
1655 \\
1655\end{array}$ \\
\hline & $\begin{array}{l}\text { IMUTH } \\
\text { GREES }\end{array}$ & $\begin{array}{l}\text { ME IGHT } \\
\text { METERS }\end{array}$ & $\begin{array}{l}\text { EXPUSURE } \\
\text { CH-SECICU.M } \\
\times 1 O E+6\end{array}$ & $\begin{array}{l}F / D \\
\text { SEC/CU.A }\end{array}$ & $\begin{array}{l}\text { EUAO } \\
\text { I/SU.M }\end{array}$ & $\begin{array}{l}\text { UISTANCE } \\
\text { ME ILRS }\end{array}$ \\
\hline & $\begin{array}{l}132.5 \\
132.5 \\
132.5 \\
132.5 \\
132.5 \\
132.5 \\
132.5\end{array}$ & $\begin{array}{l}.2 \\
.4 \\
.4 \\
1.3 \\
1.6 \\
3.1 \\
0.2\end{array}$ & $\begin{array}{r}47 \\
50 \\
21 \\
113 \\
64 \\
74 \\
123\end{array}$ & $\begin{array}{l}2.074 E-0 A \\
2.245 F-00 \\
4.2 H 4 L-69 \\
5.021 F-08 \\
2.840 E-08 \\
3.471 E-00 \\
5.455 E-68\end{array}$ & $\begin{array}{l}0.704 t-08 \\
4.431[-0 b \\
3.699 t-08 \\
2.109 t-07 \\
1.201 \mathrm{t}-0 \mathrm{1} \\
1.456 E-07 \\
2.291 E-0 i\end{array}$ & $\begin{array}{l}1665 \\
1065 \\
1665 \\
1665 \\
1065 \\
1065 \\
1665\end{array}$ \\
\hline & $\begin{array}{l}132.5 \\
132.5 \\
132.5 \\
132.5 \\
132.5 \\
132.5 \\
132.5 \\
132.5 \\
132.5 \\
132.5 \\
132.5 \\
132.5\end{array}$ & $\begin{array}{l}9.3 \\
12.4 \\
15.5 \\
18.6 \\
21.7 \\
24.8 \\
31.0 \\
37.8 \\
43.4 \\
49.6 \\
55.8 \\
62.0\end{array}$ & $\begin{array}{l}86 \\
103 \\
119 \\
135 \\
147 \\
134 \\
156 \\
05 \\
73 \\
57 \\
33 \\
78\end{array}$ & $\begin{array}{l}3.83 U E-U 8 \\
4.57 U E-06 \\
5.277 E-06 \\
5.973 E-08 \\
6.502 E-08 \\
6.100 E-08 \\
5.720 E-08 \\
3.762 t-08 \\
3.271 t-38 \\
2.5511-08 \\
1.4611-08 \\
3.214 E-0.8\end{array}$ & $\begin{array}{l}1.6 C 4 E-U 7 \\
1.91 .9 E-07 \\
2.216 t-07 \\
2.509 t-07 \\
2.731 t-07 \\
2.563 E-U 7 \\
2.406 t-07 \\
1.50 U E-U 7 \\
1.353 t-07 \\
1.072 t-U 7 \\
6.135 t-0 A \\
1.346 t-07\end{array}$ & $\begin{array}{l}1065 \\
1665 \\
1665 \\
1065 \\
1065 \\
1065 \\
1665 \\
1665 \\
1665 \\
1665 \\
1665 \\
1665\end{array}$ \\
\hline
\end{tabular}

TLIWER UATA FILLLIH....

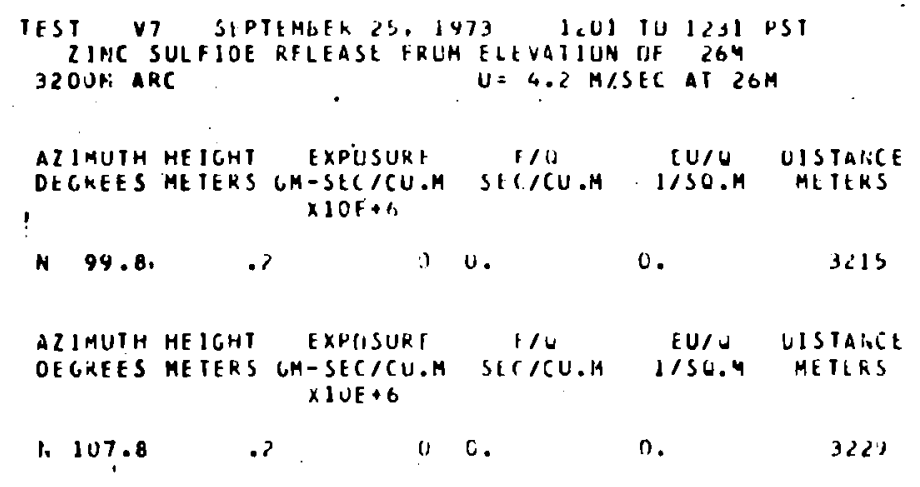




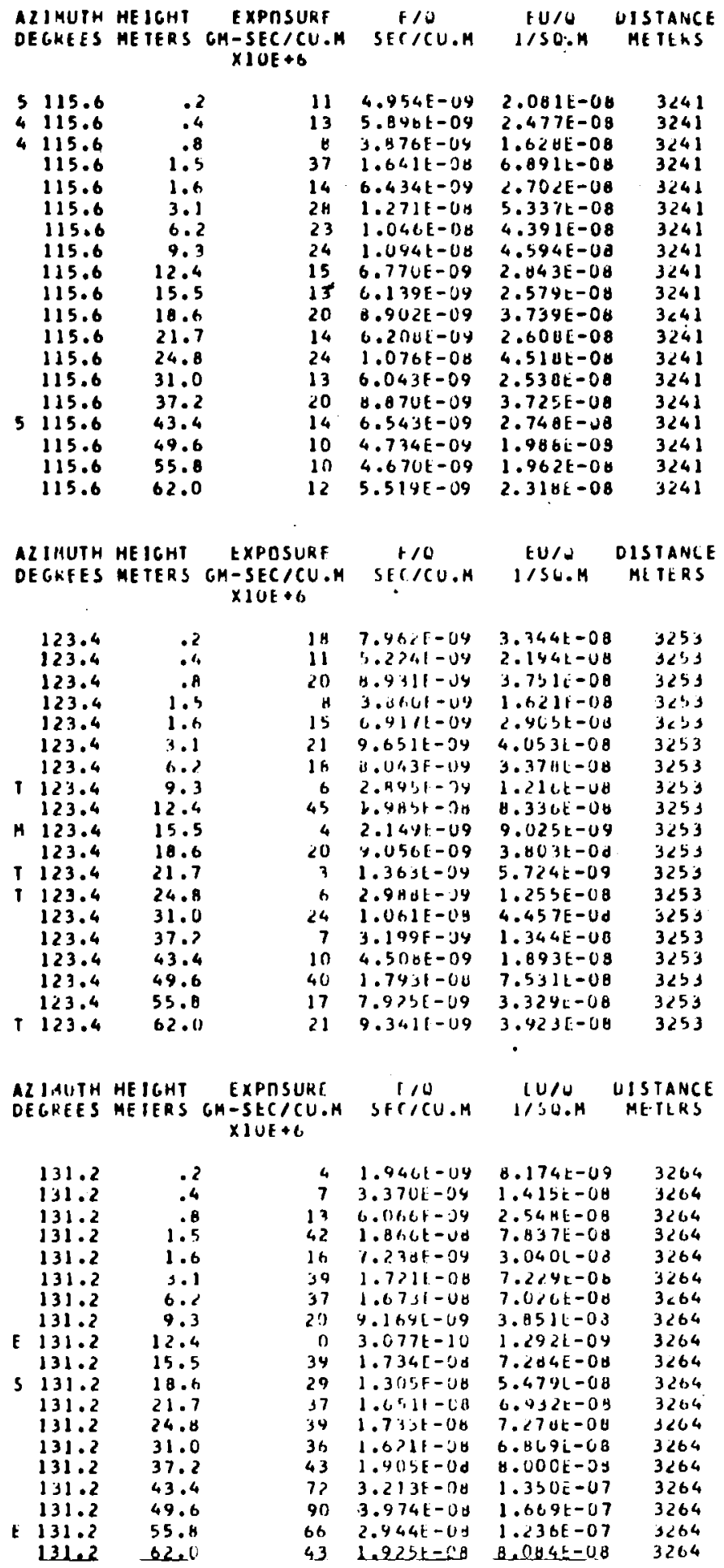




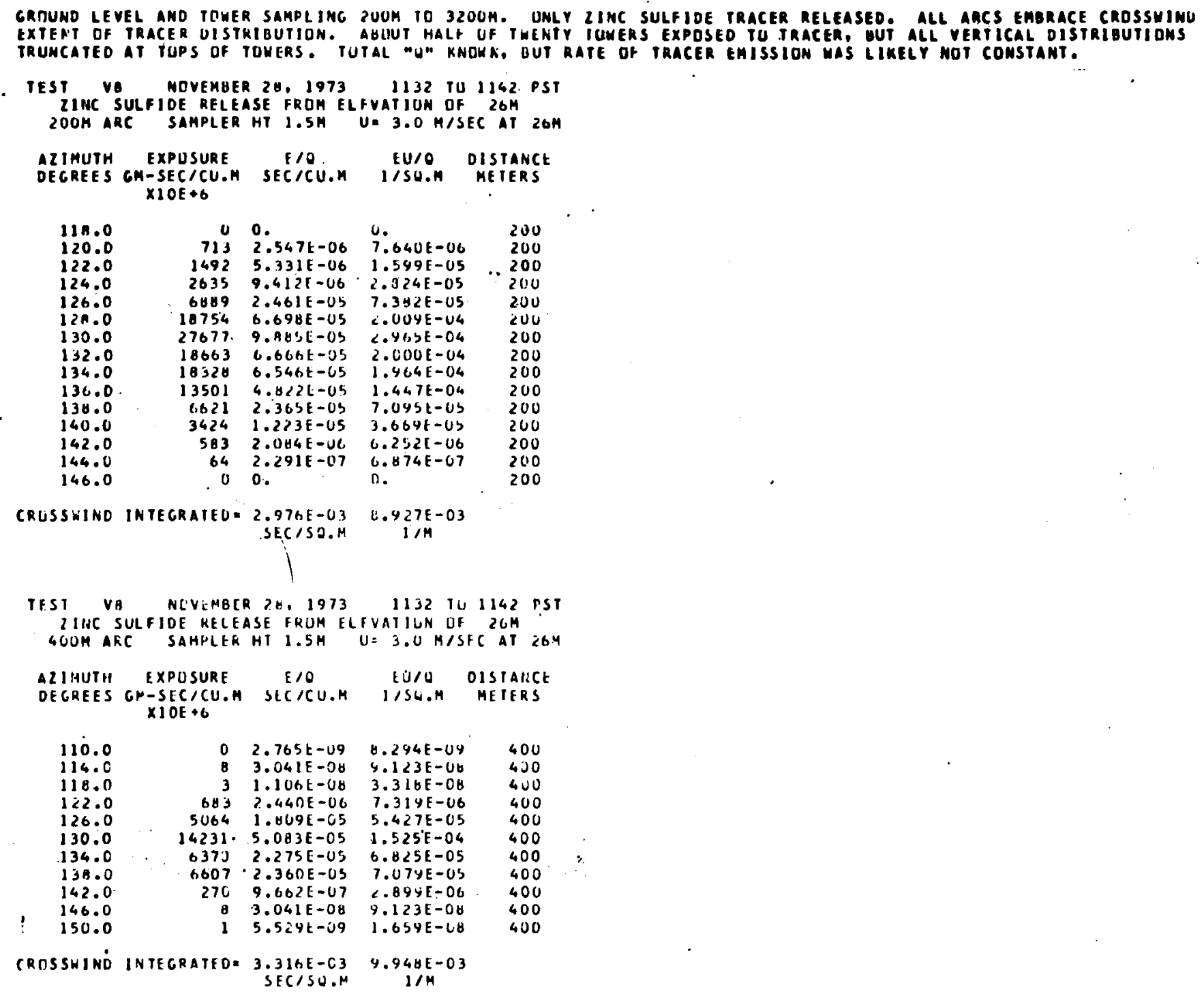




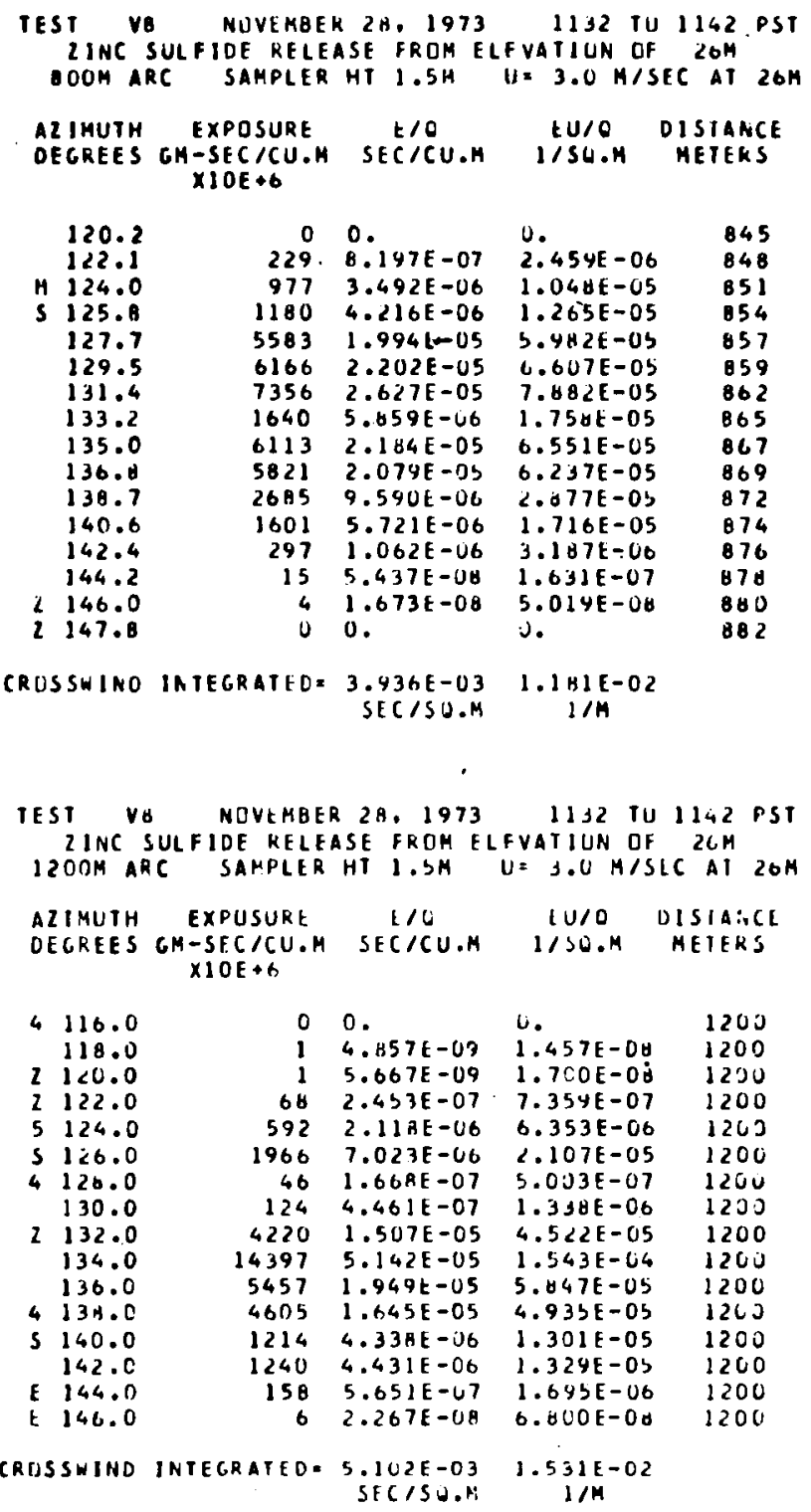




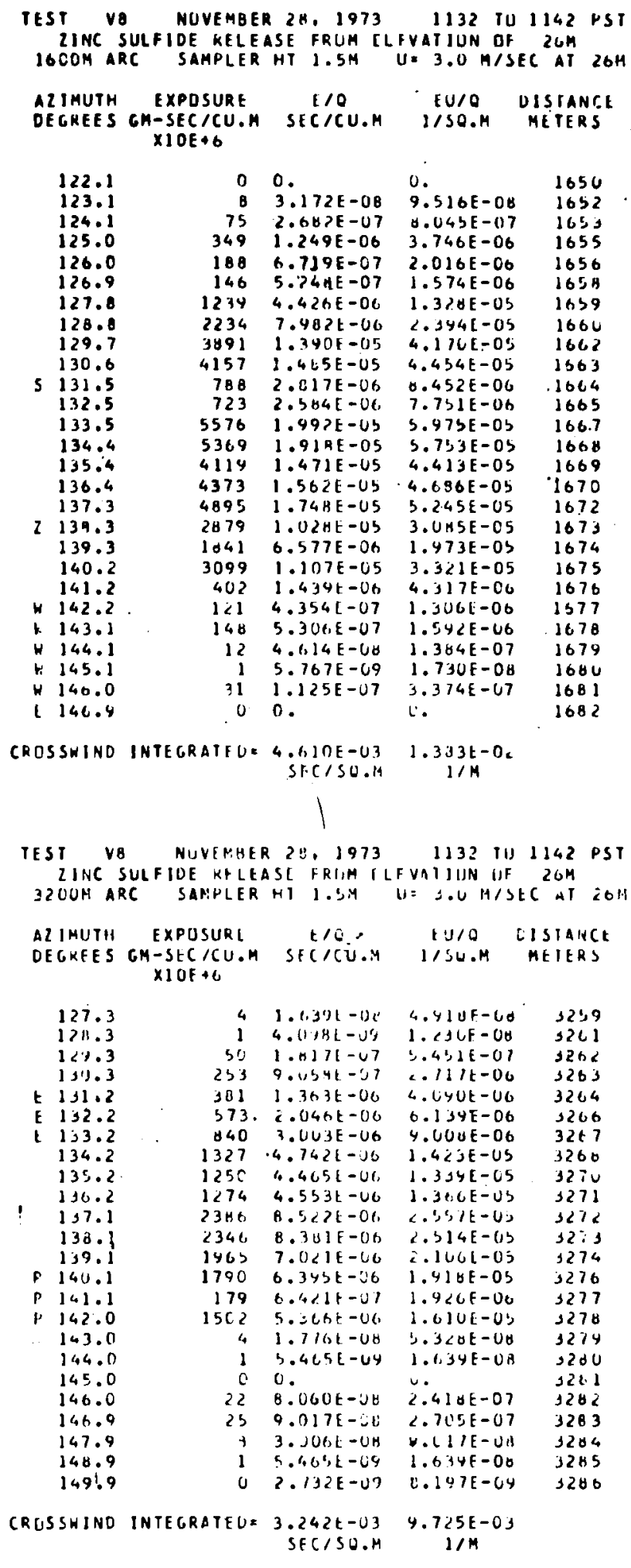


TOWER DATA FRLLOW....

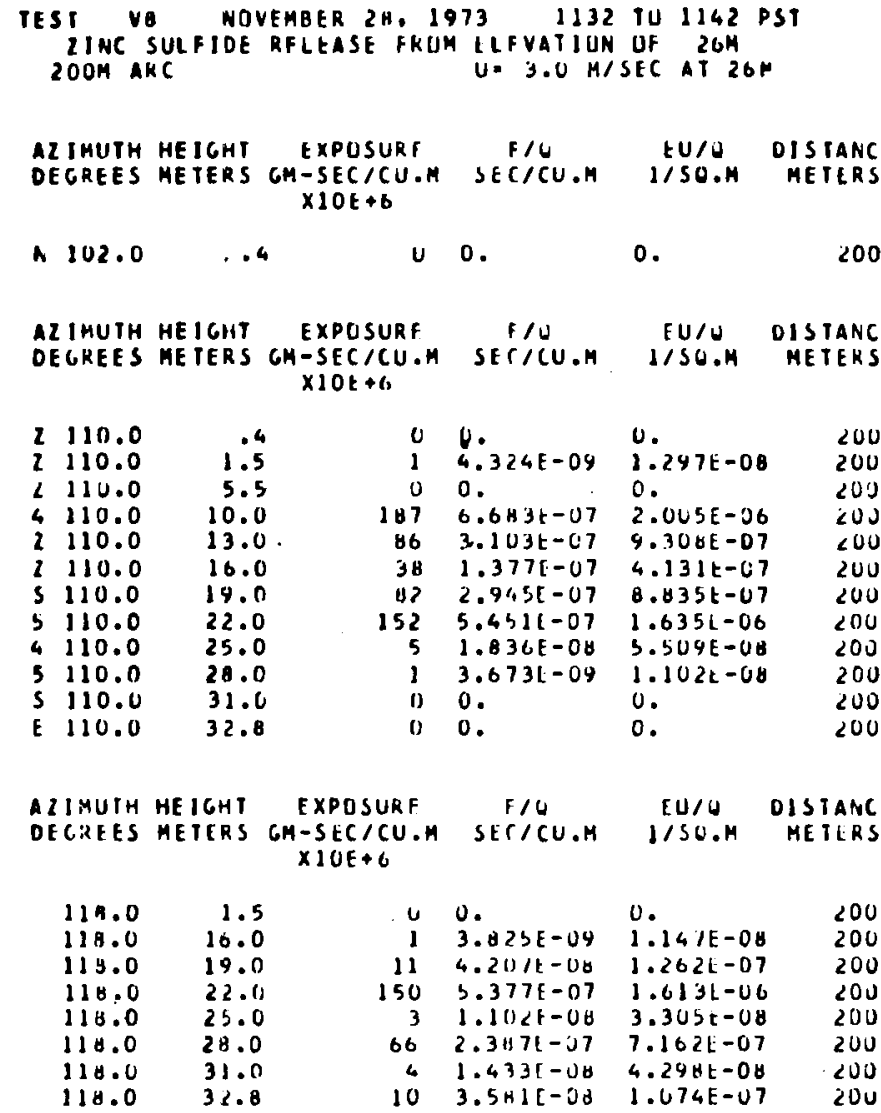

AIIMUTH HEIGHT EXPOSUKF $1 / 0$ EU/J UISTANCE DECREES METERS CM-SEC/RU.M SICICU.M IISO.M METLRS $\times 10 E+6$

\begin{tabular}{|c|c|c|c|c|c|}
\hline 126.0 & .4 & 1304 & 5.6 SYE = 16 & $1.694 t=05$ & 200 \\
\hline 126.0 & .8 & $283 t$ & $1.014 t-U b$ & $3.042 t-05$ & 200 \\
\hline $1<6.0$ & 1.5 & 6869 & $2.4611-05$ & $7.362 t-05$ & 200 \\
\hline 126.0 & 2.5 & 7224 & $2.5+2 t-09$ & $7.745 i-05$ & 200 \\
\hline 126.0 & 4.0 & 9542 & $3.400 \mathrm{t}-05$ & $1.022 t-04$ & 200 \\
\hline $\begin{array}{l}126.0 \\
126.0\end{array}$ & $\begin{array}{l}5.5 \\
7.0\end{array}$ & $\begin{array}{r}8916 \\
13046\end{array}$ & $\begin{array}{l}3.1 H 1 \mathrm{t}-\mathrm{US} \\
4.654 \mathrm{Y}-\mathrm{US}\end{array}$ & $\begin{array}{l}4.343 t-65 \\
1.396 t-04\end{array}$ & $\begin{array}{l}200 \\
200\end{array}$ \\
\hline 126.0 & 4.5 & 16330 & $5.8121-63$ & $1.750 t-04$ & 200 \\
\hline 126.0 & 10.0 & 26733 & $9.565 E-05$ & $2.864 E-C 4$ & 200 \\
\hline 126.0 & 13.0 & $\begin{array}{l}34477 \\
37879\end{array}$ & $1.231 E-44$ & $\begin{array}{l}3.694 E-C 4 \\
4-09-04-04\end{array}$ & 200 \\
\hline 126.0 & 19.0 & 36301 & $1.246 \mathrm{E}-\mathrm{U} 4$ & $3.0845-04$ & 2001 \\
\hline 126.0 & 22.0 & 43209 & $1.543 \mathrm{E}-014$ & $4.630 \mathrm{t}-\mathrm{C} 4$ & $<00$ \\
\hline 126.0 & 25.0 & 61156 & $2.1 B 4 E-04$ & $6.552 \mathrm{t}-04$ & $\angle 00$ \\
\hline 126.0 & 28.0 & $464<8$ & $1.676 t-04$ & $5.026 E-04$ & 200 \\
\hline & 31.0 & 34554 & $1.234 \mathrm{E}=04$ & $3.70<E-04$ & 200 \\
\hline 126.0 & 32.6 & 46424 & $1.7411-04$ & $5.242 t-04$ & 200 \\
\hline
\end{tabular}

ALIMUTH HEIGHT EXPDSULE I/O EU/W UISTANGE DECKEES METERS GM-SLC/CU.M SIC/CU.M I/SU.M MEIERS

$$
X \perp O E+h
$$

\begin{tabular}{|c|c|c|c|c|c|}
\hline 134.0 & .4 & 14029 & $5.0105-65$ & $1.5 \cup 3 t-04$ & $\angle 00$ \\
\hline 134.0 & .8 & 14757 & $5.270 \mathrm{E}-05$ & $1.501 t=04$ & 200 \\
\hline 134.0 & 1.5 & $10.4 c^{\prime \prime}$ & $6.5461-35$ & $1.934 t-04$ & $\angle D O$ \\
\hline 134.0 & 2.5 & $16+27$ & $5.9131-35$ & $1.771 \mathrm{t}-\mathrm{C} 4$ & 200 \\
\hline 134.0 & 4.0 & 11366 & $4.04 .5[-J 5$ & $1.214 \mathrm{t}-04$ & $\angle U J$ \\
\hline 134.0 & 5.5 & 14030 & $3.34, u t-U 5$ & $1.602 L-04$ & $\angle 00$ \\
\hline $\begin{array}{l}134.0 \\
134.0 \\
134.0\end{array}$ & $\begin{array}{r}7.0 \\
8.5 \\
10.0\end{array}$ & $\begin{array}{l}22734 \\
17972 \\
27045\end{array}$ & $\begin{array}{l}3.14<5-1,3 \\
6.419 F-05 \\
1.0711-05\end{array}$ & $\begin{array}{l}2.443 t-04 \\
1.926 t-04 \\
2.36<t=04\end{array}$ & $\begin{array}{l}200 \\
200 \\
200\end{array}$ \\
\hline $\begin{array}{l}134.0 \\
134.0\end{array}$ & $\begin{array}{l}13.0 \\
16.0 \\
10.0\end{array}$ & $\begin{array}{l}29002 \\
30533 \\
37184\end{array}$ & $\begin{array}{l}1.076 L-04 \\
1.376 E-04\end{array}$ & $\begin{array}{l}3.114 E-04 \\
4.124 t-04 \\
3.464 t-04\end{array}$ & $\begin{array}{l}\angle 00 \\
200 \\
200\end{array}$ \\
\hline $\begin{array}{l}134.0 \\
134.0\end{array}$ & $\begin{array}{l}19.0 \\
22.0\end{array}$ & $\begin{array}{l}37188 \\
42446\end{array}$ & $\begin{array}{l}1.376 t-04 \\
1.536 t-04\end{array}$ & $\begin{array}{l}3.464 i-C 4 \\
4.548 E-04\end{array}$ & $\begin{array}{l}200 \\
200\end{array}$ \\
\hline $\begin{array}{l}134.0 \\
134.0\end{array}$ & $\begin{array}{l}25.0 \\
28.0\end{array}$ & $\begin{array}{l}111119 \\
110223\end{array}$ & $\begin{array}{l}3.969 E-04 \\
3.937 E-04\end{array}$ & $\begin{array}{l}1.191 \mathrm{t}-03 \\
1.181 \mathrm{t}-03\end{array}$ & $\begin{array}{l}200 \\
200\end{array}$ \\
\hline $\begin{array}{l}134.0 \\
134.0\end{array}$ & $\begin{array}{l}31.0 \\
32.8\end{array}$ & $\begin{array}{l}45469 \\
61823\end{array}$ & $\begin{array}{l}1.642 E-04 \\
2.208 E-04\end{array}$ & $\begin{array}{l}4.927 t-04 \\
6.624 E-04\end{array}$ & $\begin{array}{l}200 \\
200\end{array}$ \\
\hline
\end{tabular}


TOWEK DATA FILLUH....

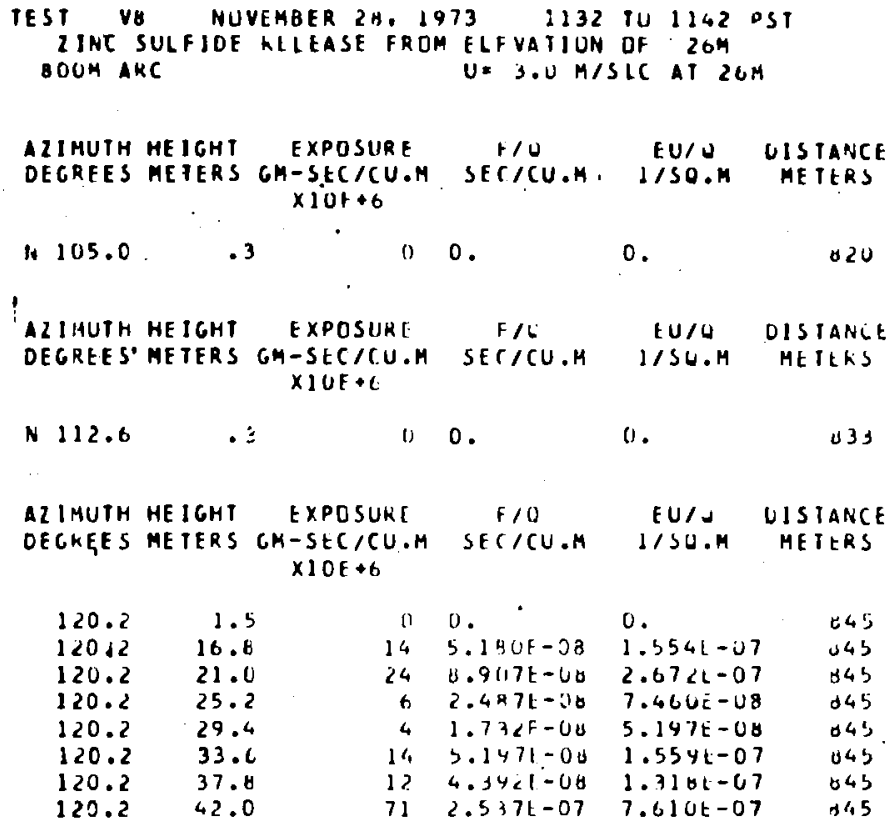

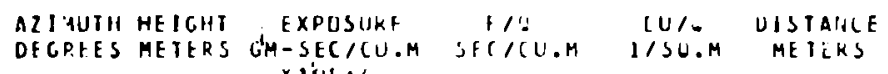
$\times 1 \mathrm{i} t+1$

\begin{tabular}{|c|c|c|c|c|c|c|}
\hline 127.7 & .3 & & 544 in & $1.14 .21-035$ & $6.4<71-u s$ & (1) \\
\hline 127.7 & .'s & & $4: 46$ & $1.411-24$ & $4.3 b c t-05$ & $0>8$ \\
\hline $1<7.7$ & 1.1 & & $50: 3$ & 1. HI J1-us & $5.406 t-65$ & של \\
\hline 127.7 & 1.3 & & 5563 & $1.944 \mathrm{t}-0.5$ & $3.482 .-15$ & แ」 7 \\
\hline 127.7 & 2.1 & & 5475 & $1.41,(1-65$ & $3.067 i-05$ & abl \\
\hline 127.7 & 4.2 & 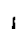 & $4 H 116$ & $1.71 / 1-05$ & 3.1, & I \\
\hline $1<7.7$ & 6.3 & 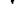 & 4652 & $1.0611-35$ & $4.48 \div L-0 B$ & d引 \\
\hline 127.7 & 8.4 & & 4262 & $1.3 / 2 t-J 5$ & $4.5661-05$ & 1 \\
\hline 127.7 & 10.3 & & 1329 & $4.740 \mathrm{E}-\mathrm{CO}$ & $1.424 E-U 5$ & 051 \\
\hline 127.7 & 12.6 & & 4231 & $1.518 E-05$ & $4.535 t-03$ & 057 \\
\hline 127 & 14.7 & & 9047 & $3.231 \mathrm{E}-0.0$ & $9.644 t-05$ & טל \\
\hline 127.7 & 16.6 & & 4209 & $1.2641-05$ & $4.5111-4 b$ & 437 \\
\hline 127.7 & 21.0 & & 3337 & $1.19<[-05$ & $3.576 t-05$ & 057 \\
\hline 127.7 & 25.2 & & 2402 & $3.0011 F-06$ & $2.500 t-05$ & 537 \\
\hline .7 & $\angle 9.4$ & & 1 jox & $\therefore$ ghet $-v 0$ & $1.61 \cup L-05$ & as 7 \\
\hline 127.7 & 33.1 & & 304,4 & לנ-נה & $3.206 \mathrm{t}-03$ & טל \\
\hline 127.7 & 37.6 & & $3<11$ & $3.00 i-1,6$ & $1.05 \cup L=65$ & obl \\
\hline & 42.0 & & 1445 & $\therefore 1 t ; 1-i 6$ & $1.344 i-63$ & os 7 \\
\hline
\end{tabular}

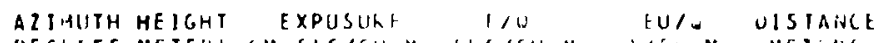

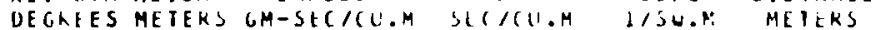

$$
\begin{aligned}
& x \perp 0 i+t \text {. }
\end{aligned}
$$

\begin{tabular}{|c|c|c|c|c|c|c|}
\hline & 135.0 & .3 & 0140 & $2.9075-05$ & $0.722 t-45$ & 067 \\
\hline & 135.6 & .5 & $170 \mathrm{C}$ & t. $.35 t t-06$ & $1.401:-05$ & 467 \\
\hline & 135.0 & 1.1 & 7032 & $2.7201-: 13$ & $0.177 t-05$ & 361 \\
\hline & 133.0 & 1.5 & 6113 & $2.1+4 i-63$ & $0.351 t-05$ & 061 \\
\hline & 135.0 & 2.1 & 4673 & $1.60 \times t-05$ & $3.007 i-05$ & 067 \\
\hline & 135.0 & 4.2 & 7035 & 2.74 ot -05 & $8.345 t-05$ & 367 \\
\hline & 135.0 & 6.3 & 968 & $3.436 E=36$ & $1.031 t-05$ & 267 \\
\hline & 135.0 & 8.4 & 1343 & $4.79 \mathrm{ct}=40$ & $1.43 y_{L}-0.5$ & 067 \\
\hline & 135.0 & 10.5 & 1405 & $5.021 E-00$ & 1. Sut -05 & 067 \\
\hline & 135.0 & 12.6 & 1413 & $5.048 E-06$ & $1.314 L-05$ & 001 \\
\hline & 133.0 & 14.7 & 1500 & $5.64 c t-0 c$ & $1.694 t-15$ & ye 7 \\
\hline M & 135.0 & 16.8 & 750 & $2.701 E-00$ & B. $10<5-06$ & 067 \\
\hline & 135.0 & 21.0 & 1107 & $3.94, t-00$ & $1.186 t-05$ & 867 \\
\hline$M$ & 135.0 & 25.2 & 712 & $2.544 E-20$ & $7.631[-06$ & 867 \\
\hline H & 135.0 & 29.4 & 749 & $2.670 \mathrm{E}-\mathrm{U} 6$ & $8.014 t-06$ & do 7 \\
\hline M & 135.0 & 33.6 & sus & $1.805 E-016$ & $3.413 t-06$ & 867 \\
\hline M & 135.0 & 37.8 & 526 & $1.8 \leqslant \angle E-66$ & $5.645 t-06$ & 867 \\
\hline & & & 736 & $2.702 E=06$ & $6.10 \mathrm{st}-\mathrm{Cb}$ & 867 \\
\hline
\end{tabular}


TOWER DATA FLLEN....

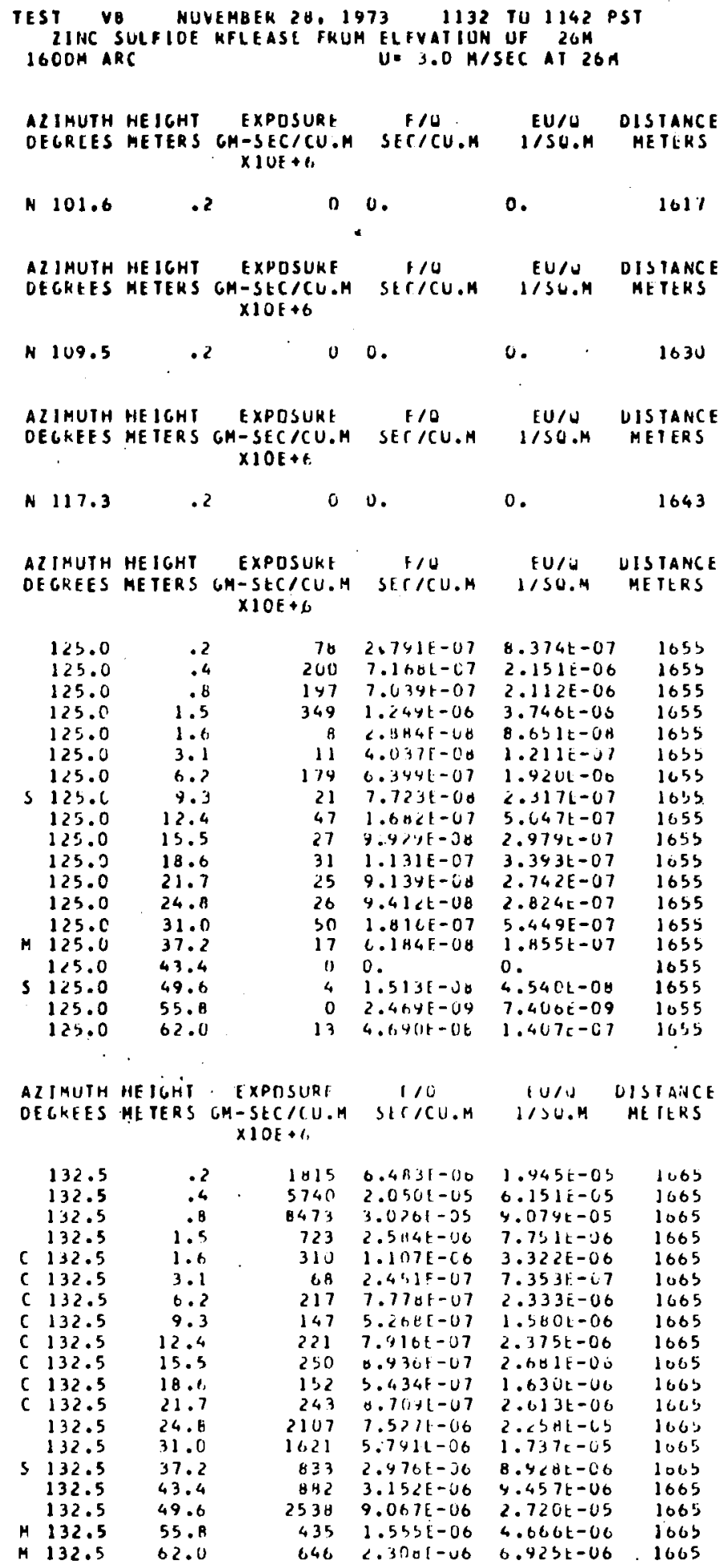


TUWFR DATA FDLOW...

TEST. VO NOVEMBEH 28, $1973 \quad 1132$ TU 1142 PST 2 INC SULFIOE RELEASE FRUM ELPVAIIUN UT $20 M$ 32 COM ARC $U=3.0 \mathrm{M} / \mathrm{SEC}$ AT $26 \mathrm{~m}$

\begin{tabular}{|c|c|c|c|c|c|c|c|}
\hline \multicolumn{2}{|c|}{$\begin{array}{l}\text { AZ IHUTH } \\
\text { DEGREES }\end{array}$} & $\begin{array}{l}\text { HEISHT } \\
\text { NE IRES }\end{array}$ & \multicolumn{2}{|c|}{$\begin{array}{c}\text { EXPOSURE } \\
\text { GH-SEC /CU.H } \\
\times 1 O E+6\end{array}$} & \multirow[t]{2}{*}{$\begin{array}{c}\text { F/O } \\
\text { Serreu.n }\end{array}$} & $\begin{array}{l}\text { EU/O } \\
\text { I/SO.M }\end{array}$ & $\begin{array}{l}\text { UISTANCE } \\
\text { METERS }\end{array}$ \\
\hline$\Lambda$ & 99.6 & .2 & & 0 & & 0 & $3<13$ \\
\hline & $\begin{array}{l}\text { J MUTH: } \\
\text { GKEES }\end{array}$ & $\begin{array}{l}\text { HE DCHT } \\
\text { ME IEAS } \\
\text { - }\end{array}$ & $\begin{array}{r}\text { EXPC } \\
6 H-S E C \\
\times 10 \%\end{array}$ & CU.M & $\begin{array}{c}\text { H O } \\
\text { SEC/CU.M }\end{array}$ & $\begin{array}{l}\text { tu/u } \\
1 / 50.4\end{array}$ & $\begin{array}{l}\text { UIS TANCE } \\
\text { HE IEKS }\end{array}$ \\
\hline N & 107.0 & .7 & & (1) & $u$. & 0 & $3<23$ \\
\hline & $\begin{array}{l}\text { JHUTH } \\
\text { CKEES }\end{array}$ & $\begin{array}{l}\text { HE IGHI } \\
\text { MEIERS }\end{array}$ & $\begin{array}{r}\text { EXP } \\
6 \mathrm{~A}-5 \mathrm{E} \\
\times 10\end{array}$ & $\begin{array}{l}\text { UKF } \\
\text { CU.H } \\
6\end{array}$ & $\begin{array}{c}F / U \\
S E C / C U . M \\
.\end{array}$ & $\begin{array}{l}{[U / 2} \\
1 / S \cup . M\end{array}$ & $\begin{array}{l}\text { UISTANCE } \\
\text { HETERS }\end{array}$ \\
\hline N & $1154^{6}$ & .2 & & 0) & 0. & 0 & $3<41$ \\
\hline & $\begin{array}{l}\text { IIUTH } \\
\text { EREES }\end{array}$ & $\begin{array}{l}\text { HE ICHI } \\
\text { ME DERS }\end{array}$ & $\begin{array}{r}E \times P 0 \\
6 m-3 t 0 \\
\times 10\end{array}$ & $\begin{array}{l}\text { IIRE } \\
\text { CU.M }\end{array}$ & $\begin{array}{c}1 / 0 \\
\text { Se } / 1 C U . H\end{array}$ & $\begin{array}{l}\text { EU/L } \\
1 / S O . M\end{array}$ & $\begin{array}{c}\text { DISIANCE } \\
\text { HETERS }\end{array}$ \\
\hline N & 123.4 & .2 & & 0 & 0. & 0. & $3 \times 3$ \\
\hline & $\begin{array}{l}\text { I YUTH } \\
\text { CHEES }\end{array}$ & $\begin{array}{l}\text { HEIGHT } \\
\text { MEIERS }\end{array}$ & $\begin{array}{r}\text { IEXPL } \\
6 A-5 L \\
\times 10\end{array}$ & $\begin{array}{l}\text { JRI } \\
\text { CU.A }\end{array}$ & $\begin{array}{c}F / 0 \\
\text { sfr. }\end{array}$ & $\begin{array}{l}E U / U \\
1 / S W \cdot r\end{array}$ & $\begin{array}{l}\text { WISTANCE } \\
\text { METERS }\end{array}$ \\
\hline $\begin{array}{l}\text { L } \\
\text { H } \\
\text { L }\end{array}$ & $\begin{array}{l}131.2 \\
131.2 \\
131.2 \\
131.2 \\
131.2 \\
131.2 \\
131.2 \\
131.2 \\
131.2 \\
131.2 \\
131.2 \\
131.2 \\
131.2 \\
131.2 \\
131.2 \\
131.2 \\
131.2 \\
131.2 \\
131.2\end{array}$ & $\begin{array}{r}.2 \\
.4 \\
.6 \\
1.5 \\
1.6 \\
3.1 \\
6.2 \\
9.3 \\
12.4 \\
15.5 \\
18.6 \\
21.7 \\
24.8 \\
31.0 \\
37.2 \\
43.4 \\
49.4 \\
55.8 \\
62.0\end{array}$ & $\begin{array}{l}3 \\
4 \\
5 \\
6 \\
\\
2 \\
3 \\
4 \\
5 \\
6 \\
4 \\
4 \\
4 \\
4 \\
4 \\
5\end{array}$ & $\begin{array}{r}33 \\
346 \\
106 \\
181 \\
16 \\
230 \\
434 \\
172 \\
42 \\
97 \\
113 \\
1 \\
2 \\
0 \\
1 \\
121 \\
40 \\
13 \\
67\end{array}$ & 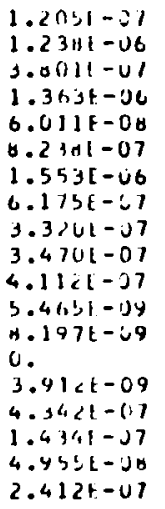 & 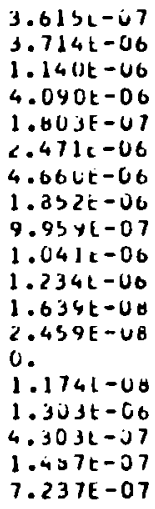 & $\begin{array}{l}3264 \\
3264 \\
3264 \\
3264 \\
3264 \\
3264 \\
3264 \\
3264 \\
3264 \\
3204 \\
3264 \\
3264 \\
3264 \\
3264 \\
3264 \\
3264 \\
3264 \\
3264 \\
3264\end{array}$ \\
\hline
\end{tabular}


APPENDIX B

DIFFUSION SUMMARIES 


$$
\text { B-1 }
$$


GREUAD-LEVEL AND TOWER SAMPLING 200M TO 3200M. INVERSION OF 3F DEG (2M TO 15M) AT O445: ISOTHERMAL AT O5I5. ALL ARCS EMBRACE CROSSWIND EXTENT OF TRACER DISTRIBUTION--EXCEPT MINUR EXTRAPOLATION AT NORTH END OF $200 M$ ARC. VERTICAL DISTRIBUTIUNS OF TRAGER ON ALL 20 TOWERS: PEAK VALUES MEASURED. BUT TRUNCATED AT TOP IN ALL CASES.

\begin{tabular}{|c|c|c|c|c|c|c|c|c|c|}
\hline $\begin{array}{l}\text { TEST } \\
\text { ZINC } \\
\text { SAMPLER }\end{array}$ & $\begin{array}{l}\text { DI } \\
\text { SULFIDE } \\
\text { HT } 1.5 \mathrm{M}\end{array}$ & $\begin{array}{l}\text { JULY } \\
\text { RELFASE }\end{array}$ & 7. 196 & $\begin{array}{l}7 \quad 0445 \\
\text { ELEVATIOI } \\
U=1.2 \mathrm{M} / \mathrm{S}\end{array}$ & $\begin{array}{l}\text { TU } 051 \\
\text { N LF } \\
\text { SEC AT }\end{array}$ & $\begin{array}{l}5 \text { PST } \\
2 M\end{array}$ & & $\therefore$ & \\
\hline $\begin{array}{l}\text { DISTANCE } \\
\text { FROM }\end{array}$ & $--\operatorname{CBOS}$ & $\begin{array}{l}\text { SWINDED } \\
\text { STAND }\end{array}$ & $\begin{array}{l}\text { SIRIBU } \\
\text {-COEEE }\end{array}$ & $\begin{array}{l}\text { IILN- } \\
\text { ICIENI_- }\end{array}$ & THREE & MAXIMUM EX & POSURES & $\begin{array}{l}\text { CROSS } \\
\text { INIEGRAIE }\end{array}$ & $\begin{array}{l}\text { WIND } \\
\text { D_YALUES }\end{array}$ \\
\hline SDURCE & MEAN & DEV & SKEW & KUR T & AZIMUTH & $E / 0$ & $E U / O$ & $C I \mid E / Q)$ & $C I(E U / Q)$ \\
\hline METERS & DEG & DEG, & & & OEG & $S E C / C U . M$ & $1 / 50 . M$ & SEC/SQ.M & $1 / M$ \\
\hline 200 & 99.6 & 12.23 & .154 & 2.894 & $\begin{array}{r}98.0 \\
102.0 \\
100.0\end{array}$ & $\begin{array}{l}2.77 E-04 \\
2.73 E-04 \\
2.45 E-04\end{array}$ & $\begin{array}{l}3.33 E-04 \\
3.28 E-04 \\
2.94 E-04\end{array}$ & $2.43 E-02$ & $2.91 E-02$ \\
\hline 800 & 105.8 & 7.57 & 1.244 & 5.923 & $\begin{array}{l}104.0 \\
102.0 \\
106.0\end{array}$ & $\begin{array}{l}9.46 \mathrm{E}-05 \\
8.18 \mathrm{E}-05 \\
7.57 \mathrm{E}-05\end{array}$ & $\begin{array}{l}1.13 E-04 \\
9.82 E-05 \\
9.08 E-05\end{array}$ & $1.68 \mathrm{E}-02$ & $2.01 E-02$ \\
\hline 1600 & 108.7 & 7.64 & 1.183 & 5.502 & $\begin{array}{l}103.0 \\
102.0 \\
106.0\end{array}$ & $\begin{array}{l}2.54 E-05 \\
2.47 E-05 \\
2.31 E-05\end{array}$ & $\begin{array}{l}3.04 E-05 \\
2.96 E-05 \\
2.78 E-05\end{array}$ & $1.03 E-02$ & $1.24 E-02$ \\
\hline 3200 & 108.1 & 8.59 & 1.338 & 5.505 & $\begin{array}{l}101.0 \\
102.0 \\
100.0\end{array}$ & $\begin{array}{l}6.76 E-06 \\
5.85 E-06 \\
5.20 E-06\end{array}$ & $\begin{array}{l}8.12 E-06 \\
7.03 E-06 \\
6.23 E-06\end{array}$ & $4.00 E-03$ & $4.80 E-03$ \\
\hline - & INL & CATES UA & NCERT & INTIES IN & DATA. & & & & \\
\hline & $X \quad I N$ & ATES & AL IU & OR INCOM & ETE. & A. & & & \\
\hline
\end{tabular}


TEST DI JULY 7, 1967 6445 T'J OSI5 PST

FLUGRESCEIN RELEASE FROM ELEVATILN OF LY

\begin{tabular}{|c|c|c|c|c|c|c|c|c|c|}
\hline \multirow{2}{*}{$\begin{array}{l}\text { DISTANCE } \\
\text { FROH } \\
\text { SLURCE }\end{array}$} & \multicolumn{4}{|c|}{$\begin{array}{l}\text {-CRLSSWIND_DISIRIBUIIGL } \\
\text { STAND -COEEEICLENI- }\end{array}$} & \multicolumn{3}{|c|}{ THREE MAXIMUM EXPLSURES } & \multicolumn{2}{|c|}{$\begin{array}{c}\text { CKUSSWIND } \\
\text { LUIEERAIEQ_KALULS_ }\end{array}$} \\
\hline & MEAN & OEV & SKEW & KURT & ALIMUTH & $E / J$ & EU/O & $C I(E / N)$ & CIIEU/O) \\
\hline$\cdots--\cdots$ & $\cdots-\infty$ & $--\infty-\infty$ & $---\cdots$ & ------ & ------ & -- & $\cdots-\cdots-\cdots$ & $---\infty \cdot--$ & - \\
\hline METTERS & DEG & OEG & & & DES & $S[C / C U \cdot M$ & $1 / S U . M$ & SEC/SW.M & $1 / M$ \\
\hline . & & & & & & & & & \\
\hline$\because 200$ & 100.1 & 11.97 & .113 & 2.832 & $\begin{array}{r}98.0 \\
96.0 \\
102.0\end{array}$ & $\begin{array}{l}4.42 E-04 \\
3.34 t-04 \\
3.44 t-04\end{array}$ & $\begin{array}{l}5.31 t-04 \\
4.25 L-04 \\
4.13 L-04\end{array}$ & $3.32 t-02$ & $3.90 \mathrm{E}-02$ \\
\hline$\triangle C O$ & 105.7 & 7.40 & 1.290 & 5.778 & $\begin{array}{l}134.0 \\
106.0 \\
102.0\end{array}$ & $\begin{array}{l}9.09 t-U 5 \\
y .05 t-05 \\
y .51 E-U 5\end{array}$ & $\begin{array}{l}1.19 t-U 4 \\
1.19 E-U 4 \\
1.14 t-04\end{array}$ & $1.64 t-32$ & $2.2 U E-02$ \\
\hline 1600 & 107.2 & 8.08 & 1.289 & $\left.5.23^{\prime}\right)$ & $\begin{array}{r}100.0 \\
99.0 \\
103.0\end{array}$ & $\begin{array}{l}5.42 t-j 5 \\
3.46 t-J 5 \\
2.20 E-U 5\end{array}$ & $\begin{array}{l}0.30 t-U S \\
4.16 E-U S \\
2.65 t-U S\end{array}$ & $1.0 \exists t-v 2$ & $1.31 \mathrm{E}-02$ \\
\hline 3200 & 108.1 & B. 24 & 1.250 & 5.451 & $\begin{array}{l}101.0 \\
102.0 \\
10 J .0\end{array}$ & $\begin{array}{l}6.12 E-66 \\
3.12 L-J 6 \\
4.97 t--66\end{array}$ & $\begin{array}{l}7.34 L-0 b \\
6.14 t-6 b \\
b .97 t-60\end{array}$ & $3.03 E-03$ & $4.6 \cup E-0.3$ \\
\hline
\end{tabular}

$?$ INOICATFS UNCERTAINTIES IN UATA.

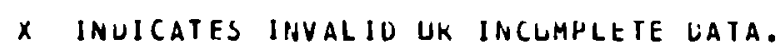


GROUND LEVEL AND TOWER SAMPLING 20OM TO 3200M. ALL ARCS CONTAIN CROSSWINO EXTENT OF TRACER DISTRIBUTIONS. VERTICAL DISTRIBUTION OF TRAKER ON MOST TUWERS. CDNCENTRATIONS FALL TO BACKGROUND BELOW TOPS OF MOST TOWERS. STRONG INVERSION THRDUGH THE ENTIRE DEPTH OF THE $122 \mathrm{M}$ TUWER. STRONG RESERVATIUNS ON "Q" FOR BOTH TRACERS.

\begin{tabular}{|c|c|c|c|c|c|c|c|c|c|}
\hline $\begin{array}{l}\text { TEST } \\
\text { ZINC } \\
\text { SAMPLER }\end{array}$ & $\begin{array}{l}\text { D2 } \\
\text { SULFIDE } \\
\text { HT } 1.5 \mathrm{M}\end{array}$ & $\begin{array}{l}\text { JULY } 1 \\
\text { RELEASE }\end{array}$ & $\begin{array}{l}1 \text { P } 196 \\
\text { FROM }\end{array}$ & $\begin{array}{l}7 \\
\text { ELEVAIII } \\
U=1.9 \mathrm{MI}\end{array}$ & $\begin{array}{lll}O & T U & 042 \\
N & O F & 2 \\
S E C & A T\end{array}$ & $\begin{array}{l}0 \text { PST } \\
M \\
Z M\end{array}$ & & & \\
\hline $\begin{array}{l}\text { DI STANCE } \\
\text { FRLIM }\end{array}$ & \multicolumn{4}{|c|}{$\begin{array}{l}\text { CBOSSWIND_DISIBIBUIIDN- } \\
\text { STAND COEEEICIENI_ }\end{array}$} & THREE & \multicolumn{2}{|c|}{ MAXIMUM EXPOSURES } & \multicolumn{2}{|c|}{$\begin{array}{l}\text { CROSSWIND } \\
\text { IAIEGBAIEO_YALUES- }\end{array}$} \\
\hline SUURCE & MEAN & DEV & SKEW & KUR T & AZIMUTH & $E / O$ & $E U / Q$ & $C I(E / O)$ & $C I(E U / O)$ \\
\hline------- & ---- & $-\cdots--$ &.----- & ----- & $\cdots----$ & -------- & $---\cdots--$ & $---\cdots-c$ & --- \\
\hline METERS & DEC & DEG & & & DEG & SEC/CU.M & $1 / 50 . M$ & SEC/SO.M & $1 / M$ \\
\hline 200 & 102.2 & 8.63 & -.193 & 1.750 & $\begin{array}{r}108.0 \\
110.0 \\
94.0\end{array}$ & $\begin{array}{l}3.49 \mathrm{E}-04 ? \\
3.20 \mathrm{E}-\mathrm{U} 4 ? \\
2.46 \mathrm{E}-04 ?\end{array}$ & $\begin{array}{l}6.63 E-04 ? \\
6.08 E-04 ? \\
4.66 E-04 ?\end{array}$ & $2.09 E-02 ?$ & $3.97 E-027$ \\
\hline 200 & 101.6 & 8.59 & .192 & 1.750 & $\begin{array}{r}92.0 \\
94.0 \\
100.0\end{array}$ & $\begin{array}{l}7.56 E-05 ? \\
6.72 E-05 ? \\
5.62 E-05 ?\end{array}$ & $\begin{array}{l}1.44 \mathrm{E}-04 ? \\
1.28 \mathrm{E}-04 ? \\
1.11 \mathrm{E}-04 ?\end{array}$ & $1.79 E-02 ?$ & $3.41 E-02 ?$ \\
\hline 1600 & 103.7 & 6.80 & .221 & 1.801 & $\begin{array}{l}97.0 \\
96.0 \\
98.0\end{array}$ & $\begin{array}{l}2.84 E-05 ? \\
2.01 E-05 ? \\
2.48 E-05 ?\end{array}$ & $\begin{array}{l}5.40 E-05 ? \\
5.34 E-05 ? \\
4.72 E-05 ?\end{array}$ & $1.10 E-02 ?$ & $2.09 E-02 ?$ \\
\hline 3200 & 107.7 & 4.74 & .187 & 1.996 & $\begin{array}{l}104.0 \\
103.0 \\
105.0\end{array}$ & $\begin{array}{l}6.14 E-06 ? \\
5.88 t-06 ? \\
4.56 t-06 ?\end{array}$ & $\begin{array}{l}1.17 E-05 ? \\
1.12 E-05 ? \\
3.66 E-06 ?\end{array}$ & $3.50 E-03 ?$ & $6.65 E-03 ?$ \\
\hline
\end{tabular}

$?$ INDICATES UNCERTAINTIES IN DATA.

X INDICATES INVALIO DR INCCMPLETE DATA. 
DATA FRDM THE LABORATURY ASSESSMENT FOR FLUORESCEIN WAS LOST. HOHEVER. RESULTS FROM THAT ASSESMENT HAD GEEN GRAPHED. THE FLUDRESCEIN DATA THAT FOLLOW EVQLVE FROM THESE GRAPHS.

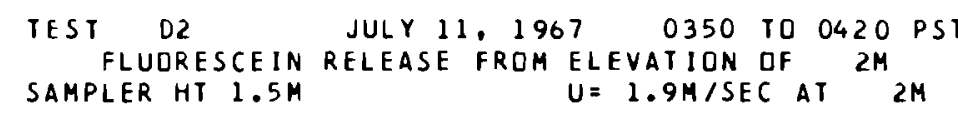

\begin{tabular}{|c|c|c|c|c|c|c|c|c|c|}
\hline \multirow{2}{*}{$\begin{array}{l}\text { DISTANCE } \\
\text { FROM } \\
\text { SOURCE }\end{array}$} & \multicolumn{4}{|c|}{$\begin{array}{l}\text { STAND -COEEFICIENI- } \\
\text { STANDISIBIBUIION }\end{array}$} & \multicolumn{3}{|c|}{ THREE MAXIMUM EXPOSURES } & \multicolumn{2}{|c|}{$\begin{array}{l}\text { CROSSWIND } \\
\text { INIEGRAIEQ_YALUES- }\end{array}$} \\
\hline & MEAN & DEV & SKEW & KURT & AZIMUTH & $E / O$ & $E U / O$ & $C I(E / O)$ & $C I(E \cup / Q)$ \\
\hline & & & & & & & & & \\
\hline METERS & DEG & DEG & & & DEG & SEC/CU.M & $1 / 50 . M$ & SEC/SO.M & $1 / M$ \\
\hline 200 & 101.9 & 8.37 & -.152 & 1.749 & $\begin{array}{r}108.0 \\
110.0 \\
92.0\end{array}$ & $\begin{array}{l}4.82 E-04 ? \\
4.16 E-04 ? \\
3.94 E-04 ?\end{array}$ & $\begin{array}{l}9.16 \mathrm{E}-04 ? \\
7.91 \mathrm{E}-04 ? \\
7.48 \mathrm{E}-04 ?\end{array}$ & $2.75 E-02 ?$ & $5.22 \mathrm{E}-02 ?$ \\
\hline 800 & 101.0 & 8.33 & .295 & 1.886 & $\begin{array}{l}92.0 \\
94.0 \\
96.0\end{array}$ & $\begin{array}{l}9.91 \mathrm{E}-05 ? \\
8.19 \mathrm{E}-05 ? \\
7.27 \mathrm{E}-05 ?\end{array}$ & $\begin{array}{l}1.88 E-04 ? \\
1.56 E-04 ? \\
1.38 E-04 ?\end{array}$ & $2.21 E-02 ?$ & $4.21 E-02 ?$ \\
\hline 1600 & 103.7 & 6.75 & .220 & 1.838 & $\begin{array}{l}97.0 \\
98.0 \\
95.0\end{array}$ & $\begin{array}{l}3.24 E-05 ? \\
2.77 E-05 ? \\
2.58 E-05 ?\end{array}$ & $\begin{array}{l}6.15 E-05 ? \\
5.27 E-05 ? \\
4.89 E-05 ?\end{array}$ & $1.18 \mathrm{E}-02 ?$ & $2.23 E-02 ?$ \\
\hline 3200 & 108.1 & 4.67 & .121 & 2.098 & $\begin{array}{l}104.0 \\
110.0 \\
109.0\end{array}$ & $\begin{array}{l}5.28 E-06 ? \\
4.82 E-06 ? \\
4.69 E-06 ?\end{array}$ & $\begin{array}{l}1.00 E-05 ? \\
9.16 E-06 ? \\
8.91 E-06 ?\end{array}$ & $3.43 E-03 ?$ & $6.52 E-03 ?$ \\
\hline
\end{tabular}

? INDICATES UNCERTAINTIES IN DATA.

X INDICATES INVALID OR INCOMPLETE DATA. 
CRLUNO LEVEL ANO TOWEK SAMPLING 2 UOM TO $3200 M$.

2UOM ARC EXIRAPULATED AT BOTH EDGES. FLAT ORं MULTI-MODE CRCSSNIND DISTRIBUTIONS ON ALL ARCS. INVALIU SAMPLES ABOVE 4.2M LEVEL ON TOWER AT 122 DEG ON BOUM ARC. VERTICAL UISTRIGUTIUN OF IRACERS LN ALL 20 TUWERS: PEAK VALUES MEASUREO. BUT TRUNCATEU AT TOP IN ALL CASES.

\begin{tabular}{|c|c|c|c|c|c|c|c|c|c|}
\hline $\begin{array}{l}\text { TEST L } \\
\text { ZINC } \\
\text { SAMPLER }\end{array}$ & $\begin{array}{l}\text { US } \\
\text { SULFIUE } \\
\text { HT } 1.5 \mathrm{M}\end{array}$ & $\begin{array}{r}\text { JULY } 1 \\
\text { RELFASE }\end{array}$ & $\begin{array}{r}13,1967 \\
\text { E FROM EL } \\
U\end{array}$ & $\begin{array}{r}0400 \\
\text { LEVATIUN } \\
=2.0 \mathrm{M} / \mathrm{S}\end{array}$ & $\begin{array}{l}\text { TU U } 430 \\
\text { UF } 21 \\
\text { SEC AT }\end{array}$ & $\begin{array}{l}0 \text { PST } \\
2 M\end{array}$ & ! & & \\
\hline $\begin{array}{l}\text { DISTANCE } \\
\text { FRCM }\end{array}$ & \multicolumn{4}{|c|}{$\begin{array}{c}\text { CRLSSNINDELISIBIBUIICIN- } \\
\text { STANU COEEEICIENI- }\end{array}$} & \multicolumn{3}{|c|}{ THREE MAXIMUM EXPOSURES } & \multirow{2}{*}{\multicolumn{2}{|c|}{$\begin{array}{l}\text { CROSSWIND } \\
\text { INIEGBAIED_YALUES- } \\
\text { CI (E/O) CIIEU/QI }\end{array}$}} \\
\hline SOURCE & MEAN & OEV & SKEW & KURT & AZ IMUTH & $E / Q$ & $\mathrm{EU} / \mathrm{O}$ & & \\
\hline$\cdots-\cdots$ & ----- & ------ & ----- & ------ & ----- & $----\infty$ & $---\cdot ?--$ & $-\cdots--$ & ------- \\
\hline METERS & DFG & UEG, & & & DEG & SEC/CU.M & $1 / 5 Q . M$ & SEC/SU.M & $1 / M$ \\
\hline 200 & $106.6 ?$ & $32.29 ?$ & $.657 ?$ & $2.014 ?$ & $\begin{array}{l}72.0 \\
74.0 \\
32.0\end{array}$ & $\begin{array}{l}8.29 t-05 \\
8.00 E-0.5 \\
7.52 E-U 5\end{array}$ & $\begin{array}{l}2.15 E-04 \\
2.08 E-04 \\
1.95 E-04\end{array}$ & $1.22 \mathrm{E}-02 ?$ & $3.16, E-02 ?$ \\
\hline 800 & 118.7 & 24.17 & -.089 & 1.597 & $\begin{array}{l}86.0 \\
08.0 \\
90.0\end{array}$ & $\begin{array}{l}1.22 E-U 5 \\
1.02 E=05 \\
7.74 E-U 6\end{array}$ & $\begin{array}{l}3.17 E-05 \\
2.66 E-05 \\
2.01 E-05\end{array}$ & $5.00 E-03$ & $1.3 \cup E-02$ \\
\hline 1600 & 118.3 & 17.53 & .312 & 1.818 & $\begin{array}{l}111.0 \\
108.0 \\
112.0\end{array}$ & $\begin{array}{l}5.00 E-06 \\
4.49 E-06 \\
4.49 E-06\end{array}$ & $\begin{array}{l}1.3 U E-05 \\
1.17 E-05 \\
1.17 E-05\end{array}$ & $4.76 E-03$ & $1.24 E-02$ \\
\hline 3200 & 117.1 & $15.07^{\circ}$ & .079 & 1.831 & $\begin{array}{l}96.0 \\
Y 5.0 \\
y 7.0\end{array}$ & $\begin{array}{l}1.89 E-J 6 \\
1.68 E-06 \\
1.67 E-66\end{array}$ & $\begin{array}{l}4.91 E-06 \\
4.36 E-06 \\
4.34 E-06\end{array}$ & $\begin{array}{c}2.67 E-03 \\
.\end{array}$ & $6.94 E-03$ \\
\hline
\end{tabular}

? INDICATES UNCERTAINTIES IN DATA.

X INOICATES INVAL ID DR INCOMPLETE DATA. 


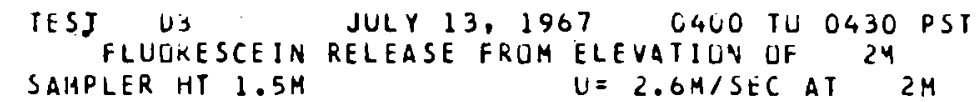

\begin{tabular}{|c|c|c|c|c|c|c|c|c|c|}
\hline $\begin{array}{l}\text { CISTANCE } \\
\text { FROAG }\end{array}$ & - CBOS & $\begin{array}{l}\text { SHIND_D } \\
\text { STANO }\end{array}$ & $\begin{array}{l}\text { ISIR } \perp B U I \\
\text {-COEEEI }\end{array}$ & $\begin{array}{l}\text { IDW } \\
\text { CIENI_- }\end{array}$ & & MAXIMUM E & POSURES & $\begin{array}{l}\text { CKUSS } \\
\text { INIEGBAIE }\end{array}$ & $\begin{array}{l}\text { IND } \\
\text { _KALUES_ }\end{array}$ \\
\hline SOURCE & MEAN & OEV & SKEW. & KURT & AZIMUTA & $E / C$ & $E \cup / Q$ & $C I(E N)$ & CIIEU/O) \\
\hline------- & ---- & ----- & ----- & ----- & ------ & ----- & ------ & -------4 & ------ \\
\hline METERS & DEG & DEG & & & UEC & SEC/CU.M & $1 / \mathrm{SW} \cdot \mathrm{M}$ & SEC/SG.M & $1 / M$ \\
\hline 200 & $102.1 ?$ & $28.71 ?$ & $.899 ?$ & $2.579 ?$ & $\begin{array}{l}78.0 \\
92.0 \\
74.0\end{array}$ & $\begin{array}{l}1.16 E-U 4 \\
1.09 E-J 4 \\
1.05 E-04\end{array}$ & $\begin{array}{l}3.01 E-04 \\
2.04 E-04 \\
2.73 E-04\end{array}$ & $1.43 E-02 ?$ & $3.7 \angle E-02 ?$ \\
\hline 800 & 110.2 & 23.60 & -.087 & 3.685 & $\begin{array}{r}126.0 \\
06.0 \\
68.0\end{array}$ & $\begin{array}{l}1.56 t-05 \\
1.49 t-J 5 \\
1.36 t-05\end{array}$ & $\begin{array}{l}4 . \cup 5 t-05 \\
3.80 t-05 \\
3.60 t-i 5\end{array}$ & $0.74 E-03$ & $1.75 E-02$ \\
\hline 1600 & 117.0 & 17.46 & .413 & 1.904 & $\begin{array}{l}98.0 \\
97.0 \\
y 6.0\end{array}$ & $\begin{array}{l}7.42 E-U 6 \\
5.42 E-U 6 \\
4.05 E-06\end{array}$ & $\begin{array}{l}1.93 t-J 5 \\
1.41 t-05 \\
1.26 t-0 b\end{array}$ & $4.95 t-03$ & $1.29 E-02$ \\
\hline \pm 200 & 116.1 & 15.00 & .184 & 1.827 & $\begin{array}{l}95.0 \\
96.0 \\
97.0\end{array}$ & $\begin{array}{l}1.59 E-J 6 \\
1.55 E-16 \\
1.35 E-j 6\end{array}$ & $\begin{array}{l}4.13 E-00 \\
4.02 E-06 \\
3.32 E-06\end{array}$ & $2.26 E-03$ & $5.87 \mathrm{E}-03$ \\
\hline
\end{tabular}

? INDICATES UNCERTAINTIES IN DATA.

x INDicates INVALio or INCUMPlete vata. 
GRDUND LEVEL AND TOWER SAMPLING 200M TO 3200M. ALL GRDUND LEVEL SAMPLING TRUNCAIED SIGNIFICANTLY DN NDRTH. TEST TERMINATED AFTER 16 MINUTES BECAUSE SURFACE WIND SPEED DRUPPED TL CALM.

TRACER DN MCST TOWERS, BUT FREQUENTLY LIGHT AND SPDTIY.

\begin{tabular}{|c|c|c|c|c|c|c|c|c|c|}
\hline $\begin{array}{l}\text { TEST D } \\
\text { ZINC } \\
\text { SAMPLER }\end{array}$ & $\begin{array}{l}\text { D4 } \\
\text { SULF IDE } \\
\text { HT } 1.5 M\end{array}$ & $\begin{array}{l}\text { AUGUST } \\
\text { RELFASE }\end{array}$ & $\begin{array}{r}1967 \\
\text { FROM E } \\
\text { U }\end{array}$ & $\begin{aligned} & 0405 \\
& \text { LEVATIUI } \\
&= 1.4 \mathrm{M} / \mathrm{S}\end{aligned}$ & $\begin{array}{l}\text { IU } 0421 \\
\text { OF } \\
\text { SEC AT }\end{array}$ & $\begin{array}{l}1 \text { PST } \\
2 M\end{array}$ & & & \\
\hline $\begin{array}{l}\text { DISTANCE } \\
\text { FKLIM }\end{array}$ & \multicolumn{4}{|c|}{-CQUSSWIND_DISIBIGUIJUA } & THREE & \multicolumn{2}{|c|}{ MAXIMUM EXPUSURES } & \multicolumn{2}{|c|}{$\begin{array}{l}\text { CROSSWIND } \\
\text { INIESBAIED_XALUES }\end{array}$} \\
\hline SUURCE & MEAN & DI: $V$ & SKEW & KUR I & $\wedge Z I M U T H$ & $F / U$ & $E U / 0$ & $C I(E / U)$ & CIIEU/O) \\
\hline$\cdots-\cdots-\infty$ & ----- & ----- & ----- & $-\cdots--$ & 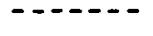 & $---\cdots-\cdot$ & $\cdots-\cdots$ & 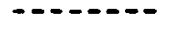 & $-\infty-\infty-\infty$ \\
\hline METERS & DEG & DEG & & & DEG & SEC/CU.M & $1 / 50 . M$ & $S E C / S O . M$ & $1 / M$ \\
\hline 200 & $85.8 x$ & $5.58 x$ & $-1.042 x$ & $4.638 x$ & $\begin{array}{l}68.0 \\
06.0 \\
90.0\end{array}$ & $\begin{array}{l}3.12 E-04 \\
3.04 E-04 \\
2.73 E-04\end{array}$ & $\begin{array}{l}5.21 E-04 \\
4.26 E-04 \\
3.83 E-04\end{array}$ & $1.23 E-02 x$ & $1.72 E-02 x$ \\
\hline 800 & $77.7 x$ & $6.27 x$ & $.893 x$ & $3.770 x$ & $\begin{array}{l}70.0 ? \\
76.0 ? \\
74.0 ?\end{array}$ & $\begin{array}{l}1.23 E-05 ? \\
1.11 E-05 ? \\
9.91 E-06 ?\end{array}$ & $\begin{array}{l}1.73 E-05 ? \\
1.55 E-05 ? \\
1.39 E-05 ?\end{array}$ & $2.24 E-03 x$ & $3.13 E-03 x$ \\
\hline 1600 & $80.7 x$ & $6.23 x$ & $.271 x$ & $2.343 x$ & $\begin{array}{l}\text { d5.0? } \\
15.0 ? \\
\text { d1.0? }\end{array}$ & $\begin{array}{l}8.24 E-06 ? \\
5.84 E-06 ? \\
5.03 t-06 ?\end{array}$ & $\begin{array}{l}1.15 E-05 ? \\
8.17 E-06 ? \\
0.16 t-06 ?\end{array}$ & $2.75 E-03 x$ & $3.85 E-03 x$ \\
\hline 3200 & $80.4 x$ & $5.05 x$ & $.301 x$ & $2.552 x$ & $\begin{array}{l}41.0 ? \\
\$ 4.0 ? \\
15.0 ?\end{array}$ & $\begin{array}{l}7.34 E-07 ? \\
6.461-07 ? \\
6.091-47 ?\end{array}$ & $\begin{array}{l}1.03 t-06 ? \\
4.74 t-01 ? \\
4.64 t-017\end{array}$ & $6.45 E-04 X$ & $9.03 E-04 X$ \\
\hline
\end{tabular}

$?$ INDICATES UNCERTAINTIES IN DATA.

X INDICATES INVALIO OR INCUMPLETE DATA. 


\begin{tabular}{|c|c|c|c|c|c|c|c|c|c|}
\hline $\begin{array}{l}\text { TEST } \\
\text { FLUI } \\
\text { SAMPLER }\end{array}$ & $\begin{array}{l}4 \\
\text { RESCEIN } \\
\text { HT } 1.5 \mathrm{M}\end{array}$ & $\begin{array}{l}\text { AUGUST } \\
\text { RELEASE }\end{array}$ & $\begin{array}{l}\text { 8. } 1967 \\
\text { FRUM }\end{array}$ & $\begin{array}{l}7 \quad 040 \\
E L F V A T I U R \\
J=1.4 \mathrm{M} /\end{array}$ & 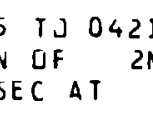 & $\begin{array}{l}\frac{1}{M} P S T \\
2 M\end{array}$ & & & \\
\hline $\begin{array}{l}\text { UISIANCE } \\
\text { FRUM }\end{array}$ & & $\begin{array}{l}S=1 Q D-01 \\
\text { STANO }\end{array}$ & $\begin{array}{l}\text { SIBLAUI } \\
\text { - COEEEJ }\end{array}$ & ICIENI- & THREE & MAXIMUM EXP & OSURES & $\begin{array}{l}\text { CRUSS } \\
\text { INIELBAIE }\end{array}$ & $\begin{array}{l}\text { IND } \\
\text { L_XALULS_ }\end{array}$ \\
\hline SUURCE & MEAN & DE V & SKEW & KURT & AZIMUTAS & i / & $\mathrm{t} u / u$ & $(1)(t / \omega)$ & Cl(t) \\
\hline$---\infty$ & $-\cdots-$ & $---n$ & $-\cdots--$ & ----- & ----- & ------- & ------- & ----- & 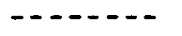 \\
\hline HETERS & DEC & DEG & & & DEG & $S E C / C U . M$ & $1 / S G . M$ & SEC/SJ.M & $1 / M$ \\
\hline 200 & $86.0 x$ & 4. $7 u x$ & $\begin{array}{c}-1.035 x \\
.\end{array}$ & $x 5.560 x$ & $\begin{array}{l}36.0 \\
58.0 \\
04.0\end{array}$ & $\begin{array}{l}9.63 E-J 4 \\
6.63 E-J 4 \\
0.35 E-U 4\end{array}$ & $\begin{array}{l}1.35 t-03 \\
1.21 t-03 \\
8.09 t-04\end{array}$ & $2.90 t-3<x$ & $4.17 E-U 2 X$ \\
\hline $80 \mathrm{~J}$ & $78.5 x$ & $6.44 x$ & $.690 x$ & $x 3.124 x$ & $\begin{array}{l}78.0 ? \\
70.0 ? \\
76.0 ?\end{array}$ & $\begin{array}{l}1.48 c-J 5 ? \\
1.32 t-05 ? \\
1.22 c-05 ?\end{array}$ & $\begin{array}{l}2.07 t-U 5 ? \\
1.84 t-U 5 ? \\
1.70 t-U 5 ?\end{array}$ & $2.31 E-03 x$ & 3. Y SE - O3X \\
\hline 1600 & $80.6 x$ & $6.28 x$ & $.247\rangle$ & $x>242 x$ & $\begin{array}{l}00.0 ? \\
75.0 ? \\
01.0 ?\end{array}$ & $\begin{array}{l}6.07 E-06 ? \\
5.96 E-J 6 ? \\
5.05 E-16 ?\end{array}$ & $\begin{array}{l}0.5 \cup t-66 ? \\
t .35 c-06 ? \\
0.14 t-66 ?\end{array}$ & $2.06 c-03 x$ & $4.01 E-63 x$ \\
\hline 3200 & $80.1 x$ & $6.20 x$ & .5742 & $x 2.963 x$ & $\begin{array}{l}81.0 ? \\
70.0 ? \\
90.0 ?\end{array}$ & $\begin{array}{l}8.01 E-\cup 7 ? \\
0.15 L-\cup 7 ? \\
7.41 E-J 7 ?\end{array}$ & $\begin{array}{l}1.21 E-66 ? \\
1.14 t-60 ? \\
1.11 t-60 ?\end{array}$ & $6.55 i-04 x$ & $9.17 E-04 x$ \\
\hline
\end{tabular}

$?$ INUICATES UNCERTAINTIES IN OATA.

X IndicatFs inval io or incumplete lata. 
CKRUNU LEVEL ANL TOKER SAMPLING AT ZOCM AIVO AJOM LRLY. AFTER RLLATIVELY HINUK EATKAHOLATILN ON NOKTH, B.UTH

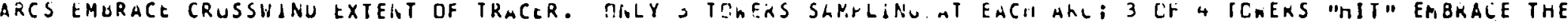
VFRIICAL EXIENT OF IRACER. DIRECIIUN SHEAR WITH HEIGHI IS UBVIUUS FRUM TUKER SAMPLES.

IEST C1 SEPIEMJEK 15,1907 UCUU TU JCIS PST KKYYTUN-BS KELEASE FRUM ELEVATILN UF
EK HT 1.5H SAMPLEK HT $1.5 \mathrm{H}$

\begin{tabular}{|c|c|c|}
\hline UISTANCE & _ CROSSUINO_ & $S I B+B U I \perp O H$ \\
\hline SDURLE & $M E A N$ & SKEW \\
\hline
\end{tabular}

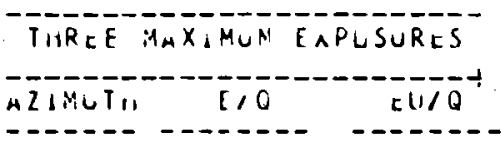

METERS

UES DEG

200

144.

$5.65 \quad-.191 \quad 2.3<3$

\lrcorner E

$د F L / L U . M$

$1 / 56.14$

$10 R .0 \quad 2.111-03 \quad 2.96 E-v 3$

$110.0 \quad 1.94 L-U 3 \quad 2.72 t-63$

$1 \cup 6.0 \quad 1.69 k-u 3 \quad k .36 t-v ?$

deu

104.6

$5.18 \quad-.249 \quad 2.3<0$

$103.01 .336-04$

$106.01 .03 E-v 4$

$1: 0.01 .7 E_{L}-44$

$2.57 \mathrm{E}-v 4$

2. $50 \mathrm{c}-14$

$2.502-64$

$?$ INLICATES UNCERTAINTIES IN DATA.

$x$ imuicates INYALIU uR IIICJMPLETL Lata.

CKossinino

LAIEGBAIEL_KALULS

CIIL/U) CIItU/O)

$S E C / S H . M \quad I / M$

$1.05 E-01 \cdot 1.47 E-01$

3.77E-02 5.2 $2 E-02$ 
GRQUNO LEVEL AND TOWER SAMPLING AT 2004 AVD $80 J M$ ONLY. AFTER RATHER BOLD EXIRAPOLATION ON SOUTH END OF $20 O M$ ARC, BDTH ARCS EMBRACE CROSSWIND EXTENT DF IRACER. ONLY 3 TOWERS SAMPLING AT EACH ARC, AND DNLY 3 DF THESE IVTEREEPT TRACER. VINE JF THE TONERS EYBRACE THE VERTICAL EXTENT OF THE PLUME JURIVS THIS JNSTABLE ZELEASE.

$$
\begin{aligned}
& \text { TEST C? DCTJZER 17, 1957 J302 TJ J817 JST } \\
& \begin{array}{l}
\text { KRYJTJV-85 RELEASE FRJY ELEVATION OF IM } \\
\text { SAMPLER HT 1.54 }
\end{array}
\end{aligned}
$$

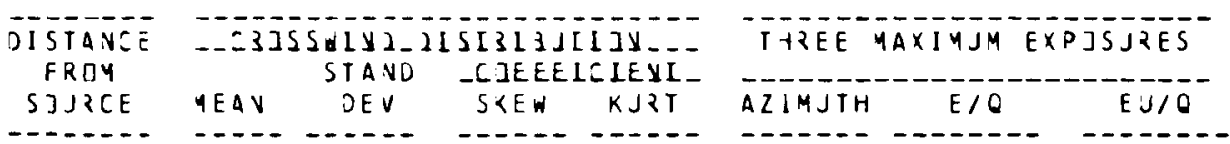

METERS DEG JES DEG SEC/CU.M $1 / 50.4$

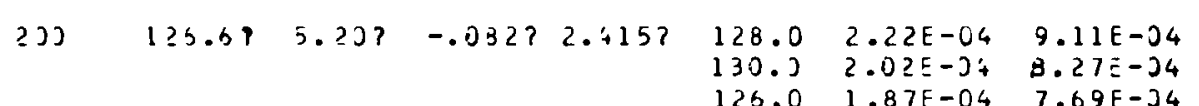

$803 \quad 126.5 \quad 2.59 \quad-.078 \quad 2.553 \quad 125.3 \quad 1.33 E-05 \quad 7.63 E-05$

$\begin{array}{lll}125.3 & 1.33 E-03 & 7.63 E-05 \\ 128.3 & 1.75 E-05 & 7.23 E-35\end{array}$

$124.0 \quad 1.12 E-05 \quad 4.58 E-35$

CROSTSTIND

IUIEGBALED-XALUES.

(IIIIJ) CI $(E J / 2)$

(1)

SEC/SQ.M I/M

$1.05 E-32 ?+.35 E-32 ?$

$1.85 E-03 \quad 7.62 E-03$

? INOICATES JVCERTAIVIIES IN DATA.

r indicates ivvalio jo incouplete oata. 
GRDUND LEVEL AND TOWER SAMPLING AT 230.4 AND BOOY ONLY. BOTH ARCS SEVERELY TRUNCATED AT SDUTH END. ONLY 3 T JWERS SAYPLING AT EACH ARC: 5 JFTHE 6 TOWERS INTERCEPT TRACER. NO TOWER SOMPLETELY EMBRACES THE VERTICAL EXTENT OF TRAEER JURING THIS ZELIASE IVTJ AV JVSTABLE ATMOSPHERE.

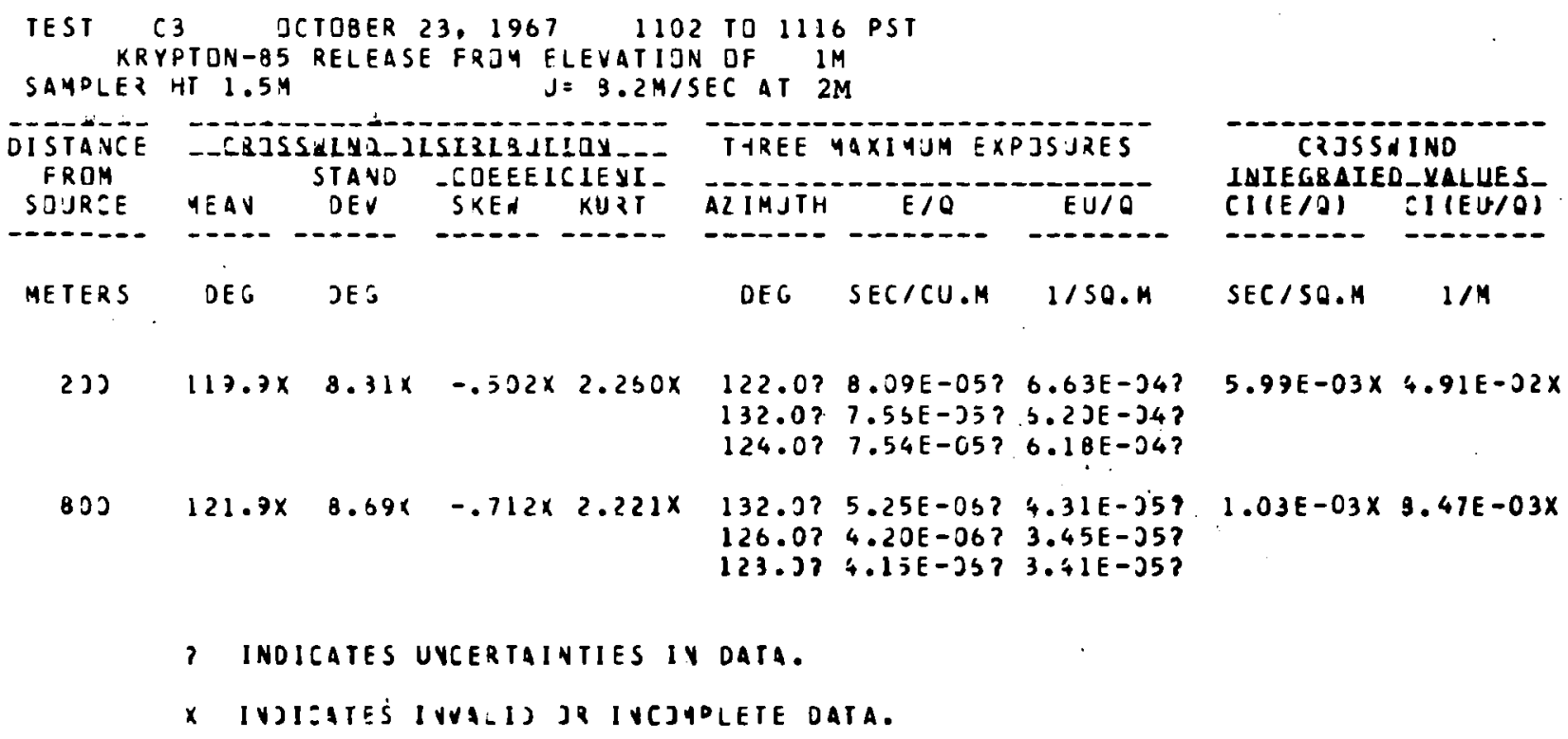

7 INDICATES UYCERTAINTIES IV DATA.

$x$ IVJIEATES IVVALIJ JP IVCJPLLTE DATA.

CZJSSNIND INIEGRAIED YALUES-
CI IE/O) CI (EU/O)

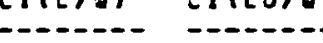

SEC/SQ.H I/M

$5.97 E-03 X+.91 E-02 X$ 


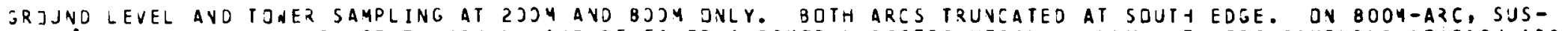
PECT FURTHER SAYPLIVG JY SJJTA WJJID TAVE ZEVEALED A BIMODAL DISTRIBUTION. ONLY 3 TOWERS SAMPLING AT EACA IRE. FOUR TOWERS INTEREEPTES IRAEER DJRINJ THIS ZEL DASE INTO AN UNSTABLE ATMOSPHERE.

$$
\begin{aligned}
& \text { TEST C4 JCTOBER 24, } 1967 \quad 1104 \text { TO } 1114 \text { PST } \\
& \text { KRYPTON-85 RELEASE FRJY ELEVATIJN OF I4 } \\
& \text { SAYPLEZ HI } 1.5 \mathrm{M} \text { JE } 4.14 / S E C \text { AT } 2 \mathrm{M}
\end{aligned}
$$

\begin{tabular}{|c|c|c|c|c|c|c|c|c|c|}
\hline $\begin{array}{l}\text { DISTAVCE } \\
\text { FRJY }\end{array}$ & \multicolumn{4}{|c|}{ 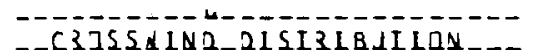 } & \multicolumn{3}{|c|}{ THREE MAXIMUM EXPOSURES } & \multicolumn{2}{|c|}{ CROSSWIND } \\
\hline SOURCE & YEAN & JEV & S<EW & $K J>T$ & $4214 J 1 t$ & $E / O$ & $E J / 2$ & $C I(E / Q)$ & $C I(E \cup / Q)$ \\
\hline------ & ---- & ----- & ------ & ------ & ----- & -------- & ------ & ------ & --- \\
\hline YETEZS & DEO & DEG & & & DEG & SEC/CU.M & $1 / S Q .4$ & $S E C / 52.4$ & $1 / 4$ \\
\hline 200 & $121.6 x$ & $6.00 x$ & $-.112 x$ & $2.205 x$ & $\begin{array}{l}122.0 \\
120.0 \\
124.0\end{array}$ & $\begin{array}{l}1.04 E-04 \\
9.80 E-05 \\
9.77 E-J 5\end{array}$ & $\begin{array}{l}4.27 E-04 \\
4.02 E-04 \\
4.01 E-04\end{array}$ & $\begin{array}{c}6.05 E-03 x \\
\vdots\end{array}$ & $2.48 E-02 x$ \\
\hline 30 & $1 \geq 0 . \therefore x$ & $4.33 x$ & $.747 x$ & $3.248 x$ & $\begin{array}{l}118.0 \\
125.0 ? \\
116.02\end{array}$ & $\begin{array}{l}6.30 E-06 \\
4.33 E-5 S ? \\
4.02 E-06 ?\end{array}$ & $\begin{array}{l}2.53 E-J 5 \\
1.7 J E-J 5 ? \\
1.65 E-05 ?\end{array}$ & $7 . J 1 \varepsilon-J \div x$ & $2.87 E-33 X$ \\
\hline
\end{tabular}

? INDICATES UNCERTAINTIES IN DATA.

X IVOIZATES. IVVALID JR IVEJYPLETE DATA. 
GRIJUNU LEVEL AND TOWER SAMPLING AT 200M AND 8OCM LNLY. KRYPTON RELEASED ABDUT 2M TO RIGHT (FACING DOHNHIND) AND IM BELOW ZNS RELEASE POINT. BOTH ARCS EMBRACE THE CKDSSWIND DISTRIBUTION OF TRACER B BOTH TRACER DISTRIBUTIONS EXTENDED ABOVE TOWER TOPS, BUT MAXIMUM CUNCENTRATIONS WERE GENERALLY BELUW TUWER TUPS.

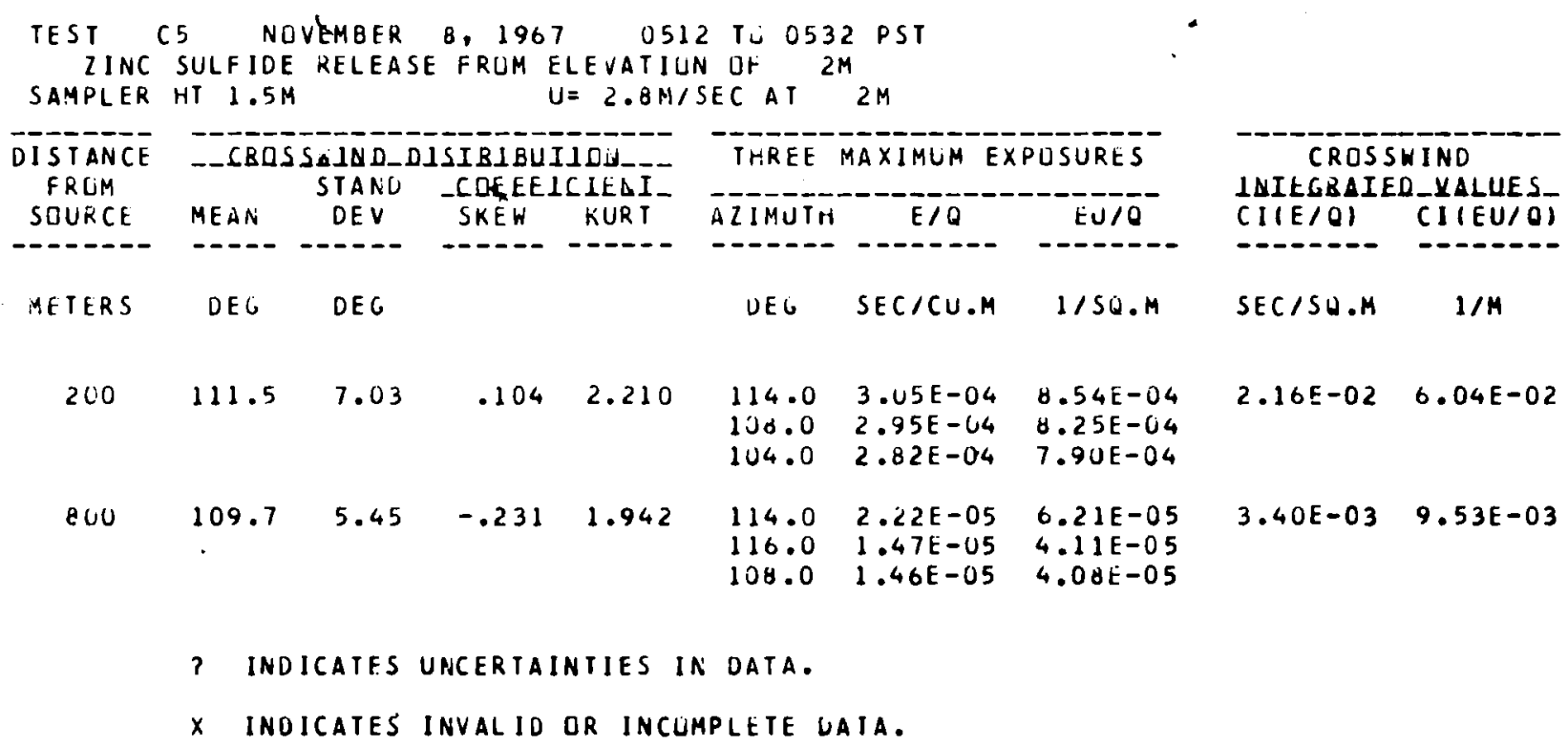


TEST C5 NUVEMOEK 8, 967 O512 TU 0532 P I KKYPTUN-ES KELFASE FRUM ELEVAIIUN OF IM

SAMPLEK HT $1.5 \mathrm{H}$

$U=\bar{Z} \cdot$. QRi/SEC AI

DISTANCE -CBOSSAINDEDSIBIBUIDDU-

SIAI:U - LOEEEILIEAI

DEV SKEW KJRI

TURLE MAXIMUA EAPLSUFES

TIMUTH

-

DEC SECICU.M I/SO.M

MEIFRS

UEC

$<n$

111.5

6.74

$.067 \quad 2.117$

$114.0 \quad 2.32 \mathrm{t}-44$

$6.41 t-v 4$

800

$113.2 \quad 5.74 \quad-.769 \quad 2.699$

$\begin{array}{lll}113.0 & 3.2 C L-v 5 & 2.96 \mathrm{~L}-u 5 \\ 116.0 & 2.70 \mathrm{~L}-\mathrm{-5} & 7.57 \mathrm{~L}-\mathrm{J}\end{array}$

$116.0 \quad 2.70 \mathrm{~L}-\mathrm{vS} \quad 7.57 \mathrm{~L}-\mathrm{v}$

$114.0 \quad 1.98 \mathrm{c}-\mathrm{us} \quad 5.54 t-j 5$
Cocissing

IUILGSAIEL_VALULS

CIIE/A) CIIEU/O)

SEC/SO.M $\quad$ I/M

$1.54 E-0<\quad 4.3 \angle E-C \angle$

$4.53 E-0, \quad 1.27 E-0<$

$?$ INUICATES UNCEKTAINTIES IN DATA

x INUICATFS INVALIL UR INELMPLETL jaIA. 


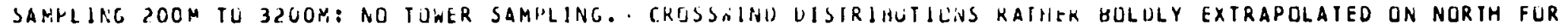

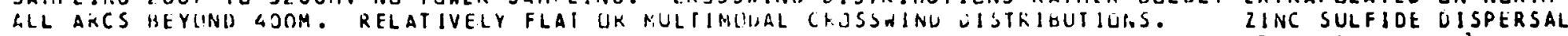

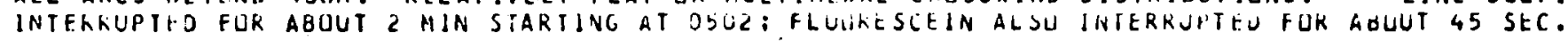

\begin{tabular}{|c|c|c|c|c|c|c|c|c|c|}
\hline & \multicolumn{2}{|c|}{$\begin{array}{c}\text { Cogsingad } \\
\text { STARU }\end{array}$} & \multirow{2}{*}{\multicolumn{2}{|c|}{$\begin{array}{l}\text { ISIRIGULLU } \\
\text { - } L E E E \perp C \perp L I-\end{array}$}} & rinkE & \multicolumn{2}{|c|}{ AAXIMUA EXPUSUNES } & \multirow{2}{*}{\multicolumn{2}{|c|}{$\begin{array}{l}\text { CKUSSWIND } \\
\text { 1DILGQAIEL_YALUES }\end{array}$}} \\
\hline & $M F i N$ & UEV & & $K \cup R T$ & AZIMUTH & $E / 0$ & E $\cup 10$ & & \\
\hline 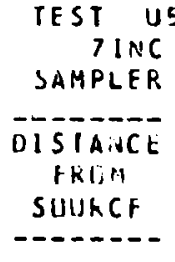 & $\cdots+$ & $\cdots+$ & $\cdots+-$ & 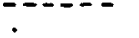 & -..- & $\ldots$ & & & \\
\hline MEIERS & DEG & $D E G$ & & & JEC & SEC/CU.M & $1 / 50 . M$ & SECASE.M & $1 / M$ \\
\hline 200 & $55.9 ?$ & $15.26 ?$ & $-.964 ?$ & $<.440 ?$ & $\begin{array}{l}46.0 ? \\
70.0 ? \\
74.0 ?\end{array}$ & $\begin{array}{l}0.09 t-U 5 ? \\
5.87 t-U 5 ? \\
5.83 E-\cup 5 ?\end{array}$ & $\begin{array}{l}1.10 E-04 ? \\
1.06 E-64 ? \\
1.05 E-04 ?\end{array}$ & $0.18 E-03 ?$ & $1.47 E-02 ?$ \\
\hline 300 & 52.5 & 17.10 & $-.23 y$ & 2.302 & $\begin{array}{l}40.0 \\
44.0 \\
04.0\end{array}$ & $\begin{array}{l}5.02 \mathrm{E}-\mathrm{US} \\
4.39 \mathrm{~L}-\mathrm{US} \\
3.02 \mathrm{E}-\mathrm{US}\end{array}$ & $\begin{array}{l}9.03 t-05 \\
8.26 t-05 \\
0.52 E-05\end{array}$ & $6.511-0.3$ & $1.53 E-02$ \\
\hline 400 & 52.4 & 16.46 & -.247 & 2.312 & $\begin{array}{l}45.0 \\
50.0 \\
54.0\end{array}$ & $\begin{array}{l}4.70 t-U 5 \\
3.09 i-U 5 \\
2.93 t-v S\end{array}$ & $\begin{array}{l}\forall .46 t-05 \\
3.57 t-65 \\
3.27 t-65\end{array}$ & $t .37 t-03$ & $1.51 \mathrm{E}-02$ \\
\hline 500 & $\begin{array}{r}53.7 ? \\
\quad . \cdot\end{array}$ & $15.07 ?$ & -.3727 & 2.5253 & $\begin{array}{l}74.0 \\
36.0 \\
32.0\end{array}$ & $\begin{array}{l}3.73 t-U b \\
3.34 i-U b \\
3.44 c-65\end{array}$ & $\begin{array}{l}7.07 t-U 5 \\
0.37 t-U S \\
6.2 U t-U S\end{array}$ & $1.03 t-02 ?$ & $1.86 E-02 ?$ \\
\hline 600 & $51.9 ?$ & $13.39 ?$ & $-.34 . ?$ & $2.700 ?$ & $\begin{array}{l}46.0 \\
26.0 \\
54.0\end{array}$ & $\begin{array}{l}3.74 t-u b \\
3.24 t-U 5 \\
2.37 t-U 5\end{array}$ & $\begin{array}{l}6.74 i-J 5 \\
5.03 i-U S \\
5.17 t-U S\end{array}$ & $9.93 E-03 ?$ & $1.79 E-02 ?$ \\
\hline 700 & 51.97 & $14.24 ?$ & $-.145 ?$ & $2.459 ?$ & $\begin{array}{l}52.0 \\
44.0 \\
-0.0\end{array}$ & $\begin{array}{l}2.37 t-u 5 \\
2.32 t-u 5 \\
2.00 t-u 5\end{array}$ & $\begin{array}{l}4.27 E-U S \\
4.16 E-O S \\
4 . U 7 E-U S\end{array}$ & $9.72 E-03 ?$ & $1.7 \mathrm{SE}-02 ?$ \\
\hline o::0 & $51.3 ?$ & $13.03 ?$ & $-.184 ?$ & 2.5378 & $\begin{array}{l}44.0 \\
46.0 \\
32.0\end{array}$ & $\begin{array}{l}2.04 t-15 \\
2.13 t-U 5 \\
2.11 L-U 5\end{array}$ & $\begin{array}{l}4.76 t-05 \\
4.55 t-05 \\
3.74 t-05\end{array}$ & $9.1 \forall E-03 ?$ & $\begin{array}{c}1.65 E-0.2 ? \\
.\end{array}$ \\
\hline 1200 & $49.7 ?$ & 13.027 & $-.03 ! ?$ & $2.773 ?$ & $\begin{array}{l}48.0 \\
50.0 \\
32.0\end{array}$ & $\begin{array}{l}1.74 t-v 5 \\
1.04 t-05 \\
8.14 t-06\end{array}$ & $\begin{array}{l}3.14 E-05 \\
2.96 E-U 5 \\
1.47 E-U 5\end{array}$ & $4.96 E-039$ & $8.93 E-03 ?$ \\
\hline 1600 & $49.4 ?$ & $13.08 ?$ & $-.087 ?$ & $2.429 ?$ & $\begin{array}{l}45.0 \\
50.0 \\
46.0\end{array}$ & $\begin{array}{l}7.96 E-U 6 \\
6.40 \mathrm{c}-\mathrm{VO} \\
4.67 \mathrm{E}-\mathrm{VO}\end{array}$ & $\begin{array}{l}1.44 t-U 5 \\
1.15 t-U 5 \\
6.40 t-06\end{array}$ & $4.30 E-03 ?$ & $7.73 E-03 ?$ \\
\hline 3200 & 49.27 & $10.16 ?$ & $-.197 ?$ & $2.508 ?$ & $\begin{array}{l}50.0 ? \\
22.0 ? \\
48.0 ?\end{array}$ & $\begin{array}{l}1.45 E-06 ? \\
1.45 L-00 ? \\
1.34 t-06 ?\end{array}$ & $\begin{array}{l}2.61 t-06 ? \\
2.611-06 ? \\
2.41 t-06 ?\end{array}$ & $1.87 t-03 ?$ & $3.37 E-03 ?$ \\
\hline
\end{tabular}




\begin{tabular}{|c|c|c|c|c|c|c|c|c|c|}
\hline $\begin{array}{l}\text { UISTAIICE } \\
\text { FHLiA }\end{array}$ & \multicolumn{4}{|c|}{ 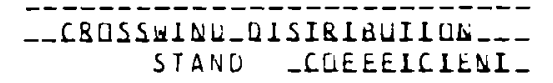 } & thkE & \multicolumn{2}{|c|}{ YAXIYUMA EXPLSURES } & \multicolumn{2}{|c|}{ 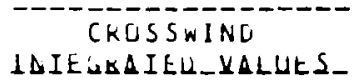 } \\
\hline sllukCt & $M E \wedge N$ & DFV & $S K E W$ & KURI & ARIMUTH & $i / b$ & $E \cup / Q$ & (\IE/) & $(I \mid E \cup / 0)$ \\
\hline.------ & ---- & ------ & ----- & ------ & ----- & -------- & ------- & 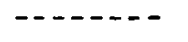 & -------- \\
\hline MEIERS & DEG & DEC & & & UEC & $S t C . / C U . M$ & $1 / 5 \mathrm{~J} . \mathrm{M}$ & SEC/SW.IA & $1 / M$ \\
\hline 200 & $45.9 ?$ & $23.947 ?$ & .0837 & $1.58 \mathrm{C} ?$ & $\begin{array}{l}18.0 ? \\
22.0 ? \\
62.0 ?\end{array}$ & $\begin{array}{l}0.3 y=-J 7 ? \\
3.7 y t-J 7 ? \\
2.13 E-j 7 ?\end{array}$ & $\begin{array}{l}2.96 L-06 ? \\
1.03 E-C 6 ? \\
9.16 E-27 ?\end{array}$ & 3 . dut $-u b ?$ & $1.67 t-04 ?$ \\
\hline 301$)$ & 54.5 & 22.47 & -.386 & 1.737 & $\begin{array}{l}76.6 \\
24.6 \\
60.0\end{array}$ & $\begin{array}{l}0.13 E-J 7 \\
0.07 E-U 7 \\
\text { b.06E-U7 }\end{array}$ & $\begin{array}{l}3.52 t-00 \\
2.07 t-i 0 \\
2.50 t-0 t\end{array}$ & $1.30 t-04$ & $3.59 E-64$ \\
\hline 400 & 55.5 & 16.96 & -.761 & 3.194 & $\begin{array}{l}53.0 \\
34.0 \\
62.0\end{array}$ & $\begin{array}{l}1.27 t-36 \\
7.32 t-37 \\
7.1 C \mathrm{CL}-67\end{array}$ & $\begin{array}{l}5.47 t-66 \\
3.15 t-66 \\
3.65 E-66\end{array}$ & $1.71 F-U 4$ & $7.35 E-04$ \\
\hline büo & $00.4 x$ & $9.87 x$ & $.049 x$ & $2.792 x$ & $\begin{array}{l}56.3 \\
52.0 \\
00.0\end{array}$ & $\begin{array}{l}3.54 E-J 6 \\
3.3 r E-U 6 \\
2.53 E-06\end{array}$ & $\begin{array}{l}1.52 E-05 \\
1.33 i-05 \\
1.11 L-05\end{array}$ & $5.76 E-04 x$ & $2.4 d E-03 x$ \\
\hline 600 & $59.9 x$ & $8.77 x$ & $-.178 x$ & $2.232 x$ & $\begin{array}{l}62.0 \\
54.1 \\
66.0\end{array}$ & $\begin{array}{l}4.221-36 \\
3.171-06 \\
2.36 t-36\end{array}$ & $\begin{array}{l}1.01 t-u 5 \\
1.36 t-05 \\
1.01 t-45\end{array}$ & $6.0<E-04 x$ & $2.84 E-03 x$ \\
\hline 7013 & $61.3 x$ & $8.60 x$ & $.305 x$ & $2.720 x$ & $\begin{array}{l}00.0 \\
04.0 \\
56.0\end{array}$ & $\begin{array}{l}5.113 t-116 \\
3.67 t-06 \\
3.54 t-166\end{array}$ & $\begin{array}{l}2.16 t-0 b \\
1.58 t-05 \\
1.52 t-0 b\end{array}$ & $1.03 t-03 x$ & $4.66 E-03 X$ \\
\hline$\triangle 00$ & $59.4 x$ & $9.91 \times$ & $.400 x$ & $2.390 x$ & $\begin{array}{l}58.0 \\
35.0 \\
07.0\end{array}$ & $\begin{array}{l}u .12 t-j t \\
4.22 t-v 6 \\
2.32 t-46\end{array}$ & $\begin{array}{l}2.03 i-U 5 \\
1.81 L-05 \\
9.545-06\end{array}$ & $1.05 k-03 x$ & $4.51 E-03 X$ \\
\hline $120 \mathrm{~J}$ & $61.0 x$ & $8.23 x$ & $.403 x$ & $3.072 x$ & $\begin{array}{l}52.0 \\
=6.00 \\
60.0\end{array}$ & $\begin{array}{l}7 . Y 1 E-U C \\
1.2 Y E-U 6 \\
7.1: E-36\end{array}$ & $\begin{array}{l}3.13 E-v b \\
3.27 t-v 3 \\
3.09 i-U b\end{array}$ & $2.68 t-03 x$ & 1. $15 E-02 x$ \\
\hline 1600 & $57.9 x$ & $5.44 x$ & $.996 x$ & $4.397 x$ & $\begin{array}{l}0.0 .0 \\
5.09 \\
02.0\end{array}$ & $\begin{array}{l}8.3 i t-v 6 \\
3.12 t-j 6 \\
3.16 t-36\end{array}$ & $\begin{array}{l}3.41 E-U 5 \\
3.43 E-05 \\
3.40 E-U 5\end{array}$ & 2 . $3 y t-0) x$ & 1. $C 3 E-0<x$ \\
\hline 3200 & $65.3 x$ & $4.52 x$ & $.7 y \Delta \lambda$ & $? .38+x$ & $\begin{array}{l}60.0 ? \\
62.0 ? \\
64.01 ?\end{array}$ & $\begin{array}{l}2.41 t-46 ? \\
1.47 t-46 ? \\
1.47 c-56 ?\end{array}$ & $\begin{array}{l}4.44 t-v 0 ? \\
8.4 B L-16 ? \\
8.43 t-00 ?\end{array}$ & $1.26 t-03 x$ & $5.40 E-03 X$ \\
\hline
\end{tabular}

$?$ INUICATFS UNCERTAINTIES IiV JaIA.

x inuicatfos invalio lo incuaplete liata. 
SAMPLING 4 OUM TO $3200 M$ : NO TOKER SAMPLIMG.

TKACEK OISTRIBUTIUN EXTENCS BEYOND SAMPLERS AT ZZUUH. AIND IU LESSEK EXTENI AT IGCUM. ZNS EXTKAPOLATED AT 3700M. STRTING LUH-LEVEL IEMPFRATURE INVERSILIV.

\begin{tabular}{|c|c|c|c|c|c|c|c|c|c|}
\hline $\begin{array}{l}\text { TEST US } \\
\text { TINC } \\
\text { SAMFLER }\end{array}$ & $\begin{array}{l}7 \\
\text { SIILFIDE } \\
H T 1.5 \text { i }\end{array}$ & $\begin{array}{l}\text { AUGUST } \\
\text { RELLFASE }\end{array}$ & 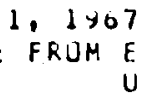 & $\begin{array}{l}\quad 645 \\
E L E V A T I L I \\
J=1.5 \mathrm{H} /\end{array}$ & $\begin{array}{l}\text { TU USCL } \\
\text { Ut } 2 \text { C } \\
\text { EC AT }\end{array}$ & $\begin{array}{l}U P S T \\
2 M\end{array}$ & & & \\
\hline $\begin{array}{l}\text { UISTANCE } \\
\text { FKIIM }\end{array}$ & - CBOS & $\begin{array}{l}\text { SIIND_DI } \\
\text { STANi }\end{array}$ & $\begin{array}{l}\text { SIBLBUI } \\
\text {-CIEEEL }\end{array}$ & 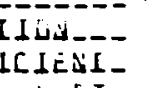 & THKEE & MAXIMUM EX & PUSURES & $\begin{array}{r}\text { CROSS } \\
\text { LUIEGXAIES }\end{array}$ & $\begin{array}{l}\text { INO } \\
\text { Z_YALUES }\end{array}$ \\
\hline SOURCF & NEAN & DEV & SKEK' & KukT & AZLAUTH & $t / 0$ & $E \cup / O$ & CI(E/U) & $(I) c u / 4)$ \\
\hline METFKS & DEG & DEC & & & UES & SECICU.M & 1/SU.K & $S L C / S U . M$ & $1 / M$ \\
\hline 400 & 102.4 & 6.01 & -.714 & 3.137 & $\begin{aligned} 100.0 \\
102.0 \\
54.0\end{aligned}$ & $\begin{array}{l}3.13 E-64 \\
2.10 t-v 4 \\
1.34 E-64\end{array}$ & $\begin{array}{l}4.64 c-v 4 \\
3.16[-04 \\
2.01 E-v 4\end{array}$ & $2.53 t-02$ & 3.J $\triangle E-02$ \\
\hline 6010 & 103.0 & 5.13 & -.401 & 4.413 & $\begin{array}{l}106.0 \\
1020.0 \\
70.0\end{array}$ & $\begin{array}{l}3.14 t-64 \\
1.46 t-64 \\
1.60 t-64\end{array}$ & $\begin{array}{l}4.11 t-04 \\
2.34 t-64 \\
2.34 t-0.4\end{array}$ & $\begin{array}{c}3.67 E-02 \\
?\end{array}$ & $5.50 E-02$ \\
\hline $7 ن$ & 105.0 & 3.65 & -1.062 & 5.514 & $\begin{array}{l}100.0 \\
104.0 \\
160.0\end{array}$ & $\begin{array}{l}3.47 L-J 4 \\
2.01 t-l 4 \\
1.45 t-v 4\end{array}$ & $\begin{array}{l}5.35 L-j 4 \\
4.21 i-04 \\
2.18 E-04\end{array}$ & $4.23 \mathrm{E}-02$ & $6.35 E-02$ \\
\hline 300 & 104.2 & 5.25 & -.500 & 3.027 & $\begin{array}{l}100.0 \\
100.0 \\
109.0\end{array}$ & $\begin{array}{l}1.53 c-U 4 \\
8.3 U t-U 5 \\
7.3 O E-U 5\end{array}$ & $\begin{array}{l}2.29 t-U 4 \\
1.25 E-U 4 \\
1.14 E-U 4\end{array}$ & $1.99 E-02$ & $2.94 E-02$ \\
\hline 1200 & 106.3 & 4.15 & -1.500 & 12.276 & $\begin{array}{l}100.0 \\
110.0 \\
106.0\end{array}$ & $\begin{array}{l}9.76 t-45 \\
7.16 L-65 \\
3.75 t-45\end{array}$ & $\begin{array}{l}1.4 c, t-U 4 \\
1.07 t-j 4 \\
\text { d. OJE-JS }\end{array}$ & $1.65 E-02$ & $2.4 d E-02$ \\
\hline 1600 & 106.1 & 4. AO & -2.497 & 14.753 & $\begin{array}{l}104.0 \\
116.0 \\
103.0\end{array}$ & $\begin{array}{l}3.46 E-65 \\
3.45 t-65 \\
2.47 t-65\end{array}$ & $\begin{array}{l}5.20 t-05 \\
3.17 t-U 5 \\
4.45 t-U 5\end{array}$ & $8.75 E-03$ & $1.31 E-02$ \\
\hline 3200 & $104.0 ?$ & $10.11 ?$ & $-.122 ?$ & $1.520 ?$ & $\begin{array}{l}114.0 \\
110.0 \% \\
90.0\end{array}$ & $\begin{array}{l}2.46 t-v b \\
2.0 C E-J 67 \\
1.0 \angle E-U 6\end{array}$ & $\begin{array}{l}3.04 t-06 \\
2.49 E-06 ? \\
2.73 c-46\end{array}$ & $2.11 E-03 ?$ & $3.16 E-03 ?$ \\
\hline
\end{tabular}

$?$ INDICATFS UNCERIAINTIES IL UATA.

x inuicates invalio or incumpllte vaia. 


\begin{tabular}{|c|c|c|c|c|c|c|c|c|c|}
\hline \multirow{3}{*}{$\begin{array}{l}\text { TEST US } \\
\text { FLUE } \\
\text { SAMPLER } \\
\text { LISTAICE } \\
\text { FKEI } \\
\text { SCUPCE }\end{array}$} & $\begin{array}{l}7 \\
\text { KESCEINA } \\
\text { HT } 1.5 \mathrm{M}\end{array}$ & $\begin{array}{l}\text { AUGUST } \\
\text { RELEASE }\end{array}$ & $\begin{array}{r}1.196 .7 \\
\text { FROM E } \\
U\end{array}$ & $\begin{array}{r}0450 \\
\text { LFVATILN } \\
=3.4 \mathrm{M} / \mathrm{S}\end{array}$ & $\begin{array}{l}\text { TO } 05 \angle 0 \\
\text { UF } 20 \mathrm{~T} \\
S C_{\text {AI }}\end{array}$ & $\begin{array}{l}0 \text { PST } \\
M \\
26 \mathrm{M}\end{array}$ & & \multirow{2}{*}{\multicolumn{2}{|c|}{$\begin{array}{l}\text { CKOSSWINU } \\
\text { INIELBAIEZ_YALUES_ }\end{array}$}} \\
\hline & \multicolumn{4}{|c|}{ 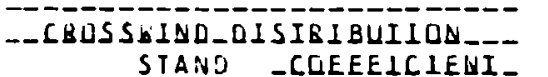 } & \multicolumn{3}{|c|}{ THREE MAXIMUM EXPUSUKLS } & & \\
\hline & MEAN & DEV & SKEW & KURT & AZIMUTA & $E / 0$ & $E \cup / O$ & $C I(E / U):$ & CIIEU/QI \\
\hline :4t TERS & DEG & CEC & & & DEC & SEC/CU.M & $1 / 50 . M$ & $S E C / S U . M$ & $1 / M$ \\
\hline 400 & $107.3 ?$ & $11.17 ?$ & $-.802 ?$ & $2.926 ?$ & $\begin{array}{l}118.0 ? \\
166.0 ? \\
114.0 ?\end{array}$ & $\begin{array}{l}1.55 E-67 ? \\
1.36 E-67 ? \\
6.17 E-08 ?\end{array}$ & $\begin{array}{l}2.29 c-07 ? \\
4.61 E-67 ? \\
2.10 E-67 ?\end{array}$ & $1.77 \mathrm{E}-05 ?$ & $6.02 E-05 ?$ \\
\hline 640 & $103.5 ?$ & $5.48 ?$ & $.342 ?$ & $4.125 ?$ & $\begin{array}{r}106.0 ? \\
102.0 ? \\
98.0 ?\end{array}$ & $\begin{array}{l}6.7 Y E-U Q ? \\
4.06 E-U 3 ? \\
2.0 \angle E-U 8 ?\end{array}$ & $\begin{array}{l}2.31 t-07 ? \\
1.58 t-07 ? \\
0.896-00 ?\end{array}$ & $0.09 t-06 ?$ ? & $2.75 E-05 ?$ \\
\hline 760 & 95.0 & 16.09 & -.812 & 2.363 & $\begin{aligned} 108.0 \\
76.0 \\
104.0\end{aligned}$ & $\begin{array}{l}1.36 E-J 7 \\
8.60 E-J 8 \\
6.33 E-U 8\end{array}$ & $\begin{array}{l}4.61 t-07 \\
2.99 E-07 \\
2.15 t-07\end{array}$ & $2.30 E-05$ & $7.83 E-05$ \\
\hline $\mathrm{HCO}$ & 104.4 & 9.90 & -1.581 & $7.74 \mathrm{~b}$ & $\begin{array}{l}106.0 \\
100.0 \\
109.0\end{array}$ & $\begin{array}{l}\text { 6.UUE-UD } \\
5.1 d E-J E \\
3.34 E-U \theta\end{array}$ & $\begin{array}{l}2.04 t-07 \\
1.76 t-07 \\
1.20 t-67\end{array}$ & $1.09 t-0 s$ & $3.7 \cup[-0,5$ \\
\hline 1200 & 109.6 & 7.49 & -1.786 & 0.643 & $\begin{array}{l}110.0 \\
112.0 \\
108.0\end{array}$ & $\begin{array}{l}5.01 E-U 7 \\
3.54 E-U 7 \\
2.9 \cup E-U 7\end{array}$ & $\begin{array}{l}1.70 t-66 \\
1.20 t-6.6 \\
9.04 E-U 7\end{array}$ & $1.02 E-04$ & $3.46 E-04$ \\
\hline 1600 & $111.1 ?$ & $5.79 ?$ & $-2.942 ?$ & 19.6737 & $\begin{array}{l}110.0 \\
112.0 \\
114.0\end{array}$ & $\begin{array}{l}1.36 t-06 \\
1.27 t-06 \\
6.73 t-0 ?\end{array}$ & $\begin{array}{l}4.64 t-06 \\
4.31 t-06 \\
2.36 t-06\end{array}$ & $3.11 E-04 ?$ & $1.06 E-03 ?$ \\
\hline 3200 & $108.7 x$ & $0.28 x$ & $-1.558 x$ & $4.147 x$ & $\begin{array}{l}114.0 ? \\
112.0 ? \\
110.0 ?\end{array}$ & 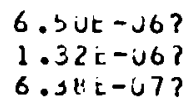 & $\begin{array}{l}2.21 t-05 ? \\
4.48 t-06 ? \\
2.17 t-46 ?\end{array}$ & $1.34 E-03 x$ & $4.55 E-03 x$ \\
\hline
\end{tabular}

? INDICATES UNCERTAINTIES IN UATA.

$x$ INDICATES INVALIU OR INCLMPLETE iata. 


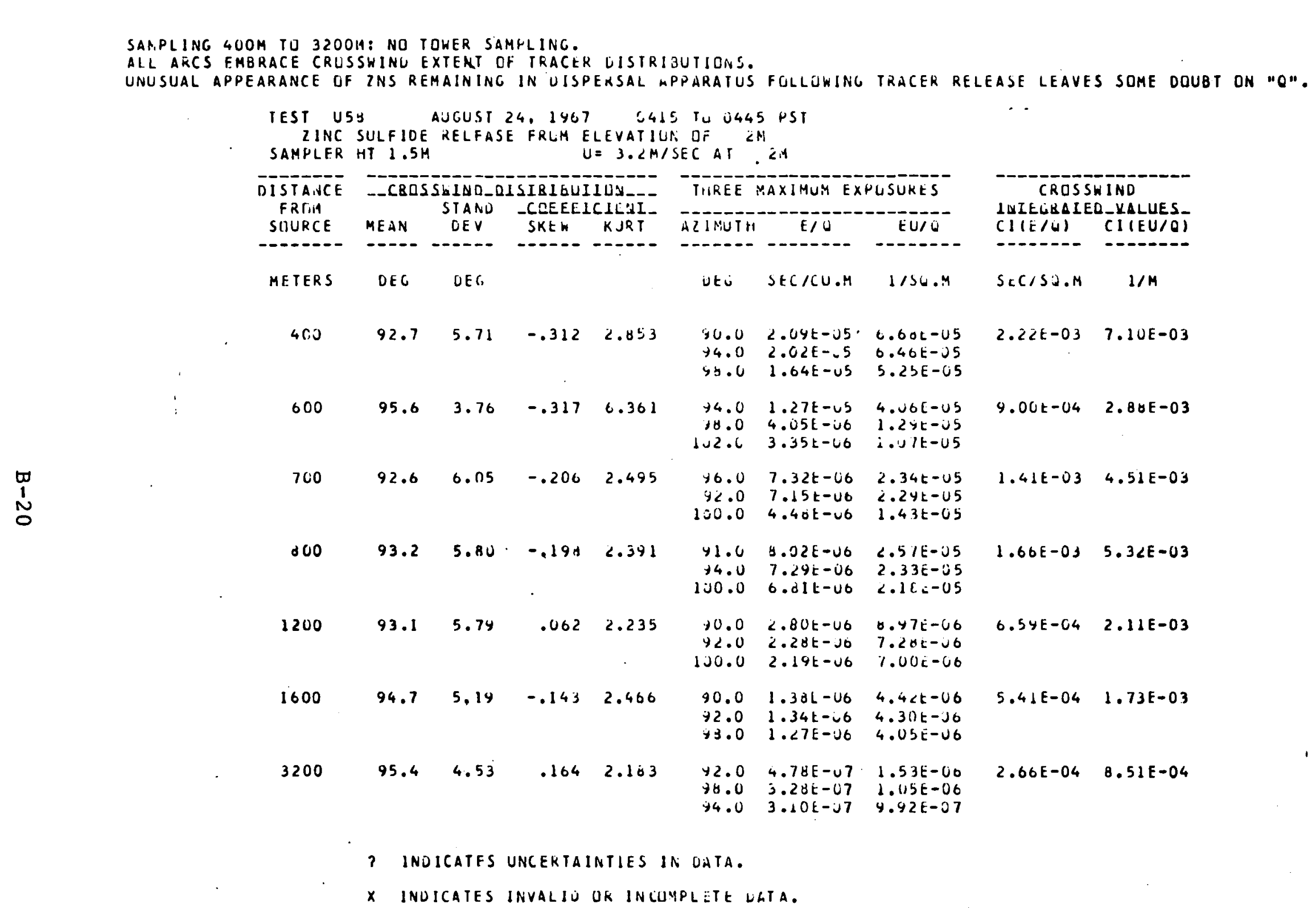


TEST USB AUGUST 24. $1967 \quad 0415$ TU 0445 PST

FLUURESCEIN RELEASE FROM ELFVATION OF ZOOY

$$
\text { SAMPLER HT } 1.5 \mathrm{M} \quad U=5.6 \mathrm{M} / \mathrm{SEC} \text { AT } 26 \mathrm{M}
$$

\begin{tabular}{|c|c|c|c|c|c|c|c|c|c|}
\hline \multirow{2}{*}{$\begin{array}{l}\text { DISTAIVCE } \\
\text { FREVA } \\
\text { SCURCE }\end{array}$} & \multicolumn{4}{|c|}{ 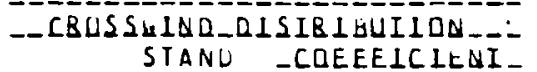 } & \multicolumn{3}{|c|}{ THREE YAXIMUMU EXPUSURES } & \multicolumn{2}{|c|}{$\begin{array}{l}\text { CRUSSWIND } \\
\text { LQIECQAIEL_KALUES_ }\end{array}$} \\
\hline & MEAN & DEV & SKEW & KURT & ALINUTH & $E / 0$ & $E \cup / 0$ & $C I(E / J)$ & CIIEU/O) \\
\hline---- & ---- & ------ & ------ & ------ & ------- & $-\cdots---$ & -------- & -------- & ------- \\
\hline METERS & DEG & DEG & & & UEG & $S E L / C U . M$ & $1 / \mathrm{SOH} \cdot \mathrm{M}$ & SEC/SW.M & $1 / M$ \\
\hline 400 & 94.4 & 5.75 & -.057 & 7.593 & $\begin{array}{l}94.0 \\
9 ? .0 \\
90.0\end{array}$ & $\begin{array}{l}8.05 i-1) 6 \\
7.765-06 \\
5.458-10\end{array}$ & $\begin{array}{l}4.51 t-05 \\
4.31 E-05 \\
3.31 E-05\end{array}$ & $8.751-1) 4$ & $4.91 t-03$ \\
\hline 000 & 95.0 & 4.67 & .200 & 2.266 & $\begin{array}{r}94.0 \\
70.0 \\
102.0\end{array}$ & $\begin{array}{l}1.1 C E-J 5 \\
7.70 E-j 6 \\
5.95 E-U 6\end{array}$ & $\begin{array}{l}6.17 E-U 5 \\
4.31 E-U S \\
3.33 t-U 5\end{array}$ & $1.3 \sqcup E-03$ & $7.54 E-03$ \\
\hline 700 & 94.8 & 4.81 & .205 & 2.606 & $\begin{array}{r}92.0 \\
76.0 \\
100.0\end{array}$ & $\begin{array}{l}1.2 t E-J 5 \\
9.97 L-06 \\
6.65 t--66\end{array}$ & $\begin{array}{l}7.06 t-u b \\
5.39 L-u 5 \\
3.72 a-u 5\end{array}$ & $1.86 t-03$ & $1: 04 E-02$ \\
\hline 800 & 94.9 & 5.54 & -.322 & 7.580 & $\begin{array}{r}94.0 \\
91.0 \\
100.0\end{array}$ & $\begin{array}{l}y .62 i-J 6 \\
9.27 L-J 6 \\
9 .<7 E-06\end{array}$ & $\begin{array}{l}5.39 L-05 \\
5.19 L-U S \\
5.19 L-U 5\end{array}$ & $2.07 t-03$ & $1.16 E-02$ \\
\hline $12 \mathrm{co}$ & 94.9 & 5.03 & -.173 & 2.606 & $\begin{array}{l}92.0 \\
90.0 \\
94.0\end{array}$ & $\begin{array}{l}7.03 E-00 \\
5.75 E-20 \\
5.56 t-06\end{array}$ & $\begin{array}{l}3.94 E-J 5 \\
3.32 E-U 5 \\
3.12 E-C S\end{array}$ & $1.65 t-0 j$ & $9.27 E-03$ \\
\hline 1600 & 95.0 & 4.56 & -.027 & 7.464 & $\begin{array}{l}92.0 \\
94.0 \\
95.0\end{array}$ & $\begin{array}{l}5.7 U L-U C \\
5.36 L-J 0 \\
4.83 L-J 6\end{array}$ & $\begin{array}{l}3.22 i-U 5 \\
3.12 L-i j \\
i .73 i-i j\end{array}$ & $1.04 \dot{E}-0 j$ & $1.0 s t-0<$ \\
\hline 3200 & 96.7 & 4.42 & -.056 & 2.173 & $\begin{array}{r}94.0 \\
96.0 \\
102.0\end{array}$ & $\begin{array}{l}1.93 t-v 6 \\
1.37 t-v 6 \\
1.47 t-v 6\end{array}$ & $\begin{array}{l}1.00 t-l j \\
0.00 L-L O \\
0.23 L-U L\end{array}$ & $1.13 E-03$ & $0.3 \angle E-03$ \\
\hline
\end{tabular}

? INDICATFS UNCERTAINTIES 1!, DATA.

- $x$ indicates inValid do mircuiplete uata. 
SAMPLI:IG JOOM TU 12AUJM, NO TUIER SAMPLING.

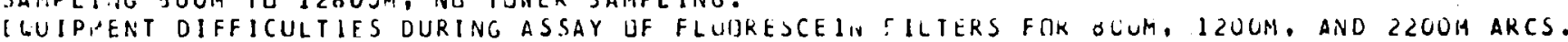

500014 AKC TRUINCATEL ON NURTH. 700014 ANU $12000 M$ AKC DATA EXTRAPULATEO SLILIITLY TU NLKTH.

\begin{tabular}{|c|c|c|c|c|c|c|c|c|c|}
\hline $\begin{array}{l}\text { IEST US } \\
\text { IINC } \\
\text { SAMFLFR }\end{array}$ & $\begin{array}{l}9 \\
\text { SULFIDE. } \\
\text { IIT } 1 . S N\end{array}$ & $\begin{array}{l}\text { JUNE } \\
\text { KELFASE }\end{array}$ & $=$ FRROME & $\begin{array}{r}321 \\
\text { LEVATILi } \\
=3.4 \mathrm{MI}\end{array}$ & $\begin{array}{l}\text { IU } 223 \\
\text { UF } 20 \\
\text { SEC AI }\end{array}$ & $\begin{array}{l}2 \text { PDT } \\
2614 \\
2619\end{array}$ & & & \\
\hline $\begin{array}{l}\text { DISTANCE } \\
\text { FETH }\end{array}$ & \multicolumn{4}{|c|}{ 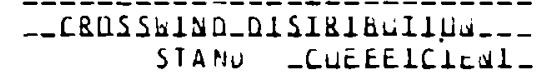 } & THREE & \multicolumn{2}{|c|}{ MAXIMUM EXPUSUKES } & \multicolumn{2}{|c|}{$\begin{array}{l}\text { CROSSWIND } \\
\text { IVIEGSAIEO_KALUES- }\end{array}$} \\
\hline SIJUhCE & MELN & UE V & SKEn & $K J R T$ & $A Z I, A U T H$ & $E / U$ & $E \cup /, 0$ & $C I(E / U)$ & $C|| E U / 0)$ \\
\hline$-\cdots---$ & ---- & ----- & $-\cdots--$ & ----- & ----- & ------- & $------i$ & ------- & -----.- \\
\hline METFRS & DEC & DEG & & & utu & SECACU.M & $1 / 30 \cdot M$ & $S E C / S O . H$ & $1 / M$ \\
\hline 400 & 76.1 & $8.5 i$ & .277 & 2.524 & $\begin{array}{l}76.0 \\
70.0 \\
79.0\end{array}$ & $\begin{array}{l}1.39 t-u 5 \\
1.33 t-j 5 \\
1.23 E-U 5\end{array}$ & $\begin{array}{l}4.73 t-05 \\
4.51 t-05 \\
4.10 t-05\end{array}$ & $4.5 L E-03$ & $1.53 E-02$ \\
\hline 1200 & 77.3 & 8.19 & .253 & 2.518 & $\begin{array}{l}72.0 \\
70.0 \\
74.0\end{array}$ & $\begin{array}{l}8.34 E-06 \\
7.77 t-66 \\
7.56 t-06\end{array}$ & $\begin{array}{l}2.04 t-U S \\
2.04 t-U 5 \\
2.53 t-U 5\end{array}$ & $3.71 E-03$ & $1.26 E-02$ \\
\hline 1600 & 79.1 & $8.66^{\circ}$ & .473 & 2.643 & $\begin{array}{l}74.0 \\
72.0 \\
10.0\end{array}$ & $\begin{array}{l}6.44 E-U 6 \\
6.41 E-V 6 \\
6.30 E-U 6\end{array}$ & $\begin{array}{l}2.30 e-\cup 5 \\
2.1 j t-\cup 5 \\
2.17 t-U 5\end{array}$ & $3.7 \forall E-03$ & $1.26 E-02$ \\
\hline 2200 & 79.7 & 8.30 & .650 & 2.808 & $\begin{array}{l}73.0 \\
74.0 \\
72.0\end{array}$ & $\begin{array}{l}4.39 t-06 \\
4.06 t-06 \\
3.56 t-66\end{array}$ & $\begin{array}{l}1.49 . t-U j \\
1.30 E-U 5 \\
1.20 t-U 5\end{array}$ & $2.59 E-U 3$ & $8.8<E-U 3$ \\
\hline 3200 & 84.3 & 7.64 & .643 & 3.119 & $\begin{array}{l}13.8 \\
13.7 \\
84.8\end{array}$ & $\begin{array}{l}\angle .10 E-v 6 \\
1.03 L-U 6 \\
1.58 E-U 6\end{array}$ & $\begin{array}{l}7.13 t-06 \\
5.35 i-06 \\
5.365-06\end{array}$ & $1.58 E-03$ & $5.37 E-03$ \\
\hline 5000 & $94.0 x$ & $7.52 x$ & $.199 x$ & $1.914 x$ & $\begin{array}{r}70.0 \\
100.0 \\
34.0\end{array}$ & $\begin{array}{l}1.00 t-U 6 \\
6.34 E-07 \\
4.10 E-67\end{array}$ & $\begin{array}{l}3.41 t-06 \\
2.15 E-06 \\
1.34 t-06\end{array}$ & $7.17 E-04 X$ & $2.44 E-03 x$ \\
\hline 7000 & 90.6 & 7.71 & .410 & 2.961 & $\begin{array}{l}92.0 \\
34.0 \\
30.0\end{array}$ & $\begin{array}{l}4.62 E-07 \\
2.54 E-07 \\
1.58 E-07\end{array}$ & $\begin{array}{l}1.64 \mathrm{E}-06 \\
8.41 \mathrm{E}-U 7 \\
5.37 E-07\end{array}$ & $3.96 E-04$ & $1.35 E-03$ \\
\hline 12800 & 99.1 & 4.32 & .071 & 2.517 & $\begin{array}{r}100.0 \\
101.0 \\
90.0\end{array}$ & $\begin{array}{l}0.27 t-08 \\
4.68 t-68 \\
4.53 t-0 t\end{array}$ & $\begin{array}{l}2.13 E-U 7 \\
1.54 E-U 7 \\
11.54 E-U 7\end{array}$ & $1.03 E-64$ & $3.4 Y E-O 4$ \\
\hline
\end{tabular}

? INOICATFS UNCERIAINTIES IR: UAIA.

x inUicates INVALiU GK inCuitrlete vata. 


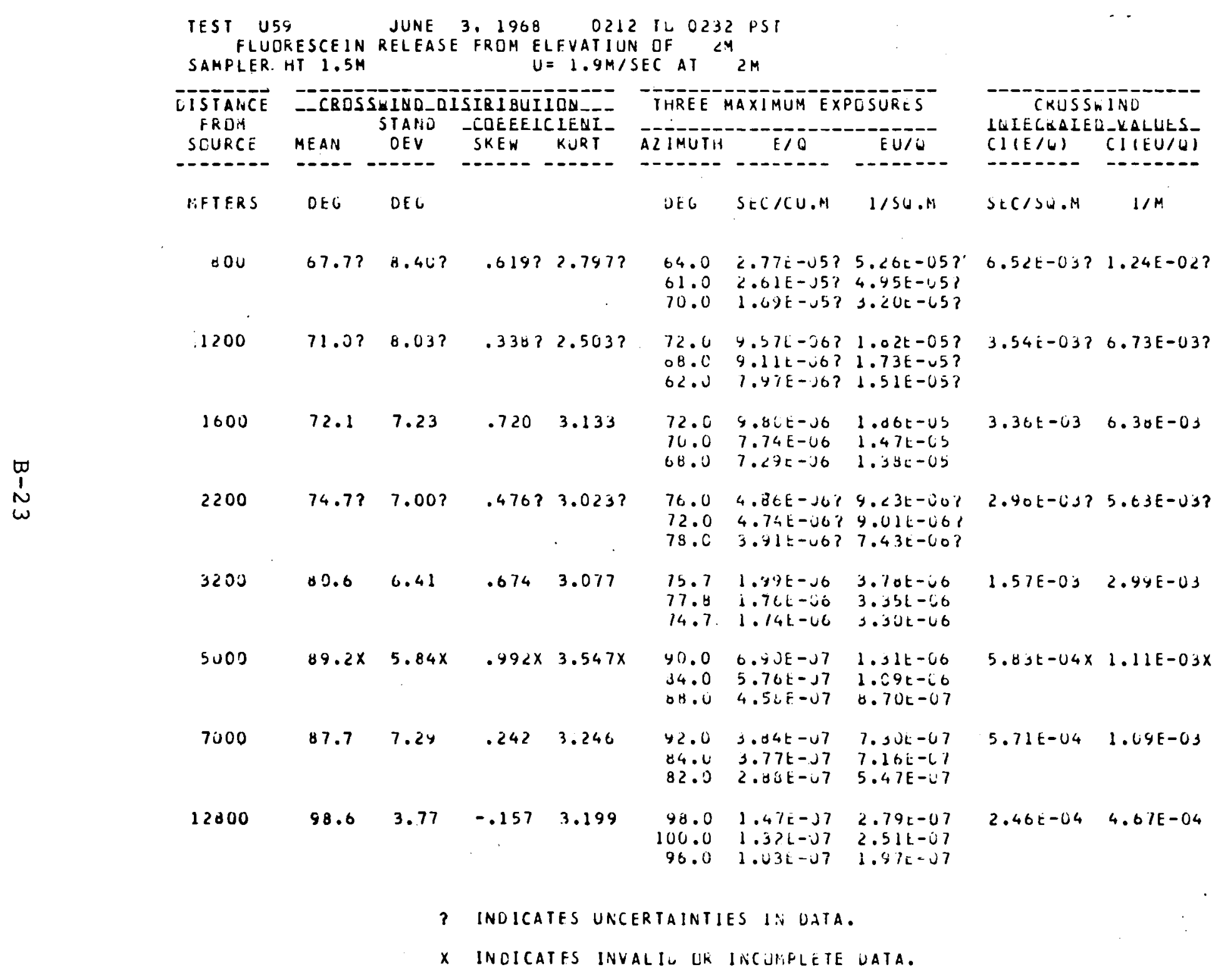


SAMPLIH:G A0OM TL I2Y00M, NI TUWER SAMPLING. MUCH DUSS UN FILTERS. MANY FIELD PROBLEMS, MINIMAL EFFORT MADE TO

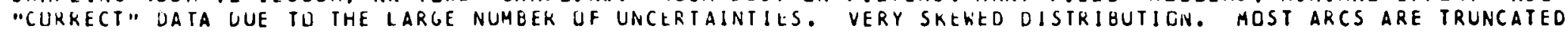
LN NURTH ENU.

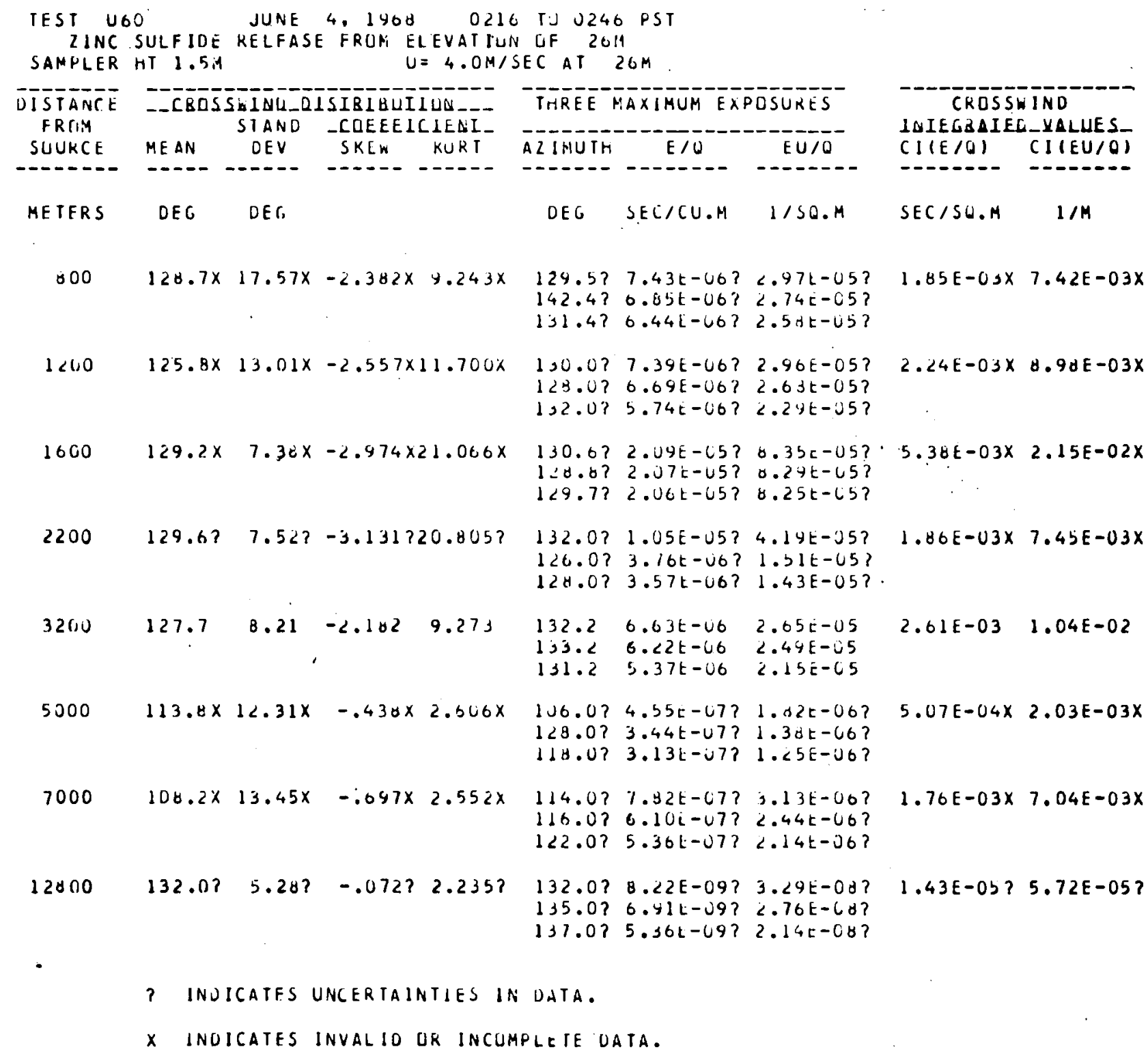




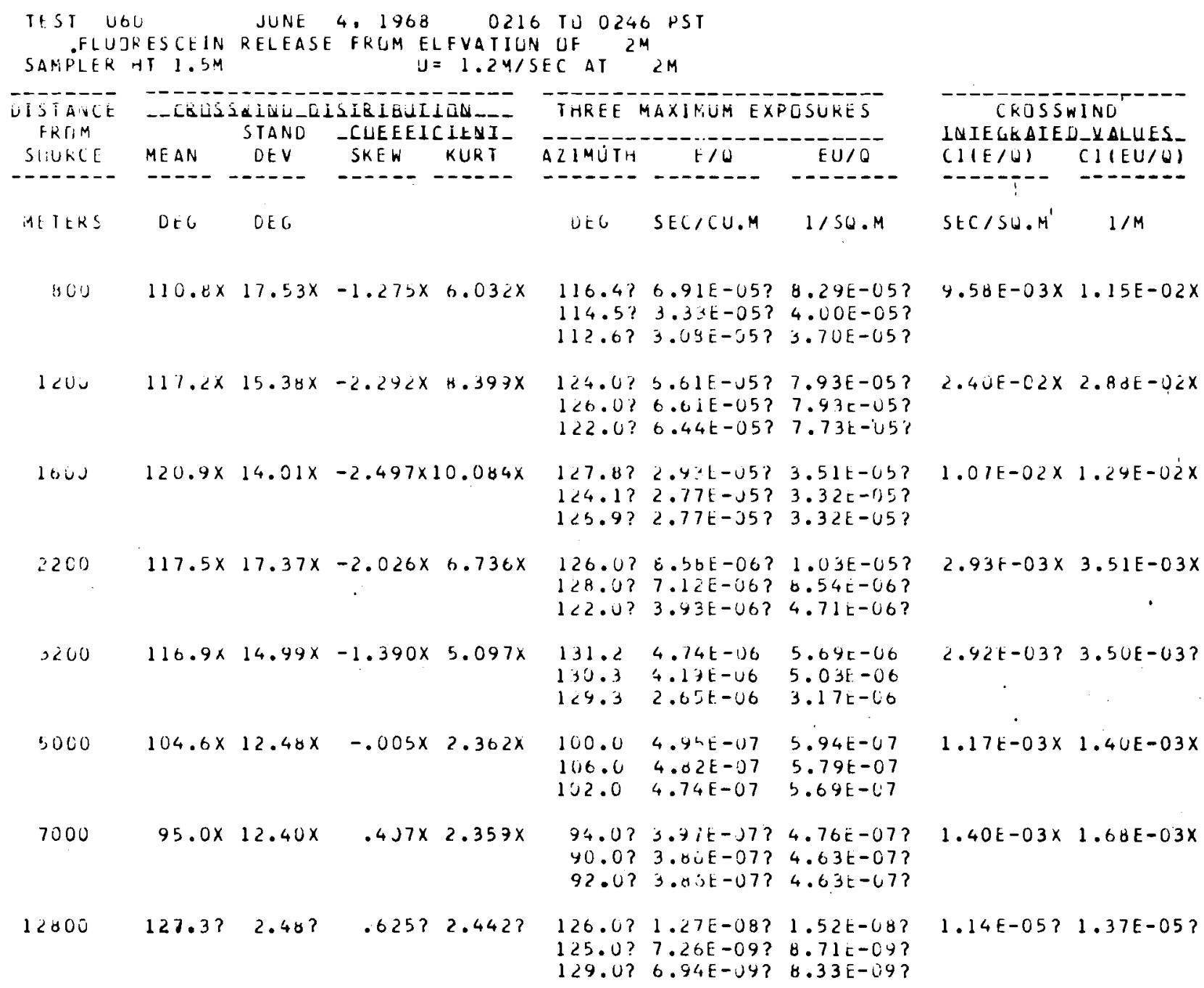

$126.0 ? 1.27 E-08 ? \quad 1.52 E-08 ?$ $125.0 ? 7.26 \mathrm{E}-09 ? 8.71 \mathrm{~L}-04 ?$ $129.0 ? 6.94 E-U 4 ? 6.33 E-09$ ?

\section{CKOSSWIND \\ IQIEGEDIED_ZALUES (IIE/U) CI(EU/U)}

$S E C / S U \cdot M^{\prime} \quad 1 / M$

$9.58 E-03 \times 1.15 E-02 x$

$2.4 U E-02 \times 2.80 E-0 \angle X$

$1.07 \mathrm{E}-02 \times 1.29 \mathrm{E}-02 \times$

$2.935-03 \times 3.51 E-03 x$

$2.92 E-03 ? 3.5 U E-03 ?$

$1.17 k-03 \times 1.4 U E-03 X$

$1.40 E-03 \times 1.68 E-03 x$ $1.14 E-05 ? 1.37 E-05 ?$

$?$ INUICATES UNCERTAINTIES IN DATA.

x indicatFs ivValio jR ivcjuplete data. 
SAMPL IIG BOOM TU 12 OOUM, NO TUWER SAMPLING. ALL ARCS EMBRACE CRUSSHINU EXTENT OF TRACER. NIIT EIUUGH TRACEK SAMPLED IN 5 UOOM ARC TU CUNFIDEIUILY INTERPULATE OUESTIGNABLE DATA PUINTS. THIRTY MIN OF ZINC SULFIDE OISPERSAL, IS MIN UF FL QURESCEIN DISPEKSAL.

\begin{tabular}{|c|c|c|c|c|c|c|c|c|c|}
\hline $\begin{array}{l}\text { TEST UG } \\
\text { ZINC } \\
\text { SAMPLER }\end{array}$ & $\begin{array}{l}1 \\
\text { SULFIDE } \\
H T 1.5 M\end{array}$ & $\begin{array}{l}\text { JUNE } \\
\text { KELFASE }\end{array}$ & $\begin{array}{r}\text { 6. } 1966 \\
F R L M E \\
J\end{array}$ & $\begin{aligned} & \text { UICE } \\
& \text { LEVAIILR } \\
&=8.5 \mathrm{HI} S\end{aligned}$ & $\begin{array}{l}\text { TU } 0136 \\
\text { UF } 201 \\
\text { EC AT }\end{array}$ & $\begin{array}{l}6 P S T \\
P B \\
26 M\end{array}$ & & & \\
\hline $\begin{array}{l}\text { UISTANCE } \\
\text { FRIIM }\end{array}$ & - cRoss & $\begin{array}{l}\text { SWINDEA } \\
\text { STAND }\end{array}$ & $\begin{array}{l}\text { SIRIEUI } \\
\text { CDEEEI }\end{array}$ & CIESI- & THREE & MAXIMUM EXF & PUSUKES & $\begin{array}{r}\text { CROSSW } \\
\text { IAIEGSAIED }\end{array}$ & $\begin{array}{l}\text { INO } \\
\text { YYLUES_- }\end{array}$ \\
\hline STURCF & MEAN & DEV & SKE & KUR T & AZIMUTH & $E / \omega$ & EUIU & $C](E / 0)$ & $C I(E U / Q)$ \\
\hline$--1---$ & ---- & $-\cdots$ & ---- & $---\cdots$ & $-\cdots \cdots-$ & 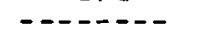 & ------- & $-\cdots--$ & 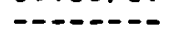 \\
\hline METEKS & DEU & DEC & & & DEG & $S E C / C U . M$ & $1 / S 0 . M$ & SEC/SQ.M & $1 / M$ \\
\hline sno & $112.7 ?$ & $2.74 ?$ & $-.106 ?$ & $3.140 ?$ & $\begin{array}{l}112.6 \\
114.5 ? \\
110.7\end{array}$ & $\begin{array}{l}1.25 t-45 \\
1.25 E-U 5 ? \\
1.04 t-05\end{array}$ & $\begin{array}{l}1.07 i-04 \\
1.06 t-04 ? \\
8.30 E-05\end{array}$ & $1.39 t-03 ?$ & $1.1 \triangle E-02 ?$ \\
\hline 1200 & 112.3 & 2.79 & -.024 & 2.863 & $\begin{array}{l}112.0 \\
114.0 \\
110.0\end{array}$ & $\begin{array}{l}6.14 t-06 \\
5.53 E-06 \\
4.3 C t-06\end{array}$ & $\begin{array}{l}5.22 t-05 \\
4.7 C E-05 \\
3.66 E-05\end{array}$ & $9.33 E-04$ & $7.93 E-03$ \\
\hline 1600 & 111.6 & 2.51 & -.115 & $2.8<y$ & $\begin{array}{l}111.4 \\
112.4 \\
113.4\end{array}$ & $\begin{array}{l}4.46 E-U 6 \\
4.41 E-06 \\
3.71 t-06\end{array}$ & $\begin{array}{l}3.79 E-05 \\
3.75 E-05 \\
3.16 E-05\end{array}$ & $7.97 E-04$ & $6.77 E-03$ \\
\hline 2200 & 110.6 & 2.71 & .387 & 3.050 & $\begin{array}{l}110.0 \\
112.0 \\
106.0\end{array}$ & $\begin{array}{l}3.09 t-06 \\
2.21 t-16 \\
2.10 t-06\end{array}$ & $\begin{array}{l}2.63 \dot{c}-\cup 5 \\
1.0 \partial E-\cup 5 \\
1.7 \partial E-\cup 5\end{array}$ & $6.7 U E-04$ & $5.75 E-03$ \\
\hline 3200 & 109.6 & 2.20 & .064 & 2.454 & $\begin{array}{l}1 \cup 5.8 \\
1 \cup 9.7 \\
1 \cup 7.8\end{array}$ & $\begin{array}{l}1.01 E-06 \\
9.60 E-U 7 \\
0.79 E-U 7\end{array}$ & $\begin{array}{l}8.59 E-U 6 \\
0.16 E-U 6 \\
7.47 E-06\end{array}$ & $3.54 \mathrm{E}-04$ & $3.01 E-03$ \\
\hline 5000 & $109.4 ?$ & $1.95 x$ & $-.763 x$ & $4.719 x$ & $\begin{array}{l}110.0 ? \\
106.0 ? \\
112.0 ?\end{array}$ & $\begin{array}{l}3.58 t-07 ? \\
6.74 t-U 8 ? \\
3.11 t-C 8 ?\end{array}$ & $\begin{array}{l}3.04 t-06 ? \\
5.73 t-07 ? \\
2.65 t-07 ?\end{array}$ & $8.79 E-05 X$ & $7.47 E-04 X$ \\
\hline 7000 & $109.6 ?$ & 2.017 & $.490 ?$ & $3.56 u ?$ & $\begin{array}{l}110.0 ? \\
100.0 \\
112.0\end{array}$ & $\begin{array}{l}1.46 E-U 7 \\
1.18 t-07 \\
4.16 E-00\end{array}$ & $\begin{array}{l}1.24 E-06 \\
1.00 E-06 \\
3.54 t-07\end{array}$ & $8.56 E-05$ & $7.27 E-04$ \\
\hline 128010 & 111.7 & 1.82 & -.114 & 3.291 & $\begin{array}{l}112.0 \\
113.0 \\
111.0\end{array}$ & $\begin{array}{l}4 . \cup 9 E-\cup 8 \\
3.32 t-\cup 8 \\
2.47 E-U 8\end{array}$ & $\begin{array}{l}3.47 E-07 \\
2.02 E-07 \\
2.53 t-07\end{array}$ & $3.56 E-05$ & $3.03 E-04$ \\
\hline
\end{tabular}

? INOICATES UNCERTAINTIES IN UATA.

X INDICATFS INVALID OK INCONPLETE DATA. 


\begin{tabular}{|c|c|c|c|c|c|c|c|c|c|}
\hline $\begin{array}{l}\text { TEST UE } \\
\text { FLUU } \\
\text { SAUFIER }\end{array}$ & $\begin{array}{l}1 \\
\text { XESCEIN } \\
\text { HT } 1.5 M\end{array}$ & $\begin{array}{l}\text { JUTE } \\
\text { KELEASE }\end{array}$ & 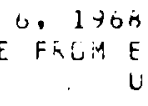 & $\begin{array}{l}\text { UFVISE } \\
\text { LFVTIUS } \\
=3.214\end{array}$ & 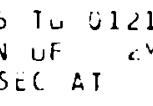 & 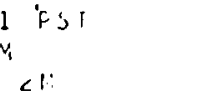 & & & \\
\hline $\begin{array}{l}\text { LISTA CE } \\
\text { FRII, }\end{array}$ & -- GELS. & $\begin{array}{l}\text { SRLSLEL } \\
\text { STANE }\end{array}$ & $\begin{array}{l}\text { ILIBIOUI } \\
\text {-COEEEI }\end{array}$ & $\begin{array}{l}\text { IUix-- } \\
\text { CIEVI- }\end{array}$ & TIREE & 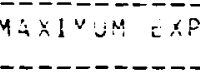 & USUR:S & $\begin{array}{l}\text { CRUSS } \\
\text { LUIÉSAIES }\end{array}$ & D_YALUtad- \\
\hline SIURCE & N.EAN & OEV & SKE iv & KURT & $A Z I M U I N$ & $1 / 0$ & $E U / O$ & $C I(E / O)$ & $C I(E U / U)$ \\
\hline 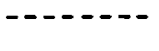 & ---- & ----- & ------ & ------ & ------ & ------- & $---\cdots$ & $\because-\cdots---$ & ------- \\
\hline$\because E$ TERS & DEG & OES. & & & UEG & SSEC/CU. IT & $1 / 50.9$ & $S E C / S O M$ & $1 / M$ \\
\hline ocos & 110.3 & 2.50 & .073 & 3.343 & $\begin{array}{l}1 \cup 8.0 \\
110.7 \\
112.0\end{array}$ & $\begin{array}{l}3.4 \geqslant t-J 5 \\
3.23 t-35 \\
2.14 t-35\end{array}$ & $\begin{array}{l}1.79:-1,4 \\
1.67 t-64 \\
1.11 t-64\end{array}$ & $3.1 J E-0.3$ & $1.61 E-02$ \\
\hline 1200 & 109.4 & 2.43 & .546 & 5.353 & $\begin{array}{l}168.0 \\
110.0 \\
112.0\end{array}$ & $\begin{array}{l}0.44 t-26 \\
3.43 t-20 \\
5 . \angle 7 t-j 6\end{array}$ & $\begin{array}{l}4.04 t-1.5 \\
4.411-6.5 \\
2.741-0.5\end{array}$ & $1.18 E-U 3$ & $6.1 \supset E-03$ \\
\hline 1600 & 109.3 & 2.30 & $.06 \pi$ & 3.355 & $\begin{array}{l}109.5 \\
108.5 \\
111.4\end{array}$ & $\begin{array}{l}7.6 x-30 \\
7.21 L-j 0 \\
6.7 y 6-v 3\end{array}$ & $\begin{array}{l}3 . y h-u j \\
3.7 h-6 s \\
3.31-0 b\end{array}$ & $1.29 E-\cup 3$ & $6.6 y E-U 3$ \\
\hline 2259 & 106.9 & 1.60 & .491 & 7.322 & $\begin{array}{l}123.0 \\
111.0 \\
112.0\end{array}$ & $\begin{array}{l}4.0 j t-j t \\
3.1 .1 t-2 t \\
5.26 \bar{t}-J 7\end{array}$ & 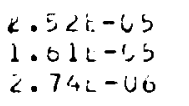 & $6.9 \cup E-04$ & 3.5 yt -03 \\
\hline 3200 & 107.9 & 2.06 & .264 & $\begin{array}{c}? .945 \\
:\end{array}$ & $\begin{array}{l}1 \cup 6.8 \\
1 \cup 7.8 \\
1 \cup 3.8\end{array}$ & $\begin{array}{l}1.42 t-16 \\
1.15 t-66 \\
1.22 t-36\end{array}$ & $\begin{array}{l}7.37 t-v i \\
6.14 t-06 \\
5.32 t-66\end{array}$ & $3.61 t-c 4$ & $1.00 \mathrm{E}-\mathrm{O} 3$ \\
\hline 5000 & $107.3 ?$ & $2.23 x$ & $.503 x$ & $3.450 x$ & $\begin{array}{l}106.0 ? \\
110.0 ? \\
104.0 ?\end{array}$ & $\begin{array}{l}3.69 \mathrm{E}-\mathrm{U}\} \\
2.56 \mathrm{E}-\mathrm{J} ? \\
7.21 \mathrm{t}-\mathrm{J} ?\end{array}$ & $\begin{array}{l}1.92 L-06 ? \\
1.34 t-06 ? \\
3.75 t-177 ?\end{array}$ & $0.3 y t-05 x$ & $4.36 E-04 X$ \\
\hline 7.1$)(100$ & 108.9 & 2.07 & -.166 & 3.663 & $\begin{array}{l}100.0 \\
110.0 \\
106.0\end{array}$ & $\begin{array}{l}3.03 E-U 7 \\
3.03 E-\cup 7 \\
1.32 E-J 7\end{array}$ & $\begin{array}{l}1.54 t-v 6 \\
1.58 c-06 \\
6.04 t-07\end{array}$ & $2.14 E-04$ & $1.11 \mathrm{E}-03$ \\
\hline 12800 & 110.7 & 1.88 & .064 & 2.699 & $\begin{array}{l}113.0 \\
109.0 \\
110.0\end{array}$ & $\begin{array}{l}8.28 \mathrm{E}-j 0 \\
6.96 E-U 8 \\
6.5 \angle L-60\end{array}$ & $\begin{array}{l}4.31 t-07 \\
3.62 t-67 \\
3.33 t-67\end{array}$ & $7.32 E-05$ & $4.06 E-04$ \\
\hline
\end{tabular}

? INDICATFS UVCERTAINTIES IN UATA.

X INOICATFS INVALIU UR INCUMFLETE LATA. 
SAM.PLING YOOM TU 1280 GM, NG TOHER SAMPLING. ALL AKCS EHHALCEU CRUSSWINU EXTENT UF IRACER.

ZINC SULFIDE DISPERSEL FIRR 30 MIN, UISPEKSAL UIFFICULTIES RLSTRICTEU FLUURESCEIN DISPERSAL TO 19.5 MIN.

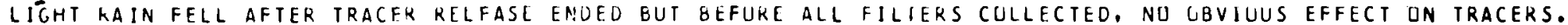

\begin{tabular}{|c|c|c|c|c|c|c|c|c|c|}
\hline \multirow{2}{*}{$\begin{array}{l}\text { DISTANCE } \\
\text { FRFY. } \\
\text { SOURCE }\end{array}$} & \multicolumn{4}{|c|}{$\begin{array}{l}\text {-CEDSSWINDODISIRIGUIION- } \\
\text { STAND _COEEICIENI- }\end{array}$} & \multirow{2}{*}{$\begin{array}{l}\text { THEEE } \\
\text { AZIMUTH }\end{array}$} & \multicolumn{2}{|c|}{ MAXIMUM EXPOSURES } & \multicolumn{2}{|c|}{$\begin{array}{c}\text { CROS SWIND } \\
\text { INIEGSAIED_YALUES }\end{array}$} \\
\hline & MEAN & DEV & SKEW & KURT & & $E / Q$ & EU/O & CIIE/O) & CI $(E U / O)$ \\
\hline METERS & DEG & UEG & & & UEG & SEL/CU.M & 1/SO.M & SEC/SG.M & $1 / M$ \\
\hline 800 & 108.5 & 4.39 & $.15 \theta$ & 2.620 & $\begin{array}{l}106.9 \\
108.8 \\
105.0\end{array}$ & $\begin{array}{l}1.0 H E-U 5 \\
1.06 E-05 \\
4.24 E-06\end{array}$ & $\begin{array}{l}9.08 E-05 \\
8.87 E-05 \\
7.76 E-05\end{array}$ & $1.76 E-03$ & $1.48 E-02$ \\
\hline 1260 & 108.8 & $4.0 d$ & .169 & 2.613 & $\begin{array}{l}108.0 \\
106.0 \\
110.0\end{array}$ & $\begin{array}{l}3.27 t-06 \\
4.28 t-06 \\
3.68 t-06\end{array}$ & $\begin{array}{l}4.43 E-05 \\
3.60 E-05 \\
3.09 t-05\end{array}$ & $1.06 \mathrm{E}-03$ & $8.88 E-03$ \\
\hline 1600 & 109.4 & 3.77 & .086 & 2.548 & $\begin{array}{l}1 \cup 9.5 \\
1 J 5.5 \\
108.5\end{array}$ & $\begin{array}{l}2.93 t-06 \\
1.74 t-v 6 \\
1.14 t-v 6\end{array}$ & $\begin{array}{l}2.46 t-U 5 \\
1.49 E-U 5 \\
1.46 t-05\end{array}$ & $5.69 t-04$ & $4.78 E-03$ \\
\hline 2200 & 109.6 & 3.61 & .204 & 2.742 & $\begin{array}{l}110.0 \\
109.0 \\
106.0\end{array}$ & $\begin{array}{l}1.72 t-66 \\
1.71 E-06 \\
1.25 E-06\end{array}$ & $\begin{array}{l}1.45 E-05 \\
1.44 E-05 \\
1.05 E-05\end{array}$ & $6.27 E-04$ & $5.27 E-03$ \\
\hline 3200 & 110.0 & 3.18 & -.043 & 2.277 & $\begin{array}{l}112.7 \\
110.7 \\
106.8\end{array}$ & $\begin{array}{l}1 . \angle 3 E-U 6 \\
4.38 E-U 7 \\
9.79 E-07\end{array}$ & $\begin{array}{l}1.03 t-05 \\
8.3 U E-06 \\
8.22 E-06\end{array}$ & $5.27 E-04$ & $4.43 E-03$ \\
\hline 5000 & 110.5 & 2.54 & .000 & 2.983 & $\begin{array}{l}110.0 \\
112.0 \\
108.0\end{array}$ & $\begin{array}{l}6.17 t-U 7 \\
5.72 E-U 7 \\
3.06 t-U 7\end{array}$ & $\begin{array}{l}5.19 E-06 \\
4.81 E-0 E \\
3.24 E-06\end{array}$ & $3.63 E-04$ & $3.05 E-03$ \\
\hline 7000 & 110.9 & 2.26 & -.165 & 4.848 & $\begin{array}{l}112.0 \\
110.0 \\
114.0\end{array}$ & $\begin{array}{l}2.75 E-07 \\
2.66 E-07 \\
1.07 E-U 7\end{array}$ & $\begin{array}{l}2.31 E-06 \\
2.23 E-06 \\
9.02 E-07\end{array}$ & $1.95 E-04$ & $1.64 E-03$ \\
\hline 12800 & 111.4 & 1.34 & -.192 & 4.116 & $\begin{array}{l}111.0 \\
112.0 \\
110.0\end{array}$ & $\begin{array}{l}2 . y 4 E-U 7 \\
2.12 t-07 \\
1.38 t-U 7\end{array}$ & $\begin{array}{l}2.47 E-06 \\
1.78 E-06 \\
1.16 E-06\end{array}$ & $1.98 E-04$ & $1.67 E-03$ \\
\hline
\end{tabular}

$?$ inUicatfs UnCERTAinties in data.

$x$ indicates invalid or incumplete uata. 


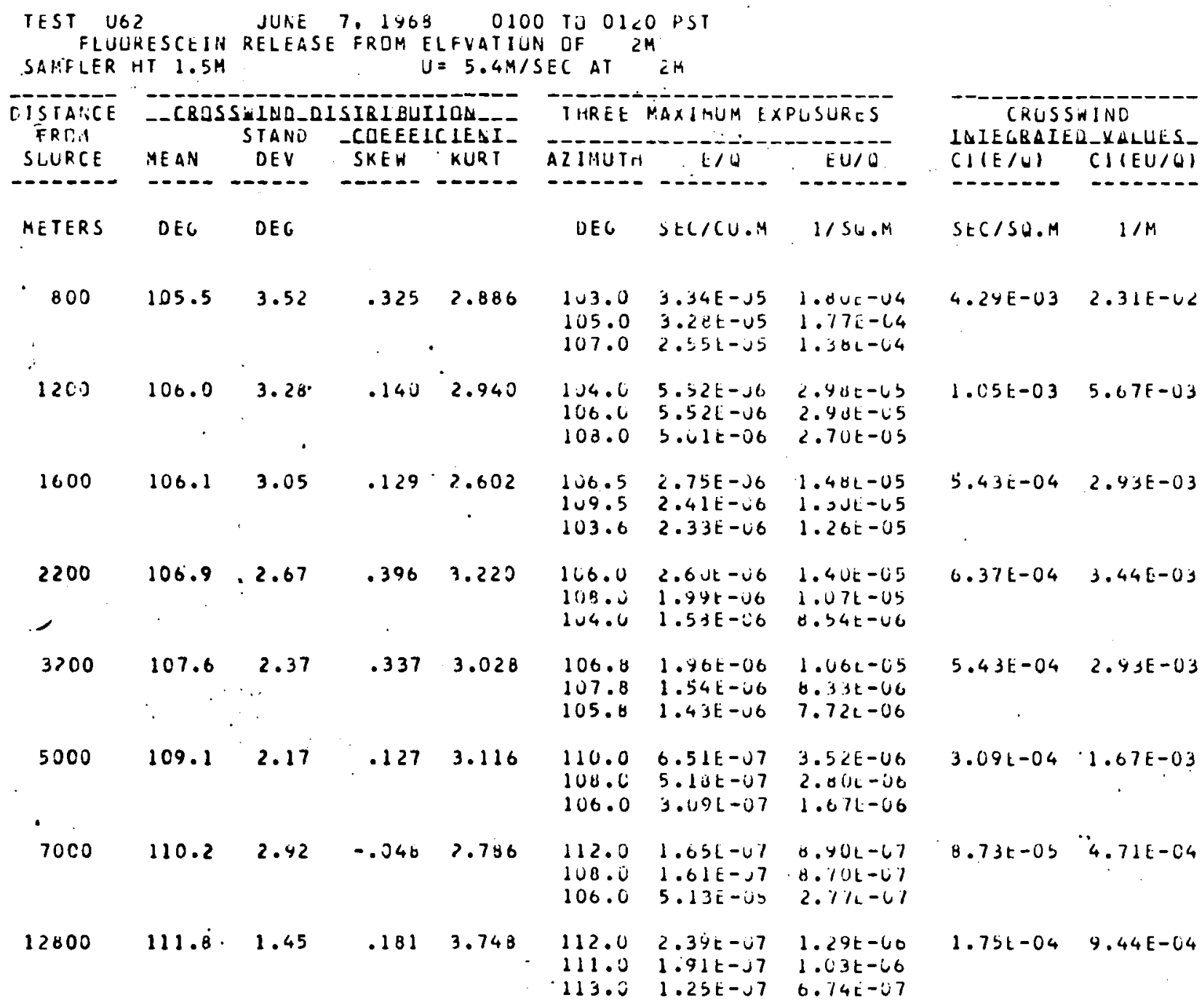

? INUICATES UNGERIAINTIES IN UATA.

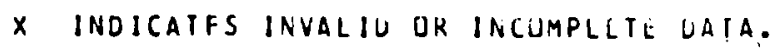


SARPLING 4OOM TO 12 OCUH, NU TUWER SAMPLING.

2I IVC SULFIDE DISPERSEU FUK 3 O MIN, OISPERSAL OIFFICULIIES RESTRICIEU FLULRESCEIN UISPERSAL IO 25 MIN. 2200 AND 12800 MRCS TRUNCATED MIV NERTH: $12800 M$ UATA EXIKAFQLATEL SLIGHTLY.

\begin{tabular}{|c|c|c|c|c|c|c|c|c|c|}
\hline $\begin{array}{l}\text { TEST UG } \\
\text { ZINC } \\
\text { SAMPLER }\end{array}$ & $\begin{array}{l}3 \\
\text { SULFIOE } \\
H T \quad 1.5 \mathrm{H}\end{array}$ & $\begin{array}{l}\text { JUNE } 2 \\
\text { KELFASE }\end{array}$ & 4. 1968 & $\begin{aligned} & 0100 \\
\text { LEVAIIUI } & =0.0 \mathrm{M} / \mathrm{S}\end{aligned}$ & $\begin{array}{l}\text { TU } U 130 \\
\text { UE } 20 \mathrm{AT}\end{array}$ & $\begin{array}{l}0 \text { PST } \\
M \quad 6 M \\
26 M\end{array}$ & & & \\
\hline $\begin{array}{l}\text { DISTANCE } \\
\text { FRUM }\end{array}$ & \multicolumn{4}{|c|}{$\begin{array}{l}\text { STESSINDEDISIBIBUI IDU - } \\
\text { STAND CUEEEICIENI- }\end{array}$} & THREE & \multicolumn{2}{|c|}{ MAXIMUM EXPOSUKES } & \multicolumn{2}{|c|}{$\begin{array}{l}\text { CRISSWINI } \\
\text { INIEGBAIED_YALUES. }\end{array}$} \\
\hline SDURCE & MEAN & UEV & SKEW & $K \cup R T$ & A $21 \mathrm{MUTH}$ & $E / Q$ & EU/O & $C I(E / Q)$ & (IIEU/Q) \\
\hline $\begin{array}{c}1 \\
----1\end{array}$ & $-\cdots-$ & --- & ----- & $-\cdots$ & $-\cdots--$ & $-\cdots---$ & ------ & ------- & ------- \\
\hline METFRS & DEG & DEG & & 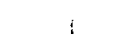 & UEO & $S E C / C U . M$ & $1 / S O . M$ & $S E C / S U . M$ & $1 / M$ \\
\hline 400 & 109.0 & 2.66 & -.442 & 3.667 & $\begin{array}{l}110.0 \\
106.0 \\
114.0\end{array}$ & $\begin{array}{l}2.57 E-U 5 \\
1.07 E-05 \\
3.53 E-U 6\end{array}$ & $\begin{array}{l}1.54 t-04 \\
6.44 t-05 \\
2.12 E-65\end{array}$ & $1.10 E-03$ & $6.96 E-03$ \\
\hline 800 & 109.3 & 2.49 & -.198 & 2.755 & $\begin{array}{l}108.8 \\
110.7 \\
112.6:\end{array}$ & $\begin{array}{l}4.42 t-U 5 \\
3.20 E-U 5 \\
2.55 L-05\end{array}$ & $\begin{array}{l}2.65 t-134 \\
1.92 E-04 \\
1.53 t-U 4\end{array}$ & $3.94 E-U 3$ & $2.37 E-02$ \\
\hline 1200 & 109.2 & 2.11 & -.060 & 2.791 & $\begin{array}{l}110.0 \\
108.0 \\
112.0\end{array}$ & $\begin{array}{l}2.79 t-U 5 \\
2.78 t-05 \\
1.49 t-U 5\end{array}$ & $\begin{array}{l}1.67 t-04 \\
1.67 t-04 \\
6.43 t-05\end{array}$ & $3.54 t-03$ & $2.13 E-02$ \\
\hline 1600 & 109.2 & 1.97 & .007 & 2.664 & $\begin{array}{l}108.5 \\
110.4 \\
107.5\end{array}$ & $\begin{array}{l}2.19 t-05 \\
2.14 t-05 \\
1.62 t-05\end{array}$ & $\begin{array}{l}1.31 t-04 \\
1.28 t-04 \\
5.73 t-05\end{array}$ & $3.11 t-03$ & $1.87 E-02$ \\
\hline 2200 & $110.4 x$ & $\frac{.91 x}{:}$ & $2.105 x$ & $6.974 x$ & $\begin{array}{l}110.0 x \\
112.0 x \\
114.0 x\end{array}$ & $\begin{array}{l}1.52 t-05 x \\
3.18 t-0 t x \\
3.50 E-v 7 x\end{array}$ & $\begin{array}{l}9.13 t-05 x \\
1.91 t-45 x \\
2.13 t-06 x\end{array}$ & $1.44 E-03 x$ & $8.64 E-03 x$ \\
\hline 3200 & $108.6 ?$ & $2.32 ?$ & $-1.310 ?$ & 6.2787 & $\begin{array}{l}108: 8 ? \\
109.7 ? \\
107.8 ?\end{array}$ & $\begin{array}{l}0.74 t-U 6 ? \\
8.39 \mathrm{E}-16 ? \\
6.78 \mathrm{E}-06 ?\end{array}$ & $\begin{array}{l}5.24 t-U 5 ? \\
5.03 t-U 5 ? \\
4.07 E-05 ?\end{array}$ & $2.37 E-03 ?$ & $1.42 E-02 ?$ \\
\hline 5000 & 106.6 & 3.20 & -.448 & 2.805 & $\begin{array}{l}106.0 \\
100.0 \\
110.0\end{array}$ & $\begin{array}{l}1.24 t-v 6 \\
1.22 t-06 \\
1.09 t-06\end{array}$ & $\begin{array}{l}7.40 t-66 \\
7.30 E-06 \\
6.52 t-06\end{array}$ & $9.03 E-04$ & $5.42 E-03$ \\
\hline 7000 & 105.9 & 3.64 & -.446 & 2.533 & $\begin{array}{l}108.0 \\
1 \cup 4.0 \\
110.0\end{array}$ & $\begin{array}{l}8.12 E-67 \\
6.94 E-07 \\
5.11 E-07\end{array}$ & $\begin{array}{l}4.67 E-00 \\
4.17 E-06 \\
3.06 E-66\end{array}$ & $6.92 E-04$ & $4.15 E-03$ \\
\hline 12000 & 105.8 & 4.89 & -.852 & 4.642 & $\begin{array}{l}1 \cup 7.0 \\
1 \cup 8.0 \\
1 \cup 9.0\end{array}$ & $\begin{array}{l}8.63 E-U 7 \\
\forall .28 E-07 \\
5.58 E-07\end{array}$ & $\begin{array}{l}5.1 \Delta E-06 \\
4.97 E-06 \\
3.35 E-06\end{array}$ & $1.08 \mathrm{E}-03$ & $6.51 E-03$ \\
\hline
\end{tabular}

$?$ INUICATES UNCERTAINTIES IN DATA.

X INOICATFS INVALID DR INCUMPLETE DATA. 


\begin{tabular}{|c|c|c|c|c|c|c|c|c|c|}
\hline $\begin{array}{l}\text { DISTAIICE } \\
\text { FRGM }\end{array}$ & \multicolumn{4}{|c|}{$\begin{array}{l}\text {-CROSSUINOAQISIRIGUIIDA- } \\
\text { STAND COEEEICIENI- }\end{array}$} & \multicolumn{3}{|c|}{ THREE MAXIMUM EXPLSURES } & \multicolumn{2}{|c|}{$\begin{array}{l}\text { CRUSSWIIU } \\
\text { LULEGRAIED_KALUES. }\end{array}$} \\
\hline SUURCE & MEAN & DEV & SKEW & KUR T & AZIMUTH & $E / 0$ & $\bar{E} \cup / 0$ & $E(E / \theta)$ & Cl(EU/O) \\
\hline------ & ---- & ----- & $-\infty---$ & ----- & ------ & ------- & ------ & -------- & ------- \\
\hline METERS & DEC & DEC & & & DEG & SEC/CU.M & $1 / \sin \cdot M$ & SECISO.M & $1 / \mathrm{Hi}$ \\
\hline 400 & 106.5 & 3.24 & $\begin{array}{l}-.579 \\
.\end{array}$ & 4.514 & $\begin{array}{l}106.0 \\
110.0 \\
102.0\end{array}$ & $\begin{array}{l}2.31 E-04 \\
1.36 E-04 \\
4.75 i-05\end{array}$ & $\begin{array}{l}t .94 t-C 4 \\
4.09 t-04 . \\
1.42 E-04\end{array}$ & $1.23 E-02$ & $3.7 \cup E-02$ \\
\hline $8 C 0$ & 107.3 & 2.37 & -.464 & 3.294 & $\begin{array}{l}108.8 \\
106.9 \\
105.0\end{array}$ & $\begin{array}{l}1 . \angle 0 E-04 \\
1.13 E-04 \\
5.09 E-0.5\end{array}$ & $\begin{array}{l}3.61 t-04 \\
3.39 t-04 \\
1.53 i-64\end{array}$ & $1.00 E-02$ & $3.01 t-0<$ \\
\hline 1200 & 107.5 & 2.11 & -.190 & 2.822 & $\begin{array}{l}108.0 \\
106.0 \\
110.0\end{array}$ & $\begin{array}{l}3.64 E-J 5 \\
2.44 E-U 5 \\
2.11 E-J 5\end{array}$ & $\begin{array}{l}1.09 L-U 4 \\
7.32 L-U 5 \\
6.33 t-U 5\end{array}$ & $4.05 E-03$ & $1.22 E-02$ \\
\hline 1600 & 107.8 & 1.84 & -.082 & 3.630 & $\begin{array}{l}107.5 \\
100.5 \\
106.5\end{array}$ & $\begin{array}{l}2.52 E-U 5 \\
2.44 E-U 5 \\
2.12 E-U 5\end{array}$ & $\begin{array}{l}7.57 t-05 \\
7.33 t-05 \\
0.35 t-05\end{array}$ & $3.37 E-03$ & $1.01 t-02$ \\
\hline 2200 & $110.1 x$ & $.54 x$ & $3.796 x$ & $118.330 x$ & $\begin{array}{l}110.0 x \\
112.0 x \\
114.0 x\end{array}$ & $\begin{array}{l}4.21 t-46 x \\
3.03 t-47 x \\
1.06 t-46 x\end{array}$ & $\begin{array}{l}1.26 t-j 5 x \\
9.08 E-07 x \\
3.18 t-08 x\end{array}$ & $3.47 E-04 X$ & $1.04 E-03 X$ \\
\hline 3200 & 106.8 & 2.96 & -1.410 & 5.840 & $\begin{array}{l}107.6 \\
106.8 \\
108.8\end{array}$ & $\begin{array}{l}5.96 \dot{E}-36 \\
4.97 \dot{E}-i 16 \\
4.36 t-36\end{array}$ & $\begin{array}{l}1.79 t-05 \\
1.49 E-05 \\
1.46 t-05\end{array}$ & $1.6 Y E-03$ & $5.07 t-03$ \\
\hline 5000 & 105.2 & 3.54 & -.651 & 3.144 & $\begin{array}{l}108.0 \\
106.0 \\
104.0\end{array}$ & $\begin{array}{l}1.12 E-06 \\
1.01 E-06 \\
7.15 E-J 7\end{array}$ & $\begin{array}{l}3.37 c-06 \\
3.03 c-06 \\
2.15 E-06\end{array}$ & $7.35 E-04$ & $2.21 E-U 3$ \\
\hline 7000 & 104.1 & 3.88 & -.186 & 2.351 & $\begin{array}{l}104.0 \\
106.0 \\
108.0\end{array}$ & $\begin{array}{l}5.61 E-U 7 \\
4.91 E-C 7 \\
4.10 E-U 7\end{array}$ & $\begin{array}{l}1.68 t-06 \\
1.47 t-06 \\
1.23 t-06\end{array}$ & $6.56 E-04$ & $1.97 E-03$ \\
\hline 12800 & 104.6 & 4.53 & -.826 & 4.184 & $\begin{array}{l}107.0 \\
168.0 \\
106.0\end{array}$ & $\begin{array}{l}4.44 E-J 7 \\
3.03 E-j 7 \\
3.70 E-07\end{array}$ & $\begin{array}{l}1.33 E-06 \\
1.13 E-06 \\
1.11 E-06\end{array}$ & $6.35 E-04$ & $1.91 E-03$ \\
\hline
\end{tabular}

$?$ INDICATES UYCERTAINTIES IN DATA.

x inoigates invalio gr incumplete lata. 


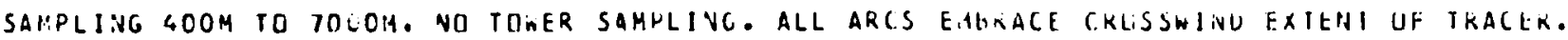

ZIA.C SULFIOE DISPERSEO FIR 30 MIN, DISPERSAL UIFFICULIIFS GESIHICIEL FLUIIRESCEIN LISPERSAL TO 20 MIN.

LARGF HIND OIRECIIINN SHEAR BETWEFN TRAEER RELEASE LEVELS. TKACEK UISIKIBJIIJHS SKCVEL LUNG TAIL TII THE INOKTH.

$$
\text { IEST U64 JJVE } 25,1468 \text { } 5347 \text { Tं } 0417 \text { PST }
$$

IINC SULFIOE KELFASE FRJY ELEVATILIN UL $26 \mathrm{M}$

$$
\text { SAMPLER HT } 1.5 \mathrm{MH} \text { UE 5.3M/SEC AI } 20 \mathrm{M}
$$

\begin{tabular}{|c|c|c|c|c|c|c|c|c|c|}
\hline $\begin{array}{l}\text { DISTANCE } \\
\text { FRMM }\end{array}$ & $-C 325$ & $\begin{array}{l}\text { Sa'LUA_D } \\
\text { STANU }\end{array}$ & $\begin{array}{l}\text { LSIBIEII } \\
\text {-CUEEE }\end{array}$ & $\begin{array}{l}\text { LIUE- } \\
\text { ICIENI- }\end{array}$ & 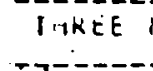 & MAXIMUIA EX & Pusures & $\begin{array}{r}\text { CKUSSH } \\
\text { IUILGKAIE }\end{array}$ & $\begin{array}{l}\text { INI } \\
\text { YALUES }\end{array}$ \\
\hline SDURCF & MEAN & DEV & SKEW & KURं। & $4 Z, Y J T H$ & $E / G$ & EJ/O & $(1(1) / U)$ & $(1(E) / 0)$ \\
\hline 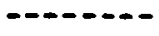 & $\cdots---$ & $\cdots--$ & $-\cdots-$ & $-\cdots-\infty$ & $---\infty-$ & $-\cdots--\cdots$ & $-\ldots, \ldots$ & $\ldots \ldots$ & $\cdots \cdots$ \\
\hline METERS & DEG & DEC, & & & UEU & SEC/CU.H & $1 / S W \cdot M$ & $S t C / S A . H$ & - $\quad 1 / M$ \\
\hline 400 & 111.7 & 4.60 & -1.793 & $10 .+50$ & $\begin{array}{l}114.0 \\
110.0 \\
106.0\end{array}$ & $\begin{array}{l}3.2+t-20 \\
2.02 t-16 \\
3.21 t-67\end{array}$ & $\begin{array}{l}1.72 E-05 \\
1 . J 6 E-35 \\
2.76 t-1,6\end{array}$ & $1.83 t-04$ & $9.7 \angle E-04$ \\
\hline 900 & 112.5 & 5.05 & -.417 & 2.776 & $\begin{array}{l}114.5 \\
112.6 \\
116.4\end{array}$ & $\begin{array}{l}5.32 E-16 \\
4.43 L-16 \\
4.37 E-16\end{array}$ & $\begin{array}{l}2.02 \ddot{E}-05 \\
2.35 E-05 \\
2.32 t-J 5\end{array}$ & $8.5<E-04$ & $4.5 \angle E-03$ \\
\hline 1200 & 113.1 & $\begin{array}{c}4.98 \\
.\end{array}$ & -.426 & 3.026 & $\begin{array}{l}114.0 \\
112.0 \\
110.0\end{array}$ & $\begin{array}{l}6.66 E-U 6 \\
4.75 E-U 6 \\
4.42 E-v 6\end{array}$ & $\begin{array}{r}3.53 E-65 \\
2.52 E-J 5 \\
2.3+E-35\end{array}$ & $1.40 E-03$ & $7.44 E-03$ \\
\hline 1600 & 114.4 & 5.55 & -.439 & 2.694 & $\begin{array}{l}1<1.2 \\
116.3 \\
116.2\end{array}$ & $\begin{array}{l}4.94 t-v 0 \\
4.531-v 6 \\
4.58 t-v 6\end{array}$ & $\begin{array}{l}2.62 t-v 5 \\
2.43 t-U b \\
2.43 t-U b\end{array}$ & $1.78 E-03$ & $9.42 F=0.3$ \\
\hline 2200 & 111.6 & 3.94 & .860 & 5.545 & $\begin{array}{l}110.0 \\
112.0 \\
104.0\end{array}$ & $\begin{array}{l}5.06 E-J 6 \\
1.52 L-v 6 \\
1.03 t-v 6\end{array}$ & $\begin{array}{l}2.68 t-J 5 \\
8.03 t-66 \\
5.44 t-66\end{array}$ & $8.0 У E-J 4$ & $4.2 J E-03$ \\
\hline 3200 & 120.1 & 5.51 & -.355 & 2.718 & $\begin{array}{l}113.7 \\
125.4 \\
116.6\end{array}$ & $\begin{array}{l}3.17 t-v 6 \\
2.83 t-v 6 \\
2.57 t-v 6\end{array}$ & $\begin{array}{l}1.69 E-05 \\
1.53 E-35 \\
1.36 E-U S\end{array}$ & $1.67 E-03$ & $8.86 E-03$ \\
\hline 5000 & 126.9 & 3.58 & -3.267 & 21.874 & $\begin{array}{l}1<0.0 \\
130.0 \\
1<4.0\end{array}$ & $\begin{array}{l}2.63 L-v b \\
7.66 i-v 7 \\
7.47 \mathrm{~L}-u 7\end{array}$ & $\begin{array}{l}1.56 t-05 \\
4.06 E-36 \\
3.96 t-60\end{array}$ & $9.56 t-04$ & $5.06 E-03$ \\
\hline 7000 & 128.97 & $3.31 ?$ & $\begin{array}{c}-2.572 \\
.\end{array}$ & $? 26.357 ?$ & $\begin{array}{l}120.07 \\
130.0 ? \\
132.0 ?\end{array}$ & $\begin{array}{l}3.40 E-06 ? \\
3.07 L-06 ? \\
1.12 E-06 ?\end{array}$ & $\begin{array}{l}1.04 E-05 ? \\
1.63 E-05 ? \\
3.94 E-06 ?\end{array}$ & $2.36 E-03 X$ & 1. $26 E-02 X$ \\
\hline
\end{tabular}

$?$ ivolcatfs uncertainties in Uata.

$x$ indicates inVal io or incumplete lata. 


\begin{tabular}{|c|c|c|c|c|c|c|c|c|c|}
\hline $\begin{array}{l}\text { TEST UE } \\
\text { FLUUL } \\
\text { SAHPIER }\end{array}$ & $\begin{array}{l}4 \\
\text { RESCEIS } \\
\text { HT } 1.5:\end{array}$ & $\begin{array}{l}\text { JUNE } \\
\text { RELEAS }\end{array}$ & $\begin{array}{l}25,196 \\
\text { E FROM }\end{array}$ & $\begin{array}{r}034 \\
\text { FVATIL } \\
=1.6 \mathrm{M}\end{array}$ & $\begin{array}{l}\text { TU } 040 \\
\text { OF } \\
\text { EC AT }\end{array}$ & $\begin{array}{l}7 \text { FST } \\
2 M\end{array}$ & & & \\
\hline \multirow{2}{*}{$\begin{array}{l}\text { WISTANCE } \\
\text { FKCH } \\
\text { SEURCE }\end{array}$} & \multicolumn{4}{|c|}{$\begin{array}{l}\text {-CROSSHIADEDISIBIEUIION } \\
\text { STAND -COEEEICIENI- }\end{array}$} & THREE & \multicolumn{2}{|c|}{ MAXIMUA EXPOSURES } & \multicolumn{2}{|c|}{$\begin{array}{l}\text { CROSSWINU } \\
\text { LUIEEBAIED_HALULZ_ }\end{array}$} \\
\hline & MEAN & DEV & SKEH & KUKT & AZIMUTH & $E / 0$ & $t U / 0$ & & CI $(E U / U)$ \\
\hline$-\infty,-\infty,-\infty$ & $---\infty$ & $-----\infty$ & $-\cdots--$ & ------ & $------\infty$ & ------- & $----0-0$ & 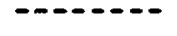 & ------- \\
\hline HETERS & DEG & IDEG & & . & DEG & $S E C / C U . M$ & $1 / S U . M$ & $S E C / S O . M$ & $1 / M$ \\
\hline $4 \% 0$ & 90.2 & 5.11 & .414 & 3.877 & $\begin{array}{l}90.0 \\
94.0 \\
86.0\end{array}$ & $\begin{array}{l}1.71 E-14 \\
1.06 E-04 \\
1.00 L-04\end{array}$ & $\begin{array}{l}2.74 E-J 4 \\
1.69 t-04 \\
1.60=-04\end{array}$ & $1.40 \mathrm{E}-02$ & $2.24 E-02$ \\
\hline 800 & 96.6 & 6.89 & -.229 & 2.205 & $\begin{array}{r}97.1 \\
91.0 \\
101.0\end{array}$ & $\begin{array}{l}5.54 E-j 5 \\
5.38 E-35 \\
3.26 E-J 5 i\end{array}$ & $\begin{array}{l}0.07 E-U 5 \\
0.01 E-U 5 \\
0.25 E-U b\end{array}$ & $1.5 \angle E-J 2$ & $2.43 E-U<$ \\
\hline 1200 & 95.7 & 6.13 & .205 & 2.339 & $\begin{array}{l}94.0 \\
92.0 \\
96.0\end{array}$ & $\begin{array}{l}2.90 E-U 5 \\
2.79 E-U 5 \\
2.79 E-U 5\end{array}$ & $\begin{array}{l}4.63 t-0 b \\
4.46 t-05 \\
4.40 t-15\end{array}$ & $1.07 E-02$ & $1.72 \mathrm{E}-02$ \\
\hline 1600 & 98.6 & 5.39 & .310 & 2.568 & $\begin{array}{l}94.6 \\
93.6 \\
95.6\end{array}$ & $\begin{array}{l}2.64 E-15 \\
2.18 E-05 \\
2.32 E-35\end{array}$ & $\begin{array}{r}4 . \angle \angle E-U b \\
13.50 E-05 \\
3.39 E-65\end{array}$ & $8.30 E-03$ & $1.33 E-U 2$ \\
\hline 2200 & $\begin{array}{c}101.7 \\
.\end{array}$ & 4.95 & .176 & 3.767 & $\begin{array}{r}100.0 \\
102.0 \\
96.0\end{array}$ & $\begin{array}{l}7.52 E-10 \\
4.26 E-06 \\
2.50 c-00\end{array}$ & $\begin{array}{l}1.2 U E-05 \\
6.01 E-06 \\
4.011-06\end{array}$ & $2.12 E-03$ & $3.30 E-03$ \\
\hline $32 \cap 0$ & 110.4 & 4.7 .9 & -1.140 & 6.141 & $\begin{array}{l}113.7 \\
112.7 \\
109.7\end{array}$ & $\begin{array}{l}4.76 E-.06 \\
3.77 E-06 . \\
2.46 E-06\end{array}$ & $\begin{array}{l}7.62 E-06 \\
6.35 E-C 6 \\
3.97 t-C 6\end{array}$ & $1.70 E-03$ & $2.72 \mathrm{E}-0 \mathrm{3}$ \\
\hline 5000 & 117.6 & 6.81 & -.891 & 4.500 & $\begin{array}{l}120.0 \\
122.0 \\
118.0\end{array}$ & $\begin{array}{l}7.85 E-J 7 \\
0.01 E-07 \\
5.56 E-U 7\end{array}$ & $\begin{array}{l}1.26 E-06 \\
9.02 E-07 \\
8.93 E-07\end{array}$ & $7.35 \mathrm{E}-04$ & $1.18 E-03$ \\
\hline 7000 & 123.7 & 7.09 & -1.788 & 6.823 & $\begin{array}{l}126.0 \\
128.0 \\
130.0\end{array}$ & $\begin{array}{l}1.13 E-U 6 \\
8.73 E-U 7 \\
6.11 E-U 7\end{array}$ & $\begin{array}{l}1.82 t-06 \\
1.40 E-06 \\
9.77 E-07\end{array}$ & $1.11 E-03$ & $1.7 \partial E-03$ \\
\hline
\end{tabular}

$?$ INOICATES UNCERTAINTIES IN CATA.

X INDICATES INVALIO OR INCUMPlete lata. 


\begin{tabular}{|c|c|c|c|c|c|c|c|c|c|}
\hline $\begin{array}{l}\text { IEST US } \\
\text { ZINC } \\
\text { SAMPLER }\end{array}$ & $\begin{array}{l}5 \\
\text { SULF } 10 E \\
\text { HT } 1.5 \mathrm{M}\end{array}$ & $\begin{array}{l}\text { JUNE } \\
\text { RELFASE }\end{array}$ & $\begin{array}{r}27,1769 \\
E \text { FRJH EL } \\
U=\end{array}$ & $\begin{array}{r}0332 \\
E V A I I U N \\
=5.5 \mathrm{M} / \mathrm{S}\end{array}$ & $\begin{array}{l}\text { IJ O4L? } \\
\text { DF } 26 \mathrm{M} \\
\text { SC A }\end{array}$ & $\begin{array}{l}2 \text { PST } \\
26,4\end{array}$ & & & \\
\hline $\begin{array}{l}\text { CISTANCE } \\
\text { FROY }\end{array}$ & $-\cos 25$ & $\begin{array}{l}\text { SEINDEDI } \\
\text { SIANU }\end{array}$ & $\begin{array}{l}\text { ISIBIEUII } \\
\text {-COEEEIC }\end{array}$ & CIENI- & TAFEE । & VAXIMUM EX & PLSURES & $\begin{array}{l}\text { CRUSSW } \\
\text { IAIEGKAIED }\end{array}$ & $\begin{array}{l}\text { WINO } \\
\text { DALUES_- }\end{array}$ \\
\hline SOURCE & MEAN & DEV & SKEW & KURT & AZIMUTH & $E / O$ & $E \cup / O$ & $(I(E / J)$ & $C .1(E U / O)$ \\
\hline------ & $\cdots--$ & ------ & $--\cdots--$ & $-\cdots-\cdots$ & $--\infty-\infty$ & $\cdots-\cdots$ & $\cdots-\cdots--$ & $---\cdots-\cdots$ & ------- \\
\hline HETFRS & DEG & OEG & & & UE G & $S E C / C U . M$ & $1 / S \cdot . M$ & $5 \in C / 5 D_{0} 14$ & $1 / M$ \\
\hline . & & & & & & & & & \\
\hline 400 & $78.7 ?$ & $10.53 ?$ & $.232 ?$ & $1.504 ?$ & $\begin{array}{l}70.0 ? \\
90.0 ? \\
74.0 ?\end{array}$ & $\begin{array}{l}6.46 t-06 ? \\
4.40 t-0 C ? \\
3.34 t-06 ?\end{array}$ & $\begin{array}{l}3.55 t-05 ? \\
2.42 t-05 ? \\
1.04 t-05 ?\end{array}$ & $6.35 E-04 ?$ & $3.4^{\prime}, E-03 ?$ \\
\hline 800 & 81.3 & 10.38 & -.039 & 1.560 & $\begin{array}{l}91.0 \\
70.0 \\
73.0\end{array}$ & $\begin{array}{l}1.16 t-65 \\
1.11 E-65 \\
1.10 E-05\end{array}$ & $\begin{array}{l}0.46 t-05 \\
t .12 t-05 \\
0.03 t-05\end{array}$ & $3.07 E-03$ & $1.6 Y E-U 2$ \\
\hline 1200 & 80.7 & 10.06 & -.245 & 1.559 & $\begin{array}{l}90.0 \\
58.0 \\
72.0\end{array}$ & $\begin{array}{l}9.23 E-06 \\
0.76 c-66 \\
c . \angle 5 L-06\end{array}$ & $\begin{array}{l}t .37 E-j 5 \\
4.82 E-U 5 \\
3.44 t-U 5\end{array}$ & $2.93 E-03$ & $1.61 E-02$ \\
\hline 1600 & 30.5 & 11.03 & -.176 & $1.43 \mathrm{~J}$ & $\begin{array}{l}71.0 \\
92.0 \\
88.0\end{array}$ & $\begin{array}{l}3.74 E-46 \\
3.58 t-v 6 \\
3.15 E-46\end{array}$ & $\begin{array}{l}2.0 \pm t-65 \\
1.97 E-U 5 \\
1.73 E-05\end{array}$ & $1.64 E-03$ & $9.03 E-03$ \\
\hline 2200 & 77.7 & 10.63 & -.172 & $1.6 \times 2$ & $\begin{array}{l}86.0 \\
90.0 \\
63.0\end{array}$ & $\begin{array}{l}1.13 E-06 \\
1 . U 7 E-06 \\
9.30 E-U 7\end{array}$ & $\begin{array}{l}6.47 L-06 \\
5.86 t-66 \\
5.12 E-36\end{array}$ & $7.38 E-04$ & $4.06 E-03$ \\
\hline 3200 & $82.5 ?$ & 7.717 & $-1.180 x$ & $4.060 x$ & $\begin{array}{l}87.0 \\
52.0 \\
02.8\end{array}$ & $\begin{array}{l}2.21 L-C 6 \\
1.36 E-\cup 6 \\
1.33 E-\angle 6\end{array}$ & $\begin{array}{l}1.22 E-U 5 \\
1.09 E-05 \\
7.33 E-06\end{array}$ & $1.24 E-03 ?$ & $6.84 E-03 ?$ \\
\hline 5000 & $85.9 x$ & $2.99 x$ & $.724 x$ & $4.094 x$ & $\begin{array}{l}36.0 ? \\
82.0 ? \\
84.0 ?\end{array}$ & $\begin{array}{l}4.61 E-\cup 7 ? \\
3.53 E-U 7 ? \\
3.23 E-U 7 ?\end{array}$ & $\begin{array}{l}2.54 t-06 ? \\
2.16 t-06 ? \\
1.77 t-06 ?\end{array}$ & $2.92 E-04 X$ & $1.61 E-03 x$ \\
\hline 7000 & $82.6 x$ & $3.66 x$ & $1.188 x$ & $5.604 x$ & $\begin{array}{l}82.0 ? \\
76.0 ? \\
90.0 ?\end{array}$ & $\begin{array}{l}6.15 t-67 ? \\
6.21 t-06 ? \\
5.36 t-06 ?\end{array}$ & $\begin{array}{l}3.30 t-U 6 ? \\
3.41 E-07 ? \\
2.96 t-07 ?\end{array}$ & $2.11 E-04 x$ & $1.16 E-03 x$ \\
\hline 12800 & $93.2 x$ & $1.96 x$ & $.231 x$ & $1.551 x$ & $\begin{array}{l}y 1.0 x \\
y 2.0 x \\
y 6.0 x\end{array}$ & $\begin{array}{l}4.47 E-U 8 X \\
3.45 E-U 8 X \\
2.65 E-06 x\end{array}$ & $\begin{array}{l}2.46 t-\cup 7 x \\
1.90 t-07 x \\
1.40 t-07 x\end{array}$ & $3.40 t-05 x$ & $1.87 E-04 x$ \\
\hline & INOI & ICATES & CERTAIN & TIES IV & JATA. & & & & \\
\hline & 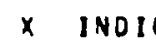 & & & & & & & & \\
\hline
\end{tabular}




\begin{tabular}{|c|c|c|c|c|c|c|c|c|c|}
\hline $\begin{array}{l}\text { TEST UE } \\
\text { FLUI } \\
\text { SAMPLER }\end{array}$ & $\begin{array}{l}\text { R } \\
\text { RESCEIN } \\
\text { HT } 1.5 \mathrm{H}\end{array}$ & $\begin{array}{l}\text { JUNE } 2 \\
\text { RELEASE }\end{array}$ & $\begin{array}{r}7.1968 \\
\text { FROM EL } \\
U=\end{array}$ & $\begin{array}{r}0 \$ 32 \\
\text { EVATIUN } \\
\quad 3.0 A / S\end{array}$ & $\begin{array}{l}2 \text { TU O4UZ } \\
N \text { J } \\
\text { SEC AT }\end{array}$ & $\begin{array}{l}2+S T \\
M M\end{array}$ & & \multirow{2}{*}{\multicolumn{2}{|c|}{$\begin{array}{l}\text { CRUSSWINL } \\
\text { LUIEGBAIEL_YALUES_ }\end{array}$}} \\
\hline $\begin{array}{l}\text { CISTAICE } \\
\text { FRL:Y }\end{array}$ & \multicolumn{4}{|c|}{$\begin{array}{l}\text { CBCSSYIULEISIRIEUIIDA } \\
\text { STANO -COEEEICIENI_- }\end{array}$} & THREE & \multicolumn{2}{|c|}{ MAXIMUN EXPLSURES } & & \\
\hline SOUKCE & MEAN & DEV & SKEW & KURT & ALINUTI1 & 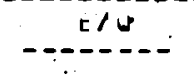 & tU/6 & 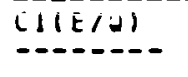 & CIIEU/Gi \\
\hline MEIERS & $D E G$ & DEG & & & DEC & SEC/CU.M & $1 / 5 W . M$ & SEC/SU.M & $1 / M$ \\
\hline $\begin{array}{c}400 \\
\vdots\end{array}$ & 73.47 & $11.10 ?$ & .5617 & 1.9067 & $\begin{array}{l}66.0 ? \\
70.0 ? \\
02.0 ?\end{array}$ & $\begin{array}{l}3.06 t-U 5 ? \\
2.43 t-u 5 ? \\
2.01 t-05 ?\end{array}$ & $\begin{array}{l}9.19 t-05 i \\
0.80 t-U 5 ? \\
7.02 i-05 ?\end{array}$ & $4.26 t-0.17$ & $1.20 t-02 ?$ \\
\hline 800 & 78.3 & 10.51 & -.195 & 1.534 & $\begin{array}{l}88.0 \\
91.0 \\
65.0\end{array}$ & $\begin{array}{l}2.93 E-.15 \\
1.90 E-U 5 \\
1.89 E-U 5\end{array}$ & $\begin{array}{l}0.00 t-i s \\
5.07 c-i s \\
5.67 t-05\end{array}$ & $6.59[-43$ & $1.40 E-U 2$ \\
\hline 1200 & 79.1 & 10.90 & -.462 & 1.735 & $\begin{array}{l}85.0 \\
66.0 \\
90.0\end{array}$ & $\begin{array}{l}1.64 E-05 \\
1.24 E-J 5 \\
1.04 E-05\end{array}$ & $\begin{array}{l}4.91 t-U 5 \\
3.71 t-05 \\
3.27 t-05\end{array}$ & $4.09 t-03$ & $1 . \angle \triangle S E-02$ \\
\hline 1600 & $77 . .1$ & 11.66 &. .151 & 1.579 & $\begin{array}{l}06.0 \\
08.0 \\
90.0\end{array}$ & $\begin{array}{l}5.11 E-06 \\
5.11 E-1.16 \\
4.2 C E-J 6\end{array}$ & $\begin{array}{l}1.33 t-05 \\
1.53 t-0 s \\
1.26 E-05\end{array}$ & $2.34 t-03$ & $7.13 t-43$ \\
\hline$\angle 200$ & 75.2 & 11.37 & -.231 & 1.734 & $\begin{array}{l}84.0 \\
86.0 \\
64.0\end{array}$ & $\begin{array}{l}2.61 E-06 \\
2.37 E-16 \\
2.1 \forall t-30\end{array}$ & $\begin{array}{l}7.82 t-06 \\
7.11 t-06 \\
6.55 t-v 6\end{array}$ & $1.54 E-03$ & $4.01 E-03$ \\
\hline 3200 & $80.07^{\circ}$ & $9.30 ?$ & $-1.251 x$ & $3.669 x$ & $\begin{array}{l}64.0 \\
86.0 \\
61.0\end{array}$ & $\begin{array}{l}1.95 E-06 \\
1.57 E-06 \\
1.41 E-06\end{array}$ & $\begin{array}{l}5.05 t-06 \\
4.72 t-06 \\
4.24 t-06\end{array}$ & $1.2 \cup E-03 ?$ & $3.59 E-03 ?$ \\
\hline 5000 & $84.7 x$ & $2.33 x$ & $.834 x$ & $3.301 x$ & $\begin{array}{l}84.0 ? \\
82.0 ? \\
86.0 ?\end{array}$ & $\begin{array}{l}9.26 E-J 7 ? \\
6.11 E-07 ? \\
4.92 E-137 ?\end{array}$ & $\begin{array}{l}2.78 t-v 6 ? \\
1.83 L-v 6 ? \\
1.43 t-v 6 ?\end{array}$ & $3.92 E-C 4 X$ & . I J OE-OSX \\
\hline 7000 & B 3.2X. & $3.61 x$ & $.616 x$ & $4.999 x$ & $\begin{array}{l}82.0 ? \\
34.0 ? \\
66.0 ?\end{array}$ & $\begin{array}{l}4.53 E-U 7 ? \\
2.90 E-J 7 ? \\
1.71 E-J 7 ?\end{array}$ & $\begin{array}{l}1.30 t-i 6 ? \\
8.69 t-07 ? \\
5.14 t-07 ?\end{array}$ & $2.92 E-04 X$ & $8.77 E-04 x$ \\
\hline 12600 & $92.7 x$ & $1.71 x$ & $.710 x$ & $2.143 x$ & $\begin{array}{l}91.0 x \\
92.0 x \\
94.0 x\end{array}$ & $\begin{array}{l}7.67 E-0 \Delta x \\
5.61 E-0 t x \\
3.17 E-U \forall x\end{array}$ & $\begin{array}{l}2.30 t-07 x \\
1.68 E-07 x \\
9.505-08 x\end{array}$ & $4.80 E-05 x$ & $1.46 E-04 x$ \\
\hline
\end{tabular}

$?$ INUICATES UNCERTAINTIES IN CATA.

x indicates inValio or inlumpleté data. 


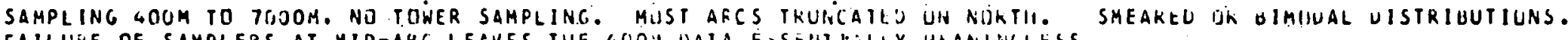

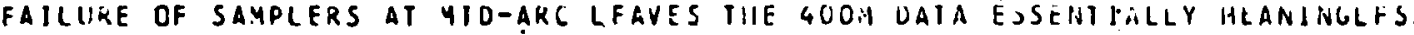

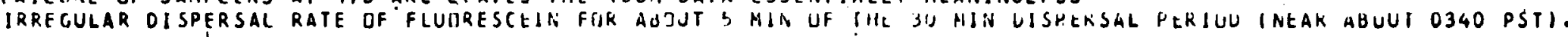

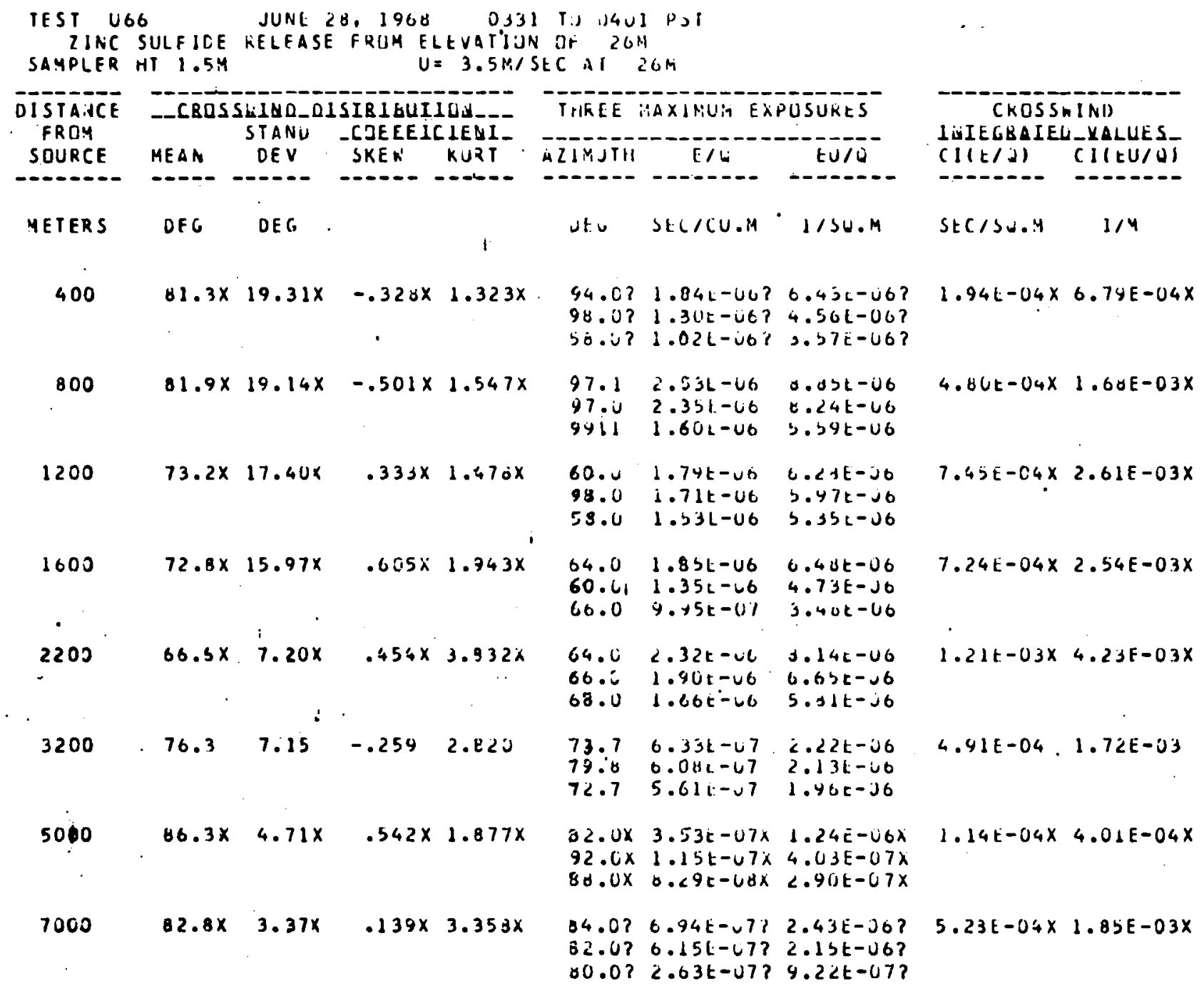

$?$ indicates uncertainties in vata.

$x$ inoicates invalio dé incugplete vaia. 


\begin{tabular}{|c|c|c|c|c|c|c|c|c|c|}
\hline $\begin{array}{l}\text { TEST US } \\
\text { FLUL } \\
\text { SH.SPLER }\end{array}$ & $\begin{array}{l}6 \\
\text { HESSCEIN } \\
\text { HT } 1.5 .4\end{array}$ & $\begin{array}{l}\text { JUPE } 28 \\
\text { RELEASE }\end{array}$ & $\begin{array}{r}\forall, 1968 \\
\text { FRO } H^{-1} E L \\
U=\end{array}$ & $\begin{array}{r}0331 \\
\text { FVATION } \\
1.4 M / 5\end{array}$ & $\begin{array}{l}1 \text { IU U4UI } \\
\text { LEF } \\
\text { SEL AT }\end{array}$ & 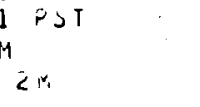 & & & \\
\hline $\begin{array}{l}\text { ISTAUCE } \\
\text { FELA }\end{array}$ & -CBOSS & $\begin{array}{l}0100-015 \\
\text { STAlU }\end{array}$ & $\begin{array}{l}\text { SIEIBUIL } \\
\text { - CLEEEIS }\end{array}$ & UISI- & THKEE & AXXIVUU EXP & PLSUKES & $\begin{array}{l}\text { incssw } \\
\text { dOILEAItL }\end{array}$ & $\begin{array}{l}\text { INU } \\
\text { A_YLLUES_ }\end{array}$ \\
\hline SI.LRCE & MEAN & DEV & SKEN & KURT & ALIMUIH & $c / 2$ & EU/l & $(d(c / \omega)$ & $(1) E U / Q)$ \\
\hline$-\cdots-1$ & $\cdots+-$ & - & $-\cdots---$ & -...- & ------ & $-\cdots-\cdots$ & $-\cdots-1 .-1$ & 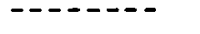 & ---- \\
\hline METERS & $D E C$ & DEC & & & UEG & $S \in C / C J \cdot H i$ & $1 / 50 . M$ & $S E C I S W . M$ & $i / A$ \\
\hline 400 & $77.5 x$ & $18.53 x$ & $-.236 x$ & $1.037 x$ & $\begin{array}{l}94.0 ? \\
\text { Yo.2? } \\
74.6 ?\end{array}$ & $\begin{array}{l}\text { S.UCt-US? } \\
2 . \forall U L-J 5 ? \\
2 . b y t-J 5 ?\end{array}$ & $\begin{array}{l}7.035-65 ? \\
4.30 L-65 ? \\
3.0<6-65 ?\end{array}$ & $5.74 t-63 x$ & $0.04 E-03 X$ \\
\hline$P C O$ & $64.3 x$ & $20.94 x$ & $.425 x$ & $1.510 x$ & $\begin{array}{l}49.0 ? \\
43.0 ? \\
40.0 ?\end{array}$ & $\begin{array}{l}1.1<E-U 5 ? \\
1.55 E-U 5 ? \\
1.36 t-1,5 ?\end{array}$ & $\begin{array}{l}\angle .4 U L-U>? \\
2.17 L-U b ? \\
1.94 L-U 5 ?\end{array}$ & $5.04 E-03 x$ & 7. $89 E-03 X$ \\
\hline 120,0 & $t 0.4 x$ & $12.20 x$ & $1.572 x$ & $4.57 d x$ & $\begin{array}{l}56.0 \\
54.0 \\
28.0\end{array}$ & $\begin{array}{l}1.14 t-U 5 \\
1.05 t-J 5 \\
1.07 t-U 5\end{array}$ & $\begin{array}{l}1 . b y c-l b \\
1 . b 3 t-U b \\
1.3 j E-6 b\end{array}$ & $3.4 y i-03 x$ & 4. EYE-U3X \\
\hline 1660 & $58.6 \times$ & $10.26 x$ & $2.221 x$ & $8.273 x$ & $\begin{array}{l}56 . c \\
22.0 \\
20.0\end{array}$ & $\begin{array}{l}0.42 E-.16 \\
1.14 L-10 \\
6.43 L-16\end{array}$ & 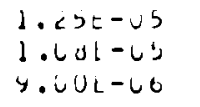 & $3.00 \mathrm{E}-\mathrm{U} 3 x$ & $4.2 \cup E-03 X$ \\
\hline $2<00$ & $58.6 x$ & $7.13 x$ & $.018 x$ & $3.482 x$ & $\begin{array}{l}56.0 \\
24.0 \\
50.0\end{array}$ & $\begin{array}{l}4.3 j t-j 6 \\
4.43 i-j 0 \\
4.34 t-j 6\end{array}$ & $\begin{array}{l}0.3 \cup t-v 6 \\
0.20 t-v 6 \\
0.10 t-06\end{array}$ & $2.916-03 x$ & $4.07 E-03 X$ \\
\hline 3200 & 66.0 & 7.23 & .393 & 2.947 & $\begin{array}{l}02.0 \\
04.0 \\
00.0\end{array}$ & $\begin{array}{l}9.23 t-J 7 \\
3.32 E-U 7 \\
8.52 t-j 7\end{array}$ & $\begin{array}{l}1.29 t-16 \\
1.19 t-196 \\
1.19 c-46\end{array}$ & $3.05 t-04$ & 1. ISE-OS \\
\hline 5000 & $34.6 x$ & $3.85 x$ & $1.310 x$ & $3.757 x$ & $\begin{array}{l}02.0 x \\
36.0 \hat{x} \\
90.0 x\end{array}$ & $\begin{array}{l}9.8 v t-u B i \\
2 . v 5 c-J 3 x \\
1.34 E-J u x\end{array}$ & $\begin{array}{l}1.37 t-07 x \\
2.07 t-j O x \\
2.16 E-U \Delta x\end{array}$ & $2.53 t-0 b x$ & $3.54 \mathrm{E}-0>\mathrm{X}$ \\
\hline 70100 & $81.0 x$ & $3.77 x$ & $\begin{array}{c}.587 x \\
.\end{array}$ & $3.300 x$ & $\begin{array}{l}02.0 ? \\
04.0 ? \\
76.0 ?\end{array}$ & $\begin{array}{l}1.07 L-J 7 ? \\
1.4 \pi E-J 7 ? \\
1.39 E-J 7 ?\end{array}$ & $\begin{array}{l}\text { C.34L-U7? } \\
\text { L.U3L-U7? } \\
1.44 L-U 7 ?\end{array}$ & $1.72 t-04 x$ & $2.411-04 x$ \\
\hline & & $?$ & $\begin{array}{l}\text { INUICATH } \\
\text { INUICAT }\end{array}$ & $\begin{array}{l}\text { ES UNCE } \\
\text { FS INVA }\end{array}$ & $\begin{array}{l}\text { RIAINIIE } \\
\text { ALIU.OK I }\end{array}$ & $\begin{array}{l}\text { ES IN UATA. } \\
\text { INCUMPLETL }\end{array}$ & LAIA. & & \\
\hline
\end{tabular}




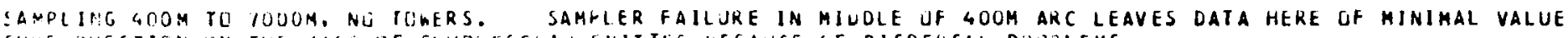

EVI:SUM AND 700ON ARCS TRUNCATEU UN VURTH

\begin{tabular}{|c|c|c|c|c|c|c|c|c|c|}
\hline $\begin{array}{l}\text { TEST UG } \\
\text { IINC } \\
\text { SAMPLFR }\end{array}$ & $\begin{array}{l}67 \\
\text { SULF IOE } \\
\text { HT } 1.5 .9\end{array}$ & $\begin{array}{l}\text { JJTY } 1: \\
\text { RELFASE }\end{array}$ & $\begin{array}{r}15,1968 \\
E F K U M E L\end{array}$ & $\begin{array}{l}J 313 \\
J=4.4 \mathrm{H} / S\end{array}$ & 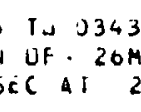 & $\begin{array}{l}3 \text { PSI } \\
M \\
26 M\end{array}$ & & & \\
\hline $\begin{array}{l}\text { DISTANCE } \\
\text { FRIM }\end{array}$ & \multicolumn{4}{|c|}{ 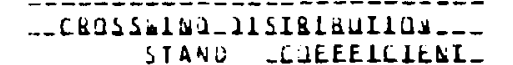 } & TISKEE & \multicolumn{2}{|c|}{ MAXJMUM EXPUSURES } & \multicolumn{2}{|c|}{$\begin{array}{l}\text { CKOSSWINU } \\
\text { INIEGBAIEO_YLUES }\end{array}$} \\
\hline SUUKCF & $V_{\text {IEAid }}$ & UEV & SKEW & KUR T & ALIRUIH & $E / 0$ & EU/O & (IItル) & CIIEU/O) \\
\hline - & $\cdots \cdots$ & $\cdots$ & $\ldots .$. & $\cdots$ & $\cdots$ & $-\cdots$ & $\rightarrow$ & & \\
\hline METIRS & ort & $O E Q$ & & & DÉG & $S E C / C U . M$ & 1/So.m & SEC/SU.M & $1 / M$ \\
\hline $40 \mathrm{~J}$ & A. $9.3 x$ & $6.40 x$ & $.304 x$ & $2.1 \times 4 x$ & $\begin{array}{l}36.0 x \\
94.0 x \\
02.0 x\end{array}$ & $\begin{array}{l}1 . \cot -05 x \\
y .56 t=00 x \\
d .32 t-60 x\end{array}$ & $\begin{array}{l}4.42 E-U 5 x \\
4.21 E-J 5 x \\
3.06 t-05 x\end{array}$ & $1.16 E-03 x$ & $5.1<E-03 x$ \\
\hline 400 & 07.0 & 7.46 & .101 & 1.543 & $\begin{array}{l}92.0 \\
79.0 \\
17.1\end{array}$ & $\begin{array}{l}1.77 t-u 5 \\
1.27 t-u 5 \\
0.03 t-v t\end{array}$ & $\begin{array}{l}7.00 L-U S \\
5.00 E-U S \\
3.09 E-U S\end{array}$ & $3 . C O E-03$ & $1.35 E-02$ \\
\hline 1260 & 65.4 & 7.44 & .308 & 1.739 & $\begin{array}{l}78.0 \\
80.0 \\
8<.0\end{array}$ & $\begin{array}{l}1.23 t-05 \\
1.20 t-05 \\
0.75 t-00\end{array}$ & $\begin{array}{l}3.41 t-05 \\
5.31 t-U 5 \\
3.84 t-65\end{array}$ & $3.45 E-03$ & $1.5<E-02$ \\
\hline 16000 & $39 . ?$ & 8. 54 & -.514 & 1.775 & $\begin{array}{l}3.0 \\
1.9 .1 \\
\times 4.0\end{array}$ & $\begin{array}{l}1.78 t-80 \\
3.94 t-50 \\
4.78 t-00\end{array}$ & $\begin{array}{l}3.42 t-J 5 \\
2.01 t-05 \\
2.1\} c-05\end{array}$ & $2.45 E-03$ & $1.03 E-02$ \\
\hline $22 n 0$ & B 3.11 & 8.117 & .517 & 1.775 & $\begin{array}{l}18.0 \\
10.0 \\
81 \% .0\end{array}$ & $\begin{array}{l}3.16 E-J 6 \\
3.03 t-v 6 \\
2.56 t-v 6\end{array}$ & $\begin{array}{l}1.34 t-v 5 \\
1.33 t-05 \\
1.13 t-05\end{array}$ & $1.71 \mathrm{E}-03$ & $7.5<E-U 3$ \\
\hline 1200 & 03.1 & 7.94 & .447 & 1.711 & $\begin{array}{l}15.7 \\
14.7 \\
70.6\end{array}$ & $\begin{array}{l}4.73 t-60 \\
4.01 t-66 \\
4.33 t-06\end{array}$ & $\begin{array}{l}2.08 t-05 \\
2.03 t-U 5 \\
1 . S 9 c-U b\end{array}$ & $4.07 E-01$ & $1.7 y t-0 z$ \\
\hline 5060 & $86 .<x$ & $5.10 x$ & $1.041 x$ & $2.455 x$ & $\begin{array}{l}06.0 x \\
06.0 x \\
34.0 x\end{array}$ & $\begin{array}{l}1.00 t-66 x \\
6.08 t-u 7 x \\
3.0 d t-07 x\end{array}$ & $\begin{array}{l}0.14 E-06 x \\
3.03 E-06 x \\
1.35 E-30 x\end{array}$ & $0.65 E-04 x$ & $2.93 E-03 X$ \\
\hline 7000 & t]. $.9 x$ & $5.74 x$ & $1.257 x$ & 3.0786 & $\begin{array}{l}78.0 x \\
02.0 x \\
76.0 x\end{array}$ & $\begin{array}{l}1.61 t-06 x \\
1.02 L-06 x \\
0.10 t-07 x\end{array}$ & $\begin{array}{l}3.07 t-06 x \\
4.47 E-06 x \\
3.57 t-06 x\end{array}$ & $1.22 E-03 x$ & $5.30 E-03 x$ \\
\hline & 7 & CATFS & En & NTIES & DATA. & & & & \\
\hline & $x \quad I N$ & KCATS & NVAL IU & INCE & $\vdots$ TE & ATA. & & & \\
\hline
\end{tabular}




\begin{tabular}{|c|c|c|c|c|c|c|c|c|c|}
\hline $\begin{array}{l}\text { TEST UG } \\
\text { FLUU } \\
\text { SAMPLER }\end{array}$ & $\begin{array}{l}7 \\
\text { RESCEIN } \\
\text { HT } 1.5 \mathrm{H}\end{array}$ & $\begin{array}{r}\text { JULY } 1 \\
\text { RELEASE }\end{array}$ & $\begin{array}{l}\text { 5, } 1968 \\
\text { FROK EL }\end{array}$ & $\begin{array}{r}0313 \\
\text { LEVATIDH } \\
=2.3 \mathrm{H} / \mathrm{S}\end{array}$ & $\begin{array}{l}\text { TO } 0343 \\
\text { UF } \\
\text { SEC AT }\end{array}$ & $\begin{array}{l}3 \text { PST } \\
2 \mathrm{~A} \\
2 \mathrm{C}\end{array}$ & & & \\
\hline DISTANCE & & $\begin{array}{l}\text { SHIND-DI } \\
\text { STAND }\end{array}$ & SIRIBUIJ & ION- & THREE M & MAXIMUM EXP & POSURES & CROSS & IND \\
\hline SEUKCE & MEAN & DEV & SKEN & KURT & AZIMUTH & E/O & $E \bar{O}$ & CIIE/O) & CIIEU/O) \\
\hline$-\cdots$ & ---- & -....- & $-\ldots-\infty$ & $-\ldots$ & ---..- & ....... & $\ldots-\ldots$ & $-\ldots-\infty-$ & $-\ldots-\ldots$ \\
\hline METERS & DEG & DEG & & & DEG & SEC/CU.M & $1 / \mathrm{SO} . \mathrm{M}$ & SEC/SQ.M & $1 / m$ \\
\hline 400 & $77.0 ?$ & $5.60 ?$ & $1.412 ?$ & $4.934 ?$ & $\begin{array}{l}74.0 ? \\
78.0 ? \\
82.0 ?\end{array}$ & $\begin{array}{l}1.14 E-05 ? \\
5.22 E-36 ? \\
2.39 E-06 ?\end{array}$ & $\begin{array}{l}2.63 E-05 ? \\
1.20 E-05 ? \\
5.50 E-06 ?\end{array}$ & $6.71 t-04 ?$ & $1.54 E-03 ?$ \\
\hline 800 & 78.5 & 5.54 & 1.720 & 6.080 & $\begin{array}{l}76.0 \\
79.0 \\
73.0\end{array}$ & $\begin{array}{l}5.21 \mathrm{E}-06 ? \\
3.45 \mathrm{E}-06 ? \\
3.14 \mathrm{E}-06 ?\end{array}$ & $\begin{array}{l}1.20 E-05 ? \\
7.94 E-06 ? \\
7.23 E-06 ?\end{array}$ & $0.36 E-04 ?$ & $1.46 E-03 ?$ \\
\hline 1200 & 77.6 & 4.67 & 2.127 & 7.996 & $\begin{array}{l}76.0 \\
78.0 \\
74.0\end{array}$ & $\begin{array}{l}2.27 E-06 ? \\
1.50 E-06 ? \\
1.37 E-06 ?\end{array}$ & $\begin{array}{l}5.21 E-06 ? \\
3.44 E-06 ? \\
3.15 E-06 ?\end{array}$ & $2.90 E-04 ?$ & $6.67 E-04 ?$ \\
\hline 1600 & 77.1 & 6.23 & 1.826 & 5.648 & $\begin{array}{l}74.0 \\
76.0 \\
72.0\end{array}$ & $\begin{array}{l}1.71 E-06 ? \\
9.11 E-07 ? \\
8.34 E-47 ?\end{array}$ & $\begin{array}{l}3.93 E-06 ? \\
2.09 E-06 ? \\
1.92 E-06 ?\end{array}$ & $2.86 E-04 ?$ & $6.63 E-04 ?$ \\
\hline 2260 & 76.2 & 4.97 & 2.303 & 9.050 & $\begin{array}{l}74.0 \\
76.0 \\
72.0\end{array}$ & $\begin{array}{l}7.33 E-J 7 ? \\
5.55 E-j 7 ? \\
4.00 E-J 7 ?\end{array}$ & $\begin{array}{l}1.69 E-06 ? \\
1.20 E-06 ? \\
1.06 E-66 ?\end{array}$ & $1.09 E-04 ?$ & $4.35 E-04 ?$ \\
\hline 2200 & 76.0 & 4.69 & 1.964 & 8.887 & $\begin{array}{l}72.7 \\
74.7 \\
75.7\end{array}$ & $\begin{array}{l}5.45 E-07 ? \\
5.45 E-U 7 ? \\
5.07 E-U 7 ?\end{array}$ & $\begin{array}{l}1.25 E-06 ? \\
1.25 E-06 ? \\
1.17 E-06 ?\end{array}$ & $2.64 E-042$ & $+6.08 E-04 ?$ \\
\hline 5000 & $85.0 x$ & $4.25 x$ & $1.610 x$ & $4.470 x$ & $\begin{array}{l}82.0 x \\
84.0 x \\
06.0 x\end{array}$ & $\begin{array}{l}7.69 E-08 x \\
4.52 E-08 x \\
1.97 E-08 x\end{array}$ & $\begin{array}{l}1.77 E-07 x \\
1.04 E-07 x \\
4.52 E-08 x\end{array}$ & $2.81 E-05 x$ & $6.46 E-05 x$ \\
\hline 7000 & $79.0 x$ & $3.03 x$ & $1.671 x$ & $8.145 x$ & $\begin{array}{l}76.0 x \\
78.0 x \\
80.0 x\end{array}$ & $\begin{array}{l}1.44 E-07 x \\
1.18 E-07 x \\
9.81 E-08 x\end{array}$ & $\begin{array}{l}3.32 E-07 x \\
2.70 E-07 x \\
2.26 E-07 x\end{array}$ & $1.19 E-04 x$ & $2.74 E-04 X$ \\
\hline & & ? & INDICAT & TES UNC & A INTIE & ES IN DATA, & , & & \\
\hline & & $x$ & INDICAT & TES & JO OR I & I NGORPLETE & OATA. & & \\
\hline
\end{tabular}


SAMPLINC FRLM 4004 TO 12930 M. NO TJWER SAMPLINS. BIMUDAL DISTRIBUTIONS.

ALL ARCS EMARACE CRCUSSHIND EXTENT JF TRACER EXCEPT 70JOM ARC. ALSU. ALTHOUGH $12800 M$ ARC DROPS TD BACKGROUND VALUES AT NORTH FXTREMITY TF SAMHLINC. THERE MAY HAVE BEEN MDKE TKACER NOKTH OF THE SAMPLERS.

\begin{tabular}{|c|c|c|c|c|c|c|c|c|c|}
\hline $\begin{array}{l}\text { TEST US } \\
\text { ZIHC } \\
\text { SAMPLER }\end{array}$ & $\begin{array}{l}\text { SH } \\
\text { SULFIDE } \\
\text { HT } 1.5 M\end{array}$ & $\begin{array}{l}\text { JULY I } \\
\text { RELFASE }\end{array}$ & $\begin{array}{r}16,1965 \\
\text { EROM E }\end{array}$ & $\begin{aligned} & 031 \\
\text { LEVATIS } & =4.7 \mathrm{MI}\end{aligned}$ & 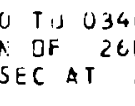 & $\begin{array}{l}0 \text { PST } \\
\text { MOM }\end{array}$ & & & \\
\hline $\begin{array}{l}\text { DISTANCF } \\
\text { FREM }\end{array}$ & \multicolumn{4}{|c|}{$\begin{array}{c}\text { CEOSSUINQDISIBIEUIIEA- } \\
\text { STAMU GJEEEICIEYI- }\end{array}$} & TríkE & \multicolumn{2}{|c|}{ MAXIMUM EXPESURES } & \multicolumn{2}{|c|}{$\begin{array}{l}\text { CROSSWIND } \\
\text { IAIEGBAIES_YALUES }\end{array}$} \\
\hline SLIUKCF & $A, E A$ A & DEV & $5 K E N$ & KURT & $A Z I M U T H$ & $E / C$ & EU/O & CIIE/W) & CIIEU/O) \\
\hline$---1-\cdots$ & ----- & 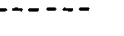 & $\cdots$ & ---1 & 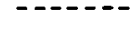 & 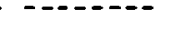 & $\cdots$ & $--0-0-0$ & -- \\
\hline :AETFRS & DEC & DE, & & & JE, & SEC/CU.M & $1 / 50 . \mathrm{M}$ & $S E C / S O . M$. & $1 / M$ \\
\hline 40 & 104.4 & 9.20 & -.440 & 1.534 & $\begin{array}{r}110.0 \\
114.0 \\
74.0\end{array}$ & $\begin{array}{l}1.09 E-U 5 \\
7.70 E-U t \\
7.06 E-i 6\end{array}$ & $\begin{array}{l}0.90 E-05 \\
3.62 E-65 \\
3.60 E-i 5\end{array}$ & $1.51 E-03$ & $7.08 E-0 \mathrm{~s}$ \\
\hline 300 & 10.7 & 6.42 & -.945 & 2.726 & $\begin{array}{l}11.5 .7 \\
11.88 \\
112.6\end{array}$ & $\begin{array}{l}4.32 E-05 \\
3.46 E-U 5 \\
3.12 E-65\end{array}$ & $\begin{array}{l}2.03 c-i 4 \\
1.02 c-64 \\
1.47 t-64\end{array}$ & $5.31 E-03$ & $2.50 E-02$ \\
\hline $12^{2 \cdot 0}$ & 125.7 & $7 \cdot 3$ & $-.73 v$ & 2.141 & 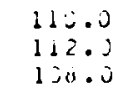 & $\begin{array}{l}2 .+4 t-45 \\
2.19 t-45 \\
2.41 E-45\end{array}$ & $\begin{array}{l}i .38 t-j 4 \\
1.03 E-j 4 \\
6.61 c-05\end{array}$ & $5.05 E-C 3$ & $2.36 E-02$ \\
\hline 1500 & 125.7 & 5.54 & -.053 & $2.3>2$ & $\begin{array}{l}110.4 \\
119.5 \\
111.4\end{array}$ & $\begin{array}{l}\therefore .19 E-U 5 \\
2.03 E-U 5 \\
i .09 E-l 5\end{array}$ & $\begin{array}{l}1.63 t-04 \\
7.55 E-05 \\
0.07 E-05\end{array}$ & $4.95 E-63$ & $2.3<E-0\rangle$ \\
\hline 2200 & $105.7 ?$ & $t .00 ?$ &.$- \nexists 1 \cup ?$ & $2.343 ?$ & $\begin{array}{l}110.0 ? \\
100.0 \\
112.0\end{array}$ & $\begin{array}{l}6.79 t-16 ? \\
4.45 t-v 6 \\
2.23 t-v 6\end{array}$ & $\begin{array}{l}3.1 Y E-C S ? \\
2 . J Y E-05 \\
1.35 E-35\end{array}$ & $1.64 t-03 ?$ & $8.66 E-03 ?$ \\
\hline$\therefore 2 i c$ & 105.5 & 6.25 & -.540 & 2.006 & $\begin{array}{l}110.7 \\
111.7 \\
109.7\end{array}$ & $\begin{array}{l}9.7 y E-v 6 \\
9.33 t-66 \\
6.28 E-66\end{array}$ & $\begin{array}{l}4.60 t-05 \\
4.39 c-05 \\
2.75 E-J 5\end{array}$ & $4.25 k-03$ & 2. CUE -02 \\
\hline 5900 & 106.0 & $6.1 y$ & -.575 & 2.768 & $\begin{array}{l}112.0 \\
1060 \\
110.0\end{array}$ & $\begin{array}{l}2.03 E-2 t \\
4.49 t-27 \\
0.54 t-67\end{array}$ & $\begin{array}{l}9.55 E-j 6 \\
4.64 E-66 \\
4.01 E-66\end{array}$ & $1.42 E-03$ & $6.65 E-03$ \\
\hline 7500 & $122.9 x$ & $7.2<x$ & $-.360 x$ & 3.5001 & $\begin{array}{l}1,6.0 \\
1.4 .0 \\
102.0\end{array}$ & $\begin{array}{l}9.15 E-37 \\
9.19 E--7 \\
0.35 E-07\end{array}$ & $\begin{array}{l}4.32 E-36 \\
+.32 E-36 \\
3.22 E-06\end{array}$ & $1.43 E-03 x$ & $0.71 E-03 x$ \\
\hline $12+c 0$ & $95.7 ?$ & $2.07 ?$ & $-.14 \equiv ?$ & $3.280 ?$ & $\begin{array}{l}+6.0 ? \\
75.0 ? \\
92.0 ?\end{array}$ & $\begin{array}{l}1.4 C E-U 0 ? \\
8.53 E-U 9 ? \\
7.36 E-U 9 ?\end{array}$ & $\begin{array}{l}0.50 E-08 ? \\
4.01 E-08 ? \\
3.32 E-0 d ?\end{array}$ & $1.06 E-05 ?$ & $4.97 E-05 ?$ \\
\hline
\end{tabular}

? InLicatFs UnCERtainties Iin data.

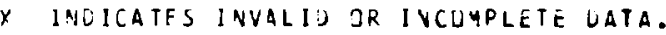




\begin{tabular}{|c|c|c|c|c|c|c|c|c|c|}
\hline \multirow{2}{*}{$\begin{array}{l}\text { DISTANCE } \\
\text { FKGAM } \\
\text { SHURCE }\end{array}$} & \multicolumn{2}{|c|}{$\begin{array}{c}\text { CBOSSWILLOEI } \\
\text { SIANU }\end{array}$} & \multicolumn{2}{|c|}{$\begin{array}{l}\text { ISIRIEUIION } \\
\text { COEEEICIEUI }\end{array}$} & THREE & MAXIMUN & EXPUSURES & \multicolumn{2}{|c|}{$\begin{array}{l}\text { CROSSWIMD } \\
\text { INIEGRAIEO_YALUES }\end{array}$} \\
\hline & MEAN & DE & SKEW & KURT & ALIMUIH & E/O & $E U / O$ & CIIE/U) & CIIEU/OI \\
\hline MEIERS & DEG & DEG & & & DEG & SEC/CU.H & $1 / 54 . n$ & SECISO.H & $1 / M$ \\
\hline 400 & 104.3 & 8.51 & -1.154 & 3.141 & $\begin{array}{l}110.0 \\
106.0 \\
114.0\end{array}$ & $\begin{array}{l}1.63 k-04 \\
1.30 E-04 \\
4.48 E-05\end{array}$ & $\begin{array}{l}3.91 t-64 \\
3.13 E-04 \\
1.08 t-64\end{array}$ & $1.32 E-02$ & $3.18 E-02$ \\
\hline a: & 105.1 & 7.19 & -1.294 & 3.556 & $\begin{array}{l}160.8 \\
106.8 \\
110.7\end{array}$ & $\begin{array}{l}7.42 E-U 5 \\
6.30 E-05 \\
4.50 E-05\end{array}$ & $\begin{array}{l}1.78 E-04 \\
1.51 E-64 \\
1.08 E-04\end{array}$ & $8.76 t-03$ & $2.10 \mathrm{E}-02 \mathrm{~L}$ \\
\hline 1260 & 102.3 & $H .20$ & -.570 & $1.76 \mathrm{~s}$ & $\begin{array}{l}108.0 \\
110.0 \\
106.0\end{array}$ & $\begin{array}{l}2.69 E-05 \\
2.23 E-05 \\
1.06 E-05\end{array}$ & $\begin{array}{l}6.45 E-U 5 \\
5.36 E-U 5 \\
2.54 E-05\end{array}$ & $4.96 E-03$ & $1.19 \varepsilon-02$ \\
\hline 1600 & 104.5 & 7.09 & -.899 & 2.413 & $\begin{array}{l}108.5 \\
109.5 \\
110.4\end{array}$ & $\begin{array}{l}2.01 E-05 \\
1.32 E-05 \\
1.25 E-05\end{array}$ & $\begin{array}{l}4.83 E-05 \\
3.17 E-65 \\
3.01 E-65\end{array}$ & $3.59 E-03$ & $0.63 E-03$ \\
\hline 2200 & 102.1 & 7.77 & $-.47 y$ & 1.642 & $\begin{array}{r}108.0 \\
110.0 \\
92.0\end{array}$ & $\begin{array}{l}7.67 E-06 \\
4.72 E-06 \\
2.77 E-06\end{array}$ & $\begin{array}{l}1.89 E-05 \\
1.13 E-05 \\
6.65 E-06\end{array}$ & $2.40 E-03$ & $5.77 E-03$ \\
\hline 3200 & 103.3 & 7.40 & -.570 & $1.90 \mathrm{~L}$ & $\begin{array}{l}109.7 \\
110.7 \\
108.8\end{array}$ & $\begin{array}{l}5.36 E-06 \\
4.05 E-06 \\
3.74 E-06\end{array}$ & $\begin{array}{l}1.29 E-U 5 \\
1.16 E-05 \\
8.98 E-06\end{array}$ & $2.54 E-03$ & $6.10 F-03$ \\
\hline 5004 & 104.2 & 7.18 & -.456 & 1.850 & $\begin{array}{r}110.0 \\
112.0 \\
94.0\end{array}$ & $\begin{array}{l}4.05 E-06 \\
3.66 E-06 \\
1.09 E-06\end{array}$ & $\begin{array}{l}9.72 E-06 \\
8.77 E-06 \\
4.54 E-66\end{array}$ & $3.78 E-03$ & $9.07 E-03$ \\
\hline 7000 & $10<.0 x$ & $0.16 x$ & $-.563 x$ & $2.656 x$ & $\begin{array}{l}110.0 \\
108.0 \\
100.0\end{array}$ & $\begin{array}{l}0.42 E-07 \\
5.95 E-07 \\
4.62 E-07\end{array}$ & $\begin{array}{l}2.02 E-06 \\
2.43 E-06 \\
1.11 E-06\end{array}$ & $1.32 E-03 x$ & $3.17 E-03 x$ \\
\hline 12600 & 95.77 & $1.80 ?$ & -.4727 & $2.863 ?$ & $\begin{array}{l}96.0 ? \\
94.0 ? \\
97.0 ?\end{array}$ & $\begin{array}{l}1.04 E-08 ? \\
7.73 E-09 ? \\
5.47 E-09 ?\end{array}$ & $\begin{array}{l}2.49 E-08 ? \\
1.86 E-08 ? \\
1.43 E-08 ?\end{array}$ & $8.74 E-06 ?$ & $2.10 E-05 ?$ \\
\hline & & $?$ & $\begin{array}{l}\text { INOICAT } \\
\text { INOICAT }\end{array}$ & $\begin{array}{l}\text { IES UNC } \\
\text { IES INV }\end{array}$ & $\begin{array}{l}\text { ERTAIATIE } \\
\text { ALIO UR I }\end{array}$ & $\begin{array}{l}\text { ES IN UATA. } \\
\text { IN CURPLETE }\end{array}$ & DATA. & & \\
\hline
\end{tabular}


SAMPLING FREM 400M TD $12800 M$. NO TOWER SAMPLING. ONLY ZINC SULFIDE TRACER RELEASED THIS DATE.

TRACEG APPEARS CTNTAINED WITHIN SAMPLERS TO A DISTANCE OF 5OOUM, DISTRIBUTION SKEWED STRONGLY TO SOUTH.

TRUACATED DISTRIBUTION JN 7ODOM ARC AND SIGNIFICANT DUST ON $1280 O \mathrm{M}$ ARC MAKE THESE ARC DATA QUESTIONABLE.

\begin{tabular}{|c|c|c|c|c|c|c|c|c|c|}
\hline \multirow{3}{*}{$\begin{array}{l}\text { TEST UG } \\
\text { ZINC } \\
\text { SAMPLER } \\
\text { DISTANCE } \\
\text { FROM } \\
\text { SCURCE }\end{array}$} & $\begin{array}{l}\text { 69 } \\
\text { SLLFIDE } \\
\text { HT } 1.5 M\end{array}$ & $\begin{array}{r}J U 1 Y \\
\text { RELEAS }\end{array}$ & JUIY 16,1968 & $\begin{array}{r}0118 \\
\text { LEVATION } \\
=\quad+.7 M / S\end{array}$ & $\begin{array}{lll}B & T D & 14 \\
N & O F & 26 \\
\text { SEC AT }\end{array}$ & $\begin{array}{l}8 \text { PST } \\
26 M \\
26\end{array}$ & & & \\
\hline & \multicolumn{4}{|c|}{-_CEISSLIND_QLSIBIBULIOA } & THREE & \multicolumn{2}{|c|}{ MAXIMUM EXPUSURES } & \multicolumn{2}{|c|}{$\begin{array}{c}\text { CROSSWIND } \\
\text { INIEGRAIED_YALUES_ }\end{array}$} \\
\hline & MEAN & DEV & SKEW & KURT & AZIMUTH & $E / 0$ & EU/O & $C I(E / O)$ & CII EU/O) \\
\hline & ( & - & & & & & 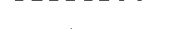 & & \\
\hline METERS & CEG & $D E C$ & & & DEG & SEC/CU.M & $1 / \mathrm{SO} . \mathrm{M}$ & SEC/SG.M & $1 / M$ \\
\hline $4 C 0$ & 97.4 & 7.25 & ل & 2.101 & $\begin{array}{l}74.0 \\
102.0 \\
100.0\end{array}$ & $\begin{array}{l}1.07 E-65 \\
1.42 E-15 \\
1.42 E-65\end{array}$ & $\begin{array}{l}8.18 E-35 \\
6.96 c-05 \\
6.94 E-05\end{array}$ & $2.35 E-03$ & $1.1 S E-02$ \\
\hline$\Leftrightarrow 20$ & 100.5 & 6.50 & $-.<4<$ & 2.143 & $\begin{array}{l}135.0 \\
103.0 \\
100.0\end{array}$ & $\begin{array}{l}1.08 c-u 5 \\
1.77 E-v 5 \\
1.73 t-v 5\end{array}$ & $\begin{array}{l}9.20 E-05 \\
0.68 E-j 5 \\
8.50 E-03\end{array}$ & $5.05 E-03$ & $2.40 E-02$ \\
\hline 1203 & 97.3 & 5.75 & .177 & 1.324 & $\begin{array}{l}34.0 \\
32.0 \\
76.0\end{array}$ & $\begin{array}{l}\text { 1. U5E- }-05 \\
0.98 t-v 0 \\
0.75 E-v 6\end{array}$ & $\begin{array}{l}5.16 E-0 S \\
4.40 E-05 \\
4.2 Y E-05\end{array}$ & $3.51 E-03$ & $1.72 E-02$ \\
\hline 1600 & 101.4 & $t .1:$ & .074 & 1.835 & $\begin{array}{r}y 6.6 \\
y 4.6 \\
109.5\end{array}$ & $\begin{array}{l}6.05 E-66 \\
0.74 t-06 \\
6.51 t-66\end{array}$ & $\begin{array}{l}3.36 E-U 5 \\
3.30 E-U 5 \\
3.19 E-05\end{array}$ & $3.17 \mathrm{E}-03$ & $1.55 E-02$ \\
\hline 226,0 & $103.6 ?$ & $5.31 ?$ & $-.204 ?$ & 1.9528 & $\begin{array}{l}78.0 \\
110.0 ? \\
168.0\end{array}$ & $\begin{array}{l}3.66 E-06 \\
3.79 E-06 ? \\
1.73 E-06\end{array}$ & $\begin{array}{l}1.89 E-U 5 \\
1.86 E-U 5 ? \\
8.46 E-U 6\end{array}$ & $1.24 E-03 ?$ & $6.00 E-03 ?$ \\
\hline $32 \div 0$ & $10 j .0$ & 5.35 & .213 & 1.788 & $\begin{array}{r}109.7 \\
100.8 \\
96.8\end{array}$ & $\begin{array}{l}3.75 E-\cup 6 \\
2.97 E-v 6 \\
2.85 E-U 6\end{array}$ & $\begin{array}{l}1.04 E-J 5 \\
1.46 E-05 \\
1.40 E-05\end{array}$ & $2.44 E-03$ & $1.2 J E-02$ \\
\hline 5000 & $1: 3.6$ & 3.82 & .363 & 2.242 & $\begin{array}{l}130.0 \\
102.0 \\
106.0\end{array}$ & $\begin{array}{l}1.17 E-U 6 \\
\forall .92 E-U 7 \\
7.87 E-U 7\end{array}$ & $\begin{array}{l}5.73 E-06 \\
4.37 E-06 \\
3.86 E-06\end{array}$ & $7.34 E-04$ & $3.60 E-03$ \\
\hline 7000 & $105.5 x$ & $5.53 x$ & $3.235 \times 1$ & $16.731 x$ & $\begin{array}{l}106.0 \\
104.0 \\
108.0\end{array}$ & $\begin{array}{l}1.24 E-U 6 \\
1.04 E-V 6 \\
1.01 E-66\end{array}$ & $\begin{array}{l}6.07 \bar{E}-06 \\
5.08 E-06 \\
4.95 E-06\end{array}$ & $1.32 E-03 X$ & $6.44 E-03 X^{-}$ \\
\hline 12000 & $126.3 ?$ & $10.16 ?$ & $-.321 ?$ & $3.759 ?$ & $\begin{array}{l}122.0 ? \\
125.0 ? \\
124.0 ?\end{array}$ & $\begin{array}{l}4.59 E-08 ? \\
3.15 E-C 8 ? \\
2.77 E-C B ?\end{array}$ & $\begin{array}{l}2.25 E-07 ? \\
1.54 E-07 ? \\
1.36 E-07 ?\end{array}$ & $\begin{array}{c}9.08 E-05 ? \\
.\end{array}$ & $4.45 E-04 ?$ \\
\hline & $\begin{array}{l}? \quad \text { INOI } \\
\times \quad \text { INCI }\end{array}$ & $\begin{array}{l}\text { ICATFS } \\
\text { ICATES }\end{array}$ & $\begin{array}{l}\text { NCERTAIN } \\
\text { NVALIO D }\end{array}$ & $\begin{array}{l}\text { VIES IN } \\
\text { OR INCOM }\end{array}$ & $\begin{array}{l}\text { OATA. } \\
\text { APLETE D }\end{array}$ & ATA. & & & \\
\hline
\end{tabular}




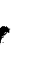

?

$r$ 
SAMPLING 4OOM TO $12800 \mathrm{M}$, NO TOMER SAMPLING. OISTRIBUTION IENOS TOMARO BIMODAL.

ALL ARCS EXCEPT 700OM EMBRACE CROSSHIND EXTEMT OF BOTH TRACERS. THE SKEWING OF 7OOOM DATA TO THE SOUTH IS MOT READILY EXPLAINABLE.

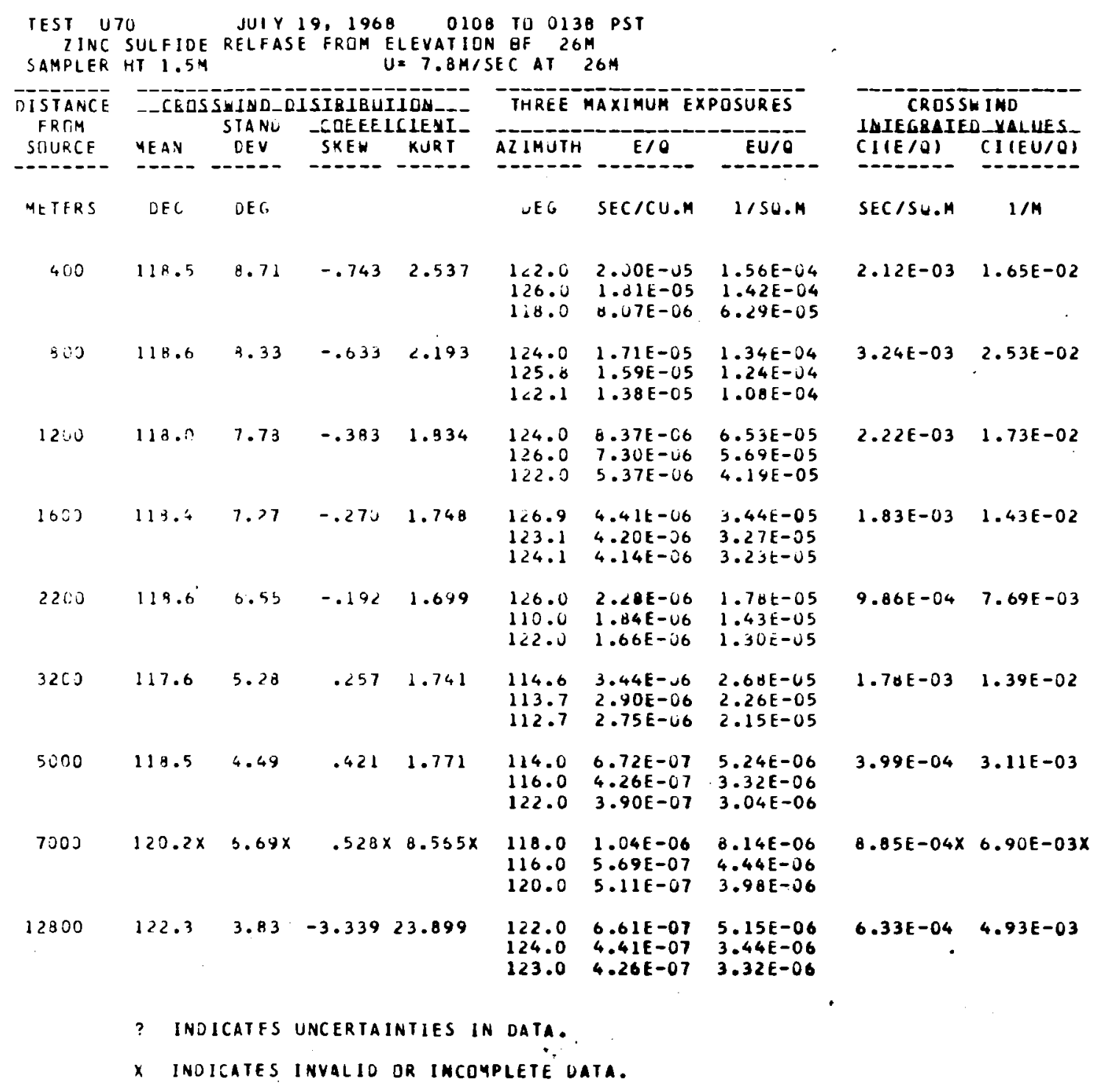




\begin{tabular}{|c|c|c|c|c|c|c|c|c|c|}
\hline \multicolumn{2}{|c|}{$\begin{array}{l}\text { TEST UTO } \\
\text { FLUDRESCE IN } \\
\text { SAMPLER HI } 1.5 \mathrm{M}\end{array}$} & \multirow{2}{*}{$\begin{array}{l}\text { JULY I } \\
\text { RELEASE } \\
\text { SEIMD }\end{array}$} & $\begin{array}{l}19,1968 \\
\text { E FRUA EL }\end{array}$ & $\begin{aligned} & 010 \\
\text { LEVATIUI } & \\
= & 4.8 \mathrm{HI}\end{aligned}$ & $\begin{array}{l}\text { OU } 0138 \\
\text { N OF } \\
\text { SEC AI }\end{array}$ & PST & & & \\
\hline $\begin{array}{l}\text { DISTANCE } \\
\text { FROM }\end{array}$ & & & $\begin{array}{l}\text { ISIRI RUI } \\
\text { COEEEI }\end{array}$ & CIENI- & THREE & MAXIMUA EX & PCSURES & IULEGRAIE & DIMO \\
\hline SUURCE & ME AN & OEV & SKEW & KURT & AZ IMUTH & $E / O$ & $E \cup / 0$ & $C 1(E / O)$ & CIIEU/OI \\
\hline METERS & $D E G$ & DEG & & & DEG & SEC/CU.M & $1 / 50 . \mathrm{H}$ & SEC/SO.N & $1 / H$ \\
\hline 400 & 119.6 & 8.23 & -1.003 & 3.208 & $\begin{array}{l}122.0 \\
1<6.0 \\
118.0\end{array}$ & $\begin{array}{l}6.45 E-05 \\
5.73 E-05 \\
2.44 E-05\end{array}$ & $\begin{array}{l}3.69 E-04 \\
2.75 E-14 \\
1.41 E-04\end{array}$ & $0.30 E-03$ & $3.05 E-02$ \\
\hline 800 & 119.4 & 8.36 & -.924 & 2.643 & $\begin{array}{l}124.0 \\
125.0 \\
122.1\end{array}$ & $\begin{array}{l}3.06 E-U 5 \\
2.06 E-U 5 \\
2.23 E-U 5\end{array}$ & $\begin{array}{l}1.47 E-04 \\
1.37 t-64 \\
1.07 t-04\end{array}$ & $4.99 t-03$ & $2.39 E-02$ \\
\hline 1200 & 119.9 & 7.55 & -.845 & 2.543 & $\begin{array}{l}126.0 \\
124.0 \\
122.0\end{array}$ & $\begin{array}{l}8.70 E-06 \\
6.91 E-16 \\
5.57 E-06\end{array}$ & $\begin{array}{l}4.17 E-05 \\
3.32 E-05 \\
2.6 \forall t-05\end{array}$ & $1.85 E-03$ & $0.07 E-03$ \\
\hline 1600 & 118.8 & $7.5 \mathrm{~J}$ & -.441 & 1.847 & $\begin{array}{l}126.0 \\
126.9 \\
124.1\end{array}$ & $\begin{array}{l}4.74 E-36 \\
4.36 E-06 \\
3.49 E-06\end{array}$ & $\begin{array}{l}2.27 E-05 \\
2.09 E-05 \\
1.67 E-05\end{array}$ & $1.43 E-03$ & $0.86 E-03$ \\
\hline 2200 & 118.6 & 6.54 & -.219 & 1.775 & $\begin{array}{l}126.0 \\
124.0 \\
1<2.0\end{array}$ & $\begin{array}{l}1.49 E-06 \\
1.39 E-06 \\
1.15 E-06\end{array}$ & $\begin{array}{l}7.17 E-06 \\
6.67 t-06 \\
5.50 t-06\end{array}$ & $7.37 E-04$ & $3.54 E-03$ \\
\hline 3200 & 118.3 & 5.47 & -.001 & 1.655 & $\begin{array}{l}114.6 \\
123.4 \\
113.7\end{array}$ & $\begin{array}{l}1.77 E-06 \\
1.52 E-06 \\
1.45 E-06\end{array}$ & $\begin{array}{l}8.50 E-06 \\
7.31 E-06 \\
6.96 E-06\end{array}$ & $1.11 E-03$ & $5.34 E-03$ \\
\hline 3000 & 119.0 & 4.43 & .172 & 1.761 & $\begin{array}{l}114.0 \\
122.0 \\
116.0\end{array}$ & $\begin{array}{l}7.45 E-07 \\
6.00 E-07 \\
5.52 E-07\end{array}$ & $\begin{array}{l}3.58 E-06 \\
2.80 E-06 \\
2.65 E-06\end{array}$ & $5.70 E-04$ & $2.73 E-03$ \\
\hline 7000 & $120.2 x$ & $6.21 x$ & $-.339 x$ & $0.545 x$ & $\begin{array}{l}118.0 \\
116.0 \\
124.0\end{array}$ & $\begin{array}{l}5.10 E-U 7 \\
4.16 E-U 7 \\
3.5 \Delta E-07\end{array}$ & $\begin{array}{l}2.45 E-06 \\
2.00 E-06 \\
1.72 E-06\end{array}$ & $6.31 E-04 x$ & $3.03 E-03 x$ \\
\hline 12000 & 122.0 & 3.03 & -1.136 & 7.921 & $\begin{array}{l}122.0 \\
124.0 \\
125.0\end{array}$ & $\begin{array}{l}\text { 5. } 00 E-07 \\
4.56 E-07 \\
3.48 E-07\end{array}$ & $\begin{array}{l}2.40 E-06 \\
2.19 E-06 \\
1.67 E-66\end{array}$ & $6.62 E-04$ & $3.18 E-03$ \\
\hline
\end{tabular}

$?$ INOICATES UNCERTAIMTIES IN DATA.

$x$ indicates invalio ar incumplete data. 
SAMPLING 400M TO $12800 \mathrm{M}$; NO TOWER SAMPLING. ZNS DISPERSED FOR 30 MIN: FLUDRESCEIN DISPERSED ONLY LAST 15 MIN. ARCS DUT IO 3200 M EMERACE CROSSHIND EXTENT OF TRACER, 50OOM, 700OM AND $12800 M$ ARCS TRUNCATED ON NORTH.

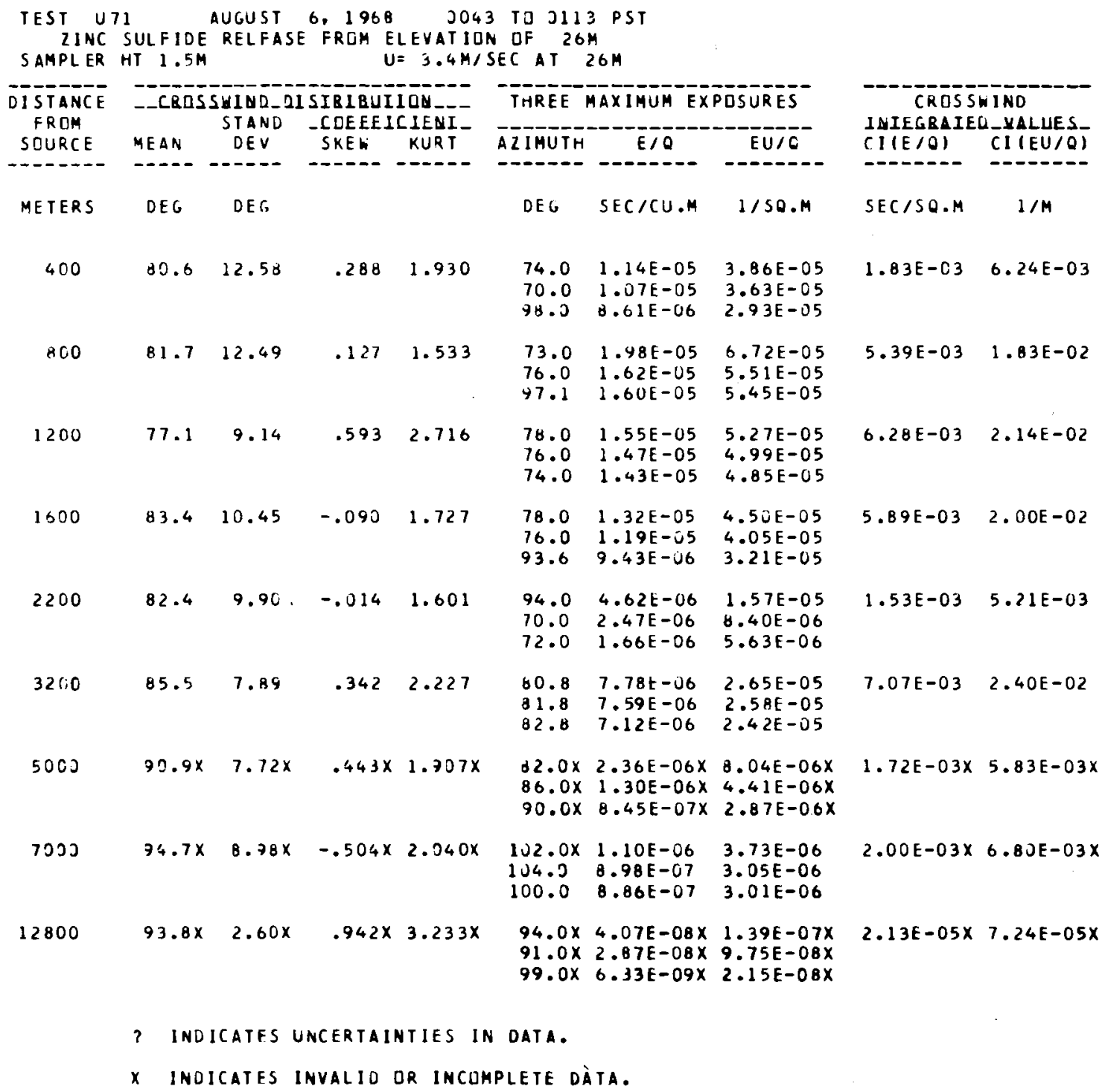


IEST U71 AUGUST 6, 1960 0058 TU 0113 PST

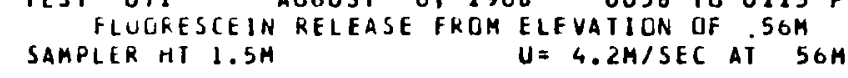

\begin{tabular}{|c|c|c|c|c|c|c|c|c|c|}
\hline \multirow{2}{*}{$\begin{array}{l}\text { UISTAIVCE } \\
\text { FRCA } \\
\text { SNUKCE }\end{array}$} & \multicolumn{4}{|c|}{-CRUSSUIU⿴囗DISIRIBUIION } & \multicolumn{3}{|c|}{ THREE MAXIMUA EXPOSURES } & \multicolumn{2}{|c|}{ CROSSWIND } \\
\hline & MEAN & DEV & SKEN & KUR I & AL IAUTH & E/O & EU/O & $C I(E / 0)$ & CIIEU/O) \\
\hline METERS & DEG & DEC & & & UEG & SEC/CU.M & I/SU.M & SEC/SO.N & $1 / H$ \\
\hline 400 & 78.7 & 8.66 & .736 & 5.242 & $\begin{array}{l}02.0 \\
06.0 \\
66.0\end{array}$ & $\begin{array}{l}2.21 E-06 \\
2.07 E-.06 \\
1.47 E-U 6\end{array}$ & $\begin{array}{l}9.26 t-06 \\
8.68 c-06 \\
6.17 E-06\end{array}$ & $2.70 t-04$ & $1.14 E-03$ \\
\hline$\triangle C_{U}$ & $\square 0.2$ & 8.00 & 1.065 & 4.771 & $\begin{array}{l}82.0 \\
79.0 \\
73.0\end{array}$ & $\begin{array}{l}0.02 E-06 \\
3.97 E-06 \\
4.06 E-J 6\end{array}$ & $\begin{array}{l}3.62 E-05 \\
2.51 E-05 \\
2.04 E-05\end{array}$ & $1.55 E-03$ & $6.51 E-03$ \\
\hline $12 \mathrm{co}$ & 01.0 & 7.34 & .630 & 3.763 & $\begin{array}{l}84.0 \\
00.0 \\
82.0\end{array}$ & $\begin{array}{l}7.89 E-06 \\
7.20 E-06 \\
0.54 E-J 6\end{array}$ & $\begin{array}{l}3.31 E-05 \\
3.06 E-05 \\
2.74 E-05\end{array}$ & $2.24 k-03$ & $9.42 t-03$ \\
\hline 1600 & 07.3 & 9.03 & .103 & 1.990 & $\begin{array}{l}26.0 \\
76.0 \\
04.0\end{array}$ & $\begin{array}{l}6.04 E-U 6 \\
5.00 E-V 6 \\
5.0 C E-U 6\end{array}$ & $\begin{array}{l}2.54 E-U S \\
2.43 E-U S \\
2.43 E-0 S\end{array}$ & $2.66 t-03$ & $1.12 \mathrm{E}-02$ \\
\hline 2200 & 86.3 & 7.59 & .432 & 3.370 & $\begin{array}{l}04.0 \\
92.0 \\
02.0\end{array}$ & $\begin{array}{l}2.31 E-06 \\
2.11 E-36 \\
1.53 E-06\end{array}$ & $\begin{array}{l}9.60 E-06 \\
8.8 \forall E-06 \\
6.63 E-06\end{array}$ & $1.13 E-03$ & $4.75 t-03$ \\
\hline 3200 & 09.3 & 7.49 & .750 & 3.121 & $\begin{array}{l}87.8 \\
82.8 \\
04.6\end{array}$ & $\begin{array}{l}5.00 E-06 \\
4.24 E-06 \\
4.24 E-06\end{array}$ & $\begin{array}{l}2.10 E-05 \\
1.78 E-05 \\
1.78 E-05\end{array}$ & $3.6 \forall E-03$ & $1.55 E-02$ \\
\hline 5000 & $91.6 x$ & $7.11 x$ & $.611 x$ & $2.961 x$ & $\begin{array}{l}90.0 ? \\
00.0 ? \\
06.0 ?\end{array}$ & $\begin{array}{l}1.11 E-06 ? \\
9.98 E-07 ? \\
0.25 E-07 ?\end{array}$ & $\begin{array}{l}4.66 E-06 ? \\
4.19 \mathrm{E}-06 ? \\
3.46 \mathrm{E}-06 ?\end{array}$ & $1.16 E-03 x$ & $4.87 E-03 x$ \\
\hline 700 & $92.0 x$ & $8.72 x$ & $.174 x$ & $2.680 x$ & $\begin{array}{l}90.0 \\
92.0 \\
88.0\end{array}$ & $\begin{array}{l}4.52 E-U 7 \\
3.49 E-U 7 \\
3.59 E-07\end{array}$ & $\begin{array}{l}1.90 E-06 \\
1.68-06 \\
1.510-00\end{array}$ & $1.04 E-03 x$ & $4.35 E-03 x$ \\
\hline 12000 & $93.6 x$ & $2.80 x$ & $1.641 x$ & $6.331 x$ & $\begin{array}{l}91.0 \\
94.0 x \\
92.0 x\end{array}$ & $\begin{array}{l}6.72 E-08 x \\
5.03 E-08 x \\
3.55 E-08 x\end{array}$ & $\begin{array}{l}2.82 E-07 x \\
2.11 E-07 x \\
1.49 E-07 x\end{array}$ & $5.50 E-0.5 x$ & $2.31 E-04 X$ \\
\hline
\end{tabular}

$?$ INOICATES UNCERTAINTIES IN DATA.

x indicates invalio or incohplete data. 
SAMPLING 400M TO 7000M: NO TOWER SAMPLING. ZNS UISPERSAL FOR 30 MIN: FLUURESCEIN DISPERSED UNLY LAST 20 MIN. DISTRIBUTIONS ARE BIMLOAL DR MULTIMODAL.

ALL ARCS EMBRACE CRUSSKINU EXTENT OF TRACER.

\begin{tabular}{|c|c|c|c|c|c|c|c|c|c|}
\hline \multirow{2}{*}{$\begin{array}{l}\text { DISTANCE } \\
\text { FROM } \\
\text { SOURCE }\end{array}$} & \multicolumn{2}{|c|}{$\begin{array}{c}--\operatorname{CBLSSLINO21} \\
\text { STANO }\end{array}$} & \multicolumn{2}{|c|}{$\begin{array}{l}\text { ISIRIEUIIOA-- } \\
\text {-COEEEICIEUI_- }\end{array}$} & \multirow{2}{*}{$\begin{array}{l}\text { THREE } \\
\text { AZIMUTH }\end{array}$} & \multicolumn{2}{|c|}{ MAXIMUM EXPQSURES } & \multicolumn{2}{|c|}{$\begin{array}{c}\text { CROSSWIND } \\
\text { INIEGRAIED_YALUES }\end{array}$} \\
\hline & ME AN & DEV & SKEW & KUR T & & $E / O$ & EU/O & CI(E/O) & CI $(E U / O)$ \\
\hline METERS & DEG & DES, & & & UEG & SEC/CU.M & $1 / 50 . M$ & SEC/SO.M & $1 / M$ \\
\hline 400 & 116.7 & 8.92 & -1.249 & 4.497 & $\begin{array}{l}122.0 \\
118.0 \\
126.0\end{array}$ & $\begin{array}{l}1.32 E-05 \\
1.02 E-05 \\
7.02 E-06\end{array}$ & $\begin{array}{l}5.79 E-05 \\
4.50 E-05 \\
3.09 E-05\end{array}$ & $1.36 E-03$ & $5.94 E-03$ \\
\hline 800 & 119.2 & 8.93 & -1.673 & 5.769 & $\begin{array}{l}125.8 \\
124.0 \\
122.1\end{array}$ & $\begin{array}{l}2.10 E-05 \\
1.01 E-05 \\
1.54 E-0.5\end{array}$ & $\begin{array}{l}4.26 E-05 \\
7.06 E-05 \\
6.76 E-0.5\end{array}$ & $\begin{array}{c}3.37 E-03 \\
\ddots\end{array}$ & $1.40 E-02$ \\
\hline 1200 & 117.6 & 10.71 & -1.310 & 4.025 & $\begin{array}{l}126.0 \\
124.0 \\
128.0\end{array}$ & $\begin{array}{l}7.60 E-06 \\
6.47 E-06 \\
3.11 E-06\end{array}$ & $\begin{array}{l}3.34 E-05 \\
2.84 E-05 \\
1.37 E-05\end{array}$ & $1.75 \mathrm{E}-03$ & 7.60 E-03 \\
\hline 1600 & 119.9 & 9.39 & -1.389 & 4.208 & $\begin{array}{l}126.9 \\
126.0 \\
127.8\end{array}$ & $\begin{array}{l}1.68 E-U 5 \\
1.46 E-U 5 \\
1.37 E-U 5\end{array}$ & $\begin{array}{l}7.41 E-05 \\
6.44 E-05 \\
6.05 E-05\end{array}$ & $3.74 E-03$ & $1.65 E-02$ \\
\hline 2200 & 122.6 & 7.16 & -1.684 & 5.330 & $\begin{array}{l}126.0 \\
128.0 \\
124.0\end{array}$ & $\begin{array}{l}6.49 E-06 \\
\forall .24 E-\cup 6 \\
2.74 E-J 6\end{array}$ & $\begin{array}{l}3.74 E-05 \\
3.62 E-65 \\
1.20 E-05\end{array}$ & $2.19 E-03$ & $9.64 E-03$ \\
\hline 3200 & 121.5 & 7.37 & -1.337 & 3.767 & $\begin{array}{l}127.3 \\
126.3 \\
125.4\end{array}$ & $\begin{array}{l}5.06 t-06 \\
5.27 t-06 \\
4.07 t-06\end{array}$ & $\begin{array}{l}2.5 \mathrm{E}-05 \\
2.32 \mathrm{E}-05 \\
1.79 \mathrm{E}-05\end{array}$ & $2.25 E-03$ & 9.9 UE -03 \\
\hline 5000 & 123.1 & 5.26 & -1.313 & 4.072 & $\begin{array}{l}1<6.0 \\
1<0.0 \\
120.0\end{array}$ & $\begin{array}{l}1.13 E-06 \\
6.77 E-07 \\
3.02 E-07\end{array}$ & $\begin{array}{l}4.99 E-06 \\
2.98 E-06 \\
1.65 E-06\end{array}$ & $6.04 E-04$ & $2.66 E-03$ \\
\hline 7000 & 125.0 & 5.02 & -.475 & 2.266 & $\begin{array}{l}126.0 \\
128.0 \\
130.0\end{array}$ & $\begin{array}{l}9.62 E-07 \\
5.41 E-U 7 \\
4.07 E-U 7\end{array}$ & $\begin{array}{l}3.79 E-06 \\
2.3 \forall E-06 \\
1.7 y E-06\end{array}$ & $8.77 E-04$ & $3.86 E-03$ \\
\hline & $? \quad 1$ & ATES & ERT & TIES & DATA. & & & & \\
\hline & $X$ IND & ICATES & 10 & 0 & ETE DA & ATA. & & & \\
\hline
\end{tabular}




\begin{tabular}{|c|c|c|c|c|c|c|c|c|c|}
\hline \multicolumn{2}{|c|}{$\begin{array}{l}\text { TEST UTI } \\
\text { FLUGKESCEIN } \\
\text { SAMPLER HI } 1.5 \mathrm{SH}\end{array}$} & $\begin{array}{l}\text { AUGUSI } \\
\text { RELEASE }\end{array}$ & \multicolumn{2}{|c|}{$\begin{array}{r}\text { 6, 1960 } \\
\text { E FROMELFVAIION } \\
U=4.2 \mathrm{~S} / \mathrm{S}\end{array}$} & $\begin{array}{ll}T u & 0113 \\
0 F & 56 \mathrm{M}\end{array}$ & $\begin{array}{l}3 \text { PSI } \\
4 \\
564\end{array}$ & & \\
\hline $\begin{array}{l}\text { USTAIVCE } \\
\text { FRDA }\end{array}$ & -CQUSS & $\begin{array}{l}\text { SHINDDD } \\
\text { SIAND }\end{array}$ & $\begin{array}{l}\text { LSIRIBUI } \\
\text {-CDEEEI }\end{array}$ & ICIENI- & THREE & MAXIHUM EXP & OSURES & $\begin{array}{l}\text { CROSS } \\
\text { IUIEGRAIE }\end{array}$ & $\begin{array}{l}\text { IND } \\
\text { D_KALUES }\end{array}$ \\
\hline SnURCE & MEAN & DEV & SKEW & KUR T & ALIMUTH & $E / O$ & EU/O & Cl(E/O) & CIIEU/OI \\
\hline 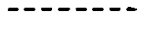 & $--\cdots-$ & ----- & ------ & ------ & $---\cdot--$ & - & 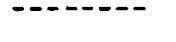 & - - & 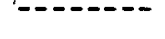 \\
\hline MEIERS & DEL & DEC & & & UEC & SEC/CU.H & $1 / 50 . M$ & SECISO.H & $1 / M$ \\
\hline 400 & 78.7 & 0.66 & .736 & 5.242 & $\begin{array}{l}02.0 \\
06.0 \\
66.0\end{array}$ & $\begin{array}{l}2.21 E-06 \\
2.07 E-36 \\
1.47 E-06\end{array}$ & $\begin{array}{l}9.26 t-06 \\
8.68 t-06 \\
6.17 E-06\end{array}$ & $2.70 t-04$ & $1.14 E-03$ \\
\hline$\Delta C U$ & 00.2 & 8.06 & 1.065 & 4.771 & $\begin{array}{l}82.0 \\
79.0 \\
73.0\end{array}$ & $\begin{array}{l}0.62 E-06 \\
5.97 E-06 \\
4.06 E-j 6\end{array}$ & $\begin{array}{l}3.62 E-05 \\
2.51 E-05 \\
2.04 i-05\end{array}$ & $1.55 t-03$ & $6.51 E-03$ \\
\hline 1200 & 01.0 & 7.34 & .630 & 3.763 & $\begin{array}{l}84.0 \\
80.0 \\
02.0\end{array}$ & $\begin{array}{l}7.89 E-00 \\
7.28 E-00 \\
6.54 E-06\end{array}$ & $\begin{array}{l}3.31 E-05 \\
3.06 t-05 \\
2.74 E-05\end{array}$ & $2.24 t-03$ & $9.42 E-03$ \\
\hline 1000 & 67.3 & 9.03 & .103 & 1.990 & $\begin{array}{l}06.0 \\
78.0 \\
04.0\end{array}$ & $\begin{array}{l}6.04 E-U 6 \\
5.00 E-U 6 \\
5.06 E-U 6\end{array}$ & $\begin{array}{l}2.54 t-05 \\
2.43 E-05 \\
2.43 t-05\end{array}$ & $2.66 t-03$ & $1.12 \mathrm{E}-02$ \\
\hline 2200 & 86.3 & 7.59 & .432 & 3.070 & $\begin{array}{l}44.0 \\
90.0 \\
d 2.0\end{array}$ & $\begin{array}{l}2.31 E-06 \\
2.11 E-1,6 \\
1.5 \partial E-06\end{array}$ & $\begin{array}{l}9.68 E-U 6 \\
8.88 t-06 \\
6.63 E-46\end{array}$ & $1.13 E-03$ & $4.75 t-03$ \\
\hline 3260 & 89.3 & 7.49 & .756 & 3.121 & $\begin{array}{l}d 7.8 \\
82.8 \\
84.8\end{array}$ & $\begin{array}{l}5.00 E-16 \\
4.24 E-06 \\
4.24 E-06\end{array}$ & $\begin{array}{l}2.10 E-05 \\
1.70 E-05 \\
1.70 E-05\end{array}$ & $3.60 t-03$ & $1.55 E-02$ \\
\hline 5000 & $91.6 x$ & $7.11 x$ & $.011 x$ & $2.961 x$ & $\begin{array}{l}90.0 ? \\
80.0 ? \\
06.0 ?\end{array}$ & $\begin{array}{l}1.11 E-06 ? \\
9.98 E-07 ? \\
0.25 E-07 ?\end{array}$ & $\begin{array}{l}4.66 \mathrm{E}-06 ? \\
4.19 \mathrm{E}-06 ? \\
3.46 \mathrm{E}-06 ?\end{array}$ & $1.16 E-03 x$ & $4.87 E-03 x$ \\
\hline 7000 & $92.8 x$ & $8.72 x$ & $.174 x$ & $2.680 x$ & $\begin{array}{l}90.0 \\
92.0 \\
98.0\end{array}$ & $\begin{array}{l}4.52 E-U 7 \\
3.49 E-07 \\
3.59 E-07\end{array}$ & $\begin{array}{l}1.90 E-06 \\
1.68 F-06 \\
1.510-06\end{array}$ & $1.04 E-03 x$ & $4.35 E-03 x$ \\
\hline 12000 & $93.6 x$ & $2.80 x$ & $1.641 x$ & $6.331 x$ & $\begin{array}{l}91.0 \\
94.0 x \\
92.0 x\end{array}$ & $\begin{array}{l}6.72 E-08 x \\
5.03 E-38 x \\
3.55 E-08 x\end{array}$ & $\begin{array}{l}2.82 E-07 x \\
2.11 E-07 x \\
1.49 E-07 x\end{array}$ & $5.50 E-05 x$ & $2.31 E-04 X$ \\
\hline
\end{tabular}

$?$ INDICATES UNCERTAINTIES IN DATA.

X Inoicates invalio dR incomplete data. 
SAMPLING 400M TO 7000M: NO TOHER SAMPLING. ZNS UISPERSAL FOR 30 MIN: FLUORESCEIN DISPERSED UNLY LAST 20 MIN. CISTRIBUTIONS ARE BIMUDAL TR MULTIMDOAL.

ALL AKCS EMBKACE CROSSKINU EXTENT OF TRACER.

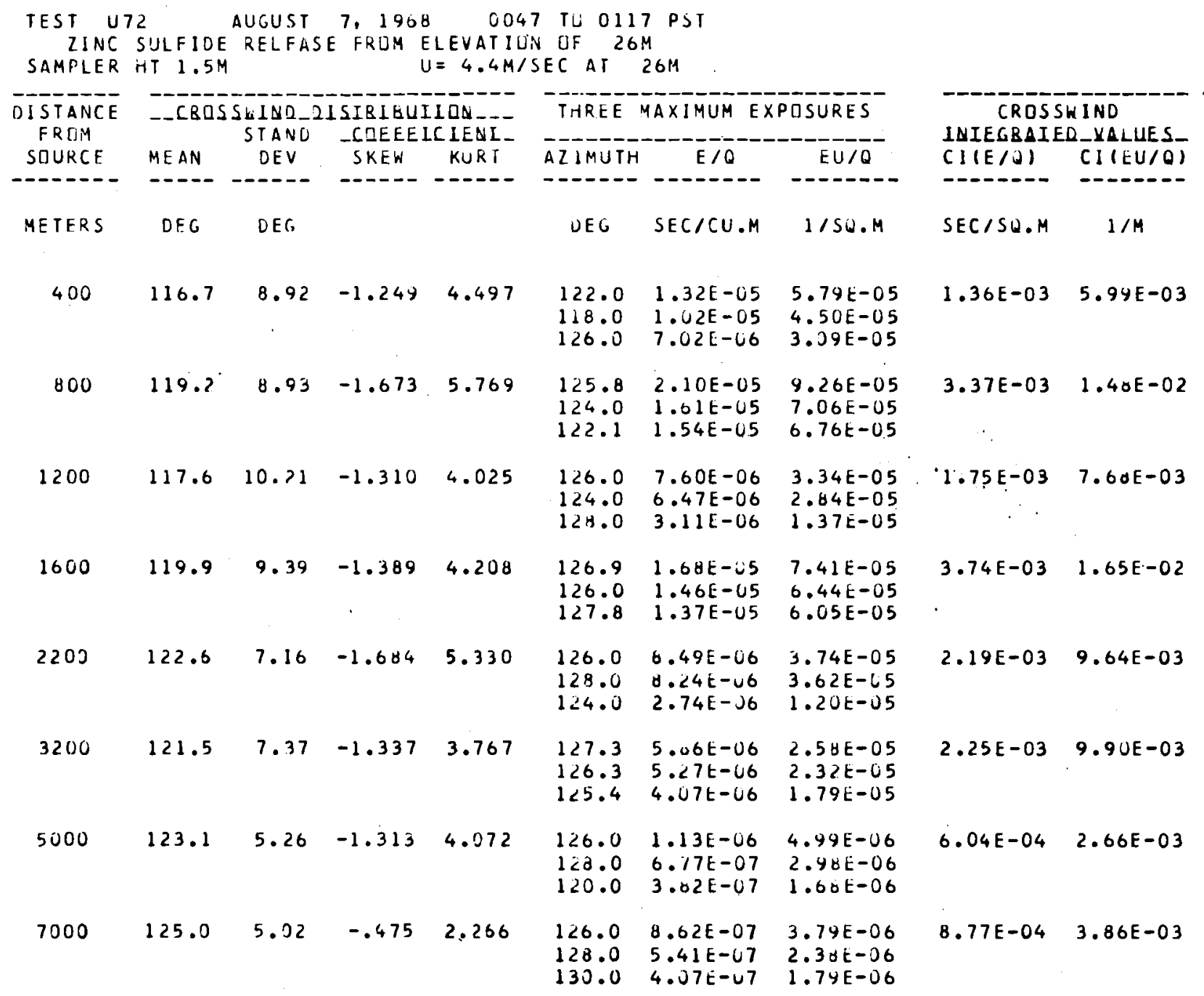

$?$ INDICATES UNCERTAINTIES IN DATA.

$x$ indicates invalio dor incumplete data. 


\begin{tabular}{|c|c|c|c|c|c|c|c|c|c|}
\hline $\begin{array}{l}\text { TEST U7 } \\
\text { PZUD } \\
\text { SAMPLER }\end{array}$ & $\begin{array}{l}2 \\
\text { RESCE IN } \\
\text { HT } 1.5 \mathrm{M}\end{array}$ & $\begin{array}{l}\text { AUGUST } \\
\text { RELEASE }\end{array}$ & $\begin{array}{l}7.1968 \\
\text { FROM }\end{array}$ & $\begin{array}{l}\quad 00 \\
\text { LFVATI } \\
=6.0 \mathrm{H}\end{array}$ & $\begin{array}{l}\text { TO } 0117 \\
\text { DF } 561 \\
\text { EC AT } 5\end{array}$ & $\begin{array}{l}7 \text { PST } \\
564\end{array}$ & & & \\
\hline \multirow{2}{*}{$\begin{array}{l}\text { DISTANCE } \\
\text { FROM. } \\
\text { SLUKCE }\end{array}$} & \multicolumn{4}{|c|}{-CROSSHIND_DISIR DUIILN- } & THREE & \multicolumn{2}{|c|}{ MAXIMUM EXPOSURES } & \multicolumn{2}{|c|}{$\begin{array}{l}\text { CROSSHINO } \\
\text { IHIECRAIEO }\end{array}$} \\
\hline & MEAN & DEV & SKEW & KURT & AL IMUTH & $E / 0$ & $E \cup / O$ & $C I(E / O)$ & CIIEU/O) \\
\hline & & & & & & & & & \\
\hline HETERS & DEG & DEC & & & DEG & SEC/CU.H & $1 / 50 \cdot 14$ & SEC/SO.M & $1 / A$ \\
\hline 400 & 113.9 & 2.36 & -5.994 & 67.399 & $\begin{array}{c}114.0 \\
122.0 \\
90.0\end{array}$ & $\begin{array}{l}1.36 E-06 \\
1.91 E-U 8 \\
7.66 E-09\end{array}$ & $\begin{array}{l}8.15 E-06 \\
1.15 E-07 \\
4.60 E-08\end{array}$ & $3.93 E-05$ & $2.36 E-04$ \\
\hline oćo & 121.7 & 4.72 & -.859 & 5.222 & $\begin{array}{l}122 \cdot 1 \\
124 \cdot 0 \\
118.3\end{array}$ & $\begin{array}{l}4.92 E-J 6 \\
4.40 E-J 6 \\
2.20 E-06\end{array}$ & $\begin{array}{l}2.95 E-05 \\
2.64 E-05 \\
1.32 E-05\end{array}$ & $6.21 E-04$ & $3.73 E-03$ \\
\hline 1200 & 122.1 & 6.26 & -1.721 & 7.896 & $\begin{array}{l}124.0 \\
128.0 \\
126.0\end{array}$ & $\begin{array}{l}2.23 E-06 \\
2.06 E-j 6 \\
1.74 E-06\end{array}$ & $\begin{array}{l}1.34 E-05 \\
1.23 E-05 \\
1.16 E-65\end{array}$ & $5.87 E-04$ & $3.52 E-03$ \\
\hline 1600 & 122.2 & 7.49 & -1.544 & 5.098 & $\begin{array}{l}128.8 \\
122.1 \\
121.2\end{array}$ & $\begin{array}{l}4.97 E-46 \\
3.91 t-06 \\
3.09 t-26\end{array}$ & $\begin{array}{l}2.90 t-05 \\
2.30 t-05 \\
2.151-05\end{array}$ & $1.24 E-03$ & $7.43 E-03$ \\
\hline 2200 & 123.8 & 5.08 & -1.867 & 7.206 & $\begin{array}{l}126.0 \\
128.0 \\
122.0\end{array}$ & $\begin{array}{l}1.01 t-06 \\
1.67 E-06 \\
1.41 E-06\end{array}$ & $\begin{array}{l}1.06 t-U S \\
1.0 U t-05 \\
8.46 t-06\end{array}$ & $6.34 L-04$ & $3.95 t-03$ \\
\hline 3200 & 125.0 & 4.48 & -2.044 & 7.520 & $\begin{array}{l}127.3 \\
126.3 \\
128.3\end{array}$ & $\begin{array}{l}4.69 E-06 \\
3.64 E-06 \\
2.71 E-36\end{array}$ & $\begin{array}{l}2.94 E-05 \\
2.19 E-05 \\
1.62 E-05\end{array}$ & $1.28 E-03$ & 7. TUE-03 \\
\hline 5000 & 126.2 & 4.11 & -1.854 & 7.014 & $\begin{array}{l}128.0 \\
130.0 \\
126.0\end{array}$ & $\begin{array}{l}8.85 E-U 7 \\
8.24 E-J 7 \\
7.63 E-07\end{array}$ & $\begin{array}{l}5.31 E-06 \\
4.94 E-06 \\
4.58 E-06\end{array}$ & $5.68 E-04$ & $3.41 E-03$ \\
\hline 7000 & 127.5 & 3.64 & -1.080 & 4.641 & $\begin{array}{l}126.0 \\
128.0 \\
130.0\end{array}$ & $\begin{array}{l}1.01 E-06 \\
5.58 E-07 \\
5.03 E-07\end{array}$ & $\begin{array}{l}6.04 E-06 \\
3.35 E-06 \\
3.02 E-06\end{array}$ & $7.26 E-04$ & $4.36 E-03$ \\
\hline
\end{tabular}

7 Indicates uncertainties in data.

$x$ inoicates invalid or incumplete data. 
SAMPLING 400M TO $12800 M$; NO TOWER SAMPLING. ALL ARCS EMBRACE CROSSWIND EXTENT DF TRACER DISTRIBUTIDN.

ZNS DISPERSAL FOR 30 CONTINUDUS MIN. FLUORESCEIN UN FIRST 5 MIN, DFF 10 MIN. THEN ON. IS MIN. IFLUDRESCEIN DN AT O140PST. OFF AT O145PST. DN AT OIS5PST AND DFF AGAIN AT OZIOPST.) FLULRESCEIN DISPERSAL DEVICE PRDBLEMS.

\begin{tabular}{|c|c|c|c|c|c|c|c|c|c|}
\hline $\begin{array}{l}\text { TEST UT } \\
\text { ZINC } \\
\text { SAMPLER }\end{array}$ & $\begin{array}{l}73 \\
\text { SULFIDE } \\
\text { HT } 1.5 \mathrm{M}\end{array}$ & $\begin{array}{l}\text { AUGUST } \\
\text { RELFASE }\end{array}$ & $\begin{array}{r}8,1968 \\
\text { FROM E }\end{array}$ & $\begin{array}{r}014 \\
\text { LEVATIO } \\
=5.5 \mathrm{M} /\end{array}$ & $\begin{array}{l}\text { Tu } 0216 \\
\text { DF } 261 \\
\text { SEC A T }\end{array}$ & $\begin{array}{l}O P S T \\
M \\
26 M\end{array}$ & & & \\
\hline \multirow{2}{*}{$\begin{array}{l}\text { OISTANCE } \\
\text { FRQM } \\
\text { SOURCE }\end{array}$} & \multicolumn{4}{|c|}{$\begin{array}{l}\text {-CROSSUIND_DISIRIBUILUA- } \\
\text { STAND - COEEEICIENI- }\end{array}$} & THREE & \multicolumn{2}{|c|}{ MAXIMUM EXPUSURES } & \multicolumn{2}{|c|}{$\begin{array}{l}\text { CROSSWIND } \\
\text { INIEGBAIEO_YALUES_- }\end{array}$} \\
\hline & MEAN & DEV & SKEW & KURT & AZIMUTH & $E / Q$ & EU/U & $C I(E / O)$ & $C I(E U / O)$ \\
\hline------ & ---- & ---- & $-\cdots--$ & ---- & ----- & $--\cdots--$ & $---\cdots$ & ------- & $----\infty$ \\
\hline METERS & DEG & DEG & & & DEG & $S E C / C U \cdot M$ & $1 / \mathrm{SO} . \mathrm{M}$ & SEC/SO.M & $1 / M$ \\
\hline 400 & 122.2 & 6.15 & .117 & 2.069 & $\begin{array}{l}118.0 \\
126.0 \\
122.0\end{array}$ & $\begin{array}{l}1.25 E-05 \\
1.16 E-U 5 \\
1.06 E-05\end{array}$ & $\begin{array}{l}6.88 E-05 \\
6.36 E-05 \\
5.81 E-05\end{array}$ & $1.60 E-03$ & $8.82 E-03$ \\
\hline 800 & 122.3 & 5.06 & -.149 & 2.437 & $\begin{array}{l}122.1 \\
124.0 \\
125.8\end{array}$ & $\begin{array}{l}1.57 E-05 \\
1.44 E-U 5 \\
1.42 E-65\end{array}$ & $\begin{array}{l}8.65 E-05 \\
7.94 E-05 \\
7.83 E-05\end{array}$ & $2.94 E-03$ & $1.64 E-02$ \\
\hline 1200 & 121.6 & 3.94 & -.049 & 2.577 & $\begin{array}{l}120.0 \\
126.0 \\
118.0\end{array}$ & $\begin{array}{l}4.08 E-06 \\
3.06 E-06 \\
3.55 E-06\end{array}$ & $\begin{array}{l}2.24 E-05 \\
2.12 E-05 \\
1.95 E-05\end{array}$ & $9.01 E-04$ & $4.95 E-03$ \\
\hline 1600 & 122.1 & 3.56 & -.194 & 2.523 & $\begin{array}{l}125.0 \\
121.2 \\
122.1\end{array}$ & $\begin{array}{l}1.41 E-05 \\
1.00 t-U 5 \\
1.00 E-05\end{array}$ & $\begin{array}{l}7.73 E-05 \\
5.96 E-05 \\
5.51 E-05\end{array}$ & $2.90 E-03$ & $1.60 E-02$ \\
\hline 2200 & 121.72 & 2.917 & .2027 & 3.0977 & $\begin{array}{l}122.0 \\
120.07 \\
126.0\end{array}$ & $\begin{array}{l}2.34 E-U 6 \\
2.31 t-06 ? \\
9.52 E-U 7\end{array}$ & $\begin{array}{l}1.29 E-05 \\
1.27 E-057 \\
5.24 E-06\end{array}$ & $6.13 E-04 ?$ & $3.37 E-03 ?$ \\
\hline 3200 & 121.4 & 2.50 & -.097 & 2.876 & $\begin{array}{l}120.5 \\
121.5 \\
122.4\end{array}$ & $\begin{array}{l}4.46 E-U 6 \\
4 . \cup 8 E-16 \\
3.45 E-U 6\end{array}$ & $\begin{array}{l}2.45 E-05 \\
2.24 E-05 \\
2.17 E-65\end{array}$ & $1.56 E-03$ & $8.58 E-03$ \\
\hline 5000 & 121.5 & 2.04 & -.169 & 3.822 & $\begin{array}{l}122.0 \\
120.0 \\
118.0\end{array}$ & $\begin{array}{l}8.78 E-07 \\
1.99 E-07 \\
1.94 E-07\end{array}$ & $\begin{array}{l}4.03 E-06 \\
1.09 E-06 \\
1.07 E-06\end{array}$ & $2.54 E-04$ & $1.40 E-03$ \\
\hline 7000 & 121.1 & 1.47 & .161 & 4.064 & $\begin{array}{l}122.0 \\
120.0 \\
124.0\end{array}$ & $\begin{array}{l}1.66 E-06 \\
1.59 E-06 \\
1.99 E-07\end{array}$ & $\begin{array}{l}9.13 E-06 \\
8.76 E-06 \\
1.09 E-06\end{array}$ & $9.12 E-04$ & $5.02 E-03$ \\
\hline 12800 & 118.7 & 2.94 & .748 & 3.713 & $\begin{array}{l}1: 7.0 \\
118.0 \\
116.0\end{array}$ & $\begin{array}{l}3.70 E-07 \\
1.09 E-07 \\
7.59 E-08\end{array}$ & $\begin{array}{l}2.03 E-06 \\
5.93 E-07 \\
4.17 E-07\end{array}$ & $2.24 E-04$ & $1.23 E-03$ \\
\hline
\end{tabular}

? INDICATFS UNCERTAINTIES IN DATA.

$x$ INDICATES INVALID DR INCOMPLE. JATA. 


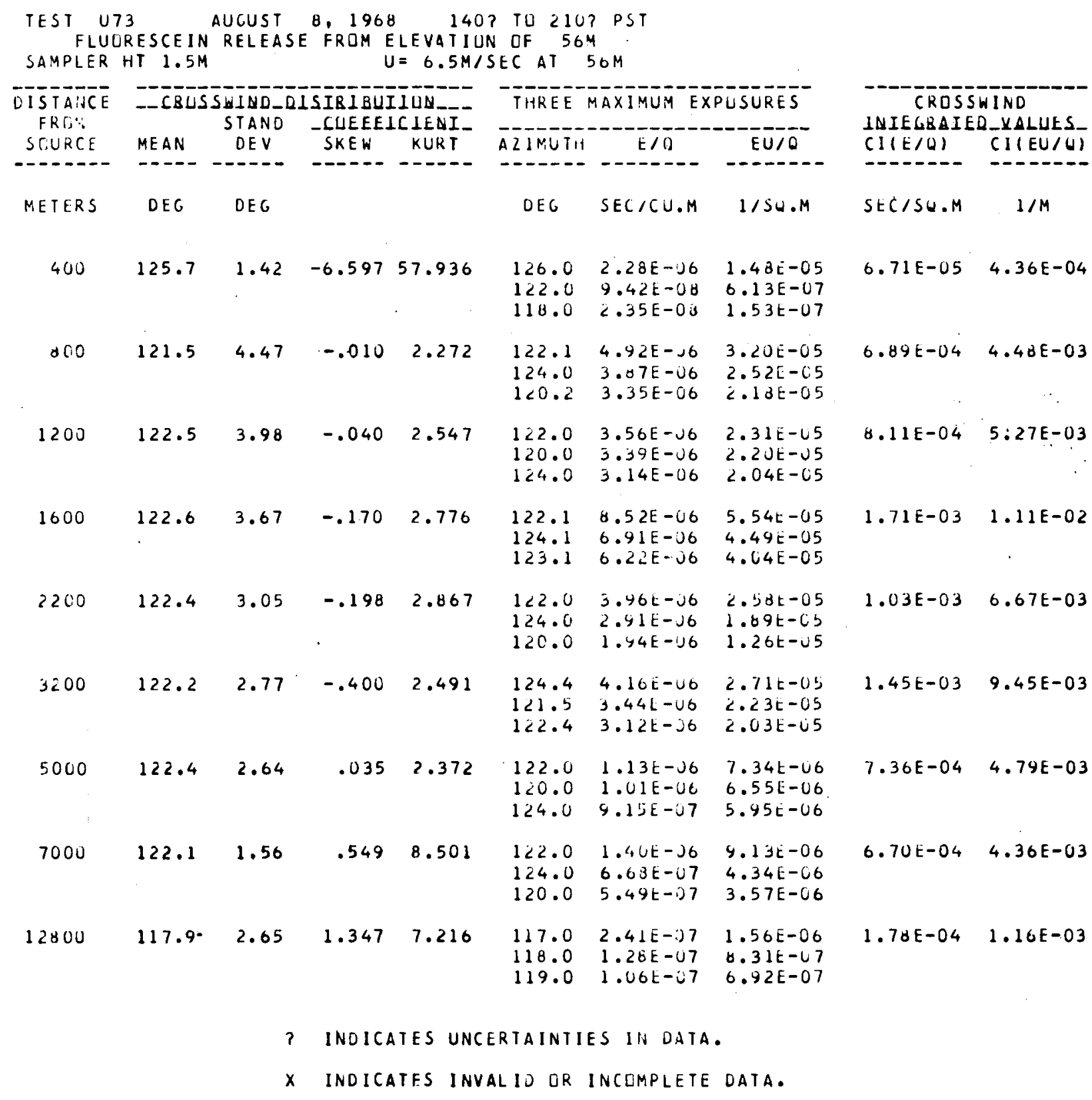


SAHPLING 4 OOM TO 7OOOM. NO TUKER SAMPLING. TRACEK SKEWED TO NIIRTH.

DIL AKCS EMBRACE CROSSWINO EXTENT OF TRACER.

\begin{tabular}{|c|c|c|c|c|c|c|c|c|c|}
\hline $\begin{array}{l}\text { TEST UT } \\
\text { QINC } \\
\text { SAMPLER }\end{array}$ & $\begin{array}{l}14 \\
\text { SULFIDE } \\
\text { HT } 1.5 \mathrm{M}\end{array}$ & $\begin{array}{l}\text { AUGUST } 2 \\
\text { RELFASE }\end{array}$ & $\begin{array}{l}29,1961 \\
\text { E FRUM }\end{array}$ & $\begin{array}{l}\text { OLEVATIL } \\
U=4.6 \mathrm{MI}\end{array}$ & $\begin{array}{l}\text { TU } 010 \\
\text { OF } 26 \\
\text { SEC AT }\end{array}$ & $\begin{array}{l}9 \text { PST } \\
M \\
26 M\end{array}$ & 1 & & \\
\hline $\begin{array}{l}\text { DISTAIVCE } \\
\text { FRLMM }\end{array}$ & \multicolumn{4}{|c|}{ 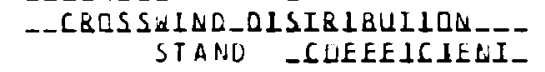 } & \multirow{2}{*}{$\begin{array}{l}\text { THREE } \\
\text { AZIMUTH }\end{array}$} & \multicolumn{2}{|c|}{ MAXIMUM EXPOSURES } & \multicolumn{2}{|c|}{$\begin{array}{l}\text { CROSSWIND } \\
\text { INIEGBAIED_YALUES- }\end{array}$} \\
\hline SDURCF. & MEAN & DEV & SKEW & KURT & & $E / O$ & $E U / 0$ & $C I(E / U)$ & $C I(E \cup / Q)$ \\
\hline------ & ---- & ---- & $\cdots--$ & $---\infty$ & 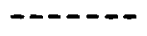 & 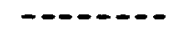 & $-0--4-0$ & -------- & $-----0--$ \\
\hline MFTERS & DFC & DEG & & & DEG & SFCACU.M & $1 / 50 . M$ & SEC/SO.M & $1 / M$ \\
\hline 400 & 111.6 & 4.18 & -.230 & 3.083 & $\begin{array}{l}110.0 \\
114.0 \\
118.0\end{array}$ & $\begin{array}{l}2.17 E-06 \\
1.49 E-06 \\
9.38 E-J 7\end{array}$ & $\begin{array}{l}9.96 E-06 \\
6.85 E-06 \\
4.31 E-06\end{array}$ & $1.56 E-04$ & $7.16 E-04$ \\
\hline 000 & 110.9 & 4.50 & -.487 & 2.513 & $\begin{array}{l}114.5 \\
110.7 \\
108.8\end{array}$ & $\begin{array}{l}2.17 E-05 \\
1.22 E-05 \\
1.18 E-05\end{array}$ & $\begin{array}{l}9.97 E-05 \\
5.63 E-05 \\
5.43 E-05\end{array}$ & $2.50 E-03$ & $1.15 E-0 ?$ \\
\hline 1200 & 109.7 & 4.18 & -.220 & 2.339 & $\begin{array}{l}114.0 \\
118.0 \\
112.0\end{array}$ & $\begin{array}{l}8.76 t-\cup 6 \\
8.47 t-06 \\
7.47 t-16\end{array}$ & $\begin{array}{l}4.03 t-05 \\
3.04 E-C 5 \\
3.44 E-05\end{array}$ & $2.17 E-03$ & $9.9 y E-03$ \\
\hline 1600 & 110.9 & 3.54 & -.536 & 2.894 & $\begin{array}{l}113.4 \\
114.4 \\
110.4\end{array}$ & $\begin{array}{l}1.79 E-U 5 \\
1.40 E-U 5 \\
1.13 E-U 5\end{array}$ & $\begin{array}{l}0.25 E-05 \\
6.44 E-05 \\
5.22 E-05\end{array}$ & $3.40 E-03$ & $1.56 E-02$ \\
\hline 2200 & 109.7 & 3.31 & -.299 & 4.280 & $\begin{array}{l}108.0 \\
110.0 ? \\
112.0\end{array}$ & $\begin{array}{l}3.64 E-06 \\
3.47 E-06 ? \\
2.94 E-U 6\end{array}$ & $\begin{array}{l}1.60 t-05 \\
1.60 E-057 \\
1.35 t-05\end{array}$ & $1.10 E-03$ & $5.04 E-03$ \\
\hline 3200 & 109.8 & 5.67 & -.825 & 3.153 & $\begin{array}{l}115.6 \\
114.6 \\
108.87\end{array}$ & $\begin{array}{l}2.76 E-U 6 \\
2.27 E-16 \\
2.0 B E-06 ?\end{array}$ & $\begin{array}{l}1.27 E-05 \\
1.04 E-05 \\
9.57 t-06 ?\end{array}$ & $1.42 \mathrm{E}-0.3$ & $6.53 E-03$ \\
\hline 5000 & 108.3 & 5. 38 & -.749 & 3.749 & $\begin{array}{l}108.0 \\
114.0 \\
106.0\end{array}$ & $\begin{array}{l}1.07 E-06 \\
1.21 E-06 \\
9.75 E-U 7\end{array}$ & $\begin{array}{l}8.59 E-06 \\
5.57 E-06 \\
4.49 E-06\end{array}$ & $1.12 \mathrm{E}-03$ & $5.14 E-03$ \\
\hline 7000 & 108.8 & 4.47 & -.578 & 4.667 & $\begin{array}{l}110.0 \\
108.0 \\
106.0\end{array}$ & $\begin{array}{l}7 . U 1 t-07 \\
1.14 t-07 \\
9.25 E-U 8\end{array}$ & $\begin{array}{l}3.23 E-U 6 \\
5.25 E-07 \\
4.25 E-07\end{array}$ & $3.05 E-04$ & $1.40 E-03$ \\
\hline
\end{tabular}

$?$ INDICATES UNCERTAINTIES IN DATA.

$X$ inOICATES INVALID DR InCDMPLETE UATA. 


\begin{tabular}{|c|c|c|c|c|c|c|c|c|c|}
\hline $\begin{array}{l}\text { TEST UT } \\
\text { FLUU } \\
\text { SAMPLER }\end{array}$ & $\begin{array}{l}4 \\
\text { RESCEIN } \\
\text { HT } 1.5 \mathrm{M}\end{array}$ & $\begin{array}{l}\text { AUGUST } \\
\text { RELEAS }\end{array}$ & $\begin{array}{l}29,196 \\
\text { E FROM }\end{array}$ & $\begin{array}{l}003 \\
J \text { LFVATID } \\
J=6.2 \mathrm{H}\end{array}$ & $\begin{array}{l}9 \text { TO } 0100 \\
\text { N DF } 561 \\
\text { SEC AT }\end{array}$ & $\begin{array}{l}9 \text { PST } \\
56 M\end{array}$ & & & \\
\hline $\begin{array}{l}\text { DISTANCE } \\
\text { FRDY }\end{array}$ & -CBDS & $\begin{array}{l}\text { SHIND D. } \\
\text { STANU }\end{array}$ & $\begin{array}{l}\text { ISIRIBU. } \\
\text {-COEEE. }\end{array}$ & LIENI- & AHRE & MAXIHUM EX & POSURES & $\begin{array}{l}\text { CROS } \\
\text { INIELRAIE }\end{array}$ & $\begin{array}{l}\text { IND } \\
\text { DYYLUES }\end{array}$ \\
\hline SUURCE & MEAN & DEV & SKEW & KURT & AZIMUTH & $E / O$ & $E U / O$ & $C I(E / O)$ & $C I(E U / Q)$ \\
\hline-------- & ----- & ----- & ----- & ----- & ------ & ------ & ------- & ------- &.------ \\
\hline METERS & DEG & DEG & & & DEG & $S E C / C U . M$ & $1 / S O \cdot M$ & $S E C / S U . M$ & $1 / M$ \\
\hline 400 & 98.6 & 11.16 & .527 & 1.806 & $\begin{array}{l}90.0 \\
86.0 \\
94.0\end{array}$ & $\begin{array}{l}1.36 E-09 \\
1.32 E-U 9 \\
1.29 E-09\end{array}$ & $\begin{array}{l}8.43 E-09 \\
8.20 E-09 \\
7.97 E-09\end{array}$ & $1.95 E-07$ & $1.21 E-06$ \\
\hline 800 & 116.4 & 1.75 & .740 & 3.496 & $\begin{array}{l}116.4 \\
114.5 \\
118.3\end{array}$ & $\begin{array}{l}1.14 E-05 \\
1.03 E-05 \\
6.64 E-06\end{array}$ & $\begin{array}{l}\text { 7. U9E-05 } \\
6.40 E-05 \\
4.11 E-05\end{array}$ & $8.32 E-04$ & $5.16 E-03$ \\
\hline 1200 & 116.1 & 1.76 & -.040 & 3.000 & $\begin{array}{l}116.0 \\
118.0 \\
114.0\end{array}$ & $\begin{array}{l}5.38 E-U 6 \\
4.55 E-U 6 \\
4.16 E-ن 6\end{array}$ & $\begin{array}{l}3.34 E-05 \\
2.62 E-05 \\
2.58 E-05\end{array}$ & $6.14 E-04$ & $3.81 E-03$ \\
\hline 1600 & 116.6 & 1.64 & -.161 & 3.539 & $\begin{array}{l}116.3 \\
117.3 \\
115.3\end{array}$ & $\begin{array}{l}1.86 E-U 5 \\
1.12 E-U 5 \\
1.02 E-U 5\end{array}$ & $\begin{array}{l}1.16 E-04 \\
6.94 E-05 \\
6.33 E-05\end{array}$ & $1.72 t-03$ & $1.06 E-02$ \\
\hline 2200 & 116.9 & 1.64 & .240 & 4.300 & $\begin{array}{l}116.0 \\
118.0 \\
120.0\end{array}$ & $\begin{array}{l}4.23 E-U 6 \\
3.02 E-06 \\
0.7 U E-U 7\end{array}$ & $\begin{array}{l}2.63 E-05 \\
1.07 E-05 \\
5.40 E-06\end{array}$ & $6.79 E-04$ & $4.21 \mathrm{E}-03$ \\
\hline 3200 & 115.8 & 2.78 & -1.062 & 4.843 & $\begin{array}{l}117.6 \\
115.6 \\
118.6\end{array}$ & $\begin{array}{l}3.06 E-06 \\
2.42 E-06 \\
1.41 E-J 6\end{array}$ & $\begin{array}{l}1.91 E-05 \\
1.5 U E-05 \\
1.1 \mathrm{dE}-05\end{array}$ & $0.31 E-04$ & $5.15 E-03$ \\
\hline 5000 & 115.8 & 3.25 & -.684 & 4.495 & $\begin{array}{l}118.0 \\
116.0 \\
114.0\end{array}$ & $\begin{array}{l}1.16 E-06 \\
1.13 E-06 \\
1.10 E-06\end{array}$ & $\begin{array}{l}7.19 E-U 6 \\
6.99 E-06 \\
6.79 E-66\end{array}$ & $8.73 E-04$ & $5.41 E-03$ \\
\hline 7000 & 115.3 & 2.52 & -.455 & 3.899 & $\begin{array}{l}116.0 \\
114.0 \\
110.0\end{array}$ & $\begin{array}{l}5.81 E-J 7 \\
5.36 E-J 7 \\
3.13 E-0.7\end{array}$ & $\begin{array}{l}3.6 U E-06 \\
3.32 E-06 \\
1.94 E-06\end{array}$ & $4.47 E-04$ & $2.77 E-03$ \\
\hline
\end{tabular}

$?$ INOICATES UNCERTAINTIES IN DATA.

X INUICATES INVALIO OK INCOMPLEIE DATA. 
SAMPLING 4OOM TO $12800 M$, BUT NO TRACER LBSERVED AT $12000 M$. NO TOWER SAMPLING.

5000 PA ANO $7000 M$ ARCS TRUNCATED ON NDRTH. WINU SPEED MEASURED AT 12BOUM ARC AVERAGED ABUUT O.2 M/SEC AT I.5M ELEVATICN. RELATIVELY LARGE DIRECTION SHEAR HITH ELEVATION IABCUT 20 DEGRES FREM ISM TO GIMI.

\begin{tabular}{|c|c|c|c|c|c|c|c|c|c|}
\hline $\begin{array}{c}\text { TEST UT } \\
\text { ZINC } \\
\text { SAMFLER }\end{array}$ & $\begin{array}{l}5 \\
\text { SULFIDE } \\
\text { HT } 1.5 \mathrm{M}\end{array}$ & $\begin{array}{l}\text { AUGUST } 3 \\
\text { RELFASE }\end{array}$ & $\begin{array}{r}0,1968 \\
U R O M U\end{array}$ & $\begin{array}{r}\text { COS } \\
\text { LEVATILI } \\
=2.9 \mathrm{M} / \mathrm{S}\end{array}$ & $\begin{array}{l}\text { TU OIO } \\
\text { OF } 26 \% \\
\text { EAT }\end{array}$ & $\begin{array}{l}1 \text { PST } \\
26 M\end{array}$ & & & \\
\hline $\begin{array}{l}\text { DISTANCE } \\
\text { FRUM }\end{array}$ & \multicolumn{4}{|c|}{$\begin{array}{l}\text { CBOSSKINDODISIRIEUIIDU- } \\
\text { STAND COEEEICIENI- }\end{array}$} & THREE & \multicolumn{2}{|c|}{ MAXIMUM EXPQSURES } & \multicolumn{2}{|c|}{$\begin{array}{l}\text { CRISSWIND } \\
\text { INIEGRAIED_YALUES_ }\end{array}$} \\
\hline SOURCE & MEAN & CEV & SKE H & KUR I & ALIMUTH & $E / G$ & $\mathrm{EU} / \mathrm{Q}$ & $C I(E / O)$ & CI (EU/O) \\
\hline METERS & DEG & DEG, & & & DEG & SEC/CU.M & $1 / 50 . \mathrm{H}$ & SEC/SO.M & $1 / H$ \\
\hline 400 & 77.1 & 5.15 & 1.106 & 3.052 & $\begin{array}{l}74.0 \\
86.0 \\
78.0\end{array}$ & $\begin{array}{l}4.76 E-06 \\
1.10 E-06 \\
1.06 E-06\end{array}$ & $\begin{array}{l}1.38 E-05 \\
3.18 E-06 \\
3.07 E-06\end{array}$ & $2.21 E-04$ & $6.41 E-04$ \\
\hline 800 & 80.57 & $4.94 ?$ & $-.397 ?$ & $3.434 ?$ & $\begin{array}{l}42.0 ? \\
79.0 ? \\
45.0\end{array}$ & $\begin{array}{l}3.23 E-06 ? \\
2.5 \forall E-06 ? \\
1.61 E-06\end{array}$ & $\begin{array}{l}9.37 E-06 ? \\
7.49 E-06 ? \\
4.68 E-06\end{array}$ & $4.52 E-04 ?$ & $1.31 E-03 ?$ \\
\hline 1200 & 78.7 & 5.21 & .230 & 2.565 & $\begin{array}{l}76.0 \\
80.0 \\
32.0\end{array}$ & $\begin{array}{l}3.96 E-06 \\
3.39 E-06 \\
2.29 E-06\end{array}$ & $\begin{array}{l}1.15 E-05 \\
9.84 E-06 \\
6.65 E-06\end{array}$ & $.9 .02 E-04$ & $2.62 E-03$ \\
\hline 1600 & 79.2 & 6.92 & .244 & 1.851 & $\begin{array}{l}72.0 \\
80.0 \\
38.0\end{array}$ & $\begin{array}{l}1.50 E-06 \\
1.08 E-06 \\
8.68 E-07\end{array}$ & $\begin{array}{l}4.36 E-06 \\
3.13 E-N 6 \\
2.52 E-06\end{array}$ & $3.92 E-04$ & $1.14 E-03$ \\
\hline 2200 & 79.1 & 4.97 & -.589 & 2.830 & $\begin{array}{l}82.0 \\
72.0 \\
14.0\end{array}$ & $\begin{array}{l}2.36 E-U 6 \\
7.16 E-U 7 \\
5.36 E-U 7\end{array}$ & $\begin{array}{l}6.84 E-06 \\
2.0 \forall E-06 \\
1.5 U E-06\end{array}$ & $3.70 E-04$ & $1.07 E-03$ \\
\hline 3200 & 78.5 & 3.28 & -.430 & 4.001 & $\begin{array}{l}78.6 \\
00.8 \\
79.8\end{array}$ & $\begin{array}{l}2.93 t-v 6 \\
2.72 t-v 6 \\
1.75 t-v 6\end{array}$ & $\begin{array}{l}8.40 t-06 \\
7.89 t-06 \\
5.00 t-06\end{array}$ & $8.05 E-04$ & $2.33 E-03$ \\
\hline 5000 & $83.6 x$ & $.91 x$ & $-.438 x$ & $4.731 x$ & $\begin{array}{l}04.0 ? \\
32.0 ? \\
36.0 ?\end{array}$ & $\begin{array}{l}7.37 t-U 7 ? \\
1.95 t-07 ? \\
2.16 E-08 ?\end{array}$ & $\begin{array}{l}2.14 E-0 E ? \\
5.67 E-07 ? \\
0.25 E-08 ?\end{array}$ & $1.54 E-04 X$ & $4.4 \forall E-04 X$ \\
\hline 7000 & $80.3 x$ & $2.45 x$ & $.063 x$ & $2.455 x$ & $\begin{array}{l}80.0 ? \\
84.0 ? \\
76.0 ?\end{array}$ & $\begin{array}{l}9.01 E-06 ? \\
3.19 E-08 ? \\
2.08 E-06 ?\end{array}$ & $\begin{array}{l}2.61 E-07 ? \\
1.10 E-07 ? \\
6.03 E-08 ?\end{array}$ & $3.72 E-05 X$ & $1.08 E-04 X$ \\
\hline 12800 & $0.0 x$ & $0.00 x$ & $0.000 x$ & $0.000 x$ & $\begin{array}{l}0.0 x \\
0.0 x \\
0.0 x\end{array}$ & $\begin{array}{l}0 . \\
0 . \\
0 .\end{array}$ & $\begin{array}{l}0 . \\
0 . \\
0 .\end{array}$ & 0. & 0. \\
\hline
\end{tabular}

? INDICATES UNCERTAINTIES IN DATA.

$X$ INDICATFS INVALID OR INCUMPLETE DATA. 


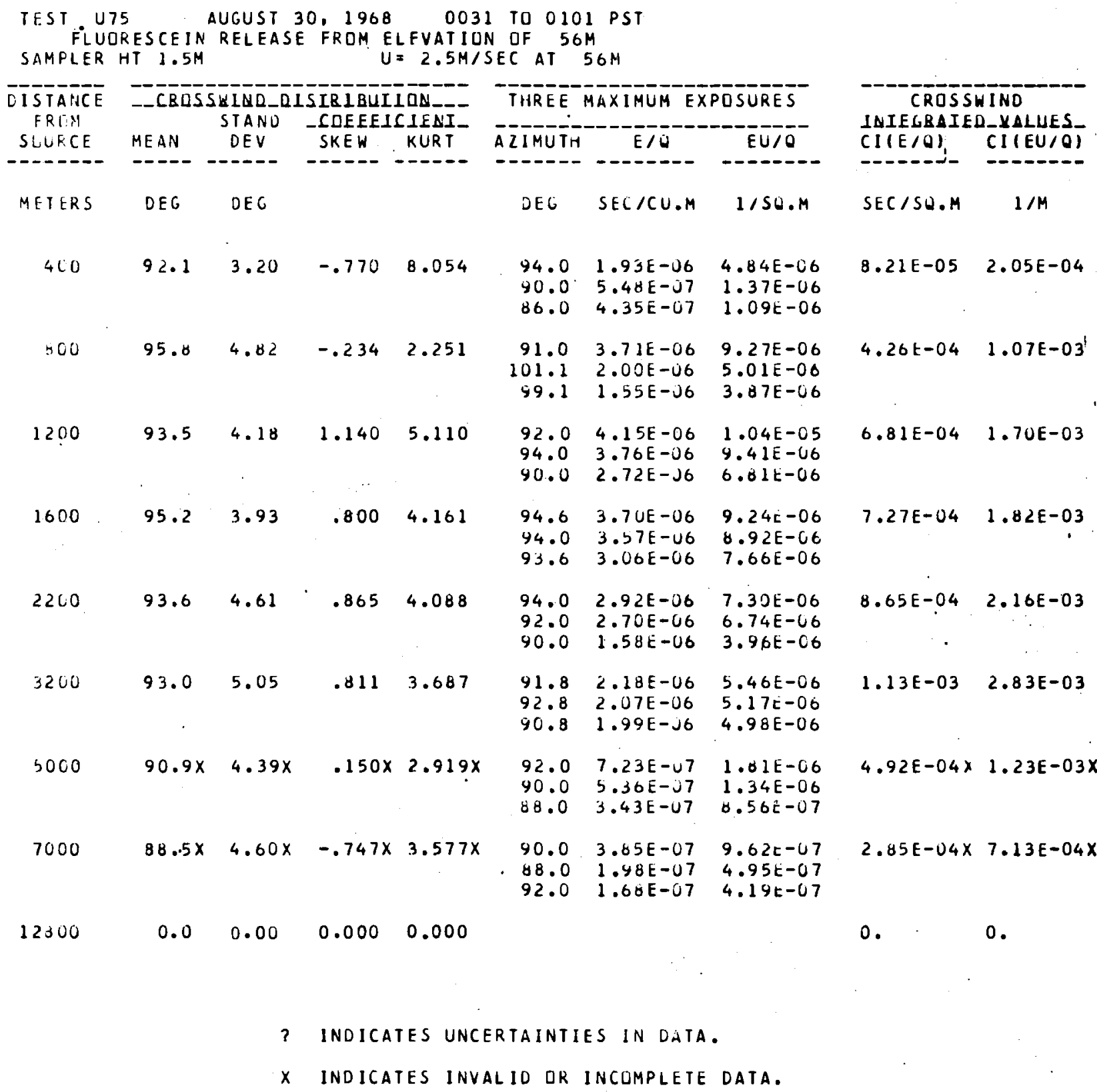


SAMPLING $400 M$ TO 7000M., NO TOWER SAMPLING.

$5000 M$ AND $7000 M$ ARCS TRUNCATED ON NORTH.

\begin{tabular}{|c|c|c|c|c|c|c|c|c|c|}
\hline \multirow{2}{*}{$\begin{array}{l}\text { DISTANCE } \\
\text { FRUM } \\
\text { SOURCE }\end{array}$} & \multicolumn{4}{|c|}{ 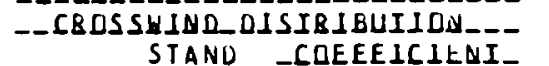 } & \multirow{2}{*}{ AIIREE } & \multicolumn{2}{|c|}{ MAXIMUM EXPOSURES } & \multicolumn{2}{|c|}{$\begin{array}{l}\text { CROSSWIND } \\
\text { INIEGBAIEQ_YALUES }\end{array}$} \\
\hline & MEAN & DEV & SKE WK & KUR T & & $E / 0$ & EU/O & $C I(E / 0)$ & $C \perp(E U / O)$ \\
\hline METERS & DEG & DEG & & & UEE & SEC/CU.M & $1 / 50 . M$ & SEC/SO.M & $1 / M$ \\
\hline 400 & 81.5 & 6.53 & -.164 & 1.821 & $\begin{array}{l}86.0 \\
74.0 \\
90.0\end{array}$ & $\begin{array}{l}9.35 E-06 \\
8.77 E-06 \\
6.42 E-06\end{array}$ & $\begin{array}{l}6.08 E-05 \\
5.70 E-05 \\
4.43 E-05\end{array}$ & $1.08 E-03$ & $7.03 E-03$ \\
\hline 800 & 77.8 & 6.64 & .459 & 1.757 & $\begin{array}{l}73.0 \\
70.0 \\
05.0\end{array}$ & $\begin{array}{l}1.71 E-05 \\
7.52 E-06 \\
7.29 E-06\end{array}$ & $\begin{array}{l}1.11 E-04 \\
4.89 E-05 \\
4.74 E-05\end{array}$ & $2.14 E-03$ & $1.39 E-02$ \\
\hline 1200 & 76.7 & 6.52 & .649 & 1.953 & $\begin{array}{l}72.0 \\
74.0 \\
70.0\end{array}$ & $\begin{array}{l}1.20 E-U 5 \\
7.49 E-06 \\
7.42 E-06\end{array}$ & $\begin{array}{l}7.82 E-05 \\
4.87 E-05 \\
4.82 E-05\end{array}$ & $2.00 E-03$ & $1.30 E-02$ \\
\hline 1600 & 75.6 & 5.92 & .901 & 2.466 & $\begin{array}{l}72.0 \\
70.0 \\
74.0\end{array}$ & $\begin{array}{l}1.17 E-05 \\
7.76 E-06 \\
5.06 E-66\end{array}$ & $\begin{array}{l}7.64 E-05 \\
5.04 E-05 \\
3.81 E-05\end{array}$ & $2.28 \mathrm{E}-03$ & $1.40 E-02$ \\
\hline 2200 & 73.2 & 4.22 & 1.589 & 4.973 & $\begin{array}{l}72.0 \\
70.0 \\
76.0\end{array}$ & $\begin{array}{l}5.13 E-06 \\
4.17 E-06 \\
1.19 E-66\end{array}$ & $\begin{array}{l}3.34 E-05 \\
2.71 E-05 \\
7.76 E-06\end{array}$ & $1.08 E-03$ & $7.01 E-03$ \\
\hline 3200 & 75.0 & 3.77 & .780 & 3.534 & $\begin{array}{l}73.7 \\
72.7 \\
74.7\end{array}$ & $\begin{array}{l}4.42 E-06 \\
3.67 E-06 \\
3.28 E-06\end{array}$ & $\begin{array}{l}2.87 E-05 \\
2.38 E-05 \\
2.13 E-05\end{array}$ & $1.71 \mathrm{E}-03$ & $1.11 E-02$ \\
\hline 5000 & $83.3 x$ & $3.06 x$ & $3.641 \times 1$ & $17.101 x$ & $\begin{array}{l}82.0 x \\
34.0 x \\
30.0 x\end{array}$ & $\begin{array}{l}1.34 E-07 X \\
3.94 t-08 X \\
4.43 E-69 x\end{array}$ & $\begin{array}{l}8.64 E-U 7 X \\
2.50 E-U 7 X \\
2.08 E-08 X\end{array}$ & $3.05 E-05 X$ & $1.98 E-04 X$ \\
\hline 7000 & $80.2 x$ & $3.07 x$ & $.795 x$ & $4.118 x$ & $\begin{array}{l}80.0 x \\
82.0 x \\
76.0 x\end{array}$ & $\begin{array}{l}1.61 E-\cup 6 x \\
1.38 E-66 x \\
9.31 E-U 7 x\end{array}$ & $\begin{array}{l}1.05 E-05 X \\
9.00 E-06 X \\
6.05 E-06 X\end{array}$ & $1.24 E-03 x$ & $8.05 E-03 x$ \\
\hline & $?$ INOI & CATES & CERTAIN & NTIES IN & DATA. & & & $\cdot$ & \\
\hline & $x$ INO I & ICATES & NVAL 100 & OR INCUMF & PLETE DA & ATA. & & . & \\
\hline
\end{tabular}




\begin{tabular}{|c|c|c|c|c|c|c|c|c|c|}
\hline $\begin{array}{l}\text { DISTANCE } \\
\text { FROM }\end{array}$ & \multicolumn{2}{|c|}{$\begin{array}{l}\text {-CBOSSUINQ_DI } \\
\text { STANO }\end{array}$} & $\begin{array}{l}\text { ISIRIBUI } \\
\text { _COEEEI }\end{array}$ & LCLENI- & THREE & \multicolumn{2}{|c|}{ MAXIMUM EXPOSURES } & \multicolumn{2}{|c|}{$\begin{array}{c}\text { CRDSSWIND } \\
\text { INIEGRAIED_YALUES_ }\end{array}$} \\
\hline SIURCE & MEAN & DEV & SKEW & KURT & AZIMUTH & $E / Q$ & $E \cup / O$ & $C I(E / O)$ & $C I(E U / O)$ \\
\hline------ & ---- & $0---$ & ----- & $----\cdots$ & ------ & $-0-----1$ & $---1--0$ & -------0 & -------- \\
\hline METERS & DEG & OEC & & & DEG & $S E C / C U, M$ & $1 / S \theta \cdot M$ & SEC/SO.M & $1 / M$ \\
\hline 400 & 87.47 & $6.8<?$ & $2.928 ?$ & $12.197 ?$ & $\begin{array}{r}86.0 ? \\
82.0 ? \\
110.0 ?\end{array}$ & $\begin{array}{l}2.84 E-07 ? \\
2.58 E-08 ? \\
1.4 \angle E-08 ?\end{array}$ & $\begin{array}{l}2.02 E-06 ? \\
1.83 E-07 ? \\
1.01 E-07 ?\end{array}$ & $9.66 E-06 ?$ & $6.86 E-05 ?$ \\
\hline 800 & 82.5 & 6.18 & $\begin{array}{c}-.379 \\
.\end{array}$ & 1.675. & $\begin{array}{l}88.0 \\
76.0 \\
85.0\end{array}$ & $\begin{array}{l}3.65 E-06 \\
2.47 E-06 \\
1.45 E-06\end{array}$ & $\begin{array}{l}2.59 E-05 \\
1.76 E-05 \\
1.38 E-05\end{array}$ & $4.60 E-04$ & $3.27 E-03$ \\
\hline 1200 & $81.6 ?$ & $5.93 ?$ & $-.239 ?$ & $? 1.703 ?$ & $\begin{array}{l}86.0 ? \\
28.0 ? \\
74.0 ?\end{array}$ & $\left\{\begin{array}{l}4.98 E-06 ? \\
3.53 E-06 ? \\
2.65 E-06 ?\end{array}\right.$ & $\begin{array}{l}3.53 E-05 ? \\
2.51 t-05 ? \\
1.88 E-05 ?\end{array}$ & $1.01 E-03 ?$ & $7.20 E-037$ \\
\hline 1600 & 80.6 & 5.56 & .060 & 1.657 & $\begin{array}{l}76.0 \\
66.0 \\
88.0\end{array}$ & $\begin{array}{l}4.21 E-06 \\
3.32 E-06 \\
2.63 E-06\end{array}$ & $\begin{array}{l}2.99 E-05 \\
2.36 E-05 \\
1.87 E-05\end{array}$ & $1.21 E-03$ & $8.6 \cup E-03$ \\
\hline 2200 & 78.2 & 4.61 & $\cdot .429$ & 2.131 & $\begin{array}{l}76.0 \\
78.0 \\
74.0\end{array}$ & $\begin{array}{l}2.29 E-06 \\
1.00 E-U 6 \\
1.59 E-U 6\end{array}$ & $\begin{array}{l}1.63 E-05 \\
1.28 E-05 \\
1.13 E-05\end{array}$ & $8.19 E-04$ & $5.82 E-03$ \\
\hline 3200 & 78.2 & 3.83 & .464 & 2.298 & $\begin{array}{l}76.8 \\
75.7 \\
74.7\end{array}$ & $\begin{array}{l}2.75 E-\cup 6 \\
2.50 E-06 \\
2.42 E-06\end{array}$ & $\begin{array}{l}1.95 E-05 \\
1.77 E-05 \\
1.72 E-05\end{array}$ & $1.33 E-03$ & $9.46 E-03$ \\
\hline 5000 & $82.2 x$. & $.63 x$ & $3.355 x$ & $16.778 x$ & $\begin{array}{l}82.0 ? \\
84.0 ? \\
86.0 ?\end{array}$ & $\begin{array}{l}5.06[-07 ? \\
5.91 E-06 ? \\
2.02 E-09 ?\end{array}$ & $\begin{array}{l}4.16 t-06 ? \\
4.20 E-07 ? \\
1.44 E-00 ?\end{array}$ & $1.05 E-04 x$ & $7.43 E-04 x$ \\
\hline 7000 & 82.8 & 2.74 & .510 & 3.436 & $\begin{array}{l}82.0 \\
84.0 \\
80.0\end{array}$ & $\begin{array}{l}1.12 E-06 \\
7.57 E-07 \\
6.35 E-07\end{array}$ & $\begin{array}{l}7.96 E-06 \\
5.37 E-06 \\
4.51 E-06\end{array}$ & $7.71 E-04$ & $5.47 E-03$ \\
\hline
\end{tabular}

$?$ INDICATES UNCERTAINTIES IN DATA.

X INDICATES INVALIU OR INCOMPLETE DATA. 
SAMPLING 4UOM TO $12800 M$. NO TOWER SAMPLING. EXTKEME SHIFT IN PLUME CENIERLINE LUCATION STARTING ABUUT I6OOH. THE PLUME CENTERLINE SHIFT CAUSED TRUNCATION UR "IMISSED PLUME" ON $1200,3200,5000,7000$ AND $12800 M$; ARCS. IL UIJEESCEIN WAS NOT ASSAYED FAR FINOUGH NUKTH ON THE $1600 M$ ARC TO $G E$ SURE OF TRUNCATION.

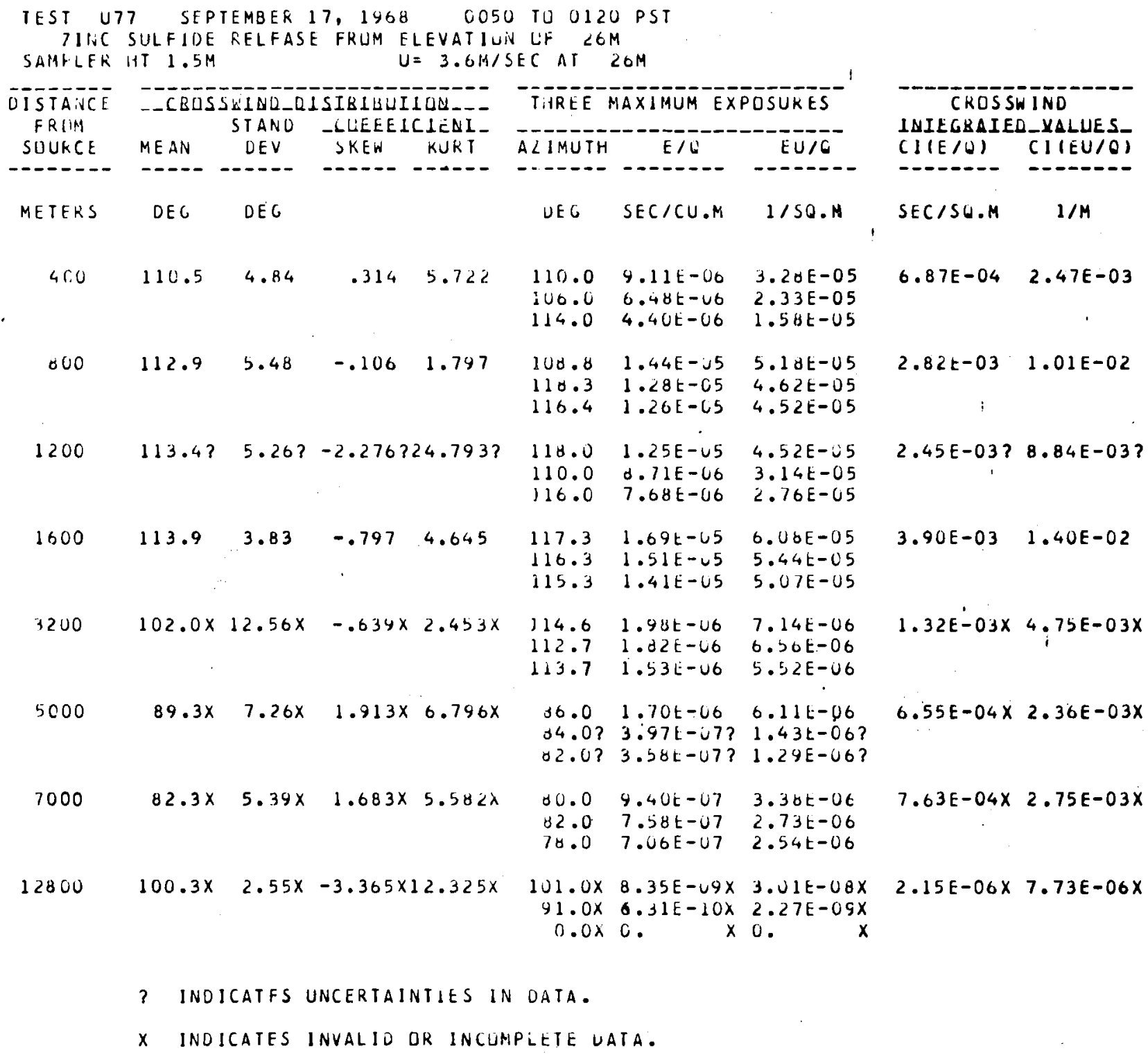
$0.0 \times 0 . \times \times 0$.

CKOSSWIND

INIEGRAIED_YALUES

CIIE/O) CIIEU/O

SEC/SO.M $\quad 1 / M$

$6.87 E-04 \quad 2.47 E-03$

$2.82 t-03 \quad 1.01 E-02$

$2.45 E-03 ? 8.84 E-03 ?$

$3.90 E-03 \quad 1.40 E-02$

1. $32 E-03 \times 4.75 E-03 X$

$6.55 E-04 \times 2.36 E-03 X$

$7.63 E-04 \times 2.75 E-03 X$

$2.15 E-06 \times 7.73 E-06 X$

? INOICATFS UNCERTAINTIES IN DATA.

$X$ INDICATES INVALIO OR INCUMPLETE UATA. 


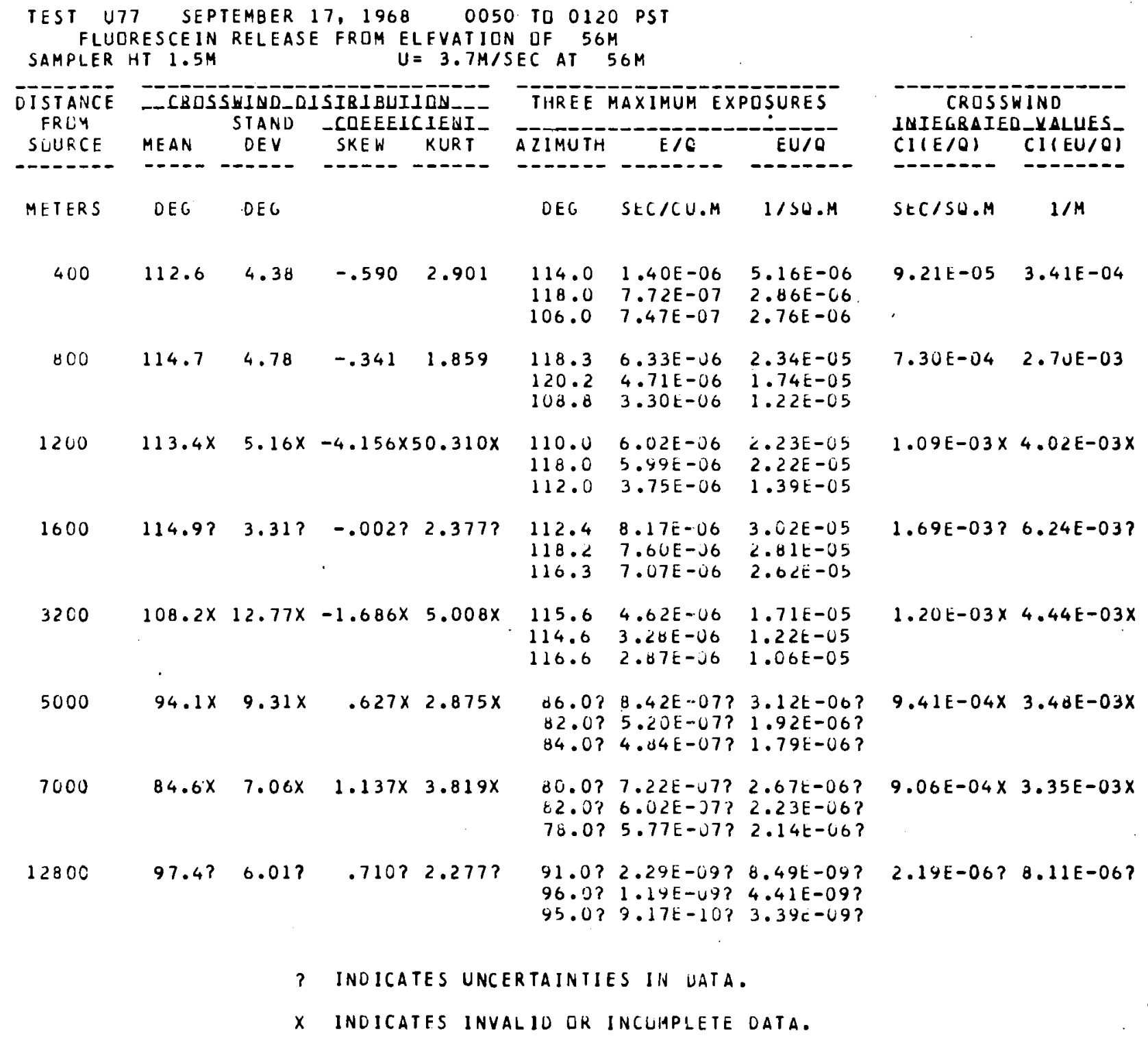

$x$ INOICATFS INVALIO OR INCUIAPLETE DATA. 
SAMPLING 4OOM TO 7UOOM. NO TOWER SAMPLING. ALL ARCS EMBRACE CRUSSWIND EXTENT UF TRACER.

TRACE OF RAIN FELL DURING TRACER RELEASE. UP TO 0.03 INCHES UF RAIN FELL DURING PICKUP OF SAMPLES: ALTHOUGH MANY FILTERS WERE VERY WET. THE EFFECTS OF THIS "WASHING" ARE UNCERTAIN.

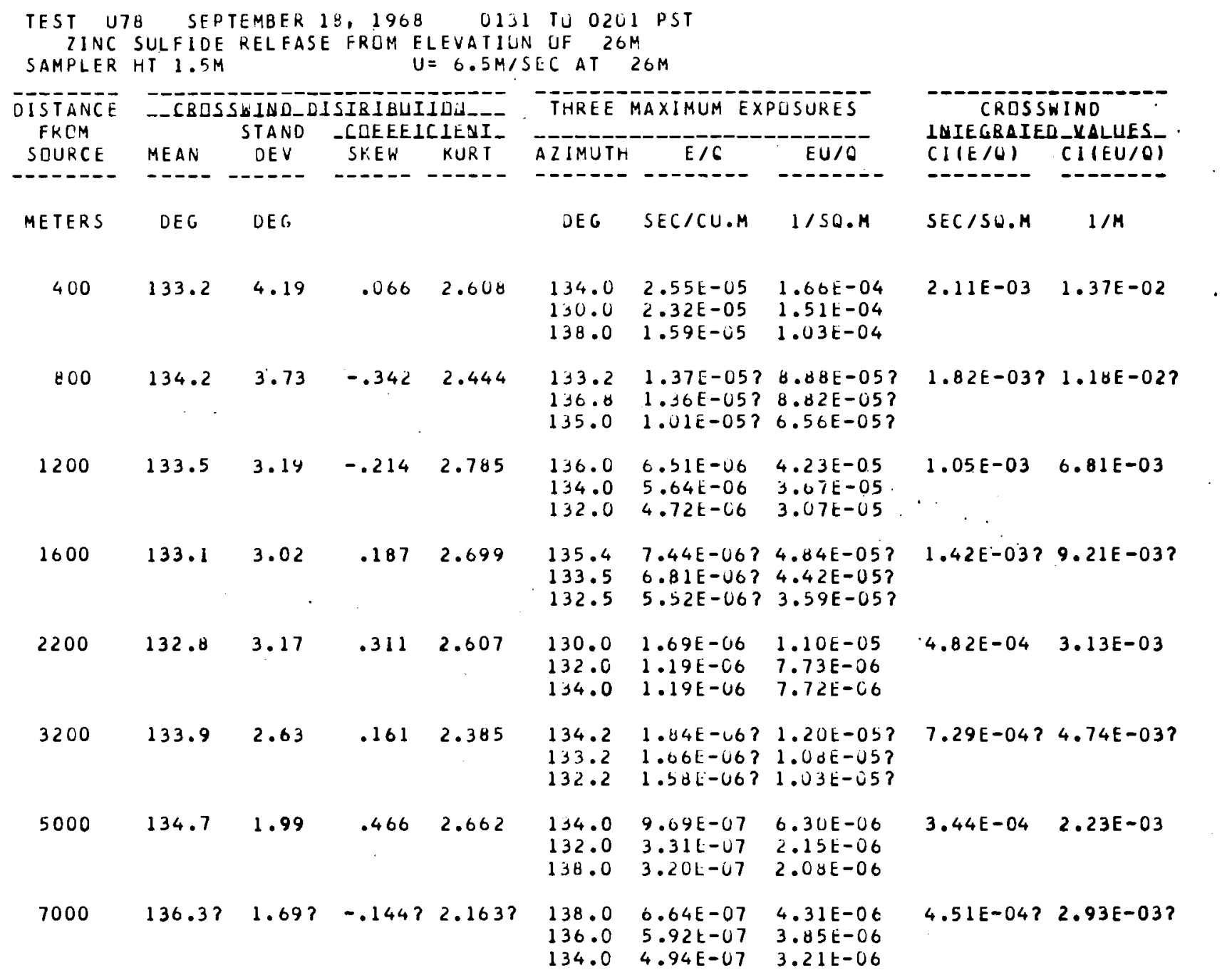

$?$ INDICATES UNCERTAINTIES IN DATA.

X INDICATES INVALIO DR INCGMPLETE DATA. 
TEST U78 SEPTEMBER 18, 1968 0131 T0 0201 PST

FLUDRESCEIN RELEASE FROM ELFVATIUN DF $56 \mathrm{M}$

SAMPEER HT $1.5 H \quad U=7.3 \mathrm{M} / \mathrm{SEC}$ AT $56 \mathrm{M}$

\begin{tabular}{|c|c|c|c|c|c|c|c|c|c|}
\hline \multirow{2}{*}{$\begin{array}{l}\text { UISTANCE } \\
\text { FRLUAI } \\
\text { SLUKCE }\end{array}$} & \multicolumn{4}{|c|}{-CECSSWINDEDISIBIBUIION- } & \multicolumn{3}{|c|}{ THREE MAXIM } & \multicolumn{2}{|c|}{$\begin{array}{l}\text { CROSSWINO } \\
\text { INIEGSAIED_YALUES }\end{array}$} \\
\hline & MEAN & DEV & SKEW & KURI & AZIHUTH & $E / 0$ & $E U / Q$ & $C l(E / O)$ & $C I(E U / Q)$ \\
\hline & & & & & & & & & \\
\hline METERS & DEC & DEC & & & DEC & $S E C / C U . M$ & $1 / 50 . M$ & $S E C / S Q . M !$ & $1 / M$ \\
\hline 400 & 133.1 & 2.88 & -.484 & 4.389 & $\begin{array}{l}134.0 \\
130.0 \\
138.0\end{array}$ & $\begin{array}{l}3.46 E-06 \\
1.26 E-06 \\
5.70 E-07\end{array}$ & $\begin{array}{l}2.53 E-05 \\
9.23 E-06 \\
4.16 E-06\end{array}$ & $1.57 E-04$ & $1.15 E-03$ \\
\hline 800 & 132.5 & 2.63 & .327 & 3.467 & $\begin{array}{l}133.2 \\
131.4 \\
129.5\end{array}$ & $\begin{array}{l}2.61 E-U 5 \\
1.62 E-05 \\
1.38 E-U 5\end{array}$ & $\begin{array}{l}1.91 E-04 \\
1.12 E-04 \\
1.01 E-0.4\end{array}$ & $2.10 E-03$ & $1.53 E-02$ \\
\hline 1200 & 132.7 & 2.83 & .122 & 2.718 & $\begin{array}{l}132.0 \\
134.0 \\
130.0\end{array}$ & $\begin{array}{l}5.77 E-06 \\
4.73 E-06 \\
4.46 E-J 6\end{array}$ & $\begin{array}{l}4.21 E-0.05 \\
3.45 E-05 \\
3.26 E-05\end{array}$ & $9.13 E-04$ & $6.66 E-03$ \\
\hline 1600 & 133.7 & 2.83 & -.396 & 2.460 & $\begin{array}{l}136.4 \\
133.5 \\
134.4\end{array}$ & $\begin{array}{l}9.40 E-06 \\
5.06 E-06 \\
5.06 E-06\end{array}$ & $\begin{array}{l}6.66 E-05 \\
3.70 E-05 \\
3.70 E-05\end{array}$ & $1.28 E-03$ & $9 \cdot 34 E-03$ \\
\hline 2200 & 133.0 & 2.75 & .347 & 2.633 & $\begin{array}{l}132.0 \\
130.0 \\
136.0\end{array}$ & $\begin{array}{l}2.35 E-J 6 \\
2.28 E-06 \\
1.87 E-36\end{array}$ & $\begin{array}{l}1.72 E-05 \\
1.67 t-05 \\
1.36 E-05\end{array}$ & $\begin{array}{c}7.07 E-04 \\
.\end{array}$ & $5.16 E-03$ \\
\hline 3200 & 133.3 & 2.35 & -.062 & 2.746 & $\begin{array}{l}133.2 \\
131.2 \\
134.2\end{array}$ & $\begin{array}{l}1.55 E-U 6 \\
1.28 E-U 6 \\
1.10 E-U 6\end{array}$ & $\begin{array}{l}1.1 \cdot 3 E-05 \\
9.31 E-06 \\
8.05 E-06\end{array}$ & $4.67 E-04$ & $3.4 \cdot 1 E-03$ \\
\hline 5000 & 134.6 & 1.68 & -.057 & 2.466 & $\begin{array}{l}134.0 \\
136.0 \\
132.0\end{array}$ & $\begin{array}{l}5.51 E-U 7 \\
5.51 E-J 7 \\
2.511-U 7\end{array}$ & $\begin{array}{l}4.02 E-06 \\
4.02 E-U 6 \\
1.84 E-06\end{array}$ & $2.51 E-04$ & $1.83 E-03$. \\
\hline 7000 & 136.3 & 1.48 & -.117 & 2.172 & $\begin{array}{l}136.0 \\
138.0 \\
134.0\end{array}$ & $\begin{array}{l}3.02 E-07 \\
2.16 E-07 \\
1.3 U E-U 7\end{array}$ & $\begin{array}{l}2.20 t-06 \\
1.57 t-06 \\
9.49 E-07\end{array}$ & $1.66 E-04$ & $1.21 E-03$ \\
\hline
\end{tabular}

? INDICATES UNCERTAINTIES IN CATA.

$X$ INDICATES INVALIU OR INCUMPLETE DATA. 
SAMPLING 400M TO $12800 M$. NO TOWER:5AMPLING.

SAMPLING ARC AT $2200 \mathrm{M}$ WAS NOT ACTIVATED.

ALL ARCS EMBRACE CROSSWINO EXTENT OF TRACER.

\begin{tabular}{|c|c|c|c|c|c|c|c|c|c|}
\hline \multirow{2}{*}{$\begin{array}{l}\text { DISTANCE } \\
\text { FRUM } \\
\text { SDURCE }\end{array}$} & \multicolumn{4}{|c|}{ _CBOSSWIND_DISIRIBUIIDV̈_- } & THREE & \multicolumn{2}{|c|}{ MAXIMUM EXPOSURES } & \multicolumn{2}{|c|}{$\begin{array}{l}\text { CROSSWIND } \\
\text { INIEGBAIED_YALUES }\end{array}$} \\
\hline & MEAN & DEV & SKE i & KUR T & AZI I U TH & $E / 0$ & $E U / 0$ & $C(I E / O)$ & CI (EU/O) \\
\hline----- & ---- & ----- & ----- & ----- & ----- & ------- & ----- & ------- & $----n$ \\
\hline METERS & DEG & DEG & & & UEO & SEC/CU.M & $1 / \mathrm{SO} . \mathrm{M}$ & SEC/SW.M & $1 / M$ \\
\hline 400 & 128.9 & 3.20 & .735 & 2.719 & $\begin{array}{l}126.0 \\
130.0 \\
134.0\end{array}$ & $\begin{array}{l}1.85 E-U 5 \\
1.44 E-C 5 \\
6.30 E-06\end{array}$ & $\begin{array}{l}9.45 E-05 \\
7.34 E-05 \\
3.21 E-05\end{array}$ & $1.12 E-03$ & $5.72 E-03$ \\
\hline 300 & 128.5 & 2.79 & .265 & 2.861 & $\begin{array}{l}127.7 \\
129.5 \\
125.8\end{array}$ & $\begin{array}{l}4.109 E-05 \\
2.35 E-05 \\
1.90 E-U 5\end{array}$ & $\begin{array}{l}2.09 E-04 \\
1.2 U E-U 4 \\
9.6 U E-05\end{array}$ & $3.38 E-03$ & $1.72 E-02$ \\
\hline 1200 & 128.6 & 2.30 & .306 & 3.190 & $\begin{array}{l}128.0 \\
150.0 \\
120.0\end{array}$ & $\begin{array}{l}2.29 E-U 5 \\
1.50 E-U 5 \\
1.30 E-05\end{array}$ & $\begin{array}{l}1.17 E-04 \\
7.64 E-05 \\
6.65 E-05\end{array}$ & $2.61 E-03$ & $1.33 E-02$ \\
\hline 1600 & 128.8 & 2.10 & .001 & 2.850 & $\begin{array}{l}128.8 \\
127.8 \\
129.7\end{array}$ & $\begin{array}{l}1.67 E-U 5 \\
1.63 E-U 5 \\
1.52 E-\cup 5\end{array}$ & $\begin{array}{l}9.52 E-U 5 \\
8.32 E-05 \\
7.75 E-05\end{array}$ & $2.88 E-03$ & $1.47 E-02$ \\
\hline 3200 & 126.1 & 2.31 & -.350 & 3.016 & $\begin{array}{l}1<6.3 \\
1<7.3 \\
125.4\end{array}$ & $\begin{array}{l}5.97 E-v 6 \\
5.74 t-16 \\
3.74 \mathrm{~L}-U 6\end{array}$ & $\begin{array}{l}3.05 E-05 \\
2.93 t-05 \\
1.44 t-05\end{array}$ & $1.57 E-03$ & $8.02 E-03$ \\
\hline 5000 & 120.8 & 2.08 & -.863 & 3.915 & $\begin{array}{l}1<2.0 \\
120.0 \\
118.0\end{array}$ & $\begin{array}{l}3.19 E-06 \\
1.25 E-06 \\
7.94 E-07\end{array}$ & $\begin{array}{l}1.63 E-05 \\
0.36 E-06 \\
4.05 E-06\end{array}$ & $1.02 E-03$ & $5.22 \mathrm{E}-03$ \\
\hline 7000 & 119.9 & 2.80 & -.354 & 3.132 & $\begin{array}{l}120.0 \\
122.0 \\
118.0\end{array}$ & $\begin{array}{l}1.57 E-06 \\
1.31 E-06 \\
8.21 E-U 7\end{array}$ & $\begin{array}{l}8.03 E-06 \\
6.69 E-06 \\
4.19 E-06\end{array}$ & $1.24 E-03$ & $6.35 E-03$ \\
\hline 12800 & 112.1 & 3.58 & -.261 & 2.490 & $\begin{array}{l}112.0 \\
110.0 \\
113.0\end{array}$ & $\begin{array}{l}1.10 E-07 \\
1.01 E-07 \\
9.52 E-08\end{array}$ & $\begin{array}{l}5.63 t-07 \\
5.16 E-07 \\
4.66 E-07\end{array}$ & $1.98 E-04$ & $1.01 E-03$ \\
\hline
\end{tabular}

$?$ INDICATES UNCERTAINTIES IN DATA.

X INDICATES INVAL IO UR INCUMPLETE UATA. 
TEST U79 SEPTEMBER 20, $1968 \quad 0027$ TU 0057 PST FLUORESCEIN RELEASE FROM ELEVATION DF $50 \mathrm{M}$ SAMPLER HT $1.5 \mathrm{M} \quad U=6.6 \mathrm{M} / \mathrm{SEC}$ AT $56 \mathrm{M}$

\begin{tabular}{|c|c|c|c|c|c|c|c|c|c|}
\hline $\begin{array}{l}\text { DISTANCE } \\
\text { FRUM }\end{array}$ & $-C B D$ & $\begin{array}{l}\text { ISHINQDO } \\
\text { STAND }\end{array}$ & $\begin{array}{l}\text { LSIRIBU } \\
\text { - CDEEE }\end{array}$ & IOA- & THREE & MAXIMUM E & OSURES & $\begin{array}{r}\text { CROS } \\
\text { INIEGRAI. }\end{array}$ & $\begin{array}{l}\text { WIND } \\
\text { D_YALUES }\end{array}$ \\
\hline SUURCE & MEAN & DEV & SKEW & KUR T & AZIMUTH & $E / Q$ & EU/O & $C I(E / Q)$ & CIIEU/OI \\
\hline METERS & DEG & DEG & & & DEG & SEC/CU.M & $1 / S a \cdot M$ & SEC/SO.M & $1 / M$ \\
\hline 400 & 139.0 & 9.18 & .368 & 1.956 & $\begin{array}{l}134.0 \\
154.0 \\
142.0\end{array}$ & $\begin{array}{l}5.04 E-09 \\
2.22 E-U 9 \\
1.64 E-09\end{array}$ & $\begin{array}{l}3.32 E-08 \\
1.47 E-08 \\
1.08 E-08\end{array}$ & $3.96 E-07$ & $2.61 E-06$ \\
\hline 800 & 130.3 & 2.32 & -.102 & 4.582 & $\begin{array}{l}129.5 \\
131.4 \\
127.7\end{array}$ & $\begin{array}{l}7.05 E-06 \\
5.37 E-06 \\
3.90 E-06\end{array}$ & $\begin{array}{l}4.65 E-05 \\
3.54 E-05 \\
2.57 E-05\end{array}$ & $5.61 E-04$ & $3.70 E-03$ \\
\hline 1200 & 130.2 & 1.57 & -.566 & 5.172 & $\begin{array}{l}130.0 \\
132.0 \\
128.0\end{array}$ & $\begin{array}{l}7.91 E-06 \\
2.94 E-06 \\
1.28 E-06\end{array}$ & $\begin{array}{l}5.22 E-05 \\
1.94 E-05 \\
8.44 E-06\end{array}$ & $5.44 E-04$ & $3.59 E-03$ \\
\hline 1600 & 130.5 & 1.68 & -.210 & 3.561 & $\begin{array}{l}130.6 \\
131.5 \\
129.7\end{array}$ & $\begin{array}{l}7.34 E-06 \\
6.09 E-06 \\
4.83 E-06\end{array}$ & $\begin{array}{l}4.84 E-05 \\
4.02 E-05 \\
3.19 E-05\end{array}$ & $7.38 E-04$ & $4.87 E-0.3$ \\
\hline 3200 & 129.0 & 1.59 & -.241 & 3.465 & $\begin{array}{l}129.3 \\
130.3 \\
127.3\end{array}$ & $\begin{array}{l}3.32 E-06 \\
2.51 E-06 \\
2.02 E-06\end{array}$ & $\begin{array}{l}2.19 E-05 \\
1.66 E-05 \\
1.34 E-05\end{array}$ & $6.81 E-04$ & $4,49 E-03$ \\
\hline 5000 & 124.6 & 2.83 & -.582 & 3.316 & $\begin{array}{l}126.0 \\
122.0 \\
124.0\end{array}$ & $\begin{array}{l}1.02 E-J 6 \\
5.19 E-07 \\
3.63 E-07\end{array}$ & $\begin{array}{l}6.74 E-06 \\
3.43 E-66 \\
2.39 E-06\end{array}$ & $4.28 E-04$ & $2.83 E-03$ \\
\hline 7000 & 124.0 & 2.59 & -.625 & 3.524 & $\begin{array}{l}126.0 \\
124.0 \\
122.0\end{array}$ & $\begin{array}{l}0.73 E-07 \\
8.06 E-07 \\
5.12 E-07\end{array}$ & $\begin{array}{l}5.79 E-06 \\
5.32 E-06 \\
3.38 E-06\end{array}$ & $6.95 E-04$ & $4.59 E-03$ \\
\hline 12800 & 115.2 & 3.73 & -.608 & 3.246 & $\begin{array}{l}115.0 \\
117.0 \\
114.0\end{array}$ & $\begin{array}{l}2.25 E-07 \\
2.25 E-U 7 \\
2.08 E-07\end{array}$ & $\begin{array}{l}1.48 E-06 \\
1.48 E-06 \\
1.37 E-06\end{array}$ & $4.09 E-04$ & $2.70 \mathrm{E}-03$ \\
\hline
\end{tabular}

? INOICATES UNCERTAINTIES IN DATA.

$X$ indicates inVALIU OR INCUMPLETE UATA. 
SAMPLING 400M TO 32U0M. NO TUWER SAMPLING. ALL ARCS TRUNCATED FUR 2NS RELEASE FROM 26M. SOME ARCS TRUNCATED FUR FLUURESCEIN KELEASE FROM 56M. TRACER "SMEARED" WIDELY IN CROSSWIND DIRECTION. THERMALLY VERY,STABLE ATMESPHERE. EQUIPMENT DIFFICULTIES UURING FLUDRESCEIIN ASSAY.

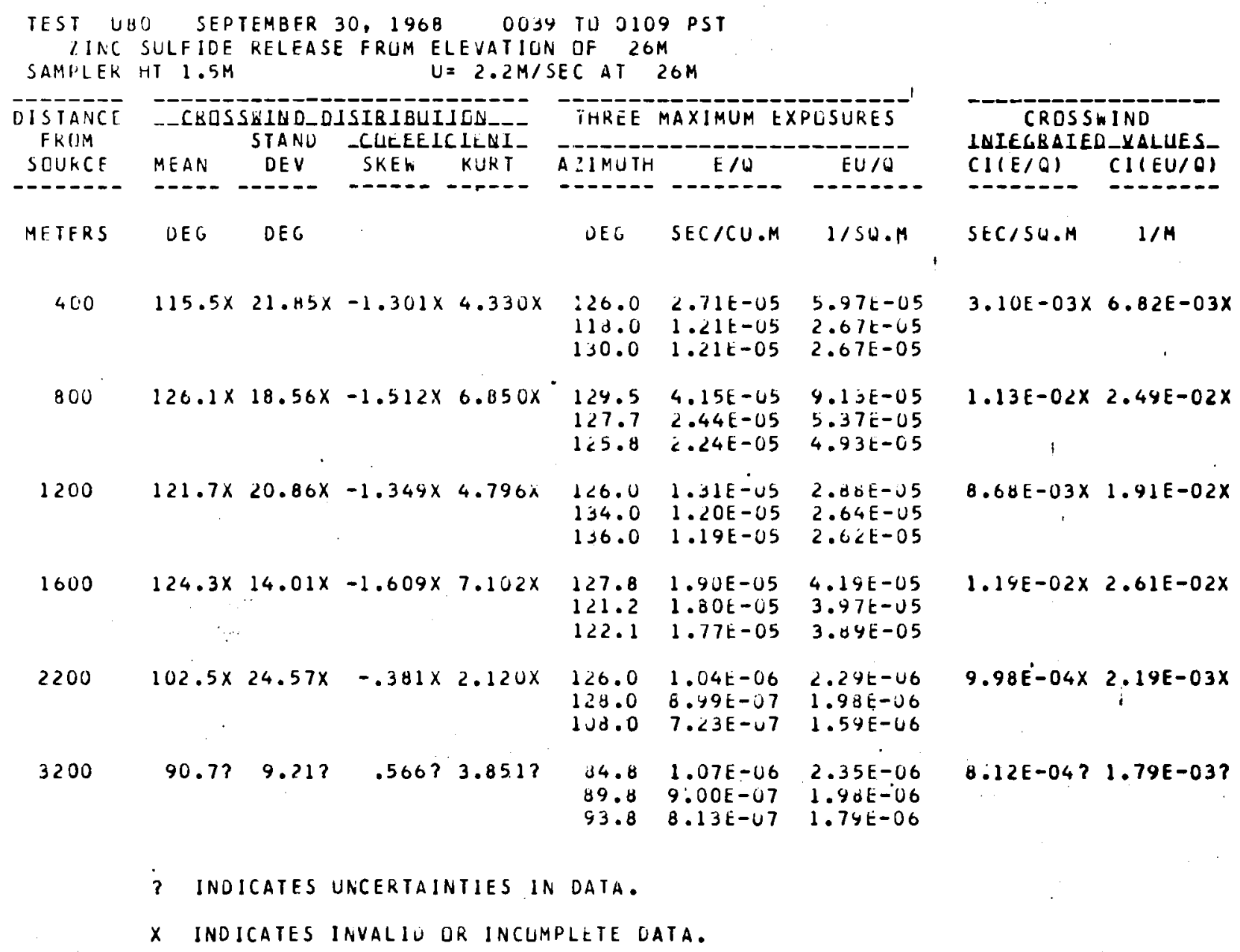




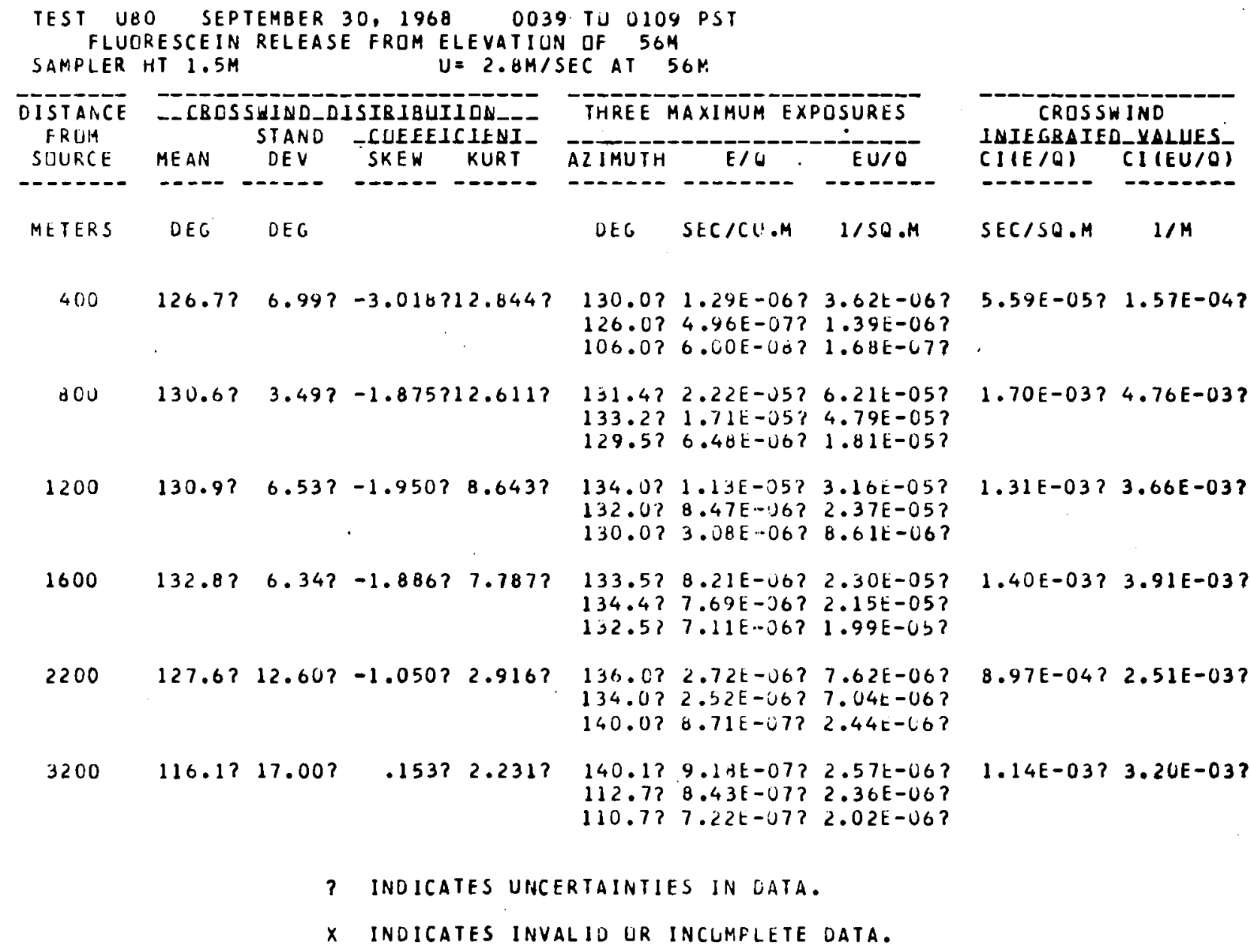

CROSSWIND

INIEGRAIED_YALUES_

CIIE/Q) CI (EU/O)

C.-.-- - - - - -

SEC/SO.M I/M

$1.70 E-03 ? 4.76 E-03 ?$

$1.31 E-03 ? 3.66 E-03 ?$

$1.40 E-03 ? 3.91 E-03 ?$

$8.97 E-04 ? 2.51 E-03 ?$

$1.14 E-03 ? 3.2 U E-03 ?$ 
SANPLING 4OOM TO $1280 U M$. NO TOWER SAMPLING. ALL ARCS EMBRACE ESSENTIALLY ENTIRE CROSSWIND EXTENT OF TRACER. HIGH UUST LEVEL DN FILTERS EXPOSED AT 50OOM, 7UUOM AND I2OOUM. IUUST DEGRADES CONFIDENCE IN TRACER ASSAY -ESPECIALLY ZNS.) LARCE WIND DIRECTIUN VARIANCE FLR RELATIVELY HIGH WIND SPEEU.

\begin{tabular}{|c|c|c|c|c|c|c|c|c|c|}
\hline $\begin{array}{l}\text { TEST UE } \\
\text { ZINC } \\
\text { SAMPLER }\end{array}$ & $\begin{array}{ll}1 & O C \\
\text { SULFIDE } \\
H T & 1.514\end{array}$ & $\begin{array}{l}\text { TOBER } \\
\text { RELFASE }\end{array}$ & $\begin{aligned} 1, & 1968 \\
\text { FRUM } & E \\
& U\end{aligned}$ & $\begin{array}{l}8 \quad 0111 \\
E L E V A I I L N \\
U=5.7 \mathrm{M} / \mathrm{S}\end{array}$ & $\begin{array}{l}\text { TU } 0141 \\
\text { UF } 26 \mathrm{P} \\
\text { EC AT }\end{array}$ & $\begin{array}{l}1 \text { PST } \\
\text { M } \\
26 M\end{array}$ & & & \\
\hline $\begin{array}{l}\text { UISTAIVE } \\
\text { FROM }\end{array}$ & \multicolumn{4}{|c|}{-CBOSSWINDODSIRIBUIICAV- } & THREE & \multicolumn{2}{|c|}{ MAXIMUM EXPOSURES } & \multicolumn{2}{|c|}{ CROSSHIND } \\
\hline SOURCF. & MEAN & CEV & SKEK' & KURT & AZIM!JTH & $E / C$ & $E \cup / 0$ & $C I(E / W)$ & $C I(E U / Q)$ \\
\hline$-\cdots-\infty$ & $-\cdots-$ & ---- & $-\cdots--$ & $-\cdots--$ & $---\cdot-\cdot$ & 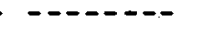 & $\cdots-\cdots$ & 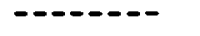 & $-\cdots----$ \\
\hline METERS & DEG & DEG & & & LE U & SEC/CU.M & $1 / S O \cdot M$ & SEC/SU.M & $1 / M$ \\
\hline 400 & $111.6 ?$ & $12.04 ?$ & $-.699 ?$ & $3.871 ?$ & $\begin{array}{l}114.0 ? \\
110.0 \\
110.0 ?\end{array}$ & $\begin{array}{l}9.51 t-06 ? \\
9.23 t-06 \\
8.01 t-06 ?\end{array}$ & $\begin{array}{l}5.42 E-05 ? \\
5.26 E-05 \\
5.02 E-05 ?\end{array}$ & $1.97 E-03 ?$ & $1.13 E-02 ?$ \\
\hline aco & 116.1 & 7.97 & -1.044 & 5.856 & $\begin{array}{l}118.3 \\
116.4 \\
114.5\end{array}$ & $\begin{array}{l}1.70 E-U S \\
1.41 E-05 \\
9.49 \mathrm{E}-U 6\end{array}$ & $\begin{array}{l}9.67 t-05 \\
0.01 E-05 \\
5.7 U E-05\end{array}$ & $3.01 E-03$ & $1.72 E-02$ \\
\hline 1200 & 116.2 & 6.26 & -1.431 & 6.631 & $\begin{array}{l}116.0 ? \\
118.0 \\
114.0\end{array}$ & $\begin{array}{l}7.08 E-06 ? \\
6.58 E-U 6 \\
3.67 E-U 6\end{array}$ & $\begin{array}{l}4.03 E-05 ? \\
3.15 E-05 \\
2.09 L-05\end{array}$ & $\begin{array}{c}1.43 E-03 \\
\cdots\end{array}$ & $8.16 E-03$ \\
\hline 1600 & 116.2 & 4.79 & -.973 & 4.892 & $\begin{array}{l}116.3 \\
117.3 \\
115.3\end{array}$ & $\begin{array}{l}6.15 E-06 \\
6.33 E-66 \\
5.28 E-66\end{array}$ & $\begin{array}{l}3.51 E-05 \\
3.43 E-05 \\
3.01 E-05\end{array}$ & $1.56 E-03$ & $8.91 E-03$ \\
\hline 2200 & 115.7 & 4.17 & -.550 & 3.592 & $\begin{array}{l}118.0 \\
114.0 \\
116.0\end{array}$ & $\begin{array}{l}4.35 E-07 \\
2.35 E-U 7 \\
2.12 E-U 7\end{array}$ & $\begin{array}{l}2.48 E-06 \\
1.34 E-06 \\
1.21 E-06\end{array}$ & $1: 15 E-04$ & $6.53 E-04$ \\
\hline 3200 & 115.8 & 3.46 & -.394 & 3.194 & $\begin{array}{l}116.6 \\
117.6 \\
115.6\end{array}$ & $\begin{array}{l}1.16 E-06 \\
1.05 E-06 \\
1.02 E-06\end{array}$ & $\begin{array}{l}6.02 E-06 \\
5 . y 9 E-06 \\
5.82 t-06\end{array}$ & $5.73 E-04$ & $3.27 E-03$ \\
\hline 5000 & $115.6 ?$ & $2.69 ?$ & $-.080 ?$ & $2.932 ?$ & $\begin{array}{l}114.0 ? \\
118.0 ? \\
116.0 ?\end{array}$ & $\begin{array}{l}6.50 E-08 ? \\
5.99 E-08 ? \\
5.32 E-08 ?\end{array}$ & $\begin{array}{l}3.70 E-07 ? \\
3.41 E-07 ? \\
3.03 E-07 ?\end{array}$ & $4.06 E-05 ?$ & $2.32 E-04 ?$ \\
\hline 7000 & $114.7 ?$ & $3.79 ?$ & $-.091 ?$ & $2.999 ?$ & $\begin{array}{l}116.0 ? \\
114.0 ? \\
112.0 ?\end{array}$ & $\begin{array}{l}2.13 t-07 ? \\
2 . U 2 t-47 ? \\
1.17 t-07 ?\end{array}$ & $\begin{array}{l}1.22 E-06 ? \\
1.15 E-06 ? \\
7.82 E-07 ?\end{array}$ & $2.36 E-04 ?$ & $1.35 E-03 ?$ \\
\hline $\begin{array}{c}12800 \\
?\end{array}$ & $112.6 ?$ & $4.09 ?$ & $-.295 ?$ & $2.405 ?$ & $\begin{array}{l}111.0 ? \\
112.0 ? \\
110.0 ?\end{array}$ & $\begin{array}{l}2.77 t-\cup 8 ? \\
2.76 E-\cup 8 ? \\
2.74 E-\cup 8 ?\end{array}$ & $\begin{array}{l}1.53 E-07 ? \\
1.57 E-07 ? \\
1.56 E-07 ?\end{array}$ & $7.82 E-05 ?$ & $4.46 E-04 ?$ \\
\hline & IND IC & CATES & CERTAIN & NTIES IN & DATA. & & & & \\
\hline & II & ES & 00 & OR I & $E$ & A. & & & \\
\hline
\end{tabular}




\begin{tabular}{|c|c|c|c|c|c|c|c|c|c|}
\hline $\begin{array}{l}\text { TEST UB } \\
\text { FLUD } \\
\text { SAMPLER }\end{array}$ & $\begin{array}{l}\text { I } \\
\text { QESCEIN } \\
\text { HT } 1.5 \mathrm{H}\end{array}$ & $\begin{array}{l}\text { CTOBER } \\
\text { RELEAS }\end{array}$ & E FROM 196 & $\begin{array}{r}011 \\
\text { LEVATIL } \\
=7.011\end{array}$ & $\begin{array}{l}\text { TD } 014 \\
\text { QF } 56 \\
\text { EC AT }\end{array}$ & $\begin{array}{l}1 \text { PST } \\
M \\
56 M\end{array}$ & & & 1 \\
\hline $\begin{array}{l}\text { CISTANCE } \\
\text { FKCH }\end{array}$ & \multicolumn{4}{|c|}{$\begin{array}{l}\text { CROSSHIND OISIRIBUIIDN } \\
\text { STANU -COEEEICIENI- }\end{array}$} & THREE & \multicolumn{2}{|c|}{ MAXIMUM EXPOSURES } & \multicolumn{2}{|c|}{$\begin{array}{l}\text { CROSSHIND } \\
\text { INIEGKAIEU_KALUES_' }\end{array}$} \\
\hline SITURCE & MEAN & DEV & SKEW & KUR T & AZ IMUTH & . $E / G$ & $E \cup / 0$ & $C I(E / U)$ & $C I(E U / O)$ \\
\hline------- & ---- & 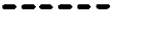 & -- &.--- & -------- & -- & ---- & -- & \\
\hline METERS & DEC & DEG & & & DEC & $S E C / C U . M$ & $1 / 5 O . M$ & SEC/SU.M & $4 / M$ \\
\hline 400 & 115.9 & 11.76 & .829 & 2.791 & $\begin{array}{l}110.0 \\
138.0 \\
114.0\end{array}$ & $\begin{array}{l}3.60 E-06 \\
2.04 E-J 6 \\
1.02 E-J 6\end{array}$ & $\begin{array}{l}2.52 E-05 \\
1.43 E-05 \\
1.27 E-C 5\end{array}$ & $3.46 E-04$ & $2.42 E-03$ \\
\hline HOO & 114.0 & 6.23 & .462 & 3.478 & $\begin{array}{l}114.5 \\
112.6 \\
108.8\end{array}$ & $\begin{array}{l}6.52 E-U 6 \\
5.76 t-U 6 \\
4.96 E-\cup 6\end{array}$ & $\begin{array}{l}4.56 E-05 \\
4.04 E-05 \\
3.47 t-05\end{array}$ & $1.11 t-03$ & $7.78 E-03$ \\
\hline 1200 & 116.4 & 5.24 & .313 & 3.363 & $\begin{array}{l}118.0 \\
114.0 \\
112.0\end{array}$ & $\begin{array}{l}4.26 E-U 6 \\
3.59 E-06 \\
2.32 E-06\end{array}$ & $\begin{array}{l}2.98 E-05 \\
2.51 E-05 \\
1.62 E-05\end{array}$ & $8.44 E-04$ & $5.91 E-03$ \\
\hline 1600 & 117.2 & 3.53 & .314 & 3.518 & $\begin{array}{l}114.4 \\
118.2 \\
115.3\end{array}$ & $\begin{array}{l}7.61 E-J 6 \\
6.61 E-06 \\
6.25 E-J E\end{array}$ & $\begin{array}{l}5.32 E-05 \\
4.63 E-65 \\
4.40 t-05\end{array}$ & $1.30 E-03$ & $9.11 E-03$ \\
\hline 2200 & 117.8 & 3.43 & .140 & 3.482 & $\begin{array}{l}116.0 \\
118.0 \\
122.0\end{array}$ & $\begin{array}{l}1.49 E-J 6 \\
1.33 E-J 6 \\
6.57 E-07\end{array}$ & $\begin{array}{l}1.04 E-05 \\
9.29 E-00 \\
4.00 t-06\end{array}$ & $3.65 E-04$ & $2.55 E-03$ \\
\hline 3200 & 118.1 & 3.84 & .151 & 2.773 & $\begin{array}{l}116.6 \\
117.6 \\
114.6\end{array}$ & $\begin{array}{l}2.02 E-U 6 \\
1.28 E-U 6 \\
1.16 E-U 6\end{array}$ & $\begin{array}{l}1.41 E-05 \\
8.94 E-06 \\
8.15 E-06\end{array}$ & $7.08 E-04$ & $.4 .96 E-03$ \\
\hline 5000 & 117.3 & 4.22 & .392 & 2.253 & $\begin{array}{l}116.0 \\
114.0 \\
112.0\end{array}$ & $\begin{array}{l}\text { 4. CUE-U7 } \\
2.69 E-U 7 \\
1.96 t-U 7\end{array}$ & $\begin{array}{l}2.80 E-06 \\
1.8 d E-06 \\
1.37 E-66\end{array}$ & $2.69 t-04$ & $1.88 E-03$ \\
\hline 7000 & 116.0 & 4.52 & .145 & 2.744 & $\begin{array}{l}118.0 \\
116.0 \\
114.0\end{array}$ & $\begin{array}{l}5 . \cup 3 E-U 7 \\
4.2 \cup E-J 7 \\
3.80 E-J 7\end{array}$ & $\begin{array}{l}3.52 E-06 \\
2.94 E-06 \\
2.66 t-06\end{array}$ & $6.65 E-04$ & $4.65 E-03$ \\
\hline 12000 & 113.9 & 4.96 & .357 & 4.408 & $\begin{array}{l}117.0 \\
116.0 \\
110.0\end{array}$ & $\begin{array}{l}1.04 E-07 \\
1.30 E-07 \\
1.12 E-07\end{array}$ & $\begin{array}{l}1.29 E-06 \\
9.13 E-U 7 \\
7.01 E-07\end{array}$ & $3.16 k-04$ & $2.21 E-03$ \\
\hline
\end{tabular}

7 INDicates uncertainties in cata.

$X$ indicates inValid or incumplete data. 
SAMPLING $400 \mathrm{M}$ TO $12800 \mathrm{M}$. NO TOWEK SAMPLING. ALL ARCS EMBRACE CRUSSWIND EXTENT UF TRACER. NU FLUDRESCEIN DETECTEO AT $400 M$ ARC. HIGH DUST LFVEL LN FILIERS LIN 5000,7000 ANO $1280 D M$ ARCS. IDUST DEGRADES CONFIOENCE IN TRACER ASSAY -- PARTICULARLY ZNS.) LARGE OIRECTILN SHEAR. PLUME CENTERLINE SHIFTS LUCATION STARTING AT 32OOM.

\begin{tabular}{|c|c|c|c|c|c|c|c|c|c|}
\hline $\begin{array}{r}\text { TEST U } \\
\text { ZINC } \\
\text { SAMPLER }\end{array}$ & $\begin{array}{lr}82 & 0 \\
\text { SULF IOE } \\
\text { HT } 1.5 M\end{array}$ & $\begin{array}{l}\text { CTOBER } \\
\text { RELFASE }\end{array}$ & $=2,1968$ & $\begin{array}{l}8 \quad 011 \\
E L E V A T I O \\
U=4.7 \mathrm{k} /\end{array}$ & $\begin{array}{l}1 \text { TU } 014 \\
N \text { OF } 261 \\
\text { SEC AT }\end{array}$ & $\begin{array}{l}1 \text { PST } \\
26 M\end{array}$ & & & \\
\hline \multirow{2}{*}{$\begin{array}{l}\text { DISTAIVCE } \\
\text { FRDM } \\
\text { SOURCE }\end{array}$} & \multicolumn{4}{|c|}{$\begin{array}{l}\text {-CBOSSHIND_DISIBIBUIION- } \\
\text { STAND -COEEEICIEUI_- }\end{array}$} & THREE & \multicolumn{2}{|c|}{ MAXIMUM EXPOSURES } & \multicolumn{2}{|c|}{$\begin{array}{l}\text { CROSSWINO } \\
\text { INIEGRAIED_KALUES }\end{array}$} \\
\hline & MEAN & DEV & SKEW & KURT & ALIMUTH & $E / Q$ & EU/O & $C I(E / C)$ & $C 1(E U / Q)$ \\
\hline METERS & OEG & DEG & & & OEG & $S E C / C U, M$ & $1 / 50 . M$ & $S E C / S O \cdot M$ & $1 / M$ \\
\hline 400 & 115.2 & 4.90 & -.835 & .3 .149 & $\begin{array}{l}118.0 \\
114.0 \\
110.0\end{array}$ & $\begin{array}{l}2.93 E-06 \\
1.27 E-06 \\
9.04 E-07\end{array}$ & $\begin{array}{l}1.38 E-05 \\
5.95 E-06 \\
4.25 E-06\end{array}$ & $1.83 E-04$ & $8.59 E-04$ \\
\hline 800 & 115.6 & 4.41 & -.679 & 3.852 & $\begin{array}{l}118.3 \\
114.5 \\
116.4\end{array}$ & $\begin{array}{l}1.36 E-05 \\
1.22 E-05 \\
1.07 E-05\end{array}$ & $\begin{array}{l}6.41 E-05 \\
5.73 E-05 \\
5.01 E-05\end{array}$ & $1.90 E-03$ & $8.93 E-03$ \\
\hline 1200 & $\begin{array}{l}113.9 \\
+\end{array}$ & 4.53 & -.863 & 4.477 & $\begin{array}{l}114.0 \\
116.0 \\
118.0\end{array}$ & $\begin{array}{l}9.51 E-06 \\
8.58 E-06 ? \\
7.38 E-06\end{array}$ & $\begin{array}{l}4.47 E-05 \\
4.03 E-05 ? \\
3.47 E-05\end{array}$ & $1.87 E-03$ & $8.79 E-03$ \\
\hline 1600 & 113.8 & 5.07 & -.467 & 3.116 & $\begin{array}{l}117.3 \\
113.4 \\
115.3\end{array}$ & $\begin{array}{l}8.20 E-06 \\
8.17 t-06 \\
7.37 E-06\end{array}$ & $\begin{array}{l}3.85 E-05 \\
3.84 E-05 \\
3.46 E-05\end{array}$ & $2.86 E-03$ & $1.34 E-02$ \\
\hline 2200 & 113.4 & 4.70 & -.226 & 3.937 & $\begin{array}{l}116.0 \\
114.0 \\
112.0\end{array}$ & $\begin{array}{l}1.3 \square E-06 \\
1.31 E-06 \\
8.40 E-07 ?\end{array}$ & $\begin{array}{l}6.49 E-06 \\
6.17 E-06 \\
3.95 E-06 ?\end{array}$ & $4.48 E-04$ & $2.11 E-03$ \\
\hline 3200 & 118.1 & 5.78 & -.398 & 2.327 & $\begin{array}{l}119.5 \\
113.7 \\
120.5\end{array}$ & $\begin{array}{l}3.41 E-06 \\
2.90 E-06 \\
2.56 E-06\end{array}$ & $\begin{array}{l}1.60 E-05 \\
1.36 E-05 \\
1.20 E-05\end{array}$ & $1.64 E-03$ & $7.70 E-03$ \\
\hline 5000 & 120.9 & 5.14 & -.087 & 2.282 & $\begin{array}{l}116.0 \\
126.0 \\
124.0\end{array}$ & $\begin{array}{l}7.71 E-U 7 \\
7.57 E-U 7 \\
5.38 E-07\end{array}$ & $\begin{array}{l}3.63 E-06 \\
3.56 E-06 \\
2.53 E-06\end{array}$ & $7.21 E-04$ & $3.39 E-03$ \\
\hline 7000 & 129.1 & 5.60 & -.691 & 2.727 & $\begin{array}{l}134.0 \\
132.0 \\
130.0\end{array}$ & $\begin{array}{l}1.70 E-06 \\
1.32 E-06 ? \\
1.08 E-06\end{array}$ & $\begin{array}{l}8.00 E-06 \\
6.21 E-06 ? \\
5.05 E-06\end{array}$ & $2.15 E-03$ & $1.01 E-02$ \\
\hline 12.800 & 130.9 & 2.14 & -.608 & 4.068 & $\begin{array}{l}133.0 \\
132.0 \\
130.0\end{array}$ & $\begin{array}{l}9.07 E-07 \\
5.04 E-07 \\
4.56 E-07 ?\end{array}$ & $\begin{array}{l}4.26 E-06 \\
2.75 E-06 \\
2.14 E-06 ?\end{array}$ & $7.35 E-04$ & $3.45 E-03$ \\
\hline
\end{tabular}

? INDICATES UNCERTAINTIES IN DATA.

$x$ INDICATES INVAL ID DR INCOMPLETE DATA. 
$\begin{array}{llllll}\text { TEST UB2 OCTDBER } 2,1968 \text { TO } & 111 & 0141 & \text { PST }\end{array}$ FLURRESCEIN RELEASE FROM ELFVATION DF $56 \mathrm{M}$

SAMPLER HT $1.5 \mathrm{M} \quad U=4.6 \mathrm{M} / \mathrm{SEC}$ AT $56 \mathrm{M}$

\begin{tabular}{|c|c|c|c|c|c|c|c|c|c|}
\hline $\begin{array}{l}\text { DISTANCE } \\
\text { FRTM }\end{array}$ & _CROS & $\begin{array}{l}\text { SHIUD_D } \\
\text { STAND }\end{array}$ & $\begin{array}{l}\text { ISIRIBUI } \\
\text {-COEEE }\end{array}$ & IDN- & THREE & MAXIMUM EX & OSURES & $\begin{array}{l}\text { CRUSS } \\
\text { INIEGRAIE }\end{array}$ & $\begin{array}{l}\text { IND } \\
\text { D_YALUES. }\end{array}$ \\
\hline SOURCE & MEAN & DEV & SKEW & KURT & AZIMUTH & $E / O$ & $E U / Q$ & $C I(E / Q)$ & $C I(E U / Q)$ \\
\hline$-\cdots+--$ & ---- & $-\cdots-\infty$ & $--\infty-\infty$ & $-\cdots-\infty$ & ------ & ------ & ------ & $---\cdots---$ & -- \\
\hline METFRS & DEG & DEG & & & DEG & SEC/CU.M & $1 / 50 . M$ & SEC/SO.M & $1 / M$ \\
\hline 400 & 0.0 & 0.00 & 0.000 & 0.000 & $\begin{array}{l}0.0 \\
0.0 \\
0.0\end{array}$ & $\begin{array}{l}0 . \\
0 . \\
0 .\end{array}$ & $\begin{array}{l}0 . \\
0 . \\
0 .\end{array}$ & 0 & 0 \\
\hline 800 & 123.1 & 2.00 & -.855 & 6.066 & $\begin{array}{l}122.1 \\
125.8 \\
124.0\end{array}$ & $\begin{array}{l}3.05 E-06 \\
1.28 E-06 \\
1.20 E-06\end{array}$ & $\begin{array}{l}1.40 E-05 \\
5.8 B E-06 \\
5.52 E-06\end{array}$ & $1.63 E-04$ & $7.50 \mathrm{E}-04$ \\
\hline 1200 & 124.0 & 2.98 & .090 & 3.358 & $\begin{array}{l}124.0 \\
126.0 \\
120.0\end{array}$ & $\begin{array}{l}2.54 E-06 \\
2.22 E-06 \\
1.19 E-06\end{array}$ & $\begin{array}{l}1.17 E-05 \\
1.0>E-05 \\
5.46 E-06\end{array}$ & $3.40 E-04$ & $1.57 \mathrm{E}-03$ \\
\hline 1600 & 123.6 & 3.23 & .768 & 3.799 & $\begin{array}{l}123.1 \\
124.1 \\
122.1\end{array}$ & $\begin{array}{l}7.06 E-06 \\
4.65 E-36 \\
3.96 E-06\end{array}$ & $\begin{array}{l}3.25 E-05 \\
2.14 E-05 \\
1.82 E-05\end{array}$ & $8.66 E-04$ & $3.98 \mathrm{E}-03$ \\
\hline 2200 & 124.5 & 3.62 & .372 & 3.016 & $\begin{array}{l}124.0 \\
126.0 \\
122.0\end{array}$ & $\begin{array}{l}1.89 E-06 \\
1.61 E-06 \\
1.58 E-06\end{array}$ & $\begin{array}{l}8.72 E-06 \\
7.42 E-06 \\
7.25 E-06\end{array}$ & $6.17 E-04$ & $2.84 E-03$ \\
\hline 3200 & 125.8 & 4.80 & .060 & 2.877 & $\begin{array}{l}123.4 \\
125.4 \\
124.4\end{array}$ & $\begin{array}{l}2.02 E-06 \\
2.02 E-06 \\
1.64 E-06\end{array}$ & $\begin{array}{l}9.31 E-06 \\
9.31 E-06 \\
7.52 E-06\end{array}$ & $1.11 \mathrm{E}-03$ & $5.12 E-03$ \\
\hline 5000 & 128.7 & 6.14 & -.421 & 2.951 & $\begin{array}{l}128.0 \\
136.0 \\
126.0\end{array}$ & $\begin{array}{l}3.49 E-.07 \\
3.35 E-07 \\
2.73 E-07\end{array}$ & $\begin{array}{l}1.60 E-06 \\
1.54 E-06 \\
1.26 E-06\end{array}$ & $3.93 E-04$ & $1.81 E-03$ \\
\hline 7000 & 132.6 & 4.94 & -.219 & 3.865 & $\begin{array}{l}134.0 \\
130.0 \\
136.0\end{array}$ & $\begin{array}{l}6.16 E-07 \\
3.01 E-07 \\
2.67 E-07\end{array}$ & $\begin{array}{l}2.83 E-06 \\
1.38 E-06 \\
1.23 E-06\end{array}$ & $5.40 E-04$ & $2.49 E-03$ \\
\hline 12800 & 134.6 & 4.40 & .640 & 3.244 & $\begin{array}{l}133.0 \\
134.0 \\
132.0\end{array}$ & $\begin{array}{l}3.08 E-07 \\
2.96 E-07 \\
2.73 E-07\end{array}$ & $\begin{array}{l}1.41 E-06 \\
1.36 E-06 \\
1.26 E-06\end{array}$ & $6.47 E-04$ & $2 .-98 E-03$ \\
\hline
\end{tabular}

$?$ INDICATES UNCERTAINTIES IN DATA.

x INDICATES INVALID OR INCQMPLETE data. 
SAMPLING 400M TO 7000M. NO TOWER SAMPLING. ALL ARCS EMBRACE CROSSWIND EXTENT OF TRACER. CENTERLINES DF FLUDRESCEIN AND ZINC SULFIDE PLUMES AT SIGNIFICANTLY DIFFERENT OIREC.TIUNS. THERMALLY VERY STABLE ATMDSPHERE. LARGE DIRECTIDN SHEAR WITH HEIGHT.

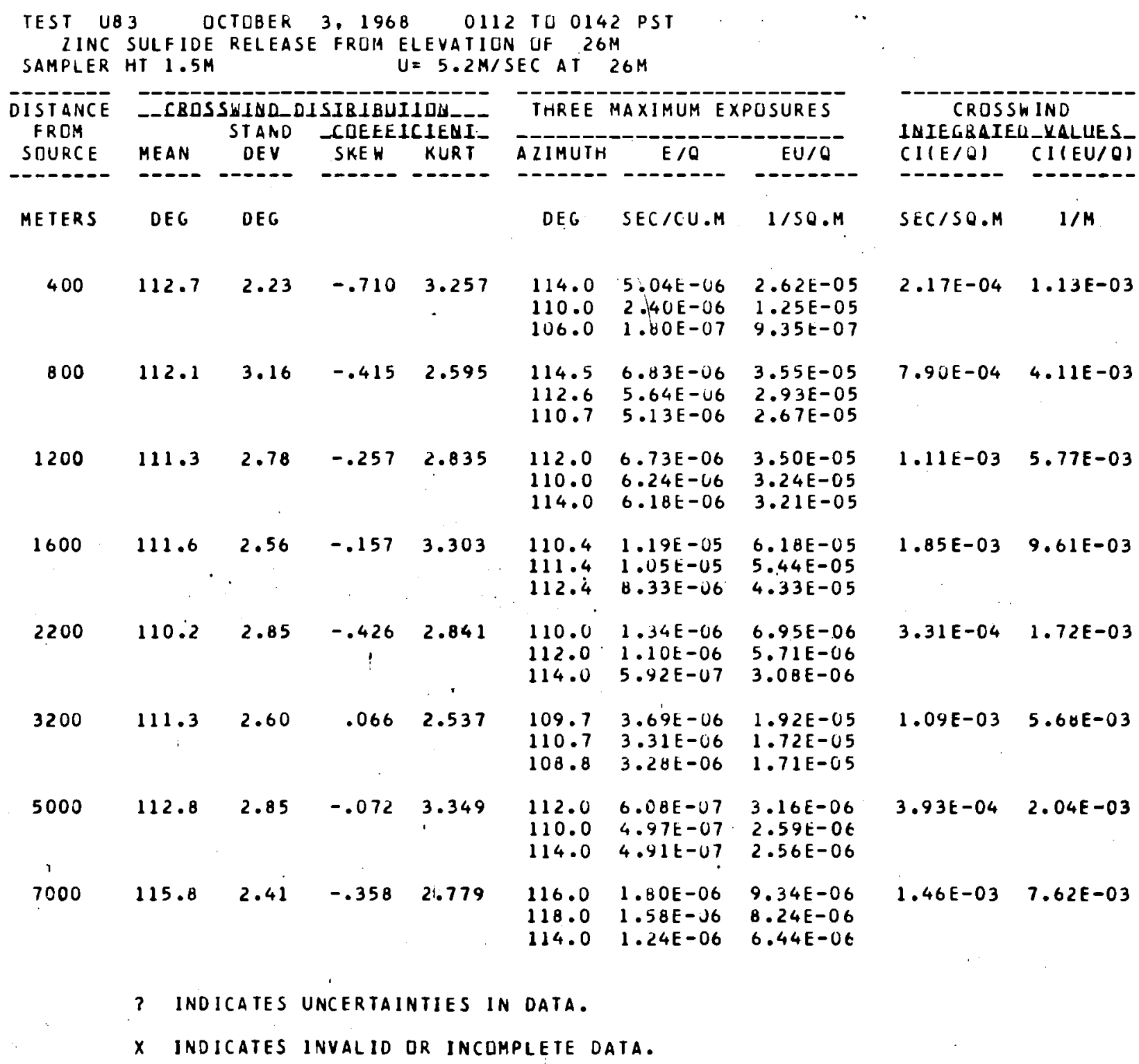




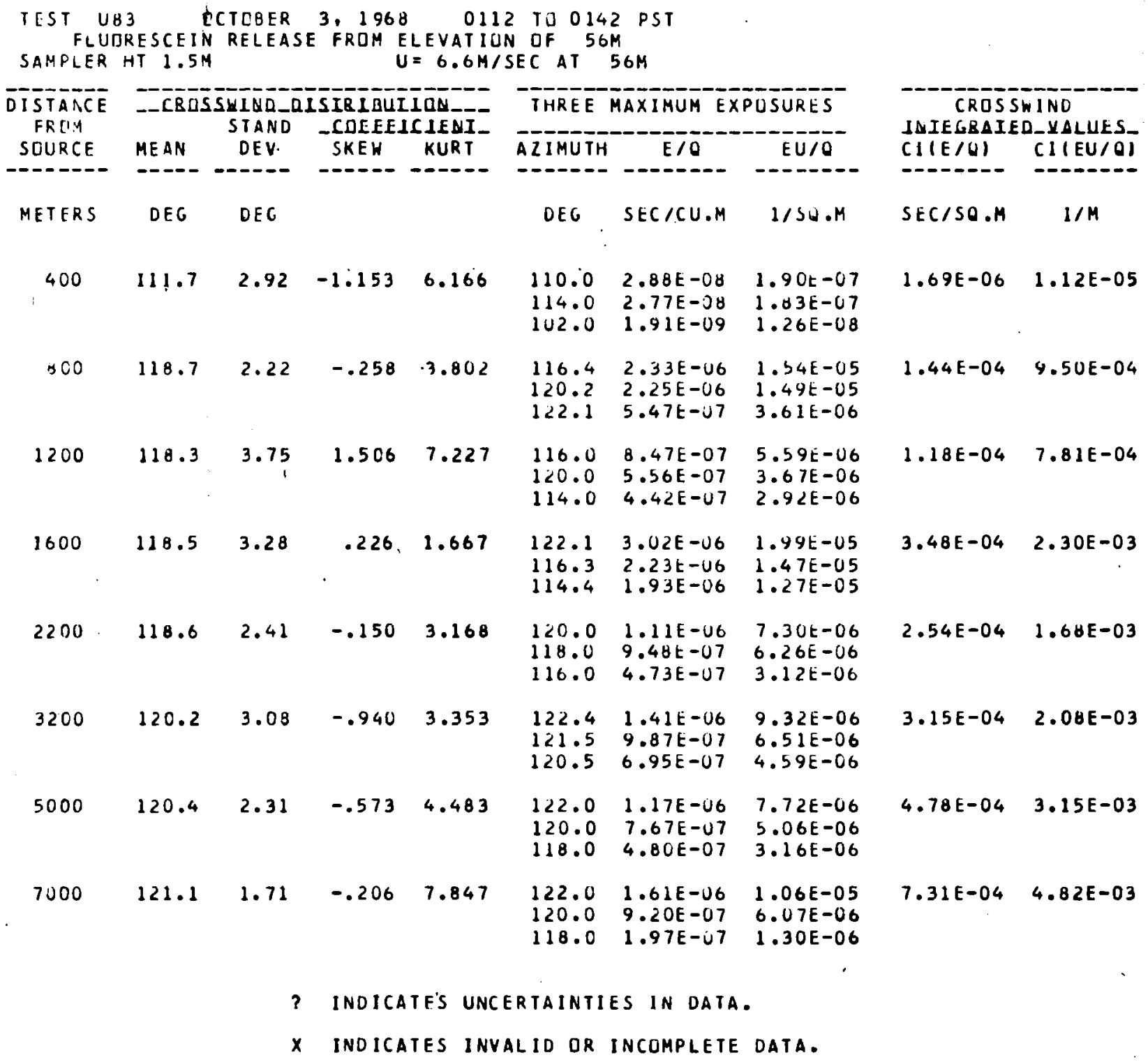


SAMPLING $400 M$ TO $12000 M$ : NO TOHER SAMPLING. UNLY ZNS TRACER WAS DISPERSED. NO TRACER OBSERVED ON $40 O M$ OR GOOM ARCS. WITH THE EXCEPTION OF THE 12800 M ARC. ALL ARCS EXPDSEO TO TRACER EMBRACED THE ENTIRE CROSSWIND CISTRIBUTION.

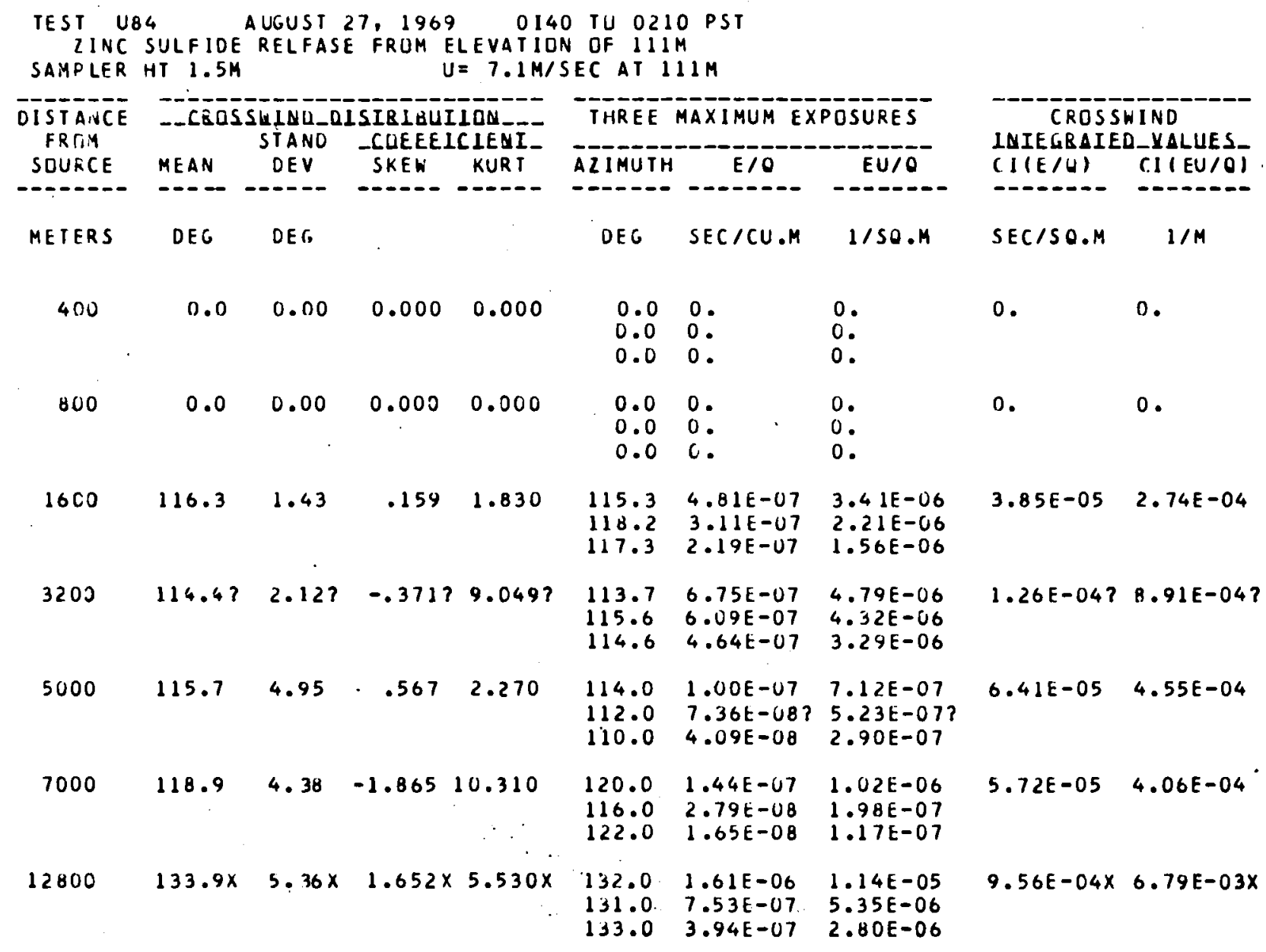

$?$ INOICATES UNCERTAINTIES IN DATA.

X INOICATFS INVALIO OR INCOMPLETE data. 


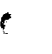

.

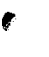

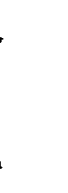

$\vartheta$ 
SAMPLING $400 M$ TO $12800 M$ : ND TOHER SAMPLING.

ALL ARCS EMBRACE CROSSKIND EXTENT OF TRACER DISTRIBUTIONS. NU ZNS TRACEK OBSERVFD UN 4 OOM ARC. SPORADIC FLULRESCEIN DISPERSAL DURING FIRST 10 MIN. UF TEST; IK OURING LAST 20 HIN.

TEST UBS AUGUST 28,19690154 TL 0224 PST

ZINC SULFIOE RELFASE FROM ELEVATION OF IIIM

SAMPLER HT $1.5 \mathrm{M} \quad U=9.7 \mathrm{M} / \mathrm{SEC}$ AT $111 \mathrm{M}$

\begin{tabular}{|c|c|c|c|c|c|c|c|c|c|}
\hline $\begin{array}{l}\text { DISTANCE } \\
\text { FRGM }\end{array}$ & -cesas & $\begin{array}{l}\text { SILNO_D } \\
\text { STAND }\end{array}$ & $\begin{array}{l}\text { SIRIOUI } \\
\text {-CDEEE }\end{array}$ & CIENI- & THREE & 4AXIMUM EX & PUSURES & $\begin{array}{l}\text { CRUSS } \\
\text { INIELKAIE }\end{array}$ & $\begin{array}{l}\text { INU } \\
\text { 2_YALUES_ }\end{array}$ \\
\hline SOURCE & KEAN & DEV & SKEN & KURT & AZIMUTH & $E / O$ & $E U / G$ & $C I(E / U)$ & (IIEU/U) \\
\hline METERS & DEG & DEG & & & DEC & SEC/CU.M & 1/SQ.M & $\mathrm{SEC} / \mathrm{SW} \cdot \mathrm{H}$ & $1 / M$ \\
\hline 400 & 0.0 & 0.00 & 0.000 & 0.000 & $\begin{array}{l}0.0 \\
0.0 \\
0.0\end{array}$ & $\begin{array}{l}0 . \\
0 . \\
0 .\end{array}$ & $\begin{array}{l}0 . \\
0 . \\
0 .\end{array}$ & 0. & 0 . \\
\hline 800 & 107.9 & 3.98 & .208 & 2.458 & $\begin{array}{l}105.0 \\
106.9 \\
110.7\end{array}$ & $\begin{array}{l}7.49 E-U 8 \\
6.69 E-08 \\
6.59 E-08\end{array}$ & $\begin{array}{l}7.26 E-07 \\
6.4 Y E-07 \\
6.39 E-J 7\end{array}$ & $9.54 \mathrm{E}-06$ & $9.25 E-05$. \\
\hline 1600 & 107.6 & 4.89 & .336 & 2.225 & $\begin{array}{l}106.5 \\
100.6 \\
107.5\end{array}$ & $\begin{array}{l}1.04 E-06 \\
6.01 E-07 \\
5.47 E-U 7\end{array}$ & $\begin{array}{l}1.01 E-05 \\
6.41 E-06 \\
5.3 \mathrm{iE}-06\end{array}$ & $2.18 t-04$ & 2.11E-03 \\
\hline 3200 & 108.4 & 3.65 & -.134 & 2.196 & $\begin{array}{l}106.5 \\
109.7 \\
107.6\end{array}$ & $\begin{array}{l}0.10 E-C 7 \\
6.47 E-07 \\
6.40 E-07\end{array}$ & $\begin{array}{l}7.03 E-J 6 \\
6.20 E-06 \\
6.20 E-06\end{array}$ & $4.03 t-04$ & $3.91 E-\cup 3$ \\
\hline 5000 & 139.0 & 2.77 & .815 & 3.177 & $\begin{array}{l}108.0 \\
106.0 \\
110.0\end{array}$ & $\begin{array}{l}5.31 E-077 \\
4.34 L-J 7 \\
4.03 E-07\end{array}$ & $\begin{array}{l}5.73 E-U 6 ? \\
4.21 E-U 6 \\
3.90 E-06\end{array}$ & $3.09 E-04$ & $3.00 E-03$ \\
\hline 7000 & 111.07 & 4. $37 ?$ & $.774 ?$ & $2.806 ?$ & $\begin{array}{l}104.0 ? \\
106.0 ? \\
110.0 ?\end{array}$ & $\begin{array}{l}3.07 t-08 ? \\
2.79 E-08 ? \\
2.34 E-06 ?\end{array}$ & $\begin{array}{l}2 . y \forall E-07 ? \\
2.71 \mathrm{E}-07 ? \\
2.27 \mathrm{E}-07 ?\end{array}$ & $3.44 t-05 ?$ & $3.34 E-04 ?$ \\
\hline 12800 & 114.5 & 2.26 & -.097 & 1.847 & $\begin{array}{l}116.0 \\
112.0 \\
117.0\end{array}$ & $\begin{array}{l}7.24 E-08 \\
6.22 E-08 \\
4.3 B E-08\end{array}$ & $\begin{array}{l}6.42 E-07 \\
6.03 E-07 \\
4.25 E-07\end{array}$ & $7.94 E-05$ & $7.71 E-04$ \\
\hline
\end{tabular}

$?$ INDICATFS UNCERTAINTIES IN DATA.

X INUICATFS INVALIO OR INCUMPLETE DATA. 


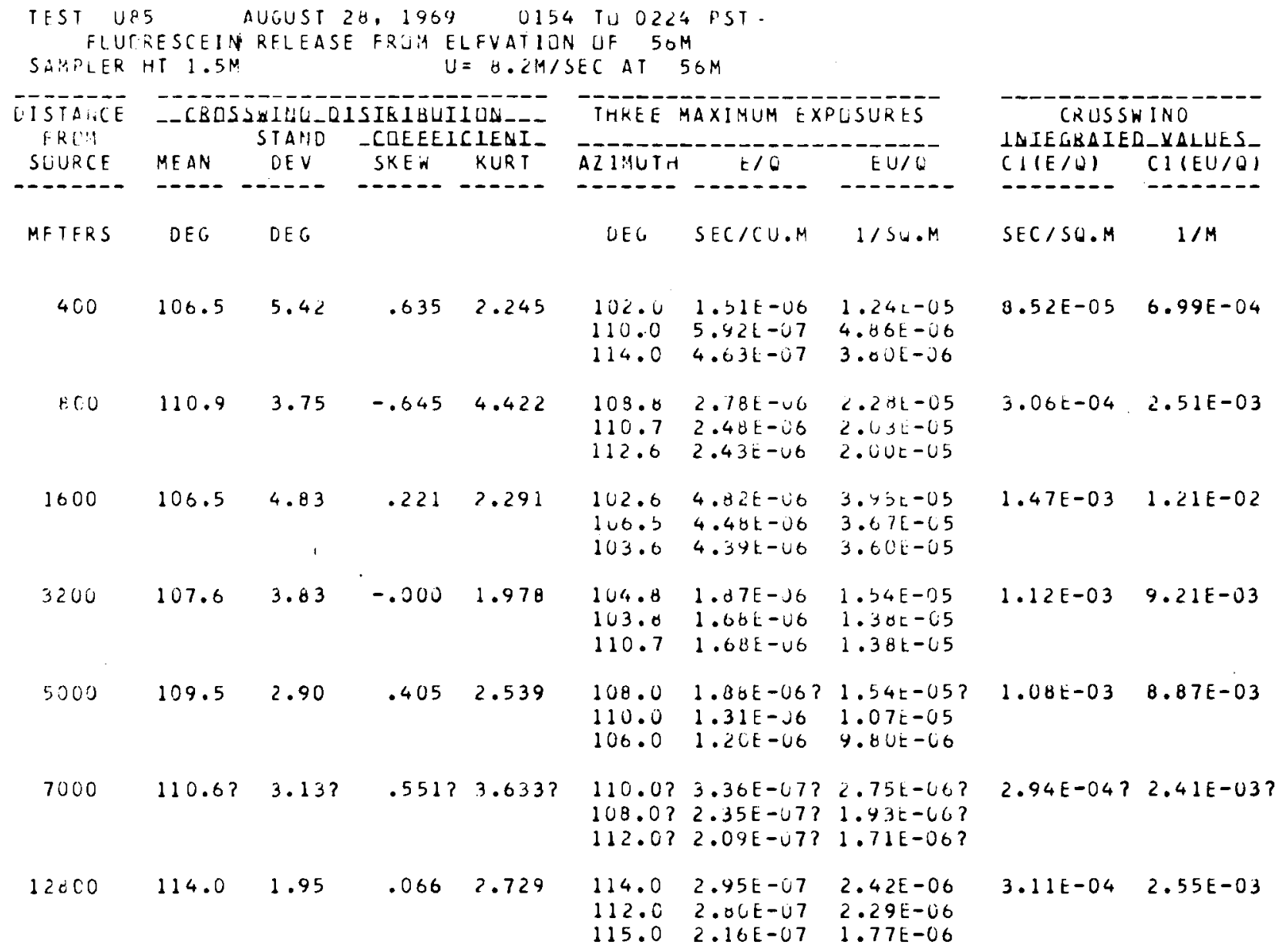

$?$ INOICATFS UNCERTAINTIES IN DATA.

x indicates invalio or incemplete uata. 


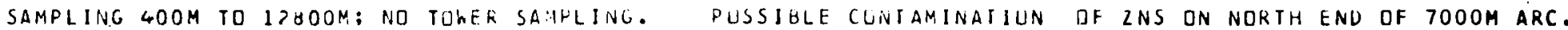
ARCS GENERALLY EMURACE CROSSWINU EXTLRT LiF TKACEK. NU LNS OBSERVED ON 4 UOM OR $80 O M$ ARCS.

FISSSIBLE FLUORESCEIA CONTAMINATIITN ON $40 \mathrm{U}$ ANU ULU:i ARCS.

\begin{tabular}{|c|c|c|c|c|c|c|c|c|c|}
\hline \multirow{2}{*}{$\begin{array}{l}\text { DISTAUCE } \\
\text { FRTS } \\
\text { SOURCE }\end{array}$} & \multicolumn{4}{|c|}{-CBDSSUINDAISIRICUIIUY } & \multicolumn{3}{|c|}{ THREE MAXIMUVA EXPUSUKES } & \multirow{2}{*}{\multicolumn{2}{|c|}{$\begin{array}{l}\text { CRUSSW IND } \\
\text { JWIELBAIEL_VALUES }\end{array}$}} \\
\hline & MEAN & $\begin{array}{l}\text { STAND } \\
\text { DEV }\end{array}$ & $\begin{array}{l}\text {-CDEEE } \\
\text { SKEW }\end{array}$ & $\begin{array}{l}\text { CIESI- } \\
\text { KURT }\end{array}$ & AZIMUTH & ENO & -EU/U & & \\
\hline METERS & DEG & DEG, & & & OEG & SEC/CU,M & $1 / 50 . M$ & StC/50.M & $1 / M$ \\
\hline 4000 & 0.0 & $0 . n 0$ & 0.000 & 0.000 & $\begin{array}{l}0.0 \\
0.0 \\
0.0\end{array}$ & $\begin{array}{l}0.0 \\
0.0 \\
0.0\end{array}$ & $\begin{array}{l}0.0 \\
0.0 \\
0.0\end{array}$ & 0. & 0 . \\
\hline 90. & 0.0 & 0.00 & 0.000 & 0.300 & $\begin{array}{l}0.0 \\
0.0 \\
0.0\end{array}$ & $\begin{array}{l}0.0 \\
0.0 \\
0.0\end{array}$ & $\begin{array}{l}0.0 \\
0.0 \\
0.0\end{array}$ & 0. & $n$. \\
\hline 1600 & 108.9 & 3.47 & .688 & 1.827 & $\begin{array}{l}105.5 \\
114.4 \\
136.5\end{array}$ & $\begin{array}{l}3.41 E-O \theta \\
3.26 k-U t \\
3.11 E-U \theta\end{array}$ & $\begin{array}{l}2.11 E-07 \\
2.02 E-07 \\
1.93 E-07\end{array}$ & 3. y3t $t-00$ & $2.44 E-05$ \\
\hline 3200 & 110.4 & 2.56. & -1.060 & $3.9 \div 5$ & $\begin{array}{l}111.7 \\
113.7 \\
110.7\end{array}$ & $\begin{array}{l}5.37 E-U 7 \\
1.74 E-U 7 \\
1.7 u L-U 7\end{array}$ & $\begin{array}{l}3.33 E-06 \\
1.2 U E-06 \\
1.03 E-06\end{array}$ & $7.72 E-05$ & $4.79 E-04$ \\
\hline 5000 & 107.3 & 4.09 & -.336 & 2.656 & $\begin{array}{l}\text { iue.0 } \\
110.0 \\
106.0\end{array}$ & $\begin{array}{l}5.401-U t \\
2.47 t-U H \\
2.26 t-U \theta\end{array}$ & $\begin{array}{l}3.35 E-67 \\
1.5 J E-07 \\
1.4 U E-07\end{array}$ & $2.90 \mathrm{E}-05$ & $1.80 F-04$ \\
\hline 7000 & $106.2 ?$ & 0.727 & -2.584 & $11.079 ?$ & $\begin{array}{l}100.0 \\
104.0 \\
110.0\end{array}$ & $\begin{array}{l}2.16 E-07 \\
9.45 E-08 \\
6.5 B t-08\end{array}$ & $\begin{array}{l}1.34 E-06 \\
5.06 E-07 \\
5.32 E-07\end{array}$ & $1.29 E-04 ?$ & $7.99 E-04 ?$ \\
\hline 12800 & 111.8 & 2.36 & $-2.4<5$ & 12.9 .72 & $\begin{array}{l}113.0 \\
.110 .0 \\
112.0\end{array}$ & $\begin{array}{l}4.49 E-07 \\
8.15 E-08 \\
7.03 E-0 B\end{array}$ & $\begin{array}{l}2.79 E-06 \\
5.05 E-07 \\
4.36 E-07\end{array}$ & $1.56 E-04$ & $9.66 \mathrm{E}-04$ \\
\hline
\end{tabular}

? INUICATFS UYCERTAIVIIES IN CATA.

$x$ indicatfs invalid of incuitplete data. 


\begin{tabular}{|c|c|c|c|c|c|c|c|c|c|}
\hline $\begin{array}{l}\text { TEST UB } \\
\text { FLUUC } \\
\text { SANFLER }\end{array}$ & $\begin{array}{l}\text { ¿ } \\
\text { RESCEIN } \\
\text { HT } 1.5 \mu\end{array}$ & $\begin{array}{l}\text { AUGUST } \\
\text { RELEAS }\end{array}$ & $\begin{array}{r}29,1969 \\
E \text { FRUM EL } \\
U\end{array}$ & $\begin{array}{r}\text { OCSE } \\
\text { LEVATIOA } \\
=4.9 \mathrm{M} / \mathrm{S}\end{array}$ & $\begin{array}{l}\text { TU } 012 \\
\text { UF } 50 \\
\text { EC AT }\end{array}$ & $\begin{array}{l}6 \text { PST } \\
M \\
56 M\end{array}$ & & & \\
\hline $\begin{array}{l}\text { CISTAIYCE } \\
\text { FREV }\end{array}$ & CBOS & $\begin{array}{l}\text { SAIVICD } \\
\text { STAND }\end{array}$ & $\begin{array}{l}\text { ISIBIBUI } \\
\text {-COEEEI }\end{array}$ & CIENI & THREE & MAXIMUM EX & POSURES & $\begin{array}{l}\text { CRUSS } \\
\text { INIEGRAIE }\end{array}$ & $\begin{array}{l}\text { WIND } \\
\text { O_YALUES_ }\end{array}$ \\
\hline SDURCE & MEAN & DEV & SKEW & KURT & AZIMUTH & $E / O$ & $\mathrm{EU} / \mathrm{O}$ & $(I(E / 0)$ & $(I(E) / O)$ \\
\hline$--\cdots---$ & ---- & $-\cdots-\cdots$ & ----- & $---\cdots$ & $\cdots-\cdots$ & $\cdots-\cdots---$ & $----\cdots--$ & 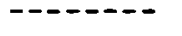 & $-\ldots-\cdots$ \\
\hline BETERS & EEG & DEC & & & DEG & SEC/CU.M & $1 / S G \cdot M$ & SEC/SQ.M & $1 / M$ \\
\hline 400 & $97.0 ?$ & $5.81 ?$ & 2.01671 & $11.919 ?$ & $\begin{array}{r}94.0 ? \\
98.0 ? \\
118.0 ?\end{array}$ & $\begin{array}{l}2.42 t-06 ? \\
2.10 E-U 6 ? \\
1.64 E-07 ?\end{array}$ & $\begin{array}{l}1.19 E-05 ? \\
1.03 E-05 ? \\
8.04 E-07 ?\end{array}$ & $1.37 t-04 ?$ & $6.71 E-04 ?$ \\
\hline 800 & $99.8 ?$ & 4.0d? & $3.679 ?$ & $20.957 ?$ & $\begin{array}{r}97.1 ? \\
99.1 ? \\
101.1 ?\end{array}$ & $\begin{array}{l}4.02 E-06 ? \\
2.01 E-66 ? \\
2.61 E-66 ?\end{array}$ & $\begin{array}{l}1.47 c-05 ? \\
1.38 t-05 ? \\
1.28 t-05 ?\end{array}$ & $2.94 E-04 ?$ & $1.44 \mathrm{E}-03 ?$ \\
\hline 1600 & $97.3 x$ & $5.58 x$ & $.472 x$ & $4.896 x$ & $\begin{array}{l}98.6 \\
99.6 \\
96.6\end{array}$ & $\begin{array}{l}5.57 E-06 \\
4.31 E-06 \\
4.11 E-06\end{array}$ & $\begin{array}{l}2.73 E-05 \\
2.11 E-05 \\
2.01 E-65\end{array}$ & $1.40 E-03 x$ & $6.84 E-03 X$ \\
\hline 3200 & 101.4 & 5.04 & -.270 & 3.129 & $\begin{array}{r}101.8 \\
99.8 \\
98.8\end{array}$ & $\begin{array}{l}2.94 E-v 6 \\
2.49 E-j 6 \\
2.36 E-j 6\end{array}$ & $\begin{array}{l}1.44 t-05 \\
1.22 t-65 \\
1.16 t-05\end{array}$ & $1.63 E-03$ & $9.01 E-03$ \\
\hline 5000 & 102.8 & 4.13 & -.285 & 7.843 & $\begin{array}{l}106.0 \\
100.0 \\
102.0\end{array}$ & $\begin{array}{l}1.11 E-06 \\
9.24 E-07 \\
0.93 E-07\end{array}$ & $\begin{array}{l}5.44 E-06 \\
4.53 E-06 \\
4.38 t-06\end{array}$ & $8.49 E-04$ & $4.16 E-03$ \\
\hline 7000 & 102.4 & 4.14 & -.293 & 2.731 & $\begin{array}{l}104.0 . \\
102.0 \\
100.0\end{array}$ & $\begin{array}{l}6.46 E-J 7 \\
5.54 E-07 \\
5.07 E-U 7\end{array}$ & $\begin{array}{l}3.17 i-U 6 \\
2.71 c-06 \\
2.49 E-v 6\end{array}$ & $7.30 E-04$ & $3.5 / E-03$ \\
\hline 12800 & 107.5 & 4.51 & -.269 & 6.582 & $\begin{array}{l}109.0 \\
110.0 \\
107.0\end{array}$ & $\begin{array}{l}4.09 E-07 \\
3.60 E-07 \\
2.45 E-07\end{array}$ & $\begin{array}{l}2.01 E-06 \\
1.06 \overline{0}-06 \\
1.20 E-06\end{array}$ & $5.30 E-04$ & $2.60 E-03$ \\
\hline
\end{tabular}

$?$ INDICATES UNCERTAINTIES IN CATA.

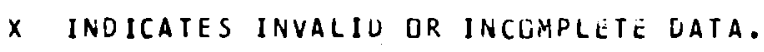


SAMPLIAG 400 H TO $12860 M$ PLUS UNE TCLEK UN 320 UM-AKC. TRACEK DISTRIGUTIUIN IS ERRAIIG UN ALL ARCS, PARTIALLY

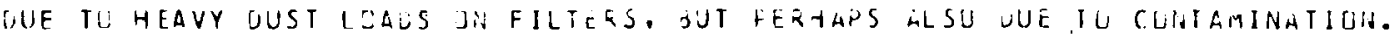

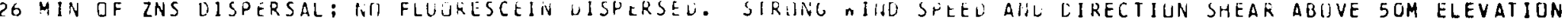

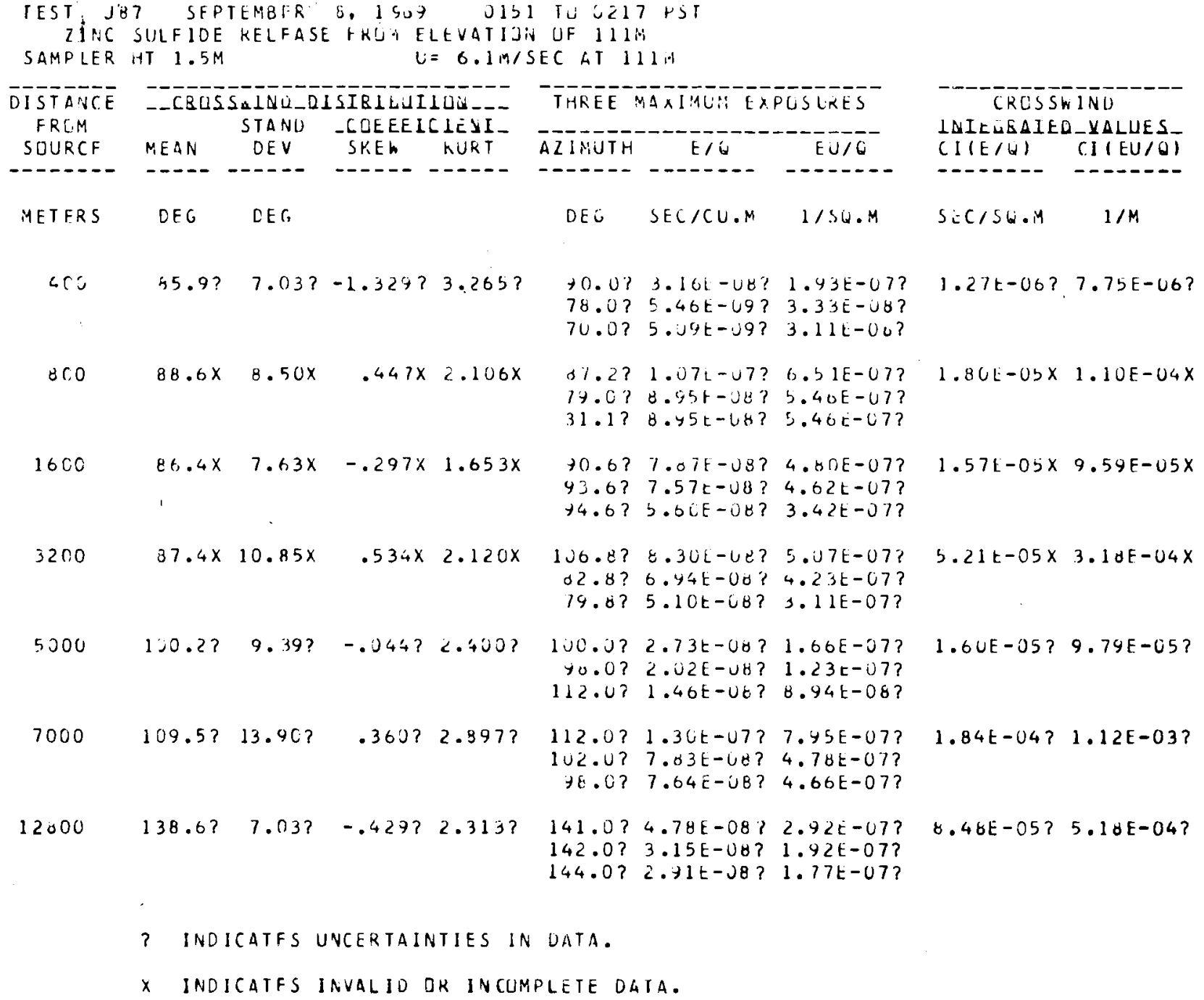

$S=C / 56.19 \quad 1 / M$

$1.27 t-06 ? 7.75 E-06 ?$

1.8UL-USX 1.10E-U4X

$1.57 t-05 \times 9.59 E-05 X$

$5.21 t-05 \times 3.10 E-04 x$

$1.6 U E-05 ? 9.79 E-05 ?$

$1.84 t-04 ? 1.12 E-03 ?$

$8.46 E-05 ? 5.10 E-04 ?$

$?$ INDICATFS UNCERTAINTIES IN DATA.

$x$ INDICATFS INVALID DK INCUMPLETE data. 
B-79 
SAMPLING 400M TO $12800 M$ : THWER SAMPLING AT 32UOM. 115.6 OEG. NU ZNS TRACER UBSERVEU CLUSER THAN $3200 M$ ARC. ANO DUST AND SAMPLFR FAILURE CAUSE) PRDBLEYS WHERE TRACER WAS FLUNU. FOK FLUURESCEIN, TRACER ON OO 49 , OFF 0055, ON AGAIN 0132, ANO FINALLY OFF O142. THIS TEST WAS MARGINALLY SUCCESSFUL. ESPECIALLY FUR FLUDRESCEIN.

\begin{tabular}{|c|c|c|c|c|c|c|c|c|c|}
\hline $\begin{array}{l}\text { TEST J } 8 \\
\text { ZINC } \\
\text { SAMPLER }\end{array}$ & $\begin{array}{ll}38 & \text { SEP } \\
\text { SULF IDE } \\
\text { HT } 1.5 \mathrm{M}\end{array}$ & $\begin{array}{l}\text { TEMBFR } 1 \\
\text { RELFASE }\end{array}$ & $\begin{aligned} 0 . & 1969 \\
\text { FRJM E } & \end{aligned}$ & $\begin{aligned} & 013 \\
& \text { LEVAIIIS } \\
&=6.4 \mathrm{MI}\end{aligned}$ & 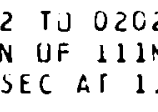 & $\begin{array}{l}2 \text { PST } \\
11 \mathrm{~N}\end{array}$ & & & \\
\hline $\begin{array}{l}\text { DISTAVCE } \\
\text { FRTM }\end{array}$ & \multicolumn{4}{|c|}{ 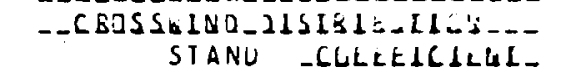 } & \multicolumn{3}{|c|}{ 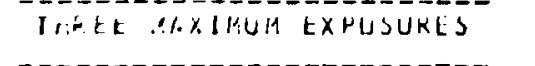 } & \multirow{2}{*}{\multicolumn{2}{|c|}{$\begin{array}{l}\text { CROSSWINU } \\
\text { INIELBAIED YALUES }\end{array}$}} \\
\hline SOJACE & MEAN & UEV & SKEW & KURT & A $2114 U \mathrm{TH}$ & $E / U$ & $i \omega / 0$ & & \\
\hline & 然 & (1) & 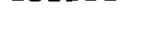 & $\ldots$ & ------- & $-\cdots$ & -------- & $-2----1$ & - \\
\hline METERS & DEC & DES & & & $D E G$ & $S E C / C U \cdot M$ & $1 / 50 . M$ & SEC/SO.M & $1 / M$ \\
\hline 400 & 0.0 & 0.00 & 0.000 & 0.000 & $\begin{array}{l}j . u \\
0 . u \\
\vdots . v\end{array}$ & $\begin{array}{l}\text { U. } \\
\text { i. } \\
ن .0\end{array}$ & $\begin{array}{l}u . u \\
0.0 \\
3.0\end{array}$ & G. & 0 . \\
\hline 8110 & 0.0 & 0.00 & 0.000 & 0.000 & $\begin{array}{l}0.0 \\
0.0 \\
0.0\end{array}$ & $\begin{array}{l}0.0 \\
0.6 \\
0.6\end{array}$ & $\begin{array}{l}c .0 \\
i . i j \\
j .0\end{array}$ & 0. & u. \\
\hline 1600 & 0.0 & 0.00 & 0.000 & 0.000 & $\begin{array}{l}2.3 \\
0.0 \\
0.0\end{array}$ & $\begin{array}{l}j .0 \\
0.0 \\
0.0\end{array}$ & $\begin{array}{l}u .0 \\
0.0 \\
0.0\end{array}$ & 0. & 0. \\
\hline 3200 & $120.2 ?$ & $1.80 ?$ & $.08 \mathrm{~J} ?$ & $1.365 ?$ & $\begin{array}{l}119.5 ? \\
122.4 ? \\
117.6 ?\end{array}$ & $\begin{array}{l}6.65 t-u 8 ? \\
b .23 t-00 ? \\
2.661-08 ?\end{array}$ & $\begin{array}{l}\text { 4. 26E-J7? } \\
3.3 \zeta E-U 7 ? \\
1.7 U E-U 7 ?\end{array}$ & $7.94 E-06 ?$ & $5.00 E-05 ?$ \\
\hline 5000 & $121.7 ?$ & $2.48 ?$ & $1.044 ?$ & $2.287 ?$ & $\begin{array}{l}120.0 ? \\
126.0 ? \\
122.0 ?\end{array}$ & $\begin{array}{l}4.02 E-J 6 ? \\
1.49 \mathrm{~A}-08 ? \\
6.61 \mathrm{~L}-\mathrm{U} 9 ?\end{array}$ & $\begin{array}{l}2.57 E-07 ? \\
9.50 E-08 ? \\
5.51 E-08 ?\end{array}$ & $1.11 \mathrm{E}-05 ?$ & $7.13 E-05 ?$ \\
\hline 7000 & $120.2 ?$ & $1.63 ?$ & $.433 ?$ & $5.273 ?$ & $\begin{array}{l}120.0 ? \\
124.0 ? \\
116.0 ?\end{array}$ & $\begin{array}{l}5.03 t-08 ? \\
6.73 t-09 ? \\
2.96 t-09 ?\end{array}$ & $\begin{array}{l}3.22 L-U 7 ? \\
4.30 E-0 d ? \\
1.84 t-U B ?\end{array}$ & $1.62 t-05 ?$ & $1.04 E-04 ?$ \\
\hline 12800 & $122.0 ?$ & $1.53 ?$ & $-.738 ?$ & $5.085 ?$ & $\begin{array}{l}122.0 ? \\
123.0 ? \\
121.0 ?\end{array}$ & $\begin{array}{l}3.61 E-08 ? \\
1.30 E-U B ? \\
1.18 E-08 ?\end{array}$ & $\begin{array}{l}2.31 E-07 ? \\
8.35 E-08 ? \\
7.58 E-08 ?\end{array}$ & $1.85 t-05 ?$ & $1.19 E-04 ?$ \\
\hline
\end{tabular}

$?$ INDICATES UNCERTAINTIES IN DATA.

$x$ IHOICATFS INVAlid or incGimplete data. 


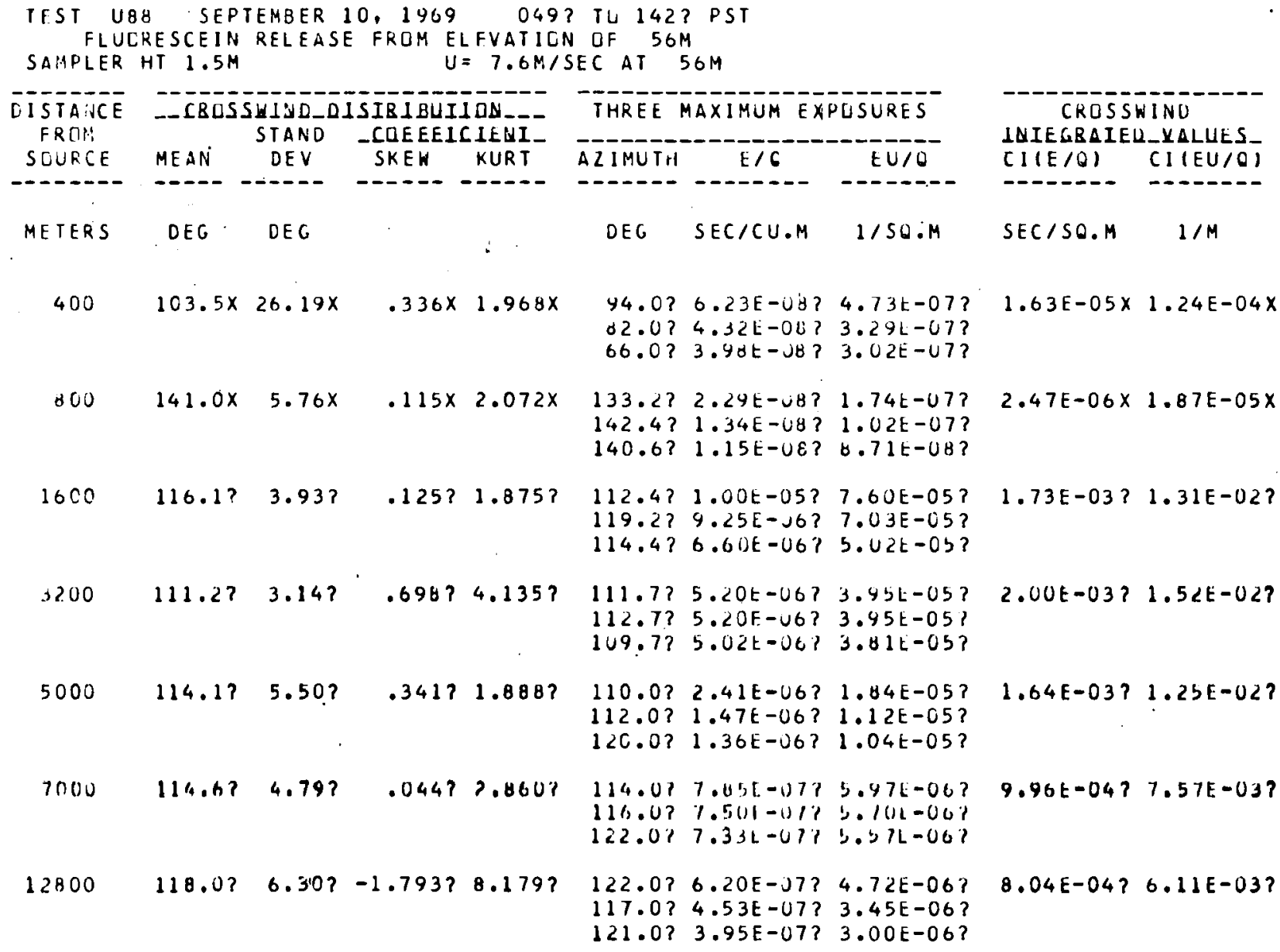

प4.0? 6.23E-U日? 4.73t-07? $02.074 .32 E-0 Q ? 3.29 L-07 ?$ $66.0 ? 3.90 t-J 8 ? 3.02 E-U 7 ?$

$1.63 E-05 \times 1.24 E-04 X$

$133.2 ? 2.29 E-U 8 ? 1.74 t-U 7 ?$ $142.4 ? 1.34 E-08 ? 1.02 E-07 ?$ $140.6 ? 1.15 E-U E ? \quad 6.71 E-U B$ ?

$\begin{array}{rrr}800 \quad 141.0 \times \quad 5.76 \times \quad .115 \times 2.072 \times \quad 133.2 ? & 2.29 E-U 8 ? 1.74 E-U 7 ? \\ & & 142.4 ? 1.34 E-U 8 ? 1.02 E-07 ? \\ & 140.6 ? 1.15 E-U 8 ? 6.71 E-08 ?\end{array}$

$1600 \quad 116.1 ? \quad 3.93 ? \quad .125 ? 1.875 ? \quad 112.4 ? 1.00 E-05 ? 7.60 E-05 ?$ $119.2 ? 9.25 E-J 6 ? 7.03 E-05 ?$ $114.4 ? 6.60 E-06 ? 5.02 t-03$ ?

$3200 \quad 111.27 \quad 3.14 ? \quad .696 ? 4.135 ? \quad 111.7 ? 5.20 t-06 ? 3.95 \mathrm{t}-05 ?$ $112.7 ? 5.20 \mathrm{~F}-\cup 6 ? 3.95 \mathrm{E}-05$ ? $1 \cup 9.7 ? 5.02 t-06 ? 3.01 t-05$ ?

$5000 \quad 114.1 ? 5.50 ? \quad .341 ? 1.888 ? \quad 110.0 ? 2.41 E-06 ? 1.84 E-05 ?$ $112.0 ? 1.47 \mathrm{E}-06 ? 1.12 \mathrm{E}-05 ?$ $120.0 ? 1.36 E-06 ? 1.04 t-05$ ?

$7000 \quad 114.6 ? 4.79 ? \quad .044 ? 2.860 ? 114.007 .056-07 ? 5.476-06 ?$ $116.0 ? 7.5(11-017 \% .161-06 ?$ $122.077 .33 \mathrm{~L}-07 \mathrm{P}$,.ל $\mathrm{L} \mathrm{L}-06$ ?

$12800 \quad 118.0 ? \quad 6.310 ?-1.793 ? 8.179 ? \quad 122.0 ? 6.20 E-37 ? \quad 4.72 E-06 ?$ $117.0 ? 4.53 E-07 ? 3.45 E-06$ ? $117.0 ? 4.53 E-07 ?$
$121.0 ? \quad 3.95 E-07 ?$
$1.00 E-06 ?$

$2.47 E-06 \times 1.87 E-05 X$

$1.73 E-03 ? 1.31 E-02 ?$

$2.00 E-03 ? 1.52 E-027$

$1.64 E-03 ? 1.25 E-02 ?$

$9.96 t-04 ? 7.57 E-037$

$8.04 E-04 ? 6.11 E-03 ?$

$?$ INOICATFS UINCERTAINTIES IN DATA.

$X$ INOICATES INVALID DR INCOMPLETE DATA. 
SAMPLINC $400 M$ TC IZEOOM. SAMPLING AT DNLY OAE TCNER, BUT NL TRACER THEREON . NO ZNS ON $40 O M$ OR BOOM ARCS. ZINC SULFIDE TRACER EMISRACEO DY CRUSSWINO EXTENT LF ARCS. FLUUKESCEIN CRLSSHIND UISTRIBUTIUNS SEEM TO EXTEND OFF, EDGES DF ARCS FREQUENTIY, BUT CUNTAMINATIUN IS PUSSIBILITY. NEITHER IRACER OBSERVED AT I2BOOM ARC.

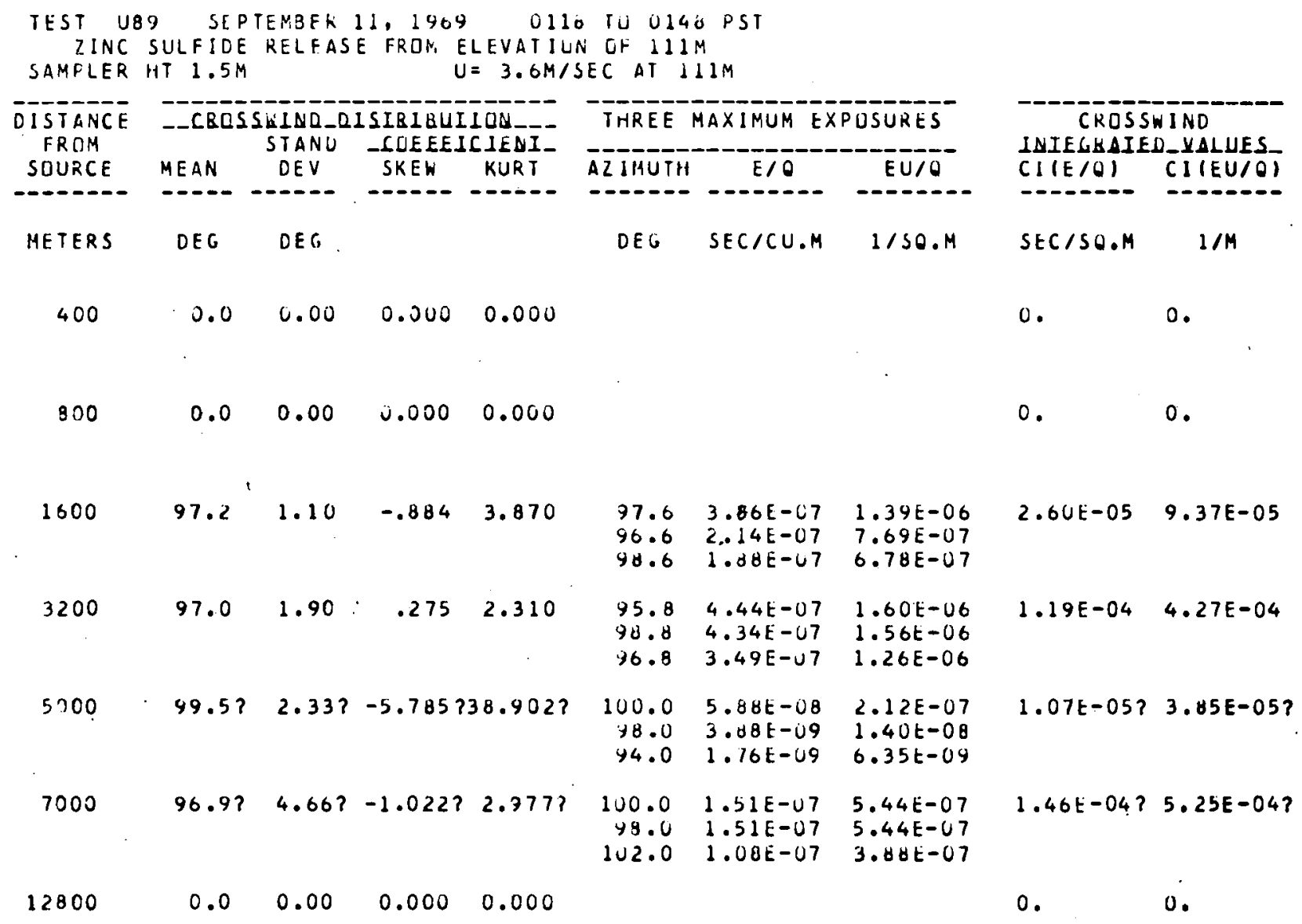

? INDICATES UNCERTAINTIES IN DATA.

X INOICATES INVALIU UR INCUMPLETE DATA. 


\begin{tabular}{|c|c|c|c|c|c|c|c|c|c|}
\hline \multirow{2}{*}{$\begin{array}{l}\text { UISTANCE } \\
\text { FROH } \\
\text { SOURCE }\end{array}$} & \multicolumn{2}{|c|}{$\begin{array}{l}\text {-CBOSSEIUD-D. } \\
\text { STAND }\end{array}$} & \multicolumn{2}{|c|}{$\begin{array}{l}\text { ISIRIBUIIDN- } \\
\text {-COEEEISIENI_- }\end{array}$} & THREE & \multicolumn{2}{|c|}{ MAXIMUM EXPUSURES } & \multicolumn{2}{|c|}{$\begin{array}{l}\text { CROS SWIND } \\
\text { INIEGBAIED_YALUES_ }\end{array}$} \\
\hline & MEAN & DEV & SKEW & KURT & AZIMUTH & $E / Q$ & $E \cup / 0$ & $C I(E / Q)$ & $C I(E \cup / Q)$ \\
\hline METERS & $D E G$ & DEG & & & DEG & $S E C / C U . M$ & $1 /$ SO.M & SEC/SO.M & $1 / M$ \\
\hline .400 & $92.9 ?$ & $13.94 ?$ & $-1.632 ?$ & $4.341 ?$ & $\begin{array}{r}98.0 ? \\
102.0 \% \\
62.0 ?\end{array}$ & $\begin{array}{l}2.65 E-06 ? \\
7.96 E-07 ? \\
6.03 E-07 ?\end{array}$ & $\begin{array}{l}7.96 E-06 ? \\
2.39 E-06 ? \\
2.05 E-06 ?\end{array}$ & $1.16 \mathrm{E}-04 ?$ & $3.48 E-04 ?$ \\
\hline 800 & $100.6 ?$ & $3.56 ?$ & $.775 ?$ & $3.866 ?$ & $\begin{array}{r}99.1 ? \\
101.1 ? \\
108.8 ?\end{array}$ & $\begin{array}{l}5.08 E-06 ? \\
2.47 E-06 ? \\
0.75 E-07 ?\end{array}$ & $\begin{array}{l}1.52 t-05 ? \\
7.42 t-06 ? \\
2.62 t-06 ?\end{array}$ & $2.93 E-04 ?$ & $8.80 E-04 ?$ \\
\hline 1600 & $98.3 ?$ & $2.88 ?$ & $-1.503 ?$ & $5.253 ?$ & $\begin{array}{r}100.6 \\
98.6 \\
97.6\end{array}$ & $\begin{array}{l}3.13 E-06 \\
2.90 E-U 6 \\
2.16 E-06\end{array}$ & $\begin{array}{l}9.38 E-06 \\
8.71 E-06 \\
6.47 E-06\end{array}$ & $4.29 E-04 ?$ & $1.29 E-03 ?$ \\
\hline 3200 & $93.6 ?$ & $5.85 ?$ & $-1.880 ?$ & $6.450 ?$ & $\begin{array}{l}95.8 \\
96.8 \\
94.8\end{array}$ & $\begin{array}{l}2.43 E-06 \\
2.27 E-06 \\
1.57 E-06\end{array}$ & $\begin{array}{l}7.28 E-06 \\
6.82 E-06 \\
4.70 E-06\end{array}$ & $7.62 \mathrm{E}-04 ?$ & $2.29 E-03 ?$ \\
\hline 5000 & $94.9 ?$ & $5.42 ?$ & $-.120 ?$ & $4.350 ?$ & $\begin{array}{r}100.0 \\
96.0 \\
94.0\end{array}$ & $\begin{array}{l}2.61 E-07 \\
2.46 E-07 \\
1.62 E-07\end{array}$ & $\begin{array}{l}8.42 E-07 \\
7.36 E-07 \\
4.86 E-07\end{array}$ & $2.11 E-04 ?$ & $6.33 E-04 ?$ \\
\hline 7000 & $94.6 ?$ & $5.14 ?$ & $-.285 ?$ & $1.988 z$ & $\begin{array}{r}90.0 \\
98.0 \\
100.0\end{array}$ & $\begin{array}{l}4.30 E-J 7 \\
3.15 E-07 \\
2.41 E-07\end{array}$ & $\begin{array}{l}1.29 E-06 \\
9.46 E-07 \\
7.23 E-07\end{array}$ & $3.89 E-04 ?$ & $1.17 E-03 ?$ \\
\hline $128 \mathrm{co}$ & 0.0 & 0.00 & 0.000 & 0.000 & & & & 0. & 0 . \\
\hline
\end{tabular}

$?$ INUICATES UNCERTAINTIES IN DATA.

$x$ INDICATES INVALIO OR INCOMPLETE DATA. 
GROUND LEVEL SAMPLING 40OM TO 12BOOM: SAYPLING ON JNE TUWER'AT $3200 M$. NU ZNS UBSERVED ON 4OOM. 8OOM OR $1280 O M$ ARCS. ZNS PLUME LIKELY OFF NGRTH EOGE OF 70JOM ANO $12 \%$ JUM ARC S.

FLUORESCEIN DISPERSAL FUR MNLY 4 MIN. INUS. SIGIJIFICANCE DF FLUCIRESCEIN MEASUREMENTS IS MINIMAL.

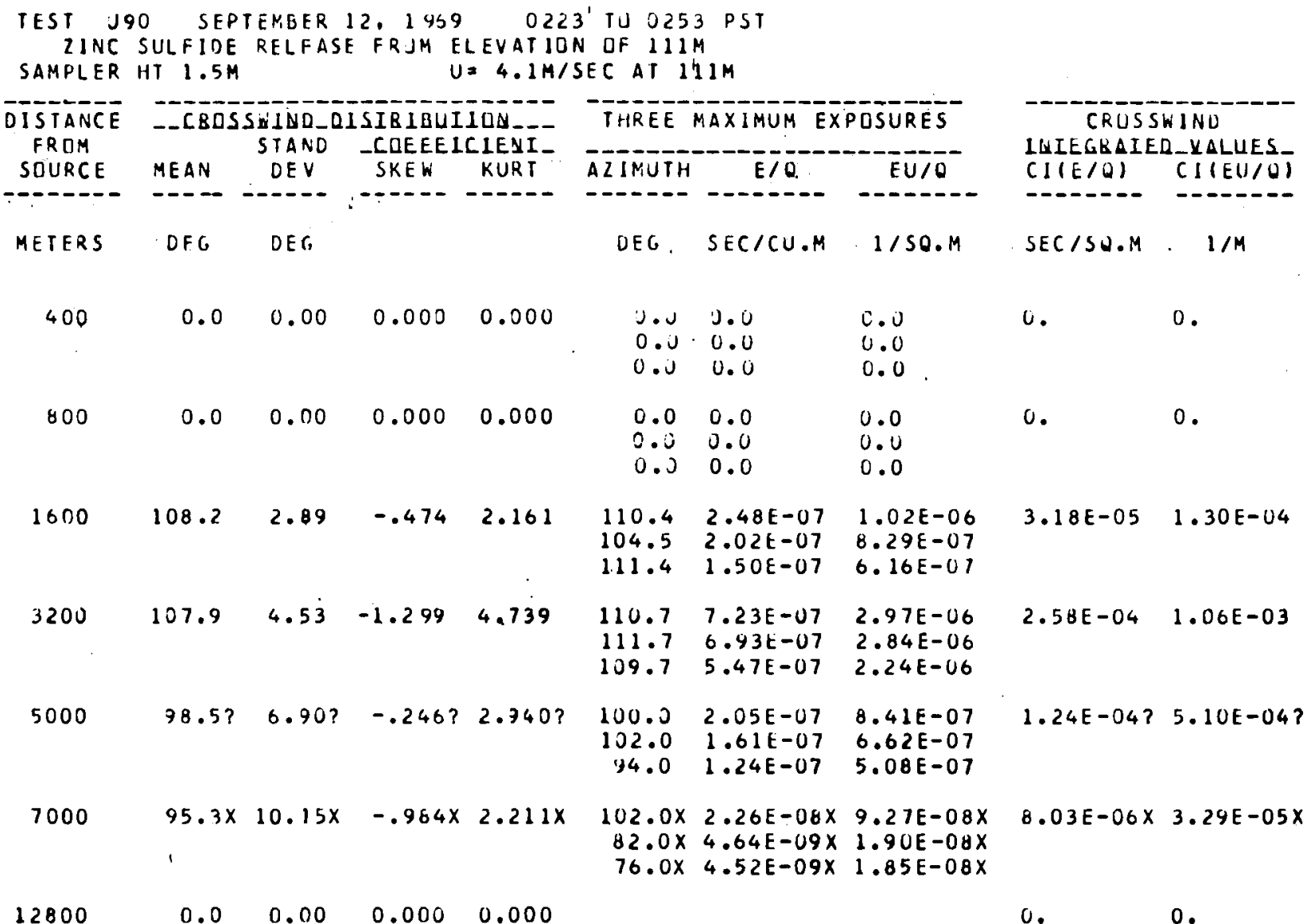

? INDICATFS UNCERTAINTIES IN DATA.

X INDICATFS INVALID DR INCOMPLETE DATA. 


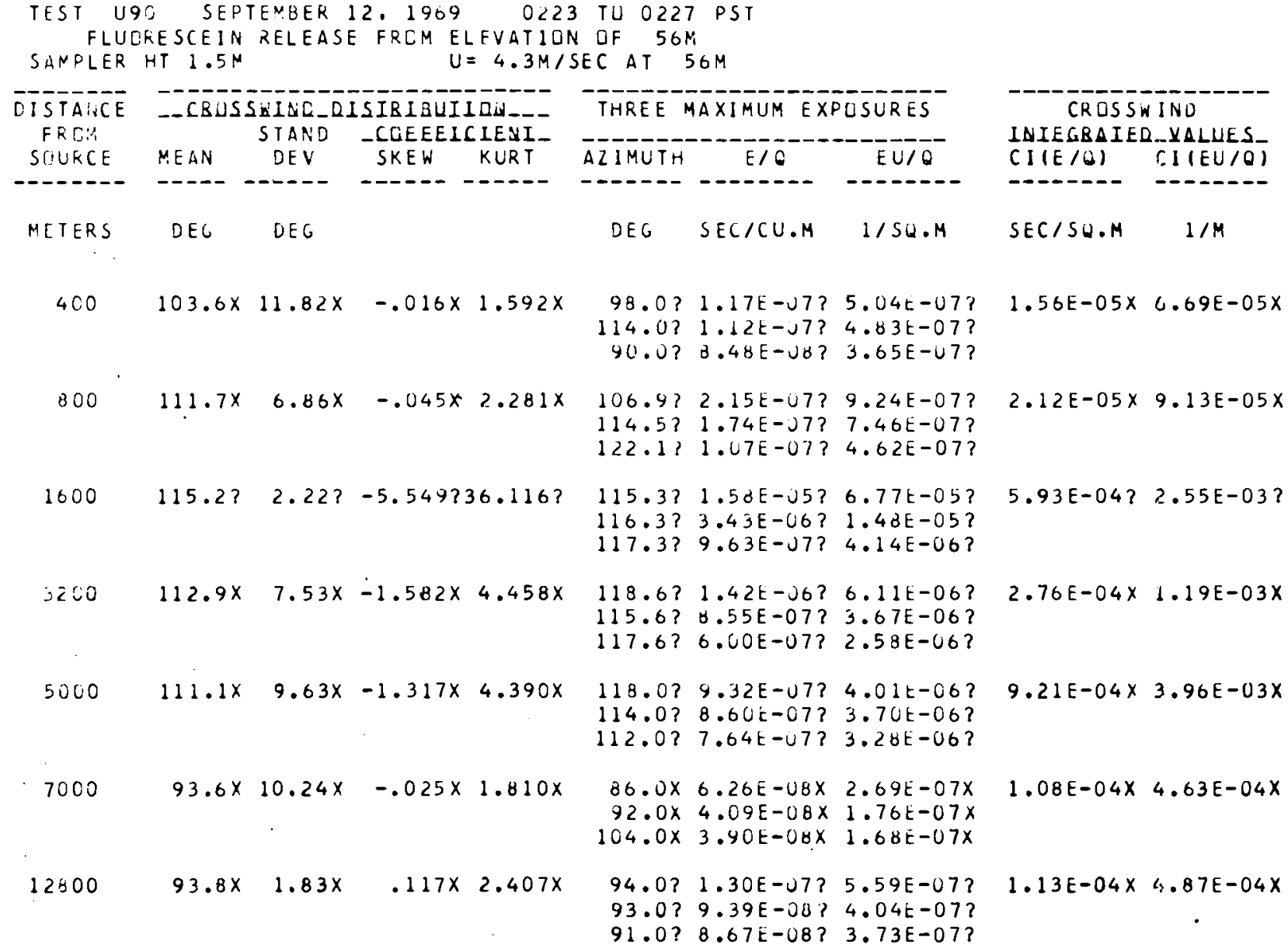

$12400 \quad 93.8 \times \quad 1.83 \times \quad .117 \times 2.407 \times \quad 94.0 ? \quad 1.30 E-07 ? \quad 5.59 E-07 ?$ $93.0 ? 9.39 E-00 ? 4.04 E-07 ?$ $91.0 ? 8.67 \mathrm{E}-08$ ? $3.73 \mathrm{E}-07$ ?

? INDICATES UNCERTAINTIES IN DATA.

$X$ Indicates InVALIU oR incumplete data. 
GROUND LEVEL SAMPLING 40OM TO 12BUOM. ONE TURER SAMPLING ONLY AT 32OUM, 115.6 DEG.

NO 2 INC SULFIDE IBSERVEU UN $400 M$ ARC. HEAVY DUST ON $12800 M$ LEAVES UNCERTAINTIES IN ASSAY OF ZINC SULFIDE. LARGE WIND OIRECTION SHEAR (BACKINJ) ABJVE 30.4 ELEVATION.

w
$\vdots$
$\infty$
$\infty$

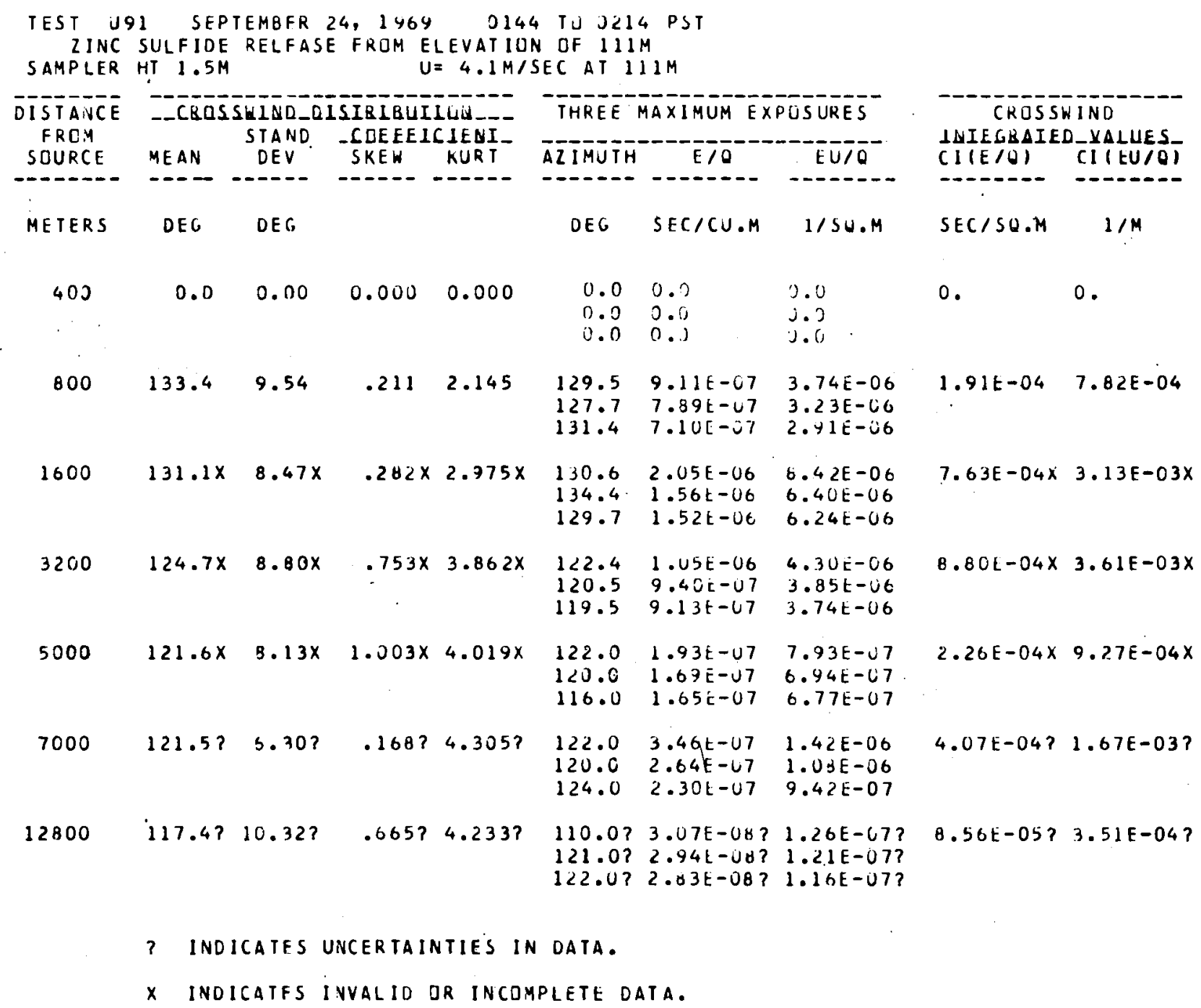




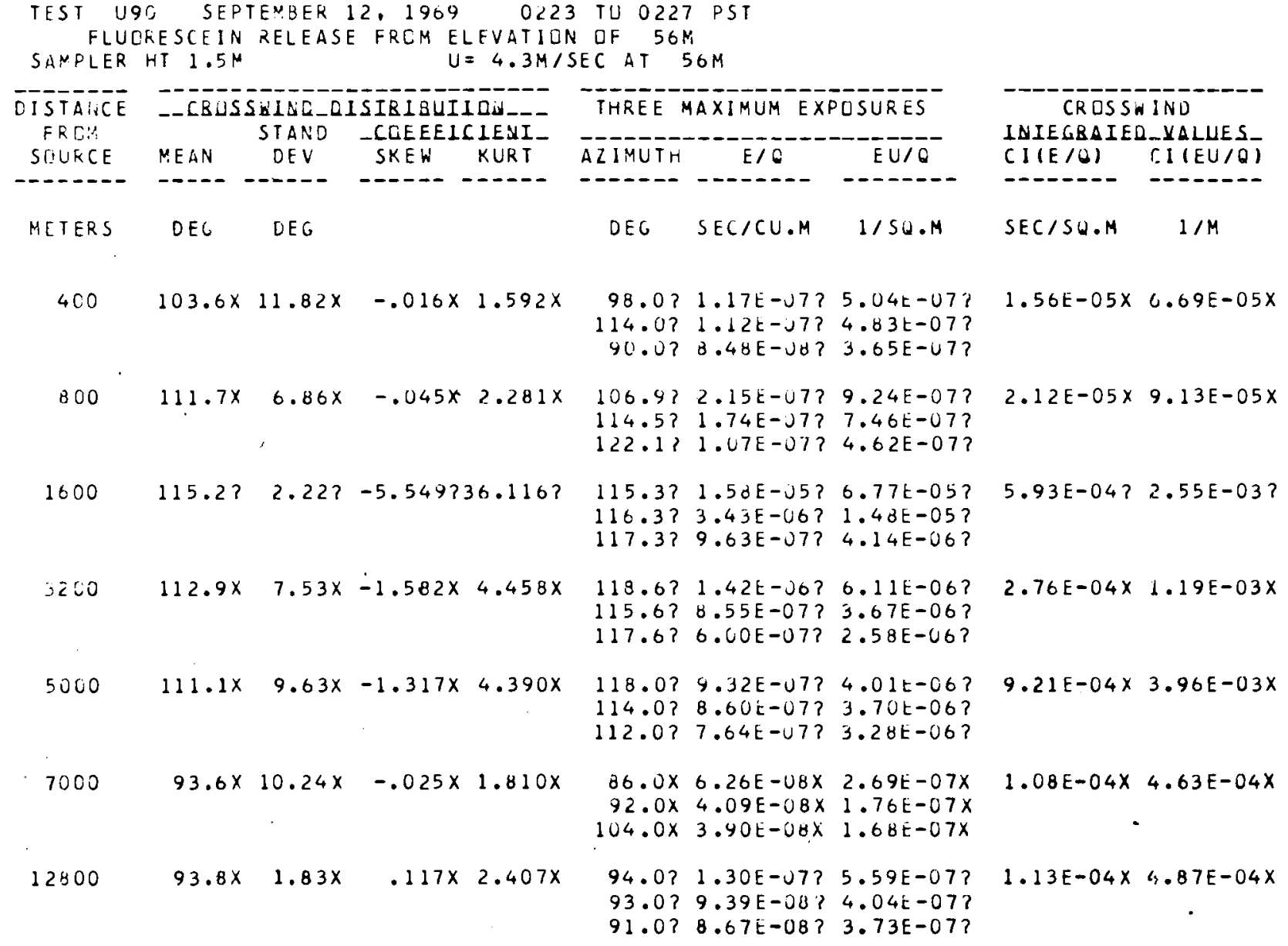

? INDICAIES UNCERTAINTIES IN DATA.

$x$ indicates inValid or incumplete data. 
GRQUND LEVEL SAMPLING 4OOM TO $12800 M$. UNE TUNER SAMPLING ONLY AT $320 U M, 115.6$ DEG

NO ZINC SULFIDE TIBSERVEU UN $400 M$ ARC. HEAVY OUST ON $1280 O M$ LEAVES UNCERTAINTIES IN ASSAY OF ZINC SULFIDE. LARGE WINO DIRECTION SHEAR (BACKINS) ABJVE 30.1 ELEVATION.

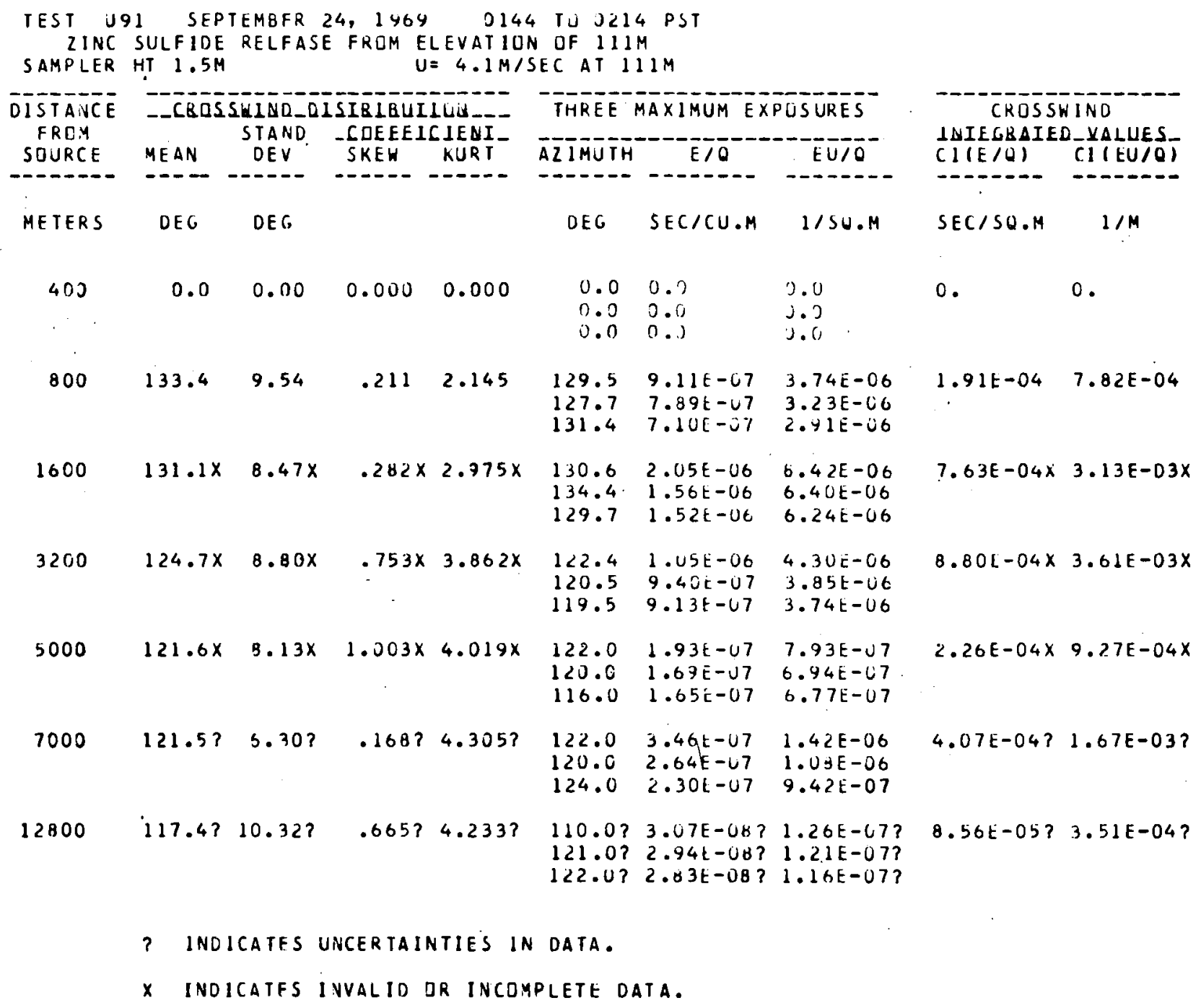


TEST U91 SEPTEMBER 24, $1969 \quad 0144$ TU 0214 PST

FLUORESCEIN RELEASE FROM ELEVATION LF $50 M$

$$
\text { SAMPLER HT 1.5M U U }
$$

\begin{tabular}{|c|c|c|c|c|c|c|c|c|c|}
\hline \multirow{2}{*}{$\begin{array}{l}\text { DISTAICE } \\
\text { FKLUA } \\
\text { SIJURCE }\end{array}$} & \multicolumn{4}{|c|}{-CROSSAIUD_RISIRIBUIICA- - } & \multicolumn{3}{|c|}{ THREE MAXIMUM EXPOSURES } & \multicolumn{2}{|c|}{$\begin{array}{l}\text { CRDSSWIND } \\
\text { 1NIEGBAIED_Y LUES }\end{array}$} \\
\hline & MEAN & DEV & SKE' & KURT & AZIMUTH & $E / Q$ & $E \cup / Q$ & $(1 / E / Q)$ & CI (EU/Q) \\
\hline 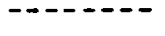 & $\cdots-$ & $--\infty---$ & $----n$ & ----- & $\cdots-\cdots$ & $-\cdots-1$ & $-\cdots-1--1$ & ------ & 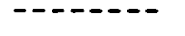 \\
\hline METERS & DEC & DEG & & & DEG & $S E C / C U . M$ & $1 / S 0 . M$ & $S E C / S O \cdot M$ & $1 / M$ \\
\hline 400 & 129.3 & 6.94 & -1.158 & 3.391 & $\begin{array}{l}134.0 \\
130.0 \\
126.0\end{array}$ & $\begin{array}{l}6.29 E-07 \\
2.01 E-U 7 \\
1.44 E-U 7\end{array}$ & $\begin{array}{l}2.39 t-06 \\
7.64 t-07 \\
5.49 E-07\end{array}$ & $3.40 E-05$ & $\therefore .29 E-04$ \\
\hline 800 & 136.6 & 8.60 & -.512 & 2.826 & $\begin{array}{l}140.6 \\
142.4 \\
136.9\end{array}$ & $\begin{array}{l}3 . \cup 7 E-\cup 6 \\
2.63 E-06 \\
2.25 t-\cup 6\end{array}$ & $\begin{array}{l}1.17 E-05 \\
9.98 E-06 \\
8.56 E-06\end{array}$ & $6.76 E-04$ & $2.57 E-03$ \\
\hline 1600 & 137.7 & 8.90 & .012 & $2: 198$ & $\begin{array}{l}136.4 \\
131.5 \\
136.3\end{array}$ & $\begin{array}{l}2.73 E-U 6 \\
2.65 E-J 6 \\
2.56 E-J 6\end{array}$ & $\begin{array}{l}1.04 t-05 \\
1.01 t-05 \\
9.73 E-06\end{array}$ & $1.86 E-03$ & $7.05 E-03$ \\
\hline 3200 & $133.6 x$ & $9.01 x$ & $.021 x$ & $2.147 x$ & $\begin{array}{l}140.1 \\
139.1 \\
125.4\end{array}$ & $\begin{array}{l}1.14 E-J 6 \\
1.12 E-J 6 \\
1.10 E-06\end{array}$ & $\begin{array}{l}4.33 t-06 \\
4.26 t-06 \\
4.19 t-06\end{array}$ & $1.53 E-03 x$ & $5.82 E-03 x$ \\
\hline 5000 & $131.7 \times$ & $7.82 x$ & $.021 x$ & 2. $398 x$ & $\begin{array}{l}132.0 \\
128.0 \\
134.0\end{array}$ & $\begin{array}{l}1.04 E-06 \\
9.33 E-07 \\
9.20 E-07\end{array}$ & $\begin{array}{l}3.93 t-06 \\
3.62 t-06 \\
3.50 t-06\end{array}$ & $1.68 E-03 x$ & t. $39 E-03 x$ \\
\hline 7000 & $128.4 x$ & $6.83 \times$ & $.360 x$ & $2.286 x$ & $\begin{array}{l}124.0 \\
126.0 \\
122.0\end{array}$ & $\begin{array}{l}3.76 E-07 \\
7.12 E-07 \\
5.63 E-07\end{array}$ & $\begin{array}{l}3.33 E-06 \\
2.71 E-06 \\
2.14 E-06\end{array}$ & $1.16 E-03 x$ & $4.42 E-03 x$ \\
\hline 12800 & 120.0 & 10.45 & .518 & 3.193 & $\begin{array}{l}122.0 \\
119.0 \\
118.0\end{array}$ & $\begin{array}{l}1.07 E-U 7 \\
1.82 E-U 7 \\
1.66 E-U 7\end{array}$ & $\begin{array}{l}7.12 E-07 \\
6.92 E-07 \\
6.33 E-07\end{array}$ & $8.00 E-04$ & $3.04 E-03$ \\
\hline
\end{tabular}

$?$ INDICATES UNCERTAINTIES IN DATA.

X INDICATES INVALID DR INCOMPLETE UATA. 
CROUNO LEVEL SAMPLING 400M TO $12803 \mathrm{M}$ : WITH PUSSIBLE EXCEPTIUN OF FLUDRESCEIN AT $12800 \mathrm{M}$. ALL ARCS EMBRACE THE CRUSSWIND EXTENT JF THE TRACER. TRACER DISTRIBUTIONS EXTEND ABUUE TOP DF THE SINGLE SAMPLING TOWER.

NI ZINC SULFIDE DOSERVED ON 4OOM. 9OJM UR $1600 M$ ARCS.

\begin{tabular}{|c|c|c|c|c|c|c|c|c|c|}
\hline \multirow{2}{*}{$\begin{array}{l}\text { DISTANCE } \\
\text { FROM } \\
\text { SOURCE }\end{array}$} & \multicolumn{4}{|c|}{$\begin{array}{l}\text {-CBOSSUIND_QISIRIBUIIOA-- } \\
\text { STAND -COEEEICIEUI- }\end{array}$} & \multirow{2}{*}{$\begin{array}{l}\text { THREE } \\
\text { AZIMUTH }\end{array}$} & \multicolumn{2}{|c|}{ MAXIMUM EXPOSURES } & \multicolumn{2}{|c|}{$\begin{array}{c}\text { CRDSSW INO } \\
\text { INIEGRAIED_YALUES }\end{array}$} \\
\hline & MEAN & DEV & SKEW & KUR T & & $E / 0$ & EU/O & $C(1 E / O)$ & CI $(E U / Q)$ \\
\hline METERS & DEG & DEG & & & DEG & SEC/CU.M & 1/SO.M & SEC/SO.M & $1 / M$ \\
\hline 400 & 0.0 & 0.00 & 0.000 & 0.300 & $\begin{array}{l}0.0 \\
r .0 \\
r .0\end{array}$ & $\begin{array}{l}0.1 \\
0.0 \\
0.0\end{array}$ & $\begin{array}{l}0.0 \\
0.0 \\
0.0\end{array}$ & 0. & 0. \\
\hline 800 & 0.0 & 0.00 & 0.000 & 0.000 & $\begin{array}{l}0.0 \\
\therefore .0 \\
0.0\end{array}$ & $\begin{array}{l}0.0 \\
0.0 \\
1) .0\end{array}$ & $\begin{array}{l}0.0 \\
0.0 \\
0.0\end{array}$ & 0. & 0. \\
\hline 1600 & 0.0 & 0.00 & 0.000 & 0.000 & $\begin{array}{l}. .0 \\
i .0 \\
1.0\end{array}$ & $\begin{array}{l}3.3 \\
0.0 \\
0.0\end{array}$ & $\begin{array}{l}0.0 \\
3.0 \\
0.0\end{array}$ & 0. & 0 . \\
\hline 3200 & 117.8 & 2.92 & 1.768 & 8.754 & $\begin{array}{l}119.5 \\
114.6 \\
116.6\end{array}$ & $\begin{array}{l}2.18 E-07 \\
1.28 E-07 \\
9.53 E-64\end{array}$ & $\begin{array}{l}1.18 E-06 \\
6.91 E-07 \\
5.15 E-07\end{array}$ & $3.87 E-05$ & $2.09 E-04$ \\
\hline 5000 & 119.0 & $\begin{array}{l}3.24 \\
1\end{array}$ & -.613 & 2.910 & $\begin{array}{l}122.0 \\
120.0 \\
118.0\end{array}$ & $\begin{array}{l}1.17 E-U 7 \\
6.58 E-0 B \\
4.59 E-U 8\end{array}$ & $\begin{array}{l}6.34 E-07 \\
3.55 E-07 \\
2.48 E-07\end{array}$ & $5.41 E-05$ & $2.92 E-04$ \\
\hline 7000 & 118.9 & 2.19 & -.372 & 2.713 & $\begin{array}{l}118.0 \\
120.0 \\
122.0\end{array}$ & $\begin{array}{l}2.37 E-U 7 \\
2.34 E-U 7 \\
1.28 E-07\end{array}$ & $\begin{array}{l}1.2 \partial E-06 \\
1.26 E-06 \\
6.43 E-07\end{array}$ & $1.84 E-04$ & $9.94 E-04$ \\
\hline 12800 & 113.2 & 3.48 & .530 & 3.583 & $\begin{array}{l}114.0 \\
108.0 \\
113.0\end{array}$ & $\begin{array}{l}8.65 E-09 \\
7.60 E-09 \\
7.60 E-J 9\end{array}$ & $\begin{array}{l}4.67 E-08 \\
4.10 E-08 \\
4.10 E-08\end{array}$ & $1.45 E-05$ & $7.85 E-05$ \\
\hline
\end{tabular}

$?$ INDICATFS UNCERTAINTIES IN DATA.

X indicates invaliu or incQmplete data. 


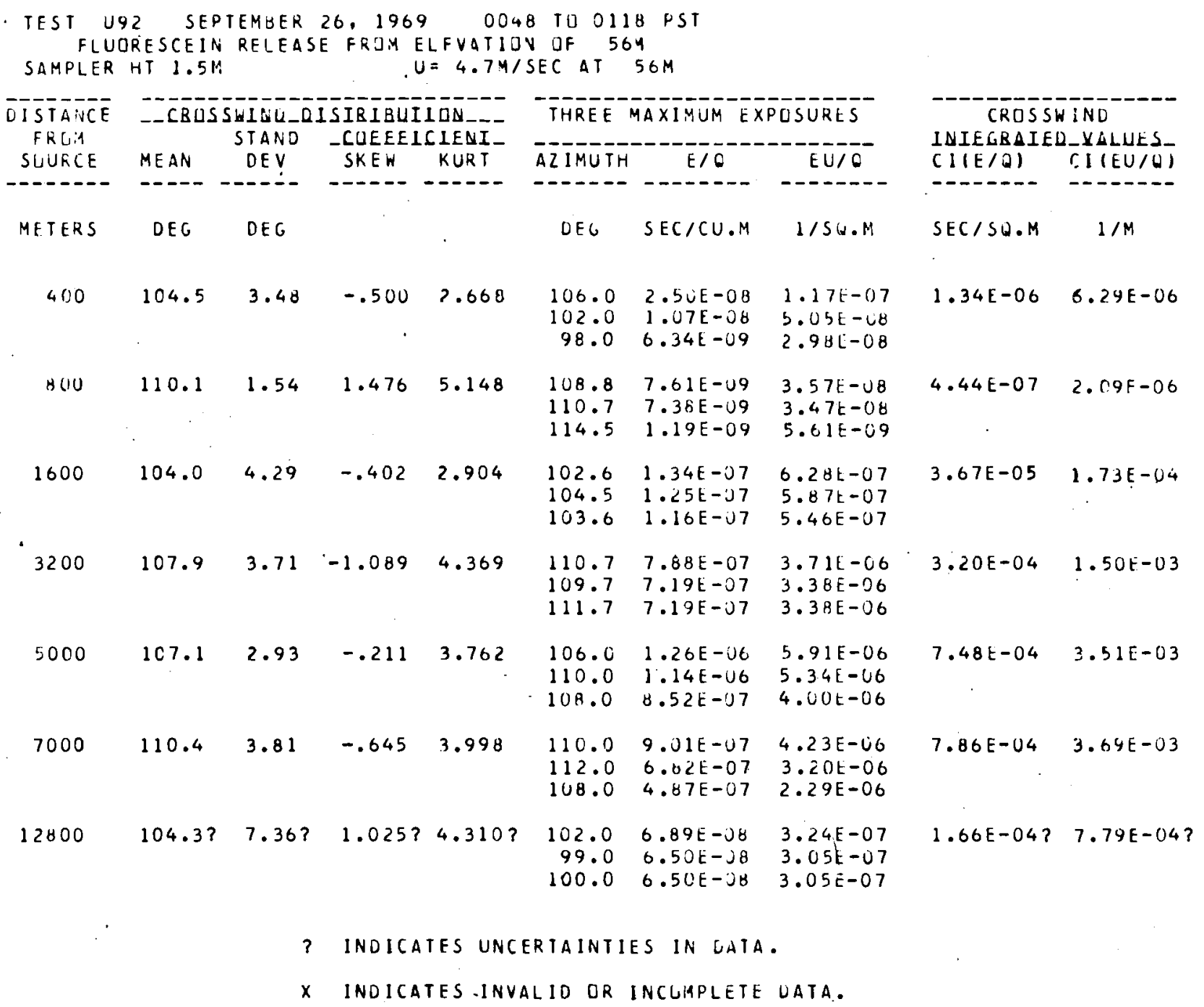


GRUUNO LEVEL AND TOWER SAMPLING 20OM TO 320UM. ALL ARCS EMBRACE CKOSSHIND EXTENT UF TRACER DISTRIBUTIONS. ALTHOUGH TRACERS TRUNCATEO AT TOPS OF TUWERS, EXTRAPDLATION IS OISTINCT POSSIBILITY ON THIS TEST.

RELATIVELY SMALL AMOUNT GF RHODAMINE DISPERSEU LEAVES CUNSIDERABLE UNCERTAINTIESIN INUIVIDUAL EXPOSURE VALUES.

\begin{tabular}{|c|c|c|c|c|c|c|c|c|c|}
\hline $\begin{array}{l}\text { TEST } \\
\text { ZINC } \\
\text { SAMPLER }\end{array}$ & $\begin{array}{l}\text { V1 } \\
\text { SULF IDE } \\
\text { HT } 1.5 \mathrm{M}\end{array}$ & $\begin{array}{l}\text { CTOBFR } 2 \\
\text { RELFASE }\end{array}$ & $\begin{array}{l}26,197 \\
E \text { FROM }\end{array}$ & $\begin{array}{l}2 \\
\text { ELEVATID } \\
U=5.8 \mathrm{M}\end{array}$ & $\begin{array}{l}\text { TU } 100 \\
\text { OF } 26 \\
\text { SEC AT }\end{array}$ & $\begin{array}{l}0 \text { PST } \\
26 M\end{array}$ & & & \\
\hline $\begin{array}{l}\text { DISTANCE } \\
\text { FROM }\end{array}$ & \multicolumn{4}{|c|}{-CBUSSWINO_DISIBIBUIIDN } & THREE & \multicolumn{2}{|c|}{ MAXIMUM EXPOSURES } & \multirow{2}{*}{\multicolumn{2}{|c|}{$\begin{array}{l}\text { CROSSWIND } \\
\text { IUIELRAIED_YALUES }\end{array}$}} \\
\hline SOUKCE & MEAN & DEV & SKEH & KURT & AZIMUT.H & $\mathrm{E} / \mathrm{O}$ & $E \cup / 0$ & & \\
\hline$-\cdots--\infty$ & --- & $--\infty-\infty$ & $---\cdot-$ & ----- & $----n$ & $---\cdots--$ & $-\cdots-\infty$ & $---\infty-\infty$ & $\cdots \cdots$ \\
\hline METERS & DEG & DEC, & & & DEG & SEC/CU.M & $1 / S Q \cdot M$ & SEC/SQ.M & $1 / M$ \\
\hline 200 & 111.8 & 7.29 & .561 & 3.618 & $\begin{array}{l}112.0 \\
114.0 \\
110.0\end{array}$ & $\begin{array}{l}7.06 E-06 \\
6.55 E-06 \\
5.90 E-06\end{array}$ & $\begin{array}{l}4.10 E-05 \\
3.80 E-05 \\
3.42 E-05\end{array}$ & $3.46 E-04$ & $2.01 E-03$ \\
\hline .400 & 113.9 & $\begin{array}{r}7.76 \\
:\end{array}$ & -.090 & 2.800 & $\begin{array}{l}114.0 \\
118.0 \\
106.0\end{array}$ & $\begin{array}{l}2.32 E-06 \\
1.72 E-06 \\
1.15 E-06\end{array}$ & $\begin{array}{l}1.34 E-05 \\
9.96 E-06 \\
6.67 E-06\end{array}$ & $2.60 E-04$ & $1.51 E-03$ \\
\hline 800 & 114.6 & 6.91 & -.175 & 2.349 & $\begin{array}{l}118.3 \\
114.5 \\
122.1\end{array}$ & $\begin{array}{l}1.60 E-06 \\
1.46 E-06 \\
1.42 E-06\end{array}$ & $\begin{array}{l}9.30 E-06 \\
6.50 E-06 \\
8.24 E-06\end{array}$ & $4.06 E-04$ & $2.35 E-03$ \\
\hline 1200 & $114: 1$ & 6.40 & .104 & 2.685 & $\begin{array}{l}116.0 \\
112.0 \\
108.0\end{array}$ & $\begin{array}{l}8.16 E-07 \\
7.26 E-U 7 \\
6.47 E-67\end{array}$ & $\begin{array}{l}4.73 E-06 \\
4.21 E-06 \\
3.75 E-06\end{array}$ & $2.40 E-04$ & $1.39 E-03$ \\
\hline 1600 & 114.8 & 6.83 & -.072 & 2.333 & $\begin{array}{l}109.5 \\
116.3 \\
117.3\end{array}$ & $\begin{array}{l}4.11 E-07 \\
4.02 E-07 \\
3.39 E-07\end{array}$ & $\begin{array}{l}2.38 E-06 \\
2.33 E-06 \\
1.97 E-06\end{array}$ & $1.60 E-04$ & $9.26 E-04$ \\
\hline $2200^{-}$ & 116.1 & 6.82 & -.037 & 2.207 & $\begin{array}{l}110.0 \\
116.0 \\
124.0\end{array}$ & $\begin{array}{l}1.36 E-07 \\
1.18 E-07 . \\
8.98 E-08\end{array}$ & $\begin{array}{l}7.88 E-07 \\
6.82 E-07 \\
5.21 E-07\end{array}$ & $6.69 E-05$ & $3.88 E-04$ \\
\hline 3200 & 114.5 & 6.22 & -.067 & 2.373 & $\begin{array}{l}113.7 \\
109.7 \\
117.6\end{array}$ & $\begin{array}{l}1.43 E-07 \\
1.30 E-07 \\
1.24 E-07\end{array}$ & $\begin{array}{l}8.32 E-07 \\
7.52 E-07 \\
7.18 E-07\end{array}$ & $1.17 E-04$ & $6.77 E-04$ \\
\hline
\end{tabular}

? INOICATES UNCERTAINTIES IN DATA.

X INDICates inValio or InCOMPlete data. 
TEST VI OCTOBER 26, 1972 0930 TU 1000 PST RHUDAMINE 8 RELEASE FRDM ELFVATIUN DF $26 \mathrm{M}$ SAHPLER HT $1.5 \mathrm{M} \quad U=5.8 \mathrm{M} / \mathrm{SEC}$ AT $26 \mathrm{M}$

\begin{tabular}{|c|c|c|c|c|c|c|c|c|c|}
\hline $\begin{array}{l}\text { DISTANCE } \\
\text { FRLA }\end{array}$ & $-C B L$ & $\begin{array}{l}\text { SSRIUD_D } \\
\text { STAND }\end{array}$ & $\begin{array}{l}\text { ISIBIBU } \\
\text { - CDEEE }\end{array}$ & CIELI- & THREE & MAXIVUM EX & PCSUKES & $\begin{array}{r}\text { CRUS } \\
\text { INIEGRAIE }\end{array}$ & $\begin{array}{l}\text { WINO } \\
\text { 2-YALUES }\end{array}$ \\
\hline SLURCE & MEAN & DEV & SKEW & KURT & AZIMUTH & $E / C$ & $E \cup / O$ & $C I(E / Q)$ & $(1(E) / Q)$ \\
\hline$----n$ & ---- & $-\cdots-$ & $-\ldots--$ & ---- & 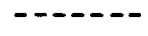 & $-\ldots-n$ & $-\cdots---$ & $-\cdots---$ & $--n-\infty$ \\
\hline METERS & DEG & DEG & & & UEG & SEC/CU.M & $1 / \mathrm{Su} . \mathrm{M}$ & SEC/SO.M & $1 / M$ \\
\hline 200 & 110.5 & 6.69 & .287 & 4.045 & $\begin{array}{l}106.0 \\
110.0 \\
112.0\end{array}$ & $\begin{array}{l}5.18 E-06 \\
2.72 E-06 \\
2.51 E-06\end{array}$ & $\begin{array}{l}3.00 t-05 \\
1.58 E-05 \\
1.46 E-0 S\end{array}$ & $1.60 E-04$ & $9.30 E-04$ \\
\hline 400 & 112.6 & 7.87 & -.121 & 2.146 & $\begin{array}{l}118.0 \\
114.0 \\
106.0\end{array}$ & $\begin{array}{l}3.53 E-06 \\
3.62 E-06 \\
3.21 E-06\end{array}$ & $\begin{array}{l}2.28 E-05 \\
2.10 E-05 \\
1.86 E-05\end{array}$ & $5.68 E-04$ & $3.29 E-03$ \\
\hline$\triangle C O$ & 113.1 & 7.11 & .039 & 2.087 & $\begin{array}{l}114.5 \\
108.8 \\
106.9\end{array}$ & $\begin{array}{l}1.15 E-06 \\
1.09 E-06 \\
9.47 E-07\end{array}$ & $\begin{array}{l}6.69 E-06 \\
6.29 E-06 \\
5.49 E-06\end{array}$ & $2.79 E-04$ & $1.62 E-03$ \\
\hline 1200 & 114.2 & 7.12 & -.133 & 2.271 & $\begin{array}{l}120.0 \\
118.0 \\
114.0\end{array}$ & $\begin{array}{l}6.00 E-07 \\
5.65 E-J 7 \\
4.96 E-07\end{array}$ & $\begin{array}{l}3.48 E-06 \\
3.28 E-06 \\
2.88 E-06\end{array}$ & $2.10 E-04$ & $1.22 E-03$ \\
\hline 1600 & 114.7 & 8.46 & -.180 & 2.074 & $\begin{array}{l}122.1 \\
121.2 \\
115.3\end{array}$ & $\begin{array}{l}3.52 E-07 \\
2.25 E-07 \\
2.04 E-07\end{array}$ & $\begin{array}{l}2.04 E-C 6 \\
1.30 t-06 \\
1.19 E-06\end{array}$ & $1.24 E-04$ & $7.20 E-04$ \\
\hline 2200 & 115.7 & 6.33 & -.105 & 7.124 & $\begin{array}{l}118.0 \\
116.0 \\
120.0\end{array}$ & $\begin{array}{l}2.72 E-07 \\
2.0 C E-07 \\
1.70 E-07\end{array}$ & $\begin{array}{l}1.58 E-06 \\
1.16 E-06 \\
9.83 E-C 7\end{array}$ & $1.36 E-04$ & $7.87 E-04$ \\
\hline 3200 & 118.5 & 7.14 & -.275 & 2.349 & $\begin{array}{l}111.7 \\
119.5 \\
122.4\end{array}$ & $\begin{array}{l}6.16 E-08 \\
5.55 E-08 \\
5.24 E-08\end{array}$ & $\begin{array}{l}3.57 \mathrm{E}-07 \\
3.22 \mathrm{E}-07 \\
3.04 \mathrm{E}-07\end{array}$ & $4.12 \mathrm{E}-05$ & $2.39 E-04$ \\
\hline
\end{tabular}

? INUICATFS UNCERTAINTIES IN OATA.

X INDICATFS INVALIO UR INCOMPLETE UATA. 
GROUNO LEVEL AND TOWER SAMPLING 20OM TO 3200M. FOLLUWING SOME EXTRAPQLATION AT SUUTH EDGE. ALL ARCS EMBRACE THE CROSSWIND EXTENT OF TRACER DISTRIBUTIUNS. 13 OF 20 TLWERS "HIT" BY TRACERS: ALL VERTICAL DISTRIBUTIONS

TRUNCATED AT TOP. ALTHO ND RAIN OURING TRACER RELEASE, PRIUR RAIN LEFT FILTERS WET; EFFECT, IF ANY, UNKNOHN.

\begin{tabular}{|c|c|c|c|c|c|c|c|c|c|}
\hline $\begin{array}{l}\text { TEST } \\
\text { ZINC } \\
\text { SAMPLER }\end{array}$ & $\begin{array}{l}V 2 \quad N D \\
\text { SULF IDE } \\
\text { HT } 1.5 \mathrm{H}\end{array}$ & $\begin{array}{l}\text { VEMBFR } \\
\text { RELEASE } \\
\text {. }\end{array}$ & $\begin{array}{l}9.197 \\
\text { FRDM }\end{array}$ & $\begin{array}{l}2103 \\
E L E V A T I 0 \\
U=4.4 \mathrm{M}\end{array}$ & $\begin{array}{lll}0 & T U & 110 \\
N & U F & 26 \\
S E C & A T\end{array}$ & $\begin{array}{l}0 \text { PST } \\
26 M\end{array}$ & & & \\
\hline $\begin{array}{l}\text { DISTANCE } \\
\text { FROM }\end{array}$ & \multicolumn{4}{|c|}{$\begin{array}{l}\text { CBOSSWIND_DISIBIBUIIQN } \\
\text { STAND -COEEEICIEAI_- }\end{array}$} & THREE & \multicolumn{2}{|c|}{ MAXIMUM EXPUSURES } & \multirow{2}{*}{\multicolumn{2}{|c|}{$\begin{array}{l}\text { CROSSWINO } \\
\text { INIEGBAIED_YALUES }\end{array}$}} \\
\hline SOURCE & MEAN & DEV & SKEV & KUR T & AZIMUTH & $E / Q$ & $E U / O$ & & CI(EU/O) \\
\hline 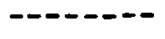 & $-\cdots$ & $-\cdots--$ & $\cdots-\cdots$ & 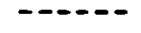 & -- & -- & - & -..-- & -- \\
\hline METERS & DEG & DEG & & & UEG & SEC/CU.M & $1 / 50 \cdot M$ & SEC/SO.M & $1 / M$ \\
\hline 200 & 135.2 & 11.43 & -.229 & 2.150 & $\begin{array}{l}144.0 \\
132.0 \\
130.0\end{array}$ & $\begin{array}{l}2 . \angle 1 E-05 \\
1.28 E-05 \\
1.17 E-05\end{array}$ & $\begin{array}{l}9.71 E-05 \\
5.61 E-05 \\
5.13 E-05\end{array}$ & $1.21 \mathrm{E}-03$ & $5.31 E-03$ \\
\hline 400 & 136.0 & 10.89 & -.180 & 2.016 & $\begin{array}{l}146.0 \\
142.0 \\
130.0\end{array}$ & $\begin{array}{l}1.79 E-U 5 \\
1.76 E-U 5 \\
1.47 E-05\end{array}$ & $\begin{array}{l}7.86 E-05 \\
7.73 E-05 \\
6.45 E-05\end{array}$ & $3.25 E-03$ & $1.43 E-02$ \\
\hline 800 & 137.6 & 10.19 & -.367 & 2.026 & $\begin{array}{l}144.2 \\
147.8 \\
146.0\end{array}$ & $\begin{array}{l}1.00 E-05 \\
8.05 E-06 \\
7.28 E-06\end{array}$ & $\begin{array}{l}4.42 E-05 \\
3.54 E-05 \\
3.20 E-05\end{array}$ & $2.81 E-03$ & $1.24 \mathrm{E}-02$ \\
\hline 1200 & 136.7 & 9.51 & -.106 & 2.080 & $\begin{array}{l}144.0 \\
138.0 \\
126.0\end{array}$ & $\begin{array}{l}4.19 E-06 \\
3.45 E-06 \\
3.24 E-06\end{array}$ & $\begin{array}{l}1.85 E-05 \\
1.52 E-05 \\
1.43 E-05\end{array}$ & $1.77 E-03$ & $7.81 \mathrm{E}-03$ \\
\hline 1600 & 138.6 & 9.05 & -.168 & 2.159 & $\begin{array}{l}144.1 \\
146.0 \\
147.9\end{array}$ & $\begin{array}{l}1.96 E-06 \\
1.96 E-06 \\
1.89 E-06\end{array}$ & $\begin{array}{l}8.61 E-06 \\
8.60 E-06 \\
8.31 E-06\end{array}$ & $1.28 E-03$ & $5.62 E-03$ \\
\hline 2200 & 139.0 & 8.75 & -.181 & 2.127 & $\begin{array}{l}138.0 \\
128.0 \\
140.0\end{array}$ & $\begin{array}{l}1.28 E-06 \\
1.27 E-06 \\
1.26 E-06\end{array}$ & $\begin{array}{l}5.64 E-06 \\
5.60 E-06 \\
5.53 E-06\end{array}$ & $1.08 E-03$ & $4.75 E-03$ \\
\hline 3200 & 140.1 & 8.94 & -.244 & 1.816 & $\begin{array}{l}148.9 \\
147.9 \\
146.9\end{array}$ & $\begin{array}{l}1.22 E-06 \\
1.207 E-06 \\
9.26 E-07\end{array}$ & $\begin{array}{l}5.35 E-06 \\
4.70 E-06 \\
4.07 E-06\end{array}$ & $9.82 \mathrm{E}-04$ & $4 \cdot 32 E-03$ \\
\hline
\end{tabular}

$?$ INDICATES UNCERTAINTIES IN OATA.

X INDICATES INVALid OR INCUMPLETE DATA. 
TEST V2 NEVEMEER, 7,1972 1030 Tİ 11 DUO PST

PMCDAMINE B RELEASE FROM ELFVATION UF $26 \mathrm{M}$

$$
U=4.4 \mathrm{M} / \mathrm{SEC} \text { AT } 26 \mathrm{M}
$$

\begin{tabular}{|c|c|c|c|c|c|c|c|c|c|}
\hline $\begin{array}{l}\text { DISTAIICE } \\
\text { FRIMM }\end{array}$ & \multicolumn{4}{|c|}{$\begin{array}{l}\text {-CBOSSWINDEDISIRIEUIION } \\
\text { SIAND -COEEEICIENI- }\end{array}$} & \multicolumn{3}{|c|}{ THIREE MAXIMUM EXPUSURES } & \multicolumn{2}{|c|}{$\begin{array}{l}\text { CROSSWINC } \\
\text { IAIELOEAIED_YALUES- }\end{array}$} \\
\hline SIIIRCE. & MEAN & DEV & SKEW & KURT & ALIMUTiA & $E / 6$ & $E U / O$ & $(1 / E / U)$ & $C I(E U / O)$ \\
\hline------- & ---- & $-\cdots--$ & ----- & $----\cdots$ & ------- & $-\cdots--\cdots$ & '-------- & $-\cdots-\infty$ & $--\infty-\cdots--$ \\
\hline METERS & DEG & DEG & & & DEG & $S E C / C U . M$ & $1 / S W \cdot M$ & $S E C / S Q \cdot M$ & $1 / M$ \\
\hline 200 & 130.1 & 10.67 & .134 & 1.947 & $\begin{array}{l}122.0 \\
126.0 \\
144.0\end{array}$ & $\begin{array}{l}5.64 E-U 6 \\
5.64 E-06 \\
3.69 t-j 6\end{array}$ & $\begin{array}{l}2.48 E-05 \\
2.48 E-05 \\
1.62 E-U 5\end{array}$ & $4.01 E-04$ & $1.77 E-03$ \\
\hline 400 & 134.4 & 10.75 & -.058 & 1.853 & $\begin{array}{l}146.0 \\
130.0 \\
142.3\end{array}$ & $\begin{array}{l}1.2 \angle E-U 5 \\
1.13 E-U 5 \\
9.9 \forall E-U 6\end{array}$ & $\begin{array}{l}5.38 E-U 5 \\
4.99 E-U 5 \\
3.95 E-U 5\end{array}$ & $2.24 E-03$ & $9.84 E-03$ \\
\hline ro & 135.2 & 10.18 & -.039 & 1.842 & $\begin{array}{l}144.2 \\
146.0 \\
124.0\end{array}$ & $\begin{array}{l}1.01 E-05 \\
7.63 \mathrm{E}-06 \\
7.20 \mathrm{E}-06\end{array}$ & $\begin{array}{l}4.43 t-05 \\
3.36 t-05 \\
3.20 t-05\end{array}$ & $2.92 E-03$ & $1.2 \forall E-02$ \\
\hline 1200 & $136.6^{\prime}$ & 9.77 & -.076 & 1.901 & $\begin{array}{l}128.0 \\
148.0 \\
130.0\end{array}$ & $\begin{array}{l}3.67 E-\cup 6 \\
3.69 E-06 \\
3.43 E-06\end{array}$ & $\begin{array}{l}1.70 E-05 \\
1.62 E-05 \\
1.51 E-05\end{array}$ & $1.76 E-03$ & $7.75 E-03$ \\
\hline 1600 & 138.3 & 9.09 & -.279 & 2.073 & $\begin{array}{l}143.1 \\
144.1 \\
146.9\end{array}$ & $\begin{array}{l}2.50 E-06 \\
2.50 E-06 \\
2.06 E-06\end{array}$ & $\begin{array}{l}1.10 E-05 \\
1.10 E-05 \\
9.05 E-06\end{array}$ & $1.37 E-03$ & $6.03 E-03$ \\
\hline 2200 & 138.5 & 8.45 & -.140 & 2.125 & $\begin{array}{l}142.0 \\
138.0 \\
140.0\end{array}$ & $\begin{array}{l}1.76 E-06 \\
1.44 E-06 \\
1.30 E-36\end{array}$ & $\begin{array}{l}7.73 E-06 \\
6.36 E-06 \\
5.74 E-06\end{array}$ & $1.20 E-03$ & $5.30 E-03$ \\
\hline 3200 & 140.1 & 8.41 & -.347 & 1.872 & $\begin{array}{l}148.9 \\
147.9 \\
146.0\end{array}$ & $\begin{array}{l}1.10 E-06 \\
1.09 E-06 \\
8.83 E-07\end{array}$ & $\begin{array}{l}4.85 E-06 \\
4.7 \forall E-06 \\
3.89 E-06\end{array}$ & $9.01 E-04$ & $3.96 E-03$ \\
\hline
\end{tabular}

$?$ INDICATES UNCERTAINTIES IN DATA.

$X$ INDICATFS INVALID DR INCOMPLETE DATA. 
HR-H5 SAMPLINC AT 200 , BOO ANO $1600 M$; TUWER SAMPLING AI 200 AND BUOM. ALL CROSSIHNU UISTRIBUTIONS TRUNCATED LN SCIUTH. SIX DF TEN TOWERS "HIT", BUT DISTRIBUTIONS TRUNCATLE AT TCP: MAY FAVE BEEN TRACER ON TUWER AT BOOM, 112.6 DEG. BUT VERY NEAR BACKGROUND. LOW VGLTAGES ON MANY BOUM SENSOKS MAY HAVELEAD TD UNDERESTIMATES OF XIQ.

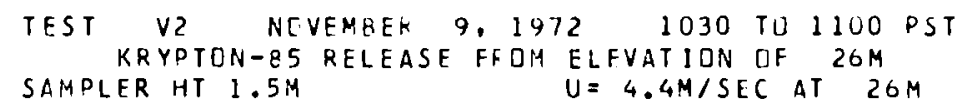

\begin{tabular}{|c|c|c|c|c|c|c|c|c|c|}
\hline $\begin{array}{l}\text { DISTANCE } \\
\text { FROA }\end{array}$ & \multicolumn{4}{|c|}{ 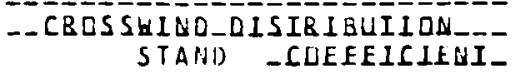 } & \multicolumn{3}{|c|}{ THREE MAXIMUM EXPUSURES } & \multicolumn{2}{|c|}{$\begin{array}{l}\text { CRUSSWIND } \\
\text { INIEGRAIED_YALUES- }\end{array}$} \\
\hline SOUURCE & MEAN & DEV & SKEW & KURT & AZIMUTH & $E / O$ & $E \cup / Q$ & $C I(E / U)$ & $C I(E U / O)$ \\
\hline------- & & $--\cdots$ & 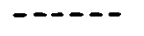 & ---- & ----- & $-\cdots-.--$ & 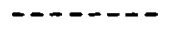 & $-\ldots-n$ & --- \\
\hline METERS & DEG & DEG & & & UEG & $S E C / C U . M$ & $1 / S Q \cdot M$ & SEC/SO.M & $1 / M$ \\
\hline 200 & $131.3 x$ & $10.72 x$ & $-.123 x$ & $1.944 x$ & $\begin{array}{l}128.0 ? \\
144.0 ? \\
122.0 ?\end{array}$ & $\begin{array}{l}5.8 \cup E-06 ? \\
5.15 E-06 ? \\
5.11 E-06 ?\end{array}$ & $\begin{array}{l}2.55 t-05 ? \\
2.26 E-05 ? \\
2.25 E-05 ?\end{array}$ & $5.27 E-04 x$ & $2.32 E-03 x$ \\
\hline $800 x$ & $123.4 x$ & $6.86 x$ & $-.058 x$ & $1.650 x$ & $\begin{array}{l}125.8 x \\
122.1 x \\
133.2 x\end{array}$ & $\begin{array}{l}1.96 E-06 X \\
1.69 E-36 X \\
1.67 E-06 X\end{array}$ & $\begin{array}{l}6.72 E-06 x \\
7.42 E-06 x \\
7.34 E-06 X\end{array}$ & $3.53 E-04 X$ & 1. $.55 E-03 x$ \\
\hline $1600 x$ & $122.1 x$ & $3.09 x$ & $-1.191 x$ & $3.553 x$ & $\begin{array}{l}125.0 x \\
123.1 x \\
121.2 x\end{array}$ & $\begin{array}{l}2.22 E-06 X \\
1.23 E-06 x \\
9.29 E-J 7 X\end{array}$ & $\begin{array}{l}9.70 t-06 x \\
5.42 E-06 x \\
4.09 t-06 x\end{array}$ & $2.02 E-04 X$ & $8.90 E-04 X$ \\
\hline
\end{tabular}

$?$ INDICATES UNCERTAINTIES IN DATA.

$X$ INDICATES INVALIO OR INCOMPLETE DATA. 


$$
\text { B }-95
$$


GRLIUNO LEVEL AND TONER SAMPLING ZUOM TO 32COM. MUST ARCS TRUNCATED ON SOUTH ENU. BUT CENTERLINE ALHAYS CBSERVED. MOST VERTICAL DISTRIBUTIGIS TRUNCATED AT TUPS LF TOWERS.

\begin{tabular}{|c|c|c|c|c|c|c|c|c|c|}
\hline $\begin{array}{l}\text { DISTANCE } \\
\text { FRCHM }\end{array}$ & \multicolumn{4}{|c|}{$\begin{array}{l}\text {-CBUSSNIND_DISIBIBUIIDN } \\
\text { STANO CLEEEICLENI- }\end{array}$} & \multirow{2}{*}{ AHREE } & \multicolumn{2}{|c|}{ MAXIMUM EXPOSURES } & \multirow{2}{*}{\multicolumn{2}{|c|}{$\begin{array}{l}\text { CROSSWIND } \\
\text { INIEGBAIEL_KALUES- }\end{array}$}} \\
\hline SUURCE & $M E A N$ & DEV & SKEW & KURT & & $E / 0$ & $E U / O$ & & \\
\hline METERS & DEG & DEG & & & UEG & SEL/CU.M & $1 / 50 . M$ & $S E C / S Q . M$ & $1 / M$ \\
\hline 200 & $140.3 x$ & $9.24 x$ & $-.284 x$ & $2.670 x$ & $\begin{array}{l}142.0 \\
144.0 \\
140.0\end{array}$ & $\begin{array}{l}3.56 E-U 5 \\
3.46 E-U 5 \\
3.42 E-U 5\end{array}$ & $\begin{array}{l}1.50 E-U 4 \\
1.45 E-04 \\
1.44 E-04\end{array}$ & $2.44 E-03 x$ & $1.02 E-02 x$ \\
\hline 400 & $140.7 x$ & $6.96 x$ & $-.293 x$ & $2.752 x$ & $\begin{array}{l}138.0 \\
146.0 \\
142.0\end{array}$ & $\begin{array}{l}2.37 E-U 5 \\
1.85 t-U 5 \\
1.57 E-U 5\end{array}$ & $\begin{array}{l}9.9 S E-05 \\
7.75 E-05 \\
6.61 t-05\end{array}$ & $2.48 E-03 x$ & $1.04 E-02 X$ \\
\hline 900 & $143.0 ?$ & $7.37 ?$ & $.143 ?$ & $2.394 ?$ & $\begin{array}{l}136.8 \\
147.8 \\
144.2\end{array}$ & $\begin{array}{l}5.78 E-U 6 \\
4.39 E-06 \\
4.72 E-U 6\end{array}$ & $\begin{array}{l}2.43 E-05 \\
2.05 E-05 \\
1.98 E-05\end{array}$ & $1.42 E-03 ?$ & $5.95 E-03 ?$ \\
\hline 1200 & $142.0 x$ & $6.71 x$ & $.462 x$ & $2.417 x$ & $\begin{array}{l}136.0 \\
138.0 \\
142.0\end{array}$ & $\begin{array}{l}1.50 E-06 \\
1.48 E-06 \\
1.18 E-66\end{array}$ & $\begin{array}{l}6.32 E-06 . \\
6.23 E-06 \\
4.94 E-06\end{array}$ & $4.27 t-04 x$ & 1. $79 E-03 x$ \\
\hline 1600 & $142.1 x$ & $6.07 x$ & $.174 x$ & $2.377 x$ & $\begin{array}{l}138.3 \\
144.1 \\
140.2\end{array}$ & $\begin{array}{l}1.11 E-06 \\
1.06 E-06 \\
1.06 E-06\end{array}$ & $\begin{array}{l}4.68 E-06 \\
4.52 E-06 \\
4.44 E-06\end{array}$ & $5.03 E-04 X$ & $2.11 E-03 x$ \\
\hline 2200 & $143.4 \times$ & $6.24 x$ & $.358 x$ & $2.508 x$ & $\begin{array}{l}140.0 \\
132.0 \\
142.0\end{array}$ & $\begin{array}{l}3.66 E-07 \\
3.39 E-07 \\
3.18 E-U 7\end{array}$ & $\begin{array}{l}1.54 E-06 \\
1.42 E-06 \\
1.34 E-06\end{array}$ & $2.04 E-04 x$ & $8.56 E-04 X$ \\
\hline 3200 & 142.7 & 5.28 & -.174 & 2.383 & $\begin{array}{l}143.0 \\
139.1 \\
144.0\end{array}$ & $\begin{array}{l}2.15 E-U 7 \\
1\{07 E-U 7 \\
1.81 E-07\end{array}$ & $\begin{array}{l}9.05 E-07 \\
7.05 E-U 7 \\
7.62 E-07\end{array}$ & $1.53 E-04$ & $6.41 E-04$ \\
\hline
\end{tabular}

$?$ INDICATES UNCERTAINTIES IN OATA.

$X$ INDICATES INVALID OR INCOMPLETE DATA. 


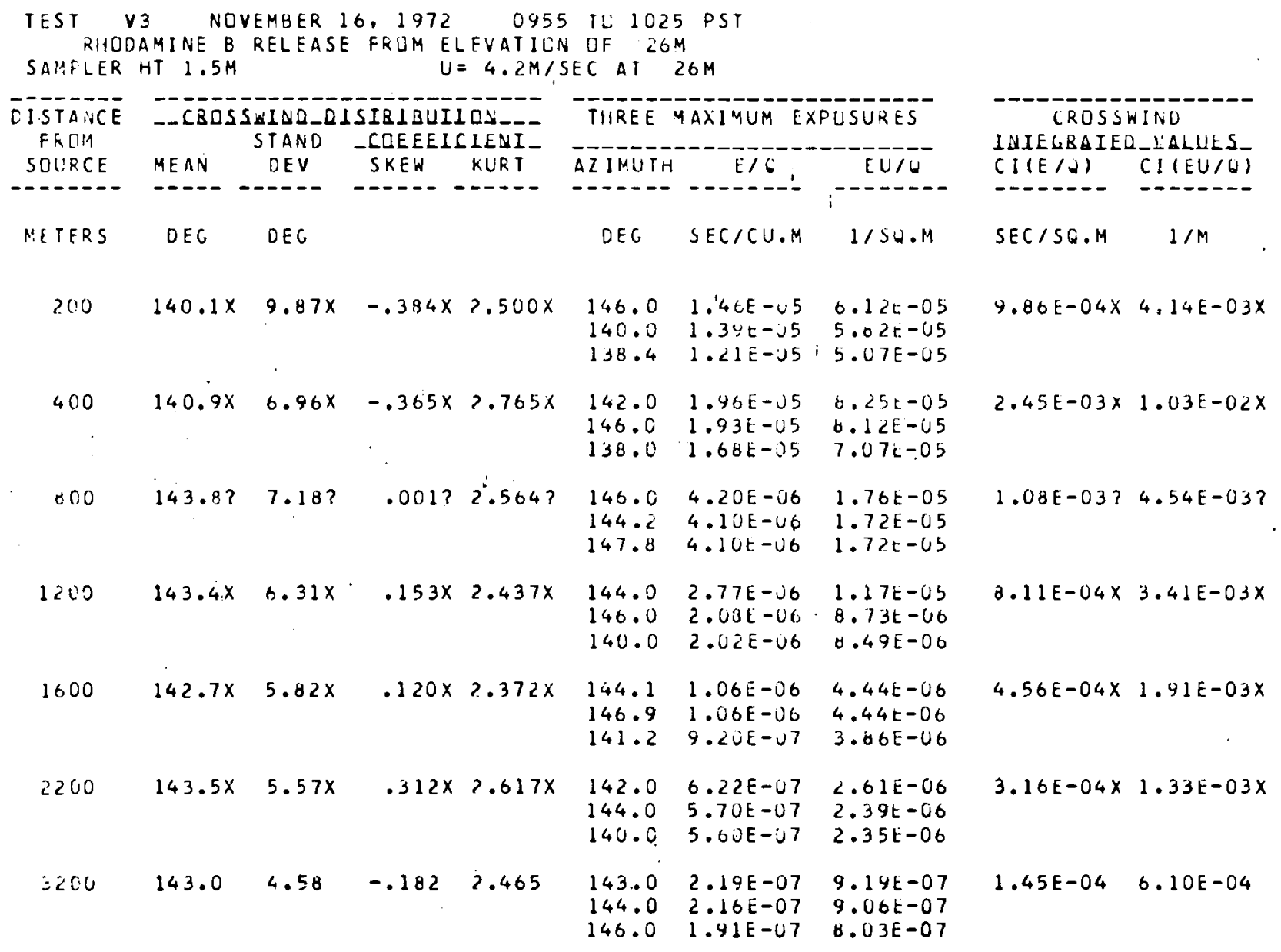

? INUICATES UNCERTAINTIES IN DATA.

$x$ indicates inValid UR INCUMplete uata. 
CROUNO LEVEL AND TOMER SAMPLING 200M TO 1600M - WITH AUUITICNAL TWO TOWERS SAMPLING AT $3200 M$.

ALL ARCS EMBRACE CRUSSWIND EXTENT CF TRACER. TOWER SAMPLES TRUNCATED AT TOP. ABOUT 9 CM SNOW DEPTH DN GRCUNO DURING EXPERIMENT. THERMALLY STABLE IN LOWEST GM. BUT NEUTRAL ABOVE.

\begin{tabular}{|c|c|c|c|c|c|c|c|c|c|}
\hline $\begin{array}{l}\text { DISTANCE } \\
\text { FRUM }\end{array}$ & \multicolumn{4}{|c|}{ 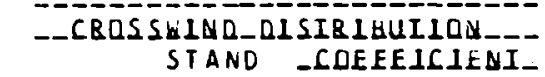 } & \multirow[t]{2}{*}{ THREE } & \multicolumn{2}{|c|}{ MAXIMUM EXPOSURES } & \multicolumn{2}{|c|}{$\begin{array}{l}\text { CRUSSWINO } \\
\text { INIEGBAIED_YALUES }\end{array}$} \\
\hline SOURCE & MEAN & DEV & SKEW & KURT & & $E / O$ & $E \cup / Q$ & $C I(E / Q)$ & CIIEU/O) \\
\hline METERS & DEG & DEG & & & UEG & $S E C / C U, M$ & $1 / \mathrm{SQ} \cdot \mathrm{M}$ & SEC/SU.M & $1 / M$ \\
\hline 200 & 132.7 & 5.14 & 2.042 & B. 701 & $\begin{array}{l}133.0 \\
134.0 \\
132.0\end{array}$ & $\begin{array}{l}6.90 E-06 \\
3.00 E-06 \\
1.70 E-06\end{array}$ & $\begin{array}{l}1.79 E-05 \\
7.80 E-0 t \\
4.41 E-06\end{array}$ & $1.12 E-04$ & $2.91 E-04$ \\
\hline 800 & 137.1 & 7.62 & .057 & 1.869 & $\begin{array}{l}138.7 \\
129.5 \\
127.7\end{array}$ & $\begin{array}{l}1.94 E-05 \\
1.22 E-05 \\
1.20 E-05\end{array}$ & $\begin{array}{l}5.05 E-05 \\
3.16 E-05 \\
3.13 E-05\end{array}$ & $3.64 E-03$ & $9.46 E-03$ \\
\hline 1600 & 136.7 & 6.64 & -.536 & 2.935 & $\begin{array}{l}138.3 \\
137.3 \\
140.2\end{array}$ & $\begin{array}{l}1.19 E-05 \\
1.07 E-05 \\
8.28 E-06\end{array}$ & $\begin{array}{l}3.10 E-05 \\
2.79 E-05 \\
2.15 E-05\end{array}$ & $3.21 E-03$ & $8.34 E-03$ \\
\hline
\end{tabular}

$?$ INDICATES UNCERTAINTIES IN OATA.

$X$ INDICATES INVALIO OR INCUMPLETE DATA. 


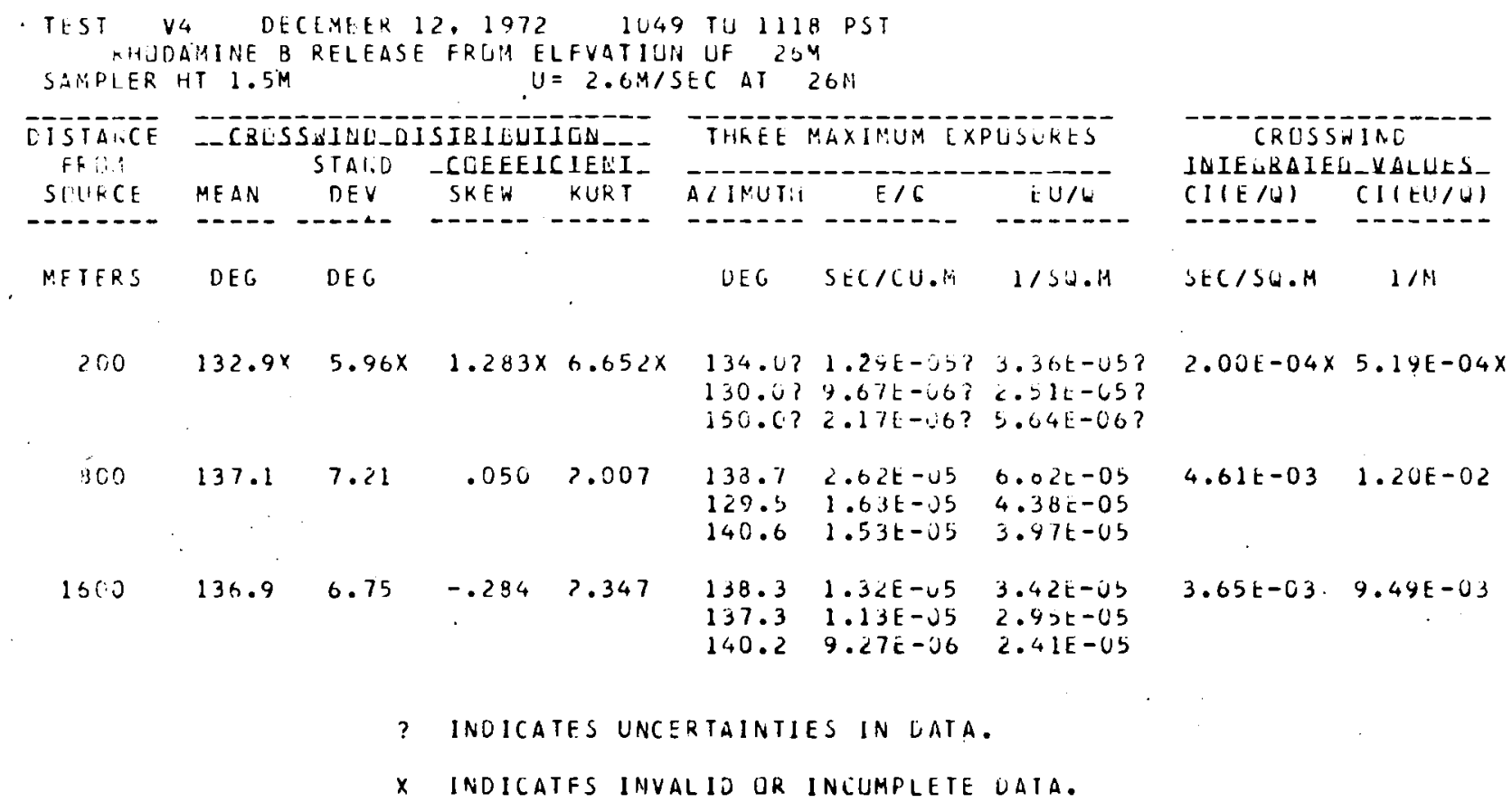


GRTIUNO LEVEL ANO TOWER SAMPLING $200 M$ TO $3200 \mathrm{i}$. ALL ARCS EMBRACE CRUSSWINU EXTEINT OF DISTRIBUTIUN.

14 TOWERS "HIT" BY TRACER: ALTHCUGH UISTRIBUTIONS TRUNCATEO AT TOPS UF TUWER.S. EXTRAPOLATION WOULO BE

REASONABLE. VERTICAL CRISS SECTICNS THRQUGH TOWER ARCS SHOWS SIGIIFICANT OIRECTIUN SHEAR OF TRACERS WITH HT.

\begin{tabular}{|c|c|c|c|c|c|c|c|c|c|}
\hline $\begin{array}{l}\text { TEST V } \\
\text { IINC } \\
\text { SAMPLER }\end{array}$ & $\begin{array}{l}5 \quad \text { SEP } \\
\text { SULFIDE } \\
\text { HT } 1.5 \mathrm{M}\end{array}$ & $\begin{array}{l}\text { TEMBFR } \\
\text { RELFASE }\end{array}$ & E. 197 & $\begin{array}{l}3 \quad 050 \\
E L E V A T I C \\
U=4.3 M\end{array}$ & $\begin{array}{lll}1 & \text { TU } & 053 \\
N & \text { OF } & 261 \\
\text { SEC AT }\end{array}$ & $\begin{array}{l}1 \text { PST } \\
26 M\end{array}$ & & & \\
\hline \multirow{2}{*}{$\begin{array}{l}\text { DISTANCE } \\
\text { FRDM } \\
\text { SUURCE }\end{array}$} & \multicolumn{4}{|c|}{$\begin{array}{l}\text { CBOSSWIND_DISIRIBUIICN } \\
\text { STAND -COEEEICIENI- }\end{array}$} & THREE & \multicolumn{2}{|c|}{ MAXIMUM EXPOSURES } & \multicolumn{2}{|c|}{$\begin{array}{c}\text { CROSSW INU } \\
\text { IUIEGBAIEU_YALUES_ }\end{array}$} \\
\hline & MEAN & DEV & SKEH & KURT & AZIMUIH & $E / 0$ & $E U / O$ & $C I(E / U)$ & $C I(E U / O)$ \\
\hline 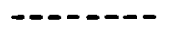 & ---- & ------ & ----- & ----- & $----1-$ & -------- & ------- & ------- & ------ \\
\hline METERS & DEG & DEG & & & UEG & $S E C / C U . M$ & $1 / 50.14$ & SEC/SQ.M & $1 / M$ \\
\hline 200 & 102.5 & 6.67 & -.057 & 2.666 & $\begin{array}{r}104.0 \\
106.0 \\
96.0\end{array}$ & $\begin{array}{l}2.03 E-06 \\
1.91 E-06 \\
1.43 E-06\end{array}$ & $\begin{array}{l}8.71 E-06 \\
8.21 E-06 \\
6.14 E-06\end{array}$ & $9.72 E-05$ & $4.1 \forall E-04$ \\
\hline 400 & 101.6 & $\begin{array}{c}6.54 \\
:\end{array}$ & -.081 & 2.554 & $\begin{array}{r}9 B .0 \\
110.0 \\
102.0\end{array}$ & $\begin{array}{l}4.72 E-U 6 \\
2.68 E-U 6 \\
2.27 E-06\end{array}$ & $\begin{array}{l}2.03 E-05 \\
1.15 E-05 \\
9.74 E-06\end{array}$ & $3.86 E-04$ & $1.60 E-03$ \\
\hline$\theta 00$ & 101.5 & 6.03 & -.532 & 2.851 & $\begin{array}{l}103.0 \\
105.0 \\
106.9\end{array}$ & $\begin{array}{l}4.49 E-06 \\
8.58 E-U 6 \\
8.04 E-16\end{array}$ & $\begin{array}{l}4.08 E-05 \\
3.69 E-05 \\
3.46 E-05\end{array}$ & $1.84 E-03$ & $7.93 E-03$ \\
\hline 1200 & 100.7 & 5.92 & -.012 & 2.710 & $\begin{array}{r}102.0 \\
100.0 \\
98.0\end{array}$ & $\begin{array}{l}6.77 E-06 \\
5.24 E-06 \\
4.01 E-06\end{array}$ & $\begin{array}{l}2.91 E-05 \\
2.25 E-05 \\
2.07 E-05\end{array}$ & $1.67 E-03$ & $7.16 E-03$ \\
\hline 1600 & 102.3 & 5.93 & -.029 & 2.466 & $\begin{array}{r}101.6 \\
99.6 \\
104.5\end{array}$ & $\begin{array}{l}4.28 E-06 \\
4.11 E-06 \\
3.57 E-06\end{array}$ & $\begin{array}{l}1.84 E-05 \\
1.77 E-05 \\
1.54 E-05\end{array}$ & $1.40 E-03$ & $6.00 E-03$ \\
\hline $3200^{\circ}$ & 109.8 & 4.49 &.-.508 & 2.963 & $\begin{array}{l}108.8 \\
109.7 \\
111.7\end{array}$ & $\begin{array}{l}3.42 E-06 \\
3.33 E-06 \\
3.06 E-06\end{array}$ & $\begin{array}{l}1.47 E-05 \\
1.43 E-05 \\
1.31 E-05\end{array}$ & $1.85 E-03$ & $7.96 E-03$ \\
\hline
\end{tabular}

? INDICATES UNCERTAINTIES IN DATA.

$X$ INDICATES INVALID DR INCOMPLETE OATA. 
TEST VS SEPTEMEER $5,1973 \quad 0501$ TU 0531 PST P.HUDAMINE B RELEASE FRDM ELEVATIUN GF $26 \mathrm{M}$ SAMPLER HT $1.5 \mathrm{M}$ U $U=4.3 \mathrm{M} / \mathrm{SEC}$ AT $26 \mathrm{i1}$

\begin{tabular}{|c|c|c|c|c|c|c|c|c|c|}
\hline \multirow{2}{*}{$\begin{array}{l}\text { QISTANCE } \\
\text { FKLAA } \\
\text { SLURCE }\end{array}$} & \multicolumn{4}{|c|}{-CBOSSYINQ_DISIRIBUIIOA } & \multicolumn{3}{|c|}{ THREE MAXIMUM EXPUSURES } & \multicolumn{2}{|c|}{$\begin{array}{l}\text { CRDSSWINO } \\
\text { INIEGBAIED_YALUES }\end{array}$} \\
\hline & MEAN & DEV & SKEW & KURT & ALIMUTH & $E / 0$ & EU/Q, & $C I(E / Q)$ & Ci(EU/O) \\
\hline 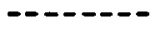 & 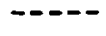 & ---- & $---\cdots$ & ---- & $-\cdots--$ & - n-.... & 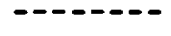 & 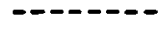 & $\cdots$ \\
\hline METERS & DEG & DEG & & & OEG & SEC/CU.M & $1 / S Q \cdot M$ & SEC/SO.M & $1 / M$ \\
\hline 200 & 101.8 & 6.78 & .370 & 2.908 & $\begin{array}{r}100.0 \\
98.0 \\
102.0\end{array}$ & $\begin{array}{l}1.26 E-06 \\
9.54 E-07 \\
8.09 E-07\end{array}$ & $\begin{array}{l}5.42 E-06 \\
4.10 E-06 \\
3.48 E-06\end{array}$ & $5.35 E-05$ & $2.30 E-04$ \\
\hline 400 & 98.9 & 6.65 & .018 & 2.536 & $\begin{array}{r}98.0 \\
102.0 \\
94.0\end{array}$ & $\begin{array}{l}3.93 E-U 6 \\
3.4 U E-06 \\
3.19 E-06\end{array}$ & $\begin{array}{l}1.69 \mathrm{E}-05 \\
1.46 \mathrm{E}-05 \\
1.37 \mathrm{E}-05\end{array}$ & $4.92 E-04$ & $2.12 \mathrm{E}-03$ \\
\hline 300 & 100.0 & 6.57 & -.282 & 2.360 & $\begin{array}{l}103.0 \\
101.1 \\
165.0\end{array}$ & $\begin{array}{l}6.19 E-06 \\
6.02 E-06 \\
6.02 E-06\end{array}$ & $\begin{array}{l}2.66 E-05 \\
2.59 E-05 \\
2.59 E-05\end{array}$ & $1.58 E-03$ & $6.78 E-03$ \\
\hline 1200 & 99.7 & 6.23 & -.067 & 2.671 & $\begin{array}{r}102.0 \\
100.0 \\
98.0\end{array}$ & $\begin{array}{l}4.01 E-06 \\
3.91 E-06 \\
3.22 E-06\end{array}$ & $\begin{array}{l}1.72 E-05 \\
1.68 E-05 \\
1.39 E-05\end{array}$ & $1.25 E-03$ & $5.36 E-03$ \\
\hline 1600 & 101.5 & 6.24 & -.083 & 2.356 & $\begin{array}{l}102.6 \\
103.6 \\
101.6\end{array}$ & $\begin{array}{l}3.97 E-U 6 \\
3.85 E-06 \\
3.51 E-06\end{array}$ & $\begin{array}{l}1.71 E-05 \\
1.66 E-05 \\
1.51 E-05\end{array}$ & $1.66 E-03$ & $7.16 E-03$ \\
\hline 2200 & 108.0 & 5.15 & -.195 & 2.299 & $\begin{array}{l}106.8 \\
111.7 \\
105.8\end{array}$ & $\begin{array}{l}1.59 E-06 \\
1.59 E-06 \\
1.53 E-06\end{array}$ & $\begin{array}{l}6.62 E-06 \\
6.82 E-06 \\
6.60 E-06\end{array}$ & $1.15 E-03$ & $4.94 E-03$ \\
\hline
\end{tabular}

? INUICATES UNCERTAINTIES IN UATA.

$X$ INDICATES INVALID OR INCOMPLETE LATA. 
KRYPTCN-85 SAMELING AT 200M, 80OM ANO 16OOM ONLY: TOWER SAMPLING ONLY AT 2UOM AND 8OOM. ALL THREE "GROUNDLEVEL" ARCS TRUNCATED TIN NURTH. ALTHUUGH 6 LF IO OPERAIING TUWERS "MIT" BY KRYPTUN. TRACER DISTRIBUTIONS ARE TRUNCATED AT TOPS.

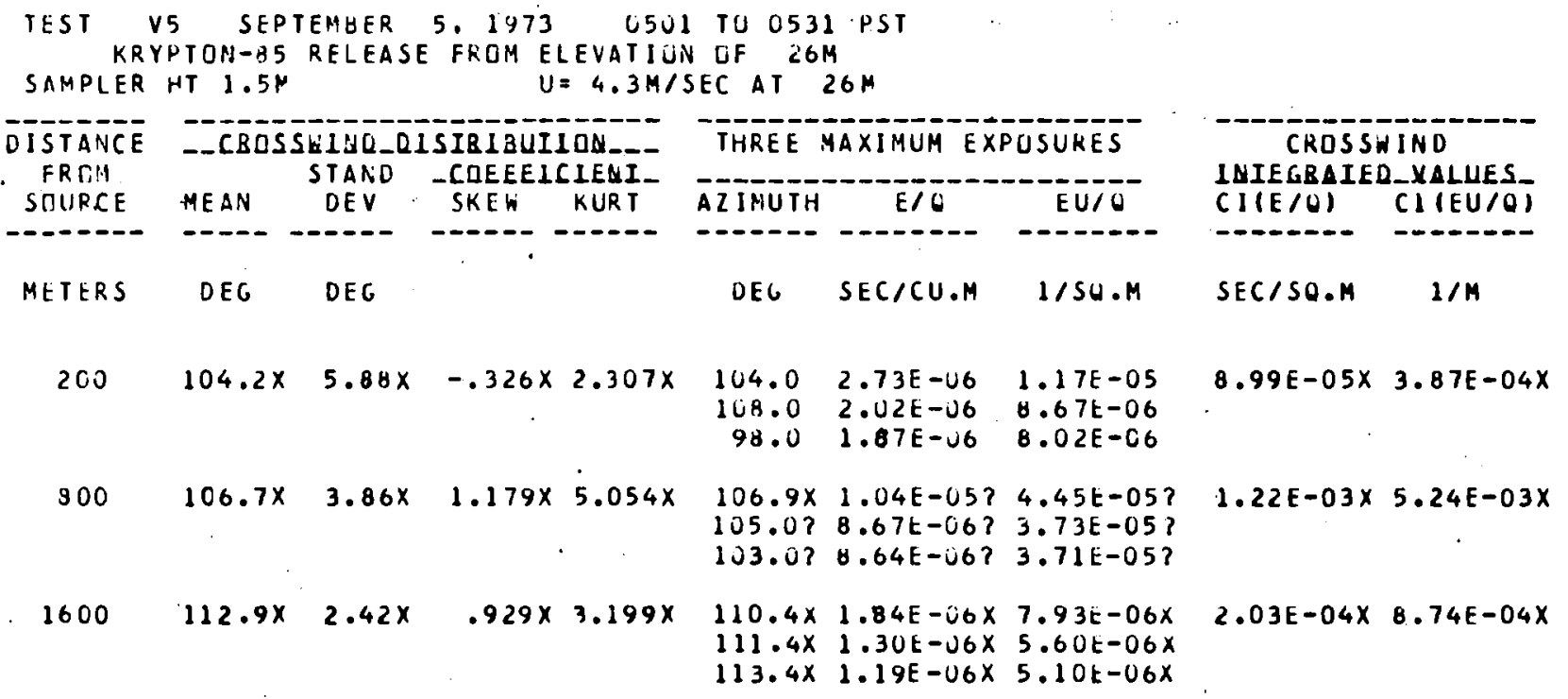

$?$ INDICATES UNCERTAINTIES IN DATA.

$x$ indicates INVAL ID DR INCOMPlete dATA. 


$$
\text { B-103 }
$$




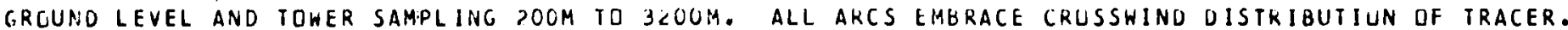
TURERS TRUNCATE TDP OF VERTICAL DISTKIBUTION. BUT EXTRAPILLATIUN WUULD NUT BE UNREASUNABLE.

\begin{tabular}{|c|c|c|c|c|c|c|c|c|c|}
\hline \multirow{2}{*}{$\begin{array}{l}\text { DISTANCE } \\
\text { FRCM } \\
\text { SOURCE }\end{array}$} & \multicolumn{4}{|c|}{ 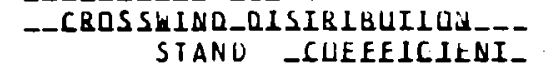 } & \multirow{2}{*}{$\begin{array}{l}\text { THREE } \\
\text { ALIMUTH }\end{array}$} & \multicolumn{2}{|c|}{ MAXIMUM EXPOSURES } & \multicolumn{2}{|c|}{$\begin{array}{l}\text { CKOSSHIND } \\
\text { INIEGBAIED_YALUES }\end{array}$} \\
\hline & MEAN & DEV & SKEW & KURT & & $E / C$ & EU/O & CIIE/UI & CI $(E U / Q)$ \\
\hline METERS & DEG & DEG & & & DEG & SEC/CU.M & $1 / 50 . M$ & SEC/SO.M & $1 / M$ \\
\hline 200 & 117.8 & 4.29 & .249 & 2.316 & $\begin{array}{l}114.0^{i} \\
116.0 \\
118.0\end{array}$ & $\begin{array}{l}6.50 E-06 \\
6.37 E-06 \\
5.57 E-06\end{array}$ & $\begin{array}{l}4.16 E-05 \\
4.07 E-05 \\
3.57 E-05\end{array}$ & $2.60 E-04$ & $1.67 E-03$ \\
\hline 400 & 118.5 & 4.65 & .165 & $2.753^{\circ}$ & $\begin{array}{l}114.0 \\
122.0 \\
118.0\end{array}$ & $\begin{array}{l}5.48 E-06 \\
4.49 E-06 \\
2.70 E-06\end{array}$ & $\begin{array}{l}3.51 E-05 \\
2.87 E-05 \\
1.73 E-05\end{array}$ & $4.19 E-04$ & $\begin{array}{c}2.68 E-03 \\
.\end{array}$ \\
\hline 800 & 119.1 & 3.90 & .215 & 2.104 & $\begin{array}{l}116.4 \\
118.3 \\
114.5\end{array}$ & $\begin{array}{l}1.53 \mathrm{E}-05 \\
1.50 \mathrm{E}-05 \\
1.20 \mathrm{E}-05\end{array}$ & $\begin{array}{l}9.00 E-05 \\
9.62 E-05 \\
7.70 E-05\end{array}$ & $2.31 E-03$ & $1.4 \forall E-02$ \\
\hline 1200 & 118.8 & 3.45 & .292 & 2.204 & $\begin{array}{l}118.0 \\
116.0 \\
122.0\end{array}$ & $\begin{array}{l}1.24 E-U 5 \\
1.21 E-05 \\
7.62 E-U 6\end{array}$ & $\begin{array}{l}7.93 E-05 \\
7.72 E-05 \\
4.88 E-05\end{array}$ & $2.27 E-03$ & $1.45 E-02$ \\
\hline 1600 & 119.1 & 3.27 & .223 & 2.100 & $\begin{array}{l}117.3 \\
118.2 \\
115.3\end{array}$ & $\begin{array}{l}1.12 E-0 b \\
9.34 E-06 \\
3.79 E-06\end{array}$ & $\begin{array}{l}7.16 E-05 \\
5.98 E-05 \\
5.63 E-05\end{array}$ & $2.39 E-03$ & $1.53 \mathrm{E}-02$ \\
\hline 3200 & .118 .8 & 2.70 & .036 & 2.185 & $\begin{array}{l}118.6 \\
116.6 \\
120.5\end{array}$ & $\begin{array}{l}4.19 E-06 \\
3.95 E-06 \\
3.49 E-06\end{array}$ & $\begin{array}{l}2.68 E-05 \\
2.53 E-05 \\
2.49 E-05\end{array}$ & $1.8 B E-03$ & $1.20 E-02$ \\
\hline
\end{tabular}

$?$ INDICATES UNCERTAINTIES IN DATA.

$x$ indicates invalio or.incomplete data. 


\begin{tabular}{|c|c|c|c|c|c|c|c|c|c|}
\hline \multirow{2}{*}{$\begin{array}{l}\text { DISTACEE } \\
\text { FRTEA } \\
\text { SUURCE }\end{array}$} & \multicolumn{4}{|c|}{$\begin{array}{l}\text {-CBOSSLISD DISIB IDUIICN } \\
\text { STAND -COEEEICIEAI_ }\end{array}$} & \multicolumn{3}{|c|}{ THREE MAXIMUA EXPUSURES } & \multicolumn{2}{|c|}{$\begin{array}{l}\text { CKOSSWIND } \\
\text { INIEGBAIEQ NOLUES }\end{array}$} \\
\hline & MEAN & DEV & SKEW & KURT & ALIMUTH & $E / O$ & $E \cup / Q$ & CIIE/OI & CI(EU/O) \\
\hline METERS & DEG & DEG & & & DEG & SEC/CU.M & $1 / S\lrcorner . M$ & SEC/SO.M & $1 / M$ \\
\hline 200 & 120.0 & 4.32 & -.096 & 2.278 & $\begin{array}{l}122.0 \\
124.0 \\
116.0\end{array}$ & $\begin{array}{l}6.31 E-07 \\
5.41 E-07 \\
5.27 E-U 7\end{array}$ & $\begin{array}{l}4.04 E-06 \\
3.46 E-06 \\
3.37 E-06\end{array}$ & $2.37 E-05$ & $1.52 E-04$ \\
\hline 800 & 119.3 & 3.83 & .142 & 2.094 & $\begin{array}{l}118.3 \\
114.5 \\
120.2\end{array}$ & $\begin{array}{l}9.89 E-06 \\
9.67 E-06 \\
6.41 E-06\end{array}$ & $\begin{array}{l}6.33 t-05 \\
6.19 E-05 \\
4.10 E-05\end{array}$ & $1.23 E-03$ & $7.89 E-03$ \\
\hline 1200 & 119.8 & 4.07 & -.404 & 1.918 & $\begin{array}{l}114.0 \\
120.0 \\
122.0\end{array}$ & $\begin{array}{l}6.92 E-06 \\
6.81 E-06 \\
6.81 E-06\end{array}$ & $\begin{array}{l}4.43 E-05 \\
4.36 E-05 \\
4.36 E-05\end{array}$ & $1.35 E-03$ & $8.62 E-03$ \\
\hline 1600 & 118.8 & 3.32 & .365 & 2.117 & $\begin{array}{l}116.3 \\
117.3 \\
115.3\end{array}$ & $\begin{array}{l}9.14 E-06 \\
8.05 E-06 \\
8.42 E-06\end{array}$ & $\begin{array}{l}5.35 E-05 \\
5.66 E-05 \\
5.39 E-05\end{array}$ & $2.08 E-03$ & $1.33 E-02$ \\
\hline 3200 & 110.2 & 2.62 & .254 & 2.295 & $\begin{array}{l}116.6 \\
117.6 \\
115.6\end{array}$ & $\begin{array}{l}4.98 E-06 \\
4.72 E-06 \\
4.54 E-06\end{array}$ & $\begin{array}{l}3.19 E-05 \\
3.02 E-05 \\
2.94 E-05\end{array}$ & $2.03 E-03$ & $1.30 E-02$ \\
\hline
\end{tabular}

? INDACATES UNCERTAIMTIES IN DATA.

$x$ INDICATES INYALID OR INCGMPLETE data. 
KRYPTFN-85 SAMPLING AT 200H, 800M ANO 160UM DILY: TUWER SAMPLING AT 200M AND BOOM ONLY. BOTH 200M AND 800M "GRIILIND-LEVEL" ARCS EMBRACE CROSSWINL EXTENT UF TRACER (AS UOES 16OOM ARC AFTER MUDEST EXTRAPOLATION).

T'H.EE MICDLE TOWERS AT BOTH $200 \mathrm{M}$ ANO OOUM INTERCEPT TRACER; PEAK CONCENTRATION SAMPLED. BUT TRUNCATION AT TOP.

TEST V6 SEPTEMBER 13, 1973 0516 TU C546 PST

KRYPTON-85 RELEASE FROM ELEVATIUN OF $26 \mathrm{M}$

SAMPLER HT $1.5 \mathrm{M} U=6.4 \mathrm{M} / \mathrm{SEC}$ AT $26 \mathrm{~N}$

\begin{tabular}{|c|c|c|c|c|c|c|c|c|c|}
\hline $\begin{array}{l}\text { DISTANCE } \\
\text { - FRTIA }\end{array}$ & \multicolumn{4}{|c|}{$\begin{array}{c}\text {-CBQSSHINO_DISIRIBUIILN-- } \\
\text { STAND -CDEEEICIENI- }\end{array}$} & \multicolumn{3}{|c|}{ THREE MAXIMUM EXPLSURES } & \multicolumn{2}{|c|}{$\begin{array}{c}\text { CRUSSWINJ } \\
\text { INIEGRAIED_Y YLUES_ }\end{array}$} \\
\hline SUURCE & MEAN & DEV & SKEW & KUR T & A ZIMUTH & $E / Q$ & EU/O & CIIE/O) & CIIEU/O) \\
\hline METERS & DEG & DEC & & & DEG & SEC/CU.M & $1 / 50 . M$ & $S E C / 5 O . M$ & $1 / M$ \\
\hline 200 & 117.3 & 5.74 & -.000 & $\begin{array}{l}2.516 \\
.\end{array}$ & $\begin{array}{l}116.0 \\
114.0 \\
122.0\end{array}$ & $\begin{array}{l}2.29 E-06 \\
2.26 E-06 \\
2.24 E-06\end{array}$ & $\begin{array}{l}1.47 E-05 \\
1.45 E-05 \\
1.43 E-05\end{array}$ & $1.22 E-04$ & $7.8 \cup E-04$ \\
\hline 800 & 119.1 & 4.21 & .150 & 2.608 & $\begin{array}{l}118.3 \\
116.4 ? \\
120.2\end{array}$ & $\begin{array}{l}1.15 E-05 \\
1.05 E-05 ? \\
9.97 E-06\end{array}$ & $\begin{array}{l}7.38 t-05 \\
6.73 E-05 ? \\
6.38 E-05\end{array}$ & $1.91 \mathrm{E}-03$ & $1.22 E-02$ \\
\hline 1600 & $119.3 ?$ & $3.44 ?$ & $.229 ?$ & $2.278 ?$ & $\begin{array}{l}117.3 \\
116.3 \\
118.2\end{array}$ & $\begin{array}{l}1.00 E-05 \\
9.31 E-06 \\
8.94 E-06\end{array}$ & $\begin{array}{l}6.41 E-05 \\
5.96 E-05 \\
5.72 E-05\end{array}$ & $2.41 t-03 ?$ & $1.54 E-02 ?$ \\
\hline
\end{tabular}

?. INDICATES UNCERTAINTIES IN DATA.

$X$ INOICATES INVALID OR INCOMPLETE DATA. 
B-107 


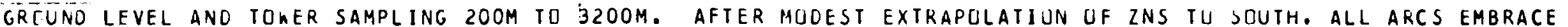
THE CROSSHINC EXTENT OF THIS TRACER. EIGHTEEN DF TWENTY TOWERS "HIT" IN THIS TEST UURING UNSTABLE CONDITIONS, BUt ALL DISTRIBUTIONS TRUNCATED AT TDPS OF TUNEKS. MUST UNSTABLE CONOITIONS UF THIS FIELD SERIES.

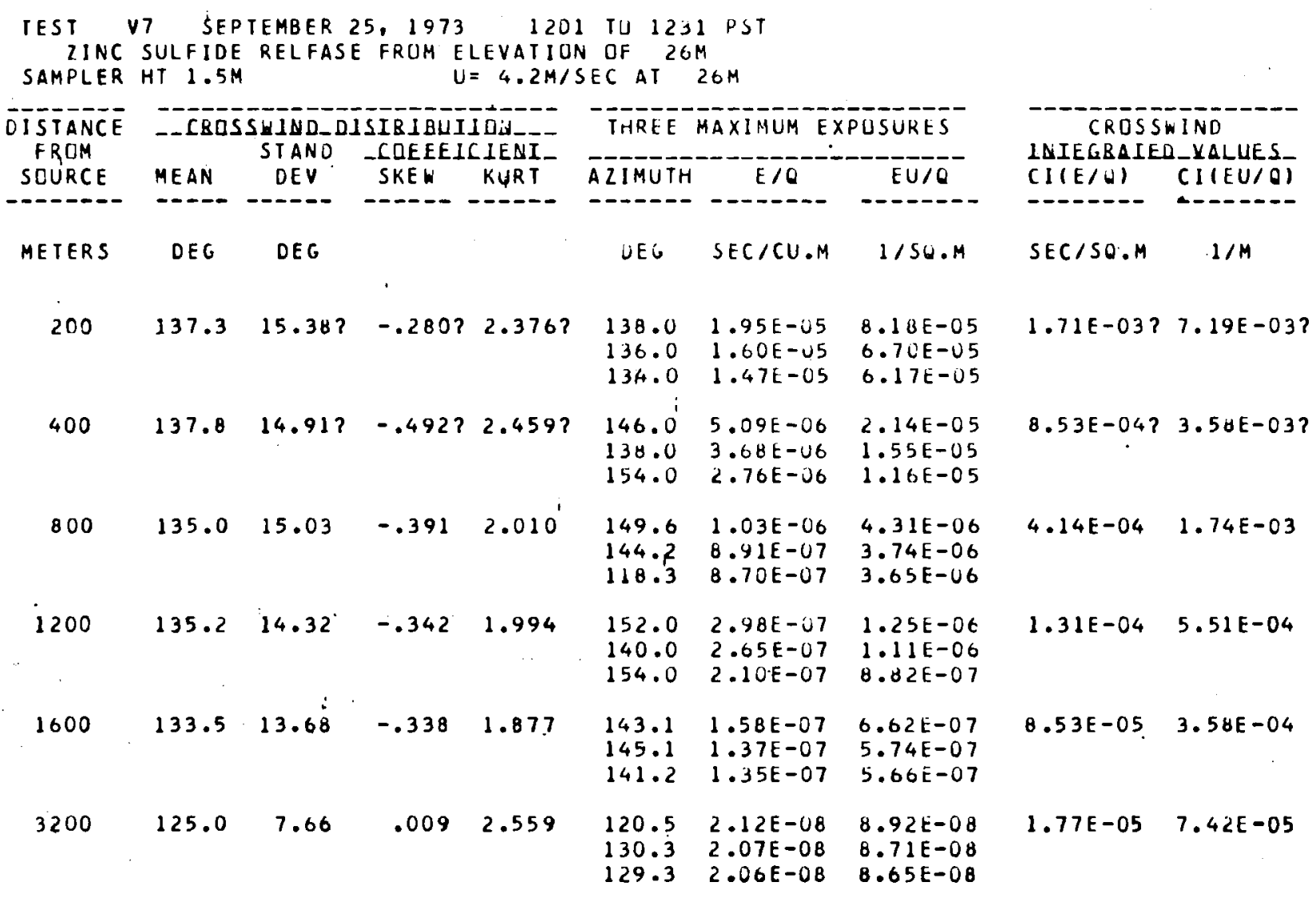

$?$ inOICATES UNCERTAinties in data.

$x$ INDICATES INVALIO OR INCDMPLETE DATA. 
ALTHGUGH SAMPLING AT 200. 300 ANI 1600M. DIFFUSIUN WAS SL GREAT THAT KRYPTON DEYECTED WITH CONFIOENCE ONLY AT 20OM. ALL 5 TOGERS AT $200 M$ HIT AY TKACER BUT TRUNCATED BADLY AT TOP IN ALL CASES. GROUNO LEVEL SAMPLING ALSO TRUNCATEO AT SOUTH. ESTIMATE AT 122 UEG BASCD CA SIMILARITY GETWEEN ZNS AND KRTPTUN CROSSWIND DISTRIBUTIONS.

'TEST VT SEPTEMBER 25, $1973 \quad 1201$ TJ 1231 PST

KRYPTON-85 RELEASE FROH ELFVATIUN OF $26 \mathrm{M}$

SAMPLER HT $1.5 \mathrm{~N}: U=4.2 \mathrm{M} / \mathrm{SEC}$ AT $26 \mathrm{M}$

\begin{tabular}{|c|c|c|c|c|c|c|c|c|c|}
\hline $\begin{array}{l}\text { DISTANCE } \\
\text { FRCII }\end{array}$ & $-C B D S$ & $\begin{array}{l}\text { SHIND_D } \\
\text { STAND }\end{array}$ & $\begin{array}{l}\text { SIRIBUI } \\
\text {-CUEEEI }\end{array}$ & CIENI & THREE M & $A A X I M U M E$ & USURES & $\begin{array}{l}\text { CROSS } \\
\text { LNIEGRAIED }\end{array}$ & $\begin{array}{l}\text { INO } \\
\text { YALUES }\end{array}$ \\
\hline SCURCE & MEAN & DEV & SKEW & KURT & AZ IMUTH & $E / 6$ & $E \cup / O$ & $C I(E / O)$ & CI $(E \cup / Q)$ \\
\hline$\cdots$ & ---- & $\ldots-\cdots$ & $-\cdots-$ & $---\cdots$ & $\cdots \cdots$ & $\ldots \ldots$ & $\cdots \cdots$ & 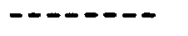 & $\therefore--\infty-\infty$ \\
\hline METERS & DEG & DEG & & & DEG & SEC/CU.M & $1 / S W \cdot M^{\prime}$ & SEC/SO.M & $1 / M$ \\
\hline 200 & $132.0 x$ & $11.86 x$ & $-.704 x$ & $2.472 x$ & $\begin{array}{l}138.0 \\
136.0 \\
142.0\end{array}$ & $\begin{array}{l}2.04 E-05 \\
1.95 E-05 \\
1.02 E-05\end{array}$ & $\begin{array}{l}8.55 E-05 \\
8.20 E-05 \\
7.64 E-05\end{array}$ & $1.36 E-03 x$ & $5.71 E-03 X$ \\
\hline
\end{tabular}

$?$ INDICATES UNCERTAINTIES IN DATA.

X INDICATES INVAL IO OR INCOMPLETE DATA. 
GRIUNU LEVEL AND TOHER SAMPLING 200M TO $3200 \mathrm{M}$. DNLY LINC SULFIDE TRACER RELEASED. ALL ARCS EMBRACE CROSSWINO EXTFNT OF TRACER DISTRIBUTIDN. ABDUT HALF UF TWENTY IUWERS EXPUSED TO TRACER, EUT ALL VERTICAL DISTRIBUTIDNS TRUNCATEO AT TUPS IIF IUWERS. FCTAL "W" KNOWN, BUT RATE OF TRACER EMISSION WAS LIKELY NOT CONSTANT.

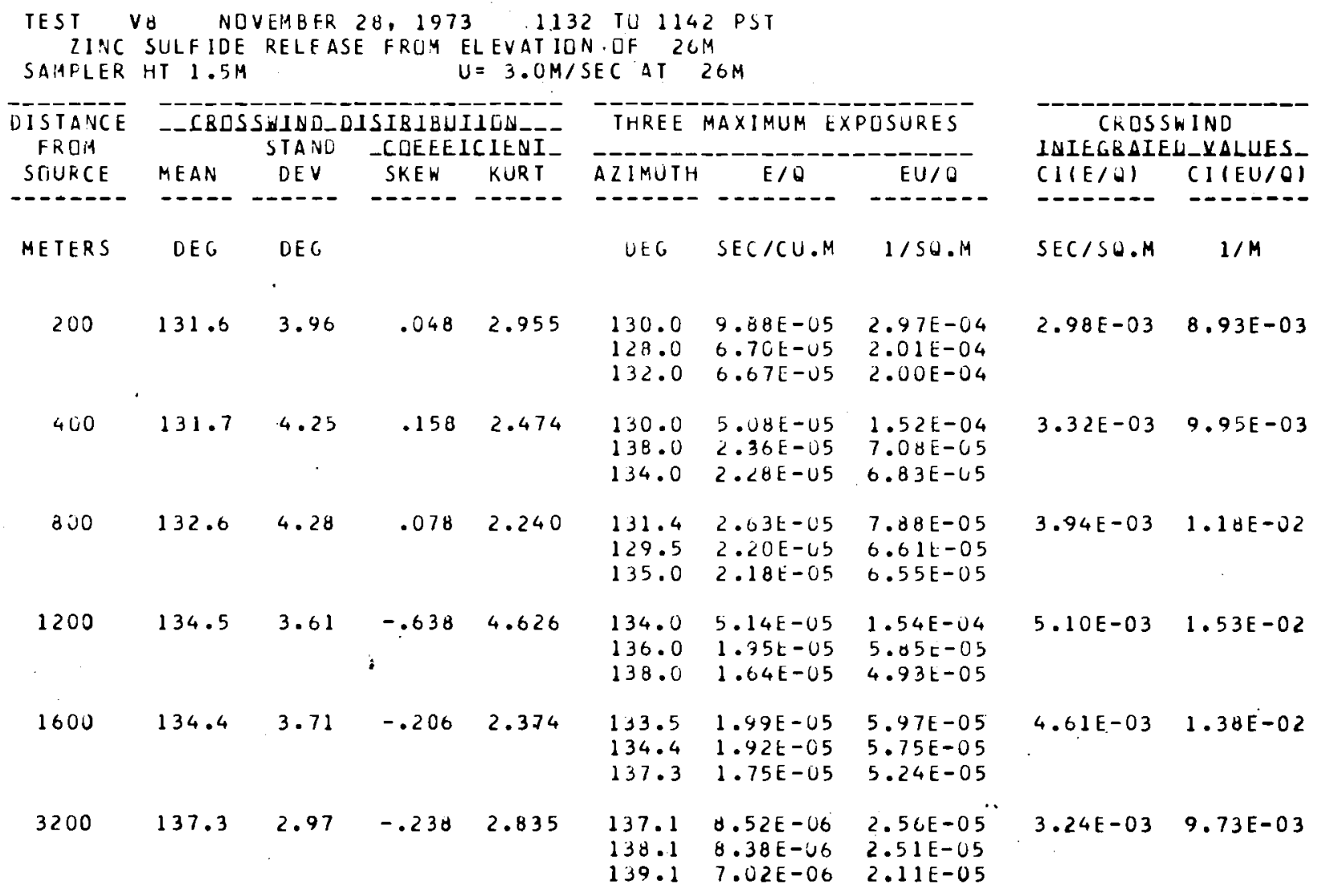

$?$ INUICATES UNCERTAINTIES IN DATA.

X INDicates inVAlio UR incomplete dAiA. 
APPENDIX C

METEOROLOGICAL DATA 
Missing data in Appendix $\mathrm{C}$ are indicated by 999.

C-1 
TABLE C-1. TEMPERATURE IDEG FI FQR PERIOU DF TRACER RELEASE.

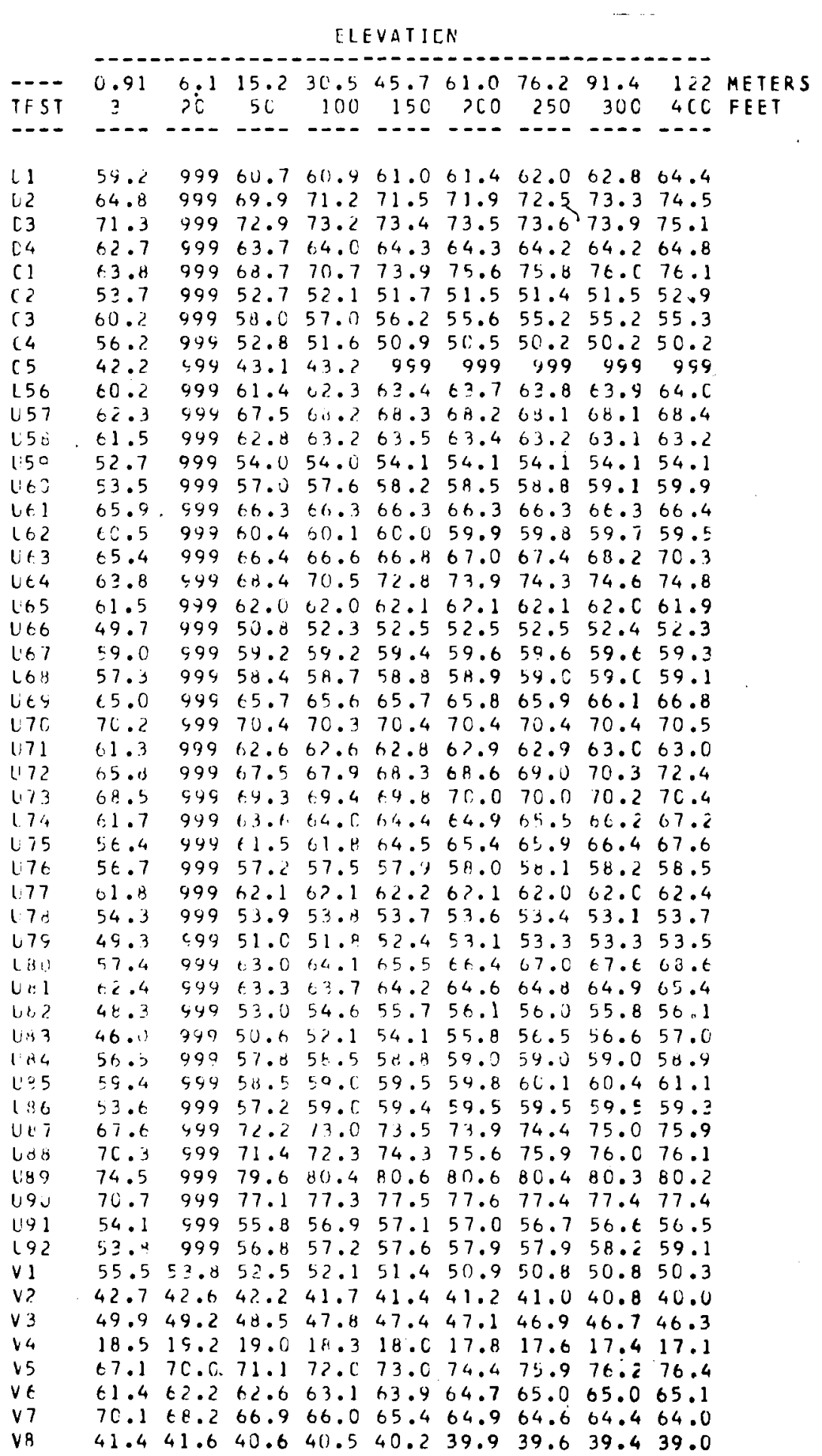




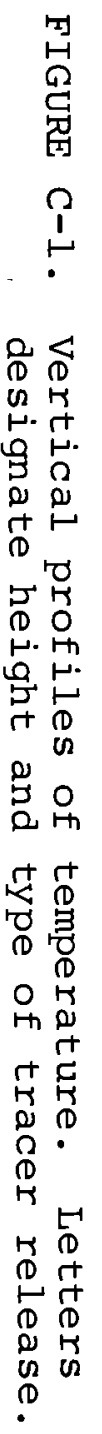
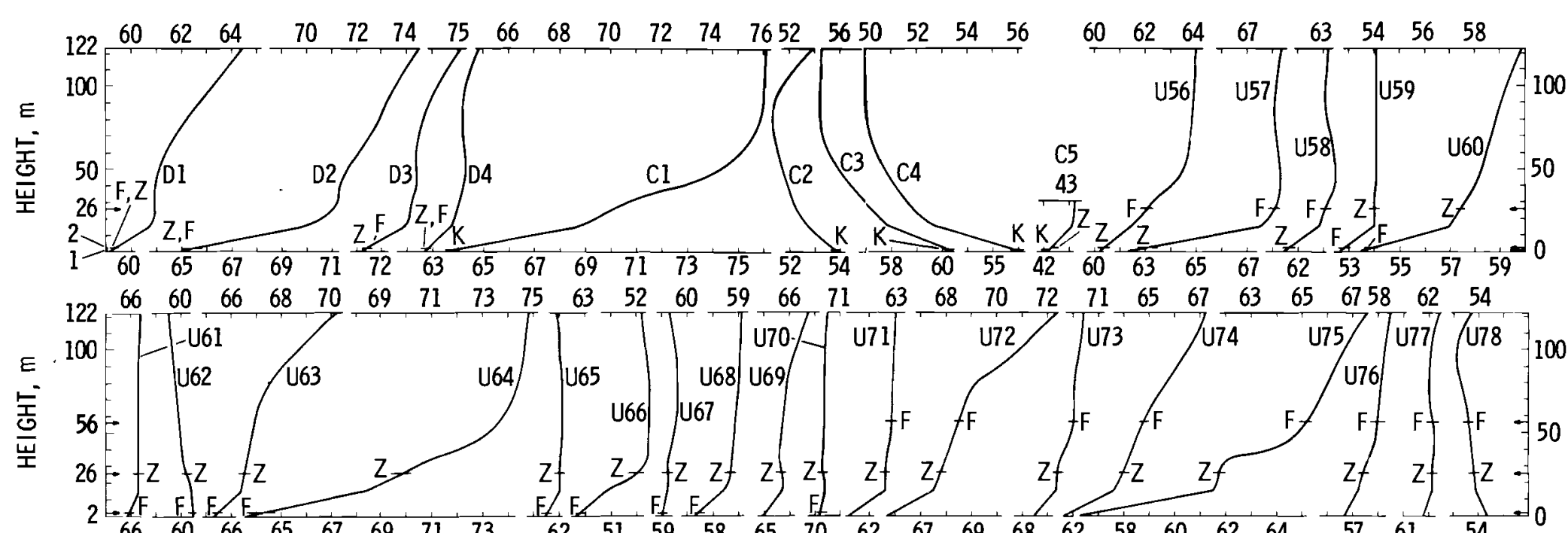

i $\quad \stackrel{0}{5} \circ$

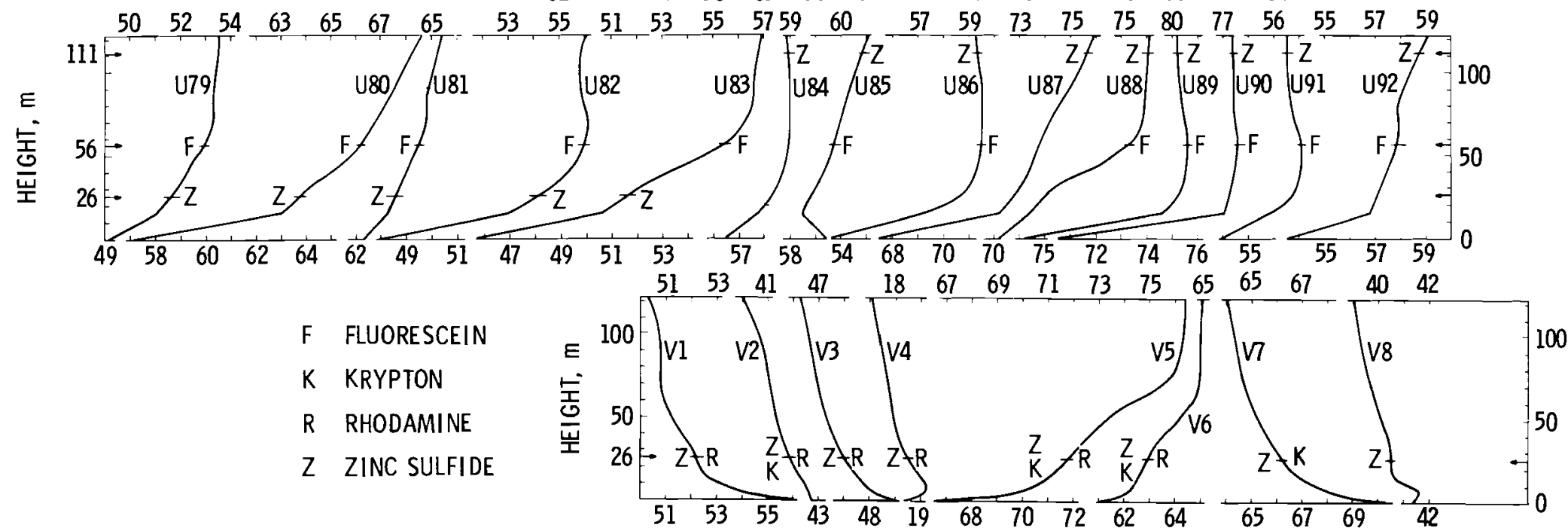

TEMPERATURE, DEGREES FAHRENHEIT 
TABLE C-2. HIND SPEED (H/SEC) FIR PERIOD DF TRACER RELEASE.

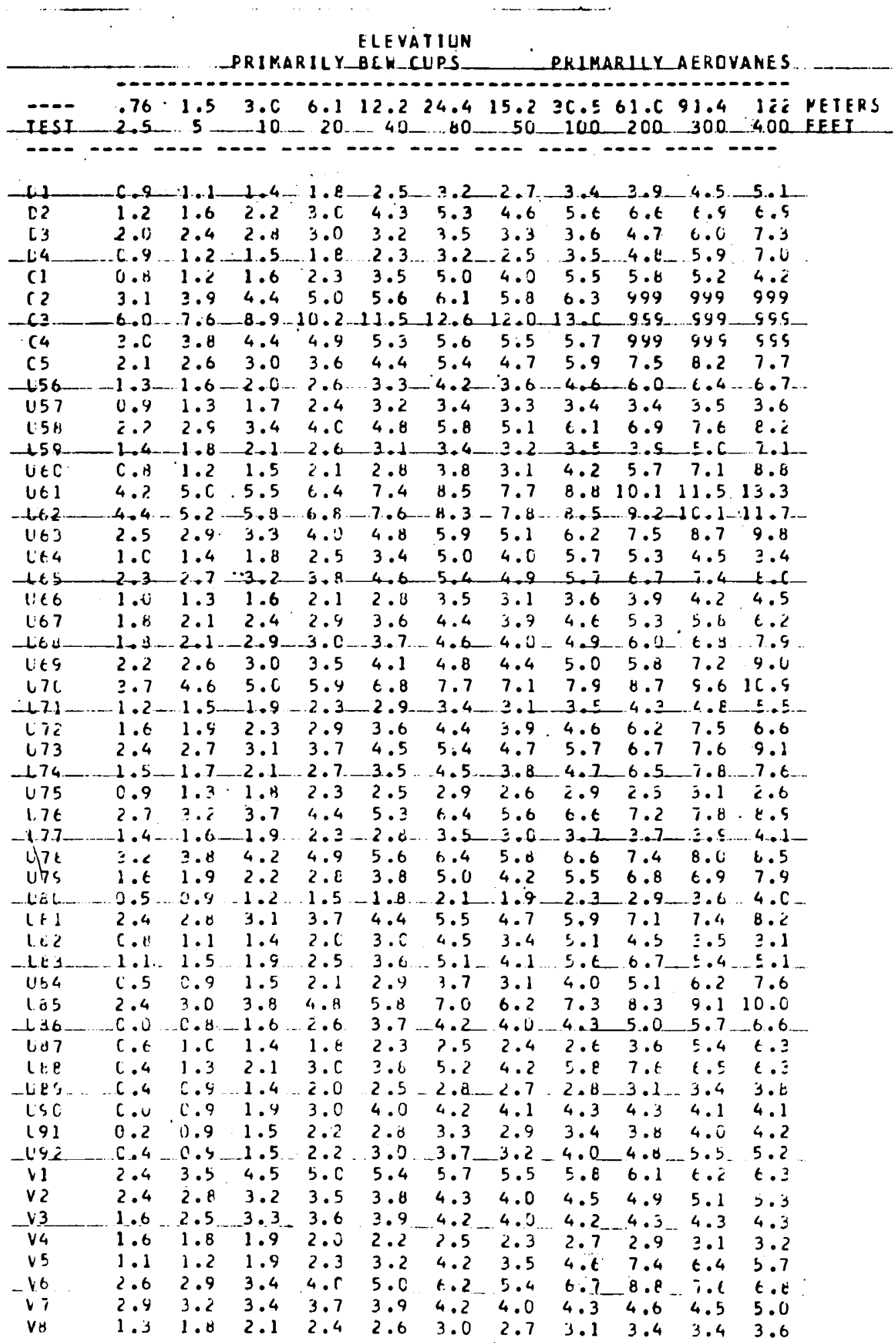




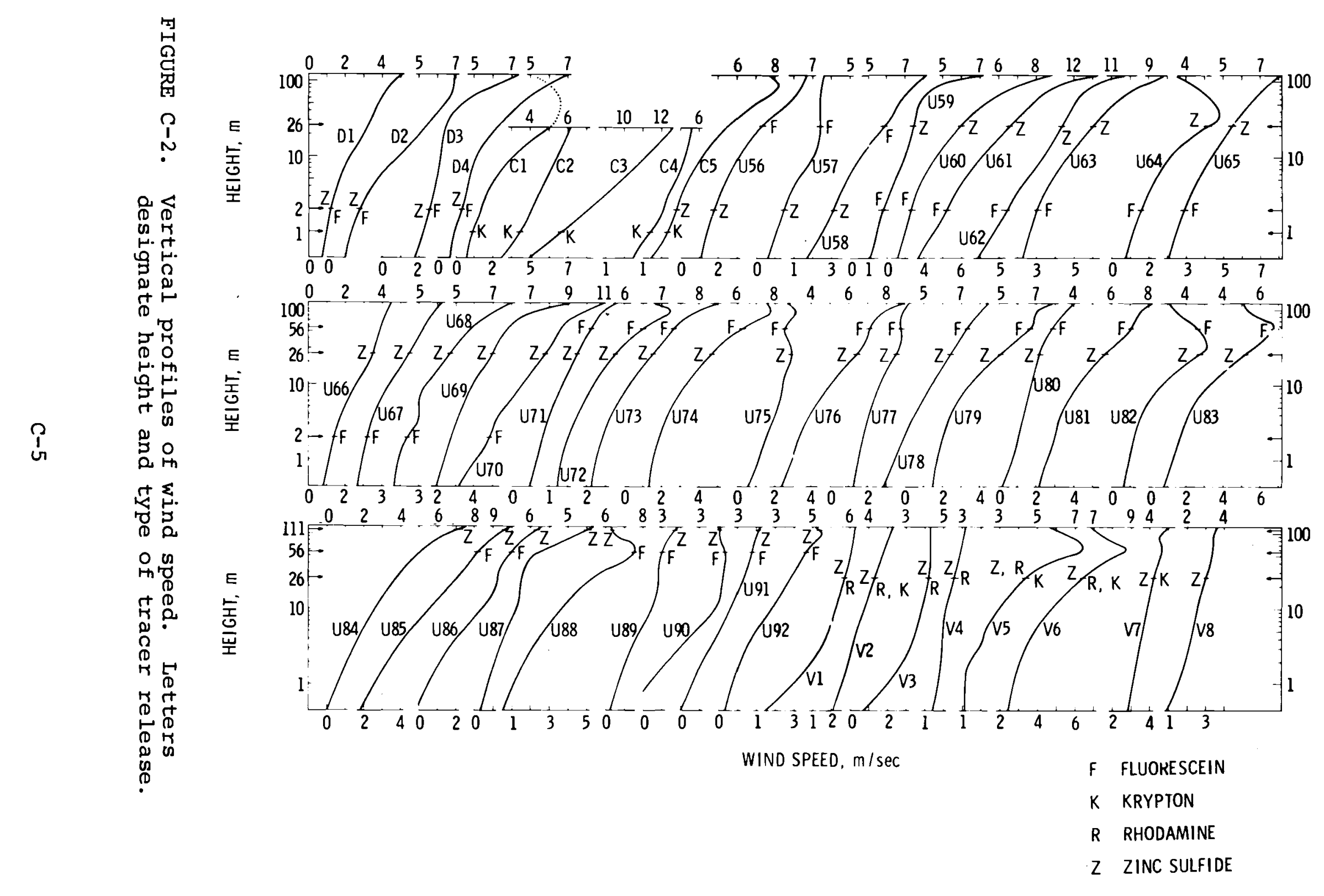


TABLE C-3. WIND DIRECTIINN (DEG) FDR PERIOD OF TRACER RELEASE.

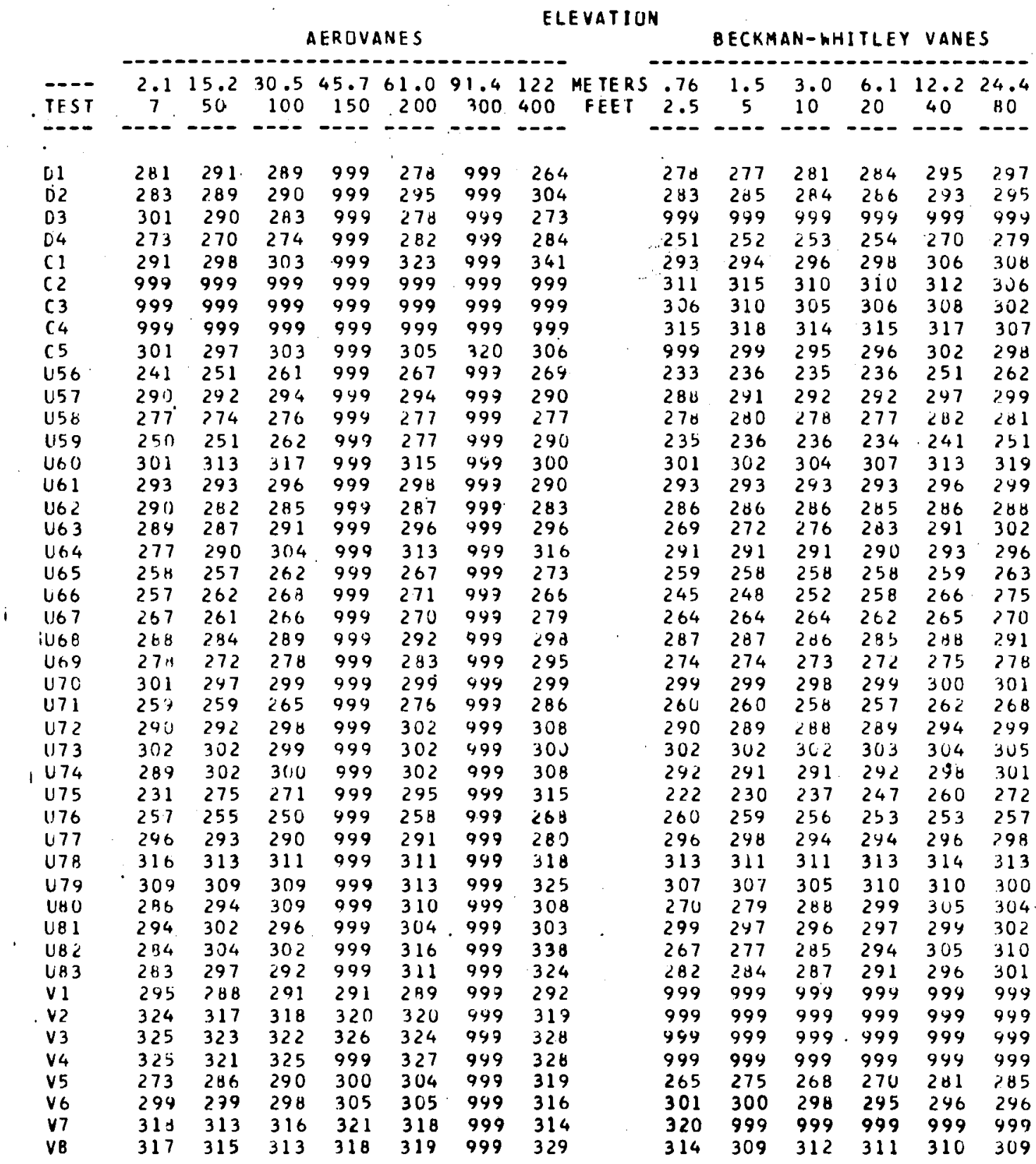


TAELF C-3. WIND DIKECTIMN (I)EG) FUK PERIUD DF TRACER RELEASF (CUITINUEO).

\begin{tabular}{|c|c|c|c|c|c|c|c|c|c|c|c|c|c|c|}
\hline & \multicolumn{14}{|c|}{ ELEVAT IUN } \\
\hline & \multicolumn{8}{|c|}{ AERDVANFS } & \multicolumn{6}{|c|}{ BECKMAN-WHITLEY VANES } \\
\hline & --- & -- & -- & & ---- & 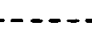 & 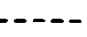 & & - & & & & & 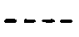 \\
\hline---- & 2.1 & 15.2 & 30.5 & 45.7 & 61.0 & 91.4 & 122 & MEIERS & 2 & 4 & 8 & 16 & 32 & 46 \\
\hline$T+S T$ & 7 & 50 & 100 & 150 & 200 & 300 & 400 & FEE T & 6.6 & 13.1 & $26 \cdot 2$ & 52.5 & 105 & 157 \\
\hline---- & --- & ---- & ---- & --- & --- & ---- & --- & & ---- & ---- & --- & --- & ---- & --- \\
\hline 484 & 288 & 293 & 304 & 939 & 300 & 307 & 300 & & 267 & 273 & 277 & 289 & 939 & 999 \\
\hline 185 & 290 & 289 & 295 & 999 & 291 & 298 & 287 & & 275 & 290 & 282 & 289 & 999 & $<48$ \\
\hline U86 & 254 & 266 & 286 & 279 & 999 & 798 & 291 & & 249 & 251 & 257 & 275 & 999 & 306 \\
\hline UA 7 & 216 & 238 & 248 & 260 & 270 & 799 & 301 & & 180 & 117 & 184 & 149 & 256 & 205 \\
\hline U8d & 287 & 294 & 288 & 295 & 305 & 308 & 309 & & 273 & 275 & 279 & 290 & 298 & 306 \\
\hline U 89 & 242 & 204 & 279 & 265 & 268 & 270 & 271 & & 237 & 247 & 263 & 286 & 283 & 284 \\
\hline$U \rightarrow C^{\circ}$ & 283 & 287 & 288 & 280 & 281 & 785 & 288 & & 280 & 281 & 284 & 293 & 297 & 298 \\
\hline U91 & 325 & 328 & 333 & 315 & 310 & 297 & 285 & & 288 & 290 & 294 & 304 & 309 & 310 \\
\hline $\mathrm{l} 92$ & 269 & 285 & 296 & 294 & 300 & 304 & 305 & & 253 & 260 & 270 & 285 & 298 & 305 \\
\hline
\end{tabular}




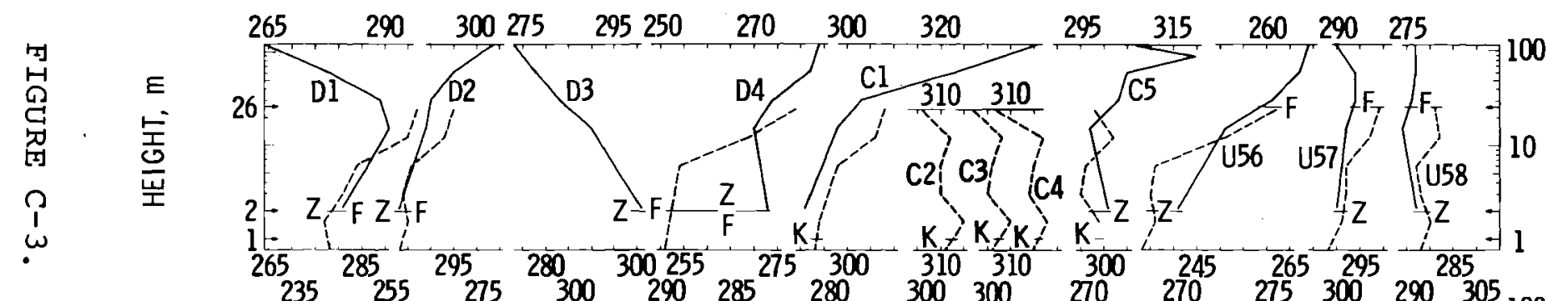

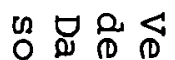

包骨.

造员

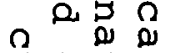

苟苗苗

范

路贾

a

(1)

每

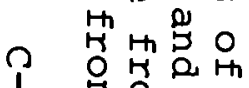

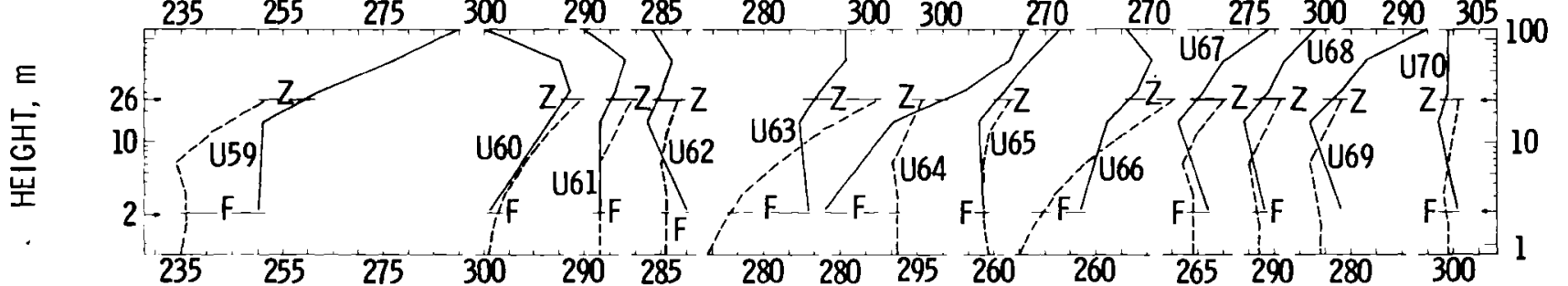

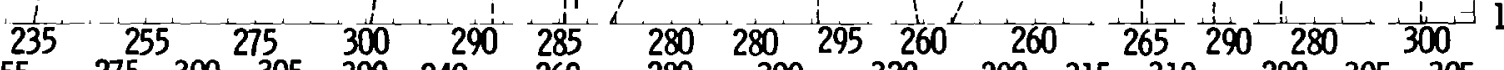

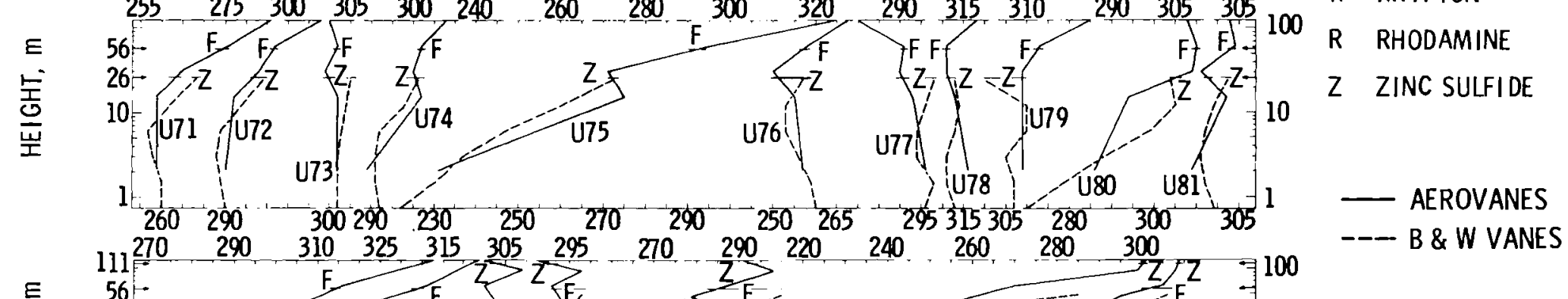

舫

必点

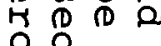

주요.

点点

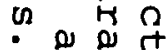

突

ल 80

$\sum_{5}$

a. 0

䓃䓃

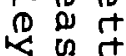

$<\frac{0}{0}$

迥

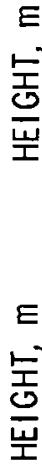

F FLUORESCEIN

KRYPTON
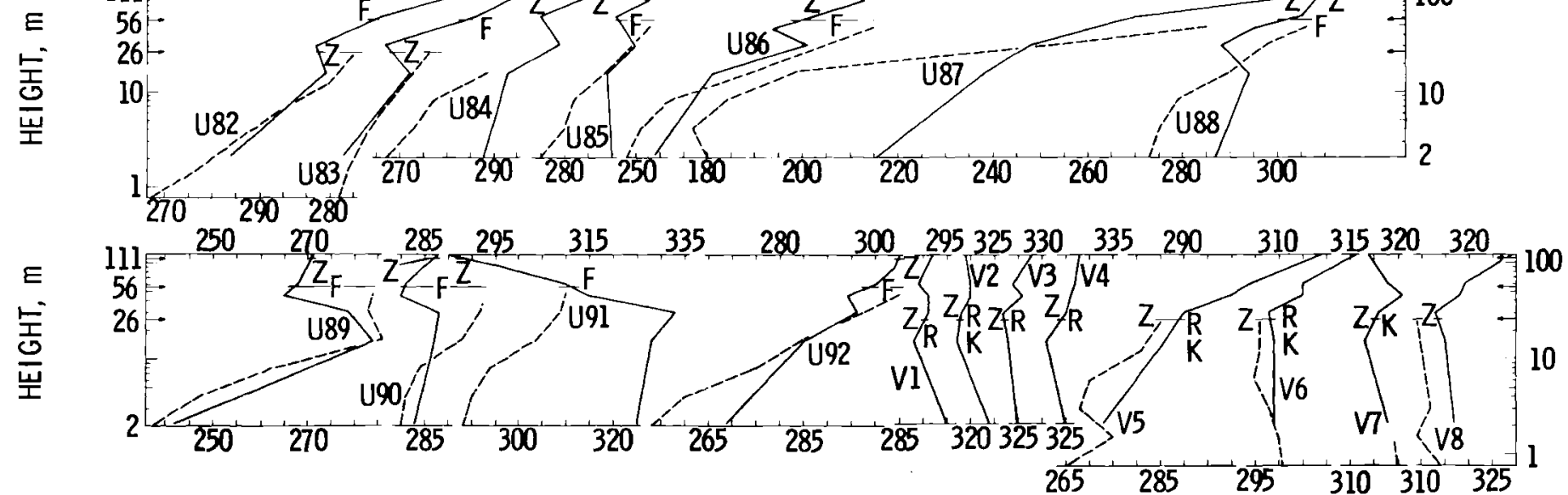

WIND DIRECTION, degrees 
TABLE C-4. HIND DIRECTIINN STANDARO DEVIATIUN (DEG) FOR PERITD OF TRACER RELEASE.

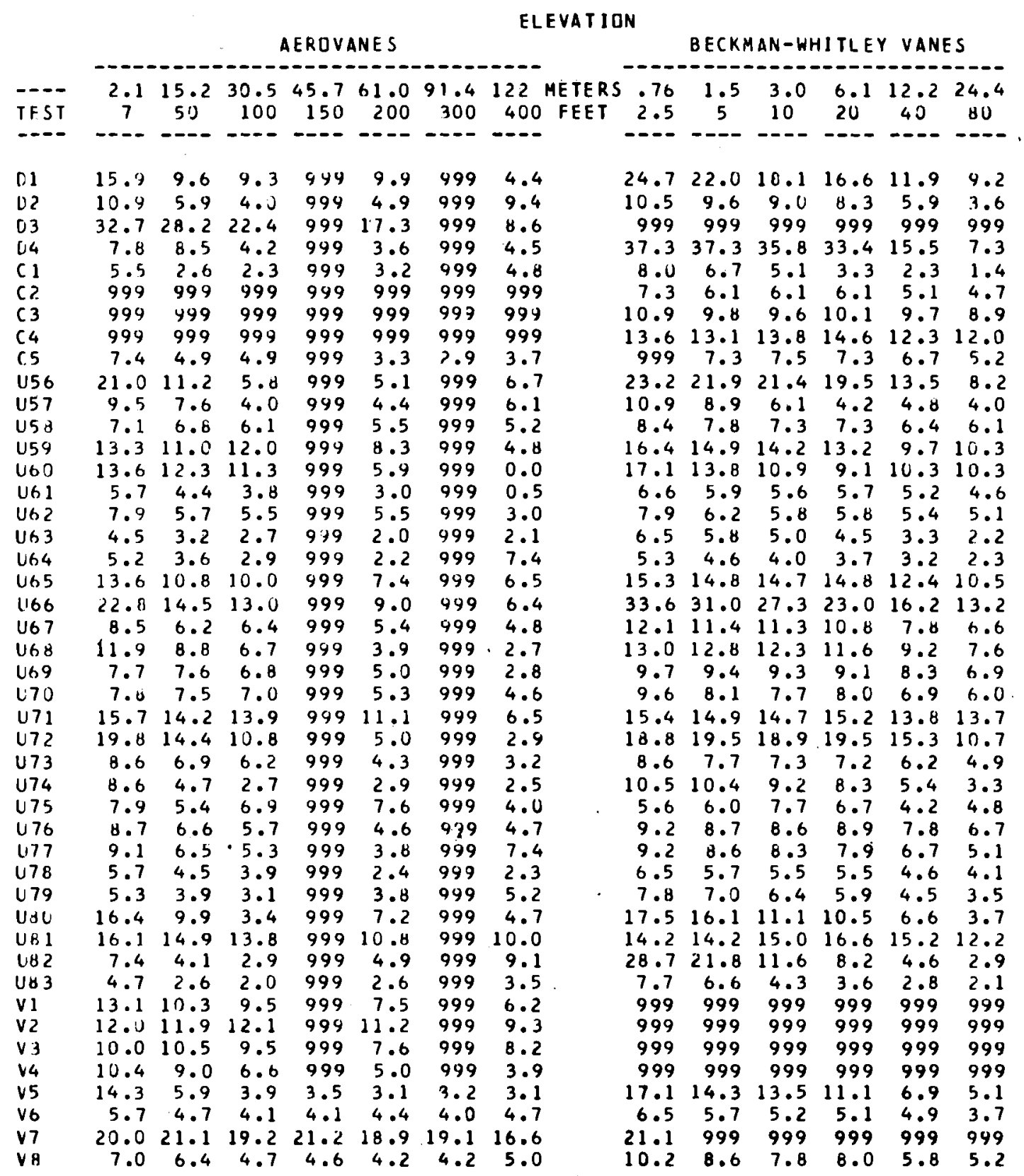


TABLE C-4. HIND DIRECTION STANDARO DEVIATION IOEGI FUR PERITO OF TRACER RELEASE ICONTINUEDI.

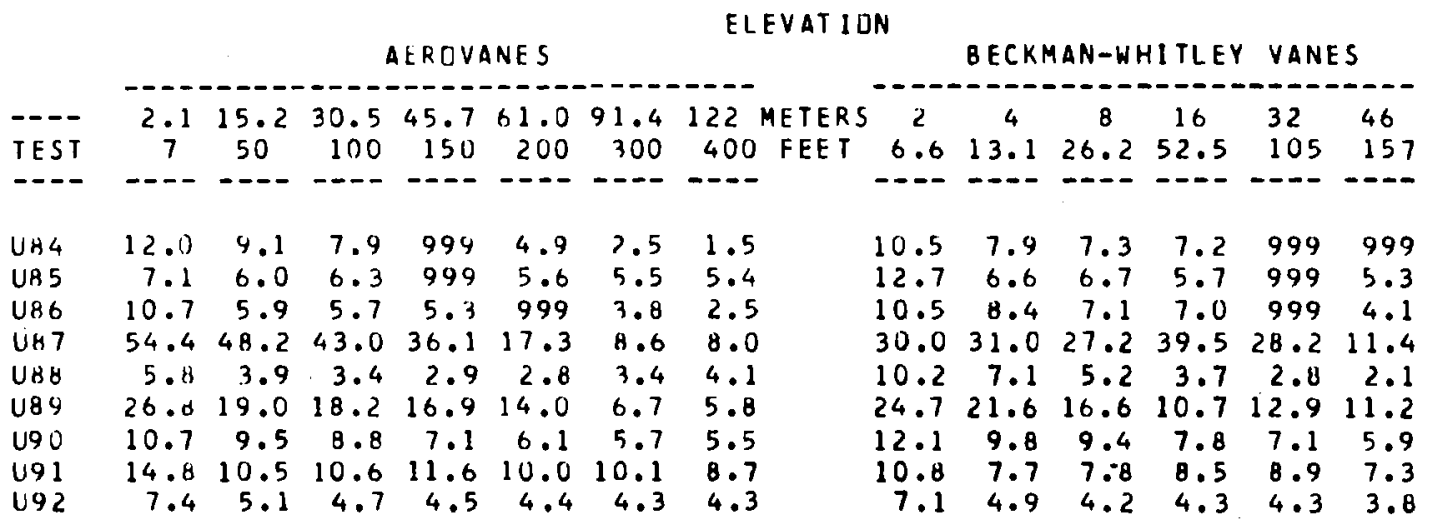




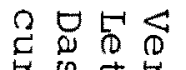

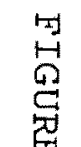

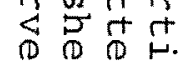

o $\Omega$ ह ?

(1)

$+\stackrel{0}{\leftarrow} \mathrm{H}$

의

उ。㐘

1
0

$? \begin{aligned} & 3 \\ & 0\end{aligned}$

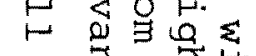

吕㟧苛占

$\therefore \Omega$

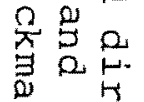

$5+\stackrel{0}{0}$

क त

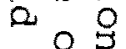

$\sum \stackrel{0}{3}$

点.

त.

(1)

$<\quad r$

点

苗苗

$\therefore 05$

ज

0.

茴?
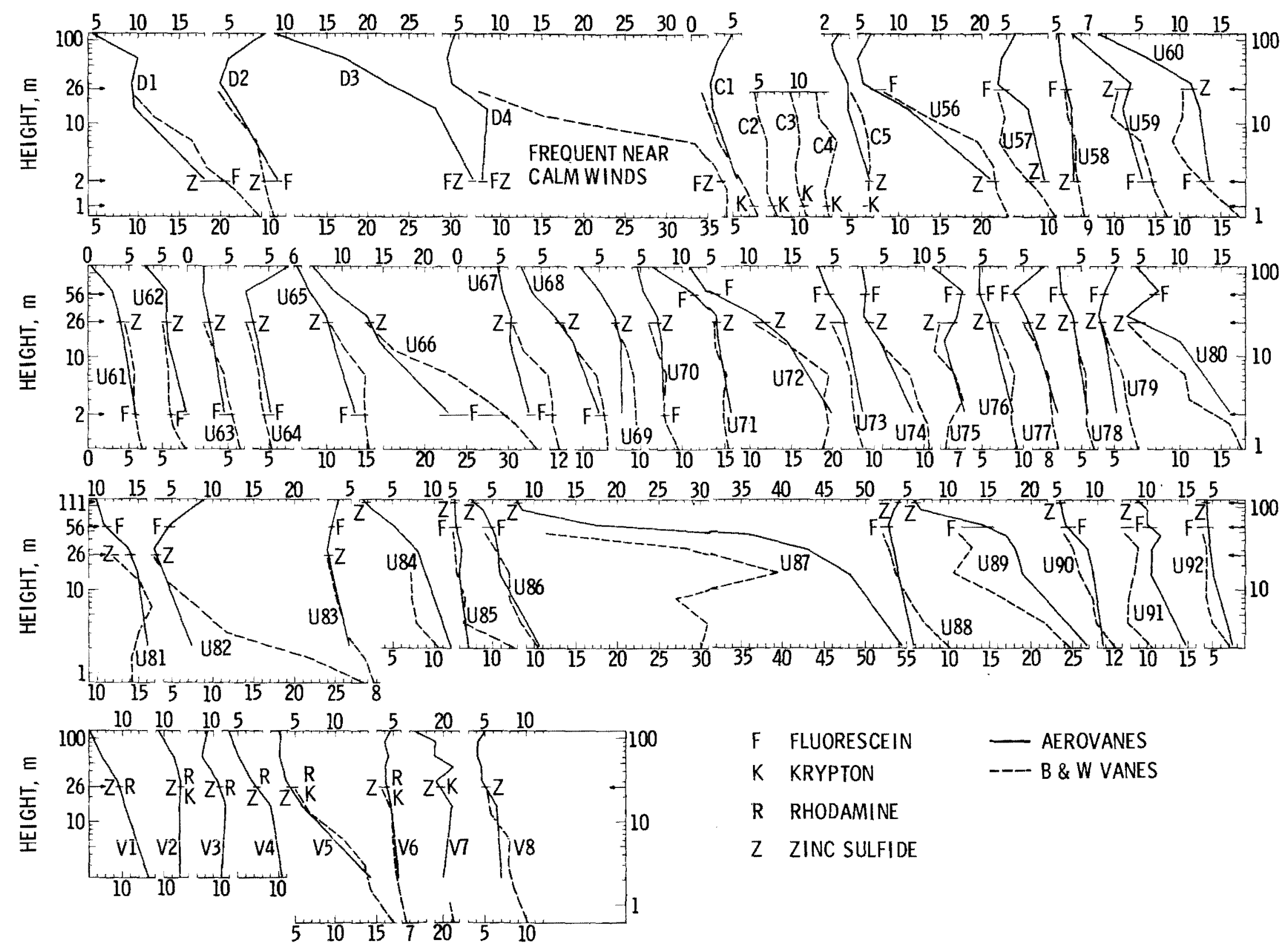

F FLUORESCEIN

K KRYPTON

- AEROVANES

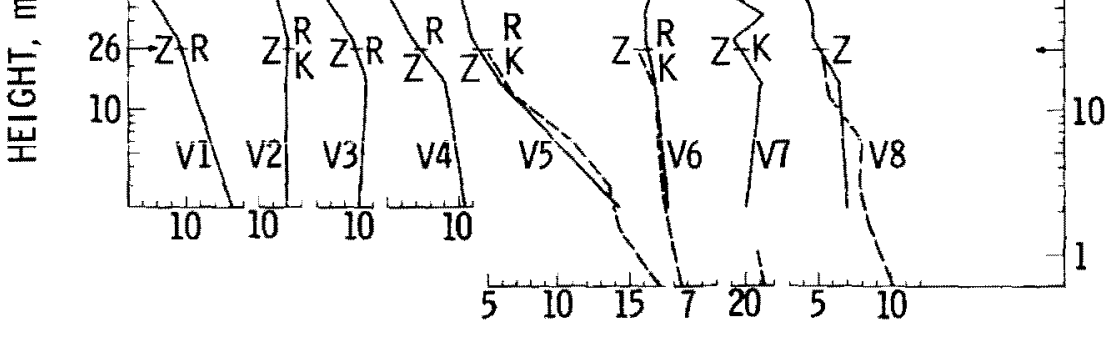

$\mathrm{R}$ RHODAMINE

$\sigma_{\theta}$, degrees

Z ZINC SULFIDE 

DISTRIBUTION 


\section{DISTRIBUTION}

№. of

Copies

OFFSITE
No. of

Copies

\section{1}

1 A. A. Churm

DOE Chicago Patent Group 9800 S. Cass Avenue

Argonne, IL 60439

225 DOE Technical Information Center

263 Robert Wade

Division of Technical Information and Document Control

U. S. NRC

Washington, DC 20555

S. Barr

University of California

Los Alamos Scientific Laboratory

P. O. Box 1663

Los Alamos, NM 87544

F. I. Badgley

Department of Atmospheric Sciences University of Washington

Seattle, Washington 98105

D. S. Ballantine

Division of Biomedical and Environmental Research

U. S. DOE

Washington, DC 20545

R. W. Beadle

Division of Biomedical and

Environmental Research

U. S. DOE

Washington, DC 20545
G. H, Clark

Health Physics Research Section A.A.E.C. Research Establishment Private Mailbag, Sutherland New South Wales 2232

AUSTRALIA

A. C. Chamberlain

United Kingdam Atomic Energy Authority

Harwell, Didoott, Berkshire

ENGLAND

Todd Crawford

Envirommental Analysis Planning

Savannah River Plant Operations Office

E. I. duPont de Nemours \& Co.

Aiken, S. C. 29801

J. W. Deardorf

NCAR

Boulder, $\infty 80303$

C. R. Dickson

Air Resources Laboratory

$\mathrm{N} O \mathrm{~A}$ A

Idaho Falls, Idaho 83401

A. Nelson Dingle

Department of Atmospheric \& Oceanic Sciences

The University of Michigan

4072 E. Engineering Building

Ann Arbor, Michigan 48104 
No. of

Copies

R. R. Draxler

NOAA Air Resources Laboratories

Silver Springs, Maryland 20910

Frankl in A. Gifford, Jr.

Director, Atmospheric Turbulence \& Diffusion Laboratory

ESSA, P. O. Box E

Oak Ridge, TN 37830

G. A. Briggs

Atmospheric Turbulence and Diffusion Laboratory

NOAA, Oak Ridge, TN 37830

J. T. Goll

Hydrology-Meteorology Branch

USNRC

Washington, DC 20555

B. A. Egan

Environmental Research and Technology, Inc.

696 Virginia Road

Concord, MA 01742

A. E. J. Eggleton

Atomic Energy Research

Establishment

Harwe 1 1, Oxfordshire

OXIIORB, ENGLAND

William P. Elliott

Air Resources Laboratories

NOAA

8060 13th Street

Silver Springs, MD 20910

Environmental Protection Agency ATTN: Mail Stop NCCM-5

Research Triangle Park, NC 27711
No. of

Copies

Rudolf J. Engelmann

Environmental Research Laboratories

National Oceanic and Atmospheric Administration

Boulder, Colorado 80302

Paul Frenzen

Argonne National Laboratory

9700 South Cass Avenue

Argonne, IL 60439

Steve Hanna

Atmospheric Turbulence and

Diffusion Laboratory

NOAA, P. O. Box E

Oak Ridge, TN 37830

D. A. Haugen

Wave Propagation Laboratory

NOAA

Boulder, CO 80303

Bruce B. Hicks

Argonne National Laboratories

D0181, 9700 S. Cass

Argonne, IL 60439

J. Z. Holl and

National Oceanic and Atmospheric

Administration

North Bethesda Office Center

11420 Rockville Heights

Rockville, MD 20852

W. E. Johnson

Atmospheric Sciences Laboratory

SRI International

Men lo Park, CA 94025

J. B. Knox

University of California

Lawrence Livermore Laboratory

P. 0. Box 808 (L-42)

Livermore, California 94550 
No. of

Copies

W. S. Lewellen

Aeronautical Research Associates of Princeton, Inc.

50 Washington Road

Princeton, NJ 07060

E. H. Markee, Jr.

Hydro-Meteorology Branch

USNRC

Washington, DC 20555

Paul Michae1

Brookhaven National Laboratories

Meteorology Department

Associated Universities, Inc.

Upton, Long Island, NY 11973

H. A. Panofsky

Department of Meteorology

The Pennsylvania State University

University Park, PA 16802

F. Pasquili

Meteorological Office

Met. 0.14

London Road

Brackne1 1, Berkshire, U. K.

\section{A. Roffman}

Westinghouse Environmental Systems Department

P. 0. Box 1899

Pittsburgh, PA 15230

K. F. Rosanski

Florida Power and Light Co.

P. 0. Box 013100

Miami, FL 33101

R. N. Sachdev

Air Monitoring Section

Bhabha Atomic Research Centre

Trombay, Bombay, 400-085, INDIA

Donald L. Shearer

TRC of New England

125 Sials Deane Highway

Wethersfield, CT 06109
No. of

Copies

D. H. Slade

Division of Biomedical and Environmental Research

U. S. DOE

Washington, DC 20545

W. G. N. Slinn

Atmospheric Sciences Department

Oregon State University

Corval1is, Oregon 97331

M. E. Smith

Meteorological Evaluation Services, Inc.

Amityville, NY 11701

D. B. Turner

Meteorology \& Assessment Div.

U. S. EPA

Research Triangle Park, NC 27711

I. Van der Hoven

Air Resources Laboratory

National Oceanic and Atmospheric Administration

8060 13th Street

Silver Spring, MD 20910

K. J. Vogt

Kernforschungsanlage Julich

Gmb $H$

Jul ich, Bendesrepublik

Deutschland

A. H. Weber

Environmental Transport Div.

Bldg. 773-12 A

Savannah River Laboratories

Aiken, SC 29801

M. A. Wolf

Atmospheric Sciences Department

Oregon State University

Corvallis, Oregon 97331 
NNo. of

Copies

ONSITE

1

DOE, Richland Gerations Office

W. Lei

H. E. Ransom

57

Battelle Northwest

R. L. Conley

J. C. Doran

R. L. Drake

C. E. Elderkin

J. J. Fuquay

R. K. Hadlock

J. M. Hales

T. W. Horst

P. W. Nickola (36)

H. M. Parker

D. C. Powell

J. V. Ramsdell

W. F. Sandusky

G. A. Sehmel

C. L. Simpson

E. C. Watson

L. L. Wendell

Technical Information (5)

Technical Publications 